WSRC-TR-95-0146-2

Unclassified

\title{
F-AREA SEEPAGE BASINS GROUNDWATER MONITORING \\ REPORT (U)
}

\section{FIRST AND SECOND QUARTERS 1995}

Volume I

Publication Date: September 1995

Authorized Derivative Classifier and Reviewing Official:

UNCLASSIFIED

Does Not Contain Unclassified

Controlled Nuclear Information

Westinghouse Savannah River Company

Savannah River Site

Aiken, SC 29808

\section{MASTER}

LISTRIBUTIONOFTHIS DOCUMENT IS UNLIMIED 


\section{DISCLAIMER}

This report was prepared as an account of work sponsored by an agency of the United States Government. Neither the United States Government nor any agency thereof, nor any of their employees, makes any warranty, express or implied, or assumes any legal liability or responsibility for the accuracy, completeness, or usefulness of any information, apparatus, product, or process disclosed, or represents that its use would not infringe privately owned rights. Reference herein to any specific commercial product, process, or service by trade name, trademark, manufacturer, or otherwise does not necessarily constitute or imply its endorsement, recommendation, or favoring by the United States Government or any agency thereof. The views and opinions of authors expressed herein do not necessarily state or reflect those of the United States Government or any agency thereof.

This report has been reproduced directly from the best available copy.

Available to DOE and DOE contractors from the Office of Scientific and Technical Information, P.O. Box 62, Oak Ridge, TN 37831; prices available from (615) 576-8401.

Available to the public from the National Technical Information Service, U.S. Department of Commerce; 5285 Port Royal Road, Springfield, VA 22161. 
WSRC-TR-95-0146-2

Unclassified

\section{F-AREA SEEPAGE BASINS GROUNDWATER MONITORING REPORT (U)}

FIRST AND SECOND QUARTERS 1995

\section{Volume I}

Publication Date: September 1995

Key Words

aluminum

FSB wells

nitrate

tritium

zinc

Authorized Derivative Classifier and Reviewing Official:

UNCLASSIFIED

Does Not Contain Unclassified

Controlled Nuclear Information

Westinghouse Savannah River Company

Savannah River Site

Aiken, SC 29808 
WSRC-TR-95-0146-2

Unclassified

THIS PAGE LEFT BLANK INTENTIONALLY. 


\section{Abstract}

Groundwater at the F-Area Seepage Basins (FASB) is monitored in compliance with Module III, Section C, of South Carolina Hazardous Waste Permit SC1-890-008-989, effective November 2, 1992. The monitoring well network is composed of 86 FSB wells and well HSB 85A. These wells are screened in the three hydrostratigraphic units that make up the uppermost aquifer beneath the FASB. A detailed description of the uppermost aquifer is included in the Resource Conservation and Recovery Act Part B post-closure care permit application for the F-Area Hazardous Waste Management Facility submitted to the South Carolina Department of Health and Environmental Control (SCDHEC) in December 1990. Data from 9 FSL wells are included in this report only to provide additional information for this area; the FSL wells are not part of Permit SC1-890-008-989.

Monitoring results are compared to the SCDHEC Groundwater Protection Standard (GWPS), which is specified in the approved F-Area Seepage Basins Part B permit (November 1992). Historically and currently, gross alpha, nitrate, nonvolatile beta, and tritium are among the primary constituents to exceed standards. Numerous other radionuclides and hazardous constituents also exceeded the GWPS in the groundwater at the FASB during the first half of 1995, notably aluminum, iodine-129, $\mathrm{pH}$, strontium-90, and zinc. The elevated constituents are found primarily in Aquifer Zone $\|_{1} B_{2}$ (Water Table) and Aquifer Zone IIB (Barnwell/McBean) wells. However, several Aquifer Unit IIA (Congaree) wells also contain elevated levels of constituents.

Isoconcentration/isoactivity maps included in this report indicate both the concentration/activity and extent of the primary contaminants in each of the three hydrostratigraphic units. Geologic cross sections indicate both the extent and depth of contamination of the primary contaminants in all of the hydrostratigraphic units during the first half of 1995. Water-level maps indicate that the groundwater flow rates and directions at the FASB have remained relatively constant since the basins ceased to be active in 1988 . 
THIS PAGE LEFT BLANK INTENTIONALLY. 


\section{Contents}

Volume I

Abstract

List of Figures $\ldots \ldots \ldots \ldots \ldots \ldots \ldots \ldots \ldots \ldots \ldots \ldots \ldots \ldots \ldots \ldots$

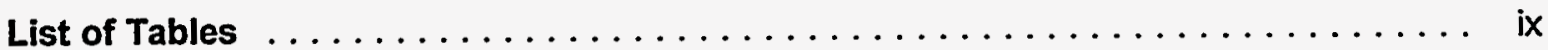

Executive Summary $\ldots \ldots \ldots \ldots \ldots \ldots \ldots \ldots \ldots \ldots \ldots \ldots \ldots \ldots$

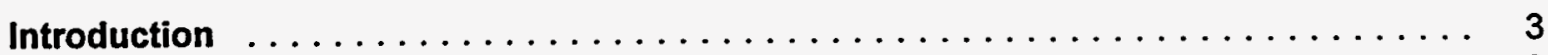

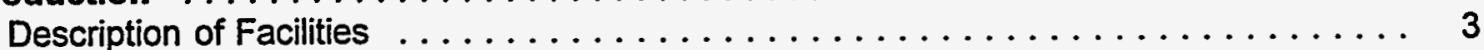

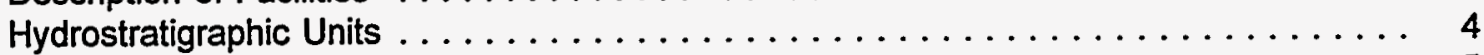

Monitoring Well Nomenclature $\ldots \ldots \ldots \ldots \ldots \ldots \ldots \ldots \ldots \ldots \ldots \ldots$

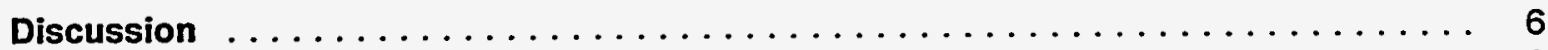

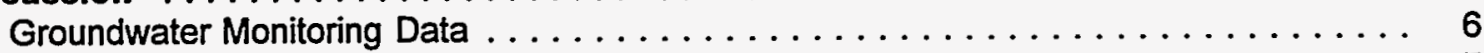

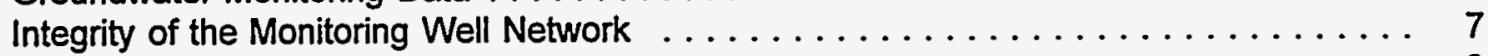

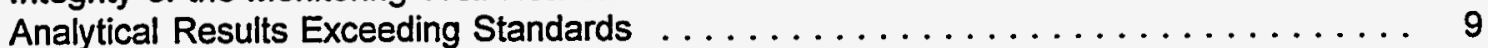

Maps and Time Series Plots . . . . . . . . . . . . . . . . . . . . 12

Water Levels . . . . . . . . . . . . . . . . . . . . . . . . . . 12

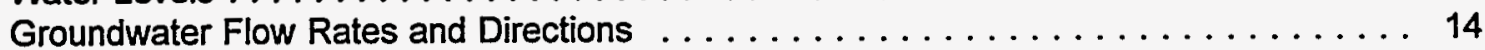

Upgradient and Background Results $\ldots \ldots \ldots \ldots \ldots \ldots \ldots \ldots \ldots \ldots \ldots \ldots$

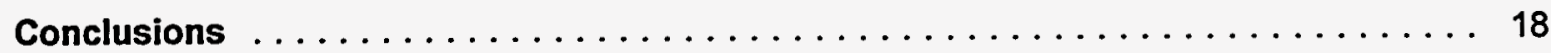

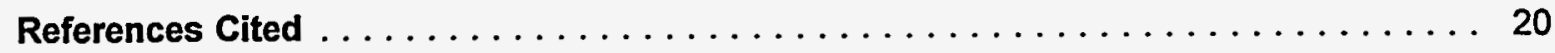

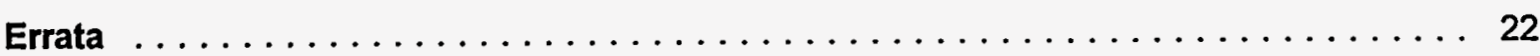

Appendix A-Standards $\ldots \ldots \ldots \ldots \ldots \ldots \ldots \ldots \ldots \ldots \ldots \ldots \ldots \ldots \ldots \ldots \ldots \ldots$

Appendix B-Flagging Criteria $\ldots \ldots \ldots \ldots \ldots \ldots \ldots \ldots \ldots \ldots \ldots \ldots \ldots \ldots$

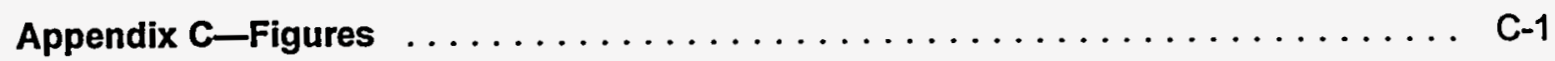

Appendix $\mathrm{D}-$ Groundwater Monitoring Results Tables $\ldots \ldots \ldots \ldots \ldots \ldots \ldots$

Appendix E-Data Quality/Usability Assessment $\ldots \ldots \ldots \ldots \ldots \ldots \ldots \ldots \ldots$

Appendix F-Time Series Plots $\ldots \ldots \ldots \ldots \ldots \ldots \ldots \ldots \ldots \ldots \ldots \ldots \ldots \ldots \ldots$

Appendix G-Hydrographs $\ldots \ldots \ldots \ldots \ldots \ldots \ldots \ldots \ldots \ldots \ldots \ldots \ldots \ldots$ 
Volume II

General Separations Area Water-Elevation Maps for the F-and H-Area Seepage Basins Groundwater Monitoring Reports 


\section{List of Figures}

Page

1. Location of the F-Area Seepage Basins at the Savannah River Site $\ldots \ldots \ldots \ldots$ C-3

2. Location of the Groundwater Monitoring Wells at the F-Area Seepage Basins $\ldots \ldots$ C-4

3. Hydrostratigraphic Nomenclature $\ldots \ldots \ldots \ldots \ldots \ldots \ldots \ldots \ldots \ldots \ldots \ldots$

4. Regional Correlation of Hydrostratigraphic and Lithostratigraphic

5. Location of Aquifer Zone $\| 1_{2}$ (Water Table) Monitoring Wells at the F-Area Seepage

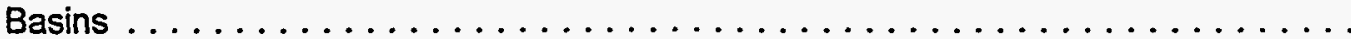

6. Location of Aquifer Zone $\|_{1}$ (Barnwell/McBean) Monitoring Wells at the F-Area Seepage Basins

7. Location of Aquifer Unit IIA (Congaree) Monitoring Wells at the F-Area Seepage Basins

8. Cadmium Concentrations in Aquifer Zone $\|_{2} B_{2}$ (Water Table) at the F-Area Seepage Basins, First Quarter 1995 .

9. Cadmium Concentrations in Aquifer Zone $\|_{1}$ (Barnwell/McBean) at the F-Area Seepage Basins, First Quarter 1995

10. Cadmium Concentrations in Aquifer Unit IIA (Congaree) at the F-Area Seepage Basins, First Quarter 1995 .

11. Gross Alpha Activities in Aquifer Zone $\| B_{2}$ (Water Table) at the F-Area Seepage Basins, First Quarter 1995 .

12. Gross Alpha Activities in Aquifer Zone $\|_{1} B_{1}$ (Barnwell/McBean) at the F-Area Seepage Basins, First Quarter 1995.

13. Gross Alpha Activities in Aquifer Unit IIA (Congaree) at the F-Area Seepage Basins, First Quarter 1995

14. Lead Concentrations in Aquifer Zone $\|_{2}$ (Water Table) at the F-Area Seepage Basins, First Quarter 1995

15. Lead Concentrations in Aquifer Zone IIB (Barnwell/McBean) at the F-Area Seepage Basins, First Quarter 1995 .

16. Lead Concentrations in Aquifer Unit IIA (Congaree) at the F-Area Seepage Basins, First Quarter 1995 
17. Nitrate Concentrations in Aquifer Zone $\mathrm{IIB}_{2}$ (Water Table) at the F-Area Seepage

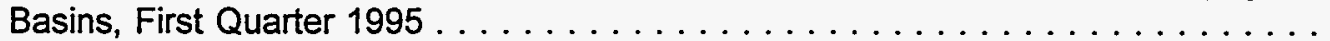

18. Nitrate Concentrations in Aquifer Zone IIB ${ }_{1}$ (Barnwell/McBean) at the F-Area Seepage Basins, First Quarter 1995

19. Nitrate Concentrations in Aquifer Unit IIA (Congaree) at the F-Area Seepage Basins, First Quarter 1995

20. Nonvolatile Beta Activities in Aquifer Zone $\| B_{2}$ (Water Table) at the F-Area Seepage Basins, First Quarter 1995

21. Nonvolatile Beta Activities in Aquifer Zone IIB (Barnwell/McBean) at the F-Area Seepage Basins, First Quarter 1995

22. Nonvolatile Beta Activities in Aquifer Unit IIA (Congaree) at the F-Area Seepage Basins, First Quarter 1995 . . . . . . . . . . . . . . . . . . . . . . . . .

23. Radium-226 Activities in Aquifer Zone $\| \mathrm{B}_{2}$ (Water Table) at the F-Area Seepage Basins, First Quarter 1995

24. Radium-226 Activities in Aquifer Zone $\|_{1} B_{1}$ (Barnwell/McBean) at the F-Area Seepage Basins, First Quarter 1995 .

25. Radium-226 Activities in Aquifer Unit IIA (Congaree) at the F-Area Seepage Basins, First Quarter 1995

26. Radium-228 Activities in Aquifer Zone $\| \mathrm{B}_{2}$ (Water Table) at the F-Area Seepage Basins, First Quarter 1995

27. Radium-228 Activities in Aquifer Zone $\|_{1} B_{1}$ (Barnwell/McBean) at the F-Area Seepage Basins, First Quarter 1995 .

28. Radium-228 Activities in Aquifer Unit IIA (Congaree) at the F-Area Seepage Basins, First Quarter 1995

29. Tritium Activities in Aquifer Zone $\mathrm{IIB}_{2}$ (Water Table) at the F-Area Seepage Basins, First Quarter 1995

30. Tritium Activities in Aquifer Zone $\mathrm{IIB}_{1}$ (Barnwell/McBean) at the F-Area Seepage Basins, First Quarter 1995

31. Tritium Activities in Aquifer Unit IIA (Congaree) at the F-Area Seepage Basins, First Quarter 1995

32. Piezometric Surface Map of Aquifer Zone $\mathrm{IIB}_{2}$ (Water Table) at the F-Area Seepage Basins, First Quarter 1995

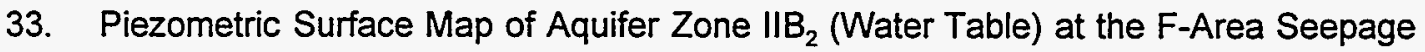
Basins, Second Quarter 1995 
34. Potentiometric Surface Map of Aquifer Zone IIB, (Barnwell/McBean) at the F-Area Seepage Basins, First Quarter $1995 \ldots \ldots \ldots \ldots \ldots \ldots \ldots \ldots$. . . . . . . . . . . . .

35. Potentiometric Surface Map of Aquifer Zone IIB (Barnwell/McBean) at the F-Area Seepage Basins, Second Quarter 1995

36. Potentiometric Surface Map of Aquifer Unit IIA (Congaree) at the F-Area Seepage Basins, First Quarter 1995

37. Potentiometric Surface Map of Aquifer Unit IIA (Congaree) at the F-Area Seepage

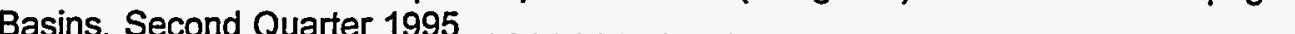

38. $\mathrm{pH}$ Values in Aquifer Zone $\mathrm{IB}_{2}$ (Water Table) at the F-Area Seepage Basins, First Quarter 1995

39. pH Values in Aquifer Zone IIB (Barnwell/McBean) at the F-Area Seepage Basins, First Quarter 1995

40. $\mathrm{pH}$ Values in Aquifer Unit IIA (Congaree) at the F-Area Seepage Basins, First Quarter 1995

41. Specific Conductance in Aquifer Zone $\mathrm{IIB}_{2}$ (Water Table) at the F-Area Seepage Basins, First Quarter 1995

42. Specific Conductance in Aquifer Zone $\mathrm{IIB}_{1}$ (Barnwell/McBean) at the F-Area Seepage Basins, First Quarter 1995 .

43. Specific Conductance in Aquifer Unit IIA (Congaree) at the F-Area Seepage Basins, First Quarter 1995

\section{List of Tables}

1. Maximum Levels of Constituents Exceeding the Groundwater Protection

Standards

2. Maximum Levels of Constituents Exceeding the SRS Flag 2 Criteria

3. Groundwater Monitoring Results for Individual Wells 
WSRC-TR-95-0146-2

Unclassified

THIS PAGE LEFT BLANK INTENTIONALLY. 


\section{Executive Summary}

The groundwater in the uppermost aquifer beneath the F-Area Seepage Basins (FASB) at the Savannah River Site (SRS) is monitored for various hazardous and radioactive constituents as required by Module III, Section C, of South Carolina Hazardous Waste Permit SC1-890-008-989, effective November 2, 1992 (SCDHEC, 1992). Currently, 86 wells of the FSB well series and well HSB 85A are monitored quarterly for alkalinity, cadmium, gross alpha, lead, nitrate, nonvolatile beta, $\mathrm{pH}$, specific conductance, tritium, and water elevation. Selected wells are monitored semi-annually for constituents with a Groundwater Protection Standard (GWPS), and all wells are monitored annually for GWPS constituents. GWPS constituents include selected heavy metals, indicator parameters, radionuclides, and volatile organics. This report describes the groundwater monitoring results and indicates those results that exceeded the GWPS and SRS flagging criteria during first and second quarters 1995. Data from $9 \mathrm{FSL}$ wells are included only to provide additional information on the area; the FSL wells are not part of Permit SC1-890-008-989. Groundwater monitoring reports are submitted to the South Carolina Department of Health and Environmental Control (SCDHEC) semiannually as required by Permit SC1-890-008-989.

Thirty-three FSB wells and 1 FSL well were not sampled during second quarter 1995 because they were added to the Purge Water Containment (PWC) Program. During the first half of 1995, the following parameters exceeded the GWPS in FSB wells at the FASB: aluminum, americium241, barium, bis(2-ethylhexyl) phthalate, cadmium, cesium-137, cobalt, copper, curium-243/244, curium-245/246, cyanide, dichloromethane, gross alpha, iodine-129, lead, mercury, nickel, nitrate, nonvolatile beta, $\mathrm{pH}$, plutonium-238, radium-226, radium-228, silver, strontium-89 ${ }^{1}$, strontium-90, thorium-234, trichloroethylene, trichlorofluoromethane, tritium, uranium-233/234, uranium-235, uranium-238, and zinc.

Aluminum, gross alpha, iodine-129, nitrate, nonvolatile beta, $\mathrm{pH}$, strontium-90, tritium, and zinc were elevated most frequently, exceeding GWPS in 35 or more FSB wells each. In $74(86 \%)$ of the 86 FSB wells, pH exceeded the GWPS, most often exceeding the acidic standard. Barium, bis(2-ethylhexyl) phthalate, cesium-137, curium-245/246, cyanide, dichloromethane, lead, mercury, plutonium-238, silver, trichloroethylene, and trichlorofluoromethane were elevated less frequently, exceeding GWPS in 5 or fewer FSB wells each. Elevated levels of aluminum, nitrate, $\mathrm{pH}$, trichlorofluoromethane, tritium, and zinc were found in one or more of the upgradient FSB wells.

Elevated constituents were found primarily in Aquifer Zone $\| \mathrm{B}_{2}$ (Water Table) and Aquifer Zone $\| \mathrm{B}_{1}$ (Barnwell/McBean). However, gross alpha, iodine-129, nitrate, $\mathrm{pH}$, and tritium exceeded the GWPS in one or more of the FSB wells in Aquifer Unit IIA (Congaree).

The following parameters exceeded the GWPS in FSL wells in F Area during the first half of 1995: aluminum, gross alpha, lead, nitrate, nonvolatile beta, $\mathrm{pH}$, trichloroethylene, trichlorofluoromethane, tritium, and zinc.

Groundwater flow directions beneath the FASB in Aquifer Zone IIB ${ }_{2}$ (Water Table) and Aquifer Zone IIB, (Barnwell/McBean) are generally south and south to southeast toward Fourmile Branch (using universal transverse Mercator coordinates). Flow in Aquifer Unit IIA (Congaree) is generally northwest toward Upper Three Runs Creek. Flow directions and rates have remained 
relatively constant in recent history; however, previous descriptions may appear to differ because SRS grid coordinates were used prior to 1993. 


\section{Introduction}

\section{Description of Facilities}

The three F-Area Seepage Basins (FASB) are located in the central part of the Savannah River Site (SRS) (Figures 1 and 2, Appendix C) within the Separations Area. The following description outlines important events in the history of the FASB:

- The FASB began operating in 1955 to replace the Old F-Area Seepage Basin. These unlined, hydraulically connected basins received wastewater containing low-level radioactive constituents and chemicals primarily from the F-Area Separations Facility. The main sources for this wastewater included cooling water from the tritium facilities, nitric acid recovery overheads, general purpose evaporator overheads, other process evaporator overheads, and retention basin transfers (Heffner and Exploration Resources, 1991).

- The Resource Conservation and Recovery Act (RCRA) Part A post-closure care permit application was submitted to the South Carolina Department of Health and Environmental Control (SCDHEC) on September 29, 1980, and the Part B permit application was submitted on February 6, 1985.

- In Administrative Consent Order 85-70-SW (November 7, 1985), the U.S. Department of Energy (DOE) agreed to comply with the South Carolina Hazardous Waste Management Regulations (SCHWMR) (SCDHEC, 1990) and to continue quarterly groundwater monitoring at the FASB.

- In Settlement Agreement 86-52-SW (June 20, 1986), SCDHEC required DOE to submit groundwater monitoring reports to address groundwater contamination at the FASB.

- On May 1, 1987, Settlement Agreement 87-27-SW became effective. In this agreement, SCDHEC required DOE to submit a revised Part B post-closure care permit application by April 15, 1988, to address a Notice of Deficiency issued by SCDHEC on November 21, 1985.

- DOE submitted a revised Part B post-closure care permit application on April 15,1988 , in compliance with Settlement Agreement 87-27-SW.

- Under the Consent Decree of June 1, 1988 (Natural Resources Defense Council et al., Civil Action 1:85-2583-6, U.S. District Court, District of South Carolina, Aiken Division), the FASB was placed on Interim Status.

- Discharge of wastewater to the FASB was discontinued on November 7, 1988.

- SCDHEC terminated Interim Status for the FASB on November 8, 1988.

- On December 3, 1990, DOE submitted a revised Part B post-closure care permit application to SCDHEC that included a plan for groundwater remediation (WSRC, 1990). 
- Closure caps for the FASB were completed on January 4, 1991. These low permeability caps prevent infiltration of rainwater through the basin sediments and minimize migration of contaminants into the groundwater.

- A revised Part B post-closure care permit application was submitted to SCDHEC on January 22, 1991.

- The basins were certified closed April 26, 1991, in accordance with SCHWMR (SCDHEC, 1990).

- In March 1992, a revised Part B post-closure care permit application (WSRC, 1992a) was submitted to SCDHEC.

- SCDHEC issued a modification with conditions to permit SC1-890-008-989 on September 30, 1992 (SCDHEC, 1992). The permit, effective November 2, 1992, requires submittal of a detailed corrective action plan for groundwater remediation at the FASB.

- On February 15, 1994, SCDHEC issued a modification to permit SC1-890-008-989 that changed the groundwater sampling schedule to quarterly monitoring of all wells for selected field and analytical parameters, semi-annual monitoring of selected wells for all constituents with a Groundwater Protection Standard (GWPS), and annual monitoring of all wells for all constituents with a GWPS.

The Environmental Protection Department/Environmental Monitoring Section (EPD/EMS) monitors the groundwater beneath the FASB as part of the SRS Groundwater Monitoring Program. The Environmental Restoration Department provides semiannual reports to SCDHEC as required by South Carolina Hazardous Waste Permit SC1-890-008-989, effective November 2, 1992 (SCDHEC, 1992).

\section{Hydrostratigraphic Units}

Historically, groundwater monitoring reports for the FASB have used the nomenclature Water Table, Barnwell, McBean, and Congaree to identify water-bearing units. However, an interim alphanumeric system developed by Aadland and Bledsoe (1990) (Figure 3, Appendix C) defines these units at SRS using hydrostratigraphic rather than lithostratigraphic designations. Figure 4 (Appendix C) shows a correlation of these designations. For clarity, this report uses the newer nomenclature and also includes the older names used in earlier reports. The FASB RCRA Part B post-closure care permit application (WSRC, 1992a), submitted March 1992, includes an indepth explanation of the new nomenclature.

The FASB well network monitors three distinct hydrostratigraphic units in the uppermost aquifer beneath the facility: Aquifer Zone $\| B_{2}$ (Water Table), which is supported by Confining Zone $I I B_{1}-I I B_{2}$ (Tan Clay); the poorly confined Aquifer Zone IIB ${ }_{1}$ (Barnwell/McBean); and the semi-confined Aquifer Unit IIA (Congaree), which is separated from the overlying Aquifer Zone IIB (Barn- $^{-}$ well/McBean) by Confining Unit IIA-IIB (Green Clay). The base of the uppermost aquifer is defined as the uppermost bed of Confining System I-II (Ellenton Formation), which lies approximately $300 \mathrm{ft}$ below the surface of the area. The FASB RCRA Part B post-closure care permit application includes a detailed description of the geologic and hydrogeologic systems at the FASB. 


\section{Monitoring Well Nomenclature}

Generally, groundwater monitoring wells are designated with an abbreviated waste management unit prefix (e.g., FSB), a number (1 through 999), and a suffix $(A, B, C$, or D) which indicates the hydrostratigraphic unit being monitored. At the FASB, if the suffix is followed by a $D$, the well monitors Aquifer Zone $\|_{1} B_{2}$ (Water Table); if it is followed by a $C$, the well monitors Aquifer Zone $\mathrm{IIB}_{1}$ (Barnwell/McBean); and if it is followed by an $A$ or $B$, the well monitors the lower or upper portion of Aquifer Unit IIA (Congaree), respectively. An $R$ designation following the $A, B, C$, or $D$ suffix indicates a replacement well.

There are exceptions to this nomenclature. For example, wells FSB 76, 77, 78, and 79 monitor Aquifer Zone IIB ${ }_{2}$ (Water Table), and wells FSB 96AR, 97A, 98AR, 99A, 100A, 101A, 112A, $113 \mathrm{~A}, 114 \mathrm{~A}$, and $120 \mathrm{~A}$ monitor the upper portion of Aquifer Unit IIA (Congaree). 
WSRC-TR-95-0146-2

Unclassified

\section{Discussion}

\section{Groundwater Monitoring Data}

The sampling procedure (WSRC, 1992b) for pumped wells requires evacuation of a minimum of two well volumes and stabilization of $\mathrm{pH}$, specific conductance, and turbidity prior to sample collection. Stability for each parameter is established when a minimum of three successive measurements, taken at 10-minute intervals, are within 10 percent of each other. If a well pumps dry before two well volumes are purged or before stabilization is achieved, it must be revisited within 24 hours for the data to be considered part of a single sampling event. On the second visit within 24 hours, samples are taken without purging or stability measurements; thus, these samples may not be representative of the groundwater quality.

Variable-speed pumps are installed in wells at the FASB that have had a history of elevated metals. Samples from these wells are collected at a slower rate to minimize turbidity, which has been associated with elevated metals levels. Results from previous SRS studies indicate decreased aluminum and iron concentrations as well as lower turbidity values for samples from wells with this kind of pump. Currently, wells FSB 77, 78, 78C, 79, 79C, 88D, 89D, 90D, 91C, 91D, 92C, 92D, 93D, 94C, 97C, 97D, 98C, 98D, 102C, 104D, 105C, 105DR, 106C, 107D, and $110 \mathrm{D}$, and wells FSL 1D, 2D, 3D, 4D, 5D, 6D, 7D, 8D, and 9D have variable-speed pumps.

During first and second quarters 1995, samples from wells at the FASB were analyzed for field indicator parameters, gross alpha, certain heavy metals, nitrate, nonvolatile beta, tritium, and other radioactive and nonradioactive constituents. Analytical results that exceeded the GWPS (Appendix A) or the SRS flagging criteria (Appendix B) are presented in this report. The GWPS is based on standards listed in section 264.94 of SCHWMR (SCDHEC, 1990), practical quantitation limits identified in Appendix IX of SCHWMR or established by the contract laboratories, interim use of primary and secondary drinking water standards (EPA, 1992a; 1992b), and the SRS flagging criteria (Appendix B). The SRS flagging criteria are based on following standards:

- the Safe Drinking Water Act final Primary Drinking Water Standards (PDWS) established by the U.S. Environmental Protection Agency (EPA)

- proposed PDWS

- Secondary Drinking Water Standards

- method detection limits.

For simplicity, results that either equaled or exceeded standards are described as elevated or as exceeding standards or above standards.

The SCDHEC permit requires that all radionuclides except gross alpha, nonvolatile beta, total radium (radium-226 and radium-228), and tritium be compared to activities found in SRS background wells. The studies to obtain background activities are not completed; thus, SRS Flag 2 criteria are used in this report as interim standards for radionuclides requiring background activity standards. 
Currently, EPD/EMS does not provide analyses for several required radionuclides. Americium243 , nickel-63, plutonium-241, and plutonium-242 activities are not determined because their determinations require difficult methodologies which yield questionable results. In this report, the activities of these radionuclides were calculated as ratios of measured activities of chemically similar radionuclides. The activity ratios are as follows: americium-243:curium-244 $\approx 3.0 \mathrm{E}-03$; nickel-63:cobalt- $60 \approx 1.0 \mathrm{E}+00$; plutonium-241:plutonium-239 $\approx 4.0 \mathrm{E}+00$; and plutonium-242: plutonium-239 $\approx 2.0 \mathrm{E}-05$ (Environmental Restoration Department memorandum WSRC-RP-930723 from H. P. Holcomb to C. M. Lewis, May 14, 1993). Curium-244 and plutonium-239 activities currently are not determined by the analytical laboratory. Therefore, curium-243/244 and plutonium-239/240 activities were used to calculate the activities of americium-243, plutonium241 , and plutonium-242. Detection limit values for curium-243/244, cobalt-60, and plutonium$239 / 240$ were treated as real numbers in calculations. Results calculated from detection limit values are preceded by less than $(<)$ signs in the data tables.

Also, total radium (radium-226 and radium-228) and curium-246, required by the SCDHEC permit, are not determined by the analytical laboratory. Total radium activities are not calculated for this report because of problems inherent in summing the numerous detection limit values obtained for radium-226 and radium-228. Individual results for radium-226 and radium-228, which were analyzed for first quarter only, are reported to meet this requirement. Curium-245/246 activities are reported as an upper limit for curium-246 activities.

The activities for gross alpha and nonvolatile beta can not be compared with the summed activities of the individual radionuclides. The difference between the counts for gross alpha and the sum of the counts for the individual alpha emitters (Am-241, Cm-243/244, Ra-226, Th-228, Th$230, U-233 / 234, U-235, U-238$ ) is usually plus or minus 50 percent. The difference between the counts for nonvolatile beta and the sum of the counts for the individual beta emitters (Co-60, Cs137, I-129, Ra-228, Sr-89, Sr-90, Tc-99, Th-234) is up to two orders of magnitude. The discrepancies are the result of different counting techniques. The techniques used to determine the activities of the individual radionuclides are quantitative; however, the technique used to determine gross alpha and nonvolatile beta activities has the following problems:

- Corrections must be made in equipment calibration and counting to compensate for the amount of solids in the sample.

- Low-energy beta emitters are counted less efficiently because they are shielded by solids. Tc-99, a low-energy beta emitter, generally makes up at least 80 percent of the summed activities.

- Gross alpha and nonvolatile beta activities are counted simultaneously in the same sample, resulting in count interference.

Permit conditions IIIC.H.10.b.vi, vii, ix, and $x$, which are specific requirements for a corrective action program, are not discussed in this report because corrective action has not yet begun at the FASB.

\section{Integrity of the Monitoring Well Network}

The current groundwater monitoring well network at the FASB (Figure 2, Appendix C) is composed of the following:

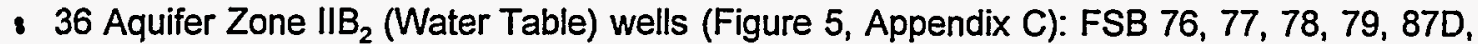
88D, 89D, 90D, 91D, 92D, 93D, 94DR, 95DR, 97D, 98D, 99D, 104D, 105DR, 106D, 107D, 
108D, 109D, 110D, 111D, 112D, 113D, 114D, 115D, 116D, 117D, 118D, 119D, 120D, 121DR, 122D, and 123D

- 32 Aquifer Zone IIB (Barnwell/McBean) wells (Figure 6, Appendix C): FSB 76C, 78C, 79C, 87C, 88C, 89C, 90C, 91C, 92C, 93C, 94C, 95CR, 97C, 98C, 99C, 102C, 103C, 104C, 105C, $106 \mathrm{C}, 107 \mathrm{C}, 110 \mathrm{C}, 111 \mathrm{C}, 112 \mathrm{C}, 113 \mathrm{C}, 114 \mathrm{C}, 115 \mathrm{C}, 116 \mathrm{C}, 120 \mathrm{C}, 121 \mathrm{C}, 122 \mathrm{C}$, and 123C

- 19 Aquifer Unit IIA (Congaree) wells (Figure 7, Appendix C): FSB 76B, 78B, 79B, 87B, 96AR, 97A, 98AR, 99A, 100A, 101A, 112A, 113A, 114A, and 120A and HSB 85A in the upper portion; FSB 76A, 78A, 79A, and 87A in the lower portion

- 9 additional Aquifer Zone $\|_{1} B_{2}$ (Water Table) wells (Figure 5, Appendix C): FSL 1D, 2D, 3D, $4 D, 5 D, 6 D, 7 D, 8 D$, and 9D

The designated background wells are FSB 76C and 108D and HSB 85A. Compared to the remaining upgradient FSB wells, these wells have lower constituent concentrations and activities in the units where they are screened as well as in adjacent units. They have been approved by SCDHEC as background wells.

The designated point-of-compliance (POC) wells are as follows:

- Aquifer Zone $\|_{3}$ (Water Table): FSB 88D, 89D, 90D, 91D, 92D, 93D, 94DR, 95DR, 97D, 98D, 99D, and 109D

- Aquifer Zone $\|_{1}$ (Barnwell/McBean): FSB 88C, 89C, 90C, 91C, 92C, 93C, 94C, 95CR, 97C, $98 \mathrm{C}$, and $99 \mathrm{C}$

- Aquifer Unit IIA (Congaree): FSB 96AR, 97A, 98AR, 99A, 100A, and 101A

SRS has a program in place to rehabilitate or replace wells that do not produce representative samples from the units being monitored. A complete record of well installations, replacements, and abandonments at the FASB is found in the EPD/EMS well inventory (EPD/EMS, 1995). The most recent well construction activity for FSB wells occurred during 1991. All FSL wells were installed in 1992.

Table 3 (Appendix D) lists the number of well volumes purged from each of the FASB monitoring wells during first and second quarters 1995 and provides sampling codes $(1, P, X$, etc. as defined in the front section of Appendix D) that describe unusual sampling events. Unusual sampling events occurred as follows:

- Wells that went dry during purging and were revisited within 24 hours $(X)$ : FSB 78C, 87D, 91C, 94C, 97C, 97D, 98D, 108D, 109D, 113A, 113C, 115C, 115D, 116D, 119D, 120D, and 121DR and FSL 1D, 2D, 3D, 4D, 5D, and 7D during first quarter, and wells FSB106D, 108D, 109D, 113A, 113C, 115C, 116D, 120D, and 121DR and FSL 1D, 2D, 3D, 4D, and 5D during second quarter.

- Wells that went dry during sampling, there was insufficient water to collect all samples (I): FSB 90D, 93D, and 106D during first quarter. These wells went dry after field analysis and did not yield enough water for complete laboratory analysis. SRS currently is evaluating the performance of well FSB106D. 
- Wells that were inaccessible or had mechanical failure that prevented sampling (P): FSB 88D during first quarter. This problem is currently being addressed.

- Wells that were not sampled during second quarter 1995 because they were added to the Purge Water Containment (PWC) Program (W): FSB 77, 78, 78C, 79, 79C, 88D, 89D, 90D, 91C, 91D, 92C, 92D, 93D, 94C, 94DR, 95CR, 95DR, 97C, 97D, 98C, 98D, 102C, 104D, 105C, 105DR, 106C, 107D, 110D, 112C, 112D, 115D, 117D, and 119D and well FSL 7D. SRS is currently implementing the PWC Program as stated in the approved InvestigationDerived Waste Management Plan (WSRC, 1994). It is anticipated that these wells will be sampled during fourth quarter 1995.

\section{Analytical Results Exceeding Standards}

Results for analytes that exceeded the GWPS (see Appendix A) during first and second quarters 1995 are summarized in Table 1 (Appendix D) and in the discussion below.

\section{Aquifer Zone IIB ${ }_{2}$ (Water Table), First Quarter 1995}

Thirty-five of $36 \mathrm{FSB}$ wells and all $9 \mathrm{FSL}$ wells in Aquifer Zone IIB ${ }_{2}$ (Water Table) exhibited one or more parameters that exceeded the GWPS during first quarter 1995. Laboratory pH measurements for 35 FSB wells exceeded the GWPS; laboratory pH was not measured for the FSL wells. Aluminum was elevated in 29 FSB wells, with a maximum concentration of $96,000 \mu \mathrm{g} / \mathrm{L}$ in well FSB110D, and in $8 \mathrm{FSL}$ wells, with a maximum concentration of $2,600 \mu \mathrm{g} / \mathrm{L}$ in well FSL 9D. Zinc was elevated in $28 \mathrm{FSB}$ wells, with a maximum concentration of $720 \mu \mathrm{g} / \mathrm{L}$ in well FSB106D, and was not analyzed in FSL wells. Tritium was elevated in $24 \mathrm{FSB}$ wells, with a maximum activity of $1.9 \mathrm{E}+04 \mathrm{pCi} / \mathrm{mL}$ in well $\mathrm{FSB} 110 \mathrm{D}$, and in $7 \mathrm{FSL}$ wells, with a maximum activity of $1.9 \mathrm{E}+03 \mathrm{pCi} / \mathrm{mL}$ in well FSL 7D. Gross alpha was elevated in $21 \mathrm{FSB}$ wells, with a maximum activity of $2.5 \mathrm{E}+03 \mathrm{pCi} / \mathrm{L}$ in well FSB 78 , and in $2 \mathrm{FSL}$ wells, with a maximum activity of $8.1 E+01 \mathrm{pCi} / L$ in well FSL 9D. lodine-129 was elevated in $21 \mathrm{FSB}$ wells, with a maximum activity of $2.9 \mathrm{E}+02 \mathrm{pCi} / \mathrm{L}$ in well $\mathrm{FSB} 78$, and was not analyzed in FSL wells.

Nitrate was elevated in $20 \mathrm{FSB}$ wells, with a maximum concentration of $380,000 \mu \mathrm{g} / \mathrm{L}$ in well FSB 77, and in $6 \mathrm{FSL}$ wells, with a maximum concentration of $370,000 \mu \mathrm{g} / \mathrm{L}$ in well FSL 1D. Nonvolatile beta was elevated in $20 \mathrm{FSB}$ wells, with a maximum activity of $3.1 \mathrm{E}+03 \mathrm{pCi} / \mathrm{L}$ in wells FSB 78 and 112D, and in $3 \mathrm{FSL}$ wells, with a maximum activity of $8.5 \mathrm{E}+02 \mathrm{pCi} / \mathrm{L}$ in well FSL 5D.

Strontium-90 was elevated in $20 \mathrm{FSB}$ wells, with a maximum activity of $2.1 \mathrm{E}+03 \mathrm{pCi} / \mathrm{L}$ in well FSB 78 . Uranium-238 was elevated in 19 FSB wells, with a maximum activity of 1.3E+03 pCi/L in wells FSB 94DR and 110D. Uranium-233/234 was elevated in $18 \mathrm{FSB}$ wells, with a maximum activity of $8.8 \mathrm{E}+02 \mathrm{pCi} / \mathrm{L}$ in well FSB119D. Americium-241 was elevated in 14 FSB wells, and curium-243/244, radium-228, and uranium-235 were elevated in 11 FSB wells each. None of these constituents were analyzed in FSL wells during first quarter 1995.

Copper was elevated in 9 FSB wells, and strontium- $89^{1}$ and thorium-234 were elevated in 7 FSB wells each. Cadmium was elevated in 5 FSB wells, and radium-226 was elevated in 4 FSB wells. The aforementioned constituents were not analyzed in FSL wells. Mercury was elevated in 4 FSB and no FSL wells, and cesium-137 and cobalt were elevated in 3 FSB wells each but were not analyzed in FSL wells. 
Lead was elevated in 2 FSB and 2 FSL wells, and bis(2-ethylhexyl) phthalate, curium$245 / 246$, cyanide, and nickel were elevated in 2 FSB wells each but were not analyzed in FSL wells. Barium and trichlorofluoromethane were elevated in 1 FSB well each and were not analyzed in FSL wells. Trichloroethylene was not elevated in any of the FSB wells but was elevated in $2 \mathrm{FSL}$ wells.

\section{Aquifer Zone IIB, (Barnwell/McBean), First Quarter 1995}

All 32 Aquifer Zone $\|_{1} B_{1}$ (Barnwell/McBean) wells exhibited one or more parameters that exceeded the GWPS. No FSL wells are located in Aquifer Zone IIB ${ }_{1}$ (Barnwell/McBean). Laboratory $\mathrm{pH}$ measurements exceeded the GWPS in $31 \mathrm{FSB}$ wells. Tritium was elevated in 24 FSB wells, with a maximum activity of $1.4 \mathrm{E}+04 \mathrm{pCi} / \mathrm{mL}$ in well FSB 95CR. lodine-129 was elevated in $21 \mathrm{FSB}$ wells, with a maximum activity of $2.3 \mathrm{E}+02 \mathrm{pCi} / \mathrm{L}$ in well $\mathrm{FSB} 95 \mathrm{CR}$.

Nitrate was elevated in $20 \mathrm{FSB}$ wells, with a maximum concentration of $250,000 \mu \mathrm{g} / \mathrm{L}$ in well FSB 94C. Aluminum was elevated in 19 FSB wells, with a maximum concentration of $77,000 \mu \mathrm{g} / \mathrm{L}$ in well FSB 97C. Zinc was elevated in $17 \mathrm{FSB}$ wells, with a maximum concentration of $590 \mu \mathrm{g} / \mathrm{L}$ in well FSB 78C. Nonvolatile beta and strontium-90 were elevated in 15 FSB wells each. Nonvolatile beta had a maximum activity of $2.5 \mathrm{E}+03 \mathrm{pCi} / \mathrm{L}$ in well FSB $79 \mathrm{C}$, and strontium-90 had a maximum activity of $1.2 \mathrm{E}+03 \mathrm{pCi} / \mathrm{L}$ in well FSB 95CR.

Gross alpha was elevated in 14 FSB wells, radium-226 was elevated in 9 FSB wells, and cobalt was elevated in 8 FSB wells. Cadmium, uranium-233/234, and uranium-238 were elevated in 7 FSB wells each. Americium-241, curium-243/244, nickel, radium-228, strontium- $89^{1}$, and uranium-235 were elevated in 5 FSB wells each, and copper was elevated in 3 FSB wells. Trichloroethylene and trichlorofluoromethane were elevated in 2 FSB wells each, and dichloromethane and plutonium-238 were elevated in $1 \mathrm{FSB}$ well each.

\section{Upper portion of Aquifer Unit IIA (Congaree), First Quarter 1995}

Thirteen of the 15 wells in the upper portion of Aquifer Unit IIA (Congaree) exhibited one or more parameters that exceeded the GWPS. No FSL wells are located in the upper portion of Aquifer Unit IIA (Congaree). Tritium was elevated in $10 \mathrm{FSB}$ wells, with a maximum activity of $2.7 \mathrm{E}+02 \mathrm{pCi} / \mathrm{mL}$ in well FSB 78B. Aluminum was elevated in $4 \mathrm{FSB}$ wells, and iodine129 was elevated in 3 FSB wells. Laboratory measurements of pH exceeded the GWPS in 3 FSB wells. Nitrate was elevated in 1 FSB well.

\section{Lower portion of Aquifer Unit IIA (Congaree), First Quarter 1995}

All four of the FSB wells in the lower portion of Aquifer Unit IIA (Congaree) had laboratory pH measurements that exceeded the GWPS. No FSL wells are located in the lower portion of Aquifer Unit IIA (Congaree).

\section{Aquifer Zone IIB ${ }_{2}$ (Water Table), Second Quarter 1995}

During second quarter 1995, 21 of the 36 FSB wells and 1 of the 9 FSL wells in Aquifer Zone IIB ${ }_{2}$ (Water Table) were not sampled because they were part of the PWC Program. The remaining wells were sampled for a reduced number of constituents. Fifteen FSB wells and $8 \mathrm{FSL}$ wells in Aquifer Zone $\mathrm{IB}_{2}$ (Water Table) exhibited one or more parameters that exceeded the GWPS during second quarter 1995. Laboratory pH measurements were elevated in $15 \mathrm{FSB}$ wells and $7 \mathrm{FSL}$ wells. 
Tritium was elevated in $5 \mathrm{FSB}$ wells, with a maximum activity of $4.8 \mathrm{E}+02 \mathrm{pCi} / \mathrm{mL}$ in well FSB118D, and in $6 \mathrm{FSL}$ wells, with a maximum activity of $6.4 \mathrm{E}+02 \mathrm{pCi} / \mathrm{mL}$ in well FSL $5 \mathrm{D}$. Gross alpha was elevated in $3 \mathrm{FSB}$ wells and $1 \mathrm{FSL}$ well. Lead and nonvolatile beta were elevated in $1 \mathrm{FSB}$ well and $2 \mathrm{FSL}$ wells each. Silver was elevated in $1 \mathrm{FSB}$ well and no FSL wells. Nitrate was elevated in no FSB wells and 3 FSL wells.

Zinc was elevated in 4 FSL wells, trichlorofluoromethane was elevated in $3 \mathrm{FSL}$ wells, and trichloroethylene was elevated in $1 \mathrm{FSL}$ well. These constituents were not analyzed in FSB wells.

\section{Aquifer Zone IIB, (Barnwell/McBean), Second Quarter 1995}

During second quarter 1995, 12 of the 32 FSB wells in Aquifer Zone $\| B_{1}$ (Barnwell/McBean) were not sampled because they were part of the PWC Program. The remaining wells were sampled for a reduced number of constituents. No FSL wells are located in Aquifer Zone $I I B_{1}$ (Barnwell/McBean). Twenty FSB wells in Aquifer Zone IIB, (Barnwell/McBean) had one or more parameters that exceeded the GWPS. Laboratory $\mathrm{pH}$ measurements exceeded the GWPS in 20 FSB wells.

Tritium was elevated in $11 \mathrm{FSB}$ wells, with a maximum activity of $1.7 \mathrm{E}+03 \mathrm{pCi} / \mathrm{mL}$ in well FSB122C. Nitrate was elevated in 8 FSB wells, nonvolatile beta was elevated in 3 FSB wells, and gross alpha was elevated in 2 FSB wells.

\section{Upper portion of Aquifer Unit IIA (Congaree), Second Quarter 1995}

Ten of the $15 \mathrm{FSB}$ wells in the upper portion of Aquifer Unit IIA (Congaree) exhibited one or more parameters that exceeded the GWPS. No FSL wells are located in the upper portion of Aquifer Unit IIA (Congaree). Tritium was elevated in 8 FSB wells, with a maximum activity of $4.9 \mathrm{E}+02 \mathrm{pCi} / \mathrm{mL}$ in well FSB114A. Laboratory $\mathrm{pH}$ measurements exceeded the GWPS in 3 FSB wells. Gross alpha and nitrate were elevated in 1 FSB well each.

\section{Lower portion of Aquifer Unit IIA (Congaree), Second Quarter 1995}

Laboratory measurements of pH exceeded the GWPS in all 4 FSB wells in the lower portion of Aquifer Unit IIA (Congaree). No FSL wells are located in this aquifer unit.

Results for analytes that exceeded the SRS Flag 2 criteria (Appendix B) during first and second quarters 1995 are summarized in Table 2 (Appendix D).

Table 3 (Appendix D) shows field water quality results, water elevations at sampling time, and laboratory analytical data as required in permit section IIIC.H.10.b.i. Table 3 also identifies the results that received modifiers (which help define laboratory accuracy and precision) or that exceeded EPA-approved holding times, the GWPS, or SRS flagging criteria during first and second quarters 1995. Constituent results in Table 3 that appear to equal the GWPS but are not marked in the ST column (exceeded GWPS) are below the GWPS in the database. Database results, which are the results compared to the GWPS, are entered with more significant digits than the results given in this report. Apparent discrepancies are due to the rounding of reported results.

In addition to the results tables, Appendix $D$ provides definitions of the abbreviations and the modifiers used in the results tables as well as descriptions of holding times, data rounding, and data qualification practices. Appendix E provides a general assessment of the quality and usability of EPD/EMS data. 


\section{Maps and Time Series Plots}

Isoconcentration maps for cadmium, gross alpha, lead, nitrate, nonvolatile beta, radium-226, radium-228, and tritium in the hydrostratigraphic units during first quarter 1995 are presented in Figures 8 through 31 (Appendix $\mathrm{C}$ ). Isoconcentration contouring for radium-226 and -228 maps for second quarter 1995 was not possible because of these constituents were not scheduled for sampling. Only first quarter 1995 data were used for all constituents because some of the wells in the General Separations Area were not sampled second quarter 1995. Piezometric and potentiometric surface maps for the monitored water-bearing units during first and second quarters 1995 are located in Figures 32 through 37 (Appendix C). Levels of pH and specific conductance in the three hydrostratigraphic units during first quarter 1995 are shown in Figures 38 through 43 (Appendix C).

Large-scale ( 1 in. $=900 \mathrm{ft}$ ) isoconcentration maps of cadmium, gross alpha, lead, nitrate, nonvolatile beta, radium-226, radium-228, and tritium for first quarter 1995 , as required by section IIIC.H.10.b.iv of the permit, and large-scale piezometric and potentiometric maps for first quarter 1995, as required by section IIIC.H.10.b.v of the permit, are located in Volume II of this report.

Synchronous water elevations were used to construct the small- and large-scale piezometric and potentiometric maps and cross sections. Synchronous water elevations are water-elevation measurements taken over as short a time period as possible; thus, other field measurements and sampling for laboratory analyses are not conducted during the time these measurements are made. This short time period allows the production of steady-state piezometric and potentiometric maps by minimizing the variation in water elevations that could occur, for example, if some elevations were measured before a large rain and the remaining elevations were measured after the rain. Synchronous water elevations were measured on March 27 during first quarter 1995 and on June 15 and 16 during second quarter 1995.

Appendix $F$ contains time series plots for cadmium, gross alpha, lead, nitrate, nonvolatile beta, radium-226, radium-228, and tritium for selected FSB wells as required in section IIIC.H.10.b.iii of the permit. These wells were selected to delineate the extent of the constituent distribution, to monitor the migration of the constituents, and to follow trends of constituents present at high levels.

\section{Water Levels}

Rainfall data for the period of this report, required by section IIIC.H.10.b.viii of the permit, are presented below. Rainfall per month in F Area (200-F) for the first half of 1995 is compared with the SRS average monthly rainfall for the same period and to the SRS historical average monthly rainfall. 
Rainfall (in.) during First and Second Quarters of 1995

\begin{tabular}{|lccc|}
\hline & $\begin{array}{c}\text { F-Area (200-F) } \\
\text { Rainfall/Month } \\
(1995)\end{array}$ & $\begin{array}{c}\text { SRS Average } \\
\text { RainfallMonth } \\
(1995)\end{array}$ & $\begin{array}{c}\text { SRS Average } \\
\text { Rainfall/Month } \\
\text { (historical) }\end{array}$ \\
\hline January & 5.97 & 6.95 & 4.30 \\
February & 7.50 & 7.46 & 4.46 \\
March & 0.83 & 5.28 & 4.90 \\
April & 0.93 & 4.28 & 3.35 \\
May & 2.10 & 3.78 & 3.86 \\
June & 12.47 & 4.58 & 4.48 \\
Six-month total & 29.80 & 32.33 & 25.35 \\
\hline
\end{tabular}

Hydrographs showing the water elevations for the FSB wells, required by section IIIC.H.10. b.ii of the permit, are provided in Appendix G. Hydrograph data include synchronous measurements and measurements taken during sampling. Average water elevations in the three hydrostratigraphic units beneath the FASB for the past four quarters are shown in the following table.

\section{Average Water Elevations (ft $\mathrm{msl}$ ) in the Hydrostratigraphic Units beneath the FASB}

\begin{tabular}{|lllll|}
\hline Unit & $3 \mathrm{Q} 94$ & $4 \mathrm{Q} 94$ & $1 \mathrm{Q} 95$ & $2 \mathrm{Q} 95$ \\
\hline Aquifer Zone $\mathrm{IIB}_{2}$ (Water Table) & 211.78 & 211.66 & 212.56 & 211.94 \\
Aquifer Zone IIB, (Barnwell/McBean) & 205.23 & 204.73 & 206.13 & 206.15 \\
Aquifer Unit IIA (Congaree, upper portion) & 153.90 & 153.78 & $154.19^{\mathrm{a}}$ & 154.51 \\
Aquifer Unit IIA (Congaree, lower portion) & 155.39 & 155.60 & 156.51 & 156.51 \\
\hline
\end{tabular}

a The water-level measurement for well FSB113A for first quarter 1995 was anomalous and was not used in average water-level calculations.

During first quarter 1995, the average water elevation in Aquifer Zone $\mathrm{IIB}_{2}$ (Water Table) rose $0.90 \mathrm{ft}$ compared to fourth quarter 1994; the average water level in Aquifer Zone IIB $_{1}$ (Barnwell/ McBean) rose $1.4 \mathrm{ft}$; the average water level in the upper portion of Aquifer Unit IIA (Congaree) rose $0.41 \mathrm{ft}$; and the average water level in the lower portion of Aquifer Unit IIA (Congaree) rose $0.91 \mathrm{ft}$.

During second quarter 1995, the average water elevation in Aquifer Zone $\|_{1} B_{2}$ (Water Table) declined $0.62 \mathrm{ft}$ compared to first quarter 1995; the average water level in Aquifer Zone $\| \mathrm{B}_{1}$ (Barnwell/McBean) rose $0.02 \mathrm{ft}$; the average water level in the upper portion of Aquifer Unit IIA (Congaree) rose $0.32 \mathrm{ft}$; and the average water level in the lower portion of Aquifer Unit IIA (Congaree) remained the same.

A consistent vertical head relationship exists among the hydrostratigraphic units monitored at the FASB (WSRC, 1992a, Section E). Flow potential is downward from Aquifer Zone $11 B_{2}$ (Water Table) to Aquifer Zone IIB 1 (Barnwell/McBean) and downward from Aquifer Zone IIB, (Barnwell/ McBean) to Aquifer Unit IIA (Congaree). The heterogeneous nature of the confining zone that 
separates Aquifer Zone $\| B_{2}$ (Water Table) from Aquifer Zone IIB (Barnwell/McBean) may influence vertical flow rates between these two units. A head reversal exists between the lower and upper portions of Aquifer Unit IIA (Congaree) at the FASB, which indicates the potential for upward flow within the aquifer. This vertical head relationship among the different monitored units has remained essentially unchanged through past quarters.

\section{Groundwater Flow Rates and Directions}

Piezometric and potentiometric maps for the monitored water-bearing units (Figures 32 through 37. Appendix C) illustrate flow patterns beneath the FASB for first and second quarters 1995. Large-scale piezometric and potentiometric maps for each hydrostratigraphic unit during first quarter 1995, required by section IIIC.H.10.b.v of the permit, are located in Volume II of this report. Calculations for Aquifer Unit IIA (Congaree) were derived using wells in the upper portion of this unit.

In the past, when SRS grid coordinates were used, flows in Aquifer Zone $\| \mathrm{B}_{2}$ (Water Table) and Aquifer Zone IIB ${ }_{1}$ (Barnwell/McBean) were generally reported as south or south-southwest toward Fourmile Branch. Using universal transverse Mercator (UTM) coordinates, flow in Aquifer Zone $\| \mathrm{B}_{2}$ (Water Table) was south or south to southeast and flow in Aquifer Zone IIB, (Barnwell/ McBean) was south or south to southeast during first and second quarters 1995. Flow in Aquifer Unit IIA (Congaree) was northwest toward Upper Three Runs Creek, consistent with the direction reported in previous quarters.

Horizontal flow rate estimates for the hydrostratigraphic units during first and second quarters are provided in the following table.

\section{Horizontal Groundwater Flow Rates (ft/year) in the Hydrostratigraphic Units beneath the} FASB

\begin{tabular}{|lll|}
\hline Unit & $1 \mathrm{Q} 95$ & $2 \mathrm{Q} 95$ \\
\hline Aquifer Zone IIB ${ }_{2}$ (Water Table) & $88-110$ & $95-110$ \\
Aquifer Zone IIB (Barnwell/McBean) & $84-210$ & $73-180$ \\
Aquifer Unit IIA (Congaree) & 550 & 620 \\
\hline
\end{tabular}

Horizontal flow rate calculations provide estimates of the transport rate for constituents originating from the FASB. Flow rates in Aquifer Zone $\|_{1} B_{2}$ (Water Table) and Aquifer Zone $\|_{1} B_{1}$ (Barnwell/McBean) are calculated along two flow paths (designated flow paths $A$ and $B$ ) to characterize the approximate maximum and minimum groundwater flow rates within these units in areas downgradient from the basins. The flow rate in Aquifer Unit IIA (Congaree) is calculated along a single flow path because the flow rate within this unit is generally more uniform than in the overlying units. Flow rates are estimated using the following equation:

$$
\text { Flow (ft/day) }=\frac{\text { Hydraulic Conductivity (ft/day) }}{\text { Porosity (unitless) }} \times \frac{d h(\mathrm{ft})}{d l(\mathrm{ft})}
$$

The hydraulic conductivity (Kh) constant for Aquifer Zone $\mathrm{IIB}_{2}$ (Water Table) is $10 \mathrm{ft} /$ day; for Aquifer Zone $\|_{1} B_{1}$ (Barnwell/McBean), $\mathrm{Kh}$ is $10.4 \mathrm{ft} /$ day; and for Aquifer Unit IIA (Congaree), $\mathrm{Kh}$ is $65 \mathrm{ft} /$ day (Geraghty \& Miller, 1990). An effective porosity value of 20 percent is used for each hydrostratigraphic unit (Jaegge et al., 1987). The value $d h$ is the difference in head, and $d l$ is the length of the flow path. 
Flow path length is calculated to the nearest $100 \mathrm{ft}$. Flow rate per day is calculated to two significant digits, then multiplied by 365 and rounded to two significant digits for the flow rate per year. Flow rate estimates vary depending on the vertical gradient between wells, the size of the area under consideration, the number of data points, and the length and location of the flow path. Because these calculations are based on inferred or estimated parameters, flow rate estimates should be considered accurate to an order of magnitude only.

Groundwater flow rates were calculated for first quarter 1995 as follows:

- Aquifer Zone $\mathrm{IIB}_{2}$ (Water Table) (Figure 32, Appendix C)

Flow path $\mathrm{A}$

$\frac{10}{0.20} \times \frac{10}{2,100} \approx 0.24 \mathrm{ft} /$ day

$0.24 \mathrm{ft} /$ day $\times 365$ days $\approx 88 \mathrm{ft} /$ year

Flow path $B$

$\frac{10}{0.20} \times \frac{12}{2,050} \approx 0.29 \mathrm{ft} / \mathrm{day}$

$0.29 \mathrm{ft} /$ day $\times 365$ days $\approx 110 \mathrm{ft} /$ year

- Aquifer Zone $\|_{1}$ (Barnwell/McBean) (Figure 34, Appendix C)

Flow path $A$

$\frac{10.4}{0.20} \times \frac{12}{2,700} \approx 0.23 \mathrm{ft} /$ day

$0.23 \mathrm{ft} /$ day $\times 365$ days $\approx 84$ ftlyear

Flow path B

$\frac{10.4}{0.20} \times \frac{16}{1,450} \approx 0.57 \mathrm{ft} /$ day

$0.57 \mathrm{ft} /$ day $\times 365$ days $\approx 210 \mathrm{ft} /$ year

- Aquifer Unit IIA (Congaree) (Figure 36, Appendix C)

$$
\frac{65}{0.20} \times \frac{7}{1,500} \approx 1.5 \mathrm{ft} / \mathrm{day}
$$

$1.5 \mathrm{ft} /$ day $\times 365$ days $\approx 550 \mathrm{ft} /$ year 
Groundwater flow rates were calculated for second quarter 1995 as follows:

- Aquifer Zone $\mathrm{IIB}_{2}$ (Water Table) (Figure 33, Appendix C)

Flow path $\mathrm{A}$

$\frac{10}{0.20} \times \frac{10}{1,950} \approx 0.26 \mathrm{ft} / \mathrm{day}$

$0.26 \mathrm{ft} /$ day $\times 365$ days $\approx 95 \mathrm{ft} /$ year

Flow path B

$\frac{10}{0.20} \times \frac{10}{1,600} \approx 0.31 \mathrm{ft} /$ day

$0.31 \mathrm{ft} /$ day $\times 365$ days $\approx 110 \mathrm{ft} /$ year

- Aquifer Zone IIB 1 (Barnwell/McBean) (Figure 35, Appendix C)

Flow path $A$

$\frac{10.4}{0.20} \times \frac{10}{2,600} \approx 0.20 \mathrm{ft} / \mathrm{day}$

$0.20 \mathrm{ft} /$ day $\times 365$ days $\approx 73 \mathrm{ft} /$ year

Flow path $B$

$\frac{10.4}{0.20} \times \frac{14}{1,500} \approx 0.49 \mathrm{ft} /$ day

$0.49 \mathrm{ft} /$ day $\times 365$ days $\approx 180 \mathrm{ft} /$ year

- Aquifer Unit IIA (Congaree) (Figure 37, Appendix C)

$\frac{65}{0.20} \times \frac{7}{1,350} \approx 1.7 \mathrm{ft} / \mathrm{day}$

$1.7 \mathrm{ft} /$ day $\times 365$ days $\approx 620 \mathrm{ft} /$ year

\section{Upgradient and Background Results}

Wells FSB108D and 109D are the upgradient wells for Aquifer Zone $\|_{2}$ (Water Table) (Figure 5, Appendix C). Although well FSB 76 lies upgradient of the facility, it is not considered an upgradient well because it is downgradient of a collapsed process sewer line that has contaminated the groundwater. Well FSB $76 \mathrm{C}$ is the upgradient well for Aquifer Zone $\| \mathrm{B}_{1}$ (Barnwell/McBean) (Figure 6, Appendix C). Wells FSB 78B, 79B, 112A, and 113A are upgradient wells for the upper 
portion of Aquifer Unit IIA (Congaree), and wells FSB 78A and 79A are upgradient wells for the lower portion of Aquifer Unit IIA (Congaree) (Figure 7, Appendix C).

During the first half of 1995, upgradient wells FSB 78B, 108D, 109D, and 113A contained elevated levels of aluminum. Upgradient wells FSB 76C, 78A, 79A, 108D, 109D, and 113A exhibited $\mathrm{pH}$ values that exceeded the GWPS. Upgradient wells FSB108D and 109D contained elevated levels of zinc. Upgradient well FSB 78B contained elevated levels of nitrate. Upgradient wells FSB 78B, 79B, 109D, 112A, and 113A contained elevated levels of tritium. Upgradient well FSB $76 \mathrm{C}$ also contained an elevated level of trichlorofluoromethane.

Wells FSB 76C and 108D and well HSB 85A are the designated background wells for the FASB. Aluminum, $\mathrm{pH}$, trichlorofluoromethane, and zinc were the only parameters exceeding GWPS in one or more of the background wells during the first half of 1995. 


\section{Conclusions}

The groundwater at the FASB contains elevated levels of radionuclides and chemicals from 30 years of receiving contaminated wastewater from the F-Area Separations Area. During the first half of 1995, 34 parameters exceeded the GWPS in wells at the FASB. Elevated constituents occurred in 84 of the 86 FSB wells monitoring the FASB and in all 9 FSL monitoring wells.

Aluminum and zinc were the primary metals that exceeded the GWPS during the first half of 1995. Aluminum occurred at a maximum concentration of $96,000 \mu \mathrm{g} / \mathrm{L}$ in well FSB110D during first quarter 1995, and zinc was found at a maximum concentration of $720 \mu \mathrm{g} / \mathrm{L}$ in well FSB106D during first quarter 1995. Barium, cadmium, cobalt, copper, cyanide, lead, mercury, nickel, and silver also exceeded the GWPS in one or more FSB wells during the first half of 1995.

Nitrate and $\mathrm{pH}$, which are monitored along with aluminum as water-quality indicator parameters, exceeded the GWPS in numerous FSB wells during the first half of 1995. Most of the pH results exceeded the acidic standard of $6.8 \mathrm{pH}$ units. Bis(2-ethylhexyl) phthalate, dichloromethane (a common laboratory contaminant), trichloroethylene, and trichlorofluoromethane exceeded GWPS in 3 or fewer FSB wells each during the first half of 1995.

During the first half of 1995 , americium-241, curium-243/244, gross alpha, iodine-129, nonvolatile beta, radium-228, strontium-90, tritium, uranium-233/234, uranium-235, and uranium-238 were the primary radionuclides detected at elevated levels at the FASB, each occurring in 16 or more FSB wells during at least one quarter. These constituents' maximum activities, which were all detected during first quarter 1995, were as follows: americium-241 at $9.9 \mathrm{E}+01 \mathrm{pCi} / \mathrm{L}$ in well FSB110D, curium-243/244 at 1.6E+02 pCi/L in well FSB 94DR, gross alpha at 2.5E+03 pCi/L in well FSB 78 , iodine -129 at $2.9 \mathrm{E}+02 \mathrm{pCi} / \mathrm{L}$ in well $\mathrm{FSB} 78$, nonvolatile beta at $3.1 \mathrm{E}+03 \mathrm{pCi} / \mathrm{L}$ in wells FSB 78 and $112 \mathrm{D}$, radium-228 at $2.5 \mathrm{E}+02 \mathrm{pCi} / \mathrm{L}$ in well FSB $95 \mathrm{CR}$, strontium-90 at $2.1 \mathrm{E}+03 \mathrm{pCi} / \mathrm{L}$ in well FSB 78 , tritium at $1.9 \mathrm{E}+04 \mathrm{pCi} / \mathrm{mL}$ in well $\mathrm{FSB} 110 \mathrm{D}$, uranium-233/234 at $8.8 \mathrm{E}+02 \mathrm{pCi} / \mathrm{L}$ in well FSB119D, uranium-235 at 4.6E+01 pCi/L in well FSB119D, and uranium238 at $1.3 \mathrm{E}+03 \mathrm{pCi} / \mathrm{L}$ in wells FSB $94 \mathrm{DR}$ and $110 \mathrm{D}$. Elevated activities of cesium-137, curium$245 / 246$, plutonium-238, radium-226, strontium- $89^{1}$, and thorium-234 also were detected during this period.

The constituents that occurred most frequently in FSB wells during the first half of 1995, with the addition of strontium-90, are the same as those that occurred most frequently during the second half of 1994. During the second half of 1994, aluminum, gross alpha, iodine-129, nitrate, nonvolatile beta, tritium, and zinc were elevated in 34 or more FSB wells each. These same constituents were elevated in 35 or more FSB wells each during the first half of 1995.

As in previous quarters, elevated constituents were found primarily in Aquifer Zone $\mathrm{IB}_{2}$ (Water Table) and Aquifer Zone IIB, (Barnwell/McBean). Also as in previous quarters, several constituents at levels exceeding the GWPS occurred in one or more FSB wells in Aquifer Unit IIA (Congaree), indicating that vertical pathways into the deeper water-bearing units exist at the FASB. A current groundwater flow model for the Separations Area indicates that the vertical component beneath this area is important as a constituent pathway (GeoTrans, Inc., 1992).

During the first half of 1995, upgradient FSB wells contained elevated levels of aluminum, nitrate, $\mathrm{pH}$, trichlorofluoromethane, tritium, or zinc. Downgradient FSB wells contained elevated levels of 
numerous metals and radionuclides and several other constituents. Generally, constituents found in wells upgradient of a waste management unit are considered to be from a source other than the waste management unit; constituents found in downgradient wells but not in upgradient wells at a waste management unit are considered products of the waste management unit.

The following constituents exceeded the GWPS in FSL wells during the first half of 1995: aluminum, gross alpha, lead, nitrate, nonvolatile beta, $\mathrm{pH}$, trichloroethylene, trichlorofluoromethane, tritium, and zinc.

Groundwater flow directions beneath the FASB have remained relatively unchanged for several years. Flow in Aquifer Zone $\|_{1} B_{2}$ (Water Table) and Aquifer Zone IIB (Barnwell/McBean) is generally south to southeast toward Fourmile Branch (using UTM coordinates). Flow in Aquifer Unit IIA (Congaree) is generally northwest toward Upper Three Runs Creek.

The estimated groundwater flow rate during first quarter 1995 ranged from approximately 88 to $110 \mathrm{ft} / \mathrm{year}$ and from approximately 95 to $110 \mathrm{ft} / \mathrm{year}$ in Aquifer Zone $\mathrm{IlB}_{2}$ (Water Table). The flow rate in Aquifer Zone IIB, (Barnwell/McBean) ranged from approximately 84 to $210 \mathrm{ft} / y e a r$ during first quarter 1995 and from approximately 73 to 180 ft/year during second quarter 1995. The flow rate in Aquifer Unit IIA (Congaree) was estimated at $550 \mathrm{ft} / \mathrm{year}$ during first quarter 1995 and $620 \mathrm{ft} /$ year during second quarter 1995.

\section{Endnote}

'WSRC has documented that strontium-89 is not present in detectable quantities on the site based on the last known operations date for processes that produced the radionuclide and the relative half-lives of strontium-89 and strontium-90 (Environmental Restoration Department memorandum WSRC-RP-93-1060 from H. P. Holcomb to M. A. Ebra, Strontium-89-an unnecessary contaminant of concern in SRS environmental samples (U), July 28,1993$)$. Positive results reported for strontium-89 in SRS environmental samples are considered to originate from error in the analytical method. 
Aadland, R. K., and H.W. Bledsoe, 1990. Classification of Hydrostratigraphic Units at the Savannah River Site, South Carolina, WSRC-RP-90-987. Westinghouse Savannah River Company, Savannah River Site, Aiken, SC.

EPA (Environmental Protection Agency), 1992a. National Primary Drinking Water Regulations. Code of Federal Regulations, Title 40, Part 141, pp. 589-729. Washington, DC.

EPA (Environmental Protection Agency), 1992b. National Secondary Drinking Water Regulations. Code of Federal Regulations, Title 40, Part 143, pp. 772-776. Washington, DC.

EPD/EMS (Environmental Protection Department/Environmental Monitoring Section), 1995. Environmental Protection Department's Well Inventory (through the fourth quarter of 1994), ESH-EMS-940519. Westinghouse Savannah River Company, Savannah River Site, Aiken, SC.

GeoTrans, inc., 1992. Groundwater Flow Model for the General Separations Area, Savannah River Site. Prepared for Westinghouse Savannah River Company, Environmental Restoration Department, Savannah River Site, Aiken, SC.

Geraghty \& Miller, Inc., 1990. Evaluation of Integrated Waste Facility Closure Capping on Ground-Water Flow and Solute Transport in General Separations Area, Savannah River Site: Flow Model and Particle-Tracking Analysis, Final Report. Prepared by Geraghty \& Miller Modeling Group for Westinghouse Savannah River Company, Waste Management Technology, Savannah River Site, Aiken, SC.

Heffner, J. D., and Exploration Resources, Inc., 1991. Technical Summary of Groundwater Quality Protection Program at the Savannah River Site (1952-1986), Volume I-Site Geohydrology and Waste Sites, DPSP-88-1002. Westinghouse Savannah River Company, Savannah River Site, Aiken, SC.

Jaegge, W. J., N. L. Kolb, B. B. Looney, I. W. Marine, O. A. Towler, and J. R. Cook, 1987. Radioactive Waste Burial Grounds, DPST-85-694. Savannah River Laboratory, E. I. du Pont de Nemours \& Company, Aiken, SC.

SCDHEC (South Carolina Department of Health and Environmental Control), 1990. South Carolina Hazardous Waste Management Regulations; R.61-79.124, .260 through .266, .268, and .270; November 1990. Columbia, SC.

SCDHEC (South Carolina Department of Health and Environmental Control), 1992. Hazardous Waste Permit Modification, Permit No. SC1-890-008-989. Office of Environmental Quality Control, Bureau of Solid and Hazardous Waste Management, Columbia, SC.

WSRC (Westinghouse Savannah River Company), 1990. Application for a Hazardous Waste Part B Post-Closure Care Permit. Volume IV. F-Area Hazardous Waste Management Facility. Revision 2, December 3, 1990. Savannah River Site, Aiken, SC. 
WSRC (Westinghouse Savannah River Company), 1992a. Application for a Hazardous Waste Part B Post-Closure Care Permit. Volume IV. F-Area Hazardous Waste Management Facility. Revision 3, March 13, 1992. Savannah River Site, Aiken, SC.

WSRC (Westinghouse Savannah River Company), 1992b. Sampling Groundwater Monitoring Wells, Hydrogeologic Data Collection Procedures and Specifications, Manual 3Q5, Rev. 1, Chapter 15. Savannah River Site, Aiken, SC.

WSRC (Westinghouse Savannah River Company), 1994. Investigation-Derived Waste Management Plan, WSRC-RP-94-1227, Revision 2. Savannah River Site, Aiken, SC. 
First and Second Quarters 1994:

- Page C-31, Figure 29: Wells FSB110D and 112D were omitted from the $10,000 \mathrm{pCi} / \mathrm{mL}$ contour. See the third and fourth quarters 1994 report (WSRC-TR-94-0487) for the corrected map.

- Appendix F: The time series plot for gross alpha activities for well FSB117D was omitted inadvertently from the report. The plot is included on page 24 of the third and fourth quarters 1994 report (WSRC-TR-94-0487).

Third and Fourth Quarters 1994:

- Modifiers for the following analyses in Table 3 (Appendix D) were not assigned correctly for some wells as follows: laboratory $\mathrm{pH}$ values should have had holding time indicators and measured radionuclides with a result of $0.0 E+00$ preceded by a less than $(<)$ sign should have had UI modifiers instead of the less than sign.

- Volume II: The large isoconcentration maps included with the report had the incorrect document numbers listed in the map legends. The correct document numbers are WSRCTR-94-0487 and WSRC-TR-94-0488, F-Area and H-Area, respectively. 
WSRC-TR-95-0146-2

Unclassified

\title{
Appendix A
}

\author{
Standards
}


THIS PAGE LEFT BLANK INTENTIONALLY. 


\section{Table 1. Groundwater Protection Standards}

Established for the F-Area Seepage Basins by the September 30,1992, modification of South Carolina Hazardous Waste Permit SC1-890-008-989.
Analyte
Unit
Concentration/Activity Limit

SCHWMR 264.94 Table 1 Hazardous Constituents

$\begin{array}{lll}\text { Arsenic } & \boldsymbol{\mu} \mathrm{g} / \mathrm{L} & 50 \\ \text { Barium } & \boldsymbol{\mu g} / \mathrm{L} & 1,000 \\ \text { Cadmium } & \boldsymbol{\mu g} / \mathrm{L} & 10 \\ \text { Chromium } & \boldsymbol{\mu g} / \mathrm{L} & 50 \\ \text { Lead } & \boldsymbol{\mu g} / \mathrm{L} & 50 \\ \text { Mercury } & \boldsymbol{\mu g} / \mathrm{L} & 2 \\ \text { Selenium } & \boldsymbol{\mu g} / \mathrm{L} & 10 \\ \text { Silver } & \boldsymbol{\mu g} / \mathrm{L} & 50\end{array}$

SCHWMR 261 Appendix VIII/264 Appendix IX Hazardous Constituents

$\begin{array}{lll}\text { Antimony } & \boldsymbol{\mu} g / \mathrm{L} & 30 \\ \text { Benzene } & \boldsymbol{\mu g} / \mathrm{L} & 5 \\ \text { Cobalt } & \boldsymbol{\mu g} / \mathrm{L} & 70 \\ \text { Copper } & \boldsymbol{\mu g} / \mathrm{L} & 60 \\ \text { Cyanide } & \boldsymbol{\mu g} / \mathrm{L} & 5^{\mathrm{a}} \\ \text { Nickel } & \boldsymbol{\mu} \mathrm{g} / \mathrm{L} & 50 \\ \text { Phenols } & \boldsymbol{\mu} & 5 \\ \text { Tetrachloroethylene } & \boldsymbol{\mu g} / \mathrm{L} & 5 \\ \text { Thallium } & \boldsymbol{\mu g} / \mathrm{L} & 10 \\ \text { Trichloroethylene } & \boldsymbol{\mu g} / \mathrm{L} & 5 \\ \text { Trichlorofluoromethane } & \boldsymbol{\mu g} / \mathrm{L} & 5 \\ \text { Vanadium } & \boldsymbol{\mu g} / \mathrm{L} & 50 \\ \text { Zinc } & \boldsymbol{\mu g} / \mathrm{L} & 80\end{array}$

Water Quality Indicator Parameters

Aluminum

Nitrate

$\mathrm{pH}$

Common Laboratory Contaminants

Bis(2-ethylhexyl) phthalate

Dichloromethane

Radiological Indicator Parameters

Gross alpha

Nonvolatile beta

$\mathrm{pCi} / \mathrm{L}$

$\mathrm{pCi} / \mathrm{L}$

$\mu g / L$

$\mu \mathrm{g} / \mathrm{L}$

10

5

Measured Radionuclides ${ }^{\mathrm{e}}$

Actinium-228

Americium-241

Cesium-137

Cobalt-60

$\begin{array}{ll}\mu g / L & 50-200^{c} \\ \mu g / L & 10,000 \\ \mathrm{pH} & 6.8-8.5\end{array}$

.8-8.5 
Analyte

Curium-242

Curium-243/244

Curium-245/246

Curium-246

lodine-129

Plutonium-238

Plutonium-239/240

Radium-226

Radium-228

Radium, total (Radium-226 and -228)

Strontium-89

Strontium-90

Technetium-99

Thorium-228

Thorium-230

Thorium-232

Thorium-234

Tritium

Uranium-233/234

Uranium-235

Uranium-238

\section{Calculated Radionuclides ${ }^{f}$}

Americium-243

Nickel-63

Plutonium-241

Plutonium-242

\section{Unit}

$\mathrm{pCi} / \mathrm{L}$

pCill

pCi/L

pCill

$\mathrm{pCi} / \mathrm{L}$

$\mathrm{pCi} / \mathrm{L}$

pCill

$\mathrm{pCi} / \mathrm{L}$

pCi/L

pCi/L

pCi/L

pCi/L

pCi/L

pCi/L

pCill

pCill

pCill

pCi/mL

pCill

pCill

pCi/L

$\begin{array}{ll}\text { pCi/L } & 6.37 E+00 \\ \text { pCi/L } & 5 E+01 \\ \text { pCi/L } & 6.26 E+01 \\ \text { pCi/L } & 6.54 E+01\end{array}$

$1.33 E+02$
$8.3 E+00$
$6.23 E+00$
$6.27 E+00$
$1 E+00$
$7.02 E+00$
$6.21 E+01$
$2 E+01$
$2 E+01$
$5 E+00$
$2 E+01$
$8 E+00$
$9 E+02$
$1.25 E+02$
$7.92 E+01$
$8.8 E+01$
$4.01 E+02$
$2.0 E+01$
$1.38 E+01$
$1.45 E+01$
$1.46 E+01$

1.33E+02

$6.23 E+00$

$6.27 E+00$

$1 E+00$

$6.21 \mathrm{E}+01$

$2 \mathrm{E}+01$

2E+01

$5 E+00$

2E+01

$8 \mathrm{E}+00$

$9 \mathrm{E}+02$

1.25E+02

7.92E+01

4.01E+02

2.0E+01

$1.45 \mathrm{E}+01$

1.46 E+ 01

$6.54 E+01$
Concentration/Activity Limit

a The suggested analytical method for cyanide in Appendix IX (EPA, 1992) is 9010, with a PQL of $40 \mu \mathrm{g} / \mathrm{L}$; however, the method specified in the SRS contract with the analytical laboratory for analyzing cyanide in the Appendix IX suite is 9012 , with a PQL of $5 \mu \mathrm{g} / \mathrm{L}$.

b Phenols is not an Appendix VIII or IX constituent. The method specified in the SRS contract with the analytical laboratory for analyzing phenols is 420.2 , with a PQL of $5 \mu \mathrm{g} / \mathrm{L}$.

c SRS takes a conservative approach by flagging aluminum results at the bottom of this range.

d This is the screening level above which providers of public drinking water should perform analyses for specific man-made radionuclides. The standard for the total dose equivalent from all such radionuclides is 4 mrem per year.

e The activity limits used in this report are final or proposed drinking water standards. When radionuclide analyses are combined, the lower standard of the two isotopes is used. The GWPS is based on background levels, not drinking water standards. A study to establish such levels at SRS is being conducted.

$f$ The activities of these radionuclides were calculated as ratios of measured activities of other radionuclides. The activity ratios are as follows: americium-243/curium-243/244 $\approx 3 E-03$; nickel-63/cobalt-60 $\approx 1$; plutonium-241/plutonium-239/240 $\approx 4$; and plutonium-242/plutonium-239/240 $\approx 2 E-05$. 


\section{References Cited}

EPA (U.S. Environmental Protection Agency), 1992. Appendix IX-Ground-Water Monitoring List. Code of Federal Regulations, Title 40, Part 264, pp. 324-330. Washington, DC.

SCDHEC (South Carolina Department of Health and Environmental Control), 1992 (modification effective date). Hazardous Waste Permit SC1-890-008-989. Office of Environmental Quality Control, Bureau of Solid and Hazardous Waste Management, Columbia, SC. 
Table 2. Final Primary Drinking Water Standards

Analyte
Antimony
Arsenic
Asbestos
Barium
Benzene
Benzo[a]pyrene
Beryllium
Bis(2-ethylhexyl) phthalate
Bromodichloromethane
Bromoform
2-sec-Butyl-4,6-dinitrophenol
Cadmium
Carbon tetrachloride
Chlordane
Chlorobenzene
Chloroethene (Vinyl chloride)
Chloroform
Chromium
Copper
Cyanide
Dibromochloromethane
Dibromochloropropane
1,2-Dibromoethane (Ethylene dibromide)
1,2-Dichlorobenzene
1,4-Dichlorobenzene
1,2-Dichloroethane
1,1-Dichloroethene
1,2-Dichloroethene
cis-1,2-Dichloroethene
trans-1,2-Dichloroethene
Dichloromethane (Methylene chloride)
2,4-Dichlorophenoxyacetic acid
1,2-Dichloropropane
Pndrin
Pentacholatile betac
Ethylbenzene
Fluoride
Gross alphab
Heptachlor
Heptachlor epoxide
Hexachlorobenzene
Hexachlorocyclopentadiene
Lead
Lindane
Mercury
Methoxychlor
Nickel

\begin{tabular}{|c|c|}
\hline Unit & Level \\
\hline$\mu \mathrm{g} / \mathrm{L}$ & 6 \\
\hline$\mu \mathrm{g} / \mathrm{L}$ & 50 \\
\hline Fibers/L & $7,000,000$ \\
\hline$\mu \mathrm{g} / \mathrm{L}$ & 2,000 \\
\hline$\mu g / L$ & 5 \\
\hline$\mu g / L$ & 0.2 \\
\hline$\mu g / L$ & 4 \\
\hline$\mu g / L$ & 6 \\
\hline$\mu \mathrm{g} / \mathrm{L}$ & $100^{a}$ \\
\hline$\mu \mathrm{g} / \mathrm{L}$ & $100^{a}$ \\
\hline$\mu \mathrm{g} / \mathrm{L}$ & 7 \\
\hline$\mu g / L$ & 5 \\
\hline$\mu g / L$ & 5 \\
\hline$\mu g / L$ & 2 \\
\hline$\mu \mathrm{g} / \mathrm{L}$ & 100 \\
\hline$\mu g / L$ & 2 \\
\hline$\mu g / L$ & $100^{a}$ \\
\hline$\mu g / L$ & 100 \\
\hline$\mu \mathrm{g} / \mathrm{L}$ & 1,300 \\
\hline$\mu \mathrm{g} / \mathrm{L}$ & 200 \\
\hline$\mu g / L$ & $100^{a}$ \\
\hline$\mu \mathrm{g} / \mathrm{L}$ & 0.2 \\
\hline$\mu g / L$ & 0.05 \\
\hline$\mu g / L$ & 600 \\
\hline$\mu \mathrm{g} / \mathrm{L}$ & 75 \\
\hline$\mu \mathrm{g} / \mathrm{L}$ & 5 \\
\hline$\mu \mathrm{g} / \mathrm{h}$ & 7 \\
\hline$\mu g / L$ & 50 \\
\hline$\mu \mathrm{g} / \mathrm{L}$ & 70 \\
\hline$\mu \mathrm{g} / \mathrm{L}$ & 100 \\
\hline$\mu \mathrm{g} / \mathrm{L}$ & 5 \\
\hline$\mu \mathrm{g} / \mathrm{L}$ & 70 \\
\hline$\mu \mathrm{g} / \mathrm{L}$ & 5 \\
\hline$\mu \mathrm{g} / \mathrm{L}$ & 2 \\
\hline$\mu \mathrm{g} / \mathrm{L}$ & 700 \\
\hline$\mu g / L$ & 4,000 \\
\hline $\mathrm{pCi} / \mathrm{L}$ & $1.5 E+01$ \\
\hline$\mu g / L$ & 0.4 \\
\hline$\mu g / L$ & 0.2 \\
\hline$\mu g / L$ & 1 \\
\hline$\mu g / L$ & 50 \\
\hline$\mu g / L$ & 50 \\
\hline$\mu \mathrm{g} / \mathrm{L}$ & 0.2 \\
\hline$\mu \mathrm{g} / \mathrm{L}$ & 2 \\
\hline$\mu \mathrm{g} / \mathrm{L}$ & 40 \\
\hline$\mu \mathrm{g} / \mathrm{L}$ & 100 \\
\hline$\mu \mathrm{g} / \mathrm{L}$ & 10,000 \\
\hline$\mu g / L$ & 10,000 \\
\hline$\mu \mathrm{g} / \mathrm{L}$ & 1,000 \\
\hline $\mathrm{pCi} / \mathrm{L}$ & $5 E+01$ \\
\hline$\mu g / L$ & 0.5 \\
\hline$\mu g / L$ & 1 \\
\hline$\mu g / L$ & 50 \\
\hline
\end{tabular}

\begin{tabular}{|c|c|}
\hline Status & Source \\
\hline Final & EPA, 1992b \\
\hline Final & EPA, 1992a \\
\hline Final & EPA, 1992a \\
\hline Final & EPA, 1992a \\
\hline Final & EPA, 1992a \\
\hline Final & EPA, 1992b \\
\hline Final & EPA, 1992b \\
\hline Final & EPA, $1992 b$ \\
\hline Final & EPA, 1992a \\
\hline Final & EPA, 1992a \\
\hline Final & EPA, $1992 b$ \\
\hline Final & EPA, 1992a \\
\hline Final & EPA, $1992 a$ \\
\hline Final & EPA, 1992a \\
\hline Final & EPA, 1992a \\
\hline Final & EPA, 1992a \\
\hline Final & EPA, 1992a \\
\hline Final & EPA, 1992a \\
\hline Final & EPA, 1993 \\
\hline Final & EPA, 1992b \\
\hline Final & EPA, 1992a \\
\hline Final & EPA, 1992a \\
\hline Final & EPA, 1992a \\
\hline Final & EPA, 1992a \\
\hline Final & EPA, 1992a \\
\hline Final & EPA, $1992 a$ \\
\hline Final & EPA, 1992a \\
\hline Final & EPA, $1992 b$ \\
\hline Final & EPA, 1992a \\
\hline Final & EPA, 1992a \\
\hline Final & EPA, 1992b \\
\hline Final & EPA, 1992a \\
\hline Final & EPA, 1992a \\
\hline Final & EPA, 1992b \\
\hline Final & EPA, 1992a \\
\hline Final & EPA, 1992a \\
\hline Final & EPA, 1992a \\
\hline Final & EPA, 1992a \\
\hline Final & EPA, 1992a \\
\hline Final & EPA, 1992b \\
\hline Final & EPA, 1992b \\
\hline Final & SCDHEC, 198 \\
\hline Final & EPA, 1992a \\
\hline Final & EPA, 1992a \\
\hline Final & EPA, 1992a \\
\hline Final & EPA, 1992b \\
\hline Final & EPA, 1992a \\
\hline Final & EPA, 1992a \\
\hline Final & EPA, 1992a \\
\hline Final & EPA, 1977 \\
\hline Final & EPA, 1992a \\
\hline Final & EPA, $1992 a$ \\
\hline Final & EPA, $1992 a$ \\
\hline
\end{tabular}




\begin{tabular}{|c|c|c|c|c|}
\hline Analyte & Unit & Level & $\underline{\text { Status }}$ & Source \\
\hline Strontium-89/90 ${ }^{e}$ & pCill & $8 E+00$ & Final & EPA, 1992a \\
\hline Strontium-90 & pCill & $8 E+00$ & Final & EPA, 1992a \\
\hline Styrene & $\mu \mathrm{g} / \mathrm{L}$ & 100 & Final & EPA, 1992a \\
\hline $2,3,7,8-T C D D$ & $\mu \mathrm{rg} / \mathrm{L}$ & 0.00003 & Final & EPA, 1992b \\
\hline Tetrachloroethylene & $\mu g / L$ & 5 & Final & EPA, 1992a \\
\hline Thallium & $\mu \mathrm{g} / \mathrm{L}$ & 2 & Final & EPA, 1992b \\
\hline Toluene & $\mu g / L$ & 1,000 & Final & EPA, 1992a \\
\hline Total trihalomethanes & $\mu g / L$ & 100 & Final & EPA, 1992a \\
\hline Toxaphene & $\mu g / L$ & 3 & Final & EPA, 1992 \\
\hline 2,4,5-TP (Silvex) & $\mu \mathrm{g} / \mathrm{L}$ & 50 & Final & EPA, 1992 \\
\hline 1,2,4-Trichlorobenzene & $\mu g / L$ & 70 & Final & EPA, 1992b \\
\hline 1,1,1-Trichloroethane & $\mu g / L$ & 200 & Final & EPA, 1992 \\
\hline 1,1,2-Trichloroethane & $\mu \mathrm{g} / \mathrm{L}$ & 5 & Final & EPA, 1992 \\
\hline Trichloroethylene & $\mu g / L$ & 5 & Final & EPA, 1992a \\
\hline Tritium & $\mathrm{pCi} / \mathrm{mL}$ & $2 E+01$ & Final & EPA, 1992a \\
\hline Xylenes & $\mu \mathrm{g} / \mathrm{L}$ & 10,000 & Final & EPA, 1992 \\
\hline
\end{tabular}

a This value is the drinking water standard for total trihalomethanes (the sum of bromodichloromethane, bromoform, chloroform, and dibromochloromethane).

b The standard given is for gross alpha including radium-226 but excluding radon and uranium.

c This is the screening level above which providers of public drinking water should perform analyses for specific man-made radionuclides. The standard for the total dose equivalent from all such radionuclides is 4 mrem per year.

d Analyses were conducted in 1994 for the following: РCB 1016, РСB 1221, РCB 1232, РСB 1242 , PCB 1248, PCB 1254, and PCB 1260.

e For double radionuclide analyses where each separate radionuclide has its own standard, the more stringent standard is used.

\section{References Cited}

EPA (U.S. Environmental Protection Agency), 1977. National Interim Primary Drinking Water Regulations, EPA-570/9-76-003. Washington, DC.

EPA (U.S. Environmental Protection Agency), 1992a. National Primary Drinking Water Regulations, Code of Federal Regulations, Title 40, Part 141, pp. 589-729. Washington, DC.

EPA (U.S. Environmental Protection Agency), 1992b. National Primary Drinking Water Regulations-Synthetic Organic Chemicals and Inorganic Chemicals; National Primary Drinking Water Regulations Implementation. Federal Register, July 17, 1992, pp. 31776-31849.

Washington, DC.

EPA (U.S. Environmental Protection Agency), 1993. National Primary Drinking Water Regulations, Code of Federal Regulations, Title 40, Part 141, pp. 592-732. Washington, DC.

SCDHEC (South Carolina Department of Health and Environmental Control), 1981. State Primary Drinking Water Regulations, R.61-58.5. Columbia, SC. 
WSRC-TR-95-0146-2

Unclassified

THIS PAGE LEFT BLANK INTENTIONALLY. 
WSRC-TR-95-0146-2

Unclassified

\section{Appendix B}

\section{Flagging Criteria}


WSRC-TR-95-0146-2

Unclassified

THIS PAGE LEFT BLANK INTENTIONALLY. 


\section{Flagging Criteria}

The Savannah River Site Environmental Protection Department/Environmental Monitoring Section (EPD/EMS) flagging criteria are as follows:

- Flag 2 criteria for constituents equal the Safe Drinking Water Act (SDWA) final Primary Drinking Water Standards (PDWS), the SDWA proposed PDWS, or the SDWA Secondary Drinking Water Standards (SDWS). If a constituent does not have a drinking water standard, the Flag 2 criterion equals 10 times the method detection limit (MDL) calculated as the 90th percentile detection limit obtained recently by one of the primary analytical laboratories.

- Flag 1 criteria for constituents equal one-half of the final PDWS, one-half the proposed PDWS, or one-half the SDWS. If a constituent does not have a drinking water standard, the Flag 1 criterion equals 5 times the MDL calculated as the 90th percentile detection limit obtained recently by one of the primary analytical laboratories.

- Flag 0 criteria are assigned to constituent levels below Flag 1 criteria, constituent levels below the sample detection limits, or constituents having no flagging criteria.

The following parameters are exceptions to the flagging rules:

- EPD/EMS sets flagging criteria for $\mathrm{pH}$ and specific conductance. No flags are set for alkalinity, calcium, carbonate, magnesium, potassium, silica, sodium, total dissolved solids, total phosphates (as P), and total phosphorus. Analyses for these parameters are conducted as part of the biennial comprehensive analyses or by special request.

- Aesthetic parameters such as color, corrosivity, Eh, odor, surfactants, and turbidity are not assigned flagging criteria but are analyzed by special request.

- Common laboratory contaminants and cleaners such as dichloromethane, ketones, phthalates, and toluene are not assigned flagging criteria unless they have PDWS. These constituents are analyzed by special request.

Analyte

Acenaphthene

Acenaphthylene

Acetone

Acetonitrile (Methyl cyanide)

Acetophenone

2-Acetylaminofluorene

Acrolein

Acrylonitrile

Actinium-228

Alachlor

Aldicarb $^{b}$

Aldicarb sulfone ${ }^{b}$

Aldicarb sulfoxide ${ }^{b}$

Aldrin

Alkalinity (as $\mathrm{CaCO}_{3}$ )

Allyl chloride

Aluminum

Aluminum, dissolved

Aluminum, total recoverable

\begin{tabular}{llll} 
Unit & Flag 1 & Flag 2 \\
\cline { 3 - 3 } & & \\
$\mu \mathrm{g} / \mathrm{L}$ & 50 & 100 \\
$\mu \mathrm{g} / \mathrm{L}$ & 50 & 100 \\
$\mu \mathrm{g} / \mathrm{L}$ & 500 & 1,000 \\
$\mu \mathrm{g} / \mathrm{L}$ & 500 & 1,000 \\
$\mu \mathrm{g} / \mathrm{L}$ & 50 & 100 \\
$\mu \mathrm{g} / \mathrm{L}$ & 50 & 100 \\
$\mu \mathrm{g} / \mathrm{L}$ & 100 & 200 \\
$\mu \mathrm{g} / \mathrm{L}$ & 100 & 200 \\
$\mathrm{pCi} / \mathrm{L}$ & $1.64 \mathrm{E}+03$ & $3.27 \mathrm{E}+03$ \\
$\mu \mathrm{g} / \mathrm{L}$ & 1 & 2 \\
$\mu \mathrm{g} / \mathrm{L}$ & 1.5 & 3 \\
$\mu \mathrm{g} / \mathrm{L}$ & 1 & 2 \\
$\mu \mathrm{g} / \mathrm{L}$ & 2 & 4 \\
$\mu \mathrm{g} / \mathrm{L}$ & 0.25 & 0.5 \\
& No flag & No flag \\
$\mu \mathrm{g} / \mathrm{L}$ & 250 & 500 \\
$\mu \mathrm{g} / \mathrm{L}$ & 25 & 50 \\
$\mu \mathrm{g} / \mathrm{L}$ & 25 & 50 \\
$\mu \mathrm{g} / \mathrm{L}$ & 25 & 50
\end{tabular}

Source $^{a}$

EPA Method 8270

EPA Method 8270

EPA Method 8240

EPA Method 8240

EPA Method 8270

EPA Method 8270

EPA Method 8240

EPA Method 8240

Proposed PDWS (EPA, 1991)

Final PDWS (EPA, 1993a)

Final PDWS (EPA, 1993a)

Final PDWS (EPA, 1993a)

Final PDWS (EPA, 1993a)

EPA Method 8080

Set by EPD/EMS

EPA Method 8240

SDWS (EPA, 1993b)

SDWS (EPA, 1993b)

SDWS (EPA, 1993b) 
Analyte

Americium-241

Americium-243

4-Aminobiphenyl

Ammonia

Ammonia nitrogen

Aniline

Anthracene

Antimony

Antimony, dissolved

Antimony, total recoverable

Antimony-125

Aramite

Arsenic

Arsenic, dissolved

Arsenic, total recoverable

Asbestos

Atrazine

Azobenzene

Barium

Barium, dissolved

Barium, total recoverable

Barium $-140^{c}$

Benzene

alpha-Benzene hexachloride

beta-Benzene hexachloride

delta-Benzene hexachloride

Benzidine

Benzo[a]anthracene

Benzo[b]fiuoranthene

Benzo[k]fluoranthene

Benzoic acid

Benzo[g, $h$,$] perylene$

Benzo[a]pyrene

1,4-Benzoquinone

Benzyl alcohol

Beryllium

Beryllium, dissolved

Beryllium, total recoverable

Beryllium-7

Bis(2-chloroethoxy) methane

Bis(2-chloroethyl) ether

Bis(2-chloroisopropyl) ether

Bis(chloromethyl) ether

Bis(2-ethylhexyl) phthalate

Bismuth-214

Boron

Boron, dissolved

Boron, total recoverable

Bromide

Bromodichloromethane

Bromoform

Bromomethane (Methyl bromide)

4-Bromophenyl phenyl ether

Butylbenzyl phthalate

2-sec-Butyl-4,6-dinitrophenol

\begin{tabular}{|c|c|c|c|}
\hline Unit & Flag 1 & Flag 2 & Source \\
\hline $\mathrm{pCi} / \mathrm{L}$ & 3.17E+00 & $6.34 E+00$ & Proposed PDWS (EPA, 1991) \\
\hline $\mathrm{pCi} / \mathrm{L}$ & $3.19 E+00$ & 6.37E+00 & Proposed PDWS (EPA, 1991) \\
\hline$\mu g / L$ & 50 & 100 & EPA Method 8270 \\
\hline$\mu \mathrm{g} / \mathrm{L}$ & 500 & 1,000 & APHA Method 417B \\
\hline$\mu g / L$ & 500 & 1,000 & EPA Method 350.1 \\
\hline$\mu \mathrm{g} / \mathrm{L}$ & 50 & 100 & EPA Method 8270 \\
\hline$\mu g / L$ & 50 & 100 & EPA Method 8270 \\
\hline$\mu g / L$ & 3 & 6 & Final PDWS (EPA, 1993a) \\
\hline$\mu g / L$ & 3 & 6 & Final PDWS (EPA, 1993a) \\
\hline$\mu g / L$ & 3 & 6 & Final PDWS (EPA, 1993a) \\
\hline pCi/L. & $1.5 E+02$ & $3 E+02$ & Interim Final PDWS (EPA, 1977) \\
\hline$\mu g / L$ & 50 & 100 & EPA Method 8270 \\
\hline$\mu g / L$ & 25 & 50 & Final PDWS (EPA, 1993a) \\
\hline$\mu g / L$ & 25 & 50 & Final PDWS (EPA, 1993a) \\
\hline$\mu \mathrm{g} / \mathrm{L}$ & 25 & 50 & Final PDWS (EPA, 1993a) \\
\hline Fibers/L & $3,500,000$ & $7,000,000$ & Final PDWS (EPA, 1993a) \\
\hline$\mu g / L$ & 1.5 & 3 & Final PDWS (EPA, 1993a) \\
\hline$\mu \mathrm{g} / \mathrm{L}$ & 50 & 100 & EPA Method 625 \\
\hline$\mu g / L$ & 1,000 & 2,000 & Final PDWS (EPA, 1993a) \\
\hline$\mu \mathrm{g} / \mathrm{L}$ & 1,000 & 2,000 & Final PDWS (EPA, 1993a) \\
\hline$\mu g / L$ & 1,000 & 2,000 & Final PDWS (EPA, 1993a) \\
\hline $\mathrm{pCi} / \mathrm{L}$ & $4.5 E+01$ & $9 E+01$ & Interim Final PDWS (EPA, 1977) \\
\hline$\mu \mathrm{g} / \mathrm{L}$ & 2.5 & 5 & Final PDWS (EPA, 1993a) \\
\hline$\mu g / L$ & 0.25 & 0.5 & EPA Method 8080 \\
\hline$\mu g / L$ & 0.25 & 0.5 & EPA Method 8080 \\
\hline$\mu g / L$ & 0.25 & 0.5 & EPA Method 8080 \\
\hline$\mu g / L$ & 250 & 500 & EPA Method 8270 \\
\hline$\mu g / L$ & 0.05 & 0.1 & Proposed PDWS (EPA, 1990) \\
\hline$\mu g / L$ & 0.1 & 0.2 & Proposed PDWS (EPA, 1990) \\
\hline$\mu g / L$ & 0.1 & 0.2 & Proposed PDWS (EPA, 1990) \\
\hline$\mu \mathrm{g} / \mathrm{L}$ & 250 & 500 & EPA Method 8270 \\
\hline$\mu g / L$ & 50 & 100 & EPA Method 8270 \\
\hline$\mu \mathrm{g} / \mathrm{L}$ & 0.1 & 0.2 & Final PDWS (EPA, 1993a) \\
\hline$\mu g / L$ & 50 & 100 & EPA Method 8270 \\
\hline$\mu g / L$ & 50 & 100 & EPA Method 8270 \\
\hline$\mu g / L$ & 2 & 4 & Final PDWS (EPA, 1993a) \\
\hline$\mu \mathrm{g} / \mathrm{L}$ & 2 & 4 & Final PDWS (EPA, 1993a) \\
\hline$\mu g / L$ & 2 & 4 & Final PDWS (EPA, 1993a) \\
\hline $\mathrm{pCi}$ & $3 E+03$ & $6 E+03$ & Interim Final PDWS (EPA, 1977) \\
\hline$\mu g / L$ & 50 & 100 & EPA Method 8270 \\
\hline$\mu \mathrm{g} / \mathrm{L}$ & 50 & 100 & EPA Method 8270 \\
\hline$\mu \mathrm{g} / \mathrm{L}$ & 50 & 100 & EPA Method 8270 \\
\hline$\mu \mathrm{g} / \mathrm{L}$ & 50 & 100 & EPA Method 8270 \\
\hline$\mu g / L$ & 3 & 6 & Final PDWS (EPA, 1993a) \\
\hline $\mathrm{pCi} / \mathrm{L}$ & $9.4 E+03$ & $1.89 E+04$ & Proposed PDWS (EPA, 1991) \\
\hline$\mu \mathrm{g} / \mathrm{L}$ & 150 & 300 & EPA Method 6010 \\
\hline$\mu g / L$ & 150 & 300 & EPA Method 6010 \\
\hline$\mu \mathrm{g} / \mathrm{L}$ & 150 & 300 & EPA Method 6010 \\
\hline$\mu \mathrm{g} / \mathrm{L}$ & 5,000 & 10,000 & EPA Method 300.0 \\
\hline$\mu g / L$ & 50 & 100 & Final PDWS (EPA, 1993a) \\
\hline$\mu \mathrm{g} / \mathrm{L}$ & 50 & 100 & Final PDWS (EPA, 1993a) \\
\hline$\mu g / L$ & 5 & 10 & EPA Method 8240 \\
\hline$\mu g / L$ & 50 & 100 & EPA Method 8270 \\
\hline & No flag & No flag & Set by EPD/EMS \\
\hline$\mu \mathrm{g} / \mathrm{L}$ & 3.5 & 7 & Final PDWS (EPA, 1993a) \\
\hline
\end{tabular}




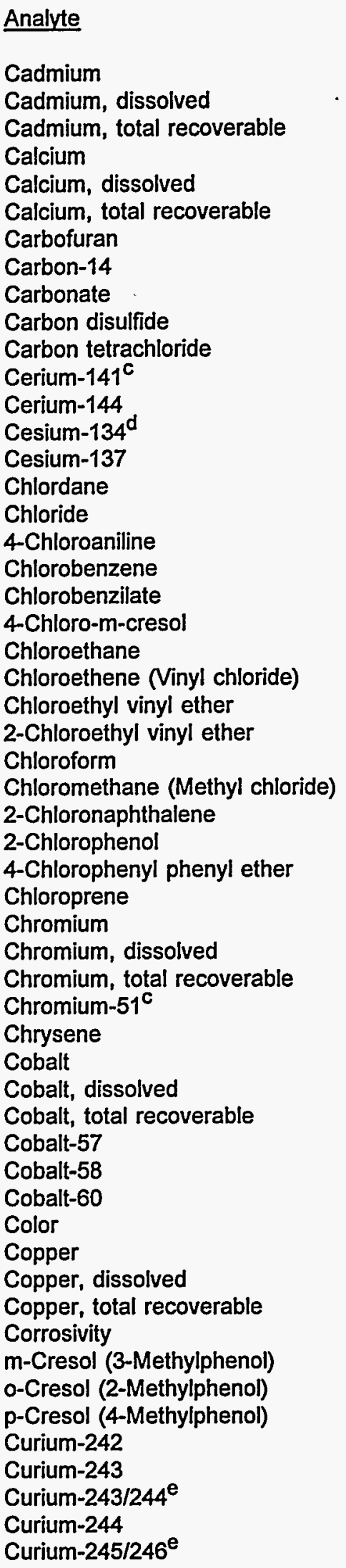

\begin{tabular}{|c|c|c|}
\hline Unit & Flag 1 & Flag 2 \\
\hline$\mu g / L$ & $2.5^{\circ}$ & 5 \\
\hline$\mu g / L$ & 2.5 & 5 \\
\hline$\mu g / L$ & 2.5 & 5 \\
\hline & No flag & No flag \\
\hline & No flag & No flag \\
\hline & No flag & No flag \\
\hline$\mu g / L$ & 20 & 40 \\
\hline $\mathrm{pCi} / \mathrm{L}$ & $1 E+03$ & $2 E+03$ \\
\hline & No flag & No flag \\
\hline$\mu g / L$ & 5 & 10 \\
\hline$\mu g / L$ & 2.5 & 5 \\
\hline $\mathrm{pCi} / \mathrm{L}$ & $1.5 \mathrm{E}+02$ & $3 E+02$ \\
\hline $\mathrm{pCi} / \mathrm{L}$ & $1.31 E+02$ & $2.61 E+02$ \\
\hline $\mathrm{pCi} / \mathrm{L}$ & 4.07E+01 & $8.13 E+01$ \\
\hline $\mathrm{pCi} / \mathrm{L}$ & $1 E+02$ & $2 E+02$ \\
\hline$\mu g / L$ & 1 & 2 \\
\hline$\mu g / L$ & 125,000 & 250,000 \\
\hline$\mu g / L$ & 50 & 100 \\
\hline$\mu g / L$ & 50 & 100 \\
\hline$\mu g / L$ & 50 & 100 \\
\hline$\mu \mathrm{g} / \mathrm{L}$ & 50 & 100 \\
\hline$\mu \mathrm{g} / \mathrm{L}$ & 5 & 10 \\
\hline$\mu g / L$ & 1 & 2 \\
\hline$\mu g / L$ & 5 & 10 \\
\hline$\mu g / L$ & 5 & 10 \\
\hline$\mu \mathrm{g} / \mathrm{L}$ & 50 & 100 \\
\hline$\mu g / L$ & 5 & 10 \\
\hline$\mu \mathrm{g} / \mathrm{L}$ & 50 & 100 \\
\hline$\mu g / L$ & 50 & 100 \\
\hline$\mu g / L$ & 50 & 100 \\
\hline$\mu \mathrm{g} / \mathrm{L}$ & 1,000 & 2,000 \\
\hline$\mu g / L$ & 50 & 100 \\
\hline$\mu g / L$ & 50 & 100 \\
\hline$\mu g / L$ & 50 & 100 \\
\hline $\mathrm{pCi} / \mathrm{L}$ & $3 E+03$ & $6 E+03$ \\
\hline$\mu g / L$ & 0.1 & 0.2 \\
\hline$\mu g / L$ & 20 & 40 \\
\hline$\mu g / L$ & 20 & 40 \\
\hline$\mu g / L$ & 20 & 40 \\
\hline pCi/L & 5E+02 & $1 E+03$ \\
\hline pCi/L & $4.5 E+03$ & $9 E+03$ \\
\hline $\mathrm{pCi} / \mathrm{L}$ & $5 E+01$ & $1 E+02$ \\
\hline & No flag & No flag \\
\hline$\mu g / L$ & 500 & 1,000 \\
\hline$\mu g / L$ & 500 & 1,000 \\
\hline$\mu g / L$ & 500 & 1,000 \\
\hline & No flag & No flag \\
\hline$\mu g / L$ & 50 & 100 \\
\hline$\mu g / L$ & 50 & 100 \\
\hline$\mu g / L$ & 50 & 100 \\
\hline $\mathrm{pCi} / \mathrm{L}$ & $6.65 E+01$ & $1.33 E+02$ \\
\hline $\mathrm{pCi} / \mathrm{L}$ & 4.15E+00 & $8.3 E+00$ \\
\hline pCi/L & 4.15E+00 & 8.3E+00 \\
\hline pCi/L & 4.92E+00 & 9.84E+00 \\
\hline pCi/L & 3.12E+00 & $6.23 E+00$ \\
\hline
\end{tabular}

\section{Source}

Final PDWS (EPA, 1993a)

Final PDWS (EPA, 1993a)

Final PDWS (EPA, 1993a)

Set by EPD/EMS

Set by EPD/EMS

Set by EPD/EMS

Final PDWS (EPA, 1993a)

Interim Final PDWS (EPA, 1977)

Set by EPD/EMS

EPA Method 8240

Final PDWS (EPA, 1993a)

Interim Final PDWS (EPA, 1977)

Proposed PDWS (EPA, 1991)

Proposed PDWS (EPA, 1991)

Interim Final PDWS (EPA, 1977)

Final PDWS (EPA, 1993a)

SDWS (EPA, 1993b)

EPA Method 8270

Final PDWS (EPA, 1993a)

EPA Method 8270

EPA Method 8270

EPA Method 8240

Final PDWS (EPA, 1993a)

EPA Method 8240

EPA Method 8240

Final PDWS (EPA, 1993a)

EPA Method 8240

EPA Method 8240

EPA Method 8270

EPA Method 8270

EPA Method 8240

Final PDWS (EPA, 1993a)

Final PDWS (EPA, 1993a)

Final PDWS (EPA, 1993a)

Interim Final PDWS (EPA, 1977)

Proposed PDWS (EPA, 1990)

EPA Method 6010

EPA Method 6010

EPA Method 6010

Interim Final PDWS (EPA, 1977)

Interim Final PDWS (EPA, 1977)

Interim Final PDWS (EPA, 1977)

Set by EPD/EMS

Final PDWS (SCDHEC, 1981)

Final PDWS (SCDHEC, 1981)

Final PDWS (SCDHEC, 1981)

Set by EPD/EMS

EPA Method 8270

EPA Method 8270

EPA Method 8270

Proposed PDWS (EPA, 1991)

Proposed PDWS (EPA, 1991)

Proposed PDWS (EPA, 1991)

Proposed PDWS (EPA, 1991)

Proposed PDWS (EPA, 1991) 


Analyte
Curium-246
Cyanide
Dalapon
p,p'-DDD
p,p'-DDE
p,p'-DDT
Diallate
Dibenz[a,h]anthracene
Dibenzofuran
Dibromochloromethane
1,2-Dibromo-3-chloropropane
1,2-Dibromoethane
Dibromomethane
(Methylene bromide)
Di-n-butyl phthalate
1,2-Dichlorobenzene
1,3-Dichlorobenzene
1,4-Dichlorobenzene
3,3'-Dichlorobenzidine
trans-1,4-Dichloro-2-butene
Dichlorodifluoromethane
1,1-Dichloroethane
1,2-Dichloroethane
1,1-Dichloroethylene
1,2-Dichloroethylene
cis-1,2-Dichloroethylene
trans-1,2-Dichloroethylene
Dichloromethane
2,4-Dichlorophenol
2,6-Dichlorophenol
2,4-Dichlorophenoxyacetic acid
1,2-Dichloropropane
cis-1,3-Dichloropropene
trans-1,3-Dichloropropene
Dieldrin
Di(2-ethylhexyl) adipate
Diethyl phthalate
Dimethoate
p-Dimethylaminoazobenzene
p-(Dimethylamino)ethylbenzene
7,12-Dimethylbenz[a]anthracene
3,3'-Dimethylbenzidine
a,a-Dimethylphenethylamine
2,4-Dimethyl phenol
Dimethyl phthalate
1,3-Dinitrobenzene
Diquat dibromide
Dissolved organic carbon

\begin{tabular}{|c|c|c|c|}
\hline Unit & Flag 1 & Flag 2 & Source \\
\hline $\mathrm{pCi} / \mathrm{L}$ & $3.14 E+00$ & $6.27 E+00$ & Proposed PDWS (EPA, 1991) \\
\hline$\mu g / L$ & 100 & 200 & Final PDWS (EPA, 1993a) \\
\hline$\mu g / L$ & 100 & 200 & Final PDWS (EPA, 1993a) \\
\hline$\mu g / L$ & 0.5 & 1 & EPA Method 8080 \\
\hline$\mu g / L$ & 0.5 & 1 & EPA Method 8080 \\
\hline$\mu g / L$ & 0.5 & 1 & EPA Method 8080 \\
\hline$\mu g / L$ & 50 & 100 & EPA Method 8270 \\
\hline$\mu g / L$ & 0.15 & 0.3 & Proposed PDWS (EPA, 1990) \\
\hline$\mu g / L$ & 50 & 100 & EPA Method 8270 \\
\hline$\mu g / L$ & 50 & 100 & Final PDWS (EPA, 1993a) \\
\hline$\mu g / L$ & 0.1 & 0.2 & Final PDWS (EPA, 1993a) \\
\hline$\mu g / L$ & 0.025 & 0.05 & Final PDWS (EPA, 1993a) \\
\hline \multirow[t]{2}{*}{$\mu g / L$} & 5 & 10 & EPA Method 8240 \\
\hline & No flag & No flag & Set by EPD/EMS \\
\hline$\mu g / L$ & 300 & 600 & Final PDWS (EPA, 1993a) \\
\hline$\mu \mathrm{g} / \mathrm{L}$ & 50 & 100 & EPA Method 8270 \\
\hline$\mu g / L$ & 37.5 & 75 & Final PDWS (EPA, 1993a) \\
\hline$\mu g / L$ & 50 & 100 & EPA Method 8270 \\
\hline$\mu g / L$ & 150 & 300 & EPA Method 8240 \\
\hline$\mu g / L$ & 5 & 10 & EPA Method 8240 \\
\hline$\mu g / L$ & 5 & 10 & EPA Method 8240 \\
\hline$\mu g / L$ & 2.5 & 5 & Final PDWS (EPA, 1993a) \\
\hline$\mu g / L$ & 3.5 & 7 & Final PDWS (EPA, 1993a) \\
\hline$\mu g / L$ & 25 & 50 & Final PDWS (EPA, 1993a) \\
\hline$\mu \mathrm{g} / \mathrm{L}$ & 35 & 70 & Final PDWS (EPA, 1993a) \\
\hline$\mu g / L$ & 50 & 100 & Final PDWS (EPA, 1993a) \\
\hline$\mu \mathrm{g} / \mathrm{L}$ & 2.5 & 5 & Final PDWS (EPA, 1993a) \\
\hline$\mu g / L$ & 50 & 100 & EPA Method 8270 \\
\hline$\mu g / L$ & 50 & 100 & EPA Method 8270 \\
\hline$\mu g / L$ & 35 & 70 & Final PDWS (EPA, 1993a) \\
\hline$\mu g / L$ & 2.5 & 5 & Final PDWS (EPA, 1993a) \\
\hline$\mu g / L$ & 5 & 10 & EPA Method 8240 \\
\hline$\mu \mathrm{g} / \mathrm{L}$ & 5 & 10 & EPA Method 8240 \\
\hline$\mu \mathrm{g} / \mathrm{L}$ & 2.5 & 5 & EPA Method 8080 \\
\hline$\mu \mathrm{g} / \mathrm{L}$ & 200 & 400 & Final PDWS (EPA, 1993a) \\
\hline & No flag & No flag & Set by EPD/EMS \\
\hline$\mu \mathrm{g} / \mathrm{L}$ & 50 & 100 & EPA Method 8270 \\
\hline$\mu g / L$ & 50 & 100 & EPA Method 8270 \\
\hline$\mu g / L$ & 50 & 100 & EPA Method 8270 \\
\hline$\mu g / L$ & 50 & 100 & EPA Method 8270 \\
\hline$\mu \mathrm{g} / \mathrm{L}$ & 50 & 100 & EPA Method 8270 \\
\hline$\mu \mathrm{g} / \mathrm{L}$ & 50 & 100 & EPA Method 8270 \\
\hline$\mu g / L$ & 50 & 100 & EPA Method 8270 \\
\hline & No flag & No flag & Set by EPD/EMS \\
\hline$\mu g / L$ & 50 & 100 & EPA Method 8270 \\
\hline$\mu \mathrm{g} / \mathrm{L}$ & 250 & 500 & EPA Method 8270 \\
\hline$\mu \mathrm{g} / \mathrm{L}$ & 50 & 100 & EPA Method 8270 \\
\hline$\mu \mathrm{g} / \mathrm{L}$ & 50 & 100 & EPA Method 8270 \\
\hline & No flag & No flag & Set by EPD/EMS \\
\hline$\mu g / L$ & 50 & 100 & EPA Method 8270 \\
\hline$\mu g / L$ & 50 & 100 & EPA Method 8270 \\
\hline$\mu g / L$ & 50 & 100 & EPA Method 8270 \\
\hline$\mu \mathrm{g} / \mathrm{L}$ & 10 & 20 & Final PDWS (EPA, 1993a) \\
\hline & 5,000 & 10,000 & EPA Method 9060 \\
\hline
\end{tabular}


Analyte

Disulfoton

Eh

Endosulfan I

Endosulfan II

Endosulfan sulfate

Endothall ${ }^{b}$

Endrin

Endrin aldehyde

Endrin ketone

Ethylbenzene

Ethyl methacrylate

Ethyl methanesulfonate

Europium-152

Europium-154

Europium-155

Famphur

Fluoranthene

Fluorene

Fluoride

Glyphosate $^{b}$

Gross alpha

Heptachlor

Heptachlor epoxide

Heptachlorodibenzo-p-dioxin isomers

1,2,3,4,6,7,8-HPCDD

Heptachlorodibenzo-p-furan

isomers

1,2,3,4,6,7,8-HPCDF

Hexachlorobenzene

Hexachlorobutadiene

Hexachlorocyclopentadiene

Hexachlorodibenzo-p-dioxin

isomers

$1,2,3,4,7,8-H \times C D D$

Hexachlorodibenzo-p-furan isomers

$1,2,3,4,7,8-H X C D F$

Hexachloroethane

Hexachlorophene

Hexachloropropene

2-Hexanone

Indeno[1,2,3-c, c] pyrene

lodine

lodine-129

lodine-131 ${ }^{c}$

lodomethane (Methyl iodide)

Iron

Iron, dissolved

Iron, total recoverable

Iron- $55^{\mathrm{C}}$

Iron-59

Isobutyl alcohol

Isodrin

Isophorone

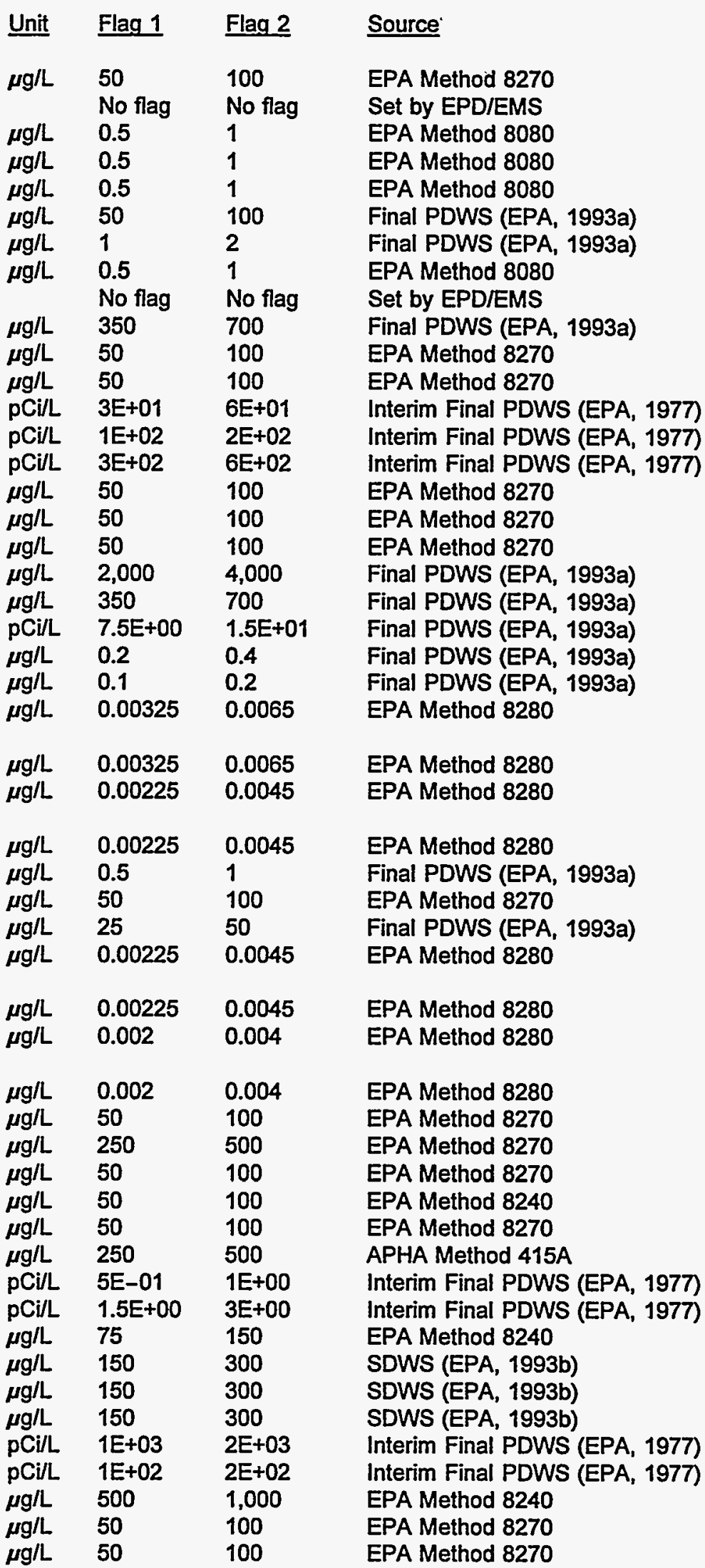


Analyte

Isosafrole

Kepone

Lanthanum-140 ${ }^{c}$

Lead

Lead, dissolved

Lead, total recoverable

Lead-212

Lindane

Lithium

Lithium, dissolved

Lithium, total recoverable

Magnesium

Magnesium, dissolved

Magnesium, total recoverable

Manganese

Manganese, dissolved

Manganese, total recoverable

Manganese-54

Mercury

Mercury, dissolved

Mercury, total recoverable

Methacrylonitrile

Methapyrilene

Methoxychlor

3-Methylcholanthrene

2-Methyl-4,6-dinitrophenol

Methyl ethyl ketone

Methyl isobutyl ketone

Methyl methacrylate

Methyl methanesulfonate

2-Methylnaphthalene

Molybdenum

Molybdenum, dissolved

Molybdenum, total recoverable

Naphthalene

1,4-Naphthoquinone

1-Naphthylamine

2-Naphthylamine

Neptunium-237

Nickel

Nickel, dissolved

Nickel, total recoverable

Nickel- $59^{\mathrm{C}}$

Nickel-63 ${ }^{\mathrm{C}}$

Niobium-95

Nitrate as nitrogen

Nitrate-nitrite as nitrogen

Nitrite as nitrogen

m-Nitroaniline

o-Nitroaniline

p-Nitroaniline

Nitrobenzene

Nitrogen by Kjeldahi method

2-Nitrophenol

4-Nitrophenol

\begin{tabular}{|c|c|c|}
\hline Unit & Flag 1 & Flag 2 \\
\hline$\mu g / L$ & 50 & 100 \\
\hline$\mu \mathrm{g} / \mathrm{L}$ & 50 & 100 \\
\hline $\mathrm{pCi} / \mathrm{L}$ & $3 E+01$ & $6 E+01$ \\
\hline$\mu \mathrm{g} / \mathrm{L}$ & 25 & 50 \\
\hline$\mu \mathrm{g} / \mathrm{L}$ & 25 & 50 \\
\hline$\mu \mathrm{g} / \mathrm{L}$ & 25 & 50 \\
\hline $\mathrm{pCi} / \mathrm{L}$ & $6.2 E+01$ & $1.23 E+02$ \\
\hline$\mu g / L$ & 0.1 & 0.2 \\
\hline$\mu g / L$ & 25 & 50 \\
\hline$\mu g / L$ & 25 & 50 \\
\hline$\mu \mathrm{g} / \mathrm{L}$ & 25 & 50 \\
\hline & No flag & No flag \\
\hline & $\begin{array}{l}\text { No flag } \\
\text { No flag }\end{array}$ & $\begin{array}{l}\text { No flag } \\
\text { No flag }\end{array}$ \\
\hline & 25 & 50 \\
\hline & 25 & 50 \\
\hline & 25 & 50 \\
\hline $\mathrm{pCi} / \mathrm{L}$ & $1.5 \mathrm{E}+02$ & $3 E+02$ \\
\hline & 1 & 2 \\
\hline & 1 & 2 \\
\hline$\mu g / L$ & 1 & 2 \\
\hline$\mu \mathrm{g} / \mathrm{L}$ & 250 & 500 \\
\hline$\mu \mathrm{g} / \mathrm{L}$ & 50 & 100 \\
\hline$\mu g / L$ & 20 & 40 \\
\hline$\mu g / L$ & 50 & 100 \\
\hline$\mu \mathrm{g} / \mathrm{L}$ & 250 & 500 \\
\hline & No flag & No flag \\
\hline & No flag & No flag \\
\hline$\mu \mathrm{s}$ & 50 & 100 \\
\hline$\mu g / L$ & 50 & 100 \\
\hline g/L & 50 & 100 \\
\hline$\mu \mathrm{g} / \mathrm{L}$ & 250 & 500 \\
\hline$\mu \mathrm{g} / \mathrm{L}$ & 250 & 500 \\
\hline / $/ \mathrm{L}$ & 250 & 500 \\
\hline$\mu \mathrm{g} / \mathrm{L}$ & 50 & 100 \\
\hline$\mu \mathrm{g} / \mathrm{L}$ & 50 & 100 \\
\hline g/L & 50 & 100 \\
\hline$\mu \mathrm{g} / \mathrm{L}$ & 50 & 100 \\
\hline $\mathrm{pCi} / \mathrm{L}$ & $3.53 E+00$ & $7.06 E+00$ \\
\hline$\mu g / L$ & 50 & 100 \\
\hline$\mu g / L$ & 50 & 100 \\
\hline$\mu g / L$ & 50 & 100 \\
\hline $\mathrm{pCi} / \mathrm{L}$ & $1.5 \mathrm{E}+02$ & $3 E+02$ \\
\hline pCi/L & $2.5 \mathrm{E}+01$ & $5 E+01$ \\
\hline $\mathrm{pCi} / \mathrm{L}$ & $1.5 \mathrm{E}+02$ & 3.E+02 \\
\hline$\mu \mathrm{g} / \mathrm{L}$ & 5,000 & 10,000 \\
\hline$\mu g / L$ & 5,000 & 10,000 \\
\hline$\mu g / L$ & 500 & 1,000 \\
\hline & 50 & 100 \\
\hline$\mu \mathrm{g} / \mathrm{L}$ & 50 & 100 \\
\hline$\mu \mathrm{g} / \mathrm{L}$ & 50 & 100 \\
\hline$\mu g / L$ & 50 & 100 \\
\hline & 500 & 1,000 \\
\hline$\mu \mathrm{g} / \mathrm{L}$ & 50 & 100 \\
\hline & 50 & 100 \\
\hline
\end{tabular}

Source

EPA Method 8270

EPA Method 8270

Interim Final PDWS (EPA, 1977)

Final PDWS (SCDHEC, 1981)

Final PDWS (SCDHEC, 1981)

Final PDWS (SCDHEC, 1981)

Proposed PDWS (EPA, 1991)

Final PDWS (EPA, 1993a)

EPA Method 6010

EPA Method 6010

EPA Method 6010

Set by EPD/EMS

Set by EPD/EMS

Set by EPD/EMS

SDWS (EPA, 1993b)

SDWS (EPA, 1993b)

SDWS (EPA, 1993b)

Interim Final PDWS (EPA, 1977)

Final PDWS (EPA, 1993a)

Final PDWS (EPA, 1993a)

Final PDWS (EPA, 1993a)

EPA Method 8240

EPA Method 8270

Final PDWS (EPA, 1993a)

EPA Method 8270

EPA Method 8270

Set by EPD/EMS

Set by EPD/EMS

EPA Method 8270

EPA Method 8270

EPA Method 8270

EPA Method 6010

EPA Method 6010

EPA Method 6010

EPA Method 8270

EPA Method 8270

EPA Method 8270

EPA Method 8270

Proposed PDWS (EPA, 1991)

Final PDWS (EPA, 1993a)

Final PDWS (EPA, 1993a)

Final PDWS (EPA, 1993a)

Interim Final PDWS (EPA, 1977)

Interim Final PDWS (EPA, 1977)

Interim Final PDWS (EPA, 1977)

Final PDWS (EPA, 1993a)

Final PDWS (EPA, 1993a)

Final PDWS (EPA, 1993a)

EPA Method 8270

EPA Method 8270

EPA Method 8270

EPA Method 8270

EPA Method 351.2

EPA Method 8270

EPA Method 8270 


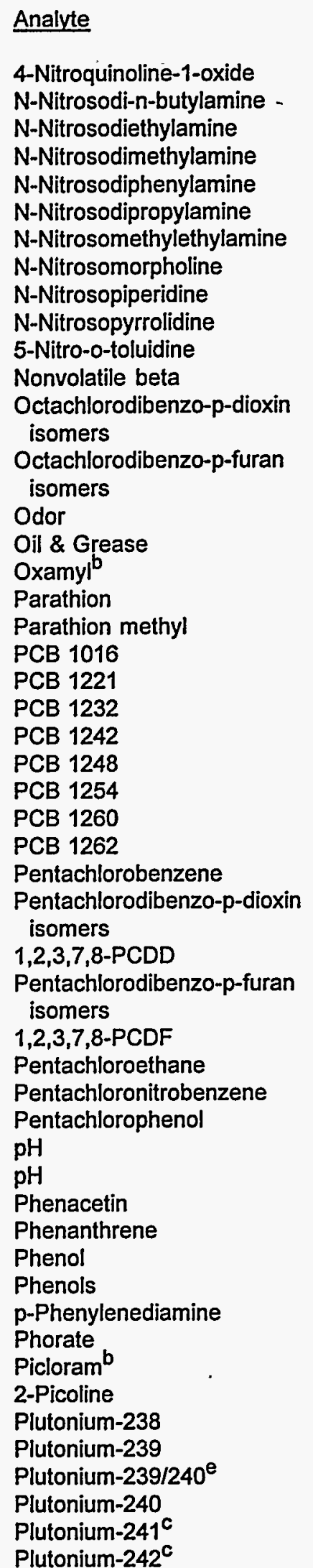

\begin{tabular}{|c|c|c|c|}
\hline Unit & Flag 1 & Flag 2 & Source \\
\hline$\mu g / L$ & 50 & 100 & EPA Method 8270 \\
\hline$\mu \mathrm{g} / \mathrm{L}$ & 50 & 100 & EPA Method 8270 \\
\hline$\mu g / L$ & 50 & 100 & EPA Method 8270 \\
\hline$\mu \mathrm{g} / \mathrm{L}$ & 50 & 100 & EPA Method 8270 \\
\hline$\mu \mathrm{g} / \mathrm{L}$ & 50 & 100 & EPA Method 8270 \\
\hline$\mu g / L$ & 50 & 100 & EPA Method 8270 \\
\hline$\mu g / L$ & 50 & 100 & EPA Method 8270 \\
\hline$\mu g / L$ & 50 & 100 & EPA Method 8270 \\
\hline$\mu \mathrm{g} / \mathrm{L}$ & 50 & 100 & EPA Method 8270 \\
\hline$\mu g / L$ & 50 & 100 & EPA Method 8270 \\
\hline$\mu g / L$ & 50 & 100 & EPA Method 8270 \\
\hline $\mathrm{pCi} / \mathrm{L}$ & 2.5E+01 & $5 E+01$ & Interim Final PDWS (EPA, 1977) \\
\hline$\mu g / L$ & 0.005 & 0.01 & EPA Method 8280 \\
\hline$\mu g / L$ & 0.005 & 0.01 & EPA Method 8280 \\
\hline & No flag & No flag & Set by EPD/EMS \\
\hline$\mu g / L$ & 5,000 & 10,000 & EPA Method 413.1 \\
\hline$\mu g / L$ & 100 & 200 & Final PDWS (EPA, 1993a) \\
\hline$\mu g / L$ & 0.25 & 0.5 & EPA Method 8080 \\
\hline$\mu g / L$ & 0.25 & 0.5 & EPA Method 8080 \\
\hline$\mu g / L$ & 0.25 & 0.5 & Final PDWS (EPA, 1993a) \\
\hline$\mu g / L$ & 0.25 & 0.5 & Final PDWS (EPA, 1993a) \\
\hline$\mu g / L$ & 0.25 & 0.5 & Final PDWS (EPA, 1993a) \\
\hline$\mu g / L$ & 0.25 & 0.5 & Final PDWS (EPA, 1993a) \\
\hline$\mu g / L$ & 0.25 & 0.5 & Final PDWS (EPA, 1993a) \\
\hline$\mu g / L$ & 0.25 & 0.5 & Final PDWS (EPA, 1993a) \\
\hline$\mu g / L$ & 0.25 & 0.5 & Final PDWS (EPA, 1993a) \\
\hline$\mu \mathrm{g} / \mathrm{L}$ & 0.25 & 0.5 & Final PDWS (EPA, 1993a) \\
\hline$\mu g / L$ & 50 & 100 & EPA Method 8270 \\
\hline$\mu \mathrm{g} / \mathrm{L}$ & 0.00275 & 0.0055 & EPA Method 8280 \\
\hline$\mu g / L$ & 0.00275 & 0.0055 & EPA Method 8280 \\
\hline$\mu g / L$ & 0.00275 & 0.0055 & EPA Method 8280 \\
\hline$\mu g / L$ & 0.00275 & 0.0055 & EPA Method 8280 \\
\hline$\mu g / L$ & 50 & 100 & EPA Method 8270 \\
\hline$\mu g / L$ & 50 & 100 & EPA Method 8270 \\
\hline$\mu \mathrm{g} / \mathrm{L}$ & 0.5 & 1 & Final PDWS (EPA, 1993a) \\
\hline $\mathrm{pH}$ & 8 & 10 & Set by EPD/EMS \\
\hline $\mathrm{pH}$ & 4 & 3 & Set by EPD/EMS \\
\hline$\mu \mathrm{g} / \mathrm{L}$ & 50 & 100 & EPA Method 8270 \\
\hline$\mu g / L$ & 50 & 100 & EPA Method 8270 \\
\hline$\mu \mathrm{g} / \mathrm{L}$ & 50 & 100 & EPA Method 8270 \\
\hline$\mu g / L$ & 25 & 50 & EPA Method 420.1 \\
\hline$\mu \mathrm{g} / \mathrm{L}$ & 50 & 100 & EPA Method 8270 \\
\hline$\mu \mathrm{g} / \mathrm{L}$ & 0.5 & 1 & EPA Method 8080 \\
\hline$\mu g / L$ & 250 & 500 & Final PDWS (EPA, 1993a) \\
\hline$\mu g / L$ & 50 & 100 & EPA Method 8270 \\
\hline $\mathrm{pCi} / \mathrm{L}$ & $3.51 E+00$ & 7.02E+00 & Proposed PDWS (EPA, 1991) \\
\hline $\mathrm{pCi} / \mathrm{L}$ & 3.11E+01 & $6.21 E+01$ & Proposed PDWS (EPA, 1991) \\
\hline $\mathrm{pCi} / \mathrm{L}$ & 3.11E+01 & $6.21 \mathrm{E}+01$ & Proposed PDWS (EPA, 1991) \\
\hline pCi/L & 3.11E+01 & $6.22 E+01$ & Proposed PDWS (EPA, 1991) \\
\hline $\mathrm{pCi} / \mathrm{L}$ & 3.13E+01 & $6.26 \mathrm{E}+01$ & Proposed PDWS (EPA, 1991) \\
\hline pCi/L & $3.27 E+01$ & $6.54 E+01$ & Proposed PDWS (EPA, 1991) \\
\hline
\end{tabular}


Analyte

Potassium

Potassium, dissolved

Potassium, total recoverable

Potassium-40

Promethium-144

Promethium-146

Promethium-147

Pronamid

Propionitrile

Pyrene

Pyridine

Radium (alpha-emitting) ${ }^{\ddagger}$

Radium-226

Radium-228

Radon-222

Ruthenium-103c

Ruthenium-106

Safrole

Selenium

Selenium, dissolved

Selenium, total recoverable

Silica

Silica, dissolved

Silica, total recoverable

Silver

Silver, dissolved

Silver, total recoverable

Simazine ${ }^{b}$

Sodium

Sodium, dissolved

Sodium, total recoverable

Sodium-22

Specific conductance

Strontium-89

Strontium-89/90 ${ }^{\mathrm{e}}$

Strontium-90

Styrene

Sulfate

Sulfide

Sulfotepp

Surfactants

2,3,7,8-TCDD

$2,3,7,8-T C D F$

Technetium-99

1,2,4,5-Tetrachlorobenzene

Tetrachlorodibenzo-p-dioxin isomers

Tetrachlorodibenzo-p-furan isomers

1,1,1,2-Tetrachloroethane

1,1,2,2-Tetrachloroethane

Tetrachloroethylene

2,3,4,6-Tetrachlorophenol

Thallium

Thallium, dissolved

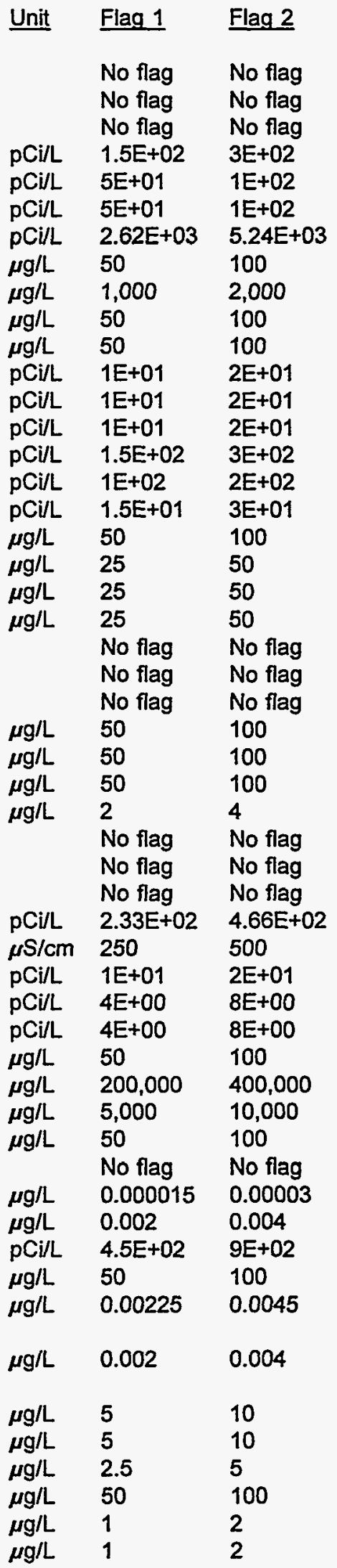

Source

Set by EPD/EMS

Set by EPD/EMS

Set by EPD/EMS

Proposed PDWS (EPA, 1986)

EPA Method 901.1

EPA Method 901.1

Proposed PDWS (EPA, 1991)

EPA Method 8270

EPA Method 8240

EPA Method 8270

EPA Method 8270

Proposed PDWS (EPA, 1991)

Proposed PDWS (EPA, 1991)

Proposed PDWS (EPA, 1991)

Proposed PDWS (EPA, 1991)

Interim Final PDWS (EPA, 1977)

Interim Final PDWS (EPA, 1977)

EPA Method 8270

Final PDWS (EPA, 1993a)

Final PDWS (EPA, 1993a)

Final PDWS (EPA, 1993a)

Set by EPD/EMS

Set by EPD/EMS

Set by EPD/EMS

SDWS (EPA, 1993b)

SDWS (EPA, 1993b)

SDWS (EPA, 1993b)

Final PDWS (EPA, 1993a)

Set by EPD/EMS

Set by EPD/EMS

Set by EPD/EMS

Proposed PDWS (EPA, 1991)

Set by EPD/EMS

Interim Final PDWS (EPA, 1977)

Final PDWS (EPA, 1993a)

Final PDWS (EPA, 1993a)

Final PDWS (EPA, 1993a)

Proposed PDWS (EPA, 1990)

EPA Method 9030

EPA Method 8270

Set by EPD/EMS

Final PDWS (EPA, 1993a)

EPA Method 8280

Interim Final PDWS (EPA, 1977)

EPA Method 8270

EPA Method 8280

EPA Method 8280

EPA Method 8240

EPA Method 8240

Final PDWS (EPA, 1993a)

EPA Method 8270

Final PDWS (EPA, 1993a)

Final PDWS (EPA, 1993a) 
Analyte

Thallium, total recoverable

Thionazin

Thorium-228

Thorium-230

Thorium-232

Thorium-234

Tin

Tin, dissolved

Tin, total recoverable

Tin- $113^{c}$

Toluene

o-Toluidine

Total carbon

Total coliform

Total dissolved solids

Total hydrocarbons

Total inorganic carbon

Total organic carbon

Total organic halogens

Total organic nitrogen

Total petroleum hydrocarbons

Total phosphates (as P)

Total phosphorus

Toxaphene

2,4,5-TP (Silvex)

Tributyl phosphate

1,2,4-Trichlorobenzene

1,1,1-Trichloroethane

1,1,2-Trichloroethane

Trichloroethylene

Trichlorofluoromethane

2,4,5-Trichlorophenol

2,4,6-Trichlorophenol

2,4,5-Trichlorophenoxyacetic acid

1,2,3-Trichloropropane

$0,0,0$-Triethyl phosphorothioate

1,3,5-Trinitrobenzene

Tritium

Turbidity 9

Uranium

Uranium, dissolved

Uranium, total recoverable

Uranium alpha activity

Uranium-233/234 ${ }^{\mathrm{e}}$

Uranium-234

Uranium-235

Uranium-238

Vanadium

Vanadium, dissolved

Vanadium, total recoverable

Vinyl acetate

Xylenes

Yttrium-88

Zinc

Zinc, dissolved

\begin{tabular}{|c|c|c|}
\hline Jnit & Flag 1 & Flag 2 \\
\hline$\mu \mathrm{g} / \mathrm{L}$ & 1 & 2 \\
\hline$\mu g / L$ & 50 & 100 \\
\hline & $6.25 \mathrm{E}+01$ & $1.25 \mathrm{E}+02$ \\
\hline $\mathrm{Ci} / \mathrm{L}$ & $3.96 \mathrm{E}+01$ & $7.92 E+01$ \\
\hline $\mathrm{pCi} / \mathrm{L}$ & $4.4 E+01$ & $8.8 \mathrm{E}+01$ \\
\hline pCill & $2 E+02$ & $4.01 E+02$ \\
\hline$\mu \mathrm{g} / \mathrm{L}$ & 10 & 20 \\
\hline$\mu \mathrm{g} / \mathrm{L}$ & 10 & 20 \\
\hline$\mu g / L$ & 10 & 20 \\
\hline pCi/L & $1.5 E+02$ & $3 E+02$ \\
\hline$\mu \mathrm{g} / \mathrm{L}$ & 500 & 1,000 \\
\hline$g / L$ & 50 & 100 \\
\hline$g / L$ & 5,000 & 10,000 \\
\hline & 0 & 0 \\
\hline & No flag & No flag \\
\hline$g / L$ & 5,000 & 10,000 \\
\hline$\mu \mathrm{g} / \mathrm{L}$ & 5,000 & 10,000 \\
\hline$\mu g / L$ & 5,000 & 10,000 \\
\hline$g / L$ & 25 & 50 \\
\hline$\mu \mathrm{g} / \mathrm{L}$ & 500 & 1,000 \\
\hline$\mu \mathrm{g} / \mathrm{L}$ & 5,000 & 10,000 \\
\hline & No flag & No flag \\
\hline & No flag & No flag \\
\hline$g / L$ & 1.5 & 3 \\
\hline$\mu \mathrm{g} / \mathrm{L}$ & 25 & 50 \\
\hline$\mu \mathrm{g} / \mathrm{L}$ & 50 & 100 \\
\hline$\mu g / L$ & 35 & 70 \\
\hline$\mu g / L$ & 100 & 200 \\
\hline$\mu g / L$ & 2.5 & 5 \\
\hline$\mu \mathrm{g} / \mathrm{L}$ & 2.5 & 5 \\
\hline$\mu g / L$ & 5 & 10 \\
\hline$\mu g / L$ & 50 & 100 \\
\hline$\mu g / L$ & 50 & 100 \\
\hline$\mu g / L$ & 2.5 & 5 \\
\hline$\mu \mathrm{g} / \mathrm{L}$ & 5 & 10 \\
\hline$\mu g / L$ & 50 & 100 \\
\hline$\mu g / L$ & 50 & 100 \\
\hline pCi/mL & $\begin{array}{l}1 E+01 \\
\text { No flag }\end{array}$ & $\begin{array}{l}2 E+01 \\
\text { No flaq }\end{array}$ \\
\hline$\mu g / L$ & 10 & 20 \\
\hline$\mu \mathrm{g} / \mathrm{L}$ & 10 & 20 \\
\hline g/L & 10 & 20 \\
\hline Cill & $1.5 E+01$ & $3 E+01$ \\
\hline pCill & $6.9 E+00$ & $1.38 E+01$ \\
\hline $\mathrm{pCi} / \mathrm{L}$ & $6.95 \mathrm{E}+00$ & $1.39 E+01$ \\
\hline pCi/L & $7.25 E+00$ & $1.45 E+01$ \\
\hline pCill & $7.3 E+00$ & $1.46 E+01$ \\
\hline & 40 & 80 \\
\hline & 40 & 80 \\
\hline$\mu \mathrm{g} / \mathrm{L}$ & 40 & 80 \\
\hline$\mu g / L$ & 5 & 10 \\
\hline (1) & 5,000 & 10,000 \\
\hline iil & $5 E+01$ & $1 E+02$ \\
\hline & 2,500 & 5,000 \\
\hline & 2,500 & 5,000 \\
\hline
\end{tabular}

Source

Final PDWS (EPA, 1993a)

EPA Method 8270

Proposed PDWS (EPA, 1991)

Proposed PDWS (EPA, 1991)

Proposed PDWS (EPA, 1991)

Proposed PDWS (EPA, 1991)

EPA Method 282.2

EPA Method 282.2

EPA Method 282.2

Interim Final PDWS (EPA, 1977)

Final PDWS (EPA, 1993a)

EPA Method 8270

EPA Method 9060

Final PDWS (EPA, 1993a)

Set by EPD/EMS

EPA Method 418.1

EPA Method 9060

EPA Method 9060

EPA Method 9020

APHA Method 420

EPA Method 418.1

Set by EPD/EMS

Set by EPD/EMS

Final PDWS (EPA, 1993a)

Final PDWS (EPA, 1993a)

EPA Method 8270

Final PDWS (EPA, 1993a)

Final PDWS (EPA, 1993a)

Final PDWS (EPA, 1993a)

Final PDWS (EPA, 1993a)

EPA Method 8240

EPA Method 8270

EPA Method 8270

EPA Method 8150

EPA Method 8240

EPA Method 8270

EPA Method 8270

Final PDWS (EPA, 1993a)

Set by EPD/EMS

Proposed PDWS (EPA, 1991)

Proposed PDWS (EPA, 1991)

Proposed PDWS (EPA, 1991)

Proposed PDWS (EPA, 1991)

Proposed PDWS (EPA, 1991)

Proposed PDWS (EPA, 1991)

Proposed PDWS (EPA, 1991)

Proposed PDWS (EPA, 1991)

EPA Method 6010

EPA Method 6010

EPA Method 6010

EPA Method 8240

Final PDWS (EPA, 1993a)

EPA Method 901.1

SDWS (EPA, 1993b)

SDWS (EPA, 1993b) 
Analyte

Zinc, total recoverable

Zinc-65

Zirconium-95 ${ }^{\mathrm{c}}$

Zirconium/Niobium-95 ${ }^{\mathrm{C}}$

\begin{tabular}{|c|c|c|}
\hline Unit & Flag 1 & Flag 2 \\
\hline$\mu \mathrm{g} / \mathrm{L}$ & 2,500 & 5,000 \\
\hline $\mathrm{pCi} / \mathrm{L}$ & $1.5 E+02$ & $3 E+02$ \\
\hline $\mathrm{pCi} / \mathrm{L}$ & $1 E+02$ & $2 E+02$ \\
\hline $\mathrm{pCi} / \mathrm{L}$ & $1 E+02$ & $2 E+02$ \\
\hline
\end{tabular}

Source

SDWS (EPA, 1993b)

Interim Final PDWS (EPA, 1977)

Interim Final PDWS (EPA, 1977)

Interim Final PDWS (EPA, 1977)

a References for methods are in Appendix E; references for dated sources are at the end of this appendix.

b EMS is currently unable to perform this analysis.

c EMS discontinued monitoring this radionuclide because it is inappropriate for the SRS Groundwater Monitoring Program.

d EPD/EMS set this flagging criterion using the 1991 proposed PDWS because the final PDWS in 1977 may have been in error.

e For double radionuclide analyses where each separate radionuclide has its own standard, the more stringent standard is used.

$f$ The applied standard is for radium-226.

$g$ The primary maximum contaminant level range for turbidity is 1-5 NTU, which is inappropriate for the SRS Groundwater Monitoring Program.

\section{References Cited}

EPA (U.S. Environmental Protection Agency), 1977. National Interim Primary Drinking Water Regulations, EPA-570/9-76-003. Washington, DC.

EPA (U.S. Environmental Protection Agency), 1986. Water Pollution Control; National Primary Drinking Water Regulations, Radionuclides (Proposed). Federal Register, September 30, 1986, pp. 34835-34862. Washington, DC.

EPA (U.S. Environmental Protection Agency), 1990. National Primary and Secondary Drinking Water Regulations; Synthetic Organic Chemicals and Inorganic Chemicals (Proposed Rule). Federal Register, July 25, 1990, pp. 30369-30448. Washington, DC.

EPA (U.S. Environmental Protection Agency), 1991. National Primary Drinking Water Regulations; Radionuclides; Proposed Rule. Federal Register, July 18, 1991, pp. 33052-33127. Washington, DC.

EPA (U.S. Environmental Protection Agency), 1993a. National Primary Drinking Water Regulations. Code of Federal Regulations, Title 40, Part 141, pp. 592-732. Washington, DC.

EPA (U.S. Environmental Protection Agency), 1993b. National Secondary Drinking Water Regulations. Code of Federal Regulations, Title 40, Part 143, pp. 774-777. Washington, DC.

SCDHEC (South Carolina Department of Health and Environmental Control), 1981. State Primary Drinking Water Regulations, R.61-58.5. Columbia, SC. 
WSRC-TR-95-0146-2

Unclassified

\section{Appendix C}

Figures 
WSRC-TR-95-0146-2

Unclassified

THIS PAGE LEFT BLANK INTENTIONALLY. 


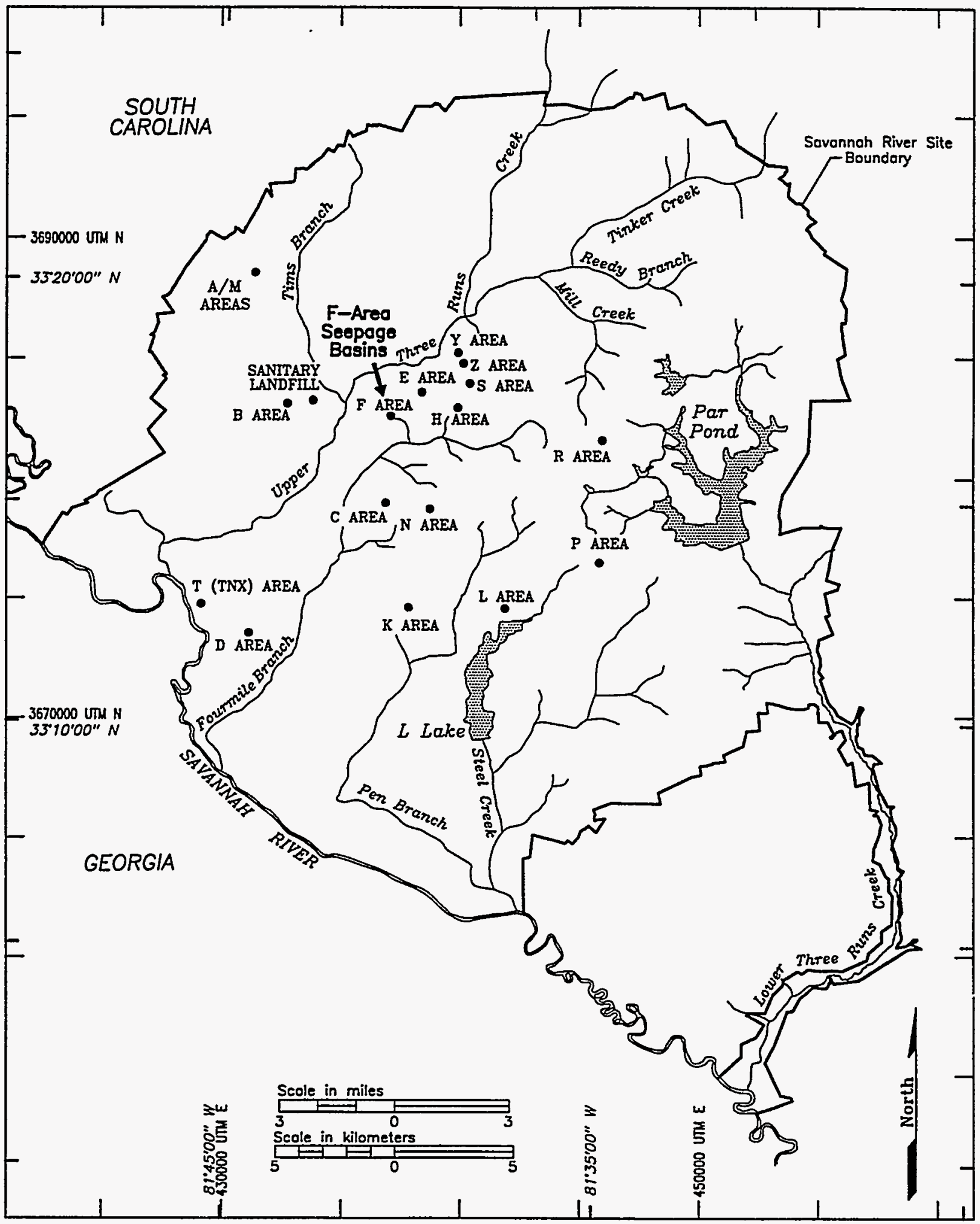

Figure 1. Location of the F-Area Seepage Basins at the Savannah River Site 


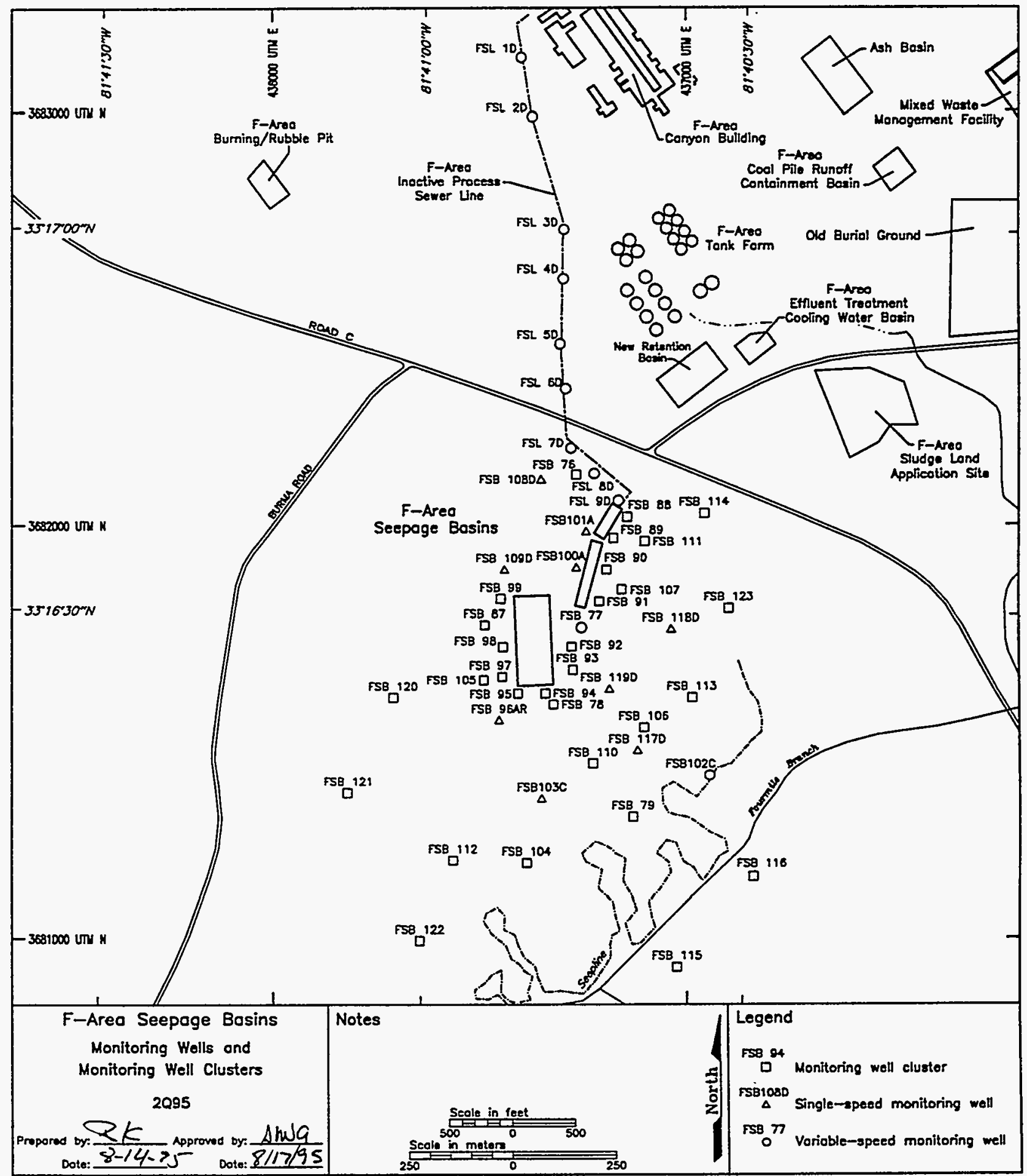

Figure 2. Location of the Groundwater Monitoring Wells at the F-Area Seepage Basins 


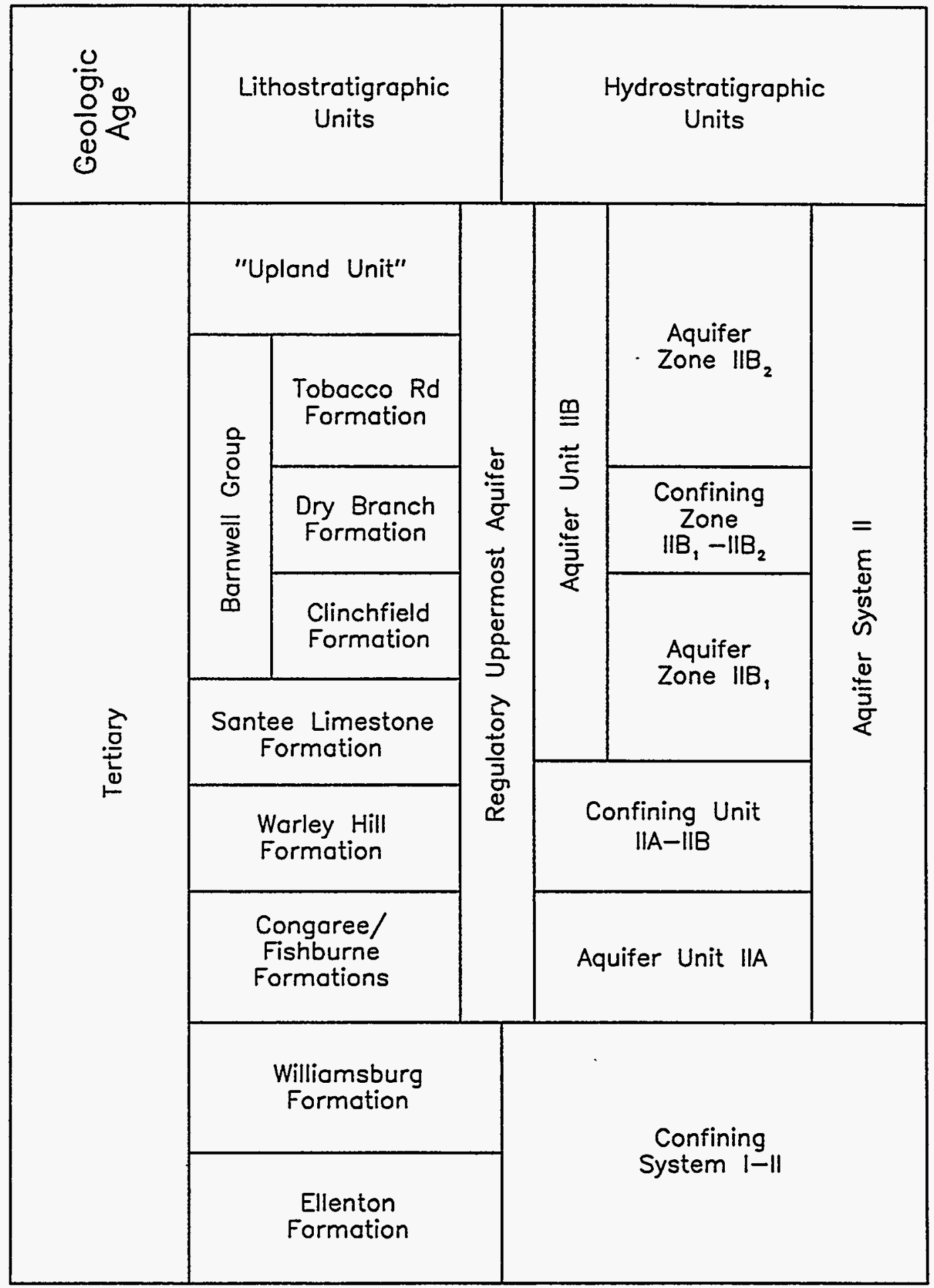

Figure 3. Hydrostratigraphic Nomenclature 


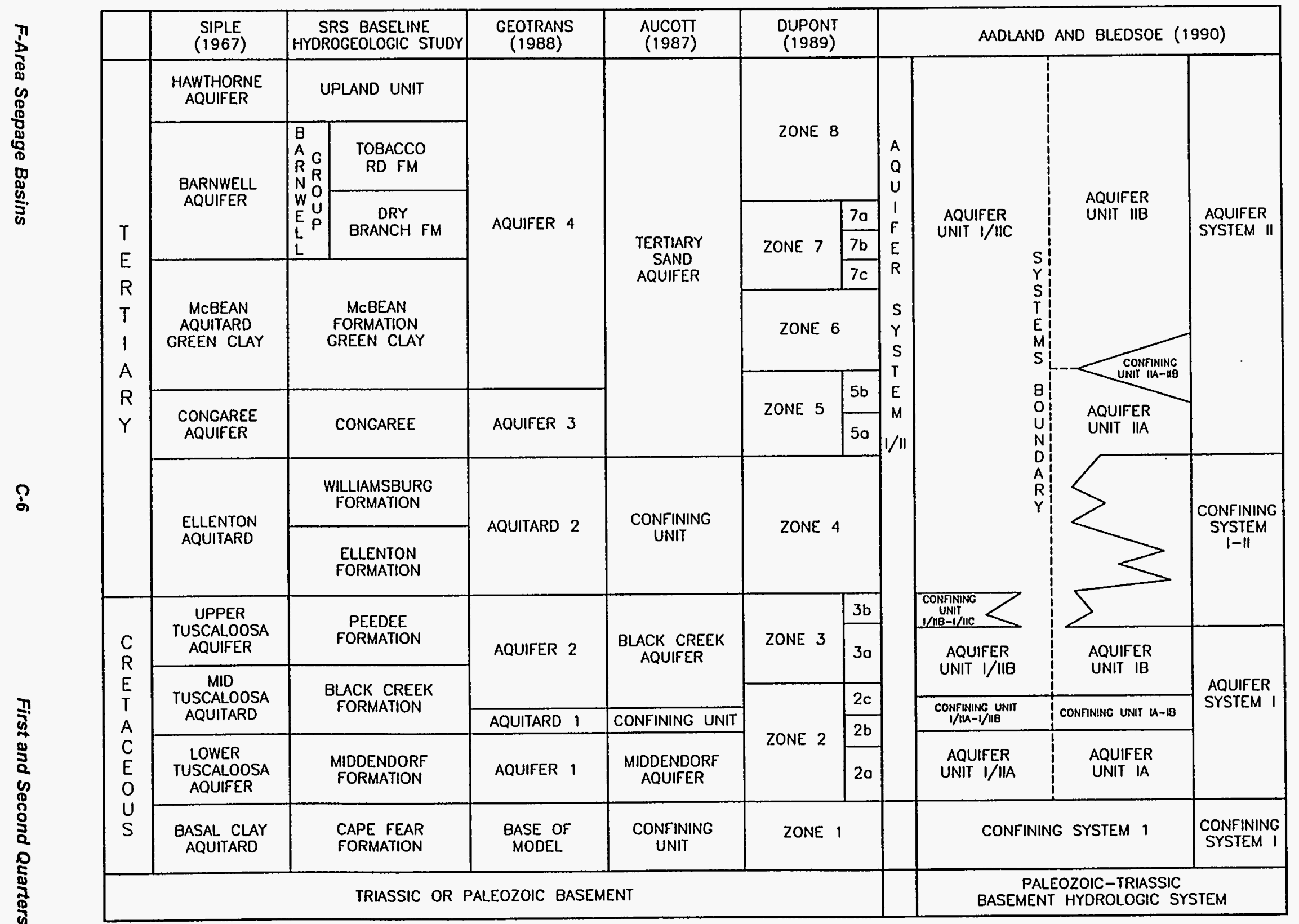

Figure 4. Regional Correlation of Hydrostratigraphic and Lithostratigraphic Nomenclatures 


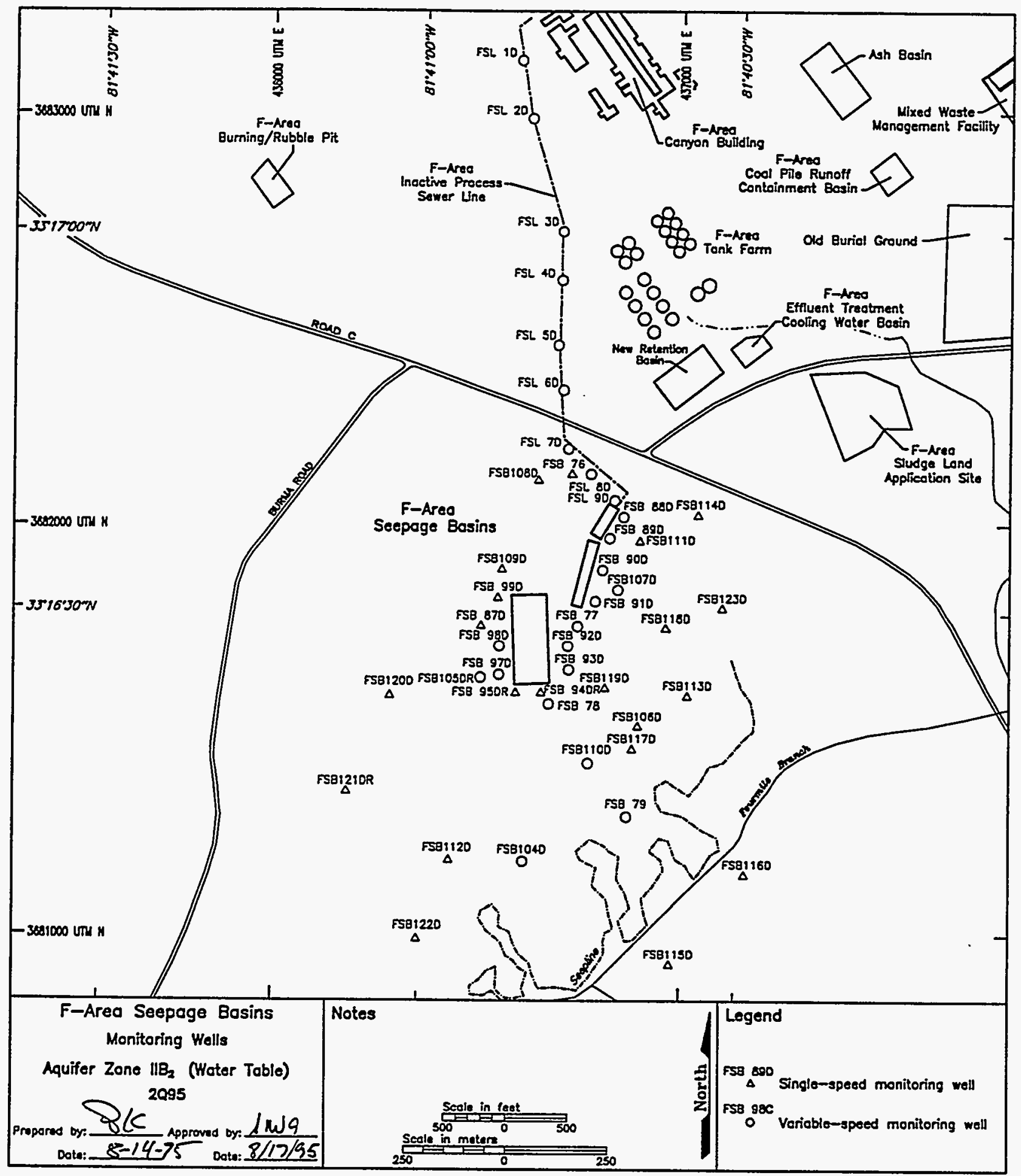

Figure 5. Location of Aquifer Zone $\mathrm{IIB}_{2}$ (Water Table) Monitoring Wells at the F-Area Seepage Basins 


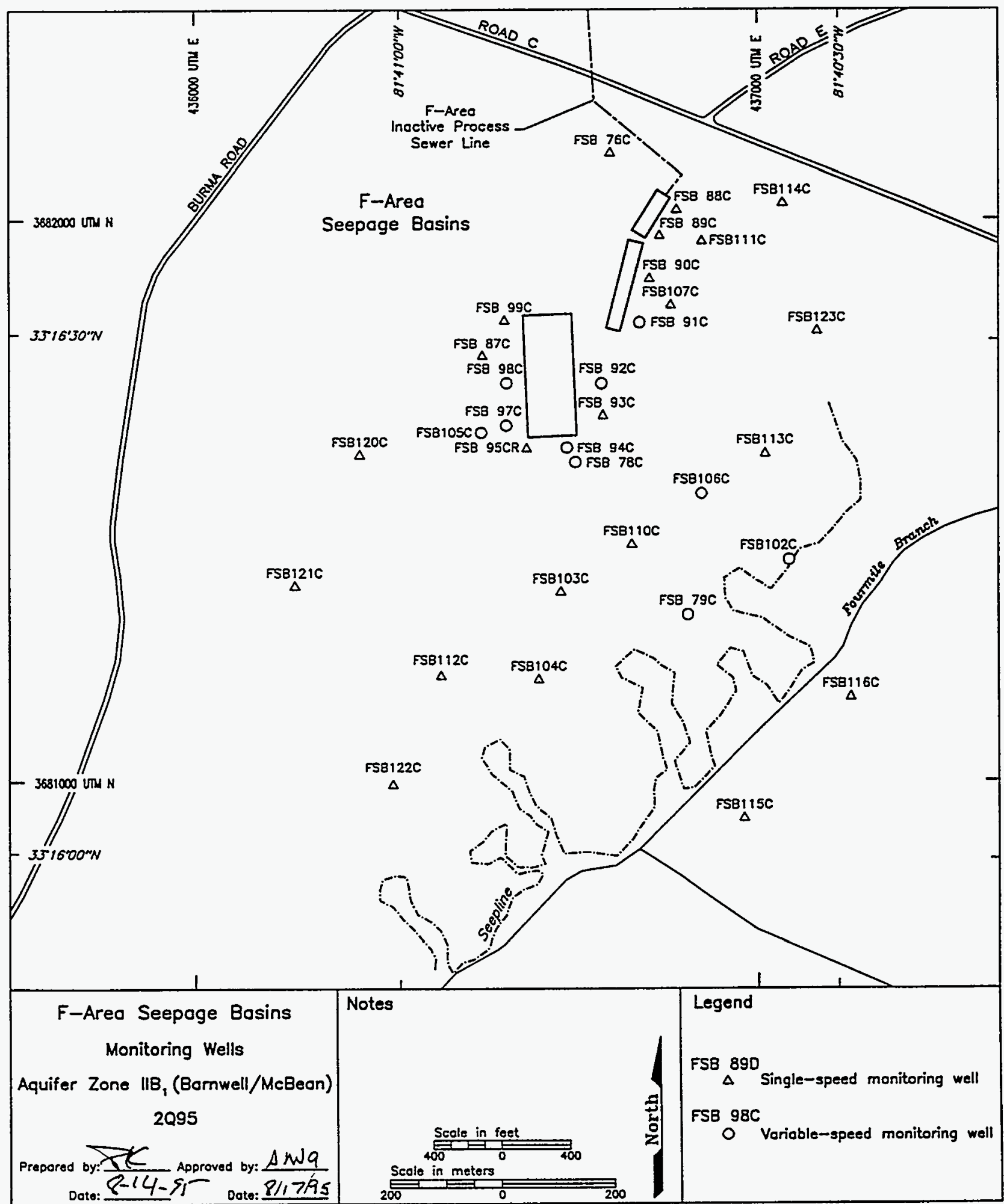

Figure 6. Location of Aquifer Zone $\|_{1} B_{1}$ (Barnwell/McBean) Monitoring Wells at the F-Area Seepage Basins 


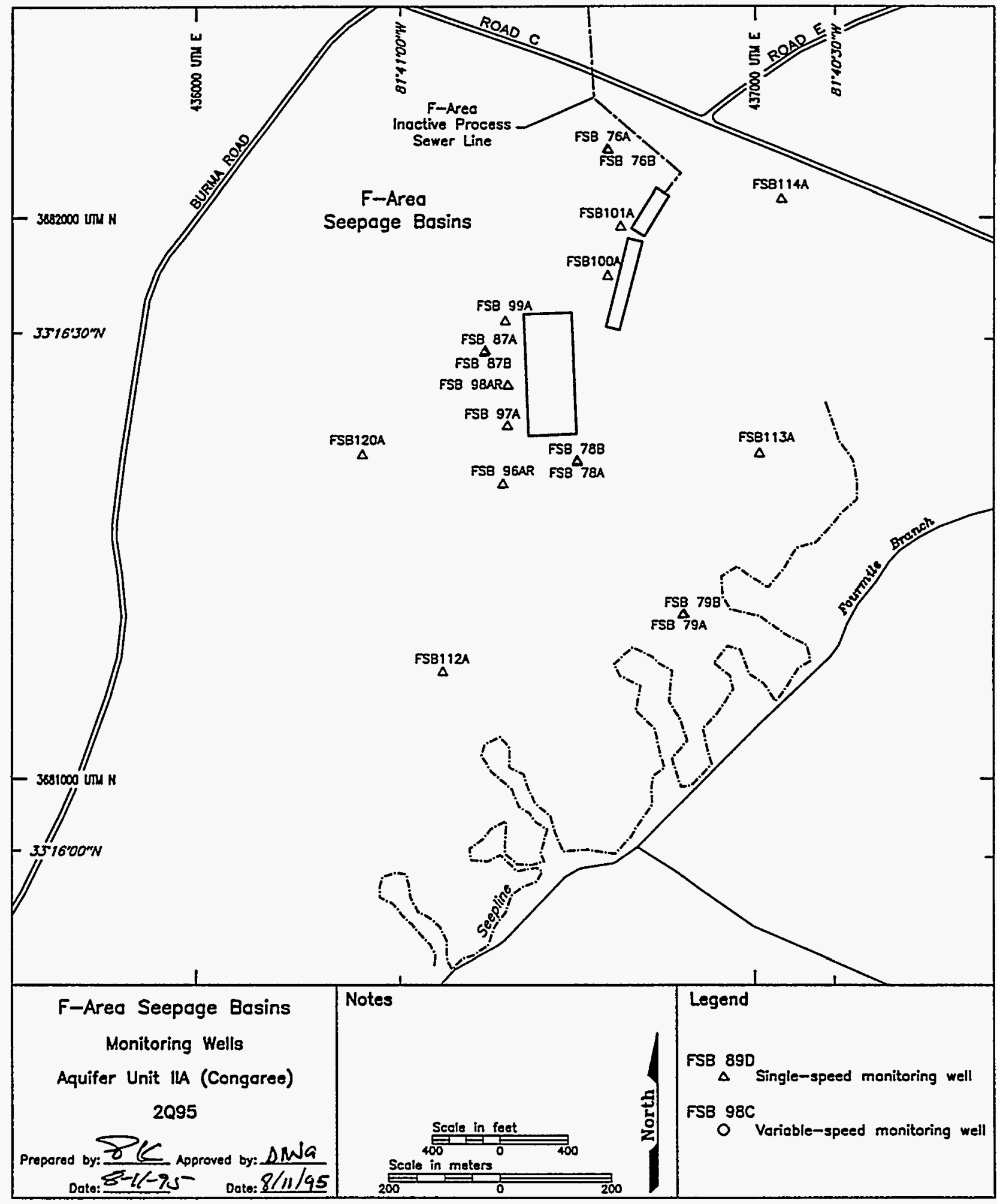

Figure 7. Location of Aquifer Unit IIA (Congaree) Monitoring Wells at the F-Area Seepage Basins 


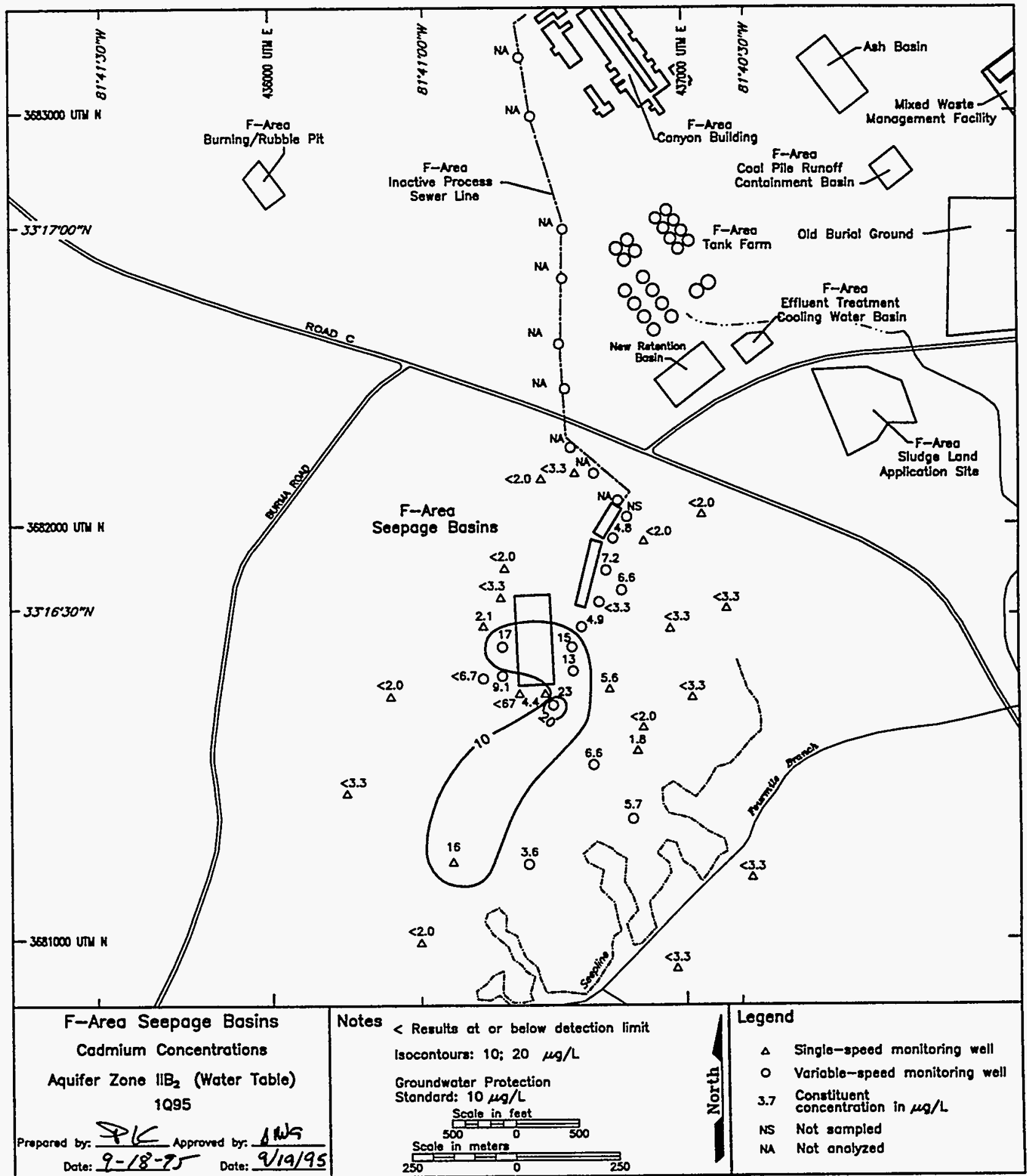

Figure 8. Cadmium Concentrations in Aquifer Zone $\mathrm{IIB}_{2}$ (Water Table) at the F-Area Seepage Basins, First Quarter 1995 


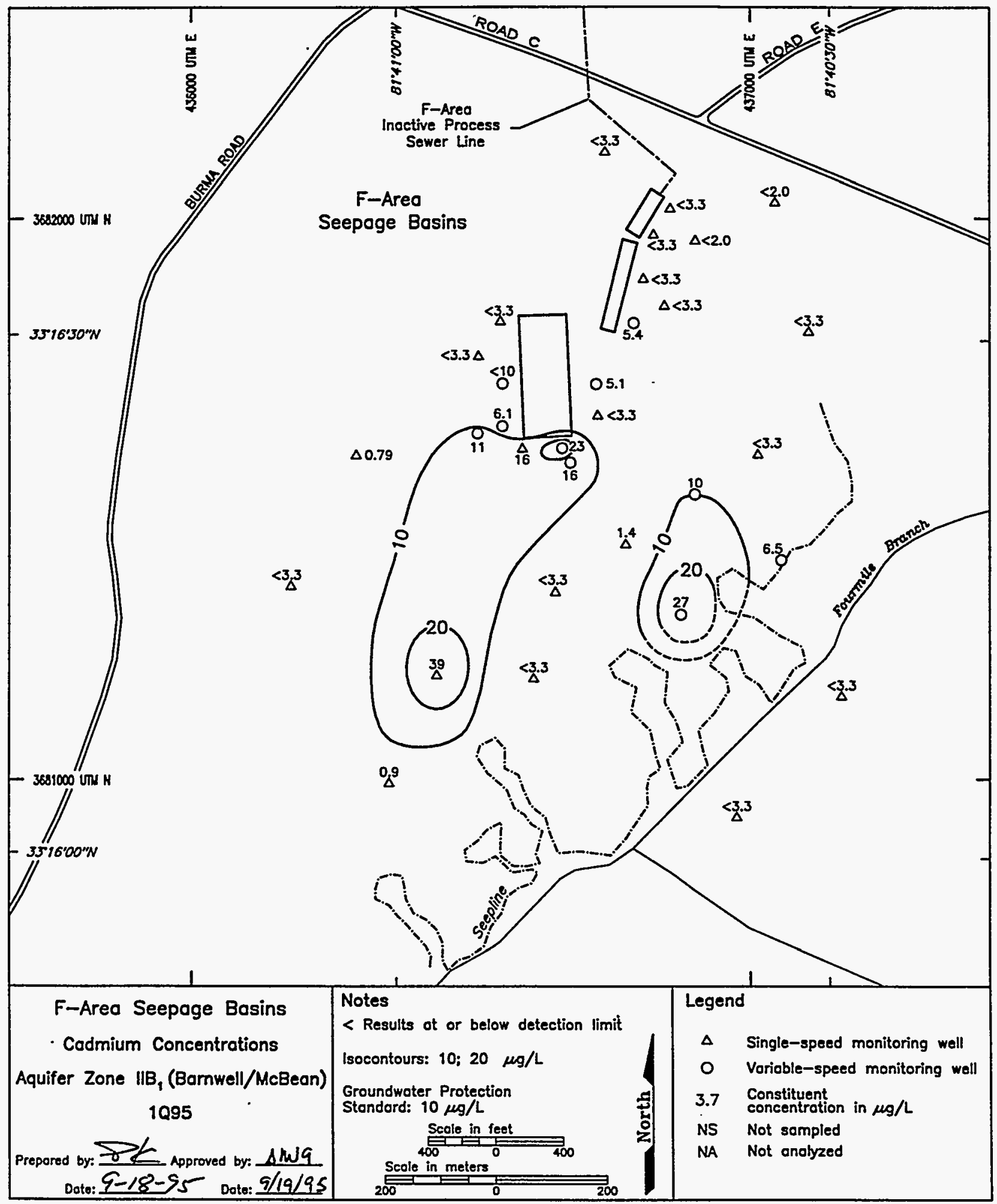

Figure 9. Cadmium Concentrations in Aquifer Zone IIB, (Barnwell/McBean) at the F-Area Seepage Basins, First Quarter 1995 


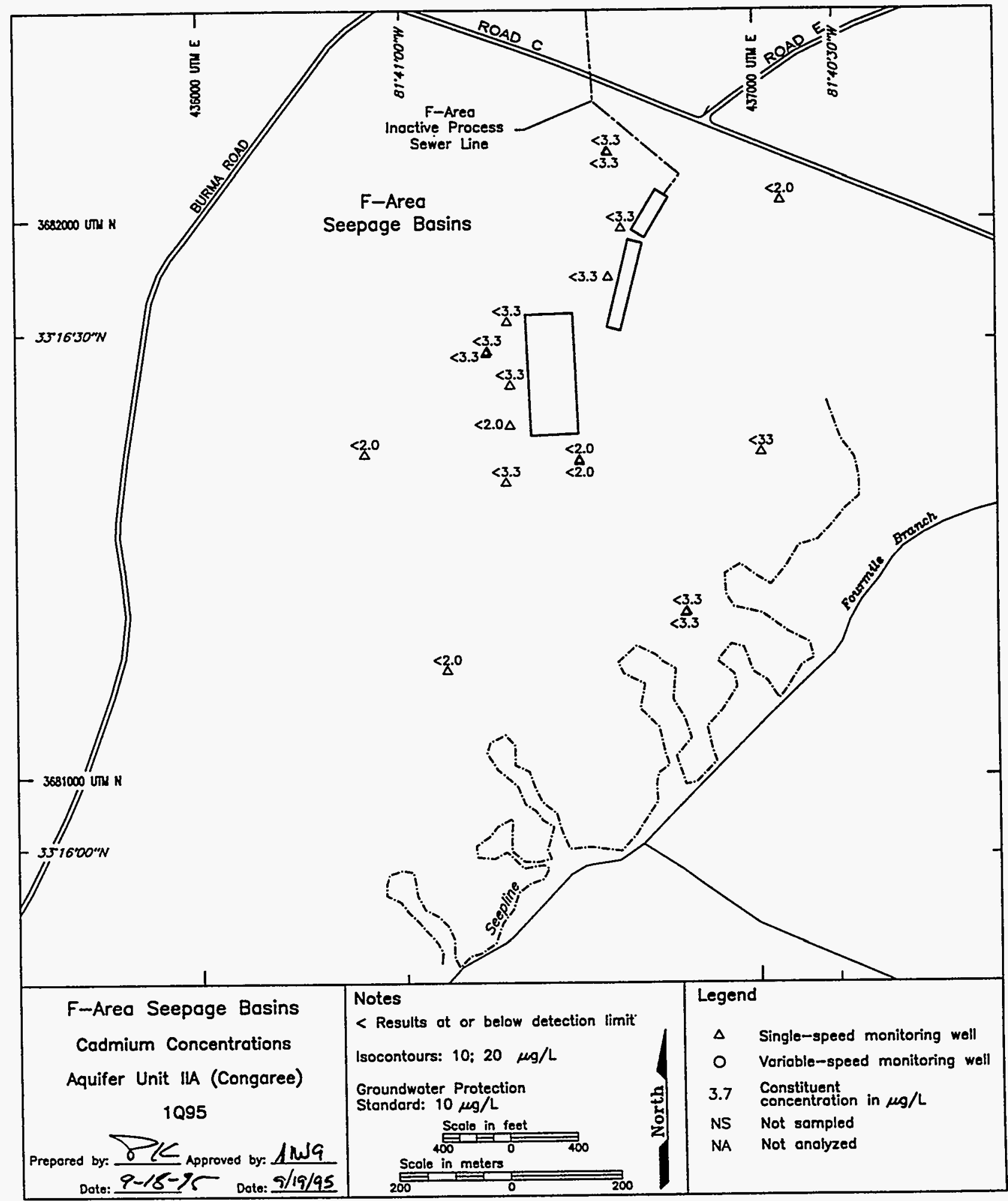

Figure 10. Cadmium Concentrations in Aquifer Unit IIA (Congaree) at the F-Area Seepage Basins, First Quarter 1995 


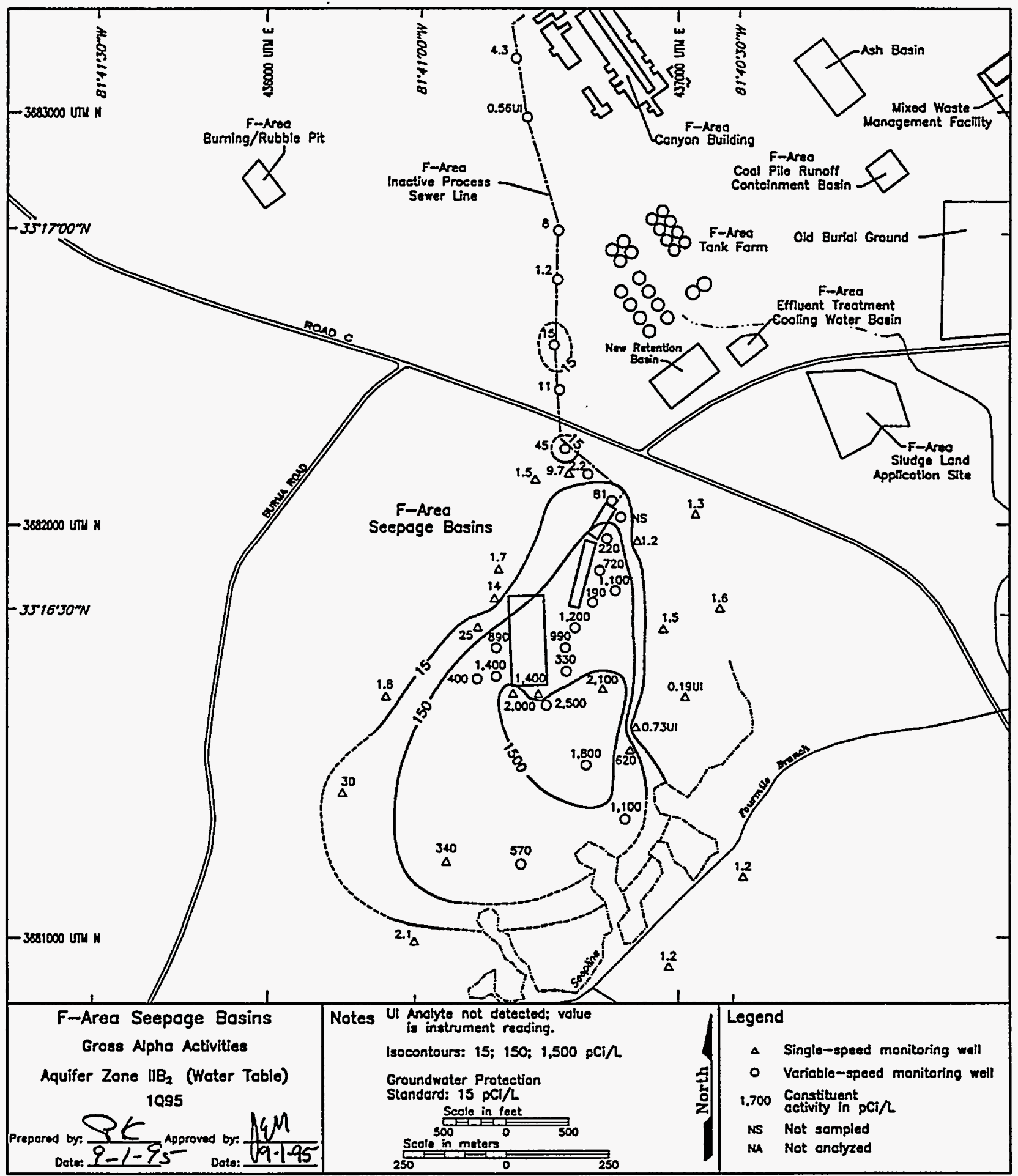

Figure 11. Gross Alpha Activities in Aquifer Zone $\mathrm{IIB}_{2}$ (Water Table) at the F-Area Seepage Basins, First Quarter 1995 


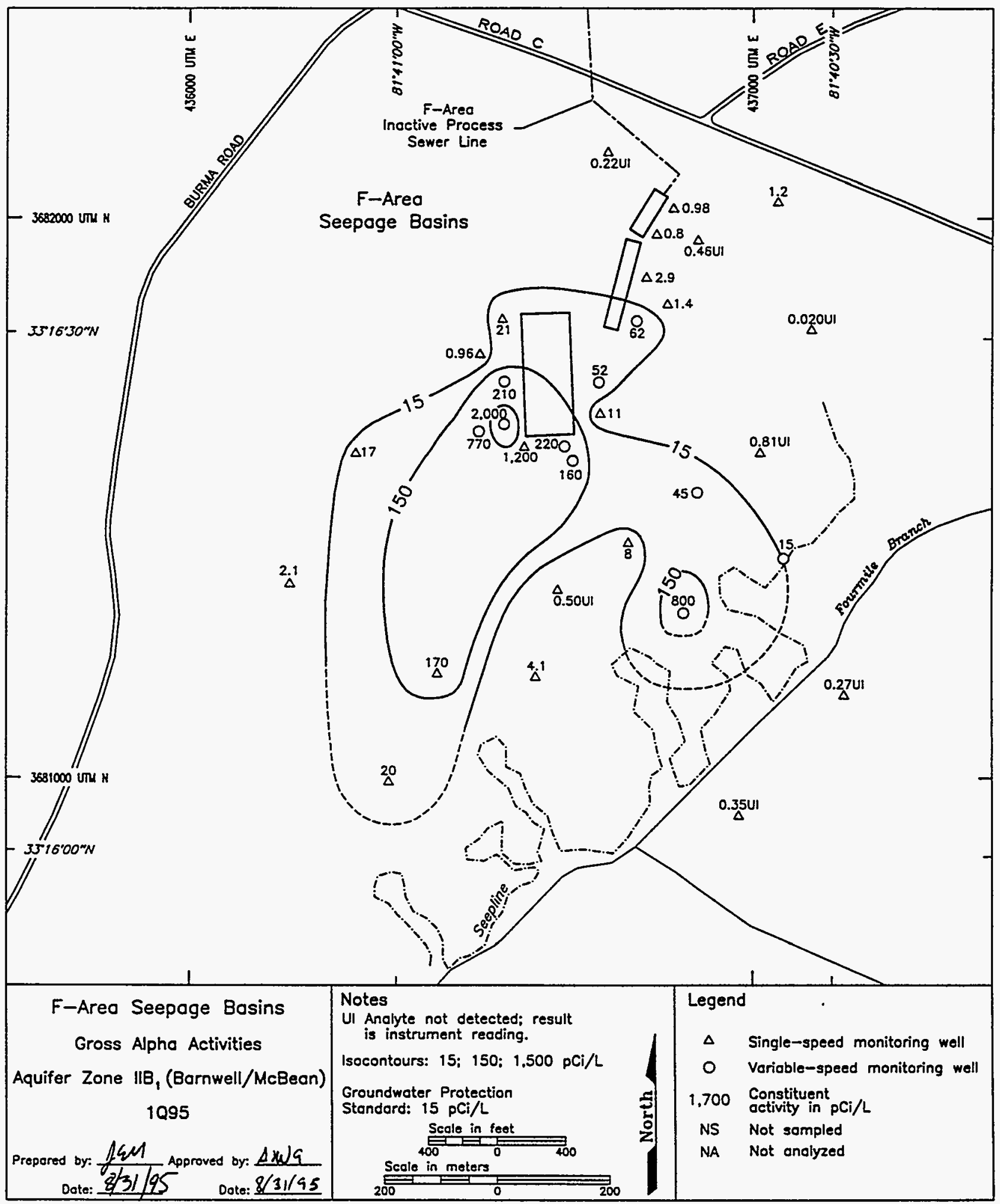

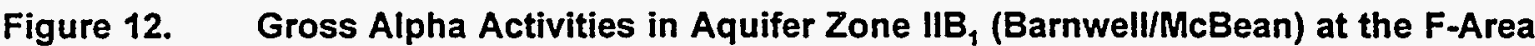
Seepage Basins, First Quarter 1995 


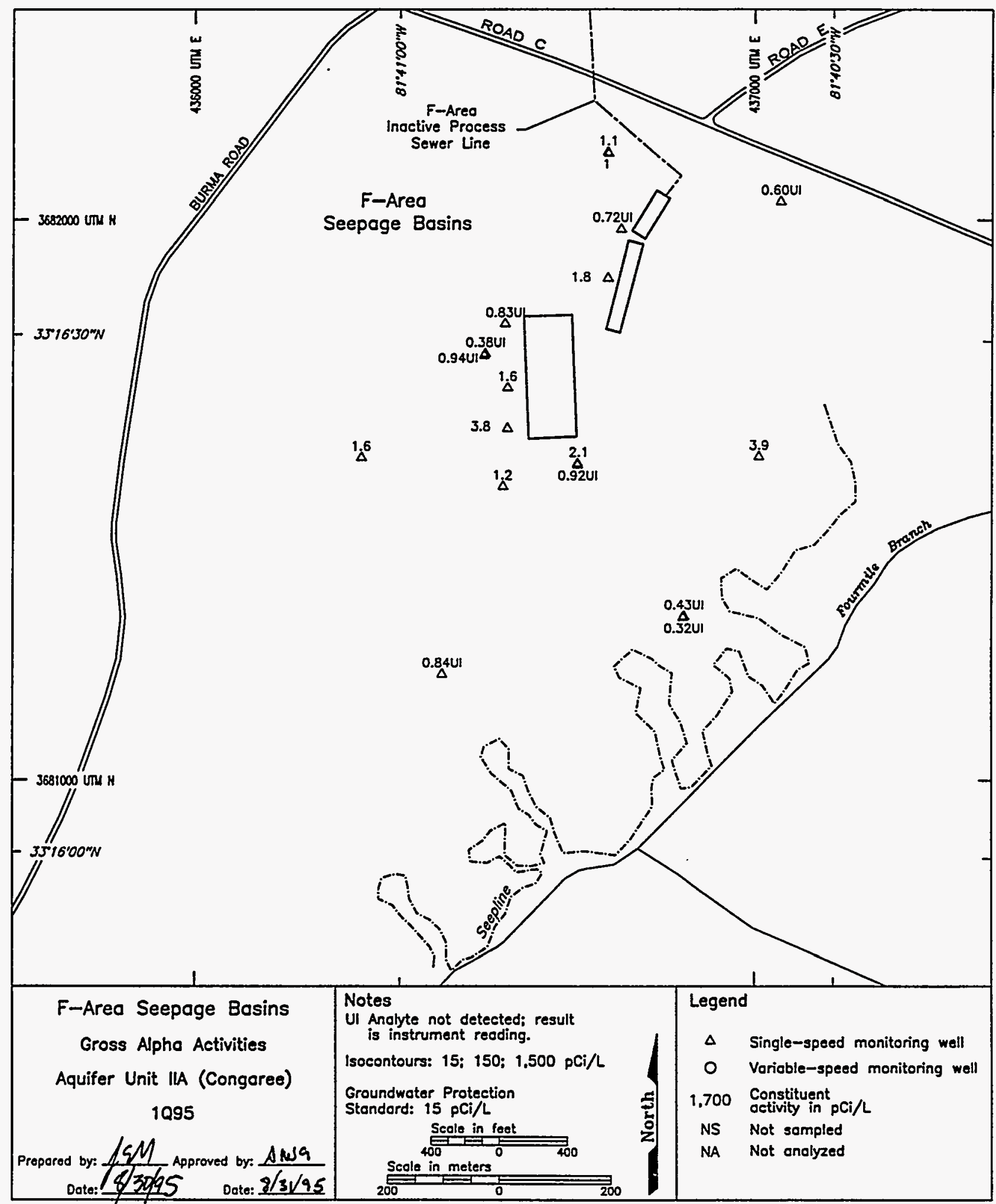

Figure 13. Gross Alpha Activities in Aquifer Unit IIA (Congaree) at the F-Area Seepage Basins, First Quarter 1995 


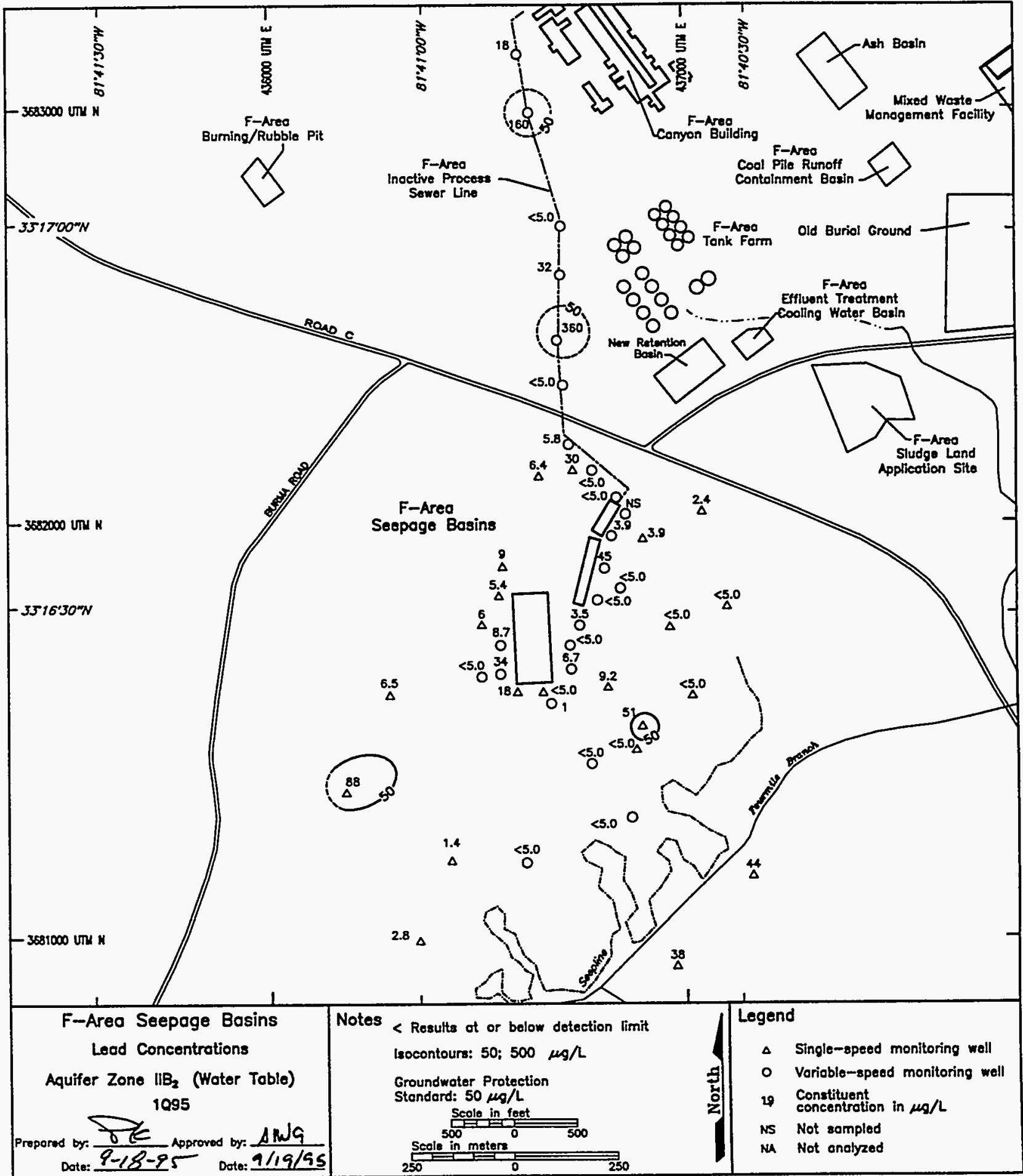

Figure 14. Lead Concentrations in Aquifer Zone $\|_{2} B_{2}$ (Water Table) at the F-Area Seepage Basins, First Quarter 1995 


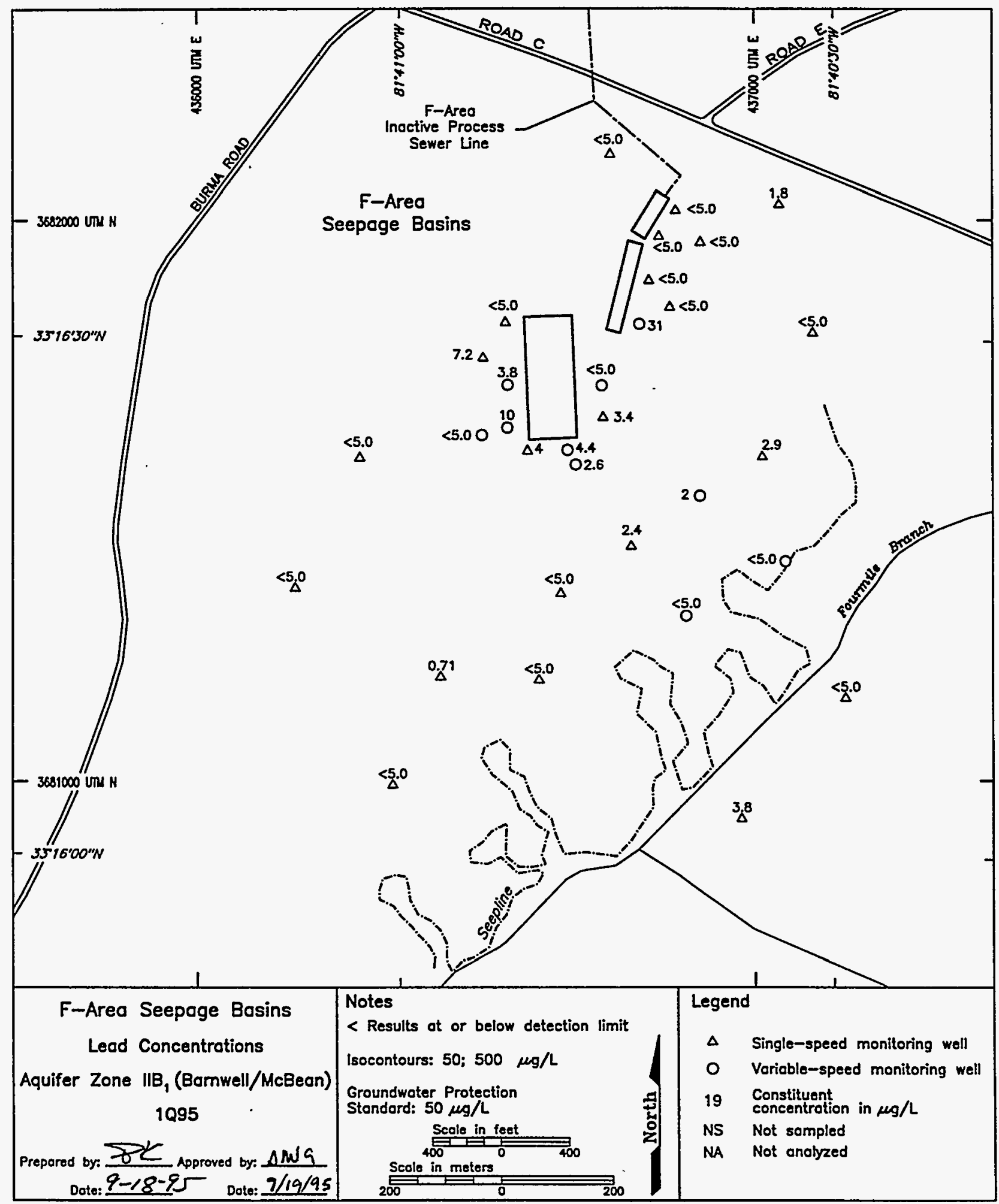

Figure 15. Lead Concentrations in Aquifer Zone $\|_{1} B_{1}$ (Barnwell/McBean) at the F-Area Seepage Basins, First Quarter 1995 


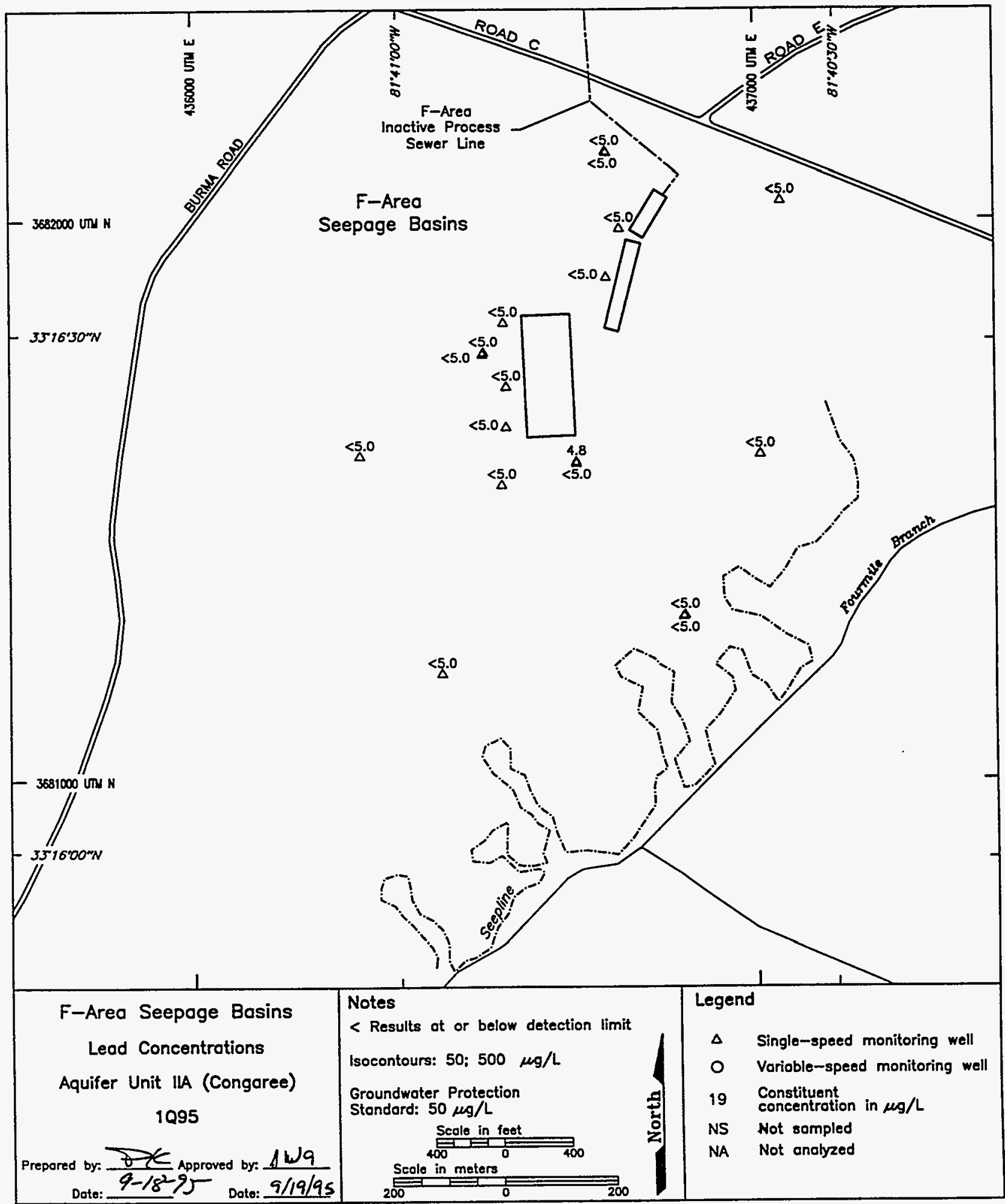

Figure 16. Lead Concentrations in Aquifer Unit IIA (Congaree) at the F-Area Seepage Basins, First Quarter 1995 


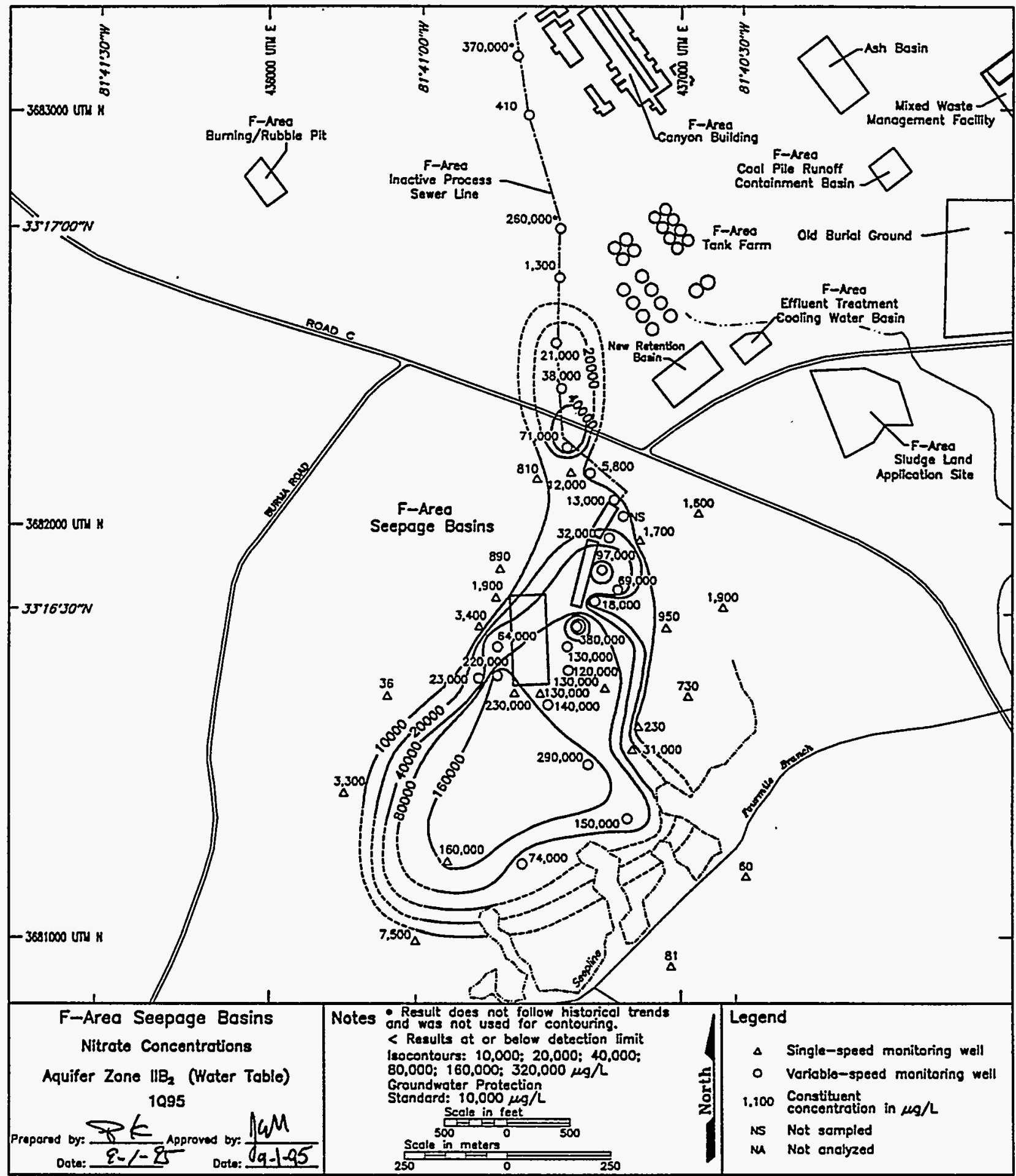

Figure 17. Nitrate Concentrations in Aquifer Zone $\|_{2} B_{2}$ (Water Table) at the F-Area Seepage Basins, First Quarter 1995 


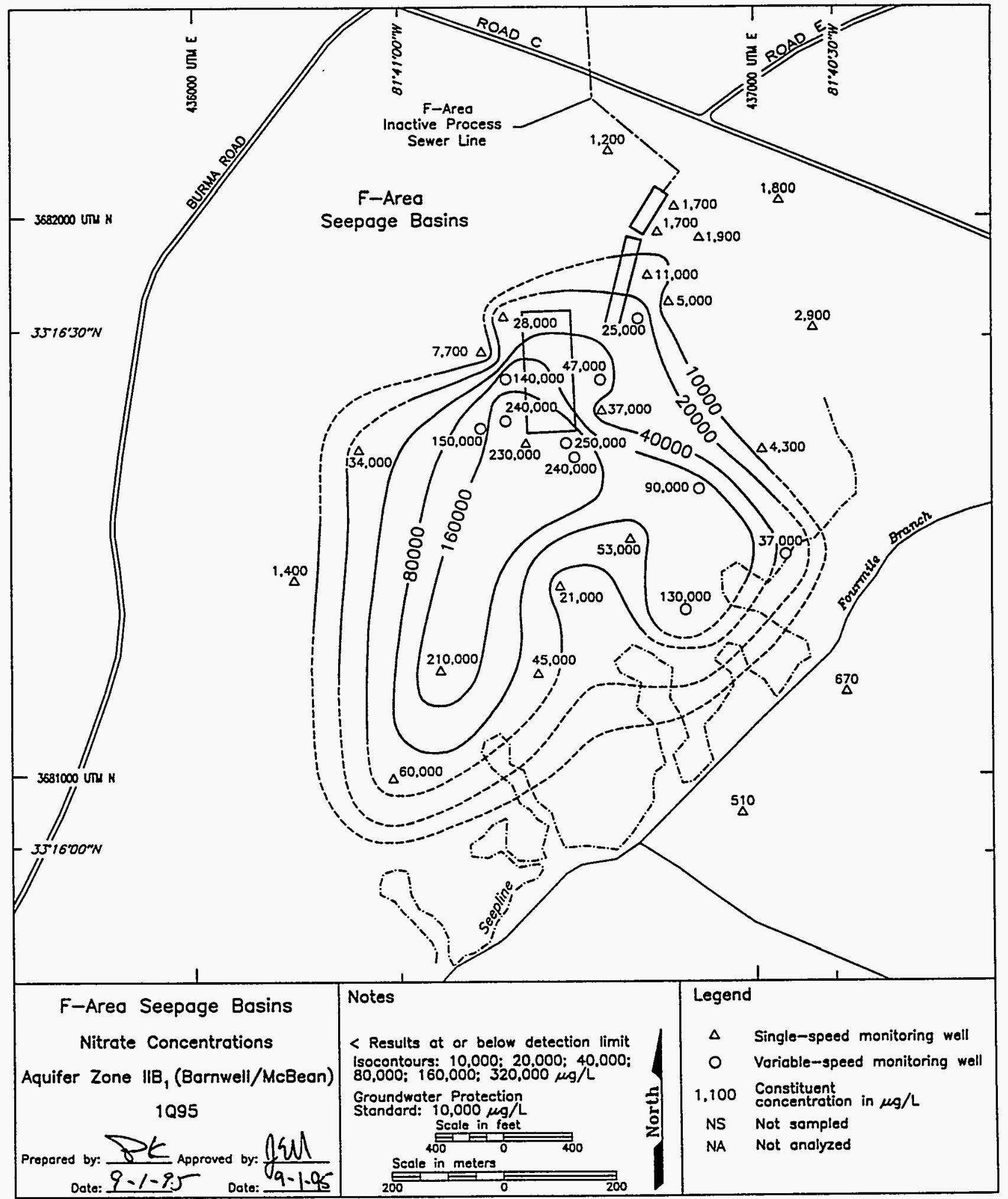

Figure 18. Nitrate Concentrations in Aquifer Zone IIB, (Barnwell/McBean) at the F-Area Seepage Basins, First Quarter 1995 


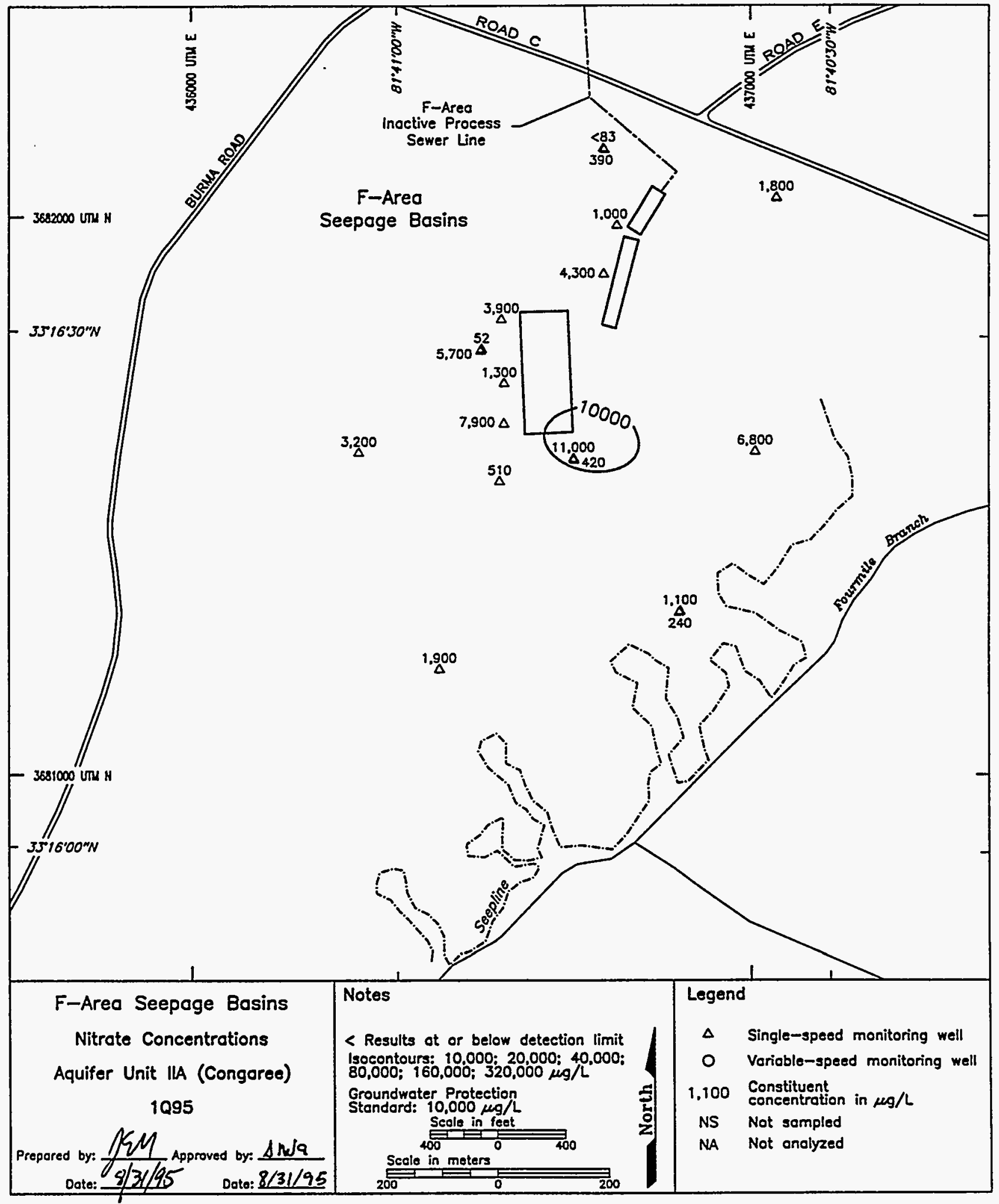

Figure 19. Nitrate Concentrations in Aquifer Unit IIA (Congaree) at the F-Area Seepage Basins, First Quarter 1995 


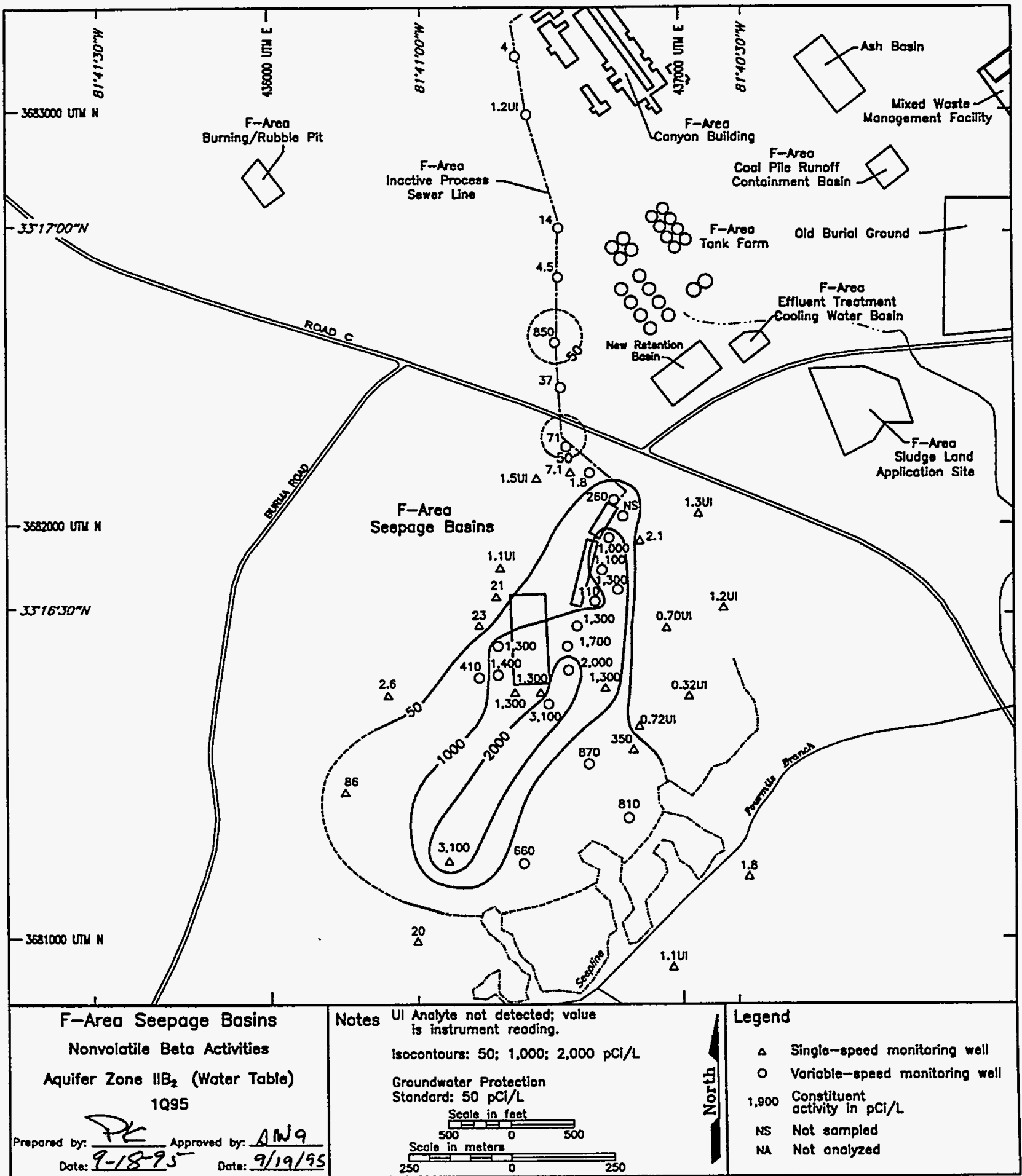

Figure 20. Nonvolatile Beta Activities in Aquifer Zone $\mathrm{IIB}_{2}$ (Water Table) at the F-Area Seepage Basins, First Quarter 1995 


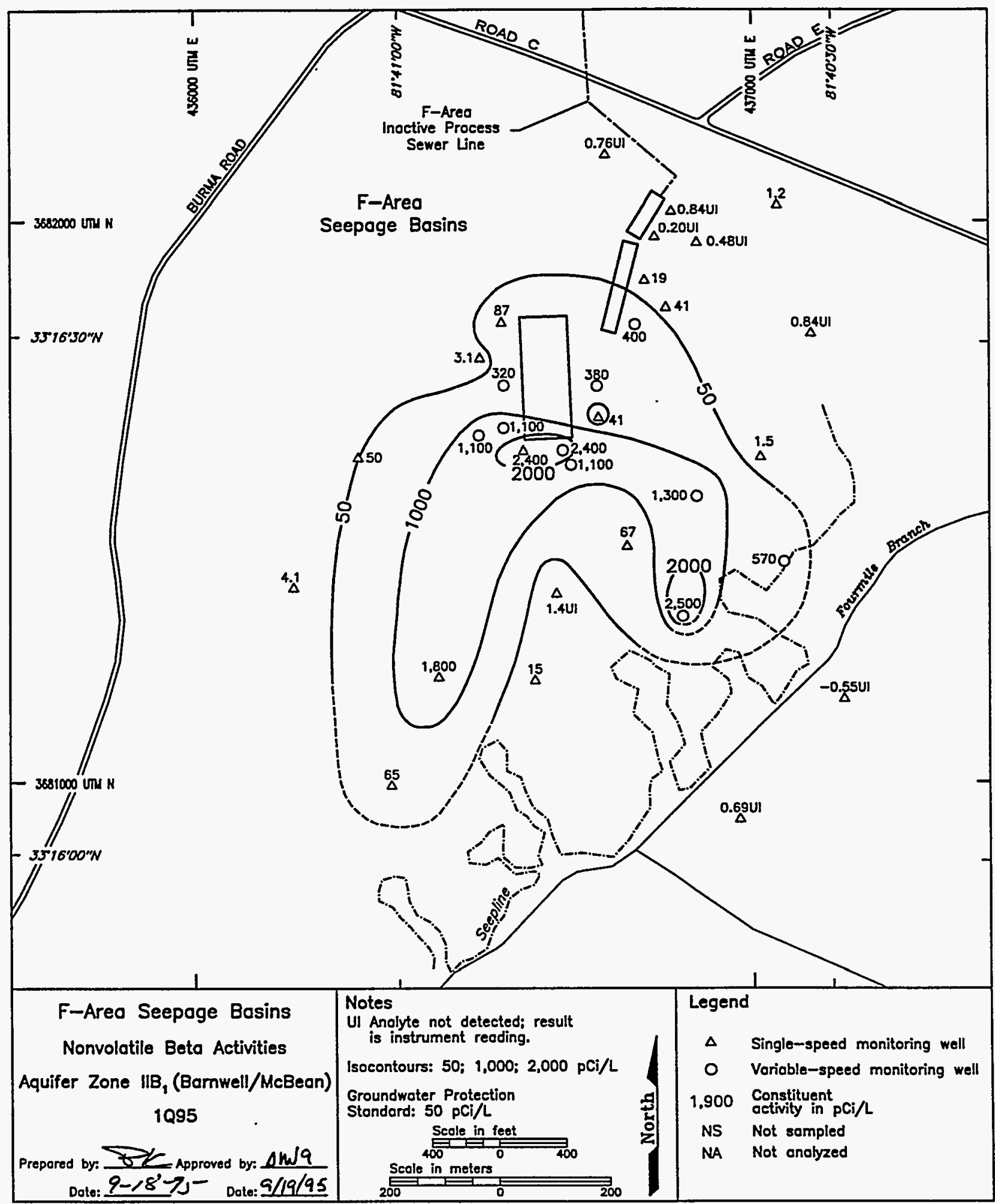

Figure 21. Nonvolatile Beta Activities in Aquifer Zone $\|_{1} B_{1}$ (Barnwell/McBean) at the F-Area Seepage Basins, First Quarter 1995 


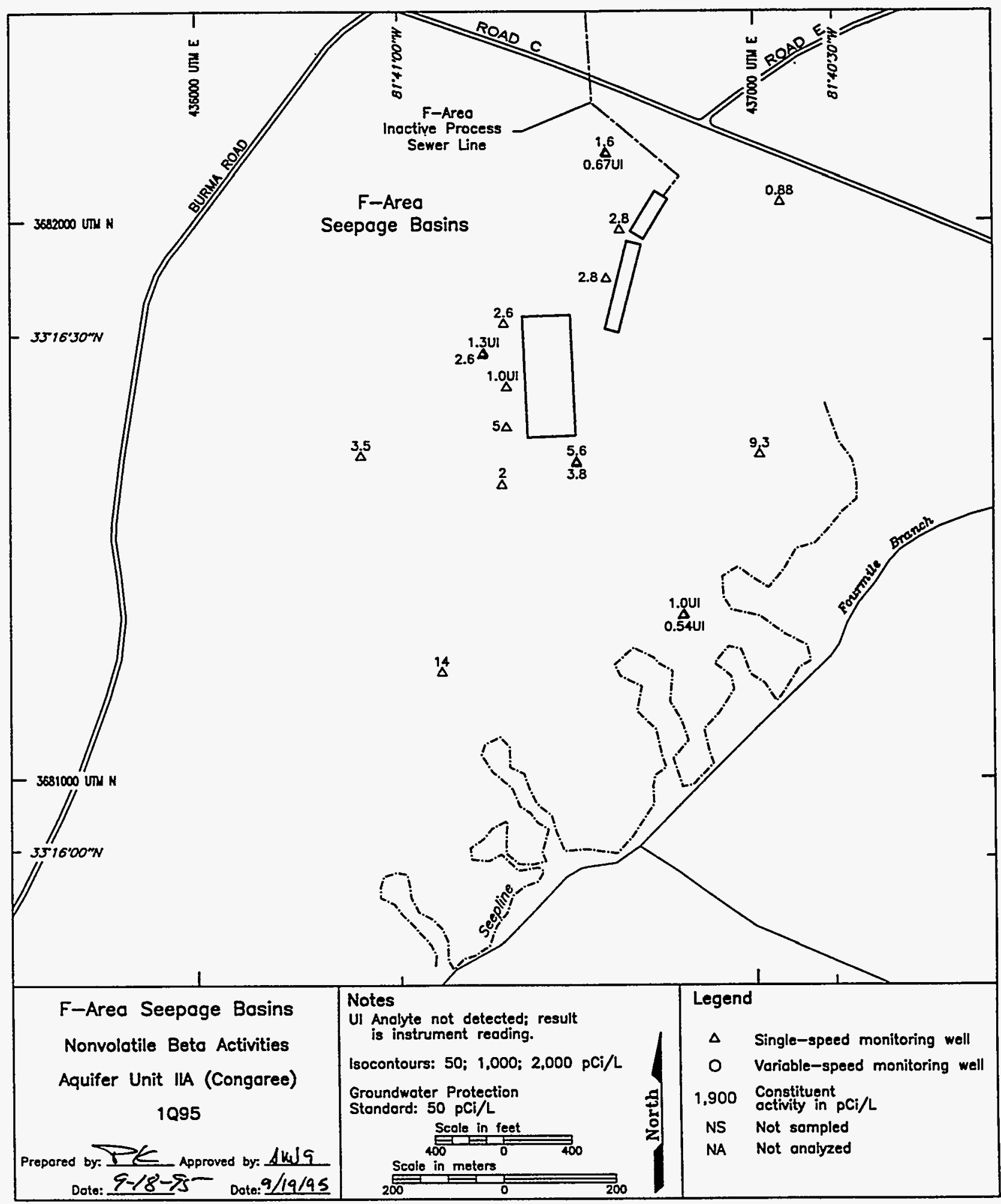

Figure 22. Nonvolatile Beta Activities in Aquifer Unit IIA (Congaree) at the F-Area Seepage Basins, First Quarter 1995 


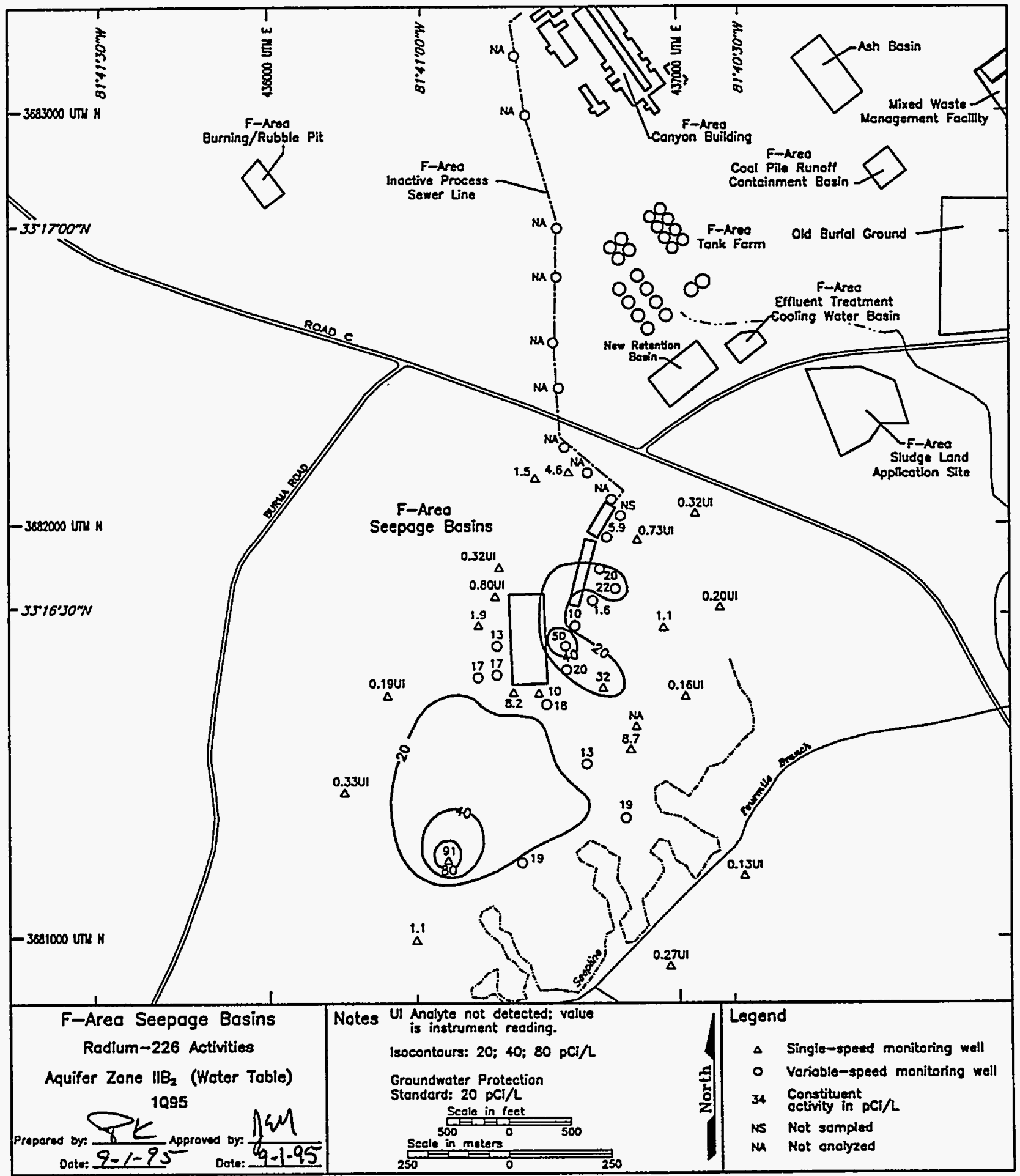

Figure 23. Radium-226 Activities in Aquifer Zone $\mathrm{IIB}_{2}$ (Water Table) at the F-Area Seepage Basins, First Quarter 1995 


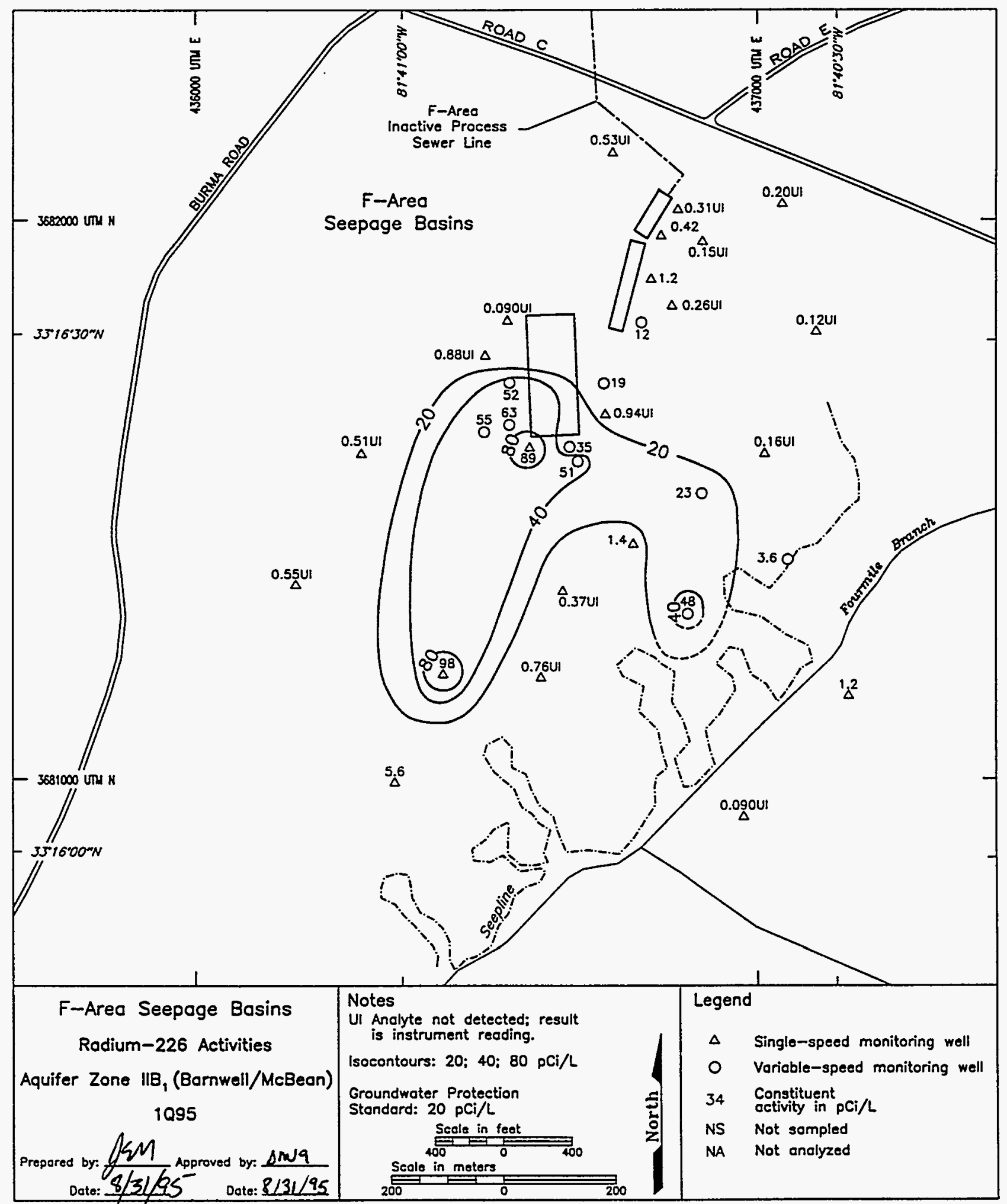

Figure 24. Radium-226 Activities in Aquifer Zone IIB, (Barnwell/McBean) at the F-Area Seepage Basins, First Quarter 1995 


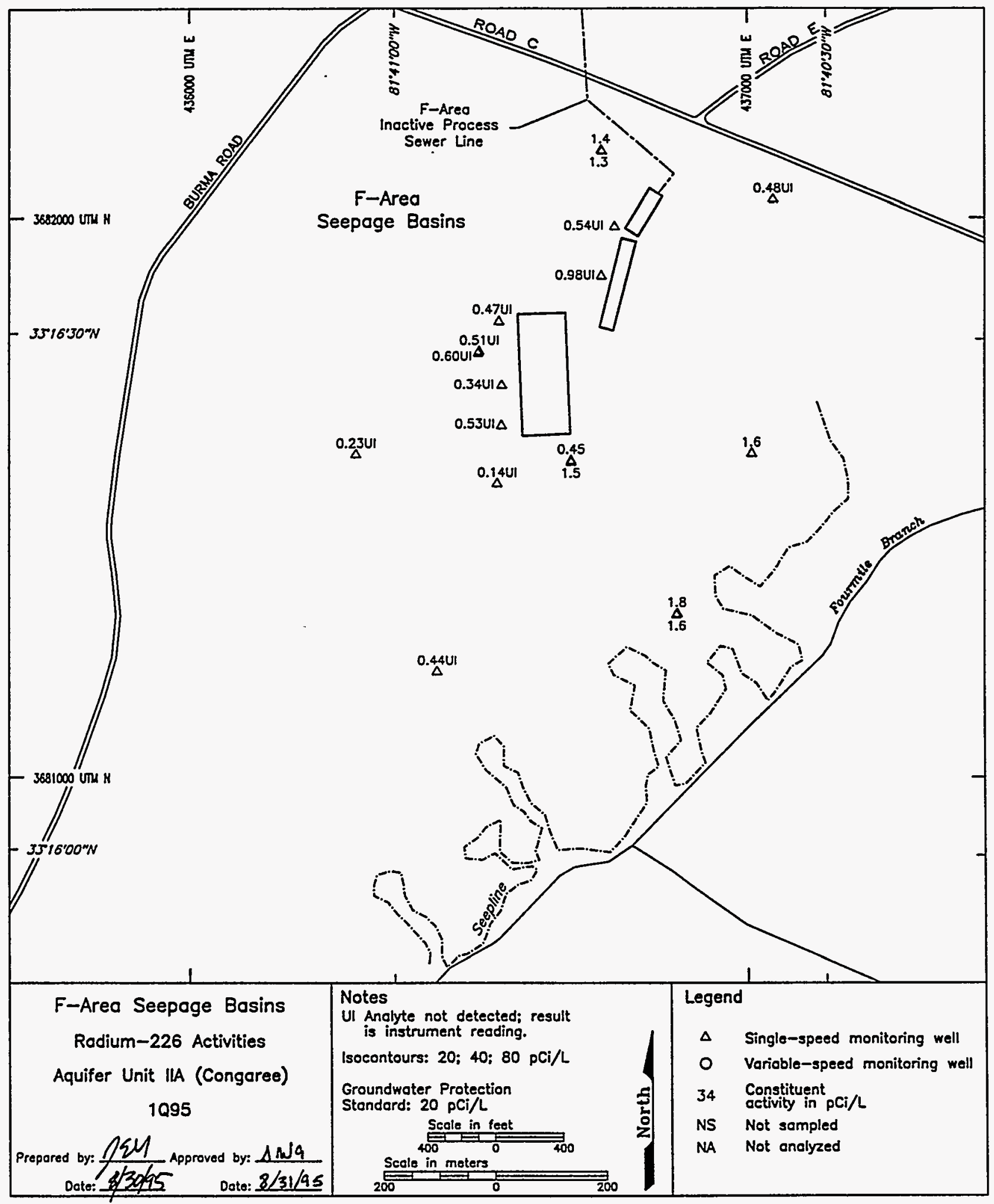

Figure 25. Radium-226 Activities in Aquifer Unit IIA (Congaree) at the F-Area Seepage Basins, First Quarter 1995 


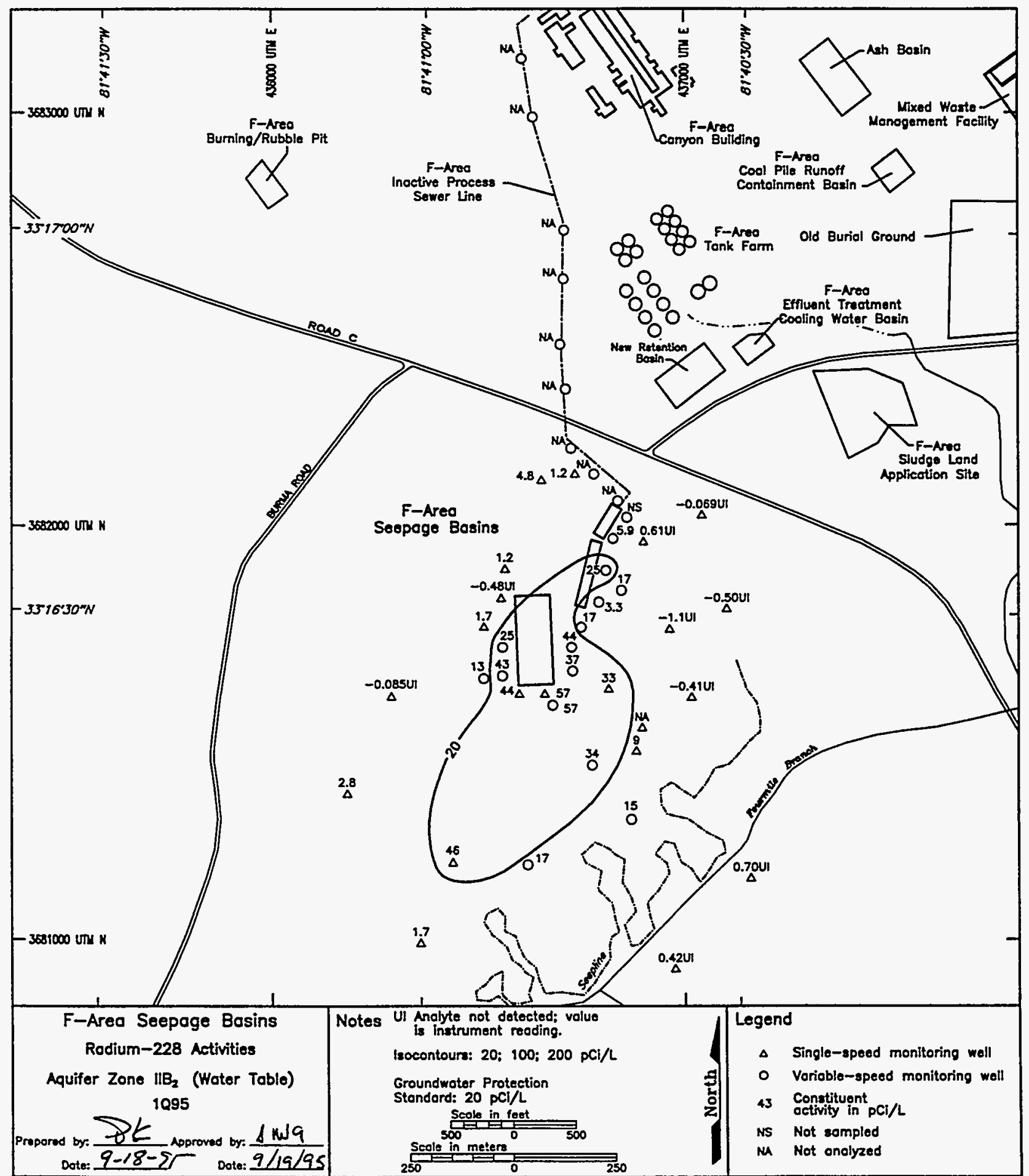

Figure 26. Radium-228 Activities in Aquifer Zone $\|_{2} B_{2}$ (Water Table) at the F-Area Seepage Basins, First Quarter 1995 


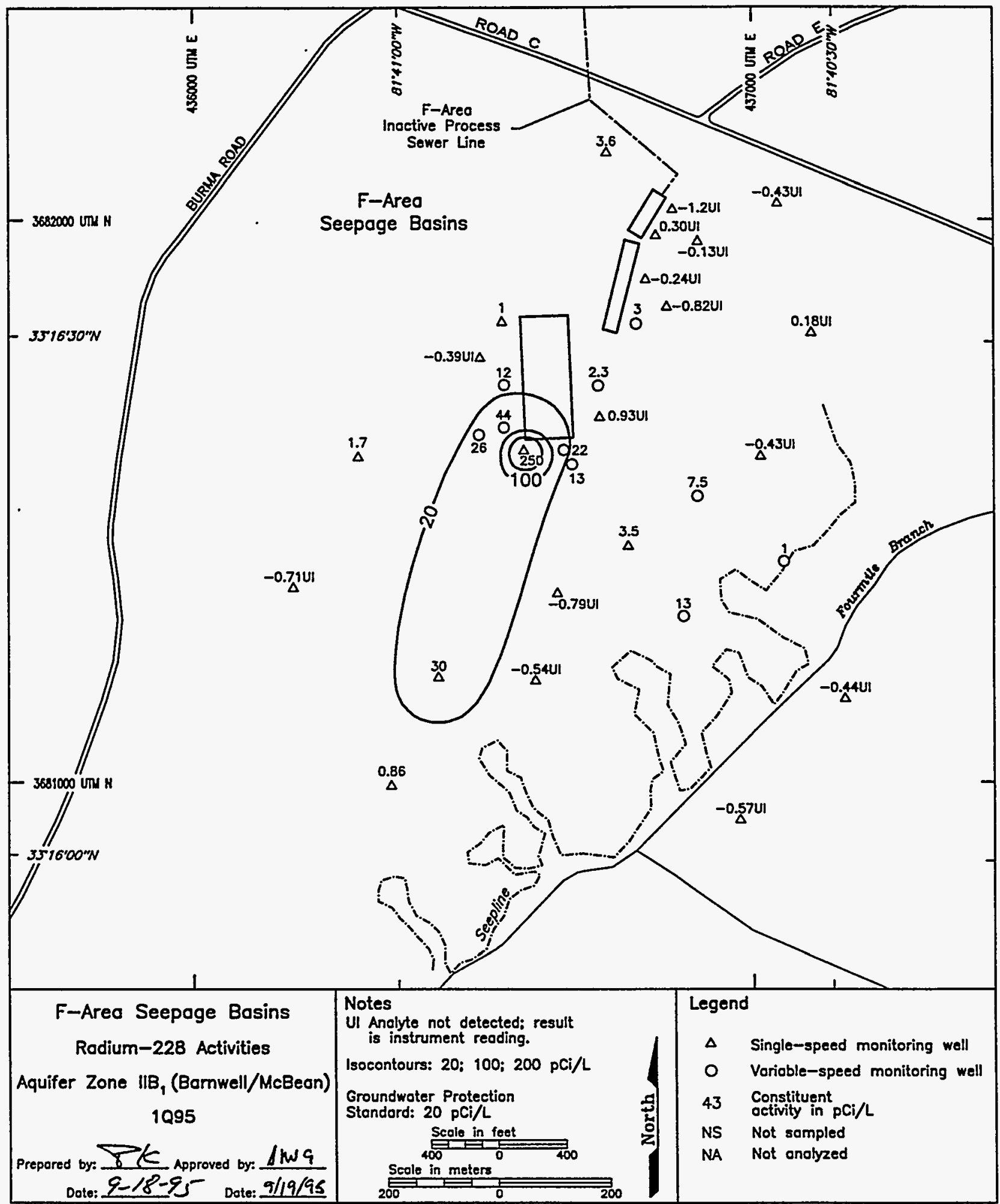

Figure 27. Radium-228 Activities in Aquifer Zone $\mathrm{IIB}_{1}$ (Barnwell/McBean) at the F-Area Seepage Basins, First Quarter 1995 


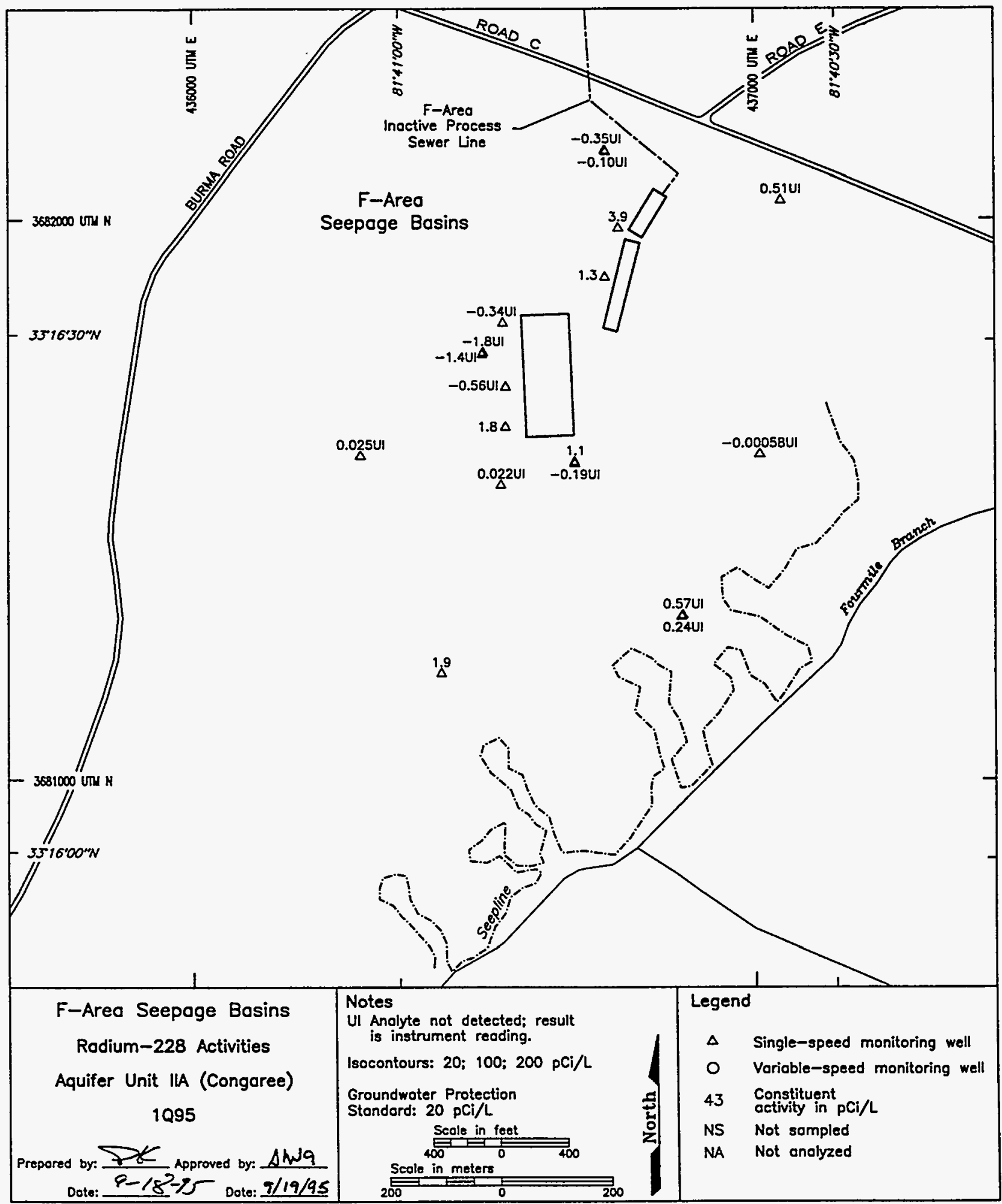

Figure 28. Radium-228 Activities in Aquifer Unit IIA (Congaree) at the F-Area Seepage Basins, First Quarter 1995 
S66 L dəرeno łS!!d

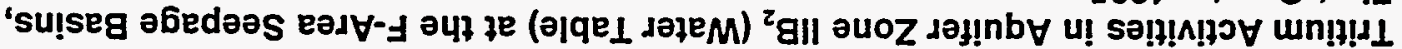

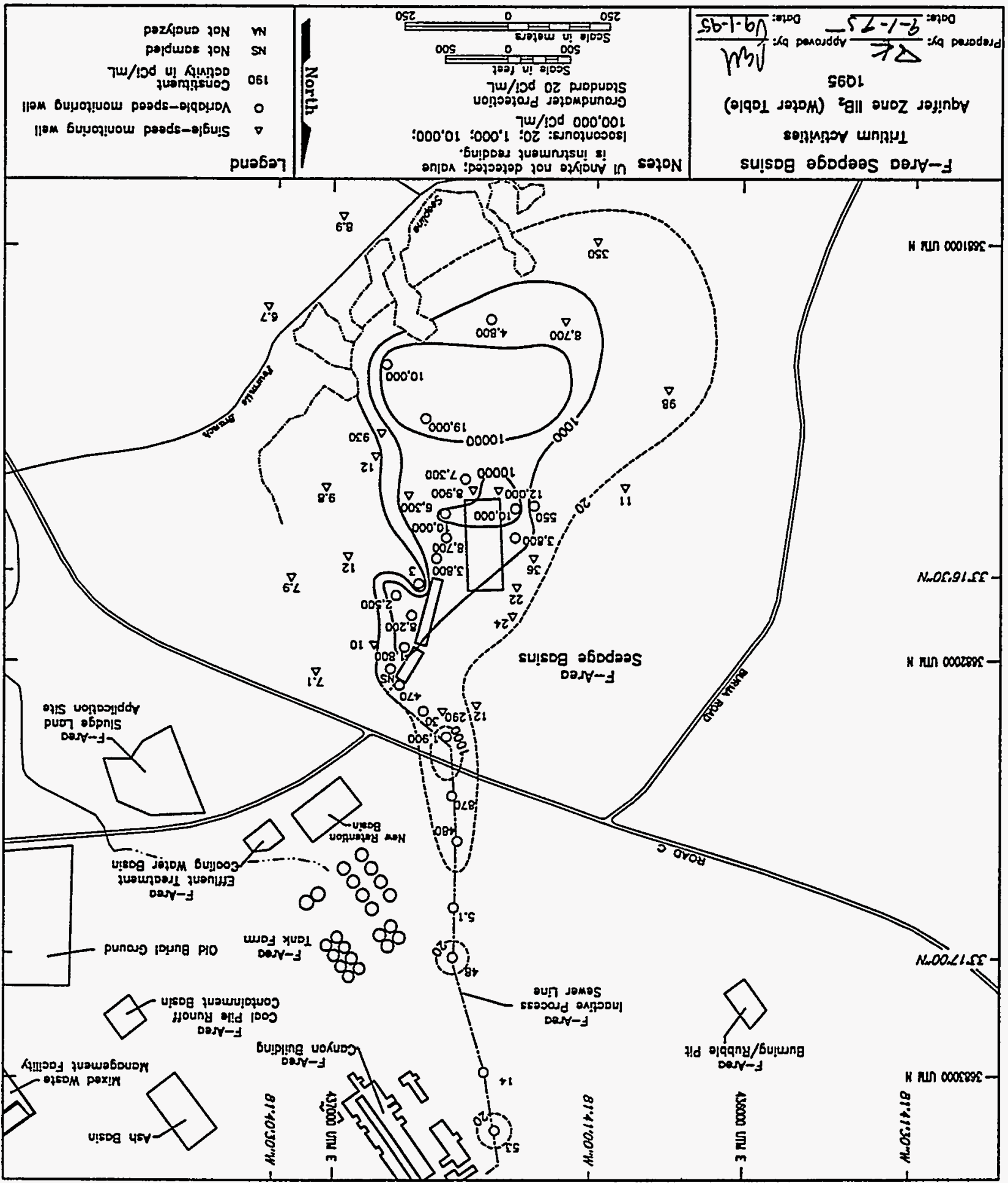




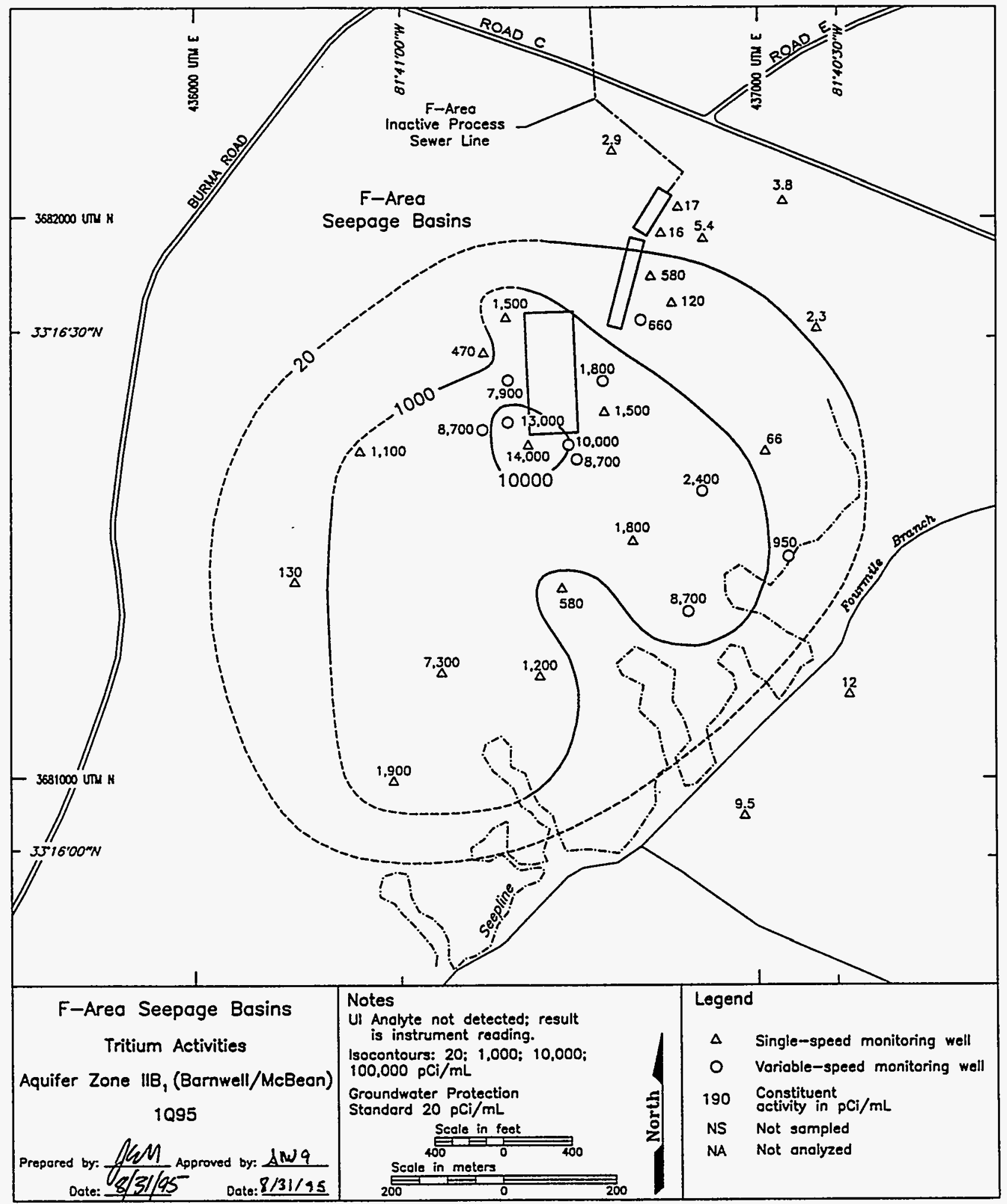

Figure 30. Tritium Activities in Aquifer Zone IIB, (Barnwell/McBean) at the F-Area Seepage Basins, First Quarter 1995 


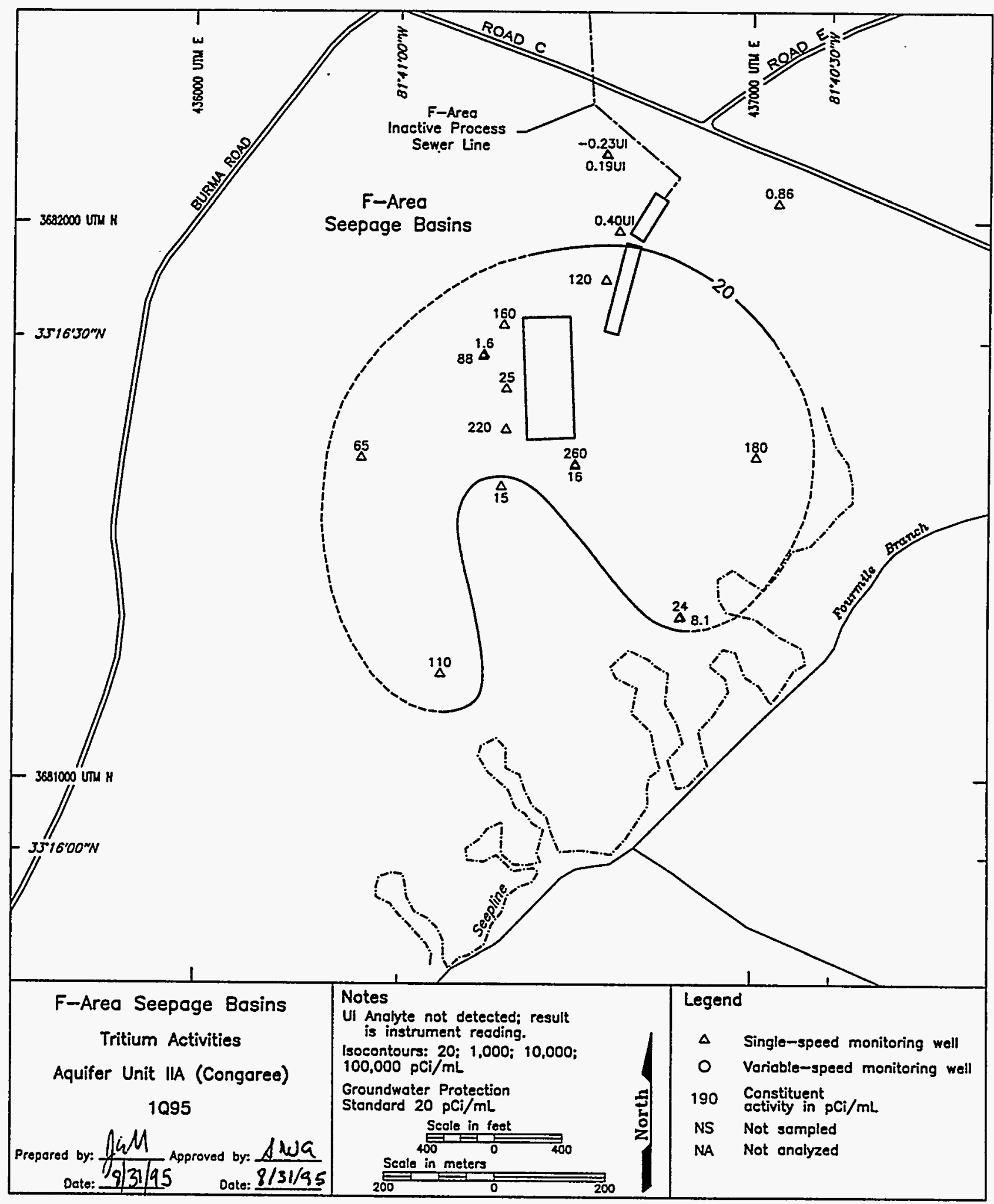

Figure 31. Tritium Activities in Aquifer Unit IIA (Congaree) at the F-Area Seepage Basins, First Quarter 1995 


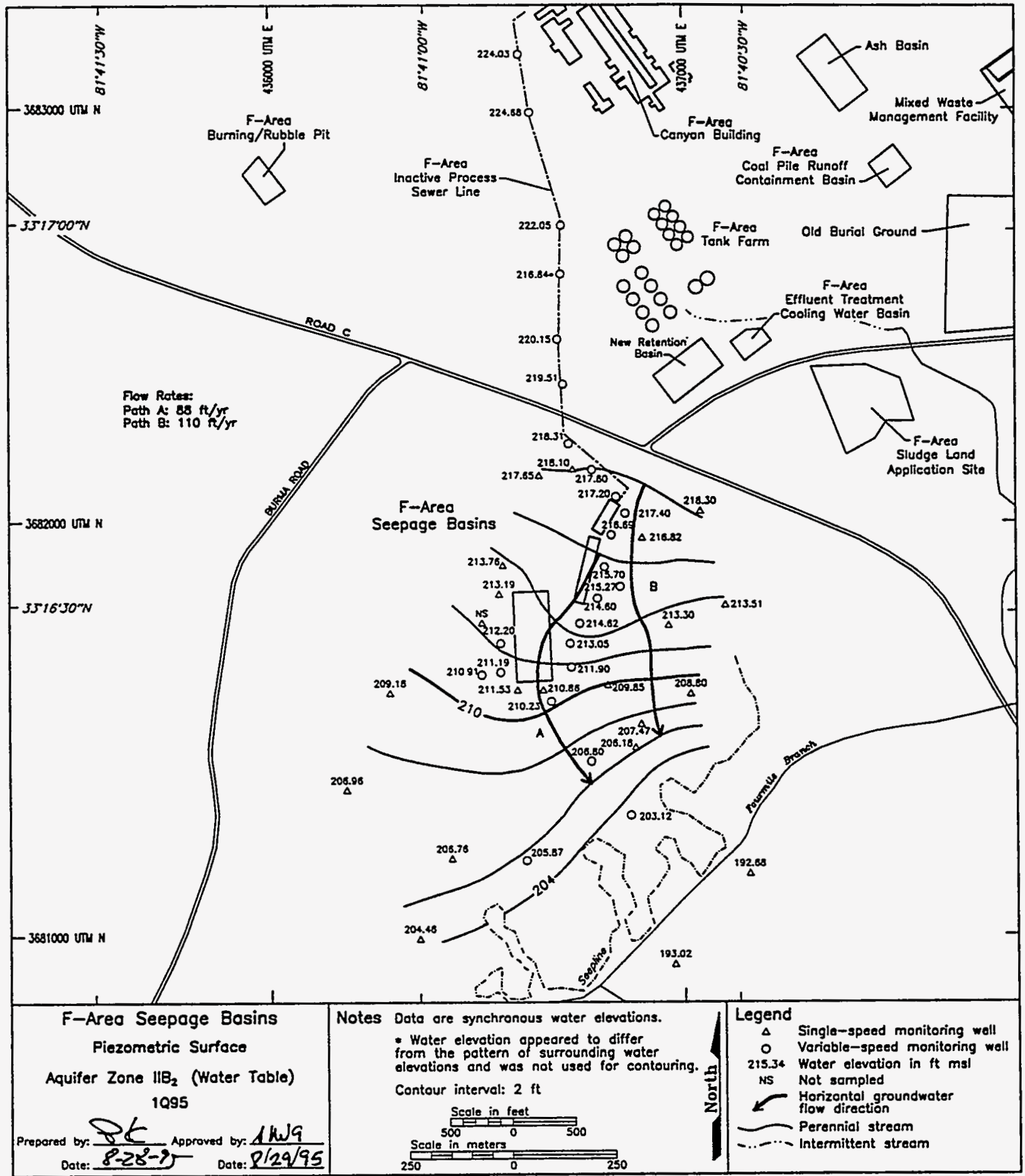

Figure 32. Piezometric Surface Map of Aquifer Zone $\|_{2} B_{2}$ (Water Table) at the F-Area Seepage Basins, First Quarter 1995 


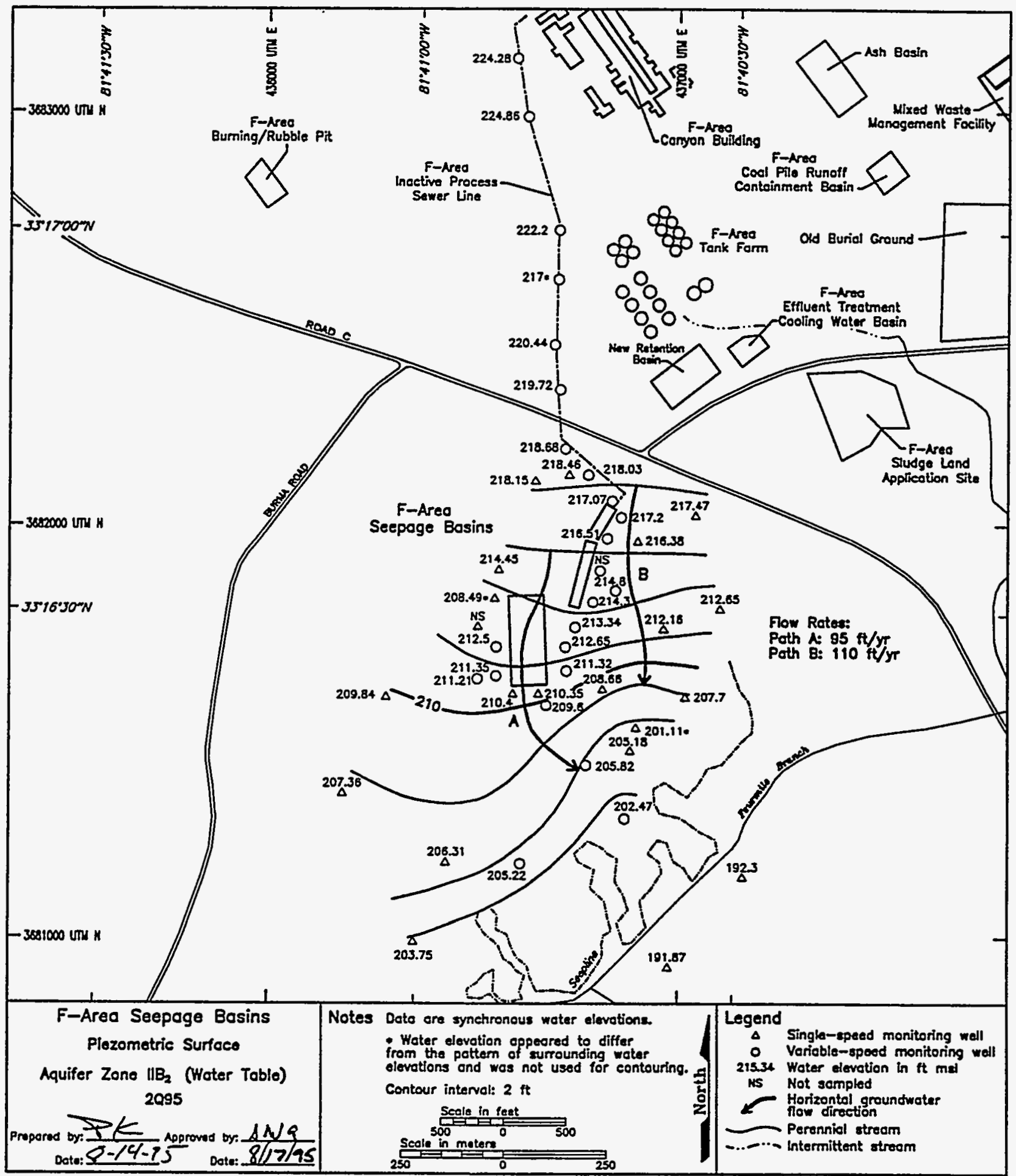

Figure 33. Piezometric Surface Map of Aquifer Zone $\|_{2} B_{2}$ (Water Table) at the F-Area Seepage Basins, Second Quarter 1995 


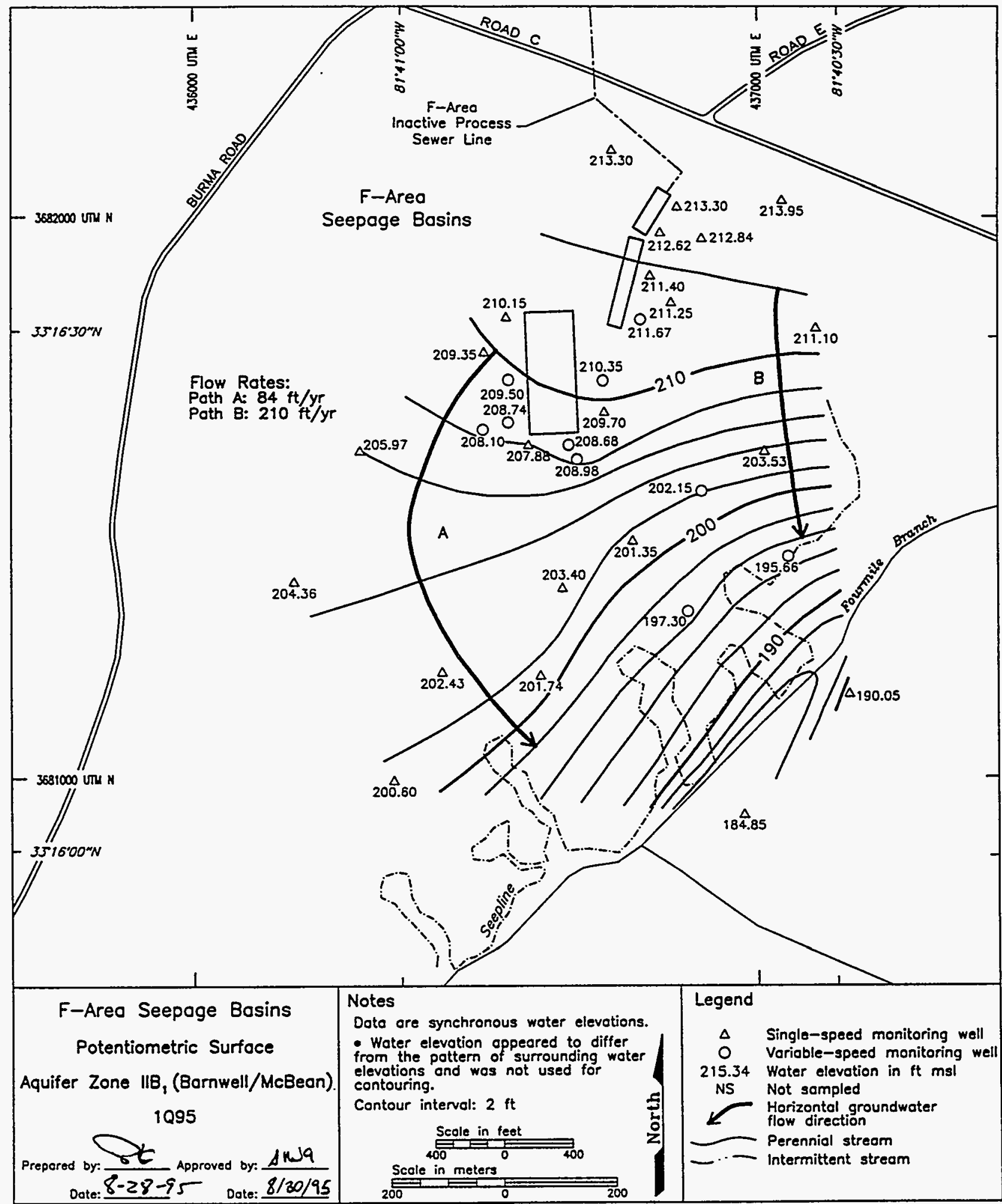

Figure 34. Potentiometric Surface Map of Aquifer Zone $\|_{1} B_{1}$ (Barnwell/McBean) at the F-Area Seepage Basins, First Quarter 1995 


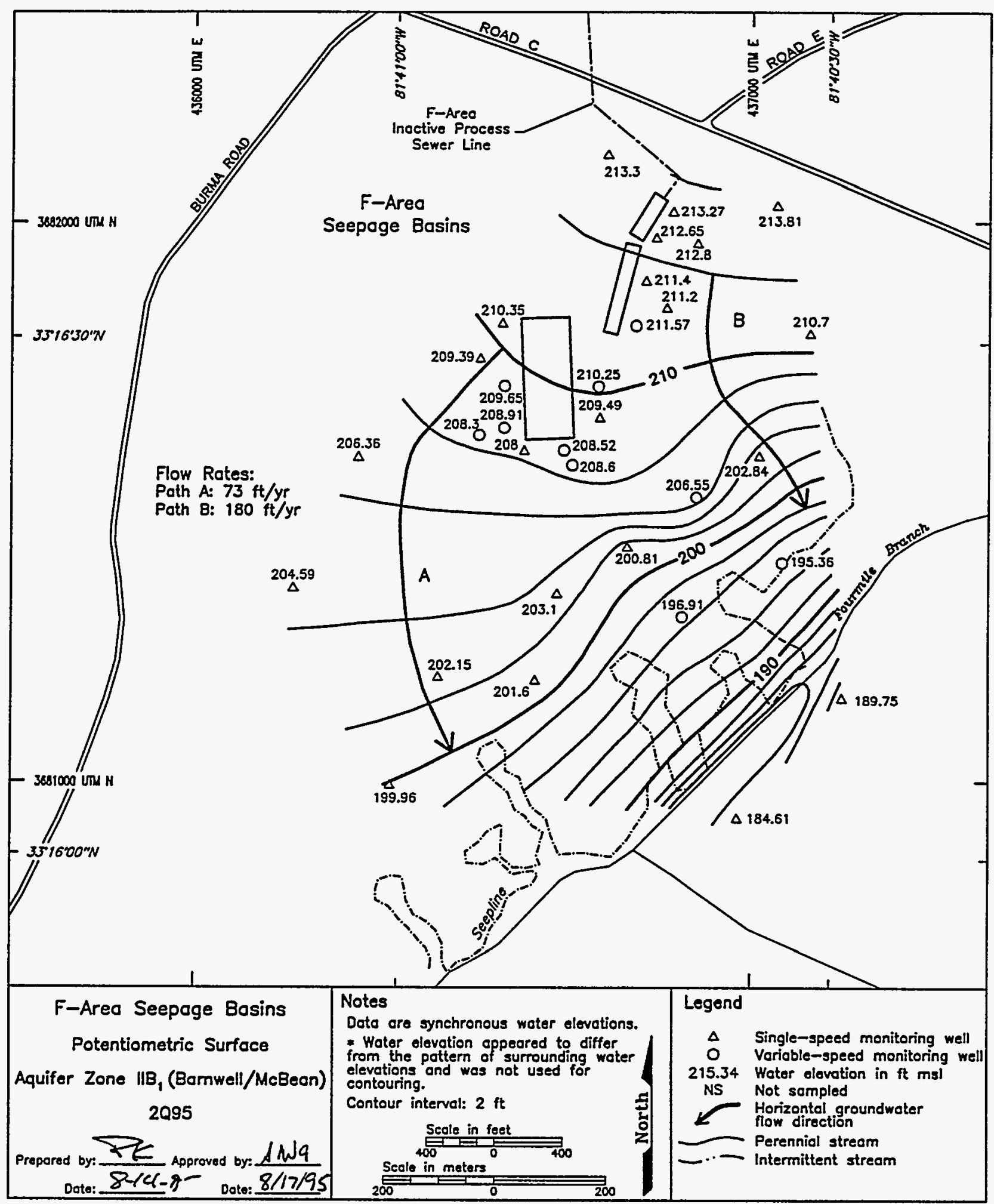

Figure 35. Potentiometric Surface Map of Aquifer Zone IIB, (Barnwell/McBean) at the F-Area Seepage Basins, Second Quarter 1995 


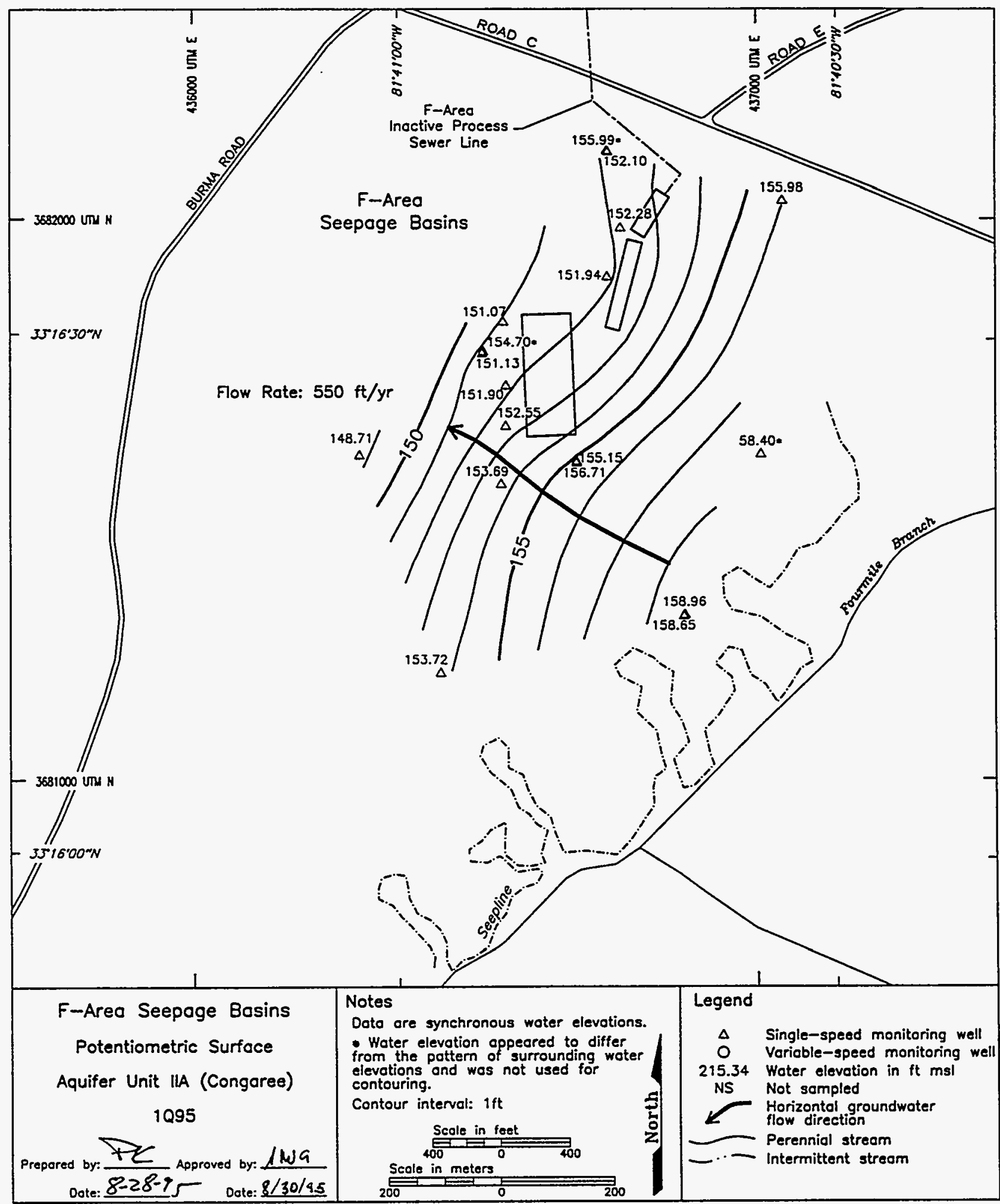

Figure 36. Potentiometric Surface Map of Aquifer Unit IIA (Congaree) at the F-Area Seepage Basins, First Quarter 1995 


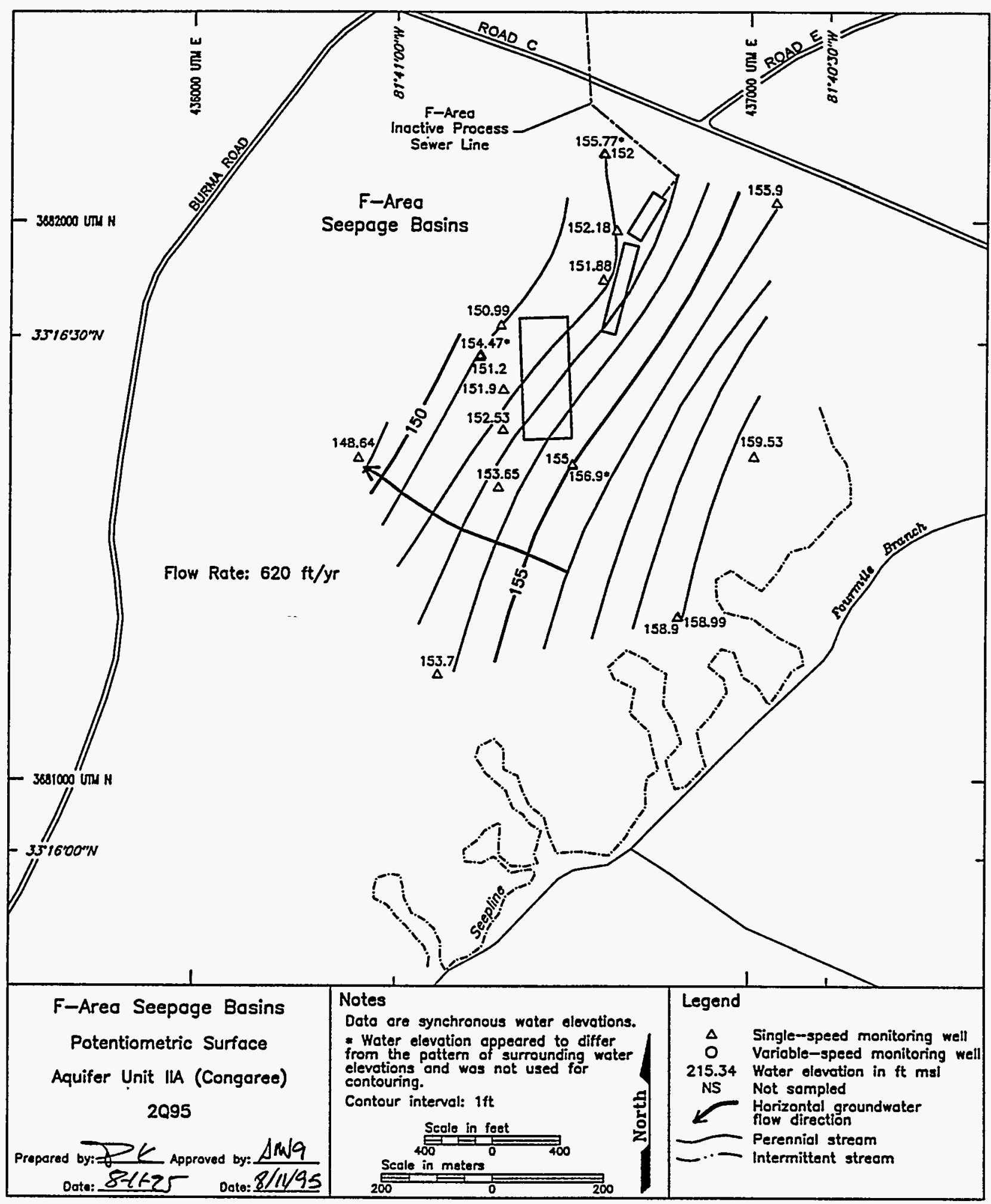

Figure 37. Potentiometric Surface Map of Aquifer Unit llA (Congaree) at the F-Area Seepage Basins, Second Quarter 1995 


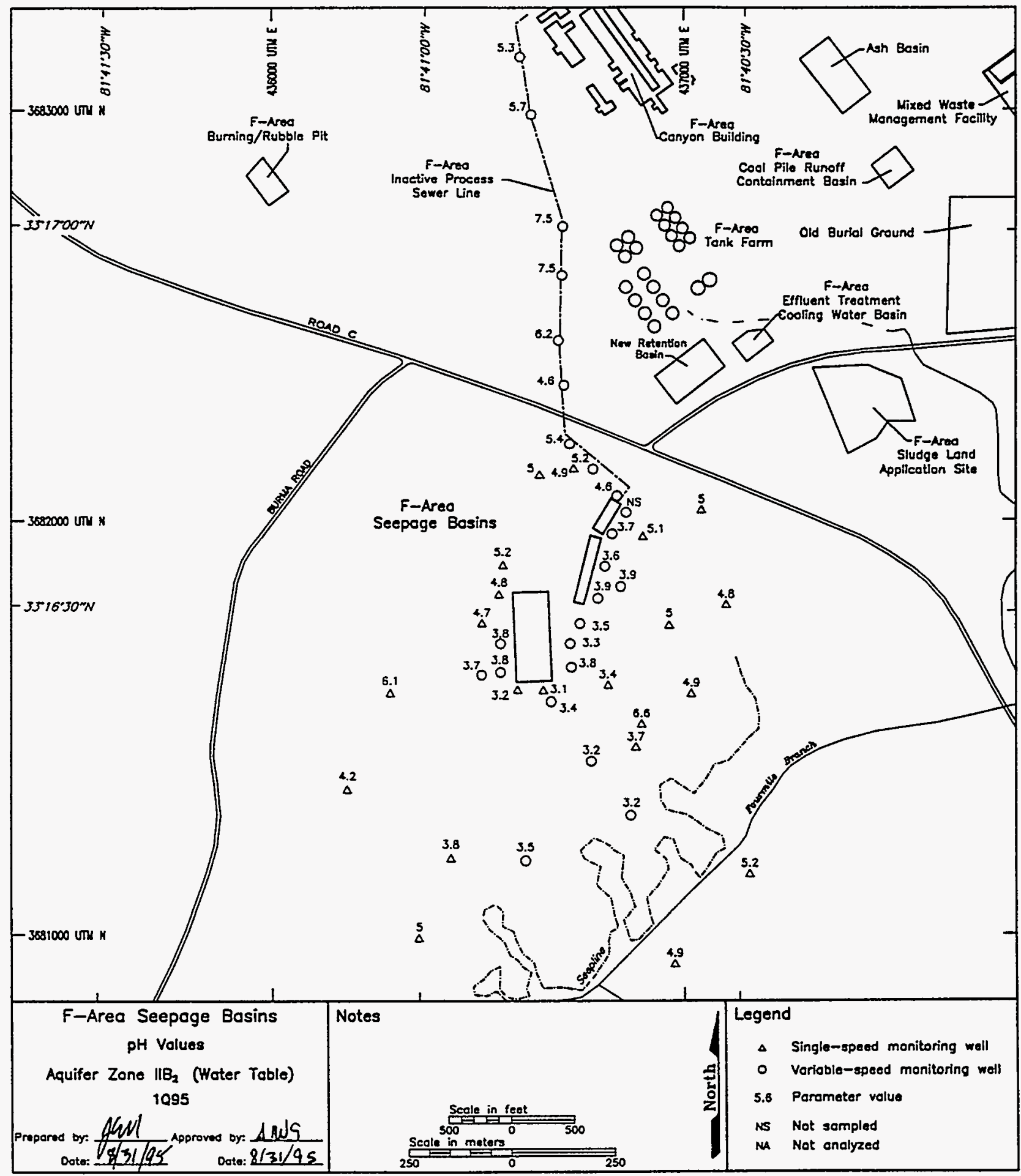

Figure 38. $\quad \mathrm{pH}$ Values in Aquifer Zone $\|_{1} B_{2}$ (Water Table) at the F-Area Seepage Basins, First Quarter 1995 


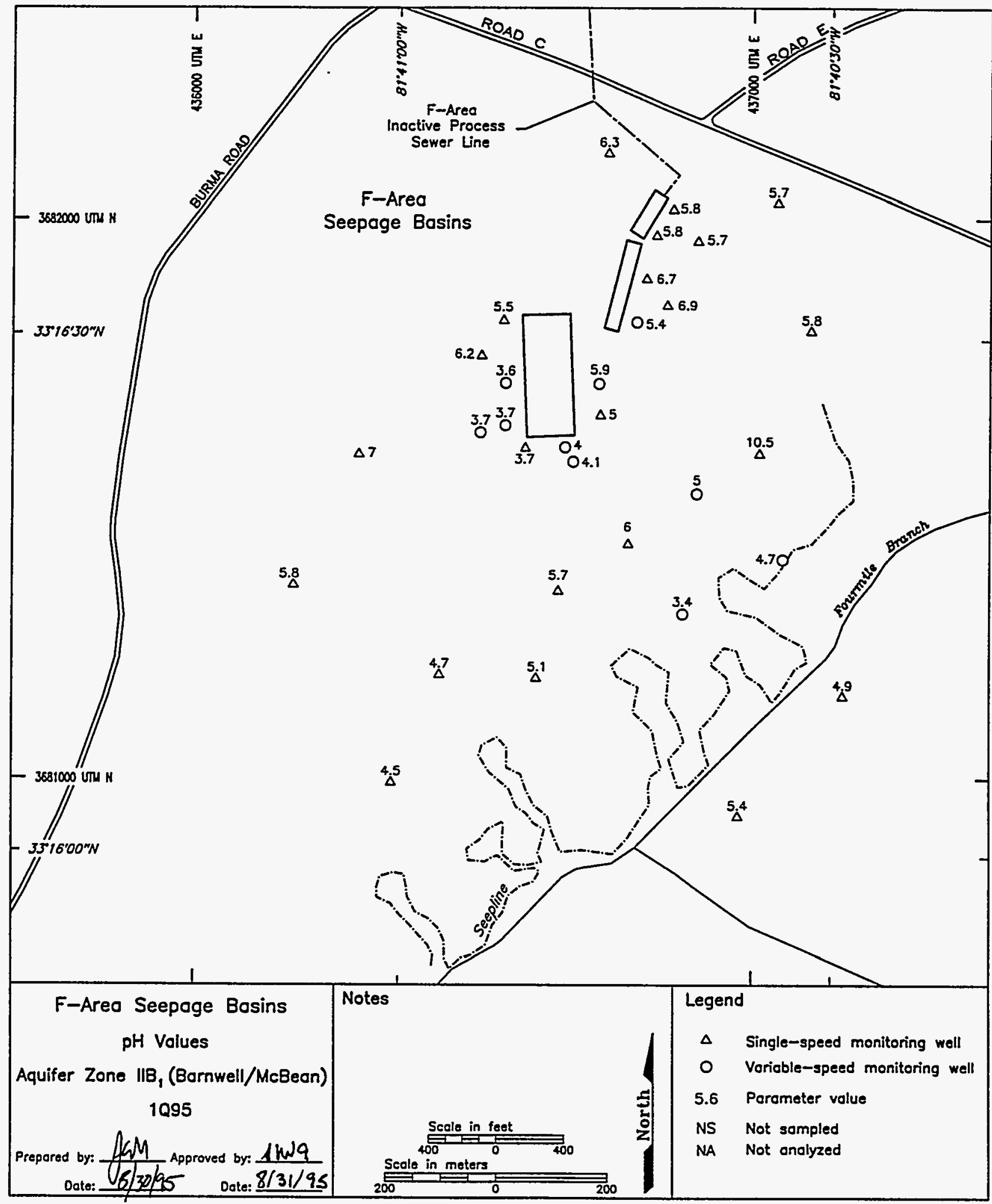

Figure 39. $\quad \mathrm{pH}$ Values in Aquifer Zone IIB, (Barnwell/McBean) at the F-Area Seepage Basins, First Quarter 1995 


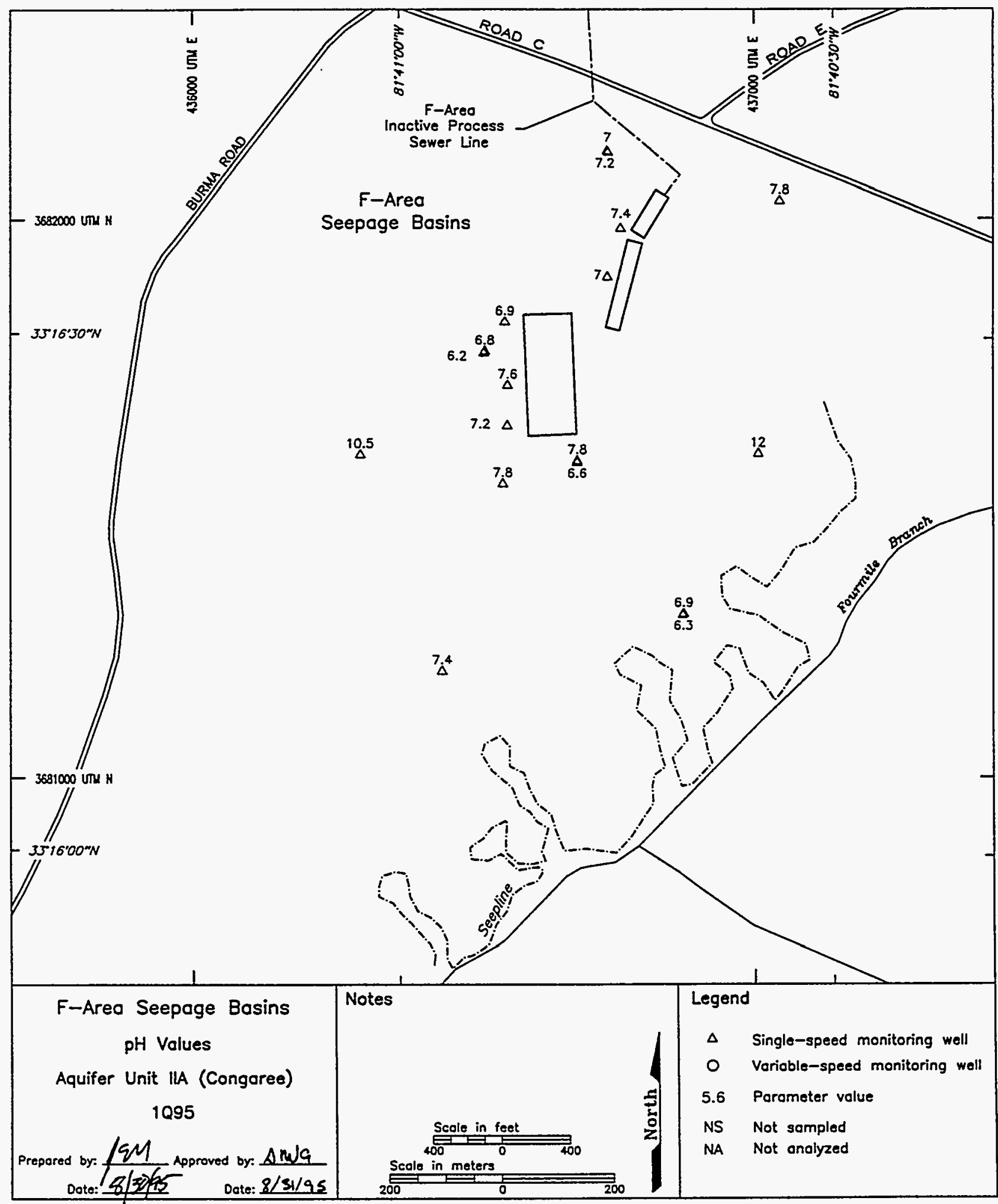

Figure 40. $\quad \mathrm{pH}$ Values in Aquifer Unit IIA (Congaree) at the F-Area Seepage Basins, First Quarter 1995 


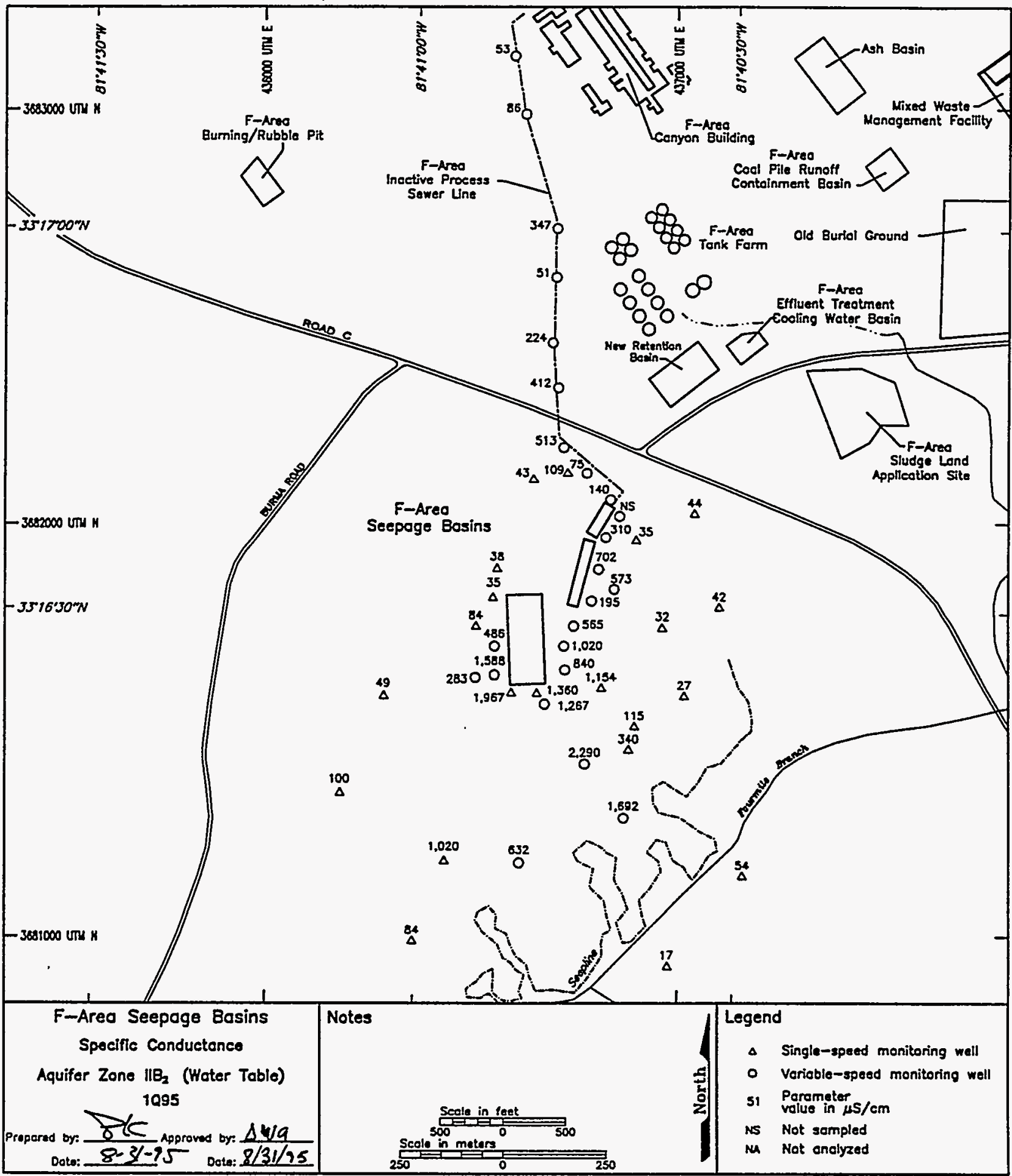

Figure 41. Specific Conductance in Aquifer Zone $\mathrm{IBB}_{2}$ (Water Table) at the F-Area Seepage Basins, First Quarter 1995 


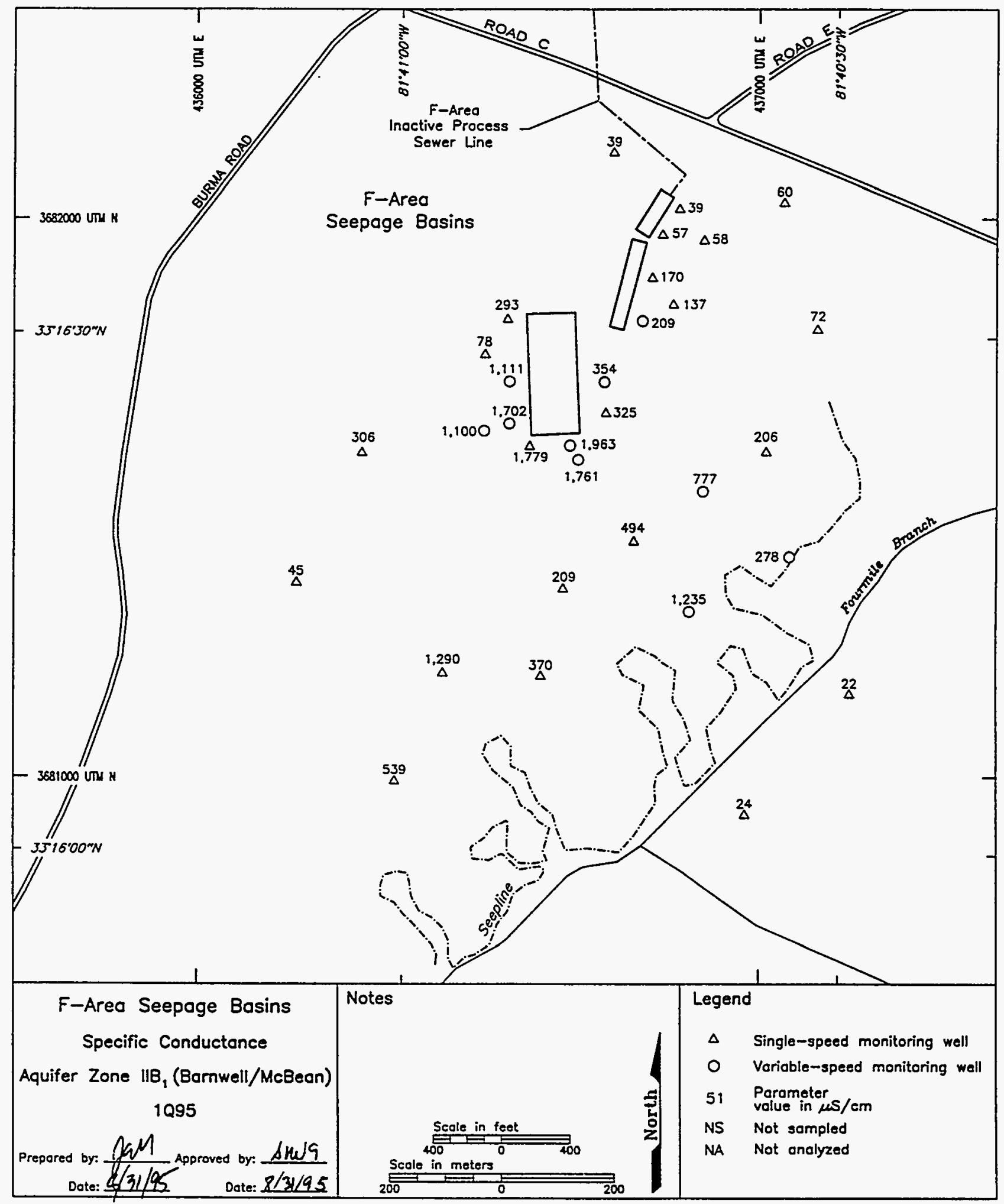

Figure 42. Specific Conductance in Aquifer Zone IIB, (Barnwell/McBean) at the F-Area Seepage Basins, First Quarter 1995 


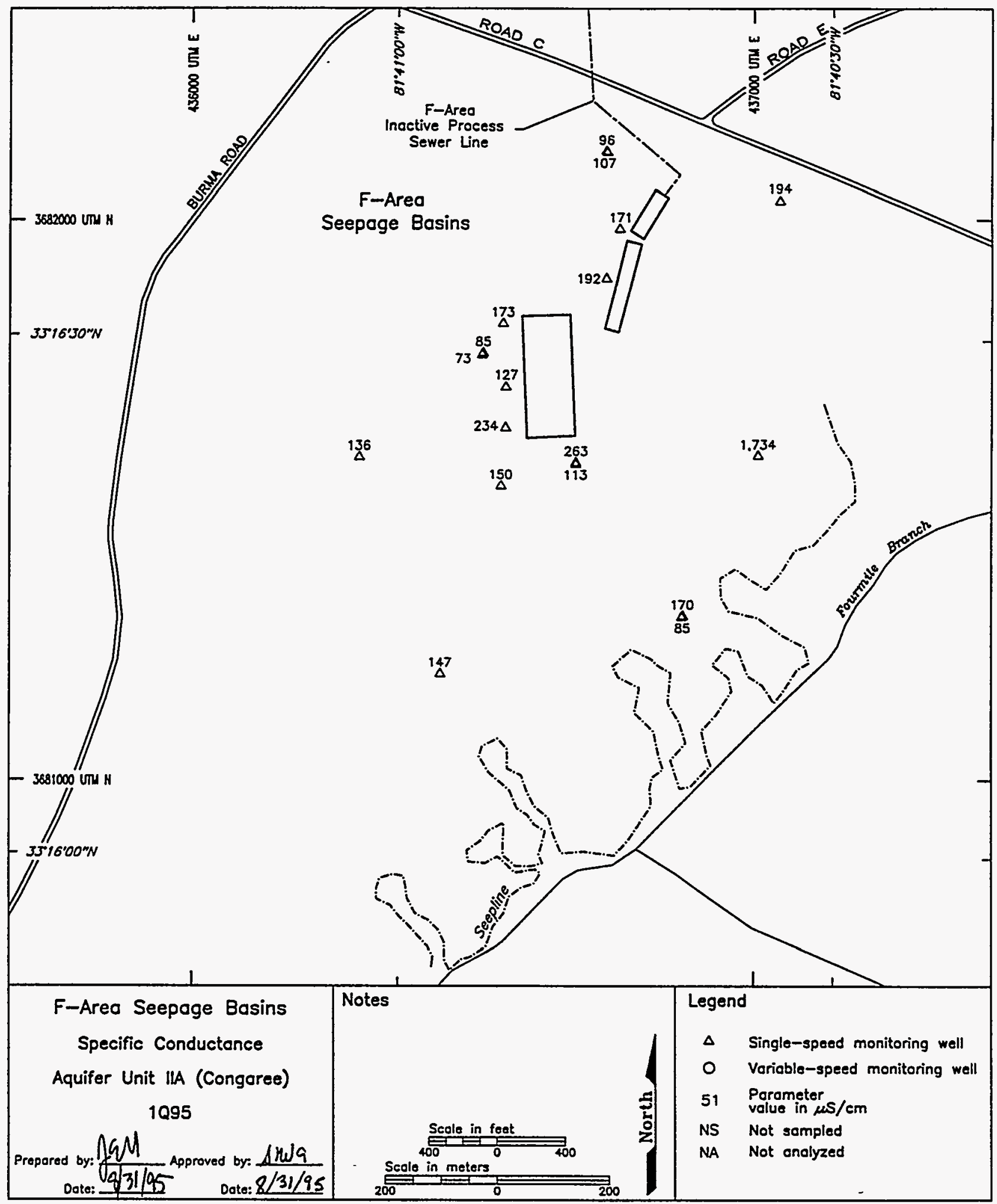

Figure 43. Specific Conductance in Aquifer Unit IIA (Congaree) at the F-Area Seepage Basins, First Quarter 1995 


\section{References}

Aadland, R. K., and H. W. Bledsoe, 1990. Classification of Hydrostratigraphic Units at the Savannah River Site, South Carolina, WSRC-RP-90-987. Westinghouse Savannah River Company, Savannah River Site, Aiken, SC.

Aucott, W. R., M. E. Davis, and G. K. Speiran, 1987. Geohydrologic Framework of the Coastal Plain Aquifers of South Carolina, U.S. Geological Survey Water-Resources Investigations Report 85-4271.

Du Pont (E. I. du Pont de Nemours \& Company), 1989. Final Safety Analysis Report of the Defense Waste Processing Facility, DPSTSA-200-10. Savannah River Plant, Aiken, SC.

GeoTrans, Inc., 1988. A Numerical Model of the Hydrogeological System Underlying the Savannah River Plant, Final report submitted to Savannah River Laboratory. GeoTrans, Inc., Sterling, VA.

Siple, G. E., 1967. Geology and Ground Water of the Savannah River Plant and Vicinity, South Carolina. Geological Survey Water-Supply Paper 1841, Reston, VA. 
WSRC-TR-95-0146-2

Unclassified

\section{Appendix D}

\section{Groundwater Monitoring Results Tables}


WSRC-TR-95-0146-2

Unclassified

THIS PAGE LEFT BLANK INTENTIONALLY. 


\section{Key to Reading the Tables}

The following abbreviations may appear in the data tables:

\section{Constituents}

\section{1,2,3,4,6,7,8-HPCDD \\ $1,2,3,4,6,7,8$-HPCDF \\ $1,2,3,4,7,8-H X C D D$ \\ $1,2,3,4,7,8-\mathrm{HXCDF}$ \\ Lindane \\ PCB}

$1,2,3,7,8-P C D D$

1,2,3,7,8-PCDF

Sp. conductance

TCDD

TCDF

\section{Laboratories}

\section{CN \\ EM}

GE and GP

SC

SP

TM

WA and WS

Sampling Codes
B
C
D
E
I
L
N
$P$
$S$
W
$x$

\section{Sampling Methods}

B

$\mathbf{P}$

\author{
1,2,3,4,6,7,8-heptachlorodibenzo-p-dioxin \\ $1,2,3,4,6,7,8$-heptachlorodibenzo-p-furan \\ $1,2,3,4,7,8$-hexachlorodibenzo-p-dioxin \\ 1,2,3,4,7,8-hexachlorodibenzo-p-furan \\ gamma-benzene hexachloride \\ polychlorinated biphenyl \\ 1,2,3,7,8-pentachlorodibenzo-p-dioxin \\ 1,2,3,7,8-pentachlorodibenzo-p-furan \\ specific conductance \\ tetrachlorodibenzo-p-dioxin \\ tetrachlorodibenzo-p-furan
}

Clemson Technical Center, Inc.

Environmental Protection Department/Environmental

Monitoring Section (EPD/EMS) Laboratory

General Engineering Laboratories, Inc.

Savannah River Technology Center

Spencer Testing Services, Inc.

TMA/Eberline

Roy F. Weston, Inc.

blank sample was collected

well was pumping continuously

well was dry

equipment blank was collected

well went dry during sampling; insufficient water to collect all samples

well went dry before sampling began; only depth to water can be determined

well was not stabilized before sampling began

inaccessibility or mechanical failure prevented sample

collection and field analysis of the water

no water in standpipe; for water-level events only

purged water must be containerized; only water-level

measurements were obtained

well went dry during purging; samples collected after well recovered

sample collected using an open-bucket bailer sample collected using a bladder pump 
Units

$\begin{array}{ll}\mathrm{mg} / \mathrm{L} & \text { milligrams per liter } \\ \mathrm{msl} & \text { mean sea level } \\ \mathrm{MSL} & \text { million structures per liter } \\ \mathrm{NTU} & \text { nephelometric turbidity unit } \\ \mathrm{pCi} / \mathrm{L} & \text { picocuries per liter } \\ \mathrm{pCi} / \mathrm{mL} & \text { picocuries per milliliter } \\ \mathrm{pH} & \mathrm{pH} \text { unit } \\ \mu \mathrm{g} / \mathrm{L} & \text { micrograms per liter } \\ \mu \mathrm{S} / \mathrm{cm} & \text { microsiemens per centimeter }\end{array}$

Other

CS
DF
E
H
Mod
PDWS
PVC
ST
TOC

sample collected using a single-speed centrifugal downhole pump sample collected using a variable-speed pump carbon steel

dilution factor column in data tables

exponential notation (e.g., 1.1E-09 $=1.1 \times 10^{-9}=$ 0.0000000011 )

holding time column in data tables

modifier column in data tables

primary drinking water standard

polyvinyl chloride

exceeded the final PDWS or screening level column in

data tables

top of casing

\section{Holding Times}

Standard analytical methods include a limit, called holding time, on the maximum elapsed time between sample collection and extraction or analysis by the laboratory. In the data tables, a large bullet $(\cdot)$ in the $H$ (holding time) column indicates that holding time was exceeded. Analyses performed beyond holding times may not yield valid results.

The South Carolina Department of Health and Environmental Control (SCDHEC) allows only 15 minutes to elapse between sampling and analysis for $\mathrm{pH}$. Thus, only field $\mathrm{pH}$ measurements can meet the holding time criterion; laboratory $\mathrm{pH}$ analyses always will exceed it.

The laboratory procedure used for the determination of specific conductance allows one day to elapse between sampling and analysis. Thus, laboratory specific conductance measurements may exceed the holding time criterion.

\section{Data Rounding}

Constituent results in analytical results tables that appear to equal the final PDWS but are not marked in the ST (exceeded the final PDWS or screening level) column are below the final PDWS in the database. Values stored in the database contain more significant digits than the reported results. Apparent discrepancies in the tables are due to the rounding of reported results. 


\section{Data Qualification}

The contract laboratories submit sample- or batch-specific quality assurance/quality control information either at the same time as analytical results or in a quarterly summary. Properly defined and used data modifiers (also referred to as qualifiers) can be a key component in assessing data usability. Modifiers designed by EPD/EMS and provided to the primary laboratories are defined below. These modifiers appear in the data tables under the column Mod. The lettered modifiers are based on EPA's STORET codes.

\section{Modifiers}

Data are not qualified. Numbers should be interpreted exactly as reported.

I

$J$

$L$

M

$\mathbf{R}$

T

U

V or V

Y

1

2

3
The value in the result field is the instrument reading, not the sample quantitation limit. Always used with the result qualifier $U$.

Value is estimated because quantitation in the sample or in associated quality control samples did not meet specifications.

Value is off-scale high. The actual value is not known but is known to be greater than the value shown.

Presence of the analyte is verified but not quantified.

Result was rejected because performance requirements in the sample analysis or associated quality control analyses were not met.

Analyte was not detected; if present, it was below the criteria for detection.

Material analyzed for but not detected. Analytical result reported is less than the sample quantitation limit.

Analyte was detected in an associated method blank.

Result was obtained from an unpreserved or improperly preserved sample. Data may not be accurate.

Result may be an underestimation of the true value due to analytical bias.

Result may be an overestimation of the true value due to analytical bias.

The associated result may be of poor precision (high variability) due to analytical bias.

Note: These are only some of the qualifiers present in the database. All modifiers associated with the data are published in the result tables of EPD/EMS' quarterly groundwater monitoring reports, the official repository of the data. 
Table 1. Maximum Levels of Constituents Exceeding the Groundwater Protection Standards

Aquifer Zone IIB $_{2}$ (Water Table)

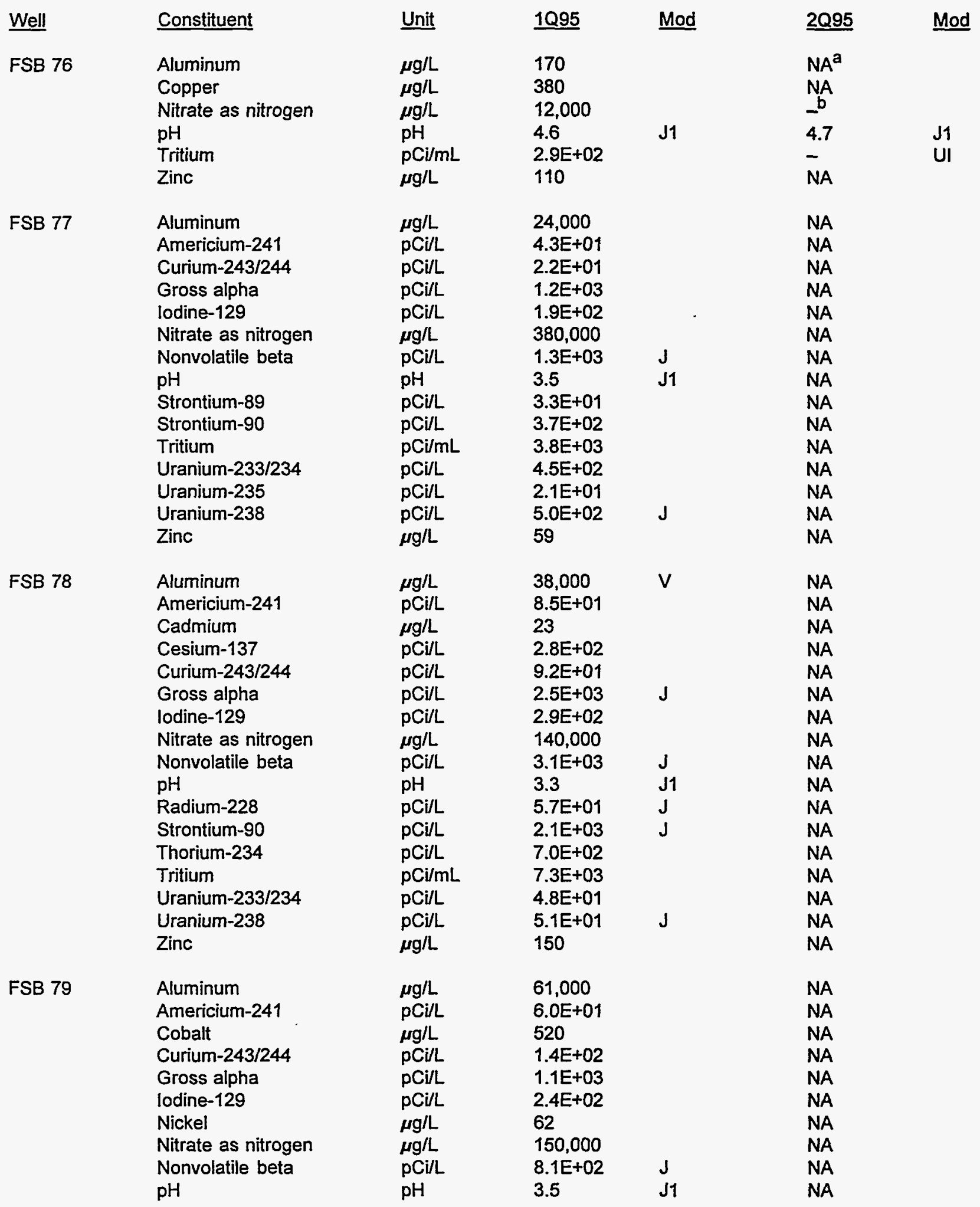




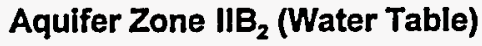

\begin{tabular}{|c|c|c|c|c|c|c|}
\hline \multirow[t]{7}{*}{ Well } & Constituent & Unit & $\underline{1 Q 95}$ & Mod & $\underline{2 Q 95}$ & Mod \\
\hline & Strontium-90 & pCi/L & $1.9 E+02$ & & NA & \\
\hline & Tritium & $\mathrm{pCi} / \mathrm{mL}$ & $1.0 E+04$ & & NA & \\
\hline & Uranium-233/234 & pCi/L & 2.3E+02 & & NA & \\
\hline & Uranium-235 & pCi/L & $1.8 E+01$ & & NA & \\
\hline & Uranium-238 & pCill & 4.7E+02 & $\mathrm{J}$ & NA & \\
\hline & Zinc & $\mu g / L$ & 140 & & NA & \\
\hline \multirow[t]{8}{*}{ FSB 87D } & Aluminum & $\mu g / L$ & 140 & & NA & \\
\hline & Copper & $\mu g / L$ & 230 & & NA & \\
\hline & Gross alpha & pCill & $2.5 E+01$ & & $5.2 E+01$ & $\mathrm{~J}$ \\
\hline & lodine-129 & pCill & $2.9 E+01$ & & NA & \\
\hline & $\mathrm{pH}$ & pH & 5.1 & J1 & 4.6 & J1 \\
\hline & Tritium & $\mathrm{pCi} / \mathrm{mL}$ & $3.6 E+01$ & & - & UI \\
\hline & Uranium-238 & pCill & $1.9 E+01$ & & NA & \\
\hline & Zinc & $\mu \mathrm{g} / \mathrm{L}$ & 62 & & NA & \\
\hline \multirow[t]{13}{*}{ FSB 89D } & Aluminum & $\mu \mathrm{g} / \mathrm{L}$ & 6,600 & & NA & \\
\hline & Americium-241 & $\mathrm{pCi} / \mathrm{L}$ & $1.1 E+01$ & & NA & \\
\hline & Gross alpha & $\mathrm{pCi} / \mathrm{L}$ & $2.2 E+02$ & & NA & \\
\hline & lodine-129 & pCi/L & $1.4 E+02$ & & NA & \\
\hline & Mercury & $\mu \mathrm{g} / \mathrm{L}$ & 4.8 & & NA & \\
\hline & Nitrate as nitrogen & $\mu g / L$ & 32,000 & & NA & \\
\hline & Nonvolatile beta & pCi/L & $1.0 E+03$ & & NA & \\
\hline & $\mathrm{pH}$ & pH & & J1 & NA & \\
\hline & Strontium-90 & pCi/L & $4.3 E+02$ & & NA & \\
\hline & Tritium & $\mathrm{pCi} / \mathrm{mL}$ & $1.8 E+03$ & & NA & \\
\hline & Uranium-233/234 & pCill & $9.1 E+01$ & & NA & \\
\hline & Uranium-238 & pCi/L & $1.1 E+02$ & & NA & \\
\hline & Zinc & $\mu g / L$ & 26 & & NA & \\
\hline \multirow[t]{15}{*}{ FSB 9OD } & Aluminum & $\mu \mathrm{g} / \mathrm{L}$ & 29,000 & & NA & \\
\hline & Americium-241 & pCill & $1.9 E+01$ & & NA & \\
\hline & Copper & $\mu \mathrm{g} / \mathrm{L}$ & 61 & & NA & \\
\hline & Gross alpha & $\mathrm{pCi} / \mathrm{L}$ & $7.2 E+02$ & $\mathbf{J}$ & NA & \\
\hline & lodine-129 & $\mathrm{pCi} / \mathrm{L}$ & $2.2 E+02$ & & NA & \\
\hline & Mercury & $\mu \mathrm{g} / \mathrm{L}$ & 14 & V & NA & \\
\hline & Nitrate as nitrogen & $\mu \mathrm{g} / \mathrm{L}$ & 97,000 & & NA & \\
\hline & Nonvolatile beta & $\mathrm{pCi} / \mathrm{L}$ & $1.1 E+03$ & J & NA & \\
\hline & pH & pH & 3.8 & $\mathrm{~J} 1$ & NA & \\
\hline & Radium-228 & pCill & $2.5 E+01$ & J & NA & \\
\hline & Strontium-90 & $\mathrm{pCi} / \mathrm{L}$ & $3.5 E+02$ & & NA & \\
\hline & Tritium & $\mathrm{pCi} / \mathrm{mL}$ & $8.2 E+03$ & & NA & \\
\hline & Uranium-233/234 & pCi/l & $3.3 E+02$ & & NA & \\
\hline & Uranium-238 & $\mathrm{pCi} / \mathrm{L}$ & $3.7 E+02$ & $\mathbf{J}$ & NA & \\
\hline & Zinc & $\mu g / L$ & 79 & & NA & \\
\hline \multirow[t]{6}{*}{ FSB 91D } & Aluminum & $\mu \mathrm{g} / \mathrm{L}$ & 7,200 & & NA & \\
\hline & Gross alpha & pCill & $1.9 E+02$ & & NA & \\
\hline & lodine-129 & $\mathrm{pCi} / \mathrm{L}$ & $2.4 E+01$ & & NA & \\
\hline & Nitrate as nitrogen & $\mu g / L$ & 18,000 & $\mathrm{~J}$ & NA & \\
\hline & Nonvolatile beta & pCill & 1.1E+02 & & NA & \\
\hline & $\begin{array}{l}\mathrm{pH} \\
\text { Strontium-90 }\end{array}$ & $\begin{array}{l}\mathrm{pH} \\
\mathrm{pCin}\end{array}$ & $\begin{array}{l}3.8 \\
2.3 F+01\end{array}$ & J1 & NA & \\
\hline
\end{tabular}


Aquifer Zone $\| \mathrm{B}_{2}$ (Water Table)

Well

FSB 92D

FSB 94DR

Constituent
Uranium-233/234
Uranium-238

Aluminum

Americium-241

Cadmium

Curium-243/244

Cyanide

Gross alpha

lodine-129

Nitrate as nitrogen

Nonvolatile beta

$\mathrm{pH}$

Radium-226

Radium-228

Strontium-89

Strontium-90

Tritium

Uranium-233/234

Uranium-238

Zinc

Aluminum

Cadmium

Gross alpha

lodine-129

Nitrate as nitrogen

Nonvolatile beta

$\mathrm{pH}$

Radium-228

Strontium-89

Strontium-90

Tritium

Uranium-233/234

Uranium-238

Zinc

Aluminum
Americium-241
Cesium-137
Copper
Curium-243/244
Gross alpha
lodine-129
Nitrate as nitrogen
Nonvolatile beta
pH
Radium-228
Strontium-89
Strontium-90
Thorium-234
Tritium
Uranium-233/234

\begin{tabular}{|c|c|c|c|}
\hline Unit & 1Q95 & Mod & 2Q95 \\
\hline $\begin{array}{l}\mathrm{pCi} / \mathrm{L} \\
\mathrm{pCi} / \mathrm{L}\end{array}$ & $\begin{array}{l}6.8 E+01 \\
1.1 E+02\end{array}$ & & $\begin{array}{l}\text { NA } \\
\text { NA }\end{array}$ \\
\hline$\mu g / L$ & 58,000 & & NA \\
\hline $\mathrm{pCi} / \mathrm{L}$ & $1.0 \mathrm{E}+01$ & & NA \\
\hline$\mu \mathrm{g} / \mathrm{L}$ & 15 & & NA \\
\hline $\mathrm{pCi} / \mathrm{L}$ & $9.0 \mathrm{E}+00$ & & NA \\
\hline$\mu \mathrm{g} / \mathrm{L}$ & 5.4 & JV & NA \\
\hline $\mathrm{pCi} / \mathrm{L}$ & $9.9 E+02$ & $J$ & NA \\
\hline pCi/L & 1.2E+02 & & NA \\
\hline$\mu g / L$ & 130,000 & & NA \\
\hline $\mathrm{pCi} / \mathrm{L}$ & 1.7E+03 & $\mathbf{J}$ & NA \\
\hline $\mathrm{pH}$ & 3.5 & J1 & NA \\
\hline $\mathrm{pCi} / \mathrm{L}$ & $5.0 \mathrm{E}+01$ & $J$ & NA \\
\hline $\mathrm{pCi} / \mathrm{L}$ & $4.4 E+01$ & $\mathrm{~J}$ & NA \\
\hline $\mathrm{pCi} / \mathrm{L}$ & $1.0 E+02$ & & NA \\
\hline $\mathrm{pCi} / \mathrm{L}$ & $3.4 E+02$ & & NA \\
\hline $\mathrm{pCi} / \mathrm{mL}$ & 8.7E+03 & & NA \\
\hline $\mathrm{pCi} / \mathrm{L}$ & $3.0 E+02$ & & NA \\
\hline $\mathrm{pCi} / \mathrm{L}$ & $3.6 E+02$ & $J$ & NA \\
\hline$\mu g / L$ & 96 & & NA \\
\hline$\mu \mathrm{g} / \mathrm{L}$ & 34,000 & & NA \\
\hline$\mu \mathrm{g} / \mathrm{L}$ & 13 & & NA \\
\hline $\mathrm{pCi} / \mathrm{L}$ & $3.3 E+02$ & J & NA \\
\hline $\mathrm{pCi} / \mathrm{L}$ & $1.0 E+02$ & & NA \\
\hline$\mu \mathrm{g} / \mathrm{L}$ & 120,000 & & NA \\
\hline $\mathrm{pCi} / \mathrm{L}$ & $2.0 \mathrm{E}+03$ & $J$ & NA \\
\hline $\mathrm{pH}$ & 3.9 & J1 & NA \\
\hline $\mathrm{pCi} / \mathrm{L}$ & $3.7 E+01$ & $J$ & NA \\
\hline $\mathrm{pCi} / \mathrm{L}$ & 2.6E+02 & & NA \\
\hline $\mathrm{pCi} / \mathrm{L}$ & 7.1E+02 & & NA \\
\hline $\mathrm{pCi} / \mathrm{mL}$ & 1.0E +04 & & NA \\
\hline $\mathrm{pCi} / \mathrm{L}$ & $5.9 E+01$ & & NA \\
\hline $\mathrm{pCi} / \mathrm{L}$ & 8.2E+01 & $\mathbf{J}$ & NA \\
\hline$\mu \mathrm{g} / \mathrm{L}$ & 62 & & NA \\
\hline$\mu g / L$ & 64,000 & & NA \\
\hline $\mathrm{pCi} / \mathrm{L}$ & $9.0 E+01$ & & NA \\
\hline $\mathrm{pCi} / \mathrm{L}$ & 2.8E+02 & & NA \\
\hline$\mu \mathrm{g} / \mathrm{L}$ & 67 & & NA \\
\hline $\mathrm{pCi} / \mathrm{L}$ & 1.6E+02 & & NA \\
\hline $\mathrm{pCi} / \mathrm{L}$ & $1.4 E+03$ & & NA \\
\hline $\mathrm{pCi} / \mathrm{L}$ & $1.4 E+02$ & & NA \\
\hline$\mu \mathrm{g} / \mathrm{L}$ & 130,000 & & NA \\
\hline $\mathrm{pCi} / \mathrm{L}$ & 1.3E+03 & $\mathbf{J}$ & NA \\
\hline $\mathrm{pH}$ & 3.3 & J1 & NA \\
\hline $\mathrm{pCi} / \mathrm{L}$ & 5.7E+01 & $\mathbf{J}$ & NA \\
\hline $\mathrm{pCi} / \mathrm{L}$ & 2.1E+01 & & NA \\
\hline $\mathrm{pCi} / \mathrm{L}$ & $2.3 E+02$ & & NA \\
\hline $\mathrm{pCi} / \mathrm{L}$ & $7.0 E+02$ & & NA \\
\hline $\mathrm{pCi} / \mathrm{mL}$ & $8.9 E+03$ & & NA \\
\hline pCi/L & $6.7 E+02$ & & NA \\
\hline
\end{tabular}


Aquifer Zone $\mathrm{IB}_{2}$ (Water Table)

\begin{tabular}{|c|c|c|c|c|c|c|}
\hline \multirow[t]{4}{*}{ Well } & Constituent & Unit & $1 \mathrm{Q95}$ & Mod & $\underline{2 Q 95}$ & Mod \\
\hline & Uranium-235 & pCill & $3.9 E+01$ & & NA & \\
\hline & Uranium-238 & $\mathrm{pCi} / \mathrm{L}$ & $1.3 E+03$ & $\mathbf{J}$ & NA & \\
\hline & Zinc & $\mu \mathrm{g} / \mathrm{L}$ & 140 & & NA & \\
\hline \multirow[t]{18}{*}{ FSB 95DR } & Aluminum & $\mu g / L$ & 47,000 & & NA & \\
\hline & Americium-241 & pCill & $8.4 E+01$ & & NA & \\
\hline & Cesium-137 & pCi/L & $4.4 E+02$ & & NA & \\
\hline & Curium-243/244 & pCill & 8.7E+01 & & NA & \\
\hline & Curium-245/246 & $\mathrm{pCi} / \mathrm{L}$ & $7.9 E+00$ & & NA & \\
\hline & Gross alpha & $\mathrm{pCi} / \mathrm{L}$ & $2.0 E+03$ & & NA & \\
\hline & lodine-129 & pCi/L & $2.3 E+02$ & & NA & \\
\hline & Nitrate as nitrogen & $\mu \mathrm{g} / \mathrm{L}$ & 230,000 & & NA & \\
\hline & Nonvolatile beta & $\mathrm{pCi} / \mathrm{L}$ & 1.3E+03 & $\mathbf{J}$ & NA & \\
\hline & $\mathrm{pH}$ & $\mathrm{pH}$ & 3.3 & J1 & NA & \\
\hline & Radium-228 & $\mathrm{pCi} / \mathrm{L}$ & $4.4 E+01$ & & NA & \\
\hline & Strontium-90 & $\mathrm{pCi} / \mathrm{L}$ & $1.5 \mathrm{E}+02$ & & NA & \\
\hline & Thorium-234 & $\mathrm{pCi} / \mathrm{L}$ & $9.5 \mathrm{E}+02$ & & NA & \\
\hline & Tritium & $\mathrm{pCi} / \mathrm{mL}$ & $1.2 E+04$ & & NA & \\
\hline & Uranium-233/234 & pCill & $4.2 E+02$ & & NA & \\
\hline & Uranium-235 & pCill & $3.0 E+01$ & & NA & \\
\hline & Uranium-238 & $\mathrm{pCi} / \mathrm{L}$ & $1.2 E+03$ & $\mathbf{J}$ & NA & \\
\hline & Zinc & $\mu \mathrm{g} / \mathrm{L}$ & 140 & & NA & \\
\hline \multirow[t]{18}{*}{ FSB 97D } & Aluminum & $\mu g / L$ & 53,000 & V & NA & \\
\hline & Americium-241 & pCill & $5.0 \mathrm{E}+01$ & & NA & \\
\hline & Barium & $\mu g / L$ & 1,200 & V & NA & \\
\hline & Cobalt & $\mu g / L$ & 81 & & NA & \\
\hline & Copper & $\mu g / L$ & 64 & & NA & \\
\hline & Curium-243/244 & pCill & $4.9 \mathrm{E}+01$ & & NA & \\
\hline & Gross alpha & pCi/L & $1.4 E+03$ & $J$ & NA & \\
\hline & lodine-129 & pCill & $7.3 E+01$ & & NA & \\
\hline & Nitrate as nitrogen & $\mu \mathrm{g} / \mathrm{L}$ & 220,000 & & NA & \\
\hline & Nonvolatile beta & pCi/L & 1.4E+03 & $\mathbf{J}$ & NA & \\
\hline & $\mathrm{pH}$ & $\mathrm{pH}$ & 3.8 & J1 & NA & \\
\hline & Radium-228 & $\mathrm{pCi} / \mathrm{L}$ & $4.3 \mathrm{E}+01$ & $J$ & NA & \\
\hline & Strontium-90 & $\mathrm{pCi} / \mathrm{L}$ & $5.9 E+02$ & $\mathbf{J}$ & NA & \\
\hline & Tritium & $\mathrm{pCi} / \mathrm{mL}$ & $1.0 \mathrm{E}+04$ & & NA & \\
\hline & Uranium-233/234 & $\mathrm{pCi} / \mathrm{L}$ & $4.5 E+02$ & & NA & \\
\hline & Uranium-235 & $\mathrm{pCi} / \mathrm{L}$ & $3.2 E+01$ & & NA & \\
\hline & Uranium-238 & pCill & $1.2 \mathrm{E}+03$ & & NA & \\
\hline & Zinc & $\mu g / L$ & 180 & & NA & \\
\hline \multirow[t]{11}{*}{ FSB 98D } & Aluminum & $\mu g / L$ & 16,000 & & NA & \\
\hline & Americium-241 & pCill & 2.3E+01 & & NA & \\
\hline & Cadmium & $\mu \mathrm{g} / \mathrm{L}$ & 17 & & NA & \\
\hline & Curium-243/244 & $\mathrm{pCi} / \mathrm{L}$ & 2.1E+01 & & NA & \\
\hline & Gross alpha & pCill & $8.9 E+02$ & $\mathbf{J}$ & NA & \\
\hline & lodine-129 & pCill & $2.5 \mathrm{E}+01$ & & NA & \\
\hline & Nitrate as nitrogen & $\mu g / L$ & 64,000 & & NA & \\
\hline & Nonvolatile beta & $\mathrm{pCi} / \mathrm{L}$ & $1.3 \mathrm{E}+03$ & $\mathbf{J}$ & NA & \\
\hline & $\mathrm{pH}$ & $\mathrm{pH}$ & 3.9 & J1 & NA & \\
\hline & Radium-228 & $\mathrm{pCi} / \mathrm{L}$ & $2.5 E+01$ & $\mathbf{J}$ & NA & \\
\hline & Strontium-89 & pCill & $1.1 E+02$ & & NA & \\
\hline
\end{tabular}


Aquifer Zone IIB $_{2}$ (Water Table)

\begin{tabular}{|c|c|c|c|c|c|c|}
\hline \multirow[t]{8}{*}{ Well } & Constituent & $\underline{\text { Unit }}$ & $1 Q 95$ & Mod & $\underline{2 Q 95}$ & Mod \\
\hline & Strontium-90 & $\mathrm{pCi} / \mathrm{L}$ & $2.8 E+02$ & & NA & \\
\hline & Thorium-234 & $\mathrm{pCi} / \mathrm{L}$ & $4.6 \mathrm{E}+02$ & & NA & \\
\hline & Tritium & $\mathrm{pCi} / \mathrm{mL}$ & $3.8 \mathrm{E}+03$ & & NA & \\
\hline & Uranium-233/234 & $\mathrm{pCi} / \mathrm{L}$ & $2.4 \mathrm{E}+02$ & & NA & \\
\hline & Uranium-235 & pCi/L & $1.5 \mathrm{E}+01$ & & NA & \\
\hline & Uranium-238 & $\mathrm{pCi} / \mathrm{L}$ & $5.3 \mathrm{E}+02$ & $J$ & NA & \\
\hline & Zinc & $\mu \mathrm{g} / \mathrm{L}$ & 96 & & NA & \\
\hline \multirow{4}{*}{ FSB 99D } & Aluminum & $\mu \mathrm{g} / \mathrm{L}$ & 190 & & NA & \\
\hline & Gross alpha & $\mathrm{pCi} / \mathrm{L}$ & - & & $1.6 E+01$ & $J$ \\
\hline & & $\mathrm{pH}$ & 4.8 & J1 & 5.3 & J1 \\
\hline & Tritium & $\mathrm{pCi} / \mathrm{mL}$ & $2.2 E+01$ & & $5.5 \mathrm{E}+01$ & \\
\hline \multirow[t]{14}{*}{ FSB104D } & Aluminum & $\mu \mathrm{g} / \mathrm{L}$ & 34,000 & V & NA & \\
\hline & Americium-241 & pCill & $1.2 E+01$ & & NA & \\
\hline & Curium-243/244 & $\mathrm{pCi} / \mathrm{L}$ & $1.1 E+01$ & & NA & \\
\hline & Gross alpha & $\mathrm{pCi} / \mathrm{L}$ & $5.7 E+02$ & & NA & \\
\hline & lodine-129 & pCill & $3.9 \mathrm{E}+01$ & & NA & \\
\hline & Nitrate as nitrogen & $\mu g / L$ & 74,000 & & NA & \\
\hline & Nonvolatile beta & pCill & $6.6 E+02$ & $J$ & NA & \\
\hline & & $\mathrm{pH}$ & & J1 & NA & \\
\hline & Strontium-89 & pCill & $2.6 E+01$ & & NA & \\
\hline & Strontium-90 & $\mathrm{pCi} / \mathrm{L}$ & $1.6 \mathrm{E}+02$ & & NA & \\
\hline & Tritium & $\mathrm{pCi} / \mathrm{mL}$ & $4.8 \mathrm{E}+03$ & & NA & \\
\hline & Uranium-233/234 & pCi/l & $2.5 \mathrm{E}+02$ & & NA & \\
\hline & Uranium-238 & $\mathrm{pCi} / \mathrm{L}$ & $3.1 E+02$ & $\mathrm{~J}$ & NA & \\
\hline & Zinc & $\mu g / L$ & 55 & & NA & \\
\hline \multirow[t]{12}{*}{ FSB105DR } & Aluminum & $\mu g / L$ & 10,000 & & NA & \\
\hline & Gross alpha & pCill & $4.0 E+02$ & & NA & \\
\hline & lodine-129 & $\mathrm{pCi} / \mathrm{L}$ & $1.7 E+01$ & & NA & \\
\hline & Nitrate as nitrogen & $\mu \mathrm{g} / \mathrm{L}$ & 23,000 & $\mathbf{J}$ & NA & \\
\hline & Nonvolatile beta & $\mathrm{pCi} / \mathrm{L}$ & 4.1E+02 & & NA & \\
\hline & $\mathrm{pH}$ & $\mathrm{pH}$ & & J1 & NA & \\
\hline & Strontium-90 & $\mathrm{pCi} / \mathrm{L}$ & $3.4 E+01$ & & NA & \\
\hline & Tritium & $\mathrm{pCi} / \mathrm{mL}$ & $5.5 \mathrm{E}+02$ & & NA & \\
\hline & Uranium-233/234 & $\mathrm{pCi} / \mathrm{L}$ & $2.2 \mathrm{E}+02$ & & NA & \\
\hline & Uranium-235 & $\mathrm{pCi} / \mathrm{L}$ & $1.6 \mathrm{E}+01$ & & NA & \\
\hline & Uranium-238 & $\mathrm{pCi} / \mathrm{L}$ & $5.6 \mathrm{E}+02$ & & NA & \\
\hline & Zinc & $\mu \mathrm{g} / \mathrm{L}$ & 54 & & NA & \\
\hline \multirow[t]{5}{*}{ FSB106D } & Aluminum & $\mu \mathrm{g} / \mathrm{L}$ & 54 & & NA & \\
\hline & Bis(2-ethylhexyl) phthalate & $\mu \mathrm{g} / \mathrm{L}$ & 190 & & NA & \\
\hline & Lead & $\mu g / L$ & 51 & & - & \\
\hline & $\mathrm{pH}$ & $\mathrm{pH}$ & 6.6 & J1 & 6.6 & J1 \\
\hline & Zinc & $\mu g / L$ & 720 & & NA & \\
\hline \multirow[t]{6}{*}{ FSB107D } & Aluminum & $\mu \mathrm{g} / \mathrm{L}$ & 15,000 & & NA & \\
\hline & Americium-241 & $\mathrm{pCi} / \mathrm{L}$ & $2.9 E+01$ & & NA & \\
\hline & Curium-243/244 & $\mathrm{pCi} / \mathrm{L}$ & $1.3 E+01$ & & NA & \\
\hline & Gross alpha & pCill & $1.1 E+03$ & $\mathrm{~J}$ & NA & \\
\hline & lodine-129 & $\mathrm{pCi} / \mathrm{L}$ & $1.6 \mathrm{E}+02$ & & NA & \\
\hline & Mercury & $\mu \mathrm{g} / \mathrm{L}$ & 5.4 & & NA & \\
\hline
\end{tabular}


Aquifer Zone $\mathrm{IIB}_{2}$ (Water Table)

\begin{tabular}{|c|c|c|c|c|c|c|}
\hline \multirow[t]{14}{*}{ Well } & Constituent & $\underline{\text { Unit }}$ & $\underline{1095}$ & Mod & 2Q95 & Mod \\
\hline & Nitrate as nitrogen & $\mu \mathrm{g} / \mathrm{L}$ & 69,000 & & NA & \\
\hline & Nonvolatile beta & $\mathrm{pCi} / \mathrm{L}$ & $1.3 E+03$ & J & NA & \\
\hline & pH & $\mathrm{pH}$ & 3.6 & $\mathrm{~J} 1$ & NA & \\
\hline & Radium-226 & $\mathrm{pCi} / \mathrm{L}$ & $2.2 E+01$ & $\mathrm{~J}$ & NA & \\
\hline & Strontium-89 & $\mathrm{pCi} / \mathrm{L}$ & $6.8 E+01$ & & NA & \\
\hline & Strontium-90 & pCill & $2.5 E+02$ & & NA & \\
\hline & Thorium-234 & $\mathrm{pCi} / \mathrm{L}$ & $5.3 E+02$ & & NA & \\
\hline & Trichlorofluoromethane & $\mu \mathrm{g} / \mathrm{L}$ & 15 & & NA & \\
\hline & Tritium & $\mathrm{pCi} / \mathrm{mL}$ & $2.5 E+03$ & & NA & \\
\hline & Uranium-233/234 & pCill & $4.3 E+02$ & & NA & \\
\hline & Uranium-235 & $\mathrm{pCi} / \mathrm{L}$ & $2.4 E+01$ & & NA & \\
\hline & Uranium-238 & $\mathrm{pCi} / \mathrm{L}$ & $5.5 E+02$ & J & NA & \\
\hline & Zinc & $\mu g / L$ & 57 & & NA & \\
\hline \multirow[t]{3}{*}{ FSB108D } & Aluminum & $\mu \mathrm{g} / \mathrm{L}$ & 120 & v & NA & \\
\hline & $\mathrm{pH}$ & $\mathrm{pH}$ & 6.1 & $\mathrm{J1}$ & 6.2 & $J 1$ \\
\hline & Zinc & $\mu g / L$ & 53 & V & NA & \\
\hline \multirow[t]{4}{*}{ FSB109D } & Aluminum & $\mu \mathrm{g} / \mathrm{L}$ & 100 & v & NA & \\
\hline & $\mathrm{pH}$ & $\mathrm{pH}$ & 6.3 & J1 & 6.2 & J1 \\
\hline & Tritium & $\mathrm{pCi} / \mathrm{mL}$ & $2.4 E+01$ & & $2.7 \mathrm{E}+01$ & \\
\hline & Zinc & $\mu g / L$ & 89 & V & NA & \\
\hline \multirow[t]{21}{*}{ FSB110D } & Aluminum & $\mu g / L$ & 96,000 & v & NA & \\
\hline & Americium-241 & pCill & $9.9 E+01$ & & NA & \\
\hline & Copper & $\mu \mathrm{g} / \mathrm{L}$ & & & NA & \\
\hline & Curium-243/244 & $\mathrm{pCi} / \mathrm{L}$ & $1.5 E+02$ & & NA & \\
\hline & Curium-245/246 & pCill & $9.9 E+01$ & & NA & \\
\hline & Cyanide & $\mu \mathrm{g} / \mathrm{L}$ & & JWVY & NA & \\
\hline & Gross alpha & pCill & $1.8 E+03$ & $\mathrm{~J}$ & NA & \\
\hline & lodine-129 & pCilL & $1.3 E+02$ & & NA & \\
\hline & Mercury & $\mu g / L$ & 4.8 & & NA & \\
\hline & Nickel & $\mu \mathrm{g} / \mathrm{L}$ & 66 & J & NA & \\
\hline & Nitrate as nitrogen & $\mu \mathrm{g} / \mathrm{L}$ & 290,000 & & NA & \\
\hline & Nonvolatile beta & pCill & 8.7E+02 & J & NA & \\
\hline & $\mathrm{pH}$ & $\mathrm{pH}$ & & J1 & NA & \\
\hline & Radium-228 & $\mathrm{pCi} / \mathrm{L}$ & $3.4 E+01$ & $\mathbf{J}$ & NA & \\
\hline & Strontium-90 & $\mathrm{pCi} / \mathrm{L}$ & $3.1 E+02$ & $\mathbf{J}$ & NA & \\
\hline & Thorium-234 & $\mathrm{pCi} / \mathrm{L}$ & $6.1 E+02$ & & NA & \\
\hline & Tritium & $\mathrm{pCi} / \mathrm{mL}$ & $1.9 E+04$ & & NA & \\
\hline & Uranium-233/234 & $\mathrm{pCi} / \mathrm{L}$ & $3.5 E+02$ & & NA & \\
\hline & Uranium-235 & $\mathrm{pCi} / \mathrm{L}$ & $2.8 E+01$ & & NA & \\
\hline & Uranium-238 & pCill & $1.3 E+03$ & $\mathbf{J}$ & NA & \\
\hline & Zinc & $\mu \mathrm{g} / \mathrm{L}$ & 230 & & NA & \\
\hline \multirow[t]{2}{*}{ FSB111D } & $\mathrm{pH}$ & pH & 5.1 & J1 & 5.2 & $\mathrm{~J}$ \\
\hline & Zinc & $\mu g / L$ & 46 & & NA & \\
\hline \multirow[t]{5}{*}{ FSB112D } & Aluminum & $\mu g / L$ & 44,000 & v & NA & \\
\hline & Cadmium & $\mu g / L$ & 16 & & NA & \\
\hline & Cobalt & $\mu \mathrm{g} / \mathrm{L}$ & 77 & & NA & \\
\hline & Copper & $\mu \mathrm{g} / \mathrm{L}$ & & & NA & \\
\hline & Gross alpha & pCill & $3.4 E+02$ & J & NA & \\
\hline
\end{tabular}


Aquifer Zone IIB $_{2}$ (Water Table)

\begin{tabular}{|c|c|c|c|c|c|c|}
\hline \multirow[t]{10}{*}{ Well } & Constituent & Unit & $1 Q 95$ & Mod & $\underline{2 Q 95}$ & Mod \\
\hline & lodine-129 & $\mathrm{pCi} / \mathrm{L}$ & $7.3 E+01$ & & NA & \\
\hline & Nitrate as nitrogen & $\mu g / L$ & 160,000 & & NA & \\
\hline & Nonvolatile beta & $\mathrm{pCi} / \mathrm{L}$ & $3.1 E+03$ & J & NA & \\
\hline & $\mathrm{pH}$ & $\mathrm{pH}$ & 3.7 & $\mathrm{J1}$ & NA & \\
\hline & Radium-226 & pCill & $9.1 E+01$ & & NA & \\
\hline & Radium-228 & $\mathrm{pCi} / \mathrm{L}$ & 4.6E+01 & $J$ & NA & \\
\hline & Strontium-90 & pCi/l & $1.2 E+03$ & J & NA & \\
\hline & Tritium & $\mathrm{pCi} / \mathrm{mL}$ & $8.8 E+03$ & & NA & \\
\hline & Zinc & $\mu g / L$ & 88 & & NA & \\
\hline FSB113D & $\mathrm{pH}$ & $\mathrm{pH}$ & 5.0 & J1 & 5.1 & 31 \\
\hline FSB114D & $\mathrm{pH}$ & $\mathrm{pH}$ & 5.1 & J1 & 5.4 & J1 \\
\hline \multirow[t]{5}{*}{ FSB115D } & Aluminum & $\mu g / L$ & 110 & & NA & \\
\hline & Bis(2-ethylhexyl) phthalate & $\mu g / L$ & 24 & & NA & \\
\hline & Copper & $\mu g / L$ & 110 & & NA & \\
\hline & $\mathrm{pH}$ & $\mathrm{pH}$ & 5.3 & J & NA & \\
\hline & Zinc & $\mu g / L$ & 120 & & NA & \\
\hline \multirow[t]{5}{*}{ FSB116D } & Aluminum & $\mu g / L$ & 160 & & NA & \\
\hline & Copper & $\mu \mathrm{g} / \mathrm{L}$ & 150 & & NA & \\
\hline & $\mathrm{pH}$ & $\mathrm{pH}$ & 5.5 & $\mathbf{J}$ & 5.3 & J1 \\
\hline & Silver & $\mu g / L$ & - & V & 73 & V \\
\hline & Zinc & $\mu g / L$ & 230 & & NA & \\
\hline \multirow[t]{11}{*}{ FSB117D } & Aluminum & $\mu g / L$ & 9,500 & & NA & \\
\hline & Gross alpha & $\mathrm{pCi} / \mathrm{L}$ & $6.2 E+02$ & $J$ & NA & \\
\hline & lodine-129 & $\mathrm{pCi} / \mathrm{L}$ & $4.2 E+01$ & & NA & \\
\hline & Nitrate as nitrogen & $\mu g / L$ & 31,000 & & NA & \\
\hline & Nonvolatile beta & $\mathrm{pCi} / \mathrm{L}$ & $3.5 E+02$ & & NA & \\
\hline & $\mathrm{pH}$ & $\mathrm{pH}$ & 3.7 & J1 & NA & \\
\hline & Strontium-90 & $\mathrm{pCi} / \mathrm{L}$ & $5.9 E+01$ & & NA & \\
\hline & Tritium & $\mathrm{pCi} / \mathrm{mL}$ & $9.3 E+02$ & & NA & \\
\hline & Uranium-233/234 & $\mathrm{pCi} / \mathrm{L}$ & $3.5 E+02$ & & NA & \\
\hline & Uranium-235 & $\mathrm{pCi} / \mathrm{L}$ & $2.0 E+01$ & & NA & \\
\hline & Uranium-238 & $\mathrm{pCi} / \mathrm{L}$ & $3.8 \mathrm{E}+02$ & & NA & \\
\hline \multirow[t]{3}{*}{ FSB118D } & $\mathrm{pH}$ & $\mathrm{pH}$ & 5.1 & $\sqrt{ } 1$ & 5.9 & J1 \\
\hline & Tritium & $\mathrm{pCi} / \mathrm{mL}$ & NA & & $4.8 E+02$ & \\
\hline & Zinc & $\mu \mathrm{g} / \mathrm{L}$ & 31 & & NA & \\
\hline \multirow[t]{11}{*}{ FSB119D } & Aluminum & $\mu \mathrm{g} / \mathrm{L}$ & 54,000 & V & NA & \\
\hline & Americium-241 & $\mathrm{pC} \mathrm{i} / \mathrm{L}$ & $1.6 E+01$ & & NA & \\
\hline & Gross alpha & $\mathrm{pCi} / \mathrm{L}$ & 2.1E+03 & J & NA & \\
\hline & lodine-129 & $\mathrm{pCi} / \mathrm{L}$ & $1.5 E+02$ & & NA & \\
\hline & Nitrate as nitrogen & $\mu g / L$ & 130,000 & & NA & \\
\hline & Nonvolatile beta & pCill & 1.3E+03 & J & NA & \\
\hline & $\mathrm{pH}$ & $\mathrm{pH}$ & 3.4 & J1 & NA & \\
\hline & Radium-226 & $\mathrm{pCi} / \mathrm{L}$ & $3.2 E+01$ & & NA & \\
\hline & Radium-228 & pCi/L & $3.3 E+01$ & $J$ & NA & \\
\hline & Strontium-90 & $\mathrm{pCi} / \mathrm{L}$ & $4.6 E+02$ & $J$ & NA & \\
\hline & Thorium-234 & pCill & $4.5 E+02$ & & NA & \\
\hline
\end{tabular}




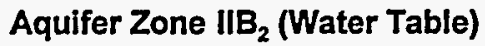

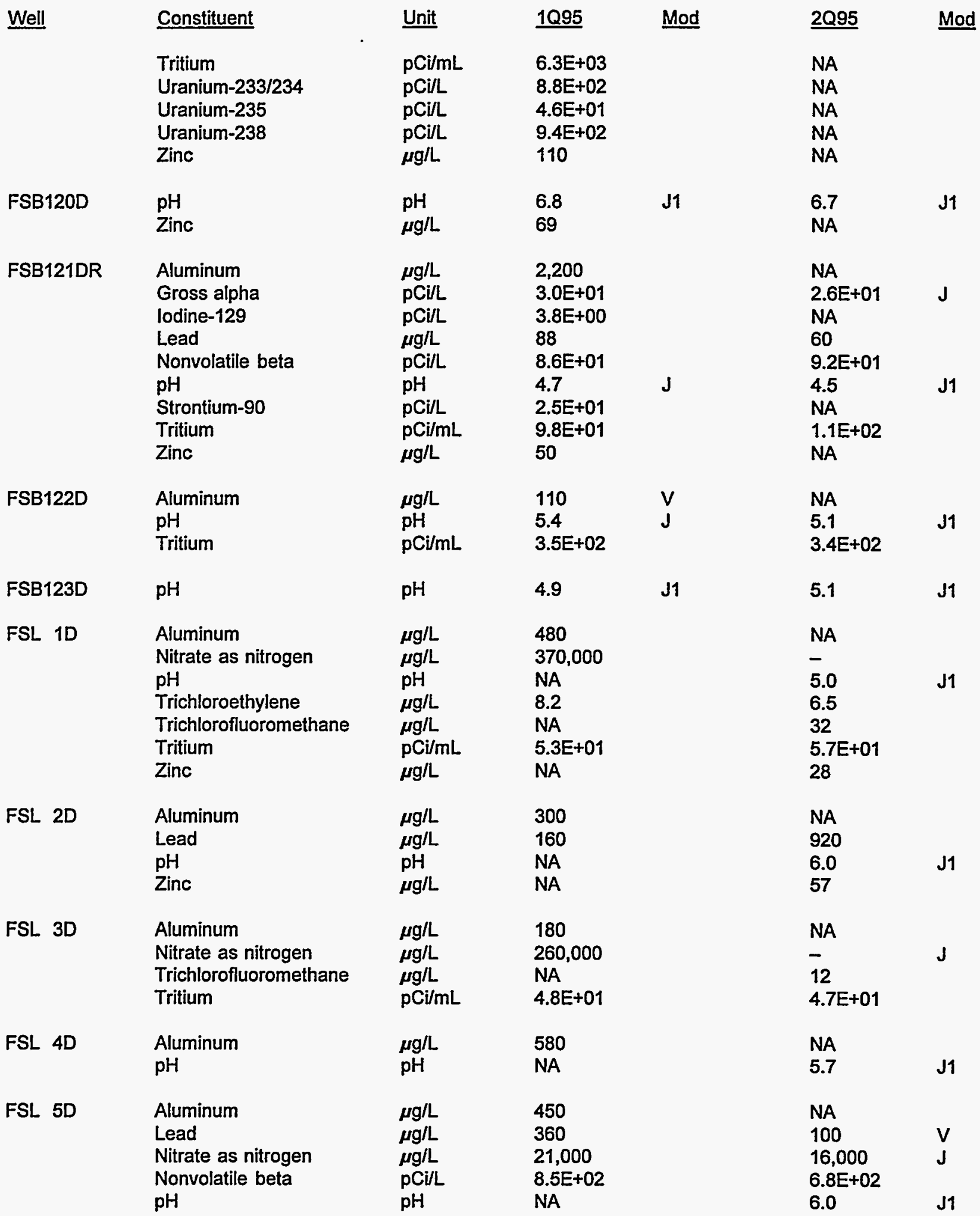


Aquifer Zone IIB $_{2}$ (Water Table)

Well

Constituent
Tritium
Zinc

FSL 6D

FSL 7D

$$
\begin{aligned}
& \text { Aluminum } \\
& \text { Gross alpha } \\
& \text { Nitrate as nitrogen } \\
& \text { Nonvolatile beta } \\
& \text { Tritium }
\end{aligned}
$$

FSL 8D

$$
\begin{aligned}
& \text { pH } \\
& \text { Trichloroethylene } \\
& \text { Tritium }
\end{aligned}
$$

FSL 9D

Aluminum
Nitrate as nitrogen
pH
Tritium

Unit
$\mathrm{pCi} / \mathrm{mL}$
$\mu \mathrm{g} / \mathrm{L}$

1095

4.8E+02

NA

420

$\underset{\mu \mathrm{g} / \mathrm{L}}{\mu \mathrm{L}}$

$\mathrm{pCi} / \mathrm{mL}$

(

NA

8.7E+02

$\mu g / L$

pCi/l

$\mu \mathrm{g} / \mathrm{L}$

pCi/L

$\mathrm{pCi} / \mathrm{mL}$

$\mathrm{pH} \quad \mathrm{NA}$

$\mu \mathrm{g} / \mathrm{L}$

$\mathrm{pCi} / \mathrm{mL}$

$\mu \mathrm{g} / \mathrm{L}$

$\mathrm{pCi} / \mathrm{L}$

$\mu \mathrm{g} / \mathrm{L}$

$\mathrm{pCi} / \mathrm{L}$

$\mathrm{pH}$

$\mu \mathrm{g} / \mathrm{L}$

$\mathrm{pCi} / \mathrm{mL}$

$\mu \mathrm{g} / \mathrm{L}$
1,200

4.5E+01

71,000

7.1E+01

$1.9 E+03$

NA

3. $0 \mathrm{E}+01$

2,600

$8.1 \mathrm{E}+01$

13,000

2. $6 \mathrm{E}+02$

NA

NA

4.7E+02

NA
Mod

$\underline{2 Q 95}$

Mod

$6.4 E+02$

26

NA

27,000

5.1

$3.1 \mathrm{E}+02$

J1

NA

NA

NA

NA

NA

5.2

$-$

$3.9 E+01$

NA

1.3E+02

14,000

$3.2 \mathrm{E}+02$

4.7

$J$

5.9

$4.0 \mathrm{E}+02$

25

$\underline{2 Q 95}$

Mod

Well

Constituent

Unit

1Q95

Mod

6.1

NA

$\mathrm{pH}$
$\mu \mathrm{g} / \mathrm{L}$

6.0

J1

16,000

16

$\mu g / L$

$\mu g / L$

$\mu \mathrm{g} / \mathrm{L}$

pCi/L

pCi/L

$\mu g / L$

$\mu \mathrm{g} / \mathrm{L}$

pCi/L

$\mathrm{pH}$

pCill

$\mathrm{pCi/L}$

$\mathrm{pCi} / \mathrm{mL}$

pCill

pCill

pCi/L

$\mu \mathrm{g} / \mathrm{L}$

300

1. $6 \mathrm{E}+02$

$1.2 \mathrm{E}+02$

120

240,000

$1.1 \mathrm{E}+03$

4.2

$5.1 \mathrm{E}+01$

$6.2 \mathrm{E}+02$

8.7E+03

$3.9 \mathrm{E}+02$

2. $4 \mathrm{E}+01$

$1.0 \mathrm{E}+03$

590

v

NA

NA

NA

NA

NA

NA

NA

NA

NA

NA

NA

NA

NA

NA

NA

NA

$\mu \mathrm{g} / \mathrm{L}$

pCi/L

39,000

2.1E+01
NA

NA 
Aquifer Zone $\| B_{1}$ (Barnwell/McBean)

\begin{tabular}{|c|c|c|c|c|c|}
\hline Well & Constituent & Unit & 1095 & Mod & $\underline{2 Q 95}$ \\
\hline & $\begin{array}{l}\text { Cadmium } \\
\text { Cobalt } \\
\text { Curium-243/244 } \\
\text { Gross alpha } \\
\text { lodine-129 } \\
\text { Nitrate as nitrogen } \\
\text { Nonvolatile beta } \\
\text { pH } \\
\text { Plutonium-238 } \\
\text { Radium-226 } \\
\text { Strontium-89 } \\
\text { Strontium-90 } \\
\text { Tritium } \\
\text { Uranium-233/234 } \\
\text { Uranium-235 } \\
\text { Uranium-238 } \\
\text { Zinc }\end{array}$ & $\begin{array}{l}\mu \mathrm{g} / \mathrm{L} \\
\mu \mathrm{g} / \mathrm{L} \\
\mathrm{pCi} / \mathrm{L} \\
\mathrm{pCi} / \mathrm{L} \\
\mathrm{pCi} / \mathrm{L} \\
\mu \mathrm{g} / \mathrm{L} \\
\mathrm{pCi/L} \\
\mathrm{pH} \\
\mathrm{pCi} / \mathrm{L} \\
\mathrm{pCi} / \mathrm{L} \\
\mathrm{pCi} / \mathrm{L} \\
\mathrm{pCi} / \mathrm{L} \\
\mathrm{pCi} / \mathrm{mL} \\
\mathrm{pCi} / \mathrm{L} \\
\mathrm{pCi} / \mathrm{L} \\
\mathrm{pCi} / \mathrm{L} \\
\mu \mathrm{g} / \mathrm{L}\end{array}$ & $\begin{array}{l}27 \\
140 \\
2.8 E+01 \\
8.0 E+02 \\
9.2 E+01 \\
130,000 \\
2.5 E+03 \\
3.7 \\
2.3 E+01 \\
4.8 E+01 \\
2.0 E+02 \\
7.2 E+02 \\
8.7 E+03 \\
2.2 E+02 \\
1.8 E+01 \\
2.3 E+02 \\
110\end{array}$ & $\begin{array}{l}\mathrm{J} \\
\mathrm{J} 1\end{array}$ & $\begin{array}{l}\text { NA } \\
\text { NA } \\
\text { NA } \\
\text { NA } \\
\text { NA } \\
\text { NA } \\
\text { NA } \\
\text { NA } \\
\text { NA } \\
\text { NA } \\
\text { NA } \\
\text { NA } \\
\text { NA } \\
\text { NA } \\
\text { NA } \\
\text { NA } \\
\text { NA }\end{array}$ \\
\hline FSB $87 C$ & $\begin{array}{l}\text { lodine-129 } \\
\text { pH } \\
\text { Tritium }\end{array}$ & $\begin{array}{l}\mathrm{pCi} / \mathrm{L} \\
\mathrm{pH} \\
\mathrm{pCi} / \mathrm{mL}\end{array}$ & $\begin{array}{l}3.3 E+00 \\
5.9 \\
4.7 E+02\end{array}$ & J1 & $\begin{array}{l}N A \\
6.1 \\
4.8 E+02\end{array}$ \\
\hline FSB $88 \mathrm{C}$ & $\mathrm{pH}$ & $\mathrm{pH}$ & 5.7 & J1 & 5.8 \\
\hline FSB 89C & $\begin{array}{l}\text { Dichloromethane } \\
\text { pH } \\
\text { Trichlorofluoromethane }\end{array}$ & $\begin{array}{l}\mu \mathrm{g} / \mathrm{L} \\
\mathrm{pH} \\
\mu \mathrm{g} / \mathrm{L}\end{array}$ & $\begin{array}{l}14 \\
5.9 \\
6.6\end{array}$ & $\begin{array}{l}W \\
\mathrm{~J}\end{array}$ & $\begin{array}{l}\text { NA } \\
6.0 \\
\text { NA }\end{array}$ \\
\hline FSB $90 \mathrm{C}$ & $\begin{array}{l}\text { Aluminum } \\
\text { Nitrate as nitrogen } \\
\text { pH } \\
\text { Tritium }\end{array}$ & $\begin{array}{l}\mu \mathrm{g} / \mathrm{L} \\
\mu \mathrm{g} / \mathrm{L} \\
\mathrm{pH} \\
\mathrm{pCi} / \mathrm{mL}\end{array}$ & $\begin{array}{l}73 \\
11,000 \\
6.8 \\
5.8 E+02\end{array}$ & $\begin{array}{l}\mathrm{J} \\
\mathrm{J} 1\end{array}$ & $\begin{array}{l}\text { NA } \\
14,000 \\
6.4 \\
6.1 E+02\end{array}$ \\
\hline FSB 91C & $\begin{array}{l}\text { Aluminum } \\
\text { Copper } \\
\text { Gross alpha } \\
\text { lodine-129 } \\
\text { Nitrate as nitrogen } \\
\text { Nonvolatile beta } \\
\text { pH } \\
\text { Strontium-90 } \\
\text { Tritium } \\
\text { Zinc }\end{array}$ & $\begin{array}{l}\mu \mathrm{g} / \mathrm{L} \\
\mu \mathrm{g} / \mathrm{L} \\
\mathrm{pCi} / \mathrm{L} \\
\mathrm{pCi} / \mathrm{L} \\
\mu \mathrm{g} / \mathrm{L} \\
\mathrm{pCi} / \mathrm{L} \\
\mathrm{pH} \\
\mathrm{pCi} / \mathrm{L} \\
\mathrm{pCi} / \mathrm{mL} \\
\mu \mathrm{g} / \mathrm{L}\end{array}$ & $\begin{array}{l}28,000 \\
99 \\
6.2 E+01 \\
4.8 E+01 \\
25,000 \\
4.0 E+02 \\
5.4 \\
1.6 E+02 \\
6.6 E+02 \\
130\end{array}$ & $\begin{array}{l}V \\
\text { J1 }\end{array}$ & $\begin{array}{l}\text { NA } \\
\text { NA } \\
\text { NA } \\
\text { NA } \\
\text { NA } \\
\text { NA } \\
\text { NA } \\
\text { NA } \\
\text { NA } \\
\text { NA }\end{array}$ \\
\hline FSB 92C & $\begin{array}{l}\text { Aluminum } \\
\text { Gross alpha } \\
\text { lodine-129 } \\
\text { Nitrate as nitrogen } \\
\text { Nonvolatile beta } \\
\text { pH } \\
\text { Strontium- } 89 \\
\text { Strontium-90 }\end{array}$ & $\begin{array}{l}\mu \mathrm{g} / \mathrm{L} \\
\mathrm{pCi} / \mathrm{L} \\
\mathrm{pCi} / \mathrm{L} \\
\mu \mathrm{g} / \mathrm{L} \\
\mathrm{pCilL} \\
\mathrm{pH} \\
\mathrm{pCi} / \mathrm{L} \\
\mathrm{pCi} / \mathrm{L}\end{array}$ & $\begin{array}{l}1,200 \\
5.2 \mathrm{E}+01 \\
1.5 \mathrm{E}+01 \\
47,000 \\
3.8 \mathrm{E}+02 \\
5.5 \\
3.1 \mathrm{E}+01 \\
9.5 \mathrm{E}+01\end{array}$ & $\begin{array}{l}J \\
\text { J1 }\end{array}$ & $\begin{array}{l}\text { NA } \\
\text { NA } \\
\text { NA } \\
\text { NA } \\
\text { NA } \\
\text { NA } \\
\text { NA } \\
\text { NA }\end{array}$ \\
\hline
\end{tabular}


Aquifer Zone IIB, (Barnwell/McBean)

\begin{tabular}{|c|c|c|c|c|c|c|}
\hline Well & Constituent & Unit & $\underline{1095}$ & Mod & 2095 & Mod \\
\hline & $\begin{array}{l}\text { Tritium } \\
\text { Zinc }\end{array}$ & $\begin{array}{l}\mathrm{pCi} / \mathrm{mL} \\
\mu \mathrm{g} / \mathrm{L}\end{array}$ & $\begin{array}{l}1.8 E+03 \\
60\end{array}$ & & $\begin{array}{l}\text { NA } \\
\text { NA }\end{array}$ & \\
\hline FSB $93 \mathrm{C}$ & lodine- 129 & $\mathrm{pCi} / \mathrm{L}$ & 1.7E+01 & & $\begin{array}{l}\text { NA } \\
46.000\end{array}$ & \\
\hline & $\mathrm{pH}$ & $\mathrm{pH}$ & & J1 & & J1 \\
\hline & Tritium & $\mathrm{pCi} / \mathrm{mL}$ & $1.5 E+03$ & & 1. $6 \mathrm{E}+03$ & \\
\hline & Zinc & $\mu \mathrm{g} / \mathrm{L}$ & 99 & & NA & \\
\hline FSB 94C & Aluminum & $\mu \mathrm{g} / \mathrm{L}$ & 46,000 & & NA & \\
\hline & Cadmium & $\mu g / L$ & 23 & $\mathrm{~J}$ & NA & \\
\hline & Cobalt & $\mu \mathrm{g} / \mathrm{L}$ & 670 & & NA & \\
\hline & Gross alpha & $\mathrm{pCi} / \mathrm{L}$ & $2.2 E+02$ & & NA & \\
\hline & lodine-129 & $\mathrm{pCi} / \mathrm{L}$ & $1.5 \mathrm{E}+02$ & & NA & \\
\hline & Nickel & $\mu g / L$ & 180 & & NA & \\
\hline & Nitrate as nitrogen & $\mu \mathrm{g} / \mathrm{L}$ & 250,000 & & NA & \\
\hline & Nonvolatile beta & $\mathrm{pCi} / \mathrm{L}$ & $2.4 E+03$ & $\mathrm{~J}$ & NA & \\
\hline & $\mathrm{pH}$ & $\mathrm{pH}$ & 4.5 & J1 & NA & \\
\hline & Radium-226 & $\mathrm{pCi} / \mathrm{L}$ & $3.5 E+01$ & $J$ & NA & \\
\hline & Radium-228 & $\mathrm{pCi} / \mathrm{L}$ & $2.2 E+01$ & $\mathrm{~J}$ & NA & \\
\hline & Strontium-90 & $\mathrm{pCi} / \mathrm{L}$ & $7.2 E+02$ & & NA & \\
\hline & Tritium & $\mathrm{pCi} / \mathrm{mL}$ & $1.0 E+04$ & & NA & \\
\hline & Uranium-233/234 & $\mathrm{pCi} / \mathrm{L}$ & $1.7 E+01$ & & NA & \\
\hline & Uranium-238 & pCill & $1.9 \mathrm{E}+01$ & $\mathrm{~J}$ & NA & \\
\hline & Zinc & $\mu \mathrm{g} / \mathrm{L}$ & 320 & & NA & \\
\hline FSB 95CR & Aluminum & $\mu \mathrm{g} / \mathrm{L}$ & 47,000 & & NA & \\
\hline & Americium-241 & $\mathrm{pCi} / \mathrm{L}$ & $1.3 E+01$ & & NA & \\
\hline & Cadmium & $\mu \mathrm{g} / \mathrm{L}$ & 16 & & NA & \\
\hline & Cobalt & $\mu g / L$ & 460 & & NA & \\
\hline & Curium-243/244 & $\mathrm{pCi} / \mathrm{L}$ & $2.6 E+01$ & & NA & \\
\hline & Gross alpha & $\mathrm{pCi} / \mathrm{L}$ & $1.2 E+03$ & & NA & \\
\hline & lodine-129 & $\mathrm{pCi} / \mathrm{L}$ & $2.3 E+02$ & & NA & \\
\hline & Nickel & $\mu g / L$ & 96 & & NA & \\
\hline & Nitrate as nitrogen & $\mu \mathrm{g} / \mathrm{L}$ & 230,000 & & NA & \\
\hline & Nonvolatile beta & pCi/l & $2.4 E+03$ & & NA & \\
\hline & $\mathrm{pH}$ & $\mathrm{pH}$ & 4.0 & $\mathrm{J1}$ & NA & \\
\hline & Radium-226 & pCi/L & $8.9 E+01$ & & NA & \\
\hline & Radium-228 & $\mathrm{pCi} / \mathrm{L}$ & $2.5 E+02$ & & NA & \\
\hline & Strontium-89 & pCi/L & $1.9 E+02$ & $\sqrt{ } 2$ & NA & \\
\hline & Strontium-90 & $\mathrm{pCi} / \mathrm{L}$ & $1.2 \mathrm{E}+03$ & & NA & \\
\hline & Tritium & $\mathrm{pCi} / \mathrm{mL}$ & $1.4 \mathrm{E}+04$ & v & NA & \\
\hline & Uranium-233/234 & $\mathrm{pCi} / \mathrm{L}$ & $3.5 E+02$ & & NA & \\
\hline & Uranium-235 & pCill & $1.8 E+01$ & $\mathbf{R}$ & NA & \\
\hline & Uranium-238 & $\mathrm{pCi} / \mathrm{L}$ & 4.4E+02 & $\mathbf{R}$ & NA & \\
\hline & Zinc & $\mu g / L$ & 310 & & NA & \\
\hline FSB 97C & Aluminum & $\mu \mathrm{g} / \mathrm{L}$ & 77,000 & v & NA & \\
\hline & Americium-241 & $\mathrm{pCi} / \mathrm{L}$ & $5.3 E+01$ & & NA & \\
\hline & Cobalt & $\mu g / L$ & 320 & & NA & \\
\hline & Copper & $\mu g / L$ & 70 & & NA & \\
\hline & Curium-243/244 & pCill & $3.7 E+01$ & & NA & \\
\hline & Gross alpha & pCill & $2.0 E+03$ & $\mathbf{J}$ & NA & \\
\hline
\end{tabular}


Aquifer Zone IIB, (Barnwell/McBean)

\begin{tabular}{|c|c|c|c|c|c|c|}
\hline \multirow[t]{15}{*}{ Well } & Constituent & Unit & $\underline{1095}$ & Mod & 2Q95 & Mod \\
\hline & lodine-129 & pCi/L & $8.6 E+01$ & & NA & \\
\hline & Nickel & $\mu \mathrm{g} / \mathrm{L}$ & 75 & & NA & \\
\hline & Nitrate as nitrogen & $\mu \mathrm{g} / \mathrm{L}$ & 240,000 & & NA & \\
\hline & Nonvolatile beta & $\mathrm{pCi} / \mathrm{L}$ & 1.1E+03 & $\mathrm{J}$ & NA & \\
\hline & $\mathrm{pH}$ & pH & 3.7 & J1 & NA & \\
\hline & Radium-226 & $\mathrm{pCi} / \mathrm{L}$ & $6.3 E+01$ & & NA & \\
\hline & Radium-228 & pCi/L & $4.4 E+01$ & $J$ & NA & \\
\hline & Strontium-90 & pCill & $3.4 E+02$ & $\mathrm{~J}$ & NA & \\
\hline & Trichloroethylene & $\mu \mathrm{g} / \mathrm{L}$ & 9.3 & & NA & \\
\hline & Tritium & $\mathrm{pC} \mathrm{i} / \mathrm{mL}$ & $1.3 E+04$ & & NA & \\
\hline & Uranium-233/234 & $\mathrm{pCi} / \mathrm{L}$ & $4.2 E+02$ & & NA & \\
\hline & Uranium-235 & pCi/L & $2.5 E+01$ & & NA & \\
\hline & Uranium-238 & pCill & $5.7 E+02$ & $J$ & NA & \\
\hline & Zinc & $\mu g / L$ & 190 & & NA & \\
\hline \multirow[t]{17}{*}{ FSB 98C } & Aluminum & $\mu \mathrm{g} / \mathrm{L}$ & 39,000 & v & NA & \\
\hline & Americium-241 & pCill & $3.4 \mathrm{E}+01$ & & NA & \\
\hline & Cobalt & $\mu \mathrm{g} / \mathrm{L}$ & 350 & & NA & \\
\hline & Copper & $\mu \mathrm{g} / \mathrm{L}$ & 72 & & NA & \\
\hline & Curium-243/244 & $\mathrm{pCi} / \mathrm{L}$ & $3.3 \mathrm{E}+01$ & & NA & \\
\hline & Gross alpha & $\mathrm{pCi} / \mathrm{L}$ & $2.1 E+02$ & & NA & \\
\hline & lodine-129 & $\mathrm{pCi} / \mathrm{L}$ & $8.5 E+01$ & & NA & \\
\hline & Nitrate as nitrogen & $\mu \mathrm{g} / \mathrm{L}$ & 140,000 & & NA & \\
\hline & Nonvolatile beta & pCill & $3.2 \mathrm{E}+02$ & $\mathrm{~J}$ & NA & \\
\hline & pH & pH & 3.4 & J1 & NA & \\
\hline & Radium-226 & pCi/L & $5.2 E+01$ & $\mathbf{J}$ & NA & \\
\hline & Strontium-90 & $\mathrm{pCi} / \mathrm{L}$ & $1.6 E+02$ & & NA & \\
\hline & Tritium & $\mathrm{pCi} / \mathrm{mL}$ & $7.9 E+03$ & & NA & \\
\hline & Uranium-233/234 & pCill & $4.5 E+02$ & & NA & \\
\hline & Uranium-235 & pCi/L & $2.3 E+01$ & & NA & \\
\hline & Uranium-238 & pCill & $6.6 \mathrm{E}+02$ & $\mathbf{J}$ & NA & \\
\hline & Zinc & $\mu \mathrm{g} / \mathrm{L}$ & 120 & & NA & \\
\hline \multirow[t]{9}{*}{ FSB 99C } & Aluminum & $\mu g / L$ & 120 & & NA & \\
\hline & Gross alpha & $\mathrm{pCi} / \mathrm{L}$ & $2.1 E+01$ & & 3.7E+01 & \\
\hline & lodine-129 & $\mathrm{pCi} / \mathrm{L}$ & $1.2 \mathrm{E}+01$ & & & \\
\hline & Nitrate as nitrogen & $\mu \mathrm{g} / \mathrm{L}$ & 28,000 & & 30,000 & \\
\hline & Nonvolatile beta & $\mathrm{pCi} / \mathrm{L}$ & $8.7 E+01$ & & $9.0 E+01$ & $\mathbf{J}$ \\
\hline & & $\mathrm{pH}$ & 5.4 & $\mathrm{~J} 1$ & 5.9 & $\mathrm{~J} 1$ \\
\hline & Strontium-90 & $\mathrm{pCi} / \mathrm{L}$ & $1.8 \mathrm{E}+01$ & & NA & \\
\hline & Trichloroethylene & $\mu \mathrm{g} / \mathrm{L}$ & $\uparrow 8$ & & NA & \\
\hline & Tritium & $\mathrm{pCi} / \mathrm{mL}$ & $1.5 E+03$ & & NA & \\
\hline \multirow[t]{9}{*}{ FSB102C } & Aluminum & $\mu \mathrm{g} / \mathrm{L}$ & 1,200 & & NA & \\
\hline & lodine-129 & $\mathrm{pCi} / \mathrm{L}$ & $2.9 E+01$ & & NA & \\
\hline & Nitrate as nitrogen & $\mu \mathrm{g} / \mathrm{L}$ & 37,000 & $\mathbf{J}$ & NA & \\
\hline & Nonvolatile beta & pCill & $5.7 E+02$ & & NA & \\
\hline & pH & $\mathrm{pH}$ & 4.5 & J1 & NA & \\
\hline & Strontium-89 & pCill & $2.3 E+01$ & & NA & \\
\hline & Strontium-90 & $\mathrm{pCi} / \mathrm{L}$ & $1.7 E+02$ & & NA & \\
\hline & Tritium & $\mathrm{pCi} / \mathrm{mL}$ & $9.5 \mathrm{E}+02$ & & NA & \\
\hline & Zinc & $\mu \mathrm{g} / \mathrm{L}$ & 39 & & NA & \\
\hline
\end{tabular}


Aquifer Zone IIB (Barnwell/McBean)

\begin{tabular}{|c|c|c|c|c|c|c|}
\hline Well & Constituent & Unit & 1095 & Mod & $2 Q 95$ & $\mathrm{Moc}$ \\
\hline FSB103C & $\begin{array}{l}\text { lodine }-129 \\
\text { Nitrate as nitrogen } \\
\text { pH } \\
\text { Tritium } \\
\text { Zinc }\end{array}$ & $\begin{array}{l}\mathrm{pCi} / \mathrm{L} \\
\mu \mathrm{g} / \mathrm{L} \\
\mathrm{pH} \\
\mathrm{pCi} / \mathrm{mL} \\
\mu \mathrm{g} / \mathrm{L}\end{array}$ & $\begin{array}{l}5.1 E+00 \\
21,000 \\
5.9 \\
5.8 E+02 \\
71\end{array}$ & J1 & $\begin{array}{l}\text { NA } \\
25,000 \\
5.8 \\
5.9 E+02 \\
\text { NA }\end{array}$ & J1 \\
\hline FSB104C & $\begin{array}{l}\text { lodine-129 } \\
\text { Nitrate as nitrogen } \\
\text { pH } \\
\text { Tritium } \\
\text { Zinc }\end{array}$ & $\begin{array}{l}\mathrm{pCi} / \mathrm{L} \\
\mu \mathrm{g} / \mathrm{L} \\
\mathrm{pH} \\
\mathrm{pCi} / \mathrm{mL} \\
\mu \mathrm{g} / \mathrm{L}\end{array}$ & $\begin{array}{l}6.0 \mathrm{E}+00 \\
45,000 \\
5.4 \\
1.2 \mathrm{E}+03 \\
53\end{array}$ & J1 & $\begin{array}{l}\text { NA } \\
43,000 \\
5.9 \\
- \\
\text { NA }\end{array}$ & J1 \\
\hline FSB105C & $\begin{array}{l}\text { Aluminum } \\
\text { Americium-241 } \\
\text { Cadmium } \\
\text { Cobalt } \\
\text { Curium-243/244 } \\
\text { Gross alpha } \\
\text { lodine-129 } \\
\text { Nitrate as nitrogen } \\
\text { Nonvolatile beta } \\
\text { pH } \\
\text { Radium-226 } \\
\text { Radium-228 } \\
\text { Strontium-89 } \\
\text { Strontium-90 } \\
\text { Tritium } \\
\text { Uranium-233/234 } \\
\text { Uranium-238 } \\
\text { Zinc }\end{array}$ & $\begin{array}{l}\mu \mathrm{g} / \mathrm{L} \\
\mathrm{pCi} / \mathrm{L} \\
\mu \mathrm{g} / \mathrm{L} \\
\mu \mathrm{g} / \mathrm{L} \\
\mathrm{pCi} / \mathrm{L} \\
\mathrm{pCi} / \mathrm{L} \\
\mathrm{pCi} / \mathrm{L} \\
\mu \mathrm{g} / \mathrm{L} \\
\mathrm{pCi} / \mathrm{L} \\
\mathrm{pH} \\
\mathrm{pCi} / \mathrm{L} \\
\mathrm{pCi} / \mathrm{L} \\
\mathrm{pCi} / \mathrm{L} \\
\mathrm{pCi} / \mathrm{L} \\
\mathrm{pCi} / \mathrm{mL} \\
\mathrm{pCi} / \mathrm{L} \\
\mathrm{pCi} / \mathrm{L} \\
\mu \mathrm{g} / \mathrm{L}\end{array}$ & $\begin{array}{l}46,000 \\
2.4 E+01 \\
11 \\
240 \\
2.1 E+01 \\
7.7 E+02 \\
6.3 E+01 \\
150,000 \\
1.1 E+03 \\
3.4 \\
5.5 E+01 \\
2.6 E+01 \\
6.0 E+01 \\
2.2 E+02 \\
8.7 E+03 \\
2.3 E+02 \\
2.5 E+02 \\
110\end{array}$ & $\begin{array}{l}\mathrm{J} \\
\mathrm{J} 1 \\
\mathrm{~J} \\
\mathrm{~J}\end{array}$ & $\begin{array}{l}\text { NA } \\
\text { NA } \\
\text { NA } \\
\text { NA } \\
\text { NA } \\
\text { NA } \\
\text { NA } \\
\text { NA } \\
\text { NA } \\
\text { NA } \\
\text { NA } \\
\text { NA } \\
\text { NA } \\
\text { NA } \\
\text { NA } \\
\text { NA } \\
\text { NA } \\
\text { NA }\end{array}$ & \\
\hline FSB106C & $\begin{array}{l}\text { Aluminum } \\
\text { Cadmium } \\
\text { Gross alpha } \\
\text { lodine-129 } \\
\text { Nitrate as nitrogen } \\
\text { Nonvolatile beta } \\
\text { pH } \\
\text { Radium-226 } \\
\text { Strontium-90 } \\
\text { Tritium } \\
\text { Zinc }\end{array}$ & $\begin{array}{l}\mu \mathrm{g} / \mathrm{L} \\
\mu \mathrm{g} / \mathrm{L} \\
\mathrm{pCi} / \mathrm{L} \\
\mathrm{pCi} / \mathrm{L} \\
\mu \mathrm{g} / \mathrm{L} \\
\mathrm{pCi} / \mathrm{L} \\
\mathrm{pH} \\
\mathrm{pCi} / \mathrm{L} \\
\mathrm{pCi} / \mathrm{L} \\
\mathrm{pCi} / \mathrm{mL} \\
\mu \mathrm{g} / \mathrm{L}\end{array}$ & $\begin{array}{l}4,300 \\
10 \\
4.5 E+01 \\
1.6 E+02 \\
90,000 \\
1.3 E+03 \\
4.7 \\
2.3 E+01 \\
4.8 E+02 \\
2.4 E+03 \\
40\end{array}$ & $\begin{array}{l}J \\
J 1\end{array}$ & $\begin{array}{l}\text { NA } \\
\text { NA } \\
\text { NA } \\
\text { NA } \\
\text { NA } \\
\text { NA } \\
\text { NA } \\
\text { NA } \\
\text { NA } \\
\text { NA } \\
\text { NA }\end{array}$ & \\
\hline FSB107C & $\begin{array}{l}\mathrm{pH} \\
\text { Strontium-90 } \\
\text { Tritium }\end{array}$ & $\begin{array}{l}\mathrm{pH} \\
\mathrm{pCi} / \mathrm{L} \\
\mathrm{pCi} / \mathrm{mL}\end{array}$ & $\begin{array}{l}- \\
1.2 E+01 \\
1.2 E+02\end{array}$ & J1 & $\begin{array}{l}6.7 \\
\text { NA } \\
1.1 E+02\end{array}$ & J1 \\
\hline FSB110C & $\begin{array}{l}\text { Aluminum } \\
\text { lodine-129 } \\
\text { Nitrate as nitrogen } \\
\text { Nonvolatile beta } \\
\text { pH } \\
\text { Strontium-90 }\end{array}$ & $\begin{array}{l}\mu \mathrm{g} / \mathrm{L} \\
\mathrm{pCi} / \mathrm{L} \\
\mu \mathrm{g} / \mathrm{L} \\
\mathrm{pCi} / \mathrm{L} \\
\mathrm{pH} \\
\mathrm{pCi} / \mathrm{L}\end{array}$ & $\begin{array}{l}100 \\
2.5 E+01 \\
55,000 \\
6.7 E+01 \\
6.0 \\
1.2 E+01\end{array}$ & J1 & $\begin{array}{l}\text { NA } \\
\text { NA } \\
53,000 \\
5.6 E+01 \\
6.1 \\
\text { NA }\end{array}$ & J1 \\
\hline
\end{tabular}


Aquifer Zone IIB, (Barnwell/McBean)

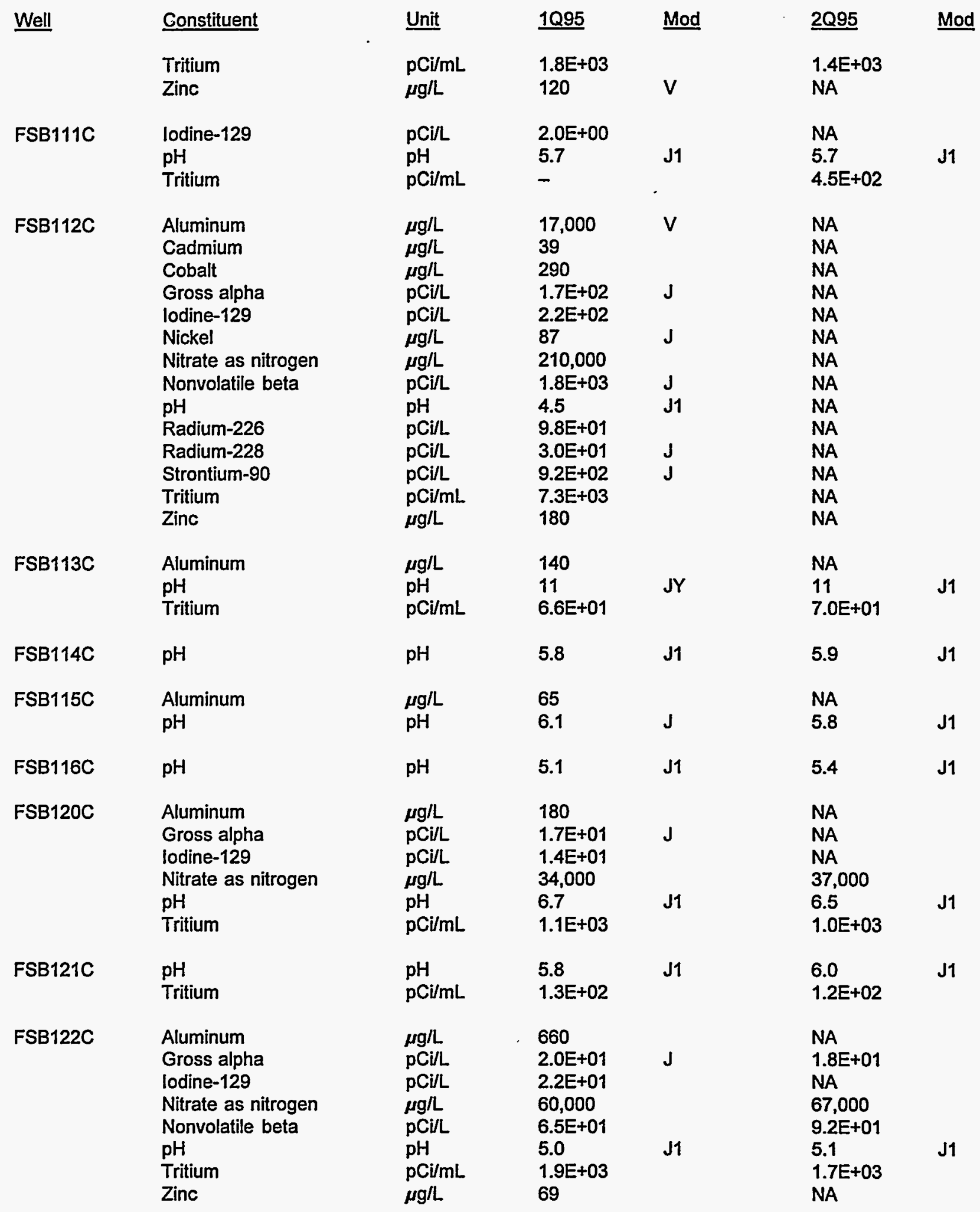


Aquifer Zone IIB (Barnwell/McBean)

\begin{tabular}{|c|c|c|c|c|c|c|}
\hline Well & Constituent & Unit & 1095 & Mod & $2 Q 95$ & Mod \\
\hline FSB123C & $\mathrm{pH}$ & $\mathrm{pH}$ & 5.9 & J1 & 6.0 & J1 \\
\hline \multicolumn{7}{|c|}{ Aquifer Unit IIA (upper Congaree) } \\
\hline Well & Constituent & Unit & 1095 & Mod & 2Q95 & Mod \\
\hline FSB 76B & lodine-129 & $\mathrm{pCi} / \mathrm{L}$ & $3.0 \mathrm{E}+00$ & & NA & \\
\hline FSB 78B & $\begin{array}{l}\text { Aluminum } \\
\text { Nitrate as nitrogen } \\
\text { Tritium }\end{array}$ & $\begin{array}{l}\mu \mathrm{g} / \mathrm{L} \\
\mu \mathrm{g} / \mathrm{L} \\
\mathrm{pCi} / \mathrm{mL}\end{array}$ & $\begin{array}{l}92 \\
11,000 \\
2.7 E+02\end{array}$ & W & $\begin{array}{l}\text { NA } \\
11,000 \\
3.0 E+02\end{array}$ & \\
\hline FSB $79 B$ & Tritium & $\mathrm{pCi} / \mathrm{mL}$ & $2.4 \mathrm{E}+01$ & & - & UI \\
\hline FSB 87B & $\begin{array}{l}\mathrm{pH} \\
\text { Tritium }\end{array}$ & $\begin{array}{l}\mathrm{pH} \\
\mathrm{pCi} / \mathrm{mL}\end{array}$ & $\begin{array}{l}6.2 \\
8.8 E+01\end{array}$ & J1 & $\begin{array}{l}6.0 \\
-\end{array}$ & $\begin{array}{l}\text { J1 } \\
\text { UI }\end{array}$ \\
\hline FSB 96AR & lodine-129 & $\mathrm{pCi} / \mathrm{L}$ & $7.5 \mathrm{E}+00$ & & NA & \\
\hline FSB 97A & $\begin{array}{l}\text { Gross alpha } \\
\text { Tritium }\end{array}$ & $\begin{array}{l}\mathrm{pCi} / \mathrm{L} \\
\mathrm{pCi} / \mathrm{mL}\end{array}$ & $\begin{array}{l}\text { NA } \\
2.2 E+02\end{array}$ & & $\begin{array}{l}1.6 E+01 \\
2.7 E+02\end{array}$ & J1 \\
\hline FSB 98AR & Tritium & $\mathrm{pCi} / \mathrm{mL}$ & $2.5 E+01$ & & - & Ul \\
\hline FSB 99A & $\begin{array}{l}\text { lodine-129 } \\
\text { Tritium }\end{array}$ & $\begin{array}{l}\mathrm{pCi} / \mathrm{L} \\
\mathrm{pCi} / \mathrm{mL}\end{array}$ & $\begin{array}{l}2.6 E+00 \\
1.6 E+02\end{array}$ & & $\begin{array}{l}\text { NA } \\
7.8 E+01\end{array}$ & \\
\hline FSB100A & Tritium & $\mathrm{pCi} / \mathrm{mL}$ & $1.2 E+02$ & & $1.3 E+02$ & \\
\hline FSB112A & Tritium & $\mathrm{pCi} / \mathrm{mL}$ & $1.1 E+02$ & & $1.6 \mathrm{E}+02$ & \\
\hline FSB113A & $\begin{array}{l}\text { Aluminum } \\
\mathrm{pH} \\
\text { Tritium }\end{array}$ & $\begin{array}{l}\mu \mathrm{g} / \mathrm{L} \\
\mathrm{pH} \\
\mathrm{pCi} / \mathrm{mL}\end{array}$ & $\begin{array}{l}2,100 \\
12 \\
1.8 E+02\end{array}$ & $J$ & $\begin{array}{l}\text { NA } \\
12 \\
1.7 E+02\end{array}$ & J1 \\
\hline FSB114A & $\begin{array}{l}\text { Aluminum } \\
\text { Tritium }\end{array}$ & $\begin{array}{l}\mu \mathrm{g} / \mathrm{L} \\
\mathrm{pCi} / \mathrm{mL}\end{array}$ & $\begin{array}{l}110 \\
-\end{array}$ & & $\begin{array}{l}\text { NA } \\
4.9 E+02\end{array}$ & \\
\hline FSB120A & $\begin{array}{l}\text { Aluminum } \\
\mathrm{pH} \\
\text { Tritium }\end{array}$ & $\begin{array}{l}\mu \mathrm{g} / \mathrm{L} \\
\mathrm{pH} \\
\mathrm{pCi} / \mathrm{mL}\end{array}$ & $\begin{array}{l}590 \\
10 \\
6.5 E+01\end{array}$ & J1 & $\begin{array}{l}\text { NA } \\
9.8 \\
2.3 E+01\end{array}$ & J1 \\
\hline HSB $85 A$ & Aluminum & $\mu \mathrm{g} / \mathrm{L}$ & - & & 85 & V \\
\hline \multicolumn{7}{|c|}{ Aquifer Unit IIA (lower Congaree) } \\
\hline Well & Constituent & Unit & $1 \mathrm{Q95}$ & Mod & $\underline{2 Q 95}$ & Mod \\
\hline FSB 76A & $\mathrm{pH}$ & $\mathrm{pH}$ & 6.7 & J1 & 6.8 & J1 \\
\hline
\end{tabular}


Aquifer Unit IIA (lower Congaree)

Well

$$
\text { Constituent }
$$

Unit

$\underline{1095}$

Mod

$\underline{2 Q 95}$

Mod

FSB 78A

$\mathrm{pH}$

$\mathrm{pH}$

6.7

J1

6.7

J1

FSB 79A pH

pH

6.4

J1

6.5

J1

FSB 87A $\mathrm{pH}$

$\mathrm{pH}$

6.5

J1

6.7

J1

Notes: This table presents the highest value for duplicate/replicate results.

The groundwater samples are unfiltered. Therefore, the results for the metals are for total recoverable metals.

Flags are established by EPD/EMS and are based on final PDWS, Secondary Drinking Water Standards, or method detection limits (see Appendix B).

a $N A=$ not analyzed.

b $-=$ analyzed but not above the GWPS. 
Table 2. Maximum Levels of Constituents Exceeding the SRS Flag 2 Criteria

Aquifer Zone IIB (Water Table)

\begin{tabular}{|c|c|c|c|c|c|}
\hline Well & Constituent & $\underline{\text { Unit }}$ & 1Q95 & Mod & $\underline{2 Q 9}$ \\
\hline \multirow[t]{3}{*}{ FSB 76} & Aluminum & $\mu \mathrm{g} / \mathrm{L}$ & 170 & & $N A^{a}$ \\
\hline & Nitrate as nitrogen & $\mu g / L$ & 12,000 & & \\
\hline & Tritium & $\mathrm{pCi} / \mathrm{mL}$ & $2.9 \mathrm{E}+02$ & & - \\
\hline \multirow[t]{13}{*}{ FSB 77} & Aluminum & $\mu g / L$ & 24,000 & & NA \\
\hline & Americium-241 & $\mathrm{pCi} / \mathrm{L}$ & 4.3E+01 & & NA \\
\hline & Curium-243/244 & $\mathrm{pCi} / \mathrm{L}$ & $2.2 E+01$ & & NA \\
\hline & Gross alpha & $\mathrm{pCi} / \mathrm{L}$ & $1.2 E+03$ & & NA \\
\hline & lodine-129 & $\mathrm{pCi} / \mathrm{L}$ & $1.9 \mathrm{E}+02$ & & NA \\
\hline & Nitrate as nitrogen & $\mu \mathrm{g} / \mathrm{L}$ & 380,000 & & NA \\
\hline & Nonvolatile beta & $\mathrm{pCi} / \mathrm{L}$ & $1.3 E+03$ & $\mathrm{~J}$ & NA \\
\hline & Strontium-89 & $\mathrm{pCi} / \mathrm{L}$ & $3.3 E+01$ & & NA \\
\hline & Strontium-90 & $\mathrm{pCi} / \mathrm{L}$ & 3.7E+02 & & NA \\
\hline & Tritium & $\mathrm{pCi} / \mathrm{mL}$ & $3.8 \mathrm{E}+03$ & & NA \\
\hline & Uranium-233/234 & $\mathrm{pCi} / \mathrm{L}$ & $4.5 E+02$ & & NA \\
\hline & Uranium-235 & $\mathrm{pCi} / \mathrm{L}$ & 2.1E+01 & & NA \\
\hline & Uranium-238 & $\mathrm{pCi} / \mathrm{L}$ & $5.0 E+02$ & $J$ & NA \\
\hline \multirow[t]{15}{*}{ FSB 78} & Aluminum & $\mu \mathrm{g} / \mathrm{L}$ & 38,000 & v & NA \\
\hline & Americium-241 & $\mathrm{pCi} / \mathrm{L}$ & $8.5 E+01$ & & NA \\
\hline & Cadmium & $\mu g / L$ & & & NA \\
\hline & Cesium-137 & pCill & $2.8 \mathrm{E}+02$ & & NA \\
\hline & Curium-243/244 & $\mathrm{pCi} / \mathrm{L}$ & $9.2 E+01$ & & NA \\
\hline & Gross alpha & $\mathrm{pCi} / \mathrm{L}$ & $2.5 E+03$ & $\mathbf{J}$ & NA \\
\hline & lodine-129 & $\mathrm{pCi} / \mathrm{L}$ & $2.9 \mathrm{E}+02$ & & NA \\
\hline & Nitrate as nitrogen & $\mu \mathrm{g} / \mathrm{L}$ & 140,000 & & NA \\
\hline & Nonvolatile beta & pCill & 3.1E+03 & $\mathrm{J}$ & NA \\
\hline & Radium-228 & $\mathrm{pCi} / \mathrm{L}$ & $5.7 E+01$ & $\mathrm{~J}$ & NA \\
\hline & Strontium-90 & $\mathrm{pCi} / \mathrm{L}$ & 2.1E+03 & $J$ & NA \\
\hline & Thorium-234 & $\mathrm{pCi} / \mathrm{L}$ & $7.0 E+02$ & & NA \\
\hline & Tritium & $\mathrm{pCi} / \mathrm{mL}$ & $7.3 E+03$ & & NA \\
\hline & Uranium-233/234 & $\mathrm{pCi} / \mathrm{L}$ & $4.8 \mathrm{E}+01$ & & NA \\
\hline & Uranium-238 & $\mathrm{pCi} / \mathrm{L}$ & $5.1 E+01$ & $\mathrm{~J}$ & NA \\
\hline \multirow[t]{14}{*}{ FSB 79} & Aluminum & $\mu \mathrm{g} / \mathrm{L}$ & 61,000 & & NA \\
\hline & Americium-241 & $\mathrm{pCi} / \mathrm{L}$ & $6.0 E+01$ & & NA \\
\hline & Cadmium & $\mu \mathrm{g} / \mathrm{L}$ & 5.7 & $J$ & NA \\
\hline & Cobalt & $\mu \mathrm{g} / \mathrm{L}$ & 520 & & NA \\
\hline & Curium-243/244 & $\mathrm{pCi} / \mathrm{L}$ & $1.4 \mathrm{E}+02$ & & NA \\
\hline & Gross alpha & $\mathrm{pCi} / \mathrm{L}$ & $1.1 \mathrm{E}+03$ & & NA \\
\hline & lodine-129 & $\mathrm{pCi} / \mathrm{L}$ & $2.4 \mathrm{E}+02$ & & NA \\
\hline & Nitrate as nitrogen & $\mu \mathrm{g} / \mathrm{L}$ & 150,000 & & NA \\
\hline & Nonvolatile beta & $\mathrm{pCi} / \mathrm{L}$ & 8.1E+02 & $J$ & NA \\
\hline & Strontium-90 & $\mathrm{pCi} / \mathrm{L}$ & $1.9 E+02$ & & NA \\
\hline & Tritium & $\mathrm{pCi} / \mathrm{mL}$ & $1.0 E+04$ & & NA \\
\hline & Uranium-233/234 & $\mathrm{pCi} / \mathrm{L}$ & 2.3E+02 & & NA \\
\hline & Uranium-235 & $\mathrm{pCi} / \mathrm{L}$ & $1.8 E+01$ & & NA \\
\hline & Uranium-238 & $\mathrm{pCi} / \mathrm{L}$ & 4.7E+02 & $\mathrm{J}$ & NA \\
\hline
\end{tabular}




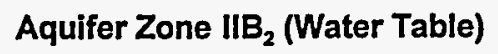

\begin{tabular}{|c|c|c|c|c|c|c|}
\hline Well & Constituent & $\underline{\text { Unit }}$ & $1 \mathrm{Q95}$ & Mod & $\underline{2 Q 95}$ & Mod \\
\hline FSB 87D & $\begin{array}{l}\text { Aluminum } \\
\text { Gross alpha } \\
\text { lodine-129 } \\
\text { Tritium } \\
\text { Uranium-238 }\end{array}$ & $\begin{array}{l}\mu \mathrm{g} / \mathrm{L} \\
\mathrm{pCi} / \mathrm{L} \\
\mathrm{pCi} / \mathrm{L} \\
\mathrm{pCi} / \mathrm{mL} \\
\mathrm{pCi} / \mathrm{L}\end{array}$ & $\begin{array}{l}140 \\
2.5 E+01 \\
2.9 E+01 \\
3.6 E+01 \\
1.9 E+01\end{array}$ & & $\begin{array}{l}N A \\
5.2 E+01 \\
N A \\
- \\
N A\end{array}$ & $\begin{array}{l}\mathrm{J} \\
\mathrm{UI}\end{array}$ \\
\hline FSB 89D & $\begin{array}{l}\text { Aluminum } \\
\text { Americium-241 } \\
\text { Gross alpha } \\
\text { lodine-129 } \\
\text { Mercury } \\
\text { Nitrate as nitrogen } \\
\text { Nonvolatile beta } \\
\text { Strontium-90 } \\
\text { Tritium } \\
\text { Uranium-233/234 } \\
\text { Uranium-238 }\end{array}$ & $\begin{array}{l}\mu \mathrm{g} / \mathrm{L} \\
\mathrm{pCi} / \mathrm{L} \\
\mathrm{pCi} / \mathrm{L} \\
\mathrm{pCi} / \mathrm{L} \\
\mu \mathrm{g} / \mathrm{L} \\
\mu \mathrm{g} / \mathrm{L} \\
\mathrm{pCi} / \mathrm{L} \\
\mathrm{pCi} / \mathrm{L} \\
\mathrm{pCi} / \mathrm{mL} \\
\mathrm{pCi} / \mathrm{L} \\
\text { pCill }\end{array}$ & $\begin{array}{l}6,600 \\
1.1 E+01 \\
2.2 E+02 \\
1.4 E+02 \\
4.8 \\
32,000 \\
1.0 E+03 \\
4.3 E+02 \\
1.8 E+03 \\
9.1 E+01 \\
1.1 E+02\end{array}$ & & $\begin{array}{l}\text { NA } \\
\text { NA } \\
\text { NA } \\
\text { NA } \\
\text { NA } \\
\text { NA } \\
\text { NA } \\
\text { NA } \\
\text { NA } \\
\text { NA } \\
\text { NA }\end{array}$ & \\
\hline FSB 90D & $\begin{array}{l}\text { Aluminum } \\
\text { Americium-241 } \\
\text { Cadmium } \\
\text { Gross alpha } \\
\text { lodine-129 } \\
\text { Mercury } \\
\text { Nitrate as nitrogen } \\
\text { Nonvolatile beta } \\
\text { Radium-228 } \\
\text { Strontium-90 } \\
\text { Tritium } \\
\text { Uranium-233/234 } \\
\text { Uranium-238 }\end{array}$ & $\begin{array}{l}\mu \mathrm{g} / \mathrm{L} \\
\mathrm{pCi/L} \\
\mu \mathrm{g} / \mathrm{L} \\
\mathrm{pCi} / \mathrm{L} \\
\mathrm{pCi} / \mathrm{L} \\
\mu \mathrm{g} / \mathrm{L} \\
\mu \mathrm{g} / \mathrm{L} \\
\mathrm{pCi/L} \\
\mathrm{pCi} / \mathrm{L} \\
\mathrm{pCi} / \mathrm{L} \\
\mathrm{pCi} / \mathrm{mL} \\
\mathrm{pCi} / \mathrm{L} \\
\text { pCi/L }\end{array}$ & $\begin{array}{l}29,000 \\
1.9 E+01 \\
7.2 \\
7.2 \mathrm{E}+02 \\
2.2 \mathrm{E}+02 \\
14 \\
97,000 \\
1.1 \mathrm{E}+03 \\
2.5 \mathrm{E}+01 \\
3.5 \mathrm{E}+02 \\
8.2 \mathrm{E}+03 \\
3.3 \mathrm{E}+02 \\
3.7 \mathrm{E}+02\end{array}$ & $\begin{array}{l}J \\
V \\
J \\
J\end{array}$ & $\begin{array}{l}\text { NA } \\
\text { NA } \\
\text { NA } \\
\text { NA } \\
\text { NA } \\
\text { NA } \\
\text { NA } \\
\text { NA } \\
\text { NA } \\
\text { NA } \\
\text { NA } \\
\text { NA } \\
\text { NA }\end{array}$ & \\
\hline FSB 91D & $\begin{array}{l}\text { Aluminum } \\
\text { Gross alpha } \\
\text { lodine-129 } \\
\text { Nitrate as nitrogen } \\
\text { Nonvolatile beta } \\
\text { Strontium-90 } \\
\text { Uranium-233/234 } \\
\text { Uranium-238 }\end{array}$ & $\begin{array}{l}\mu \mathrm{g} / \mathrm{L} \\
\mathrm{pCi} / \mathrm{L} \\
\mathrm{pCi} / \mathrm{L} \\
\mu \mathrm{g} / \mathrm{L} \\
\mathrm{pCi} / \mathrm{L} \\
\mathrm{pCi/L} \\
\mathrm{pCi} / \mathrm{L} \\
\mathrm{pCi} / \mathrm{L}\end{array}$ & $\begin{array}{l}7,200 \\
1.9 E+02 \\
2.4 E+01 \\
18,000 \\
1.1 E+02 \\
2.3 E+01 \\
6.8 E+01 \\
1.1 E+02\end{array}$ & $J$ & $\begin{array}{l}\text { NA } \\
\text { NA } \\
\text { NA } \\
\text { NA } \\
\text { NA } \\
\text { NA } \\
\text { NA } \\
\text { NA }\end{array}$ & \\
\hline FSB 92D & $\begin{array}{l}\text { Aluminum } \\
\text { Americium-241 } \\
\text { Cadmium } \\
\text { Curium-243/244 } \\
\text { Gross alpha } \\
\text { lodine-129 } \\
\text { Nitrate as nitrogen } \\
\text { Nonvolatile beta } \\
\text { Radium-226 } \\
\text { Radium-228 } \\
\text { Strontium-89 } \\
\text { Strontium-90 }\end{array}$ & $\begin{array}{l}\mu \mathrm{g} / \mathrm{L} \\
\mathrm{pCi} / \mathrm{L} \\
\mu \mathrm{g} / \mathrm{L} \\
\mathrm{pCil} / \mathrm{L} \\
\mathrm{pCi} / \mathrm{L} \\
\mathrm{pCi} / \mathrm{L} \\
\mu \mathrm{g} / \mathrm{L} \\
\mathrm{pCi} / \mathrm{L} \\
\mathrm{pCi} / \mathrm{L} \\
\mathrm{pCi} / \mathrm{L} \\
\mathrm{pCi} / \mathrm{L} \\
\mathrm{pCi} / \mathrm{L}\end{array}$ & $\begin{array}{l}58,000 \\
1.0 \mathrm{E}+01 \\
15 \\
9.0 \mathrm{E}+00 \\
9.9 \mathrm{E}+02 \\
1.2 \mathrm{E}+02 \\
130,000 \\
1.7 \mathrm{E}+03 \\
5.0 \mathrm{E}+01 \\
4.4 \mathrm{E}+01 \\
1.0 \mathrm{E}+02 \\
3.4 \mathrm{E}+02\end{array}$ & $\begin{array}{l}\mathbf{J} \\
\mathbf{J} \\
\mathbf{J}\end{array}$ & $\begin{array}{l}\text { NA } \\
\text { NA } \\
\text { NA } \\
\text { NA } \\
\text { NA } \\
\text { NA } \\
\text { NA } \\
\text { NA } \\
\text { NA } \\
\text { NA } \\
\text { NA } \\
\text { NA }\end{array}$ & \\
\hline
\end{tabular}


Aquifer Zone $\mathrm{IIB}_{2}$ (Water Table)

\begin{tabular}{|c|c|c|c|c|c|}
\hline Well & Constituent & $\underline{\text { Unit }}$ & $1 Q 95$ & Mod & $\underline{2 Q 95}$ \\
\hline & Tritium & $\mathrm{pCi} / \mathrm{mL}$ & $8.7 E+03$ & & NA \\
\hline & Uranium-233/234 & $\mathrm{pCi} / \mathrm{L}$ & $3.0 E+02$ & & NA \\
\hline & Uranium-238 & $\mathrm{pCi} / \mathrm{L}$ & $3.6 \mathrm{E}+02$ & $\mathbf{J}$ & NA \\
\hline FSB 93D & Aluminum & $\mu \mathrm{g} / \mathrm{L}$ & 34,000 & & NA \\
\hline & Cadmiúm & $\mu g / L$ & 13 & & NA \\
\hline & Gross alpha & pCi/L & $3.3 E+02$ & $\mathbf{J}$ & NA \\
\hline & lodine-129 & $\mathrm{pCi} / \mathrm{L}$ & $1.0 E+02$ & & NA \\
\hline & Nitrate as nitrogen & $\mu \mathrm{g} / \mathrm{L}$ & 120,000 & & NA \\
\hline & Nonvolatile beta & $\mathrm{pCi} / \mathrm{L}$ & $2.0 E+03$ & $\mathrm{~J}$ & NA \\
\hline & Radium-228 & $\mathrm{pCi} / \mathrm{L}$ & $3.7 E+01$ & $\mathrm{~J}$ & NA \\
\hline & Strontium-89 & pCill & $2.6 E+02$ & & NA \\
\hline & Strontium-90 & $\mathrm{pCi} / \mathrm{L}$ & $7.1 \mathrm{E}+02$ & & NA \\
\hline & Tritium & $\mathrm{pCi} / \mathrm{mL}$ & $1.0 E+04$ & . & NA \\
\hline & Uranium-233/234 & $\mathrm{pCi} / \mathrm{L}$ & $5.9 E+01$ & & NA \\
\hline & Uranium-238 & $\mathrm{pCi} / \mathrm{L}$ & $8.2 E+01$ & $\mathrm{~J}$ & NA \\
\hline FSB 94DR & Aluminum & $\mu \mathrm{g} / \mathrm{L}$ & 64,000 & & NA \\
\hline & Americium-241 & $\mathrm{pCi} / \mathrm{L}$ & $9.0 E+01$ & & NA \\
\hline & Cesium-137 & $\mathrm{pCi} / \mathrm{L}$ & $2.8 E+02$ & & NA \\
\hline & Curium-243/244 & pCill & $1.6 \mathrm{E}+02$ & & NA \\
\hline & Gross alpha & $\mathrm{pCi} / \mathrm{L}$ & $1.4 \mathrm{E}+03$ & & NA \\
\hline & lodine-129 & $\mathrm{pCi} / \mathrm{L}$ & $1.4 \mathrm{E}+02$ & & NA \\
\hline & Nitrate as nitrogen & $\mu g / L$ & 130,000 & & NA \\
\hline & Nonvolatile beta & pCi/L & $1.3 \mathrm{E}+03$ & $\mathbf{J}$ & NA \\
\hline & Radium-228 & $\mathrm{pCi} / \mathrm{L}$ & $5.7 E+01$ & $\mathbf{J}$ & NA \\
\hline & Strontium-89 & $\mathrm{pCi} / \mathrm{L}$ & 2.1E+01 & & NA \\
\hline & Strontium-90 & $\mathrm{pCi} / \mathrm{L}$ & $2.3 E+02$ & & NA \\
\hline & Thorium-234 & pCill & $7.0 E+02$ & & NA \\
\hline & Tritium & $\mathrm{pCi} / \mathrm{mL}$ & $8.9 E+03$ & & NA \\
\hline & Uranium-233/234 & $\mathrm{pCi} / \mathrm{L}$ & $6.7 E+02$ & & NA \\
\hline & Uranium-235 & $\mathrm{pCi} / \mathrm{L}$ & $3.9 E+01$ & & NA \\
\hline & Uranium-238 & $\mathrm{pCi} / \mathrm{L}$ & $1.3 \mathrm{E}+03$ & $J$ & NA \\
\hline FSB 95DR & Aluminum & $\mu g / L$ & 47,000 & & NA \\
\hline & Americium-241 & $\mathrm{pCi} / \mathrm{L}$ & 8.4E+01 & & NA \\
\hline & Cesium-137 & pCill & $4.4 E+02$ & & NA \\
\hline & Curium-243/244 & $\mathrm{pCi} / \mathrm{L}$ & 8.7E+01 & & NA \\
\hline & Curium-245/246 & $\mathrm{pCi} / \mathrm{L}$ & $7.9 E+00$ & & NA \\
\hline & Gross alpha & pCill & $2.0 E+03$ & & NA \\
\hline & lodine-129 & $\mathrm{pCi} / \mathrm{L}$ & $2.3 E+02$ & & NA \\
\hline & Nitrate as nitrogen & $\mu g / L$ & 230,000 & & NA \\
\hline & Nonvolatile beta & $\mathrm{pCi} / \mathrm{L}$ & $1.3 \mathrm{E}+03$ & $\mathrm{~J}$ & NA \\
\hline & Radium-228 & $\mathrm{pCi} / \mathrm{L}$ & $4.4 \mathrm{E}+01$ & & NA \\
\hline & Strontium-90 & $\mathrm{pCi} / \mathrm{L}$ & $1.5 \mathrm{E}+02$ & & NA \\
\hline & Thorium-234 & $\mathrm{pCi} / \mathrm{L}$ & $9.5 \mathrm{E}+02$ & & NA \\
\hline & Tritium & $\mathrm{pCi} / \mathrm{mL}$ & $1.2 \mathrm{E}+04$ & & NA \\
\hline & Uranium-233/234 & $\mathrm{pCi} / \mathrm{L}$ & $4.2 E+02$ & & NA \\
\hline & Uranium-235 & $\mathrm{pCi} / \mathrm{L}$ & $3.0 E+01$ & & NA \\
\hline & Uranium-238 & $\mathrm{pCi} / \mathrm{L}$ & $1.2 \mathrm{E}+03$ & $J$ & NA \\
\hline
\end{tabular}


Aquifer Zone $\|_{2}$ (Water Table)

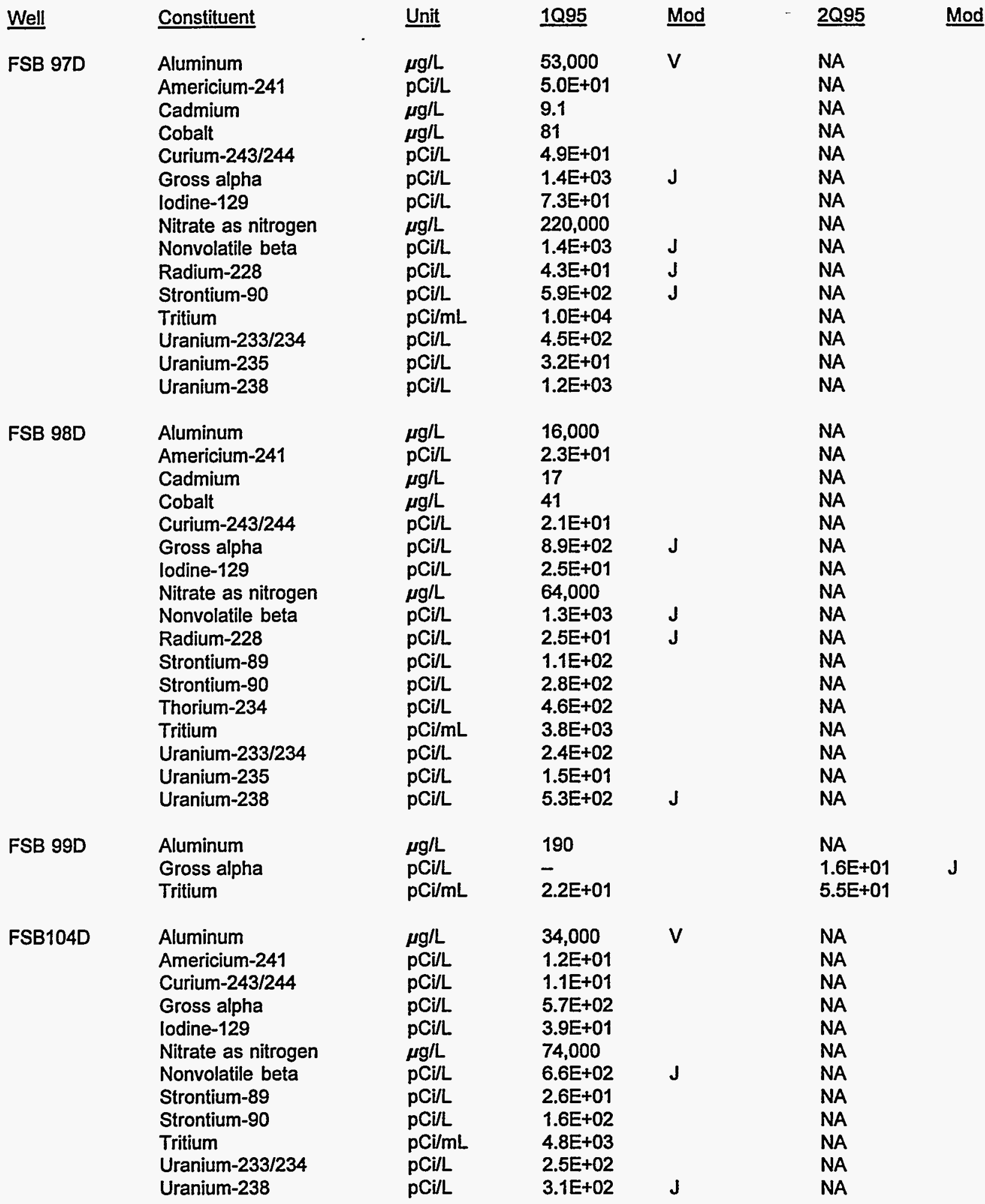


Aquifer Zone $\|_{2} B_{2}$ (Water Table)

\begin{tabular}{|c|c|c|c|c|c|}
\hline Well & Constituent & Unit & 1095 & Mod & $2 Q 95$ \\
\hline \multirow[t]{10}{*}{ FSB105DR } & Aluminum & $\mu g / L$ & 10,000 & & NA \\
\hline & Gross alpha & $\mathrm{pCi} / \mathrm{L}$ & 4.0E+02 & & NA \\
\hline & lodine-129 & $\mathrm{pCi} / \mathrm{L}$ & $1.7 E+01$ & & NA \\
\hline & Nitrate as nitrogen & $\mu g / L$ & 23,000 & $J$ & NA \\
\hline & Nonvolatile beta & $\mathrm{pCi} / \mathrm{L}$ & 4.1E+02 & & NA \\
\hline & Strontium-90 & pCill & $3.4 E+01$ & & NA \\
\hline & Tritium & $\mathrm{pCi} / \mathrm{mL}$ & $5.5 E+02$ & & NA \\
\hline & Uranium-233/234 & $\mathrm{pCi} / \mathrm{L}$ & $2.2 E+02$ & & NA \\
\hline & Uranium-235 & $\mathrm{pCi} / \mathrm{L}$ & 1.6E+01 & & NA \\
\hline & Uranium-238 & pCi/L & $5.6 E+02$ & & NA \\
\hline \multirow[t]{3}{*}{ FSB106D } & Aluminum & $\mu g / L$ & 54 & & NA \\
\hline & Bis(2-ethylhexyl) phthalate & $\mu g / L$ & 190 & & NA \\
\hline & Lead & $\mu g / L$ & 51 & & - \\
\hline \multirow[t]{18}{*}{ FSB107D } & Aluminum & $\mu g / L$ & 15,000 & & NA \\
\hline & Americium-241 & pCi/L & 2.9E+01 & & NA \\
\hline & Cadmium & $\mu g / L$ & 6.6 & & NA \\
\hline & Curium-243/244 & pCill & 1.3E+01 & & NA \\
\hline & Gross alpha & pCill & $1.1 E+03$ & $J$ & NA \\
\hline & lodine-129 & pCill & $1.6 E+02$ & & NA \\
\hline & Mercury & $\mu \mathrm{g} / \mathrm{L}$ & 5.4 & & NA \\
\hline & Nitrate as nitrogen & $\mu g / L$ & 69,000 & & NA \\
\hline & Nonvolatile beta & pCi/L & 1.3E+03 & $J$ & NA \\
\hline & Radium-226 & pCill & $2.2 E+01$ & J & NA \\
\hline & Strontium-89 & $\mathrm{pCi} / \mathrm{L}$ & $6.8 E \div 01$ & & NA \\
\hline & Strontium-90 & $\mathrm{pCi} / \mathrm{L}$ & $2.5 E+02$ & & NA \\
\hline & Thorium-234 & pCill & $5.3 E+02$ & & NA \\
\hline & Trichlorofluoromethane & $\mu g / L$ & 15 & & NA \\
\hline & Tritium & $\mathrm{pCi} / \mathrm{mL}$ & $2.5 E+03$ & & NA \\
\hline & Uranium-233/234 & $\mathrm{pCi} / \mathrm{L}$ & $4.3 E \div 02$ & & NA \\
\hline & Uranium-235 & $\mathrm{pCi} / \mathrm{L}$ & $2.4 E+01$ & & NA \\
\hline & Uranium-238 & $\mathrm{pCi} / \mathrm{L}$ & $5.5 E+02$ & $J$ & NA \\
\hline FSB108D & Aluminum & $\mu \mathrm{g} / \mathrm{L}$ & 120 & V & NA \\
\hline \multirow[t]{2}{*}{ FSB109D } & Aluminum & $\mu g / L$ & 100 & v & NA \\
\hline & Tritium & $\mathrm{pCi} / \mathrm{mL}$ & $2.4 E+01$ & & 2.7E+01 \\
\hline \multirow[t]{14}{*}{ FSB110D } & Aluminum & $\mu g / L$ & 96,000 & V & NA \\
\hline & Americium-241 & $\mathrm{pCi} / \mathrm{L}$ & $9.9 E+01$ & & NA \\
\hline & Cadmium & $\mu \mathrm{g} / \mathrm{L}$ & 6.6 & J & NA \\
\hline & Curium-243/244 & $\mathrm{pCi} / \mathrm{L}$ & $1.5 E+02$ & & NA \\
\hline & Curium-245/246 & $\mathrm{pCi} / \mathrm{L}$ & $9.9 E+01$ & & NA \\
\hline & Gross alpha & $\mathrm{pCi} / \mathrm{L}$ & $1.8 \mathrm{E}+03$ & J & NA \\
\hline & lodine-129 & $\mathrm{pCi} / \mathrm{L}$ & $1.3 E+02$ & & NA \\
\hline & Mercury & $\mu \mathrm{g} / \mathrm{L}$ & 4.8 & & NA \\
\hline & Nitrate as nitrogen & $\mu \mathrm{g} / \mathrm{L}$ & 290,000 & & NA \\
\hline & Nonvolatile beta & $\mathrm{pCi} / \mathrm{L}$ & 8.7E+02 & J & NA \\
\hline & Radium-228 & pCill & $3.4 E+01$ & $J$ & NA \\
\hline & Strontium-90 & $\mathrm{pCi} / \mathrm{L}$ & $3.1 E+02$ & $J$ & NA \\
\hline & Thorium-234 & $\mathrm{pCi} / \mathrm{L}$ & $6.1 E+02$ & & NA \\
\hline & Tritium & $\mathrm{pCi} / \mathrm{mL}$ & $1.9 E+04$ & & NA \\
\hline
\end{tabular}


Aquifer Zone $\mathrm{IIB}_{2}$ (Water Table)

\begin{tabular}{|c|c|c|c|c|c|}
\hline Well & Constituent & Unit & 1095 & Mod & $\underline{2 Q 95}$ \\
\hline & Uranium-233/234 & $\mathrm{pCi} / \mathrm{L}$ & $3.5 E+02$ & & NA \\
\hline & Uranium-235 & pCill & $2.8 E+01$ & & NA \\
\hline & Uranium-238 & pCill & 1.3E+03 & $J$ & NA \\
\hline FSB112D & Aluminum & $\mu \mathrm{g} / \mathrm{L}$ & 44,000 & V & NA \\
\hline & Cadmium & $\mu \mathrm{g} / \mathrm{L}$ & & & NA \\
\hline & Cobalt & $\mu \mathrm{g} / \mathrm{L}$ & 77 & & NA \\
\hline & Gross alpha & pCill & $3.4 E+02$ & $\mathrm{~J}$ & NA \\
\hline & lodine-129 & $\mathrm{pCi} / \mathrm{L}$ & 7.3E+01 & & NA \\
\hline & Nitrate as nitrogen & $\mu g / L$ & 160,000 & & NA \\
\hline & Nonvolatile beta & pCill & $3.1 E+03$ & $\mathrm{~J}$ & NA \\
\hline & Radium-226 & pCill & $9.1 E+01$ & & NA \\
\hline & Radium-228 & pCill & $4.6 E+01$ & $\mathbf{J}$ & NA \\
\hline & Strontium-90 & pCill & $1.2 E+03$ & $\mathrm{~J}$ & NA \\
\hline & Tritium & $\mathrm{pCi} / \mathrm{mL}$ & $8.8 E+03$ & & NA \\
\hline FSB115D & Aluminum & $\mu g / L$ & 110 & & NA \\
\hline & Bis(2-ethylhexyl) phthalate & $\mu g / L$ & 24 & & NA \\
\hline FSB116D & Aluminum & $\mu g / L$ & 160 & & NA \\
\hline FSB117D & Aluminum & $\mu \mathrm{g} / \mathrm{L}$ & 9,500 & & NA \\
\hline & Gross alpha & pCill & $6.2 E+02$ & $\mathrm{~J}$ & NA \\
\hline & lodine-129 & $\mathrm{pCi} / \mathrm{L}$ & $4.2 E+01$ & & NA \\
\hline & Nitrate as nitrogen & $\mu \mathrm{g} / \mathrm{L}$ & 31,000 & & NA \\
\hline & Nonvolatile beta & pCill & $3.5 E+02$ & & NA \\
\hline & Strontium-90 & $\mathrm{pCi} / \mathrm{L}$ & $5.9 E+01$ & & NA \\
\hline & Tritium & $\mathrm{pCi} / \mathrm{mL}$ & $9.3 E+02$ & & NA \\
\hline & Uranium-233/234 & pCill & $3.5 E+02$ & & NA \\
\hline & Uranium-235 & $\mathrm{pCi} / \mathrm{L}$ & $2.0 E+01$ & & NA \\
\hline & Uranium-238 & $\mathrm{pCi} / \mathrm{L}$ & $3.8 E+02$ & & NA \\
\hline FSB118D & Tritium & $\mathrm{pCi} / \mathrm{mL}$ & - & & $4.8 E+02$ \\
\hline FSB119D & Aluminum & $\mu g / L$ & 54,000 & v & NA \\
\hline & Americium-241 & $\mathrm{pCi} / \mathrm{L}$ & $1.6 E+01$ & & NA \\
\hline & Cadmium & $\mu \mathrm{g} / \mathrm{L}$ & 5.6 & & NA \\
\hline & Cobalt & $\mu g / L$ & 50 & & NA \\
\hline & Gross alpha & pCill & 2.1E+03 & $\mathbf{J}$ & NA \\
\hline & lodine-129 & $\mathrm{pCill}$ & $1.5 E+02$ & & NA \\
\hline & Nitrate as nitrogen & $\mu \mathrm{g} / \mathrm{L}$ & 130,000 & & NA \\
\hline & Nonvolatile beta & pCill & $1.3 E+03$ & $J$ & NA \\
\hline & Radium-226 & $\mathrm{pCi} / \mathrm{L}$ & $3.2 E+01$ & & NA \\
\hline & Radium-228 & $\mathrm{pCi} / \mathrm{L}$ & $3.3 E+01$ & $J$ & NA \\
\hline & Strontium-90 & $\mathrm{pCill}$ & $4.6 \mathrm{E}+02$ & $\mathrm{~J}$ & NA \\
\hline & Thorium-234 & pCill & $4.5 \mathrm{E}+02$ & & NA \\
\hline & Tritium & $\mathrm{pCi} / \mathrm{mL}$ & $6.3 E+03$ & & NA \\
\hline & Uranium-233/234 & pCill & $8.8 E+02$ & & NA \\
\hline & Uranium-235 & $\mathrm{pCi} / \mathrm{L}$ & 4.6E+01 & & NA \\
\hline & Uranium-238 & $\mathrm{pCi} / \mathrm{L}$ & $9.4 E+02$ & & NA \\
\hline
\end{tabular}


Aquifer Zone IIB $_{2}$ (Water Table)

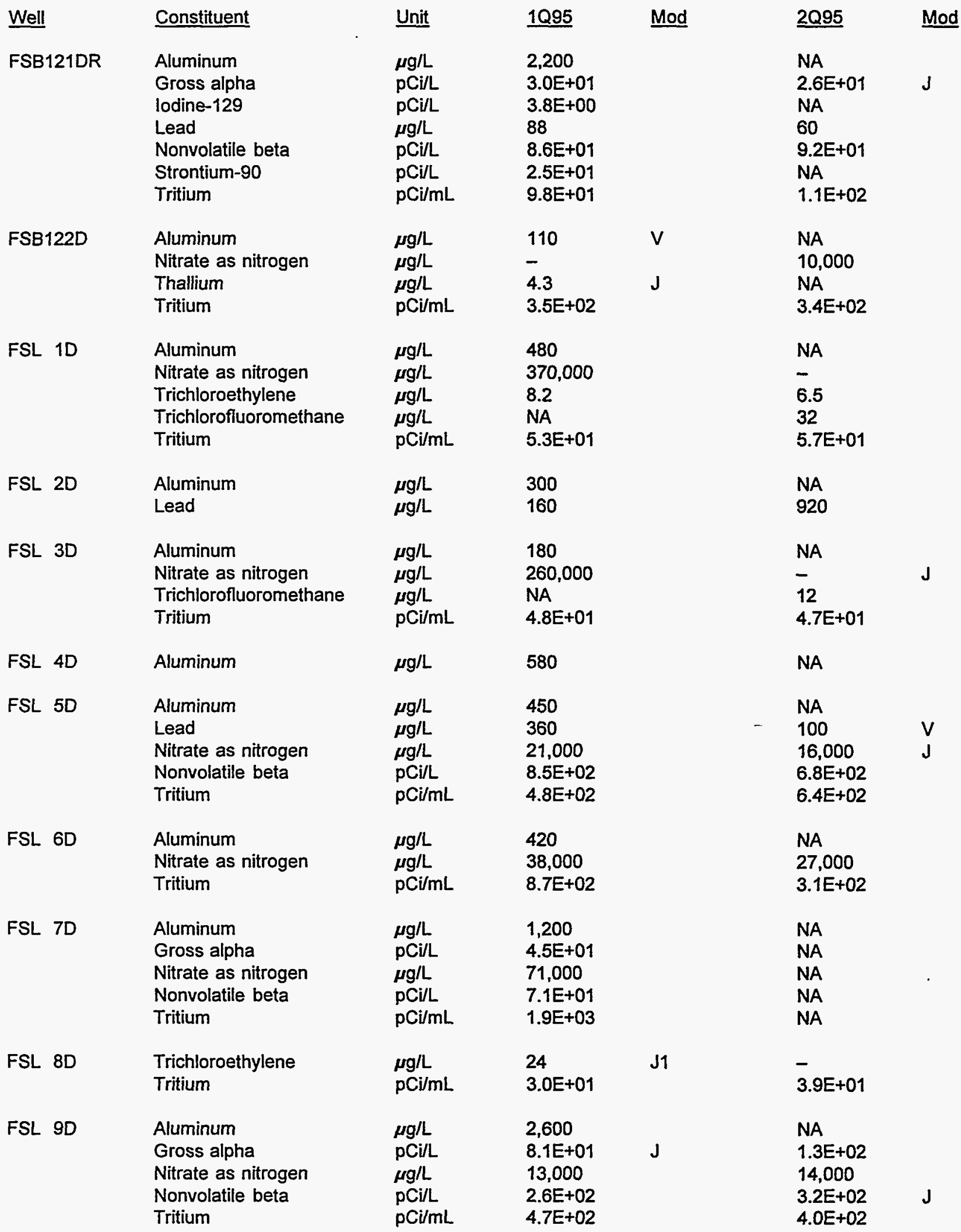


Aquifer Zone IIB, (Barnwell/McBean)

\begin{tabular}{|c|c|c|c|c|c|}
\hline Well & Constituent & Unit & 1095 & Mod & $\underline{2 Q 95}$ \\
\hline FSB $78 \mathrm{C}$ & $\begin{array}{l}\text { Aluminum } \\
\text { Cadmium } \\
\text { Cobalt } \\
\text { Gross alpha } \\
\text { lodine-129 } \\
\text { Nickel } \\
\text { Nitrate as nitrogen } \\
\text { Nonvolatile beta } \\
\text { Radium-226 } \\
\text { Strontium-90 } \\
\text { Tritium } \\
\text { Uranium-233/234 } \\
\text { Uranium-235 } \\
\text { Uranium-238 }\end{array}$ & $\begin{array}{l}\mu \mathrm{g} / \mathrm{L} \\
\mu \mathrm{g} / \mathrm{L} \\
\mu \mathrm{g} / \mathrm{L} \\
\mathrm{pCi} / \mathrm{L} \\
\mathrm{pCi} / \mathrm{L} \\
\mu \mathrm{g} / \mathrm{L} \\
\mu \mathrm{g} / \mathrm{L} \\
\mathrm{pCi/L} \\
\mathrm{pCi} / \mathrm{L} \\
\mathrm{pCi} / \mathrm{L} \\
\mathrm{pCi/mL} \\
\mathrm{pCi} / \mathrm{L} \\
\mathrm{pCi} / \mathrm{L} \\
\mathrm{pCi} / \mathrm{L}\end{array}$ & $\begin{array}{l}16,000 \\
16 \\
300 \\
1.6 E+02 \\
1.2 E+02 \\
120 \\
240,000 \\
1.1 E+03 \\
5.1 E+01 \\
6.2 E+02 \\
8.7 E+03 \\
3.9 E+02 \\
2.4 E+01 \\
1.0 E+03\end{array}$ & $\begin{array}{l}V \\
J \\
J\end{array}$ & $\begin{array}{l}\text { NA } \\
\text { NA } \\
\text { NA } \\
\text { NA } \\
\text { NA } \\
\text { NA } \\
\text { NA } \\
\text { NA } \\
\text { NA } \\
\text { NA } \\
\text { NA } \\
\text { NA } \\
\text { NA } \\
\text { NA }\end{array}$ \\
\hline FSB $79 \mathrm{C}$ & $\begin{array}{l}\text { Aluminum } \\
\text { Americium-241 } \\
\text { Cadmium } \\
\text { Cobalt } \\
\text { Curium-243/244 } \\
\text { Gross alpha } \\
\text { lodine-129 } \\
\text { Nitrate as nitrogen } \\
\text { Nonvolatile beta } \\
\text { Plutonium-238 } \\
\text { Radium-226 } \\
\text { Strontium-89 } \\
\text { Strontium-90 } \\
\text { Tritium } \\
\text { Uranium-233/234 } \\
\text { Uranium-235 } \\
\text { Uranium-238 }\end{array}$ & $\begin{array}{l}\mu g / L \\
\text { pCi/L } \\
\mu g / L \\
\mu g / L \\
\text { pCi/L } \\
\text { pCi/L } \\
\text { pCi/L } \\
\mu g / L \\
\text { pCi/L } \\
\text { pCi/L } \\
\text { pCi/L } \\
\text { pCi/L } \\
\text { pCi/L } \\
\text { pCi/mL } \\
\text { pCi/L } \\
\text { pCi/L } \\
\text { pCi/L }\end{array}$ & $\begin{array}{l}39,000 \\
2.1 E+01 \\
27 \\
140 \\
2.8 E+01 \\
8.0 E+02 \\
9.2 E+01 \\
130,000 \\
2.5 E+03 \\
2.3 E+01 \\
4.8 E+01 \\
2.0 E+02 \\
7.2 E+02 \\
8.7 E+03 \\
2.2 E+02 \\
1.8 E+01 \\
2.3 E+02\end{array}$ & $\begin{array}{l}J \\
J\end{array}$ & $\begin{array}{l}\text { NA } \\
\text { NA } \\
\text { NA } \\
\text { NA } \\
\text { NA } \\
\text { NA } \\
\text { NA } \\
\text { NA } \\
\text { NA } \\
\text { NA } \\
\text { NA } \\
\text { NA } \\
\text { NA } \\
\text { NA } \\
\text { NA } \\
\text { NA } \\
\text { NA }\end{array}$ \\
\hline FSB $87 \mathrm{C}$ & $\begin{array}{l}\text { lodine-129 } \\
\text { Tritium }\end{array}$ & $\begin{array}{l}\mathrm{pCi} / \mathrm{L} \\
\mathrm{pCi} / \mathrm{mL}\end{array}$ & $\begin{array}{l}3.3 E+00 \\
4.7 E+02\end{array}$ & & $\begin{array}{l}N A \\
4.8 E+02\end{array}$ \\
\hline FSB $89 \mathrm{C}$ & $\begin{array}{l}\text { Dichloromethane } \\
\text { Thallium }\end{array}$ & $\begin{array}{l}\mu \mathrm{g} / \mathrm{L} \\
\mu \mathrm{g} / \mathrm{L}\end{array}$ & $\begin{array}{l}14 \\
3.3\end{array}$ & $\begin{array}{l}\text { W } \\
\text { J }\end{array}$ & $\begin{array}{l}\text { NA } \\
\text { NA }\end{array}$ \\
\hline FSB 90C & $\begin{array}{l}\text { Aluminum } \\
\text { Nitrate as nitrogen } \\
\text { Tritium }\end{array}$ & $\begin{array}{l}\mu \mathrm{g} / \mathrm{L} \\
\mu \mathrm{g} / \mathrm{L} \\
\mathrm{pCi} / \mathrm{mL}\end{array}$ & $\begin{array}{l}73 \\
11,000 \\
5.8 E+02\end{array}$ & $J$ & $\begin{array}{l}N A \\
14,000 \\
6.1 E+02\end{array}$ \\
\hline FSB 91C & $\begin{array}{l}\text { Aluminum } \\
\text { Cadmium } \\
\text { Cobalt } \\
\text { Gross alpha } \\
\text { lodine-129 } \\
\text { Nitrate as nitrogen } \\
\text { Nonvolatile beta }\end{array}$ & $\begin{array}{l}\mu \mathrm{g} / \mathrm{L} \\
\mu \mathrm{g} / \mathrm{L} \\
\mu \mathrm{g} / \mathrm{L} \\
\mathrm{pCi} / \mathrm{L} \\
\mathrm{pCi} / \mathrm{L} \\
\mu \mathrm{g} / \mathrm{L} \\
\mathrm{pCi} / \mathrm{L}\end{array}$ & $\begin{array}{l}28,000 \\
5.4 \\
43 \\
6.2 E+01 \\
4.8 E+01 \\
25,000 \\
4.0 E+02\end{array}$ & $\begin{array}{l}V \\
V\end{array}$ & $\begin{array}{l}\text { NA } \\
\text { NA } \\
\text { NA } \\
\text { NA } \\
\text { NA } \\
\text { NA } \\
\text { NA }\end{array}$ \\
\hline
\end{tabular}


Aquifer Zone IIB, (BarnwelI/McBean)

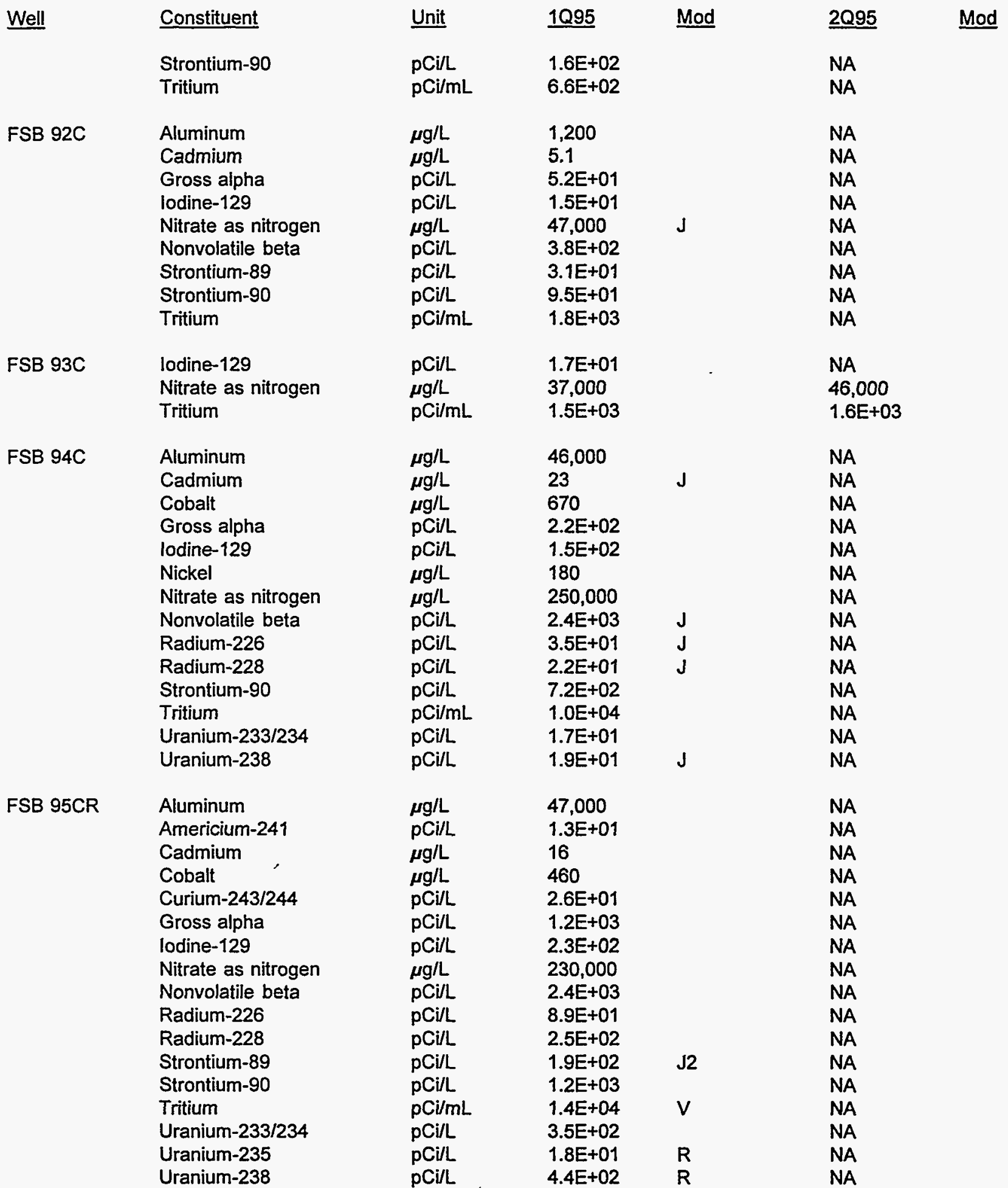


Aquifer Zone IIB ${ }_{1}$ (Barnwell/McBean)

\begin{tabular}{|c|c|c|c|c|c|c|}
\hline Well & Constituent & Unit & 1095 & Mod & $\underline{2 Q 95}$ & Mod \\
\hline \multirow[t]{16}{*}{ FSB 97C } & $\begin{array}{l}\text { Aluminum } \\
\text { Americium-241 }\end{array}$ & $\begin{array}{l}\mu g / L \\
\text { pCill }\end{array}$ & $\begin{array}{l}77,000 \\
5.3 E+01\end{array}$ & v & $\begin{array}{l}\text { NA } \\
\text { NA }\end{array}$ & \\
\hline & Cadmium & $\mu g / L$ & 6.1 & $\mathbf{J}$ & NA & \\
\hline & Cobalt & $\mu g / L$ & 320 & & NA & \\
\hline & Curium-243/244 & pCill & $3.7 E+01$ & & NA & \\
\hline & Gross alpha & pCill & $2.0 \mathrm{E}+03$ & $\mathrm{~J}$ & NA & \\
\hline & lodine-129 & $\mathrm{pCi} / \mathrm{L}$ & $8.6 E+01$ & & NA & \\
\hline & Nitrate as nitrogen & $\mu \mathrm{g} / \mathrm{L}$ & 240,000 & & NA & \\
\hline & Nonvolatile beta & pCilL & $1.1 E+03$ & $\mathbf{J}$ & NA & \\
\hline & Radium-226 & $\mathrm{pCill}$ & $6.3 E+01$ & & NA & \\
\hline & Radium-228 & $\mathrm{pCi} / \mathrm{L}$ & 4.4E+01 & $\mathbf{J}$ & NA & \\
\hline & Strontium-90 & pCill & $3.4 E+02$ & $\mathrm{~J}$ & NA & \\
\hline & Trichloroethylene & $\mu \mathrm{g} / \mathrm{L}$ & 9.3 & & NA & \\
\hline & Tritium & $\mathrm{pCi} / \mathrm{mL}$ & $1.3 E+04$ & & NA & \\
\hline & Uranium-233/234 & pCill & $4.2 E+02$ & & NA & \\
\hline & Uranium-235 & $\mathrm{pCi} / \mathrm{L}$ & $2.5 E+01$ & & NA & \\
\hline & Uranium-238 & pCill & $5.7 \mathrm{E}+02$ & $J$ & NA & \\
\hline \multirow[t]{14}{*}{ FSB 98C } & Aluminum & $\mu \mathrm{g} / \mathrm{L}$ & 39,000 & v & NA & \\
\hline & Americium-241 & $\mathrm{pCi} / \mathrm{L}$ & $3.4 \mathrm{E}+01$ & & NA & \\
\hline & Cobalt & $\mu \mathrm{g} / \mathrm{L}$ & 350 & & NA & \\
\hline & Curium-243/244 & $\mathrm{pCi} / \mathrm{L}$ & $3.3 E+01$ & & NA & \\
\hline & Gross alpha & $\mathrm{pCi} / \mathrm{L}$ & $2.1 E+02$ & & NA & \\
\hline & lodine-129 & $\mathrm{pCilL}$ & $8.5 E+01$ & & NA & \\
\hline & Nitrate as nitrogen & $\mu \mathrm{g} / \mathrm{L}$ & 140,000 & & NA & \\
\hline & Nonvolatile beta & pCill & $3.2 \mathrm{E}+02$ & $J$ & NA & \\
\hline & Radium-226 & $\mathrm{pCi} / \mathrm{L}$ & $5.2 E+01$ & $\mathbf{J}$ & NA & \\
\hline & Strontium-90 & $\mathrm{pCi} / \mathrm{L}$ & $1.6 E+02$ & & NA & \\
\hline & Tritium & $\mathrm{pCi} / \mathrm{mL}$ & $7.9 E+03$ & & NA & \\
\hline & Uranium-233/234 & $\mathrm{pCi} / \mathrm{L}$ & $4.5 E+02$ & & NA & \\
\hline & Uranium-235 & $\mathrm{pCill}$ & 2.3E+01 & & NA & \\
\hline & Uranium-238 & $\mathrm{pCi} / \mathrm{L}$ & $6.6 \mathrm{E}+02$ & $J$ & NA & \\
\hline \multirow[t]{8}{*}{ FSB 99C } & Aluminum & $\mu g / L$ & 120 & & NA & \\
\hline & Gross alpha & pCill & 2.1E+01 & & 3.7E+01 & \\
\hline & lodine-129 & pCill & $1.2 \mathrm{E}+01$ & & NA & \\
\hline & Nitrate as nitrogen & $\mu \mathrm{g} / \mathrm{L}$ & 28,000 & & 30,000 & \\
\hline & Nonvolatile beta & pCill & $8.7 E+01$ & & $9.0 E+01$ & $\mathrm{~J}$ \\
\hline & Strontium-90 & $\mathrm{pCi} / \mathrm{L}$ & $1.8 \mathrm{E}+01$ & & NA & \\
\hline & Trichloroethylene & $\mu \mathrm{g} / \mathrm{L}$ & 18 & & NA & \\
\hline & Tritium & $\mathrm{pCi} / \mathrm{mL}$ & $1.5 E+03$ & & - & \\
\hline \multirow[t]{9}{*}{ FSB102C } & Aluminum & $\mu \mathrm{g} / \mathrm{L}$ & 1,200 & & NA & \\
\hline & Cadmium & $\mu \mathrm{g} / \mathrm{L}$ & 6.5 & & NA & \\
\hline & Cobalt & $\mu \mathrm{g} / \mathrm{L}$ & 48 & & NA & \\
\hline & lodine-129 & pCill & $2.9 E+01$ & & NA & \\
\hline & Nitrate as nitrogen & $\mu \mathrm{g} / \mathrm{L}$ & 37,000 & $J$ & NA & \\
\hline & Nonvolatile beta & pCill & $5.7 \mathrm{E}+02$ & & NA & \\
\hline & Strontium-89 & $\mathrm{pCi} / \mathrm{L}$ & $2.3 E+01$ & & NA & \\
\hline & Strontium-90 & $\mathrm{pCi} / \mathrm{L}$ & $1.7 E+02$ & & NA & \\
\hline & Tritium & $\mathrm{pCi} / \mathrm{mL}$ & $9.5 E+02$ & & NA & \\
\hline
\end{tabular}


Aquifer Zone IIB, (Barnwell/McBean)

\begin{tabular}{|c|c|c|c|c|c|}
\hline Well & Constituent & $\underline{\text { Unit }}$ & 1095 & Mod & $2 Q 95$ \\
\hline FSB103C & $\begin{array}{l}\text { lodine-129 } \\
\text { Nitrate as nitrogen } \\
\text { Tritium }\end{array}$ & $\begin{array}{l}\mathrm{pCi} / \mathrm{L} \\
\mu \mathrm{g} / \mathrm{L} \\
\mathrm{pCi} / \mathrm{mL}\end{array}$ & $\begin{array}{l}5.1 E+00 \\
21,000 \\
5.8 E+02\end{array}$ & & $\begin{array}{l}\text { NA } \\
25,000 \\
5.9 E+02\end{array}$ \\
\hline FSB104C & $\begin{array}{l}\text { lodine }-129 \\
\text { Nitrate as nitrogen } \\
\text { Tritium }\end{array}$ & $\begin{array}{l}\mathrm{pCi} / \mathrm{L} \\
\mu \mathrm{g} / \mathrm{L} \\
\mathrm{pCi} / \mathrm{mL}\end{array}$ & $\begin{array}{l}6.0 E+00 \\
45,000 \\
1.2 E+03\end{array}$ & & $\begin{array}{l}\text { NA } \\
43,000 \\
-\end{array}$ \\
\hline FSB105C & $\begin{array}{l}\text { Aluminum } \\
\text { Americium-241 } \\
\text { Cadmium } \\
\text { Cobalt } \\
\text { Curium-243/244 } \\
\text { Gross alpha } \\
\text { lodine-129 } \\
\text { Nitrate as nitrogen } \\
\text { Nonvolatile beta } \\
\text { Radium-226 } \\
\text { Radium-228 } \\
\text { Strontium-89 } \\
\text { Strontium-90 } \\
\text { Tritium } \\
\text { Uranium-233/234 } \\
\text { Uranium-238 }\end{array}$ & $\begin{array}{l}\mu \mathrm{g} / \mathrm{L} \\
\mathrm{pCi} / \mathrm{L} \\
\mu \mathrm{g} / \mathrm{L} \\
\mu \mathrm{g} / \mathrm{L} \\
\mathrm{pCi} / \mathrm{L} \\
\mathrm{pCi} / \mathrm{L} \\
\mathrm{pCi} / \mathrm{L} \\
\mu \mathrm{g} / \mathrm{L} \\
\mathrm{pCi} / \mathrm{L} \\
\mathrm{pCi} / \mathrm{L} \\
\mathrm{pCi} / \mathrm{L} \\
\mathrm{pCi} / \mathrm{L} \\
\mathrm{pCi} / \mathrm{L} \\
\mathrm{pCi} / \mathrm{mL} \\
\mathrm{pCi} / \mathrm{L} \\
\mathrm{pCi} / \mathrm{L}\end{array}$ & $\begin{array}{l}46,000 \\
2.4 E+01 \\
11 \\
240 \\
2.1 E+01 \\
7.7 E+02 \\
6.3 E+01 \\
150,000 \\
1.1 E+03 \\
5.5 E+01 \\
2.6 E+01 \\
6.0 E+01 \\
2.2 E+02 \\
8.7 E+03 \\
2.3 E+02 \\
2.5 E+02\end{array}$ & $\begin{array}{l}J \\
J \\
J\end{array}$ & $\begin{array}{l}\text { NA } \\
\text { NA } \\
\text { NA } \\
\text { NA } \\
\text { NA } \\
\text { NA } \\
\text { NA } \\
\text { NA } \\
\text { NA } \\
\text { NA } \\
\text { NA } \\
\text { NA } \\
\text { NA } \\
\text { NA } \\
\text { NA } \\
\text { NA }\end{array}$ \\
\hline FSB106C & $\begin{array}{l}\text { Aluminum } \\
\text { Cadmium } \\
\text { Cobalt } \\
\text { Gross alpha } \\
\text { lodine-129 } \\
\text { Nitrate as nitrogen } \\
\text { Nonvolatile beta } \\
\text { Radium-226 } \\
\text { Strontium-90 } \\
\text { Tritium }\end{array}$ & $\begin{array}{l}\mu g / L \\
\mu g / L \\
\mu g / L \\
\mathrm{pCi} / L \\
\mathrm{pCi} / \mathrm{L} \\
\mu g / L \\
\mathrm{pCi} / \mathrm{L} \\
\mathrm{pCi} / \mathrm{L} \\
\mathrm{pCi} / \mathrm{L} \\
\mathrm{pCi} / \mathrm{mL}\end{array}$ & $\begin{array}{l}4,300 \\
10 \\
63 \\
4.5 E+01 \\
1.6 E+02 \\
90,000 \\
1.3 E+03 \\
2.3 E+01 \\
4.8 E+02 \\
2.4 E+03\end{array}$ & $\begin{array}{l}j \\
j\end{array}$ & $\begin{array}{l}\text { NA } \\
\text { NA } \\
\text { NA } \\
\text { NA } \\
\text { NA } \\
\text { NA } \\
\text { NA } \\
\text { NA } \\
\text { NA } \\
\text { NA }\end{array}$ \\
\hline FSB107C & $\begin{array}{l}\text { Strontium-90 } \\
\text { Tritium }\end{array}$ & $\begin{array}{l}\mathrm{pCi} / \mathrm{L} \\
\mathrm{pCi} / \mathrm{mL}\end{array}$ & $\begin{array}{l}1.2 E+01 \\
1.2 E+02\end{array}$ & & $\begin{array}{l}\text { NA } \\
1.1 E+02\end{array}$ \\
\hline FSB110C & $\begin{array}{l}\text { Aluminum } \\
\text { lodine-129 } \\
\text { Nitrate as nitrogen } \\
\text { Nonvolatile beta } \\
\text { Strontium-90 } \\
\text { Thallium } \\
\text { Tritium }\end{array}$ & $\begin{array}{l}\mu g / L \\
\text { pCi/L } \\
\mu g / L \\
\text { pCi/L } \\
\text { pCi/L } \\
\mu g / L \\
\text { pCi/mL }\end{array}$ & $\begin{array}{l}100 \\
2.5 E+01 \\
55,000 \\
6.7 E+01 \\
1.2 E+01 \\
4.2 \\
1.8 E+03\end{array}$ & $w$ & $\begin{array}{l}\text { NA } \\
\text { NA } \\
53,000 \\
5.6 E+01 \\
\text { NA } \\
\text { NA } \\
1.4 E+03\end{array}$ \\
\hline FSB111C & $\begin{array}{l}\text { lodine-129 } \\
\text { Tritium }\end{array}$ & $\begin{array}{l}\mathrm{pCi} / \mathrm{L} \\
\mathrm{pCi} / \mathrm{mL}\end{array}$ & $\begin{array}{l}2.0 E+00 \\
-\end{array}$ & & $\begin{array}{l}\text { NA } \\
4.5 E+02\end{array}$ \\
\hline
\end{tabular}


Aquifer Zone $\|_{1} B_{1}$ (Barnwell/McBean)

\begin{tabular}{|c|c|c|c|c|c|c|}
\hline Well & Constituent & $\underline{\text { Unit }}$ & 1095 & Mod & $\underline{2 Q 95}$ & Mod \\
\hline FSB112C & $\begin{array}{l}\text { Aluminum } \\
\text { Cadmium } \\
\text { Cobalt } \\
\text { Gross alpha } \\
\text { lodine-129 } \\
\text { Nitrate as nitrogen } \\
\text { Nonvolatile beta } \\
\text { Radium-226 } \\
\text { Radium-228 } \\
\text { Strontium-90 } \\
\text { Tritium }\end{array}$ & $\begin{array}{l}\mu \mathrm{g} / \mathrm{L} \\
\mu \mathrm{g} / \mathrm{L} \\
\mu \mathrm{g} / \mathrm{L} \\
\mathrm{pCill} \\
\mathrm{pCi} / \mathrm{L} \\
\mu \mathrm{g} / \mathrm{L} \\
\mathrm{pCi} / \mathrm{L} \\
\mathrm{pCi} / \mathrm{L} \\
\mathrm{pCi} / \mathrm{L} \\
\mathrm{pCi} / \mathrm{L} \\
\mathrm{pCi} / \mathrm{mL}\end{array}$ & $\begin{array}{l}17,000 \\
39 \\
290 \\
1.7 E+02 \\
2.2 E+02 \\
210,000 \\
1.8 E+03 \\
9.8 E+01 \\
3.0 E+01 \\
9.2 E+02 \\
7.3 E+03\end{array}$ & $\begin{array}{l}\mathbf{J} \\
\mathbf{J}\end{array}$ & $\begin{array}{l}\text { NA } \\
\text { NA } \\
\text { NA } \\
\text { NA } \\
\text { NA } \\
\text { NA } \\
\text { NA } \\
\text { NA } \\
\text { NA } \\
\text { NA } \\
\text { NA }\end{array}$ & \\
\hline FSB113C & $\begin{array}{l}\text { Aluminum } \\
\text { pH } \\
\text { Tritium }\end{array}$ & $\begin{array}{l}\mu \mathrm{g} / \mathrm{L} \\
\mathrm{pH} \\
\mathrm{pCi} / \mathrm{mL}\end{array}$ & $\begin{array}{l}140 \\
11 \\
6.6 E+01\end{array}$ & JY & $\begin{array}{l}N A \\
11 \\
7.0 E+01\end{array}$ & $\sqrt{1}$ \\
\hline FSB115C & Aluminum & $\mu g / L$ & 65 & & NA & \\
\hline FSB120C & $\begin{array}{l}\text { Aluminum } \\
\text { Gross alpha } \\
\text { lodine-129 } \\
\text { Nitrate as nitrogen } \\
\text { Tritium }\end{array}$ & $\begin{array}{l}\mu \mathrm{g} / \mathrm{L} \\
\mathrm{pCi} / \mathrm{L} \\
\mathrm{pCi} / \mathrm{L} \\
\mu \mathrm{g} / \mathrm{L} \\
\mathrm{pCi} / \mathrm{mL}\end{array}$ & $\begin{array}{l}180 \\
1.7 E+01 \\
1.4 E+01 \\
34,000 \\
1.1 E+03\end{array}$ & $J$ & $\begin{array}{l}\text { NA } \\
\overline{N A} \\
37,000 \\
1.0 E+03\end{array}$ & \\
\hline FSB121C & Tritium & $\mathrm{pCi} / \mathrm{mL}$ & $1.3 E+02$ & & $1.2 \mathrm{E}+02$ & \\
\hline FSB122C & $\begin{array}{l}\text { Aluminum } \\
\text { Gross alpha } \\
\text { lodine-129 } \\
\text { Nitrate as nitrogen } \\
\text { Nonvolatile beta } \\
\text { Tritium }\end{array}$ & $\begin{array}{l}\mu \mathrm{g} / \mathrm{L} \\
\mathrm{pCi} / \mathrm{L} \\
\mathrm{pCi} / \mathrm{L} \\
\mu \mathrm{g} / \mathrm{L} \\
\mathrm{pCi} / \mathrm{L} \\
\mathrm{pCi} / \mathrm{mL}\end{array}$ & $\begin{array}{l}660 \\
2.0 E+01 \\
2.2 E+01 \\
60,000 \\
6.5 E+01 \\
1.9 E+03\end{array}$ & J & $\begin{array}{l}\text { NA } \\
1.8 E+01 \\
\text { NA } \\
67,000 \\
9.2 E+01 \\
1.7 E+03\end{array}$ & \\
\hline \multicolumn{7}{|c|}{ Aquifer Unit IIA (upper Congaree) } \\
\hline Well & Constituent & $\underline{\text { Unit }}$ & $\underline{1 Q 95}$ & Mod & $\underline{2 Q 95}$ & Mod \\
\hline FSB 76B & lodine-129 & $\mathrm{pCi} / \mathrm{L}$ & $3.0 E+00$ & & NA & \\
\hline FSB 78B & $\begin{array}{l}\text { Aluminum } \\
\text { Nitrate as nitrogen } \\
\text { Thallium } \\
\text { Tritium }\end{array}$ & $\begin{array}{l}\mu \mathrm{g} / \mathrm{L} \\
\mu \mathrm{g} / \mathrm{L} \\
\mu \mathrm{g} / \mathrm{L} \\
\mathrm{pCi} / \mathrm{mL}\end{array}$ & $\begin{array}{l}92 \\
11,000 \\
3.6 \\
2.7 E+02\end{array}$ & $\begin{array}{l}\text { W } \\
J\end{array}$ & $\begin{array}{l}\text { NA } \\
11,000 \\
N A \\
3.0 E+02\end{array}$ & \\
\hline FSB 79B & Tritium & $\mathrm{pCi} / \mathrm{mL}$ & $2.4 E+01$ & & - & UI \\
\hline FSB 87B & Tritium & $\mathrm{pCi} / \mathrm{mL}$ & $8.8 \mathrm{E}+01$ & & - & $\mathrm{UI}$ \\
\hline FSB 96AR & lodine-129 & pCill & $7.5 E+00$ & & NA & \\
\hline
\end{tabular}


Aquifer Unit IIA (upper Congaree)

\begin{tabular}{|c|c|c|c|c|c|}
\hline Well & Constituent & Unit & $1 \mathrm{Q95}$ & Mod & $\underline{2 Q 95}$ \\
\hline FSB 97A & $\begin{array}{l}\text { Gross alpha } \\
\text { Tritium }\end{array}$ & $\begin{array}{l}\mathrm{pCi} / \mathrm{L} \\
\mathrm{pCi} / \mathrm{mL}\end{array}$ & $\overline{2.2 E}+02$ & $J$ & $\begin{array}{l}1.6 \mathrm{E}+01 \\
2.7 \mathrm{E}+02\end{array}$ \\
\hline FSB 98AR & Tritium & $\mathrm{pCi} / \mathrm{mL}$ & $2.5 E+01$ & & - \\
\hline FSB 99A & $\begin{array}{l}\text { lodine-129 } \\
\text { Tritium }\end{array}$ & $\begin{array}{l}\mathrm{pCi} / \mathrm{L} \\
\mathrm{pCi} / \mathrm{mL}\end{array}$ & $\begin{array}{l}2.6 E+00 \\
1.6 E+02\end{array}$ & & $\begin{array}{l}\text { NA } \\
7.8 E+01\end{array}$ \\
\hline FSB100A & Tritium & $\mathrm{pCi} / \mathrm{mL}$ & $1.2 E+02$ & & $1.3 E+02$ \\
\hline FSB112A & Tritium & $\mathrm{pCi} / \mathrm{mL}$ & 1.1E+02 & & 1.6E+02 \\
\hline FSB113A & $\begin{array}{l}\text { Aluminum } \\
\text { pH } \\
\text { Tritium }\end{array}$ & $\begin{array}{l}\mu \mathrm{g} / \mathrm{L} \\
\mathrm{pH} \\
\mathrm{pCi} / \mathrm{mL}\end{array}$ & $\begin{array}{l}2,100 \\
12 \\
1.8 E+02\end{array}$ & $J$ & $\begin{array}{l}\text { NA } \\
12 \\
1.7 E+02\end{array}$ \\
\hline FSB114A & $\begin{array}{l}\text { Aluminum } \\
\text { Tritium }\end{array}$ & $\begin{array}{l}\mu \mathrm{g} / \mathrm{L} \\
\mathrm{pCi} / \mathrm{mL}\end{array}$ & $\begin{array}{l}110 \\
-\end{array}$ & & $\begin{array}{l}N A \\
4.9 E+02\end{array}$ \\
\hline FSB120A & $\begin{array}{l}\text { Aluminum } \\
\text { Tritium }\end{array}$ & $\begin{array}{l}\mu \mathrm{g} / \mathrm{L} \\
\mathrm{pCi} / \mathrm{mL}\end{array}$ & $\begin{array}{l}590 \\
6.5 E+01\end{array}$ & & $\begin{array}{l}\mathrm{NA} \\
2.3 \mathrm{E}+01\end{array}$ \\
\hline HSB $85 A$ & Aluminum & $\mu g / L$ & - & & 85 \\
\hline
\end{tabular}

Notes: This table presents the highest value for duplicate/replicate results.

The groundwater samples are unfiltered. Therefore, the results for the metals are for total recoverable metals.

Flags are established by EPD/EMS and are based on final PDWS, Secondary Drinking Water Standards, or method detection limits (see Appendix B).

a $N A=$ not analyzed.

b $-=$ analyzed but not above the Flag 2 criterion. 
Table 3. Groundwater Monitoring Results for Individual Wells

\section{WELL FSB 76}

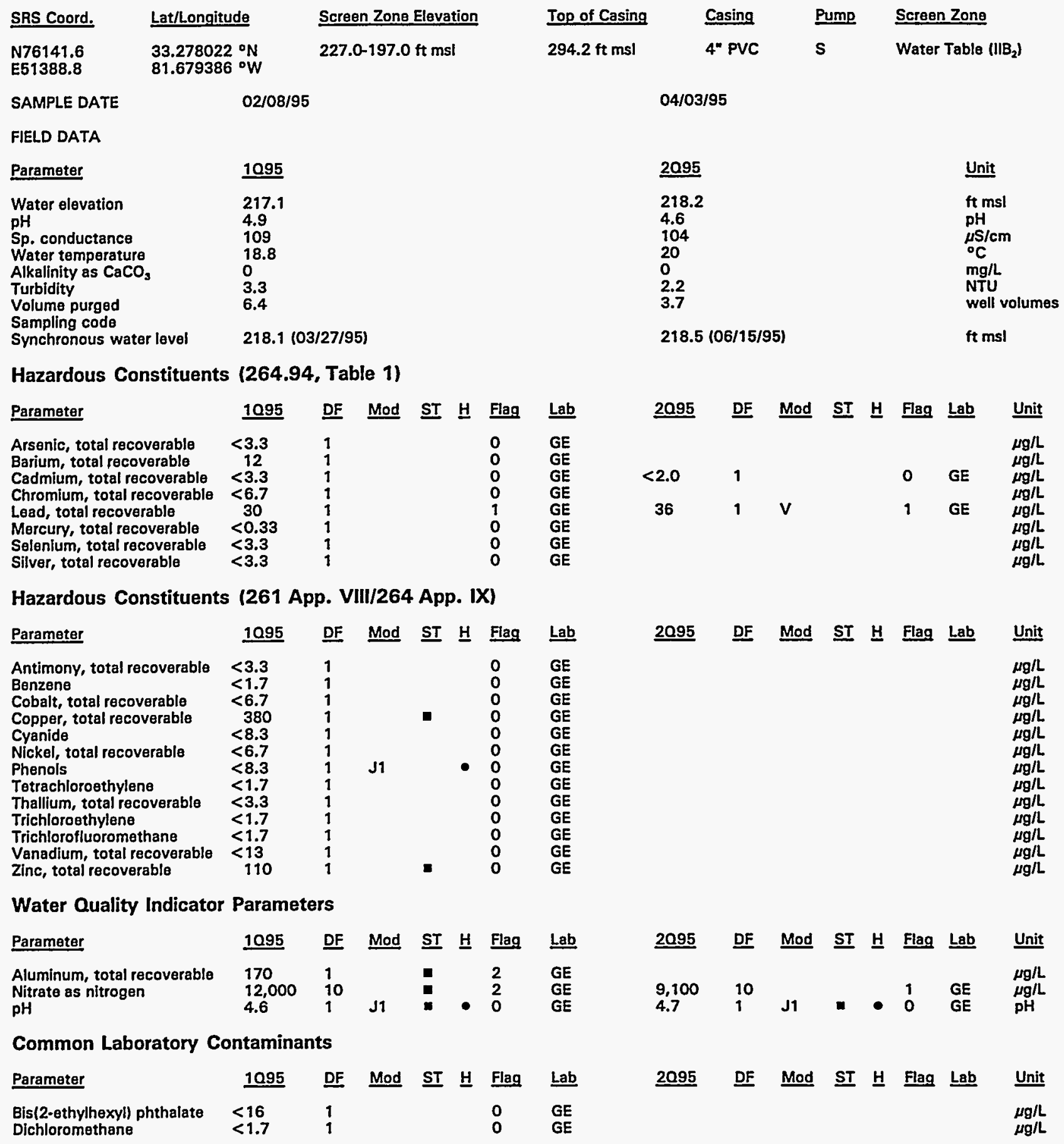

Note: See Appendix B for flagging criteria. Synchronous water levels are measured over a 3-5 day period.

- = exceeded holding time.

- = exceeded groundwater protection standard. 
WELL FSB 76 (cont.)

Radiological Indicator Parameters

\begin{tabular}{|c|c|c|c|c|c|c|c|c|c|c|c|}
\hline Parameter & 1095 & Mod & $\underline{\text { ST }} \underline{H}$ & Flag & Lab & $\underline{2095}$ & $\underline{D F}$ & Mod & $\underline{\text { ST }} \underline{H}$ & Flag & Lab \\
\hline $\begin{array}{l}\text { Gross alpha } \\
\text { Nonvolatile beta }\end{array}$ & $\begin{array}{l}9.7 E+00 \\
7.1 E+00\end{array}$ & 1 & & $\begin{array}{l}1 \\
0\end{array}$ & $\begin{array}{l}\text { GP } \\
\text { GP }\end{array}$ & $\begin{array}{l}1.2 E+01 \\
1.1 E+01\end{array}$ & 1 & $J$ & & $\begin{array}{l}1 \\
0\end{array}$ & $\begin{array}{l}\text { GP } \\
\text { GP }\end{array}$ \\
\hline
\end{tabular}

Measured Radionuclides

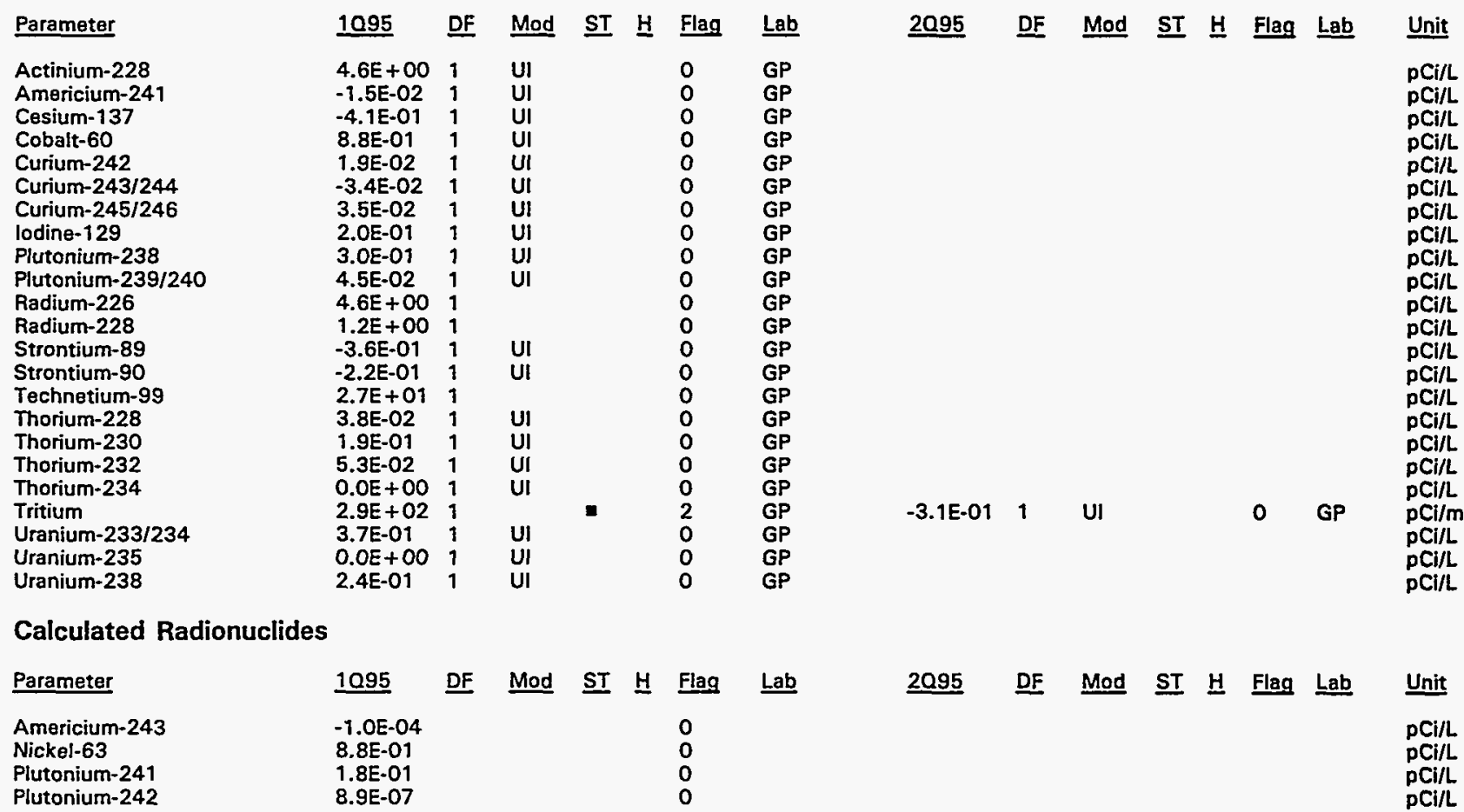

Note: See Appendix B for flagging criteria. Synchronous water levels are measured over a 3-5 day period. - = exceeded holding time.

- =xceeded groundwater protection standard. 
WELL FSB 76A

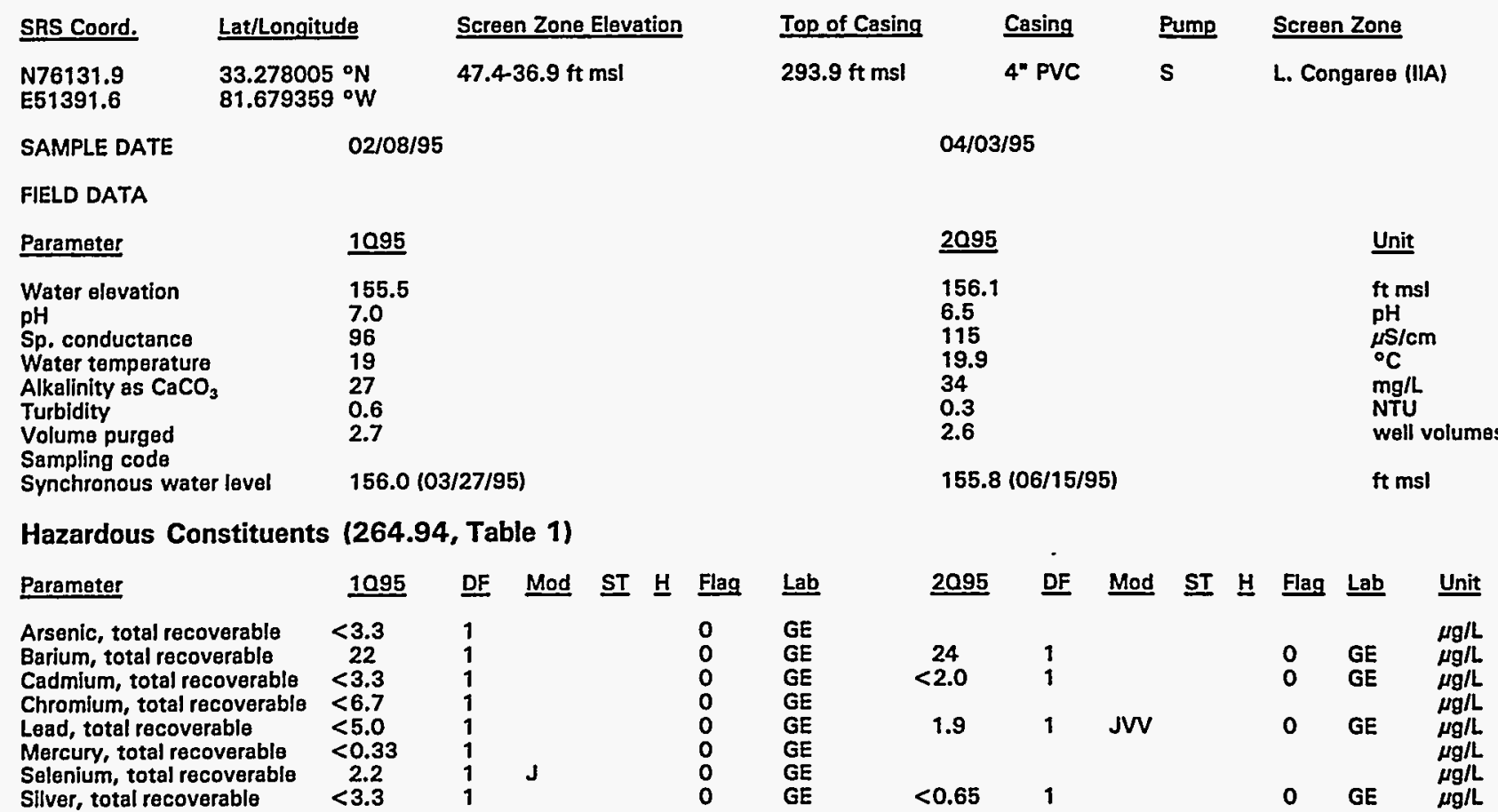

Hazardous Constituents (261 App. VIII/264 App. IX)

\begin{tabular}{|c|c|c|c|c|c|c|c|c|c|c|c|c|c|c|c|}
\hline Parameter & 1095 & DF & Mod & $\underline{S T}$ & 보 & Flag & Lab & 2095 & DF & Mod & $\underline{S T}$ & 브 & Flag & Lab & Unit \\
\hline $\begin{array}{l}\text { Antimony, total recoverable } \\
\text { Benzene } \\
\text { Cobalt, total recoverable } \\
\text { Copper, total recoverable } \\
\text { Cyanide } \\
\text { Nickel, total recoverable } \\
\text { Phenols } \\
\text { Tetrachloroethylene } \\
\text { Thallium, total recoverable } \\
\text { Trichloroethylene } \\
\text { Trichlorofluoromethane } \\
\text { Vanadium, total recoverable } \\
\text { Zinc, total recoverable }\end{array}$ & $\begin{array}{l}<3.3 \\
<1.7 \\
<6.7 \\
<6.7 \\
<8.3 \\
<6.7 \\
<8.3 \\
<1.7 \\
<3.3 \\
<1.7 \\
<1.7 \\
<13 \\
<3.3\end{array}$ & $\begin{array}{l}1 \\
1 \\
1 \\
1 \\
1 \\
1 \\
1 \\
1 \\
1 \\
1 \\
1 \\
1 \\
1\end{array}$ & $\begin{array}{l}\text { J } \\
\text { J1 }\end{array}$ & & - & $\begin{array}{l}0 \\
0 \\
0 \\
0 \\
0 \\
0 \\
0 \\
0 \\
0 \\
0 \\
0 \\
0 \\
0\end{array}$ & $\begin{array}{l}\text { GE } \\
\text { GE } \\
\text { GE } \\
\text { GE } \\
\text { GE } \\
\text { GE } \\
\text { GE } \\
\text { GE } \\
\text { GE } \\
\text { GE } \\
\text { GE } \\
\text { GE } \\
\text { GE }\end{array}$ & $<10$ & 1 & & & & 0 & GE & $\begin{array}{l}\mu g / L \\
\mu g / L \\
\mu g / L \\
\mu g / L \\
\mu g / L \\
\mu g / L \\
\mu g / L \\
\mu g / L \\
\mu g / L \\
\mu g / L \\
\mu g / L \\
\mu g / L \\
\mu g / L\end{array}$ \\
\hline \multicolumn{16}{|c|}{ Water Quality Indicator Parameters } \\
\hline Parameter & 1095 & $\underline{\text { DF }}$ & Mod & $\underline{\text { ST }}$ & $\underline{H}$ & Flag & Lab & $\underline{2095}$ & $\underline{\text { DF }}$ & Mod & $\underline{\text { ST }}$ & $\underline{\mathrm{H}}$ & Flag & $\underline{\text { Lab }}$ & Unit \\
\hline \multicolumn{16}{|c|}{ Common Laboratory Contaminants } \\
\hline Parameter & $\underline{1095}$ & $\underline{D F}$ & Mod & $\underline{\text { ST }}$ & $\underline{H}$ & Flag & Lab & $\underline{2095}$ & DF & Mod & $\underline{\mathbf{S T}}$ & $\underline{H}$ & Flag & Lab & Unit \\
\hline $\begin{array}{l}\text { Bis(2-ethylhexyl) phthalate } \\
\text { Dichloromethane }\end{array}$ & $\begin{array}{l}<17 \\
<1.7\end{array}$ & $\begin{array}{l}1 \\
1\end{array}$ & $v$ & & & $\begin{array}{l}0 \\
0\end{array}$ & $\begin{array}{l}\text { GE } \\
\text { GE }\end{array}$ & & & & & & & & $\begin{array}{l}\mu g / L \\
\mu g / L\end{array}$ \\
\hline
\end{tabular}

Note: See Appendix B for flagging criteria. Synchronous water levels are measured over a 3-5 day period.

- = exceeded holding time.

- = exceeded groundwater protection standard. 
WELL FSB 76A (cont.)

Radiological Indicator Parameters

\begin{tabular}{|c|c|c|c|c|c|c|c|c|c|c|c|c|c|c|c|}
\hline Parameter & 1095 & $\underline{\mathrm{DF}}$ & Mod & $\underline{\mathrm{ST}}$ & $\underline{\mathbf{H}}$ & Flag & $\underline{\text { Lab }}$ & $\underline{2095}$ & $\underline{\mathrm{DF}}$ & Mod & $\underline{\text { ST }}$ & $\underline{H}$ & Flag & $\underline{\text { Lab }}$ & Unit \\
\hline $\begin{array}{l}\text { Gross alpha } \\
\text { Nonvolatile beta }\end{array}$ & $\begin{array}{l}1.1 E+00 \\
1.6 E+00\end{array}$ & 1 & & & & $\begin{array}{l}0 \\
0\end{array}$ & $\begin{array}{l}\text { GP } \\
\text { GP }\end{array}$ & $\begin{array}{l}5.3 E-01 \\
4.6 E+00\end{array}$ & $\begin{array}{l}1 \\
1\end{array}$ & UJi & & & $\begin{array}{l}0 \\
0\end{array}$ & $\begin{array}{l}\text { GP } \\
\text { GP }\end{array}$ & $\begin{array}{l}\mathrm{pCi} / \mathrm{L} \\
\mathrm{pCi} / \mathrm{L}\end{array}$ \\
\hline \multicolumn{16}{|c|}{ Measured Radionuclides } \\
\hline Parameter & $\underline{1095}$ & $\underline{\mathrm{DF}}$ & Mod & $\underline{\text { ST }}$ & 브 & Flag & $\underline{\text { Lab }}$ & $\underline{2095}$ & $\underline{D F}$ & Mod & $\underline{S T}$ & $\underline{\mathrm{H}}$ & Flag & $\underline{\text { Lab }}$ & Unit \\
\hline $\begin{array}{l}\text { Actinium-228 } \\
\text { Americium-241 } \\
\text { Cesium-137 } \\
\text { Cobalt-60 } \\
\text { Curium-242 } \\
\text { Curium-243/244 } \\
\text { Curium-245/246 } \\
\text { lodine-129 } \\
\text { Plutonium-238 } \\
\text { Plutonium-239/240 } \\
\text { Radium-226 } \\
\text { Radium-228 } \\
\text { Strontium-89 } \\
\text { Strontium-90 } \\
\text { Technetium-99 } \\
\text { Thorium-228 } \\
\text { Thorium-230 } \\
\text { Thorium-232 } \\
\text { Thorium-234 } \\
\text { Tritium } \\
\text { Uranium-233/234 } \\
\text { Uranium-235 } \\
\text { Uranium-238 }\end{array}$ & $\begin{array}{l}8.1 E+00 \\
-1.0 E-02 \\
7.0 E-01 \\
-2.3 E-01 \\
0.0 E+00 \\
7.4 E-02 \\
-5.2 E-03 \\
-1.2 E-01 \\
2.4 E-01 \\
-7.9 E-03 \\
1.4 E+00 \\
-3.5 E-01 \\
-1.1 E+00 \\
-9.1 E-01 \\
5.5 E+00 \\
2.8 E-01 \\
3.7 E-01 \\
0.0 E+00 \\
0.0 E+00 \\
-2.3 E-01 \\
4.3 E-02 \\
-5.4 E-03 \\
0.0 E+00\end{array}$ & $\begin{array}{l}1 \\
1 \\
1 \\
1 \\
1 \\
1 \\
1 \\
1 \\
1 \\
1 \\
1 \\
1 \\
1 \\
1 \\
1 \\
1 \\
1 \\
1 \\
1 \\
1 \\
1 \\
1 \\
1\end{array}$ & $\begin{array}{l}\text { UI } \\
\text { UI } \\
\text { UI } \\
\text { UI } \\
\text { UI } \\
\text { UI } \\
\text { UII } \\
\text { UI } \\
\text { UI } \\
\text { UII } \\
\text { UI } \\
\text { UI } \\
\text { UI } \\
\text { UI } \\
\text { UI } \\
\text { UI } \\
\text { UI } \\
\text { UI } \\
\text { UI } \\
\text { UI } \\
\text { UI } \\
\text { UI }\end{array}$ & & & $\begin{array}{l}0 \\
0 \\
0 \\
0 \\
0 \\
0 \\
0 \\
0 \\
0 \\
0 \\
0 \\
0 \\
0 \\
0 \\
0 \\
0 \\
0 \\
0 \\
0 \\
0 \\
0 \\
0\end{array}$ & $\begin{array}{l}\text { GP } \\
\text { GP } \\
\text { GP } \\
\text { GP } \\
\text { GP } \\
\text { GP } \\
\text { GP } \\
\text { GP } \\
\text { GP } \\
\text { GP } \\
\text { GP } \\
\text { GP } \\
\text { GP } \\
\text { GP } \\
G P \\
G P \\
G P \\
G P \\
G P \\
G P \\
G P \\
G P\end{array}$ & $-1.8 \mathrm{E}-04$ & 1 & UI & & & 0 & GP & $\begin{array}{l}\mathrm{pCi} / \mathrm{L} \\
\mathrm{pCi} / \mathrm{L} \\
\mathrm{pCi} / \mathrm{L} \\
\mathrm{pCi} / \mathrm{L} \\
\mathrm{pCi} / \mathrm{L} \\
\mathrm{pCi} / \mathrm{L} \\
\mathrm{pCi/L} \\
\mathrm{pCi} / \mathrm{L} \\
\mathrm{pCi} / \mathrm{L} \\
\mathrm{pCi} / \mathrm{L} \\
\mathrm{pCi} / \mathrm{L} \\
\mathrm{pCi} / \mathrm{L} \\
\mathrm{pCi} / \mathrm{L} \\
\mathrm{pCi} / \mathrm{L} \\
\mathrm{pCi} / \mathrm{L} \\
\mathrm{pCi} / \mathrm{L} \\
\mathrm{pCi} / \mathrm{L} \\
\mathrm{pCi} / \mathrm{L} \\
\mathrm{pCi} / \mathrm{L} \\
\mathrm{pCi} / \mathrm{m} \\
\mathrm{pCi} / \mathrm{L} \\
\mathrm{pCi} / \mathrm{L} \\
\mathrm{pCi} / \mathrm{L}\end{array}$ \\
\hline \multicolumn{16}{|c|}{ Calculated Radionuclides } \\
\hline Parameter & 1095 & DF & Mod & $\underline{\mathbf{S T}}$ & 브 & Flag & $\underline{\text { Lab }}$ & $\underline{2095}$ & $\underline{D F}$ & Mod & $\underline{\text { ST }}$ & H & Flag & $\underline{\text { Lab }}$ & Unit \\
\hline $\begin{array}{l}\text { Americium-243 } \\
\text { Nickel-63 } \\
\text { Plutonium-241 } \\
\text { Plutonium-242 }\end{array}$ & $\begin{array}{l}2.2 \mathrm{E}-04 \\
-2.3 \mathrm{E}-01 \\
-3.2 \mathrm{E}-02 \\
-1.6 \mathrm{E}-07\end{array}$ & & & & & $\begin{array}{l}0 \\
0 \\
0 \\
0\end{array}$ & & & & & & & & & $\begin{array}{l}\mathrm{pCi} / \mathrm{L} \\
\mathrm{pCi} / \mathrm{L} \\
\mathrm{p} \mathrm{Ci} / \mathrm{L} \\
\mathrm{pCi} / \mathrm{L}\end{array}$ \\
\hline
\end{tabular}

Note: See Appendix B for flagging criteria. Synchronous water levels are measured over a 3-5 day period.

- = exceeded holding time.

- = exceeded groundwater protection standard. 


\section{WELL FSB 76B}

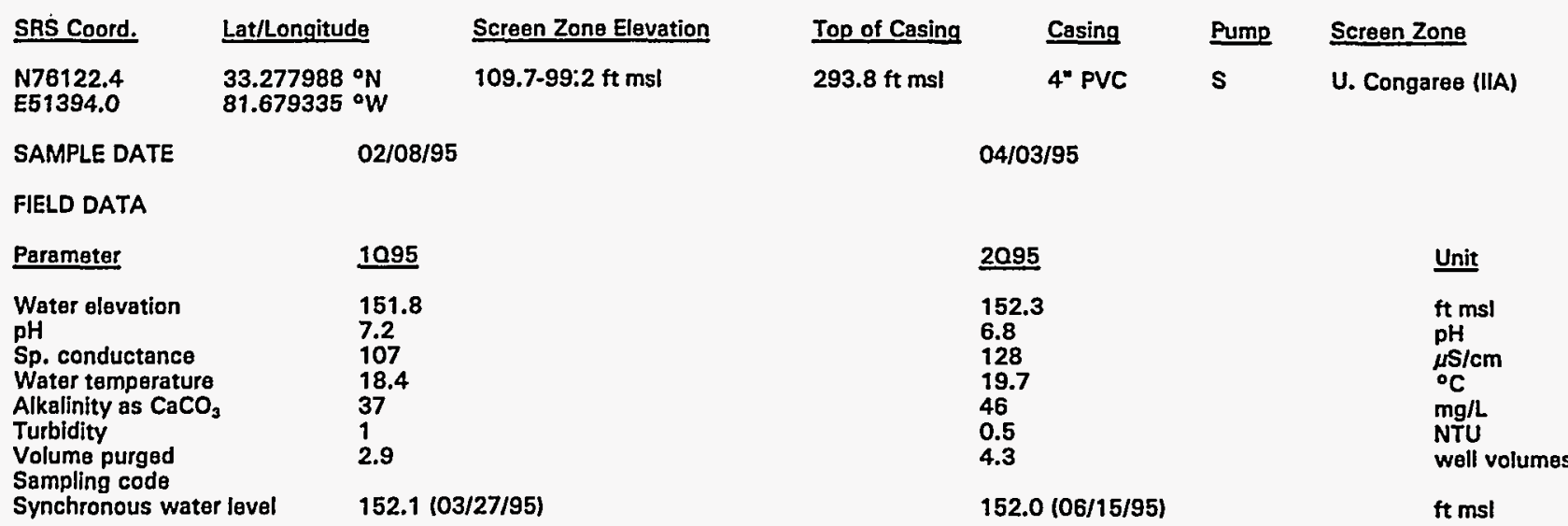

Hazardous Constituents (264.94, Table 1)

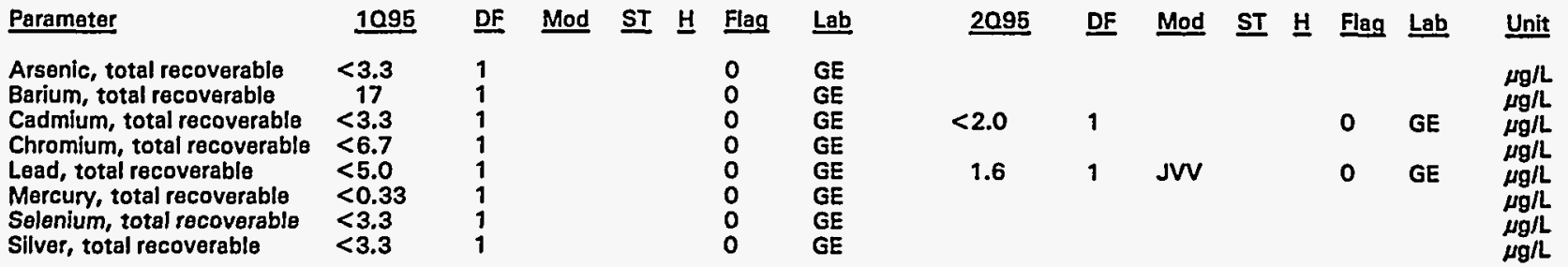

Hazardous Constituents (261 App. VIII/264 App. IX)

\begin{tabular}{|c|c|c|c|c|c|c|c|c|c|c|c|c|c|c|c|}
\hline Parameter & 1095 & $\underline{D F}$ & Mod & $\underline{S T}$ & $\underline{\text { H}}$ & Flag & $\underline{\text { Lab }}$ & $\underline{2095}$ & $\underline{D F}$ & Mod & ST & 보 & Flag & $\underline{\text { Lab }}$ & Unit \\
\hline $\begin{array}{l}\text { Antimony, total recoverable } \\
\text { Benzene } \\
\text { Cobalt, total recoverable } \\
\text { Copper, total recoverable } \\
\text { Cyanide } \\
\text { Nickel, total recoverable } \\
\text { Phenols } \\
\text { Tetrachloroethylene } \\
\text { Thallium, total recoverable } \\
\text { Trichloroethylene } \\
\text { Trichlorofluoromethane } \\
\text { Vanadium, total recoverable } \\
\text { Zinc, total recoverable }\end{array}$ & $\begin{array}{l}<3.3 \\
<1.7 \\
<6.7 \\
7.9 \\
<8.3 \\
<6.7 \\
<8.3 \\
<1.7 \\
<3.3 \\
<1.7 \\
<1.7 \\
<13 \\
<3.3\end{array}$ & $\begin{array}{l}1 \\
1 \\
1 \\
1 \\
1 \\
1 \\
1 \\
1 \\
1 \\
1 \\
1 \\
1 \\
1\end{array}$ & $\mathbf{J}$ & & & $\begin{array}{l}0 \\
0 \\
0 \\
0 \\
0 \\
0 \\
0 \\
0 \\
0 \\
0 \\
0 \\
0 \\
0\end{array}$ & $\begin{array}{l}\mathrm{GE} \\
\mathrm{GE} \\
\mathrm{GE} \\
\mathrm{GE} \\
\mathrm{GE} \\
\mathrm{GE} \\
\mathrm{GE} \\
\mathrm{GE} \\
\mathrm{GE} \\
\mathrm{GE} \\
\mathrm{GE} \\
\mathrm{GE} \\
\mathrm{GE}\end{array}$ & & & & & & & & $\begin{array}{l}\mu g / L \\
\mu g / L \\
\mu g / L \\
\mu g / L \\
\mu g / L \\
\mu g / L \\
\mu g / L \\
\mu g / L \\
\mu g / L \\
\mu g / L \\
\mu g / L \\
\mu g / L \\
\mu g / L\end{array}$ \\
\hline \multicolumn{16}{|c|}{ Water Quality Indicator Parameters } \\
\hline Parameter & 1095 & DF & Mod & ST & H & Flag & $\underline{\text { Lab }}$ & $\underline{2095}$ & $\underline{\mathrm{DF}}$ & Mod & $\underline{\text { ST }}$ & $\underline{H}$ & Flag & $\underline{\text { Lab }}$ & Unit \\
\hline $\begin{array}{l}\text { Aluminum, total recoverable } \\
\text { Nitrate as nitrogen } \\
\text { pH }\end{array}$ & $\begin{array}{l}<33 \\
390 \\
6.9\end{array}$ & $\begin{array}{l}1 \\
1 \\
1\end{array}$ & J1 & & - & $\begin{array}{l}0 \\
0 \\
0\end{array}$ & $\begin{array}{l}\mathrm{GE} \\
\mathrm{GE} \\
\mathrm{GE}\end{array}$ & $\begin{array}{l}330 \\
7.0\end{array}$ & $\begin{array}{l}1 \\
1\end{array}$ & J1 & & $\bullet$ & $\begin{array}{l}0 \\
0\end{array}$ & $\begin{array}{l}\text { GE } \\
\text { GE }\end{array}$ & $\begin{array}{l}\mu \mathrm{g} / \mathrm{L} \\
\mu \mathrm{g} / \mathrm{L} \\
\mathrm{pH}\end{array}$ \\
\hline \multicolumn{16}{|c|}{ Common Laboratory Contaminants } \\
\hline Parameter & $\underline{1095}$ & $\underline{\mathrm{DF}}$ & Mod & ST & H & Flag & Lab & $\underline{2095}$ & $\underline{\mathrm{DF}}$ & Mod & $\underline{S T}$ & $\underline{H}$ & Flag & Lab & $\underline{\text { Unit }}$ \\
\hline $\begin{array}{l}\text { Bis(2-ethylhexyl) phthalate } \\
\text { Dichloromethane }\end{array}$ & $\begin{array}{l}<16 \\
<1.7\end{array}$ & 1 & V & & & $\begin{array}{l}0 \\
0\end{array}$ & $\begin{array}{l}\text { GE } \\
\text { GE }\end{array}$ & & & & & & & & $\begin{array}{l}\mu g / L \\
\mu g / L\end{array}$ \\
\hline
\end{tabular}

Note: See Appendix B for flagging criteria. Synchronous water levels are measured over a 3-5 day period.

$-=$ exceeded holding time.

" = exceeded groundwater protection standard. 
WELL FSB $76 B$ (cont.)

Radiological Indicator Parameters

Parameter

Gross alpha

Nonvolatile beta
1095

1.0E

$\begin{array}{ll}1.0 E+00 & 1 \\ 6.7 E-01 & 1\end{array}$
Mod ST H Flag Lab

UI

$\begin{array}{ll}0 & \text { GP } \\ 0 & \text { GP }\end{array}$
1095

5.9E-01

$-1.3 \mathrm{E}-02$

9.9E-01

$1.6 \mathrm{E}-01$

$0.0 E+00$

8.6E-02

$-6.6 \mathrm{E}-03$

$3.0 E+00$

1.5E-01

$-9.6 \mathrm{E}-03$

1.3E + 00

$-1.0 \mathrm{E}-01$

$-4.4 \mathrm{E}-01$

$-2.5 \mathrm{E}-01$

$2.5 E+00$

8.1E-02

$1.8 \mathrm{E}-01$

6.2E-02

$0.0 \mathrm{E}+00$

1.9E-01

1.1E.01

4.0E-02

$-5.3 \mathrm{E}-03$

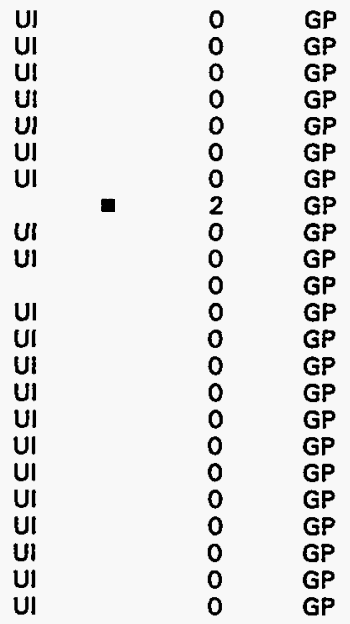

DF Mod ST H Flag

2095 DF Mod ST H Flag Lab Unit

$\begin{array}{llllll}1.6 E+\infty & 1 & \text { J } & 0 & \mathrm{GP} & \mathrm{pCi/L} \\ 1.4 E+00 & 1 & \mathrm{UI} & 0 & \mathrm{GP} & \mathrm{pCi} / \mathrm{L}\end{array}$

2095 DF Mod ST $\underline{H}$ Flag Lab Unit

$\mathrm{pCi} / \mathrm{L}$

$\mathrm{pCi} / \mathrm{L}$

$\mathrm{pCi} / \mathrm{L}$

pCi/L

PCI/

$\mathrm{PCi} / \mathrm{L}$

pCi/L

$\mathrm{pCi} / \mathrm{L}$

$\mathrm{PCI} / \mathrm{L}$

$\mathrm{pCi} / \mathrm{L}$

pCi/L

$\mathrm{pCI} / \mathrm{L}$

$\mathrm{PCi} / \mathrm{L}$

$\mathrm{pCi} / \mathrm{L}$

pCin

pCi/L

pCi/L

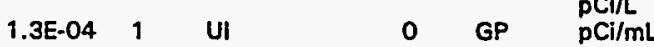

Uranium-233/234

Uranium-235

Uranium-238

Calculated Radionuclides

Parameter

Americium-243

Nickel-63

Plutonium-241

Plutonium-242
1095

2.6E-04

1.6E-01

$-3.8 \mathrm{E}-02$

$-1.9 \mathrm{E}-07$

DF Mod ST $\underline{H}$ Flag Lab

$\underline{2095}$

DF Mod ST $\underline{\text { H Flag Lab }}$

Unit

$\mathrm{pCi} / \mathrm{L}$

$\mathrm{pCi/L}$

$\mathrm{pCi} / \mathrm{h}$

pCi/L

Note: See Appendix B for flagging criteria. Synchronous water levels are measured over a 3-5 day period.

- exceeded holding time.

- exceeded groundwater protection standard. 


\section{WELL FSB 76C}

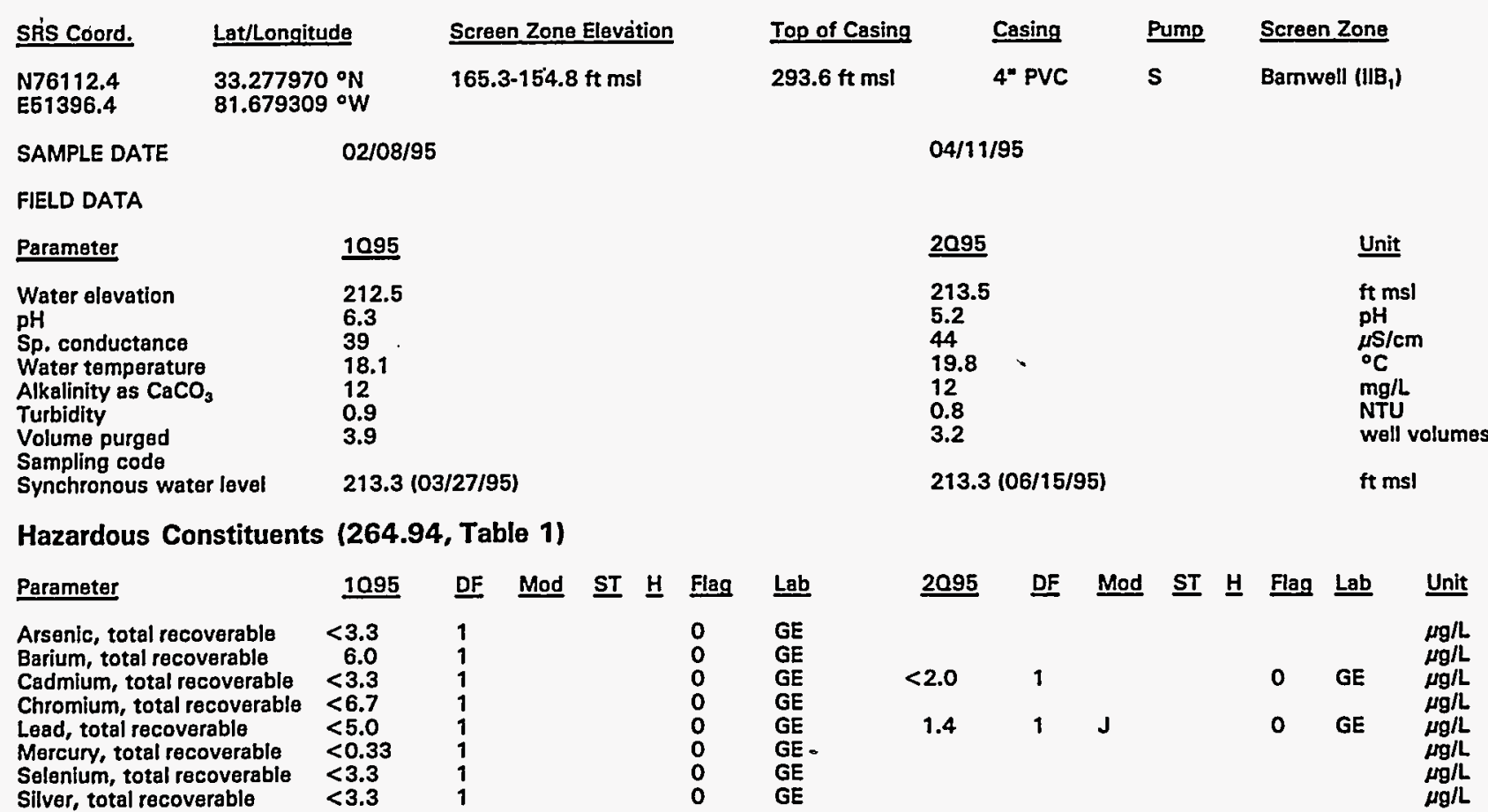

Hazardous Constituents (261 App. VIII/264 App. IX)

\begin{tabular}{|c|c|c|c|c|c|c|c|c|c|c|c|c|c|c|c|}
\hline Parameter & 1095 & $\underline{\text { DF }}$ & $\underline{\text { Mod }}$ & $\underline{\mathbf{S T}}$ & $\underline{\mathrm{H}}$ & Flag & $\underline{\text { Lab }}$ & $\underline{2095}$ & $\underline{D F}$ & Mod & ST & $\underline{\mathbf{H}}$ & Flag & $\underline{\text { Lab }}$ & Unit \\
\hline $\begin{array}{l}\text { Antimony, total recoverable } \\
\text { Benzene } \\
\text { Cobalt, total recoverable } \\
\text { Copper, total recoverable } \\
\text { Cyanide } \\
\text { Nickel, total recoverable } \\
\text { Phenols } \\
\text { Tetrachloroethylene } \\
\text { Thallium, total recoverable } \\
\text { Trichloroethylene } \\
\text { Trichlorofluoromethane } \\
\text { Vanadium, total recoverable } \\
\text { Zinc, total recoverable }\end{array}$ & $\begin{aligned}<3.3 \\
<1.7 \\
<6.7 \\
11 \\
<8.3 \\
<6.7 \\
<8.3 \\
<1.7 \\
<3.3 \\
3.0 \\
9.4 \\
<13 \\
17\end{aligned}$ & $\begin{array}{l}1 \\
1 \\
1 \\
1 \\
1 \\
1 \\
1 \\
1 \\
1 \\
1 \\
1 \\
1 \\
1\end{array}$ & $\begin{array}{l}J \\
J 1\end{array}$ & - & • & $\begin{array}{l}0 \\
0 \\
0 \\
0 \\
0 \\
0 \\
0 \\
0 \\
0 \\
1 \\
1 \\
0 \\
0\end{array}$ & $\begin{array}{l}\mathrm{GE} \\
\mathrm{GE} \\
\mathrm{GE} \\
\mathrm{GE} \\
\mathrm{GE} \\
\mathrm{GE} \\
\mathrm{GE} \\
\mathrm{GE} \\
\mathrm{GE} \\
\mathrm{GE} \\
\mathrm{GE} \\
\mathrm{GE} \\
\mathrm{GE}\end{array}$ & & & & & & & & $\begin{array}{l}\mu g / L \\
\mu g / L \\
\mu g / L \\
\mu g / L \\
\mu g / L \\
\mu g / L \\
\mu g / L \\
\mu g / L \\
\mu g / L \\
\mu g / L \\
\mu g / L \\
\mu g / L \\
\mu g / L\end{array}$ \\
\hline \multicolumn{16}{|c|}{ Water Quality Indicator Parameters } \\
\hline Parameter & 1095 & $\underline{\mathrm{DF}}$ & Mod & $\underline{\underline{S T}}$ & 브 & Flag & $\underline{\text { Lab }}$ & $\underline{2095}$ & $\underline{\text { DF }}$ & Mod & $\underline{\mathbf{S T}}$ & $\underline{H}$ & Flag & $\underline{\text { Lab }}$ & Unit \\
\hline $\begin{array}{l}\text { Aluminum, total recoverable } \\
\text { Nitrate as nitrogen } \\
\text { pH }\end{array}$ & $\begin{array}{l}<33 \\
1.200 \\
6.0\end{array}$ & $\begin{array}{l}1 \\
1 \\
1\end{array}$ & J1 & - & $\bullet$ & $\begin{array}{l}0 \\
0 \\
0\end{array}$ & $\begin{array}{l}\text { GE } \\
\text { GE } \\
\text { GE }\end{array}$ & $\begin{array}{l}1,300 \\
6.1\end{array}$ & $\begin{array}{l}1 \\
1\end{array}$ & JY & . & - & $\begin{array}{l}0 \\
0\end{array}$ & $\begin{array}{l}\text { GE } \\
W A\end{array}$ & $\underset{\mathrm{pH}}{\mu \mathrm{g} / \mathrm{L}}$ \\
\hline \multicolumn{16}{|c|}{ Common Laboratory Contaminants } \\
\hline Parameter & 1095 & DFF & Mod & $\underline{\mathbf{S T}}$ & $\underline{H}$ & Flag & $\underline{\text { Lab }}$ & $\underline{2095}$ & $\underline{D F}$ & Mod & $\underline{\mathbf{S T}}$ & 브 & Flag & Lab & $\underline{U}_{\text {nit }}$ \\
\hline $\begin{array}{l}\text { Bis(2-ethylhexyl) phthalate } \\
\text { Dichloromethane }\end{array}$ & $\begin{array}{l}<17 \\
<1.7\end{array}$ & 1 & $v$ & & & $\stackrel{0}{0}$ & $\begin{array}{l}\text { GE } \\
\text { GE }\end{array}$ & & & & & & & & $\underset{\mu g / L}{\mu g / L}$ \\
\hline
\end{tabular}

Note: See Appendix B for flagging criteria. Synchronous water levels are measured over a 3-5 day period. - = exceeded holding time.

- = exceeded groundwater protection standard. 
WELL FSB 76C (cont.)

Radiological Indicator Parameters

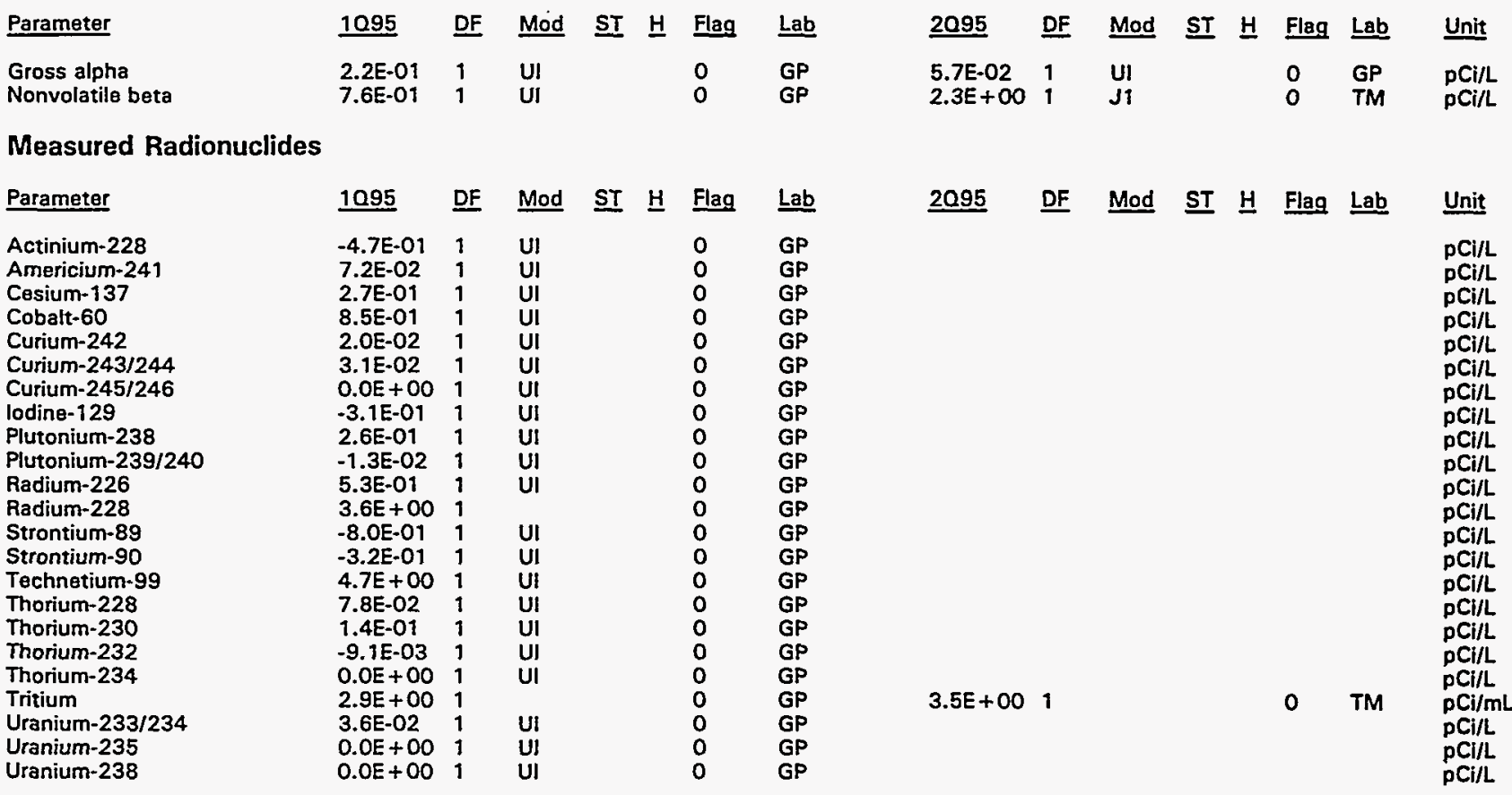

Calculated Radionuclides

Parameter

Americium-243

Nickel-63

Plutonium-241

Plutonium-242
1095

DF

9.2E-05

$8.5 \mathrm{E}-01$

$-5.2 \mathrm{E}-02$

$-2.6 \mathrm{E}-07$
DF Mod ST $\underline{\text { H Flag }} \underline{\text { Lab }}$

0

0
0
0
2095 DF Mod ST H Flag Lab Unit

$\mathrm{pCI} / \mathrm{L}$

pCi/L

pCin

Note: See Appendix B for flagging criteria. Synchronous water levels are measured over a 3-5 day period.

- = exceeded holding time.

- = exceeded groundwater protection standard. 


\section{WELL FSB 77}

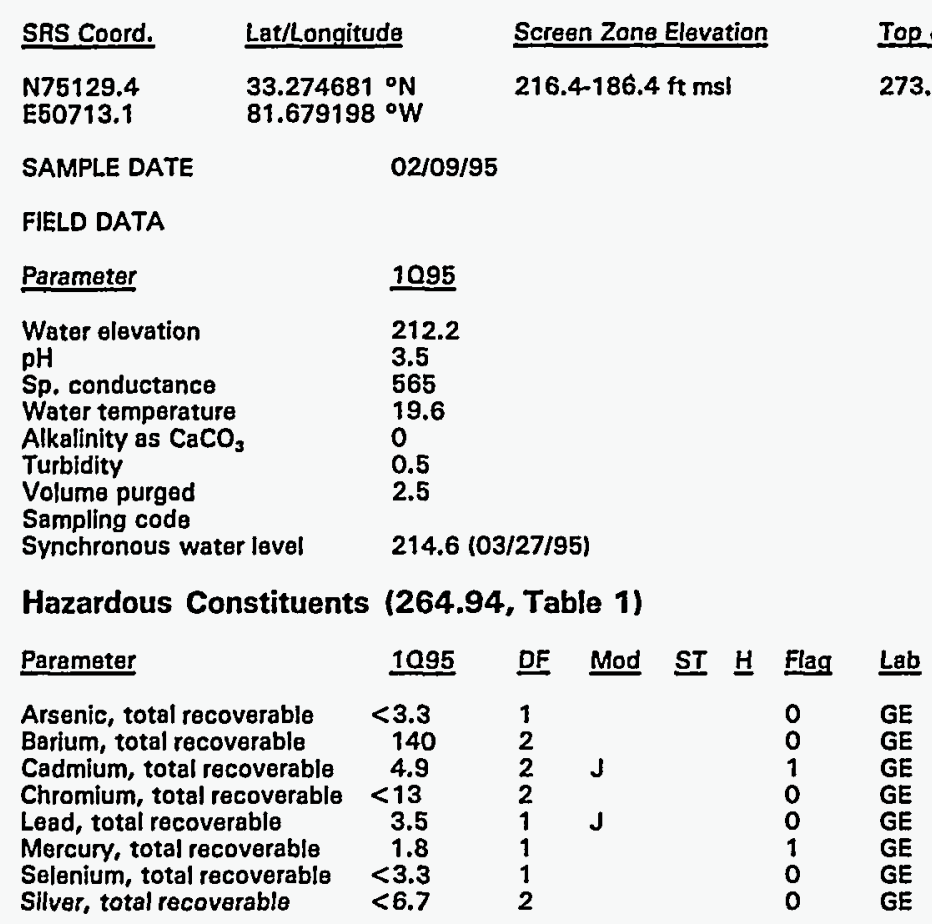

$\begin{array}{llll}\text { Top of Casing } & \text { Casing } & \text { Pump } & \text { Screon Zone } \\ 273.3 \mathrm{ft} \mathrm{msl} & 4^{\text {" PVC }} & V & \text { Water Table }\left(11 \mathrm{~B}_{2}\right)\end{array}$

$05 / 04 / 95$

2095 Unit

214.0 ft msl

it msl
pH
of $/ \mathrm{cm}$

${ }^{\circ} \mathrm{C}$

Mg/L

W

well volumes

$213.3(06 / 15 / 95) \quad$ ft ms

Hazardous Constituents (261 App. Vili/264 App. IX)

\begin{tabular}{|c|c|c|c|c|c|}
\hline Parameter & 1095 & DF & Mod & ST $\quad$ H & Flag \\
\hline $\begin{array}{l}\text { Antimony, total recoverable } \\
\text { Benzene } \\
\text { Cobalt, total recoverable } \\
\text { Copper, total recoverable } \\
\text { Cyanide } \\
\text { Nickel, total recoverable } \\
\text { Phenols } \\
\text { Tetrachloroethylene } \\
\text { Thallium, total recoverable } \\
\text { Trichloroethylene } \\
\text { Trichlorofluoromethane } \\
\text { Vanadium, total recoverable } \\
\text { Zinc, total recoverable }\end{array}$ & $\begin{aligned}<3.3 \\
<1.7 \\
<13 \\
\\
\quad 48 \\
<8.3 \\
\quad 16 \\
<8.3 \\
<1.7 \\
<3.3 \\
<1.7 \\
<1.7 \\
<27 \\
59\end{aligned}$ & $\begin{array}{l}1 \\
1 \\
2 \\
2 \\
1 \\
2 \\
1 \\
1 \\
1 \\
1 \\
1 \\
2 \\
2\end{array}$ & & n & $\begin{array}{l}0 \\
0 \\
0 \\
0 \\
0 \\
0 \\
0 \\
0 \\
0 \\
0 \\
0 \\
0 \\
0\end{array}$ \\
\hline
\end{tabular}

2095 DF Mod ST $H$ Flag Lab Unit

Water Quality Indicator Parameters

\begin{tabular}{|c|c|c|c|c|c|c|c|c|c|c|c|c|c|c|c|}
\hline Parameter & 1095 & $\underline{\mathrm{DF}}$ & Mod & $\underline{\text { ST }}$ & $\underline{\mathrm{H}}$ & Flag & $\underline{\text { Lab }}$ & $\underline{2095}$ & $\underline{\mathrm{DF}}$ & Mod & $\underline{\mathrm{ST}}$ & $\underline{H}$ & Flag & $\underline{\text { Lab }}$ & Unit \\
\hline $\begin{array}{l}\text { Aluminum, total racoverable } \\
\text { Nitrate as nitrogen } \\
\text { pH }\end{array}$ & $\begin{array}{l}24,000 \\
380,000 \\
3.5\end{array}$ & $\begin{array}{l}2 \\
400 \\
1\end{array}$ & J1 & 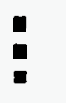 & & $\begin{array}{l}2 \\
2 \\
1\end{array}$ & $\begin{array}{l}\mathrm{GE} \\
\mathrm{GE} \\
\mathrm{GE}\end{array}$ & & & & & & & & $\begin{array}{l}\mu \mathrm{g} / \mathrm{L} \\
\mu \mathrm{g} / \mathrm{L} \\
\mathrm{pH}\end{array}$ \\
\hline \multicolumn{16}{|c|}{ Common Laboratory Contaminants } \\
\hline Parameter & 1095 & DF & Mod & $\underline{\mathbf{S T}}$ & $\underline{H}$ & Flag & $\underline{\text { Lab }}$ & $\underline{2095}$ & $\underline{\mathrm{DF}}$ & Mod & $\underline{\text { ST }}$ & $\underline{H}$ & Flag & Lab & Unit \\
\hline $\begin{array}{l}\text { Bis(2-ethylhexyl) phthalate } \\
\text { Dichloromethane }\end{array}$ & $\begin{array}{l}<17 \\
<1.7\end{array}$ & $\begin{array}{l}1 \\
1\end{array}$ & v & & & $\begin{array}{l}0 \\
0\end{array}$ & $\begin{array}{l}\mathrm{GE} \\
\mathrm{GE}\end{array}$ & & & & & & & & \\
\hline
\end{tabular}

Note: See Appendix B for flagging criteria. Synchronous water levels are measured over a 3-5 day period.

- = exceeded holding time.

- = exceeded groundwater protection standard. 
WELL FSB 77 (cont.)

Radiological Indicator Parameters

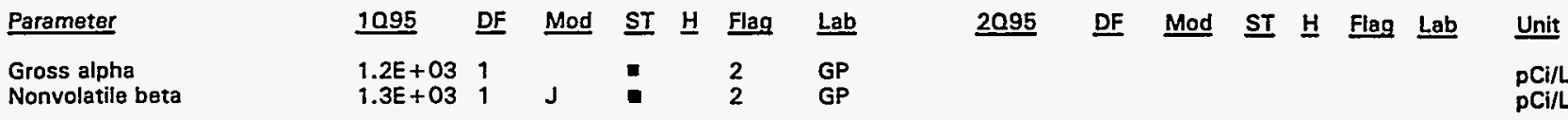

Measured Radionuclides

\section{Parameter}

Actinium-228

Americium-241

Cesium-137

Cobalt-60

Curium-242

Curium-243/244

Curium-245/246

lodine-129

Plutonium-238

Plutonium-239/240

Radium-226

Radium-228

Strontium-89

Strontium-90

Technetium-99

Thorium-228

Thorium-230

Thorium-232

Thorium-234

Tritium

Uranium-233/234

Uranium-235

Uranium-238

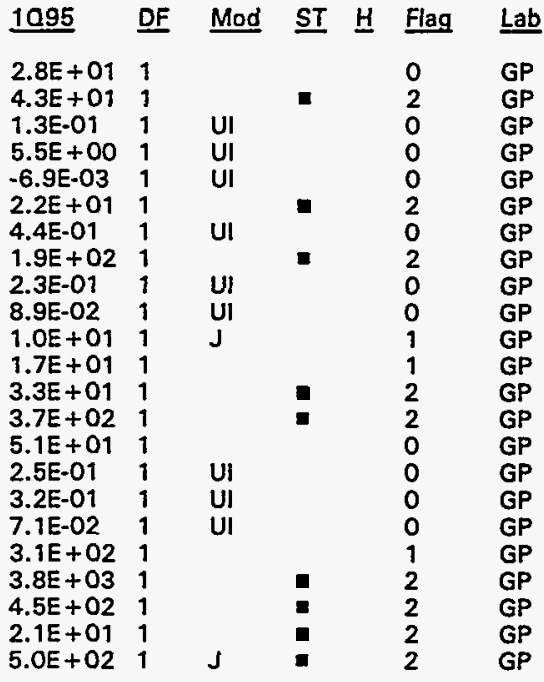

2095

DF Mod ST $\underline{H}$ Flag Lab Unit

$\mathrm{pCi}$

$\mathrm{pCi} / \mathrm{L}$

$\mathrm{pCi} / \mathrm{L}$

pCi/L

pCi/L

PCi/l

pCi/L

pCilL

pCi/L

$\mathrm{pCi} / \mathrm{L}$

pCi/L

pCill

pCi/L

$\mathrm{pCi} / \mathrm{L}$

pCi/l

$\mathrm{pCi} / \mathrm{L}$

pCi/L

pCi/L

$\mathrm{pCi} / \mathrm{mL}$

$\mathrm{pCi} / \mathrm{L}$.

$\mathrm{PCI} / \mathrm{L}$

$\mathrm{pCi} / \mathrm{L}$

Calculated Radionuclides

Parameter

Americium-243

1095

DF

Mod ST $\underline{H}$ Flag Lab

$\underline{2095}$

DF

OF Mod ST H Flag Lab

$\underline{\text { Unit }}$

$5.5 \mathrm{E}+00$

3.6E-01

Plutonium-241

Plutonium-242

$\mathrm{pCi} / \mathrm{L}$

$\mathrm{pCI} / \mathrm{L}$

$\mathrm{PCI} / \mathrm{L}$

pCi/L.

Note: See Appendix B for flagging criteria. Synchronous water levels are measured over a 3-5 day period.

$-=$ exceeded holding time.

- exceeded groundwater protection standard. 


\section{WELLL FSB 78}

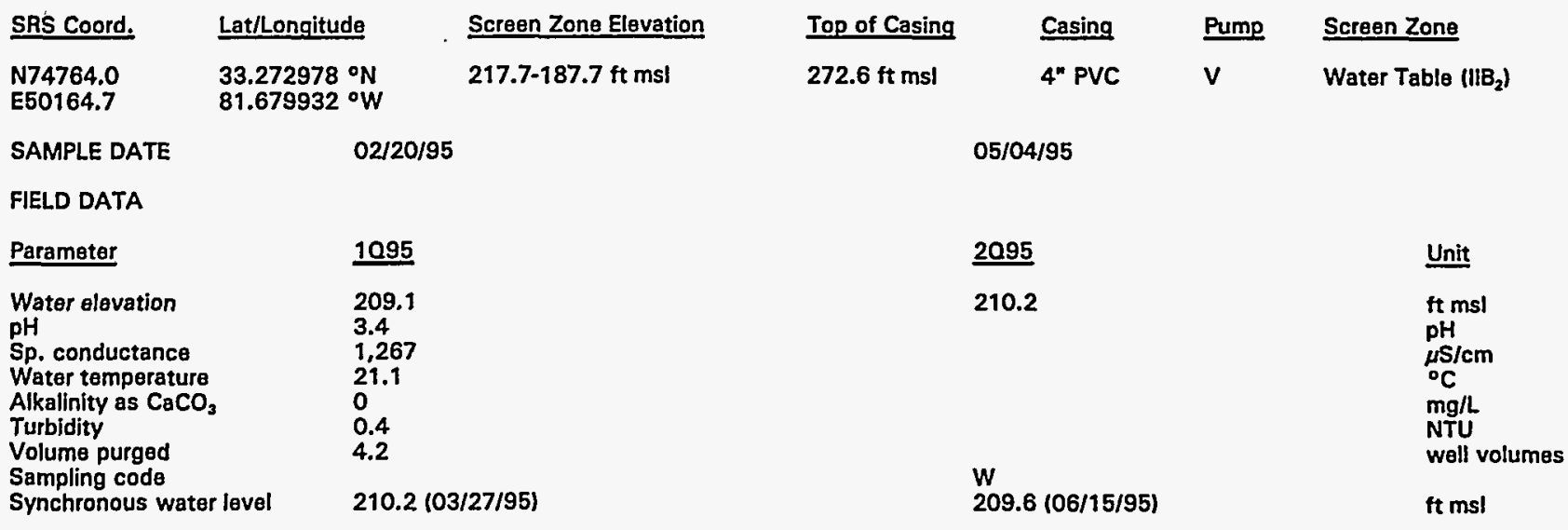

Hazardous Constituents (264.94, Table 1)

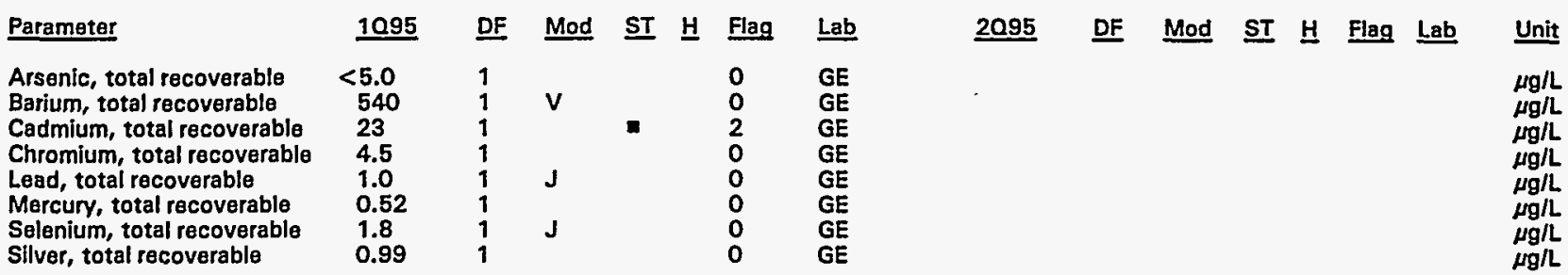

Hazardous Constituents (261 App. VIII/264 App. IX)

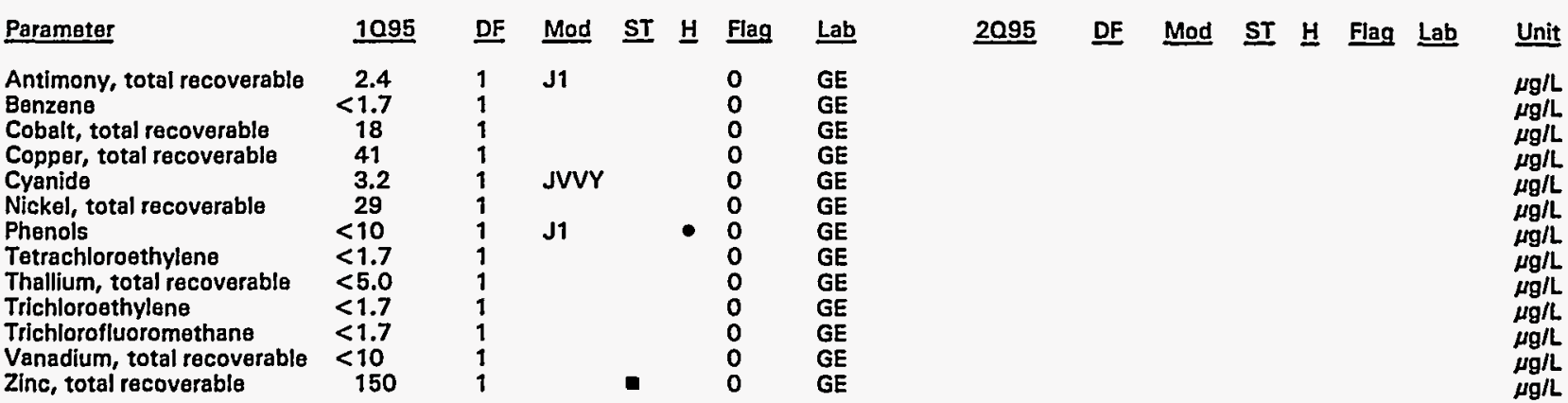

Water Quality Indicator Parameters

\begin{tabular}{|c|c|c|c|c|c|c|c|c|c|c|c|c|c|c|c|}
\hline Parameter & 1095 & $\underline{D F}$ & Mod & $\underline{\mathbf{S T}}$ & H & Flag & $\underline{\text { Lab }}$ & 2095 & DF & Mod & $\underline{\text { ST }}$ & $\underline{\mathbf{H}}$ & Flag & $\underline{\text { Lab }}$ & $\underline{\text { Unit }}$ \\
\hline $\begin{array}{l}\text { Aluminum, total recoverable } \\
\text { Nitrate as nitrogen } \\
\text { pH }\end{array}$ & $\begin{array}{l}38,000 \\
140,000 \\
3.3\end{array}$ & $\begin{array}{l}1 \\
160 \\
1\end{array}$ & $\begin{array}{l}v \\
J 1\end{array}$ & 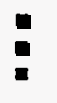 & & $\begin{array}{l}2 \\
2 \\
1\end{array}$ & $\begin{array}{l}\text { GE } \\
\mathrm{GE} \\
\mathrm{GE}\end{array}$ & & & & & & & & $\underset{\mathrm{pH}}{\mu \mathrm{g} / \mathrm{L}}$ \\
\hline \multicolumn{16}{|c|}{ Common Laboratory Contaminants } \\
\hline Parameter & 1095 & DF & Mod & $\underline{\mathbf{S T}}$ & $\underline{H}$ & Flag & $\underline{\text { Lab }}$ & 20.95 & DF & Mod & $\underline{\mathbf{S T}}$ & 브 & Flag & $\underline{\text { Lab }}$ & Unit \\
\hline $\begin{array}{l}\text { Bis(2-ethylhexyl) phthalate } \\
\text { Dichloromethane }\end{array}$ & $\begin{array}{l}<17 \\
<1.7\end{array}$ & $\begin{array}{l}1 \\
1\end{array}$ & & & & $\begin{array}{l}0 \\
0\end{array}$ & $\begin{array}{l}\text { GE } \\
\text { GE }\end{array}$ & & & & & & & & $\underset{\mu \mathrm{g} / \mathrm{L} / \mathrm{L}}{\mu}$ \\
\hline
\end{tabular}

Note: See Appendix B for flagging criteria. Synchronous water levels are measured over a 3-5 day period.

- = exceeded holding time.

- = exceeded groundwater protection standard. 
WELL FSB 78 (cont.)

Radiological Indicator Parameters

Parameter

Gross alpha Nonvolatile beta

$$
1095
$$

$2.5 E+03$

DF Mod ST H Flag Lab

$\begin{array}{llllll}2.5 E+03 & 1 & J & = & 2 & G P \\ 3.1 E+03 & 1 & J & = & 2 & G P\end{array}$

GP

Measured Radionuclides

Parameter

Actinium-228

Americium-241

Cesium-137

Cobalt -60

Curium-242

Curium-243/244

Curium-245/246

lodine-129

Plutonium-238

Plutonium-239/240

Radium-226

Radium-228

Strontium-89

Strontium-90

Technetium-99

Thorium-228

Thorium-230

Thorium-232

Thorium-234

Tritium

Uranium-233/234

Uranium-235

Uranium-238

109

DF

$8.5 \mathrm{E}+011$

2.8E + 021

4.3E + 001

5.8E-02

$9.2 \mathrm{E}+011$

$1.2 \mathrm{E}+001$

$2.9 \mathrm{E}+02$

1.5E-01

2.OE-O2

$1.8 \mathrm{E}+011$

$5.7 E+01$

$-1.2 E+031$

2.1E+O3 1

$5.5 E+011$

$1.8 \mathrm{E}+001$

6.0E-01

$-4.7 E-021$

$7.0 E+021$

$7.3 \mathrm{E}+031$

$4.8 \mathrm{E}+01$

2.7E+00 1

5.1E+ 011

Calculated Radionuclides

Parameter

Americium-243

Nickel-63

Plutonium-241

Plutonium-242
2.8E-01

$4.3 E+00$

8.OE-02

4. OE-O7
Mod ST $\underline{H}$ Flag Lab

0 GP

- 2

UI 0

$\begin{array}{lll}0 & G P \\ - & 2 & G P\end{array}$

0 GP

UI $\quad \begin{array}{lll}2 & G P \\ \text { U } & 0 & G P\end{array}$

UI 0 GP

J

UJI 0 GP

$\begin{array}{lll}2 & G P \\ 0 & 0\end{array}$

0

UI 0 GP

$\begin{array}{lll} & 0 & G P \\ & 2 & G P\end{array}$

- 2 GP

- 2 GP

$\begin{array}{llll} & 0 & G P \\ & 2 & G P\end{array}$
2095

DF Mod ST $\underline{\text { Flag Lab Unit }}$

$\mathrm{pCi} / \mathrm{L}$

$\mathrm{pCi} / \mathrm{L}$

2095 DF Mod ST $\underline{H}$ Flag Lab Unit

pCi/L

$\mathrm{pCi} / \mathrm{L}$

$\mathrm{pCl} / \mathrm{L}$

$\mathrm{pCl} / \mathrm{L}$

pCi/L

$\mathrm{pCi} / \mathrm{L}$

$\mathrm{pCi} / \mathrm{L}$

pCi/L

$\mathrm{pCi} / \mathrm{L}$

$\mathrm{pCi} / \mathrm{L}$

$\mathrm{pCi} / \mathrm{L}$

$\mathrm{pCi} / \mathrm{L}$

$\mathrm{pCl} / \mathrm{L}$

$p C i / L$

$\mathrm{pCi} / \mathrm{L}$

$\mathrm{PCI} / \mathrm{L}$

$\mathrm{pCi} / \mathrm{L}$

$\mathrm{pCi} / \mathrm{L}$

$\mathrm{pCl} / \mathrm{mL}$

$\mathrm{pCi} / \mathrm{L}$

$\mathrm{pCi} / \mathrm{L}$

$\mathrm{pCi} / \mathrm{L}$

2095

DF Mod ST $\underline{H}$ Flag Lab Unit

$\mathrm{pCi} / \mathrm{L}$

$\mathrm{pCi} / \mathrm{L}$

pCi/L

$\mathrm{pCi} / \mathrm{L}$

Note: See Appendix B for flagging criteria. Synchronous water levels are measured over a 3-5 day period.

- = exceeded holding time.

- = exceeded groundwater protection standard. 


\section{WELL FSB 78A}

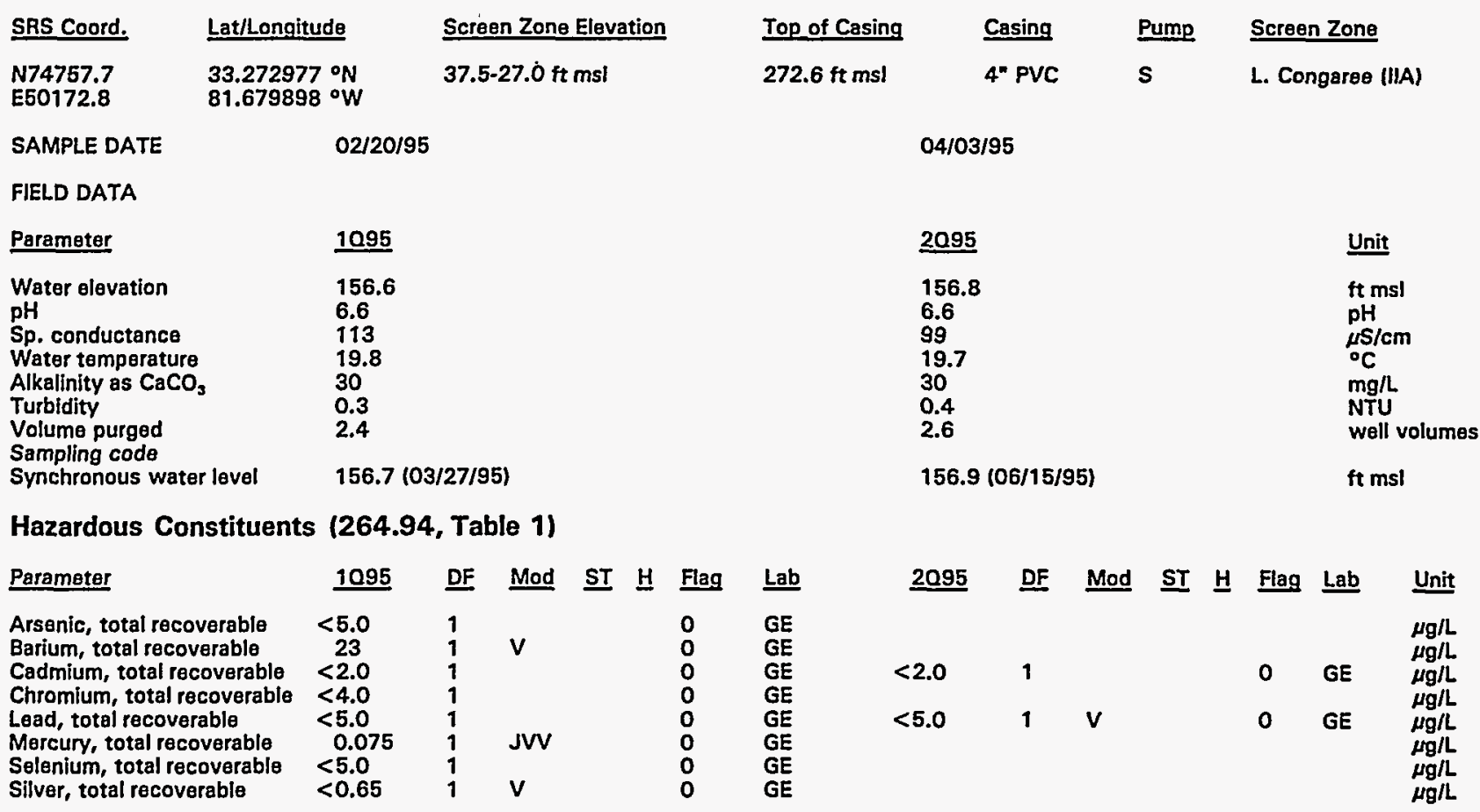

Hazardous Constituents (261 App. VIII/264 App. IX)

\begin{tabular}{|c|c|c|c|c|c|c|c|c|c|c|c|c|c|c|c|}
\hline Parameter & 1095 & DE & Mod & $\underline{\text { ST }}$ & $\underline{H}$ & Flag & $\underline{L a b}$ & $\underline{2095}$ & $\underline{\mathrm{DF}}$ & Mod & ST & 브 & Flag & $\underline{\text { Lab }}$ & Unit \\
\hline $\begin{array}{l}\text { Antimony, total recoverable } \\
\text { Benzene } \\
\text { Cobalt, total recoverable } \\
\text { Copper, total recoverable } \\
\text { Cyanide } \\
\text { Nickel, total recoverable } \\
\text { Phenols } \\
\text { Tetrachloroethylene } \\
\text { Thallium, total recoverable } \\
\text { Trichloroethylene } \\
\text { Trichlorofluoromethane } \\
\text { Vanadium, total recoverable } \\
\text { Zinc, total recoverable }\end{array}$ & $\begin{aligned}<5.0 \\
<1.7 \\
<4.0 \\
<4.0 \\
2.6 \\
<10 \\
<10 \\
<1.7 \\
<5.0 \\
<1.7 \\
<1.7 \\
<10 \\
<5.0\end{aligned}$ & $\begin{array}{l}1 \\
1 \\
1 \\
1 \\
1 \\
1 \\
1 \\
1 \\
1 \\
1 \\
1 \\
1 \\
1\end{array}$ & JVV & & & $\begin{array}{l}0 \\
0 \\
0 \\
0 \\
0 \\
0 \\
0 \\
0 \\
0 \\
0 \\
0 \\
0 \\
0\end{array}$ & $\begin{array}{l}\mathrm{GE} \\
\mathrm{GE} \\
\mathrm{GE} \\
\mathrm{GE} \\
\mathrm{GE} \\
\mathrm{GE} \\
\mathrm{GE} \\
\mathrm{GE} \\
\mathrm{GE} \\
\mathrm{GE} \\
\mathrm{GE} \\
\mathrm{GE} \\
\mathrm{GE}\end{array}$ & & & & & & & & $\begin{array}{l}\mu g / L \\
\mu g / L \\
\mu g / L \\
\mu g / L \\
\mu g / L \\
\mu g / L \\
\mu g / L \\
\mu g / L \\
\mu g / L \\
\mu g / L \\
\mu g / L \\
\mu g / L \\
\mu g / L\end{array}$ \\
\hline \multicolumn{16}{|c|}{ Water Quality Indicator Parameters } \\
\hline Parameter & 1095 & $\underline{\mathrm{DF}}$ & Mod & $\underline{\text { ST }}$ & $\underline{\mathrm{H}}$ & Flag & $\underline{\text { Lab }}$ & $\underline{2095}$ & $\underline{D F}$ & Mod & $\underline{\mathbf{S T}}$ & H & Flag & Labb & Unit \\
\hline $\begin{array}{l}\text { Aluminum, total recoverable } \\
\text { Nitrate as nitrogen } \\
\mathrm{pH}\end{array}$ & $\begin{array}{l}3.5 \\
420 \\
6.7\end{array}$ & $\begin{array}{l}1 \\
1 \\
1\end{array}$ & $\begin{array}{l}\text { J } \\
\text { J1 }\end{array}$ & $\mathbf{n}$ & - & $\begin{array}{l}0 \\
0 \\
0\end{array}$ & $\begin{array}{l}\mathrm{GE} \\
\mathrm{GE} \\
\mathrm{GE}\end{array}$ & $\begin{array}{l}210 \\
6.7\end{array}$ & $\begin{array}{l}1 \\
1\end{array}$ & J1 & - & $\bullet$ & $\begin{array}{l}0 \\
0\end{array}$ & $\begin{array}{l}\text { GE } \\
\text { GE }\end{array}$ & $\begin{array}{l}\mu \mathrm{g} / \mathrm{L} \\
\mu \mathrm{g} / \mathrm{L} \\
\mathrm{pH}\end{array}$ \\
\hline \multicolumn{16}{|c|}{ Common Laboratory Contaminants } \\
\hline Parameter & 1095 & $\underline{D F}$ & Mod & $\underline{\text { ST }}$ & $\underline{\mathrm{H}}$ & Flag & $\underline{\text { Lab }}$ & $\underline{2095}$ & $\underline{D F}$ & Mod & $\underline{\text { ST }}$ & $\underline{H}$ & Flag & Lab & Unit \\
\hline $\begin{array}{l}\text { Bis(2-ethylhexyil) phthalate } \\
\text { Dichloromethane }\end{array}$ & $\begin{array}{l}<17 \\
<1.7\end{array}$ & $\begin{array}{l}1 \\
1\end{array}$ & & & & $\begin{array}{l}0 \\
0\end{array}$ & $\begin{array}{l}\mathrm{GE} \\
\mathrm{GE}\end{array}$ & & & & & & & & $\begin{array}{l}\mu g / L \\
\mu g / L\end{array}$ \\
\hline
\end{tabular}

Note: See Appendix B for flagging criteria. Synchronous water levels are measured over a 3-5 day period.

- = exceeded holding time.

- = exceeded groundwater protection standard. 
WELL FSB 78A (cont.)

Radiological Indicator Parameters

\begin{tabular}{|c|c|c|c|c|c|c|c|c|c|c|c|c|c|}
\hline Parameter & 1095 & DF & Mod & $\underline{\text { ST }} \underline{H}$ & Flag & Lab & 20.95 & $\underline{\text { DF }}$ & Mod & $\underline{\mathbf{S T}}$ & 브 & Flag & Lab \\
\hline $\begin{array}{l}\text { Gross alpha } \\
\text { Nonvolatile beta }\end{array}$ & $\begin{array}{l}9.2 \mathrm{E}-01 \\
3.8 \mathrm{E}+00\end{array}$ & $\begin{array}{l}1 \\
1\end{array}$ & UH & & $\begin{array}{l}0 \\
0\end{array}$ & $\begin{array}{l}\text { GP } \\
\text { GP }\end{array}$ & $\begin{array}{l}7.0 E-01 \\
3.7 E+00\end{array}$ & $\begin{array}{l}1 \\
1\end{array}$ & UJI & & & $\begin{array}{l}0 \\
0\end{array}$ & $\begin{array}{l}\text { GP } \\
\text { GP }\end{array}$ \\
\hline
\end{tabular}

Measured Radionuclides

\begin{tabular}{|c|c|c|c|c|c|c|c|c|c|c|c|c|c|c|c|}
\hline Parameter & 1095 & DF & Mod & $\underline{\text { ST }}$ & $\underline{H}$ & Flag & Lab & 2095 & DF & Mod & $\underline{\text { ST }}$ & $\underline{H}$ & Flag & Lab & Unit \\
\hline $\begin{array}{l}\text { Actinium-228 } \\
\text { Americium-241 } \\
\text { Cesium-137 } \\
\text { Cobalt-60 } \\
\text { Curium-242 } \\
\text { Curium-243/244 } \\
\text { Curium-245/246 } \\
\text { lodine-129 } \\
\text { Plutonium-238 } \\
\text { Plutonium-239/240 } \\
\text { Radium-226 } \\
\text { Radium-228 } \\
\text { Strontium-89 } \\
\text { Strontium-90 } \\
\text { Technetium-99 } \\
\text { Thorium-228 } \\
\text { Thorium-230 } \\
\text { Thorium-232 } \\
\text { Thorium-234 } \\
\text { Tritium } \\
\text { Uranium-233/234 } \\
\text { Uranium-235 } \\
\text { Uranium-238 }\end{array}$ & $\begin{array}{l}5.0 E-01 \\
5.0 E-02 \\
-1.6 E-01 \\
-1.0 E+00 \\
1.2 E-02 \\
1.6 E-02 \\
-5.6 E-03 \\
2.8 E-02 \\
3.6 E-02 \\
0.0 E+00 \\
1.5 E+00 \\
-1.9 E-01 \\
3.2 E-01 \\
4.9 E-01 \\
-2.5 E+01 \\
-2.5 E-02 \\
1.2 E-01 \\
-8.2 E-03 \\
0.0 E+00 \\
1.6 E+01 \\
7.3 E-02 \\
5.2 E-03 \\
4.2 E-02\end{array}$ & $\begin{array}{l}1 \\
1 \\
1 \\
1 \\
1 \\
1 \\
1 \\
1 \\
1 \\
1 \\
1 \\
1 \\
1 \\
1 \\
1 \\
1 \\
1 \\
1 \\
1 \\
1 \\
1 \\
1 \\
1\end{array}$ & $\begin{array}{l}\text { UI } \\
\text { UI } \\
\text { UI } \\
\text { UI } \\
\text { UI } \\
\text { UI } \\
\text { UI } \\
\text { UI } \\
\text { UJI } \\
\text { UI } \\
\text { UII } \\
\text { UI } \\
\text { UI } \\
\text { UI } \\
\text { UI } \\
\text { UI } \\
\text { UI } \\
\text { UI } \\
\text { UJI }\end{array}$ & & & $\begin{array}{l}0 \\
0 \\
0 \\
0 \\
0 \\
0 \\
0 \\
0 \\
0 \\
0 \\
0 \\
0 \\
0 \\
0 \\
0 \\
0 \\
0 \\
0 \\
0 \\
1 \\
0 \\
0 \\
0\end{array}$ & $\begin{array}{l}\text { GP } \\
\text { GP } \\
\text { GP } \\
\text { GP } \\
\text { GP } \\
\text { GP } \\
\text { GP } \\
\text { GP } \\
\text { GP } \\
\text { GP } \\
\text { GP } \\
\text { GP } \\
\text { GP } \\
\text { GP } \\
\text { GP } \\
\text { GP } \\
\text { GP } \\
\text { GP } \\
\text { GP } \\
\text { GP } \\
\text { GP } \\
\text { GP } \\
\text { GP }\end{array}$ & $9.4 \mathrm{E}-03$ & 1 & UI & & & 0 & GP & 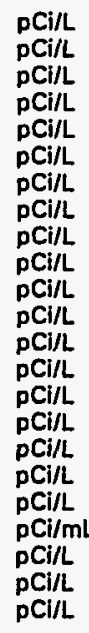 \\
\hline \multicolumn{16}{|c|}{ Calculated Radionuclides } \\
\hline Parameter & $\underline{1095}$ & $\underline{\mathrm{DF}}$ & Mod & $\underline{\mathbf{S T}}$ & $\underline{\mathrm{H}}$ & Flag & $\underline{\text { Lab }}$ & 2095 & $\underline{\mathrm{DF}}$ & Mod & $\underline{\text { ST }}$ & H & Flag & $\underline{\text { Lab }}$ & Unit \\
\hline $\begin{array}{l}\text { Americium-243 } \\
\text { Nickel-63 } \\
\text { Plutonium-241 } \\
\text { Plutonium-242 }\end{array}$ & $\begin{array}{l}4.7 E-05 \\
-1.0 E+00 \\
0.0 E+00 \\
0.0 E+00\end{array}$ & & & & & $\begin{array}{l}0 \\
0 \\
0 \\
0\end{array}$ & & & & & & & & & $\begin{array}{l}\text { pCi/l } \\
\text { pCi/L } \\
\text { pCi/L } \\
\text { pCi/L }\end{array}$ \\
\hline
\end{tabular}

Note: See Appendix 8 for flagging criteria. Synchronous water levels are measured over a 3-5 day period.

- = exceeded holding time.

- exceeded groundwater protection standard. 


\section{WELL FSB 78B}

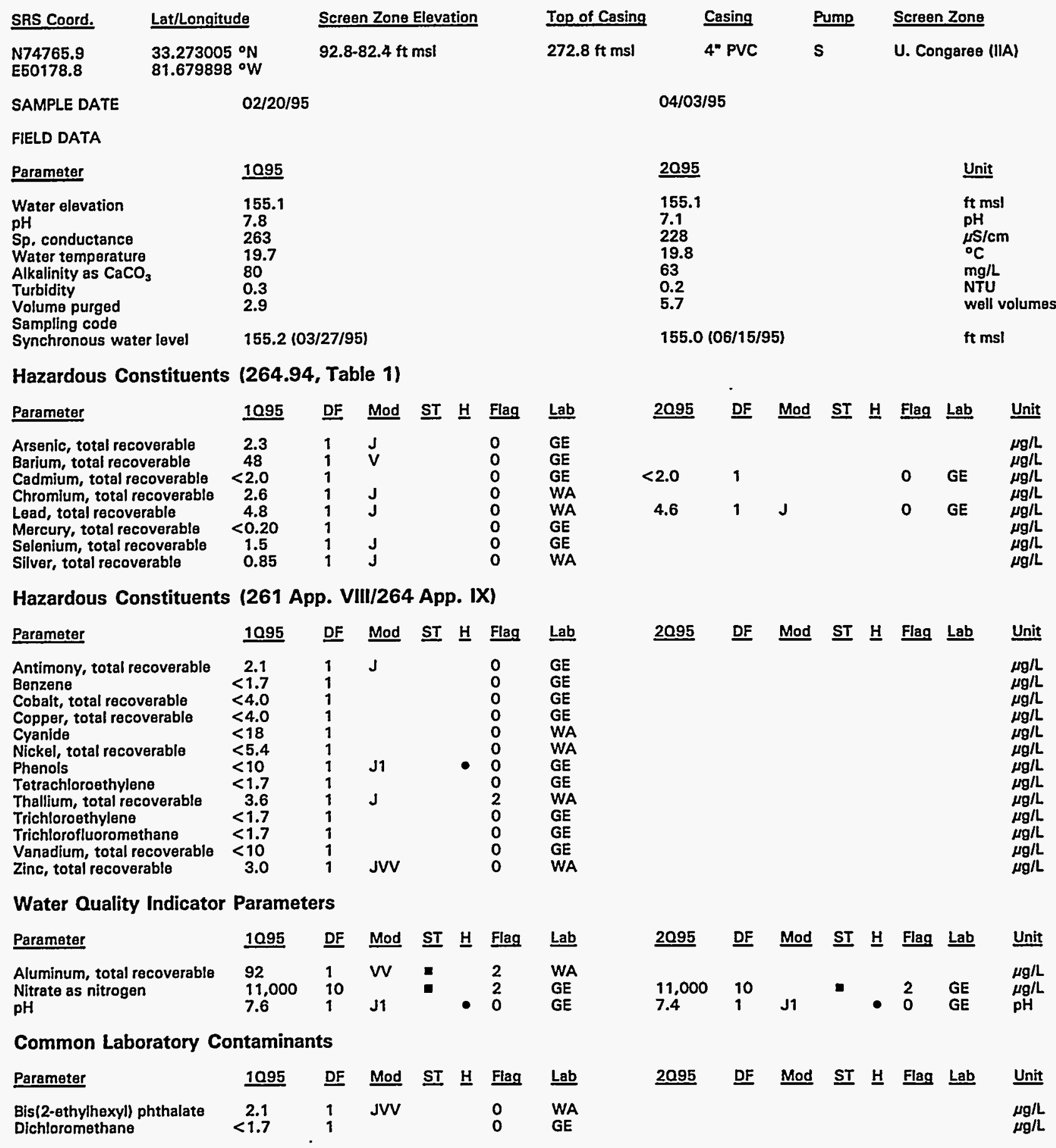

Note: See Appendix B for flagging criteria. Synchronous water levels are measured over a 3-5 day period.

- = exceeded holding time.

- = exceeded groundwater protection standard. 
WELL FSB 78B (cont.)

Radiological Indicator Parameters

\begin{tabular}{|c|c|c|c|c|c|c|c|c|c|c|c|c|c|c|}
\hline & 1095 & $\underline{\text { DF }}$ & Mod & $\underline{\text { ST }}$ & 브 & Flag & Lab & 2095 & $\underline{D F}$ & Mod & $\underline{\mathbf{S T}}$ & $\underline{H}$ & Flag & $\underline{\text { Lab }}$ \\
\hline $\begin{array}{l}\text { Gross alpha } \\
\text { Nonvolatile beta }\end{array}$ & $\begin{array}{l}2.1 E+00 \\
5.6 E+00\end{array}$ & $\begin{array}{l}1 \\
1\end{array}$ & & & & $\begin{array}{l}0 \\
0\end{array}$ & $\begin{array}{l}\text { GP } \\
\text { GP }\end{array}$ & $\begin{array}{l}1.8 E+00 \\
2.6 E+00\end{array}$ & $\begin{array}{l}1 \\
1\end{array}$ & $\mathbf{J}$ & & & $\begin{array}{l}0 \\
0\end{array}$ & $\begin{array}{l}\text { GP } \\
\text { GP }\end{array}$ \\
\hline
\end{tabular}

Measured Radionuclides

Parameter

Actinium-228

Americium-241

Cesium-137

Cobalt -60

Curium-242

Curium-243/244

Curium-245/246

lodine- 129

Plutonium-238

Plutonium-239/240

Radium-226

Radium-228

Strontium-89

Strontium-90

Technetium-99

Thorium-228

Thorium-230

Thorium-232

Thorium-234

Tritium

Uranium-233/234

Uranium-235

Uranium-238

Calculated Radionuclides

Parameter

Americium-243

Nickel-63

Plutonium-241

Plutonium-242

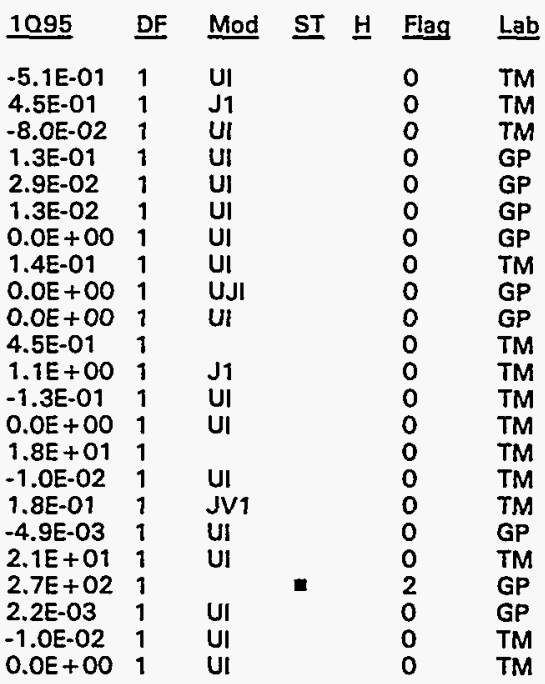

$\underline{2095}$

DF Mod ST $H$ Flag Lab Unit

TM

TM

GP

GP

TM

GP

TM

$T M$

$T M$

$T M$

GP

pCi/L

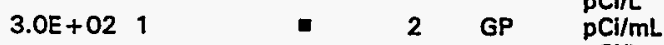

$\mathrm{pCi} / \mathrm{L}$

$\mathrm{PCi}$

$\mathrm{pCi} / \mathrm{L}$

2095 DF Mod ST $\underline{\text { H Flag Lab }}$ Unit

3.9E-05

$1.3 E-01$

$0.0 E+00$

$0.0 E+00$

DF Mod ST $H$ Flag Lab

0

0
0
0
0

$\mathrm{pCi} / \mathrm{L}$

$\mathrm{pCi} / \mathrm{L}$

$\mathrm{PC} / \mathrm{L}$

pCi/L

Note: See Appendix B for flagging criteria. Synchronous water levels are measured over a 3-5 day period.

- = exceeded holding time.

- = exceeded groundwater protection standard. 


\section{WELL FSB 78C}

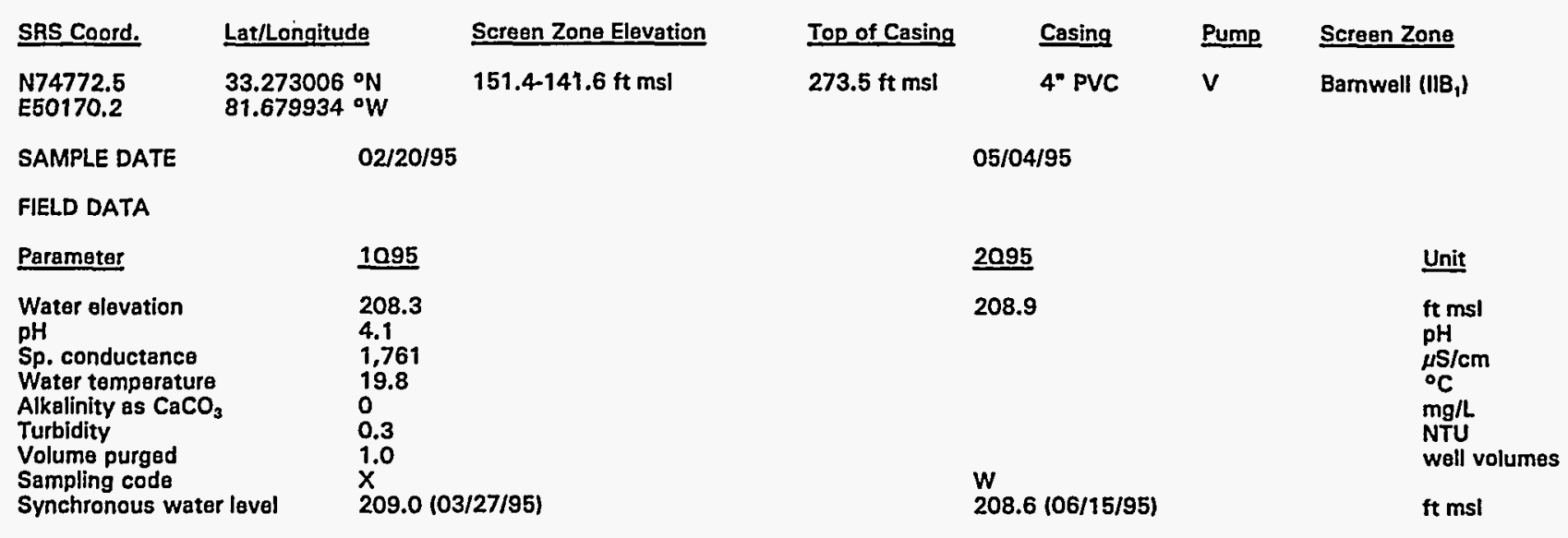

Hazardous Constituents (264.94, Table 1)

$\begin{array}{lllllllll}\text { Parameter } & 1095 & & \text { DF } & \text { Mod } & \text { ST } & \text { H } & \text { Flag } & \text { Lab } \\ \text { Arsenic, total recoverable } & 1.8 & 1 & \text { J } & & & 0 & \text { GE } \\ \text { Barium, total recoverable } & 560 & 10 & \text { V } & & & 0 & \text { GE } \\ \text { Cadmium, total recoverable } & 16 & 10 & \text { J } & & & 2 & \text { GE } \\ \text { Chromium, total recoverable } & <40 & 10 & & & 0 & \text { GE } \\ \text { Lead, total recoverable } & 2.6 & 1 & \text { J } & & 0 & \text { GE } \\ \text { Mercury, total recoverable } & <0.20 & 1 & & & 0 & \text { GE } \\ \text { Selenium, total recoverable } & <5.0 & 1 & & & 0 & \text { GE } \\ \text { Silver, total recoverable } & <6.5 & 10 & & & 0 & \text { GE }\end{array}$

2095 DF Mod ST $\underline{\text { H Flag Lab Unit }}$

Hazardous Constituents (261 App. VIll/264 App. IX)

\begin{tabular}{|c|c|c|c|c|c|c|}
\hline Parameter & 1095 & DF & Mod & $\underline{\mathrm{ST}}$ & $\underline{H}$ & Flag \\
\hline $\begin{array}{l}\text { Antimony, total recoverable } \\
\text { Benzene } \\
\text { Cobalt, total recoverable } \\
\text { Copper, total recoverable } \\
\text { Cyanide } \\
\text { Nickel, total recoverable } \\
\text { Phenols } \\
\text { Tetrachloroethylene } \\
\text { Thallium, total recoverable } \\
\text { Trichloroethylene } \\
\text { Trichlorofluoromethane } \\
\text { Vanadium, total recoverable } \\
\text { Zinc, total recoverable }\end{array}$ & $\begin{array}{c}4.3 \\
<1.7 \\
300 \\
11 \\
2.9 \\
120 \\
<10 \\
<1.7 \\
0.85 \\
<1.7 \\
<1.7 \\
<100 \\
590\end{array}$ & $\begin{array}{l}1 \\
1 \\
10 \\
10 \\
1 \\
10 \\
1 \\
1 \\
1 \\
1 \\
1 \\
10 \\
10\end{array}$ & $\begin{array}{l}\text { J1 } \\
\text { J } \\
\text { JVV } \\
\text { J1 } \\
\text { J }\end{array}$ & $\begin{array}{l}\mathbf{a} \\
\mathbf{0}\end{array}$ & • & $\begin{array}{l}1 \\
0 \\
2 \\
0 \\
0 \\
2 \\
0 \\
0 \\
0 \\
0 \\
0 \\
0 \\
0\end{array}$ \\
\hline
\end{tabular}

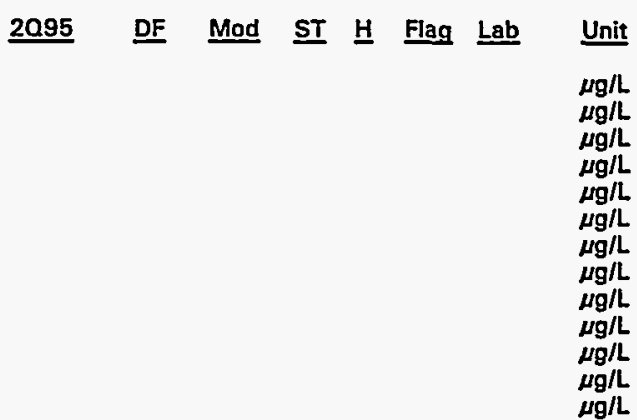

Water Quality Indicator Parameters

\begin{tabular}{|c|c|c|c|c|c|c|c|c|c|c|c|c|c|c|c|}
\hline Parameter & 1095 & $\underline{\text { DF }}$ & Mod & $\underline{\text { ST }}$ & 브 & Flag & $\underline{\mathrm{Lab}}$ & 2095 & $\underline{\text { DF }}$ & Mod & $\underline{\text { ST }}$ & $\underline{H}$ & Flag & $\underline{\text { Lab }}$ & Unit \\
\hline $\begin{array}{l}\text { Aluminum, total recoverable } \\
\text { Nitrate as nitrogen } \\
\text { pH }\end{array}$ & $\begin{array}{l}16,000 \\
240,000 \\
4.2\end{array}$ & $\begin{array}{l}10 \\
160 \\
1\end{array}$ & $\begin{array}{l}v \\
J 1\end{array}$ & 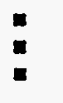 & & $\begin{array}{l}2 \\
2 \\
0\end{array}$ & $\begin{array}{l}\text { GE } \\
\text { GE } \\
\text { GE }\end{array}$ & & & & & & & & $\begin{array}{l}\mu g / L \\
\mu g / L \\
p H\end{array}$ \\
\hline \multicolumn{16}{|c|}{ Common Laboratory Contaminants } \\
\hline Parameter & 1095 & $\underline{\text { DF }}$ & Mod & $\underline{\text { ST }}$ & $\underline{\mathbf{H}}$ & Flag & $\underline{L a b}$ & 2095 & DF & Mod & $\underline{\mathbf{S T}}$ & $\underline{H}$ & Flag & Lab & Unit \\
\hline $\begin{array}{l}\text { Bis(2-ethylhexyl) phthalate } \\
\text { Dichloromethane }\end{array}$ & $<17$ & $\begin{array}{l}1 \\
1\end{array}$ & & & & $\begin{array}{l}0 \\
0\end{array}$ & $\begin{array}{l}\mathrm{GE} \\
\mathrm{GE}\end{array}$ & & & & & & & & \\
\hline
\end{tabular}

Note: See Appendix B for flagging criteria. Synchronous water levels are measured over a 3-5 day period. - = exceeded holding time.

- = exceeded groundwater protection standard. 
WELL FSB $78 C$ (cont.)

Radiological Indicator Parameters

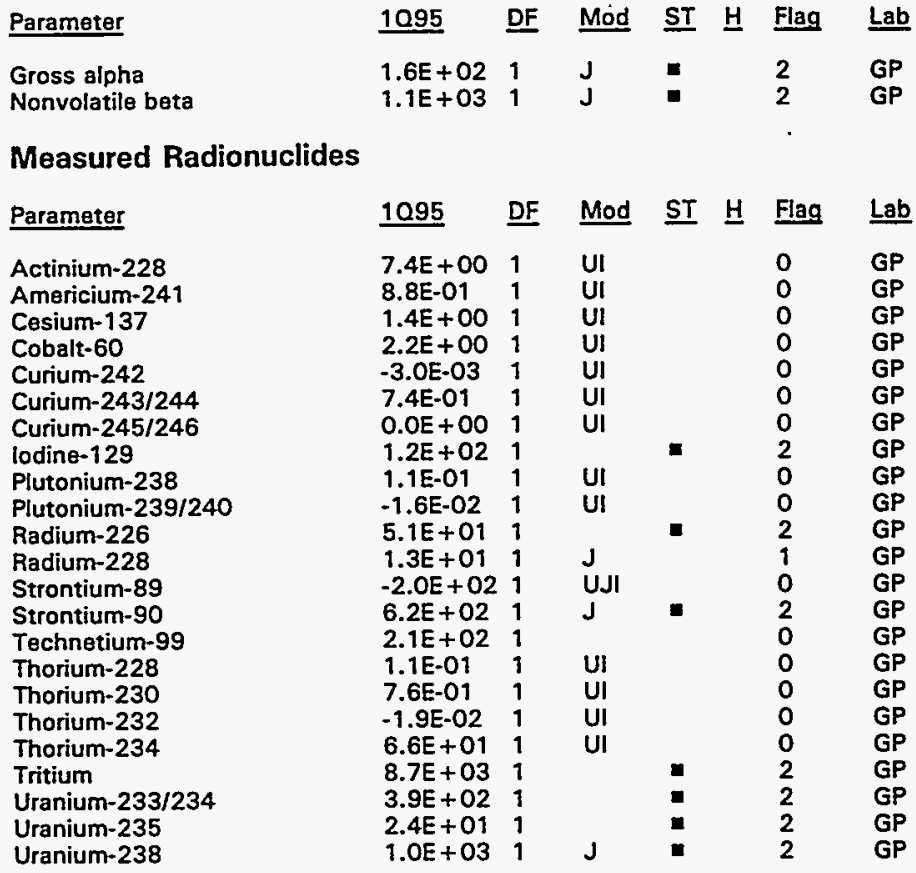

$\underline{2095}$ DF Mod ST $\underline{H}$ Flag Lab $\underline{\text { Unit }}$ $\begin{array}{ll}\mathrm{GP} & \mathrm{pCi} / \mathrm{L} \\ \mathrm{GCi} & \end{array}$

Calculated Radionuclides

\section{Parameter}

Americium-243

Nickel-63

Plutonium-241

Plutonium-242
1095

2.2E-03

$2.2 E+00$

$-6.2 \mathrm{E}-02$

$-3.1 E-07$ $\underline{\text { DF Mod }} \underline{\mathrm{ST}} \underline{\mathrm{H}}$ Flag $\underline{\text { Lab }}$

0

0
2095 DF Mod ST H Flag Lab Unit

$\mathrm{pCi} / \mathrm{L}$

pCi/L

pCi/L

$\mathrm{pCi} / \mathrm{L}$

$\mathrm{pCi} / \mathrm{L}$

pCi/L

pCi/L

$\mathrm{PCi} / \mathrm{L}$

pCilL

pCi/L

pCi/L

pCi/L

$\mathrm{pCi} / \mathrm{L}$

$\mathrm{PCi} / \mathrm{L}$

pCi/L

pCi/L

$\mathrm{pCl} / \mathrm{L}$

pCi/L

$\mathrm{pCi} / \mathrm{L}$

pCi/mL

pCi/L

$\mathrm{pCi} / \mathrm{L}$
$\mathrm{pCI} / \mathrm{L}$

$\underline{2095}$ DF Mod ST $\underline{H}$ Flag Lab Uni
$\mathrm{pCi} / \mathrm{L}$

PCI/L

PCi/L

$\mathrm{pCi} / \mathrm{L}$

Note: See Appendix B for flagging criteria. Synchronous water levels are measured over a 3-5 day period.

$\checkmark=$ exceeded holding time.

- = exceeded groundwater protection standard. 


\section{WELL FSB 79}

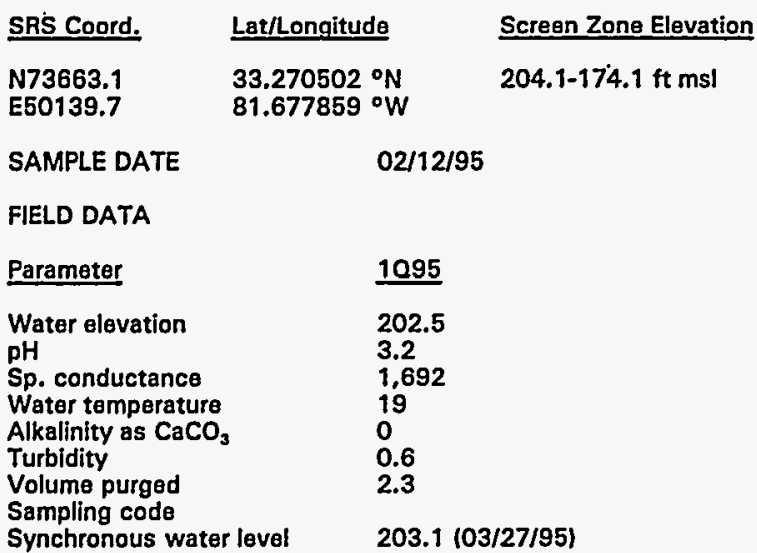

\begin{tabular}{|c|c|c|c|}
\hline Top of Casing & Casing & Pump & Screen Zorie \\
\hline $217.8 \mathrm{ft} \mathrm{msl}$ & 4" PVC & v & Water Table $\left(\mathrm{HB}_{2}\right)$ \\
\hline
\end{tabular}

Hazardous Constituents (264.94, Table 1)

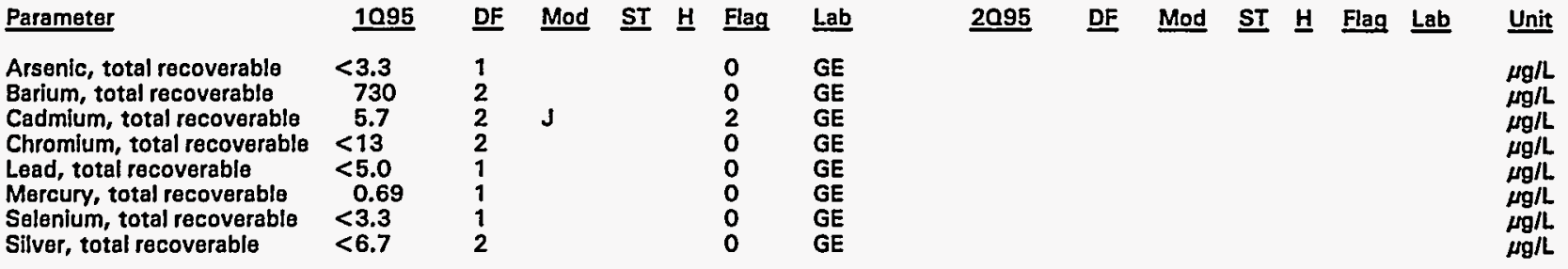

Hazardous Constituents (261 App. VIII/264 App. (X)

\begin{tabular}{|c|c|c|c|c|c|c|c|c|c|c|c|c|c|c|c|}
\hline Parameter & 10.95 & $\underline{\text { DF }}$ & Mod & $\underline{\mathbf{S T}}$ & $\underline{H}$ & Flag & $\underline{\text { Lab }}$ & $\underline{2095}$ & DF & Mod & $\underline{\mathbf{S T}}$ & 브 & Flag & $\underline{\text { Lab }}$ & Unit \\
\hline $\begin{array}{l}\text { Antimony, total recoverable } \\
\text { Benzene } \\
\text { Cobalt, total recoverable } \\
\text { Copper, total recoverable } \\
\text { Cyanide } \\
\text { Nickel, total recoverable } \\
\text { Phenols } \\
\text { Tetrachloroethylene } \\
\text { Thallium, total recoverable } \\
\text { Trichloroethylene } \\
\text { Trichlorofluoromethane } \\
\text { Vanadium, total recoverable } \\
\text { Zinc, total recoverable }\end{array}$ & $\begin{array}{l}<3.3 \\
<1.7 \\
520 \\
60 \\
<8.3 \\
62 \\
<8.3 \\
<1.7 \\
<3.3 \\
<1.7 \\
<1.7 \\
<27 \\
140\end{array}$ & $\begin{array}{l}1 \\
1 \\
2 \\
2 \\
1 \\
2 \\
1 \\
1 \\
1 \\
1 \\
1 \\
2 \\
2\end{array}$ & $\mathbf{Y}$ & $\begin{array}{l}\text { - } \\
\text { - }\end{array}$ & & $\begin{array}{l}0 \\
0 \\
2 \\
0 \\
0 \\
1 \\
0 \\
0 \\
0 \\
0 \\
0 \\
0 \\
0\end{array}$ & $\begin{array}{l}\mathrm{GE} \\
\mathrm{GE} \\
\mathrm{GE} \\
\mathrm{GE} \\
\mathrm{GE} \\
\mathrm{GE} \\
\mathrm{GE} \\
\mathrm{GE} \\
\mathrm{GE} \\
\mathrm{GE} \\
\mathrm{GE} \\
\mathrm{GE} \\
\mathrm{GE}\end{array}$ & & & & & & & & $\begin{array}{l}\mu g / L \\
\mu g / L \\
\mu g / L \\
\mu g / L \\
\mu g / L \\
\mu g / L \\
\mu g / L \\
\mu g / L \\
\mu g / L \\
\mu g / L \\
\mu g / L \\
\mu g / L \\
\mu g / L\end{array}$ \\
\hline \multicolumn{16}{|c|}{ Water Quality Indicator Parameters } \\
\hline Parameter & $\underline{1095}$ & DF & Mod & $\underline{\text { ST }}$ & $\underline{H}$ & Flag & $\underline{\text { Lab }}$ & $\underline{2095}$ & $\underline{\mathrm{DF}}$ & Mod & $\underline{\mathrm{ST}}$ & 보 & Flag & Lab & Unit \\
\hline $\begin{array}{l}\text { Aluminum, total recoverable } \\
\text { Nitrate as nitrogen } \\
\text { pH }\end{array}$ & $\begin{array}{l}61,000 \\
150,000 \\
3.5\end{array}$ & $\begin{array}{l}2 \\
160 \\
1\end{array}$ & J1 & 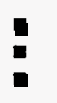 & • & $\begin{array}{l}2 \\
2 \\
1\end{array}$ & $\begin{array}{l}\text { GE } \\
\text { GE } \\
\text { GE }\end{array}$ & & & & & & & & $\begin{array}{l}\mu g / L \\
\mu g / L \\
\mathrm{pH}\end{array}$ \\
\hline \multicolumn{16}{|c|}{ Common Laboratory Contaminants } \\
\hline Parameter & 1095 & $\underline{D F}$ & Mod & $\underline{\text { ST }}$ & 브 & Flag & $\underline{\text { Lab }}$ & $\underline{2095}$ & DF & Mod & ST & 브 & Flag & $\underline{\text { Lab }}$ & Unit \\
\hline $\begin{array}{l}\text { Bis(2-ethylhexyl) phthalate } \\
\text { Dichloromethane }\end{array}$ & $\begin{array}{l}<17 \\
<1.7\end{array}$ & $\begin{array}{l}1 \\
1\end{array}$ & $v$ & & & $\begin{array}{l}0 \\
0\end{array}$ & $\begin{array}{l}\mathrm{GE} \\
\mathrm{GE}\end{array}$ & & & & & & & & \\
\hline
\end{tabular}

Note: See Appendix B for flagging criteria. Synchronaus water levels are measured over a 3-5 day period.

$-=$ exceeded holding time.

- exceeded groundwater protection standard. 
WELL FSB 79 (cont.)

Radiological Indicator Parameters

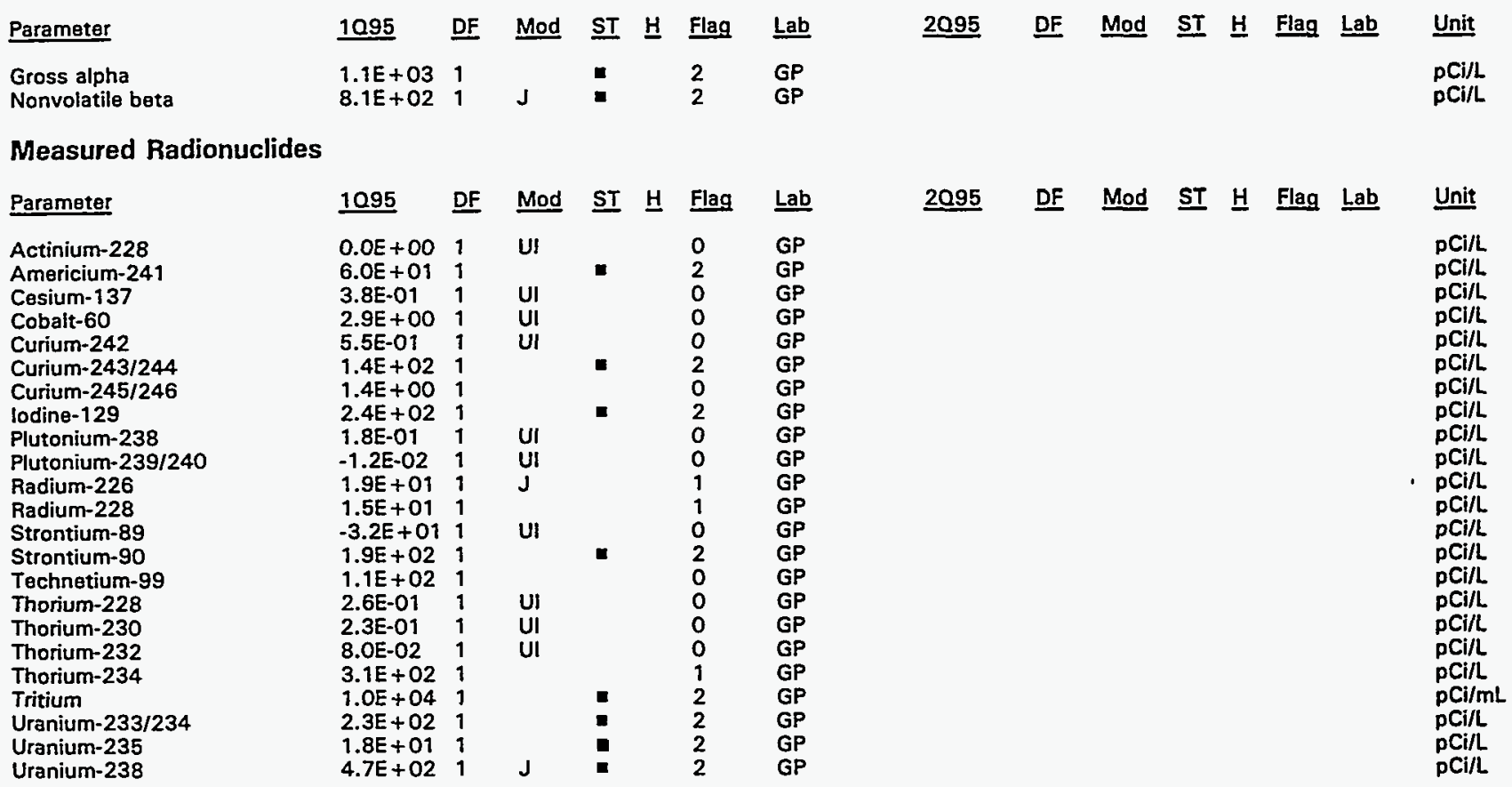

Calculated Radionuclides

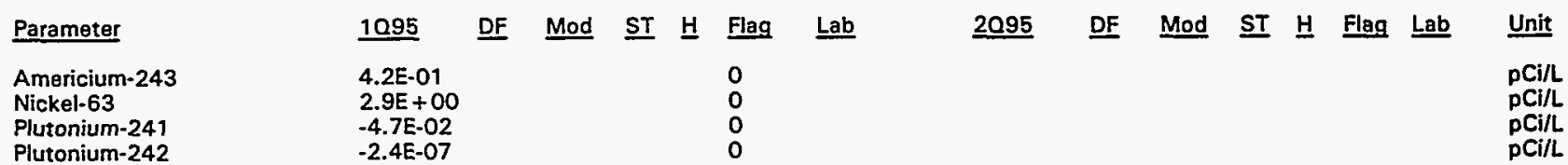

Note: See Appendix B for flagging criteria. Synchronous water levels are measured over a 3-5 day period. $-=$ exceeded holding time.

- = exceeded groundwater protection standard. 


\section{WELL FSB 79A}

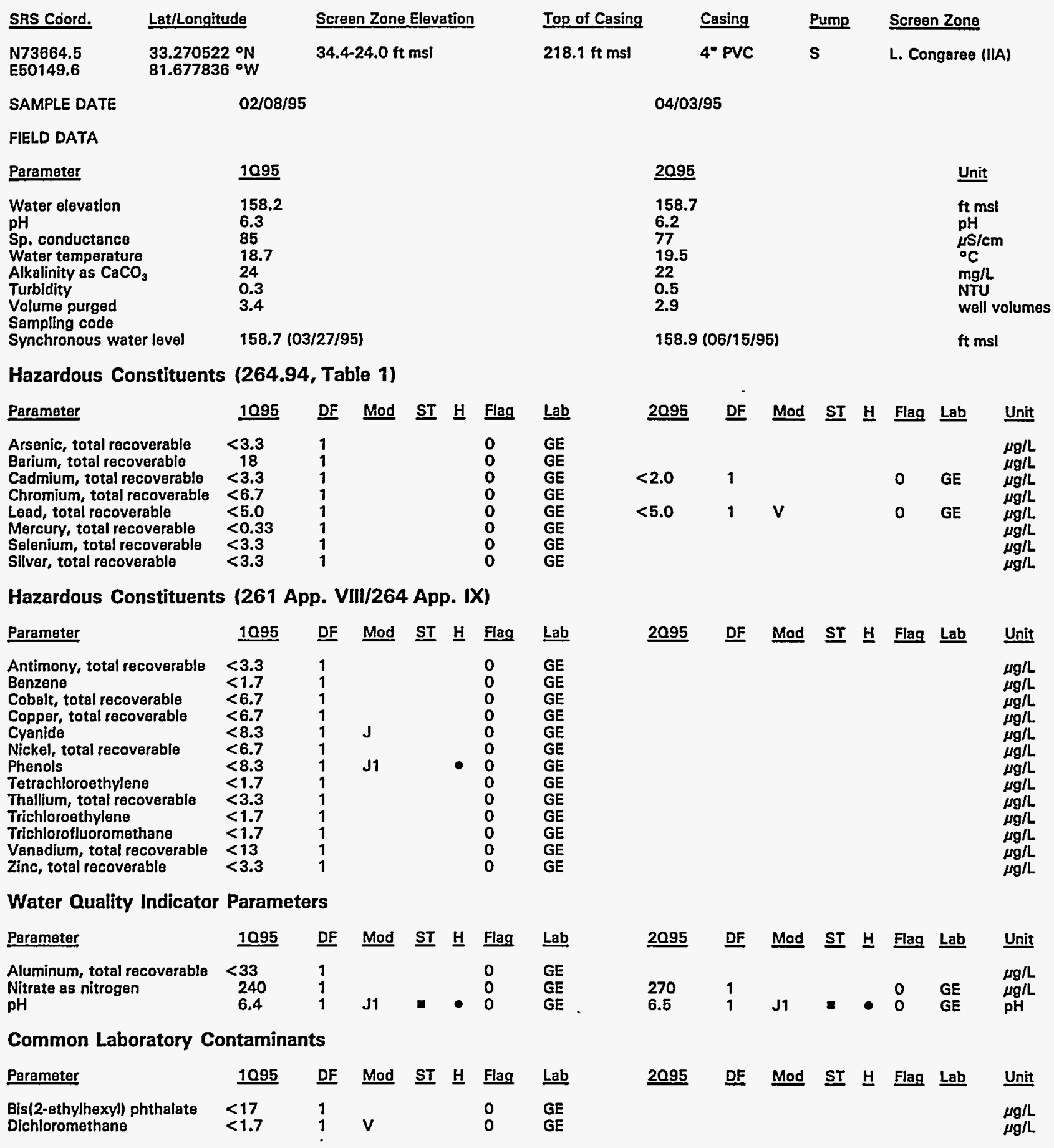

Note: See Appendix B for flagging criteria. Synchronous water levels are measured over a 3-5 day period.

- = exceeded holding time.

n = exceeded groundwater protection standard. 
WELL FSB 79A (cont.)

Radiological Indicator Parameters

\begin{tabular}{|c|c|c|c|c|c|c|c|c|c|c|c|c|c|c|}
\hline Parameter & 1095 & DF & Mod & ST & 브 & Flag & $\underline{\text { Lab }}$ & 2095 & $\underline{D F}$ & Mod & $\underline{\text { ST }} \underline{H}$ & Flag & Lab & Unit \\
\hline $\begin{array}{l}\text { Gross alpha } \\
\text { Nonvolatile beta }\end{array}$ & $\begin{array}{l}3.2 E-01 \\
5.4 E-01\end{array}$ & $\begin{array}{l}1 \\
1\end{array}$ & $\begin{array}{l}\text { UI } \\
\text { UI }\end{array}$ & & & $\begin{array}{l}0 \\
0\end{array}$ & $\begin{array}{l}\text { GP } \\
\text { GP }\end{array}$ & $\begin{array}{l}2.9 E-01 \\
2.5 E+\infty 0\end{array}$ & 1 & UJI & & $\begin{array}{l}0 \\
0\end{array}$ & $\begin{array}{l}\text { GP } \\
\text { GP }\end{array}$ & $\begin{array}{l}\mathrm{pCi} / \mathrm{L} \\
\mathrm{pCi} / \mathrm{L}\end{array}$ \\
\hline
\end{tabular}

Measured Radionuclides

\begin{tabular}{|c|c|c|c|c|c|c|c|c|c|c|c|c|c|c|c|}
\hline Parameter & 1095 & $\underline{\mathrm{DF}}$ & Mod & $\underline{\text { ST }}$ & $\underline{\mathbf{H}}$ & Fiag & $\underline{\mathrm{Lab}}$ & 2095 & $\underline{D F}$ & Mod & $\underline{\mathbf{S T}}$ & 트 & Flag & Lab & Unit \\
\hline $\begin{array}{l}\text { Actinium-228 } \\
\text { Amsricium-241 } \\
\text { Cesium-137 } \\
\text { Cobalt-60 } \\
\text { Curium-242 } \\
\text { Curium-243/244 } \\
\text { Curium-245/246 } \\
\text { lodine-129 } \\
\text { Plutonium-238 } \\
\text { Plutonium-239/240 } \\
\text { Radium-226 } \\
\text { Radium-228 } \\
\text { Strontium-89 } \\
\text { Strontium-90 } \\
\text { Technetium-99 } \\
\text { Thorium-228 } \\
\text { Thorium-230 } \\
\text { Thorium-232 } \\
\text { Thorium-234 } \\
\text { Tritium } \\
\text { Uranium-233/234 } \\
\text { Uranium-235 } \\
\text { Uranium-238 }\end{array}$ & $\begin{array}{l}4.2 \mathrm{E}+00 \\
-2.0 \mathrm{E}-02 \\
5.3 \mathrm{E}-01 \\
4.7 \mathrm{E}-01 \\
3.3 \mathrm{E}-02 \\
4.7 \mathrm{E}-02 \\
0.0 \mathrm{E}+00 \\
2.3 \mathrm{E}-01 \\
2.9 \mathrm{E}-01 \\
-1.3 \mathrm{E}-02 \\
1.6 \mathrm{E}+00 \\
2.4 \mathrm{E}-01 \\
-4.1 \mathrm{E}-01 \\
-4.4 \mathrm{E}-01 \\
1.1 \mathrm{E}+01 \\
-4.8 \mathrm{E}-02 \\
6.6 \mathrm{E}-01 \\
1.4 \mathrm{E}-01 \\
0.0 \mathrm{E}+00 \\
8.1 \mathrm{E}+00 \\
3.4 \mathrm{E}-02 \\
3.9 \mathrm{E}-02 \\
3.9 \mathrm{E}-02\end{array}$ & $\begin{array}{l}1 \\
1 \\
1 \\
1 \\
1 \\
1 \\
1 \\
1 \\
1 \\
1 \\
1 \\
1 \\
1 \\
1 \\
1 \\
1 \\
1 \\
1 \\
1 \\
1 \\
1 \\
1 \\
1\end{array}$ & 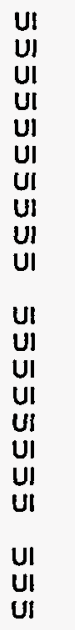 & & & $\begin{array}{l}0 \\
0 \\
0 \\
0 \\
0 \\
0 \\
0 \\
0 \\
0 \\
0 \\
0 \\
0 \\
0 \\
0 \\
0 \\
0 \\
0 \\
0 \\
0 \\
0 \\
0 \\
0 \\
0\end{array}$ & $\begin{array}{l}\text { GP } \\
\text { GP } \\
\text { GP } \\
\text { GP } \\
\text { GP } \\
\text { GP } \\
\text { GP } \\
\text { GP } \\
\text { GP } \\
\text { GP } \\
\text { GP } \\
\text { GP } \\
\text { GP } \\
\text { GP } \\
\text { GP } \\
\text { GP } \\
\text { GP } \\
\text { GP } \\
\text { GP } \\
\text { GP } \\
\text { GP } \\
\text { GP } \\
\text { GP }\end{array}$ & $1.1 E-02$ & 1 & Ui & & & 0 & GP & $\begin{array}{l}\text { pCi/L } \\
\text { pCi/L } \\
\text { pCi/L } \\
\text { pCi/L } \\
\text { pCi/L } \\
\text { pCi/L } \\
\text { pCi/L } \\
\text { pCi/L } \\
\text { pCi/L } \\
\text { pCi/L } \\
\text { pCi/L } \\
\text { pCi/L } \\
\text { pCi/L } \\
\text { pCi/L } \\
\text { pCi/L } \\
\text { pCi/L } \\
\text { pCi/L } \\
\text { pCi/L } \\
\text { pCi/L } \\
\text { pCi/m } \\
\text { pCi/L } \\
\text { pCi/L } \\
\text { pCi/L }\end{array}$ \\
\hline \multicolumn{16}{|c|}{ Calculated Radionuclides } \\
\hline Parameter & 1095 & $\underline{D F}$ & Mod & $\underline{\mathrm{ST}}$ & $\underline{H}$ & Flag & $\underline{L a b}$ & $\underline{2095}$ & $\underline{D F}$ & Mod & $\underline{\text { ST }}$ & 보 & Flag & $\underline{\text { Lab }}$ & Unit \\
\hline $\begin{array}{l}\text { Americium-243 } \\
\text { Nickel-63 } \\
\text { Plutonium-241 } \\
\text { Plutonium-242 }\end{array}$ & $\begin{array}{l}1.4 E-04 \\
4.7 E-01 \\
-5.2 E-02 \\
-2.6 E-07\end{array}$ & & & & & $\begin{array}{l}0 \\
0 \\
0 \\
0\end{array}$ & & & & & & & & & $\begin{array}{l}\mathrm{pCi} / \mathrm{L} \\
\mathrm{pCi} / \mathrm{L} \\
\mathrm{pCi} / \mathrm{L} \\
\mathrm{pCi} / \mathrm{L}\end{array}$ \\
\hline
\end{tabular}

Note: See Appendix B for flagging criteria. Synchronous water levels are measured over a 3-5 day period. - = exceeded holding time.

I = exceeded groundwater protection standard. 


\section{WELL FSB 79B}

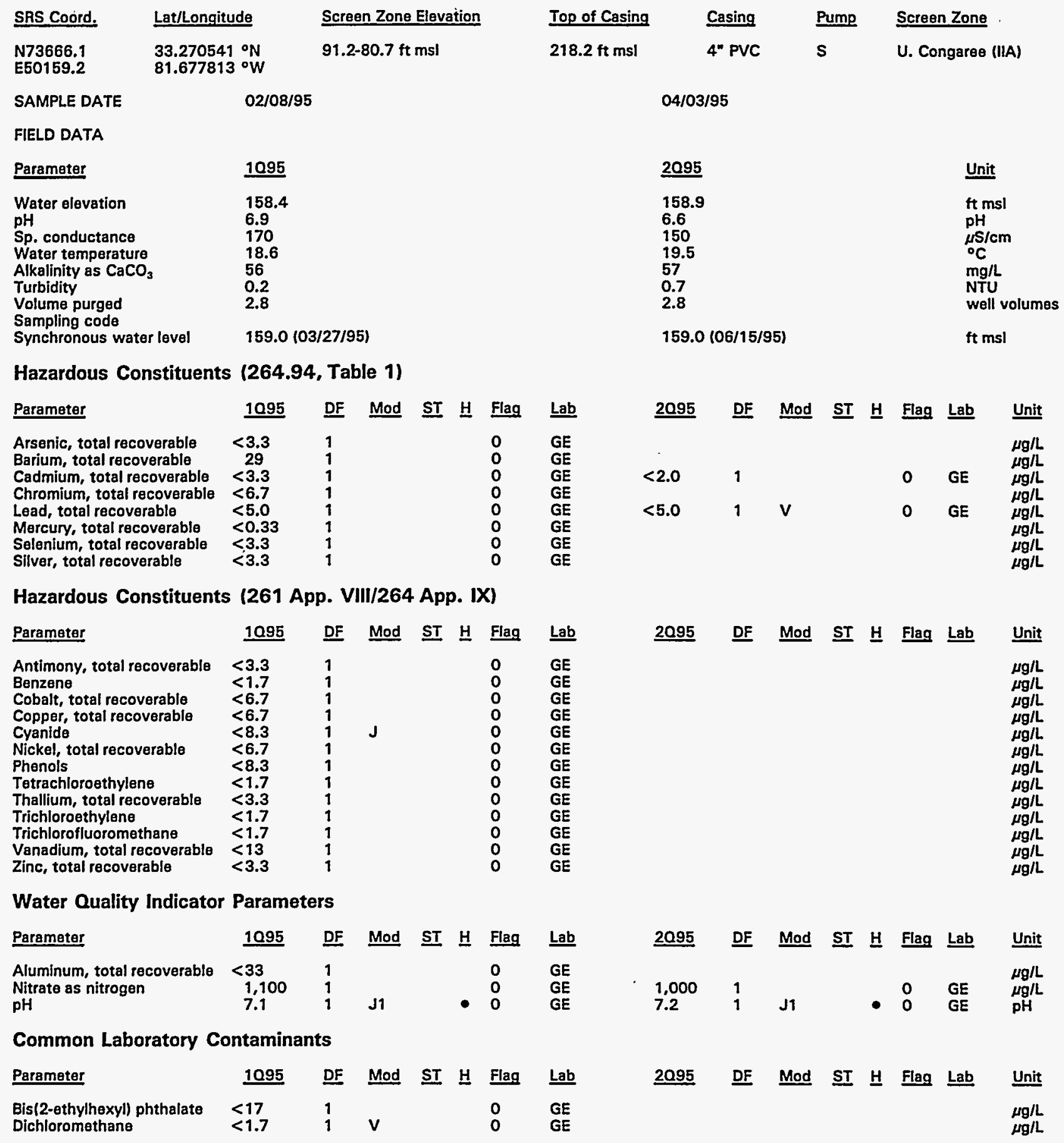

Note: See Appendix B for flagging criteria. Synchronous water levels are measured over a 3-5 day period.

- = exceeded holding time.

= exceeded groundwater protection standard. 
WELL FSB 79B (cont.)

Radiological Indicator Parameters

\begin{tabular}{|c|c|c|c|c|c|c|c|c|c|c|c|c|c|}
\hline Parameter & 1095 & DF & Mod & $\underline{\text { ST }} \underline{H}$ & Flag & Lab & $\underline{2095}$ & $\underline{\mathrm{DF}}$ & Mod & $\underline{\mathbf{S T}}$ & $\underline{\mathbf{H}}$ & Flag & Lab \\
\hline $\begin{array}{l}\text { Gross alpha } \\
\text { Nonvolatile beta }\end{array}$ & $\begin{array}{l}4.3 E-01 \\
9.9 E-01\end{array}$ & $\begin{array}{l}1 \\
1\end{array}$ & $\begin{array}{l}\text { UI } \\
\text { UI }\end{array}$ & & $\begin{array}{l}0 \\
0\end{array}$ & $\begin{array}{l}G P \\
G P\end{array}$ & $\begin{array}{l}6.7 E-01 \\
4.5 E+00\end{array}$ & $\begin{array}{l}1 \\
1\end{array}$ & UJI & & & $\begin{array}{l}0 \\
0\end{array}$ & $\begin{array}{l}\text { GP } \\
\text { GP }\end{array}$ \\
\hline
\end{tabular}

Measured Radionuclides

\begin{tabular}{|c|c|c|c|c|c|c|c|c|c|c|c|c|c|c|c|}
\hline Parameter & 1095 & DF & Mod & $\underline{\text { ST }}$ & 브 & Flag & $\underline{\text { Lab }}$ & $\underline{2095}$ & DF & Mod & $\underline{\text { ST }}$ & 브 & Flag & $\underline{\text { Lab }}$ & Unit \\
\hline $\begin{array}{l}\text { Actinium-228 } \\
\text { Ammericium-241 } \\
\text { Cesium-137 } \\
\text { Cobalt-60 } \\
\text { Curium-242 } \\
\text { Curium-243/244 } \\
\text { Curium-245/246 } \\
\text { lodine-129 } \\
\text { Plutonium-238 } \\
\text { Plutonium-239/240 } \\
\text { Radium-226 } \\
\text { Radium-228 } \\
\text { Strontium-89 } \\
\text { Strontium-90 } \\
\text { Technetium-99 } \\
\text { Thorium-228 } \\
\text { Thorium-230 } \\
\text { Thorium-232 } \\
\text { Thorium-234 } \\
\text { Tritium } \\
\text { Uranium-233/234 } \\
\text { Uranium-235 } \\
\text { Uranium-238 }\end{array}$ & $\begin{array}{l}4.0 \mathrm{E}+00 \\
8.1 \mathrm{E}-02 \\
1.7 \mathrm{E}-01 \\
3.9 \mathrm{E}-02 \\
1.5 \mathrm{E}-02 \\
2.2 \mathrm{E}-02 \\
0.0 \mathrm{E}+00 \\
8.0 \mathrm{E}-01 \\
6.6 \mathrm{E}-02 \\
2.6 \mathrm{E}-02 \\
1.8 \mathrm{E}+00 \\
5.7 \mathrm{E}-01 \\
-7.3 \mathrm{E}-01 \\
-3.7 \mathrm{E}-01 \\
8.1 \mathrm{E}+00 \\
1.0 \mathrm{E}-01 \\
3.9 \mathrm{E}-01 \\
-1.2 \mathrm{E}-02 \\
6.2 \mathrm{E}+01 \\
2.4 \mathrm{E}+01 \\
1.1 \mathrm{E}-01 \\
3.8 \mathrm{E}-02 \\
3.8 \mathrm{E}-02\end{array}$ & $\begin{array}{l}1 \\
1 \\
1 \\
1 \\
1 \\
1 \\
1 \\
1 \\
1 \\
1 \\
1 \\
1 \\
1 \\
1 \\
1 \\
1 \\
1 \\
1 \\
1 \\
1 \\
1 \\
1 \\
1\end{array}$ & 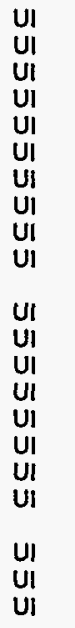 & . & & $\begin{array}{l}0 \\
0 \\
0 \\
0 \\
0 \\
0 \\
0 \\
0 \\
0 \\
0 \\
0 \\
0 \\
0 \\
0 \\
0 \\
0 \\
0 \\
0 \\
0 \\
2 \\
0 \\
0 \\
0\end{array}$ & $\begin{array}{l}\text { GP } \\
G P \\
G P \\
G P \\
G P \\
G P \\
G P \\
G P \\
G P \\
G P \\
G P \\
G P \\
G P \\
G P \\
G P \\
G P \\
G P \\
G P \\
G P \\
G P \\
G P \\
G P \\
G P\end{array}$ & $2.6 \mathrm{E}-02$ & 1 & UI & & & 0 & GP & 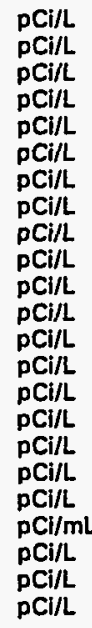 \\
\hline \multicolumn{16}{|c|}{ Calculated Radionuclides } \\
\hline Parameter & 1095 & $\underline{D F}$ & Mod & $\underline{\text { ST }}$ & $\underline{H}$ & Flag & $\underline{\text { Lab }}$ & $\underline{2095}$ & $\underline{\text { DF }}$ & Mod & ST & $\underline{H}$ & Flag & $\underline{\text { Lab }}$ & Unit \\
\hline $\begin{array}{l}\text { Americium-243 } \\
\text { Nickel-63 } \\
\text { Plutonium-241 } \\
\text { Plutonium-242 }\end{array}$ & $\begin{array}{l}6.6 \mathrm{E}-05 \\
3.9 \mathrm{E}-02 \\
1.0 \mathrm{E}-01 \\
5.2 \mathrm{E}-07\end{array}$ & & & & & $\begin{array}{l}0 \\
0 \\
0 \\
0\end{array}$ & & & & & & & & & $\begin{array}{l}\mathrm{pCi} / L \\
\mathrm{pCi} / \mathrm{L} \\
\mathrm{pCi} / \mathrm{L} \\
\mathrm{pCi} / \mathrm{L}\end{array}$ \\
\hline
\end{tabular}

Note: See Appendix B for flagging criteria. Synchronous water levels are measured over a 3-5 day period.

- = exceeded holding time.

- = exceeded groundwater protection standard. 
WELL FSB 79C

\begin{tabular}{|c|c|c|c|c|c|c|}
\hline SRS Coórd. & Lat/Longitude & Screen Zone Elevation & Top of Casing & Casing & Pump & Screen Zone \\
\hline $\begin{array}{l}\text { N73668.0 } \\
\text { E50171.3 }\end{array}$ & $\begin{array}{l}33.2705655^{\circ} \mathrm{N} \\
81.6777855^{\circ} \mathrm{W}\end{array}$ & $159.6-149.8 \mathrm{ft} \mathrm{msl}$ & $218.4 \mathrm{ft} \mathrm{msl}$ & 4" PVC & v & Barnwell (I) \\
\hline SAMPLE DATE & $02 / 0$ & & & $4 / 95$ & & \\
\hline
\end{tabular}

FIELD DATA

\begin{tabular}{|c|c|c|c|c|c|c|}
\hline Parameter & 1095 & & & & & \\
\hline $\begin{array}{l}\text { Water elevation } \\
\text { pH } \\
\text { Sp. conductance } \\
\text { Water temperature } \\
\text { Alkalinity as } \mathrm{CaCO}_{3} \\
\text { Turbidity } \\
\text { Volume purged } \\
\text { Sampling code } \\
\text { Synchronous water level }\end{array}$ & $\begin{array}{l}196.9 \\
3.4 \\
1,235 \\
18.6 \\
0 \\
0.2 \\
2.2 \\
197.3\end{array}$ & $127 / 9$ & & & & \\
\hline \multicolumn{7}{|c|}{ Hazardous Constituents (264.94, Table 1) } \\
\hline Parameter & 1095 & $\underline{D F}$ & Mod & $\underline{\text { ST }} \underline{H}$ & Flag & $\underline{\text { Lab }}$ \\
\hline $\begin{array}{l}\text { Arsenic, total recoverable } \\
\text { Barium, total recoverable } \\
\text { Cadmium, total recoverable } \\
\text { Chromium, total recoverable } \\
\text { Lead, total recoverable } \\
\text { Mercury, total recoverable } \\
\text { Selenium, total recoverable } \\
\text { Silver, total recoverable }\end{array}$ & $\begin{array}{l}<3.3 \\
530 \\
27 \\
<20 \\
<5.0 \\
0.73 \\
<3.3 \\
<10\end{array}$ & $\begin{array}{l}1 \\
3 \\
3 \\
3 \\
1 \\
1 \\
1 \\
3\end{array}$ & & $=$ & $\begin{array}{l}0 \\
0 \\
2 \\
0 \\
0 \\
0 \\
0 \\
0\end{array}$ & $\begin{array}{l}\mathrm{GE} \\
\mathrm{GE} \\
\mathrm{GE} \\
\mathrm{GE} \\
\mathrm{GE} \\
\mathrm{GE} \\
\mathrm{GE} \\
\mathrm{GE}\end{array}$ \\
\hline
\end{tabular}

\begin{tabular}{|c|c|}
\hline$\underline{2095}$ & Unit \\
\hline 196.8 & $\begin{array}{l}\mathrm{ft} \mathrm{msl} \\
\mathrm{pH} \\
\mu \mathrm{S} / \mathrm{cm} \\
{ }^{\circ} \mathrm{C} \\
\mathrm{mg} / \mathrm{L} \\
\text { NTU } \\
\text { well volumes }\end{array}$ \\
\hline $\begin{array}{l}W \\
196.9(06 / 15 / 95)\end{array}$ & $\mathrm{ft} \mathrm{msl}$ \\
\hline
\end{tabular}

Hazardous Constituents (261 App. VIII/264 App. IX)

\begin{tabular}{|c|c|c|c|c|c|c|c|c|c|c|c|c|c|c|c|}
\hline Parameter & 1095 & DF & Mod & $\underline{\mathbf{S T}}$ & 브 & Flag & $\underline{L a b}$ & 2095 & DF & Mod & $\underline{\text { ST }}$ & $\underline{H}$ & Flag & Lab & Unit \\
\hline $\begin{array}{l}\text { Antimony, total recoverable } \\
\text { Benzene } \\
\text { Cobalt, total recoverable } \\
\text { Copper, total recoverable } \\
\text { Cyanide } \\
\text { Nickel, total recoverable } \\
\text { Phenols } \\
\text { Tetrachloroethylene } \\
\text { Thallium, total recoverable } \\
\text { Trichloroethylene } \\
\text { Trichlorofluoromethane } \\
\text { Vanadium, total recoverable } \\
\text { Zinc, total recoverable }\end{array}$ & $\begin{array}{l}<3.3 \\
<1.7 \\
140 \\
42 \\
<8.3 \\
40 \\
<8.3 \\
<1.7 \\
<3.3 \\
<1.7 \\
<1.7 \\
<40 \\
110\end{array}$ & $\begin{array}{l}1 \\
1 \\
3 \\
3 \\
1 \\
1 \\
3 \\
1 \\
1 \\
1 \\
1 \\
1 \\
3 \\
3\end{array}$ & JV & - & $\bullet$ & $\begin{array}{l}0 \\
0 \\
2 \\
0 \\
0 \\
0 \\
0 \\
0 \\
0 \\
0 \\
0 \\
0 \\
0\end{array}$ & $\begin{array}{l}\mathrm{GE} \\
\mathrm{GE} \\
\mathrm{GE} \\
\mathrm{GE} \\
\mathrm{GE} \\
\mathrm{GE} \\
\mathrm{GE} \\
\mathrm{GE} \\
\mathrm{GE} \\
\mathrm{GE} \\
\mathrm{GE} \\
\mathrm{GE} \\
\mathrm{GE}\end{array}$ & & & & & & & & $\begin{array}{l}\mu \mathrm{g} / \mathrm{L} \\
\mu \mathrm{g} / \mathrm{L} \\
\mu \mathrm{g} / \mathrm{L} \\
\mu \mathrm{g} / \mathrm{L} \\
\mu \mathrm{g} / \mathrm{L} \\
\mu \mathrm{g} / \mathrm{L} \\
\mu \mathrm{g} / \mathrm{L} \\
\mu \mathrm{g} / \mathrm{L} \\
\mu \mathrm{g} / \mathrm{L} \\
\mu \mathrm{g} / \mathrm{L} \\
\mu \mathrm{g} / \mathrm{L} \\
\mu \mathrm{g} / \mathrm{L} \\
\mu \mathrm{g} / \mathrm{L}\end{array}$ \\
\hline \multicolumn{16}{|c|}{ Water Quality Indicator Parameters } \\
\hline Parameter & $\underline{1095}$ & $\underline{\text { DF }}$ & Mod & $\underline{\mathbf{S T}}$ & 브 & Flag & $\underline{\text { Lab }}$ & $\underline{2095}$ & $\underline{D F}$ & Mod & $\underline{\mathbf{S T}}$ & 보 & Flag & $\underline{L a b}$ & Unit \\
\hline $\begin{array}{l}\text { Aluminum, total recoverable } \\
\text { Nitrate as nitrogen } \\
\mathrm{pH}\end{array}$ & $\begin{array}{l}39,000 \\
130,000 \\
3.7\end{array}$ & $\begin{array}{l}3 \\
160 \\
1\end{array}$ & J1 & $=$ & • & $\begin{array}{l}2 \\
2 \\
1\end{array}$ & $\begin{array}{l}\mathrm{GE} \\
\mathrm{GE} \\
\mathrm{GE}\end{array}$ & & & & & & & & $\begin{array}{l}\mu \mathrm{g} / \mathrm{L} \\
\mu \mathrm{g} / \mathrm{L} \\
\mathrm{pH}\end{array}$ \\
\hline \multicolumn{16}{|c|}{ Common Laboratory Contaminants } \\
\hline Parameter & $\underline{1095}$ & $\underline{\text { DF }}$ & Mod & $\underline{\mathbf{S T}}$ & $\underline{H}$ & Flag & $\underline{\text { Lab }}$ & $\underline{2095}$ & $\underline{\mathrm{DF}}$ & Mod & $\underline{\mathbf{S T}}$ & $\underline{H}$ & Flag & $\underline{\text { Lab }}$ & $\underline{\text { Unit }}$ \\
\hline $\begin{array}{l}\text { Bis(2-ethylhexyl) phthalate } \\
\text { Dichloromethane }\end{array}$ & $\begin{array}{l}<17 \\
<1.7\end{array}$ & $\begin{array}{l}1 \\
1\end{array}$ & & & & $\begin{array}{l}0 \\
0\end{array}$ & $\begin{array}{l}\text { GE } \\
\text { GE }\end{array}$ & & & & & & & & $\begin{array}{l}\mu \mathrm{g} / \mathrm{L} \\
\mu \mathrm{g} / \mathrm{L}\end{array}$ \\
\hline
\end{tabular}

Note: See Appendix B for flagging criteria. Synchronous water levels are measured over a 3-5 day period.

- = exceeded holding time.

m = exceeded groundwater protection standard. 
WELL FSB $79 \mathrm{C}$ (cont.)

Radiological Indicator Parameters

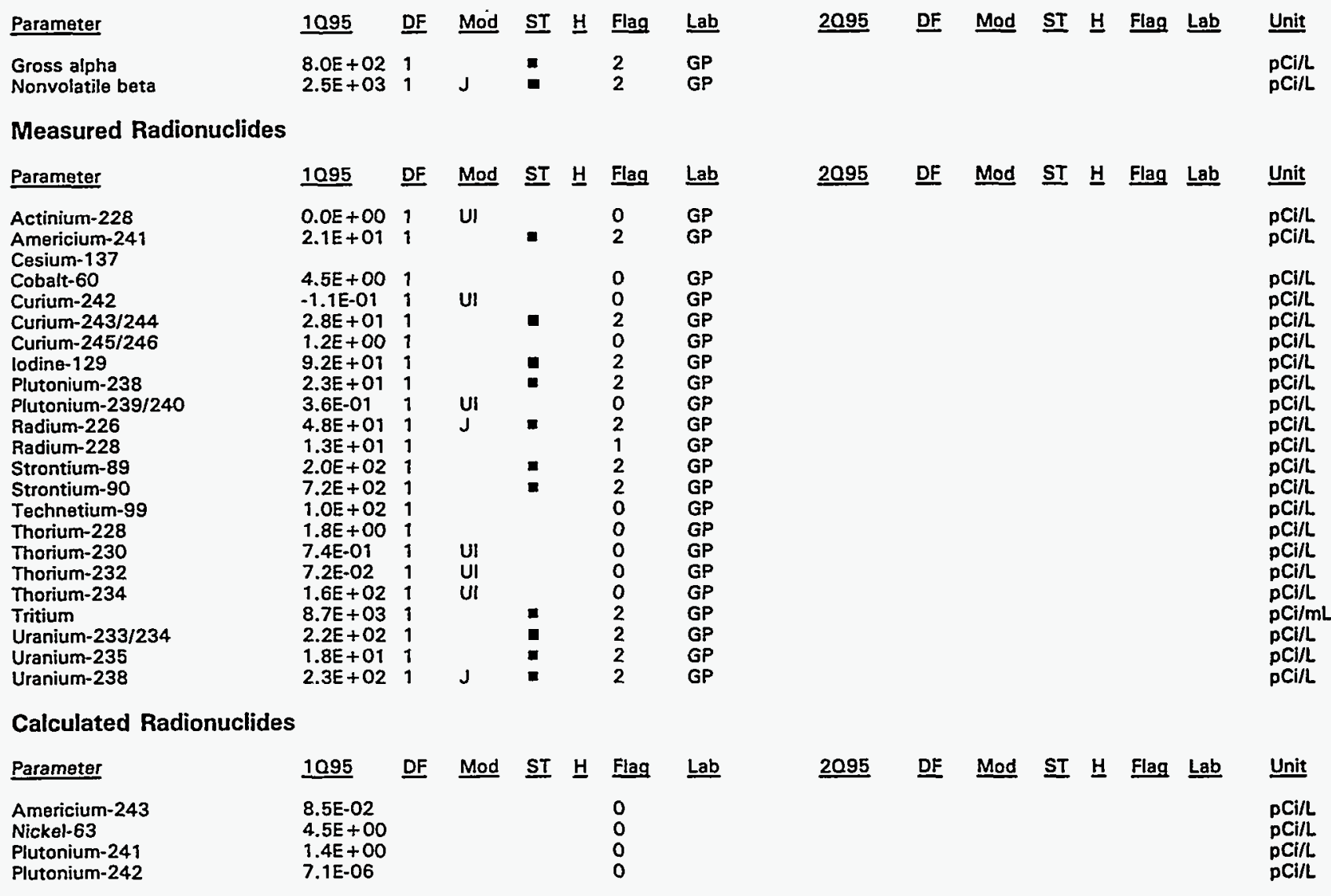

Note: See Appendix B for flagging criteria. Synchronous water levels are measured over a 3-5 day period.

- = exceeded holding time.

- = exceeded groundwater protection standard. 


\section{WELL FSB 87A}

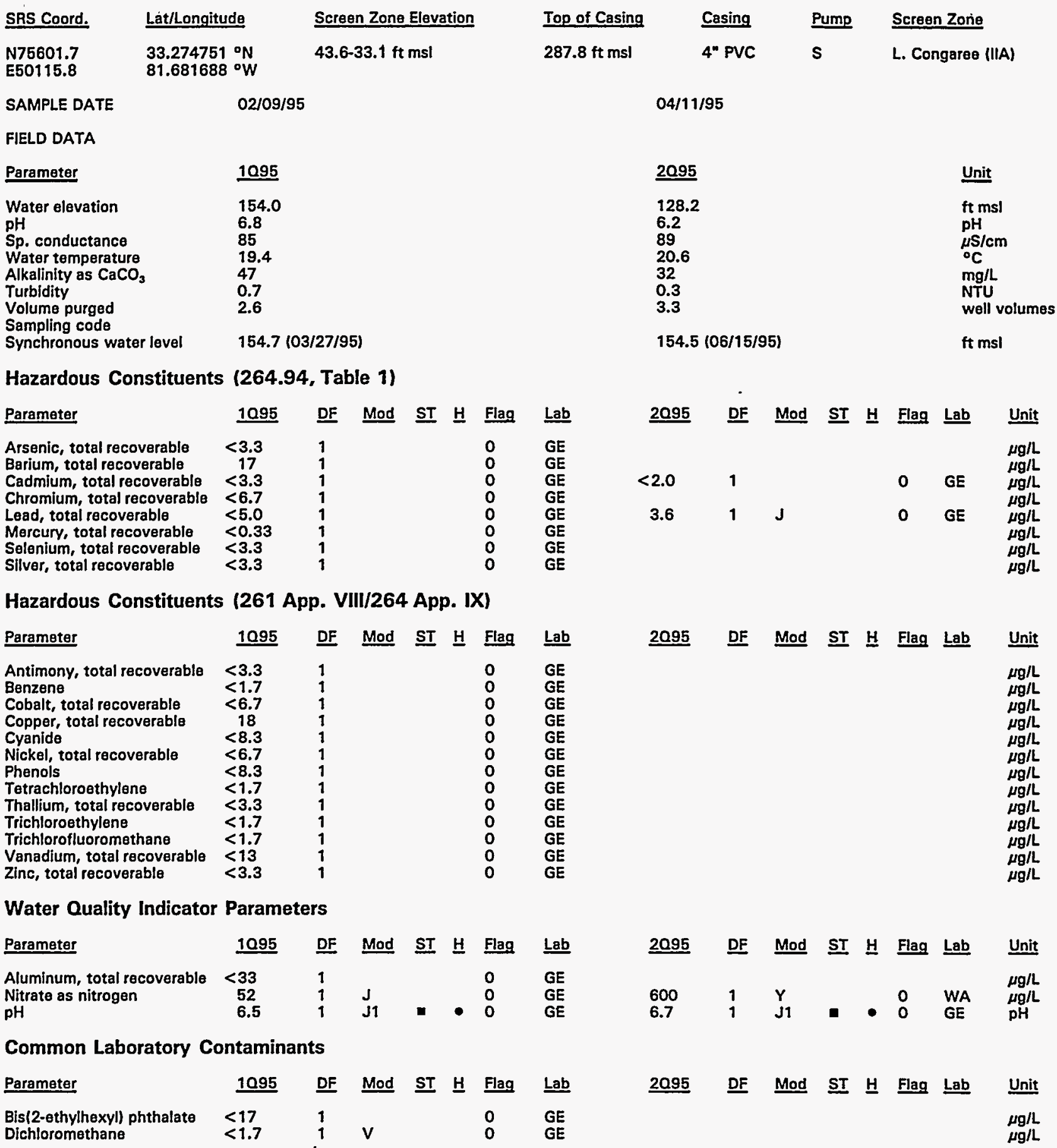

Note: See Appendix B for flagging criteria. Synchronous water levels are measured over a 3-5 day period. - = exceeded holding time.

- = exceeded groundwater protection standard. 
WELL FSB 87A (cont.)

Radiological Indicator Parameters

\begin{tabular}{|c|c|c|c|c|c|c|c|c|c|c|c|c|c|c|c|}
\hline Paramater & 1095 & $\underline{D F}$ & Mod & $\underline{\text { ST }}$ & $\underline{H}$ & Flag & $\underline{L a b}$ & $\underline{2095}$ & $\underline{\mathrm{DF}}$ & Mod & $\underline{\mathbf{S T}}$ & $\underline{\mathbf{H}}$ & Flag & $\underline{L a b}$ & Unit \\
\hline $\begin{array}{l}\text { Gross alpha } \\
\text { Nonvolatile beta }\end{array}$ & $\begin{array}{l}3.8 E-01 \\
1.3 E+00\end{array}$ & $\begin{array}{l}1 \\
1\end{array}$ & UI & & & $\begin{array}{l}0 \\
0\end{array}$ & $\begin{array}{l}\text { GP } \\
\text { GP }\end{array}$ & $\begin{array}{l}1.5 E+00 \\
2.5 E+\infty 0\end{array}$ & $\begin{array}{l}1 \\
1\end{array}$ & J1 & & & $\begin{array}{l}0 \\
0\end{array}$ & $\begin{array}{l}T M \\
T M\end{array}$ & $\begin{array}{l}\mathrm{pCi} / \mathrm{L} \\
\mathrm{pCI} / \mathrm{L}\end{array}$ \\
\hline \multicolumn{16}{|c|}{ Measured Radionuclides } \\
\hline Parameter & 1095 & DF & Mod & $\underline{\mathbf{S T}}$ & $\underline{\underline{ }}$ & Flag & $\underline{\text { Lab }}$ & $\underline{2095}$ & $\underline{\mathrm{DF}}$ & Mod & $\underline{\mathbf{S T}}$ & $\underline{H}$ & Flag & $\underline{\text { Lab }}$ & Unit \\
\hline $\begin{array}{l}\text { Actinium-228 } \\
\text { Americium-241 } \\
\text { Cesium-137 } \\
\text { Cobalt-60 } \\
\text { Curium-242 } \\
\text { Curium-243/244 } \\
\text { Curium-245/246 } \\
\text { lodine-129 } \\
\text { Plutonium-238 } \\
\text { Plutonium-239/240 } \\
\text { Radium-226 } \\
\text { Radium-228 } \\
\text { Strontium-89 } \\
\text { Strontium-90 } \\
\text { Technetium-99 } \\
\text { Thorium-228 } \\
\text { Thorium-230 } \\
\text { Thorium-232 } \\
\text { Thorium-234 } \\
\text { Tritium } \\
\text { Uranium-233/234 } \\
\text { Uranium-235 } \\
\text { Uranium-238 }\end{array}$ & $\begin{array}{l}1.4 E-01 \\
8.1 E-03 \\
-5.8 E-01 \\
-7.2 E-01 \\
-1.6 E-02 \\
2.6 E-02 \\
3.7 E-02 \\
-6.1 E-01 \\
2.3 E-02 \\
-5.9 E-03 \\
5.1 E-01 \\
-1.8 E+00 \\
-1.1 E+00 \\
-6.4 E-01 \\
1.6 E+00 \\
-7.5 E-02 \\
8.6 E-01 \\
8.7 E-02 \\
5.3 E+01 \\
1.6 E+00 \\
0.0 E+00 \\
0.0 E+00 \\
-4.6 E-03\end{array}$ & $\begin{array}{l}1 \\
1 \\
1 \\
1 \\
1 \\
1 \\
1 \\
1 \\
1 \\
1 \\
1 \\
1 \\
1 \\
1 \\
1 \\
1 \\
1 \\
1 \\
1 \\
1 \\
1 \\
1 \\
1\end{array}$ & $\begin{array}{l}\text { UI } \\
\text { UI } \\
\text { UI } \\
\text { UI } \\
\text { UI } \\
\text { UI } \\
\text { UI } \\
\text { UI } \\
\text { UI } \\
\text { UI } \\
\text { UI } \\
\text { UI } \\
\text { UI } \\
\text { UI } \\
\text { UI } \\
\text { UI } \\
\text { UI } \\
\text { UI } \\
\text { UI } \\
\text { UI } \\
\text { UI } \\
\text { UI }\end{array}$ & & & $\begin{array}{l}0 \\
0 \\
0 \\
0 \\
0 \\
0 \\
0 \\
0 \\
0 \\
0 \\
0 \\
0 \\
0 \\
0 \\
0 \\
0 \\
0 \\
0 \\
0 \\
0 \\
0 \\
0 \\
0\end{array}$ & $\begin{array}{l}\text { GP } \\
\text { GP } \\
\text { GP } \\
\text { GP } \\
\text { GP } \\
\text { GP } \\
\text { GP } \\
\text { GP } \\
\text { GP } \\
\text { GP } \\
\text { GP } \\
\text { GP } \\
\text { GP } \\
\text { GP } \\
\text { GP } \\
\text { GP } \\
\text { GP } \\
\text { GP } \\
\text { GP } \\
\text { GP } \\
\text { GP } \\
\text { GP } \\
\text { GP }\end{array}$ & $5.3 E+00$ & 1 & & & & 0 & $T M$ & 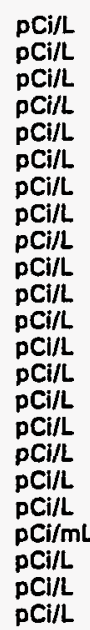 \\
\hline
\end{tabular}

\section{Calculated Radionuclides}

Parameter

Americium-243

Nickel-63

Plutonium-241

Plutonium-242
1095

7.8E-05

$-7.2 \mathrm{E}-01$

$-2.4 \mathrm{E}-02$

$-1.2 E-07$

Unit

pCi/l

pCI/L

$\mathrm{pCi} / \mathrm{L}$

pCi/L

Note: See Appendix B for flagging criteria. Synchronous water levels are measured over a 3-5 day period.

- = exceeded holding time.

- = exceeded groundwater protection standard. 
WELL FSB 87B

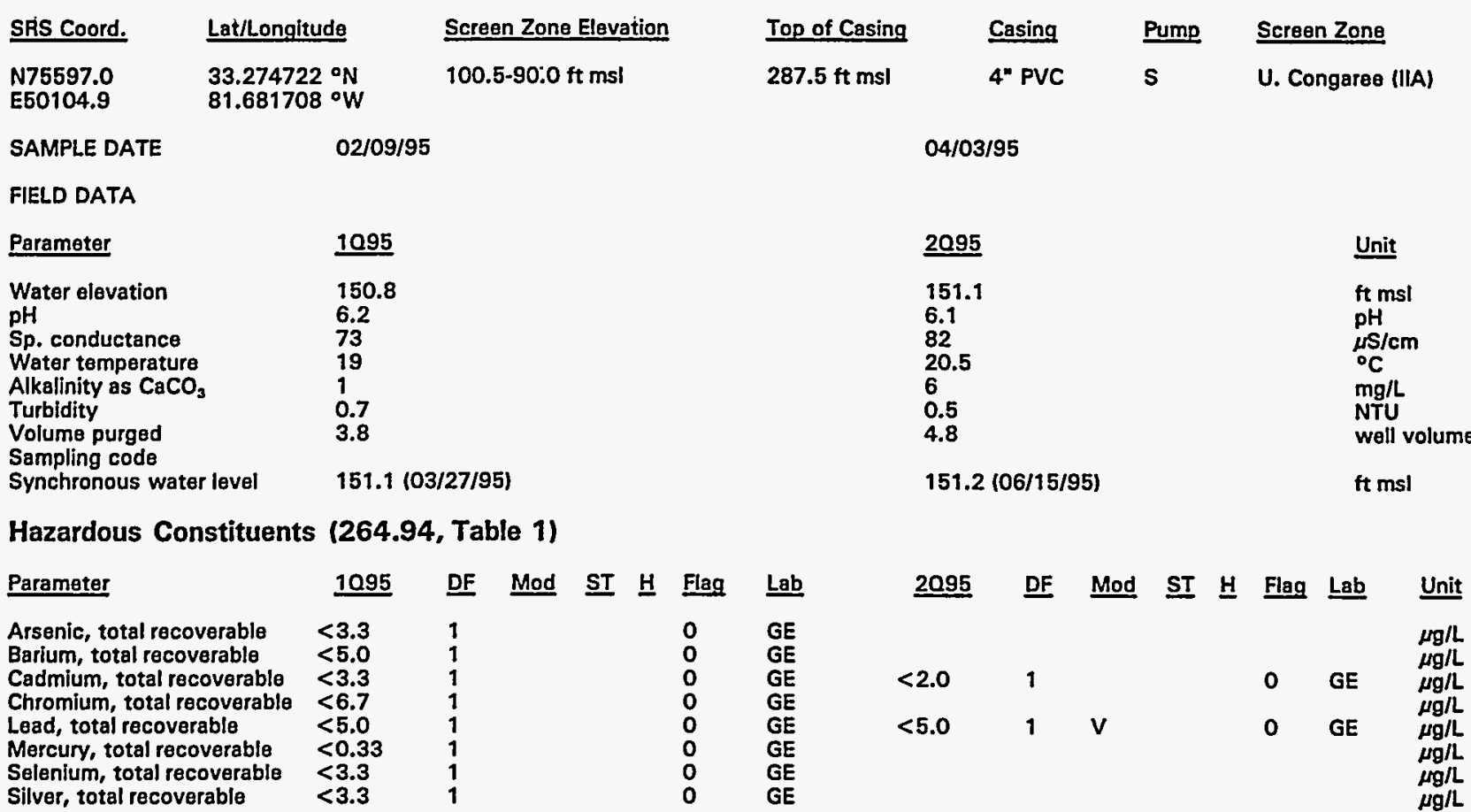

Hazardous Constituents (261 App. VIll/264 App. IX)

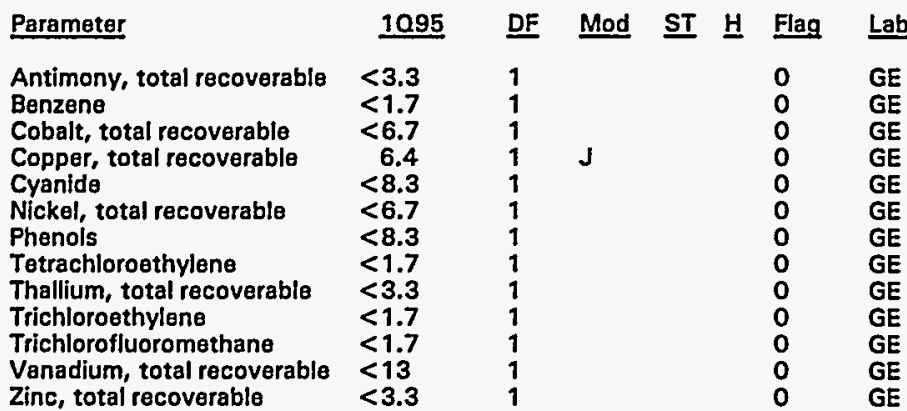

$\underline{2095}$ DF Mod ST $\underline{H} \underline{\text { Flag Lab }} \underline{\text { Unit }}$

Zinc, total recoverable $<3$

Water Quality Indicator Parameters

\begin{tabular}{|c|c|c|c|c|c|c|c|c|c|c|c|c|c|c|c|}
\hline Parameter & 1095 & $\underline{D F}$ & Mod & ST & H & Flag & $\underline{\text { Lab }}$ & $\underline{2095}$ & $\underline{D F}$ & Mod & $\underline{\mathbf{S T}}$ & 브 & Flag & $\underline{L a b}$ & Unit \\
\hline $\begin{array}{l}\text { Aluminum, total recoverable } \\
\text { Nitrate as nitrogen } \\
\text { pH }\end{array}$ & $\begin{array}{l}<33 \\
5,700 \\
6.2\end{array}$ & $\begin{array}{l}1 \\
4 \\
1\end{array}$ & J1 & a & $\bullet$ & $\begin{array}{l}0 \\
1 \\
0\end{array}$ & $\begin{array}{l}\mathrm{GE} \\
\mathrm{GE} \\
\mathrm{GE}\end{array}$ & $\begin{array}{l}5,400 \\
6.0\end{array}$ & $\begin{array}{l}4 \\
1\end{array}$ & J1 & - & • & $\begin{array}{l}1 \\
0\end{array}$ & $\begin{array}{l}\text { GE } \\
\text { GE }\end{array}$ & $\underset{\mathrm{pH}}{\mu \mathrm{g} / \mathrm{L}}$ \\
\hline \multicolumn{16}{|c|}{ Common Laboratory Contaminants } \\
\hline Parameter & $\underline{1095}$ & DF & Mod & $\underline{\text { ST }}$ & $\underline{H}$ & Flag & $\underline{\text { Lab }}$ & $\underline{2095}$ & $\underline{D F}$ & Mod & $\underline{\mathbf{S T}}$ & $\underline{H}$ & Flag & Lab & $\underline{\text { Unit }}$ \\
\hline $\begin{array}{l}\text { Bis(2-ethylhexyl) phthalate } \\
\text { Dichloromethane }\end{array}$ & $\begin{array}{l}<17 \\
<1.7\end{array}$ & 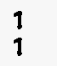 & $v$ & & & $\begin{array}{l}0 \\
0\end{array}$ & $\begin{array}{l}\text { GE } \\
\text { GE }\end{array}$ & & & & & & & & \\
\hline
\end{tabular}

Note: See Appendix B for flagging criteria. Synchronous water levels are measured over a 3-5 day period.

- = exceeded holding time.

= exceeded groundwater protection standard. 
WELL FSB 87B (cont.)

Radiological Indicator Parameters

\begin{tabular}{|c|c|c|c|c|c|c|c|c|c|c|c|c|c|c|c|}
\hline Parameter & 1095 & DF & Mod & $\underline{\text { ST }}$ & $\underline{\mathrm{H}}$ & Flag & $\underline{\text { Lab }}$ & 2095 & DF & Mod & $\underline{\text { ST }}$ & 브 & Flag & $\underline{\text { Lab }}$ & Unit \\
\hline $\begin{array}{l}\text { Gross alpha } \\
\text { Nonvolatile beta }\end{array}$ & $\begin{array}{l}9.4 E-01 \\
2.6 E+00\end{array}$ & $\begin{array}{l}1 \\
1\end{array}$ & UI & & & $\begin{array}{l}0 \\
0\end{array}$ & $\begin{array}{l}\text { GP } \\
\text { GP }\end{array}$ & $\begin{array}{l}1.1 E+\infty 0 \\
3.7 E+00\end{array}$ & $\begin{array}{l}1 \\
1\end{array}$ & $J$ & & & $\begin{array}{l}0 \\
0\end{array}$ & $\begin{array}{l}\text { GP } \\
\text { GP }\end{array}$ & $\begin{array}{l}\mathrm{pCi} / \mathrm{L} \\
\mathrm{pCi} / \mathrm{L}\end{array}$ \\
\hline \multicolumn{16}{|c|}{ Measured Radionuclides } \\
\hline Parameter & 1095 & $\underline{D F}$ & Mod & $\underline{\mathbf{S T}}$ & $\underline{H}$ & Flag & $\underline{\text { Lab }}$ & 2095 & $\underline{\text { DF }}$ & Mod & $\underline{\text { ST }}$ & $\underline{H}$ & Flag & $\underline{\text { Lab }}$ & Unit \\
\hline $\begin{array}{l}\text { Actinium-228 } \\
\text { Americium-241 } \\
\text { Cesium-137 } \\
\text { Cobalt-60 } \\
\text { Curium-242 } \\
\text { Curium-243/244 } \\
\text { Curium-245/246 } \\
\text { lodine-129 } \\
\text { Plutonium-238 } \\
\text { Plutonium-239/240 } \\
\text { Radium-226 } \\
\text { Radium-228 } \\
\text { Strontium-89 } \\
\text { Strontium-90 } \\
\text { Technetium-99 } \\
\text { Thorium-228 } \\
\text { Thorium-230 } \\
\text { Thorium-232 } \\
\text { Thorium-234 } \\
\text { Tritium } \\
\text { Uranium-233/234 } \\
\text { Uranium-235 } \\
\text { Uranium-238 }\end{array}$ & $\begin{array}{l}3.4 E+00 \\
2.0 E-02 \\
8.4 E-01 \\
6.2 E-01 \\
-2.4 E-02 \\
-4.1 E-02 \\
3.1 E-02 \\
6.4 E-01 \\
-3.8 E-02 \\
2.3 E-01 \\
6.0 E-01 \\
-1.4 E+00 \\
-3.5 E-01 \\
-2.4 E-01 \\
1.4 E+01 \\
3.6 E-03 \\
3.5 E-01 \\
7.7 E-02 \\
4.4 E+00 \\
8.8 E+01 \\
0.0 E+00 \\
-5.8 E-03 \\
4.6 E-02\end{array}$ & $\begin{array}{l}1 \\
1 \\
1 \\
1 \\
1 \\
1 \\
1 \\
1 \\
1 \\
1 \\
1 \\
1 \\
1 \\
1 \\
1 \\
1 \\
1 \\
1 \\
1 \\
1 \\
1 \\
1 \\
1\end{array}$ & $\begin{array}{l}U 1 \\
\text { UI } \\
\text { UI } \\
\text { UI } \\
U I \\
U I \\
U I \\
\text { UI } \\
\text { UI } \\
\text { UI } \\
\text { UI } \\
\text { UI } \\
\text { UI } \\
\text { UI } \\
\text { UI } \\
\text { UI } \\
\text { UI } \\
\text { UI } \\
\text { UI } \\
\text { UI } \\
\text { UI } \\
\text { UI }\end{array}$ & $=$ & & $\begin{array}{l}0 \\
0 \\
0 \\
0 \\
0 \\
0 \\
0 \\
0 \\
0 \\
0 \\
0 \\
0 \\
0 \\
0 \\
0 \\
0 \\
0 \\
0 \\
0 \\
2 \\
0 \\
0 \\
0\end{array}$ & $\begin{array}{l}\text { GP } \\
\text { GP } \\
\text { GP } \\
\text { GP } \\
\text { GP } \\
\text { GP } \\
\text { GP } \\
\text { GP } \\
\text { GP } \\
\text { GP } \\
\text { GP } \\
\text { GP } \\
\text { GP } \\
\text { GP } \\
\text { GP } \\
\text { GP } \\
\text { GP } \\
\text { GP } \\
\text { GP } \\
\text { GP } \\
\text { GP } \\
\text { GP } \\
\text { GP }\end{array}$ & $8.6 \mathrm{E}-02$ & 1 & Ul & & & 0 & GP & $\begin{array}{l}\text { pCi/L } \\
\text { pCi/L } \\
\text { pCi/L } \\
\text { pCi/L } \\
\text { pCi/L } \\
\text { pCi/L } \\
\text { pCi/L } \\
\text { pCi/L } \\
\text { pCi/L } \\
\text { pCi/L } \\
\text { pCi/L } \\
\text { pCi/L } \\
\text { pCi/L } \\
\text { pCi/L } \\
\text { pCi/L } \\
\text { pCi/L } \\
\text { pCil/L } \\
\text { pCi/L } \\
\text { pCi/L } \\
\text { pCi/m } \\
\text { pCi/L } \\
\text { pCi/L } \\
\text { pCi/L }\end{array}$ \\
\hline \multicolumn{16}{|c|}{ Calculated Radionuclides } \\
\hline Parameter & 1095 & $\underline{D F}$ & Mod & $\underline{\mathbf{S T}}$ & $\underline{H}$ & Flag & $\underline{\text { Lab }}$ & $\underline{2095}$ & $\underline{\text { DF }}$ & Mod & $\underline{\text { ST }}$ & 보 & Flag & $\underline{\text { Lab }}$ & Unit \\
\hline $\begin{array}{l}\text { Americium-243 } \\
\text { Nickel-63 } \\
\text { Plutonium-241 } \\
\text { Plutonium-242 }\end{array}$ & $\begin{array}{l}-1.2 E-04 \\
6.2 E-01 \\
9.2 E-01 \\
4.6 E-06\end{array}$ & & & & & $\begin{array}{l}0 \\
0 \\
0 \\
0\end{array}$ & & & & & & & & & $\begin{array}{l}\mathrm{pCi} / \mathrm{L} \\
\mathrm{pCi} / \mathrm{L} \\
\mathrm{pCi} / \mathrm{L} \\
\mathrm{pCi} / \mathrm{L}\end{array}$ \\
\hline
\end{tabular}

Note: See Appendix B for flagging criteria. Synchronous water levels are measured over a 3-5 day period. - = exceeded holding time.

- exceeded groundwater protection standard. 
WELL FSB 87C

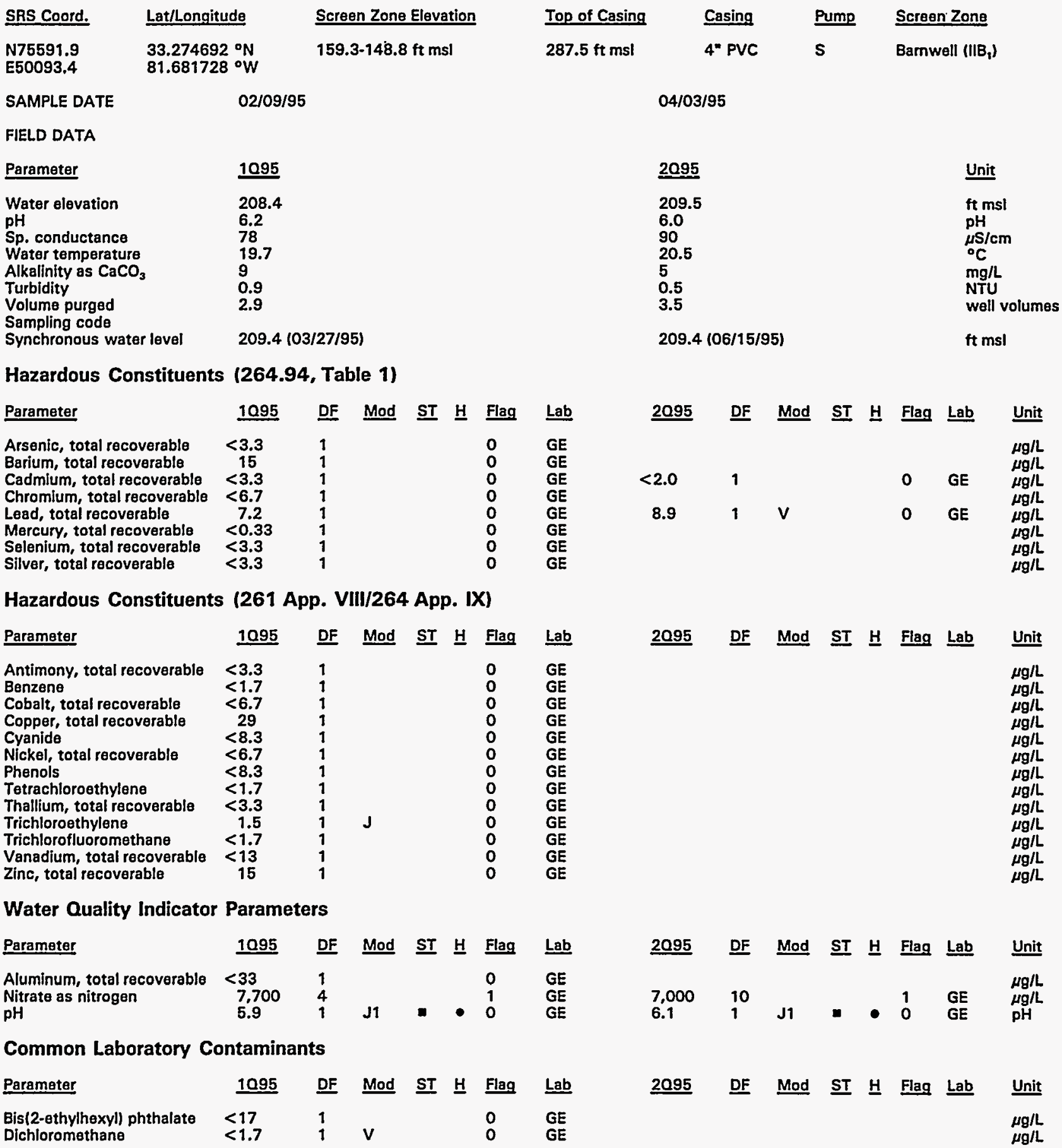

Note: See Appendix B for flagging criteria. Synchronous water levels are measured over a 3-5 day period.

- = exceeded holding time.

- = exceeded groundwater protection standard. 
WELL FSB $87 C$ (cont.)

Radiological Indicator Parameters

\begin{tabular}{|c|c|c|c|c|c|c|c|c|c|c|c|c|c|c|c|}
\hline Parameter & 1095 & DF & Mód & ST & H & Fiag & Lab & 2095 & $\underline{\mathrm{DF}}$ & Mod & $\underline{\mathrm{ST}}$ & 브 & Flag & Lab & Unit \\
\hline $\begin{array}{l}\text { Gross alpha } \\
\text { Nonvolatile beta }\end{array}$ & $\begin{array}{l}9.6 E-01 \\
3.1 E+00\end{array}$ & $\begin{array}{l}1 \\
t\end{array}$ & & & & $\begin{array}{l}0 \\
0\end{array}$ & $\begin{array}{l}\text { GP } \\
\text { GP }\end{array}$ & $\begin{array}{l}8.1 E-01 \\
2.9 E+00\end{array}$ & 1 & UI & & & $\begin{array}{l}0 \\
0\end{array}$ & $\begin{array}{l}\text { GP } \\
\text { GP }\end{array}$ & $\begin{array}{l}\mathrm{pCi} / \mathrm{L} \\
\mathrm{pCi} / \mathrm{L}\end{array}$ \\
\hline
\end{tabular}

Measured Radionuclides

$\begin{array}{llllllll}\text { Parameter } & 1095 & \text { DF } & \text { Mod } & \text { ST } & \text { Flag } & \text { Lab } \\ \text { Actinium-228 } & -3.8 E-02 & 1 & \text { UI } & & & \\ \text { Americium-241 } & -2.4 E-02 & 1 & \text { UI } & & 0 & \text { GP } \\ \text { Cesium-137 } & -1.4 E+00 & 1 & \text { UI } & & 0 & \text { GP } \\ \text { Cobalt-60 } & 1.3 E+00 & 1 & \text { UI } & & 0 & \text { GP } \\ \text { Curium-242 } & -2.1 E-02 & 1 & \text { UI } & & 0 & \text { GP } \\ \text { Curium-243/244 } & -2.6 E-02 & 1 & \text { UI } & & 0 & \text { GP } \\ \text { Curium-245/246 } & 0.0 E+00 & 1 & \text { UI } & & 0 & \text { GP } \\ \text { lodine-129 } & 3.3 E+00 & 1 & & & 2 & \text { GP } \\ \text { Plutonium-238 } & 1.2 E-01 & 1 & \text { UI } & & 0 & \text { GP } \\ \text { Plutonium-239/240 } & -6.7 E-03 & 1 & \text { UI } & & 0 & \text { GP } \\ \text { Radium-226 } & 8.8 E-01 & 1 & \text { UI } & & 0 & \text { GP } \\ \text { Radium-228 } & -3.9 E-01 & 1 & \text { UI } & & 0 & \text { GP } \\ \text { Strontium-89 } & -4.7 E-02 & 1 & \text { UI } & & 0 & \text { GP } \\ \text { Strontium-90 } & -3.1 E-01 & 1 & \text { UI } & & 0 & \text { GP } \\ \text { Technetium-99 } & 5.1 E+00 & 1 & \text { UI } & & 0 & \text { GP } \\ \text { Thorium-228 } & -2.7 E-02 & 1 & \text { UI } & & 0 & \text { GP } \\ \text { Thorium-230 } & 8.3 E-02 & 1 & \text { UI } & & 0 & \text { GP } \\ \text { Thorium-232 } & -2.0 E-02 & 1 & \text { UI } & & 0 & \text { GP } \\ \text { Thorium-234 } & 1.9 E+02 & 1 & & & 0 & \text { GP } \\ \text { Tritium } & 4.7 E+02 & 1 & & & 2 & \text { GP } \\ \text { Uranium-233/234 } & 1.1 E-01 & 1 & \text { UI } & & 0 & \text { GP } \\ \text { Uranium-235 } & 0.0 E+00 & 1 & \text { UI } & & 0 & \text { GP } \\ \text { Uranium-238 } & 0.0 E+00 & 1 & \text { UI } & & 0 & \text { GP }\end{array}$

2095 DF Mod ST $\underline{H}$ Flag Lab Unit

Calculated Radionuclides

Parameter

Americium-243

Nickel-63

Plutonium-241

Plutonium-242 $\underline{1095}$

$-7.7 E-05$

$1.3 E+00$

-2.7E-02

$-1.3 E-07$
DF Mod ST $\underline{H}$ Flag Lab

0

$\underline{2095}$

DF Mod ST $\underline{H}$ Flag Lab

Unit

$\mathrm{pCi} / \mathrm{L}$

$\mathrm{pCi} / \mathrm{L}$

$\mathrm{PCi} / \mathrm{L}$

Note: See Appendix B for flagging criteria. Synchronous water levels are measured over a $3-5$ day period.

- = exceeded holding time.

- = exceeded groundwater protection standard. 


\section{WELL FSB 87D}

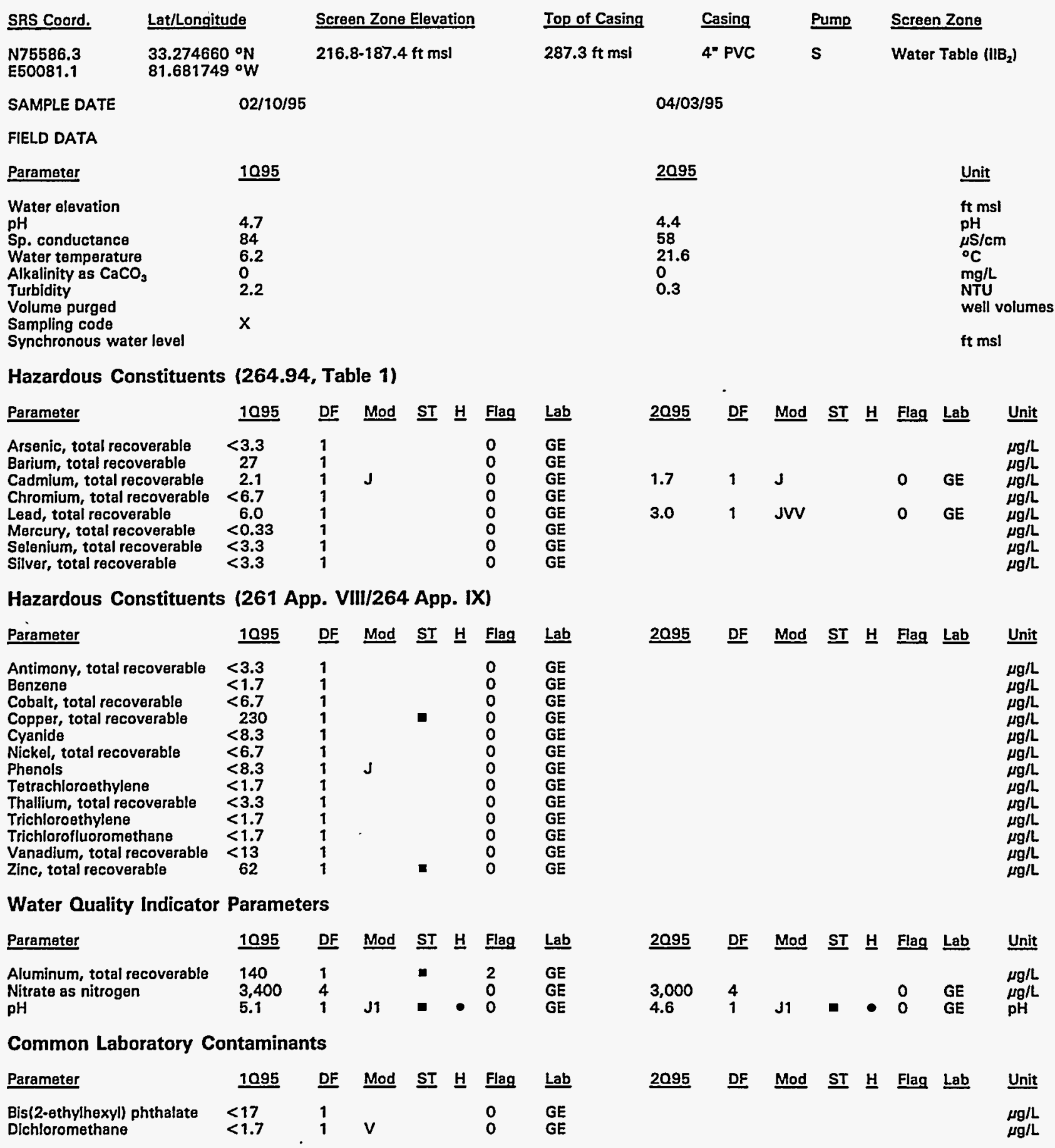

Note: See Appendix B for flagging criteria. Synchronous water levels are measured over a 3-5 day period.

- = exceeded holding time.

E = exceeded groundwater protection standard. 


\section{WELL FSB 87D (cont.)}

Radiological Indicator Parameters

\begin{tabular}{|c|c|c|c|c|c|c|c|c|c|c|c|c|c|c|c|}
\hline Parameter & 1095 & DF & Mod & ST & $\underline{\mathrm{H}}$ & Flag & $\underline{\text { Lab }}$ & 2095 & $\underline{\text { DF }}$ & Mod & $\underline{\mathbf{S T}}$ & 브 & Flag & $\underline{\text { Lab }}$ & Unit \\
\hline $\begin{array}{l}\text { Gross alpha } \\
\text { Nonvolatile beta }\end{array}$ & $\begin{array}{l}2.5 E+01 \\
2.3 E+01\end{array}$ & $\begin{array}{l}1 \\
1\end{array}$ & & - & & $\begin{array}{l}2 \\
0\end{array}$ & $\begin{array}{l}\text { GP } \\
\text { GP }\end{array}$ & $\begin{array}{l}5.2 E+01 \\
3.7 E+01\end{array}$ & $\begin{array}{l}1 \\
1\end{array}$ & $J$ & - & & $\begin{array}{l}2 \\
1\end{array}$ & $\begin{array}{l}\text { GP } \\
\text { GP }\end{array}$ & $\begin{array}{l}\mathrm{pCi} / \mathrm{L} \\
\mathrm{pCi} / \mathrm{L}\end{array}$ \\
\hline
\end{tabular}

Measured Radionuclides

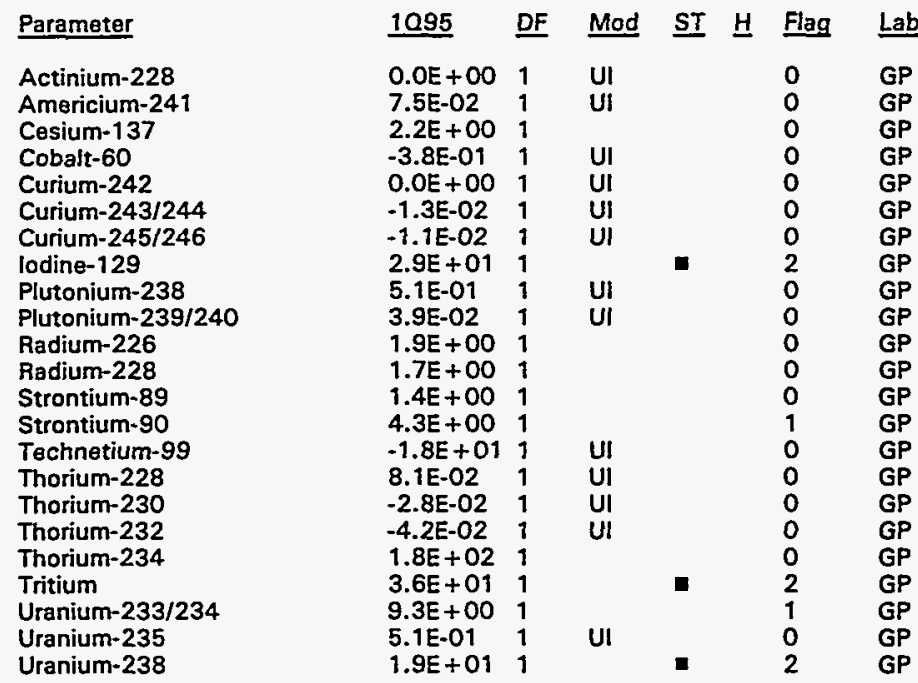

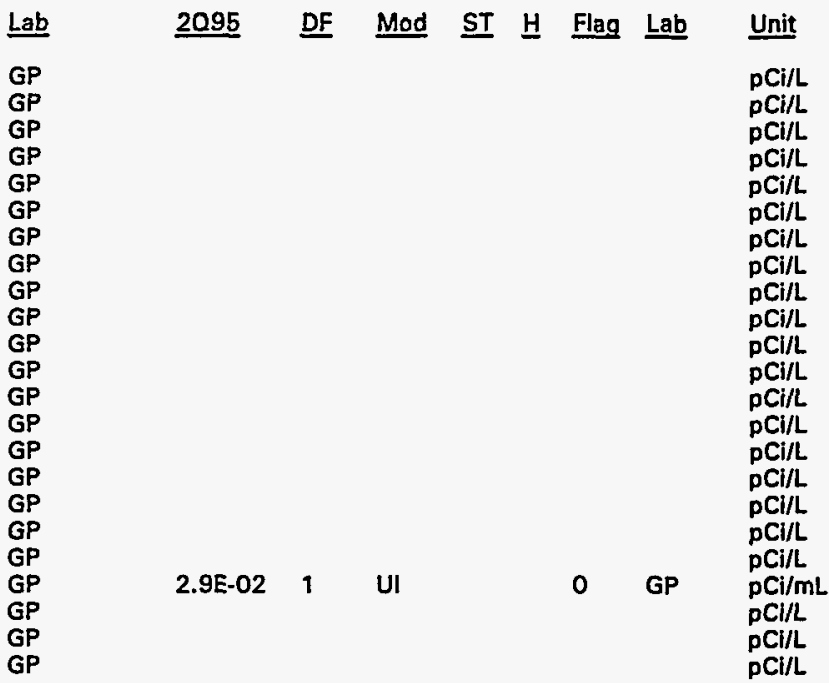

Calculated Radionuclides

\section{Parameter}

Americium-243

Nickel-63

Plutonium-24

Plutanium-242
1095 DF Mod ST $H$ Flag Lab

\section{0}

$-3.9 E-05$

3.8E-01

1.5E-01

7.7E-07 $\underline{2095}$

Unit

$\mathrm{pCi} / \mathrm{L}$

$\mathrm{pCi} / \mathrm{L}$

$\mathrm{pCl} / \mathrm{L}$

PCI/L

Note: See Appendix B for flagging criteria. Synchronous water levels are measured over a 3-5 day period.

- = exceeded holding time.

a = exceeded groundwater protection standard. 


\section{WELL FSB 88C}

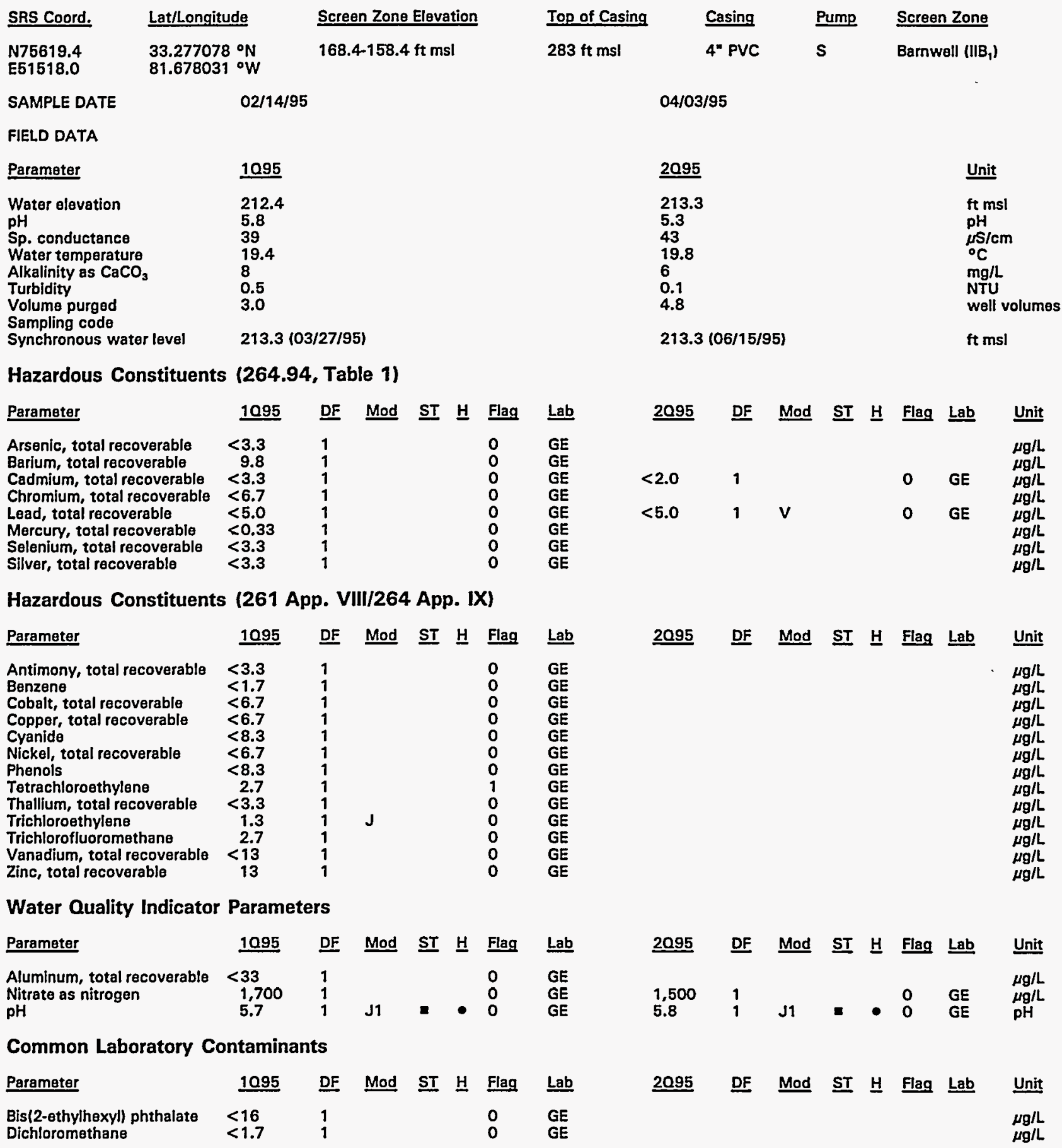

Note: See Appendix B for flagging criteria. Synchronous water levels are measured over a 3-5 day period. - = exceeded holding time.

= exceeded groundwater protection standard. 
WELL FSB 88C (cont.)

Radiological Indicator Parameters

\begin{tabular}{|c|c|c|c|c|c|c|c|c|c|c|c|c|c|c|c|}
\hline Parameter & $\underline{1095}$ & $\underline{\text { DF }}$ & Mod & $\underline{\text { ST }}$ & 브 & Flag & $\underline{\text { Lab }}$ & 2095 & DF & Mad & $\underline{\text { ST }}$ & $\underline{ }$ & Flag & Lab & Unit \\
\hline $\begin{array}{l}\text { Gross alpha } \\
\text { Nonvolatile beta }\end{array}$ & $\begin{array}{l}9.8 E-01 \\
8.4 E-01\end{array}$ & $\begin{array}{l}1 \\
1\end{array}$ & U! & & & $\begin{array}{l}0 \\
0\end{array}$ & $\begin{array}{l}\text { GP } \\
\text { GP }\end{array}$ & $\begin{array}{l}3.6 E-01 \\
6.0 E-01\end{array}$ & $\begin{array}{l}1 \\
1\end{array}$ & $\begin{array}{l}\text { UJI } \\
\text { UI }\end{array}$ & & & $\begin{array}{l}0 \\
0\end{array}$ & $\begin{array}{l}\text { GP } \\
\text { GP }\end{array}$ & $\begin{array}{l}p C i / L \\
p C i / L\end{array}$ \\
\hline \multicolumn{16}{|c|}{ Measured Radionuclides } \\
\hline Parameter & 1095 & DF & Mod & $\underline{\text { ST }}$ & 브 & Flag & $\underline{\text { Lab }}$ & 2095 & $\underline{D F}$ & Mod & $\underline{\mathbf{S T}}$ & $\underline{H}$ & Flag & Lab & Unit \\
\hline $\begin{array}{l}\text { Actinium-228 } \\
\text { Americium-241 } \\
\text { Cesium-137 } \\
\text { Cobalt-60 } \\
\text { Curium-242 } \\
\text { Curium-243/244 } \\
\text { Curium-245/246 } \\
\text { lodine-129 } \\
\text { Plutonium-238 } \\
\text { Plutonium-239/240 } \\
\text { Radium-226 } \\
\text { Radium-228 } \\
\text { Strontium-89 } \\
\text { Strontium-90 } \\
\text { Technetium-99 } \\
\text { Thorium-228 } \\
\text { Thorium-230 } \\
\text { Thorium-232 } \\
\text { Thorium-234 } \\
\text { Tritium } \\
\text { Uranium-233/234 } \\
\text { Uranium-235 } \\
\text { Uranium-238 }\end{array}$ & $\begin{array}{l}5.3 E-01 \\
8.7 E-02 \\
2.6 E+00 \\
8.3 E-01 \\
-1.4 E-02 \\
-9.9 E-03 \\
5.0 E-02 \\
-2.7 E-02 \\
-5.1 E-03 \\
0.0 E+00 \\
3.1 E-01 \\
-1.2 E+00 \\
-3.3 E-01 \\
-1.8 E-01 \\
-1.6 E+01 \\
7.5 E-02 \\
2.0 E-01 \\
0.0 E+00 \\
4.0 E+01 \\
1.7 E+01 \\
-2.4 E-02 \\
-1.6 E-02 \\
2.3 E-02\end{array}$ & $\begin{array}{l}1 \\
1 \\
1 \\
1 \\
1 \\
1 \\
1 \\
1 \\
1 \\
1 \\
1 \\
1 \\
1 \\
1 \\
1 \\
1 \\
1 \\
1 \\
1 \\
1 \\
1 \\
1 \\
1\end{array}$ & $\begin{array}{l}\text { UI } \\
\text { UI } \\
\text { UI } \\
\text { UI } \\
\text { UI } \\
\text { UI } \\
\text { UI } \\
\text { UI } \\
\text { UI } \\
\text { UI } \\
\text { UI } \\
\text { UII } \\
\text { UI } \\
\text { UI } \\
\text { UI } \\
\text { UI } \\
\text { UI } \\
\text { UI } \\
\text { UI } \\
\text { UI } \\
\text { UI } \\
\text { UI }\end{array}$ & & & $\begin{array}{l}0 \\
0 \\
0 \\
0 \\
0 \\
0 \\
0 \\
0 \\
0 \\
0 \\
0 \\
0 \\
0 \\
0 \\
0 \\
0 \\
0 \\
0 \\
0 \\
1 \\
0 \\
0 \\
0\end{array}$ & $\begin{array}{l}\text { GP } \\
\text { GP } \\
\text { GP } \\
\text { GP } \\
\text { GP } \\
\text { GP } \\
\text { GP } \\
\text { GP } \\
\text { GP } \\
\text { GP } \\
\text { GP } \\
\text { GP } \\
\text { GP } \\
\text { GP } \\
\text { GP } \\
\text { GP } \\
\text { GP } \\
\text { GP } \\
\text { GP } \\
\text { GP } \\
\text { GP } \\
\text { GP } \\
\text { GP }\end{array}$ & $1.4 E-02$ & 1 & UI & & & 0 & GP & 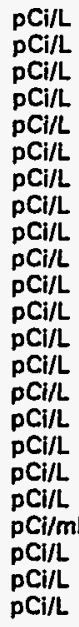 \\
\hline \multicolumn{16}{|c|}{ Calculated Radionuclides } \\
\hline Parameter & 1095 & $\underline{\text { DF }}$ & Mod & $\underline{\text { ST }}$ & $\underline{H}$ & Flag & Lab & $\underline{2095}$ & $\underline{D F}$ & Mod & $\underline{S T}$ & 브 & Flag & $\underline{\text { Lab }}$ & Unit \\
\hline $\begin{array}{l}\text { Americium-243 } \\
\text { Nickel-63 } \\
\text { Plutonium-241 } \\
\text { Plutonium-242 }\end{array}$ & $\begin{array}{l}-3.0 E-05 \\
8.3 E-01 \\
0.0 E+00 \\
0.0 E+00\end{array}$ & & & & & $\begin{array}{l}0 \\
0 \\
0 \\
0\end{array}$ & & & & & & & & & $\begin{array}{l}\mathrm{pCi} / \mathrm{L} \\
\mathrm{pCi} / \mathrm{L} \\
\mathrm{pCi} / \mathrm{L} \\
\mathrm{pCi} / \mathrm{L}\end{array}$ \\
\hline
\end{tabular}

Note: See Appendix B for flagging criteria. Synchronous water levels are measured over a 3-5 day period.

- = exceeded holding time.

- = exceeded groundwater protection standard. 


\section{WELL FSB 88D}

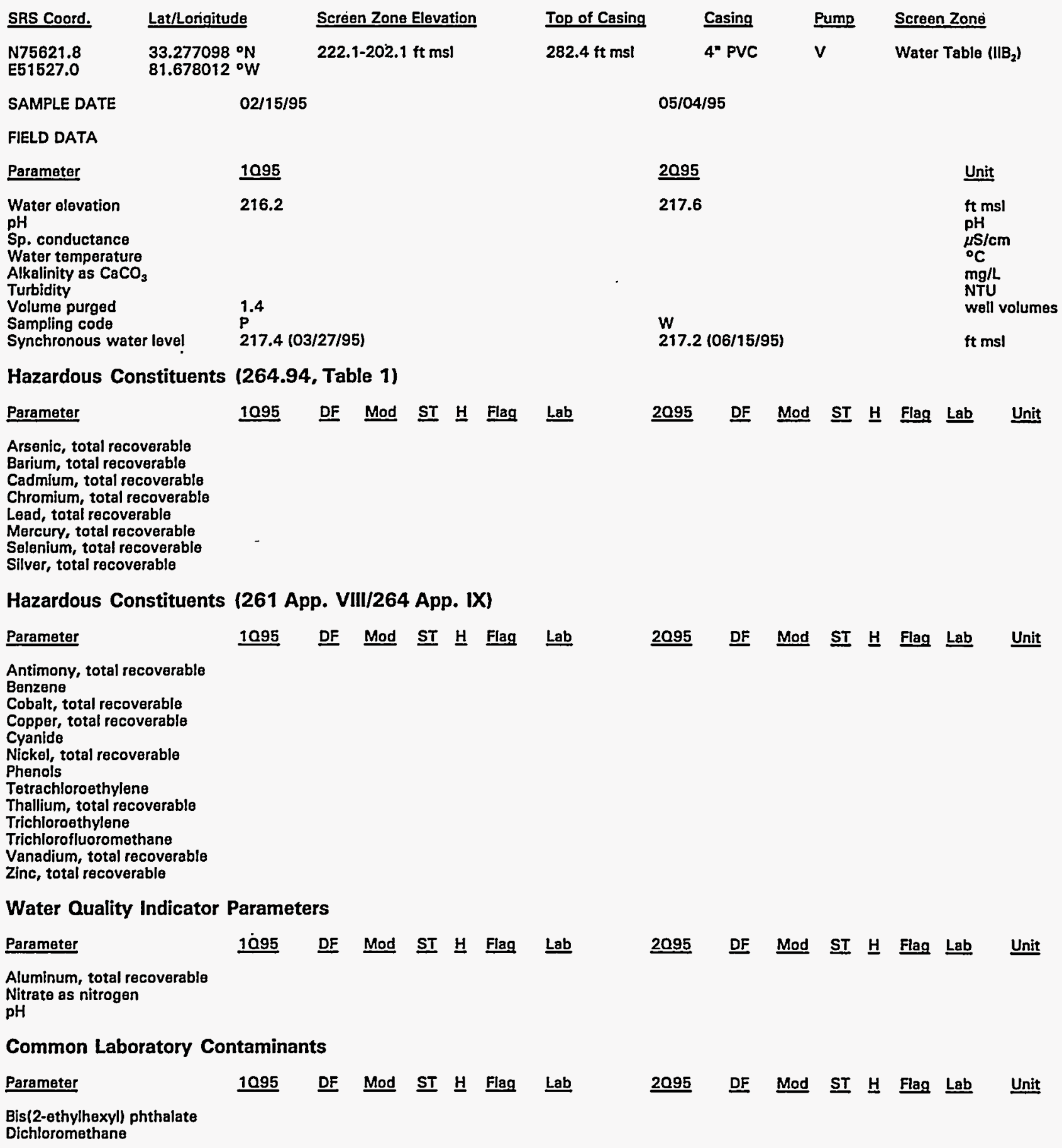

Note: See Appendix B for flagging criteria. Synchronous water levels are measured over a 3-5 day period.

- = exceeded holding time.

- = exceeded groundwater protection standard. 
WELL FSB 88D (cont.)

Radiological Indicator Parameters

Parameter

DF Mod ST $\underline{H}$ Flag Lab

$\underline{2095}$

DF Mod ST $\underline{H}$ Flag Lab

Unit

Gross alpha

Nonvolatile beta

Measured Radionuclides

Parameter

$\underline{1095}$

Actinium-228

Americium-241

Cesium-137

Cobalt-60

Curium-242

Curium-243/244

Curium-245/246

lodine-129

Plutonium-238

Plutonium-239/240

Radium-226

Radium-228

Strontium-89

Strontium-90

Technetium-99

Thorium-228

Thorium-230

Thorium-232

Thorium-234

Tritium

Uranium-233/234

Uranium-235

Uranium-238

Calculated Radionuclides

Parameter

1095

Americium-243

Nickel-63

Plutonium-241

Plutonium-242
DF Mod ST $\underline{H}$ Flag Lab $\underline{2095} \underline{\text { DF }} \underline{\text { Mod }} \underline{\text { ST }} \underline{H}$ Flag Lab $\underline{\text { Lnit }}$ 


\section{WELL FSB 89C}

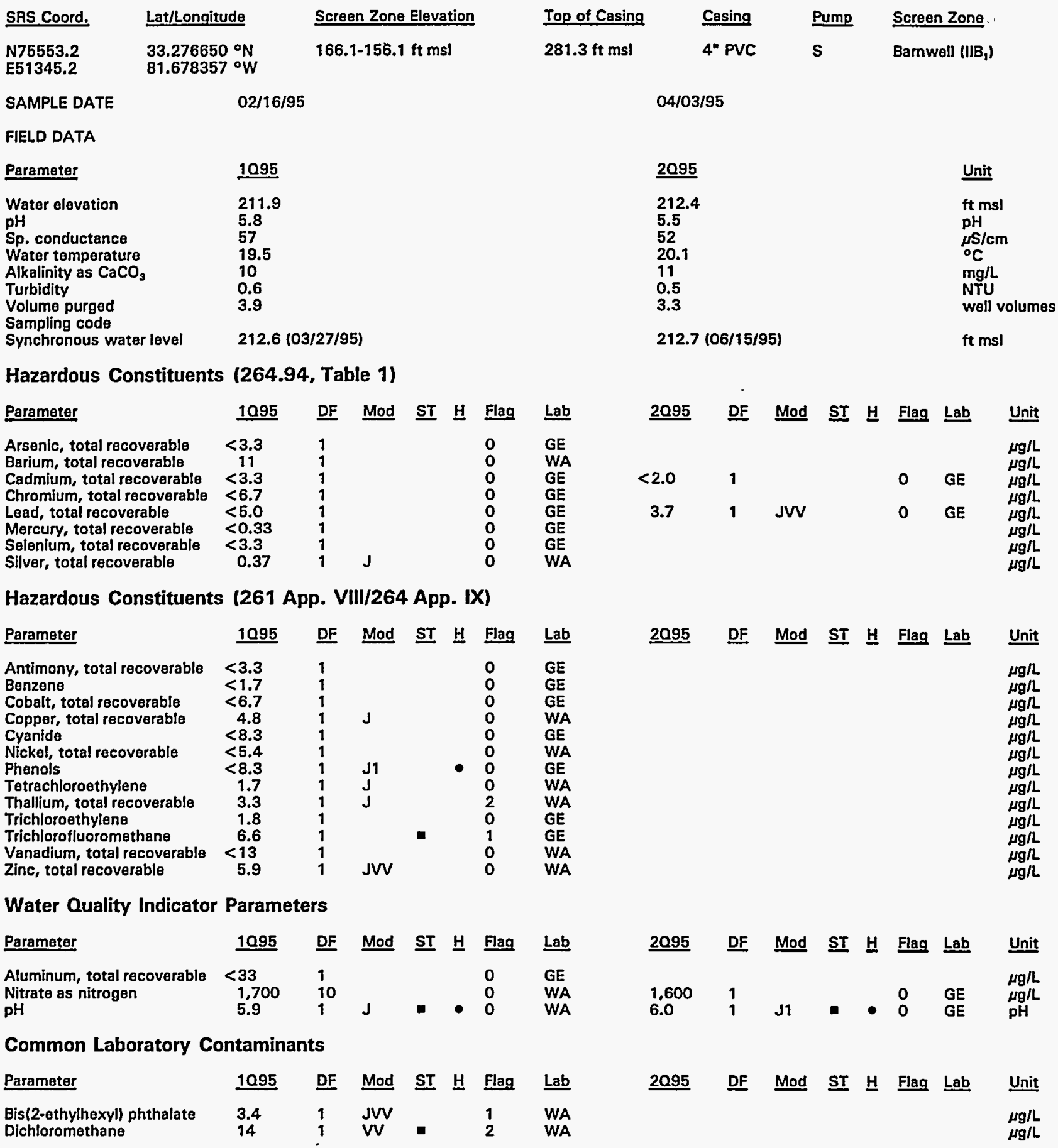

Note: See Appendix B for flagging criteria. Synchronous water levels are measured over a 3-5 day period.

$-=$ exceeded holding time.

- = exceeded groundwater protection standard. 
WELL FSB 89C (cont.)

Radiological Indicator Parameters

\begin{tabular}{|c|c|c|c|c|c|c|c|c|c|c|c|c|c|c|c|}
\hline Parameter & 1095 & DF & Mod & $\underline{\text { ST }}$ & H & Flag & $\underline{\text { Lab }}$ & $\underline{2095}$ & DF & Mod & ST & $\underline{\mathbf{H}}$ & Flag & $\underline{\text { Lab }}$ & Unit \\
\hline $\begin{array}{l}\text { Gross alpha } \\
\text { Nonvolatile beta }\end{array}$ & $\begin{array}{l}\text { 8.0E-01 } \\
2.0 \mathrm{E}-01\end{array}$ & $\begin{array}{l}1 \\
1\end{array}$ & UI & & & $\begin{array}{l}0 \\
0\end{array}$ & $\begin{array}{l}\text { TM } \\
\text { TM }\end{array}$ & $\begin{array}{l}4.2 E-01 \\
1.2 E+00\end{array}$ & $\begin{array}{l}1 \\
1\end{array}$ & $\begin{array}{l}\text { USI } \\
\text { UI }\end{array}$ & & & $\begin{array}{l}0 \\
0\end{array}$ & $\begin{array}{l}\text { GP } \\
\text { GP }\end{array}$ & $\begin{array}{l}\mathrm{pCi} / \mathrm{L} \\
\mathrm{pCi} / \mathrm{L}\end{array}$ \\
\hline
\end{tabular}

Measured Radionuclides

\begin{tabular}{|c|c|c|c|c|c|c|c|c|c|c|c|c|c|c|c|}
\hline Parameter & 1095 & $\underline{D F}$ & Mod & $\underline{\mathrm{ST}}$ & H & Flag & $\underline{L a b}$ & 2095 & $\underline{D F}$ & Mod & $\underline{\mathrm{ST}}$ & $\underline{H}$ & Flag & Lab & Unit \\
\hline $\begin{array}{l}\text { Actinium-228 } \\
\text { Amsricium-241 } \\
\text { Cesium-137 } \\
\text { Cobalt-60 } \\
\text { Curium-242 } \\
\text { Curium-243/244 } \\
\text { Curium-245/246 } \\
\text { Iodine-129 } \\
\text { Plutonium-238 } \\
\text { Plutonium-239/240 } \\
\text { Radium-226 } \\
\text { Radium-228 } \\
\text { Strontium-89 } \\
\text { Strontium-90 } \\
\text { Technetium-99 } \\
\text { Thorium-228 } \\
\text { Thorium-230 } \\
\text { Thorium-232 } \\
\text { Thorium-234 } \\
\text { Tritium } \\
\text { Uranium-233/234 } \\
\text { Uranium-235 } \\
\text { Uranium-238 }\end{array}$ & $\begin{array}{l}-7.6 E-01 \\
4.5 E-01 \\
-1.4 E-01 \\
-4.9 E-01 \\
-9.7 E-03 \\
-4.6 E-02 \\
0.0 E+00 \\
-1.1 E-01 \\
0.0 E+00 \\
-8.8 E-03 \\
4.2 E-01 \\
3.0 E-01 \\
-3.5 E-01 \\
-1.8 E-01 \\
1.1 E+00 \\
2.0 E-02 \\
1.0 E-02 \\
0.0 E+00 \\
3.4 E+02 \\
1.6 E+01 \\
2.8 E-02 \\
0.0 E+00 \\
0.0 E+00\end{array}$ & $\begin{array}{l}1 \\
1 \\
1 \\
1 \\
1 \\
1 \\
1 \\
1 \\
1 \\
1 \\
1 \\
1 \\
1 \\
1 \\
1 \\
1 \\
1 \\
1 \\
1 \\
1 \\
1 \\
1 \\
1\end{array}$ & $\begin{array}{l}\text { UI } \\
V \\
\text { UI } \\
\text { UI } \\
\text { UI } \\
\text { UI } \\
\text { UI } \\
\text { UI } \\
\text { UII } \\
\text { UI } \\
\text { UI } \\
\text { UI } \\
\text { UI } \\
\text { UI } \\
\text { UI } \\
\text { UI } \\
\text { UI } \\
\\
\text { UI } \\
\text { UI } \\
\text { UI }\end{array}$ & & & $\begin{array}{l}0 \\
0 \\
0 \\
0 \\
0 \\
0 \\
0 \\
0 \\
0 \\
0 \\
0 \\
0 \\
0 \\
0 \\
0 \\
0 \\
0 \\
0 \\
1 \\
1 \\
0 \\
0 \\
0\end{array}$ & $\begin{array}{l}T M \\
T M \\
T M \\
\text { GP } \\
\text { GP } \\
\text { GP } \\
\text { GP } \\
T M \\
\text { GP } \\
\text { GP } \\
T M \\
T M \\
T M \\
\text { GP } \\
T M \\
T M \\
T M \\
T M \\
T M \\
\text { GP } \\
T M \\
G P \\
T M \\
\text { GP }\end{array}$ & $1.6 \mathrm{E}-02$ & 1 & UI & & & 0 & GP & $\begin{array}{l}\text { pCi/L } \\
\text { pCi/L } \\
\text { pCi/L } \\
\text { pCi/L } \\
\text { pCi/L } \\
\text { pCi/L } \\
\text { pCi/L } \\
\text { pCi/L } \\
\text { pCi/L } \\
\text { pCi/L } \\
\text { pCi/L } \\
\text { pCi/L } \\
\text { pCi/L } \\
\text { pCi/L } \\
\text { pCi/L } \\
\text { pCi/L } \\
\text { pCi/L } \\
\text { pCi/L } \\
\text { pCi/L } \\
\text { pCi/mL } \\
\text { pCi/L } \\
\text { pCi/L } \\
\text { pCi/L }\end{array}$ \\
\hline \multicolumn{16}{|c|}{ Calculated Radionuclides } \\
\hline Parameter & 1095 & DF & Mod & $\underline{\text { ST }}$ & 브 & Flag & $\underline{\text { Lab }}$ & $\underline{2095}$ & DF & Mod & $\underline{S T}$ & 브 & Flag & Lab & $\underline{\text { Unit }}$ \\
\hline $\begin{array}{l}\text { Americium-243 } \\
\text { Nicke!-63 } \\
\text { Plutonium-241 } \\
\text { Plutonium-242 }\end{array}$ & $\begin{array}{l}-1.4 \mathrm{E}-04 \\
-4.9 \mathrm{E}-01 \\
-3.5 \mathrm{E}-02 \\
-1.8 \mathrm{E}-07\end{array}$ & & & & & $\begin{array}{l}0 \\
0 \\
0 \\
0\end{array}$ & & & & & & & & & $\begin{array}{l}\mathrm{pCi} / \mathrm{L} \\
\mathrm{pCi} / \mathrm{L} \\
\mathrm{pCi} / \mathrm{L} \\
\mathrm{pCi} / \mathrm{L}\end{array}$ \\
\hline
\end{tabular}

Note: See Appendix B for flagging criteria. Synchronous water levels are measured over a 3-5 day period.

- = exceeded holding time.

- = exceeded groundwater protection standard. 


\section{WELL FSB 89D}

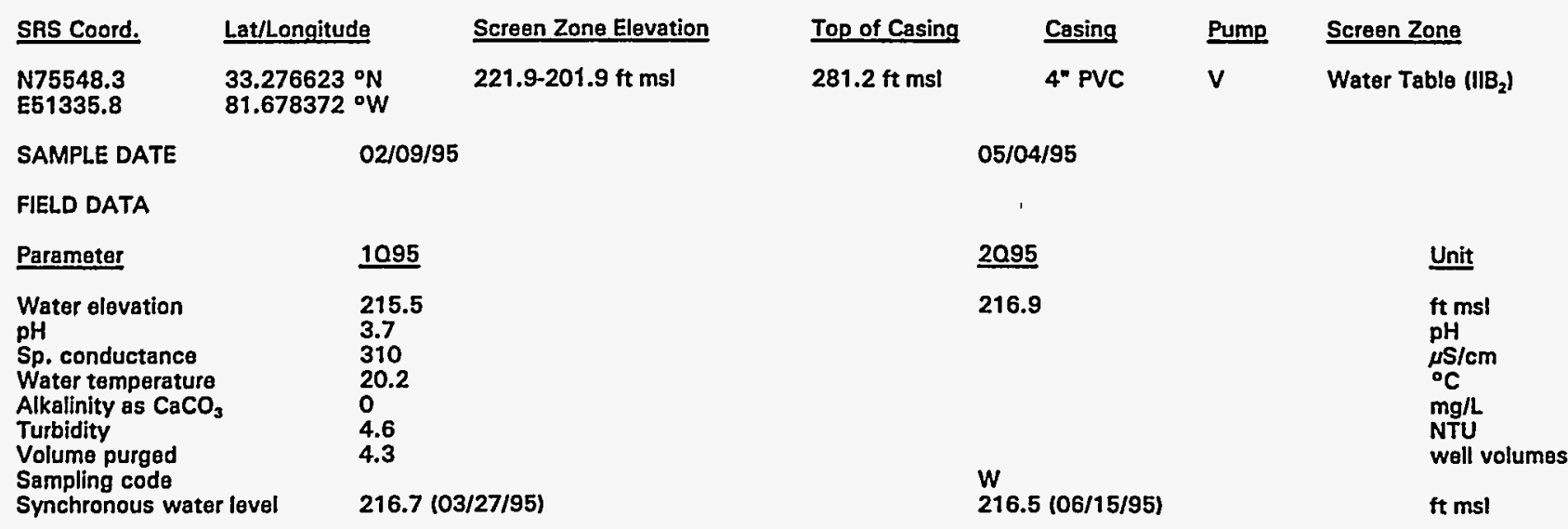

Hazardous Constituents (264.94, Table 1)

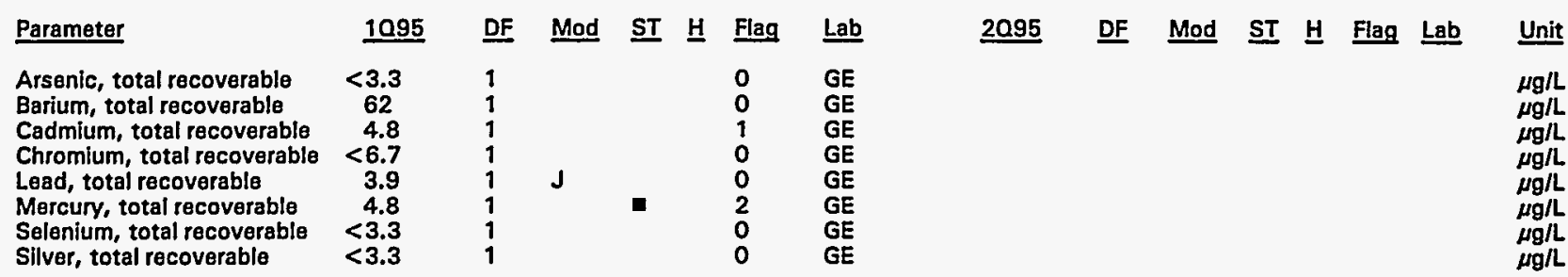

Hazardous Constituents (261 App. VIII/264 App. IX)

$\begin{array}{lccccccc}\text { Parameter } & 1095 & \text { DF } & \text { Mod } & \text { ST } & \text { Flag } & \text { Lab } \\ \text { Antimony, total recoverable } & <3.3 & 1 & & & 0 & \text { GE } \\ \text { Benzene } & <1.7 & 1 & & & 0 & \text { GE } \\ \text { Cobalt, total recoverable } & 13 & 1 & & & 0 & \text { GE } \\ \text { Copper, total recoverable } & 19 & 1 & & & 0 & \text { GE } \\ \text { Cyanide } & <8.3 & 1 & & & 0 & \text { GE } \\ \text { Nickel, total recoverable } & 9.6 & 1 & & & 0 & \text { GE } \\ \text { Phenols } & <8.3 & 1 & & & 0 & \text { GE } \\ \text { Tetrachloroethylene } & <1.7 & 1 & & & 0 & \text { GE } \\ \text { Thallium, total recoverable } & <3.3 & 1 & & & 0 & \text { GE } \\ \text { Trichloroethylene } & <1.7 & 1 & & & 0 & \text { GE } \\ \text { Trichlorofluoromethane } & 3.3 & 1 & & & 0 & \text { GE } \\ \text { Vansdium, total recoverable } & <13 & 1 & & & 0 & \text { GE } \\ \text { Zinc, total recoverable } & 26 & 1 & & & & \end{array}$

2095 DF Mod ST H Flag Lab Unit

Zinc, total recoverable

Water Quality Indicator Parameters

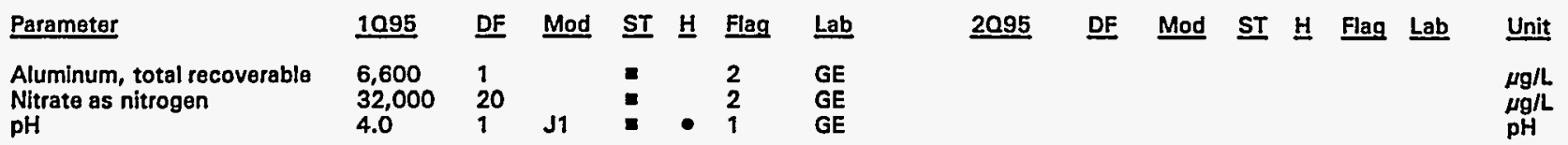

Common Laboratory Contaminants

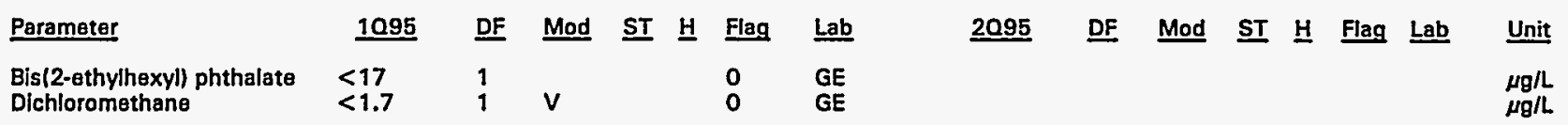

Note: See Appendix B for flagging criteria. Synchronous water levels are measured over a 3-5 day period.

- = exceeded holding time.

- = exceeded groundwater protection standard. 
WELL. FSB 89D (cont.)

Radiological Indicator Parameters

\begin{tabular}{|c|c|c|c|c|c|c|c|c|c|c|c|c|c|c|}
\hline Parameter & 1095 & DF & Mod & ST & $\underline{H}$ & Flag & $\underline{\text { Lab }}$ & 2095 & DF & Mad & $\underline{S T}$ & $\underline{H}$ & Flag & Lab \\
\hline $\begin{array}{l}\text { Gross alpha } \\
\text { Nonvolatile beta }\end{array}$ & $\begin{array}{l}2.2 E+02 \\
1.0 E+03\end{array}$ & $\begin{array}{l}1 \\
1\end{array}$ & & 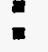 & & $\begin{array}{l}2 \\
2\end{array}$ & $\begin{array}{l}\text { GP } \\
\text { GP }\end{array}$ & & & & & & & \\
\hline
\end{tabular}

Measured Radionuclides

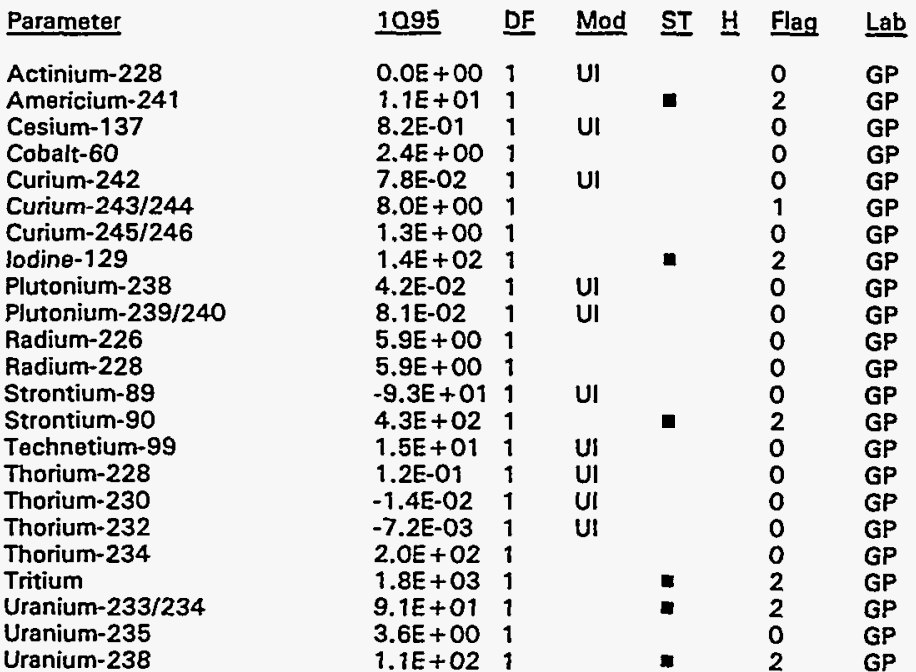

2095 DF Mod ST $\underline{H}$ Flag Lab Unit

Calculated Radionuclides

Parameter

Americium-243

Nickel-63

Plutonium-241

Plutonium-242

$\begin{array}{lc}1095 & \text { DF Mod ST } \underline{\text { H Flag Lab }} \\ 2.4 E-02 & 0 \\ 2.4 E+00 & 0 \\ 3.3 E-01 & 0 \\ 1.6 E-06 & 0\end{array}$

$\underline{\text { Unit }}$

pCi/L

pCi/L

pCi/L

pCi/L

Note: See Appendix B for flagging criteria. Synchronous water levels are measured over a 3-5 day period.

- = exceeded holding time.

= exceeded groundwater protection standard. 


\section{WELL FSB 90C}

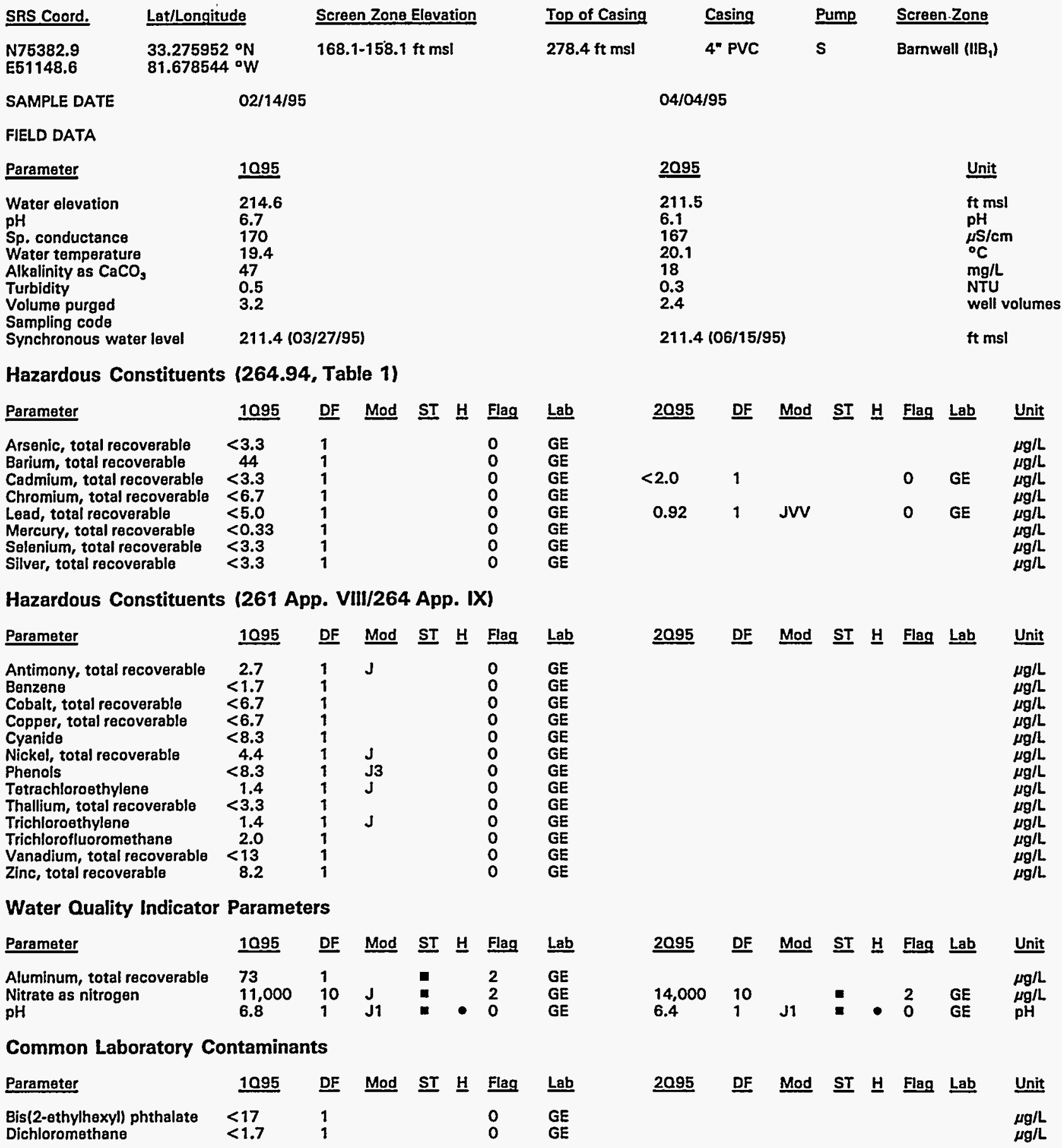

Note: See Appendix B for flagging criteria. Synchronous water levels are measured over a 3-5 day period.

- = exceeded holding time.

- = exceeded groundwater protection standard. 
WELL FSB 90C (cont.)

Radiological Indicator Parameters

\begin{tabular}{|c|c|c|c|c|c|c|c|c|c|c|c|c|c|c|c|}
\hline Parameter & 1095 & $\underline{\mathrm{DF}}$ & Mod & $\underline{\mathbf{S T}}$ & H & Flag & $\underline{\text { Lab }}$ & 2095 & $\underline{\mathrm{DF}}$ & Mod & $\underline{\mathbf{S T}}$ & H & Flag & Lab & Unit \\
\hline $\begin{array}{l}\text { Gross alpha } \\
\text { Nonvolatile beta }\end{array}$ & $\begin{array}{l}2.9 E+00 \\
1.9 E+01\end{array}$ & $\begin{array}{l}1 \\
1\end{array}$ & & & & $\begin{array}{l}0 \\
0\end{array}$ & $\begin{array}{l}\text { GP } \\
G P\end{array}$ & $\begin{array}{l}2.4 E+00 \\
1.9 E+01\end{array}$ & $\begin{array}{l}1 \\
1\end{array}$ & & & & $\begin{array}{l}0 \\
0\end{array}$ & $\begin{array}{l}\text { GP } \\
\text { GP }\end{array}$ & $\begin{array}{l}\mathrm{pCi} / \mathrm{L} \\
\mathrm{pCi} / \mathrm{L}\end{array}$ \\
\hline \multicolumn{16}{|c|}{ Measured Radionuclides } \\
\hline Parameter & 1095 & $\underline{\mathrm{DF}}$ & Mod & $\underline{\mathbf{S T}}$ & $\underline{H}$ & Flag & $\underline{\text { Lab }}$ & 2095 & $\underline{\mathrm{DF}}$ & Mod & $\underline{\text { ST }}$ & $\underline{H}$ & Flag & $\underline{\text { Lab }}$ & $\underline{\text { Unit }}$ \\
\hline $\begin{array}{l}\text { Actinium-228 } \\
\text { Americium-241 } \\
\text { Cesium-137 } \\
\text { Cobalt-60 } \\
\text { Curium-242 } \\
\text { Curium-243/244 } \\
\text { Curium-245/246 } \\
\text { lodine-129 } \\
\text { Plutonium-238 } \\
\text { Plutonium-239/240 } \\
\text { Radium-226 } \\
\text { Radium-228 } \\
\text { Strontium-89 } \\
\text { Strontium-90 } \\
\text { Technetium-99 } \\
\text { Thorium-228 } \\
\text { Thorium-230 } \\
\text { Thorium-232 } \\
\text { Thorium-234 } \\
\text { Tritium } \\
\text { Uranium-233/234 } \\
\text { Uranium-235 } \\
\text { Uranium-238 }\end{array}$ & $\begin{array}{l}3.2 E+00 \\
1.5 E-01 \\
2.2 E-01 \\
1.4 E+00 \\
0.0 E+00 \\
2.9 E-02 \\
7.6 E-02 \\
1.1 E+00 \\
3.3 E-01 \\
-9.9 E-03 \\
1.2 E+00 \\
-2.4 E-01 \\
5.0 E-01 \\
2.5 E+00 \\
4.2 E+00 \\
1.4 E-01 \\
2.0 E-01 \\
0.0 E+00 \\
8.4 E+01 \\
5.8 E+02 \\
9.7 E-02 \\
0.0 E+00 \\
4.1 E-02\end{array}$ & $\begin{array}{l}1 \\
1 \\
1 \\
1 \\
1 \\
1 \\
1 \\
1 \\
1 \\
1 \\
1 \\
1 \\
1 \\
1 \\
1 \\
1 \\
1 \\
1 \\
1 \\
1 \\
1 \\
1 \\
1\end{array}$ & 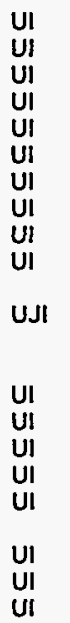 & - & & $\begin{array}{l}0 \\
0 \\
0 \\
0 \\
0 \\
0 \\
0 \\
0 \\
0 \\
0 \\
0 \\
0 \\
0 \\
0 \\
0 \\
0 \\
0 \\
0 \\
0 \\
2 \\
0 \\
0 \\
0\end{array}$ & $\begin{array}{l}\text { GP } \\
\text { GP } \\
G P \\
G P \\
G P \\
G P \\
\text { GP } \\
\text { GP } \\
\text { GP } \\
\text { GP } \\
\text { GP } \\
G P \\
G P \\
G P \\
G P \\
G P \\
G P \\
G P \\
G P \\
G P \\
G P \\
G P \\
G P\end{array}$ & $6.1 E+02$ & 1 & & - & & 2 & GP & 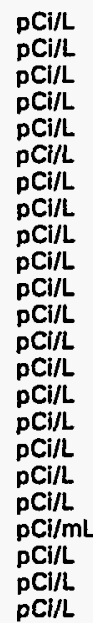 \\
\hline
\end{tabular}

Calculated Radionuclides

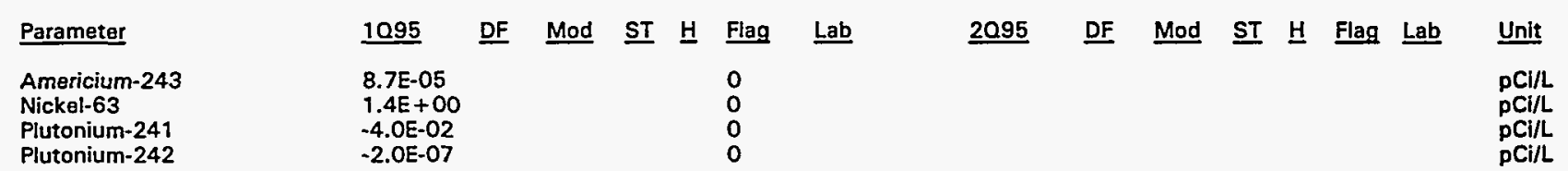

Note: See Appendix B for flagging criteria. Synchronous water levels are measured over a 3-5 day period.

$\bullet=$ exceeded holding time.

- = exceeded groundwater protection standard. 
WELL FSB 90D

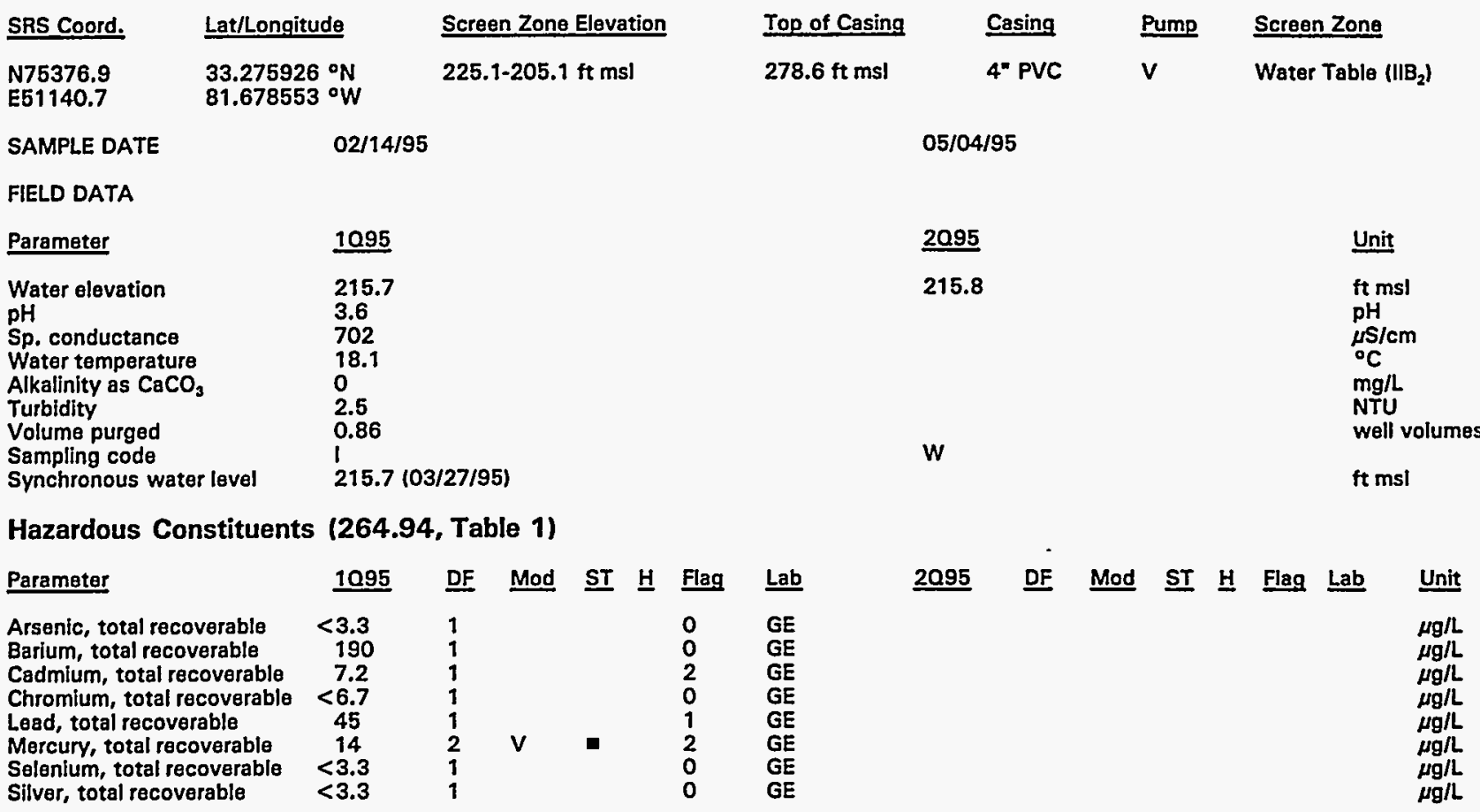

Hazardous Constituents (261 App. VIII/264 App. IX)

\begin{tabular}{|c|c|c|c|c|c|c|c|c|c|c|c|c|c|c|c|}
\hline Parameter & 1095 & DF & Mod & $\underline{\text { ST }}$ & H & Flag & $\underline{\text { Lab }}$ & $\underline{2095}$ & $\underline{D F}$ & Mod & $\underline{\text { ST }}$ & $\underline{H}$ & Flag & $\underline{\text { Lab }}$ & Unit \\
\hline $\begin{array}{l}\text { Antimony, total recoverable } \\
\text { Benzene } \\
\text { Cobalt, total recoverable } \\
\text { Copper, total recoverable } \\
\text { Cyanide } \\
\text { Nickel, total recoverable } \\
\text { Phenols } \\
\text { Tetrachloroethylene } \\
\text { Thallium, total recoverable } \\
\text { Trichloroethylene } \\
\text { Trichlorofluoromethane } \\
\text { Vanadium, total recoverable } \\
\text { Zinc, total recoverable }\end{array}$ & $\begin{aligned} & 5.4 \\
&<1.7 \\
& 13 \\
& 61 \\
&<8.3 \\
& 19 \\
&<8.3 \\
&<1.7 \\
&<3.3 \\
&<1.7 \\
&<1.7 \\
&<13 \\
& 79\end{aligned}$ & $\begin{array}{l}1 \\
1 \\
1 \\
1 \\
1 \\
1 \\
1 \\
1 \\
1 \\
1 \\
1 \\
1 \\
1\end{array}$ & $\begin{array}{l}v \\
\text { J1 }\end{array}$ & - & $\bullet$ & $\begin{array}{l}1 \\
0 \\
0 \\
0 \\
0 \\
0 \\
0 \\
0 \\
0 \\
0 \\
0 \\
0 \\
0\end{array}$ & $\begin{array}{l}\mathrm{GE} \\
\mathrm{GE} \\
\mathrm{GE} \\
\mathrm{GE} \\
\mathrm{GE} \\
\mathrm{GE} \\
\mathrm{GE} \\
\mathrm{GE} \\
\mathrm{GE} \\
\mathrm{GE} \\
\mathrm{GE} \\
\mathrm{GE} \\
\mathrm{GE}\end{array}$ & & & & & & & & $\begin{array}{l}\mu g / L \\
\mu g / L \\
\mu g / L \\
\mu g / L \\
\mu g / L \\
\mu g / L \\
\mu g / L \\
\mu g / L \\
\mu g / L \\
\mu g / L \\
\mu g / L \\
\mu g / L \\
\mu g / L\end{array}$ \\
\hline \multicolumn{16}{|c|}{ Water Quality Indicator Parameters } \\
\hline Parameter & 1095 & DF & Mod & $\underline{\mathbf{S T}}$ & $\underline{H}$ & Flag & $\underline{L a b}$ & $\underline{2095}$ & $\underline{D F}$ & Mod & $\underline{\text { ST }}$ & $\underline{H}$ & Flag & Lab & $\underline{\text { Unit }}$ \\
\hline $\begin{array}{l}\text { Aluminum, total recoverable } \\
\text { Nitrate as nitrogen } \\
\text { pH }\end{array}$ & $\begin{array}{l}29,000 \\
97,000 \\
3.8\end{array}$ & $\begin{array}{l}1 \\
80 \\
1\end{array}$ & J1 & 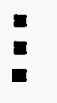 & - & $\begin{array}{l}2 \\
2 \\
1\end{array}$ & $\begin{array}{l}\mathrm{GE} \\
\mathrm{GE} \\
\mathrm{GE}\end{array}$ & & & & & & & & $\underset{\mathrm{pH}}{\mu \mathrm{g} / \mathrm{L}}$ \\
\hline \multicolumn{16}{|c|}{ Common Laboratory Contaminants } \\
\hline Parameter & 1095 & $\underline{D F}$ & Mod & $\underline{\text { ST }}$ & $\underline{H}$ & Flag & $\underline{L a b}$ & 2095 & $\underline{\mathrm{DF}}$ & Mod & $\underline{\mathbf{S T}}$ & H & Flag & $\underline{\text { Lab }}$ & Unit \\
\hline $\begin{array}{l}\text { Bis(2-ethylhexyl) phthalate } \\
\text { Dichloromethane }\end{array}$ & $\begin{array}{l}<17 \\
<1.7\end{array}$ & $\begin{array}{l}1 \\
1\end{array}$ & & & & $\begin{array}{l}0 \\
0\end{array}$ & $\begin{array}{l}\text { GE } \\
\text { GE }\end{array}$ & & & & & & & & $\begin{array}{l}\mu g / L \\
\mu \mathrm{g} / \mathrm{L}\end{array}$ \\
\hline
\end{tabular}

Note: See Appendix B for flagging criteria. Synchronous water levels are measured over a 3-5 day period.

- = exceeded holding time.

- = exceeded groundwater protection standard. 
WELL FSB 9OD (cont.)

Radiological Indicator Parameters

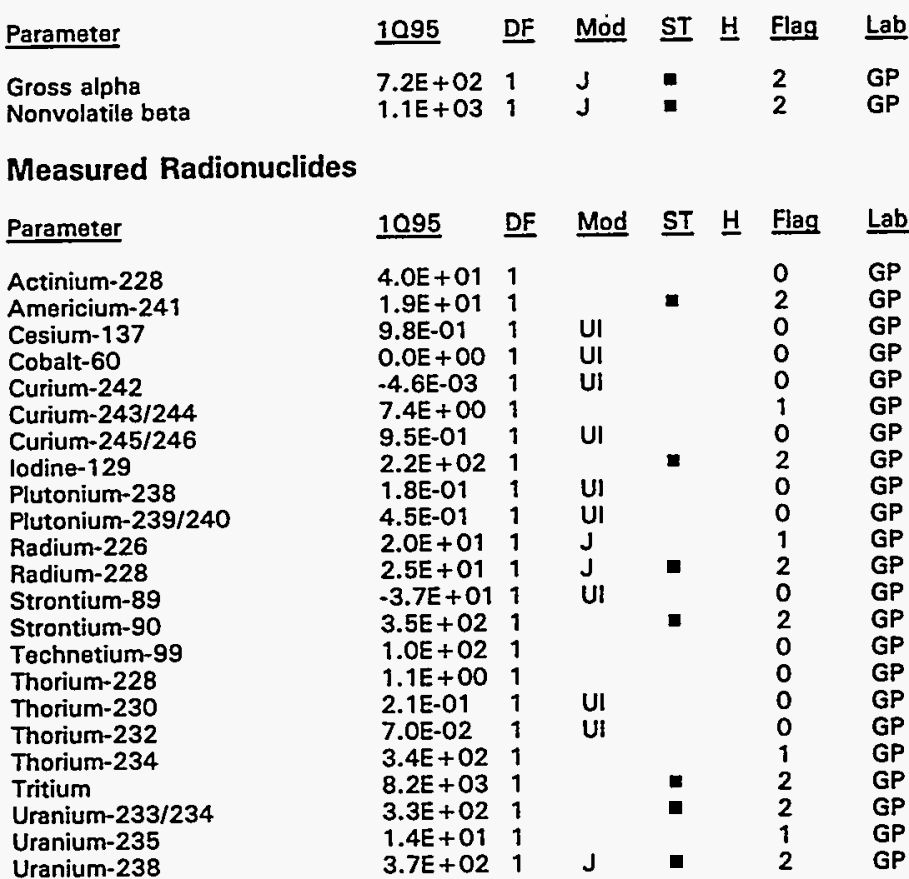

2095 DF Mod ST $\underline{H}$ Flag Lab Unit

$\mathrm{pCi} / \mathrm{L}$
$\mathrm{PCi} / \mathrm{L}$

Calculated Radionuclides

Parameter

Americium-243

Nickel-63

Plutonium-241

Plutonium-242
2.2E-02

$0.0 E+00$

$1.8 \mathrm{E}+00$

8.9E-06

pCi/L

pCi/L

pCi/L

pCi/L

$\mathrm{pCi} / \mathrm{L}$

pCi/L

$\mathrm{pCi} / \mathrm{L}$

$\mathrm{pCi} / \mathrm{L}$

$\mathrm{pCi} / \mathrm{L}$

$\mathrm{pCi} / \mathrm{L}$

pCi/L

$\mathrm{pCi} / \mathrm{L}$

pCi/L

$\mathrm{PCi} / \mathrm{L}$

PCI/L

$\mathrm{pCill}$

$\mathrm{pCl} / \mathrm{mL}$

$\mathrm{pCi} / \mathrm{L}$

$\mathrm{pCi} / \mathrm{L}$

$\mathrm{PCI} / L$

$\underline{2095}$ DF Mod $\underline{\text { ST }} \underline{\mathrm{H}}$ Flag Lab Unit

pCi/L

pCill

$\mathrm{pCi} / \mathrm{L}$

$\mathrm{pCi} / \mathrm{L}$

Note: See Appendix B for flagging criteria. Synchronous water levels are measured over a $3-5$ day period.

- = exceeded holding time.

- =xceeded groundwater protection standard. 


\section{WELL FSB 91C}

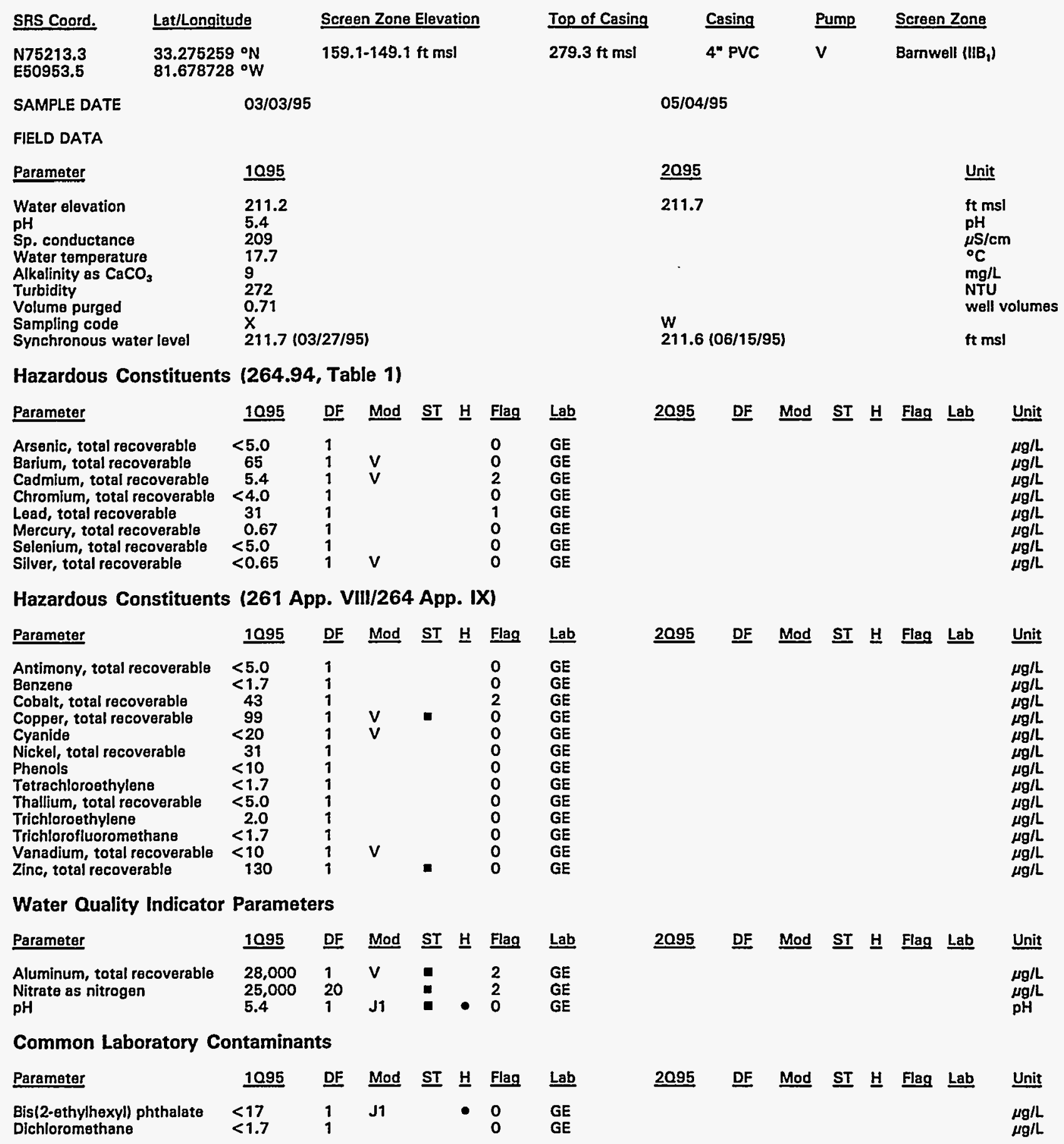

Note: See Appendix B for flagging criteria. Synchronous water levels are measured over a 3-5 day period.

- = exceeded holding time.

= exceeded groundwater protection standard. 


\section{WELL FSB $91 \mathrm{C}$ (cont.)}

Radiological Indicator Parameters

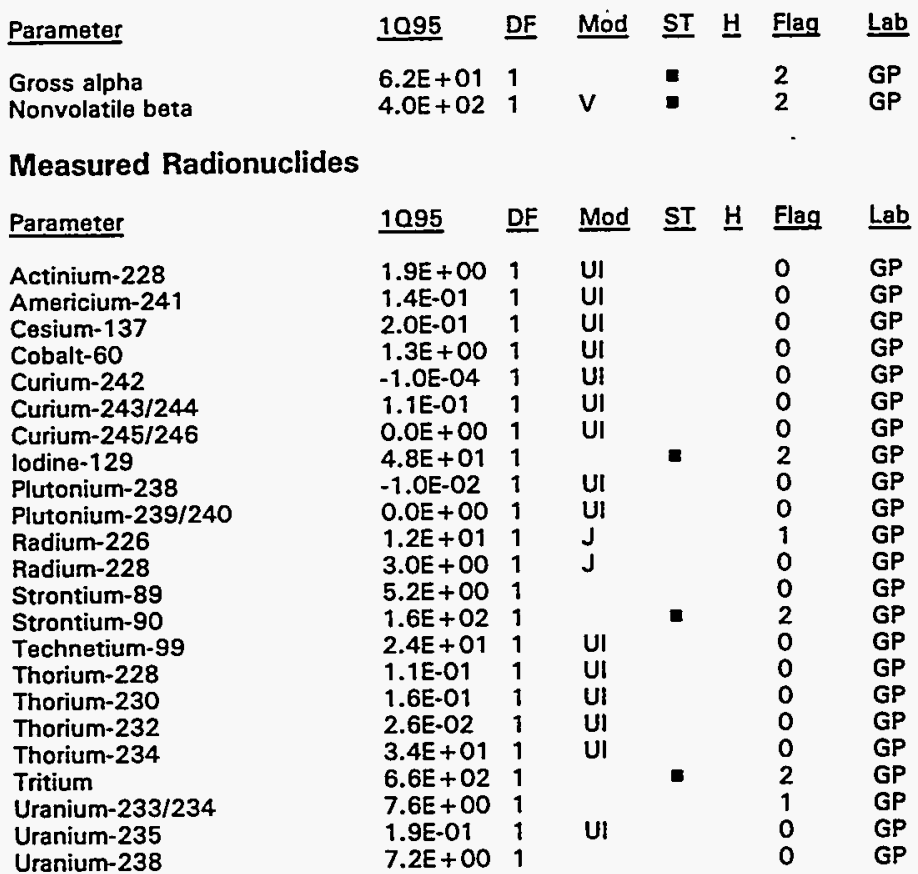

2095 DF Mod ST $\underline{H}$ Flag Lab Unit

pCi/L

2095 DF Mod ST $\underline{H}$ Flag Lab Unit

pCill

$\mathrm{pCi} / \mathrm{L}$

$\mathrm{pCi} / \mathrm{L}$

$\mathrm{pCi} / \mathrm{L}$

$\mathrm{pCi} / \mathrm{L}$

$\mathrm{pCi} / \mathrm{L}$

$\mathrm{pCi} / \mathrm{L}$

$\mathrm{pCi} / \mathrm{L}$

$\mathrm{PCi} / \mathrm{L}$

$\mathrm{pCi} / \mathrm{L}$

$\mathrm{pCi} / \mathrm{L}$

$\mathrm{pCi} / \mathrm{L}$

$\mathrm{pCi} / \mathrm{L}$

pCi/L

$\mathrm{pCi/L}$

PCi/L

pCi/L

pCi/L

pCi/L

$\mathrm{pCi} / \mathrm{mL}$

pCi/L

pCi/L

pCilL

Calculated Radionuclides

Parameter

Americium-243

Nickel-63

Plutonium-241

Plutonium-242
1095 DF Mod ST H Flag Lab

3.4E-04

$1.3 E+00$

$0.0 E+00$

$0.0 E+O 0$

0
0
0
0

Unit

pCi/L

$\mathrm{PCI} / \mathrm{L}$

$\mathrm{pCi} / \mathrm{L}$

$\mathrm{PCI} / \mathrm{L}$

Note: See Appendix B for flagging criteria. Synchronous water levels are measured over a 3-5 day period.

- = exceeded holding time.

- = exceeded groundwater protection standard. 


\section{WELL FSB 91D}

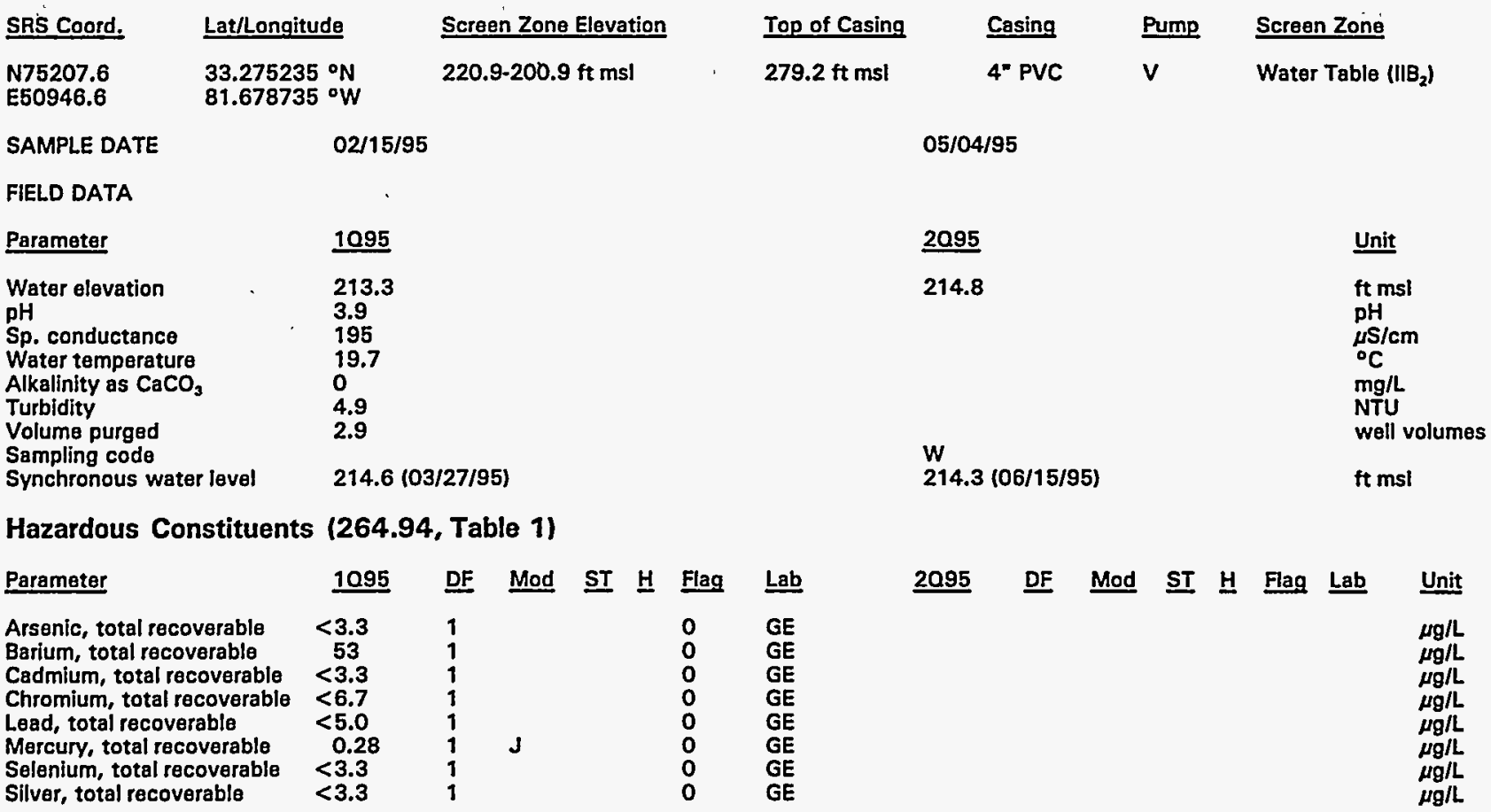

Hazardous Constituents (261 App. VIll/264 App. IX)

\begin{tabular}{|c|c|c|c|c|c|c|c|c|c|c|c|c|c|c|c|}
\hline Paramater & 1095 & DF & Mod & $\underline{\mathbf{S T}}$ & $\underline{\text { H}}$ & Flag & $\underline{\text { Lab }}$ & $\underline{2095}$ & $\underline{D F}$ & Mad & $\underline{\mathbf{S T}}$ & $\underline{\mathbf{H}}$ & Flag & $\underline{\text { Lab }}$ & Unit \\
\hline $\begin{array}{l}\text { Antimony, total recoverable } \\
\text { Benzene } \\
\text { Cobalt, total recoverable } \\
\text { Copper, total recoverable } \\
\text { Cyanide } \\
\text { Nickel, total recoverable } \\
\text { Phenols } \\
\text { Tetrachloroethylene } \\
\text { Thallium, total recoverable } \\
\text { Trichloroethylene } \\
\text { Trichlorofluoromethane } \\
\text { Vanadium, total recoverable } \\
\text { Zinc, total recoverable }\end{array}$ & $\begin{aligned}<3.3 \\
<1.7 \\
<6.7 \\
6.4 \\
<8.3 \\
<6.7 \\
<8.3 \\
<1.7 \\
<3.3 \\
<1.7 \\
<1.7 \\
<13 \\
12\end{aligned}$ & $\begin{array}{l}1 \\
1 \\
1 \\
1 \\
1 \\
1 \\
1 \\
1 \\
1 \\
1 \\
1 \\
1 \\
1\end{array}$ & J1 & & $\bullet$ & $\begin{array}{l}0 \\
0 \\
0 \\
0 \\
0 \\
0 \\
0 \\
0 \\
0 \\
0 \\
0 \\
0 \\
0\end{array}$ & $\begin{array}{l}\mathrm{GE} \\
\mathrm{GE} \\
\mathrm{GE} \\
\mathrm{GE} \\
\mathrm{GE} \\
\mathrm{GE} \\
\mathrm{GE} \\
\mathrm{GE} \\
\mathrm{GE} \\
\mathrm{GE} \\
\mathrm{GE} \\
\mathrm{GE} \\
\mathrm{GE}\end{array}$ & & & & & & & & $\begin{array}{l}\mu g / L \\
\mu g / L \\
\mu g / L \\
\mu g / L \\
\mu g / L \\
\mu g / L \\
\mu g / L \\
\mu g / L \\
\mu g / L \\
\mu g / L \\
\mu g / L \\
\mu g / L \\
\mu g / L\end{array}$ \\
\hline \multicolumn{16}{|c|}{ Water Quality Indicator Parameters } \\
\hline Parameter & 1095 & DF & Mod & $\underline{\mathbf{S T}}$ & $\underline{\text { H}}$ & Flag & $\underline{\text { Lab }}$ & $\underline{2095}$ & $\underline{D F}$ & Mod & $\underline{\mathbf{S T}}$ & $\underline{H}$ & Flag & $\underline{\text { Lab }}$ & Unit \\
\hline $\begin{array}{l}\text { Aluminum, total recoverable } \\
\text { Nitrate as nitrogen } \\
\mathrm{pH}\end{array}$ & $\begin{array}{l}7.200 \\
18,000 \\
3.8\end{array}$ & $\begin{array}{l}1 \\
10 \\
1\end{array}$ & $\begin{array}{l}\mathbf{J} \\
\mathbf{J 1}\end{array}$ & 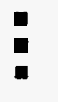 & $\bullet$ & $\begin{array}{l}2 \\
2 \\
1\end{array}$ & $\begin{array}{l}\text { GE } \\
\text { GE } \\
\text { GE }\end{array}$ & & & & & & & & $\underset{\mathrm{pH}}{\mu \mathrm{g} / \mathrm{L}}$ \\
\hline \multicolumn{16}{|c|}{ Common Laboratory Contaminants } \\
\hline Parameter & $\underline{1095}$ & $\underline{\text { DF }}$ & Mod & ST & H & Flag & $\underline{\text { Lab }}$ & $\underline{2095}$ & $\underline{\text { DF }}$ & Mod & $\underline{\mathbf{S T}}$ & 브 & Flag & $\underline{\text { Lab }}$ & Unit \\
\hline $\begin{array}{l}\text { Bis(2-ethylhexyl) phthalate } \\
\text { Dichloromethane }\end{array}$ & $\begin{array}{l}<17 \\
<1.7\end{array}$ & $\begin{array}{l}1 \\
1\end{array}$ & $v$ & & & $\begin{array}{l}0 \\
0\end{array}$ & $\begin{array}{l}\mathrm{GE} \\
\mathrm{GE}\end{array}$ & & & & & & & & $\begin{array}{l}\mu \mathrm{g} / \mathrm{L} \\
\mu \mathrm{g} / \mathrm{L}\end{array}$ \\
\hline
\end{tabular}

Note: See Appendix B for flagging criteria. Synchronous water levels are measured over a 3-5 day period.

- = exceeded holding time.

= exceeded groundwater protection standard. 
WELL FSB 91D (cont.)

Radiological Indicator Parameters

\begin{tabular}{|c|c|c|c|c|c|c|c|c|c|c|c|c|c|c|c|}
\hline Parameter & 1095 & DF & Mod & $\underline{\text { ST }}$ & H & Flag & $\underline{\text { Lab }}$ & $\underline{2095}$ & DF & Mod & $\underline{\text { ST }}$ & $\underline{H}$ & Flag & $\underline{\text { Lab }}$ & $\underline{\text { Unit }}$ \\
\hline $\begin{array}{l}\text { Gross alpha } \\
\text { Nonvolatile beta }\end{array}$ & $\begin{array}{l}1.9 E+02 \\
1.1 E+02\end{array}$ & $\begin{array}{l}1 \\
1\end{array}$ & & 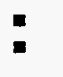 & & $\begin{array}{l}2 \\
2\end{array}$ & $\begin{array}{l}\text { GP } \\
\text { GP }\end{array}$ & & & & & & & & $\begin{array}{l}\text { pCi/L } \\
\text { pCi/L }\end{array}$ \\
\hline \multicolumn{16}{|c|}{ Measured Radionuclides } \\
\hline Parameter & 1095 & $\underline{\mathrm{DF}}$ & Mod & $\underline{\text { ST }}$ & $\underline{\mathrm{H}}$ & Fiag & $\underline{\text { Lab }}$ & $\underline{2095}$ & $\underline{\mathrm{DF}}$ & Mod & $\underline{\mathbf{S T}}$ & $\underline{H}$ & Flag & $\underline{\text { Lab }}$ & Unit \\
\hline $\begin{array}{l}\text { Actinium-228 } \\
\text { Americium-241 } \\
\text { Cesium-137 } \\
\text { Cobalt-60 } \\
\text { Curium-242 } \\
\text { Curium-243/244 } \\
\text { Curium-245/246 } \\
\text { lodine-129 } \\
\text { Plutonium-238 } \\
\text { Plutonium-239/240 } \\
\text { Radium-226 } \\
\text { Radium-228 } \\
\text { Strontium-89 } \\
\text { Strontium-90 } \\
\text { Technetium-99 } \\
\text { Thorium-228 } \\
\text { Thorium-230 } \\
\text { Thorium-232 } \\
\text { Thorium-234 } \\
\text { Tritium } \\
\text { Uranium-233/234 } \\
\text { Uranium-235 } \\
\text { Uranium-238 }\end{array}$ & $\begin{array}{l}2.7 E+00 \\
3.1 E+00 \\
1.8 E-01 \\
3.8 E-01 \\
0.0 E+00 \\
3.0 E+00 \\
2.1 E-01 \\
2.4 E+01 \\
-5.4 E-03 \\
-5.4 E-03 \\
1.6 E+00 \\
3.3 E+00 \\
1.5 E+00 \\
2.3 E+01 \\
-1.0 E+01 \\
1.4 E-01 \\
-1.0 E-02 \\
8.4 E-02 \\
5.0 E+01 \\
3.0 E+00 \\
6.8 E+01 \\
2.9 E+00 \\
1.1 E+02\end{array}$ & $\begin{array}{l}1 \\
1 \\
1 \\
1 \\
1 \\
1 \\
1 \\
1 \\
1 \\
1 \\
1 \\
1 \\
1 \\
1 \\
1 \\
1 \\
1 \\
1 \\
1 \\
1 \\
1 \\
1 \\
1\end{array}$ & $\begin{array}{l}\text { UI } \\
\text { UI } \\
\text { UI } \\
\text { UI } \\
\text { UI } \\
\text { UI } \\
\text { J } \\
\text { UI } \\
\text { UI } \\
\text { UI } \\
\text { U! } \\
\text { UI }\end{array}$ & $\begin{array}{l}= \\
\square\end{array}$ & & $\begin{array}{l}0 \\
0 \\
0 \\
0 \\
0 \\
0 \\
0 \\
2 \\
0 \\
0 \\
0 \\
0 \\
0 \\
2 \\
0 \\
0 \\
0 \\
0 \\
0 \\
0 \\
2 \\
0 \\
2\end{array}$ & $\begin{array}{l}\text { GP } \\
\text { GP } \\
\text { GP } \\
\text { GP } \\
\text { GP } \\
\text { GP } \\
\text { GP } \\
\text { GP } \\
\text { GP } \\
\text { GP } \\
\text { GP } \\
\text { GP } \\
\text { GP } \\
\text { GP } \\
\text { GP } \\
\text { GP } \\
\text { GP } \\
\text { GP } \\
\text { GP } \\
\text { GP } \\
\text { GP } \\
\text { GP } \\
\text { GP }\end{array}$ & & & & & & & & $\begin{array}{l}\mathrm{pCi} / \mathrm{L} \\
\mathrm{pCi} / \mathrm{L} \\
\mathrm{pCi} / \mathrm{L} \\
\mathrm{pCi} / \mathrm{L} \\
\mathrm{pCi} / \mathrm{L} \\
\mathrm{pCi} / \mathrm{L} \\
\mathrm{pCi} / \mathrm{L} \\
\mathrm{pCi} / \mathrm{L} \\
\mathrm{pCi} / \mathrm{L} \\
\mathrm{pCi} / \mathrm{L} \\
\mathrm{p} C i / L \\
\mathrm{pCi} / \mathrm{L} \\
\mathrm{pCi} / \mathrm{L} \\
\mathrm{pCi} / \mathrm{L} \\
\mathrm{pCi} / \mathrm{L} \\
\mathrm{pCi} / \mathrm{L} \\
\mathrm{pCi} / \mathrm{L} \\
\mathrm{pCi} / \mathrm{L} \\
\mathrm{pCi} / \mathrm{L} \\
\mathrm{pCi} / \mathrm{mL} \\
\mathrm{pCi} / \mathrm{L} \\
\mathrm{pCi} / \mathrm{L} \\
\mathrm{pCi} / \mathrm{L}\end{array}$ \\
\hline \multicolumn{16}{|c|}{ Calculated Radionuclides } \\
\hline Parameter & 1095 & DF & Mod & $\underline{\text { ST }}$ & $\underline{H}$ & Flag & $\underline{\text { Lab }}$ & $\underline{2095}$ & $\underline{\text { DF }}$ & $\underline{\text { Mad }}$ & $\underline{\mathbf{S T}}$ & $\underline{H}$ & Flag & $\underline{\text { Lab }}$ & Unit \\
\hline $\begin{array}{l}\text { Americium-243 } \\
\text { Nickel-63 } \\
\text { Plutonium-241 } \\
\text { Plutonium-242 }\end{array}$ & $\begin{array}{l}8.9 \mathrm{E}-03 \\
3.8 \mathrm{E}-01 \\
-2.2 \mathrm{E}-02 \\
-1.1 \mathrm{E}-07\end{array}$ & & & & & $\begin{array}{l}0 \\
0 \\
0 \\
0\end{array}$ & & & & & & & & & $\begin{array}{l}\text { pCi/L } \\
\text { pCi/L } \\
\text { pCi/L } \\
\text { pCi/L }\end{array}$ \\
\hline
\end{tabular}

Note: See Appendix B for flagging criteria. Synchronous water levels are measured over a 3-5 day period.

$\bullet=$ exceeded holding time.

- = exceeded groundwater protection standard. 


\section{WELL FSB 92C}

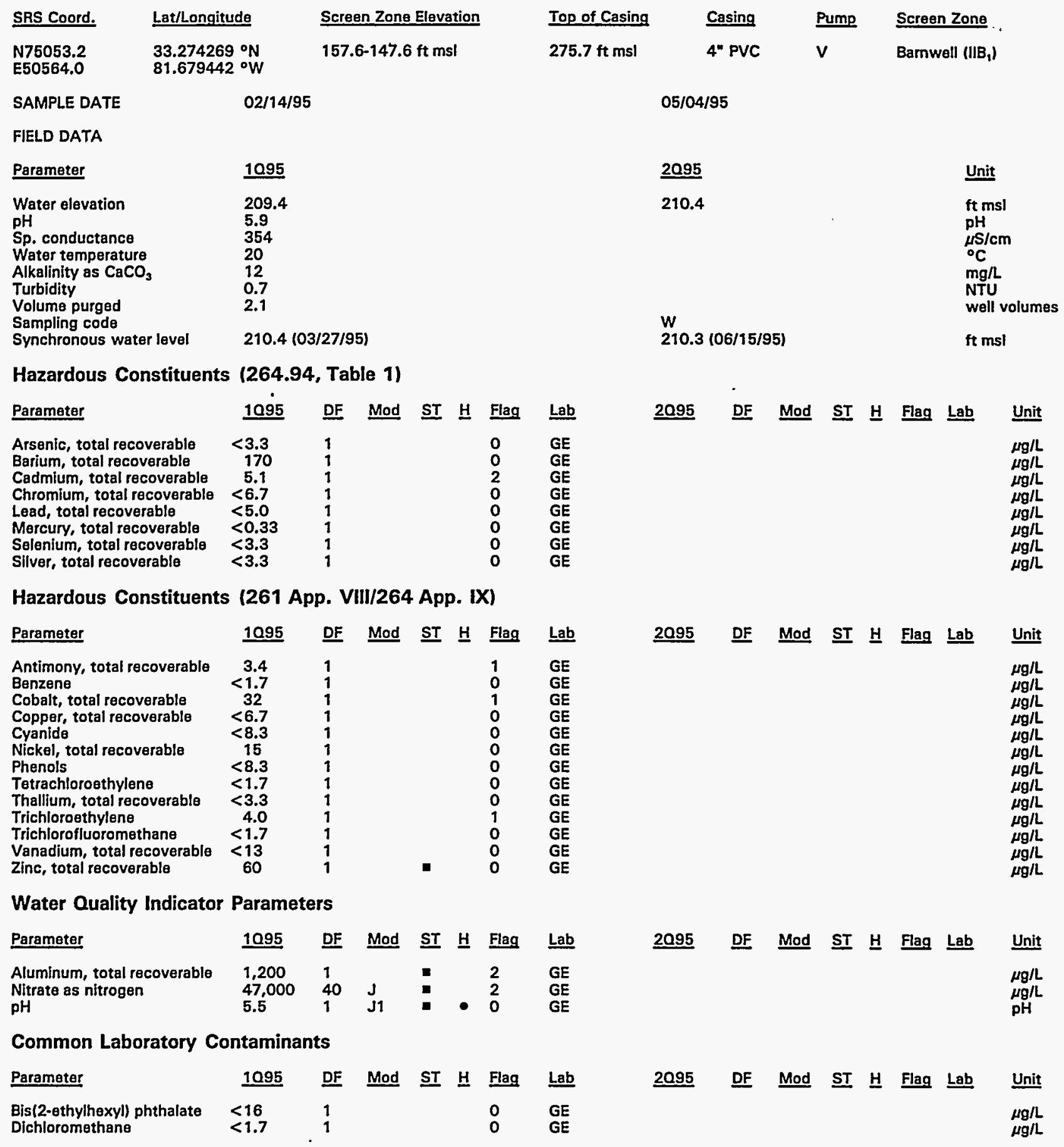

Note: See Appendix B for flagging criteria. Synchronous water levels are measured over a 3-5 day period. - = exceeded holding time.

I = exceeded groundwater protection standard. 
WELL FSB 92C (cont.)

Radiological Indicator Parameters

\begin{tabular}{|c|c|c|c|c|c|c|c|c|c|c|c|c|c|c|c|}
\hline Parameter & 1095 & DF & Mod & $\underline{\text { ST }}$ & $\underline{\mathrm{H}}$ & Flag & $\underline{\text { Lab }}$ & $\underline{2095}$ & $\underline{\mathrm{DF}}$ & Mod & $\underline{\mathbf{S T}}$ & 보 & Flag & Lab & $\underline{\text { Unit }}$ \\
\hline $\begin{array}{l}\text { Gross alpha } \\
\text { Nonvolatile beta }\end{array}$ & $\begin{array}{l}5.2 E+01 \\
3.8 E+02\end{array}$ & $\begin{array}{l}1 \\
1\end{array}$ & & $=$ & & $\begin{array}{l}2 \\
2\end{array}$ & $\begin{array}{l}\text { GP } \\
\text { GP }\end{array}$ & & & & & & & & $\begin{array}{l}\mathrm{pCi} / \mathrm{L} \\
\mathrm{pCi} / \mathrm{L}\end{array}$ \\
\hline \multicolumn{16}{|c|}{ Measured Radionuclides } \\
\hline Parameter & 1095 & $\underline{\mathrm{DF}}$ & Mod & $\underline{\mathbf{S T}}$ & $\underline{H}$ & Flag & $\underline{\text { Lab }}$ & $\underline{2095}$ & $\underline{D F}$ & Mod & $\underline{\text { ST }}$ & 브 & Flag & $\underline{L a b}$ & $\underline{\text { Unit }}$ \\
\hline $\begin{array}{l}\text { Actinium-228 } \\
\text { Americium-241 } \\
\text { Cesium-137 } \\
\text { Cobalt-60 } \\
\text { Curium-242 } \\
\text { Curium-243/244 } \\
\text { Curium-245/246 } \\
\text { lodine-129 } \\
\text { Plutonium-238 } \\
\text { Plutonium-239/240 } \\
\text { Radium-226 } \\
\text { Radium-228 } \\
\text { Strontium-89 } \\
\text { Strontium-90 } \\
\text { Technetium-99 } \\
\text { Thorium-228 } \\
\text { Thorium-230 } \\
\text { Thorium-232 } \\
\text { Thorium-234 } \\
\text { Tritium } \\
\text { Uranium-233/234 } \\
\text { Uranium-235 } \\
\text { Uranium-238 }\end{array}$ & $\begin{array}{l}1.3 E+01 \\
-5.0 E-03 \\
-2.0 E+00 \\
1.7 E+00 \\
-1.1 E-02 \\
1.2 E-01 \\
-5.0 E-03 \\
1.5 E+01 \\
2.3 E-02 \\
0.0 E+00 \\
1.9 E+01 \\
2.3 E+00 \\
3.1 E+01 \\
9.5 E+01 \\
3.8 E+01 \\
2.2 E-01 \\
4.5 E-02 \\
-1.7 E-02 \\
1.6 E+02 \\
1.8 E+03 \\
5.6 E-01 \\
9.1 E-03 \\
4.3 E-01\end{array}$ & $\begin{array}{l}1 \\
1 \\
1 \\
1 \\
1 \\
1 \\
1 \\
1 \\
1 \\
1 \\
1 \\
1 \\
1 \\
1 \\
1 \\
1 \\
1 \\
1 \\
1 \\
1 \\
1 \\
1 \\
1\end{array}$ & $\begin{array}{l}\text { UI } \\
\text { UI } \\
\text { UI } \\
\text { UI } \\
\text { UI } \\
\text { UI } \\
\text { UII } \\
\text { UI } \\
\text { UI } \\
\text { J }\end{array}$ & 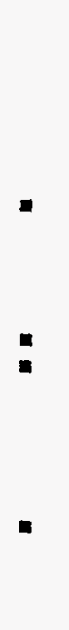 & & $\begin{array}{l}0 \\
0 \\
0 \\
0 \\
0 \\
0 \\
0 \\
2 \\
0 \\
0 \\
1 \\
0 \\
2 \\
2 \\
0 \\
0 \\
0 \\
0 \\
0 \\
2 \\
0 \\
0 \\
0\end{array}$ & $\begin{array}{l}\text { GP } \\
\text { GP } \\
\text { GP } \\
\text { GP } \\
\text { GP } \\
\text { GP } \\
\text { GP } \\
\text { GP } \\
\text { GP } \\
\text { GP } \\
\text { GP } \\
\text { GP } \\
\text { GP } \\
\text { GP } \\
\text { GP } \\
\text { GP } \\
\text { GP } \\
\text { GP } \\
\text { GP } \\
\text { GP } \\
\text { GP } \\
\text { GP } \\
\text { GP }\end{array}$ & & & & & & & & 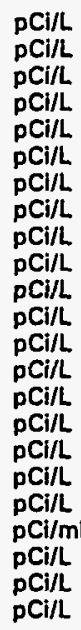 \\
\hline \multicolumn{16}{|c|}{ Calculated Radionuclides } \\
\hline Parameter & 1095 & $\underline{D F}$ & Mod & $\underline{\mathbf{S T}}$ & $\underline{H}$ & Flag & Lab & $\underline{2095}$ & DF & Mod & $\underline{\text { ST }}$ & $\underline{H}$ & Flag & $\underline{\text { Lab }}$ & Unit \\
\hline $\begin{array}{l}\text { Americium-243 } \\
\text { Nickel-63 } \\
\text { Plutonium-241 } \\
\text { Plutonium-242 }\end{array}$ & $\begin{array}{l}3.7 E-04 \\
1.7 E+00 \\
0.0 E+00 \\
0.0 E+00\end{array}$ & & & & & $\begin{array}{l}0 \\
0 \\
0 \\
0\end{array}$ & & & & & & & & & $\begin{array}{l}\mathrm{pCi} / \mathrm{L} \\
\mathrm{pCCi/L} \\
\mathrm{pCCi}^{2} \\
\mathrm{pCi/L}\end{array}$ \\
\hline
\end{tabular}

Note: See Appendix B for flagging criteria. Synchronous water levels are measured over a 3-5 day period.

- = exceeded holding time.

- = exceeded groundwater protection standard. 


\section{WELL FSB 92D}

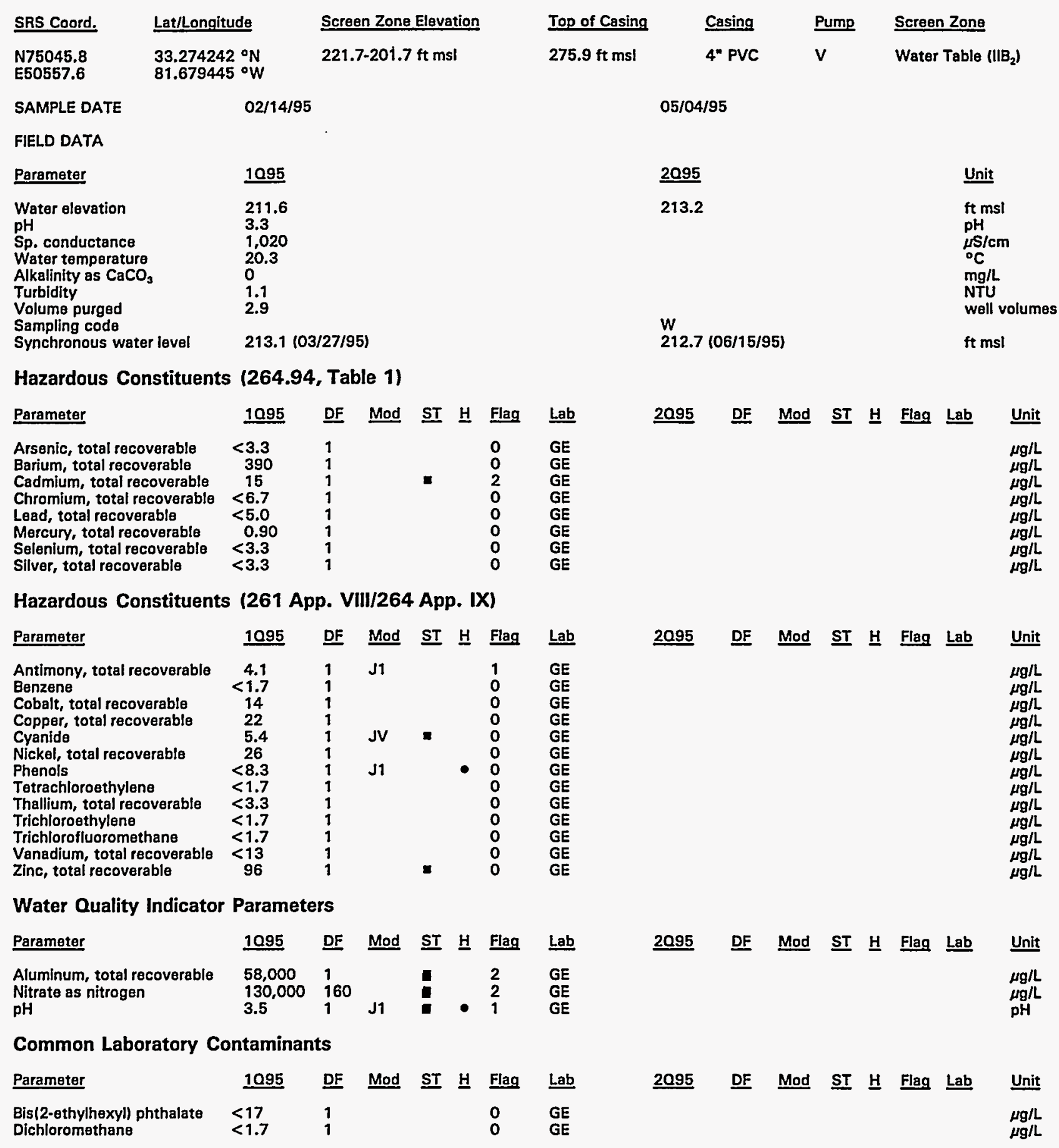

Note: See Appendix B for flagging criteria. Synchronous water levels are measured over a 3-5 day period.

- = exceeded holding time.

- exceeded groundwater protection standard. 
WELL FSB 92D (cont.)

Radiological Indicator Parameters

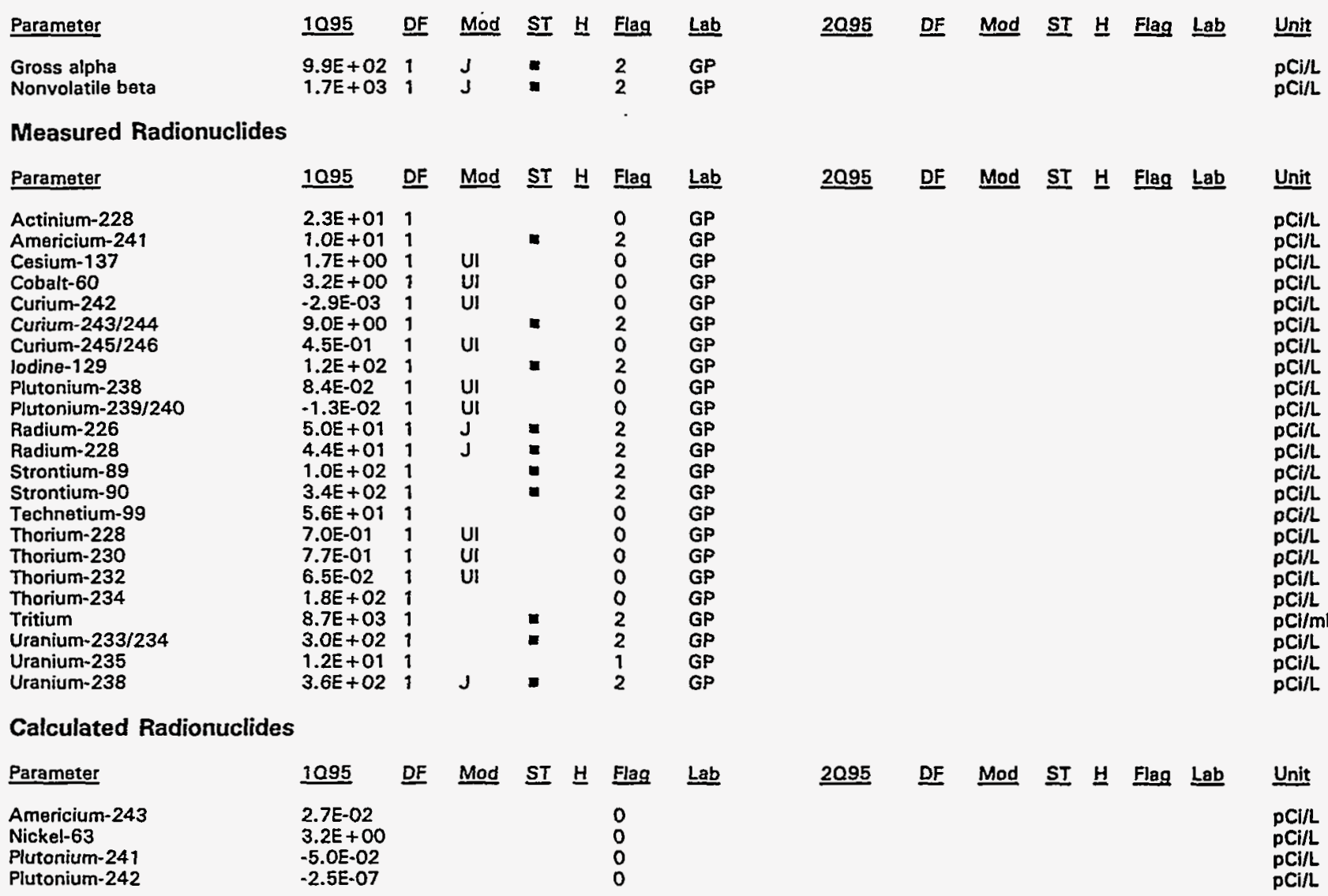

Note: See Appendix B for flagging criteria. Synchronous water levels are measured over a $3-5$ day period.

- = exceeded holding time.

- = exceeded groundwater protection standard. 


\section{WELL FSB 93C}

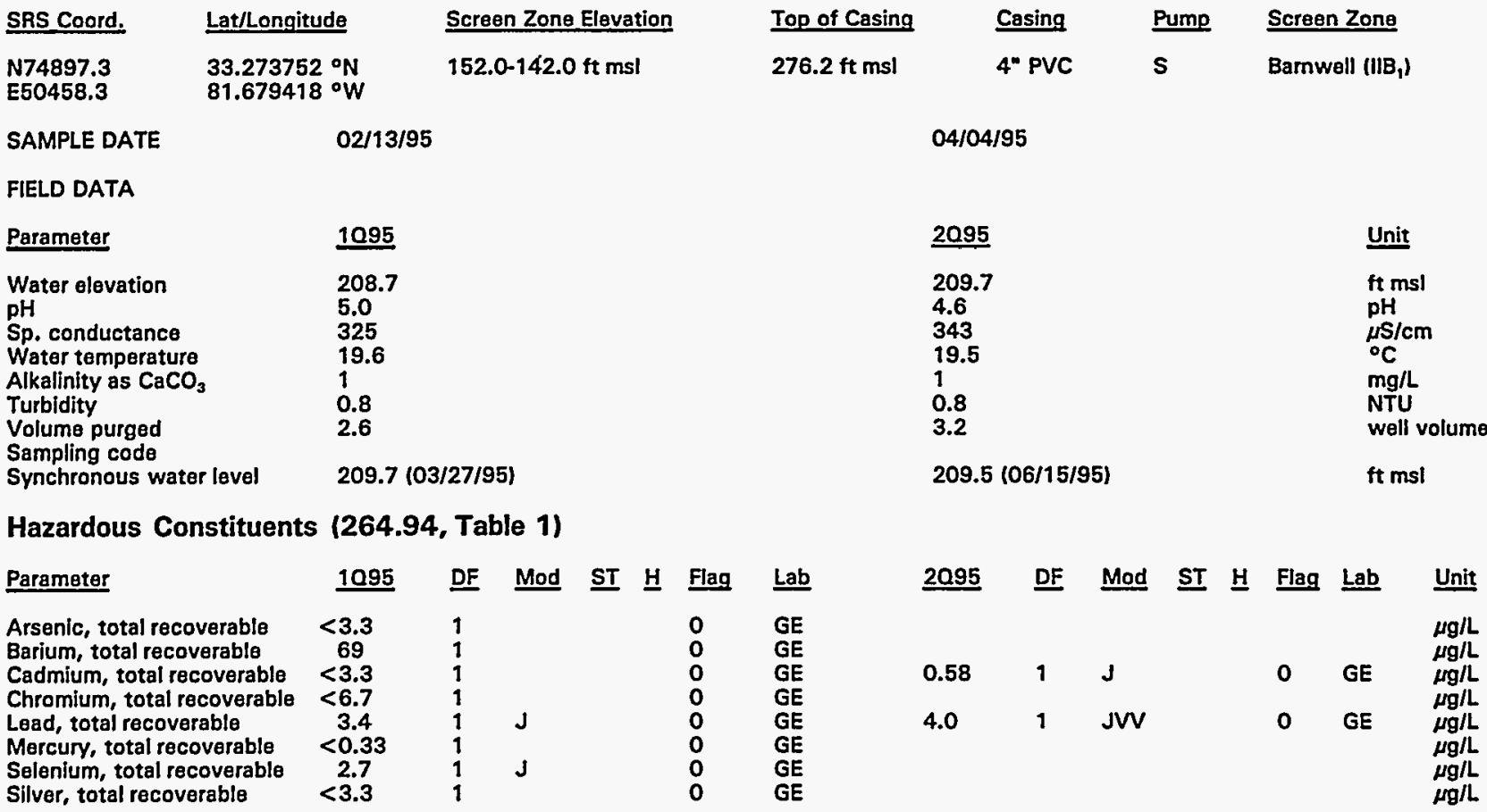

Hazardous Constituents (261 App. Vill/264 App. IX)

\begin{tabular}{|c|c|c|c|c|c|c|c|c|c|c|c|c|c|c|c|}
\hline Paramater & 1095 & DF & Mod & $\underline{\text { ST }}$ & $\underline{H}$ & Flag & $\underline{L a b}$ & 2095 & DF & Mod & ST & $\underline{H}$ & Flag & Lab & Unit \\
\hline $\begin{array}{l}\text { Antimony, total recoverable } \\
\text { Benzene } \\
\text { Cobalt, total recoverable } \\
\text { Copper, total recoverable } \\
\text { Cyanide } \\
\text { Nickel, total recoverable } \\
\text { Phenols } \\
\text { Tetrachloroethylene } \\
\text { Thallium, total recoverable } \\
\text { Trichloroethylene } \\
\text { Trichlorofluoromethane } \\
\text { Vanadium, total recoverable } \\
\text { Zinc, total recoverable }\end{array}$ & $\begin{array}{r}3.0 \\
<1.7 \\
<6.7 \\
6.3 \\
<8.3 \\
7.1 \\
<8.3 \\
<1.7 \\
<3.3 \\
1.8 \\
2.5 \\
<13 \\
99\end{array}$ & $\begin{array}{l}1 \\
1 \\
1 \\
1 \\
1 \\
1 \\
1 \\
1 \\
1 \\
1 \\
1 \\
1 \\
1\end{array}$ & $\begin{array}{l}\mathbf{J} \\
\mathbf{J}\end{array}$ & a & & $\begin{array}{l}0 \\
0 \\
0 \\
0 \\
0 \\
0 \\
0 \\
0 \\
0 \\
0 \\
0 \\
0 \\
0\end{array}$ & $\begin{array}{l}\mathrm{GE} \\
\mathrm{GE} \\
\mathrm{GE} \\
\mathrm{GE} \\
\mathrm{GE} \\
\mathrm{GE} \\
\mathrm{GE} \\
\mathrm{GE} \\
\mathrm{GE} \\
\mathrm{GE} \\
\mathrm{GE} \\
\mathrm{GE} \\
\mathrm{GE}\end{array}$ & & & & & & & & $\begin{array}{l}\mu \mathrm{g} / \mathrm{L} \\
\mu \mathrm{g} / \mathrm{L} \\
\mu \mathrm{g} / \mathrm{L} \\
\mu \mathrm{g} / \mathrm{L} \\
\mu \mathrm{g} / \mathrm{L} \\
\mu \mathrm{g} / \mathrm{L} \\
\mu \mathrm{g} / \mathrm{L} \\
\mu \mathrm{g} / \mathrm{L} \\
\mu \mathrm{g} / \mathrm{L} \\
\mu \mathrm{g} / \mathrm{L} \\
\mu \mathrm{g} / \mathrm{L} \\
\mu \mathrm{g} / \mathrm{L} \\
\mu \mathrm{g} / \mathrm{L}\end{array}$ \\
\hline \multicolumn{16}{|c|}{ Water Quality Indicator Parameters } \\
\hline Parameter & 1095 & DF & Mod & $\underline{\mathbf{S T}}$ & $\underline{H}$ & Flag & $\underline{\text { Lab }}$ & $\underline{2095}$ & DF & Mod & $\underline{\text { ST }}$ & $\underline{\mathbf{H}}$ & Flag & $\underline{\text { Lab }}$ & Unit \\
\hline $\begin{array}{l}\text { Aluminum, total recoverable } \\
\text { Nitrate as nitrogen } \\
\text { pH }\end{array}$ & $\begin{array}{l}49 \\
37,000 \\
6.8\end{array}$ & $\begin{array}{l}1 \\
40 \\
1\end{array}$ & J1 & 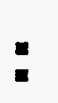 & - & $\begin{array}{l}1 \\
2 \\
0\end{array}$ & $\begin{array}{l}\text { GE } \\
\text { GE } \\
\text { GE }\end{array}$ & $\begin{array}{l}46,000 \\
5.1\end{array}$ & $\begin{array}{l}40 \\
1\end{array}$ & J1 & a & - & $\begin{array}{l}2 \\
0\end{array}$ & $\begin{array}{l}\text { GE } \\
\text { GE }\end{array}$ & $\begin{array}{l}\mu \mathrm{g} / \mathrm{L} \\
\mathrm{pH} / \mathrm{L}\end{array}$ \\
\hline \multicolumn{16}{|c|}{ Common Laboratory Contaminants } \\
\hline Parameter & 1095 & DF & Mod & $\underline{\text { ST }}$ & $\underline{H}$ & Flag & $\underline{\text { Lab }}$ & $\underline{2095}$ & $\underline{\text { DF }}$ & Mod & $\underline{\text { ST }}$ & $\underline{\mathrm{H}}$ & Flag & Lab & $\underline{\text { Unit }}$ \\
\hline $\begin{array}{l}\text { Bis(2-ethylhexyl) phthalate } \\
\text { Dichloromethane }\end{array}$ & $\begin{array}{l}<17 \\
<1.7\end{array}$ & 1 & & & & $\begin{array}{l}0 \\
0\end{array}$ & $\begin{array}{l}\text { GE } \\
\text { GE }\end{array}$ & & & & & & & & $\underset{\mu g / L}{\mu g / L}$ \\
\hline
\end{tabular}

Note: See Appendix B for flagging criteria. Synchronous water levels are measured over a 3-5 day period.

- = exceeded holding time.

- = exceeded groundwater protection standard. 
WELL FSB 93C (cont.)

Radiological Indicator Parameters

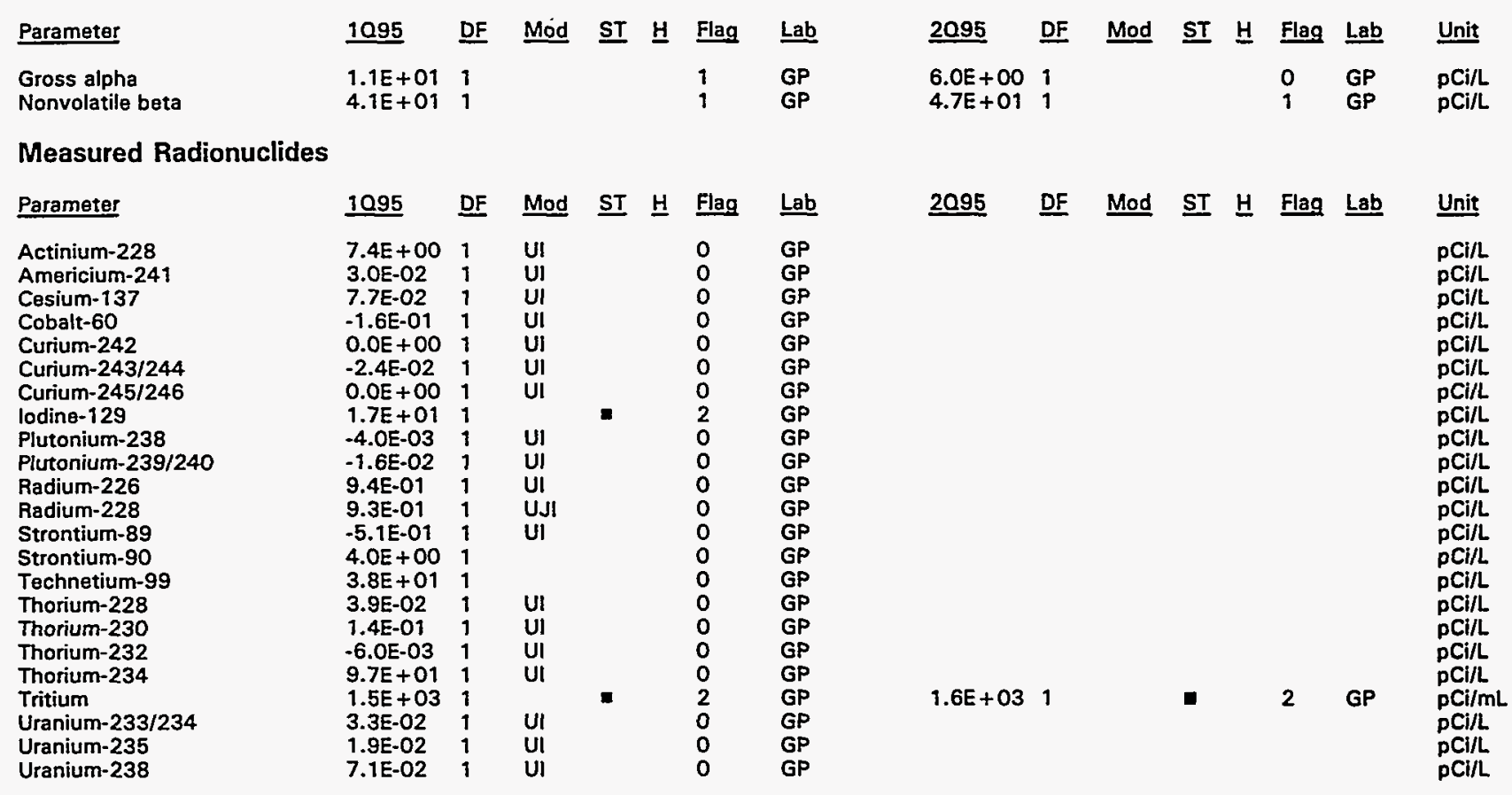

Calculated Radionuclides

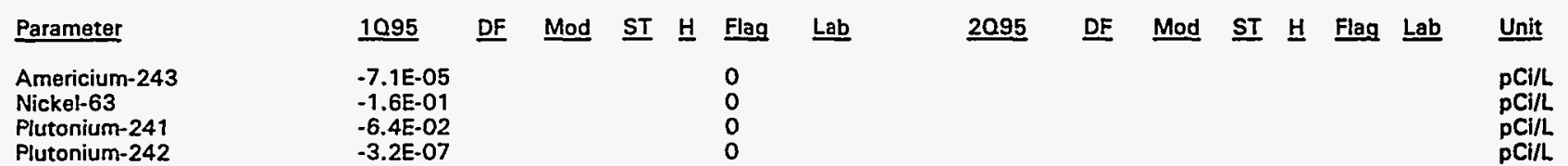

Note: See Appendix B for flagging criteria. Synchronous water levels are measured over a 3-5 day period.

- = exceeded holding time.

- = exceeded groundwater protection standard. 
WELL FSB 93D

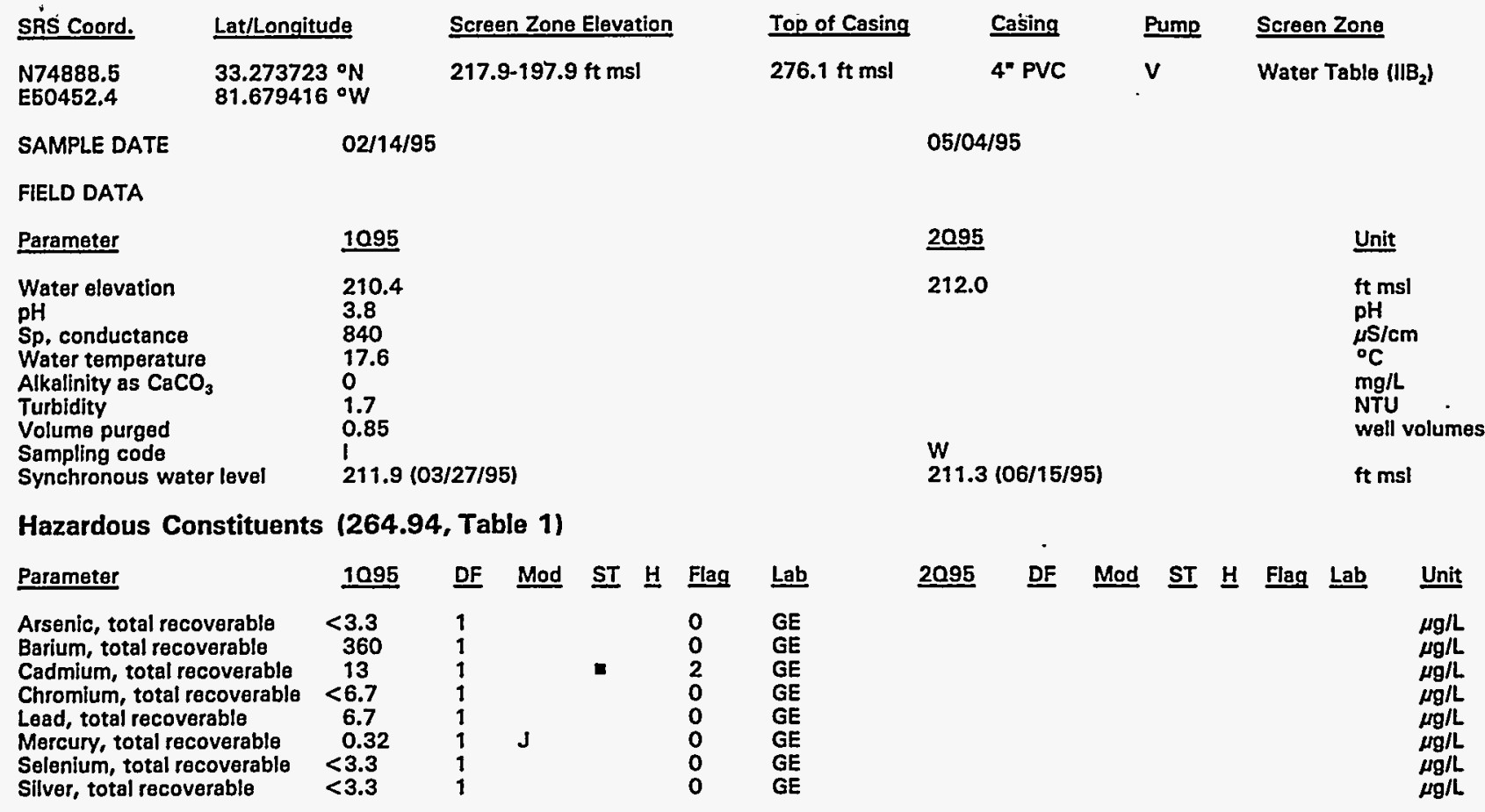

Hazardous Constituents (261 App. VIII/264 App. IX)

\begin{tabular}{|c|c|c|c|c|c|c|c|c|c|c|c|c|c|c|c|}
\hline Parameter & 1095 & DF & Mod & $\underline{\text { ST }}$ & $\underline{\mathrm{H}}$ & Flag & $\underline{\text { Lab }}$ & $\underline{2095}$ & DF & Mod & $\underline{\text { ST }}$ & $\underline{H}$ & Flag & Lab & Unit \\
\hline $\begin{array}{l}\text { Antimony, total recoverable } \\
\text { Benzene } \\
\text { Cobalt, total recoverable } \\
\text { Copper, total recoverable } \\
\text { Cyanide } \\
\text { Nickel, total recoverable } \\
\text { Phenols } \\
\text { Tetrachloroethylene } \\
\text { Thallium, total recoverable } \\
\text { Trichloroethylene } \\
\text { Trichlorofluoromethane } \\
\text { Vanadium, total recoverable } \\
\text { Zinc, total recoverable }\end{array}$ & $\begin{array}{r}4.1 \\
<1.7 \\
10 \\
31 \\
<8.3 \\
20 \\
<8.3 \\
<1.7 \\
<3.3 \\
<1.7 \\
<1.7 \\
<13 \\
62\end{array}$ & $\begin{array}{l}1 \\
1 \\
1 \\
1 \\
1 \\
1 \\
1 \\
1 \\
1 \\
1 \\
1 \\
1 \\
1\end{array}$ & $\begin{array}{l}v \\
J 1\end{array}$ & $\bullet$ & • & $\begin{array}{l}1 \\
0 \\
0 \\
0 \\
0 \\
0 \\
0 \\
0 \\
0 \\
0 \\
0 \\
0 \\
0\end{array}$ & $\begin{array}{l}\mathrm{GE} \\
\mathrm{GE} \\
\mathrm{GE} \\
\mathrm{GE} \\
\mathrm{GE} \\
\mathrm{GE} \\
\mathrm{GE} \\
\mathrm{GE} \\
\mathrm{GE} \\
\mathrm{GE} \\
\mathrm{GE} \\
\mathrm{GE} \\
\mathrm{GE}\end{array}$ & & & & & & & & $\begin{array}{l}\mu g / L \\
\mu g / L \\
\mu g / L \\
\mu g / L \\
\mu g / L \\
\mu g / L \\
\mu g / L \\
\mu g / L \\
\mu g / L \\
\mu g / L \\
\mu g / L \\
\mu g / L \\
\mu g / L\end{array}$ \\
\hline \multicolumn{16}{|c|}{ Water Quality Indicator Parameters } \\
\hline Parameter & 1095 & DF & Mod & $\underline{\text { ST }}$ & 브 & Flag & $\underline{\text { Lab }}$ & $\underline{2095}$ & $\underline{\mathrm{DF}}$ & Mod & $\underline{\text { ST }}$ & $\underline{H}$ & Flag & Lab & Unit \\
\hline $\begin{array}{l}\text { Aluminum, total recoverable } \\
\text { Nitrate as nitrogen } \\
\text { pH }\end{array}$ & $\begin{array}{l}34,000 \\
120,000 \\
3.9\end{array}$ & $\begin{array}{l}1 \\
80 \\
1\end{array}$ & J1 & 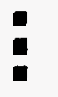 & & $\begin{array}{l}2 \\
2 \\
1\end{array}$ & $\begin{array}{l}\mathrm{GE} \\
\mathrm{GE} \\
\mathrm{GE}\end{array}$ & & & & & & & & $\begin{array}{l}\mu \mathrm{g} / \mathrm{L} \\
\mu \mathrm{g} / \mathrm{L} \\
\mathrm{pH}\end{array}$ \\
\hline \multicolumn{16}{|c|}{ Common Laboratory Contaminants } \\
\hline Parameter & 1095 & $\underline{D F}$ & Mod & $\underline{\mathbf{S T}}$ & $\underline{H}$ & Flag & $\underline{\text { Lab }}$ & $\underline{2095}$ & $\underline{\text { DF }}$ & $\underline{\text { Mod }}$ & $\underline{\text { ST }}$ & $\underline{\mathbf{H}}$ & Flag & Lab & Unit \\
\hline $\begin{array}{l}\text { Bis(2-ethylhexyl) phthalate } \\
\text { Dichloromethane }\end{array}$ & $\begin{array}{l}<16 \\
<1.7\end{array}$ & $\begin{array}{l}1 \\
1\end{array}$ & & & & $\begin{array}{l}0 \\
0\end{array}$ & $\begin{array}{l}\text { GE } \\
\text { GE }\end{array}$ & & & & & & & & $\begin{array}{l}\mu g / L \\
\mu g / L\end{array}$ \\
\hline
\end{tabular}

Note: See Appendix B for flagging criteria. Synchronous water levels are measured over a 3-5 day period.

- = exceeded holding time.

- = exceeded groundwater protection standard. 
WELL FSB 93D (cont.)

Radiological Indicator Parameters

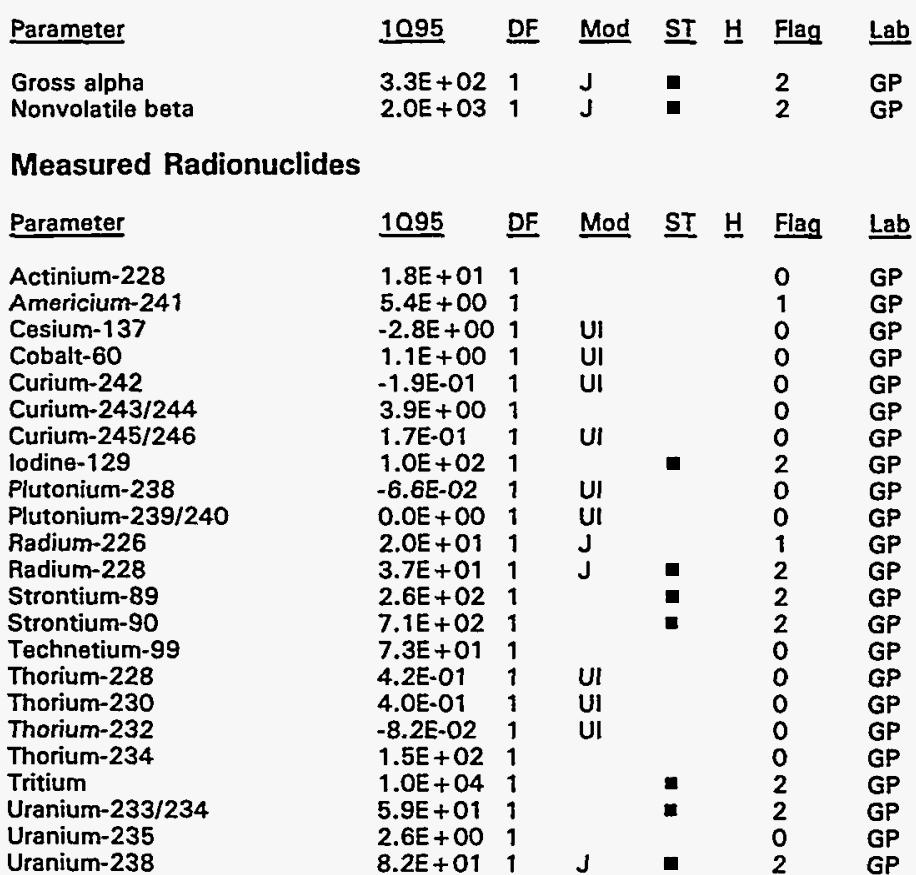

2095 DF Mod ST H Fiag Lab Unit

pCi/l

$\mathrm{pCi} / \mathrm{L}$

Calculated Radionuclides

Parameter

1095

DF Mod ST 브 Flag Lab

$\underline{2095}$

DF Mod ST $\underline{H}$ Flag Lab

Unit

Americium-243

Nickel-63

$1.2 E-02$

$1.1 E+00$

$0.0 E+00$

Plutonium-241

$O . O E+O O$

pCi/L

pCi/l

$\mathrm{pCi} / \mathrm{L}$

$\mathrm{pCi} / \mathrm{L}$

$\mathrm{pCi} / \mathrm{L}$

PCI/L

pCi/L

pCill

$\mathrm{pCi} / \mathrm{L}$

pCi/L

$\mathrm{PCI} / \mathrm{L}$

$\mathrm{PCi}$

$\mathrm{pCill}$

$\mathrm{pCi} / \mathrm{L}$

$\mathrm{pCi} / \mathrm{L}$

$\mathrm{pCI} / \mathrm{L}$

$\mathrm{pCl} / \mathrm{mL}$

$\mathrm{pCin}$

$\mathrm{pCl} / \mathrm{L}$

pCi/L

Plutonium-242

0.0

pCi/L

pCi/L

pCilL

Note: See Appendix B for flagging criteria. Synchronous water levels are measured over a 3-5 day period. - = exceeded holding time.

- = exceeded groundwater protection standard. 


\section{WELL FSB 94C}

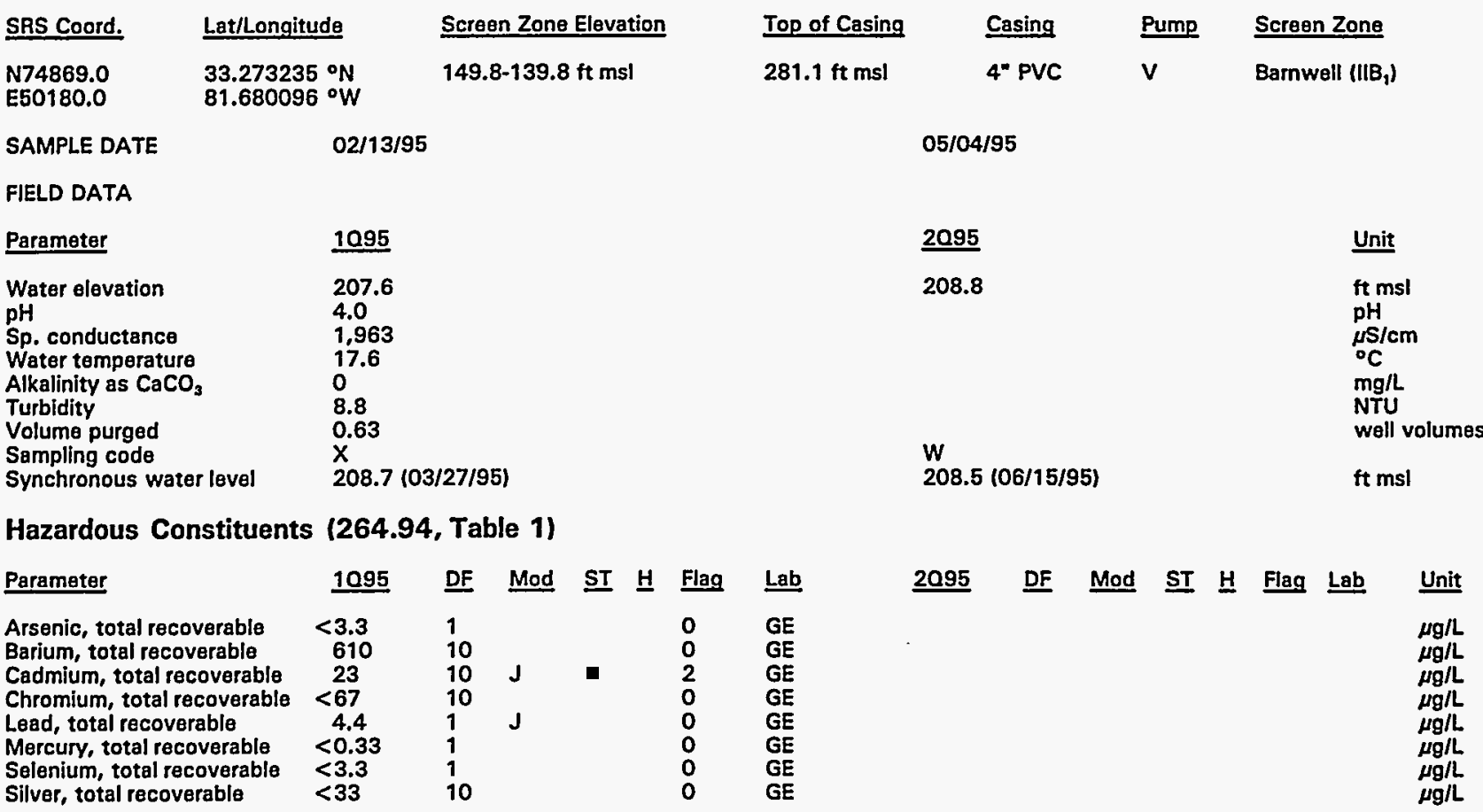

Hazardous Constituents (261 App. VIII/264 App. IX)

\begin{tabular}{|c|c|c|c|c|c|c|c|c|c|c|c|c|c|c|c|}
\hline Parameter & 1095 & $\underline{\text { DF }}$ & Mod & $\underline{\text { ST }}$ & $\underline{H}$ & Flag & $\underline{\text { Lab }}$ & $\underline{2095}$ & $\underline{D F}$ & Mod & $\underline{\text { ST }}$ & $\underline{H}$ & Flag & $\underline{\text { Lab }}$ & $\underline{\text { Unit }}$ \\
\hline $\begin{array}{l}\text { Antimony, total recoverable } \\
\text { Benzene } \\
\text { Cobalt, total recoverable } \\
\text { Copper, total recoverable } \\
\text { Cyanide } \\
\text { Nickel, total recoverable } \\
\text { Phenols } \\
\text { Tetrachloroethylene } \\
\text { Thallium, total recoverable } \\
\text { Trichloroethylene } \\
\text { Trichlorofluoromethane } \\
\text { Vanadlum, total recoverable } \\
\text { Zinc, total recoverable }\end{array}$ & $\begin{array}{r}<3.3 \\
<1.7 \\
670 \\
47 \\
<8.3 \\
180 \\
<8.3 \\
<1.7 \\
<3.3 \\
<1.7 \\
<1.7 \\
<130 \\
320\end{array}$ & $\begin{array}{l}1 \\
1 \\
10 \\
10 \\
1 \\
10 \\
1 \\
1 \\
1 \\
1 \\
1 \\
10 \\
10\end{array}$ & J1 & - & - & $\begin{array}{l}0 \\
0 \\
2 \\
0 \\
0 \\
2 \\
0 \\
0 \\
0 \\
0 \\
0 \\
0 \\
0\end{array}$ & $\begin{array}{l}\mathrm{GE} \\
\mathrm{GE} \\
\mathrm{GE} \\
\mathrm{GE} \\
\mathrm{GE} \\
\mathrm{GE} \\
\mathrm{GE} \\
\mathrm{GE} \\
\mathrm{GE} \\
\mathrm{GE} \\
\mathrm{GE} \\
\mathrm{GE} \\
\mathrm{GE}\end{array}$ & - & • & & & & & & $\begin{array}{l}\mu \mathrm{g} / \mathrm{L} \\
\mu \mathrm{g} / \mathrm{L} \\
\mu \mathrm{g} / \mathrm{L} \\
\mu \mathrm{g} / \mathrm{L} \\
\mu \mathrm{g} / \mathrm{L} \\
\mu \mathrm{g} / \mathrm{L} \\
\mu \mathrm{g} / \mathrm{L} \\
\mu \mathrm{g} / \mathrm{L} \\
\mu \mathrm{g} / \mathrm{L} \\
\mu \mathrm{g} / \mathrm{L} \\
\mu \mathrm{g} / \mathrm{L} \\
\mu \mathrm{g} / \mathrm{L} \\
\mu \mathrm{g} / \mathrm{L}\end{array}$ \\
\hline \multicolumn{16}{|c|}{ Water Quality Indicator Parameters } \\
\hline Parameter & 1095 & $\underline{D F}$ & Mod & $\underline{\mathbf{S T}}$ & $\underline{H}$ & Flag & $\underline{\text { Lab }}$ & $\underline{2095}$ & $\underline{D F}$ & Mod & $\underline{\mathbf{S T}}$ & 브 & Flag & $\underline{\text { Lab }}$ & $\underline{\text { Unit }}$ \\
\hline $\begin{array}{l}\text { Aluminum, total recoverable } \\
\text { Nitrate as nitrogen } \\
\text { pH }\end{array}$ & $\begin{array}{l}46,000 \\
250,000 \\
4.5\end{array}$ & $\begin{array}{l}10 \\
160 \\
1\end{array}$ & J1 & 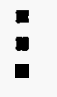 & & $\begin{array}{l}2 \\
2 \\
0\end{array}$ & $\begin{array}{l}\mathrm{GE} \\
\mathrm{GE} \\
\mathrm{GE}\end{array}$ & & & & & & & & $\underset{\mathrm{pH}}{\mu \mathrm{g} / \mathrm{L}}$ \\
\hline \multicolumn{16}{|c|}{ Common Laboratory Contaminants } \\
\hline Parameter & $\underline{1095}$ & $\underline{\mathrm{DF}}$ & Mod & $\underline{\text { ST }}$ & H & Flag & $\underline{\text { Lab }}$ & $\underline{2095}$ & DF & Mod & $\underline{\mathbf{S T}}$ & 브 & Flag & $\underline{\text { Lab }}$ & Unit \\
\hline $\begin{array}{l}\text { Bis(2-ethylhexyl) phthalate } \\
\text { Dichloromethane }\end{array}$ & $\begin{array}{l}<17 \\
<1.7\end{array}$ & $\begin{array}{l}1 \\
1\end{array}$ & & & & $\begin{array}{l}0 \\
0\end{array}$ & $\begin{array}{l}\text { GE } \\
\text { GE }\end{array}$ & & & & & & & & $\begin{array}{l}\mu g / L \\
\mu g / L\end{array}$ \\
\hline
\end{tabular}

Note: See Appendix B for flagging criteria. Synchronous water levels are measured over a 3-5 day period.

- = exceeded holding time.

- = exceeded groundwater protection standard. 
WELL FSB 94C (cont.)

Radiological Indicator Parameters

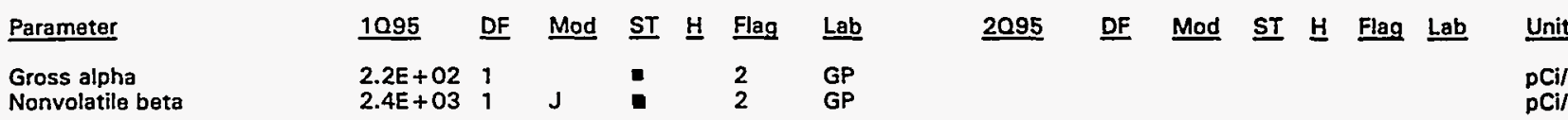

Measured Radionuclides

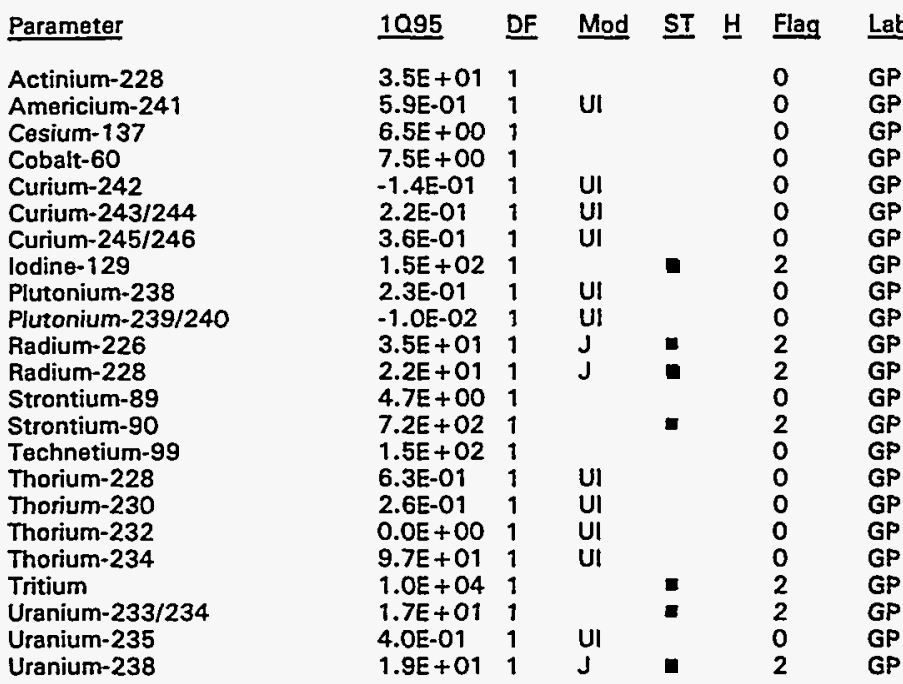

2095 DF Mod ST $\underline{H}$ Flag Lab Unit

GP
GP
GP
GP
GP
GP
GP
GP
GP
GP
GP
GP
GP
GP
GP
GP
GP
GP
GP
GP
GP
GP
GP

$\mathrm{pCi} / \mathrm{L}$

pCi/L

pCi/

$\mathrm{pCi/L}$

$\mathrm{pCi} / \mathrm{L}$

pCill

$\mathrm{pCi} / \mathrm{L}$

$\mathrm{pCi} / \mathrm{L}$

pCi/L

pCi/L

pCIh

pCi/L

pCi/l

$\mathrm{pCi} / \mathrm{L}$

pCi/L

PCill

PCi/L

$\mathrm{pCi} / \mathrm{L}$

$\mathrm{pCi} / \mathrm{L}$

pCill

Uranium-238

$1.9 E+011$

Calculated Radionuclides

Parameter

Americium-243

Nickel-63

Plutonium-241

Plutonium-242
1095

6.6E-04

$7.5 E+00$

$-4.1 \mathrm{E}-02$

$-2.0 \mathrm{E}-07$
DF Mod ST $\underline{H}$ Flag $\underline{\text { Lab }}$

0

0
0
0

Unit

pCi/h

$\mathrm{pCi} / \mathrm{L}$

pCi/L

pCI/L

Note: See Appendix B for flagging criteria. Synchronous water levels are measured over a 3-5 day period.

- = exceeded holding time.

- =xceeded groundwater protection standard. 


\section{WELL FSB 94DR}

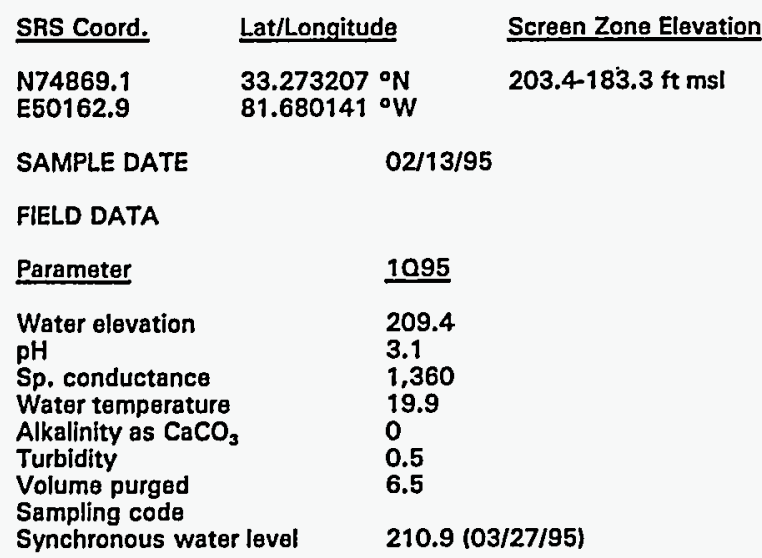

$\begin{array}{lrlll}\text { Top of Casing } & \text { Casing } & \text { Pump } & \text { Screen Zone } \\ {\mathrm{ms} !} } & \text { 4" PVC } & \mathrm{S} & \text { Water Table }\left(11 \mathrm{~B}_{2}\right) \\ & 05 / 04 / 95 & & \end{array}$

Hazardous Constituents (264.94, Table 1)

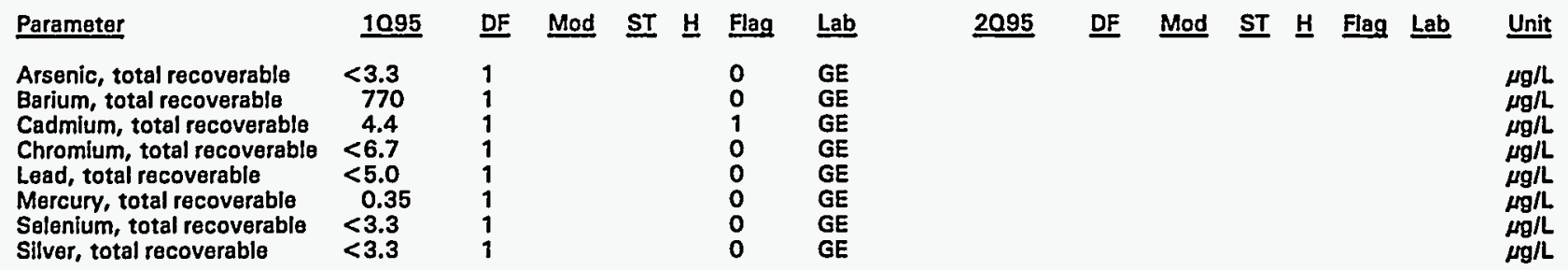

Hazardous Constituents (261 App. VIII/264 App. IX)

\begin{tabular}{|c|c|c|c|c|c|c|c|c|c|c|c|c|c|c|c|}
\hline Parameter & 1095 & DF & Mod & $\underline{\text { ST }}$ & 브 & Flag & $\underline{\text { Lab }}$ & $\underline{2095}$ & $\underline{\text { DF }}$ & Mod & $\underline{\text { ST }}$ & $\underline{H}$ & Flag & $\underline{\text { Lab }}$ & $\underline{\text { Unit }}$ \\
\hline $\begin{array}{l}\text { Antimony, total recoverable } \\
\text { Benzene } \\
\text { Cobalt, total recoverable } \\
\text { Copper, total recoverable } \\
\text { Cyanide } \\
\text { Nickel, total recoverable } \\
\text { Phenols } \\
\text { Tetrachloroethylene } \\
\text { Thallium, total recoverable } \\
\text { Trichlorosthylene } \\
\text { Trichlorofluoromethane } \\
\text { Vanadium, total recoverable } \\
\text { Zinc, total recoverable }\end{array}$ & $\begin{array}{l}<3.3 \\
<1.7 \\
35 \\
67 \\
<8.3 \\
36 \\
<8.3 \\
<1.7 \\
<3.3 \\
3.7 \\
<1.7 \\
<13 \\
140\end{array}$ & $\begin{array}{l}1 \\
1 \\
1 \\
1 \\
1 \\
1 \\
1 \\
1 \\
1 \\
1 \\
1 \\
1 \\
1 \\
1\end{array}$ & J1 & - & - & $\begin{array}{l}0 \\
0 \\
1 \\
0 \\
0 \\
0 \\
0 \\
0 \\
0 \\
1 \\
0 \\
0 \\
0\end{array}$ & $\begin{array}{l}\mathrm{GE} \\
\mathrm{GE} \\
\mathrm{GE} \\
\mathrm{GE} \\
\mathrm{GE} \\
\mathrm{GE} \\
\mathrm{GE} \\
\mathrm{GE} \\
\mathrm{GE} \\
\mathrm{GE} \\
\mathrm{GE} \\
\mathrm{GE} \\
\mathrm{GE}\end{array}$ & & & & & & & & $\begin{array}{l}\mu \mathrm{g} / \mathrm{L} \\
\mu \mathrm{g} / \mathrm{L} \\
\mu g / \mathrm{L} \\
\mu \mathrm{g} / \mathrm{L} \\
\mu \mathrm{g} / \mathrm{L} \\
\mu g / \mathrm{L} \\
\mu \mathrm{g} / \mathrm{L} \\
\mu \mathrm{g} / \mathrm{L} \\
\mu \mathrm{g} / \mathrm{L} \\
\mu \mathrm{g} / \mathrm{L} \\
\mu \mathrm{g} / \mathrm{L} \\
\mu \mathrm{g} / \mathrm{L} \\
\mu \mathrm{g} / \mathrm{L}\end{array}$ \\
\hline \multicolumn{16}{|c|}{ Water Quality Indicator Parameters } \\
\hline Parameter & 1095 & $\underline{\text { DE }}$ & Mod & $\underline{\text { ST }}$ & $\underline{H}$ & Flag & $\underline{\text { Lab }}$ & $\underline{2095}$ & $\underline{\mathrm{DF}}$ & Mod & $\underline{\text { ST }}$ & $\underline{H}$ & Flag & $\underline{\text { Lab }}$ & $\underline{\text { Unit }}$ \\
\hline $\begin{array}{l}\text { Aluminum, total recoverable } \\
\text { Nitrate as nitrogen } \\
\text { pH }\end{array}$ & $\begin{array}{l}64,000 \\
130,000 \\
3.3\end{array}$ & $\begin{array}{l}3 \\
160 \\
1\end{array}$ & J1 & 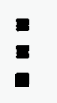 & - & $\begin{array}{l}2 \\
2 \\
1\end{array}$ & $\begin{array}{l}\text { GE } \\
\text { GE } \\
\text { GE }\end{array}$ & & & & & & & & $\underset{\mathrm{pH}}{\mu \mathrm{g} / \mathrm{L}}$ \\
\hline \multicolumn{16}{|c|}{ Common Laboratory Contaminants } \\
\hline Parameter & 1095 & $\underline{D F}$ & Mod & $\underline{\mathbf{S T}}$ & $\underline{H}$ & Flag & $\underline{\text { Lab }}$ & $\underline{2095}$ & DF & Mod & $\underline{\text { ST }}$ & $\underline{\mathrm{H}}$ & Flag & $\underline{L a b}$ & Unit \\
\hline $\begin{array}{l}\text { Bis(2-ethylhexyl) phthalate } \\
\text { Dichloromethane }\end{array}$ & $\begin{array}{l}<17 \\
<1.7\end{array}$ & $\begin{array}{l}1 \\
1\end{array}$ & & & & $\begin{array}{l}0 \\
0\end{array}$ & $\begin{array}{l}\text { GE } \\
\text { GE }\end{array}$ & & & & & & & & $\underset{\mu g / L}{\mu g / L}$ \\
\hline
\end{tabular}

Note: See Appendix B for flagging criteria. Synchronous water levels are measured over a 3-5 day period.

- = exceeded holding time.

- = exceeded groundwater protection standard. 
WELL FSB 94DR (cont.)

Radiological Indicator Parameters

\begin{tabular}{|c|c|c|c|c|c|c|c|c|c|c|c|c|c|}
\hline Parameter & 1095 & $\underline{D F}$ & Mod & $\underline{\text { ST }} \underline{H}$ & Flag & $\underline{\text { Lab }}$ & $\underline{2095}$ & DF & Mod & $\underline{\text { ST }} \underline{H}$ & Flag & $\underline{\text { Lab }}$ & $\underline{\text { Unit }}$ \\
\hline $\begin{array}{l}\text { Gross alpha } \\
\text { Nonvolatile beta }\end{array}$ & $\begin{array}{l}1.4 E+03 \\
1.3 E+03\end{array}$ & $\begin{array}{l}1 \\
1\end{array}$ & $\mathrm{~J}$ & 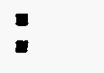 & $\begin{array}{l}2 \\
2\end{array}$ & $\begin{array}{l}\text { GP } \\
\text { GP }\end{array}$ & & & & & & & $\begin{array}{l}\mathrm{pCi} / \mathrm{L} \\
\mathrm{pCi/L}\end{array}$ \\
\hline \multicolumn{14}{|c|}{ Measured Radionuclides } \\
\hline Parameter & 1095 & $\underline{D F}$ & Mod & $\underline{\text { sT }}$ & Flag & $\underline{\text { Lab }}$ & $\underline{2095}$ & $\underline{D F}$ & Mod & $\underline{\mathrm{ST}} \underline{H}$ & Flag & $\underline{L a b}$ & $\underline{\text { Unit }}$ \\
\hline $\begin{array}{l}\text { Actinium-228 } \\
\text { Americium-241 } \\
\text { Cesium-137 } \\
\text { Cobalt-60 } \\
\text { Curium-242 } \\
\text { Curium-243/244 } \\
\text { Curium-245/246 } \\
\text { lodin-129 } \\
\text { Plutonium-238 } \\
\text { Plutonium-239/240 } \\
\text { Radium-226 } \\
\text { Radium-228 } \\
\text { Strontium-89 } \\
\text { Strontium-90 } \\
\text { Technetium-99 } \\
\text { Thorium-228 } \\
\text { Thorium-230 } \\
\text { Thorium-232 } \\
\text { Thorium-234 } \\
\text { Tritium } \\
\text { Uranium-233/234 } \\
\text { Uranium-235 } \\
\text { Uranium-238 }\end{array}$ & $\begin{array}{l}6.4 E+01 \\
9.0 E+01 \\
2.8 E+02 \\
4.0 E+00 \\
-1.4 E-01 \\
1.6 E+02 \\
3.4 E+00 \\
1.4 E+02 \\
1.1 E-01 \\
-2.3 E-02 \\
1.0 E+01 \\
5.7 E+01 \\
2.1 E+01 \\
2.3 E+02 \\
9.3 E+01 \\
3.4 E-01 \\
2.0 E-01 \\
-8.0 E-03 \\
7.0 E+02 \\
8.9 E+03 \\
6.7 E+02 \\
3.9 E+01 \\
1.3 E+03\end{array}$ & $\begin{array}{l}1 \\
1 \\
1 \\
1 \\
1 \\
1 \\
1 \\
1 \\
1 \\
1 \\
1 \\
1 \\
1 \\
1 \\
1 \\
1 \\
1 \\
1 \\
1 \\
1 \\
1 \\
1 \\
1\end{array}$ & $\begin{array}{l}\text { UI } \\
\text { UI } \\
J \\
J\end{array}$ & $\begin{array}{l}\square \\
= \\
=\end{array}$ & $\begin{array}{l}0 \\
2 \\
2 \\
0 \\
0 \\
2 \\
1 \\
2 \\
0 \\
0 \\
1 \\
2 \\
2 \\
2 \\
0 \\
0 \\
0 \\
0 \\
2 \\
2 \\
2 \\
2 \\
2\end{array}$ & $\begin{array}{l}G P \\
G P \\
G P \\
G P \\
G P \\
G P \\
G P \\
G P \\
G P \\
G P \\
G P \\
G P \\
G P \\
G P \\
G P \\
G P \\
G P \\
G P \\
G P \\
G P \\
G P \\
G P \\
G P\end{array}$ & & & & & & & $\begin{array}{l}\text { pCi/L } \\
\text { pCi/L } \\
\text { pCi/L } \\
\text { pCi/L } \\
\text { pCi/L } \\
\text { pCi/L } \\
\text { pCi/L } \\
\text { pCil/ } \\
\text { pCi/L } \\
\text { pCi/L } \\
\text { pCi/L } \\
\text { pCi/L } \\
\text { pCi/L } \\
\text { pCi/L } \\
\text { pCi/L } \\
\text { pCi/L } \\
\text { pCi/L } \\
\text { pCi/L } \\
\text { pCi/L } \\
\text { pCi/m } \\
\text { pCi/L } \\
\text { pCi } / L \\
\text { pCi/L }\end{array}$ \\
\hline
\end{tabular}

Calculated Radionuclides

Parameter

Americium-243

Nickel-63

Plutonium-241

Plutonium-242
1095

4.8E-01

$4.0 E+00$

$-9.1 E-02$

$-4.5 E-07$
DF Mod ST $H$ Flag Lab

0
0
0
0

$\underline{\text { Unit }}$

$\mathrm{pCi} / \mathrm{L}$

pCilL

$\mathrm{PC} / \mathrm{L}$

$\mathrm{pCi} / \mathrm{L}$

Note: See Appendix B for flagging criteria. Synchronous water levels are measured over a 3-5 day period.

- = exceeded holding time.

a = exceeded groundwater protection standard. 


\section{WELL FSB 95CR}

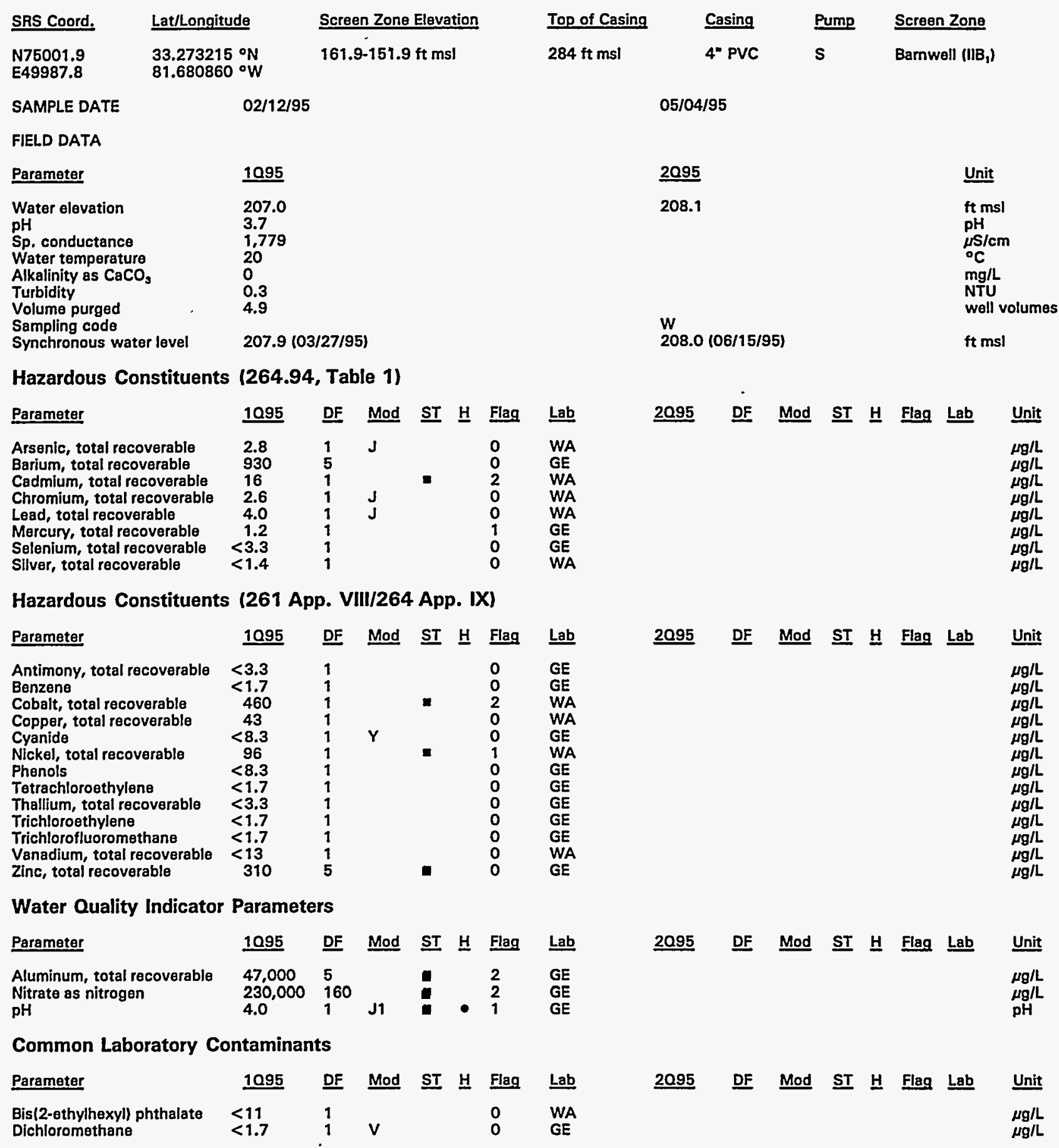

Note: See Appendix B for flagging criteria. Synchronous water levels are measured over a 3-5 day period.

- = exceeded holding time.

- = exceeded groundwater protection standard. 
WELL FSB 95CR (cont.)

Radiological Indicator Parameters

Parameter

Gross alpha

Nonvolatile beta
1.2E+03

$\begin{array}{ll}1.2 E+03 & 1 \\ 2.4 E+03 & 1\end{array}$

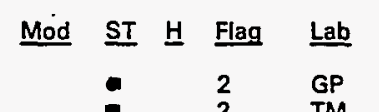

GP
TM $\underline{20.95}$

DF Mod ST $\underline{H}$ Flag Lab

Unit

Measured Radionuclides

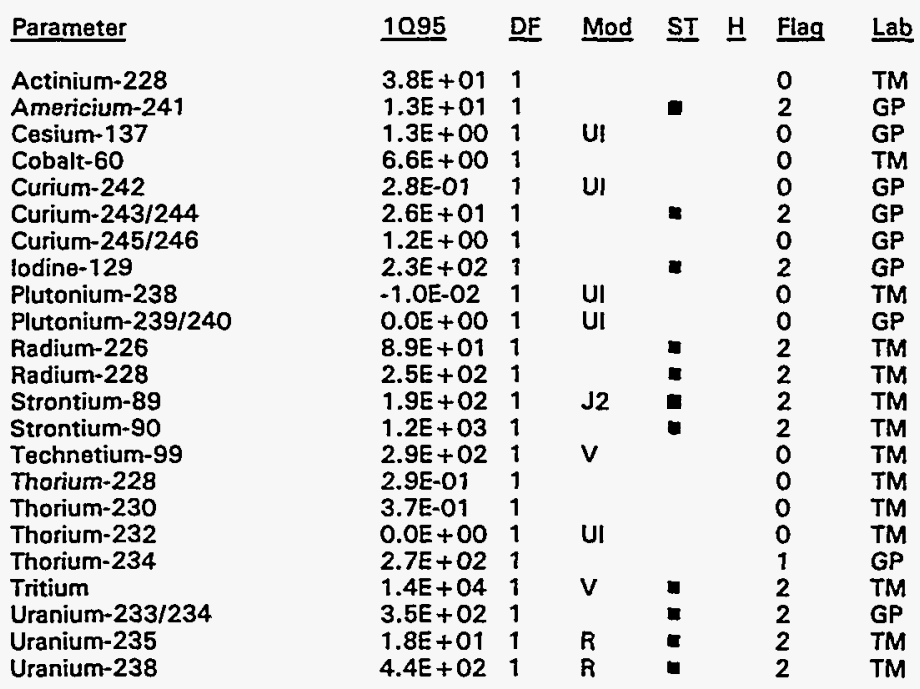

Calculated Radionuclides

Parameter

Americium-243

Nickel-63

Plutonium-241

Plutonium-242
1095

DF
Mod ST $\underline{\text { F Flag }}$
Lab

$\underline{2095}$

DF Mod ST 브 Flag Lab

Unit

$+\infty 0$

$0.0 E+00$
$0.0 E+O 0$

0
0
0
0

2095 DF Mod ST $\underline{\text { H Flag Lab Unit }}$

$\mathrm{pCi} / \mathrm{L}$

pCill

pCi/L

$\mathrm{pCi} / \mathrm{L}$

$\mathrm{pCi} / \mathrm{L}$

pCill

pCi/L

pCi/L

$\mathrm{pCi} / \mathrm{L}$

pCi/L

pCi/L

$\mathrm{pCi} / 2$

$\mathrm{pCi} / \mathrm{L}$

$\mathrm{pCi} / \mathrm{L}$

$\mathrm{pCi/L}$

$\mathrm{PCi} / \mathrm{L}$

pCi/L

pCi/L

$\mathrm{pCi} / \mathrm{L}$

PCiL

$\mathrm{pCi} / \mathrm{L}$

pCill

$\mathrm{pCi} / \mathrm{mL}$

Note: See Appendix B for flagging criteria. Synchronous water levels are measured over a 3-5 day period.

$\checkmark=$ exceeded holding time.

a = exceeded groundwater protection standard. 
WSRC-TR-95-0146-2

Unclassified

\section{WELL FSB 95DR}

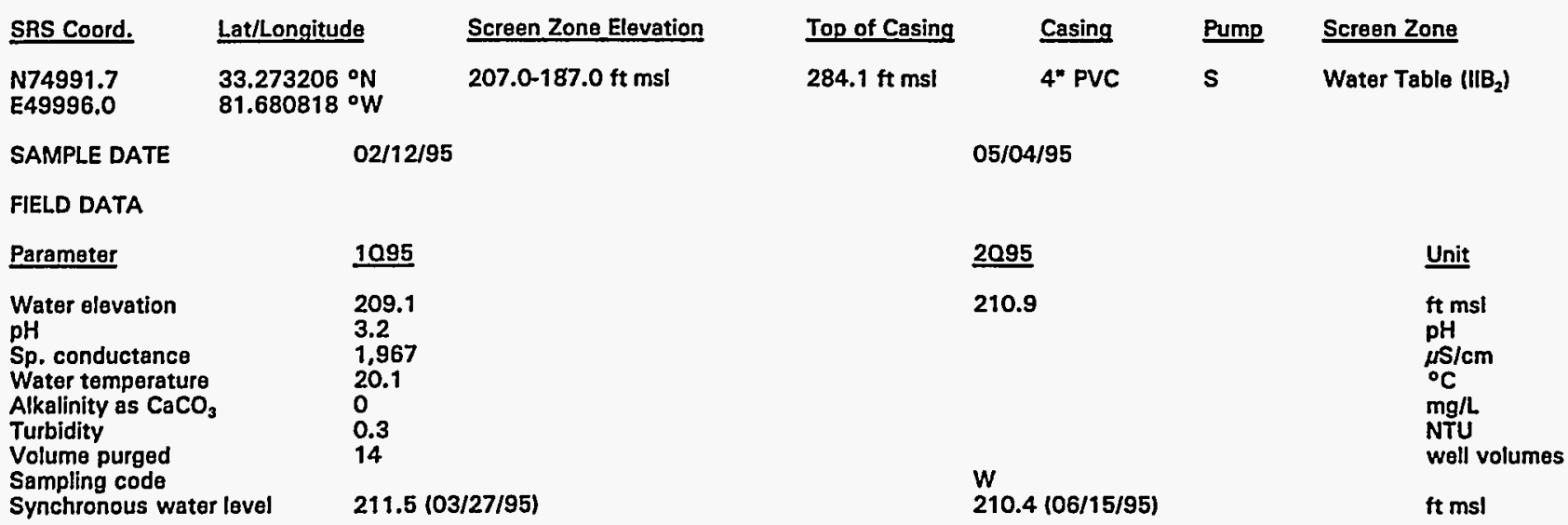

Hazardous Constituents (264.94, Table 1)

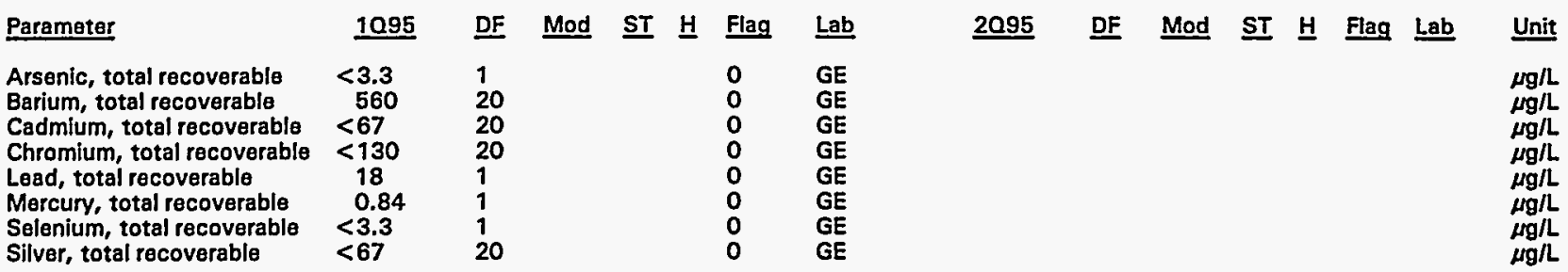

Hazardous Constituents (261 App. VIII/264 App. IX)

\begin{tabular}{|c|c|c|c|c|c|c|c|c|c|c|c|c|c|c|c|}
\hline Parameter & 1095 & $\underline{D F}$ & Mod & $\underline{S T}$ & 브 & Flag & Lab & 2095 & $\underline{\text { DF }}$ & Mod & $\underline{\mathrm{ST}}$ & $\underline{H}$ & Flag & Lab & Unit \\
\hline $\begin{array}{l}\text { Antimony, total recoverable } \\
\text { Benzene } \\
\text { Cobalt, total recoverable } \\
\text { Copper, total recoverable } \\
\text { Cyanide } \\
\text { Nickel, total recoverable } \\
\text { Phenols } \\
\text { Tetrachloroethylene } \\
\text { Thallium, total recoverable } \\
\text { Trichloroethylene } \\
\text { Trichlorofluoromethane } \\
\text { Vanadium, total recoverable } \\
\text { Zinc, total recoverable }\end{array}$ & $\begin{aligned}<3.3 \\
<1.7 \\
<130 \\
<130 \\
<8.3 \\
<130 \\
<8.3 \\
<1.7 \\
<3.3 \\
<1.7 \\
<1.7 \\
<270 \\
140\end{aligned}$ & $\begin{array}{l}1 \\
1 \\
20 \\
20 \\
1 \\
20 \\
1 \\
1 \\
1 \\
1 \\
1 \\
20 \\
20\end{array}$ & R4 & $\mathbf{m}$ & & $\begin{array}{l}0 \\
0 \\
0 \\
0 \\
0 \\
0 \\
0 \\
0 \\
0 \\
0 \\
0 \\
0 \\
0\end{array}$ & $\begin{array}{l}\text { GE } \\
\text { GE } \\
\text { GE } \\
\text { GE } \\
\text { GE } \\
\text { GE } \\
\text { GE } \\
\text { GE } \\
\text { GE } \\
\text { GE } \\
\text { GE } \\
\text { GE } \\
\text { GE }\end{array}$ & & & & & & & & $\begin{array}{l}\mu g / L \\
\mu g / L \\
\mu g / L \\
\mu g / L \\
\mu g / L \\
\mu g / L \\
\mu g / L \\
\mu g / L \\
\mu g / L \\
\mu g / L \\
\mu g / L \\
\mu g / L \\
\mu g / L\end{array}$ \\
\hline \multicolumn{16}{|c|}{ Water Quality Indicator Parameters } \\
\hline Parameter & 1095 & DF & Mod & ST & 브 & Flag & Lab & 2095 & $\underline{D F}$ & Mod & $\underline{\text { ST }}$ & $\underline{H}$ & Flag & Lab & Unit \\
\hline \multicolumn{16}{|c|}{ Common Laboratory Contaminants } \\
\hline Parameter & 1095 & $\underline{D F}$ & Mod & $\underline{\text { ST }}$ & 브 & Flag & $\underline{\text { Lab }}$ & $\underline{2095}$ & $\underline{D F}$ & Mod & $\underline{\text { ST }}$ & $\underline{H}$ & Flag & $\underline{\text { Lab }}$ & Unit \\
\hline $\begin{array}{l}\text { Bis(2-ethylhexyl) phthalate } \\
\text { Dichloromethane }\end{array}$ & $\begin{array}{l}<17 \\
<1.7\end{array}$ & $\begin{array}{l}1 \\
1\end{array}$ & & & & $\begin{array}{l}0 \\
0\end{array}$ & $\begin{array}{l}\text { GE } \\
\text { GE }\end{array}$ & & & & & & & & $\begin{array}{l}\mu g / L \\
\mu g / L\end{array}$ \\
\hline
\end{tabular}

Note: See Appendix B for flagging criteria. Synchronous water levels are measured over a 3-5 day period. - = exceeded holding time.

- = exceeded groundwater protection standard. 
WELL FSB 95DR (cont.)

Radiological Indicator Parameters

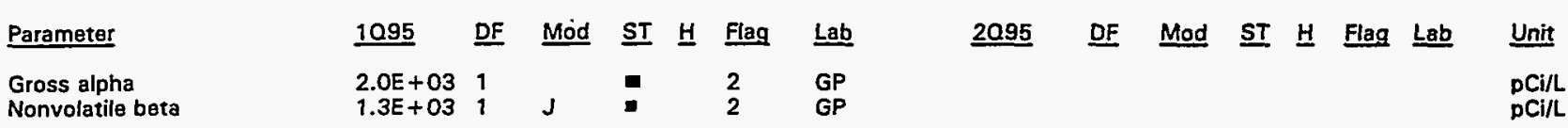

Measured Radionuclides

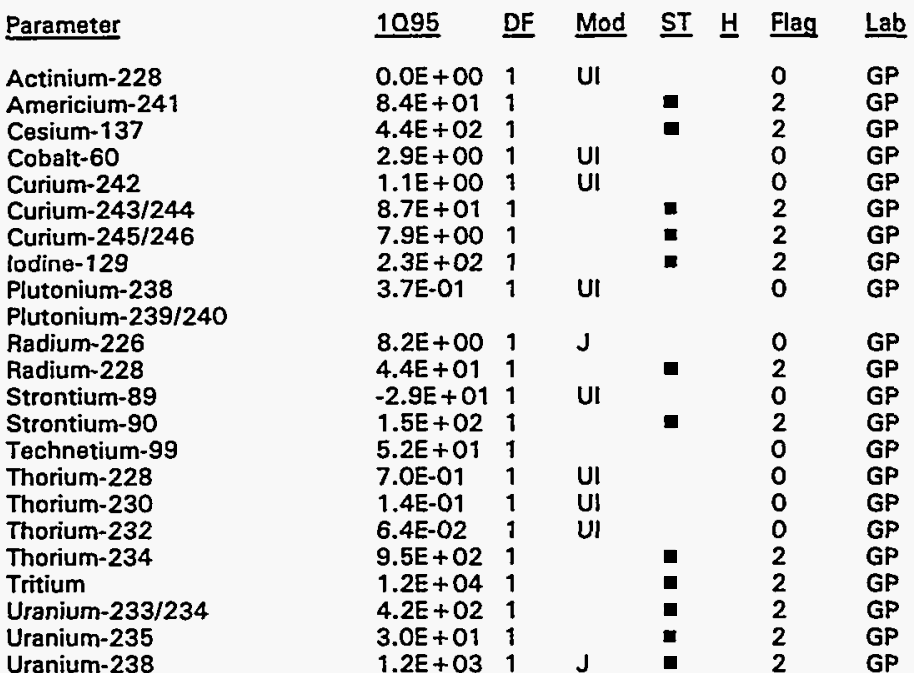

2095 DF Mod ST $\underline{H}$ Flag Lab Unit

Uranium-238

$1.2 E+03$

Calculated Radionuclides

Parameter

Americium-243

Nickel-63

Plutonium-241

Plutonium-242

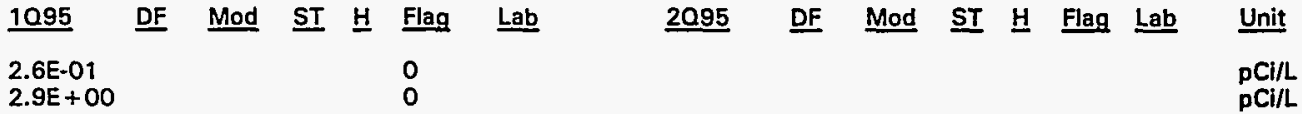

Note: See Appendix B for flagging criteria. Synchronous water levels are measured over a 3-5 day period.

- = exceeded holding time.

- = exceeded groundwater protection standard. 
WSRC-TR-95-0146-2

Unclassified

\section{WELL FSB 96AR}

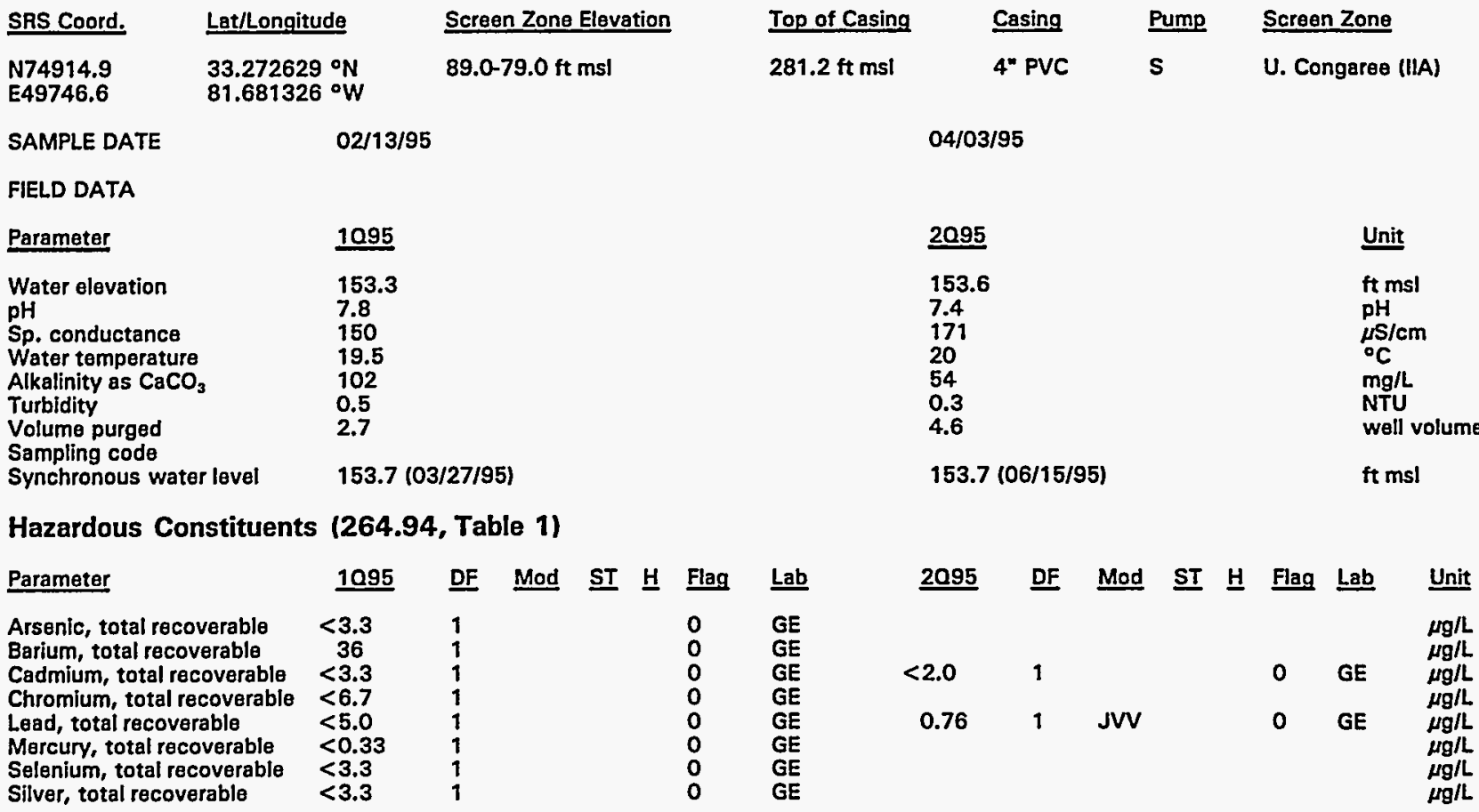

Hazardous Constituents (261 App. VIII/264 App. IX)

\begin{tabular}{|c|c|c|c|c|c|c|c|c|c|c|c|c|c|c|c|}
\hline Parameter & 1095 & DF & $\underline{\text { Mod }}$ & $\underline{\mathbf{S T}}$ & $\underline{\text { H}}$ & Flag & $\underline{\text { Lab }}$ & $\underline{2095}$ & $\underline{D F}$ & Mod & $\underline{\mathbf{S T}}$ & H & Flag & Lab & Unit \\
\hline $\begin{array}{l}\text { Antimony, total recoverable } \\
\text { Benzene } \\
\text { Cobalt, total recoverable } \\
\text { Copper, total recoverable } \\
\text { Cyanide } \\
\text { Nickel, total recoverable } \\
\text { Phenols } \\
\text { Tetrachloroethylene } \\
\text { Thallium, total recoverable } \\
\text { Trichloroethylene } \\
\text { Trichlorofluoromethane } \\
\text { Vanadium, total recoverable } \\
\text { Zinc, total recoverable }\end{array}$ & $\begin{array}{l}<3.3 \\
<1.7 \\
<6.7 \\
<6.7 \\
<8.3 \\
<6.7 \\
<8.3 \\
<1.7 \\
<3.3 \\
<1.7 \\
<1.7 \\
<13 \\
<3.3\end{array}$ & $\begin{array}{l}1 \\
1 \\
1 \\
1 \\
1 \\
1 \\
1 \\
1 \\
1 \\
1 \\
1 \\
1 \\
1\end{array}$ & $J$ & & & $\begin{array}{l}0 \\
0 \\
0 \\
0 \\
0 \\
0 \\
0 \\
0 \\
0 \\
0 \\
0 \\
0 \\
0\end{array}$ & $\begin{array}{l}\mathrm{GE} \\
\mathrm{GE} \\
\mathrm{GE} \\
\mathrm{GE} \\
\mathrm{GE} \\
\mathrm{GE} \\
\mathrm{GE} \\
\mathrm{GE} \\
\mathrm{GE} \\
\mathrm{GE} \\
\mathrm{GE} \\
\mathrm{GE} \\
\mathrm{GE}\end{array}$ & & & & & & & & $\begin{array}{l}\mu g / L \\
\mu g / L \\
\mu g / L \\
\mu g / L \\
\mu g / L \\
\mu g / L \\
\mu g / L \\
\mu g / L \\
\mu g / L \\
\mu g / L \\
\mu g / L \\
\mu g / L \\
\mu g / L\end{array}$ \\
\hline \multicolumn{15}{|c|}{ Water Quality Indicator Parameters } & \\
\hline Parameter & $\underline{1095}$ & $\underline{D F}$ & Mod & $\underline{\mathbf{S T}}$ & H & Flag & $\underline{\text { Lab }}$ & $\underline{2095}$ & $\underline{\text { DF }}$ & Mod & $\underline{\text { ST }}$ & $\underline{H}$ & Flag & Lab & \\
\hline $\begin{array}{l}\text { Aluminum, total recoverable } \\
\text { Nitrate as nitrogen } \\
\text { pH }\end{array}$ & $\begin{array}{c}<33 \\
510 \\
7.3\end{array}$ & $\begin{array}{l}1 \\
1 \\
1\end{array}$ & s1 & & - & $\begin{array}{l}0 \\
0 \\
0\end{array}$ & $\begin{array}{l}\mathrm{GE} \\
\mathrm{GE} \\
\mathrm{GE}\end{array}$ & $\begin{array}{l}340 \\
7.4\end{array}$ & $\begin{array}{l}1 \\
1\end{array}$ & J1 & & $\bullet$ & $\begin{array}{l}0 \\
0\end{array}$ & $\begin{array}{l}\text { GE } \\
\text { GE }\end{array}$ & \\
\hline \multicolumn{15}{|c|}{ Common Laboratory Contaminants } & \\
\hline Parameter & 1095 & $\underline{D F}$ & Mod & $\underline{\text { ST }}$ & H & Flag & $\underline{\text { Lab }}$ & $\underline{2095}$ & $\underline{D F}$ & Mod & $\underline{\mathbf{S T}}$ & H & Flag & $\underline{\text { Lab }}$ & Unit \\
\hline $\begin{array}{l}\text { Bis(2-othylhexyl) phthalate } \\
\text { Dichloromethane }\end{array}$ & $\begin{array}{l}<17 \\
<1.7\end{array}$ & $\begin{array}{l}1 \\
1\end{array}$ & & & & $\begin{array}{l}\mathbf{0} \\
0\end{array}$ & $\begin{array}{l}\text { GE } \\
\text { GE }\end{array}$ & & & & & & & & \\
\hline
\end{tabular}

Note: See Appendix B for flagging criteria. Synchronous water levels are measured over a 3-5 day period.

- = exceeded holding time.

m = exceeded groundwater protection standard. 
WELL FSB 96AR (cont.)

Radiological Indicator Parameters

\begin{tabular}{|c|c|c|c|c|c|c|c|c|c|c|c|c|c|c|c|}
\hline Parameter & 1095 & DF & Mod & $\underline{\text { ST }}$ & $\underline{\mathrm{H}}$ & Flag & $\underline{\text { Lab }}$ & $\underline{2095}$ & DF & Mod & $\underline{\text { ST }}$ & $\underline{H}$ & Flag & $\underline{\text { Lab }}$ & Unit \\
\hline $\begin{array}{l}\text { Gross alpha } \\
\text { Nonvolatile beta }\end{array}$ & $\begin{array}{l}1.2 E+\infty 0 \\
2.0 E+00\end{array}$ & $\begin{array}{l}1 \\
1\end{array}$ & & & & $\begin{array}{l}0 \\
0\end{array}$ & $\begin{array}{l}\text { GP } \\
\text { GP }\end{array}$ & $\begin{array}{l}1.2 E+\infty 0 \\
1.8 E+\infty 0\end{array}$ & $\begin{array}{l}1 \\
1\end{array}$ & $J$ & & & $\begin{array}{l}0 \\
0\end{array}$ & $\begin{array}{l}G P \\
G P\end{array}$ & $\begin{array}{l}\mathrm{pCi} / \mathrm{L} \\
\mathrm{pCi} / \mathrm{L}\end{array}$ \\
\hline \multicolumn{16}{|c|}{ Measured Radionuclides } \\
\hline Parameter & 1095 & $\underline{D F}$ & Mod & $\underline{\mathbf{S T}}$ & $\underline{\mathrm{H}}$ & Flag & Lab & 2095 & $\underline{\mathrm{DF}}$ & Mod & ST & $\underline{H}$ & Flag & Lab & Unit \\
\hline $\begin{array}{l}\text { Actinium-228 } \\
\text { Americium-241 } \\
\text { Cesium-137 } \\
\text { Cobalt-60 } \\
\text { Curium-242 } \\
\text { Curium-243/244 } \\
\text { Curium-245/246 } \\
\text { lodine-129 } \\
\text { Plutonium-238 } \\
\text { Plutonium-239/240 } \\
\text { Radium-226 } \\
\text { Radium-228 } \\
\text { Strontium-89 } \\
\text { Strontium-90 } \\
\text { Technetium-99 } \\
\text { Thorium-228 } \\
\text { Thorium-230 } \\
\text { Thorium-232 } \\
\text { Tharium-234 } \\
\text { Tritium } \\
\text { Uranium-233/234 } \\
\text { Uranium-235 } \\
\text { Uranium-238 }\end{array}$ & $\begin{array}{l}5.9 E+00 \\
-2.2 E-02 \\
-1.3 E-01 \\
4.3 E-01 \\
-1.4 E-02 \\
2.6 E-02 \\
0.0 E+00 \\
7.5 E+00 \\
2.6 E-02 \\
0.0 E+00 \\
1.4 E-01 \\
2.2 E-02 \\
-1.3 E+00 \\
-5.5 E-01 \\
-1.2 E+01 \\
1.7 E-01 \\
1.0 E-01 \\
0.0 E+00 \\
1.6 E+02 \\
1.5 E+01 \\
6.6 E-02 \\
3.5 E-02 \\
3.5 E-02\end{array}$ & $\begin{array}{l}1 \\
1 \\
1 \\
1 \\
1 \\
1 \\
1 \\
1 \\
1 \\
1 \\
1 \\
1 \\
1 \\
1 \\
1 \\
1 \\
1 \\
1 \\
1 \\
1 \\
1 \\
1 \\
1\end{array}$ & $\begin{array}{l}\text { UI } \\
\text { UI } \\
U I \\
\text { UI } \\
\text { UI } \\
U I \\
\text { UI } \\
\\
U I \\
U I \\
U I \\
U I \\
U I \\
U I \\
U I \\
U I \\
U I \\
U I\end{array}$ & n & & $\begin{array}{l}0 \\
0 \\
0 \\
0 \\
0 \\
0 \\
0 \\
2 \\
0 \\
0 \\
0 \\
0 \\
0 \\
0 \\
0 \\
0 \\
0 \\
0 \\
0 \\
1 \\
0 \\
0 \\
0\end{array}$ & $\begin{array}{l}\text { GP } \\
\text { GP } \\
\text { GP } \\
\text { GP } \\
\text { GP } \\
\text { GP } \\
\text { GP } \\
\text { GP } \\
\text { GP } \\
\text { GP } \\
\text { GP } \\
\text { GP } \\
\text { GP } \\
\text { GP } \\
\text { GP } \\
\text { GP } \\
\text { GP } \\
\text { GP } \\
\text { GP } \\
\text { GP } \\
\text { GP } \\
\text { GP } \\
\text { GP }\end{array}$ & $9.5 E-03$ & 1 & U1 & & & 0 & GP & 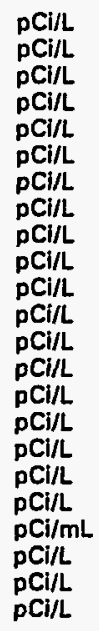 \\
\hline \multicolumn{16}{|c|}{ Calculated Radionuclides } \\
\hline Parameter & 1095 & $\underline{D F}$ & Mod & $\underline{\mathbf{S T}}$ & $\underline{H}$ & Flag & $\underline{\text { Lab }}$ & $\underline{2095}$ & $\underline{\text { DF }}$ & Mod & $\underline{\text { ST }}$ & $\underline{\text { ㅂ}}$ & Flag & $\underline{\text { Lab }}$ & Unit \\
\hline $\begin{array}{l}\text { Americium-243 } \\
\text { Nickel-63 } \\
\text { Plutonium-241 } \\
\text { Plutonium-242 }\end{array}$ & $\begin{array}{l}7.7 E-05 \\
4.3 E-01 \\
0.0 E+00 \\
0.0 E+00\end{array}$ & & & & & $\begin{array}{l}0 \\
0 \\
0 \\
0\end{array}$ & & & & & & & & & $\begin{array}{l}\mathrm{pCi} / \mathrm{L} \\
\mathrm{pCi} / \mathrm{L} \\
\mathrm{pCi} / \mathrm{L} \\
\mathrm{pCi} / \mathrm{L}\end{array}$ \\
\hline
\end{tabular}

Note: See Appendix B for flagging criteria. Synchronous water levels are measured over a 3-5 day period. - = exceeded holding time.

- =xceeded groundwater protection standard. 


\section{WELL FSB 97A}

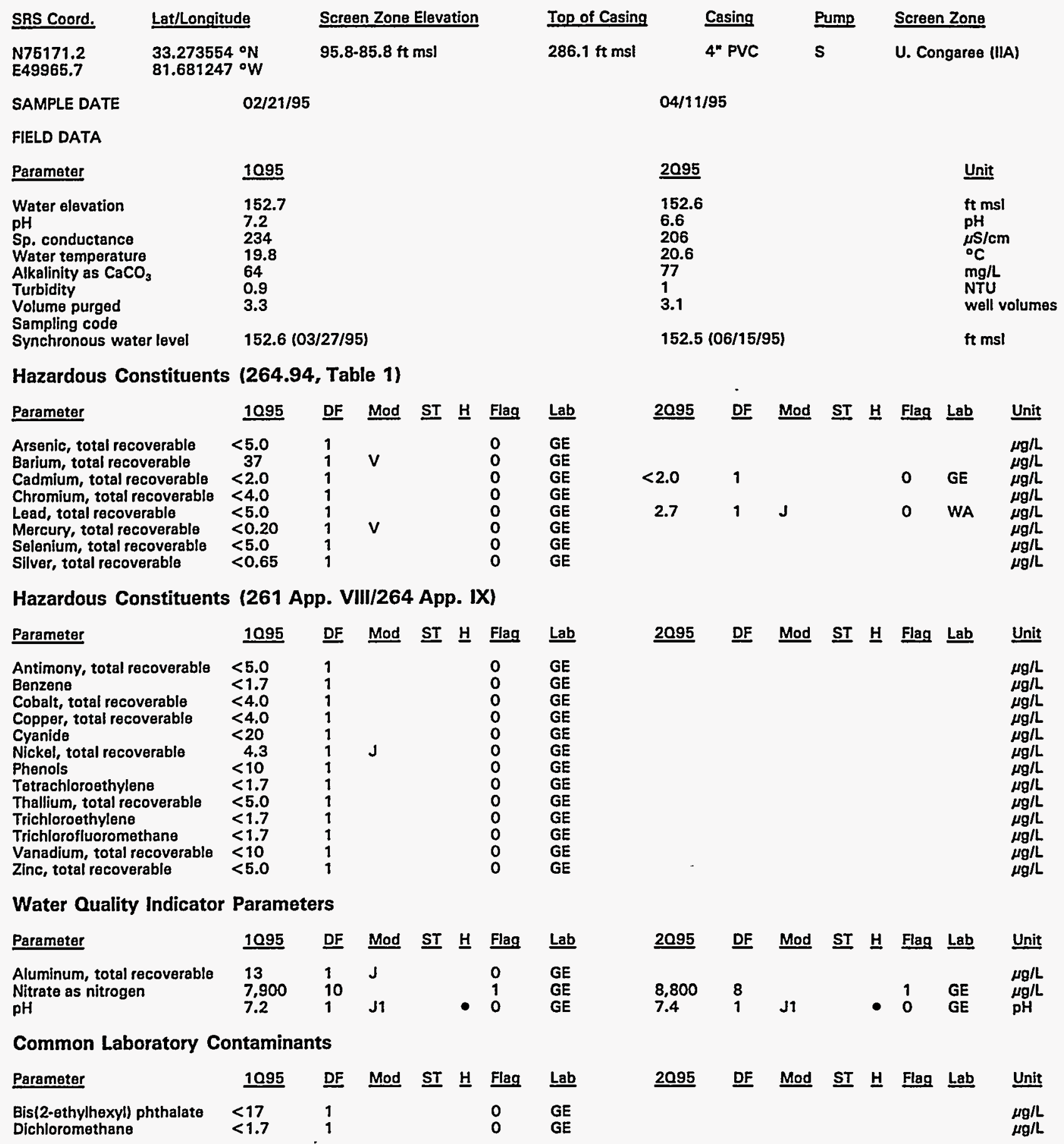

Note: See Appendix B for flagging criteria. Synchronous water levels are measured over a 3-5 day period.

- = exceeded holding time.

- = exceeded groundwater protection standard. 
WELL FSB 97A (cont.)

Radiological Indicator Parameters

\begin{tabular}{|c|c|c|c|c|c|c|}
\hline Parameter & 1095 & DF & Mo'd & $\underline{\text { ST }}$ & $\underline{H}$ & Flag \\
\hline $\begin{array}{l}\text { Gross alpha } \\
\text { Nonvolatile beta }\end{array}$ & $\begin{array}{l}3.8 E+00 \\
5.0 E+00\end{array}$ & $\begin{array}{l}1 \\
1\end{array}$ & $\jmath$ & & & $\begin{array}{l}0 \\
0\end{array}$ \\
\hline \multicolumn{7}{|c|}{ Measured Radionuclides } \\
\hline Parameter & 1095 & $\underline{\text { DF }}$ & Mod & ST & $\underline{\mathrm{H}}$ & Flag \\
\hline Actinium-228 & $6.3 E+00$ & 1 & UI & & & 0 \\
\hline Americium-241 & 2.3E-02 & 1 & UI & & & 0 \\
\hline Cesium-137 & $-6.0 E-01$ & $i$ & UI & & & 0 \\
\hline Cobalt -60 & $1.3 E+00$ & $i$ & Ui & & & 0 \\
\hline Curium-242 & $4.3 E-03$ & $i$ & UI & & & 0 \\
\hline Curium-243/244 & $-6.0 \mathrm{E}-02$ & $i$ & U! & & & 0 \\
\hline Curium-245/246 & $1.6 \mathrm{E}-02$ & 1 & U1 & & & 0 \\
\hline lodine-129 & $1.9 E+00$ & 1 & UI & & & 0 \\
\hline Plutonium-238 & 8.2E-02 & 1 & Ul & & & 0 \\
\hline Plutonium-239/240 & 8.7E-02 & 1 & Ui & & & 0 \\
\hline Radium-226 & 5.3E-01 & 1 & U] & & & 0 \\
\hline Radium-228 & $1.8 \mathrm{E}+00$ & 1 & $\mathrm{~J}$ & & & 0 \\
\hline Strontium-89 & $\begin{array}{l}-1.5 \mathrm{E}-01 \\
3 \mathrm{OE}-02\end{array}$ & 1 & UI & & & 0 \\
\hline $\begin{array}{l}\text { Strontium-90 } \\
\text { Technetium-99 }\end{array}$ & $\begin{array}{l}3.0 E-02 \\
6.1 E+00\end{array}$ & 1 & UI & & & $\begin{array}{l}0 \\
0\end{array}$ \\
\hline Thorium-228 & $-1.2 E-01$ & 1 & UI & & & 0 \\
\hline Thorium-230 & $-1.9 E-02$ & 1 & UI & & & 0 \\
\hline Thorium-232 & $-1.9 E-02$ & 1 & UI & & & 0 \\
\hline Thorium-234 & $1.2 \mathrm{E}+02$ & 1 & UI & & & 0 \\
\hline Tritium & $2.2 \mathrm{E}+\mathrm{O} 2$ & 1 & & - & & 2 \\
\hline Uranium-233/234 & 1.1E-01 & 1 & UI & & & 0 \\
\hline Uranium-235 & $0.0 E+00$ & 1 & UI & & & 0 \\
\hline Uranium-238 & $1.0 E-01$ & 1 & UI & & & 0 \\
\hline
\end{tabular}

\begin{tabular}{|c|c|c|c|c|c|c|}
\hline 2095 & DF & Mod & & 브 & Flag & Lab \\
\hline $\begin{array}{l}1.6 E+01 \\
5.7 E+00\end{array}$ & $\begin{array}{l}1 \\
1\end{array}$ & & $=$ & & $\begin{array}{l}2 \\
0\end{array}$ & $\begin{array}{l}\text { GP } \\
\text { GP }\end{array}$ \\
\hline
\end{tabular}

Uranium-238

1.OE-01 1

Lab
GP

DF Mod ST $\underline{H}$ Flag Lab Unit

GP
GP
GP
GP
GP
GP
GP
GP
GP
GP
GP
GP
GP
GP
GP
GP
GP
GP
GP
GP
GP
GP
GP

pCi/L

PCi/L

$\mathrm{pCi} / \mathrm{L}$

pCi/L

pCi/L

pCi/

pCirl

DCi/L

pCi/L

$\mathrm{pCi} / L$

pCi/L

pCi/L

pCi/L

pCi/L

$\mathrm{pCi} / L$

pCi/L

pCi/L

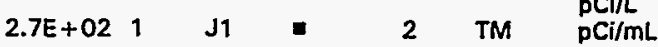

PCi/L

PCiL

$\mathrm{PCI} / \mathrm{L}$

Calculated Radionuclides

Parameter

1095 DF Mod ST H Flag

$\underline{2095}$

DF Mod ST $\underline{\text { H Flag Lab }}$

$\underline{\text { Unit }}$

Americium-243

Nickel-63

$-1.8 \mathrm{E}-04$

$1.3 E+00$

3.5E-01

Plutonium-241

1.7E-06

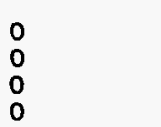

$\mathrm{Ci} / \mathrm{L}$

$\mathrm{pCl} / \mathrm{L}$

pCi/L

$\mathrm{pCi} / \mathrm{L}$

Note: See Appendix B for flagging criteria. Synchronous water levels are measured over a 3-5 day period.

- = exceeded holding time.

a = exceeded groundwater protection standard. 


\section{WELL FSB 97C}

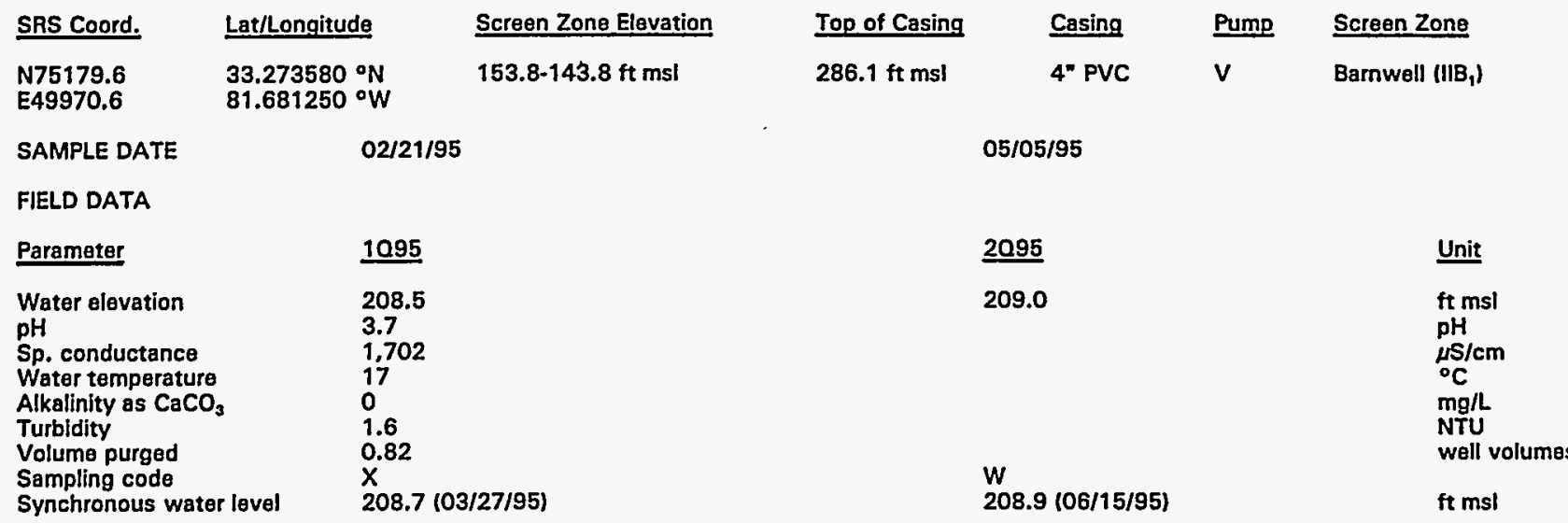

Hazardous Constituents (264.94, Table 1)

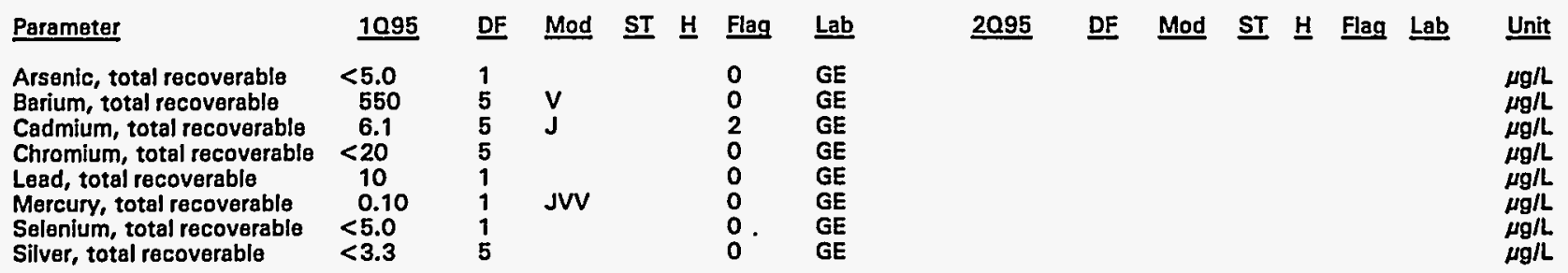

Hazardous Constituents (261 App. VIII/264 App. IX)

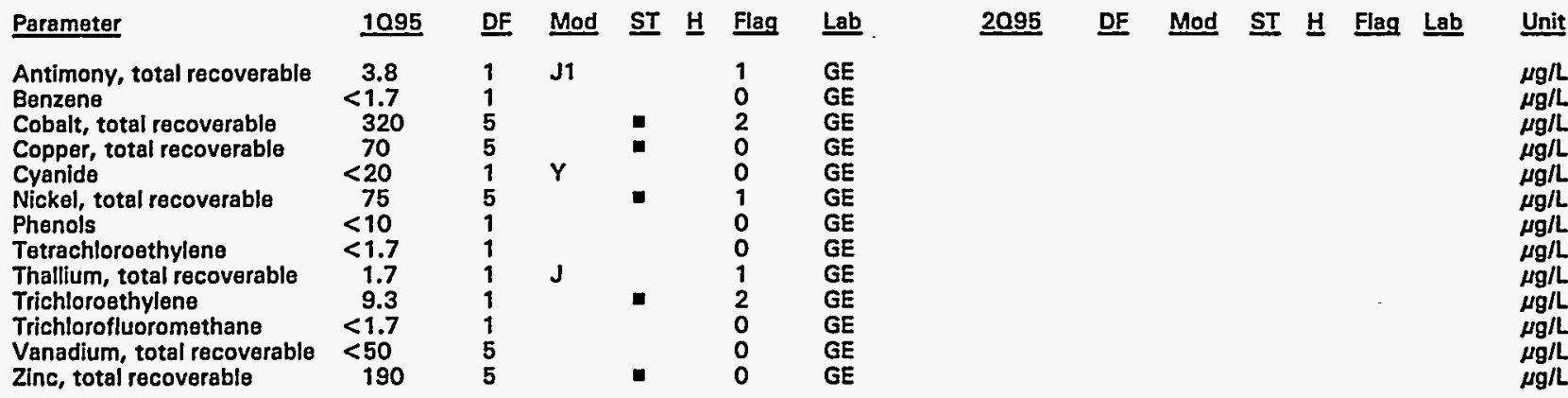

Water Quality Indicator Parameters

\begin{tabular}{|c|c|c|c|c|c|c|c|c|c|c|c|c|c|c|c|}
\hline Parameter & $\underline{1095}$ & $\underline{\mathrm{DF}}$ & Mod & $\underline{\text { ST }}$ & 브 & Flag & $\underline{\text { Lab }}$ & $\underline{2095}$ & $\underline{\mathrm{DF}}$ & Mod & $\underline{\text { ST }}$ & $\underline{\mathbf{H}}$ & Flag & $\underline{\text { Lab }}$ & Unit \\
\hline $\begin{array}{l}\text { Aluminum, total recoverable } \\
\text { Nitrate as nitrogen } \\
\text { pH }\end{array}$ & $\begin{array}{l}77,000 \\
240,000 \\
3.7\end{array}$ & $\begin{array}{l}5 \\
160 \\
1\end{array}$ & $\begin{array}{l}v \\
\mathrm{J1}\end{array}$ & : & & $\begin{array}{l}2 \\
2 \\
1\end{array}$ & $\begin{array}{l}\mathrm{GE} \\
\mathrm{GE} \\
\mathrm{GE}\end{array}$ & & & & & & & & $\begin{array}{l}\mu \mathrm{g} / \mathrm{L} \\
\mu \mathrm{g} / \mathrm{L} \\
\mathrm{pH}\end{array}$ \\
\hline \multicolumn{16}{|c|}{ Common Laboratory Contaminants } \\
\hline Parameter & $\underline{1095}$ & $\underline{\mathrm{DF}}$ & Mod & $\underline{\text { ST }}$ & $\underline{\mathbf{H}}$ & Flag & $\underline{L a b}$ & $\underline{2095}$ & $\underline{\mathrm{DF}}$ & Mod & $\underline{\mathbf{S T}}$ & $\underline{\mathbf{H}}$ & Flag & $\underline{\text { Lab }}$ & Unit \\
\hline $\begin{array}{l}\text { Bis(2-ethylhexyl) phthalate } \\
\text { Dichloromethane }\end{array}$ & $\begin{array}{l}<17 \\
<1.7\end{array}$ & $\begin{array}{l}1 \\
1\end{array}$ & & & & $\begin{array}{l}0 \\
0\end{array}$ & $\begin{array}{l}\text { GE } \\
\text { GE }\end{array}$ & & & & & & & & $\begin{array}{l}\mu \mathrm{g} / \mathrm{L} \\
\mu \mathrm{g} / \mathrm{L}\end{array}$ \\
\hline
\end{tabular}

Note: See Appendix B for flagging criteria. Synchronous water levels are measured over a 3-5 day period.

- = exceeded holding time.

- = exceeded groundwater protection standard. 
WELL FSB 97C (cont.)

Radiological Indicator Parameters

Parameter

Gross alpha

Nonvolatile beta
1095 D

2.0E $+03 \quad 1$

$1.1 \mathrm{E}+03 \quad 1$
MF

J

ST $\underline{\text { H Flag Lab }}$

GP

Measured Radionuclides

\section{Parameter}

Actinium-228

Americium-241

Cesium-137

Cobalt -60

Curium-242

Curium-243/244

Curium-245/246

lodine-129

Plutonium-238

Plutonium-239/240

Radium-226

Radium-228

Strontium-89

Strontium-90

Technetium-99

Thorium-228

Thorium-230

Thorium-232

Thorium-234

Tritium

Uranium-233/234

Uranium-235

Uranium-238

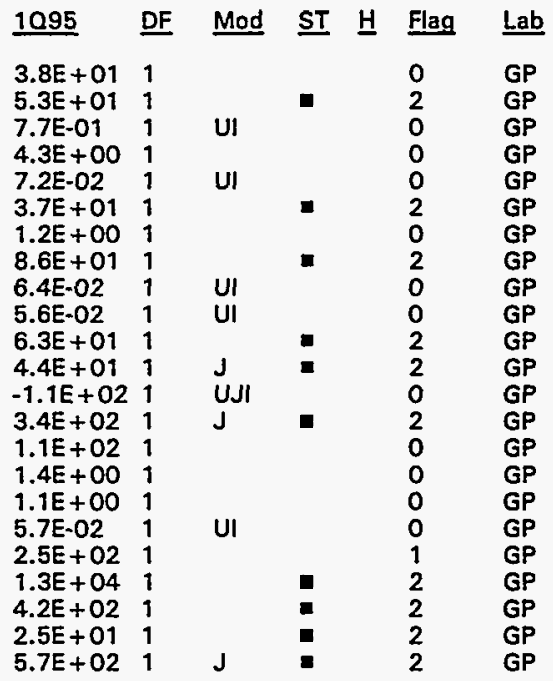

Calculated Radionuclides

Parameter

Americium-243

Nickel-63

Plutonium-241

Plutonium-242
1095

1.1E-01

4.3E +00

2.2E-01

1.1E-06
DF Mad $\underline{\text { ST }} \underline{H}$ Flag Lab $\underline{2095}$

DF Mod ST H Fiag Lab

Unit

pCi/L

pCi/L

pCi/L

2095 DF Mod ST $\underline{\text { F Flag Lab Unit }}$

pCi/L

pCi/L

pCi/L

pCi/L

pCi/L

pCill

pCi/l/

pCin

pCill

pCill

pCi/L

$\mathrm{pCi} / \mathrm{L}$

pCirL

pCill

$\mathrm{pCi} / \mathrm{L}$

PCi/L

PCi/L

pCi/L

Note: See Appendix B for flagging criteria. Synchronous water levels are measured over a 3-5 day period.

- = exceeded holding time.

- = exceeded groundwater protection standard. 
WELL FSB 97D

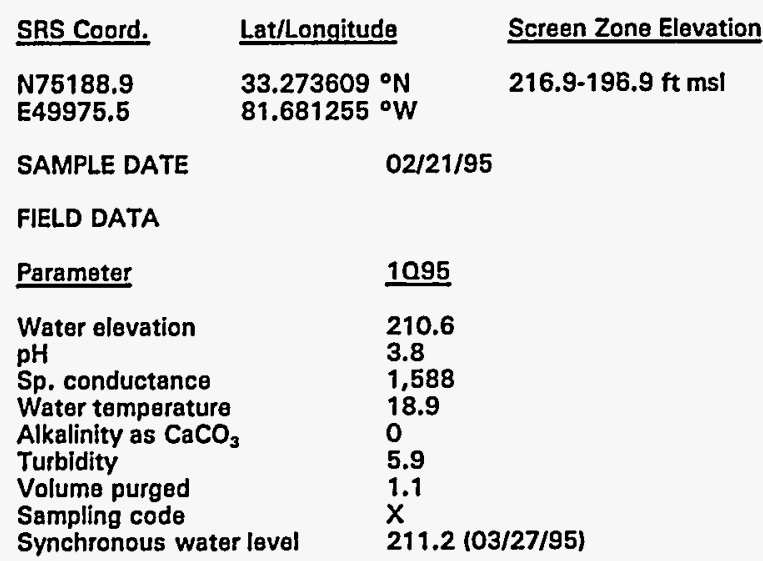

$\begin{array}{lrll}\text { Top of Casing } & \text { Casing } & \text { Pump } & \text { Screan Zone } \\ 286 \mathrm{ft} \mathrm{msl} & \text { 4" PVC } & V & \text { Water Table (IIB })_{2} \\ 05 / 04 / 95 & & \end{array}$

Hazardous Constituents (264.94, Table 1)

\begin{tabular}{|c|c|c|c|c|c|c|}
\hline Parameter & 1095 & DF & Mod & $\underline{\mathrm{ST}}$ & H & Flag \\
\hline $\begin{array}{l}\text { Arsenic, total recoverable } \\
\text { Barium, total recoverable } \\
\text { Cadmium, total recoverable } \\
\text { Chromium, total recoverable } \\
\text { Lead, total recoverable } \\
\text { Mercury, total recoverable } \\
\text { Selenium, total recoverable } \\
\text { Silver, total recoverable }\end{array}$ & $\begin{array}{c}<5.0 \\
1,200 \\
9.1 \\
<4.0 \\
34 \\
0.12 \\
<5.0 \\
2.5\end{array}$ & $\begin{array}{l}1 \\
1 \\
1 \\
1 \\
1 \\
1 \\
1 \\
1\end{array}$ & JVV & $\mathbf{\square}$ & & $\begin{array}{l}0 \\
1 \\
2 \\
0 \\
1 \\
0 \\
0 \\
0\end{array}$ \\
\hline
\end{tabular}

$\begin{array}{ll}\underline{2095} & \text { Unit } \\ 211.7 & \mathrm{ftmsl} \\ & \mathrm{pH} \\ \mu \mathrm{S} / \mathrm{cm} \\ & { }^{\circ} \mathrm{C} \\ & \mathrm{mg} / \mathrm{L} \\ & \mathrm{NTU} \\ & \text { well volumes } \\ W & \mathrm{ft} \mathrm{msl}\end{array}$

Hazardous Constituents (261 App. VIII/264 App. IX)

\begin{tabular}{|c|c|c|c|c|c|c|c|c|c|c|c|c|c|c|c|}
\hline Parameter & 1095 & DF & Mod & $\underline{\text { ST }}$ & $\underline{H}$ & Flag & $\underline{\text { Lab }}$ & $\underline{2095}$ & DF & Mod & ST & $\underline{H}$ & Flag & $\underline{\text { Lab }}$ & $\underline{\text { Unit }}$ \\
\hline $\begin{array}{l}\text { Antimony, total recoverable } \\
\text { Benzene } \\
\text { Cobalt, total recoverable } \\
\text { Copper, total recoverable } \\
\text { Cyanide } \\
\text { Nickel, total recoverable } \\
\text { Phenols } \\
\text { Tetrachloroethylene } \\
\text { Thallium, total recoverable } \\
\text { Trichloroethylene } \\
\text { Trichlorofluoromethane } \\
\text { Vanadium, total recoverable } \\
\text { Zinc, total recoverable }\end{array}$ & $\begin{array}{r}4.0 \\
<1.7 \\
81 \\
64 \\
<20 \\
47 \\
<10 \\
<1.7 \\
1.1 \\
<1.7 \\
<1.7 \\
<10 \\
180\end{array}$ & $\begin{array}{l}1 \\
1 \\
1 \\
1 \\
1 \\
1 \\
1 \\
1 \\
1 \\
1 \\
1 \\
1 \\
1\end{array}$ & J1 & 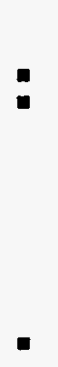 & & $\begin{array}{l}1 \\
0 \\
2 \\
0 \\
0 \\
0 \\
0 \\
0 \\
1 \\
0 \\
0 \\
0 \\
0\end{array}$ & $\begin{array}{l}\mathrm{GE} \\
\mathrm{GE} \\
\mathrm{GE} \\
\mathrm{GE} \\
\mathrm{GE} \\
\mathrm{GE} \\
\mathrm{GE} \\
\mathrm{GE} \\
\mathrm{GE} \\
\mathrm{GE} \\
\mathrm{GE} \\
\mathrm{GE} \\
\mathrm{GE}\end{array}$ & & & & & & & & $\begin{array}{l}\mu \mathrm{g} / \mathrm{L} \\
\mu \mathrm{g} / \mathrm{L} \\
\mu g / \mathrm{L} \\
\mu \mathrm{g} / \mathrm{L} \\
\mu \mathrm{g} / \mathrm{L} \\
\mu \mathrm{g} / \mathrm{L} \\
\mu \mathrm{g} / \mathrm{L} \\
\mu g / \mathrm{L} \\
\mu \mathrm{g} / \mathrm{L} \\
\mu \mathrm{g} / \mathrm{L} \\
\mu \mathrm{g} / \mathrm{L} \\
\mu \mathrm{g} / \mathrm{L} \\
\mu \mathrm{g} / \mathrm{L}\end{array}$ \\
\hline \multicolumn{16}{|c|}{ Water Quality Indicator Parameters } \\
\hline Parameter & 1095 & $\underline{D F}$ & Mod & $\underline{\text { ST }}$ & $\underline{H}$ & Flag & $\underline{\text { Lab }}$ & $\underline{2095}$ & DF & Mod & ST & $\underline{H}$ & Flag & $\underline{\text { Lab }}$ & $\underline{\text { Unit }}$ \\
\hline $\begin{array}{l}\text { Aluminum, total recoverable } \\
\text { Nitrate as nitrogen } \\
\text { pH }\end{array}$ & $\begin{array}{l}53,000 \\
220,000 \\
3.8\end{array}$ & $\begin{array}{l}10 \\
160 \\
1\end{array}$ & $\begin{array}{l}V \\
\text { J1 }\end{array}$ & 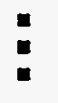 & 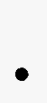 & $\begin{array}{l}2 \\
2 \\
1\end{array}$ & $\begin{array}{l}\text { GE } \\
\text { GE } \\
\text { GE }\end{array}$ & & & & & & & & $\begin{array}{l}\mu \mathrm{g} / \mathrm{L} \\
\mu \mathrm{g} / \mathrm{L} \\
\mathrm{pH}\end{array}$ \\
\hline \multicolumn{16}{|c|}{ Common Laboratory Contaminants } \\
\hline Parameter & 1095 & DF & Mod & $\underline{\text { sT }}$ & 브 & Flag & $\underline{\text { Lab }}$ & $\underline{2095}$ & $\underline{D F}$ & Mod & $\underline{\mathbf{S T}}$ & $\underline{H}$ & Flag & $\underline{\text { Lab }}$ & Unit \\
\hline $\begin{array}{l}\text { Bis(2-ethylhexyl) phthalate } \\
\text { Dichloromethane }\end{array}$ & $\begin{array}{l}<17 \\
<1.7\end{array}$ & $\begin{array}{l}1 \\
1\end{array}$ & & & & $\begin{array}{l}0 \\
0\end{array}$ & $\begin{array}{l}\mathrm{GE} \\
\mathrm{GE}\end{array}$ & & & & & & & & $\begin{array}{l}\mu g / L \\
\mu g / L\end{array}$ \\
\hline
\end{tabular}

Note: See Appendix B for flagging criteria. Synchronous water levels are measured over a 3-5 day period.

- = exceeded holding time.

- = exceeded groundwater protection standard. 
WELL FSB 97D (cont.)

Radiological Indicator Parameters

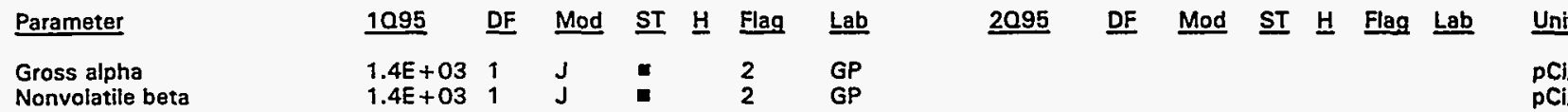

Measured Radionuclides

Parameter

Actinium-228

Americium-241

Cesium-137

Cobalt-60

Curium-242

Curium-243/244

Curium-245/246

lodine-129

Plutonium-238

Plutonium-239/240

Radium-226

Radium-228

Strontium-89

Strontium-90

Technetium-99

Thorium-228

Thorium-230

Thorium-232

Thorium-234

Tritium

Uranium-233/234

Uranium-235

Uranium-238
1095 DF Mod ST $\underline{H}$ Flag Lab

2.6E+01

5. $0 E+011$

$1.7 E+011$

2.7E + $00 \quad 1$

9.1E-02

$4.9 E+011$

2.4E + 001

$7.3 E+011$

6.1E-01

$0.0 E+001$

1.7E+01 1

$4.3 E+01$

$-2.4 \mathrm{E}+021$

$5.9 E+02$

$3.9 E+011$

$1.7 E+00$

6.9E-01

1.9E-02

$2.4 E+02$

1.OE+04 1

$4.5 \mathrm{E}+021$

$3.2 E+011$

$1.2 E+03$
2095 DF Mad ST $\underline{H}$ Flag Lab Unit

$\mathrm{pCi} / \mathrm{L}$

pCi/L

PCI/L

$\mathrm{pCi} / \mathrm{L}$

pCi/L

pCill

$\mathrm{pCi} / \mathrm{L}$

pCi/L

$\mathrm{pCi} / \mathrm{L}$

pCi/L

pCi/L

PCílL

pCi/L

$\mathrm{pCi} / \mathrm{L}$

pCi/L

pCi/L.

pCi/L

pCi/L

pCi/mL

$\mathrm{pCi} / \mathrm{L}$

pCi/L

$\mathrm{pCi} / \mathrm{L}$

Calculated Radionuclides

Parameter

DF Mod ST $\underline{H}$ Flag Lab

$\underline{2095}$

DF Mod ST $\underline{H}$ Flag Lab

Unit

$\mathrm{PCi} / \mathrm{L}$

pCi/L

PCi/L

2.7E+00

$0.0 E+00$

$0.0 E+00$

0
0
0
0

Nickel-63

Plutonium-242

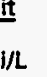

OE 


\section{WELL FSB 98AR}

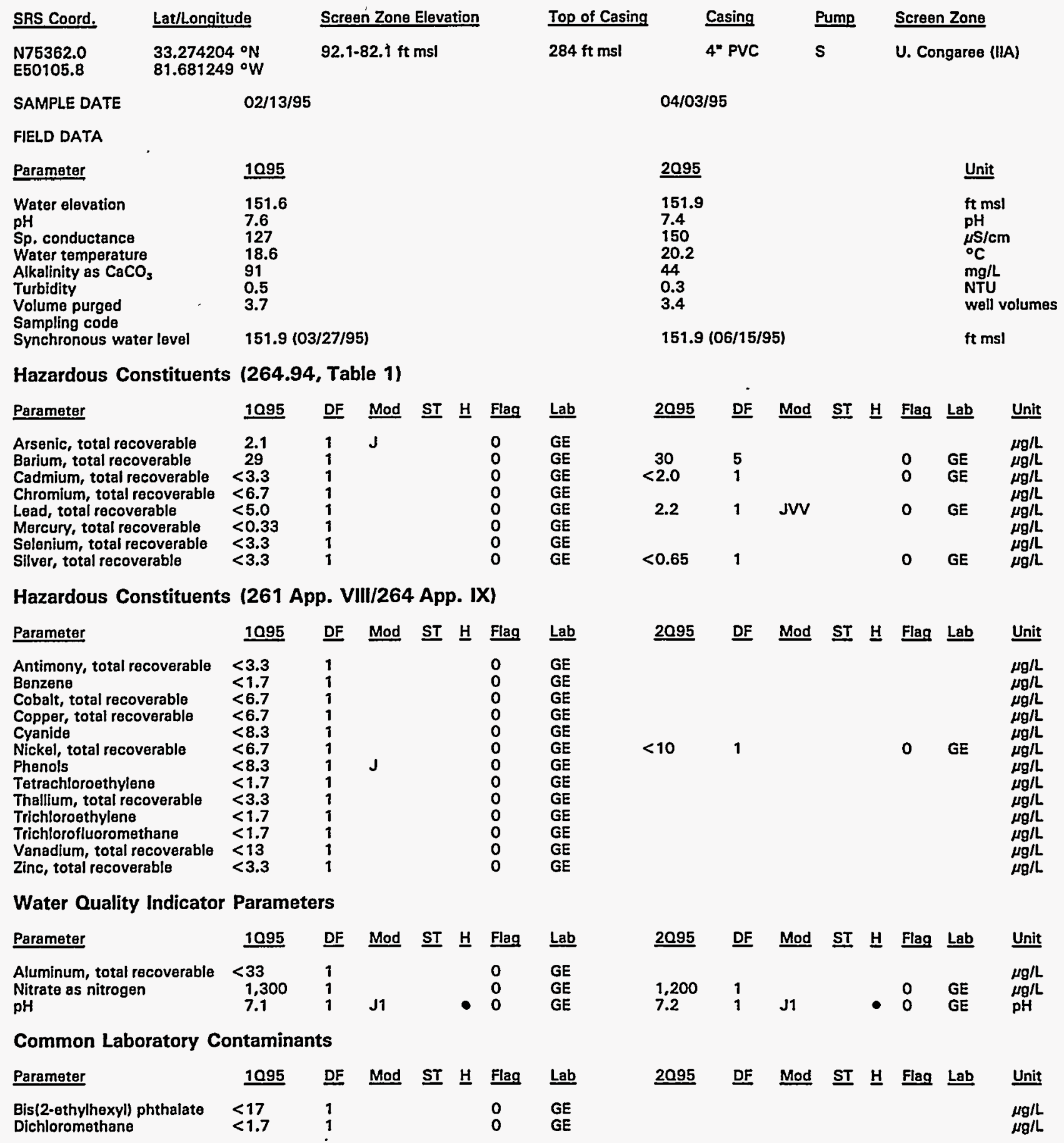

Note: See Appendix B for flagging criteria. Synchronous water levels are measured over a 3-5 day period.

$\bullet=$ exceeded holding time.

- = exceeded groundwater protection standard. 
WELL FSB 98AR (cont.)

Radiological Indicator Parameters

\begin{tabular}{|c|c|c|c|c|c|c|c|c|c|c|c|c|c|c|}
\hline Parameter & 1095 & DF & Mod & ST & ㅂ & Flag & Lab & 2095 & DF & Mod & ST $\underline{H}$ & Flag & Lab & Unit \\
\hline $\begin{array}{l}\text { Gross alpha } \\
\text { Nonvolatile beta }\end{array}$ & $\begin{array}{l}1.6 E+00 \\
1.0 E+00\end{array}$ & $\begin{array}{l}1 \\
1\end{array}$ & UI & & & $\begin{array}{l}0 \\
0\end{array}$ & $\begin{array}{l}\text { GP } \\
\text { GP }\end{array}$ & $\begin{array}{l}9.1 E-01 \\
1.3 E+\infty\end{array}$ & $\begin{array}{l}1 \\
1\end{array}$ & J & & $\begin{array}{l}0 \\
0\end{array}$ & $\begin{array}{l}\text { GP } \\
\text { GP }\end{array}$ & $\begin{array}{l}\mathrm{pCi} / \mathrm{L} \\
\mathrm{pCI} / \mathrm{L}\end{array}$ \\
\hline
\end{tabular}

Measured Radionuclides

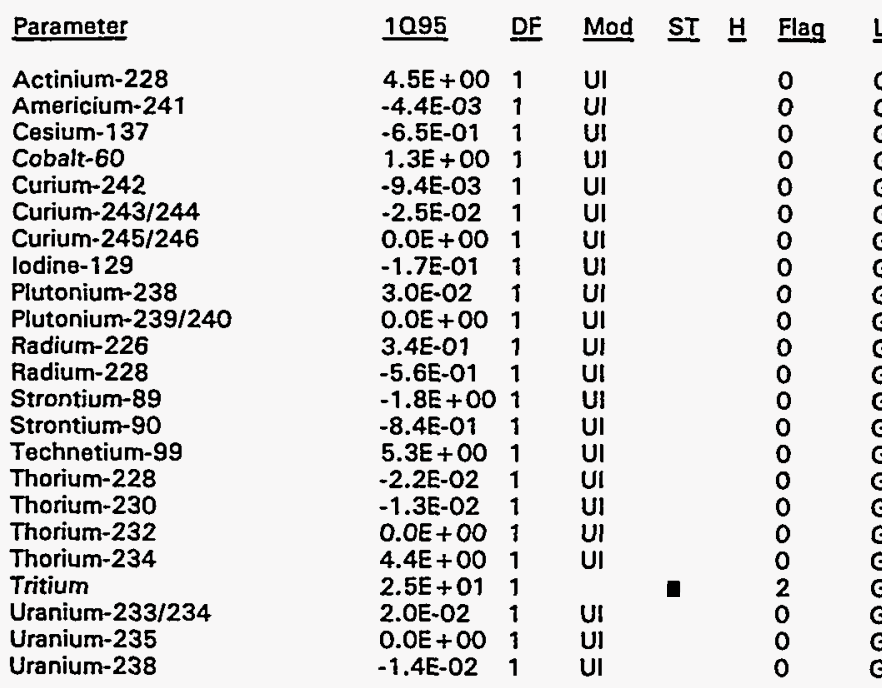

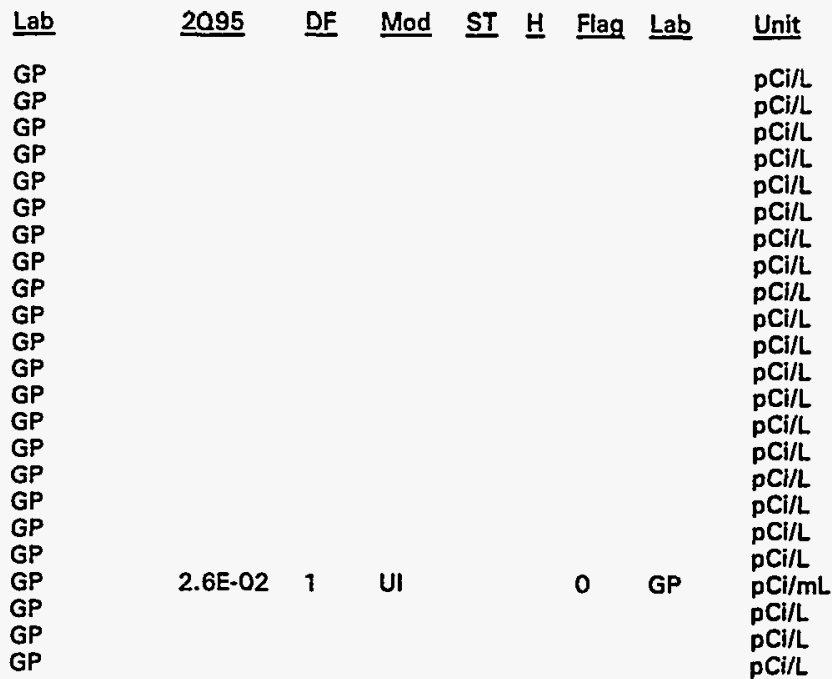

Calculated Radionuclides

Parameter

Americium-243

Nickel-63

PJutonium-241

Plutonium-242
1095 DF Mod ST H Flag Lab

$\underline{209}$
$-7.5 \mathrm{E}-05$

$1.3 E+00$

$0.0 E+00$

$0.0 E+00$
0

0 $\underline{\text { Unit }}$

pCi/L

pCi/L

pCill

Note: See Appendix B for flagging criteria. Synchronous water levels are measured over a 3-5 day period.

- = exceeded holding time.

- exceeded groundwater protection standard. 


\section{WELL FSB 98C}

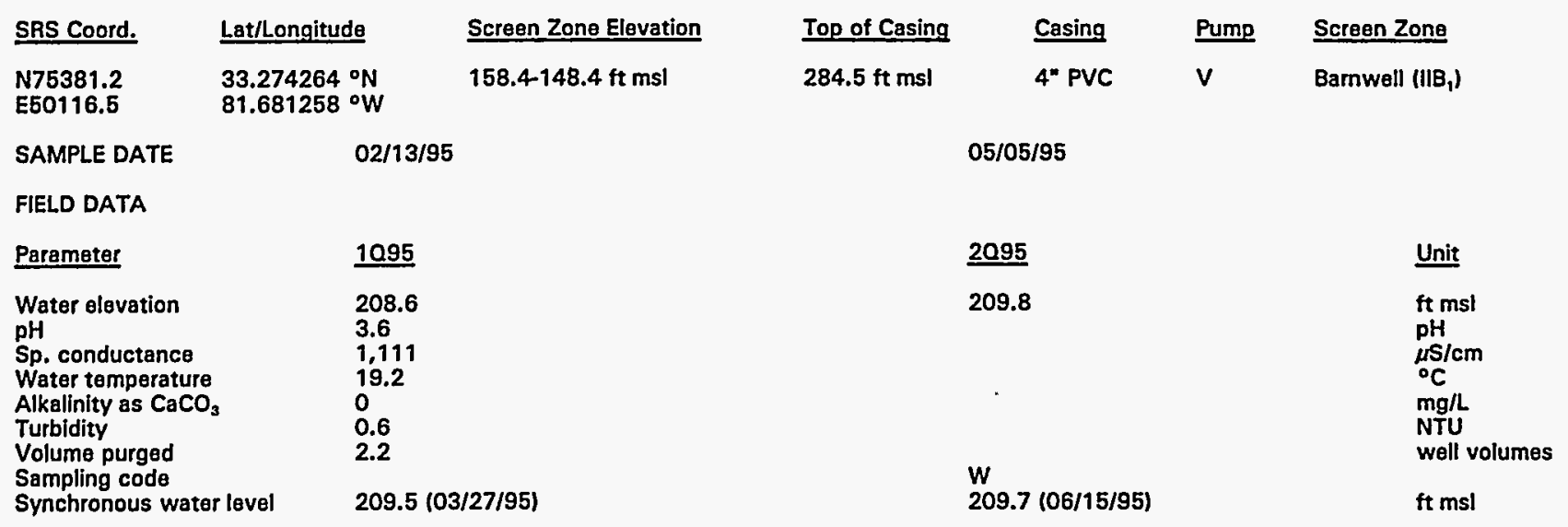

Hazardous Constituents (264.94, Table 1)

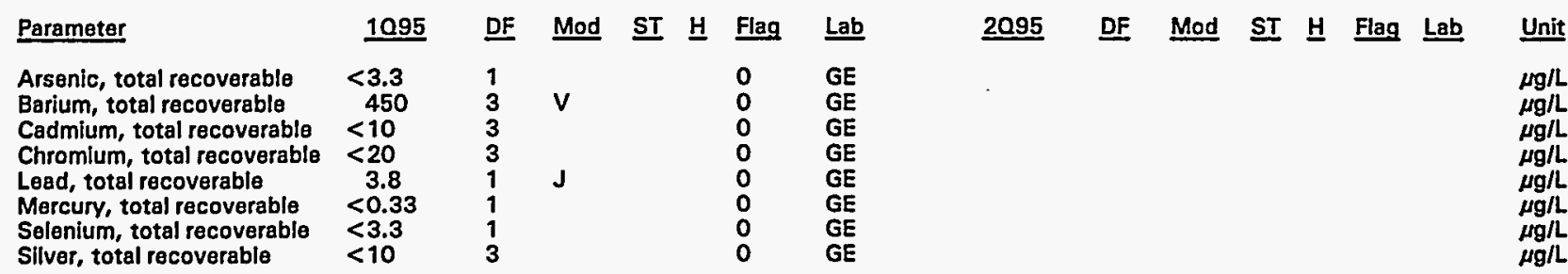

Hazardous Constituents (261 App. VIII/264 App. IX)

\begin{tabular}{|c|c|c|c|c|c|c|c|c|c|c|c|c|c|c|}
\hline Parameter & 1095 & DF & Mod & $\underline{\mathbf{S T}}$ & 보 & Flag & Lab & 2095 & DF & Mod & ST & 브 & Flag & Lab \\
\hline $\begin{array}{l}\text { Antimony, total recoverable } \\
\text { Benzene } \\
\text { Cobalt, total recoverable } \\
\text { Copper, total recoverable } \\
\text { Cyanide } \\
\text { Nickel, total recoverable } \\
\text { Phenols } \\
\text { Tetrachloroethylene } \\
\text { Thallium, total recoverable } \\
\text { Trichloroethylene } \\
\text { Trichlorofluoromethane } \\
\text { Vanadium, total recoverable } \\
\text { Zinc, total recoverable }\end{array}$ & $\begin{array}{c}<3.3 \\
<1.7 \\
350 \\
72 \\
<8.3 \\
44 \\
<8.3 \\
<1.7 \\
<3.3 \\
<1.7 \\
<1.7 \\
<40 \\
120\end{array}$ & $\begin{array}{l}1 \\
1 \\
3 \\
3 \\
1 \\
3 \\
1 \\
1 \\
1 \\
1 \\
1 \\
3 \\
3\end{array}$ & $J 1$ & $\mathbf{\square}$ & • & $\begin{array}{l}0 \\
0 \\
2 \\
0 \\
0 \\
0 \\
0 \\
0 \\
0 \\
0 \\
0 \\
0 \\
0\end{array}$ & $\begin{array}{l}\text { GE } \\
\text { GE } \\
\text { GE } \\
\text { GE } \\
\text { GE } \\
\text { GE } \\
\text { GE } \\
\text { GE } \\
\text { GE } \\
\text { GE } \\
\text { GE } \\
\text { GE } \\
\text { GE }\end{array}$ & & & & & & & \\
\hline
\end{tabular}

Water Quality Indicator Parameters

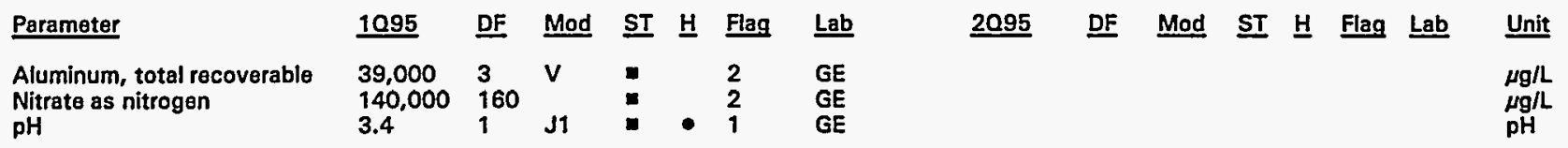

Common Laboratory Contaminants

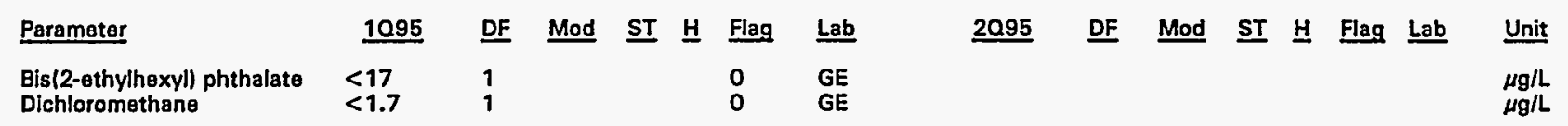

Note: See Appendix B́ for flagging criteria. Synchronous water levels are measured over a 3-5 day period.

- = exceeded holding time.

I = exceeded groundwater protection standard. 
WELL FSB 98C (cont.)

Radiological Indicator Parameters

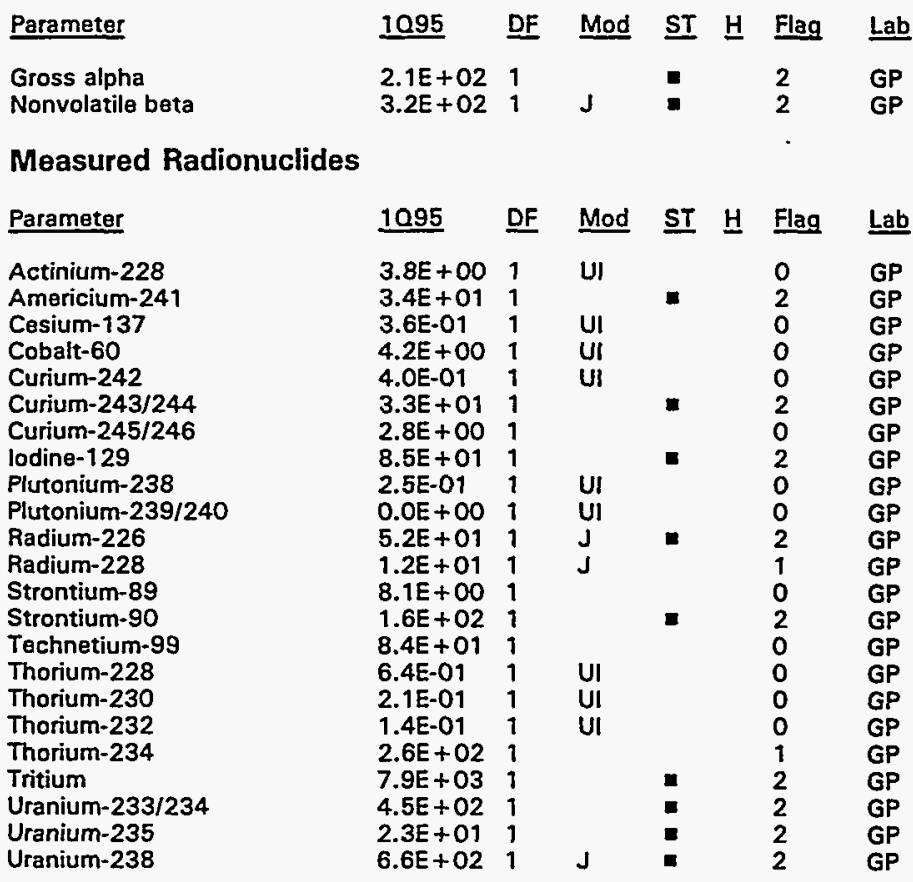

2095 DF Mod ST $\underline{H}$ Flag Lab Unit

pCI/L PCi/L

$\underline{2095}$ DF Mod ST $\underline{H}$ Flag Lab Unit

pCi/L

PCi/L

pCi/L

PCi/L

pCi/L

pCi/L

PCi/L

$\mathrm{pCi} / \mathrm{L}$

$\mathrm{pCi} / \mathrm{L}$

pCi/L

pCi/L

$\mathrm{pCi} / \mathrm{L}$

$\mathrm{pCin}$

pCi/L

$\mathrm{PCI} / \mathrm{L}$

pCi/L

$\mathrm{DCI} / \mathrm{L}$

$\mathrm{pCi} / \mathrm{L}$

DCill

pCin

$\mathrm{pCI} / \mathrm{L}$

Uranium-238

Calculated Radionuclides

Parameter

Americium-243

Nickel-63

Plutonium-241

Piutonium-242
1095

DF

Mod

Flag Lab
0
0
0
0

$\underline{2095}$

DF Mod ST $\underline{H}$ Flag Lab

Unit

$4.2 E+00$

$0.0 E+00$

$0.0 E+00$

Note: See Appendix B for flagging criteria. Synchronous water levels are measured over a 3-5 day period.

$-=$ exceeded holding time.

m = exceeded groundwater protection standard. 
WELL FSB 98D

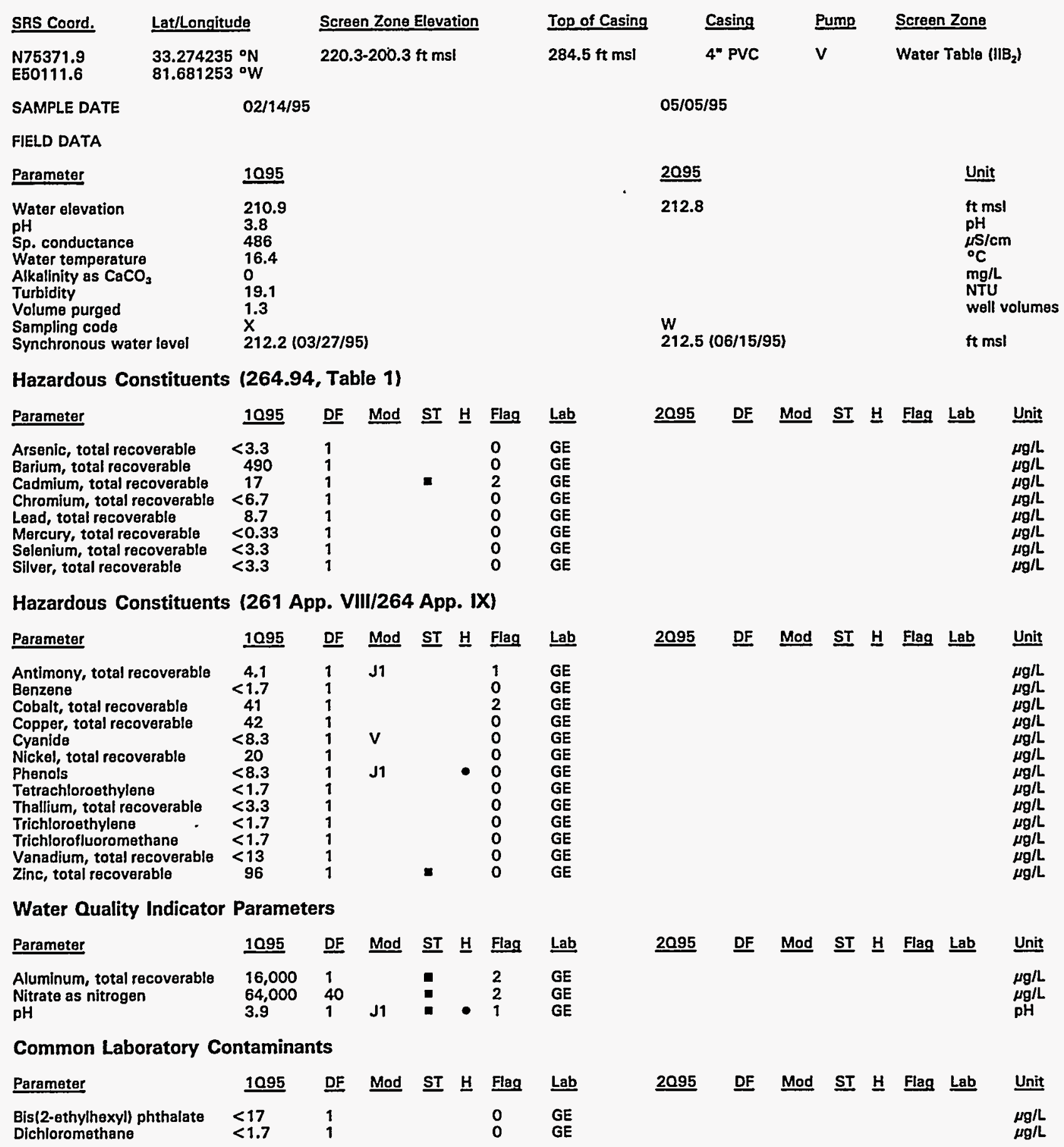

Note: See Appendix B for flagging criteria. Synchronous water levels are measured over a 3-5 day period.

- = exceeded holding time.

- = exceeded groundwater protection standard. 
WELL FSB 98D (cont.)

Radiological Indicator Parameters

\begin{tabular}{|c|c|c|c|c|c|c|c|c|c|c|c|c|c|c|c|}
\hline Parameter & $\underline{1095}$ & $\underline{\mathrm{DF}}$ & Mod & $\underline{\text { ST }}$ & $\underline{\mathrm{H}}$ & Flag & $\underline{\text { Lab }}$ & $\underline{2095}$ & $\underline{D F}$ & Mod & $\underline{\mathbf{S T}}$ & $\underline{\text { H}}$ & Flag & $\underline{\text { Lab }}$ & $\underline{\text { Unit }}$ \\
\hline $\begin{array}{l}\text { Gross alpha } \\
\text { Nonvolatile beta }\end{array}$ & $\begin{array}{l}8.9 E+02 \\
1.3 E+03\end{array}$ & $\begin{array}{l}1 \\
1\end{array}$ & $\begin{array}{l}\mathrm{J} \\
\mathrm{J}\end{array}$ & - & & $\begin{array}{l}2 \\
2\end{array}$ & $\begin{array}{l}\text { GP } \\
\text { GP }\end{array}$ & & & & & & & & $\begin{array}{l}\mathrm{pCi} / \mathrm{L} \\
\mathrm{pCi} / \mathrm{L}\end{array}$ \\
\hline \multicolumn{16}{|c|}{ Measured Radionuclides } \\
\hline Parameter & 1095 & $\underline{D F}$ & Mod & $\underline{\text { ST }}$ & $\underline{H}$ & Flag & $\underline{\text { Lab }}$ & $\underline{2095}$ & $\underline{\mathrm{DF}}$ & Mod & $\underline{\text { ST }}$ & $\underline{H}$ & Flag & $\underline{\text { Lab }}$ & $\underline{\text { Unit }}$ \\
\hline $\begin{array}{l}\text { Actinium-228 } \\
\text { Americium-241 } \\
\text { Cesium-137 } \\
\text { Cobalt-60 } \\
\text { Curium-242 } \\
\text { Curium-243/244 } \\
\text { Curium-245/246 } \\
\text { lodine-129 } \\
\text { Plutonium-238 } \\
\text { Plutonium-239/240 } \\
\text { Radium-226 } \\
\text { Radium-228 } \\
\text { Strontium-89 } \\
\text { Strontium-90 } \\
\text { Technetium-99 } \\
\text { Thorium-228 } \\
\text { Thorium-230 } \\
\text { Thorium-232 } \\
\text { Thorium-234 } \\
\text { Tritium } \\
\text { Uranium-233/234 } \\
\text { Uranium-235 } \\
\text { Uranium-238 }\end{array}$ & $\begin{array}{l}0.0 E+00 \\
2.3 E+01 \\
1.2 E+00 \\
1.3 E+00 \\
1.3 E+00 \\
2.1 E+01 \\
1.1 E+00 \\
2.5 E+01 \\
-3.3 E-02 \\
-1.1 E-02 \\
1.3 E+01 \\
2.5 E+01 \\
1.1 E+02 \\
2.8 E+02 \\
4.3 E+01 \\
5.7 E-01 \\
5.8 E-02 \\
1.5 E-01 \\
4.6 E+02 \\
3.8 E+03 \\
2.4 E+02 \\
1.5 E+01 \\
5.3 E+02\end{array}$ & $\begin{array}{l}1 \\
1 \\
1 \\
1 \\
1 \\
1 \\
1 \\
1 \\
1 \\
1 \\
1 \\
1 \\
1 \\
1 \\
1 \\
1 \\
1 \\
1 \\
1 \\
1 \\
1 \\
1 \\
1\end{array}$ & $\begin{array}{l}\text { UI } \\
\text { UI } \\
\text { UI } \\
\text { UI }\end{array}$ & $\begin{array}{l}. \\
. \\
. \\
. \\
.\end{array}$ & & $\begin{array}{l}0 \\
2 \\
0 \\
0 \\
0 \\
2 \\
0 \\
2 \\
0 \\
0 \\
1 \\
2 \\
2 \\
2 \\
0 \\
0 \\
0 \\
0 \\
2 \\
2 \\
2 \\
2 \\
2\end{array}$ & $\begin{array}{l}\text { GP } \\
\text { GP } \\
\text { GP } \\
\text { GP } \\
\text { GP } \\
\text { GP } \\
\text { GP } \\
\text { GP } \\
\text { GP } \\
\text { GP } \\
\text { GP } \\
\text { GP } \\
\text { GP } \\
\text { GP } \\
\text { GP } \\
\text { GP } \\
\text { GP } \\
\text { GP } \\
\text { GP } \\
\text { GP } \\
\text { GP } \\
\text { GP } \\
\text { GP }\end{array}$ & & & & & & & & $\begin{array}{l}\mathrm{pCi} / \mathrm{L} \\
\mathrm{pCi} / \mathrm{L} \\
\mathrm{pCi} / \mathrm{L} \\
\mathrm{pCi} / \mathrm{L} \\
\mathrm{pCi} / \mathrm{L} \\
\mathrm{pCi} / \mathrm{L} \\
\mathrm{pCi} / \mathrm{L} \\
\mathrm{pCi} / \mathrm{L} \\
\mathrm{pCi} / \mathrm{L} \\
\mathrm{pCi} / \mathrm{L} \\
\mathrm{pCi} / \mathrm{L} \\
\mathrm{pCi} / \mathrm{L} \\
\mathrm{pCi} / \mathrm{L} \\
\mathrm{pCi} / \mathrm{L} \\
\mathrm{pCi} / \mathrm{L} \\
\mathrm{pCi} / \mathrm{L} \\
\mathrm{pCi} / \mathrm{L} \\
\mathrm{pCi} / \mathrm{L} \\
\mathrm{pCi} / \mathrm{L} \\
\mathrm{pCi} / \mathrm{mL} \\
\mathrm{pCi} / \mathrm{L} \\
\mathrm{pCi} / \mathrm{L} \\
\mathrm{pCi} / \mathrm{L}\end{array}$ \\
\hline \multicolumn{16}{|c|}{ Calculated Radionuclides } \\
\hline Parameter & 1095 & $\underline{D F}$ & Mod & $\underline{\mathrm{ST}}$ & $\underline{H}$ & Flag & $\underline{\text { Lab }}$ & $\underline{2095}$ & $\underline{\mathrm{DF}}$ & Mod & $\underline{\text { ST }}$ & $\underline{H}$ & Flag & $\underline{\text { Lab }}$ & Unit \\
\hline $\begin{array}{l}\text { Americium-243 } \\
\text { Nickel-63 } \\
\text { Plutonium-241 } \\
\text { Plutonium-242 }\end{array}$ & $\begin{array}{l}6.3 E-02 \\
1.3 E+00 \\
-4.4 E-02 \\
-2.2 E-07\end{array}$ & & & & & $\begin{array}{l}0 \\
0 \\
0 \\
0\end{array}$ & & & & & & & & & $\begin{array}{l}\mathrm{pCi} / \mathrm{L} \\
\mathrm{pCC/L} \\
\mathrm{pCC/L} \\
\mathrm{pCi} / \mathrm{L}\end{array}$ \\
\hline
\end{tabular}

Note: See Appendix B for flagging criteria. Synchronous water levels are measured over a 3-5 day period.

- = exceeded holding time.

- = exceeded groundwater protection standard. 


\section{WELL FSB 99A}

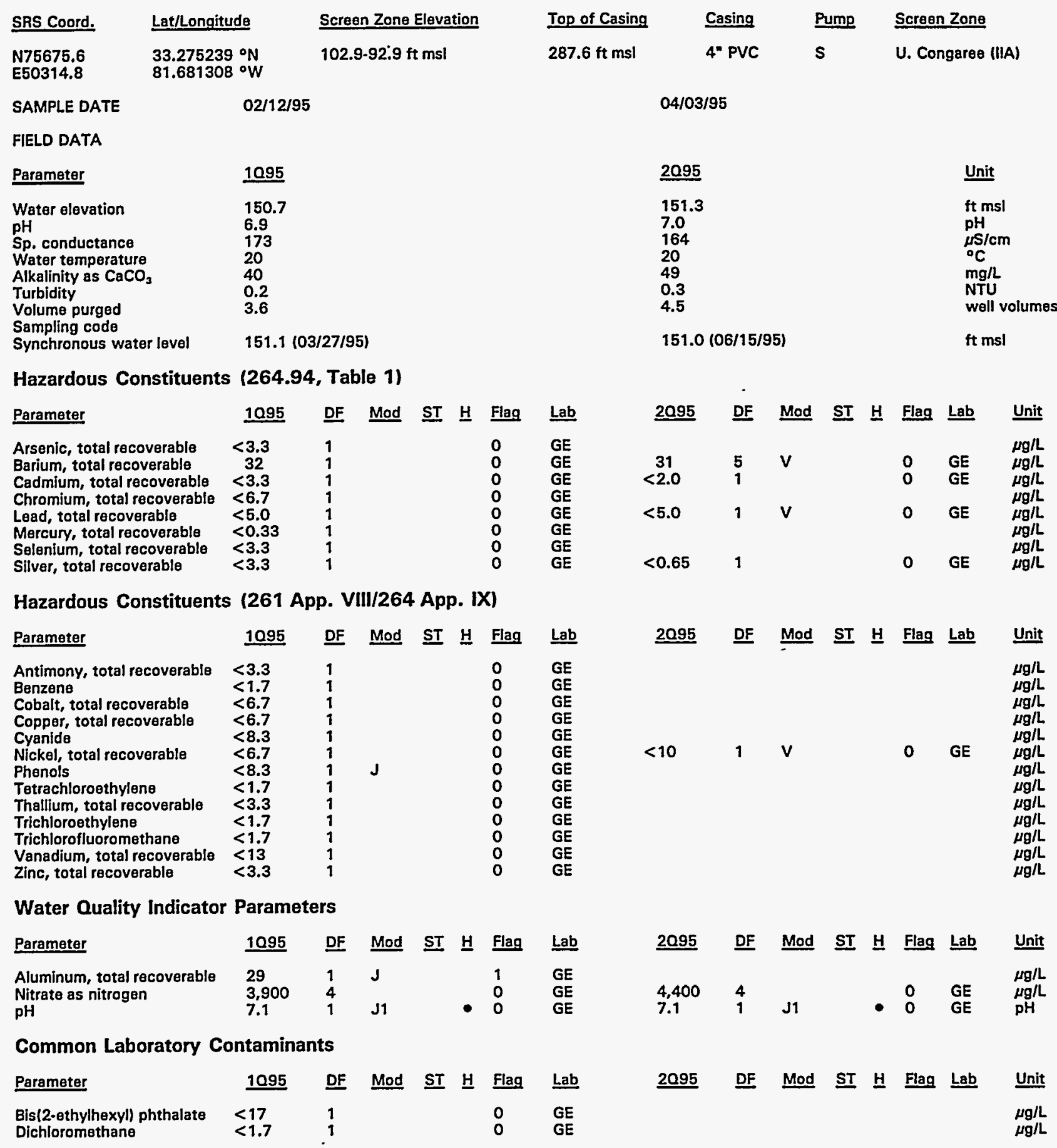

Note: See Appendix B for flagging criteria. Synchronous water levels are measured over a 3-5 day period.

$\checkmark=$ exceeded holding time.

a = exceeded groundwater protection standard. 
WELL FSB 99A (cont.)

Radiological Indicator Parameters

\begin{tabular}{|c|c|c|c|c|c|c|c|c|c|c|c|c|c|c|c|}
\hline Parameter & 1095 & DF & Mod & $\underline{\mathrm{ST}}$ & 브 & Flag & Lab & 2095 & $\underline{\text { DF }}$ & Mod & ST & $\underline{H}$ & Flag & Lab & Unit \\
\hline $\begin{array}{l}\text { Gross alpha } \\
\text { Nonvolatile beta }\end{array}$ & $\begin{array}{l}\text { 8.3E-01 } \\
2.6 E+00\end{array}$ & $\begin{array}{l}1 \\
1\end{array}$ & UI & & & $\begin{array}{l}0 \\
0\end{array}$ & $\begin{array}{l}\text { GP } \\
\text { GP }\end{array}$ & $\begin{array}{l}2.0 E+00 \\
1.8 E+00\end{array}$ & $\begin{array}{l}1 \\
1\end{array}$ & $\mathbf{J}$ & & & $\begin{array}{l}0 \\
0\end{array}$ & $\begin{array}{l}\text { GP } \\
\text { GP }\end{array}$ & $\begin{array}{l}\mathrm{pCi} / \mathrm{L} \\
\mathrm{pCi} / \mathrm{L}\end{array}$ \\
\hline \multicolumn{16}{|c|}{ Measured Radionuclides } \\
\hline Parameter & 1095 & DF & Mod & ST & $\underline{H}$ & Flag & Lab & 2095 & DF & Mod & $\underline{\mathrm{ST}}$ & $\underline{H}$ & Flag & Lab & Unit \\
\hline $\begin{array}{l}\text { Actinium-228 } \\
\text { Americium-241 } \\
\text { Cesium-137 } \\
\text { Cobalt-60 } \\
\text { Curium-242 } \\
\text { Curium-243/244 } \\
\text { Curium-245/246 } \\
\text { lodine-129 } \\
\text { Plutonium-238 } \\
\text { Plutonium-239/240 } \\
\text { Radium-226 } \\
\text { Radium-228 } \\
\text { Strontium-89 } \\
\text { Strontium-90 } \\
\text { Technetium-99 } \\
\text { Thorium-228 } \\
\text { Thorium-230 } \\
\text { Thorium-232 } \\
\text { Thorium-234 } \\
\text { Tritium } \\
\text { Uranium-233/234 } \\
\text { Uranium-235 } \\
\text { Uranium-238 }\end{array}$ & $\begin{array}{l}4.0 E+00 \\
-7.2 E-02 \\
-9.9 E-02 \\
-2.0 E-01 \\
4.5 E-02 \\
-2.8 E-02 \\
0.0 E+00 \\
2.6 E+00 \\
4.5 E-01 \\
-7.2 E-03 \\
4.7 E-01 \\
-3.4 E-01 \\
-1.6 E-01 \\
-8.9 E-01 \\
-8.0 E+00 \\
-7.8 E-02 \\
5.6 E-01 \\
-1.9 E-02 \\
1.5 E+02 \\
1.6 E+02 \\
0.0 E+00 \\
0.0 E+00 \\
2.1 E-01\end{array}$ & $\begin{array}{l}1 \\
1 \\
1 \\
1 \\
1 \\
1 \\
1 \\
1 \\
1 \\
1 \\
1 \\
1 \\
1 \\
1 \\
1 \\
1 \\
1 \\
1 \\
1 \\
1 \\
1 \\
1 \\
1\end{array}$ & $\begin{array}{l}\text { UI } \\
\text { UI } \\
\text { UI } \\
\text { UI } \\
\text { UI } \\
\text { UI } \\
\text { UI } \\
\text { UI } \\
\text { UI } \\
\text { UI } \\
\text { UI } \\
\text { UI } \\
\text { UI } \\
\text { UI } \\
\text { UI } \\
\text { UI } \\
\text { UI } \\
\text { UI } \\
\text { UI } \\
\text { UI }\end{array}$ & घ & & $\begin{array}{l}0 \\
0 \\
0 \\
0 \\
0 \\
0 \\
0 \\
2 \\
0 \\
0 \\
0 \\
0 \\
0 \\
0 \\
0 \\
0 \\
0 \\
0 \\
0 \\
2 \\
0 \\
0 \\
0\end{array}$ & $\begin{array}{l}\text { GP } \\
\text { GP } \\
\text { GP } \\
\text { GP } \\
\text { GP } \\
\text { GP } \\
\text { GP } \\
\text { GP } \\
\text { GP } \\
\text { GP } \\
\text { GP } \\
\text { GP } \\
\text { GP } \\
\text { GP } \\
\text { GP } \\
\text { GP } \\
\text { GP } \\
\text { GP } \\
\text { GP } \\
\text { GP } \\
\text { GP } \\
\text { GP } \\
\text { GP }\end{array}$ & $7.8 E+01$ & 1 & & ט & & 2 & GP & 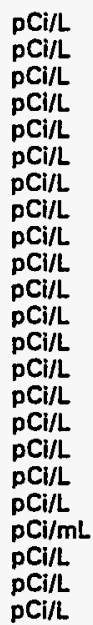 \\
\hline \multicolumn{16}{|c|}{ Calculated Radionuclides } \\
\hline Parameter & 1095 & DF & Mod & ST & $\underline{H}$ & Flag & Lab & $\underline{2095}$ & DF & Mod & ST & $\underline{H}$ & Flag & $\underline{\text { Lab }}$ & Unit \\
\hline $\begin{array}{l}\text { Americium-243 } \\
\text { Nickel-63 } \\
\text { Plutonium-241 } \\
\text { Plutonium-242 }\end{array}$ & $\begin{array}{l}-8.4 E-05 \\
-2.0 E-01 \\
-2.9 E-02 \\
-1.4 E-07\end{array}$ & & & & & $\begin{array}{l}0 \\
0 \\
0 \\
0\end{array}$ & & & & & & & & & $\begin{array}{l}\mathrm{pCi} / \mathrm{L} \\
\mathrm{pCl} / \mathrm{L} \\
\mathrm{pCi} / \mathrm{L} \\
\mathrm{pCi} / \mathrm{L}\end{array}$ \\
\hline
\end{tabular}

Note: See Appendix B for flagging criteria. Synchronous water levels are measured over a 3-5 day period.

- = exceeded holding time.

- = exceeded groundwater protection standard. 


\section{WELL FSB 99C}

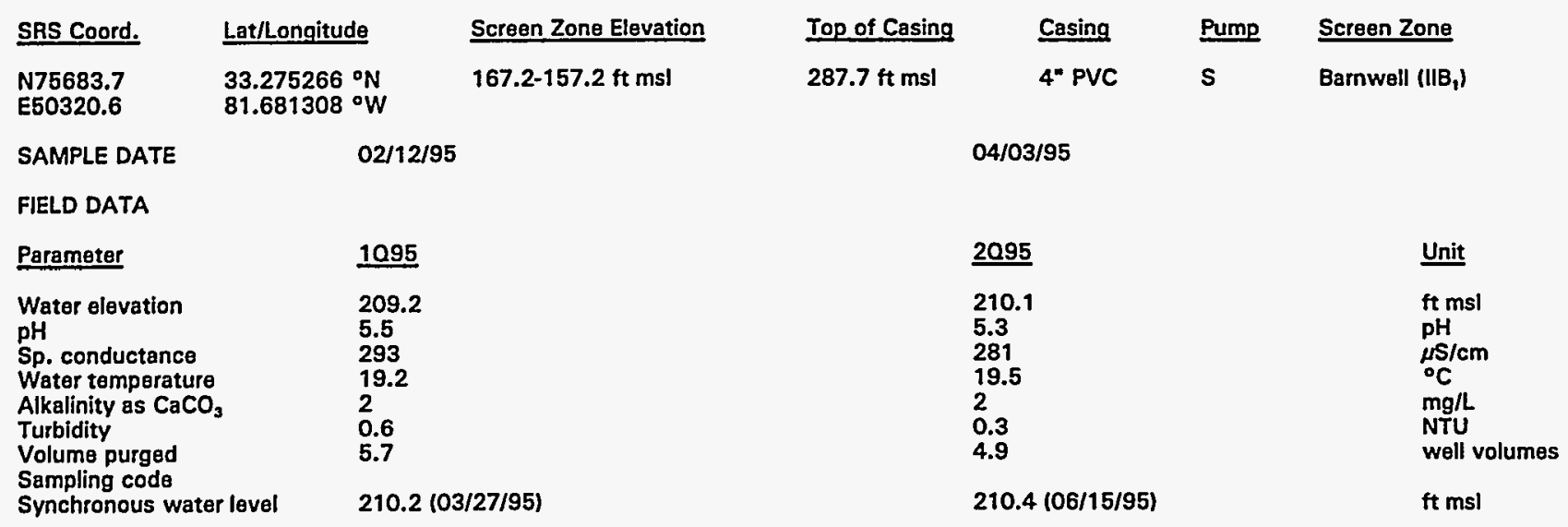

Hazardous Constituents (264.94, Table 1)

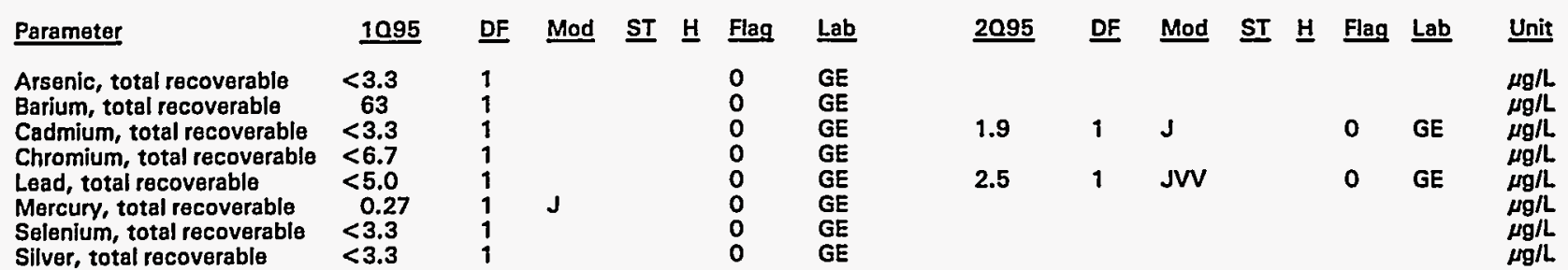

Hazardous Constituents (261 App. VIII/264 App. IX)

$\begin{array}{lccccccc}\text { Parameter } & \underline{1095} & \text { DF } & \text { Mod } & \text { ST } & \text { H } & \text { Flag } & \text { Lab } \\ \text { Antimony, total recoverable } & <3.3 & 1 & & & & 0 & \text { GE } \\ \text { Benzene } & <1.7 & 1 & & & 0 & \text { GE } \\ \text { Cobalt, total recoverable } & 5.1 & 1 & J & & 0 & \text { GE } \\ \text { Copper, total recoverable } & <6.7 & 1 & & & 0 & \text { GE } \\ \text { Cyanide } & <8.3 & 1 & & & 0 & \text { GE } \\ \text { Nickel, total recoverable } & <6.7 & 1 & & & 0 & \text { GE } \\ \text { Phenols } & <8.3 & 1 & J & & 0 & \text { GE } \\ \text { Tetrachloroethylene } & <1.7 & 1 & & & 0 & \text { GE } \\ \text { Thallium, total recoverable } & <3.3 & 1 & & & 0 & \text { GE } \\ \text { Trichlorosthylene } & 18 & 1 & & & 2 & \text { GE } \\ \text { Trichlorofluoromethane } & 2.0 & 1 & & & 0 & \text { GE } \\ \text { Vanadium, total recoverable } & <13 & 1 & & & 0 & \text { GE } \\ \text { Zinc, total recoverable } & 18 & 1 & & & 0 & \text { GE }\end{array}$

Zinc, total recoverable

18

Lab
GE
GE
GE
GE
GE
$\mathrm{GE}$
$\mathrm{GE}$
$\mathrm{GE}$
$\mathrm{GE}$
$\mathrm{GE}$
$\mathrm{GE}$
$\mathrm{GE}$

$\mu g / L$
$\mu g / L$
$\mu g / L$
$\mu g / L$
$\mu g / L$
$\mu g / L$
$\mu g / L$
$\mu g / L$
$\mu g / L$
$\mu g / L$
$\mu g / L$
$\mu g / L$
$\mu g / L$

Water Quality Indicator Parameters

\begin{tabular}{|c|c|c|c|c|c|c|c|c|c|c|c|c|c|c|c|}
\hline Parameter & 1095 & $\underline{D F}$ & Mod & $\underline{\text { ST }}$ & $\underline{H}$ & Flag & $\underline{\text { Lab }}$ & $\underline{2095}$ & $\underline{D F}$ & Mod & $\underline{\text { ST }}$ & $\underline{H}$ & Flag & $\underline{\text { Lab }}$ & $\underline{\text { Unit }}$ \\
\hline $\begin{array}{l}\text { Aluminum, total recoverable } \\
\text { Nitrate as nitrogen } \\
\text { pH }\end{array}$ & $\begin{array}{l}120 \\
28,000 \\
5.4\end{array}$ & $\begin{array}{l}1 \\
20 \\
1\end{array}$ & J1 & 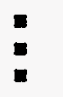 & & $\begin{array}{l}2 \\
2 \\
0\end{array}$ & $\begin{array}{l}\mathrm{GE} \\
\mathrm{GE} \\
\mathrm{GE}\end{array}$ & $\begin{array}{l}30,000 \\
5.9\end{array}$ & $\begin{array}{l}20 \\
1\end{array}$ & J1 & $=$ & & $\begin{array}{l}2 \\
0\end{array}$ & $\begin{array}{l}\text { GE } \\
\text { GE }\end{array}$ & $\begin{array}{l}\mu \mathrm{g} / \mathrm{L} \\
\mu \mathrm{g} / \mathrm{L} \\
\mathrm{pH}\end{array}$ \\
\hline \multicolumn{16}{|c|}{ Common Laboratory Contaminants } \\
\hline Parameter & 1095 & $\underline{D F}$ & Mod & $\underline{\text { ST }}$ & $\underline{H}$ & Flag & $\underline{\text { Lab }}$ & $\underline{2095}$ & DF & Mod & $\underline{\mathbf{S T}}$ & H & Flag & $\underline{\text { Lab }}$ & Unit \\
\hline $\begin{array}{l}\text { Bis(2-ethylhexyl) phthalate } \\
\text { Dichloromethane }\end{array}$ & $\begin{array}{l}<17 \\
<1.7\end{array}$ & $\begin{array}{l}1 \\
1\end{array}$ & & & & $\begin{array}{l}0 \\
0\end{array}$ & $\begin{array}{l}\mathrm{GE} \\
\mathrm{GE}\end{array}$ & & & & & & & & $\begin{array}{l}\mu g / L \\
\mu g / L\end{array}$ \\
\hline
\end{tabular}

Note: See Appendix B for flagging criteria. Synchronous water levels are measured over a 3-5 day period.

- = exceeded holding time.

E = exceeded groundwater protection standard. 
WELL FSB 99C (cont.)

Radiological Indicator Parameters

\begin{tabular}{|c|c|c|c|c|c|c|c|c|c|c|c|c|c|c|c|}
\hline Parameter & 1095 & DF & Mod & $\underline{\mathrm{ST}}$ & $\underline{H}$ & Flag & Lab & 2095 & $\underline{\text { DF }}$ & Mod & ST & 브 & Flag & $\underline{L a b}$ & Unit \\
\hline $\begin{array}{l}\text { Gross alpha } \\
\text { Nonvolatile beta }\end{array}$ & $\begin{array}{l}2.1 E+01 \\
8.7 E+01\end{array}$ & 1 & & 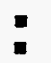 & & $\begin{array}{l}2 \\
2\end{array}$ & $\begin{array}{l}\text { GP } \\
\text { GP }\end{array}$ & $\begin{array}{l}3.7 E+01 \\
9.0 E+01\end{array}$ & $\begin{array}{l}1 \\
1\end{array}$ & $J$ & 口 & & $\begin{array}{l}2 \\
2\end{array}$ & $\begin{array}{l}\text { GP } \\
\text { GP }\end{array}$ & $\begin{array}{l}\mathrm{pCi} / \mathrm{L} \\
\mathrm{pCi} / \mathrm{L}\end{array}$ \\
\hline
\end{tabular}

Measured Radionuclides

\begin{tabular}{|c|c|c|c|c|c|c|c|c|c|c|c|c|c|c|c|}
\hline Parameter & $\underline{1095}$ & $\underline{\mathrm{DF}}$ & Mod & $\underline{\text { ST }}$ & $\underline{H}$ & Flag & $\underline{\underline{\text { Lab}}}$ & $\underline{2095}$ & $\underline{D F}$ & Mod & ST & $\underline{H}$ & Flag & $\underline{L a b}$ & Unit \\
\hline $\begin{array}{l}\text { Actinium-228 } \\
\text { Americium-241 } \\
\text { Cesium-137 } \\
\text { Cobalt-60 } \\
\text { Curium-242 } \\
\text { Curium-243/244 } \\
\text { Curium-245/246 } \\
\text { lodine-129 } \\
\text { Plutonium-238 } \\
\text { Plutonium-239/240 } \\
\text { Radium-226 } \\
\text { Radium-228 } \\
\text { Strontium-89 } \\
\text { Strontium-90 } \\
\text { Technetium-99 } \\
\text { Thorium-228 } \\
\text { Thorium-230 } \\
\text { Thorium-232 } \\
\text { Thorium-234 } \\
\text { Tritium } \\
\text { Uranium-233/234 } \\
\text { Uranium-235 } \\
\text { Uranium-238 }\end{array}$ & $\begin{array}{l}4.5 E+00 \\
2.8 E-01 \\
8.1 E-01 \\
2.4 E-01 \\
1.6 E-01 \\
-3.7 E-03 \\
0.0 E+00 \\
1.2 E+01 \\
1.9 E-02 \\
0.0 E+00 \\
9.0 E-02 \\
1.0 E+00 \\
7.1 E+00 \\
1.8 E+01 \\
9.7 E+00 \\
5.8 E-03 \\
1.1 E-01 \\
-1.7 E-02 \\
5.9 E+01 \\
1.5 E+03 \\
6.1 E+00 \\
2.1 E-01 \\
6.3 E+00\end{array}$ & $\begin{array}{l}1 \\
1 \\
1 \\
1 \\
1 \\
1 \\
1 \\
1 \\
1 \\
1 \\
1 \\
1 \\
1 \\
1 \\
1 \\
1 \\
1 \\
1 \\
1 \\
1 \\
1 \\
1 \\
1\end{array}$ & $\begin{array}{l}\text { UI } \\
\text { UI } \\
\text { UI } \\
\text { UI } \\
\text { UI } \\
\text { UI } \\
\text { UI } \\
\text { UI } \\
\text { UI } \\
\text { UI }\end{array}$ & 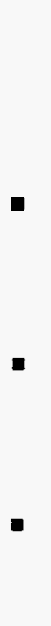 & & $\begin{array}{l}0 \\
0 \\
0 \\
0 \\
0 \\
0 \\
0 \\
2 \\
0 \\
0 \\
0 \\
0 \\
0 \\
2 \\
0 \\
0 \\
0 \\
0 \\
0 \\
2 \\
0 \\
0 \\
0\end{array}$ & $\begin{array}{l}\text { GP } \\
\text { GP } \\
\text { GP } \\
\text { GP } \\
\text { GP } \\
\text { GP } \\
\text { GP } \\
\text { GP } \\
\text { GP } \\
\text { GP } \\
\text { GP } \\
\text { GP } \\
\text { GP } \\
\text { GP } \\
\text { GP } \\
\text { GP } \\
\text { GP } \\
\text { GP } \\
\text { GP } \\
\text { GP } \\
\text { GP } \\
\text { GP } \\
\text { GP }\end{array}$ & 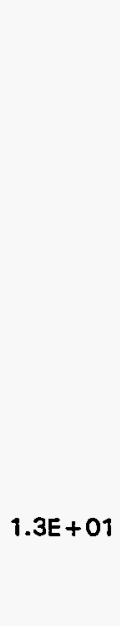 & 1 & & & & 1 & GP & $\begin{array}{l}\text { pCi/L } \\
\text { pCi/L } \\
\text { pCi/L } \\
\text { pCi/L } \\
\text { pCi/L } \\
\text { pCi/L } \\
\text { pCi/L } \\
\text { pCi/L } \\
\text { pCi/L } \\
\text { pCi/L } \\
\text { pCi/L } \\
\text { pCi/L } \\
\text { pCi/L } \\
\text { pCi/L } \\
\text { pCi/L } \\
\text { pCi/L } \\
\text { pCi/L } \\
\text { pCi/L } \\
\text { pCi/L } \\
\text { pCi/mL } \\
\text { pCi/L } \\
\text { pCi/L } \\
\text { pCi/L }\end{array}$ \\
\hline \multicolumn{16}{|c|}{ Calculated Radionuclides } \\
\hline Parameter & 1095 & DF & Mod & $\underline{\text { ST }}$ & $\underline{\mathbf{H}}$ & Flag & $\underline{\operatorname{Lab}}$ & $\underline{2095}$ & DF & Mod & ST & H & Flag & $\underline{\text { Lab }}$ & Unit \\
\hline $\begin{array}{l}\text { Americium-243 } \\
\text { Nickel-63 } \\
\text { Plutonium-241 } \\
\text { Plutonium-242 }\end{array}$ & $\begin{array}{l}-1.1 E-05 \\
2.4 E-01 \\
0.0 E+00 \\
0.0 E+00\end{array}$ & & & & & $\begin{array}{l}0 \\
0 \\
0 \\
0\end{array}$ & & & & & & & & & $\begin{array}{l}\mathrm{pCi} / \mathrm{L} \\
\mathrm{pCi} / \mathrm{L} \\
\mathrm{pCi} / \mathrm{L} \\
\mathrm{pCi} / \mathrm{L}\end{array}$ \\
\hline
\end{tabular}

Note: See Appendix B for flagging criteria. Synchronous water levels are measured over a 3-5 day period.

- = exceeded holding time.

- = exceeded groundwater protection standard. 
WELL FSB 99D

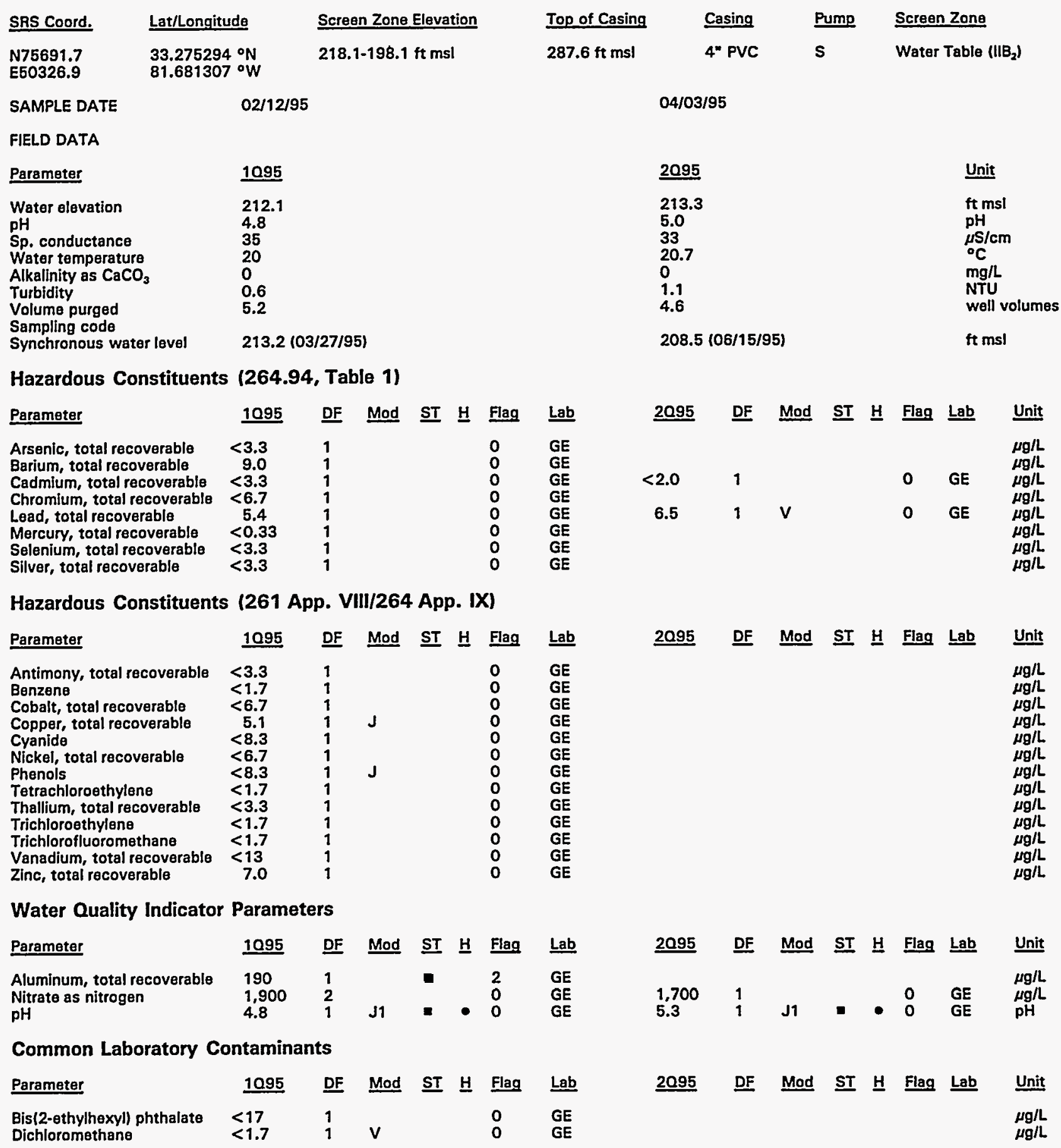

Note: See Appendix B for flagging criteria. Synchronous water levels are measured over a 3-5 day period.

$\checkmark=$ exceeded holding time.

- = exceeded groundwater protection standard. 
WELL FSB 99D (cont.)

Radiological Indicator Parameters

\begin{tabular}{|c|c|c|c|c|c|c|c|c|c|c|c|c|}
\hline Parameter & 1095 & $\underline{D F}$ & Mod & ST $\underline{H}$ & Flag & Lab & $\underline{2095}$ & $\underline{\mathrm{DF}}$ & Mod & $\underline{\text { ST }} \underline{H}$ & Fiag & Lab \\
\hline $\begin{array}{l}\text { Gross alpha } \\
\text { Nonvolatile beta }\end{array}$ & $\begin{array}{l}1.4 E+01 \\
2.1 E+01\end{array}$ & $\begin{array}{l}1 \\
1\end{array}$ & & & $\begin{array}{l}1 \\
0\end{array}$ & $\begin{array}{l}\text { GP } \\
\text { GP }\end{array}$ & $\begin{array}{l}1.6 E+0 \\
1.6 E+0\end{array}$ & 1 & $J$ & - & $\begin{array}{l}2 \\
0\end{array}$ & $\begin{array}{l}\text { GP } \\
\text { GP }\end{array}$ \\
\hline
\end{tabular}

Measured Radionuclides

\section{Parameter}

Actinium-228

Americium-241

Cesium-137

Cobalt 60

Curium-242

Curium-243/244

Curium-245/246

lodine-129

Plutonium-238

Plutonium-239/240

Radium-226

Radium-228

Strontium-89

Strontium-90

Technetium-99

Thorium-228

Thorium-230

Thorium-232

Thorium-234

Tritium

Uranium-235

Uranium-238
Uranium-233/234
1095 DF Mod ST 브 Flag Lab

$-1.2 E+001 \quad U$

8.8E-02

-2.7E-01

6.6E-01

-4.7E-03

6.9E-01

$-5.6 E-03$

9.3E-01

$-1.4 \mathrm{E}-02$

$-1.2 E-02$

8.0E-01

$-4.8 \mathrm{E}-01$

$-3.1 E-01$

$4.9 \mathrm{E}+00$

$-7.9 E+001$

$0.0 E+00$

3.0E-01

5.5E-02

$1.0 \mathrm{E}+02$

2.2E + 011

5.1E +00

2.4E-01 1

1.3E + 011

$\begin{array}{lll}\text { UI } & 0 & \text { GP } \\ \text { UI } & 0 & \text { GP }\end{array}$

UI

UII

UI

UI

UI

UI

UI

UI

UI

UI

uI

U

u

UI
Calculated Radionuclides

Parameter

Americium-243

Nickel-63

Plutonium-241

Plutonium-242
1095

2.1E-03

6.6E-01

$-4.8 \mathrm{E}-02$

$-2.4 \mathrm{E}-07$
DF Mod ST $\underline{H}$ Flag LLab

0
2095 DF Mod ST H Flag Lab Unit

$\mathrm{pCI} / \mathrm{L}$

pCi/L

$\mathrm{pCI} / \mathrm{L}$

$\mathrm{pCi} / \mathrm{L}$

$\mathrm{pCl} / \mathrm{L}$

pCi/L

$\mathrm{pCi} / \mathrm{L}$

pCi/L

pCilL

pCill

$\mathrm{pCi} / \mathrm{L}$

$\mathrm{pCi} / \mathrm{L}$

pCI/L

pCi/L

pCi/L

PCil

$\mathrm{pCi}$

$5.5 E+011$

2 GP

$\mathrm{pCi} / \mathrm{mL}$

pCi/L

pCi/L

$\mathrm{pCi} / \mathrm{L}$

$\underline{2095}$ DF Mod ST $\underline{H}$ Fiag Lab $\underline{\text { Unit }}$

$\mathrm{PCI} / \mathrm{L}$

$\mathrm{pCi/L}$

$\mathrm{pCi} / \mathrm{L}$

pCi/L

Note: See Appendix B for flagging criteria. Synchronous water levels are measured over a 3-5 day period.

- = exceeded holding time.

- = exceeded groundwater protection standard. 


\section{WELL FSB100A}

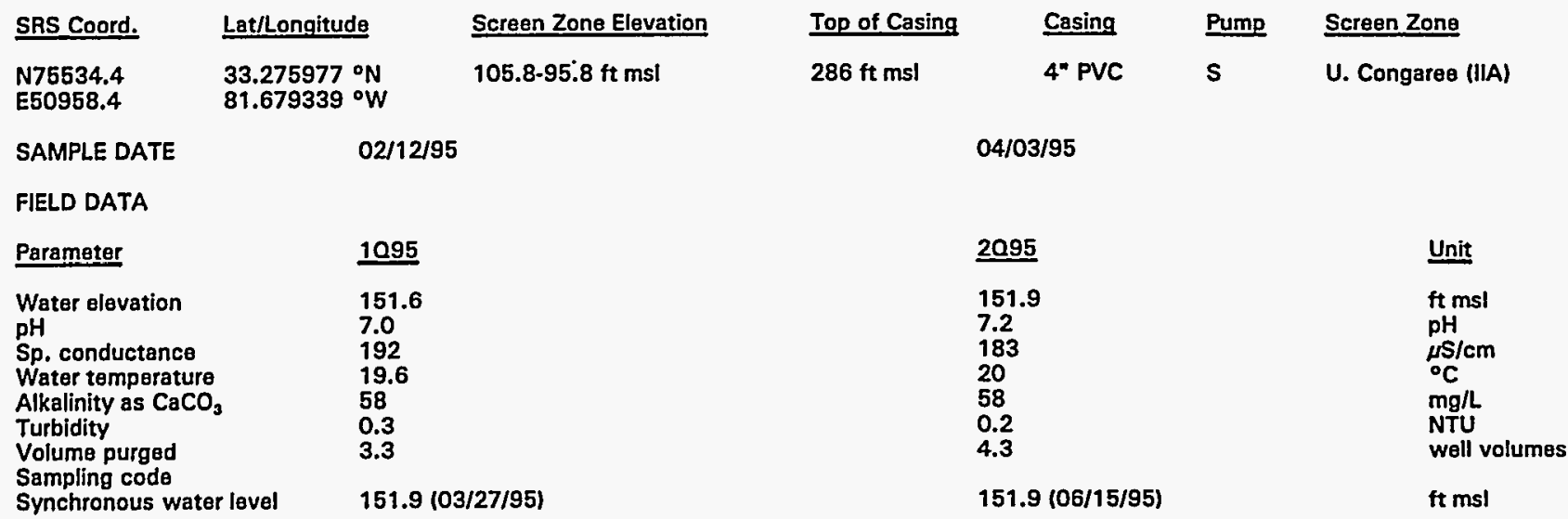

Hazardous Constituents (264.94, Table 1)

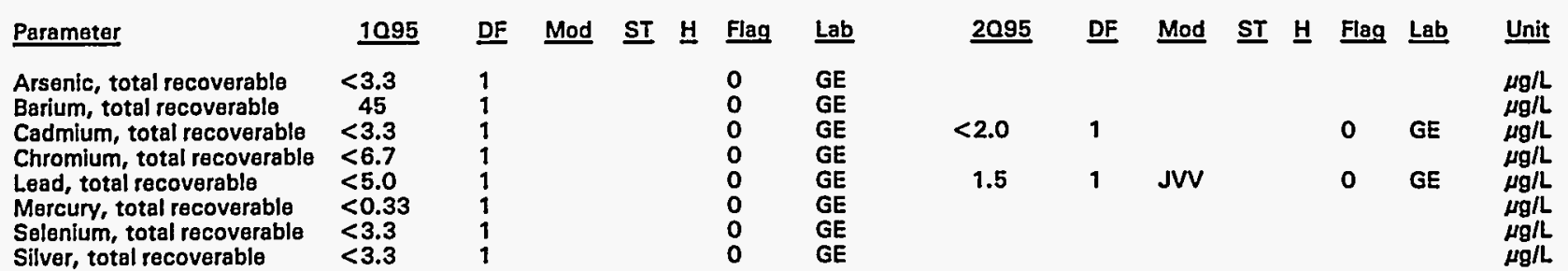

Hazardous Constituents (261 App. VIII/264 App. IX)

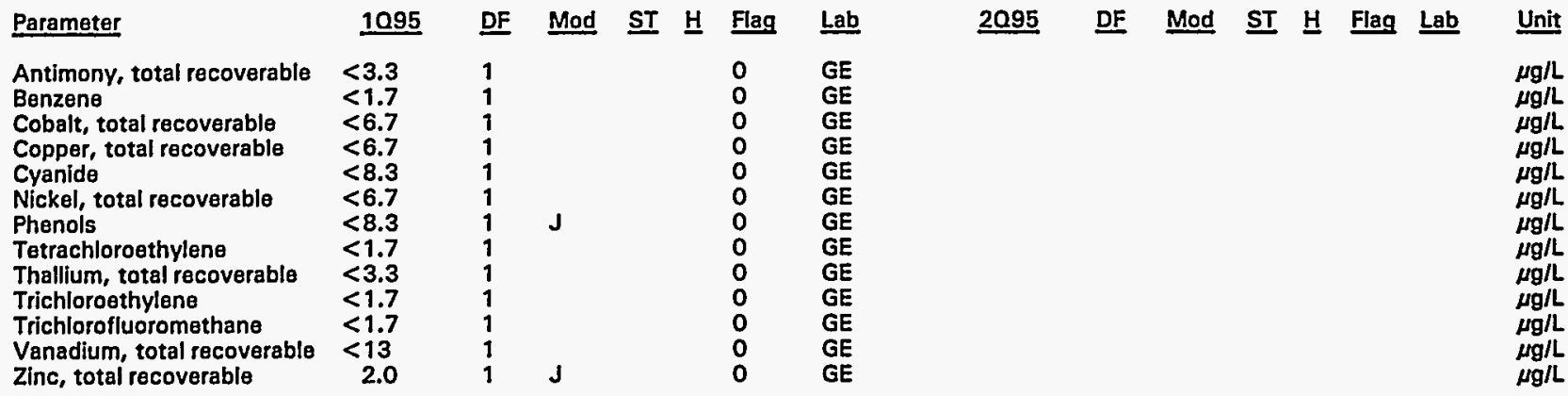

Water Quality Indicator Parameters

\begin{tabular}{|c|c|c|c|c|c|c|c|c|c|c|c|c|c|c|c|}
\hline Parameter & $\underline{1095}$ & $\underline{\mathrm{DF}}$ & Mod & $\underline{\mathrm{ST}}$ & $\underline{\mathbf{H}}$ & Flag & $\underline{\text { Lab }}$ & $\underline{2095}$ & $\underline{D F}$ & Mod & $\underline{\mathbf{S T}}$ & $\underline{H}$ & Flag & $\underline{\text { Lab }}$ & $\underline{\text { Unit }}$ \\
\hline $\begin{array}{l}\text { Aluminum, total recoverable } \\
\text { Nitrate as nitrogen } \\
\text { pH }\end{array}$ & $\begin{array}{l}<33 \\
4,300 \\
7.4\end{array}$ & $\begin{array}{l}1 \\
4 \\
1\end{array}$ & J1 & & & $\begin{array}{l}0 \\
0 \\
0\end{array}$ & $\begin{array}{l}\text { GE } \\
\text { GE } \\
\text { GE }\end{array}$ & $\begin{array}{l}4,300 \\
7.6\end{array}$ & $\begin{array}{l}4 \\
1\end{array}$ & J1 & & - & $\begin{array}{l}0 \\
0\end{array}$ & $\begin{array}{l}\text { GE } \\
\text { GE }\end{array}$ & $\begin{array}{l}\mu \mathrm{g} / \mathrm{L} \\
\mu \mathrm{g} / \mathrm{L} \\
\mathrm{pH}\end{array}$ \\
\hline \multicolumn{16}{|c|}{ Common Laboratory Contaminants } \\
\hline Parameter & 1095 & $\underline{\mathrm{DF}}$ & Mod & $\underline{\mathbf{S T}}$ & 브 & Flag & $\underline{\text { Lab }}$ & $\underline{2095}$ & $\underline{\mathrm{DF}}$ & Mod & $\underline{\mathbf{S T}}$ & $\underline{\mathbf{H}}$ & Flag & $\underline{\text { Lab }}$ & Unit \\
\hline $\begin{array}{l}\text { Bis(2-ethylhexyl) phthalate } \\
\text { Dichloromethane }\end{array}$ & $\begin{array}{l}<17 \\
<1.7\end{array}$ & 1 & $v$ & & & $\begin{array}{l}0 \\
0\end{array}$ & $\begin{array}{l}\text { GE } \\
\text { GE }\end{array}$ & & & & & & & & $\begin{array}{l}\mu g / L \\
\mu g / L\end{array}$ \\
\hline
\end{tabular}

Note: See Appendix B for flagging criteria. Synchronous water levels are measured over a 3-5 day period.

= exceeded holding time.

- = exceeded groundwater protection standard. 
WELL FSB100A (cont.)

Radiological Indicator Parameters

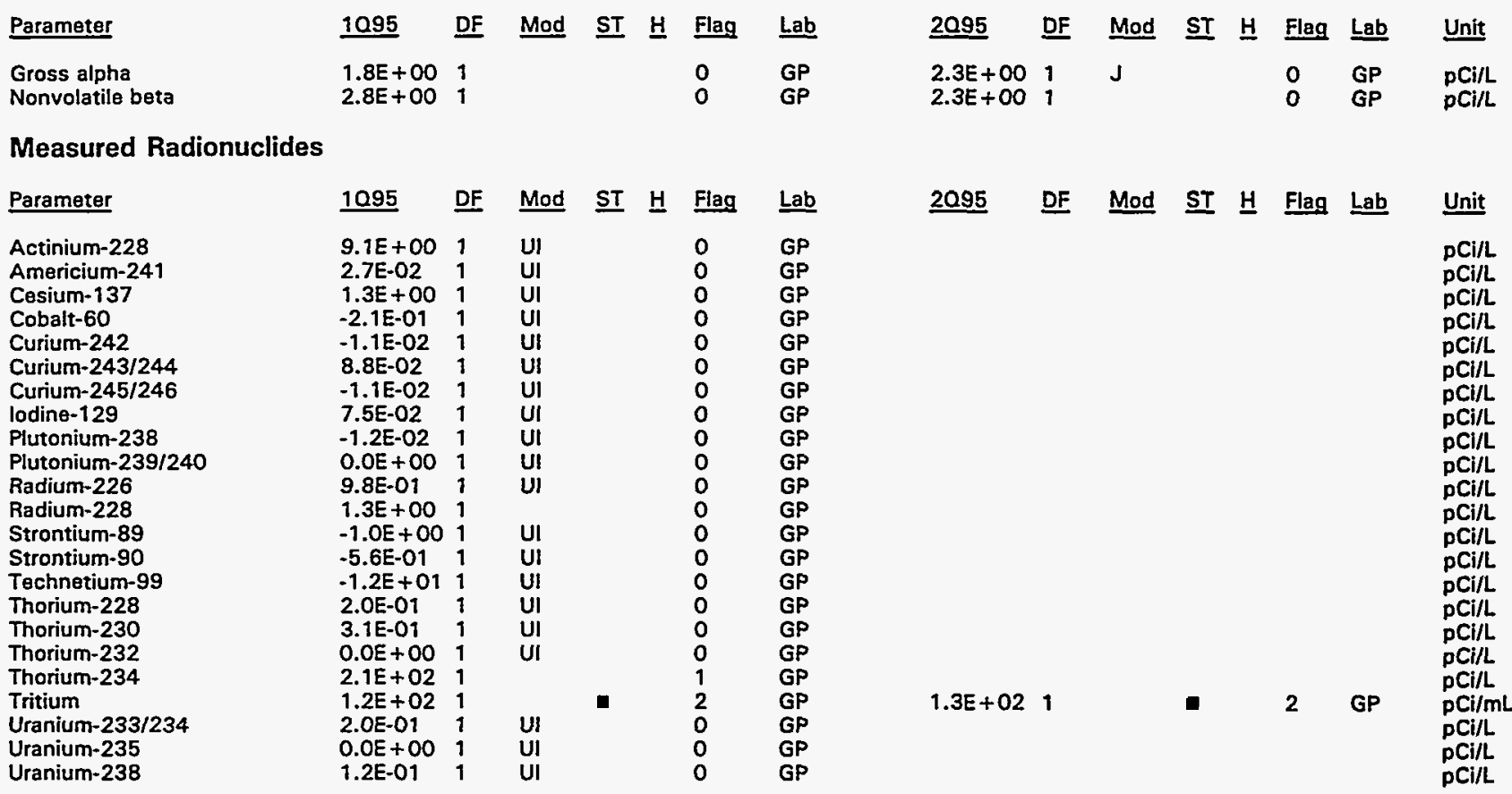

Calculated Radionuclides

Parameter

Americium-243

Nickel-63

Plutonium-241

Plutonium-242
1095

2.6E-04

-2.1E-01

$0.0 E+00$

$0.0 E+00$

DF Mod ST $\underline{H}$ Flag Lab

$\underline{2095}$

DF Mod ST $\underline{H}$ Flag Lab

Unit

0
0
0
0

pCin

$\mathrm{pCl} / \mathrm{L}$

pCi/L

Note: See Appendix B for flagging criteria. Synchronous water levels are measured over a 3-5 day period.

- = exceeded holding time.

- exceeded groundwater protection standard. 


\section{WELL FSB101A}

\begin{tabular}{|c|c|c|c|c|c|c|}
\hline SRS Coord. & Lat/Longituide & Screen Zone Elevation & Top of Casing & Casing & Pump & Screen Zone \\
\hline $\begin{array}{l}\text { N75719.0 } \\
\text { E51191.3 }\end{array}$ & $\begin{array}{l}33.276765^{\circ} \mathrm{N} \\
81.679085^{\circ} \mathrm{W}\end{array}$ & $102.9-92: 9 \mathrm{ft} \mathrm{msl}$ & $285.2 \mathrm{ft} \mathrm{msl}$ & 4" PVC & s & U. Congaree (IIA) \\
\hline SAMPLE DATE & 021 & & & 3/95 & & \\
\hline
\end{tabular}

FIELD DATA

$\begin{array}{ll}\text { Parameter } & 1095 \\ \text { Water elevation } & 152.0 \\ \text { pH } & 7.4 \\ \text { Sp. conductance } & 171 \\ \text { Water temperature } & 19.3 \\ \text { Alkalinity as } \mathrm{CaCO}_{3} & 61 \\ \text { Turbidity } & 0.2 \\ \text { Volume purged } & 3.5 \\ \text { Sampling code } & \\ \text { Synchronous water level } & 152.3(03 / 27 / 95)\end{array}$

Hazardous Constituents (264.94, Table 1)

$\begin{array}{lccccccc}\text { Parameter } & 1095 & \text { DF } & \text { Mod } & \text { ST } & \text { Flag } & \text { Lab } \\ \text { Arsenic, total recoverable } & <3.3 & 1 & & & 0 & \text { GE } \\ \text { Barium, total recoverable } & 30 & 1 & & & 0 & \text { GE } \\ \text { Cadmium, total recoverable } & <3.3 & 1 & & & 0 & \text { GE } \\ \text { Chromium, total recoverable } & <6.7 & 1 & & & 0 & \text { GE } \\ \text { Lead, total recoverable } & <5.0 & 1 & & & 0 & \text { GE } \\ \text { Mercury, total recoverable } & <0.33 & 1 & & & 0 & \text { GE } \\ \text { Selenium, total recoverable } & <3.3 & 1 & & & 0 & \text { GE } \\ \text { Silver, total recoverable } & <3.3 & 1 & & & 0 & \text { GE }\end{array}$

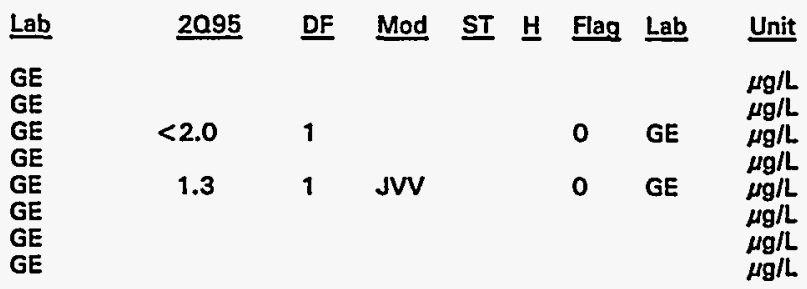

Hazardous Constituents (261 App. VIII/264 App. IX)

$\begin{array}{lccccccc}\text { Parameter } & \underline{1095} & \text { DF } & \text { Mod } & \text { ST } & \text { Flag } & \text { Lab } \\ \text { Antimony, total recoverable } & <3.3 & 1 & & & 0 & \text { GE } \\ \text { Benzene } & <1.7 & 1 & & & 0 & \text { GE } \\ \text { Cobalt, total recoverable } & <6.7 & 1 & & & 0 & \text { GE } \\ \text { Copper, total recoverable } & <6.7 & 1 & & & 0 & \text { GE } \\ \text { Cyanide } & <8.3 & 1 & & & 0 & \text { GE } \\ \text { Nickel, total recoverable } & <6.7 & 1 & & & 0 & \text { GE } \\ \text { Phenols } & <8.3 & 1 & \text { J } & & 0 & \text { GE } \\ \text { Tetrachlorosthylene } & <1.7 & 1 & & & 0 & \text { GE } \\ \text { Thallium, total recoverable } & <3.3 & 1 & & & 0 & \text { GE } \\ \text { Trichloroethylene } & <1.7 & 1 & & & 0 & \text { GE } \\ \text { Trichlorofluoromethane } & <1.7 & 1 & & & 0 & \text { GE } \\ \text { Vanadium, total recoverable } & <13 & 1 & & & 0 & \text { GE } \\ \text { Zinc, total recoverable } & <3.3 & 1 & & & 0 & \text { GE }\end{array}$

2095 DF Mod ST $\underline{H}$ Flag Lab Unit

$\mu g / L$
$\mu g / L$
$\mu g / L$
$\mu g / L$
$\mu g / L$
$\mu g / L$
$\mu g / L$
$\mu g / L$
$\mu g / L$
$\mu g / L$
$\mu g / L$
$\mu g / L$
$\mu g / L$

Water Quality Indicator Parameters

\begin{tabular}{|c|c|c|c|c|c|c|c|c|c|c|c|c|c|c|c|}
\hline Parameter & 1095 & $\underline{D F}$ & Mod & $\underline{\text { ST }}$ & $\underline{H}$ & Flag & $\underline{\text { Lab }}$ & $\underline{2095}$ & $\underline{D F}$ & Mod & ST & $\underline{H}$ & Flag & Lab & Unit \\
\hline $\begin{array}{l}\text { Aluminum, total recoverable } \\
\text { Nitrate as nitrogen } \\
\text { pH }\end{array}$ & $\begin{array}{l}<33 \\
1,000 \\
7.5\end{array}$ & $\begin{array}{l}1 \\
1 \\
1\end{array}$ & $J 1$ & & $\bullet$ & $\begin{array}{l}0 \\
0 \\
0\end{array}$ & $\begin{array}{l}\text { GE } \\
\text { GE } \\
\text { GE }\end{array}$ & $\begin{array}{l}1,000 \\
7.5\end{array}$ & $\begin{array}{l}1 \\
1\end{array}$ & 31 & & $\bullet$ & $\begin{array}{l}0 \\
0\end{array}$ & $\begin{array}{l}\text { GE } \\
\text { GE }\end{array}$ & $\begin{array}{l}\mu \mathrm{g} / \mathrm{L} \\
\mu \mathrm{g} / \mathrm{L} \\
\mathrm{pH}\end{array}$ \\
\hline \multicolumn{16}{|c|}{ Common Laboratory Contaminants } \\
\hline Parameter & 1095 & DF & Mod & $\underline{S T}$ & $\underline{H}$ & Flag & Lab & $\underline{2095}$ & $\underline{D F}$ & Mod & ST & $\underline{H}$ & Flag & Lab & Unit \\
\hline $\begin{array}{l}\text { Bis(2-ethylhexyl) phthalate } \\
\text { Dichloromethane }\end{array}$ & $\begin{array}{l}<17 \\
<1.7\end{array}$ & $\begin{array}{l}1 \\
1\end{array}$ & V & & & $\begin{array}{l}0 \\
0\end{array}$ & $\begin{array}{l}\text { GE } \\
\text { GE }\end{array}$ & & & & & & & & $\begin{array}{l}\mu \mathrm{g} / \mathrm{L} \\
\mu \mathrm{g} / \mathrm{L}\end{array}$ \\
\hline
\end{tabular}

Note: See Appendix B for flagging criteria. Synchronous water levels are measured over a 3-5 day period.

- = exceeded holding time.

- = exceeded groundwater protection standard. 
WELL FSB101A (cont.)

Radiological Indicator Parameters

\begin{tabular}{|c|c|c|c|c|c|c|c|c|c|c|c|c|c|}
\hline Parameter & 1095 & DF & Mod & $\underline{\text { ST }} \underline{H}$ & Flag & Lab & $\underline{2095}$ & DF & Mod & $\underline{\text { ST }} \underline{H}$ & Flag & Lab & Unit \\
\hline $\begin{array}{l}\text { Gross alpha } \\
\text { Nonvolatile beta }\end{array}$ & $\begin{array}{l}7.2 \mathrm{E}-01 \\
2.8 \mathrm{E}+\infty 0\end{array}$ & $\begin{array}{l}1 \\
1\end{array}$ & UI & & $\begin{array}{l}0 \\
0\end{array}$ & $\begin{array}{l}\text { GP } \\
\text { GP }\end{array}$ & $\begin{array}{l}1.3 E+\infty \\
2.8 E+\infty\end{array}$ & $\begin{array}{l}1 \\
1\end{array}$ & $J$ & & $\begin{array}{l}0 \\
0\end{array}$ & $\begin{array}{l}\text { GP } \\
\text { GP }\end{array}$ & $\mathrm{pCi} / \mathrm{L}$ \\
\hline
\end{tabular}

Measured Radionuclides

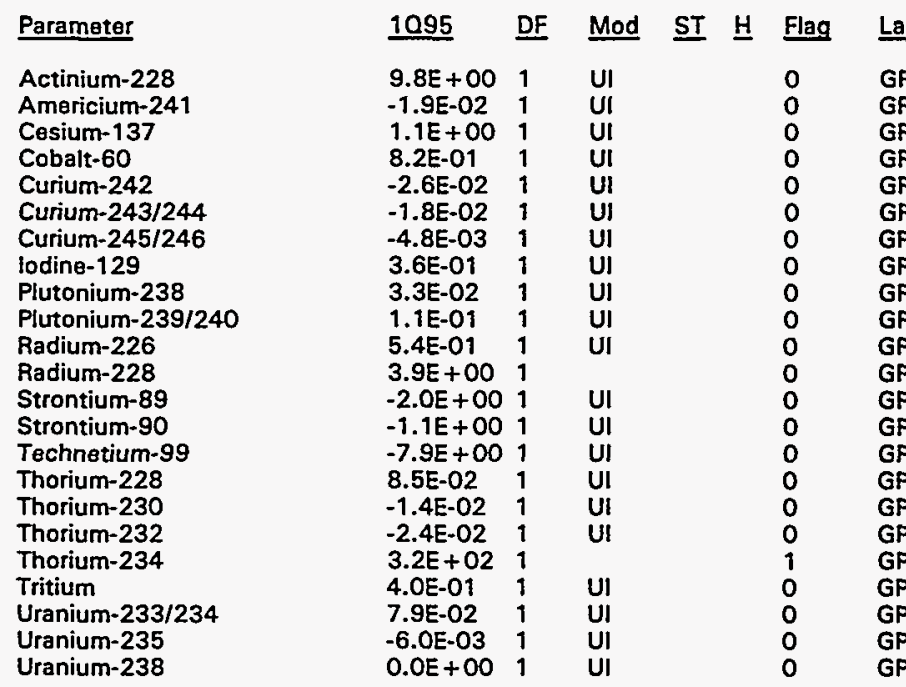

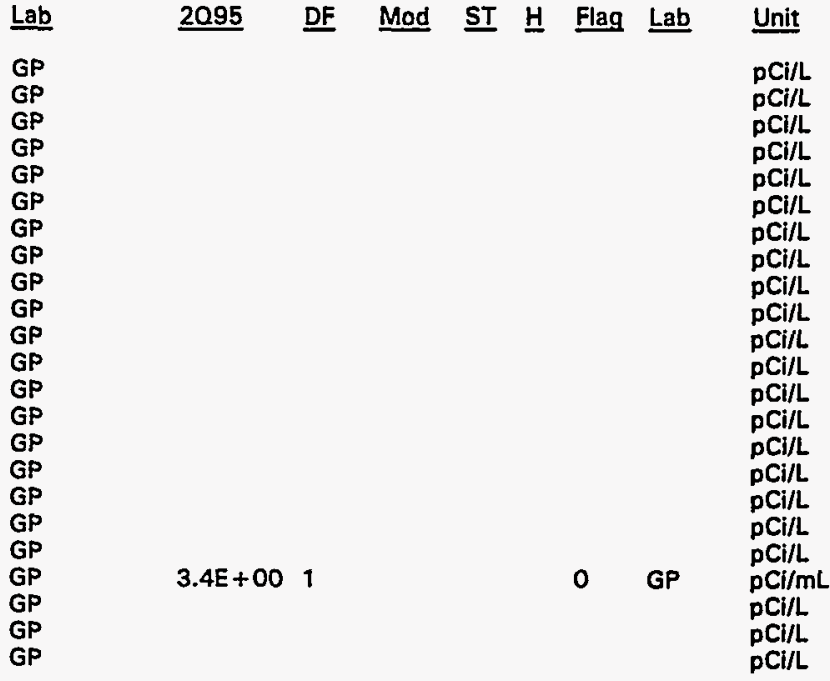

Calculated Radionuclides

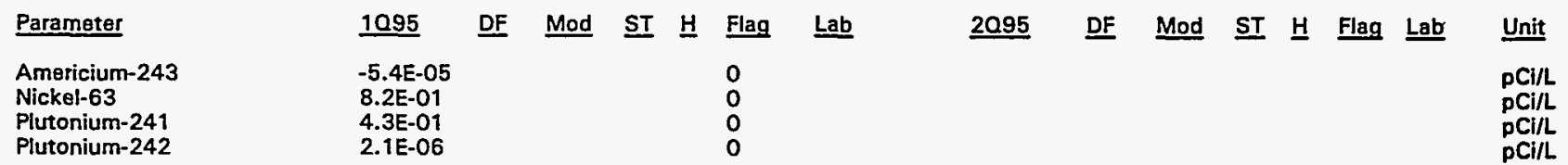

Note: See Appendix B for flagging criteria. Synchronous water levels are measured over a 3-5 day period. - = exceeded holding time.

- = exceeded groundwater protection standard. 
WELL FSB102C

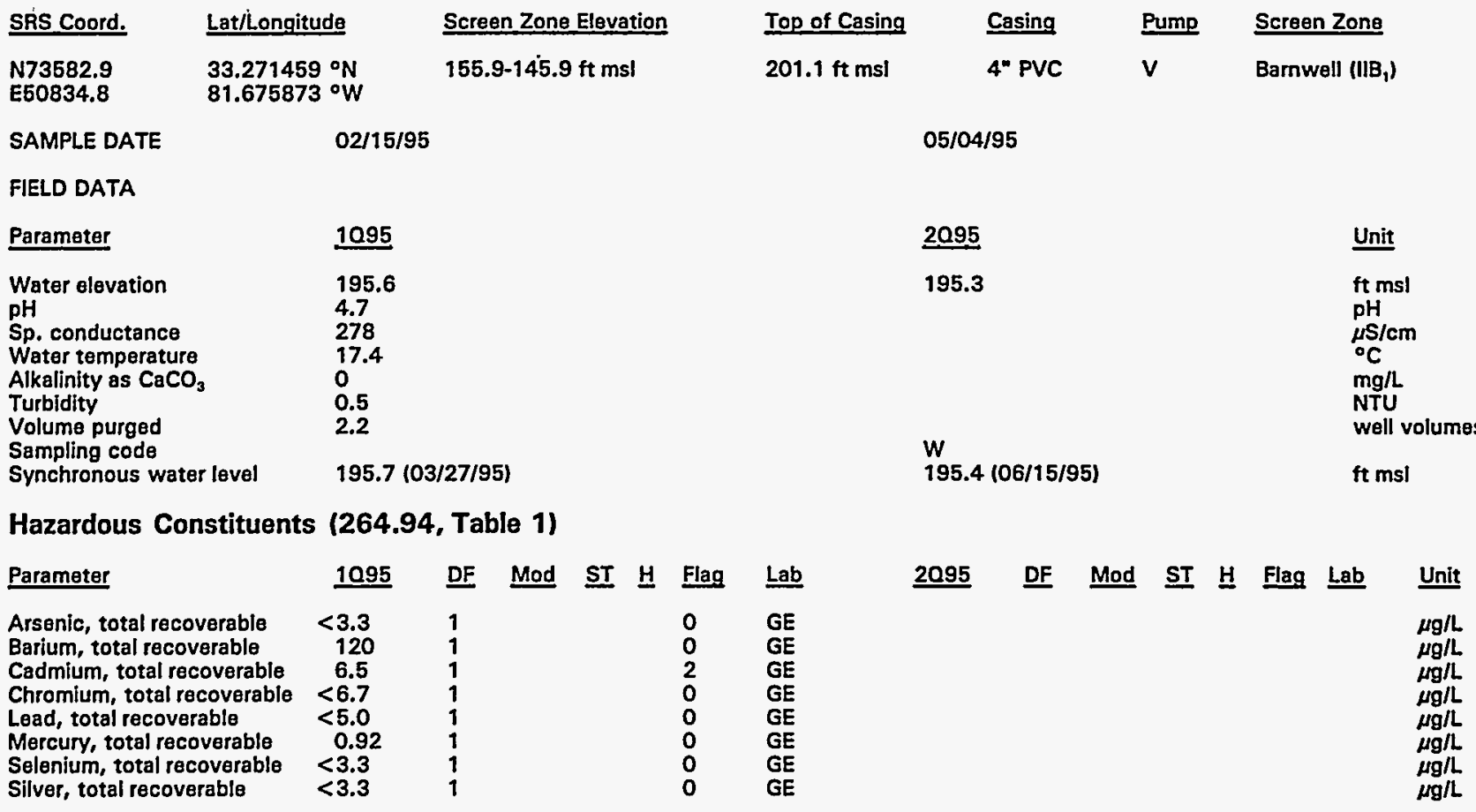

Hazardous Constituents (261 App. VIII/264 App. IX)

\begin{tabular}{|c|c|c|c|c|c|c|c|c|c|c|c|c|c|}
\hline Parameter & 1095 & $\underline{D F}$ & Mod & $\underline{\mathbf{S T}}$ & $\underline{H}$ & Flag & Lab & 2095 & $\underline{D F}$ & Mod & $\underline{\text { ST }} \underline{H}$ & Flag & $\underline{\text { Lab }}$ \\
\hline $\begin{array}{l}\text { Antimony, total recoverable } \\
\text { Benzene } \\
\text { Cobalt, total recoverable } \\
\text { Copper, total recoverable } \\
\text { Cyanide } \\
\text { Nickel, total recoverable } \\
\text { Phenols } \\
\text { Tetrachloroethylene } \\
\text { Thallium, total recoverable } \\
\text { Trichloroethylene } \\
\text { Trichlorofluoromethane } \\
\text { Vanadium, total recoverable } \\
\text { Zinc, total recoverable }\end{array}$ & $\begin{aligned} & 3.5 \\
&<1.7 \\
& 48 \\
&<6.7 \\
&<8.3 \\
& 18 \\
&<8.3 \\
&<1.7 \\
&<3.3 \\
& 1.2 \\
&<1.7 \\
&<13 \\
& 39\end{aligned}$ & $\begin{array}{l}1 \\
1 \\
1 \\
1 \\
1 \\
1 \\
1 \\
1 \\
1 \\
1 \\
1 \\
1 \\
1\end{array}$ & J1 & " & $\bullet$ & $\begin{array}{l}1 \\
0 \\
2 \\
0 \\
0 \\
0 \\
0 \\
0 \\
0 \\
0 \\
0 \\
0 \\
0\end{array}$ & $\begin{array}{l}\text { GE } \\
\text { GE } \\
\mathrm{GE} \\
\mathrm{GE} \\
\mathrm{GE} \\
\mathrm{GE} \\
\mathrm{GE} \\
\mathrm{GE} \\
\mathrm{GE} \\
\mathrm{GE} \\
\mathrm{GE} \\
\mathrm{GE} \\
\mathrm{GE}\end{array}$ & & & & & & \\
\hline \multicolumn{14}{|c|}{ Water Quality Indicator Parameters } \\
\hline Parameter & 1095 & DF & Mod & $\underline{\text { ST }}$ & $\underline{H}$ & Flag & $\underline{\text { Lab }}$ & $\underline{2095}$ & $\underline{\mathrm{DF}}$ & Mod & $\underline{\text { ST }} \underline{H}$ & Flag & $\underline{\text { Lab }}$ \\
\hline $\begin{array}{l}\text { Aluminum, total recoverable } \\
\text { Nitrate as nitrogen } \\
\text { pH }\end{array}$ & $\begin{array}{l}1,200 \\
37,000 \\
4.5\end{array}$ & $\begin{array}{l}1 \\
20 \\
1\end{array}$ & $\begin{array}{l}\mathrm{J} \\
\mathrm{J} 1\end{array}$ & 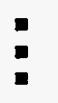 & & $\begin{array}{l}2 \\
2 \\
0\end{array}$ & $\begin{array}{l}\text { GE } \\
\text { GE } \\
\text { GE }\end{array}$ & & & & & & \\
\hline \multicolumn{14}{|c|}{ Common Laboratory Contaminants } \\
\hline Parameter & 1095 & DF & Mod & $\underline{\text { ST }}$ & $\underline{H}$ & Flag & Lab & $\underline{2095}$ & $\underline{D F}$ & Mod & $\underline{\text { ST }} \underline{H}$ & Flag & $\underline{\text { Lab }}$ \\
\hline $\begin{array}{l}\text { Bis(2-ethylhexyl) phthalate } \\
\text { Dichloromethans }\end{array}$ & $\begin{array}{l}<17 \\
<1.7\end{array}$ & $\begin{array}{l}1 \\
1\end{array}$ & v & & & $\begin{array}{l}0 \\
0\end{array}$ & $\begin{array}{l}\text { GE } \\
\text { GE }\end{array}$ & & & & & & \\
\hline
\end{tabular}

Note: See Appendix B for flagging criteria. Synchronous water levels are measured over a 3-5 day period. - = exceeded holding time.

- =xceeded groundwater protection standard. 


\section{WELL FSB102C (cont.)}

Radiological Indicator Parameters

Parameter

Gross alpha Nonvolatile beta$$
1095
$$

1.5E+01

DF Mod

$1.5 E+01$
$5.7 E+02$

ST $\underline{\text { Flag }}$ Lab

GP

GP

$\underline{2095}$

DF Mod ST $\underline{\text { H Flag Lab Unit }}$

Measured Radionuclides
Parameter

Actinium-228

Americium-241

Cesium-137

Cobalt 60

Curium-242

Curium-243/244

Curium-245/246

lodine-129

Plutonium-238

Plutonium-239/240

Radium-226

Radium-228

Strontium-89

Technetium-99

Thorium-228

Thorium-230

Thorium-232

Thorium-234

Tritium

Uranium-233/234

Uranium-235

Uranium-238
Strontium-90

$\begin{array}{lllllll}1095 & \text { DF } & \text { Mod } & \text { ST } & \text { H } & \text { Flag } & \text { Lab } \\ 4.9 E+00 & 1 & \text { UI } & & & 0 & \text { GP } \\ 0.0 E+00 & 1 & \text { UI } & & 0 & \text { GP } \\ -4.2 E-01 & 1 & \text { UI } & & 0 & \text { GP } \\ 1.4 E+00 & 1 & \text { UI } & & 0 & \text { GP } \\ -4.8 E-03 & 1 & \text { UI } & & 0 & \text { GP } \\ -4.2 E-03 & 1 & \text { UI } & & 0 & \text { GP } \\ 3.5 E-02 & 1 & \text { UI } & & 0 & \text { GP } \\ 2.9 E+01 & 1 & & & & 2 & \text { GP } \\ -7.9 E-03 & 1 & \text { UI } & & 0 & \text { GP } \\ 1.1 E-02 & 1 & \text { UI } & & 0 & \text { GP } \\ 3.6 E+00 & 1 & & & 0 & \text { GP } \\ 1.0 E+00 & 1 & \text { J } & & 0 & \text { GP } \\ 2.3 E+01 & 1 & & & 2 & \text { GP } \\ 1.7 E+02 & 1 & & & 2 & \text { GP } \\ 1.8 E+01 & 1 & \text { UI } & & 0 & \text { GP } \\ 1.3 E-01 & 1 & \text { UI } & & 0 & \text { GP } \\ -8.8 E-02 & 1 & \text { UI } & & 0 & \text { GP } \\ 2.3 E-01 & 1 & \text { UI } & & 0 & \text { GP } \\ 5.1 E+01 & 1 & \text { UI } & & 0 & \text { GP } \\ 9.5 E+02 & 1 & & & 2 & \text { GP } \\ 4.5 E-01 & 1 & \text { UI } & & 0 & \text { GP } \\ 4.3 E-02 & 1 & \text { UI } & & 0 & \text { GP } \\ 3.6 E-01 & 1 & \text { UI } & & 0 & \text { GP }\end{array}$

2095 DF Mod ST $\underline{H}$ Flag Lab Unit

pCi/L

$\mathrm{pCi} / \mathrm{L}$

$\mathrm{pCi} / \mathrm{L}$

pCi/L

PCill

$\mathrm{pCi} / \mathrm{L}$

pCi/L

$\mathrm{pCi} / \mathrm{L}$

$\mathrm{pCi} / \mathrm{L}$

$\mathrm{pCi} / \mathrm{L}$

$\mathrm{pCl} / \mathrm{L}$

pCi/L

$\mathrm{pCI} / \mathrm{L}$

$\mathrm{pCi} / \mathrm{L}$

$\mathrm{pCi} / \mathrm{L}$

$\mathrm{pCI} / \mathrm{L}$

pCi/L

pCi/L

$\mathrm{pCi} / \mathrm{mL}$

pCi/L

$\mathrm{pCi} / \mathrm{L}$

PCi/L

Calculated Radionuclides

Parameter

1095

DF Mad ST 브 Flag Lab

$\underline{2095}$

DF Mod $\underline{\text { ST }} \underline{\text { H Flag Lab }}$

Unit

$1.4 E+00$

4.4E-02

Nickel-63

4.4E-02
$2.2 \mathrm{E}-07$

\section{0}

0

$\mathrm{pCi} / \mathrm{L}$

pCi/L

pCi/L

Plutonium-241
Plutonium-242

Note: See Appendix B for flagging criteria. Synchronous water levels are measured over a 3-5 day period.

$-=$ exceeded holding time.

m = exceeded groundwater protection standard. 


\section{WELL FSB103C}

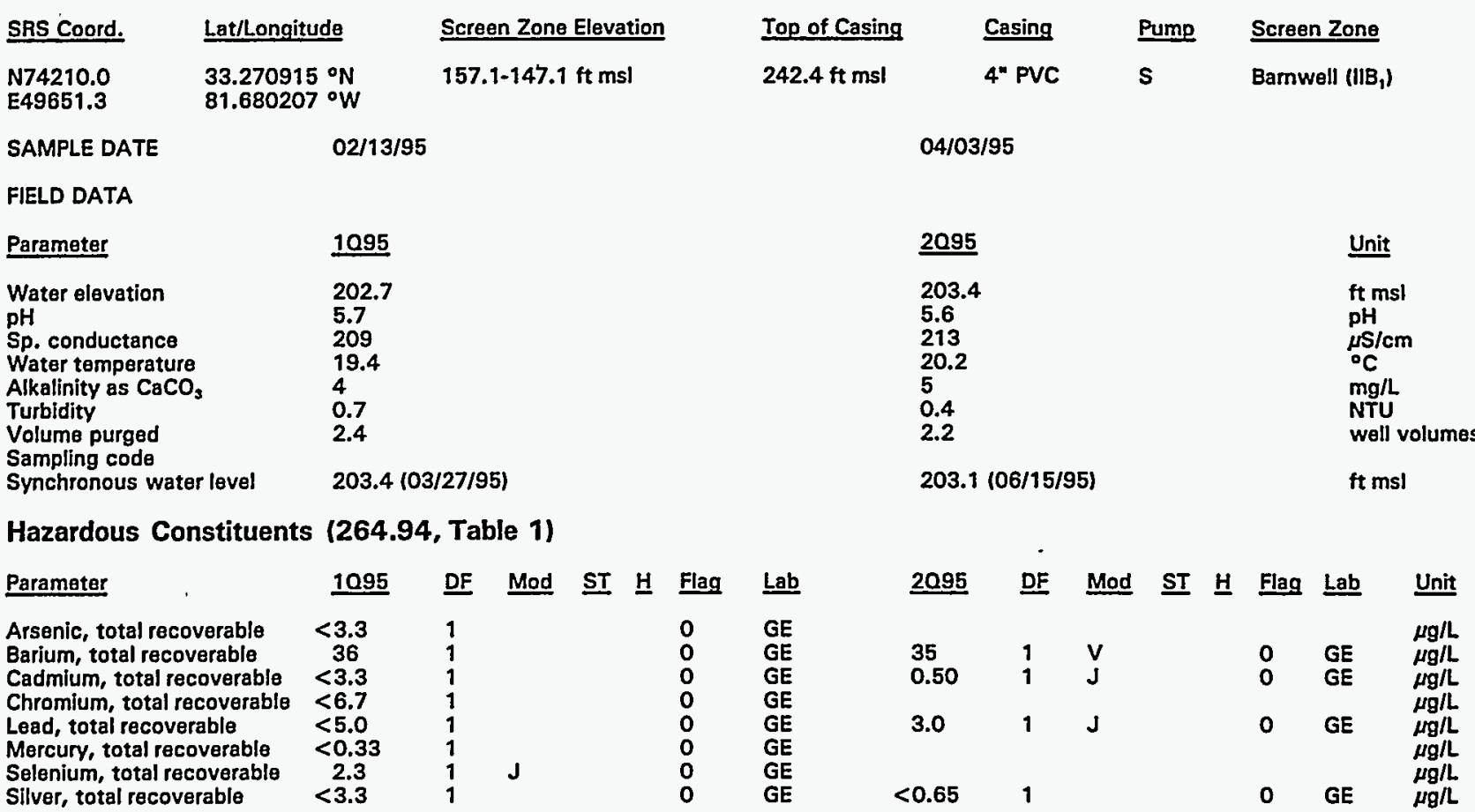

Hazardous Constituents (261 App. VIII/264 App. (X)

\begin{tabular}{|c|c|c|c|c|c|c|c|c|c|c|c|c|c|c|c|}
\hline Parameter & 1095 & DF & Mod & $\underline{\mathbf{S T}}$ & H & Flag & $\underline{\text { Lab }}$ & 2095 & DF & Mod & ST & $\underline{H}$ & Flag & $\underline{L a b}$ & Unit \\
\hline $\begin{array}{l}\text { Antimony, total recoverable } \\
\text { Benzene } \\
\text { Cobalt, total recoverable } \\
\text { Copper, total recoverable } \\
\text { Cyanide } \\
\text { Nickel, total recoverable } \\
\text { Phenols } \\
\text { Tetrachloroethylene } \\
\text { Thallium, total recoverable } \\
\text { Trichloroethylene } \\
\text { Trichlorofluoromethane } \\
\text { Vanadium, total recoverable } \\
\text { Zinc, total recoverable }\end{array}$ & $\begin{aligned} & 2.5 \\
&< 1.7 \\
&< 6.7 \\
& 11 \\
&< 8.3 \\
& 4.8 \\
&<8.3 \\
&<1.7 \\
&<3.3 \\
&<1.7 \\
&<1.7 \\
&<13 \\
& 71\end{aligned}$ & $\begin{array}{l}1 \\
1 \\
1 \\
1 \\
1 \\
1 \\
1 \\
1 \\
1 \\
1 \\
1 \\
1 \\
1\end{array}$ & $\begin{array}{l}\mathbf{J 1} \\
\mathrm{J} \\
\mathrm{J}\end{array}$ & 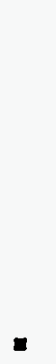 & $\bullet$ & $\begin{array}{l}0 \\
0 \\
0 \\
0 \\
0 \\
0 \\
0 \\
0 \\
0 \\
0 \\
0 \\
0 \\
0\end{array}$ & $\begin{array}{l}\text { GE } \\
\text { GE } \\
\text { GE } \\
\text { GE } \\
\text { GE } \\
\text { GE } \\
\text { GE } \\
\text { GE } \\
\text { GE } \\
\text { GE } \\
\text { GE } \\
\text { GE } \\
\text { GE }\end{array}$ & 7.9 & 1 & JVV & & & 0 & GE & $\begin{array}{l}\mu g / L \\
\mu g / L \\
\mu g / L \\
\mu g / L \\
\mu g / L \\
\mu g / L \\
\mu g / L \\
\mu g / L \\
\mu g / L \\
\mu g / L \\
\mu g / L \\
\mu g / L \\
\mu g / L\end{array}$ \\
\hline \multicolumn{16}{|c|}{ Water Quality Indicator Parameters } \\
\hline Parameter & 1095 & $\underline{D F}$ & Mod & $\underline{\text { ST }}$ & $\underline{H}$ & Flag & $\underline{\text { Lab }}$ & $\underline{2095}$ & $\underline{D F}$ & Mod & $\underline{\mathbf{S T}}$ & $\underline{H}$ & Flag & $\underline{L a b}$ & Unit \\
\hline \multicolumn{16}{|c|}{ Common Laboratory Contaminants } \\
\hline Parameter & $\underline{1095}$ & $\underline{\text { DF }}$ & Mod & $\underline{\mathrm{ST}}$ & $\underline{\mathrm{H}}$ & Flag & $\underline{\text { Lab }}$ & $\underline{2095}$ & $\underline{D F}$ & Mod & $\underline{\text { ST }}$ & $\underline{H}$ & Flag & $\underline{\text { Lab }}$ & Unit \\
\hline $\begin{array}{l}\text { Bis (2-ethylhexyl) phthalate } \\
\text { Dichloromethane }\end{array}$ & $\begin{array}{l}<17 \\
<1.7\end{array}$ & $\begin{array}{l}1 \\
1\end{array}$ & & & & $\begin{array}{l}0 \\
0\end{array}$ & $\begin{array}{l}\text { GE } \\
\text { GE }\end{array}$ & & & & & & & & $\begin{array}{l}\mu g / L \\
\mu g / L\end{array}$ \\
\hline
\end{tabular}

Note: See Appendix B for flagging criteria. Synchronous water levels are measured over a 3-5 day period. - = exceeded holding time.

$\square=$ exceeded groundwater protection standard. 
WELL FSB 103C (cont.)

Radiological Indicator Parameters

\begin{tabular}{|c|c|c|c|c|c|c|c|c|c|c|c|c|c|c|}
\hline Parameter & 1095 & DF & Mod & $\underline{\text { ST }}$ & $\underline{H}$ & Flag & Lab & 2095 & DF & Mod & $\underline{\mathrm{ST}}$ & $\underline{H}$ & Flag & Lab \\
\hline $\begin{array}{l}\text { Gross alpha } \\
\text { Nonvolatile beta }\end{array}$ & $\begin{array}{l}5.0 E-01 \\
1.4 E+00\end{array}$ & $\begin{array}{l}1 \\
1\end{array}$ & $\begin{array}{l}\text { UI } \\
\text { UI }\end{array}$ & & & $\begin{array}{l}0 \\
0\end{array}$ & $\begin{array}{l}\text { GP } \\
\text { GP }\end{array}$ & $\begin{array}{l}6.4 E-01 \\
9.5 E+00\end{array}$ & $\begin{array}{l}1 \\
1\end{array}$ & $\begin{array}{l}\text { UI } \\
\text { J }\end{array}$ & & & $\begin{array}{l}0 \\
0\end{array}$ & $\begin{array}{l}\text { GP } \\
\text { GP }\end{array}$ \\
\hline
\end{tabular}

Measured Radionuclides

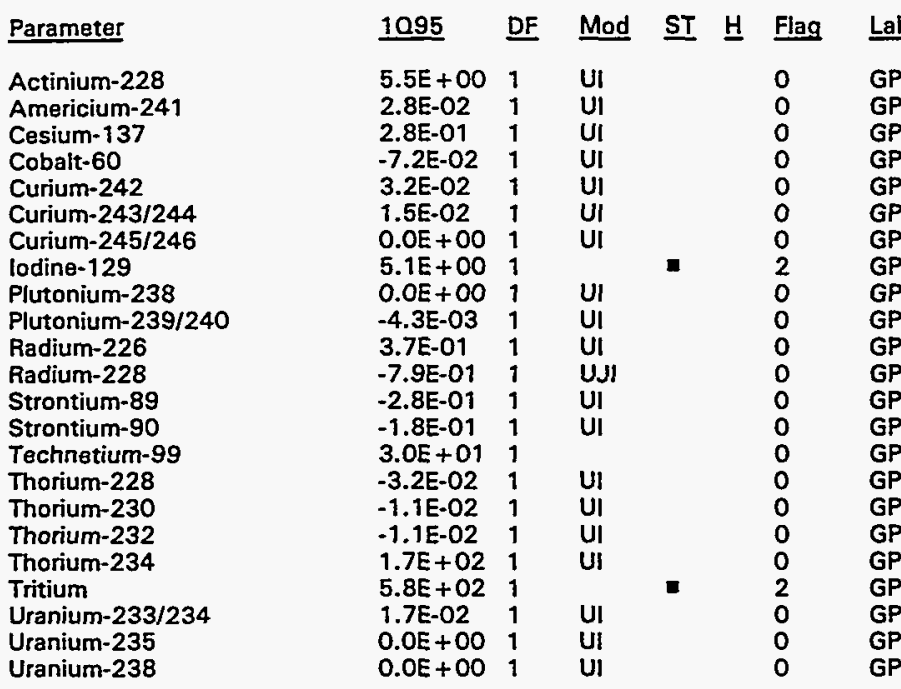

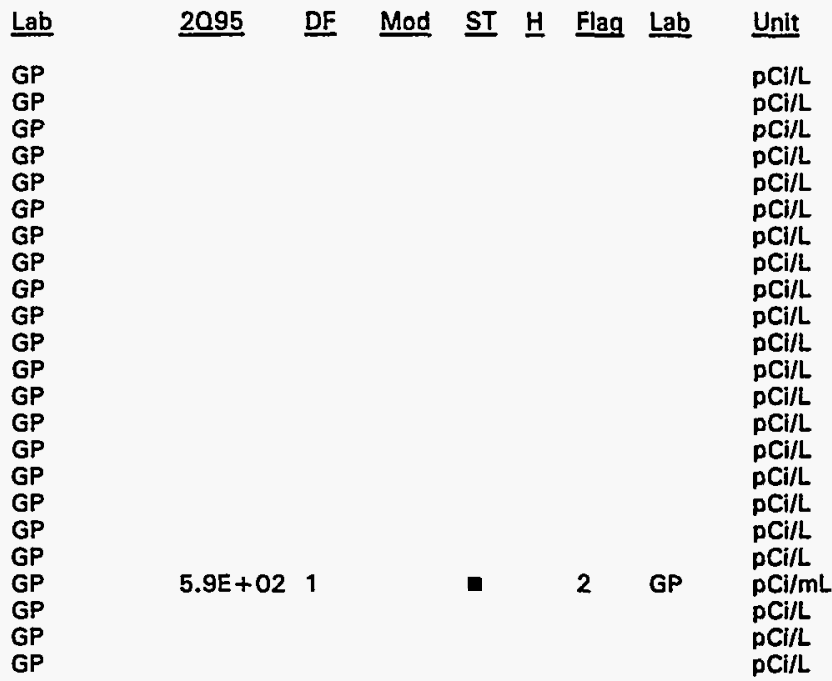

Calculated Radionuclides

Parameter

1095

DF Mod ST H Flag Lab

$\underline{2095}$

DF Mod ST $\underline{H}$ Flag Lab

Unit

Americium-243

Nickel-63

4.4E-05

$-7.2 \mathrm{E}-02$

$-1.7 \mathrm{E}-02$

Plutonium-242

$-8.7 E-08$

0
0
0
0

$\mathrm{pCI} / \mathrm{L}$

pCi/L

pCI/L

$\mathrm{PCI} / \mathrm{L}$

Note: See Appendix B for flagging criteria. Synchronous water levels are measured over a 3-5 day period.

- = exceeded holding time.

- = exceeded groundwater protection standard. 


\section{WELL FSB104C}

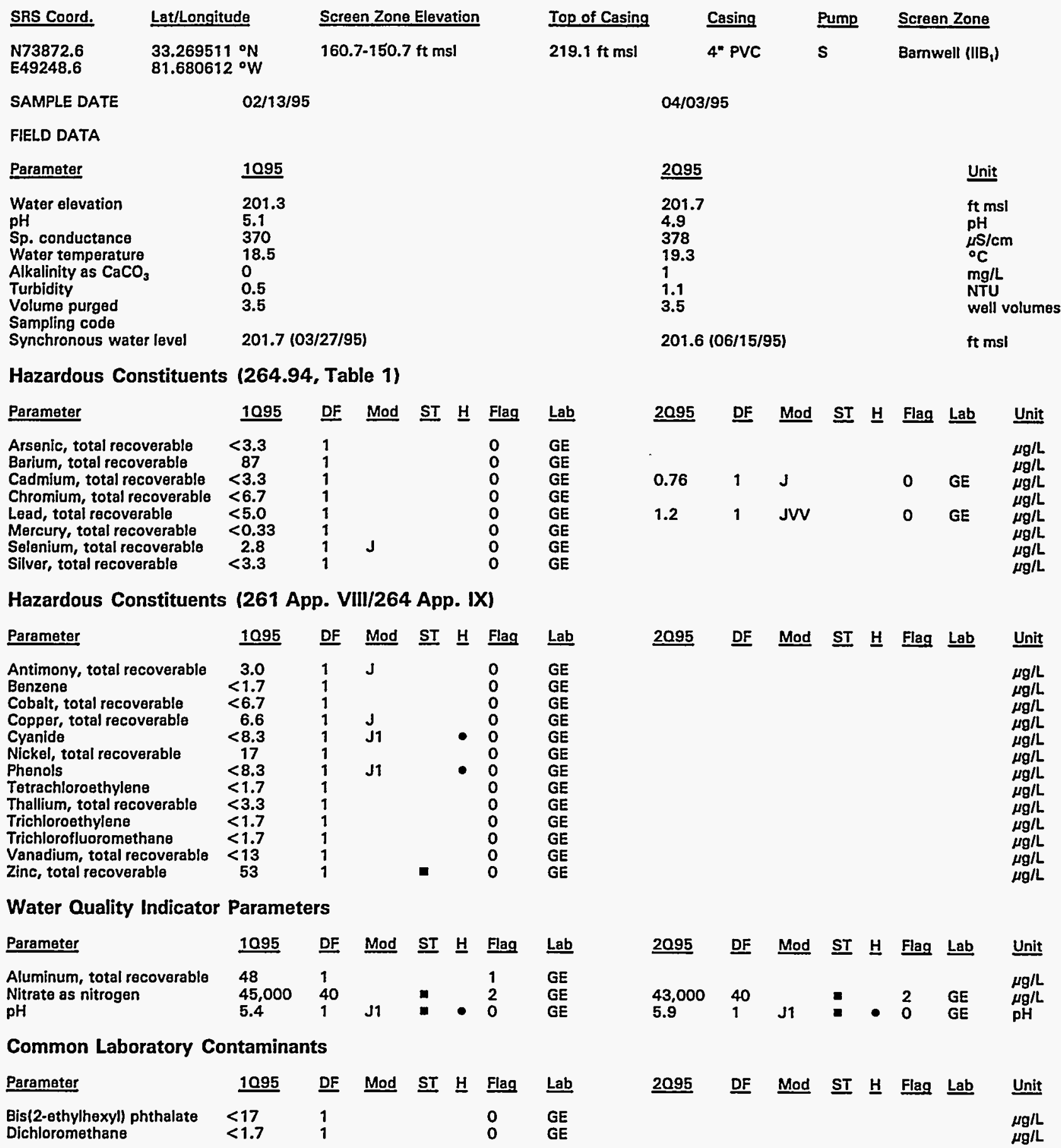

Note: See Appendix B for flagging criteria. Synchronous water levels are measured over a 3-5 day period.

- = exceeded holding time.

- = exceeded groundwater protection standard. 
WELL FSB104C (cont.)

Radiological Indicator Parameters

\begin{tabular}{|c|c|c|c|c|c|c|c|c|c|c|c|c|c|c|c|}
\hline Parameter & 1095 & DF & Mod & $\underline{\text { ST }}$ & $\underline{H}$ & Flag & Lab & $\underline{2095}$ & $\underline{D F}$ & Mod & $\underline{\text { ST }}$ & $\underline{H}$ & Flag & $\underline{\text { Lab }}$ & Unit \\
\hline $\begin{array}{l}\text { Gross alpha } \\
\text { Nonvolatile beta }\end{array}$ & $\begin{array}{l}4.1 E+00 \\
1.5 E+01\end{array}$ & $\begin{array}{l}1 \\
1\end{array}$ & & & & $\begin{array}{l}0 \\
0\end{array}$ & $\begin{array}{l}\text { GP } \\
\text { GP }\end{array}$ & $\begin{array}{l}4.5 E+00 \\
3.7 E+01\end{array}$ & $\begin{array}{l}1 \\
1\end{array}$ & $J$ & & & $\begin{array}{l}0 \\
1\end{array}$ & $\begin{array}{l}\text { GP } \\
\text { GP }\end{array}$ & $\begin{array}{l}\mathrm{pCi} / \mathrm{L} \\
\mathrm{pCi} / \mathrm{L}\end{array}$ \\
\hline \multicolumn{16}{|c|}{ Measured Radionuclides } \\
\hline Parameter & 1095 & $\underline{\mathrm{DF}}$ & Mod & $\underline{\text { ST }}$ & 브 & Flag & Lab & $\underline{2095}$ & $\underline{D F}$ & Mod & $\underline{\text { ST }}$ & $\underline{\mathrm{H}}$ & Flag & $\underline{\text { Lab }}$ & Unit \\
\hline $\begin{array}{l}\text { Actinium-228 } \\
\text { Americium-241 } \\
\text { Cesium-137 } \\
\text { Cobalt-60 } \\
\text { Curium-242 } \\
\text { Curium-243/244 } \\
\text { Curium-245/246 } \\
\text { lodine-129 } \\
\text { Plutonium-238 } \\
\text { Plutonium-239/240 } \\
\text { Radium-226 } \\
\text { Radium-228 } \\
\text { Strontium-89 } \\
\text { Strontium-90 } \\
\text { Technetium-99 } \\
\text { Thorium-228 } \\
\text { Thorium-230 } \\
\text { Thorium-232 } \\
\text { Thorium-234 } \\
\text { Tritium } \\
\text { Uranium-233/234 } \\
\text { Uranium-235 } \\
\text { Uranium-238 }\end{array}$ & $\begin{array}{l}4.3 E+00 \\
3.6 E-02 \\
-7.2 E-01 \\
-6.4 E-01 \\
-1.7 E-02 \\
-3.0 E-02 \\
-4.9 E-03 \\
6.0 E+00 \\
0.0 E+00 \\
7.1 E-02 \\
7.6 E-01 \\
-5.4 E-01 \\
1.2 E-01 \\
-2.4 E-01 \\
4.5 E+01 \\
0.0 E+00 \\
1.4 E-01 \\
5.6 E-02 \\
1.3 E+02 \\
1.2 E+03 \\
5.8 E-02 \\
-2.9 E-02 \\
-4.6 E-02\end{array}$ & $\begin{array}{l}1 \\
1 \\
1 \\
1 \\
1 \\
1 \\
1 \\
1 \\
1 \\
1 \\
1 \\
1 \\
1 \\
1 \\
1 \\
1 \\
1 \\
1 \\
1 \\
1 \\
1 \\
1 \\
1\end{array}$ & $\begin{array}{l}\text { UI } \\
\text { UI } \\
\text { UI } \\
\text { UI } \\
\text { UI } \\
\text { UI } \\
\text { UI } \\
\text { UI } \\
\text { UI } \\
\text { UI } \\
\text { UII } \\
\text { UI } \\
\text { UI } \\
\text { UI } \\
\text { UI } \\
\text { UI } \\
\text { UI } \\
\text { UI } \\
\text { UI } \\
\text { UI }\end{array}$ & - & & $\begin{array}{l}0 \\
0 \\
0 \\
0 \\
0 \\
0 \\
0 \\
2 \\
0 \\
0 \\
0 \\
0 \\
0 \\
0 \\
0 \\
0 \\
0 \\
0 \\
0 \\
2 \\
0 \\
0 \\
0\end{array}$ & $\begin{array}{l}\text { GP } \\
G P \\
G P \\
G P \\
G P \\
G P \\
G P \\
G P \\
G P \\
G P \\
G P \\
G P \\
G P \\
G P \\
G P \\
G P \\
G P \\
G P \\
G P \\
G P \\
G P \\
G P \\
G P\end{array}$ & $3.9 E+00$ & 1 & & & & 0 & GP & 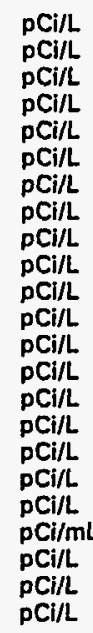 \\
\hline \multicolumn{16}{|c|}{ Calculated Radionuclides } \\
\hline Parameter & 1095 & $\underline{\mathrm{DF}}$ & Mod & $\underline{\mathrm{ST}}$ & $\underline{H}$ & Flag & $\underline{\text { Lab }}$ & $\underline{2095}$ & $\underline{D F}$ & Mod & $\underline{\text { ST }}$ & $\underline{\mathbf{H}}$ & Flag & $\underline{\text { Lab }}$ & Unit \\
\hline $\begin{array}{l}\text { Americium-243 } \\
\text { Nickel-63 } \\
\text { Plutonium-241 } \\
\text { Plutonium-242 }\end{array}$ & $\begin{array}{l}-8.9 \mathrm{E}-05 \\
-6.4 \mathrm{E}-01 \\
2.8 \mathrm{E}-01 \\
1.4 \mathrm{E}-06\end{array}$ & & & & & $\begin{array}{l}0 \\
0 \\
0 \\
0\end{array}$ & & & & & & & & & $\begin{array}{l}\mathrm{pCi} / \mathrm{L} \\
\mathrm{pCi} / \mathrm{L} \\
\mathrm{pCi} / \mathrm{L} \\
\mathrm{pCi} / \mathrm{L}\end{array}$ \\
\hline
\end{tabular}

Note: See Appendix B for flagging criteria. Synchronous water levels are measured over a 3-5 day period. - = exceeded holding time.

- = exceeded groundwater protection standard. 
WSRC-TR-95-0146-2

Unclassified

WELL FSB104D

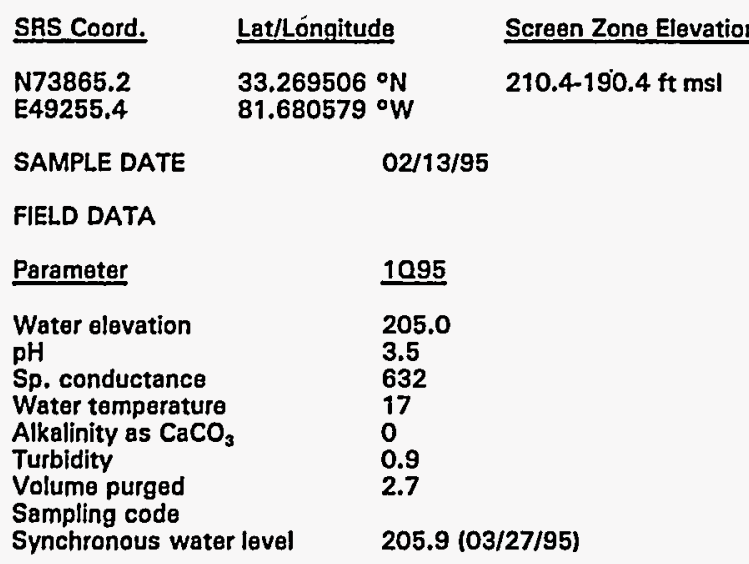

\begin{tabular}{|c|c|c|c|}
\hline Top of Casing & Casing & Pump & Screen Zone \\
\hline $219.2 \mathrm{ft} \mathrm{msl}$ & 4" PVC & v & Water Table (IIB $\left.{ }_{2}\right)$ \\
\hline
\end{tabular}

Hazardous Constituents (264.94, Table 1)

\begin{tabular}{|c|c|c|c|c|c|c|c|c|c|c|c|c|}
\hline Parameter & 1095 & DF & Mod & $\underline{S T} \underline{H}$ & Flag & Lab & 2095 & DF & Mod & $\underline{\mathrm{ST}} \underline{H}$ & Flag & $\underline{\text { Lab }}$ \\
\hline $\begin{array}{l}\text { Arsenic, total recoverable } \\
\text { Barium, total recoverable } \\
\text { Cadmium, total recoverable } \\
\text { Chromium, total recoverable } \\
\text { Lead, total recoverable } \\
\text { Mercury, total recoverable } \\
\text { Selenium, total recoverable } \\
\text { Silver, total recoverable }\end{array}$ & $\begin{array}{l}<3.3 \\
170 \\
3.6 \\
<6.7 \\
<5.0 \\
<0.33 \\
<3.3 \\
<3.3\end{array}$ & $\begin{array}{l}1 \\
1 \\
1 \\
1 \\
1 \\
1 \\
1 \\
1\end{array}$ & v & & $\begin{array}{l}0 \\
0 \\
1 \\
0 \\
0 \\
0 \\
0 \\
0\end{array}$ & $\begin{array}{l}\mathrm{GE} \\
\mathrm{GE} \\
\mathrm{GE} \\
\mathrm{GE} \\
\mathrm{GE} \\
\mathrm{GE} \\
\mathrm{GE} \\
\mathrm{GE}\end{array}$ & & & & & & - \\
\hline
\end{tabular}

Hazardous Constituents (261 App. VIII/264 App. IX)

$\begin{array}{lccccccc}\text { Parameter } & \text { 1095 } & \text { DF } & \text { Mod } & \text { SI } & \text { Flag } & \text { Lab } \\ \text { Antimony, total recoverable } & <3.3 & 1 & & & & 0 & \text { GE } \\ \text { Benzene } & <1.7 & 1 & & & 0 & \text { GE } \\ \text { Cobalt, total recoverable } & 26 & 1 & & & 1 & \text { GE } \\ \text { Copper, total recoverable } & 34 & 1 & & & 0 & \text { GE } \\ \text { Cyanide } & <8.3 & 1 & & & 0 & \text { GE } \\ \text { Nickel, total recoverable } & 18 & 1 & & & 0 & \text { GE } \\ \text { Phenols } & <8.3 & 1 & \text { J1 } & & 0 & \text { GE } \\ \text { Tetrachloroethylene } & <1.7 & 1 & & & & 0 & \text { GE } \\ \text { Thallium, total recoverable } & <3.3 & 1 & & & 0 & \text { GE } \\ \text { Trichlorosthylene } & <1.7 & 1 & & & 0 & \text { GE } \\ \text { Trichlorofluoromethane } & <1.7 & 1 & & & 0 & \text { GE } \\ \text { Vanadium, total recoverable } & <13 & 1 & & & 0 & \text { GE } \\ \text { Zinc, total recoverable } & 55 & 1 & & & 0 & \text { GE }\end{array}$

\begin{tabular}{|c|c|}
\hline 2095 & Unit \\
\hline 205.2 & $\begin{array}{l}\text { ft msl } \\
\mathrm{pH} \\
\mu \mathrm{S} / \mathrm{cm} \\
{ }^{\circ} \mathrm{C} \\
\mathrm{mg} / \mathrm{L} \\
\text { NTU } \\
\text { well volumes }\end{array}$ \\
\hline $\begin{array}{l}W \\
205.2(06 / 15 / 95)\end{array}$ & $\mathrm{ft} \mathrm{msl}$ \\
\hline
\end{tabular}

Water Quality Indicator Parameters

\begin{tabular}{|c|c|c|c|c|c|c|}
\hline Parameter & 1095 & DF & Mod & $\underline{\text { ST }}$ & 브 & Flag \\
\hline $\begin{array}{l}\text { Aluminum, total recoverable } \\
\text { Nitrate as nitrogen }\end{array}$ & $\begin{array}{l}34,000 \\
74,000\end{array}$ & $\begin{array}{l}1 \\
40\end{array}$ & & 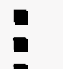 & & $\begin{array}{l}2 \\
2\end{array}$ \\
\hline
\end{tabular}

2095 DF Mod ST $\underline{H}$ Flag Lab Unit

Common Laboratory Contaminants

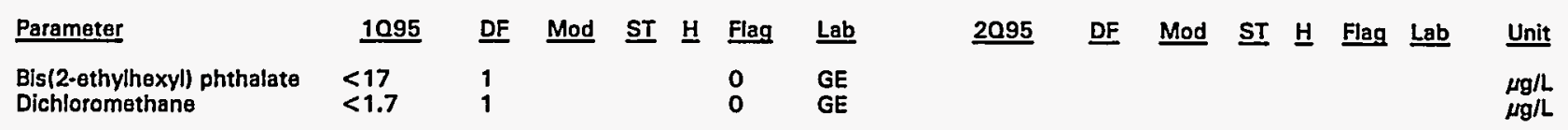

Note: See Appendix B for flagging criteria. Synchronous water levels are measured over a 3-5 day period.

- = exceeded holding time.

- exceeded groundwater protection standard. 
WELL FSB104D (cont.)

Radiological Indicator Parameters

\begin{tabular}{|c|c|c|c|c|c|c|c|c|c|c|c|c|c|c|c|}
\hline Parameter & 1095 & DF & Mod & ST & 브 & Flag & Lab & $\underline{2 Q 95}$ & DF & Mod & $\underline{\mathrm{ST}}$ & $\underline{H}$ & Flag & $\underline{\text { Lab }}$ & Unit \\
\hline $\begin{array}{l}\text { Gross alpha } \\
\text { Nonvolatile beta }\end{array}$ & $\begin{array}{l}5.7 E+02 \\
6.6 E+02\end{array}$ & $\begin{array}{l}1 \\
1\end{array}$ & J & - & & $\begin{array}{l}2 \\
2\end{array}$ & $\begin{array}{l}\text { GP } \\
\text { GP }\end{array}$ & & & & & & & & $\begin{array}{l}\mathrm{pCi} / \mathrm{L} \\
\mathrm{pCi} / \mathrm{L}\end{array}$ \\
\hline \multicolumn{16}{|c|}{ Measured Radionuclides } \\
\hline Parameter & 1095 & $\underline{D F}$ & Mod & $\underline{\text { ST }}$ & $\underline{H}$ & Flag & $\underline{\text { Lab }}$ & $\underline{2095}$ & $\underline{D F}$ & Mod & $\underline{\mathbf{S T}}$ & 븐 & Flag & $\underline{\text { Lab }}$ & Unit \\
\hline $\begin{array}{l}\text { Actinium-228 } \\
\text { Americium-241 } \\
\text { Cesium-137 } \\
\text { Cobalt-60 } \\
\text { Curium-242 } \\
\text { Curium-243/244 } \\
\text { Curium-245/246 } \\
\text { lodine-129 } \\
\text { Plutonium-238 } \\
\text { Plutonium-239/240 } \\
\text { Radium-226 } \\
\text { Radium-228 } \\
\text { Strontium-89 } \\
\text { Strontium-90 } \\
\text { Technetium-99 } \\
\text { Thorium-228 } \\
\text { Thorium-230 } \\
\text { Thorium-232 } \\
\text { Thorium-234 } \\
\text { Tritium } \\
\text { Uranium-233/234 } \\
\text { Uranium-235 } \\
\text { Uranium-238 }\end{array}$ & $\begin{array}{l}1.5 E+01 \\
1.2 E+01 \\
-1.9 E+00 \\
1.6 E+00 \\
-1.6 E-03 \\
1.1 E+01 \\
1.1 E+00 \\
3.9 E+01 \\
-1.1 E-01 \\
1.1 E-01 \\
1.9 E+01 \\
1.7 E+01 \\
2.6 E+01 \\
1.6 E+02 \\
4.2 E+01 \\
3.5 E-01 \\
2.2 E-01 \\
2.6 E-02 \\
2.4 E+02 \\
4.8 E+03 \\
2.5 E+02 \\
1.2 E+01 \\
3.1 E+02\end{array}$ & $\begin{array}{l}1 \\
1 \\
1 \\
1 \\
1 \\
1 \\
1 \\
1 \\
1 \\
1 \\
1 \\
1 \\
1 \\
1 \\
1 \\
1 \\
1 \\
1 \\
1 \\
1 \\
1 \\
1 \\
1\end{array}$ & $\begin{array}{l}\text { UI } \\
\text { UI } \\
\text { UI } \\
U ! \\
\\
U ! \\
\text { UI } \\
J \\
J\end{array}$ & $\begin{array}{l}\cdot \\
\square \\
\square \\
\square \\
\mathbf{0}\end{array}$ & & $\begin{array}{l}0 \\
2 \\
0 \\
0 \\
0 \\
2 \\
0 \\
2 \\
0 \\
0 \\
1 \\
1 \\
2 \\
2 \\
0 \\
0 \\
0 \\
0 \\
1 \\
2 \\
2 \\
1 \\
2\end{array}$ & $\begin{array}{l}\text { GP } \\
\text { GP } \\
\text { GP } \\
\text { GP } \\
\text { GP } \\
\text { GP } \\
\text { GP } \\
\text { GP } \\
\text { GP } \\
\text { GP } \\
\text { GP } \\
\text { GP } \\
\text { GP } \\
\text { GP } \\
\text { GP } \\
\text { GP } \\
\text { GP } \\
\text { GP } \\
\text { GP } \\
\text { GP } \\
\text { GP } \\
\text { GP } \\
\text { GP }\end{array}$ & & & & & & & & $\begin{array}{l}\text { pCi } / L \\
\text { pCi/L } \\
\text { pCi/L } \\
\text { pCi/L } \\
\text { pCi/L } \\
\text { pCi/L } \\
\text { pCi/L } \\
\text { pCi/L } \\
\text { pCi } / L \\
\text { pCi/L } \\
\text { pCi/L } \\
\text { pCi/L } \\
\text { pCi/L } \\
\text { pCi/L } \\
\text { pCi/L } \\
\text { pCi/L } \\
\text { pCi/L } \\
\text { pCi/L } \\
\text { pCi/L } \\
\text { pCi/mL } \\
\text { pCi/L } \\
\text { pCi/L } \\
\text { pCi/L }\end{array}$ \\
\hline \multicolumn{16}{|c|}{ Calculated Radionuclides } \\
\hline Parameter & 1095 & $\underline{\mathrm{DF}}$ & Mod & $\underline{\underline{S T}}$ & $\underline{H}$ & Flag & $\underline{\operatorname{Lab}}$ & $\underline{2095}$ & $\underline{D F}$ & Mod & $\underline{\mathrm{ST}}$ & $\underline{H}$ & Flag & Lab & Unit \\
\hline $\begin{array}{l}\text { Americium-243 } \\
\text { Nickel-63 } \\
\text { Plutonium-241 } \\
\text { Plutonium-242 }\end{array}$ & $\begin{array}{l}3.3 E-02 \\
1.6 E+00 \\
4.4 E-01 \\
2.2 E-06\end{array}$ & & & & & $\begin{array}{l}0 \\
0 \\
0 \\
0\end{array}$ & & & & & & & & & $\begin{array}{l}\mathrm{pCi} / \mathrm{L} \\
\mathrm{pCi} / \mathrm{L} \\
\mathrm{pCi} / \mathrm{L} \\
\mathrm{pCi} / \mathrm{L}\end{array}$ \\
\hline
\end{tabular}

Note: See Appendix B for flagging criteria. Synchronous water levels are measured over a 3-5 day period.

- = exceeded holding time.

- = exceeded groundwater protection standard. 


\section{WELL FSB105C}

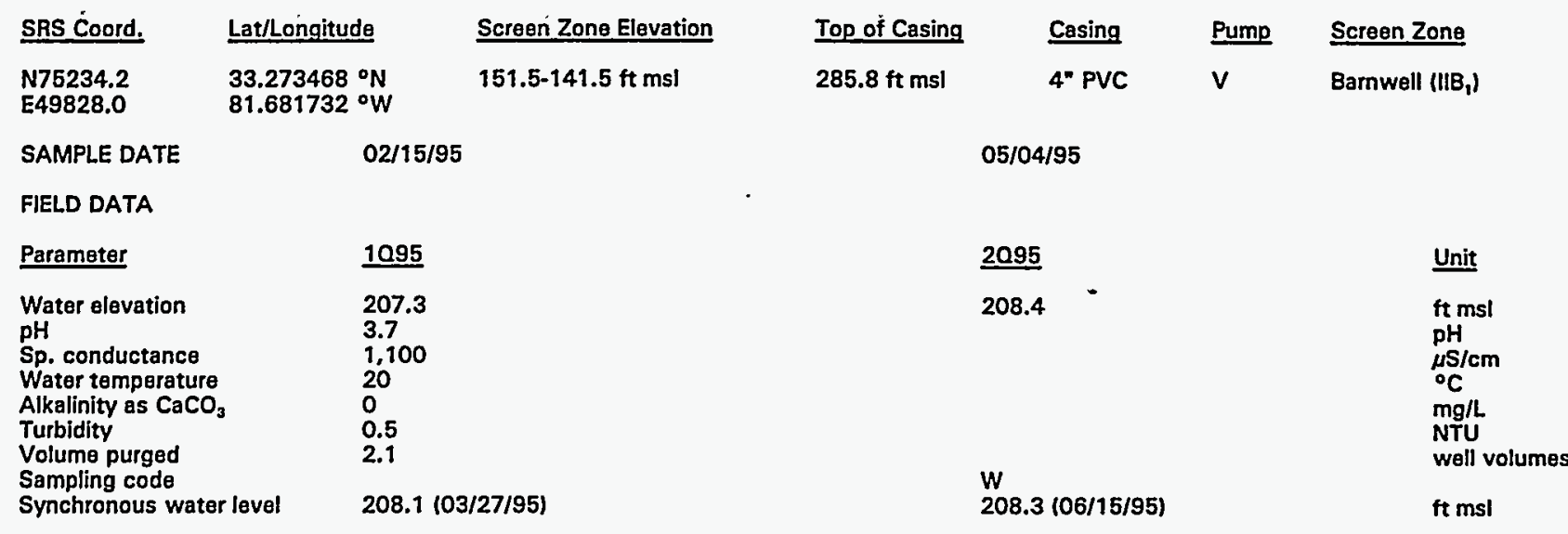

Hazardous Constituents (264.94, Table 1)

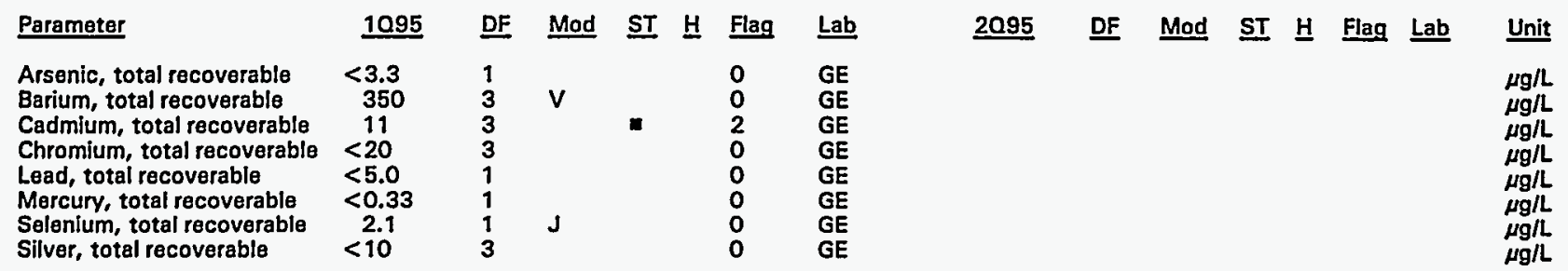

Hazardous Constituents (261 App. VIII/264 App. IX)

\begin{tabular}{|c|c|c|c|c|c|c|c|c|c|c|c|c|c|c|c|}
\hline Parameter & 1095 & DF & Mod & $\underline{\mathbf{S T}}$ & $\underline{H}$ & Flag & Lab & 2095 & $\underline{\mathrm{DF}}$ & Mod & ST & 브 & Flag & Lab & Unit \\
\hline $\begin{array}{l}\text { Antimony, total recoverable } \\
\text { Benzene } \\
\text { Cobalt, total recoverable } \\
\text { Copper, total recoverable } \\
\text { Cyanide } \\
\text { Nickel, total recoverable } \\
\text { Phenols } \\
\text { Tetrachloroethylene } \\
\text { Thallium, total recoverable } \\
\text { Trichloroethylene } \\
\text { Trichlorofluoromethane } \\
\text { Vanadium, total recoverable } \\
\text { Zinc, total recoverable }\end{array}$ & $\begin{array}{c}4.7 \\
<1.7 \\
240 \\
47 \\
<8.3 \\
45 \\
<8.3 \\
<1.7 \\
<3.3 \\
4.1 \\
<1.7 \\
<40 \\
110\end{array}$ & $\begin{array}{l}1 \\
1 \\
3 \\
3 \\
1 \\
3 \\
1 \\
1 \\
1 \\
1 \\
1 \\
3 \\
3\end{array}$ & $\begin{array}{l}\text { J1 } \\
\text { V } \\
\text { J1 }\end{array}$ & $\mathbf{u}$ & $\bullet$ & $\begin{array}{l}1 \\
0 \\
2 \\
0 \\
0 \\
0 \\
0 \\
0 \\
0 \\
1 \\
0 \\
0 \\
0\end{array}$ & $\begin{array}{l}\mathbf{G E} \\
\mathbf{G E} \\
\mathbf{G E} \\
\mathbf{G E} \\
\mathbf{G E} \\
\mathbf{G E} \\
\mathbf{G E} \\
\mathbf{G E} \\
\mathbf{G E} \\
\mathbf{G E} \\
\mathbf{G E} \\
\mathbf{G E} \\
\mathbf{G E}\end{array}$ & & & & & & & & $\begin{array}{l}\mu g / L \\
\mu g / L \\
\mu g / L \\
\mu g / L \\
\mu g / L \\
\mu g / L \\
\mu g / L \\
\mu g / L \\
\mu g / L \\
\mu g / L \\
\mu g / L \\
\mu g / L \\
\mu g / L\end{array}$ \\
\hline \multicolumn{16}{|c|}{ Water Quality Indicator Parameters } \\
\hline Parameter & 1095 & DF & Mod & $\underline{\text { ST }}$ & 브 & Flag & Lab & $\underline{2095}$ & DF & Mod & $\underline{\mathbf{S T}}$ & 브 & Flag & Lab & $\underline{\text { Unit }}$ \\
\hline \multicolumn{16}{|c|}{ Common Laboratory Contaminants } \\
\hline Parameter & $\underline{1095}$ & DF & Mod & sT & 브 & Flag & Lab & $\underline{2095}$ & DF & Mod & $\underline{\text { ST }}$ & $\underline{H}$ & Flag & Lab & Unit \\
\hline $\begin{array}{l}\text { Bis(2-ethylhexyl) phthalate } \\
\text { Dichloromethane }\end{array}$ & $\begin{array}{l}<17 \\
<1.7\end{array}$ & $\begin{array}{l}1 \\
1\end{array}$ & & & & $\begin{array}{l}0 \\
0\end{array}$ & $\begin{array}{l}\text { GE } \\
\text { GE }\end{array}$ & & & & & & & & $\begin{array}{l}\mu g / L \\
\mu g / L\end{array}$ \\
\hline
\end{tabular}

Note: See Appendix B for flagging criteria. Synchronous water levels are measured over a 3-5 day period.

$-=$ exceeded holding time.

- = exceeded groundwater protection standard. 
WELL FSB105C (cont.)

Radiological Indicator Parameters

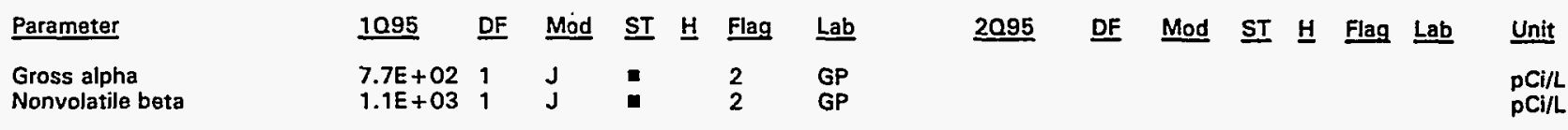

Measured Radionuclides

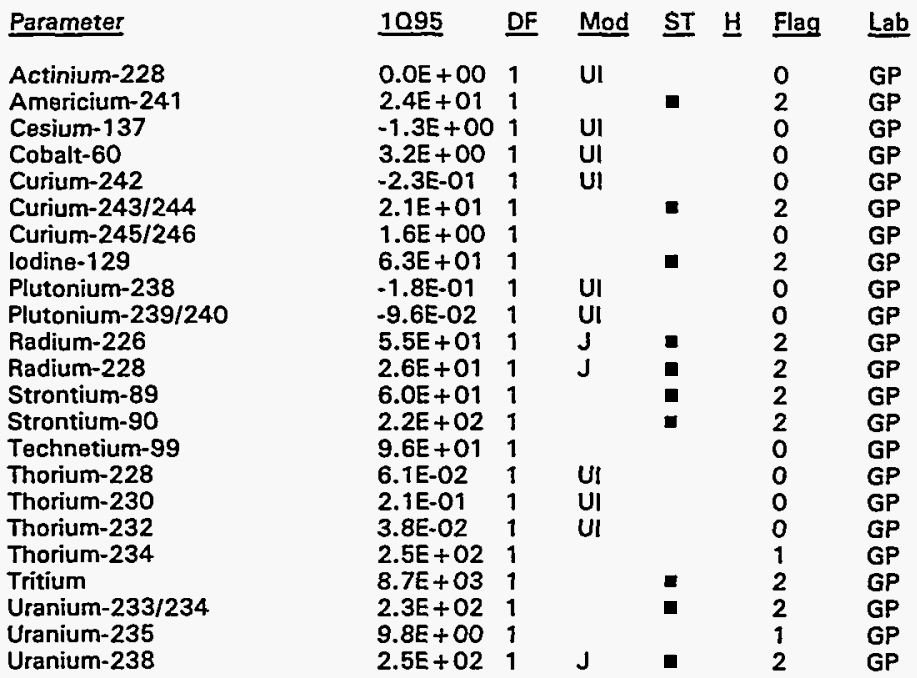

2095 DF Mod ST H Flag Lab Unit

Uranium-238

Calculated Radionuclides

Parameter

Americium-243

Nickel-63

Plutonium-241

Plutonium-242
109

6.2E-02

$3.2 E+00$

$-3.8 \mathrm{E}-01$

$-1.9 E-06$
DF Mod ST $\underline{H}$ Flag Lab

0

Unit

pCi/L

pCi/L

pCilL

Note: See Appendix B for flagging criteria. Synchronous water levels are measured over a 3-5 day period.

$\bullet=$ exceeded holding time.

- = exceeded groundwater protection standard. 


\section{WELL FSB105DR}

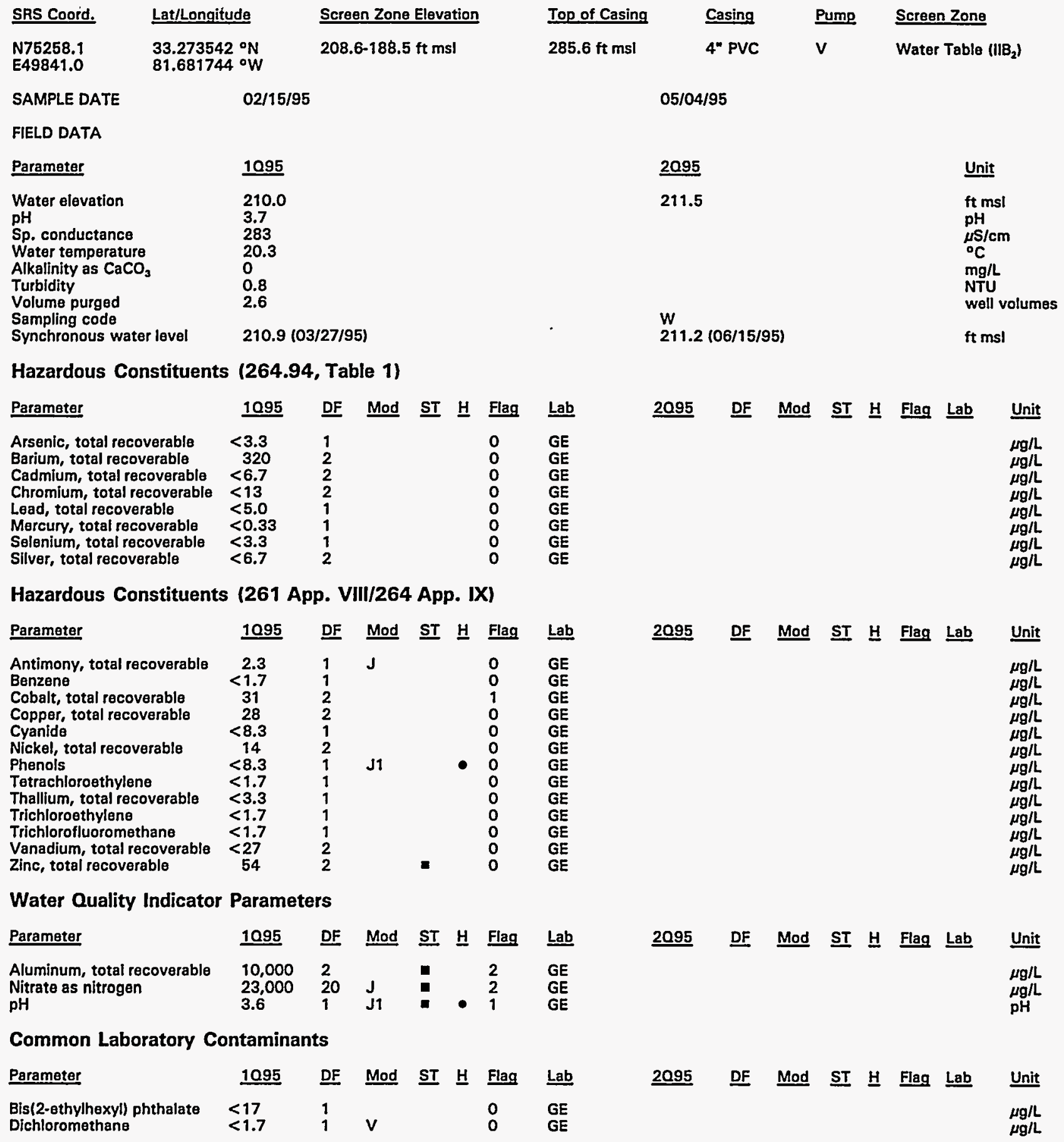

Note: See Appendix B for flagging criteria. Synchronous water levels are measured over a 3-5 day period.

- = exceeded holding time.

- = exceeded groundwater protection standard. 
WELL FSB105DR (cont.)

Radiological Indicator Parameters

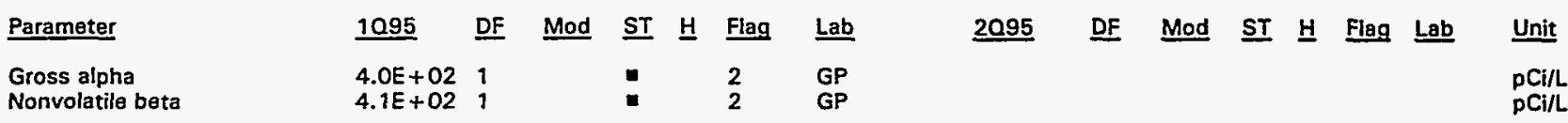

Measured Radionuclides

\begin{tabular}{|c|c|c|c|c|c|c|}
\hline Parameter & 1095 & $\underline{D F}$ & Mod & $\underline{\text { ST }} \underline{H}$ & Flag & Lab \\
\hline Actinium-228 & $5.5 E+01$ & 1 & & & 0 & GP \\
\hline Americium-241 & $3.9 E+00$ & 1 & & & 1 & GP \\
\hline Cesium-137 & $-3.4 E-01$ & 1 & UI & & 0 & GP \\
\hline Cobalt- 60 & $1.2 \mathrm{E}+00$ & 1 & Ui & & 0 & GP \\
\hline Curium-242 & $0.0 E+00$ & $i$ & UI & & 0 & GP \\
\hline Curium-243/244 & $5.0 E+00$ & 1 & & & 1 & GP \\
\hline Curium-245/246 & 8.9E-01 & 1 & UI & & 0 & GP \\
\hline lodine-129 & $1.7 E+01$ & 1 & & घ & 2 & GP \\
\hline Plutonium-238 & $3.4 \mathrm{E}-02$ & 1 & UI & & 0 & GP \\
\hline Plutonium-239/240 & 4.7E-02 & 1 & UI & & 0 & GP \\
\hline Radium-226 & $1.7 \bar{E}+01$ & 1 & & & 1 & GP \\
\hline Radium-228 & $1.3 E+01$ & 1 & $J$ & & 1 & GP \\
\hline Strontium-89 & $1.2 E+01$ & 1 & & & 1 & $\mathbf{G P}$ \\
\hline Strontium-90 & $3.4 E+01$ & 1 & & $\mathbf{a}$ & 2 & GP \\
\hline Technetium-99 & 5.3E-01 & 1 & UI & & $\overline{0}$ & GP \\
\hline Thorium-228 & 7.1E-01 & 1 & UI & & 0 & GP \\
\hline Thorium-230 & $1.9 E-02$ & 1 & UI & & 0 & GP \\
\hline Thorium-232 & $-2.2 \mathrm{E}-02$ & 1 & UI & & 0 & GP \\
\hline Thorium-234 & $2.9 E+02$ & 1 & & & 1 & GP \\
\hline Tritium & $5.5 E+02$ & 1 & & $=$ & 2 & GP \\
\hline Uranium-233/234 & $2.2 E+02$ & 1 & & 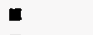 & 2 & GP \\
\hline Uranium-235 & $1.6 E+01$ & 1 & & ฮ & 2 & GP \\
\hline Uranium-238 & $5.6 E+02$ & 1 & & $\mathbf{n}$ & 2 & GP \\
\hline
\end{tabular}

Uranium-238

$5.6 E+021$

Calculated Radionuclides

Parameter

Americium-243

Nickel-63

Plutonium-241

Plutonium-242
1095 DF

Mod
$1.5 \mathrm{E}-02$

1.2E +00

1.9E-01

9.3E-07
2095 DF Mod ST $\underline{\text { H Flag Lab }} \underline{\text { Unit }}$

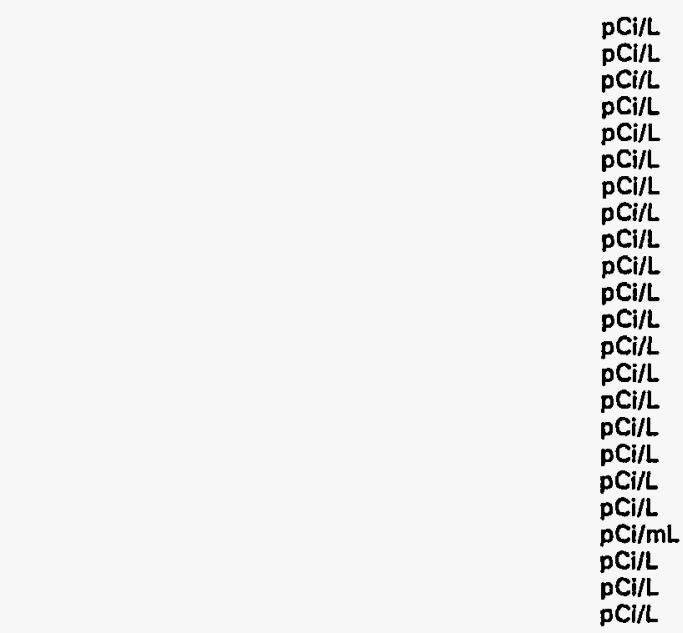

2095 DF Mod ST $\underline{H}$ Flag Lab Unit

$\mathrm{pCi} / \mathrm{L}$

$\mathrm{pCi} / \mathrm{L}$

$\mathrm{pCi} / \mathrm{L}$

Note: See Appendix B for flagging criteria. Synchronous water levels are measured over a 3-5 day period.

- = exceeded holding time.

n = exceeded groundwater protection standard. 


\section{WELL FSB106C}

\begin{tabular}{|c|c|c|c|c|c|c|}
\hline SRS Coord. & Lat/Longitude & Screen Zone Elevation & Top of Casing & Casing & Pump & Screen Zone \\
\hline $\begin{array}{l}\text { N74190.1 } \\
\text { E50651.3 }\end{array}$ & $\begin{array}{l}33.272503^{\circ} \mathrm{N} \\
81.677536^{\circ} \mathrm{W}\end{array}$ & $166.0-156.0 \mathrm{ft} \mathrm{msl}$ & $235.1 \mathrm{ft} \mathrm{msl}$ & 4" PVC & v & Barnwell ||$B_{1} \mid$ \\
\hline - ש & 02 & & & & & \\
\hline
\end{tabular}

FIELD DATA

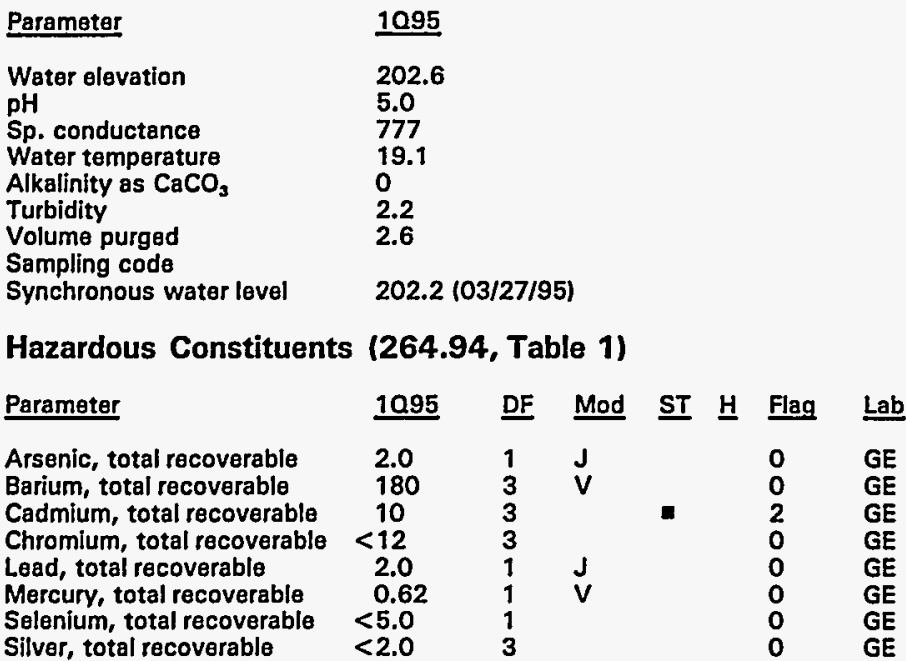

\begin{tabular}{ll}
$\underline{2095}$ & Unit \\
\hline 201.5 & $\mathrm{ft} \mathrm{msl}$ \\
& $\mathrm{pH}$ \\
$\mu \mathrm{sS} / \mathrm{cm}$ \\
& ${ }^{\circ} \mathrm{C}$ \\
& $\mathrm{mg} / \mathrm{L}$ \\
$\mathrm{NTU}$ \\
$\mathrm{WTll}$ \\
$\mathrm{W}$ & well volumes \\
$206.6(06 / 15 / 95)$ & $\mathrm{ft} \mathrm{msl}$
\end{tabular}

Hazardous Constituents (261 App. VIII/264 App. IX)

\begin{tabular}{|c|c|c|c|c|c|c|c|c|c|c|c|c|c|c|c|}
\hline Paramoter & 1095 & $\underline{\mathrm{DF}}$ & Mod & $\underline{\mathbf{S T}}$ & $\underline{\mathbf{H}}$ & Flag & $\underline{\text { Lab }}$ & $\underline{2095}$ & $\underline{\text { DF }}$ & Mod & $\underline{\mathbf{S T}}$ & 브 & Flag & $\underline{\text { Lab }}$ & Unit \\
\hline $\begin{array}{l}\text { Antimony, total recoverable } \\
\text { Benzene } \\
\text { Cobalt, total recoverable } \\
\text { Copper, total recoverable } \\
\text { Cyanide } \\
\text { Nickel, total recoverable } \\
\text { Phenols } \\
\text { Tetrachloroethylene } \\
\text { Thallium, total recoverable } \\
\text { Trichloroethylene } \\
\text { Trichlorofluoromethane } \\
\text { Vanedium, total recoverable } \\
\text { Zinc, total recoverable }\end{array}$ & $\begin{array}{r}5.1 \\
<1.7 \\
63 \\
6.6 \\
<20 \\
16 \\
<10 \\
<1.7 \\
0.96 \\
1.9 \\
<1.7 \\
<30 \\
40\end{array}$ & $\begin{array}{l}1 \\
1 \\
3 \\
3 \\
1 \\
3 \\
1 \\
1 \\
1 \\
1 \\
1 \\
3 \\
3\end{array}$ & $\begin{array}{l}J \\
Y \\
J\end{array}$ & - & & $\begin{array}{l}1 \\
0 \\
2 \\
0 \\
0 \\
0 \\
0 \\
0 \\
0 \\
0 \\
0 \\
0 \\
0\end{array}$ & $\begin{array}{l}\mathrm{GE} \\
\mathrm{GE} \\
\mathrm{GE} \\
\mathrm{GE} \\
\mathrm{GE} \\
\mathrm{GE} \\
\mathrm{GE} \\
\mathrm{GE} \\
\mathrm{GE} \\
\mathrm{GE} \\
\mathrm{GE} \\
\mathrm{GE} \\
\mathrm{GE}\end{array}$ & & & & & & & & $\begin{array}{l}\mu g / L \\
\mu g / L \\
\mu g / L \\
\mu g / L \\
\mu g / L \\
\mu g / L \\
\mu g / L \\
\mu g / L \\
\mu g / L \\
\mu g / L \\
\mu g / L \\
\mu g / L \\
\mu g / L\end{array}$ \\
\hline \multicolumn{16}{|c|}{ Water Quality Indicator Parameters } \\
\hline Parameter & 1095 & $\underline{D F}$ & Mod & $\underline{\mathbf{S T}}$ & $\underline{\mathrm{H}}$ & Flag & $\underline{\text { Lab }}$ & $\underline{2095}$ & DF & Mod & $\underline{\text { ST }}$ & $\underline{\mathbf{H}}$ & Fiag & $\underline{\text { Lab }}$ & $\underline{\text { Unit }}$ \\
\hline $\begin{array}{l}\text { Aluminum, total recoverable } \\
\text { Nitrate as nitrogen } \\
\text { pH }\end{array}$ & $\begin{array}{l}4,300 \\
90,000 \\
4.7\end{array}$ & $\begin{array}{l}3 \\
80 \\
1\end{array}$ & $\begin{array}{l}v \\
\text { J1 }\end{array}$ & 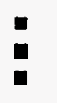 & - & $\begin{array}{l}2 \\
2 \\
0\end{array}$ & $\begin{array}{l}\text { GE } \\
\text { GE } \\
\text { GE }\end{array}$ & & & & & & & & $\begin{array}{l}\mu g / L \\
\mu g / L \\
p H\end{array}$ \\
\hline \multicolumn{16}{|c|}{ Common Laboratory Contaminants } \\
\hline Parameter & $\underline{1095}$ & $\underline{\mathrm{DF}}$ & Mod & $\underline{\text { ST }}$ & $\underline{\mathrm{H}}$ & Flag & $\underline{\text { Lab }}$ & $\underline{2095}$ & $\underline{\mathrm{DF}}$ & $\underline{\text { Mod }}$ & $\underline{\text { ST }}$ & 브 & Flag & $\underline{\text { Lab }}$ & $\underline{\text { Unit }}$ \\
\hline $\begin{array}{l}\text { Bis(2-ethylhexyl) phthalate } \\
\text { Dichloromethane }\end{array}$ & $\begin{array}{l}<17 \\
<1.7\end{array}$ & $\begin{array}{l}1 \\
1\end{array}$ & & & & $\begin{array}{l}0 \\
0\end{array}$ & $\begin{array}{l}\text { GE } \\
\text { GE }\end{array}$ & & & & & & & & $g / L$ \\
\hline
\end{tabular}

Note: See Appendix B for flagging criteria. Synchronous water levels are measured over a 3-5 day period.

- = exceeded holding time.

m = exceeded groundwater protection standard. 
WELL FSB 106C (cont.)

Radiological Indicator Parameters

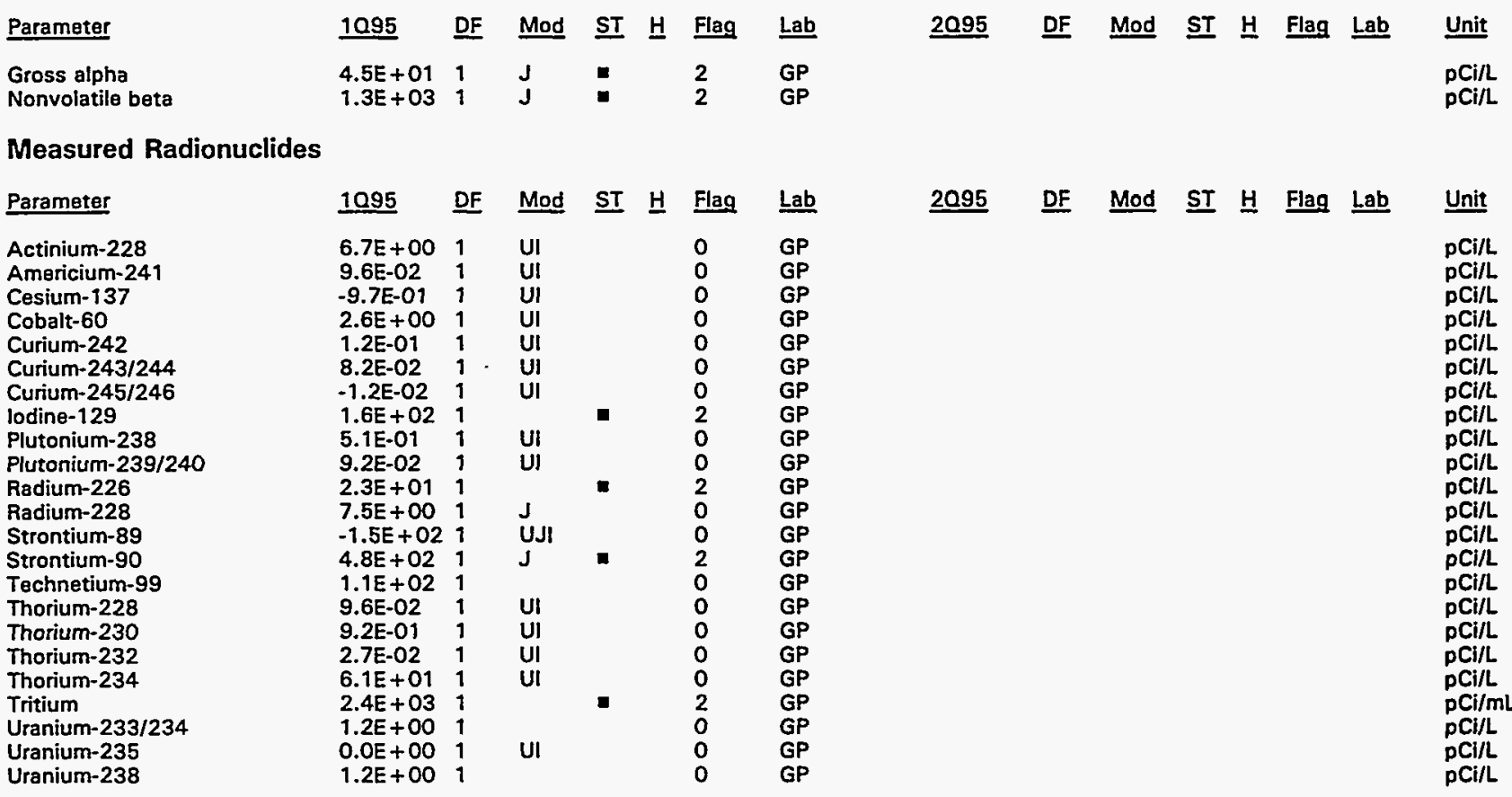

Calculated Radionuclides

Parameter

Americium-243

Nickel-63

Plutonium-241

Plutonium-242
109

2.5E-04

2. $6 E+00$

3.7E-01

1.8E-06

DF Mod ST $\underline{\text { H Flag Lab }}$

$\underline{2095}$

DF Mod ST $\underline{H}$ Flag Lab

Unit

pCi/L

pCilL

pCi/L

pCi/L

Note: See Appendix B for flagging criteria. Synchronous water levels are measured over a 3-5 day period.

- = exceeded holding time.

- = exceeded groundwater protection standard. 
WSRC-TR-95-0146-2

Unclassified

WELL FSB106D

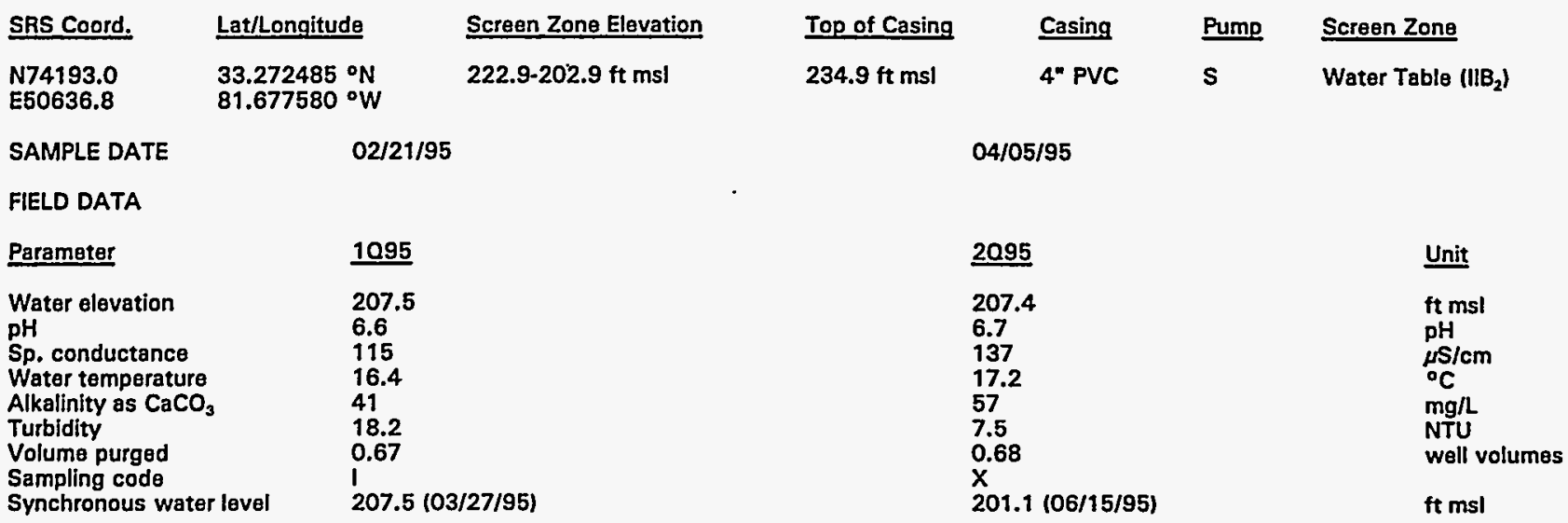

Hazardous Constituents (264.94, Table 1)

\begin{tabular}{|c|c|c|c|c|c|c|c|c|c|c|c|c|c|c|}
\hline Parameter & 1095 & DF & Mod & ST $\mathrm{H}$ & Flag & Lab & 2095 & $\underline{D F}$ & Mod & ST & $\underline{H}$ & Flag & Lab & Unit \\
\hline $\begin{array}{l}\text { Arsenic, total recoverable } \\
\text { Barium, total recoverable } \\
\text { Cadmium, total recoverable } \\
\text { Chromium, total recoverable } \\
\text { Lead, total recoverable } \\
\text { Mercury, total recoverable } \\
\text { Selenium, total recoverable } \\
\text { Silver, total recoverable }\end{array}$ & $\begin{array}{c}<5.0 \\
16 \\
<2.0 \\
<4.0 \\
51 \\
<0.20 \\
2.7 \\
<0.65\end{array}$ & $\begin{array}{l}1 \\
1 \\
1 \\
1 \\
1 \\
1 \\
1 \\
1\end{array}$ & JV & * & $\begin{array}{l}0 \\
0 \\
0 \\
0 \\
2 \\
0 \\
0 \\
0\end{array}$ & $\begin{array}{l}\text { GE } \\
\text { GE } \\
\text { GE } \\
\text { GE } \\
\text { GE } \\
\text { GE } \\
\text { GE } \\
\text { GE }\end{array}$ & $\begin{array}{r}<2.0 \\
48\end{array}$ & $\begin{array}{l}1 \\
1\end{array}$ & & & & $\begin{array}{l}0 \\
1\end{array}$ & $\begin{array}{l}\text { GE } \\
\text { GE }\end{array}$ & $\begin{array}{l}\mu \mathrm{g} / \mathrm{L} \\
\mu \mathrm{g} / \mathrm{L} \\
\mu \mathrm{g} / \mathrm{L} \\
\mu \mathrm{g} / \mathrm{L} \\
\mu \mathrm{g} / \mathrm{L} \\
\mu \mathrm{g} / \mathrm{L} \\
\mu \mathrm{g} / \mathrm{L} \\
\mu \mathrm{g} / \mathrm{L}\end{array}$ \\
\hline
\end{tabular}

Hazardous Constituents (261 App. VIII/264 App. IX)

\begin{tabular}{|c|c|c|c|c|c|c|c|c|c|c|c|c|c|c|c|}
\hline Parameter & 1095 & DF & Mod & $\underline{\mathbf{S T}}$ & $\underline{H}$ & Flag & $\underline{\text { Lab }}$ & 2095 & DF & Mod & ST & 브 & Flag & $\underline{\text { Lab }}$ & Unit \\
\hline $\begin{array}{l}\text { Antimony, total recoverable } \\
\text { Benzene } \\
\text { Cobalt, total recoverable } \\
\text { Copper, total recoverable } \\
\text { Cyanide } \\
\text { Nickel, total recoverable } \\
\text { Phenols } \\
\text { Tetrachloroethylene } \\
\text { Thallium, total recoverable } \\
\text { Trichloroethylene } \\
\text { Trichlorofluoromethane } \\
\text { Vanadium, total recoverable } \\
\text { Zinc, total recoverable }\end{array}$ & $\begin{aligned} & 3.5 \\
&<1.7 \\
&<4.0 \\
& 40 \\
&<20 \\
& 23 \\
&<10 \\
&<1.7 \\
&<5.0 \\
&<1.7 \\
&<1.7 \\
& 0.85 \\
& 720\end{aligned}$ & $\begin{array}{l}1 \\
1 \\
1 \\
1 \\
1 \\
1 \\
1 \\
1 \\
1 \\
1 \\
1 \\
1 \\
1\end{array}$ & $\mathbf{J} 1$ & - & & $\begin{array}{l}1 \\
0 \\
0 \\
0 \\
0 \\
0 \\
0 \\
0 \\
0 \\
0 \\
0 \\
0 \\
0\end{array}$ & $\begin{array}{l}\text { GE } \\
\text { GE } \\
\text { GE } \\
\text { GE } \\
\text { GE } \\
\text { GE } \\
\text { GE } \\
\text { GE } \\
\text { GE } \\
\text { GE } \\
\text { GE } \\
\text { GE } \\
\text { GE }\end{array}$ & & & & & & & & $\begin{array}{l}\mu g / L \\
\mu g / L \\
\mu g / L \\
\mu g / L \\
\mu g / L \\
\mu g / L \\
\mu g / L \\
\mu g / L \\
\mu g / L \\
\mu g / L \\
\mu g / L \\
\mu g / L \\
\mu g / L\end{array}$ \\
\hline \multicolumn{16}{|c|}{ Water Quality Indicator Parameters } \\
\hline Parameter & 1095 & $\underline{\mathrm{DF}}$ & Mod & $\underline{\text { ST }}$ & $\underline{\mathbf{H}}$ & Flag & $\underline{\text { Lab }}$ & $\underline{2095}$ & $\underline{\text { DF }}$ & Mod & $\underline{\text { ST }}$ & H & Flag & Lab & $\underline{\text { Unit }}$ \\
\hline \multicolumn{16}{|c|}{ Common Laboratory Contaminants } \\
\hline Parameter & 1095 & $\underline{\text { DF }}$ & Mod & $\underline{\mathbf{S T}}$ & 브 & Flag & $\underline{\text { Lab }}$ & 2095 & $\underline{\mathrm{DF}}$ & Mod & $\underline{\text { ST }}$ & 브 & Flag & Lab & Unit \\
\hline $\begin{array}{l}\text { Bis(2-ethylhexyl) phthalate } \\
\text { Dichloromethane }\end{array}$ & $\begin{array}{r}190 \\
<1.7\end{array}$ & $\begin{array}{l}2 \\
1\end{array}$ & & - & & $\begin{array}{l}2 \\
0\end{array}$ & $\begin{array}{l}\text { GE } \\
\text { GE }\end{array}$ & & & & & & & & $\begin{array}{l}\mu \mathrm{g} / \mathrm{L} \\
\mu \mathrm{g} / \mathrm{L}\end{array}$ \\
\hline
\end{tabular}

Note: See Appendix B for flagging criteria. Synchronous water levels are measured over a 3-5 day period.

- = exceeded holding time.

- = exceeded groundwater protection standard. 
WELL FSB106D (cont.)

Radiological Indicator Parameters

\begin{tabular}{|c|c|c|c|c|c|c|c|c|c|c|c|c|}
\hline Parameter & 1095 & $\underline{D F}$ & Mod & $\underline{\mathrm{ST}} \underline{\mathrm{H}}$ & Flag & Lab & $\underline{2095}$ & $\underline{\text { DF }}$ & $\underline{\text { Mod }}$ & $\underline{\text { ST }} \underline{H}$ & Flag & $\underline{\text { Lab }}$ \\
\hline $\begin{array}{l}\text { Gross alpha } \\
\text { Nonvolatile beta }\end{array}$ & $\begin{array}{l}7.3 \mathrm{E}-01 \\
7.2 \mathrm{E}-01\end{array}$ & $\begin{array}{l}1 \\
1\end{array}$ & $\begin{array}{l}\text { UI } \\
\text { UI }\end{array}$ & & $\begin{array}{l}0 \\
0\end{array}$ & $\begin{array}{l}\text { GP } \\
G P\end{array}$ & $\begin{array}{l}9.8 E-01 \\
2.6 E+00\end{array}$ & $\begin{array}{l}1 \\
1\end{array}$ & UJI & & $\begin{array}{l}0 \\
0\end{array}$ & $\begin{array}{l}\text { GP } \\
\text { GP }\end{array}$ \\
\hline
\end{tabular}

Measured Radionuclides

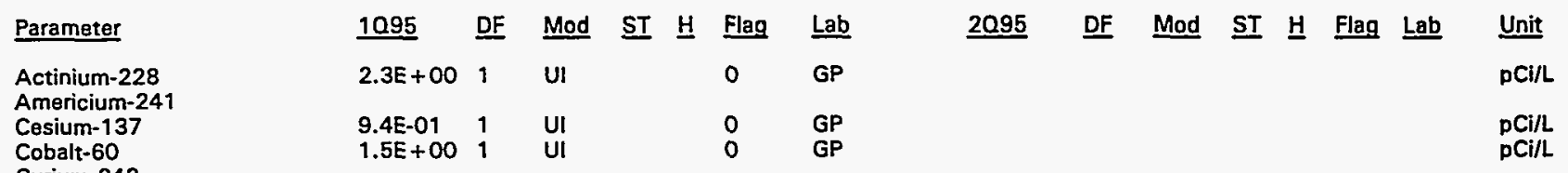

042

Curium-243/244

Curium-245/246

lodine- 129

Plutonium-238

Plutonium-239/240

Radium-226

Radium-228

Strontium-89

Strontium-90

Technetium-99

Thorium-228

Thorium-230

Thorium-232

Thorium-234

Tritium

Uranium-233/234

Uranium-235

Uranium-238

Calculated Radionuclides

\section{Parameter}

1095

DF Mod ST H Flag Lab

$\underline{2095}$

DF Mod ST $\underline{H}$ Flag Lab

Unit

Americium-243

Nickel-63

$1.5 E+00$

0

$\mathrm{pCi} / \mathrm{L}$

Plutonium-24

Plutonium-242

Note: See Appendix B for flagging criteria. Synchronous water levels are measured over a 3-5 day period.

- = exceeded holding time.

- = exceeded groundwater protection standard. 
WELL FSB107C

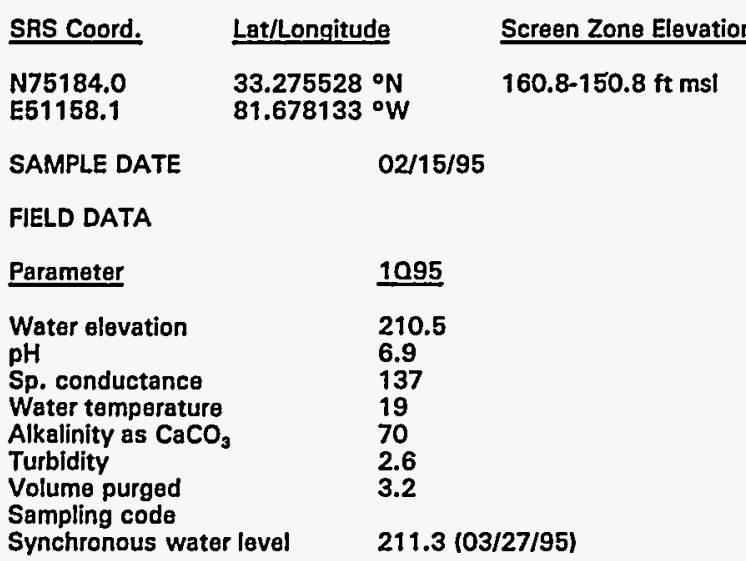

$\begin{array}{lrrrr}\text { Top of Casing } & \text { Casing } & \text { Pump } & \frac{\text { Screen Zone }}{270.9 \mathrm{ft} \mathrm{msl}} \\ 4^{- \text {PVC }} & \text { S } & \text { Barnwell (IIB, }) \\ 04 / 04 / 95 & & \end{array}$

Hazardous Constituents (264.94, Table 1)

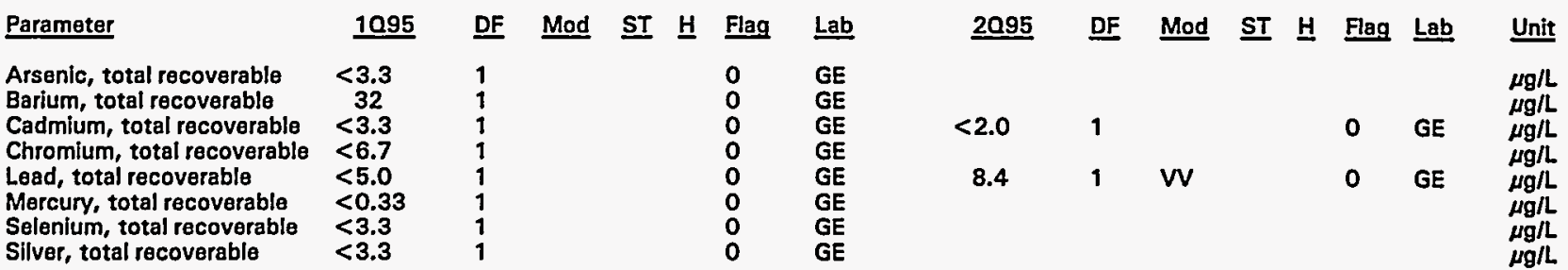

Hazardous Constituents (261 App. VIII/264 App. IX)

\begin{tabular}{|c|c|c|c|c|c|c|c|c|c|c|c|c|c|c|c|}
\hline Parameter & 1095 & $\underline{D F}$ & Mod & $\underline{\text { ST }}$ & $\underline{\mathrm{H}}$ & Flag & $\underline{\text { Lab }}$ & $\underline{2095}$ & $\underline{\text { DF }}$ & Mod & ST & $\underline{H}$ & Flag & Lab & $\underline{\text { Unit }}$ \\
\hline $\begin{array}{l}\text { Antimony, total recoverable } \\
\text { Benzene } \\
\text { Cobalt, total recoverable } \\
\text { Copper, total recoverable } \\
\text { Cyanide } \\
\text { Nickel, total recoverable } \\
\text { Phenols } \\
\text { Tetrachloroethylene } \\
\text { Thallium, total recoverable } \\
\text { Trichloroethylene } \\
\text { Trichlorofluoromethane } \\
\text { Vanadium, total recoverable } \\
\text { Zinc, total recoverable }\end{array}$ & $\begin{aligned}<3.3 \\
<1.7 \\
<6.7 \\
<6.7 \\
<8.3 \\
<6.7 \\
<8.3 \\
1.1 \\
<3.3 \\
1.4 \\
<1.7 \\
<13 \\
6.0\end{aligned}$ & $\begin{array}{l}1 \\
1 \\
1 \\
1 \\
1 \\
1 \\
1 \\
1 \\
1 \\
1 \\
1 \\
1 \\
1\end{array}$ & $\begin{array}{l}\mathbf{J} 1 \\
\mathbf{J} \\
\mathbf{J}\end{array}$ & & $\bullet$ & $\begin{array}{l}0 \\
0 \\
0 \\
0 \\
0 \\
0 \\
0 \\
0 \\
0 \\
0 \\
0 \\
0 \\
0\end{array}$ & $\begin{array}{l}\mathrm{GE} \\
\mathrm{GE} \\
\mathrm{GE} \\
\mathrm{GE} \\
\mathrm{GE} \\
\mathrm{GE} \\
\mathrm{GE} \\
\mathrm{GE} \\
\mathrm{GE} \\
\mathrm{GE} \\
\mathrm{GE} \\
\mathrm{GE} \\
\mathrm{GE}\end{array}$ & & & & & & & & $\begin{array}{l}\mu \mathrm{g} / \mathrm{L} \\
\mu \mathrm{g} / \mathrm{L} \\
\mu \mathrm{g} / \mathrm{L} \\
\mu \mathrm{g} / \mathrm{L} \\
\mu \mathrm{g} / \mathrm{L} \\
\mu \mathrm{g} / \mathrm{L} \\
\mu \mathrm{g} / \mathrm{L} \\
\mu \mathrm{g} / \mathrm{L} \\
\mu \mathrm{g} / \mathrm{L} \\
\mu \mathrm{g} / \mathrm{L} \\
\mu \mathrm{g} / \mathrm{L} \\
\mu \mathrm{g} / \mathrm{L} \\
\mu \mathrm{g} / \mathrm{L}\end{array}$ \\
\hline \multicolumn{16}{|c|}{ Water Quality Indicator Parameters } \\
\hline Parameter & 1095 & DF & Mod & ST & 보 & Flag & $\underline{\text { Lab }}$ & $\underline{2095}$ & $\underline{D F}$ & Mod & $\underline{\text { ST }}$ & H & Flag & $\underline{\text { Lab }}$ & Unit \\
\hline $\begin{array}{l}\text { Aluminum, total recoverable } \\
\text { Nitrate as nitrogen } \\
\text { pH }\end{array}$ & $\begin{array}{l}35 \\
5,000 \\
7.1\end{array}$ & $\begin{array}{l}1 \\
4 \\
1\end{array}$ & $\begin{array}{l}J \\
J 1\end{array}$ & & $\bullet$ & $\begin{array}{l}1 \\
1 \\
0\end{array}$ & $\begin{array}{l}\text { GE } \\
\text { GE } \\
\text { GE }\end{array}$ & $\begin{array}{l}5,700 \\
6.7\end{array}$ & 4 & J1 & 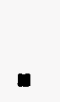 & • & $\begin{array}{l}1 \\
0\end{array}$ & $\begin{array}{l}\text { GE } \\
\text { GE }\end{array}$ & $\underset{p H}{\mu g / L}$ \\
\hline \multicolumn{16}{|c|}{ Common Laboratory Contaminants } \\
\hline Parameter & 1095 & $\underline{D F}$ & Mod & $\underline{\text { ST }}$ & $\underline{\mathrm{H}}$ & Flag & $\underline{\text { Lab }}$ & $\underline{2095}$ & $\underline{D F}$ & Mod & ST & $\underline{H}$ & Flag & $\underline{\text { Lab }}$ & $\underline{\text { Unit }}$ \\
\hline $\begin{array}{l}\text { Bis(2-ethyihexyl) phthalate } \\
\text { Dichloromethane }\end{array}$ & $\begin{array}{l}<17 \\
<1.7\end{array}$ & $\begin{array}{l}1 \\
1\end{array}$ & $v$ & & & $\begin{array}{l}0 \\
0\end{array}$ & $\begin{array}{l}\text { GE } \\
\text { GE }\end{array}$ & & & & & & & & $\begin{array}{l}\mu \mathrm{g} / \mathrm{L} \\
\mu \mathrm{g} / \mathrm{L}\end{array}$ \\
\hline
\end{tabular}

Note: See Appendix B for flagging criteria. Synchronous water levels are measured over a 3-5 day period. - = exceeded holding time.

m = exceeded groundwater protection standard. 
WELL FSB107C (cont.)

Radiological Indicator Parameters

\begin{tabular}{|c|c|c|c|c|c|c|c|c|c|c|c|c|c|c|c|}
\hline Parameter & 1095 & DF & Mod & $\underline{\text { ST }}$ & $\underline{H}$ & Flag & Lab & 2095 & DF & Mod & $\underline{\text { ST }}$ & $\underline{\mathrm{H}}$ & Fleg & $\underline{L a b}$ & Unit \\
\hline $\begin{array}{l}\text { Gross alpha } \\
\text { Nonvolatile beta }\end{array}$ & $\begin{array}{l}1.4 E+00 \\
4.1 E+01\end{array}$ & $\begin{array}{l}1 \\
1\end{array}$ & & & & $\begin{array}{l}0 \\
1\end{array}$ & $\begin{array}{l}\text { GP } \\
\text { GP }\end{array}$ & $\begin{array}{l}1.3 E+00 \\
3.9 E+01\end{array}$ & $\begin{array}{l}1 \\
1\end{array}$ & Ui & & & $\begin{array}{l}0 \\
1\end{array}$ & $\begin{array}{l}\text { GP } \\
\text { GP }\end{array}$ & $\begin{array}{l}\mathrm{pCi} / \mathrm{L} \\
\mathrm{pC} i / \mathrm{L}\end{array}$ \\
\hline
\end{tabular}

Measured Radionuclides

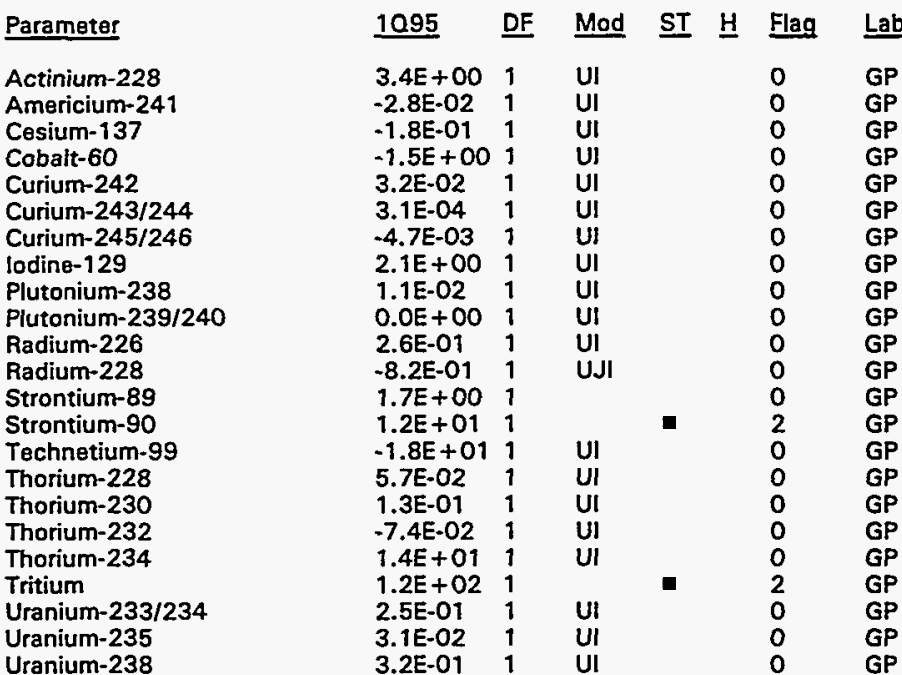

2095 DF Mod ST $\underline{H}$ Flag Lab Unit

Uranium-238

3.2E-01 1

Lab
GP
GP
GP
GP
GP
GP
GP
GP
GP
GP
GP
GP
GP
GP
GP
GP
GP
GP
GP
GP
GP
GP
GP

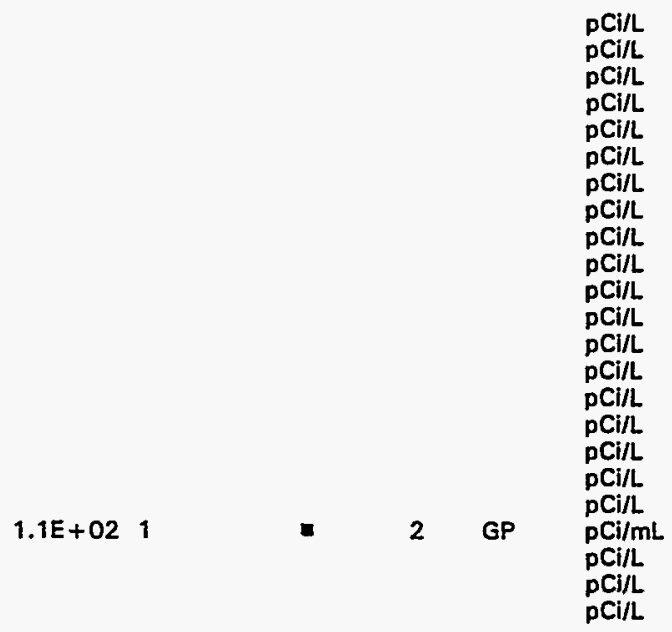

Calculated Radionuclides

\section{Parameter}

Americium-243

Nickel-63

Plutonium-241

Plutonium-242
1095

$9.4 \mathrm{E}-07$

$-1.5 \mathrm{E}+00$

$0.0 E+00$

$0.0 E+00$
DF Mod ST H Flag Lab

0

0

0
2095 DF Mod ST $\underline{\text { Flag Lab }} \underline{\underline{\text { Unit }}}$

Note: See Appendix B for flagging criteria. Synchronous water levels are measured over a 3-5 day period.

- = exceeded holding time.

- = exceeded groundwater protection standard. 
WELL FSB107D

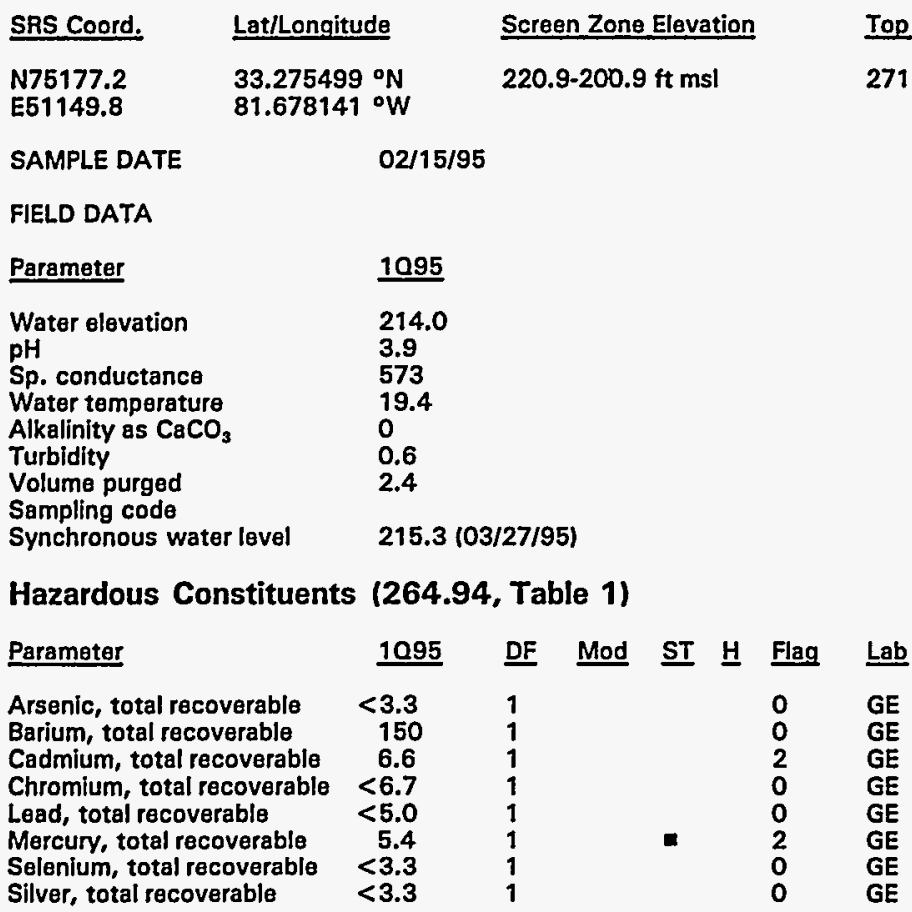

$\begin{array}{llll}\text { Top of Casing } & \text { Casing } & \text { Pump } & \text { Screen Zone } \\ 271 \mathrm{ft} \mathrm{msl} & 4^{\text {" PVC }} & \text { V } & \text { Water Table }\left(I B_{2}\right)\end{array}$

05/04/95

$\underline{2095}$

215.3

W

$214.8(06 / 15 / 95) \quad$ ft msl
Unit

tt msl

$\mathrm{pH}$

$\mu \mathrm{S} / \mathrm{cm}$

$\mathrm{mg} / \mathrm{L}$

NTU

well volumes

Hazardous Constituents (261 App. VIII/264 App. IX)

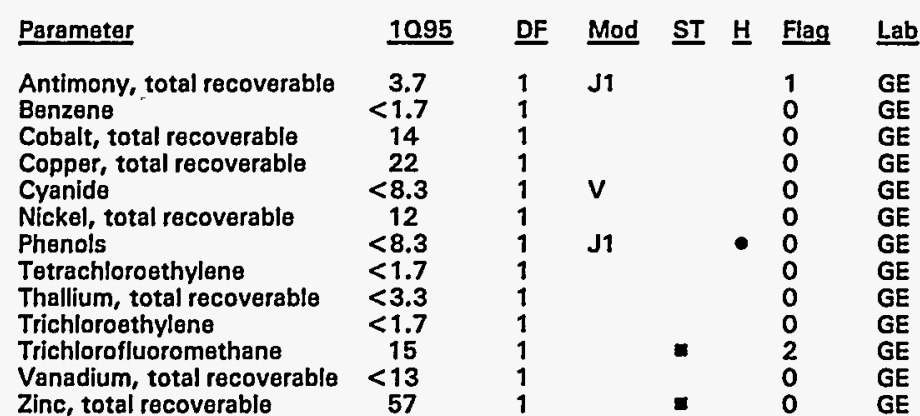

2095 DF Mod ST $\underline{H}$ Flag Lab Unit

Water Quality Indicator Parameters

\begin{tabular}{|c|c|c|c|c|c|c|c|c|c|c|c|c|c|c|c|}
\hline Parameter & 10.95 & $\underline{D F}$ & Mod & $\underline{\text { ST }}$ & $\underline{H}$ & Flag & $\underline{L a b}$ & 2095 & DF & Mod & $\underline{\text { ST }}$ & 브 & Flag & $\underline{\text { Lab }}$ & $\underline{\text { Unit }}$ \\
\hline $\begin{array}{l}\text { Aluminum, total recoverable } \\
\text { Nitrate as nitrogen } \\
\text { pH }\end{array}$ & $\begin{array}{l}15,000 \\
69,000 \\
3.6\end{array}$ & $\begin{array}{l}1 \\
40 \\
1\end{array}$ & J1 & : & $\bullet$ & $\begin{array}{l}2 \\
2 \\
1\end{array}$ & $\begin{array}{l}\text { GE } \\
\text { GE } \\
\text { GE }\end{array}$ & & & & & & & & $\underset{\mathrm{pH}}{\mu \mathrm{g} / \mathrm{L} / \mathrm{L}}$ \\
\hline \multicolumn{16}{|c|}{ Common Laboratory Contaminants } \\
\hline Parameter & 1095 & DF & Mod & $\underline{\text { ST }}$ & $\underline{H}$ & Flag & $\underline{\text { Lab }}$ & 2095 & DF & Mod & $\underline{\mathbf{S T}}$ & 브 & Flag & Lab & Unit \\
\hline $\begin{array}{l}\text { Bis(2-ethylhexyl) phthalate } \\
\text { Dichloromethane }\end{array}$ & $\begin{array}{l}<17 \\
<1.7\end{array}$ & $\begin{array}{l}1 \\
1\end{array}$ & & & & $\begin{array}{l}0 \\
0\end{array}$ & $\begin{array}{l}\mathrm{GE} \\
\mathrm{GE}\end{array}$ & & & & & & & & $\mu \mathrm{g} / \mathrm{L}$ \\
\hline
\end{tabular}

Note: See Appendix B for flagging criteria. Synchronous water levels are measured over a 3-5 day period.

- = exceeded holding time.

n = exceeded groundwater protection standard. 
WELL FSB107D (cont.)

Radiological Indicator Parameters

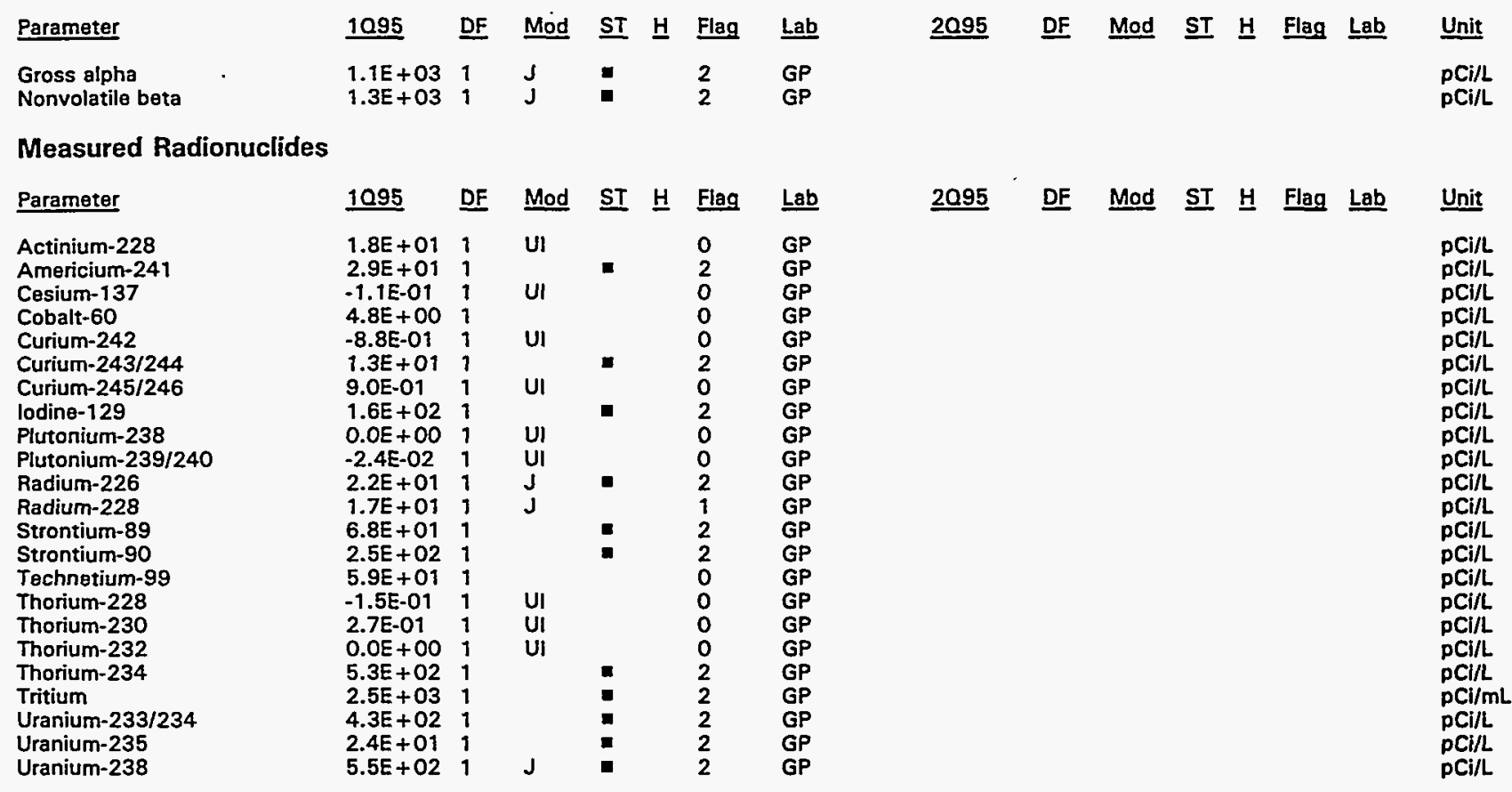

Calculated Radionuclides

1095

DF Mod ST $\underline{H}$ Flag Lab

$\underline{2095}$

DF Mod ST $\underline{\text { H Flag Lab }}$

Unit

Americium-243

Nickel-63

$3.8 \mathrm{E}-02$

$4.8 E+00$

$-9.5 \mathrm{E}-02$

Plutonium-241

$-9.5 \mathrm{E}-02$
$-4.8 \mathrm{E}-07$

0

$\mathrm{pCl} / \mathrm{L}$

$\mathrm{PCI} / \mathrm{L}$

Plutonium-242

$\mathrm{PCi} / \mathrm{L}$

Note: See Appendix B for flagging criteria. Synchronous water levels are measured over a 3-5 day period.

- = exceeded holding time.

m = exceeded groundwater protection standard. 


\section{WELL. FSB108D}

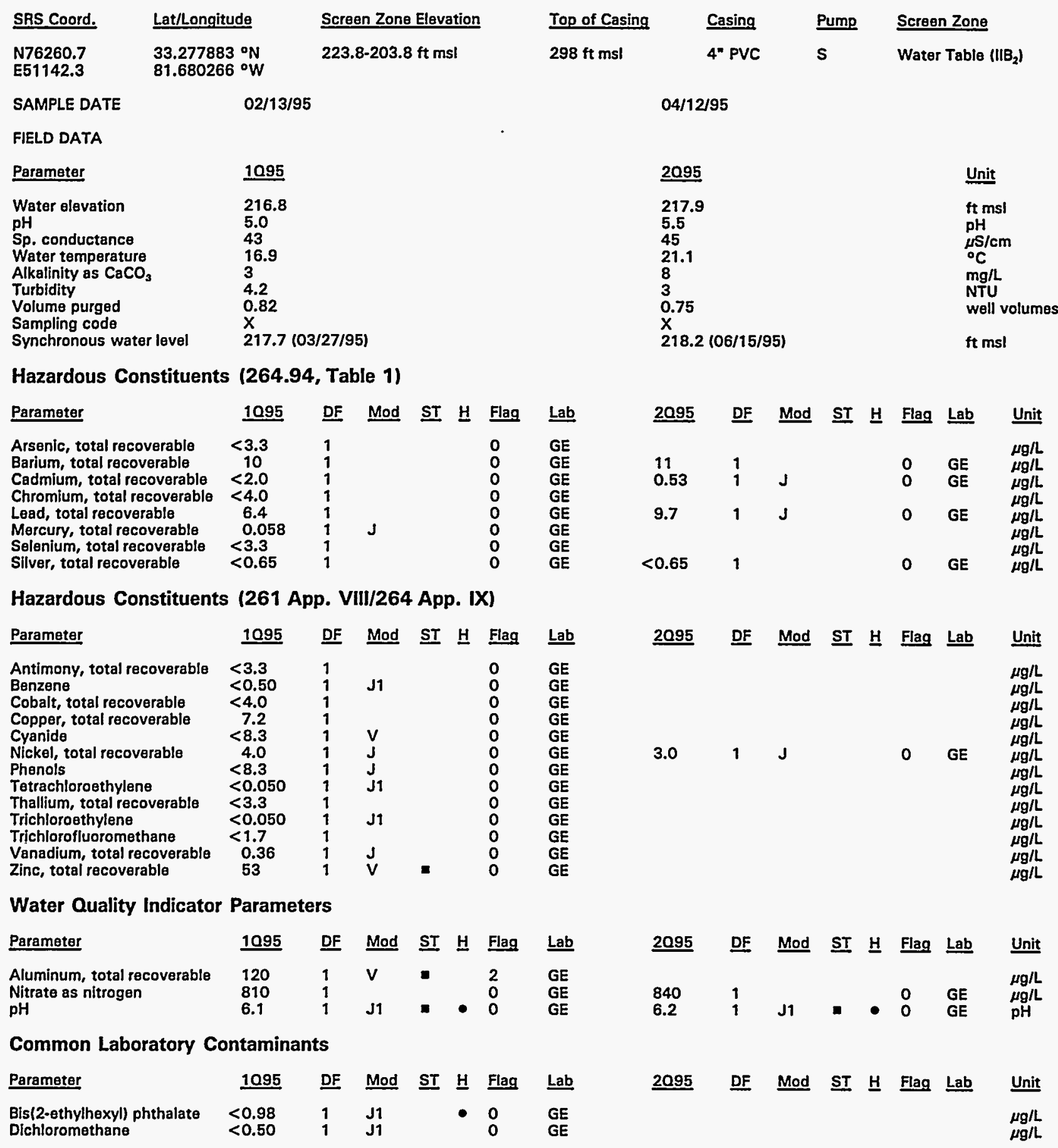

Note: See Appendix B for flagging criteria. Synchronous water levels are measured over a 3-5 day period.

- = exceeded holding time.

a = exceeded groundwater protection standard. 
WELL FSB108D (cont.)

Radiological Indicator Parameters

\begin{tabular}{|c|c|c|c|c|c|c|c|c|c|c|c|c|c|}
\hline Parameter & 1095 & DF & Mod & ST & $\underline{H}$ & Flag & $\underline{\text { Lab }}$ & $\underline{2095}$ & $\underline{\text { OF }}$ & Mod & ST $\underline{H}$ & Flag & Lab \\
\hline $\begin{array}{l}\text { Gross alpha } \\
\text { Nonvolatile beta }\end{array}$ & $\begin{array}{l}1.5 E+00 \\
1.5 E+00\end{array}$ & $\begin{array}{l}1 \\
1\end{array}$ & UI & & & $\begin{array}{l}0 \\
0\end{array}$ & $\begin{array}{l}\text { GP } \\
\text { GP }\end{array}$ & $\begin{array}{l}1.1 E+00 \\
1.7 E+00\end{array}$ & $\begin{array}{l}1 \\
1\end{array}$ & Ul & & $\begin{array}{l}0 \\
0\end{array}$ & $\begin{array}{l}\text { GP } \\
\text { GP }\end{array}$ \\
\hline
\end{tabular}

Measured Radionuclides

$\begin{array}{llllllll}\text { Parameter } & 1095 & \text { DF } & \text { Mod } & \text { ST } & \text { H } & \text { Flag } & \text { Lab } \\ \text { Actinium-228 } & -3.9 E-01 & 1 & \text { UI } & & & & \text { GP } \\ \text { Americium-241 } & -6.2 E-02 & 1 & \text { UI } & & 0 & \text { GP } \\ \text { Cesium-137 } & -2.7 E-01 & 1 & \text { UI } & & 0 & \text { GP } \\ \text { Cobalt-60 } & 6.2 E+00 & 1 & \text { UI } & & 0 & \text { GP } \\ \text { Curium-242 } & 8.5 E-02 & 1 & \text { UI } & & 0 & \text { GP } \\ \text { Curium-243/244 } & -4.8 E-02 & 1 & \text { UI } & & 0 & \text { GP } \\ \text { Curium-245/246 } & 0.0 E+00 & 1 & \text { UI } & & 0 & \text { GP } \\ \text { lodine-129 } & -3.8 E-01 & 1 & \text { UI } & & 0 & \text { GP } \\ \text { Plutonium-238 } & 1.9 E-02 & 1 & \text { UI } & & 0 & \text { GP } \\ \text { Plutonium-239/240 } & -1.3 E-02 & 1 & \text { UI } & & 0 & \text { GP } \\ \text { Radium-226 } & 1.5 E+00 & 1 & & & 0 & \text { GP } \\ \text { Radium-228 } & 4.8 E+00 & 1 & & & 0 & \text { GP } \\ \text { Strontium-89 } & -1.3 E+00 & 1 & \text { UI } & & 0 & \text { GP } \\ \text { Strontium-90 } & -6.5 E-01 & 1 & \text { UI } & & 0 & \text { GP } \\ \text { Tochnetium-99 } & -1.0 E+01 & 1 & \text { UI } & & 0 & \text { GP } \\ \text { Thorium-228 } & 7.8 E-02 & 1 & \text { UI } & & 0 & \text { GP } \\ \text { Thorium-230 } & 6.9 E-01 & 1 & \text { UI } & & 0 & \text { GP } \\ \text { Thorium-232 } & 0.0 E+00 & 1 & \text { UI } & & 0 & \text { GP } \\ \text { Thorium-234 } & 9.1 E+01 & 1 & \text { UI } & & 0 & \text { GP } \\ \text { Tritium } & 1.2 E+01 & 1 & & & 1 & \text { GP } \\ \text { Uranium-233/234 } & 8.3 E-02 & 1 & \text { UI } & & 0 & \text { GP } \\ \text { Uranium-235 } & 0.0 E+00 & 1 & \text { UI } & & 0 & \text { GP } \\ \text { Uranium-238 } & 4.2 E-02 & 1 & \text { UI } & & 0 & \text { GP }\end{array}$

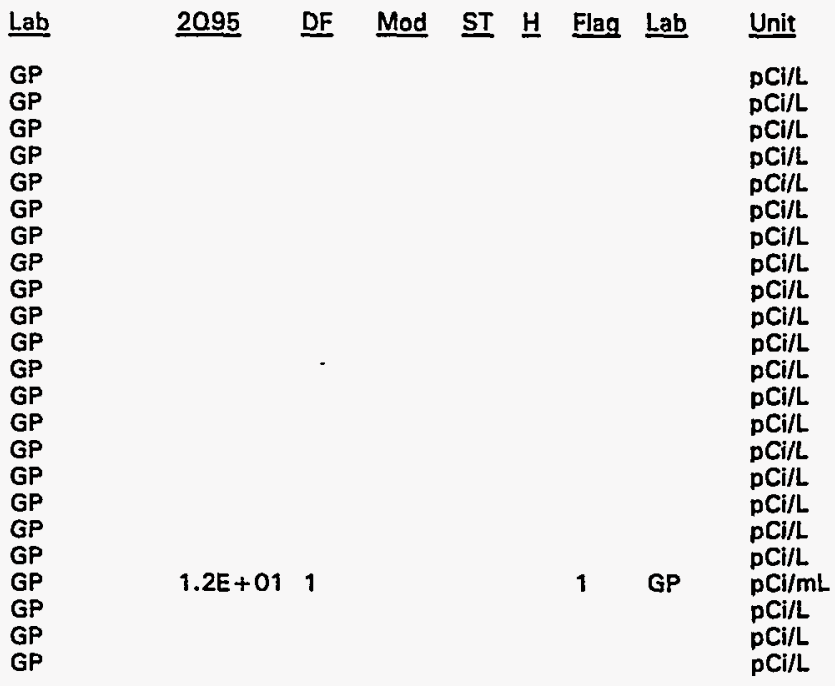

Calculated Radionuclides

Parameter

Americium-243

Nickel-63

Plutonium-241

Plutonium-242
1095

$-1.4 E-04$

$6.2 E+00$

$-5.1 \mathrm{E}-02$

$-2.5 E-07$
DF Mod ST $\underline{H}$ Flag Lab

0

0

2095 DF Mod ST H Flag Lab Unit

$\mathrm{pCi} / \mathrm{L}$

$\mathrm{pCi} / \mathrm{L}$

$\mathrm{pCi} / \mathrm{L}$

pCi/L

Note: See Appendix B for flagging criteria. Synchronous water levels are measured over a 3-5 day period.

- = exceeded holding time.

n = exceeded groundwater protection standard. 
WELL FSB109D

\begin{tabular}{|c|c|c|}
\hline SRS Coord. & Lat/Longitude & Screen Zone Elevation \\
\hline $\begin{array}{l}\text { N75855.9 } \\
\text { E50488.6 }\end{array}$ & $\begin{array}{l}33.2759211^{\circ} \mathrm{N} \\
81.681200^{\circ} \mathrm{V}\end{array}$ & $225.8-205.8 \mathrm{ft} \mathrm{msl}$ \\
\hline SAMPLE DATE & \multicolumn{2}{|c|}{$02 / 13 / 95$} \\
\hline \multicolumn{3}{|l|}{ FIELD DATA } \\
\hline Parameter & \multicolumn{2}{|c|}{1095} \\
\hline $\begin{array}{l}\text { Water elevation } \\
\text { pH } \\
\text { Sp. conductance } \\
\text { Water temperature } \\
\text { Alkalinity as CaCO, } \\
\text { Turbidity } \\
\text { Volume purged } \\
\text { Sampling code } \\
\text { Synchronous water }\end{array}$ & \multicolumn{2}{|c|}{$\begin{array}{l}212.9 \\
5.2 \\
38 \\
16.6 \\
8 \\
25.9 \\
0.86 \\
X \\
213.8(03 / 27 / 95)\end{array}$} \\
\hline
\end{tabular}

$\begin{array}{lrlll}\frac{\text { Top of Casing }}{293.1 \mathrm{fmsl}} & \frac{\text { Casing }}{4^{n} \text { PVC }} & \frac{\text { Pump }}{\mathrm{S}} & \frac{\text { Screen Zone }}{\text { Water Table (IIB })} \\ & 04 / 05 / 95 & & \end{array}$

Hazardous Constituents (264.94, Table 1)

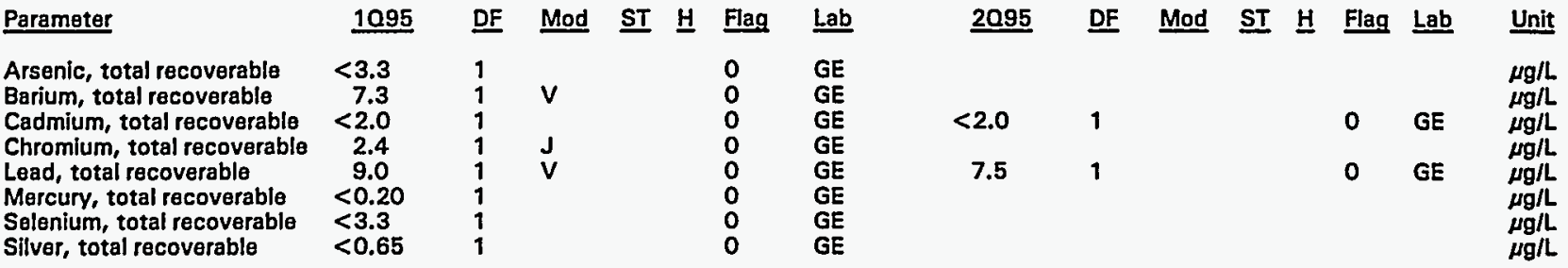

Hazardous Constituents (261 App. VIII/264 App. (X)

\begin{tabular}{|c|c|c|c|c|c|c|c|c|c|c|c|c|c|c|c|}
\hline Parameter & 1095 & DF & Mod & $\underline{\mathbf{S T}}$ & $\underline{H}$ & Flag & $\underline{\text { Lab }}$ & $\underline{2095}$ & $\underline{D F}$ & Mod & $\underline{\text { ST }}$ & $\underline{\text { H}}$ & Flag & $\underline{\text { Lab }}$ & Unit \\
\hline $\begin{array}{l}\text { Antimony, total recoverable } \\
\text { Benzene } \\
\text { Cobalt, total recoverable } \\
\text { Copper, total recoverable } \\
\text { Cyanide } \\
\text { Nickel, total recoverable } \\
\text { Phenols } \\
\text { Tetrachloroethylene } \\
\text { Thallium, total recoverable } \\
\text { Trichloroethylene } \\
\text { Trichlorofluoromethane } \\
\text { Vanadium, total recoverable } \\
\text { Zinc, total recoverable }\end{array}$ & $\begin{array}{l}<3.3 \\
<0.50 \\
0.60 \\
11 \\
<8.3 \\
4.0 \\
<8.3 \\
<0.050 \\
<3.3 \\
<0.050 \\
<1.7 \\
0.54 \\
89\end{array}$ & $\begin{array}{l}1 \\
1 \\
1 \\
1 \\
1 \\
1 \\
1 \\
1 \\
1 \\
1 \\
1 \\
1 \\
1\end{array}$ & $\begin{array}{l}\text { J1 } \\
\text { J } \\
J \\
\text { J3 } \\
\mathrm{J} 1 \\
\mathrm{~J} 1 \\
\mathrm{~J} \\
\mathrm{~V}\end{array}$ & - & & $\begin{array}{l}0 \\
0 \\
0 \\
0 \\
0 \\
0 \\
0 \\
0 \\
0 \\
0 \\
0 \\
0 \\
0\end{array}$ & $\begin{array}{l}\mathrm{GE} \\
\mathrm{GE} \\
\mathrm{GE} \\
\mathrm{GE} \\
\mathrm{GE} \\
\mathrm{GE} \\
\mathrm{GE} \\
\mathrm{GE} \\
\mathrm{GE} \\
\mathrm{GE} \\
\mathrm{GE} \\
\mathrm{GE} \\
\mathrm{GE}\end{array}$ & & & & & & & & $\begin{array}{l}\mu \mathrm{g} / \mathrm{L} \\
\mu \mathrm{g} / \mathrm{L} \\
\mu \mathrm{g} / \mathrm{L} \\
\mu \mathrm{g} / \mathrm{L} \\
\mu \mathrm{g} / \mathrm{L} \\
\mu \mathrm{g} / \mathrm{L} \\
\mu \mathrm{g} / \mathrm{L} \\
\mu \mathrm{g} / \mathrm{L} \\
\mu \mathrm{g} / \mathrm{L} \\
\mu \mathrm{g} / \mathrm{L} \\
\mu \mathrm{g} / \mathrm{L} \\
\mu \mathrm{g} / \mathrm{L} \\
\mu g / \mathrm{L}\end{array}$ \\
\hline \multicolumn{16}{|c|}{ Water Quality Indicator Parameters } \\
\hline Parameter & $\underline{1095}$ & $\underline{\mathrm{DF}}$ & Mod & $\underline{\text { ST }}$ & $\underline{H}$ & Flag & $\underline{\text { Lab }}$ & $\underline{2095}$ & $\underline{\mathrm{DF}}$ & Mad & $\underline{\mathbf{S T}}$ & $\underline{H}$ & Flag & $\underline{\text { Lab }}$ & Unit \\
\hline $\begin{array}{l}\text { Aluminum, total recoverable } \\
\text { Nitrate as nitrogen } \\
\mathrm{pH}\end{array}$ & $\begin{array}{l}100 \\
890 \\
6.3\end{array}$ & $\begin{array}{l}1 \\
1 \\
1\end{array}$ & $\begin{array}{l}v \\
\text { J1 }\end{array}$ & - & $\bullet$ & $\begin{array}{l}2 \\
0 \\
0\end{array}$ & $\begin{array}{l}\text { GE } \\
\text { GE } \\
\text { GE }\end{array}$ & $\begin{array}{l}950 \\
6.2\end{array}$ & $\begin{array}{l}1 \\
1\end{array}$ & J1 & - & $\bullet$ & $\begin{array}{l}0 \\
0\end{array}$ & $\begin{array}{l}\text { GE } \\
\text { GE }\end{array}$ & $\underset{\mathrm{pH}}{\mu \mathrm{g} / \mathrm{L}}$ \\
\hline \multicolumn{16}{|c|}{ Common Laboratory Contaminants } \\
\hline Parameter & $\underline{1095}$ & $\underline{\text { DF }}$ & Mod & $\underline{\mathbf{S T}}$ & $\underline{\mathrm{H}}$ & Flag & $\underline{\text { Lab }}$ & $\underline{2095}$ & $\underline{D F}$ & Mod & $\underline{\mathbf{S T}}$ & 브 & Flag & $\underline{\text { Lab }}$ & Unit \\
\hline $\begin{array}{l}\text { Bis(2-ethylhexyl) phthalate } \\
\text { Dichloromethane }\end{array}$ & $\begin{array}{l}<1.0 \\
<0.50\end{array}$ & 1 & $\begin{array}{l}\text { J1 } \\
\text { J1 }\end{array}$ & & $\bullet$ & $\begin{array}{l}0 \\
0\end{array}$ & $\begin{array}{l}\mathrm{GE} \\
\mathrm{GE}\end{array}$ & & & & & & & & $\mu$ \\
\hline
\end{tabular}

Note: See Appendix B for flagging criteria. Synchronous water levels are measured over a 3-5 day period.

- = exceeded holding time.

- = exceeded groundwater protection standard. 
WELL FSB109D (cont.)

Radiological Indicator Parameters

\begin{tabular}{|c|c|c|c|c|c|c|c|c|c|c|c|c|c|c|c|}
\hline Parameter & 1095 & DF & Mód & $\underline{\text { ST }}$ & $\underline{H}$ & Flag & $\underline{\text { Lab }}$ & 2095 & DF & Mod & $\underline{\text { ST }}$ & 브 & Flag & Lab & Unit \\
\hline $\begin{array}{l}\text { Gross alpha } \\
\text { Nonvolatile beta }\end{array}$ & $\begin{array}{l}1.7 E+00 \\
1.1 E+00\end{array}$ & $\begin{array}{l}1 \\
1\end{array}$ & UI & & & $\begin{array}{l}0 \\
0\end{array}$ & $\begin{array}{l}\text { GP } \\
\text { GP }\end{array}$ & $\begin{array}{l}\text { 5.7E-01 } \\
2.3 E+00\end{array}$ & $\begin{array}{l}1 \\
1\end{array}$ & UJI & & & $\begin{array}{l}0 \\
0\end{array}$ & $\begin{array}{l}\text { GP } \\
\text { GP }\end{array}$ & $\begin{array}{l}\mathrm{pCi} / \mathrm{L} \\
\mathrm{pCi} / L\end{array}$ \\
\hline
\end{tabular}

Measured Radionuclides

\begin{tabular}{|c|c|c|c|c|c|c|c|c|c|c|c|c|c|c|c|}
\hline Parameter & 1095 & DF & Mod & $\underline{S T}$ & 브 & Flag & $\underline{\mathrm{Lab}}$ & 2095 & DF & Mod & $\underline{\mathrm{ST}}$ & $\underline{H}$ & Flag & Lab & Unit \\
\hline $\begin{array}{l}\text { Actinium-228 } \\
\text { Americium-241 } \\
\text { Cesium-137 } \\
\text { Cobalt-60 } \\
\text { Curium-242 } \\
\text { Curium-243/244 } \\
\text { Curium-245/246 } \\
\text { lodine-129 } \\
\text { Plutonium-238 } \\
\text { Plutonium-239/240 } \\
\text { Radium-226 } \\
\text { Radium-228 } \\
\text { Strontium-89 } \\
\text { Strontium-90 } \\
\text { Technetium-99 } \\
\text { Thorium-228 } \\
\text { Thorium-230 } \\
\text { Thorium-232 } \\
\text { Thorium-234 } \\
\text { Tritium } \\
\text { Uranium-233/234 } \\
\text { Uranium-235 } \\
\text { Uranium-238 }\end{array}$ & $\begin{array}{l}7.7 E+00 \\
2.8 E-01 \\
7.4 E-01 \\
-2.0 E-01 \\
-1.9 E-02 \\
1.9 E-01 \\
4.3 E-02 \\
-1.5 E-01 \\
0.0 E+00 \\
3.3 E-02 \\
3.2 E-01 \\
1.2 E+00 \\
-1.1 E+00 \\
-4.4 E-01 \\
-4.4 E+00 \\
2.1 E-01 \\
1.8 E-01 \\
-2.4 E-02 \\
2.3 E+02 \\
2.4 E+01 \\
3.7 E-02 \\
-2.7 E-02 \\
-2.0 E-02\end{array}$ & $\begin{array}{l}1 \\
1 \\
1 \\
1 \\
1 \\
1 \\
1 \\
1 \\
1 \\
1 \\
1 \\
1 \\
1 \\
1 \\
1 \\
1 \\
1 \\
1 \\
1 \\
1 \\
1 \\
1 \\
1\end{array}$ & $\begin{array}{l}U I \\
U I \\
U I \\
U I \\
U I \\
U I \\
U I \\
U I \\
U I \\
U I \\
U I \\
U I \\
U I \\
\text { UI } \\
\text { UI } \\
\text { UI } \\
\text { UI } \\
\text { UI } \\
\text { UI } \\
\text { UI }\end{array}$ & 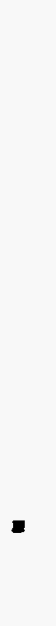 & & $\begin{array}{l}0 \\
0 \\
0 \\
0 \\
0 \\
0 \\
0 \\
0 \\
0 \\
0 \\
0 \\
0 \\
0 \\
0 \\
0 \\
0 \\
0 \\
0 \\
1 \\
2 \\
0 \\
0 \\
0\end{array}$ & $\begin{array}{l}\text { GP } \\
\text { GP } \\
\text { GP } \\
\text { GP } \\
\text { GP } \\
\text { GP } \\
\text { GP } \\
\text { GP } \\
\text { GP } \\
\text { GP } \\
\text { GP } \\
\text { GP } \\
\text { GP } \\
\text { GP } \\
\text { GP } \\
\text { GP } \\
\text { GP } \\
\text { GP } \\
\text { GP } \\
\text { GP } \\
\text { GP } \\
\text { GP } \\
\text { GP }\end{array}$ & $2.7 E+01$ & 1 & & w & & 2 & GP & $\begin{array}{l}\text { pCi/L } \\
\text { pCi/L } \\
\text { pCi/L } \\
\text { pCi/L } \\
\text { pCi/L } \\
\text { pCi/L } \\
\text { pCi/L } \\
\text { pCi/L } \\
\text { pCi/L } \\
\text { pCi/L } \\
\text { pCi/L } \\
\text { pCi/L } \\
\text { pCi/L } \\
\text { pCi/L } \\
\text { pCi/L } \\
\text { pCi/L } \\
\text { pCi/L } \\
\text { pCi/L } \\
\text { pCi/L } \\
\text { pCi/m } \\
\text { pCi/L } \\
\text { pCi/L } \\
\text { pCi/L }\end{array}$ \\
\hline \multicolumn{16}{|c|}{ Calculated Radionuclides } \\
\hline Parameter & 1095 & $\underline{D F}$ & Mod & ST & $\underline{H}$ & Flag & $\underline{\mathrm{Lab}}$ & 2095 & $\underline{D F}$ & Mod & ST & $\underline{H}$ & Flag & Lab & Unit \\
\hline $\begin{array}{l}\text { Americium-243 } \\
\text { Nickel-63 } \\
\text { Plutonium-241 } \\
\text { Plutonium-242 }\end{array}$ & $\begin{array}{l}5.7 E-04 \\
-2.0 E-01 \\
1.3 E-01 \\
6.6 E-07\end{array}$ & & & & & $\begin{array}{l}0 \\
0 \\
0 \\
0\end{array}$ & & & & & & & & & $\begin{array}{l}\mathrm{pCi} / \mathrm{L} \\
\mathrm{pCi} / \mathrm{L} \\
\mathrm{pCi} / \mathrm{L} \\
\mathrm{pCi} / \mathrm{L}\end{array}$ \\
\hline
\end{tabular}

Note: See Appendix B for flagging criteria. Synchronous water levels are measured over a 3-5 day period.

- = exceeded holding time.

- =xceeded groundwater protection standard. 


\section{WELL FSB110C}

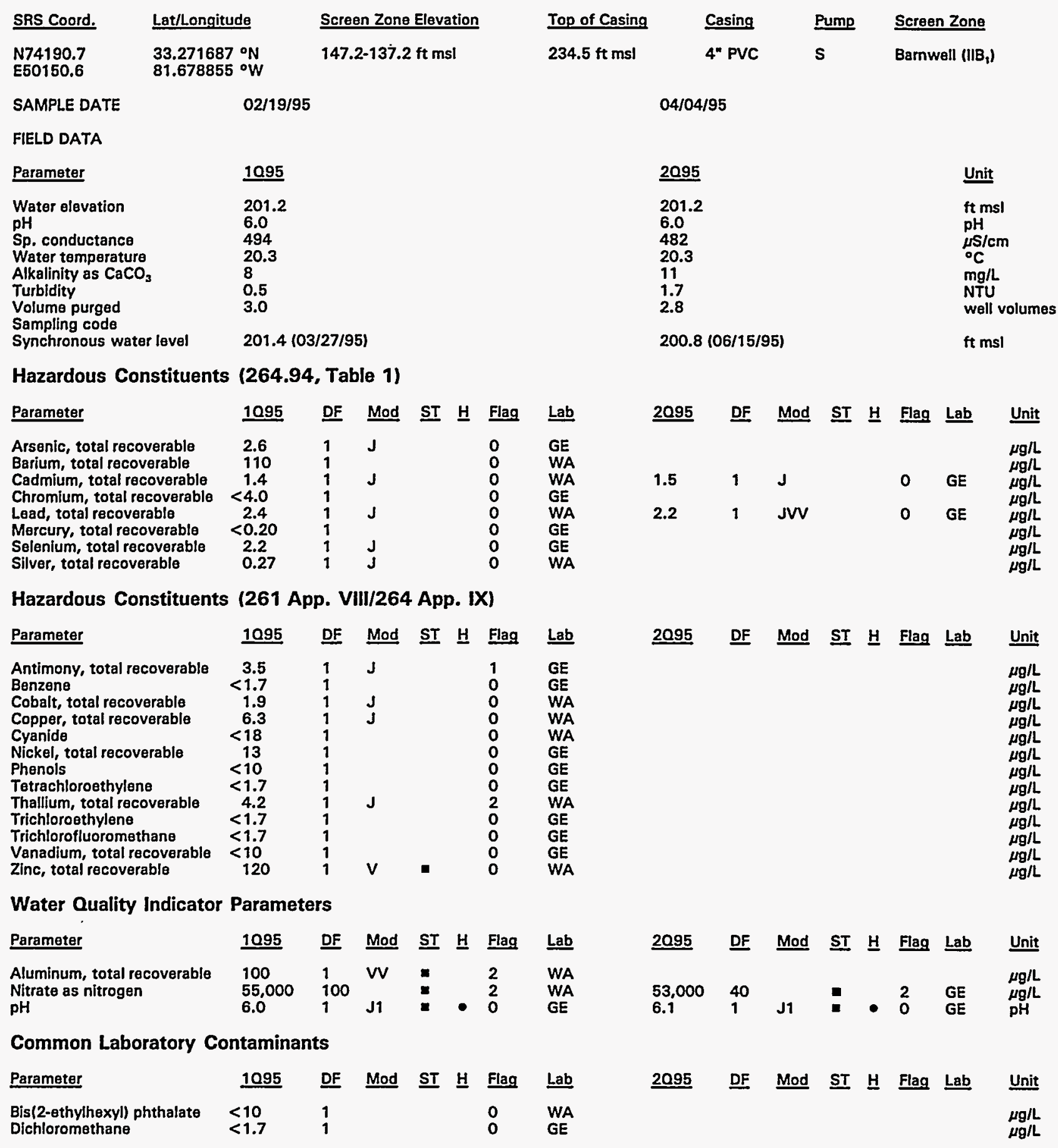

Note: See Appendix B for flagging criteria. Synchronous water levels are measured over a 3-5 day period.

$-=$ exceeded holding time.

- = exceeded groundwater protection standard. 
WELL FSB1 10C (cont.)

Radiological Indicator Parameters

\begin{tabular}{|c|c|c|c|c|c|c|c|c|c|c|c|c|c|c|c|}
\hline Parameter & 1095 & DF & Mod & $\underline{\text { ST }}$ & H & Flag & $\underline{\text { Lab }}$ & $\underline{2095}$ & $\underline{D F}$ & $\underline{\text { Mod }}$ & $\underline{\mathbf{S T}}$ & $\underline{H}$ & Flag & $\underline{\text { Lab }}$ & Unit \\
\hline $\begin{array}{l}\text { Gross alpha } \\
\text { Nonvolatile beta }\end{array}$ & $\begin{array}{l}8.0 E+00 \\
6.7 E+01\end{array}$ & $\begin{array}{l}1 \\
1\end{array}$ & & - & & $\begin{array}{l}1 \\
2\end{array}$ & $\begin{array}{l}\text { GP } \\
\text { GP }\end{array}$ & $\begin{array}{l}2.4 E+00 \\
5.6 E+01\end{array}$ & $\begin{array}{l}1 \\
1\end{array}$ & & - & & $\begin{array}{l}0 \\
2\end{array}$ & $\begin{array}{l}\text { GP } \\
\text { GP }\end{array}$ & $\begin{array}{l}\mathrm{pCi} / \mathrm{L} \\
\mathrm{pCi} / \mathrm{L}\end{array}$ \\
\hline \multicolumn{16}{|c|}{ Measured Radionuclides } \\
\hline Paramater & 1095 & $\underline{D F}$ & Mod & $\underline{\mathbf{S T}}$ & 브 & Flag & $\underline{\text { Lab }}$ & $\underline{2095}$ & $\underline{D F}$ & Mod & $\underline{\text { ST }}$ & $\underline{H}$ & Flag & $\underline{\text { Lab }}$ & Unit \\
\hline $\begin{array}{l}\text { Actinium-228 } \\
\text { Americium-241 } \\
\text { Cesium-137 } \\
\text { Cobalt-60 } \\
\text { Curium-242 } \\
\text { Curium-243/244 } \\
\text { Curium-245/246 } \\
\text { lodine-129 } \\
\text { Plutonium-238 } \\
\text { Plutonium-239/240 } \\
\text { Radium-226 } \\
\text { Radium-228 } \\
\text { Strontium-89 } \\
\text { Strontium-90 } \\
\text { Technetium-99 } \\
\text { Thorium-228 } \\
\text { Thorium-230 } \\
\text { Thorium-232 } \\
\text { Thorium-234 } \\
\text { Tritium } \\
\text { Uranium-233/234 } \\
\text { Uranium-235 } \\
\text { Uranium-238 }\end{array}$ & $\begin{array}{l}-1.5 E-01 \\
7.6 E-04 \\
1.3 E-01 \\
-4.7 E-01 \\
2.5 E-02 \\
1.4 E-02 \\
0.0 E+00 \\
2.5 E+01 \\
0.0 E+00 \\
1.9 E-02 \\
1.4 E+00 \\
3.5 E+00 \\
-5.0 E-01 \\
1.2 E+01 \\
1.2 E+02 \\
0.0 E+00 \\
1.4 E-01 \\
0.0 E+00 \\
9.8 E+01 \\
1.8 E+03 \\
5.4 E-02 \\
0.0 E+00 \\
0.0 E+00\end{array}$ & $\begin{array}{l}1 \\
1 \\
1 \\
1 \\
1 \\
1 \\
1 \\
1 \\
1 \\
1 \\
1 \\
1 \\
1 \\
1 \\
1 \\
1 \\
1 \\
1 \\
1 \\
1 \\
1 \\
1 \\
1\end{array}$ & $\begin{array}{l}\text { UI } \\
\text { UI } \\
\text { UI } \\
\text { UI } \\
\text { UI } \\
\text { UI } \\
\text { UI } \\
\text { UI } \\
\text { UI } \\
\text { J } \\
\text { UI }\end{array}$ & a & & $\begin{array}{l}0 \\
0 \\
0 \\
0 \\
0 \\
0 \\
0 \\
2 \\
0 \\
0 \\
0 \\
0 \\
0 \\
2 \\
0 \\
0 \\
0 \\
0 \\
0 \\
2 \\
0 \\
0 \\
0\end{array}$ & $\begin{array}{l}T M \\
G P \\
T M \\
G P \\
G P \\
G P \\
G P \\
G P \\
T M \\
G P \\
T M \\
T M \\
G P \\
G P \\
T M \\
G P \\
T M \\
G P \\
T M \\
T M \\
G P \\
G P \\
T M\end{array}$ & $1.4 E+03$ & 1 & & - & & 2 & GP & 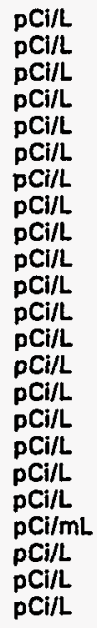 \\
\hline \multicolumn{16}{|c|}{ Calculated Radionuclides } \\
\hline Parameter & 1095 & $\underline{D F}$ & Mod & $\underline{\mathbf{S T}}$ & $\underline{H}$ & Flag & $\underline{\text { Lab }}$ & $\underline{2095}$ & $\underline{\mathrm{DF}}$ & Mod & $\underline{\mathbf{S T}}$ & $\underline{H}$ & Flag & $\underline{\text { Lab }}$ & $\underline{\text { Unit }}$ \\
\hline $\begin{array}{l}\text { Americium-243 } \\
\text { Nickel-63 } \\
\text { Plutonium-241 } \\
\text { Plutonium-242 }\end{array}$ & $\begin{array}{l}4.2 E-05 \\
-4.7 E-01 \\
7.5 E-02 \\
3.7 E-07\end{array}$ & & & & & $\begin{array}{l}0 \\
0 \\
0 \\
0\end{array}$ & & & & & & & & & $\begin{array}{l}p C i / L \\
p C i / L \\
p C i / L \\
p C i / L\end{array}$ \\
\hline
\end{tabular}

Note: See Appendix B for flagging criteria. Synchronous water levels are measured over a 3-5 day period.

- = exceeded holding time.

- = exceeded groundwater protection standard. 
WELL FSB110D

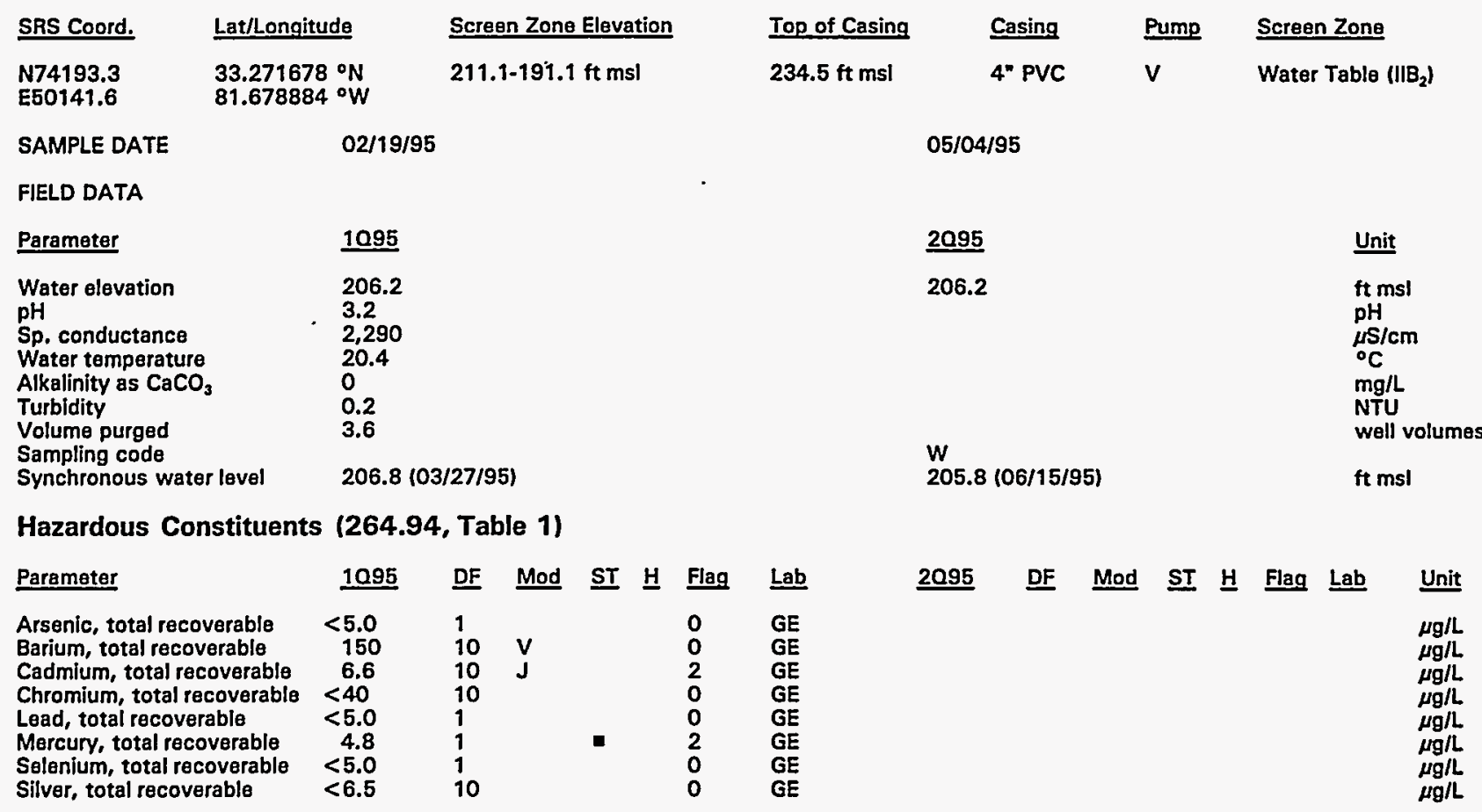

Hazardous Constituents (261 App. VIII/264 App. IX)

\begin{tabular}{|c|c|c|c|c|c|c|c|c|c|c|c|c|c|c|c|}
\hline Parameter & 1095 & $\underline{\mathrm{DF}}$ & Mod & $\underline{\underline{S T}}$ & $\underline{H}$ & Flag & $\underline{\text { Lab }}$ & $\underline{2095}$ & $\underline{\text { DF }}$ & Mod & $\underline{\text { ST }}$ & 브 & Flag & $\underline{\text { Lab }}$ & $\underline{\text { Unit }}$ \\
\hline $\begin{array}{l}\text { Antimony, total recoverable } \\
\text { Benzene } \\
\text { Cobalt, total recoverable } \\
\text { Copper, total recoverable } \\
\text { Cyanide } \\
\text { Nickel, total recoverable } \\
\text { Phenols } \\
\text { Tetrachloroethylene } \\
\text { Thallium, total recoverable } \\
\text { Trichloroethylene } \\
\text { Trichlorofluoromethane } \\
\text { Vanadium, total recoverable } \\
\text { Zinc, total recoverable }\end{array}$ & $\begin{array}{r}3.5 \\
<1.7 \\
25 \\
64 \\
8.8 \\
66 \\
<10 \\
<1.7 \\
0.75 \\
<1.7 \\
<1.7 \\
<100 \\
230\end{array}$ & $\begin{array}{l}1 \\
1 \\
10 \\
10 \\
1 \\
10 \\
1 \\
1 \\
1 \\
1 \\
1 \\
10 \\
10\end{array}$ & $\begin{array}{l}\text { J1 } \\
\text { J } \\
\text { JVVY } \\
\text { J } \\
\text { J }\end{array}$ & ! & & $\begin{array}{l}1 \\
0 \\
1 \\
0 \\
0 \\
1 \\
0 \\
0 \\
0 \\
0 \\
0 \\
0 \\
0\end{array}$ & $\begin{array}{l}\mathrm{GE} \\
\mathrm{GE} \\
\mathrm{GE} \\
\mathrm{GE} \\
\mathrm{GE} \\
\mathrm{GE} \\
\mathrm{GE} \\
\mathrm{GE} \\
\mathrm{GE} \\
\mathrm{GE} \\
\mathrm{GE} \\
\mathrm{GE} \\
\mathrm{GE}\end{array}$ & & & & & & & & $\begin{array}{l}\mu \mathrm{g} / \mathrm{L} \\
\mu g / \mathrm{L} \\
\mu \mathrm{g} / \mathrm{L} \\
\mu \mathrm{g} / \mathrm{L} \\
\mu \mathrm{g} / \mathrm{L} \\
\mu \mathrm{g} / \mathrm{L} \\
\mu \mathrm{g} / \mathrm{L} \\
\mu \mathrm{g} / \mathrm{L} \\
\mu \mathrm{g} / \mathrm{L} \\
\mu \mathrm{g} / \mathrm{L} \\
\mu \mathrm{g} / \mathrm{L} \\
\mu \mathrm{g} / \mathrm{L} \\
\mu \mathrm{g} / \mathrm{L}\end{array}$ \\
\hline \multicolumn{16}{|c|}{ Water Quality Indicator Parameters } \\
\hline Parameter & 1095 & $\underline{D F}$ & Mod & $\underline{\text { ST }}$ & $\underline{H}$ & Flag & Lab & $\underline{2095}$ & $\underline{\text { DF }}$ & Mod & $\underline{\mathbf{S T}}$ & $\underline{H}$ & Flag & $\underline{\text { Lab }}$ & $\underline{\text { Unit }}$ \\
\hline $\begin{array}{l}\text { Aluminum, total recoverable } \\
\text { Nitrate as nitrogen } \\
\text { pH }\end{array}$ & $\begin{array}{l}96,000 \\
290,000 \\
3.2\end{array}$ & $\begin{array}{l}10 \\
160 \\
1\end{array}$ & $\begin{array}{l}v \\
J 1\end{array}$ & $=$ & - & $\begin{array}{l}2 \\
2 \\
1\end{array}$ & $\begin{array}{l}\text { GE } \\
\text { GE } \\
\text { GE }\end{array}$ & & & & & & & & $\begin{array}{l}\mu \mathrm{g} / \mathrm{L} \\
\mu \mathrm{g} / \mathrm{L} \\
\mathrm{pH}\end{array}$ \\
\hline \multicolumn{16}{|c|}{ Common Laboratory Contaminants } \\
\hline Parameter & 1095 & $\underline{D F}$ & Mod & $\underline{\text { ST }}$ & $\underline{H}$ & Flag & Lab & $\underline{2095}$ & $\underline{D F}$ & Mod & $\underline{\text { ST }}$ & $\underline{H}$ & Flag & $\underline{\underline{L a b}}$ & $\underline{\text { Unit }}$ \\
\hline $\begin{array}{l}\text { Bis(2-ethylhexyi) phthalate } \\
\text { Dichloromethane }\end{array}$ & $\begin{array}{l}<17 \\
<1.7\end{array}$ & 1 & & & & $\begin{array}{l}0 \\
0\end{array}$ & $\begin{array}{l}\mathrm{GE} \\
\mathrm{GE}\end{array}$ & & & & & & & & $\mu \mathrm{g} / \mathrm{L}$ \\
\hline
\end{tabular}

Note: See Appendix B for flagging criteria. Synchronous water levels are measured over a 3-5 day period. - = exceeded holding time.

= exceeded groundwater protection standard. 
WELL FSB1 10D (cont.)

Radiological Indicator Parameters

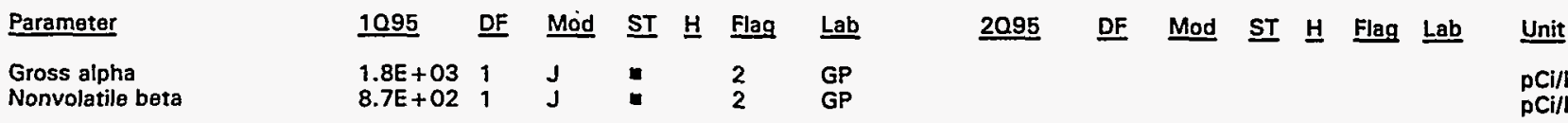

Measured Radionuclides

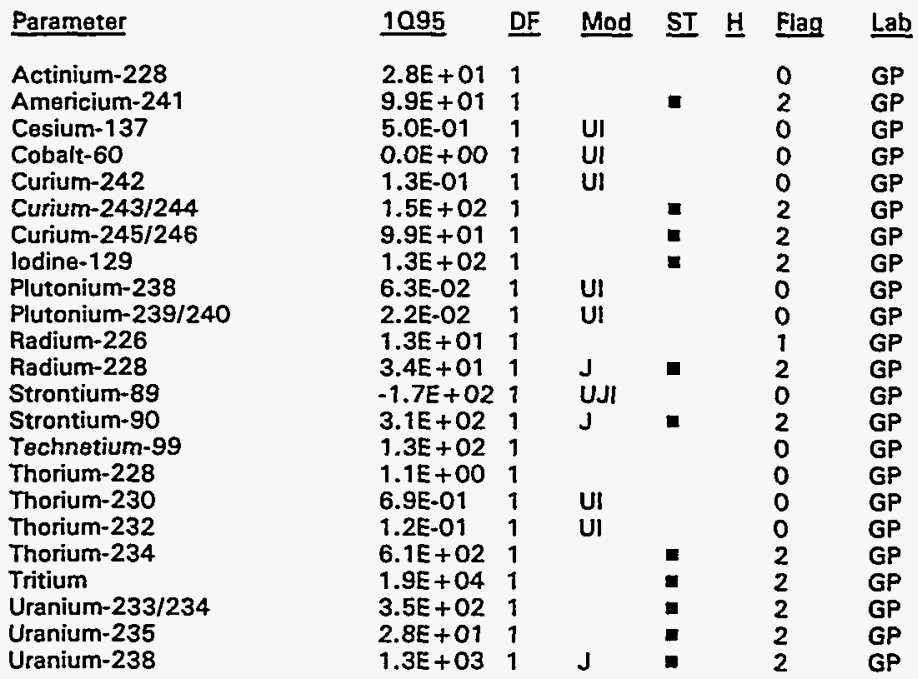

2095 DF Mod ST $\underline{H}$ Flag Lab Unit

Calculated Radionuclides

Parameter

Americium-243

Nickel-63

Plutonium-241

Plutonium-242
1095

4.5E-01

$0.0 E+\infty 0$

8.6E-02

4.3E-07

Unit

pCi/L

pCi/L

pCi/L

pCi/l

pCilL

$\mathrm{pCi} / \mathrm{L}$
$\mathrm{pCi} / \mathrm{L}$

pCi/L

pCi/L

pCill

pCi/L

pCi/L

pCi/L

pCi/L

pCi/L

$\mathrm{pCi} / \mathrm{L}$

$\mathrm{PCi} / L$

pCi/mL

$\mathrm{pCi} / \mathrm{L}$

pCi/L

$\mathrm{pCi} / L$

$\mathrm{pCi} / \mathrm{L}$

Note: See Appendix B for flagging criteria. Synchronous water levels are measured over a 3-5 day period.

- = exceeded holding time.

- =xceeded groundwater protection standard. 
WELL FSB111C

\begin{tabular}{|c|c|c|c|c|c|c|}
\hline SAS Coord. & Lat/Longitude & Screen Zone Elevation & Top of Casing & Casing & Pump & Screen Zone \\
\hline $\begin{array}{l}\text { N75383.3 } \\
\text { E51526.3 }\end{array}$ & $\begin{array}{l}33.2765699^{\circ} \mathrm{N} \\
81.677550^{\circ} \mathrm{W}\end{array}$ & $169.0-159.0 \mathrm{ft} \mathrm{ms!}$ & $276.3 \mathrm{ft} \mathrm{msl}$ & 4" PVC & $\mathbf{s}$ & Barnwell $\left(I \mid B_{1}\right)$ \\
\hline SAMPLE DATE & $02 /$ & & & //95 & & \\
\hline
\end{tabular}

FIELD DATA

\begin{tabular}{ll} 
Parameter & 1095 \\
\cline { 2 - 2 } Water elevation & 212.5 \\
pH & 5.7 \\
Sp. conductance & 58 \\
Water temperature & 19.1 \\
Alkalinity as $\mathrm{CaCO}_{3}$ & 9 \\
Turbldity & 0.4 \\
Volume purged & 4.4 \\
Sampling code & \\
Synchronous water level & 212.8 (03/27/95)
\end{tabular}

$\begin{array}{ll}\underline{2095} & \text { Unit } \\ 212.9 & \mathrm{ft} \mathrm{msl} \\ 5.2 & \mathrm{pH} \\ 51 & \mu \mathrm{SS} / \mathrm{cm} \\ 19.3 & { }^{\circ} \mathrm{c} \\ 0 & \mathrm{mg} / \mathrm{L} \\ 0.7 & \mathrm{NTU} \\ 3.8 & \text { well volumes } \\ 212.8(06 / 15 / 95) & \mathrm{ft} \mathrm{msl}\end{array}$

Hazardous Constituents (264.94, Table 1)

\begin{tabular}{|c|c|c|c|c|c|c|c|c|c|c|c|}
\hline Parameter & 1095 & $\underline{D F}$ & Mod ST $\underline{H}$ & Flag & $\underline{\text { Lab }}$ & $\underline{2095}$ & $\underline{D F}$ & Mod & $\underline{\text { ST }} \underline{H}$ & Flag & Lab \\
\hline $\begin{array}{l}\text { Arsenic, total recoverable } \\
\text { Barium, total recoverable } \\
\text { Cadmium, total recoverable } \\
\text { Chromium, total recoverable } \\
\text { Lead, total recoverable } \\
\text { Mercury, total recoverable } \\
\text { Selenlum, total recoverable } \\
\text { Silver, total recoverable }\end{array}$ & $\begin{array}{l}<5.0 \\
7.3 \\
<2.0 \\
<4.0 \\
<5.0 \\
0.082 \\
<5.0 \\
<0.65\end{array}$ & $\begin{array}{l}1 \\
1 \\
1 \\
1 \\
1 \\
1 \\
1 \\
1\end{array}$ & $\begin{array}{l}\text { JVV } \\
\mathrm{v}\end{array}$ & $\begin{array}{l}0 \\
0 \\
0 \\
0 \\
0 \\
0 \\
0 \\
0\end{array}$ & $\begin{array}{l}\mathrm{GE} \\
\mathrm{GE} \\
\mathrm{GE} \\
\mathrm{GE} \\
\mathrm{GE} \\
\mathrm{GE} \\
\mathrm{GE} \\
\mathrm{GE}\end{array}$ & $\begin{array}{r}<2.0 \\
1.7\end{array}$ & $\begin{array}{l}1 \\
1\end{array}$ & JVV & & $\begin{array}{l}0 \\
0\end{array}$ & GE \\
\hline
\end{tabular}

Hazardous Constituents (261 App. VIII/264 App. IX)

\begin{tabular}{|c|c|c|c|c|c|c|c|c|c|c|c|c|c|c|c|}
\hline Parameter & 1095 & $\underline{\mathrm{DF}}$ & Mod & $\underline{\mathbf{S T}}$ & $\underline{\mathbf{H}}$ & Flag & $\underline{\text { Lab }}$ & $\underline{2095}$ & $\underline{D F}$ & Mod & $\underline{\mathbf{S T}}$ & 븐 & Flag & $\underline{\text { Lab }}$ & $\underline{\text { Unit }}$ \\
\hline $\begin{array}{l}\text { Antimony, total recoverable } \\
\text { Benzene } \\
\text { Cobalt, total recoverable } \\
\text { Copper, total recoverable } \\
\text { Cyanide } \\
\text { Nickel, total recoverable } \\
\text { Phenols } \\
\text { Tetrachloroethylene } \\
\text { Thallium, total recoverable } \\
\text { Trichloroethylene } \\
\text { Trichlorofiuoromethane } \\
\text { Vanadium, total recoverable } \\
\text { Zinc, total recoverable }\end{array}$ & $\begin{array}{l}<5.0 \\
<1.7 \\
<4.0 \\
<4.0 \\
2.6 \\
<10 \\
<10 \\
1.4 \\
<5.0 \\
3.6 \\
<1.7 \\
0.43 \\
<5.0\end{array}$ & $\begin{array}{l}1 \\
1 \\
1 \\
1 \\
1 \\
1 \\
1 \\
1 \\
1 \\
1 \\
1 \\
1 \\
1\end{array}$ & $\begin{array}{l}\text { JVV } \\
\text { V } \\
\text { J }\end{array}$ & & & $\begin{array}{l}0 \\
0 \\
0 \\
0 \\
0 \\
0 \\
0 \\
0 \\
0 \\
1 \\
0 \\
0 \\
0\end{array}$ & $\begin{array}{l}\mathrm{GE} \\
\mathrm{GE} \\
\mathrm{GE} \\
\mathrm{GE} \\
\mathrm{GE} \\
\mathrm{GE} \\
\mathrm{GE} \\
\mathrm{GE} \\
\mathrm{GE} \\
\mathrm{GE} \\
\mathrm{GE} \\
\mathrm{GE} \\
\mathrm{GE}\end{array}$ & & & & & & & & $\begin{array}{l}\mu \mathrm{g} / \mathrm{h} \\
\mu \mathrm{g} / \mathrm{h} \\
\mu \mathrm{g} / \mathrm{h} \\
\mu \mathrm{g} / \mathrm{L} \\
\mu \mathrm{g} / \mathrm{h} \\
\mu \mathrm{g} / \mathrm{h} \\
\mu \mathrm{g} / \mathrm{L} \\
\mu \mathrm{g} / \mathrm{L} \\
\mu \mathrm{g} / \mathrm{L} \\
\mu \mathrm{g} / \mathrm{L} \\
\mu \mathrm{g} / \mathrm{h} \\
\mu \mathrm{g} / \mathrm{L} \\
\mu \mathrm{g} / \mathrm{L}\end{array}$ \\
\hline \multicolumn{16}{|c|}{ Water Quality Indicator Parameters } \\
\hline Parameter & 1095 & $\underline{D F}$ & Mod & $\underline{\mathbf{S T}}$ & $\underline{H}$ & Flag & $\underline{\text { Lab }}$ & $\underline{2095}$ & $\underline{D F}$ & Mod & $\underline{\text { ST }}$ & 브 & Flag & $\underline{\text { Lab }}$ & $\underline{\text { Unit }}$ \\
\hline $\begin{array}{l}\text { Aluminum, total recoverable } \\
\text { Nitrate as nitrogen } \\
\text { pH }\end{array}$ & $\begin{array}{l}5.6 \\
1,900 \\
5.7\end{array}$ & $\begin{array}{l}1 \\
1 \\
1\end{array}$ & $\begin{array}{l}J \\
\text { J1 }\end{array}$ & - & & $\begin{array}{l}0 \\
0 \\
0\end{array}$ & $\begin{array}{l}\text { GE } \\
\text { GE } \\
\text { GE }\end{array}$ & $\begin{array}{l}1,600 \\
5.7\end{array}$ & $\begin{array}{l}1 \\
1\end{array}$ & J1 & - & - & $\begin{array}{l}0 \\
0\end{array}$ & $\begin{array}{l}\text { GE } \\
\text { GE }\end{array}$ & $\underset{\mathrm{pH}}{\mu \mathrm{g} / \mathrm{L}}$ \\
\hline \multicolumn{16}{|c|}{ Common Laboratory Contaminants } \\
\hline Paramater & 1095 & $\underline{D F}$ & Mod & $\underline{\text { ST }}$ & $\underline{H}$ & Flag & $\underline{\underline{L a b}}$ & $\underline{2095}$ & $\underline{D F}$ & Mod & $\underline{\text { ST }}$ & 브 & Flag & $\underline{\text { Lab }}$ & $\underline{\text { Unit }}$ \\
\hline $\begin{array}{l}\text { Bis(2-ethylhexyl) phthalate } \\
\text { Dichloromethane }\end{array}$ & $\begin{array}{l}<17 \\
<1.7\end{array}$ & $\begin{array}{l}1 \\
1\end{array}$ & & & & $\begin{array}{l}0 \\
0\end{array}$ & $\begin{array}{l}\text { GE } \\
\text { GE }\end{array}$ & & & & & & & & $\underset{\mu g / L}{\mu g / L}$ \\
\hline
\end{tabular}

Note: See Appendix B for flagging criteria. Synchronous water levels are measured over a 3-5 day period. $\bullet=$ exceeded holding time.

- = exceeded groundwater protection standard. 
WELL FSB111C (cont.)

Radiological Indicator Parameters

\begin{tabular}{|c|c|c|c|c|c|c|c|c|c|c|c|c|c|c|c|}
\hline Parameter & 1095 & DF & Mod & $\underline{\text { ST }}$ & H & Flag & $\underline{\text { Lab }}$ & 2095 & DF & Mod & ST & $\underline{H}$ & Flag & Lab & Unit \\
\hline $\begin{array}{l}\text { Gross alpha } \\
\text { Nonvolatile beta }\end{array}$ & $\begin{array}{l}4.6 E-01 \\
4.8 E-01\end{array}$ & $\begin{array}{l}1 \\
1\end{array}$ & $\begin{array}{l}\text { UI } \\
\text { Ul }\end{array}$ & & & $\begin{array}{l}0 \\
0\end{array}$ & $\begin{array}{l}\text { GP } \\
\text { GP }\end{array}$ & $\begin{array}{l}3.2 E-01 \\
2.7 E-01\end{array}$ & $\begin{array}{l}1 \\
1\end{array}$ & $\begin{array}{l}\text { UI } \\
\text { UJI }\end{array}$ & & & $\begin{array}{l}0 \\
0\end{array}$ & $\begin{array}{l}\text { GP } \\
\text { GP }\end{array}$ & $\begin{array}{l}\mathrm{pCi} / \mathrm{L} \\
\mathrm{pCi} / \mathrm{L}\end{array}$ \\
\hline \multicolumn{16}{|c|}{ Measured Radionuclides } \\
\hline Parameter & 1095 & $\underline{D F}$ & Mod & $\underline{S T}$ & $\underline{H}$ & Flag & $\underline{\text { Lab }}$ & 2095 & $\underline{D F}$ & Mod & $\underline{\mathrm{ST}}$ & $\underline{H}$ & Flag & $\underline{\text { Lab }}$ & Unit \\
\hline $\begin{array}{l}\text { Actinium-228 } \\
\text { Americium-241 } \\
\text { Cesium-137 } \\
\text { Cobalt-60 } \\
\text { Curium-242 } \\
\text { Curium-243/244 } \\
\text { Curium-245/246 } \\
\text { lodine-129 } \\
\text { Plutonium-238 } \\
\text { Plutonium-239/240 } \\
\text { Radium-226 } \\
\text { Radium-228 } \\
\text { Strontium-89 } \\
\text { Strontium-90 } \\
\text { Technetium-99 } \\
\text { Thorium-228 } \\
\text { Thorium-230 } \\
\text { Thorium-232 } \\
\text { Thorium-234 } \\
\text { Tritium } \\
\text { Uranium-233/234 } \\
\text { Uranium-235 } \\
\text { Uranium-238 }\end{array}$ & $\begin{array}{l}-2.1 E+00 \\
1.3 E-01 \\
3.4 E-03 \\
1.7 E-01 \\
-2.1 E-02 \\
-1.1 E-01 \\
-9.0 E-03 \\
2.0 E+00 \\
2.7 E-01 \\
-9.7 E-03 \\
1.5 E-01 \\
-1.3 E-01 \\
-1.4 E-01 \\
-3.2 E-02 \\
-2.1 E+01 \\
2.7 E-01 \\
2.0 E-01 \\
-3.2 E-02 \\
1.1 E+02 \\
5.4 E+00 \\
6.4 E-01 \\
3.1 E-02 \\
7.1 E-01\end{array}$ & $\begin{array}{l}1 \\
1 \\
1 \\
1 \\
1 \\
1 \\
1 \\
1 \\
1 \\
1 \\
1 \\
1 \\
1 \\
1 \\
1 \\
1 \\
1 \\
1 \\
1 \\
1 \\
1 \\
1 \\
1\end{array}$ & $\begin{array}{l}\text { UI } \\
\text { UI } \\
\text { UI } \\
\text { UI } \\
\text { UI } \\
\text { UI } \\
\text { UI } \\
\text { UJI } \\
\text { UI } \\
\text { UI } \\
\text { UJI } \\
\text { UI } \\
\text { UI } \\
\text { UI } \\
\text { UI } \\
\text { UI } \\
\text { UI } \\
\text { UI } \\
\text { UI } \\
\text { UI } \\
\text { UJI }\end{array}$ & - & & $\begin{array}{l}0 \\
0 \\
0 \\
0 \\
0 \\
0 \\
0 \\
2 \\
0 \\
0 \\
0 \\
0 \\
0 \\
0 \\
0 \\
0 \\
0 \\
0 \\
0 \\
0 \\
0 \\
0 \\
0\end{array}$ & $\begin{array}{l}\text { GP } \\
\text { GP } \\
\text { GP } \\
\text { GP } \\
\text { GP } \\
\text { GP } \\
\text { GP } \\
\text { GP } \\
\text { GP } \\
\text { GP } \\
\text { GP } \\
\text { GP } \\
\text { GP } \\
\text { GP } \\
\text { GP } \\
\text { GP } \\
\text { GP } \\
\text { GP } \\
\text { GP } \\
\text { GP } \\
\text { GP } \\
\text { GP } \\
\text { GP }\end{array}$ & $4.5 E+02$ & 1 & & a & & 2 & GP & $\begin{array}{l}\text { pCi/L } \\
\text { pCi/L } \\
\text { pCi/L } \\
\text { pCi/L } \\
\text { pCi/L } \\
\text { pCi/L } \\
\text { pCi/L } \\
\text { pCi/L } \\
\text { pCi/L } \\
\text { pCi/L } \\
\text { pCi/L } \\
\text { pCi/L } \\
\text { pCi/L } \\
\text { pCi/L } \\
\text { pCi/L } \\
\text { pCi/L } \\
\text { pCi/L } \\
\text { pCi/L } \\
\text { pCi/L } \\
\text { pCi/m } \\
\text { pCi/L } \\
\text { pCi/L } \\
\text { pCi/L }\end{array}$ \\
\hline \multicolumn{16}{|c|}{ Calculated Radionuclides } \\
\hline Parameter & 1095 & $\underline{D F}$ & Mod & $\underline{\underline{S T}}$ & $\underline{H}$ & Flag & $\underline{\text { Lab }}$ & 2095 & DF & Mod & $\underline{\text { ST }}$ & $\underline{H}$ & Flag & $\underline{\text { Lab }}$ & Unit \\
\hline $\begin{array}{l}\text { Americium-243 } \\
\text { Nickel-63 } \\
\text { Plutonium-241 } \\
\text { Plutonium-242 }\end{array}$ & $\begin{array}{l}-3.3 \mathrm{E}-04 \\
1.7 \mathrm{E}-01 \\
-3.9 \mathrm{E}-02 \\
-1.9 \mathrm{E}-07\end{array}$ & & & & & $\begin{array}{l}0 \\
0 \\
0 \\
0\end{array}$ & & & & & & & & & $\begin{array}{l}\mathrm{pCi} / \mathrm{L} \\
\mathrm{pCi} / \mathrm{L} \\
\mathrm{pCi} / \mathrm{L} \\
\mathrm{pCi} / \mathrm{L}\end{array}$ \\
\hline
\end{tabular}

Note: See Appendix B for flagging criteria. Synchronous water levels are measured over a $3-5$ day period.

- = exceeded holding time.

m = exceeded groundwater protection standard. 
WELL FSB111D

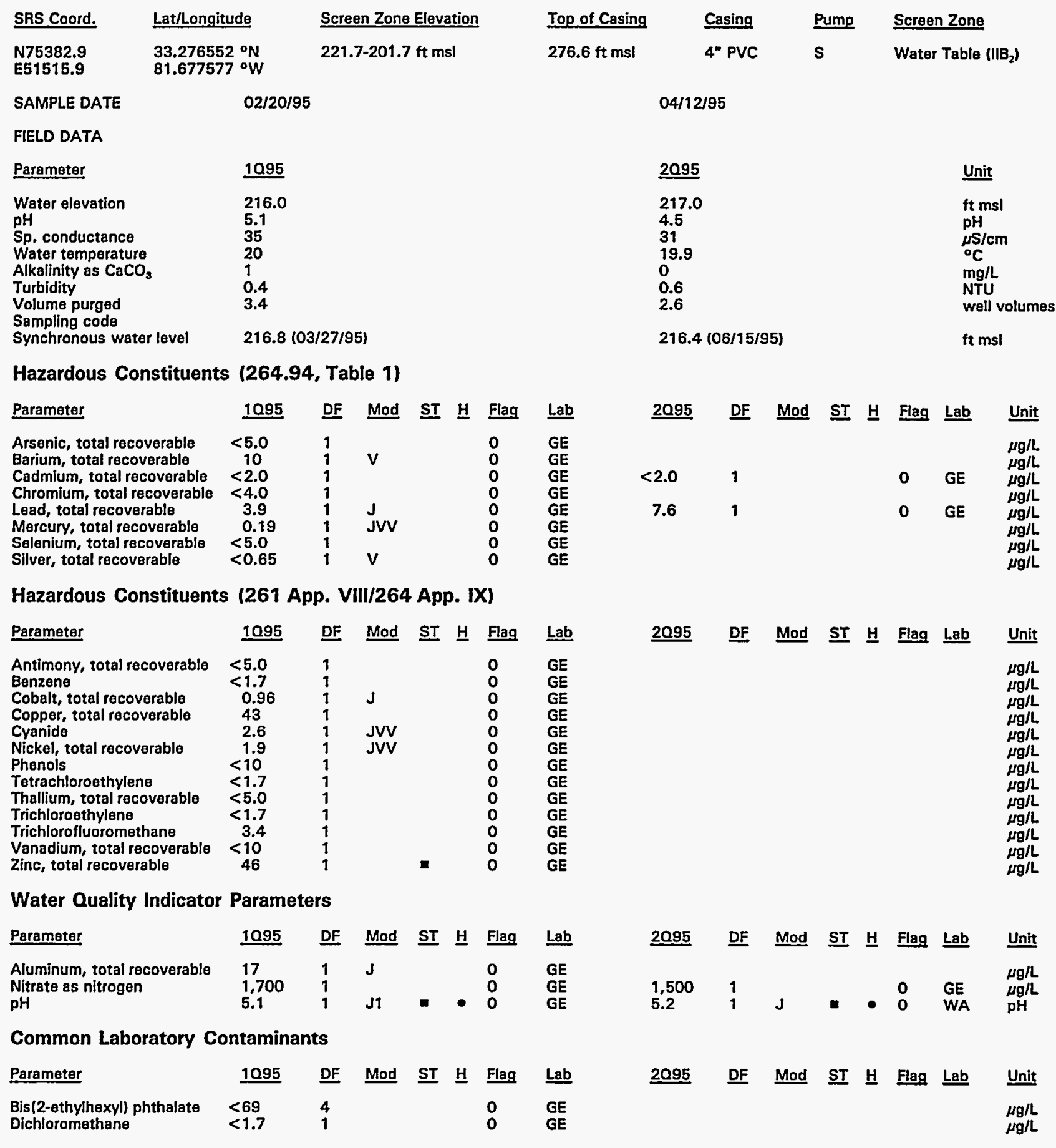

Note: See Appendix B for flagging criteria. Synchronous water levels are measured over a 3-5 day period.

- = exceeded holding time.

- = exceeded groundwater protection standard. 
WELL FSB111D (cont.)

Radiological Indicator Parameters

\begin{tabular}{|c|c|c|c|c|c|c|c|c|c|c|c|c|c|}
\hline Parameter & 1095 & $\underline{D F}$ & Mod & $\underline{\text { ST }} \underline{H}$ & Flag & $\underline{\text { Lab }}$ & 2095 & DE & Mod & $\underline{\text { ST }} \underline{H}$ & Flag & Lab & Unit \\
\hline $\begin{array}{l}\text { Gross alpha } \\
\text { Nonvolatile beta }\end{array}$ & $\begin{array}{l}1.2 E+\infty 0 \\
2.1 E+\infty 0\end{array}$ & $\begin{array}{l}1 \\
1\end{array}$ & & & $\begin{array}{l}0 \\
0\end{array}$ & $\begin{array}{l}G P \\
G P\end{array}$ & $\begin{array}{l}1.4 E+\infty 0 \\
4.7 E+\infty 0\end{array}$ & $\begin{array}{l}1 \\
1\end{array}$ & J1 & & $\begin{array}{l}0 \\
0\end{array}$ & $\underset{G P}{T M}$ & $\mathrm{pCi} / \mathrm{L}$ \\
\hline
\end{tabular}

Measured Radionuclides

\begin{tabular}{|c|c|c|c|c|c|c|c|c|c|c|c|c|c|c|c|}
\hline Parameter & 1095 & DF & Mod & $\underline{\text { ST }}$ & 보 & Flag & $\underline{\text { Lab }}$ & $\underline{2095}$ & DF & Mod & ST & H & Flag & Lab & Unit \\
\hline $\begin{array}{l}\text { Actinium-228 } \\
\text { Americium-241 } \\
\text { Cesium-137 } \\
\text { Cobalt-60 } \\
\text { Curium-242 } \\
\text { Curium-243/244 } \\
\text { Curium-245/246 } \\
\text { lodine-129 } \\
\text { Plutonium-238 } \\
\text { Plutonium-239/240 } \\
\text { Radium-226 } \\
\text { Radium-228 } \\
\text { Strontium-89 } \\
\text { Strontium-90 } \\
\text { Technetium-99 } \\
\text { Thorium-228 } \\
\text { Thorium-230 } \\
\text { Thorium-232 } \\
\text { Thorium-234 } \\
\text { Tritium } \\
\text { Uranium-233/234 } \\
\text { Uranium-235 } \\
\text { Uranium-238 }\end{array}$ & $\begin{array}{l}5.9 E+00 \\
-4.5 E-02 \\
2.3 E-01 \\
5.8 E-01 \\
4.7 E-02 \\
4.6 E-02 \\
5.0 E-02 \\
2.3 E-01 \\
5.0 E-02 \\
-5.8 E-03 \\
7.3 E-01 \\
6.1 E-01 \\
3.2 E-01 \\
6.6 E-01 \\
-1.9 E+01 \\
7.9 E-02 \\
-1.6 E-02 \\
-1.6 E-02 \\
7.4 E+00 \\
1.0 E+01 \\
5.7 E-02 \\
0.0 E+00 \\
3.8 E-02\end{array}$ & $\begin{array}{l}1 \\
1 \\
1 \\
1 \\
1 \\
1 \\
1 \\
1 \\
1 \\
1 \\
1 \\
1 \\
1 \\
1 \\
1 \\
1 \\
1 \\
1 \\
1 \\
1 \\
1 \\
1 \\
1\end{array}$ & $\begin{array}{l}\text { UI } \\
\text { UI } \\
\text { UI } \\
\text { UI } \\
\text { UI } \\
\text { UI } \\
\text { UI } \\
\text { UI } \\
\text { UII } \\
\text { UI } \\
\text { UI } \\
\text { UJI } \\
\text { UI } \\
\text { UI } \\
\text { UI } \\
\text { UI } \\
\text { UI } \\
\text { UI } \\
\text { UI } \\
\text { UJI }\end{array}$ & & & $\begin{array}{l}0 \\
0 \\
0 \\
0 \\
0 \\
0 \\
0 \\
0 \\
0 \\
0 \\
0 \\
0 \\
0 \\
0 \\
0 \\
0 \\
0 \\
0 \\
0 \\
0 \\
0 \\
0 \\
0\end{array}$ & $\begin{array}{l}\text { GP } \\
\text { GP } \\
\text { GP } \\
\text { GP } \\
\text { GP } \\
\text { GP } \\
\text { GP } \\
\text { GP } \\
\text { GP } \\
\text { GP } \\
\text { GP } \\
\text { GP } \\
\text { GP } \\
\text { GP } \\
\text { GP } \\
\text { GP } \\
\text { GP } \\
\text { GP } \\
\text { GP } \\
\text { GP } \\
\text { GP } \\
\text { GP } \\
\text { GP }\end{array}$ & $1.1 E+01$ & 1 & & & & 1 & GP & $\begin{array}{l}\text { pCi/L } \\
\text { pCi/L } \\
\text { pCi/L } \\
\text { pCi/L } \\
\text { pCi/L } \\
\text { pCi/L } \\
\text { pCi/L } \\
\text { pCi/L } \\
\text { pCi/L } \\
\text { pCi/L } \\
\text { pCi/L } \\
\text { pCi/L } \\
\text { pCi/L } \\
\text { pCi/L } \\
\text { pCi/L } \\
\text { pCi/L } \\
\text { pCi/L } \\
\text { pCi/L } \\
\text { pCi/L } \\
\text { pCi/m } \\
\text { pCi/L } \\
\text { pCi/L } \\
\text { pCi/L }\end{array}$ \\
\hline \multicolumn{16}{|c|}{ Calculated Radionuclides } \\
\hline Parameter & 1095 & DF & Mod & $\underline{\mathrm{ST}}$ & $\underline{H}$ & Flag & Lab & $\underline{2095}$ & DF & Mod & $\underline{\mathbf{S T}}$ & 브 & Flag & Lab & Unit \\
\hline $\begin{array}{l}\text { Americium-243 } \\
\text { Nickel-63 } \\
\text { Plutonium-241 } \\
\text { Plutonium-242 }\end{array}$ & $\begin{array}{l}1.4 E-04 \\
5.8 E-01 \\
-2.3 E-02 \\
-1.2 E-07\end{array}$ & & & & & $\begin{array}{l}0 \\
0 \\
0 \\
0\end{array}$ & & & & & & & & & $\begin{array}{l}\mathrm{pCi} / \mathrm{L} \\
\mathrm{pCi} / \mathrm{L} \\
\mathrm{pCi} / \mathrm{L} \\
\mathrm{pCi} / \mathrm{L}\end{array}$ \\
\hline
\end{tabular}

Note: See Appendix B for flagging criteria. Synchronous water levels are measured over a 3-5 day period.

- = exceeded holding time.

- = exceeded groundwater protection standard. 


\section{WELL FSB112A}

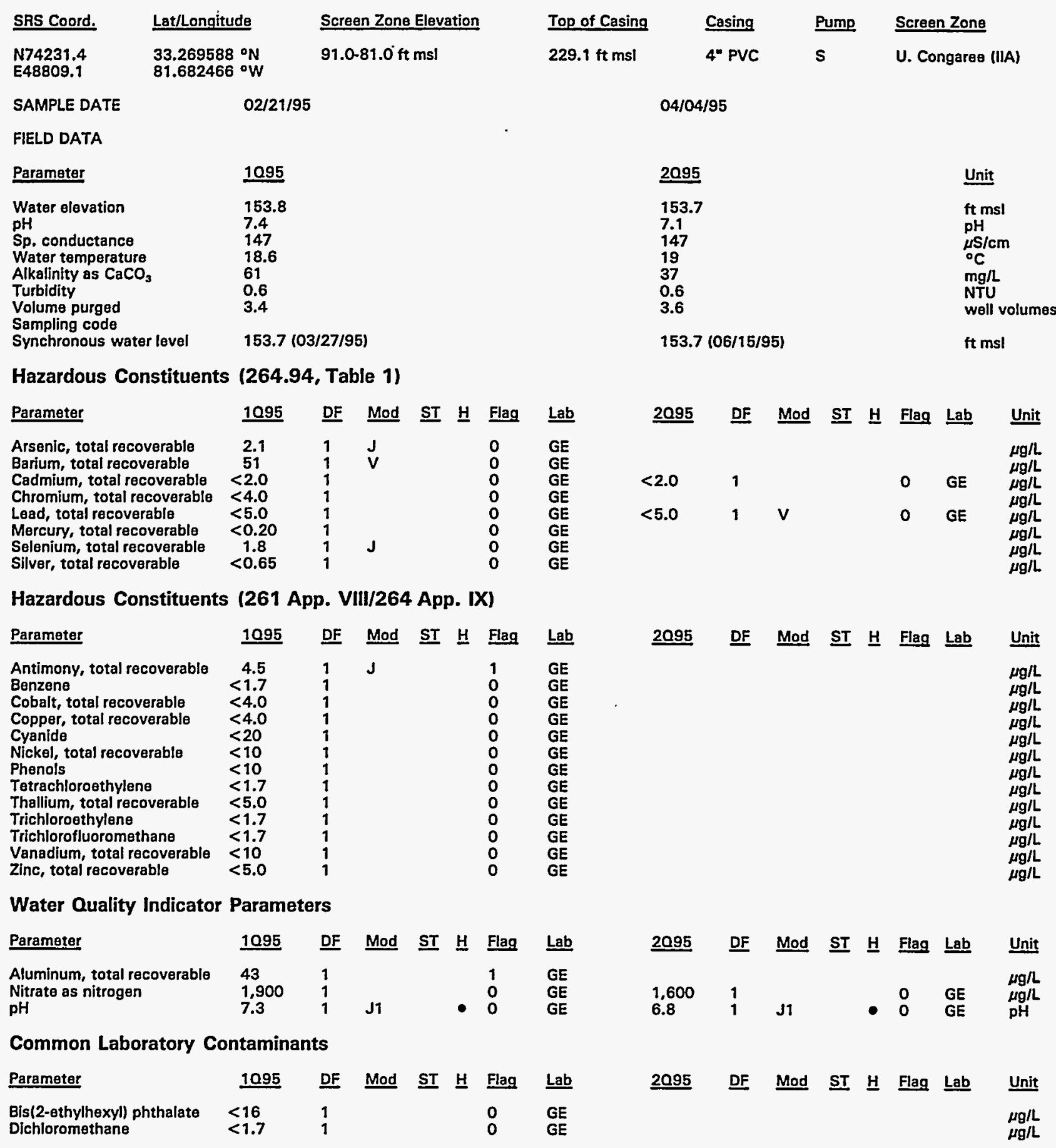

Note: See Appendix B for flagging criteria. Synchronous water levels are measured over a 3-5 day period.

- = exceeded holding time.

I = exceeded groundwater protection standard. 
WELL FSB $112 A$ (cont.)

\section{Radiological Indicator Parameters}

\begin{tabular}{|c|c|c|c|c|c|c|c|c|c|c|c|c|c|c|c|}
\hline Parameter & 1095 & DF & Mod & $\underline{\mathbf{S T}}$ & H & Flag & $\underline{\text { Lab }}$ & $\underline{2095}$ & $\underline{\mathrm{DF}}$ & Mod & $\underline{\mathbf{S T}}$ & H & Flag & $\underline{\text { Lab }}$ & $\underline{\text { Unit }}$ \\
\hline $\begin{array}{l}\text { Gross alpha } \\
\text { Nonvolatile beta }\end{array}$ & $\begin{array}{l}8.4 E-01 \\
1.4 E+01\end{array}$ & $\begin{array}{l}1 \\
1\end{array}$ & $u$ & & & $\begin{array}{l}0 \\
0\end{array}$ & $\begin{array}{l}\text { GP } \\
\text { GP }\end{array}$ & $\begin{array}{l}9.5 E-01 \\
9.7 E+00\end{array}$ & $\begin{array}{l}1 \\
1\end{array}$ & J & & & $\begin{array}{l}0 \\
0\end{array}$ & $\begin{array}{l}\text { GP } \\
\text { GP }\end{array}$ & $\begin{array}{l}\mathrm{pCi} / \mathrm{L} \\
\mathrm{pCI} / \mathrm{L}\end{array}$ \\
\hline \multicolumn{16}{|c|}{ Measured Radionuclides } \\
\hline Parameter & $\underline{1095}$ & $\underline{\mathrm{DF}}$ & Mod & $\underline{\mathbf{S T}}$ & $\underline{H}$ & Flag & $\underline{\text { Lab }}$ & $\underline{20.95}$ & DF & $\underline{\text { Mod }}$ & $\underline{\mathbf{S T}}$ & $\underline{H}$ & Flag & $\underline{\text { Lab }}$ & $\underline{\text { Unit }}$ \\
\hline $\begin{array}{l}\text { Actinium-228 } \\
\text { Americium-241 } \\
\text { Cesium-137 } \\
\text { Cobalt-60 } \\
\text { Curium-242 } \\
\text { Curium-243/244 } \\
\text { Curium-245/246 } \\
\text { lodine-129 } \\
\text { Plutonium-238 } \\
\text { Plutonium-239/240 } \\
\text { Radium-226 } \\
\text { Radium-228 } \\
\text { Strontium-89 } \\
\text { Strontium-90 } \\
\text { Technetium-99 } \\
\text { Thorium-228 } \\
\text { Thorium-230 } \\
\text { Thorium-232 } \\
\text { Thorium-234 } \\
\text { Tritium } \\
\text { Uranium-233/234 } \\
\text { Uranium-235 } \\
\text { Uranium-238 }\end{array}$ & $\begin{array}{l}3.7 E+00 \\
1.1 E-01 \\
2.3 E+00 \\
1.6 E+00 \\
0.0 E+00 \\
2.1 E-01 \\
-5.5 E-03 \\
9.9 E-01 \\
6.7 E-03 \\
-1.5 E-02 \\
4.4 E-01 \\
1.9 E+00 \\
6.3 E-01 \\
4.0 E+00 \\
-1.7 E+01 \\
6.6 E-02 \\
6.4 E-02 \\
4.7 E-02 \\
3.3 E+01 \\
1.1 E+02 \\
8.1 E-02 \\
-5.4 E-03 \\
8.1 E-02\end{array}$ & $\begin{array}{l}1 \\
1 \\
1 \\
1 \\
1 \\
1 \\
1 \\
1 \\
1 \\
1 \\
1 \\
1 \\
1 \\
1 \\
1 \\
1 \\
1 \\
1 \\
1 \\
1 \\
1 \\
1 \\
1\end{array}$ & $\begin{array}{l}\text { UI } \\
\text { UI } \\
\text { UI } \\
\text { UI } \\
\text { UI } \\
\text { UI } \\
\text { UI } \\
\text { UI } \\
\text { UJI } \\
\text { UI } \\
\text { UI } \\
J\end{array}$ & - & & $\begin{array}{l}0 \\
0 \\
0 \\
0 \\
0 \\
0 \\
0 \\
0 \\
0 \\
0 \\
0 \\
0 \\
0 \\
1 \\
0 \\
0 \\
0 \\
0 \\
0 \\
2 \\
0 \\
0 \\
0\end{array}$ & $\begin{array}{l}\text { GP } \\
\text { GP } \\
\text { GP } \\
\text { GP } \\
\text { GP } \\
\text { GP } \\
\text { GP } \\
\text { GP } \\
\text { GP } \\
\text { GP } \\
\text { GP } \\
\text { GP } \\
\text { GP } \\
\text { GP } \\
\text { GP } \\
\text { GP } \\
\text { GP } \\
\text { GP } \\
\text { GP } \\
\text { GP } \\
\text { GP } \\
\text { GP } \\
\text { GP }\end{array}$ & $1.6 E+02$ & 1 & & " & & 2 & GP & 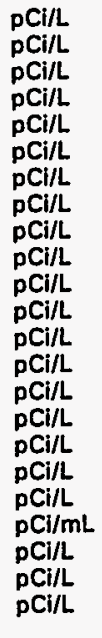 \\
\hline \multicolumn{16}{|c|}{ Calculated Radionuclides } \\
\hline Parameter & $\underline{1095}$ & $\underline{\mathrm{DF}}$ & Mod & $\underline{\text { ST }}$ & $\underline{H}$ & Flag & $\underline{\text { Lab }}$ & $\underline{2095}$ & $\underline{D F}$ & Mod & $\underline{\text { ST }}$ & 브 & Flag & $\underline{\text { Lab }}$ & Unit \\
\hline $\begin{array}{l}\text { Americium-243 } \\
\text { Nickel-63 } \\
\text { Plutonium-241 } \\
\text { Plutonium-242 }\end{array}$ & $\begin{array}{l}6.3 E-04 \\
1.6 E+00 \\
-6.0 E-02 \\
-3.0 E-07\end{array}$ & & & & & $\begin{array}{l}0 \\
0 \\
0 \\
0\end{array}$ & & & & & & & & & $\begin{array}{l}\mathrm{pCi} / \mathrm{L} \\
\mathrm{pCi} / \mathrm{L} \\
\mathrm{pCi} / \mathrm{L} \\
\mathrm{pCi} / \mathrm{L}\end{array}$ \\
\hline
\end{tabular}

Note: See Appendix B for flagging criteria. Synchronous water levels are measured over a 3-5 day period.

- = exceeded holding time.

- = exceeded groundwater protection standard. 
WELL FSB112C

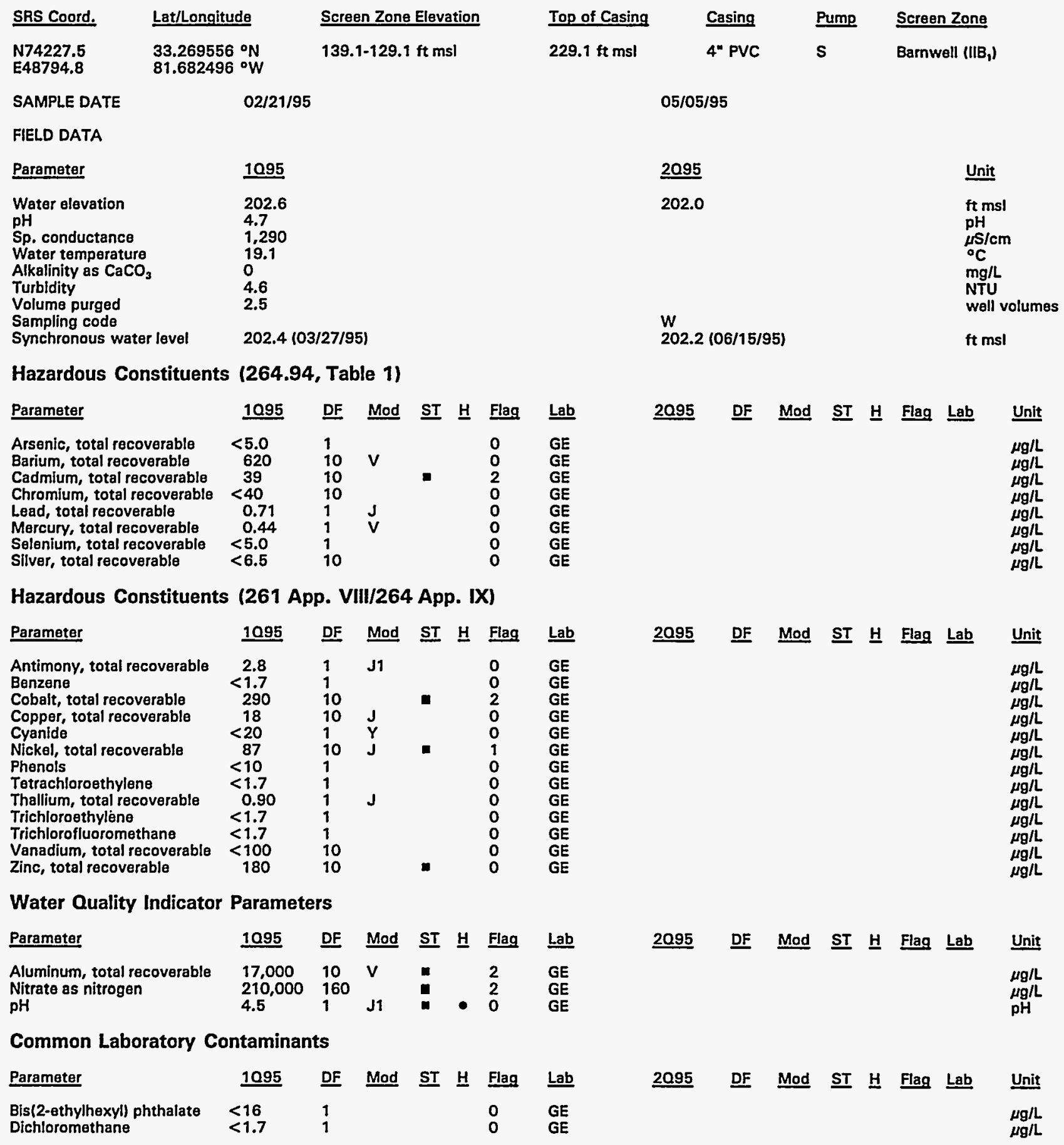

Note: See Appendix B for flagging criteria. Synchronous water levels are measured over a 3-5 day period.

- = exceeded holding time.

- = exceeded groundwater protection standard. 
WELL FSB112C (cont.)

Radiological Indicator Parameters

\begin{tabular}{|c|c|c|c|c|c|c|c|c|c|c|c|c|c|c|c|}
\hline Parameter & 1095 & $\underline{\mathrm{DF}}$ & Mod & $\underline{\underline{S T}}$ & H & Flag & $\underline{\text { Lab }}$ & $\underline{2095}$ & $\underline{D F}$ & Mod & $\underline{\mathbf{S T}}$ & $\underline{H}$ & Flag & $\underline{\text { Lab }}$ & $\underline{\text { Unit }}$ \\
\hline $\begin{array}{l}\text { Gross alpha } \\
\text { Nonvolatile beta }\end{array}$ & $\begin{array}{l}1.7 E+02 \\
1.8 E+03\end{array}$ & 1 & $\begin{array}{l}\mathrm{J} \\
\mathrm{J}\end{array}$ & 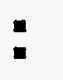 & & $\begin{array}{l}2 \\
2\end{array}$ & $\begin{array}{l}\text { GP } \\
\text { GP }\end{array}$ & & & & & & & & $\begin{array}{l}\mathrm{pCi} / \mathrm{L} \\
\mathrm{pCi} / \mathrm{L}\end{array}$ \\
\hline \multicolumn{16}{|c|}{ Measured Radionuclides } \\
\hline Parameter & 1095 & $\underline{\mathrm{DF}}$ & Mod & $\underline{\text { ST }}$ & $\underline{\mathrm{H}}$ & Flag & $\underline{\text { Lab }}$ & $\underline{2095}$ & $\underline{D F}$ & Mod & $\underline{\mathbf{S T}}$ & $\underline{\mathrm{H}}$ & Flag & $\underline{\text { Lab }}$ & Unit \\
\hline $\begin{array}{l}\text { Actinium-228 } \\
\text { Americium-241 } \\
\text { Cesium-137 } \\
\text { Cobalt-60 } \\
\text { Curium-242 } \\
\text { Curium-243/244 } \\
\text { Curium-245/246 } \\
\text { lodine-129 } \\
\text { Plutonium-238 } \\
\text { Plutonium-239/240 } \\
\text { Radium-226 } \\
\text { Radium-228 } \\
\text { Strontium-89 } \\
\text { Strontium-90 } \\
\text { Technetium-99 } \\
\text { Thorium-228 } \\
\text { Thorium-230 } \\
\text { Thorium-232 } \\
\text { Thorium-234 } \\
\text { Tritium } \\
\text { Uranium-233/234 } \\
\text { Uranium-235 } \\
\text { Uranium-238 }\end{array}$ & $\begin{array}{l}2.4 E+01 \\
7.2 E-02 \\
6.9 E-01 \\
0.0 E+00 \\
1.2 E-01 \\
1.3 E-01 \\
-1.2 E-02 \\
2.2 E+02 \\
1.7 E-01 \\
1.2 E-01 \\
9.8 E+01 \\
3.0 E+01 \\
-2.9 E+02 \\
9.2 E+02 \\
3.2 E+02 \\
8.6 E-01 \\
7.7 E-01 \\
2.4 E-02 \\
6.6 E+01 \\
7.3 E+03 \\
2.3 E+00 \\
2.1 E-01 \\
2.8 E+00\end{array}$ & $\begin{array}{l}1 \\
1 \\
1 \\
1 \\
1 \\
1 \\
1 \\
1 \\
1 \\
1 \\
1 \\
1 \\
1 \\
1 \\
1 \\
1 \\
1 \\
1 \\
1 \\
1 \\
1 \\
1 \\
1\end{array}$ & $\begin{array}{l}\text { UI } \\
\text { UI } \\
\text { UI } \\
\text { UI } \\
U I \\
\text { UI } \\
\text { UI } \\
\text { UI } \\
\text { UI } \\
J \\
\text { UJI } \\
\text { J } \\
\text { UI } \\
\text { UI } \\
\text { UI } \\
\text { UI }\end{array}$ & 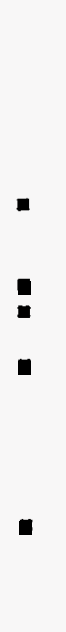 & & $\begin{array}{l}0 \\
0 \\
0 \\
0 \\
0 \\
0 \\
0 \\
2 \\
0 \\
0 \\
2 \\
2 \\
0 \\
2 \\
0 \\
0 \\
0 \\
0 \\
0 \\
2 \\
0 \\
0 \\
0\end{array}$ & $\begin{array}{l}\text { GP } \\
\text { GP } \\
\text { GP } \\
\text { GP } \\
\text { GP } \\
\text { GP } \\
\text { GP } \\
\text { GP } \\
\text { GP } \\
\text { GP } \\
\text { GP } \\
\text { GP } \\
\text { GP } \\
\text { GP } \\
\text { GP } \\
\text { GP } \\
\text { GP } \\
\text { GP } \\
\text { GP } \\
\text { GP } \\
\text { GP } \\
\text { GP } \\
\text { GP }\end{array}$ & & & & & & & & 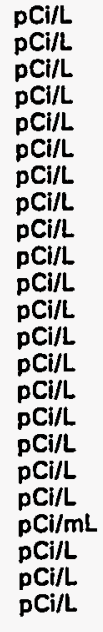 \\
\hline \multicolumn{16}{|c|}{ Calculated Radionuclides } \\
\hline Parameter & $\underline{1095}$ & $\underline{\mathrm{DF}}$ & Mod & $\underline{\mathbf{S T}}$ & $\underline{H}$ & Flag & $\underline{\text { Lab }}$ & $\underline{2095}$ & $\underline{\mathrm{DF}}$ & Mod & $\underline{\mathbf{S T}}$ & $\underline{H}$ & Flag & $\underline{\text { Lab }}$ & Unit \\
\hline $\begin{array}{l}\text { Americium-243 } \\
\text { Nickel-63 } \\
\text { Plutonium-241 } \\
\text { Plutonium-242 }\end{array}$ & $\begin{array}{l}4.0 E-04 \\
0.0 E+00 \\
4.6 E-01 \\
2.3 E-06\end{array}$ & & & & & $\begin{array}{l}0 \\
0 \\
0 \\
0\end{array}$ & & & & & & & & & $\begin{array}{l}\mathrm{pCi} / \mathrm{L} \\
\mathrm{pCi} / \mathrm{L} \\
\mathrm{pCi} / \mathrm{L} \\
\mathrm{pCi} / \mathrm{L}\end{array}$ \\
\hline
\end{tabular}

Note: See Appendix B for flagging criteria. Synchronous water levels are measured over a 3-5 day period.

- = exceeded holding time.

- = exceeded groundwater protection standard. 


\section{WELL FSB112D}

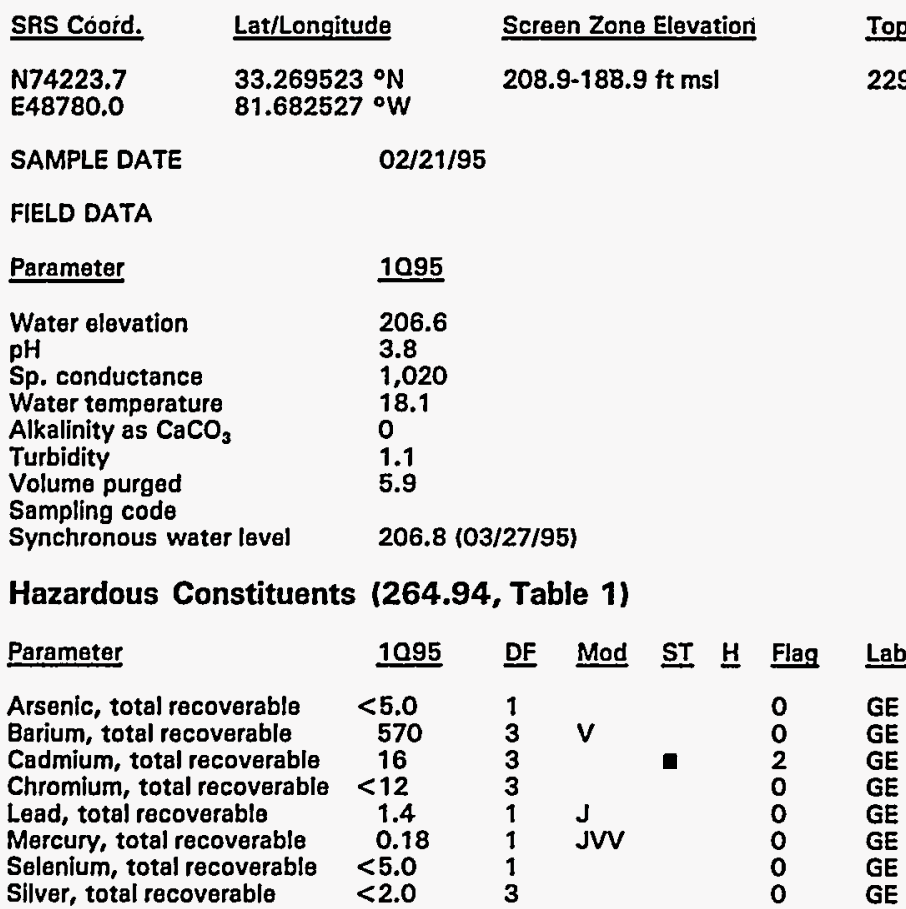

Hazardous Constituents (261 App. VIII/264 App. IX)

\begin{tabular}{|c|c|c|c|c|c|c|c|c|c|c|c|c|c|c|c|}
\hline Parameter & 1095 & DF & Mod & $\underline{\text { ST }}$ & H & Flag & $\underline{\text { Lab }}$ & $\underline{2095}$ & DF & Mod & ST & 보 & Flag & Lab & Unit \\
\hline $\begin{array}{l}\text { Antimony, total recoverable } \\
\text { Benzene } \\
\text { Cobalt, total recoverable } \\
\text { Copper, total recoverable } \\
\text { Cyanide } \\
\text { Nickel, total recoverable } \\
\text { Phenols } \\
\text { Tetrachloroethylene } \\
\text { Thallium, total recoverable } \\
\text { Trichloroethylene } \\
\text { Trichlorofluoromethane } \\
\text { Vanedium, total recoverable } \\
\text { Zinc, total recoverable }\end{array}$ & $\begin{array}{r}4.5 \\
<1.7 \\
77 \\
70 \\
<20 \\
41 \\
<10 \\
<1.7 \\
1.2 \\
<1.7 \\
<1.7 \\
<30 \\
88\end{array}$ & $\begin{array}{l}1 \\
1 \\
3 \\
3 \\
1 \\
3 \\
1 \\
1 \\
1 \\
1 \\
1 \\
3 \\
3\end{array}$ & J1 & a & & $\begin{array}{l}1 \\
0 \\
2 \\
0 \\
0 \\
0 \\
0 \\
0 \\
1 \\
0 \\
0 \\
0 \\
0\end{array}$ & $\begin{array}{l}\mathrm{GE} \\
\mathrm{GE} \\
\mathrm{GE} \\
\mathrm{GE} \\
\mathrm{GE} \\
\mathrm{GE} \\
\mathrm{GE} \\
\mathrm{GE} \\
\mathrm{GE} \\
\mathrm{GE} \\
\mathrm{GE} \\
\mathrm{GE} \\
\mathrm{GE}\end{array}$ & & & & & & & & $\begin{array}{l}\mu g / L \\
\mu g / L \\
\mu g / L \\
\mu g / L \\
\mu g / L \\
\mu g / L \\
\mu g / L \\
\mu g / L \\
\mu g / L \\
\mu g / L \\
\mu g / L \\
\mu g / L \\
\mu g / L\end{array}$ \\
\hline \multicolumn{16}{|c|}{ Water Quality Indicator Parameters } \\
\hline Parameter & 1095 & $\underline{\text { DF }}$ & Mod & $\underline{\mathbf{S T}}$ & 브 & Flag & $\underline{\text { Lab }}$ & $\underline{2095}$ & $\underline{D F}$ & Mod & $\underline{\text { ST }}$ & $\underline{H}$ & Flag & $\underline{\text { Lab }}$ & Unit \\
\hline \multicolumn{16}{|c|}{ Common Laboratory Contaminants } \\
\hline Parameter & 1095 & $\underline{D F}$ & Mod & $\underline{\text { ST }}$ & $\underline{H}$ & Fiag & $\underline{\text { Lab }}$ & $\underline{2095}$ & $\underline{D F}$ & Mod & $\underline{\text { ST }}$ & $\underline{H}$ & Flag & $\underline{\text { Lab }}$ & Unit \\
\hline $\begin{array}{l}\text { Bis(2-ethylhexyl) phthalate } \\
\text { Dichloromethane }\end{array}$ & $\begin{array}{l}<16 \\
<1.7\end{array}$ & $\begin{array}{l}1 \\
1\end{array}$ & & & & $\begin{array}{l}0 \\
0\end{array}$ & $\begin{array}{l}\text { GE } \\
\text { GE }\end{array}$ & & & & & & & & $\mu g / L$ \\
\hline
\end{tabular}

Note: See Appendix B for flagging criteria. Synchronous water levels are measured over a 3-5 day period.

- = exceeded holding time.

= exceeded groundwater protection standard. 


\section{WELL FSB112D (cont.)}

Radiological Indicator Parameters

\section{Parameter}

Gross alpha Nonvolatile beta

DQ95 Mod

$\begin{array}{ll}3.4 E+02 & 1 \\ 3.1 E+03 & 1\end{array}$

ST

2
2

Measured Radionuclides

Parameter

Actinium-228

Americium-241

Cesium-137

Cobalt -60

Curium-242

Curium-243/244

Curium-245/246

lodine-129

Plutonium-238

Plutonium-239/240

Radium-226

Radium-228

Strontium-89

Technetium-99

Thorium-228

Thorium-230

Thorium-232

Thorium-234

Tritium

Uranium-233/234

Uranium-235

Uranium-238
Strontium-90

$\begin{array}{lllllll}1095 & \text { DF } & \text { Mod } & \text { ST } & \text { H } & \text { Flag } & \text { Lab } \\ 4.6 E+01 & 1 & & & & 0 & \text { GP } \\ 4.3 E-01 & 1 & \text { UI } & & & 0 & \text { GP } \\ -6.9 E-01 & 1 & \text { UI } & & 0 & \text { GP } \\ 5.5 E+00 & 1 & & & 0 & \text { GP } \\ 4.4 E-02 & 1 & \text { UI } & & 0 & \text { GP } \\ 5.5 E-01 & 1 & \text { UI } & & 0 & \text { GP } \\ 8.1 E-02 & 1 & \text { UI } & & 0 & \text { GP } \\ 7.3 E+01 & 1 & & & & 2 & \text { GP } \\ 1.5 E-01 & 1 & \text { UI } & & 0 & \text { GP } \\ -1.6 E-02 & 1 & \text { UI } & & 0 & \text { GP } \\ 9.1 E+01 & 1 & & & 2 & \text { GP } \\ 4.6 E+01 & 1 & \text { J } & & 2 & \text { GP } \\ -4.6 E+02 & 1 & \text { UJI } & & 0 & \text { GP } \\ \text { J.2E }+03 & 1 & J & & 2 & \text { GP } \\ 5.4 E+01 & 1 & & & 0 & \text { GP } \\ 9.7 E-01 & 1 & \text { UI } & & 0 & \text { GP } \\ 7.9 E-01 & 1 & \text { UI } & & 0 & \text { GP } \\ 2.4 E-02 & 1 & \text { UI } & & 0 & \text { GP } \\ 0.0 E+00 & 1 & \text { UI } & & 0 & \text { GP } \\ 8.8 E+03 & 1 & & - & 2 & \text { GP } \\ 1.2 E+01 & 1 & & & 1 & \text { GP } \\ 2.8 E-01 & 1 & \text { UI } & & 0 & \text { GP } \\ 9.4 E+00 & 1 & & & 1 & \text { GP }\end{array}$

Calculated Radionuclides

Americium-243

Nickel-63

Plutonium-241

Plutonium-242
1095

1.6E-03

$5.5 E+00$

$-6.5 \mathrm{E}-02$

$-3.3 \mathrm{E}-07$
DF Mod ST $\underline{H}$ Flag Lab

0
0
0
0

Lab

GP

$\underline{2095}$

$\underline{2095}$

DF Mod ST $\underline{H}$ Flag Lab Unit

pCi/L

$\mathrm{pCi}$

2095 DF Mod ST $\underline{H}$ Flag Lab Unit

pCi/L

pCi/L

pCi/L

pCi/L

PCi/L

$\mathrm{PCi} / \mathrm{L}$

$\mathrm{pCi} / \mathrm{L}$

pCi/L

$\mathrm{pCi} / \mathrm{L}$

pCi/L

pCill

pCi/L

pCi/L

$\mathrm{pCi} / \mathrm{L}$

pCi/L

$\mathrm{PCi} / \mathrm{L}$

pCi/L

pCi/L

$\mathrm{pCi} / \mathrm{mL}$

pCi/L

pCi/L

$\mathrm{pCi} / \mathrm{L}$
Unit

pCi/L

$\mathrm{pCi} / \mathrm{L}$

pCi/L

$\mathrm{pCi} / \mathrm{L}$

Note: See Appendix B for flagging criteria. Synchronous water levels are measured over a 3-5 day period.

- = exceeded holding time.

- = exceeded groundwater protection standard. 


\section{WELL FSB113A}

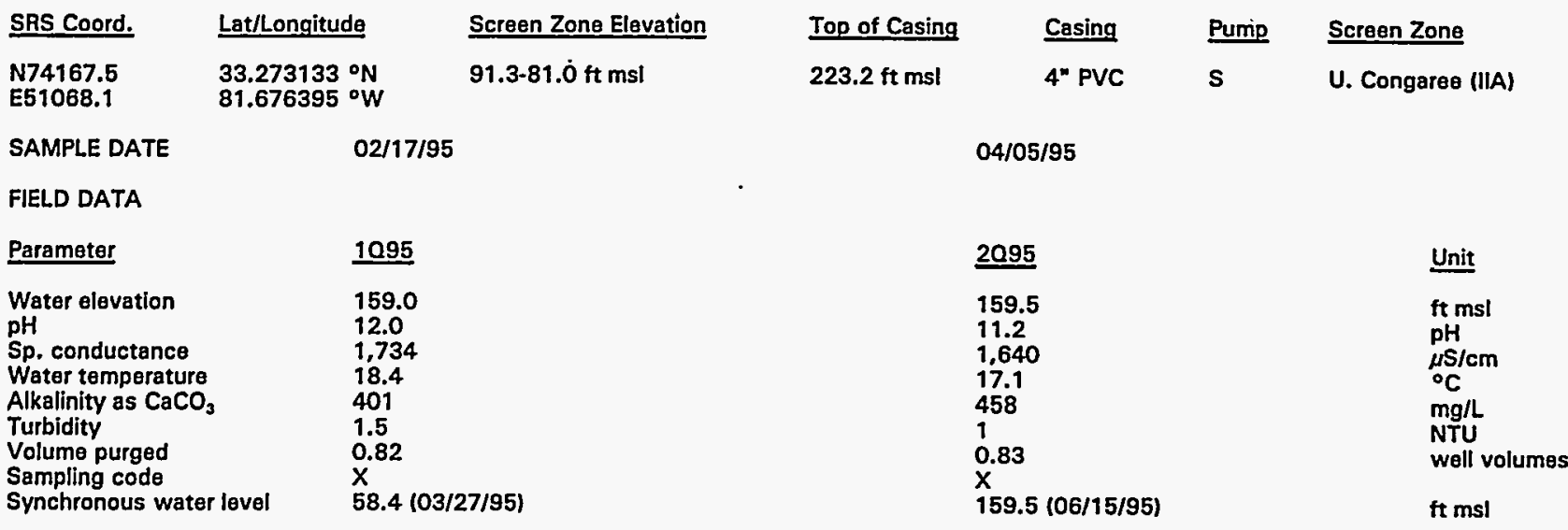

Hazardous Constituents (264.94, Table 1)

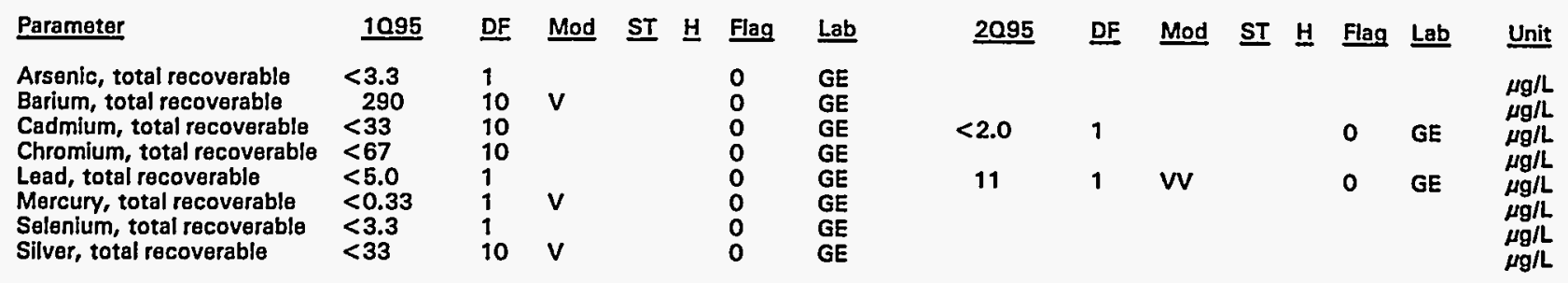

Hazardous Constituents (261 App. VIII/264 App. IX)

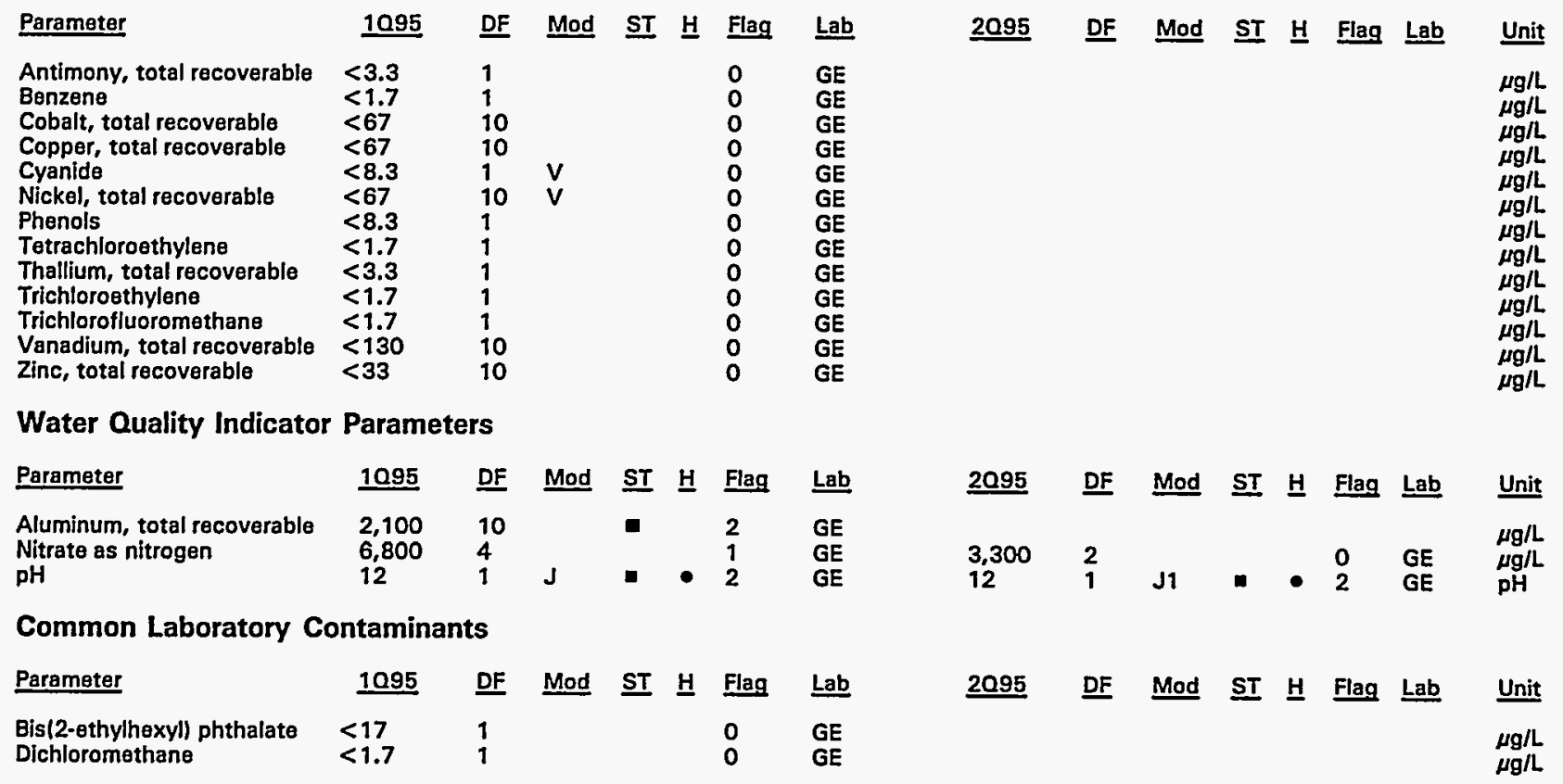

Note: See Appendix B for flagging criteria. Synchronous water levels are measured over a 3-5 day period.

- = exceeded holding time.

- = exceeded groundwater protection standard. 
WELL FSB113A (cont.)

Radiological Indicator Parameters

\begin{tabular}{|c|c|c|c|c|c|c|c|c|c|c|c|c|c|}
\hline Paramater & 1095 & DF & Mod & $\underline{\text { ST }}$ & $\underline{H}$ & Flag & $\underline{\text { Lab }}$ & $\underline{2095}$ & $\underline{D F}$ & Mod & $\underline{\mathbf{S T}} \underline{H}$ & Flag & Lab \\
\hline $\begin{array}{l}\text { Gross alpha } \\
\text { Nonvolatile beta }\end{array}$ & $\begin{array}{l}3.9 E+\infty \\
9.3 E+\infty\end{array}$ & $\begin{array}{l}1 \\
1\end{array}$ & & & & $\begin{array}{l}0 \\
0\end{array}$ & $\begin{array}{l}\text { GP } \\
\text { GP }\end{array}$ & $\begin{array}{l}3.4 E+\infty 0 \\
7.9 E+\infty 0\end{array}$ & 1 & & & $\begin{array}{l}0 \\
0\end{array}$ & $\begin{array}{l}\text { GP } \\
\text { GP }\end{array}$ \\
\hline
\end{tabular}

Measured Radionuclides

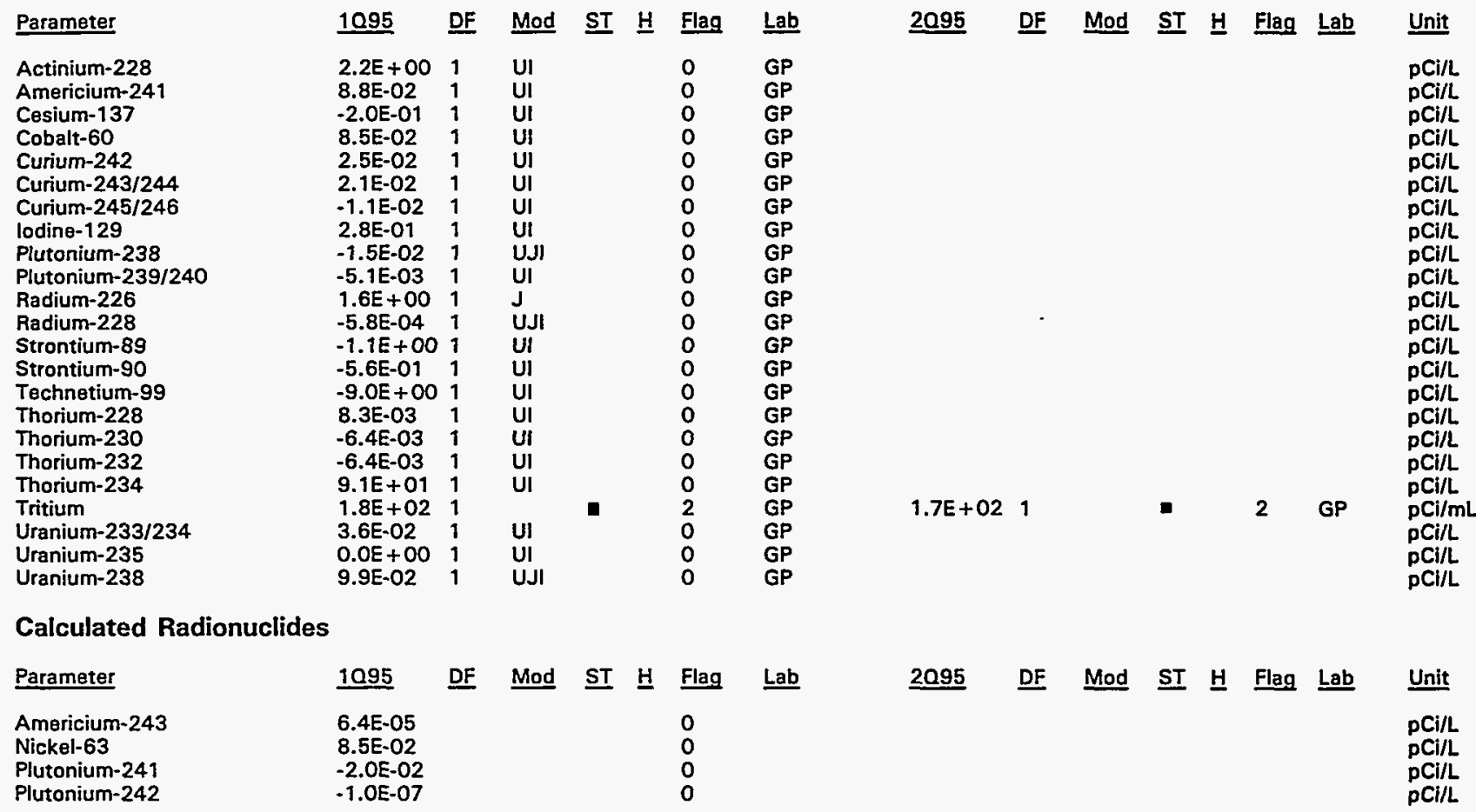

Note: See Appendix B for flagging criteria. Synchronous water levels are measured over a 3-5 day period.

- = exceeded holding time.

- = exceeded groundwater protection standard. 


\section{WELL FSB113C}

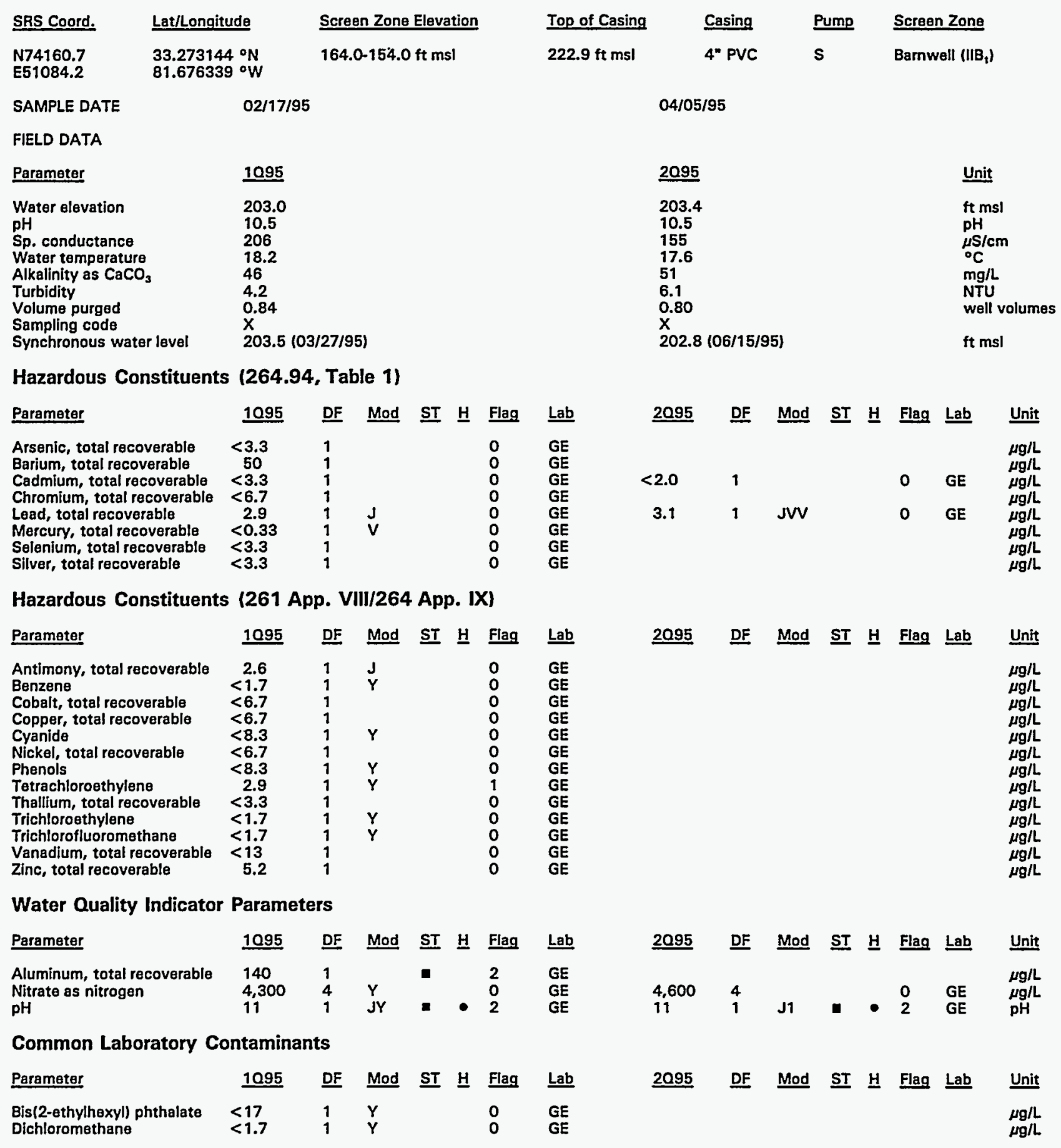

Note: See Appendix B for flagging criteria. Synchronous water levels are measured over a 3-5 day period.

- $=$ exceeded holding time.

- = exceeded groundwater protection standard. 
WELL FSB113C (cont.)

Radiological Indicator Parameters

\begin{tabular}{|c|c|c|c|c|c|c|c|c|c|c|c|c|c|c|}
\hline Parameter & 1095 & DF & Mod & $\underline{\mathrm{ST}}$ & 브 & Flag & Lab & 2095 & DF & Mod & $\underline{\text { ST }}$ & $\underline{\mathrm{H}}$ & Flag & $\underline{L a b}$ \\
\hline $\begin{array}{l}\text { Gross alpha } \\
\text { Nonvolatile beta }\end{array}$ & $\begin{array}{l}8.1 \mathrm{E}-01 \\
1.5 \mathrm{E}+00\end{array}$ & $\begin{array}{l}1 \\
1\end{array}$ & UI & & & $\begin{array}{l}0 \\
0\end{array}$ & $\begin{array}{l}\text { GP } \\
\text { GP }\end{array}$ & $\begin{array}{l}2.8 E-01 \\
1.4 E+00\end{array}$ & $\begin{array}{l}1 \\
1\end{array}$ & UI & & & $\begin{array}{l}0 \\
0\end{array}$ & $\begin{array}{l}\text { GP } \\
\text { GP }\end{array}$ \\
\hline
\end{tabular}

Measured Radionuclides

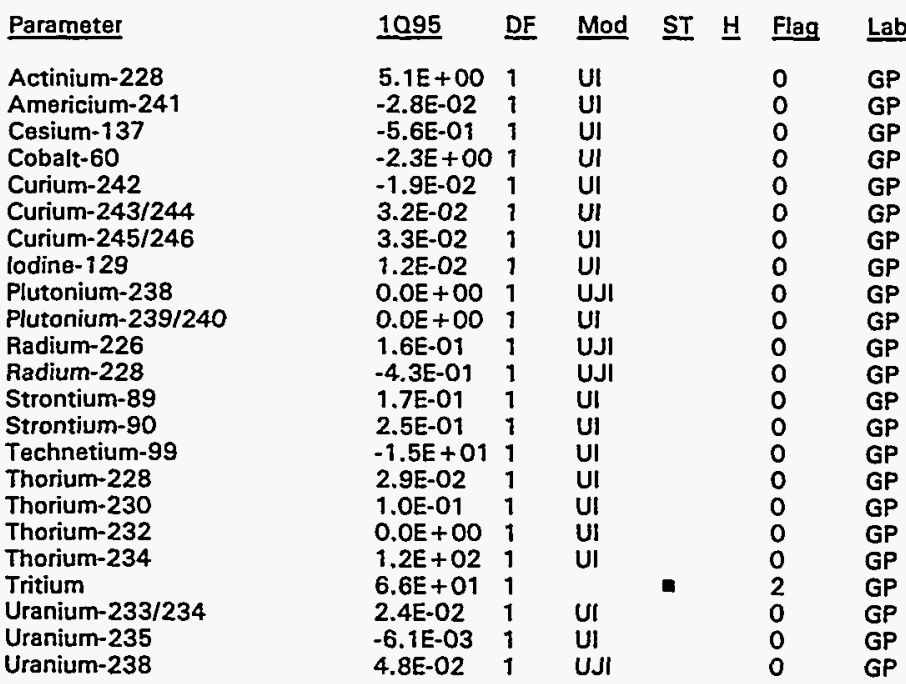

2095 DF Mod ST $\underline{H}$ Flag Lab Unit

GP

GP

GP

GP

GP

GP

GP

GP

GP

GP

GP

GP

GP

GP

$7.0 E+011$

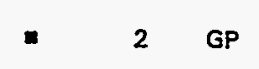

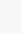

Calculated Radionuclides

Parameter

Americium-243

Nickel-63

Plutonium-241

Plutonium-242

9.7E-05

$-2.3 \mathrm{E}+00$

$0.0 E+00$

$0.0 E+00$

Flag $\quad$ Lab
0
0

DF Mod ST $\underline{H}$ Flag Lab Unit

$\mathrm{pCi} / \mathrm{L}$

$\mathrm{pCi} / \mathrm{L}$

$\mathrm{pCi} / \mathrm{L}$

$\mathrm{PCi} / \mathrm{L}$

Note: See Appendix B for flagging criteria. Synchronous water levels are measured over a 3-5 day period.

- = exceeded holding time.

a = exceeded groundwater protection standard. 
WELL FSB113D

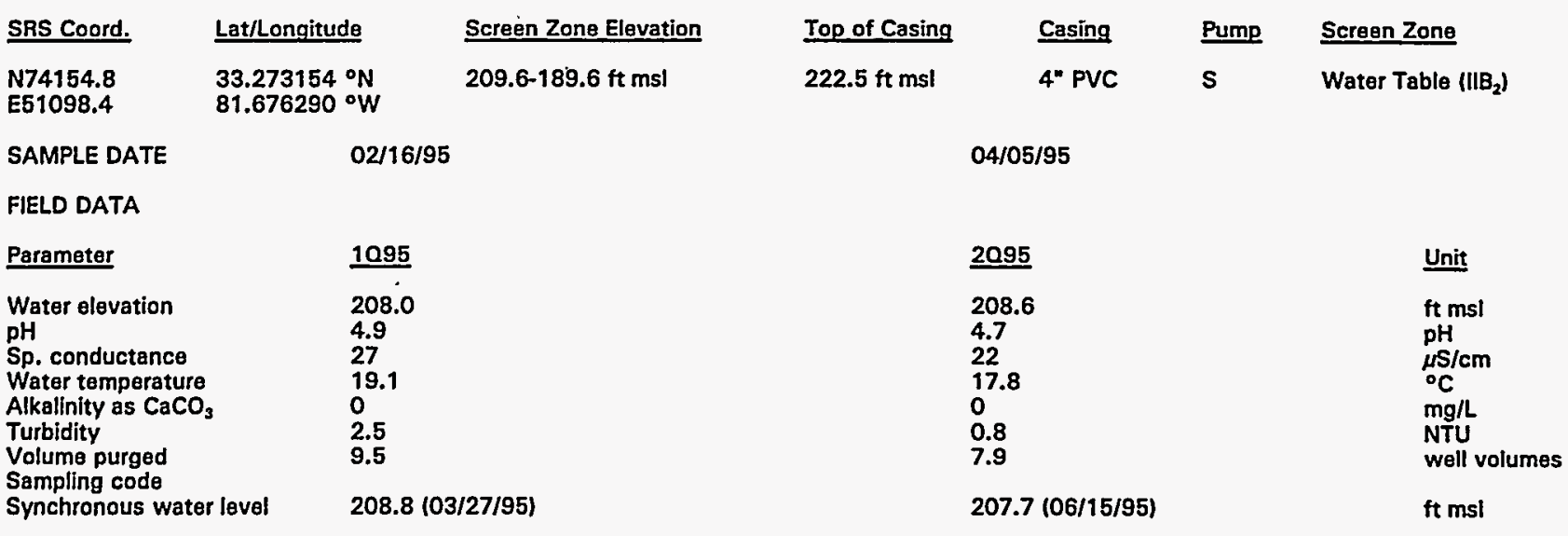

Hazardous Constituents (264.94, Table 1)

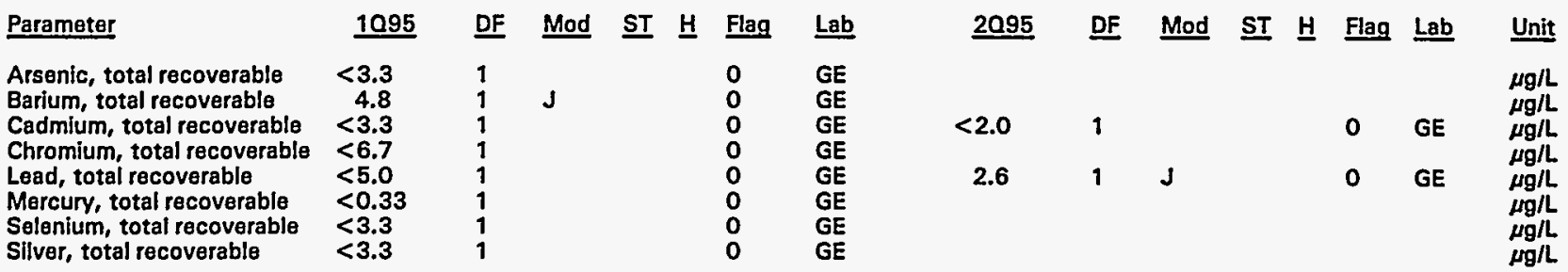

Hazardous Constituents (261 App. VIII/264 App. IX)

\begin{tabular}{|c|c|c|c|c|c|c|c|c|c|c|c|c|c|c|c|}
\hline Parameter & 1095 & $\underline{D F}$ & Mod & ST & H & Flag & Lab & 2095 & DF & Mod & $\underline{\mathbf{S T}}$ & 브 & Flag & Lab & Unit \\
\hline $\begin{array}{l}\text { Antimony, total recoverable } \\
\text { Benzene } \\
\text { Cobalt, total recoverable } \\
\text { Copper, total recoverable } \\
\text { Cyanide } \\
\text { Nickel, total recoverable } \\
\text { Phenols } \\
\text { Tetrachloroethylene } \\
\text { Thallium, total recoverable } \\
\text { Trichloroethylene } \\
\text { Trichlorofluoromethane } \\
\text { Vanadium, total recoverable } \\
\text { Zinc, total recoverable }\end{array}$ & $\begin{aligned}<3.3 \\
<1.7 \\
<6.7 \\
\\
5.7 \\
<8.3 \\
<6.7 \\
<8.3 \\
<1.7 \\
<3.3 \\
<1.7 \\
<1.7 \\
<13 \\
\quad 4.1\end{aligned}$ & $\begin{array}{l}1 \\
1 \\
1 \\
1 \\
1 \\
1 \\
1 \\
1 \\
1 \\
1 \\
1 \\
1 \\
1\end{array}$ & $J$ & & & $\begin{array}{l}0 \\
0 \\
0 \\
0 \\
0 \\
0 \\
0 \\
0 \\
0 \\
0 \\
0 \\
0 \\
0\end{array}$ & $\begin{array}{l}\text { GE } \\
\text { GE } \\
\text { GE } \\
\text { GE } \\
\text { GE } \\
\text { GE } \\
\text { GE } \\
\text { GE } \\
\text { GE } \\
\text { GE } \\
\text { GE } \\
\text { GE } \\
\text { GE }\end{array}$ & & & & & & & & $\begin{array}{l}\mu g / L \\
\mu g / L \\
\mu g / L \\
\mu g / L \\
\mu g / L \\
\mu g / L \\
\mu g / L \\
\mu g / L \\
\mu g / L \\
\mu g / L \\
\mu g / L \\
\mu g / L \\
\mu g / L\end{array}$ \\
\hline \multicolumn{16}{|c|}{ Water Quality Indicator Parameters } \\
\hline Parameter & 1095 & DF & Mod & $\underline{\underline{S T}}$ & $\underline{H}$ & Flag & Lab & $\underline{2095}$ & DF & Mod & $\underline{\mathbf{S T}}$ & $\underline{H}$ & Flag & Lab & Unit \\
\hline $\begin{array}{l}\text { Aluminum, total recoverable } \\
\text { Nitrate as nitrogen } \\
\text { pH }\end{array}$ & $\begin{array}{l}27 \\
730 \\
5.0\end{array}$ & $\begin{array}{l}1 \\
1 \\
1\end{array}$ & $\begin{array}{l}J \\
\text { Ji }\end{array}$ & $\mathbf{E}$ & $\bullet$ & $\begin{array}{l}1 \\
0 \\
0\end{array}$ & $\begin{array}{l}\text { GE } \\
\text { GE } \\
\text { GE }\end{array}$ & $\begin{array}{l}720 \\
5.1\end{array}$ & $\begin{array}{l}1 \\
1\end{array}$ & $J 1$ & - & $\bullet$ & $\begin{array}{l}0 \\
0\end{array}$ & $\begin{array}{l}\text { GE } \\
\text { GE }\end{array}$ & $\begin{array}{l}\mu g / L \\
\mu g / L \\
p H\end{array}$ \\
\hline \multicolumn{16}{|c|}{ Common Laboratory Contaminants } \\
\hline Paramater & 1095 & DF & Mod & $\underline{\text { ST }}$ & H & Flag & Lab & 2095 & DF & Mod & $\underline{\text { ST }}$ & $\underline{H}$ & Flag & Lab & Unit \\
\hline $\begin{array}{l}\text { Bis(2-ethylhexyl) phthalate } \\
\text { Dichloromethane }\end{array}$ & $\begin{array}{l}<17 \\
<1.7\end{array}$ & $\begin{array}{l}1 \\
1\end{array}$ & V & & & $\begin{array}{l}0 \\
0\end{array}$ & $\begin{array}{l}\text { GE } \\
\text { GE }\end{array}$ & & & & & & & & $\begin{array}{l}\mu g / L \\
\mu g / L\end{array}$ \\
\hline
\end{tabular}

Note: See Appendix B for flagging criteria. Synchronous water levels are measured over a 3-5 day period. - = exceeded holding time.

- =xceeded groundwater protection standard. 
WELL. FSB113D (cont.)

Radiological Indicator Parameters

\begin{tabular}{|c|c|c|c|c|c|c|c|c|c|c|c|c|c|c|c|}
\hline Parameter & 1095 & $\underline{\text { DF }}$ & Mod & $\underline{\mathbf{S T}}$ & $\underline{H}$ & Flag & $\underline{\text { Lab }}$ & $\underline{2095}$ & DF & Mod & $\underline{\mathbf{S T}}$ & $\underline{H}$ & Flag & $\underline{\text { Lab }}$ & $\underline{\text { Unit }}$ \\
\hline $\begin{array}{l}\text { Gross alpha } \\
\text { Nonvolatile beta }\end{array}$ & $\begin{array}{l}1.9 \mathrm{E}-01 \\
3.2 \mathrm{E}-01\end{array}$ & $\begin{array}{l}1 \\
1\end{array}$ & UI & & & $\begin{array}{l}0 \\
0\end{array}$ & $\begin{array}{l}\text { GP } \\
\text { GP }\end{array}$ & $\begin{array}{l}9.3 E-01 \\
9.1 E-02\end{array}$ & $\begin{array}{l}1 \\
1\end{array}$ & $J_{u}$ & & & $\begin{array}{l}0 \\
0\end{array}$ & $\begin{array}{l}\text { GP } \\
\text { GP }\end{array}$ & $\begin{array}{l}\mathrm{pCi} / \mathrm{L} \\
\mathrm{pCi} / \mathrm{L}\end{array}$ \\
\hline \multicolumn{16}{|c|}{ Measured Radionuclides } \\
\hline Parameter & 1095 & $\underline{D F}$ & $\underline{\text { Mod }}$ & $\underline{\mathbf{S T}}$ & $\underline{H}$ & Flag & Lab & $\underline{2095}$ & $\underline{\text { OF }}$ & Mod & $\underline{\text { ST }}$ & 브 & Flag & $\underline{\text { Lab }}$ & $\underline{\text { Unit }}$ \\
\hline $\begin{array}{l}\text { Actinium-228 } \\
\text { Amsricium-241 } \\
\text { Cesium-137 } \\
\text { Cobalt-60 } \\
\text { Curium-242 } \\
\text { Curium-243/244 } \\
\text { Curium-245/246 } \\
\text { lodine-129 } \\
\text { Plutonium-238 } \\
\text { Plutonium-239/240 } \\
\text { Radium-226 } \\
\text { Radium-228 } \\
\text { Strontium-89 } \\
\text { Strontium-90 } \\
\text { Technetium-99 } \\
\text { Thorium-228 } \\
\text { Thorium-230 } \\
\text { Thorium-232 } \\
\text { Thorium-234 } \\
\text { Tritium } \\
\text { Uranium-233/234 } \\
\text { Uranium-235 } \\
\text { Uranium-238 }\end{array}$ & $\begin{array}{l}4.0 E+00 \\
4.7 E-02 \\
1.1 E+00 \\
-1.2 E-01 \\
2.1 E-02 \\
1.4 E-02 \\
-4.6 E-03 \\
1.4 E+00 \\
5.8 E-04 \\
-1.1 E-02 \\
1.6 E-01 \\
-4.1 E-01 \\
-7.5 E-01 \\
-5.4 E-01 \\
-8.9 E+00 \\
7.3 E-02 \\
0.0 E+00 \\
-1.7 E-02 \\
5.1 E+01 \\
9.8 E+00 \\
7.5 E-02 \\
4.0 E-02 \\
8.5 E-02\end{array}$ & $\begin{array}{l}1 \\
1 \\
1 \\
1 \\
1 \\
1 \\
1 \\
1 \\
1 \\
1 \\
1 \\
1 \\
1 \\
1 \\
1 \\
1 \\
1 \\
1 \\
1 \\
1 \\
1 \\
1 \\
1\end{array}$ & $\begin{array}{l}\text { UI } \\
\text { UI } \\
\text { UI } \\
\text { UI } \\
\text { UI } \\
\text { UI } \\
\text { UII } \\
\text { UII } \\
\text { UI } \\
\text { UI } \\
\text { UI } \\
\text { UII } \\
\text { UI } \\
\text { UII } \\
\text { UII } \\
\text { UI } \\
\text { UI } \\
\text { UI } \\
\text { UI } \\
\text { UI } \\
\text { UII } \\
\text { UI }\end{array}$ & & & $\begin{array}{l}0 \\
0 \\
0 \\
0 \\
0 \\
0 \\
0 \\
0 \\
0 \\
0 \\
0 \\
0 \\
0 \\
0 \\
0 \\
0 \\
0 \\
0 \\
0 \\
0 \\
0 \\
0 \\
0\end{array}$ & $\begin{array}{l}\text { GP } \\
\text { GP } \\
\text { GP } \\
\text { GP } \\
\text { GP } \\
\text { GP } \\
\text { GP } \\
\text { GP } \\
\text { GP } \\
\text { GP } \\
\text { GP } \\
\text { GP } \\
\text { GP } \\
\text { GP } \\
\text { GP } \\
\text { GP } \\
\text { GP } \\
\text { GP } \\
\text { GP } \\
\text { GP } \\
\text { GP } \\
\text { GP } \\
\text { GP }\end{array}$ & $9.5 E+00$ & 1 & & & & 0 & GP & 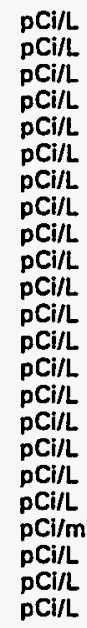 \\
\hline \multicolumn{16}{|c|}{ Calculated Radionuclides } \\
\hline Parameter & 1095 & DF & Mod & $\underline{\text { ST }}$ & $\underline{\mathrm{H}}$ & Flag & $\underline{\text { Lab }}$ & $\underline{2095}$ & $\underline{\mathrm{DF}}$ & Mad & $\underline{\text { ST }}$ & $\underline{H}$ & Flag & $\underline{\text { Lab }}$ & $\underline{\text { Unit }}$ \\
\hline $\begin{array}{l}\text { Americium-243 } \\
\text { Nickel-63 } \\
\text { Plutonium-241 } \\
\text { Plutonium-242 }\end{array}$ & $\begin{array}{l}4.3 E-05 \\
-1.2 E-01 \\
-4.4 E-02 \\
-2.2 E-07\end{array}$ & & & & & $\begin{array}{l}0 \\
0 \\
0 \\
0\end{array}$ & & & & & & & & & $\begin{array}{l}\text { pCi/L } \\
\text { pCi/L } \\
\text { pCi/L } \\
\text { pCill }\end{array}$ \\
\hline
\end{tabular}

Note: See Appendix B for flagging criteria. Synchronous water levels are measured over a 3-5 day period.

- = exceeded holding time.

- exceeded groundwater protection standard. 
WELL FSB114A

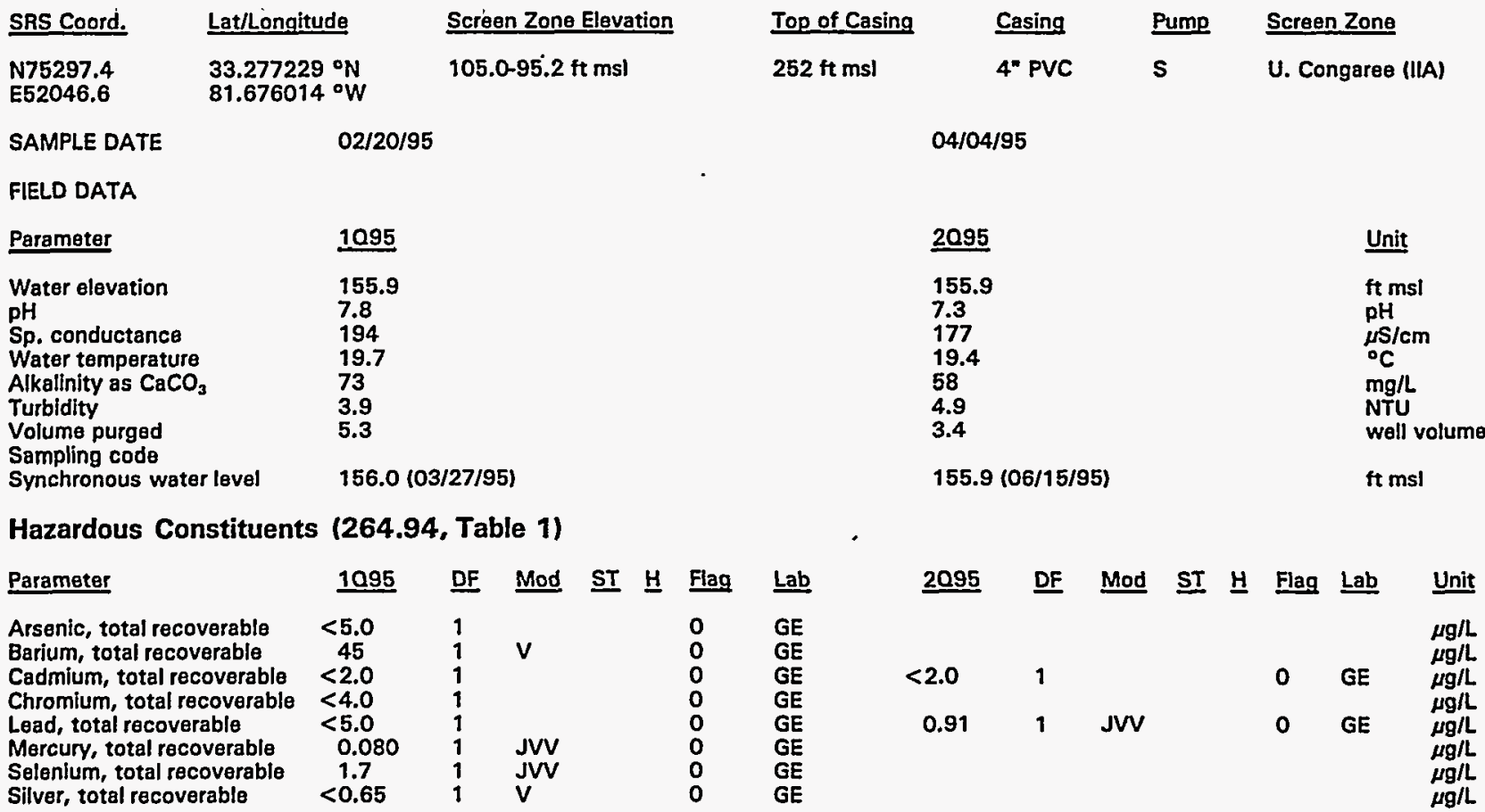

Hazardous Constituents (261 App. VIII/264 App. IX)

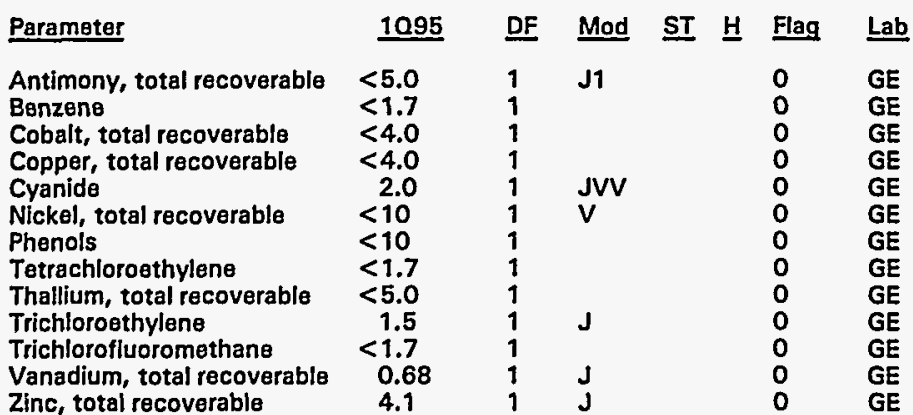

Zinc, total recoverable

Water Quality Indicator Parameters

\begin{tabular}{|c|c|c|c|c|c|c|c|c|c|c|c|c|c|c|c|}
\hline Parameter & 1095 & DF & Mod & $\underline{\mathbf{S T}}$ & H & Flag & $\underline{\text { Lab }}$ & $\underline{2095}$ & $\underline{\mathrm{DF}}$ & Mod & $\underline{\mathbf{S T}}$ & $\underline{H}$ & Flag & $\underline{\text { Lab }}$ & Unit \\
\hline $\begin{array}{l}\text { Aluminum, total recoverable } \\
\text { Nitrate as nitrogen } \\
\text { pH }\end{array}$ & $\begin{array}{l}110 \\
1,800 \\
7.7\end{array}$ & $\begin{array}{l}1 \\
1 \\
1\end{array}$ & J1 & - & & $\begin{array}{l}2 \\
0 \\
0\end{array}$ & $\begin{array}{l}\text { GE } \\
\text { GE } \\
\text { GE }\end{array}$ & $\begin{array}{l}960 \\
7.7\end{array}$ & $\begin{array}{l}1 \\
1\end{array}$ & J1 & & $\bullet$ & $\begin{array}{l}0 \\
0\end{array}$ & $\begin{array}{l}\text { GE } \\
\text { GE }\end{array}$ & $\underset{\mathrm{pH}}{\mu \mathrm{g} / \mathrm{L} / \mathrm{L}}$ \\
\hline \multicolumn{16}{|c|}{ Common Laboratory Contaminants } \\
\hline Parameter & 10.95 & DF & Mod & $\underline{\mathbf{S T}}$ & $\underline{H}$ & Flag & $\underline{\text { Lab }}$ & $\underline{2095}$ & $\underline{\text { DF }}$ & Mod & $\underline{\text { ST }}$ & $\underline{H}$ & Flag & $\underline{\text { Lab }}$ & Unit \\
\hline $\begin{array}{l}\text { Bis(2-ethylhexyl) phthalate } \\
\text { Dichloromethane }\end{array}$ & $\begin{array}{l}<17 \\
<1.7\end{array}$ & $\begin{array}{l}1 \\
1\end{array}$ & & & & $\begin{array}{l}0 \\
0\end{array}$ & $\begin{array}{l}\text { GE } \\
\text { GE }\end{array}$ & & & & & & & & \\
\hline
\end{tabular}

Note: See Appendix B for flagging criteria. Synchronous water levels are measured over a 3-5 day period. $\bullet=$ exceeded holding time.

- = exceeded groundwater protection standard. 
WELL FSB114A (cont.)

Radiological Indicator Parameters

\begin{tabular}{|c|c|c|c|c|c|c|c|c|c|c|c|c|c|c|}
\hline Parameter & 1095 & DF & Mod & ST & $\underline{\mathrm{H}}$ & Flag & Lab & 2095 & DF & Mod & ST $\underline{H}$ & Flag & Lab & Unit \\
\hline $\begin{array}{l}\text { Gross alpha } \\
\text { Nonvolatile beta }\end{array}$ & $\begin{array}{l}\text { 6.0E-01 } \\
8.8 E-01\end{array}$ & $\begin{array}{l}1 \\
1\end{array}$ & UI & & & $\begin{array}{l}0 \\
0\end{array}$ & $\begin{array}{l}\text { GP } \\
\text { GP }\end{array}$ & $\begin{array}{l}1.4 E+00 \\
1.1 E+00\end{array}$ & $\begin{array}{l}1 \\
1\end{array}$ & UJI & & $\begin{array}{l}0 \\
0\end{array}$ & $\begin{array}{l}\text { GP } \\
\text { GP }\end{array}$ & $\begin{array}{l}\mathrm{pCi} / \mathrm{L} \\
\mathrm{pCi} / \mathrm{L}\end{array}$ \\
\hline
\end{tabular}

Measured Radionuclides

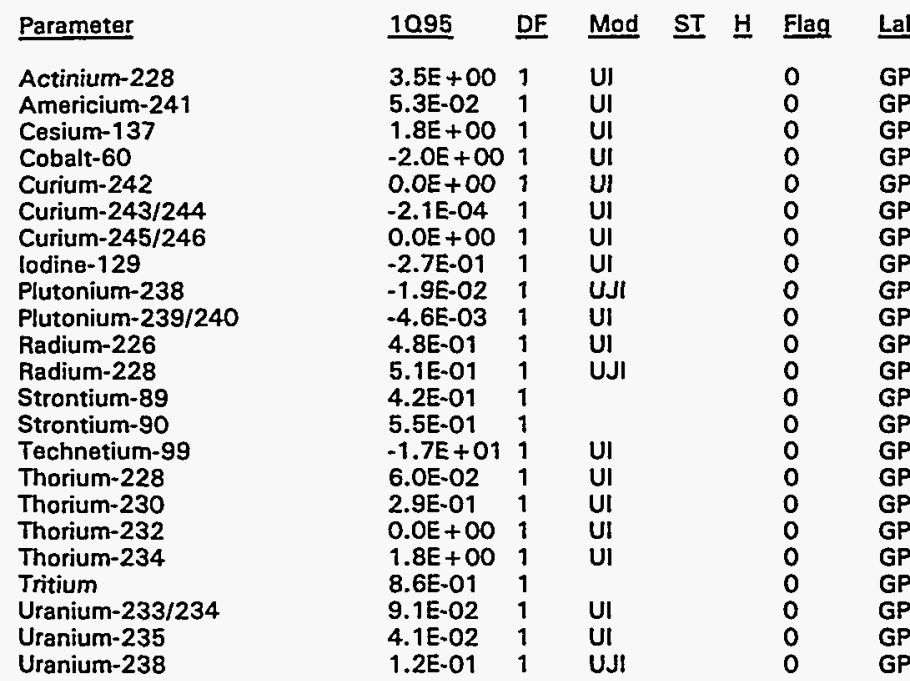

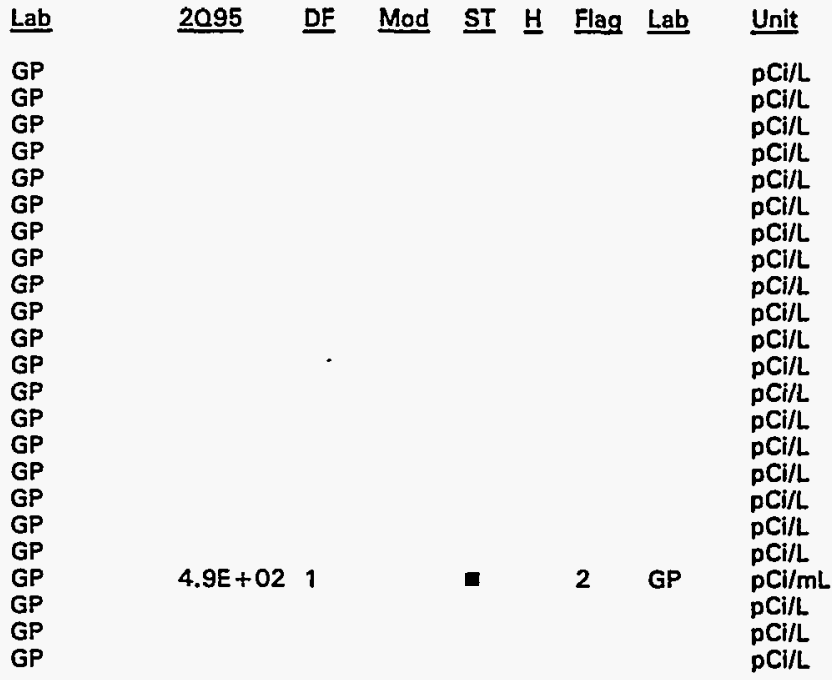

Calculated Radionuclides

Parameter

Americium-243

Nickel-63

Plutonium-241

Plutonium-242
1095 DF Mod ST H Flag Lab

0.07

$-2.0 \mathrm{E}+00$

$-1.9 \mathrm{E}-02$

$-9.3 \mathrm{E}-08$

$\underline{2095}$

DF Mod ST H Flag Lab Unit

pCi/L

pCill

$\mathrm{pCi} / \mathrm{h}$

$\mathrm{PCi} / \mathrm{L}$

Note: See Appendix B for flagging criteria. Synchronous water levels are measured over a 3-5 day period.

- = exceeded holding time.

- = exceeded groundwater protection standard. 
WELL FSB114C

\begin{tabular}{lll} 
SRS Coord. & Lat/Lóngitude & Screen Zone Elevation \\
\cline { 2 - 3 } N75288.5 & $33.277188 \circ \mathrm{N}$ & $168.0-158.0 \mathrm{ft} \mathrm{msl}$ \\
E52033.8 & $81.676030^{\circ} \mathrm{W}$ & \\
SAMPLE DATE & $02 / 20 / 95$ \\
FIELD DATA & \\
Parameter & $\underline{ }$ & \\
\hline Water elevation & 213.5 \\
pH & 5.7 \\
Sp. conductance & 60 \\
Water temperature & 19.7 \\
Alkalinity as CaCO & 8 \\
Turbidity & 0.8 \\
Volume purged & 2.9 \\
S8mpling code & \\
Synchronous water level & 214.0 (03/27/95)
\end{tabular}

$\begin{array}{rrrrr}\frac{\text { Top of Casing }}{252.2 \mathrm{ft} \mathrm{msl}} & \text { Casing } & \frac{\text { Pump }}{\text { 4" PVC }} & \text { S } & \text { Barnwell (IIB, }) \\ 04 / 04 / 95 & & \end{array}$

Hazardous Constituents (264.94, Table 1)

\begin{tabular}{|c|c|c|c|c|c|c|c|c|c|c|c|c|}
\hline Parameter & 1095 & DF & $\underline{\operatorname{Mod}}$ ST $\underline{H}$ & Flag & Lab & $\underline{2095}$ & DF & Mod & $\underline{\text { ST }} \underline{H}$ & Flag & Lab & $\underline{\text { Unit }}$ \\
\hline $\begin{array}{l}\text { Arsenic, total recoverable } \\
\text { Barium, total recoverable } \\
\text { Cadmium, total recoverable } \\
\text { Chromium, total recoverable } \\
\text { Lead, total recoverable } \\
\text { Mercury, total recoverable } \\
\text { Selenium, total recoverable } \\
\text { Silver, total recoverable }\end{array}$ & $\begin{array}{l}<5.0 \\
12 \\
<2.0 \\
<4.0 \\
1.8 \\
0.088 \\
1.5 \\
<0.65\end{array}$ & $\begin{array}{l}1 \\
1 \\
1 \\
1 \\
1 \\
1 \\
1 \\
1\end{array}$ & $\begin{array}{l}\text { J } \\
\text { JVV } \\
\text { JVV } \\
V\end{array}$ & $\begin{array}{l}0 \\
0 \\
0 \\
0 \\
0 \\
0 \\
0 \\
0\end{array}$ & $\begin{array}{l}\mathrm{GE} \\
\mathrm{GE} \\
\mathrm{GE} \\
\mathrm{GE} \\
\mathrm{GE} \\
\mathrm{GE} \\
\mathrm{GE} \\
\mathrm{GE}\end{array}$ & $\begin{array}{r}<2.0 \\
2.7\end{array}$ & 1 & JVV & & $\begin{array}{l}0 \\
0\end{array}$ & $\begin{array}{l}\text { GE } \\
\text { GE }\end{array}$ & 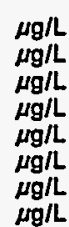 \\
\hline
\end{tabular}

Hazardous Constituents (261 App. VIII/264 App. IX)

\begin{tabular}{|c|c|c|c|c|c|c|c|c|c|c|c|c|c|c|c|}
\hline Parameter & 1095 & $\underline{D F}$ & Mod & $\underline{\text { ST }}$ & $\underline{\mathbf{H}}$ & Flag & $\underline{\text { Lab }}$ & $\underline{2095}$ & DF & Mod & $\underline{\mathbf{S T}}$ & $\underline{\mathrm{H}}$ & Flag & $\underline{\text { Lab }}$ & $\underline{\text { Unit }}$ \\
\hline $\begin{array}{l}\text { Antimony, total recoverable } \\
\text { Benzene } \\
\text { Cobalt, total recoverable } \\
\text { Copper, total recoverable } \\
\text { Cyanide } \\
\text { Nickel, total recoverable } \\
\text { Phenols } \\
\text { Tetrachloroethylene } \\
\text { Thallum, total recoverable } \\
\text { Trichloroethylene } \\
\text { Trichlorofluoromethane } \\
\text { Vanadium, total recoverable } \\
\text { Zinc, total recoverable }\end{array}$ & $\begin{array}{r}3.3 \\
<1.7 \\
<4.0 \\
1.4 \\
<20 \\
2.0 \\
<10 \\
<1.7 \\
<5.0 \\
<1.7 \\
<1.7 \\
<10 \\
7.9\end{array}$ & $\begin{array}{l}1 \\
1 \\
1 \\
1 \\
1 \\
1 \\
1 \\
1 \\
1 \\
1 \\
1 \\
1 \\
1\end{array}$ & $\begin{array}{l}\text { J1 } \\
\text { J } \\
\text { JVV }\end{array}$ & & & $\begin{array}{l}1 \\
0 \\
0 \\
0 \\
0 \\
0 \\
0 \\
0 \\
0 \\
0 \\
0 \\
0 \\
0\end{array}$ & $\begin{array}{l}\mathrm{GE} \\
\mathrm{GE} \\
\mathrm{GE} \\
\mathrm{GE} \\
\mathrm{GE} \\
\mathrm{GE} \\
\mathrm{GE} \\
\mathrm{GE} \\
\mathrm{GE} \\
\mathrm{GE} \\
\mathrm{GE} \\
\mathrm{GE} \\
\mathrm{GE}\end{array}$ & & & & & & & & $\begin{array}{l}\mu \mathrm{g} / \mathrm{L} \\
\mu \mathrm{g} / \mathrm{L} \\
\mu \mathrm{g} / \mathrm{L} \\
\mu \mathrm{g} / \mathrm{L} \\
\mu \mathrm{g} / \mathrm{L} \\
\mu \mathrm{g} / \mathrm{L} \\
\mu \mathrm{g} / \mathrm{L} \\
\mu \mathrm{g} / \mathrm{L} \\
\mu \mathrm{g} / \mathrm{L} \\
\mu \mathrm{g} / \mathrm{L} \\
\mu \mathrm{g} / \mathrm{L} \\
\mu \mathrm{g} / \mathrm{L} \\
\mu \mathrm{g} / \mathrm{L}\end{array}$ \\
\hline \multicolumn{16}{|c|}{ Water Quality Indicator Parameters } \\
\hline Parameter & 1095 & $\underline{\mathrm{DF}}$ & Mod & $\underline{\mathbf{S T}}$ & $\underline{\mathbf{H}}$ & Flag & $\underline{\text { Lab }}$ & $\underline{2095}$ & $\underline{\text { DF }}$ & Mod & $\underline{\mathbf{S T}}$ & $\underline{H}$ & Flag & $\underline{\text { Lab }}$ & $\underline{\text { Unit }}$ \\
\hline $\begin{array}{l}\text { Aluminum, total recoverable } \\
\text { Nitrate as nitrogen } \\
\text { pH }\end{array}$ & $\begin{array}{l}8.4 \\
1.800 \\
5.8\end{array}$ & $\begin{array}{l}1 \\
1 \\
1\end{array}$ & $\begin{array}{l}\text { J } \\
\text { J1 }\end{array}$ & - & & $\begin{array}{l}0 \\
0 \\
0\end{array}$ & $\begin{array}{l}\text { GE } \\
\text { GE } \\
\text { GE }\end{array}$ & $\begin{array}{l}1,700 \\
5.9\end{array}$ & $\begin{array}{l}1 \\
1\end{array}$ & J1 & - & $\bullet$ & $\begin{array}{l}0 \\
0\end{array}$ & $\begin{array}{l}\text { GE } \\
\text { GE }\end{array}$ & $\underset{\mathrm{pH}}{\mu \mathrm{g} / \mathrm{L}}$ \\
\hline \multicolumn{16}{|c|}{ Common Laboratory Contaminants } \\
\hline Parameter & 1095 & DF & Mod & $\underline{\mathbf{S T}}$ & H & Flag & $\underline{\text { Lab }}$ & 2095 & $\underline{D F}$ & Mod & ST & 브 & Flag & $\underline{\text { Lab }}$ & $\underline{\text { Unit }}$ \\
\hline $\begin{array}{l}\text { Bis(2-ethylhexyl) phthalate } \\
\text { Dichloromethane }\end{array}$ & $\begin{array}{l}<17 \\
<1.7\end{array}$ & $\begin{array}{l}1 \\
1\end{array}$ & & & & $\begin{array}{l}0 \\
0\end{array}$ & $\begin{array}{l}\text { GE } \\
\text { GE }\end{array}$ & & & & & & & & $\underset{\mu g / L}{\mu g / L}$ \\
\hline
\end{tabular}

Note: See Appendix B for flagging criteria. Synchronous water levels are measured over a 3-5 day period.

- = exceeded holding time.

- = exceeded groundwater protection standard. 
WELL FSB1 14C (cont.)

Radiological Indicator Parameters

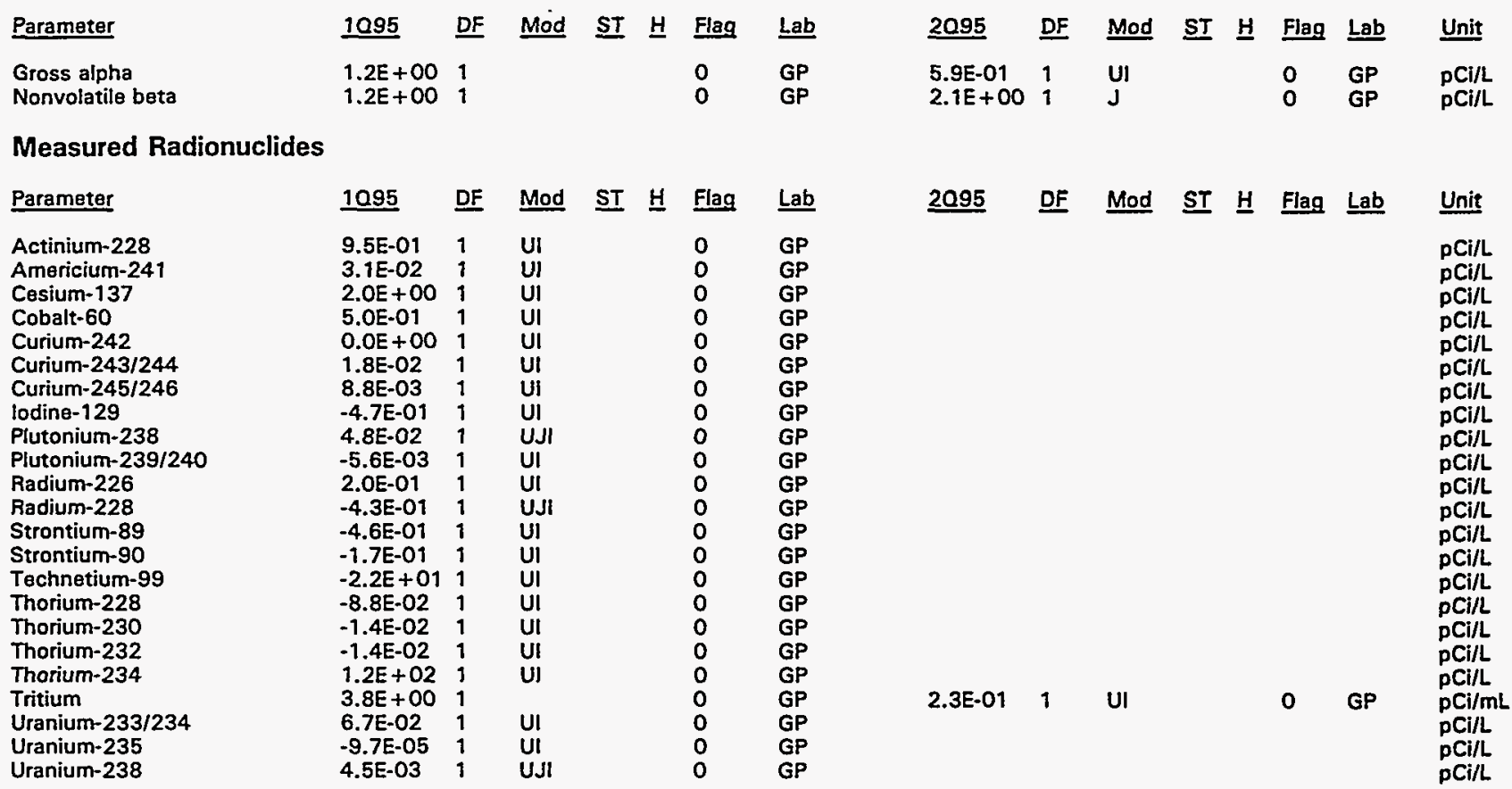

Calculated Radionuclides

Parameter

Americium-243

Nickel-63

Plutonium-241

Plutonium-242
1095

5.3E-05

5.0E-O1

$-2.2 \mathrm{E}-02$

$-1.1 E-07$
DF Mod ST $\underline{H}$ Flag Lab

$\underline{2095}$

Unit

$\mathrm{pCi/L}$

$\mathrm{PCI} / \mathrm{L}$

$\mathrm{pCill}$

$\mathrm{pCi} / \mathrm{L}$

Note: See Appendix B for flagging criteria. Synchronous water levels are measured over a 3-5 day period.

$\bullet=$ exceeded holding time.

- = exceeded groundwater protection standard. 


\section{WELL FSB114D}

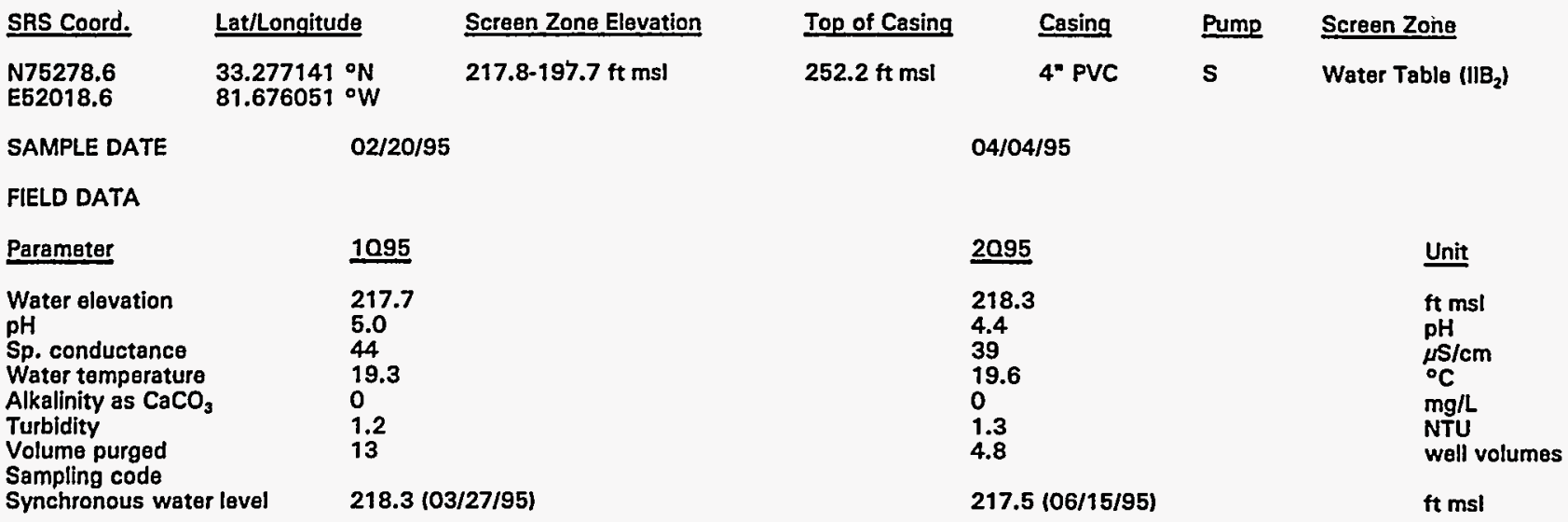

Hazardous Constituents (264.94, Table 1)

$\begin{array}{lcccccccc}\text { Parameter } & 1095 & & \text { DF } & \text { Mod } & \text { ST } & \text { H } & \text { Flag } & \text { La } \\ \text { Arsenic, total recoverable } & <5.0 & 1 & & & & 0 & \\ \text { Barium, total recoverable } & 8.6 & 1 & V & & & 0 & \text { GE } \\ \text { Cadmium, total recoverable } & <2.0 & 1 & & & 0 & \text { GE } \\ \text { Chromium, total recoverable } & <4.0 & 1 & & & 0 & \text { GE } \\ \text { Lead, total recoverable } & 2.4 & 1 & \text { J } & & 0 & \text { GE } \\ \text { Mercury, total recoverable } & 0.17 & 1 & \text { JVV } & & 0 & \text { GE } \\ \text { Selenium, total recoverable } & 1.5 & 1 & \text { JVV } & & 0 & \text { GE } \\ \text { Silver, total recoverable } & <0.65 & 1 & V & & 0 & \text { GE }\end{array}$

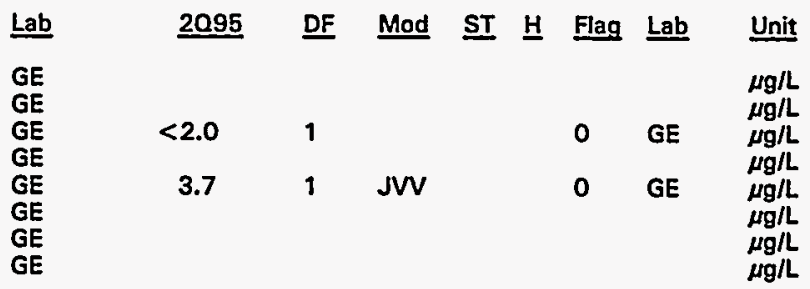

Hazardous Constituents (261 App. VIII/264 App. IX)

$\begin{array}{lcclcccc}\text { Parameter } & 1095 & \text { DF } & \text { Mod } & \text { ST } & \text { Flag } & \text { Lab } \\ \text { Antimony, total recoverable } & <5.0 & 1 & \mathrm{~J} 1 & & 0 & \text { GE } \\ \text { Benzene } & <1.7 & 1 & & & 0 & \text { GE } \\ \text { Cobalt, total recoverable } & 0.88 & 1 & \mathrm{~J} & & 0 & \mathrm{GE} \\ \text { Copper, total recoverable } & 6.8 & 1 & & & 0 & \mathrm{GE} \\ \text { Cyanide } & <20 & 1 & \mathrm{G} & & 0 & \mathrm{GE} \\ \text { Nickel, total recoverable } & <10 & 1 & \mathrm{~V} & & 0 & \mathrm{GE} \\ \text { Phenols } & <10 & 1 & \mathrm{~J} 1 & & 0 & \mathrm{GE} \\ \text { Tetrachloroethylene } & <1.7 & 1 & & & 0 & \mathrm{GE} \\ \text { Thallium, total recoverable } & <5.0 & 1 & & & 0 & \mathrm{GE} \\ \text { Trichloroethylene } & <1.7 & 1 & & & 0 & \mathrm{GE} \\ \text { Trichlorofluoromethane } & <1.7 & 1 & & & 0 & \mathrm{GE} \\ \text { Vanadium, total recoverable } & 0.45 & 1 & \mathrm{~J} & & 0 & \mathrm{GE} \\ \text { Zinc, total recoverable } & 6.0 & 1 & & & 0 & \mathrm{GE}\end{array}$

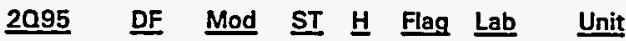

Water Quality Indicator Parameters

\begin{tabular}{|c|c|c|c|c|c|c|c|c|c|c|c|c|c|c|c|}
\hline Paramater & $\underline{1095}$ & $\underline{\text { DF }}$ & Mod & $\underline{\text { ST }}$ & $\underline{\mathrm{H}}$ & Flag & $\underline{\text { Lab }}$ & $\underline{2095}$ & $\underline{D F}$ & Mod & $\underline{\text { ST }}$ & $\underline{H}$ & Flag & $\underline{\text { Lab }}$ & $\underline{\text { Unit }}$ \\
\hline $\begin{array}{l}\text { Aluminum, total recoverable } \\
\text { Nitrate as nitrogen } \\
\text { pH }\end{array}$ & $\begin{array}{l}32 \\
1,600 \\
5.1\end{array}$ & $\begin{array}{l}1 \\
1 \\
1\end{array}$ & J1 & $=$ & & $\begin{array}{l}1 \\
0 \\
0\end{array}$ & $\begin{array}{l}\text { GE } \\
\text { GE } \\
\text { GE }\end{array}$ & $\begin{array}{l}1,200 \\
5.4\end{array}$ & $\begin{array}{l}1 \\
1\end{array}$ & J1 & - & $\bullet$ & $\begin{array}{l}0 \\
0\end{array}$ & $\begin{array}{l}\text { GE } \\
\text { GE }\end{array}$ & $\begin{array}{l}\mu \mathrm{g} / \mathrm{L} \\
\mu \mathrm{g} / \mathrm{L} \\
\mathrm{pH}\end{array}$ \\
\hline \multicolumn{16}{|c|}{ Common Laboratory Contaminants } \\
\hline Parameter & 1095 & $\underline{\text { DF }}$ & Mod & $\underline{\text { ST }}$ & $\underline{H}$ & Flag & $\underline{\text { Lab }}$ & $\underline{2095}$ & DF & Mod & $\underline{\mathbf{S T}}$ & $\underline{H}$ & Flag & $\underline{\text { Lab }}$ & $\underline{\text { Unit }}$ \\
\hline $\begin{array}{l}\text { Bis(2-ethylhexyl) phthalate } \\
\text { Dichloromethane }\end{array}$ & $\begin{array}{l}<17 \\
<1.7\end{array}$ & $\begin{array}{l}1 \\
1\end{array}$ & & & & $\begin{array}{l}0 \\
0\end{array}$ & $\begin{array}{l}\text { GE } \\
\text { GE }\end{array}$ & & & & & & & & $\begin{array}{l}\mu \mathrm{g} / \mathrm{L} \\
\mu \mathrm{g} / \mathrm{L}\end{array}$ \\
\hline
\end{tabular}

Note: See Appendix B for flagging criteria. Synchronous water levels are measured over a 3-5 day period.

- = exceeded holding time.

= exceeded groundwater protection standard. 
WELL FSB114D (cont.)

Radiological Indicator Parameters

\begin{tabular}{|c|c|c|c|c|c|c|c|c|c|c|c|c|c|c|c|}
\hline Parameter & 1095 & $\underline{D F}$ & Möd & $\underline{\mathbf{S T}}$ & $\underline{H}$ & Flag & $\underline{\text { Lab }}$ & $\underline{2095}$ & $\underline{\text { DF }}$ & Mod & $\underline{\text { ST }}$ & $\underline{H}$ & Flag & $\underline{\text { Lab }}$ & Unit \\
\hline $\begin{array}{l}\text { Gross alpha } \\
\text { Nonvolatile beta }\end{array}$ & $\begin{array}{l}1.3 E+00 \\
1.3 E+00\end{array}$ & $\begin{array}{l}1 \\
1\end{array}$ & UI & & & $\begin{array}{l}0 \\
0\end{array}$ & $\begin{array}{l}\text { GP } \\
\text { GP }\end{array}$ & $\begin{array}{l}2.8 E+00 \\
1.4 E+00\end{array}$ & $\begin{array}{l}1 \\
1\end{array}$ & J & & & $\begin{array}{l}0 \\
0\end{array}$ & $\begin{array}{l}\text { GP } \\
\text { GP }\end{array}$ & $\begin{array}{l}\text { pCi/l } \\
\text { pCi/L }\end{array}$ \\
\hline \multicolumn{16}{|c|}{ Measured Radionuclides } \\
\hline Parameter & 1095 & $\underline{\mathrm{DF}}$ & Mod & $\underline{\text { ST }}$ & $\underline{\mathbf{H}}$ & Flag & $\underline{\text { Lab }}$ & $\underline{2095}$ & DF & Mod & $\underline{\text { ST }}$ & $\underline{\mathrm{H}}$ & Flag & $\underline{\text { Lab }}$ & Unit \\
\hline $\begin{array}{l}\text { Actinium-228 } \\
\text { Americium-241 } \\
\text { Cesium-137 } \\
\text { Cobalt-60 } \\
\text { Curium-242 } \\
\text { Curium-243/244 } \\
\text { Curium-245/246 } \\
\text { lodine-129 } \\
\text { Plutonium-238 } \\
\text { Plutonium-239/240 } \\
\text { Radium-226 } \\
\text { Radium-228 } \\
\text { Strontium-89 } \\
\text { Strontium-90 } \\
\text { Technetium-99 } \\
\text { Thorium-228 } \\
\text { Thorium-230 } \\
\text { Thorium-232 } \\
\text { Thorium-234 } \\
\text { Tritium } \\
\text { Uranium-233/234 } \\
\text { Uranium-235 } \\
\text { Uranium-238 }\end{array}$ & $\begin{array}{l}7.0 E+00 \\
3.7 E-02 \\
-8.1 E-01 \\
-3.3 E-01 \\
0.0 E+00 \\
0.0 E+00 \\
0.0 E+00 \\
9.6 E-01 \\
1.7 E-03 \\
4.2 E-02 \\
3.2 E-01 \\
-6.9 E-02 \\
6.8 E-01 \\
4.8 E-01 \\
-3.0 E+01 \\
7.5 E-02 \\
8.7 E-02 \\
2.5 E-02 \\
0.0 E+00 \\
7.1 E+00 \\
-3.2 E-02 \\
-3.9 E-02 \\
-1.0 E-02\end{array}$ & $\begin{array}{l}1 \\
1 \\
1 \\
1 \\
1 \\
1 \\
1 \\
1 \\
1 \\
1 \\
1 \\
1 \\
1 \\
1 \\
1 \\
1 \\
1 \\
1 \\
1 \\
1 \\
1 \\
1 \\
1\end{array}$ & $\begin{array}{l}\text { UI } \\
\text { UI } \\
\text { UI } \\
\text { UI } \\
\text { UI } \\
\text { UI } \\
U I \\
U I \\
U I \\
U I I \\
U I \\
U I \\
\text { UIJI }\end{array}$ & & & $\begin{array}{l}0 \\
0 \\
0 \\
0 \\
0 \\
0 \\
0 \\
0 \\
0 \\
0 \\
0 \\
0 \\
0 \\
0 \\
0 \\
0 \\
0 \\
0 \\
0 \\
0 \\
0 \\
0 \\
0\end{array}$ & $\begin{array}{l}\text { GP } \\
\text { GP } \\
\text { GP } \\
\text { GP } \\
\text { GP } \\
\text { GP } \\
\text { GP } \\
\text { GP } \\
\text { GP } \\
\text { GP } \\
\text { GP } \\
\text { GP } \\
\text { GP } \\
\text { GP } \\
\text { GP } \\
\text { GP } \\
\text { GP } \\
\text { GP } \\
\text { GP } \\
\text { GP } \\
\text { GP } \\
\text { GP } \\
\text { GP }\end{array}$ & 2.3E-02 & 1 & UI & & & 0 & GP & $\begin{array}{l}\mathrm{pCi} / \mathrm{L} \\
\mathrm{pCi} / \mathrm{L} \\
\mathrm{pCi} / \mathrm{L} \\
\mathrm{pCi} / \mathrm{L} \\
\mathrm{pCi} / \mathrm{L} \\
\mathrm{pCi} / \mathrm{L} \\
\mathrm{pCi} / \mathrm{L} \\
\mathrm{pCi} / \mathrm{L} \\
\mathrm{pCi} / \mathrm{L} \\
\mathrm{pCi} / \mathrm{L} \\
\mathrm{pCi} / \mathrm{L} \\
\mathrm{pCi} / \mathrm{L} \\
\mathrm{pCi} / \mathrm{L} \\
\mathrm{pCi} / \mathrm{L} \\
\mathrm{pCi} / \mathrm{L} \\
\mathrm{pCi} / \mathrm{L} \\
\mathrm{pCi} / \mathrm{L} \\
\mathrm{pCi} / \mathrm{L} \\
\mathrm{pCi} / \mathrm{L} \\
\mathrm{pCi} / \mathrm{mL} \\
\mathrm{pCi} / \mathrm{L} \\
\mathrm{pCi} / \mathrm{L} \\
\mathrm{pCi} / \mathrm{L}\end{array}$ \\
\hline \multicolumn{16}{|c|}{ Calculated Radionuclides } \\
\hline Parameter & $\underline{10.95}$ & $\underline{\text { DF }}$ & Mod & $\underline{\mathbf{S T}}$ & $\underline{H}$ & Flag & $\underline{\text { Lab }}$ & $\underline{2095}$ & $\underline{D F}$ & Mod & ST & $\underline{\mathbf{H}}$ & Flag & $\underline{\text { Lab }}$ & $\underline{\text { Unit }}$ \\
\hline $\begin{array}{l}\text { Americium-243 } \\
\text { Nickel-63 } \\
\text { Plutonium-241 } \\
\text { Plutonium-242 }\end{array}$ & $\begin{array}{l}0.0 E+00 \\
-3.3 E-01 \\
1.7 E-01 \\
8.4 E-07\end{array}$ & & & & & $\begin{array}{l}0 \\
0 \\
0 \\
0\end{array}$ & & & & & & & & & $\begin{array}{l}\mathrm{pCi} / \mathrm{L} \\
\mathrm{pCl} / \mathrm{L} \\
\mathrm{pCi} / \mathrm{L} \\
\mathrm{pCi} / \mathrm{L}\end{array}$ \\
\hline
\end{tabular}

Note: See Appendix B for flagging criteria. Synchronous water levels are measured over a 3-5 day period.

- = exceeded holding time.

- exceeded groundwater protection standard. 


\section{WELL FSB115C}

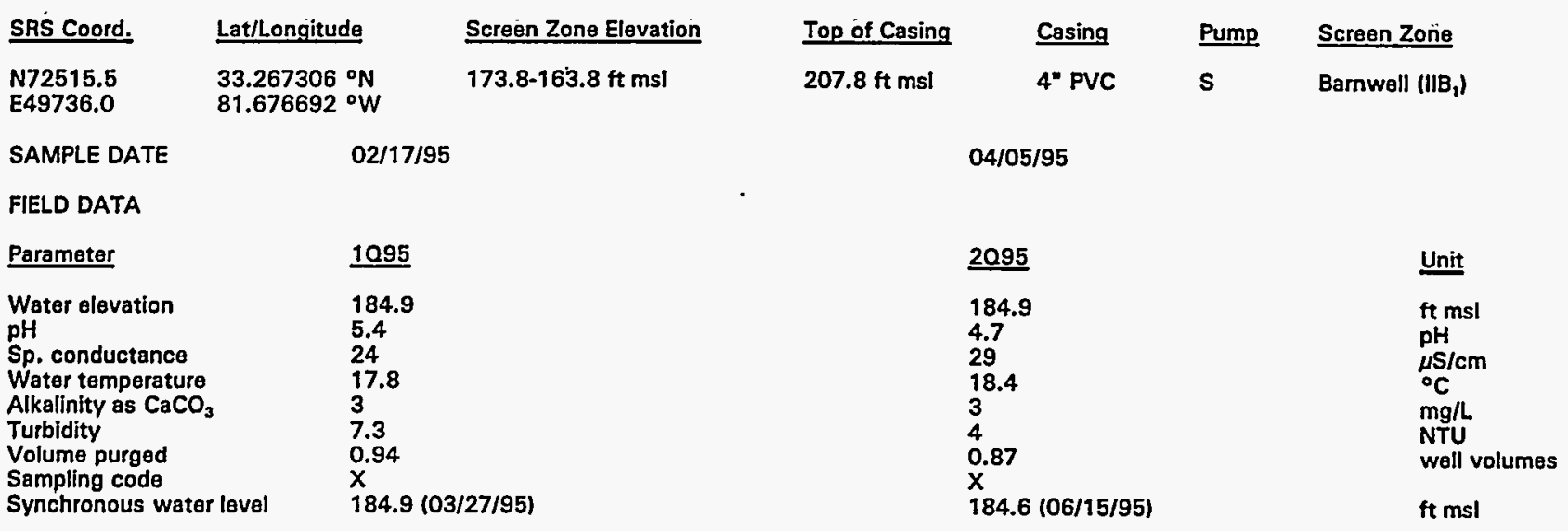

Hazardous Constituents (264.94, Table 1)

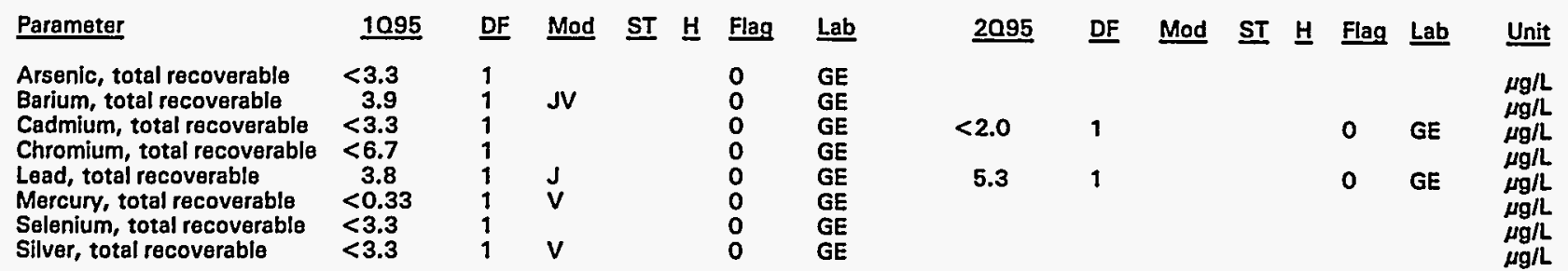

Hazardous Constituents (261 App. VIII/264 App. IX)

\begin{tabular}{|c|c|c|c|c|c|c|c|c|c|c|c|c|c|c|c|}
\hline Parameter & 1095 & $\underline{\mathrm{DF}}$ & Mod & $\underline{\mathbf{S T}}$ & $\underline{\mathrm{H}}$ & Flag & $\underline{\text { Lab }}$ & $\underline{2095}$ & $\underline{D F}$ & Mod & $\underline{\mathbf{S T}}$ & $\underline{\mathbf{H}}$ & Flag & $\underline{\text { Lab }}$ & Unit \\
\hline $\begin{array}{l}\text { Antimony, total recoverable } \\
\text { Benzene } \\
\text { Cobalt, total recoverable } \\
\text { Copper, total recoverable } \\
\text { Cyanide } \\
\text { Nickel, total recoverable } \\
\text { Phenols } \\
\text { Tetrachloroethylene } \\
\text { Thallium, total recoverable } \\
\text { Trichloroethylene } \\
\text { Trichlorofluoromethane } \\
\text { Vanadium, total recoverable } \\
\text { Zinc, total recoverable }\end{array}$ & $\begin{aligned}<3.3 \\
<1.7 \\
<6.7 \\
5.7 \\
<8.3 \\
<6.7 \\
<8.3 \\
<1.7 \\
<3.3 \\
<1.7 \\
<1.7 \\
<13 \\
13\end{aligned}$ & $\begin{array}{l}1 \\
1 \\
1 \\
1 \\
1 \\
1 \\
1 \\
1 \\
1 \\
1 \\
1 \\
1 \\
1\end{array}$ & $\begin{array}{l}J \\
v \\
V\end{array}$ & & & $\begin{array}{l}0 \\
0 \\
0 \\
0 \\
0 \\
0 \\
0 \\
0 \\
0 \\
0 \\
0 \\
0 \\
0\end{array}$ & $\begin{array}{l}\mathrm{GE} \\
\mathrm{GE} \\
\mathrm{GE} \\
\mathrm{GE} \\
\mathrm{GE} \\
\mathrm{GE} \\
\mathrm{GE} \\
\mathrm{GE} \\
\mathrm{GE} \\
\mathrm{GE} \\
\mathrm{GE} \\
\mathrm{GE} \\
\mathrm{GE}\end{array}$ & & & & & & & & $\begin{array}{l}\mu g / L \\
\mu g / L \\
\mu g / L \\
\mu g / L \\
\mu g / L \\
\mu g / L \\
\mu g / L \\
\mu g / L \\
\mu g / L \\
\mu g / L \\
\mu g / L \\
\mu g / L \\
\mu g / L\end{array}$ \\
\hline \multicolumn{16}{|c|}{ Water Quality Indicator Parameters } \\
\hline Parameter & 1095 & $\underline{D F}$ & Mod & ST & $\underline{\mathbf{H}}$ & Flag & $\underline{\text { Lab }}$ & $\underline{2095}$ & $\underline{\mathrm{DF}}$ & Mod & $\underline{\text { ST }}$ & 브 & Flag & $\underline{L a b}$ & $\underline{\text { Unit }}$ \\
\hline $\begin{array}{l}\text { Aluminum, total recoverable } \\
\text { Nitrate as nitrogen } \\
\text { pH }\end{array}$ & $\begin{array}{l}65 \\
510 \\
6.1\end{array}$ & $\begin{array}{l}1 \\
1 \\
1\end{array}$ & $J$ & - & & $\begin{array}{l}2 \\
0 \\
0\end{array}$ & $\begin{array}{l}\mathrm{GE} \\
\mathrm{GE} \\
\mathrm{GE}\end{array}$ & $\begin{array}{l}520 \\
5.8\end{array}$ & 1 & J1 & - & & $\begin{array}{l}0 \\
0\end{array}$ & $\begin{array}{l}\text { GE } \\
\text { GE }\end{array}$ & $\underset{\mathrm{pH}}{\mu \mathrm{g} / \mathrm{L}}$ \\
\hline \multicolumn{16}{|c|}{ Common Laboratory Contaminants } \\
\hline Parameter & 1095 & $\underline{D F}$ & Mod & $\underline{\text { ST }}$ & $\underline{\mathrm{H}}$ & Flag & $\underline{\text { Lab }}$ & $\underline{2095}$ & $\underline{\mathrm{DF}}$ & Mod & $\underline{\text { ST }}$ & 브 & Flag & Lab & $\underline{\text { Unit }}$ \\
\hline $\begin{array}{l}\text { Bis(2-ethylhexyl) phthalate } \\
\text { Dichloromethane }\end{array}$ & $\begin{array}{l}<17 \\
<1.7\end{array}$ & $\begin{array}{l}1 \\
1\end{array}$ & & & & $\begin{array}{l}0 \\
0\end{array}$ & $\begin{array}{l}\text { GE } \\
\text { GE }\end{array}$ & & & & & & & & $\begin{array}{l}\mu g / L \\
\mu g / L\end{array}$ \\
\hline
\end{tabular}

Note: See Appendix B for flagging criteria. Synchronous water levels are measured over a 3-5 day period.

- = exceeded holding time.

- exceeded groundwater protection standard. 
WELL. FSB115C (cont.)

Radiological Indicator Parameters

\begin{tabular}{|c|c|c|c|c|c|c|c|c|c|c|c|c|c|}
\hline Parameter & 1095 & $\underline{D F}$ & Mod & $\underline{\text { ST }} \underline{H}$ & Flag & Lab & $\underline{2095}$ & $\underline{D F}$ & Mod & ST $\underline{H}$ & Flag & $\underline{\text { Lab }}$ & $\underline{\text { Unit }}$ \\
\hline $\begin{array}{l}\text { Gross alpha } \\
\text { Nonvolatile beta }\end{array}$ & $\begin{array}{l}3.5 E-01 \\
6.9 E-01\end{array}$ & $\begin{array}{l}1 \\
1\end{array}$ & $\begin{array}{l}\text { UI } \\
\text { UI }\end{array}$ & & $\begin{array}{l}0 \\
0\end{array}$ & $\begin{array}{l}\text { GP } \\
\text { GP }\end{array}$ & $\begin{array}{l}7.1 \mathrm{E}-01 \\
4.0 \mathrm{E}-01\end{array}$ & $\begin{array}{l}1 \\
1\end{array}$ & $\begin{array}{l}\text { UJI } \\
\text { UI }\end{array}$ & & $\begin{array}{l}0 \\
0\end{array}$ & $\begin{array}{l}\text { GP } \\
\text { GP }\end{array}$ & PCi/L \\
\hline
\end{tabular}

Measured Radionuclides

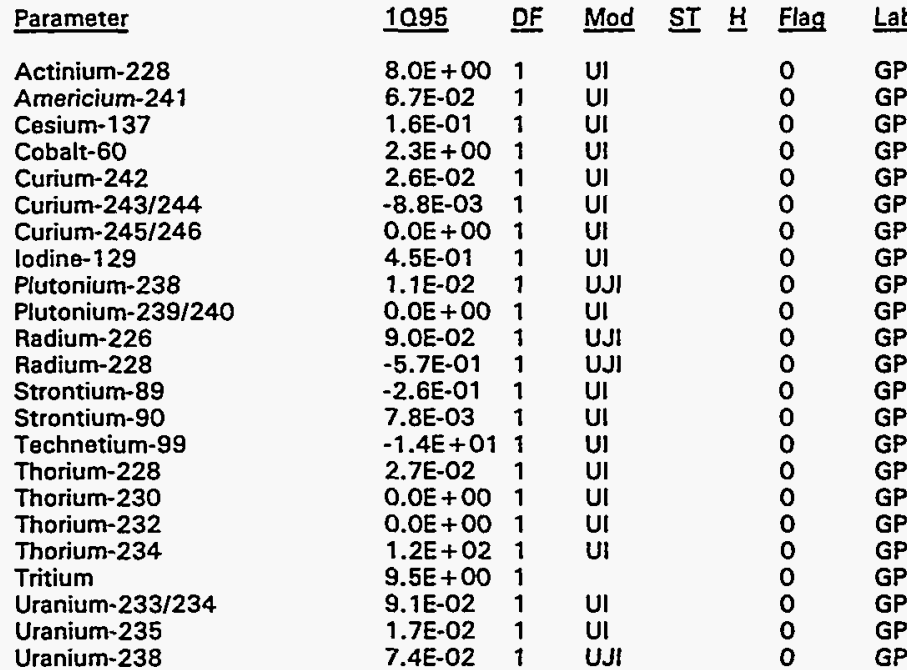

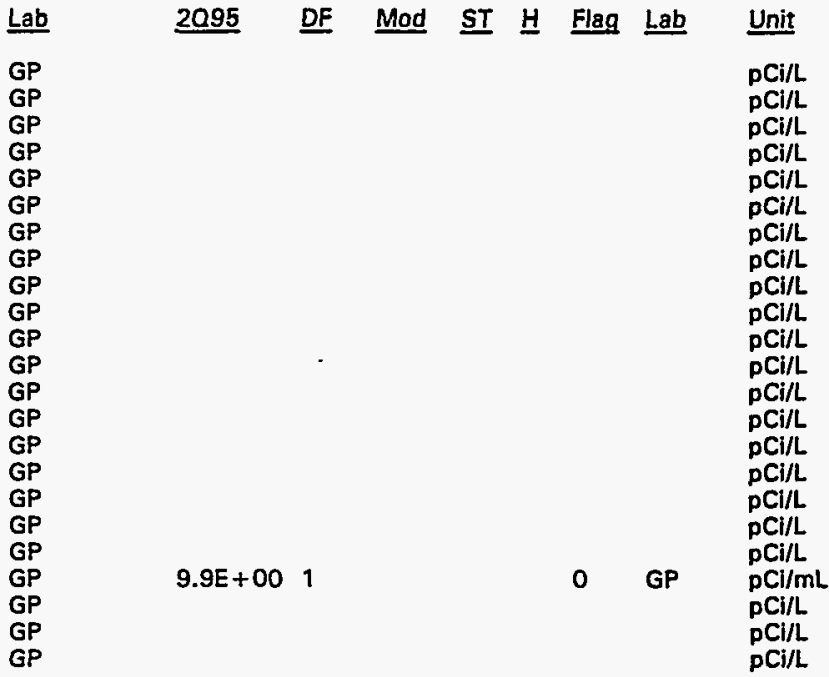

Calculated Radionuclides

\section{Parameter}

Americium-243

Nickel-63

Plutonium-241

Plutonium-242
1095

$-2.6 \mathrm{E}-05$

$2.3 E+00$

$0.0 E+00$

$0.0 E+00$
DF Mod

ST $\underline{H}$

Flag
0
0
0
0

Unit

$\mathrm{pCi} / \mathrm{L}$

$\mathrm{PCi} / \mathrm{L}$

$\mathrm{pCi} / \mathrm{L}$

pCi/L

Note: See Appendix B for flagging criteria. Synchronous water levels are measured over a 3-5 day period.

- = exceeded holding time.

- = exceeded groundwater protection standard. 


\section{WELL FSB115D}

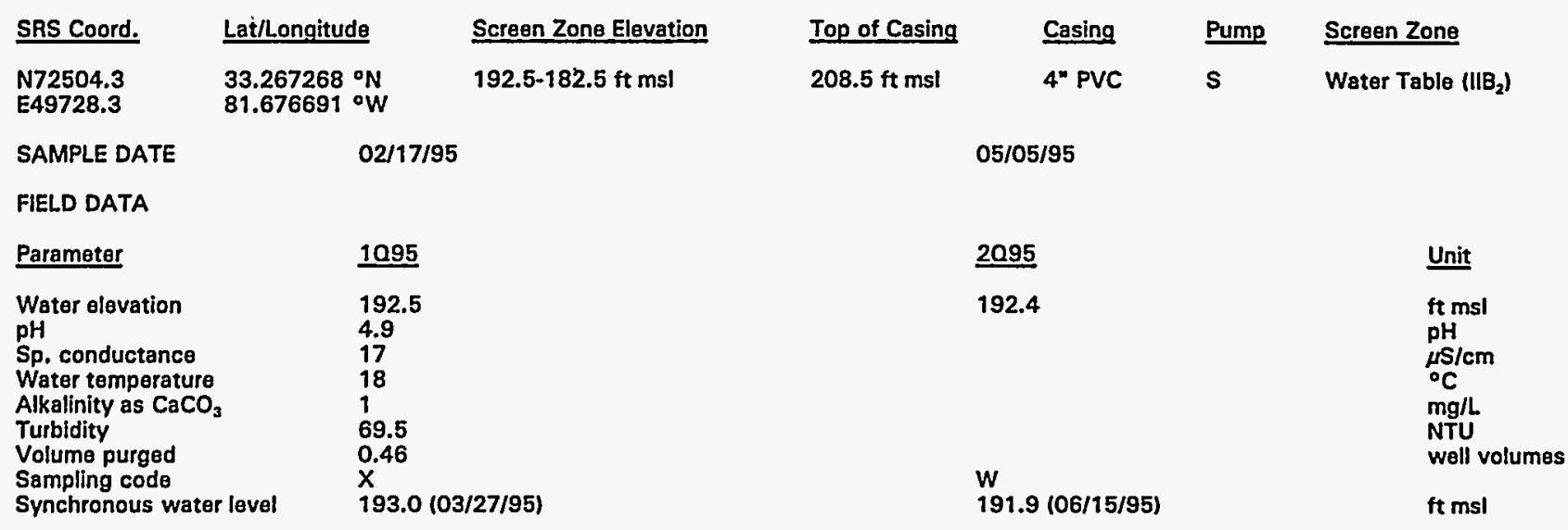

Hazardous Constituents (264.94, Table 1)

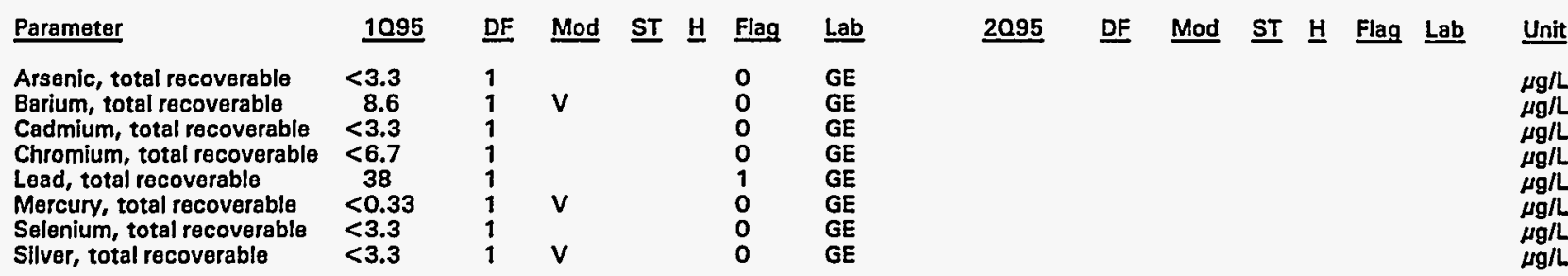

Hazardous Constituents (261 App. VIII/264 App. IX)

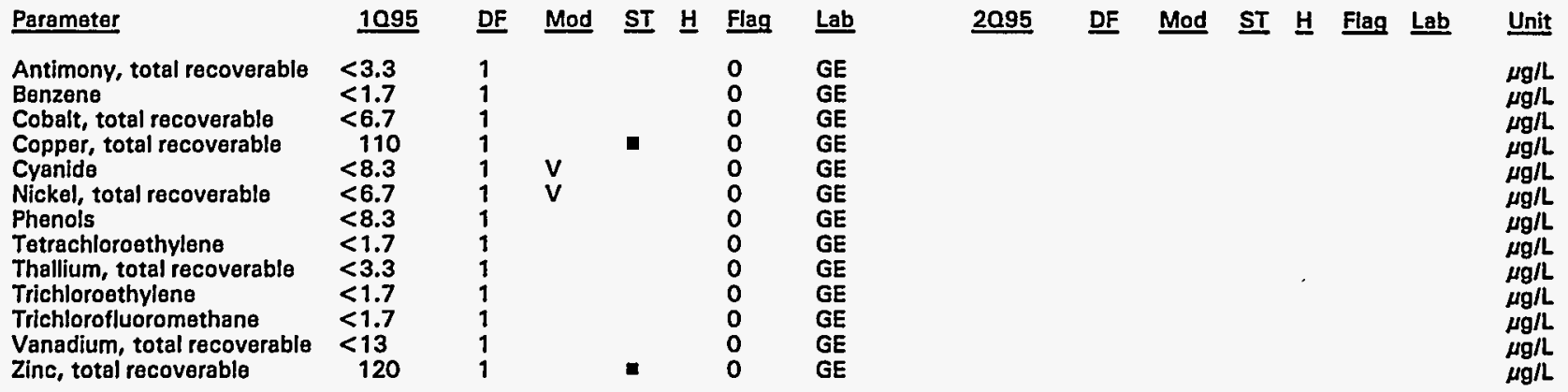

Water Quality Indicator Parameters

\begin{tabular}{|c|c|c|c|c|c|c|c|c|c|c|c|c|c|c|c|}
\hline Parameter & 1095 & DF & Mod & $\underline{\text { ST }}$ & 브 & Flag & $\underline{\text { Lab }}$ & $\underline{2095}$ & DF & Mod & $\underline{\mathbf{s T}}$ & 브 & Flag & $\underline{\text { Lab }}$ & Unit \\
\hline $\begin{array}{l}\text { Aluminum, total recoverable } \\
\text { Nitrate as nitrogen } \\
\text { pH }\end{array}$ & $\begin{array}{l}110 \\
81 \\
5.3\end{array}$ & $\begin{array}{l}1 \\
1 \\
1\end{array}$ & J & - & $\bullet$ & $\begin{array}{l}2 \\
0 \\
0\end{array}$ & $\begin{array}{l}\text { GE } \\
\text { GE } \\
\text { GE }\end{array}$ & & & & & & & & $\begin{array}{l}\mu \mathrm{g} / \mathrm{L} \\
\mu \mathrm{g} / \mathrm{L} \\
\mathrm{pH}\end{array}$ \\
\hline \multicolumn{16}{|c|}{ Common Laboratory Contaminants } \\
\hline Parameter & 1095 & $\underline{D F}$ & Mod & $\underline{\text { ST }}$ & H & Flag & $\underline{\text { Lab }}$ & 2095 & DF & Mod & $\underline{\mathbf{S T}}$ & 브 & Flag & $\underline{\text { Lab }}$ & Unit \\
\hline $\begin{array}{l}\text { Bis(2-ethylhexyl) phthalate } \\
\text { Dichloromethane }\end{array}$ & $\begin{array}{c}24 \\
<1.7\end{array}$ & $\begin{array}{l}1 \\
1\end{array}$ & & . & & $\begin{array}{l}2 \\
0\end{array}$ & $\begin{array}{l}\mathrm{GE} \\
\mathrm{GE}\end{array}$ & & & & & & & & $\begin{array}{l}\mu g^{\prime \prime L} \\
\mu g / L\end{array}$ \\
\hline
\end{tabular}

Note: See Appendix B for flagging criteria. Synchronous water levels are measured over a 3-5 day period.

$-=$ exceeded holding time.

- = exceeded groundwater protection standard. 
WELL FSB115D (cont.)

Radiological Indicator Parameters

\begin{tabular}{|c|c|c|c|c|c|c|c|c|c|c|c|c|c|c|c|}
\hline Parameter & 1095 & $\underline{\mathrm{DF}}$ & Mod & $\underline{\text { ST }}$ & 브 & Flag & Lab & 2095 & $\underline{D F}$ & Mod & $\underline{\text { ST }}$ & $\underline{\mathrm{H}}$ & Flag & $\underline{\text { Lab }}$ & Unit \\
\hline $\begin{array}{l}\text { Gross alpha } \\
\text { Nonvolatile beta }\end{array}$ & $\begin{array}{l}1.2 E+00 \\
1.1 E+00\end{array}$ & $\begin{array}{l}1 \\
1\end{array}$ & UI & & & $\begin{array}{l}0 \\
0\end{array}$ & $\begin{array}{l}\text { GP } \\
\text { GP }\end{array}$ & & & & & & & & $\begin{array}{l}\mathrm{pCi} / \mathrm{L} \\
\mathrm{pCi} / \mathrm{L}\end{array}$ \\
\hline \multicolumn{16}{|c|}{ Measured Radionuclides } \\
\hline Parameter & $\underline{1095}$ & $\underline{\text { DF }}$ & Mod & $\underline{\text { ST }}$ & $\underline{H}$ & Flag & Lab & $\underline{2095}$ & $\underline{D F}$ & Mod & $\underline{\text { ST }}$ & H & Flag & $\underline{L a b}$ & Unit \\
\hline $\begin{array}{l}\text { Actinium-228 } \\
\text { Americium-241 } \\
\text { Cesium-137 } \\
\text { Cobalt-60 } \\
\text { Curium-242 } \\
\text { Curium-243/244 } \\
\text { Curium-245/246 } \\
\text { lodine-129 } \\
\text { Plutonium-238 } \\
\text { Plutonium-239/240 } \\
\text { Radium-226 } \\
\text { Radium-228 } \\
\text { Strontium-89 } \\
\text { Strontium-90 } \\
\text { Technetium-99 } \\
\text { Thorium-228 } \\
\text { Thorium-230 } \\
\text { Thorium-232 } \\
\text { Thorium-234 } \\
\text { Tritium } \\
\text { Uranium-233/234 } \\
\text { Uranium-235 } \\
\text { Uranium-238 }\end{array}$ & $\begin{array}{l}4.8 E+00 \\
5.0 E-02 \\
-2.7 E-01 \\
-1.5 E+00 \\
-2.2 E-02 \\
-1.9 E-01 \\
0.0 E+00 \\
-7.0 E-01 \\
4.2 E-02 \\
-9.7 E-03 \\
2.7 E-01 \\
4.2 E-01 \\
4.5 E-01 \\
4.5 E-01 \\
-2.1 E+01 \\
5.0 E-02 \\
2.8 E-01 \\
-1.3 E-02 \\
2.9 E+01 \\
8.9 E+00 \\
6.8 E-02 \\
2.7 E-02 \\
5.9 E-02\end{array}$ & $\begin{array}{l}1 \\
1 \\
1 \\
1 \\
1 \\
1 \\
1 \\
1 \\
1 \\
1 \\
1 \\
1 \\
1 \\
1 \\
1 \\
1 \\
1 \\
1 \\
1 \\
1 \\
1 \\
1 \\
1\end{array}$ & $\begin{array}{l}\text { UI } \\
\text { UI } \\
\text { UI } \\
\text { UI } \\
\text { UI } \\
\text { UI } \\
\text { UI } \\
\text { UI } \\
\text { UJI } \\
\text { UI } \\
\text { UJI } \\
\text { UJI }\end{array}$ & & & $\begin{array}{l}0 \\
0 \\
0 \\
0 \\
0 \\
0 \\
0 \\
0 \\
0 \\
0 \\
0 \\
0 \\
0 \\
0 \\
0 \\
0 \\
0 \\
0 \\
0 \\
0 \\
0 \\
0 \\
0\end{array}$ & $\begin{array}{l}\text { GP } \\
G P \\
G P \\
G P \\
G P \\
G P \\
G P \\
G P \\
G P \\
G P \\
G P \\
G P \\
G P \\
G P \\
G P \\
G P \\
G P \\
G P \\
G P \\
G P \\
G P \\
G P \\
G P\end{array}$ & & & & & & & & 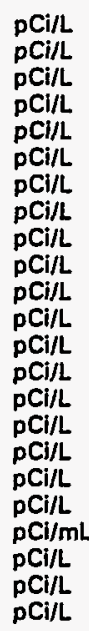 \\
\hline \multicolumn{16}{|c|}{ Calculated Radionuclides } \\
\hline Parameter & 1095 & $\underline{\mathrm{DF}}$ & Mod & $\underline{\text { ST }}$ & $\underline{H}$ & Flag & $\underline{\text { Lab }}$ & $\underline{2095}$ & $\underline{\mathrm{DF}}$ & Mod & $\underline{\mathbf{S T}}$ & 븐 & Flag & $\underline{\text { Lab }}$ & Unit \\
\hline $\begin{array}{l}\text { Americium-243 } \\
\text { Nickel-63 } \\
\text { Plutonium-241 } \\
\text { Plutonium-242 }\end{array}$ & $\begin{array}{l}-5.7 E-04 \\
-1.5 E+00 \\
-3.9 E-02 \\
-1.9 E-07\end{array}$ & & & & & $\begin{array}{l}0 \\
0 \\
0 \\
0\end{array}$ & & & & & & & & & $\begin{array}{l}p C i / L \\
p C i / L \\
p C i / L \\
p C i / L\end{array}$ \\
\hline
\end{tabular}

Note: See Appendix B for flagging criteria. Synchronous water levels are measured over a 3-5 day period.

- = exceeded holding time.

I = exceeded groundwater protection standard. 


\section{WELL FSB116C}

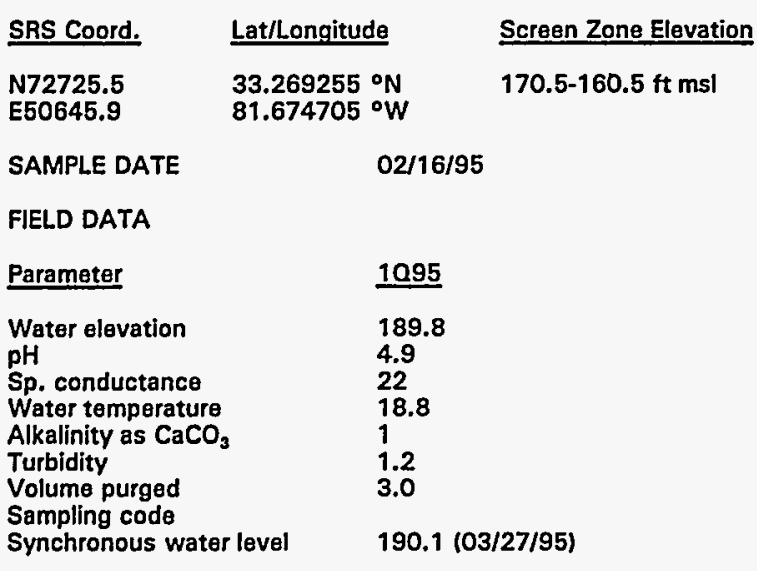

$\begin{array}{lrlll}\frac{\text { Top of Casing }}{202.5 \mathrm{ft} \mathrm{msl}} & \text { Casing } & \text { Pump } & \text { Screen Zone } \\ \text { 4" PVC } & \text { S } & \text { Barnwell (IIB,) } \\ 04 / 05 / 95 & & \end{array}$

Hazardous Constituents (264.94, Table 1)

$\begin{array}{lccccccc}\text { Parameter } & \underline{1095} & \text { DF } & \text { Mod } & \text { ST } & \text { H } & \text { Flag } & \text { Lab } \\ \text { Arsenic, total recoverable } & <3.3 & 1 & & & & 0 & \text { GE } \\ \text { Barium, total recoverable } & 4.6 & 1 & \mathrm{~J} & & & 0 & \text { GE } \\ \text { Cadmium, total recoverable } & <3.3 & 1 & & & 0 & \text { GE } \\ \text { Chromium, total recoverable } & <6.7 & 1 & & & 0 & \text { GE } \\ \text { Lead, total recoverable } & <5.0 & 1 & & & 0 & \text { GE } \\ \text { Mercury, total recoverable } & <0.33 & 1 & & & 0 & \text { GE } \\ \text { Selenium, total recoverable } & <3.3 & 1 & & & 0 & \text { GE } \\ \text { Silver, total recoverable } & <3.3 & 1 & & & 0 & \text { GE }\end{array}$

$\begin{array}{ll}\underline{2095} & \text { Unit } \\ 190.1 & \mathrm{ft} \mathrm{msl} \\ 4.5 & \mathrm{pH} \\ 20 & \mu \mathrm{S} / \mathrm{cm} \\ 18.6 & { }^{\circ} \mathrm{C} \\ 1 & \mathrm{mg} / \mathrm{h} \\ 0.5 & \mathrm{NTU} \\ 2.7 & \text { well volumes } \\ 189.8(06 / 15 / 95) & \mathrm{ft} \mathrm{msl}\end{array}$

Hazardous Constituents (261 App. VIII/264 App. IX)

\begin{tabular}{|c|c|c|c|c|c|c|c|c|c|c|c|c|c|c|c|}
\hline Parameter & 1095 & $\underline{\text { DF }}$ & Mod & ST & $\underline{\mathrm{H}}$ & Flag & $\underline{\text { Lab }}$ & $\underline{2095}$ & $\underline{D F}$ & Mod & $\underline{\mathbf{S T}}$ & $\underline{\mathbf{H}}$ & Flag & $\underline{\text { Lab }}$ & Unit \\
\hline $\begin{array}{l}\text { Antimony, total recoverable } \\
\text { Benzene } \\
\text { Cobalt, total recoverable } \\
\text { Copper, total recoverable } \\
\text { Cyanide } \\
\text { Nickel, total recoverable } \\
\text { Phenols } \\
\text { Tetrachloroethylene } \\
\text { Thallium, total recoverable } \\
\text { Trichloroethylene } \\
\text { Trichlorofluoromethane } \\
\text { Vanadium, total recoverable } \\
\text { Zinc, total recoverable }\end{array}$ & $\begin{array}{r}<3.3 \\
<1.7 \\
<6.7 \\
12 \\
<8.3 \\
<6.7 \\
<8.3 \\
<1.7 \\
<3.3 \\
<1.7 \\
<1.7 \\
<13 \\
16\end{array}$ & $\begin{array}{l}1 \\
1 \\
1 \\
1 \\
1 \\
1 \\
1 \\
1 \\
1 \\
1 \\
1 \\
1 \\
1\end{array}$ & J1 & & - & $\begin{array}{l}0 \\
0 \\
0 \\
0 \\
0 \\
0 \\
0 \\
0 \\
0 \\
0 \\
0 \\
0 \\
0\end{array}$ & $\begin{array}{l}\mathrm{GE} \\
\mathrm{GE} \\
\mathrm{GE} \\
\mathrm{GE} \\
\mathrm{GE} \\
\mathrm{GE} \\
\mathrm{GE} \\
\mathrm{GE} \\
\mathrm{GE} \\
\mathrm{GE} \\
\mathrm{GE} \\
\mathrm{GE} \\
\mathrm{GE}\end{array}$ & & & & & & & & $\begin{array}{l}\mu g / L \\
\mu g / L \\
\mu g / L \\
\mu g / L \\
\mu g / L \\
\mu g / L \\
\mu g / L \\
\mu g / L \\
\mu g / L \\
\mu g / L \\
\mu g / L \\
\mu g / L \\
\mu g / L\end{array}$ \\
\hline \multicolumn{16}{|c|}{ Water Quality Indicator Parameters } \\
\hline Parameter & 1095 & $\underline{\text { DF }}$ & Mod & $\underline{\text { ST }}$ & $\underline{\mathbf{H}}$ & Flag & $\underline{\mathrm{Lab}}$ & $\underline{2095}$ & DF & Mod & ST & H & Flag & Lab & Unit \\
\hline \multicolumn{16}{|c|}{ Common Laboratory Contaminants } \\
\hline Parameter & 1095 & $\underline{D F}$ & Mod & $\underline{\mathbf{S T}}$ & $\underline{H}$ & Flag & $\underline{\text { Lab }}$ & $\underline{2095}$ & $\underline{\text { DF }}$ & $\underline{\text { Mod }}$ & ST & $\underline{H}$ & Flag & $\underline{\text { Lab }}$ & $\underline{\text { Unit }}$ \\
\hline $\begin{array}{l}\text { Bis(2-ethylhexyl) phthalate } \\
\text { Dichloromethane }\end{array}$ & $\begin{array}{l}<17 \\
<1.7\end{array}$ & $\begin{array}{l}1 \\
1\end{array}$ & $V$ & & & $\begin{array}{l}0 \\
0\end{array}$ & $\begin{array}{l}\text { GE } \\
\text { GE }\end{array}$ & & & & & & & & $\begin{array}{l}\mu g / L \\
\mu g / L\end{array}$ \\
\hline
\end{tabular}

Note: See Appendix B for flagging criteria. Synchronous water levels are measured over a 3-5 day period. - = exceeded holding time.

m = exceeded groundwater protection standard. 
WELL FSB116C (cont.)

Radiological Indicator Parameters

\begin{tabular}{|c|c|c|c|c|c|c|c|c|c|c|c|c|c|c|c|}
\hline Parameter & 1095 & DF & Mod & $\underline{\mathbf{S T}}$ & $\underline{\mathrm{H}}$ & Flag & $\underline{\text { Lab }}$ & $\underline{2095}$ & $\underline{D F}$ & Mod & $\underline{\mathbf{S T}}$ & H & Flag & Lab & Unit \\
\hline $\begin{array}{l}\text { Gross alpha } \\
\text { Nonvolatile beta }\end{array}$ & $\begin{array}{l}2.7 E-01 \\
-5.5 E-01\end{array}$ & $\begin{array}{l}1 \\
1\end{array}$ & $\begin{array}{l}\text { UI } \\
\text { UI }\end{array}$ & & & $\begin{array}{l}0 \\
0\end{array}$ & $\begin{array}{l}\text { GP } \\
\text { GP }\end{array}$ & $\begin{array}{l}2.2 E-01 \\
3.5 E-01\end{array}$ & $\begin{array}{l}1 \\
1\end{array}$ & $\begin{array}{l}\text { UI } \\
\text { UI }\end{array}$ & & & $\begin{array}{l}0 \\
0\end{array}$ & $\begin{array}{l}\text { GP } \\
\text { GP }\end{array}$ & $\begin{array}{l}\mathrm{pCl} / \mathrm{L} \\
\mathrm{pCi} / \mathrm{L}\end{array}$ \\
\hline \multicolumn{16}{|c|}{ Measured Radionuclides } \\
\hline Parameter & 1095 & DF & Mod & ST & $\underline{\mathrm{H}}$ & Flag & $\underline{\text { Lab }}$ & $\underline{2095}$ & $\underline{D F}$ & Mod & $\underline{\mathbf{S T}}$ & H & Flag & $\underline{\text { Lab }}$ & $\underline{\text { Unit }}$ \\
\hline $\begin{array}{l}\text { Actinium-228 } \\
\text { Americium-241 } \\
\text { Cesium-137 } \\
\text { Cobalt-60 } \\
\text { Curium-242 } \\
\text { Curium-243/244 } \\
\text { Curium-245/246 } \\
\text { lodine-129 } \\
\text { Plutonium-238 } \\
\text { Plutonium-239/240 } \\
\text { Radium-226 } \\
\text { Radium-228 } \\
\text { Strontium-89 } \\
\text { Strontium-90 } \\
\text { Technetium-99 } \\
\text { Thorium-228 } \\
\text { Thorium-230 } \\
\text { Thorium-232 } \\
\text { Thorium-234 } \\
\text { Tritium } \\
\text { Uranium-233/234 } \\
\text { Uranium-235 } \\
\text { Uranium-238 }\end{array}$ & $\begin{array}{l}5.8 E+00 \\
-1.9 E-02 \\
2.7 E+00 \\
4.4 E-01 \\
2.6 E-02 \\
4.3 E-02 \\
0.0 E+00 \\
5.7 E-01 \\
-6.1 E-03 \\
-6.1 E-03 \\
1.2 E+00 \\
-4.4 E-01 \\
-5.0 E-01 \\
-2.4 E-01 \\
-2.2 E+01 \\
0.0 E+00 \\
1.3 E-01 \\
-9.0 E-03 \\
7.6 E+01 \\
1.2 E+01 \\
1.0 E-02 \\
-4.9 E-03 \\
0.0 E+00\end{array}$ & $\begin{array}{l}1 \\
1 \\
1 \\
1 \\
1 \\
1 \\
1 \\
1 \\
1 \\
1 \\
1 \\
1 \\
1 \\
1 \\
1 \\
1 \\
1 \\
1 \\
1 \\
1 \\
1 \\
1 \\
1\end{array}$ & 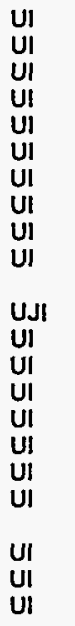 & & & $\begin{array}{l}0 \\
0 \\
0 \\
0 \\
0 \\
0 \\
0 \\
0 \\
0 \\
0 \\
0 \\
0 \\
0 \\
0 \\
0 \\
0 \\
0 \\
0 \\
0 \\
1 \\
0 \\
0 \\
0\end{array}$ & $\begin{array}{l}\text { GP } \\
\text { GP } \\
\text { GP } \\
\text { GP } \\
\text { GP } \\
\text { GP } \\
\text { GP } \\
\text { GP } \\
\text { GP } \\
\text { GP } \\
\text { GP } \\
\text { GP } \\
\text { GP } \\
\text { GP } \\
\text { GP } \\
\text { GP } \\
\text { GP } \\
\text { GP } \\
\text { GP } \\
\text { GP } \\
\text { GP } \\
\text { GP } \\
\text { GP }\end{array}$ & $1.2 E+01$ & 1 & & & & 1 & GP & $\begin{array}{l}\text { pCi/L } \\
\text { pCi/L } \\
\text { pCi/L } \\
\text { pCi/L } \\
\text { pCi/L } \\
\text { pCi/L } \\
\text { pCi/L } \\
\text { pCi/L } \\
\text { pCi/L } \\
\text { pCi/L } \\
\text { pCi/L } \\
\text { pCi/L } \\
\text { pCi/L } \\
\text { pCi/L } \\
\text { pCi/L } \\
\text { pCi/L } \\
\text { pCi/L } \\
\text { pCi/L } \\
\text { pCi/L } \\
\text { pCi/m } \\
\text { pCi/L } \\
\text { pCi/L } \\
\text { pCi/L }\end{array}$ \\
\hline \multicolumn{16}{|c|}{ Calculated Radionuclides } \\
\hline Parameter & 1095 & $\underline{\mathrm{DF}}$ & Mod & ST & $\underline{\text { H}}$ & Flag & $\underline{L a b}$ & 2095 & $\underline{D F}$ & Mod & ST & $\underline{H}$ & Flag & $\underline{\text { Lab }}$ & Unit \\
\hline $\begin{array}{l}\text { Americium-243 } \\
\text { Nickel-63 } \\
\text { Plutonium-241 } \\
\text { Plutonium-242 }\end{array}$ & $\begin{array}{l}1.3 E-04 \\
4.4 E-01 \\
-2.4 E-02 \\
-1.2 E-07\end{array}$ & & & & & $\begin{array}{l}0 \\
0 \\
0 \\
0\end{array}$ & & & & & & & & & $\begin{array}{l}\mathrm{pCi} / \mathrm{L} \\
\mathrm{pCi} / \mathrm{L} \\
\mathrm{pCi} / \mathrm{L} \\
\mathrm{pCi} / \mathrm{L}\end{array}$ \\
\hline
\end{tabular}

Note: See Appendix B for flagging criteria. Synchronous water levels are measured over a 3-5 day period.

- = exceeded holding time.

= exceeded groundwater protection standard. 
WELL FSB116D

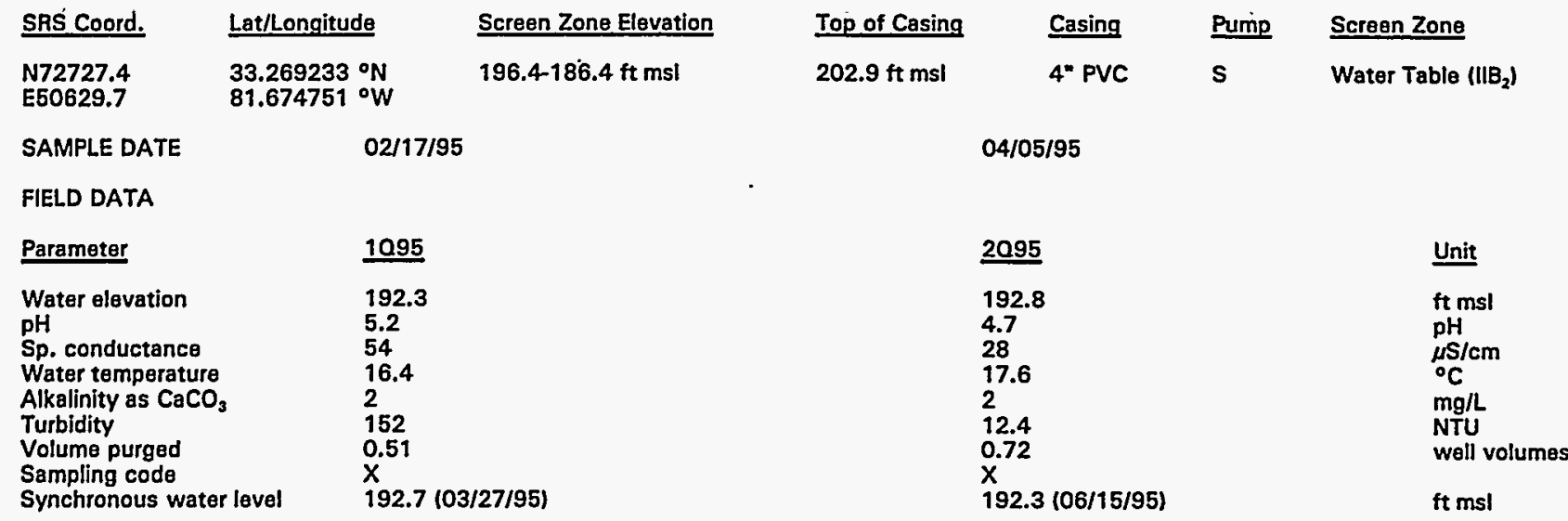

Hazardous Constituents (264.94, Table 1)

\begin{tabular}{lcccccccc} 
Parameter & 1095 & DF & Mod & ST & H & Flag & La \\
\hline Arsenic, total recoverable & $<3.3$ & 1 & & & & 0 & G \\
Barium, total recoverable & 5.7 & 1 & $V$ & & 0 & G \\
Cadmium, total recoverable & $<3.3$ & 1 & & & 0 & G \\
Chromium, total recoverable & $<6.7$ & 1 & & & 0 & GE \\
Lead, total recoverable & 44 & 1 & & & 1 & GE \\
Mercury, total recoverable & $<0.33$ & 1 & $V$ & & 0 & G \\
Selenium, total recoverable & $<3.3$ & 1 & & & 0 & GE \\
Silver, total recoverable & $<3.3$ & 1 & $V$ & & 0 & GE
\end{tabular}

Lab
$\mathrm{GE}$
$\mathrm{GE}$
$\mathrm{GE}$
$\mathrm{GE}$
$\mathrm{GE}$
$\mathrm{GE}$
$\mathrm{GE}$
$\mathrm{GE}$

\begin{tabular}{|c|c|c|c|c|c|c|}
\hline 2095 & DF & Mod & ST $H$ & Flag & Lat & Unit \\
\hline $\begin{array}{r}15 \\
<2.0\end{array}$ & $\begin{array}{l}5 \\
1\end{array}$ & v & & $\begin{array}{l}0 \\
0\end{array}$ & $\begin{array}{l}\mathrm{GE} \\
\mathrm{GE}\end{array}$ & $\mu \mathrm{g} / \mathrm{L}$ \\
\hline 36 & 1 & $v$ & & 1 & GE & $\mu \mathrm{g} / \mathrm{L}$ \\
\hline 73 & 5 & v & - & 1 & $\mathrm{GE}$ & $\mu \mathrm{g} / \mathrm{L}$ \\
\hline
\end{tabular}

Hazardous Constituents (261 App. VIII/264 App. IX)

\begin{tabular}{|c|c|c|c|c|c|c|c|c|c|c|c|c|c|c|c|}
\hline Parameter & 1095 & $\underline{D F}$ & Mod & $\underline{\text { ST }}$ & $\underline{\mathrm{H}}$ & Flag & $\underline{\text { Lab }}$ & $\underline{2095}$ & $\underline{D F}$ & Mod & $\underline{\text { ST }}$ & $\underline{H}$ & Flag & $\underline{\text { Lab }}$ & Unit \\
\hline $\begin{array}{l}\text { Antimony, total recoverable } \\
\text { Benzene } \\
\text { Cobalt, total recoverable } \\
\text { Copper, total recoverable } \\
\text { Cyanide } \\
\text { Nickel, total recoverable } \\
\text { Phenols } \\
\text { Tetrachloroethylene } \\
\text { Thallium, total recoverable } \\
\text { Trichloroethylene } \\
\text { Trichlorofluoromethane } \\
\text { Vanadium, total recoverable } \\
\text { Zinc, total recoverable }\end{array}$ & $\begin{array}{l}<3.3 \\
<1.7 \\
<6.7 \\
150 \\
<8.3 \\
<6.7 \\
<8.3 \\
<1.7 \\
<3.3 \\
<1.7 \\
<1.7 \\
<13 \\
\quad 230\end{array}$ & $\begin{array}{l}1 \\
1 \\
1 \\
1 \\
1 \\
1 \\
1 \\
1 \\
1 \\
1 \\
1 \\
1 \\
1\end{array}$ & $v$ & 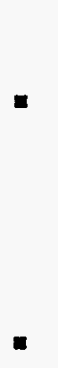 & & $\begin{array}{l}0 \\
0 \\
0 \\
0 \\
0 \\
0 \\
0 \\
0 \\
0 \\
0 \\
0 \\
0 \\
0\end{array}$ & $\begin{array}{l}\mathrm{GE} \\
\mathrm{GE} \\
\mathrm{GE} \\
\mathrm{GE} \\
\mathrm{GE} \\
\mathrm{GE} \\
\mathrm{GE} \\
\mathrm{GE} \\
\mathrm{GE} \\
\mathrm{GE} \\
\mathrm{GE} \\
\mathrm{GE} \\
\mathrm{GE}\end{array}$ & $<10$ & 1 & & & & 0 & GE & $\begin{array}{l}\mu g / L \\
\mu g / L \\
\mu g / L \\
\mu g / L \\
\mu g / L \\
\mu g / L \\
\mu g / L \\
\mu g / L \\
\mu g / L \\
\mu g / L \\
\mu g / L \\
\mu g / L \\
\mu g / L\end{array}$ \\
\hline \multicolumn{16}{|c|}{ Water Quality Indicator Parameters } \\
\hline Parameter & $\underline{1095}$ & DF & Mod & $\underline{\mathbf{S T}}$ & $\underline{H}$ & Flag & $\underline{L a b}$ & $\underline{2095}$ & DF & Mod & $\underline{\text { ST }}$ & $\underline{H}$ & Flag & $\underline{\text { Lab }}$ & Unit \\
\hline $\begin{array}{l}\text { Aluminum, total recoverable } \\
\text { Nitrate as nitrogen } \\
\text { pH }\end{array}$ & $\begin{array}{l}160 \\
60 \\
5.5\end{array}$ & $\begin{array}{l}1 \\
1 \\
1\end{array}$ & J & - & 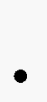 & $\begin{array}{l}2 \\
0 \\
0\end{array}$ & $\begin{array}{l}\text { GE } \\
\text { GE } \\
\text { GE }\end{array}$ & $\begin{array}{l}70 \\
5.3\end{array}$ & 1 & $\begin{array}{l}\mathrm{J} \\
\mathrm{J} 1\end{array}$ & & - & $\begin{array}{l}0 \\
0\end{array}$ & $\begin{array}{l}\text { GE } \\
\text { GE }\end{array}$ & $\begin{array}{l}\mu \mathrm{g} / \mathrm{L} \\
\mu \mathrm{g} / \mathrm{L} \\
\mathrm{pH}\end{array}$ \\
\hline \multicolumn{16}{|c|}{ Common Laboratory Contaminants } \\
\hline Parameter & 1095 & $\underline{\mathrm{DF}}$ & Mod & $\underline{\underline{S T}}$ & $\underline{H}$ & Flag & $\underline{\text { Lab }}$ & 2095 & $\underline{D F}$ & Mod & $\underline{\text { ST }}$ & 브 & Flag & $\underline{\text { Lab }}$ & Unit \\
\hline $\begin{array}{l}\text { Bis(2-ethylhexyl) phthalate } \\
\text { Dichloromethane }\end{array}$ & $\begin{array}{l}<17 \\
<1.7\end{array}$ & $\begin{array}{l}1 \\
1\end{array}$ & & & & $\begin{array}{l}0 \\
0\end{array}$ & $\begin{array}{l}\mathrm{GE} \\
\mathrm{GE}\end{array}$ & & & & & & & & $\begin{array}{c}\mu \mathrm{g} / \mathrm{L} \\
\mu \mathrm{g} / \mathrm{L}\end{array}$ \\
\hline
\end{tabular}

Note: See Appendix B for flagging criteria. Synchronous water levels are measured over a 3-5 day period. - = exceeded holding time.

- = exceeded groundwater protection standard. 
WELL FSB116D (cont.)

Radiological indicator Parameters

\begin{tabular}{|c|c|c|c|c|c|c|c|c|c|c|c|c|c|c|}
\hline Parameter & 1095 & $\underline{\mathrm{DF}}$ & Mod & ST $\underline{H}$ & Flag & Lab & 2095 & DF & Mod & $\underline{\mathbf{S T}}$ & 브 & Flag & Lab & Unit \\
\hline $\begin{array}{l}\text { Gross alpha } \\
\text { Nonvolatile beta }\end{array}$ & $\begin{array}{l}1.2 E+00 \\
1.8 E+00\end{array}$ & $\begin{array}{l}1 \\
1\end{array}$ & & & $\begin{array}{l}0 \\
0\end{array}$ & $\begin{array}{l}\text { GP } \\
\text { GP }\end{array}$ & $\begin{array}{l}9.5 E-01 \\
1.3 E+\infty 0\end{array}$ & $\begin{array}{l}1 \\
1\end{array}$ & U! & & & $\begin{array}{l}0 \\
0\end{array}$ & $\begin{array}{l}\text { GP } \\
\text { GP }\end{array}$ & $\begin{array}{l}\mathrm{pCi} / \mathrm{L} \\
\mathrm{pCj} / \mathrm{L}\end{array}$ \\
\hline
\end{tabular}

Measured Radionuclides

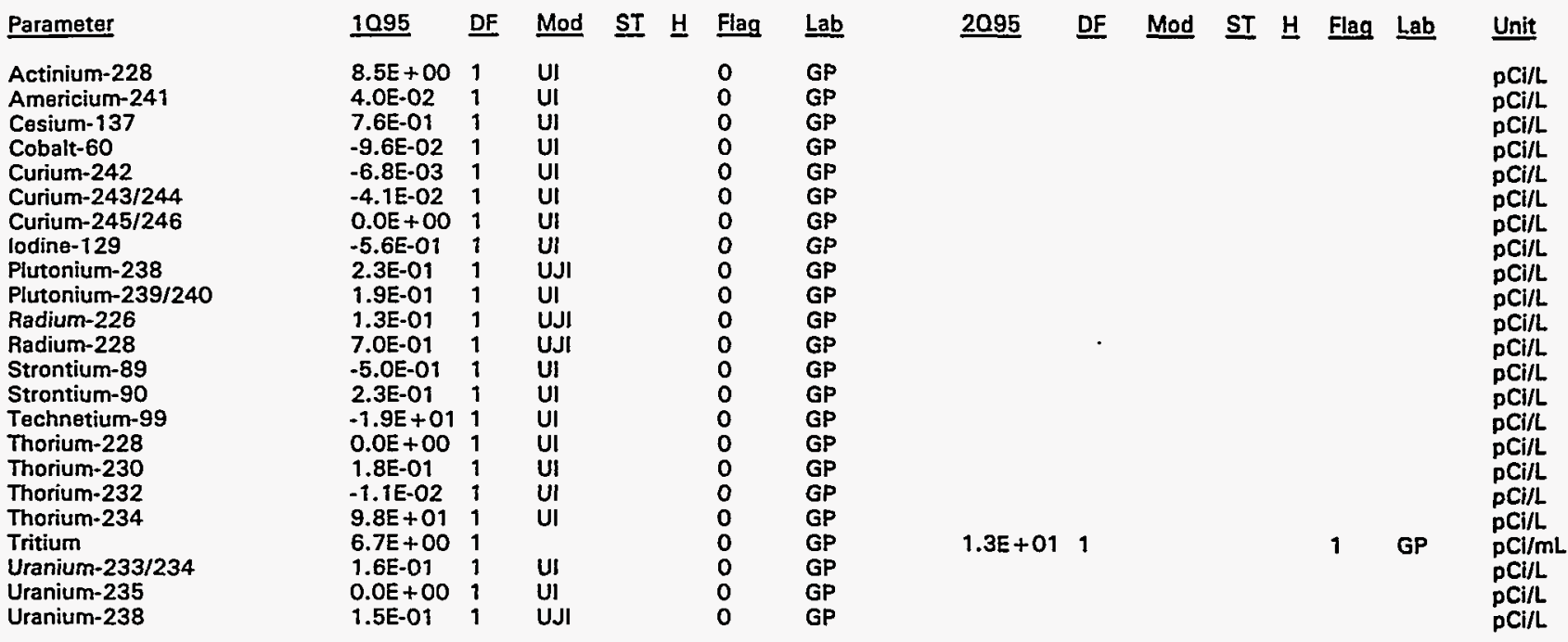

Calculated Radionuclides

Parameter

Americium-243

Nickel-63

Plutonium-241

Plutonium-242 $\underline{1095}$

$-1.2 \mathrm{E}-04$

$-9.6 \mathrm{E}-02$

7.5E-01

3.7E-O6
DF Mod ST $\underline{H}$ Flag Lab

0
0
0
0

$\underline{2095}$

DF Mod ST $\underline{H}$ Flag Lab

Unit

$\mathrm{pCi} / \mathrm{L}$

pCi/L

$\mathrm{pCl} / \mathrm{L}$

pCi/L

Note: See Appendix 8 for flagging criteria. Synchronous water levels are measured over a 3-5 day period.

- = exceeded holding time.

- = exceeded groundwater protection standard. 
WELL FSB117D

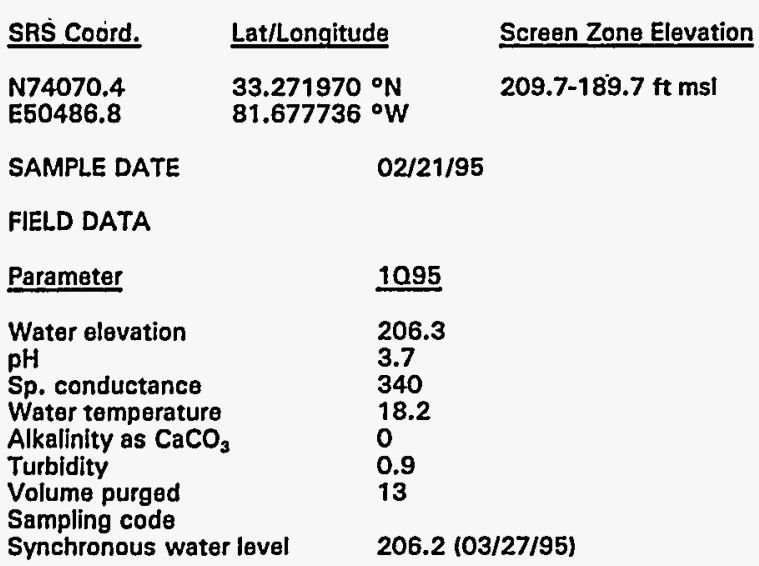

\begin{tabular}{|c|c|c|c|}
\hline Top of Casing & Casing & Pump & Screen Zone \\
\hline $230.7 \mathrm{ft} \mathrm{msl}$ & 4" PVC & $\mathbf{s}$ & Water Table $\left(\| \mathrm{B}_{2}\right)$ \\
\hline
\end{tabular}

Hazardous Constituents (264.94, Table 1)

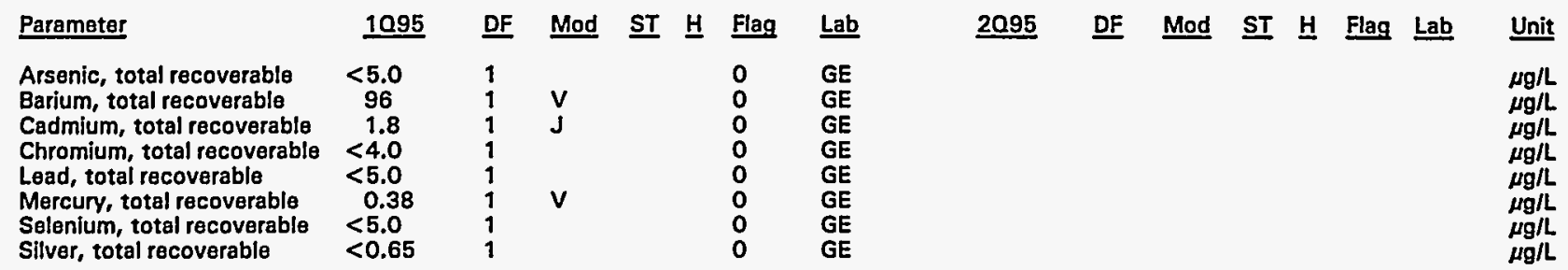

Hazardous Constituents (261 App. VIII/264 App. IX)

\begin{tabular}{|c|c|c|c|c|c|c|c|c|c|c|c|c|c|c|c|}
\hline Parameter & 1095 & $\underline{D F}$ & Mod & $\underline{\text { ST }}$ & H & Flag & $\underline{\text { Lab }}$ & $\underline{2095}$ & $\underline{\text { DF }}$ & Mod & $\underline{\mathbf{S T}}$ & H & Flag & $\underline{\text { Lab }}$ & Unit \\
\hline $\begin{array}{l}\text { Antimony, total recoverable } \\
\text { Benzene } \\
\text { Cobalt, total recoverable } \\
\text { Copper, total recoverable } \\
\text { Cyanide } \\
\text { Nickel, total recoverable } \\
\text { Phenols } \\
\text { Tetrachloroethylene } \\
\text { Thallium, total recoverable } \\
\text { Trichloroethylene } \\
\text { Trichlorofluoromethane } \\
\text { Vanadium, total recoverable } \\
\text { Zinc, total recoverable }\end{array}$ & $\begin{aligned}<5.0 \\
<1.7 \\
7.5 \\
21 \\
<20 \\
7.0 \\
<10 \\
<1.7 \\
<5.0 \\
<1.7 \\
<1.7 \\
<10 \\
14\end{aligned}$ & $\begin{array}{l}1 \\
1 \\
1 \\
1 \\
1 \\
1 \\
1 \\
1 \\
1 \\
1 \\
1 \\
1 \\
1\end{array}$ & J & & & $\begin{array}{l}0 \\
0 \\
0 \\
0 \\
0 \\
0 \\
0 \\
0 \\
0 \\
0 \\
0 \\
0 \\
0\end{array}$ & $\begin{array}{l}\mathrm{GE} \\
\mathrm{GE} \\
\mathrm{GE} \\
\mathrm{GE} \\
\mathrm{GE} \\
\mathrm{GE} \\
\mathrm{GE} \\
\mathrm{GE} \\
\mathrm{GE} \\
\mathrm{GE} \\
\mathrm{GE} \\
\mathrm{GE} \\
\mathrm{GE}\end{array}$ & & & & & & & & $\begin{array}{l}\mu g / L \\
\mu g / L \\
\mu g / L \\
\mu g / L \\
\mu g / L \\
\mu g / L \\
\mu g / L \\
\mu g / L \\
\mu g / L \\
\mu g / L \\
\mu g / L \\
\mu g / L \\
\mu g / L\end{array}$ \\
\hline \multicolumn{16}{|c|}{ Water Quality Indicator Parameters } \\
\hline Parameter & 1095 & $\underline{\mathrm{DF}}$ & Mod & $\underline{\text { ST }}$ & $\underline{\mathbf{H}}$ & Flag & $\underline{\text { Lab }}$ & $\underline{2095}$ & $\underline{\mathrm{DF}}$ & Mod & $\underline{\mathbf{S T}}$ & $\underline{\text { H}}$ & Flag & $\underline{\text { Lab }}$ & Unit \\
\hline $\begin{array}{l}\text { Aluminum, total recoverable } \\
\text { Nitrate as nitrogen } \\
\text { pH }\end{array}$ & $\begin{array}{l}9,500 \\
31,000 \\
3.7\end{array}$ & $\begin{array}{l}1 \\
20 \\
1\end{array}$ & ل1 & : & $\bullet$ & $\begin{array}{l}2 \\
2 \\
1\end{array}$ & $\begin{array}{l}\mathrm{GE} \\
\mathrm{GE} \\
\mathrm{GE}\end{array}$ & & & & & & & & $\begin{array}{l}\mu \mathrm{g} / \mathrm{L} \\
\mu \mathrm{g} / \mathrm{L} \\
\mathrm{pH}\end{array}$ \\
\hline \multicolumn{16}{|c|}{ Common Laboratory Contaminants } \\
\hline Parameter & 1095 & $\underline{\mathrm{DF}}$ & Mod & $\underline{\text { ST }}$ & H & Flag & $\underline{\text { Lab }}$ & $\underline{2095}$ & $\underline{\mathrm{DF}}$ & Mod & ST & $\underline{H}$ & Flag & Lab & Unit \\
\hline $\begin{array}{l}\text { Bis(2-ethylhexyl) phthalate } \\
\text { Dichloromethane }\end{array}$ & $\begin{array}{l}<17 \\
<1.7\end{array}$ & $\begin{array}{l}1 \\
1\end{array}$ & & & & $\begin{array}{l}0 \\
0\end{array}$ & $\begin{array}{l}\text { GE } \\
\text { GE }\end{array}$ & & & & & & & & $\begin{array}{l}\mu g / L \\
\mu g / L\end{array}$ \\
\hline
\end{tabular}

Note: See Appendix B for flagging criteria. Synchronous water levels are measured over a 3-5 day period.

- = exceeded holding time.

- exceeded groundwater protection standard. 


\section{WELL FSB117D (cont.)}

Radiological Indicator Parameters

\begin{tabular}{|c|c|c|c|c|c|c|c|c|c|c|c|c|c|}
\hline Paramater & $\underline{1095}$ & DF & $\underline{\text { Mod }}$ & ST & Flag & Lab & $\underline{2095}$ & DF & Mod & $\underline{\text { ST }} \underline{H}$ & Flag & Lab & Unit \\
\hline $\begin{array}{l}\text { Gross alpha } \\
\text { Nonvolatile beta }\end{array}$ & $\begin{array}{l}6.2 E+02 \\
3.5 E+02\end{array}$ & $\begin{array}{l}1 \\
1\end{array}$ & $J$ & 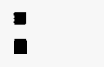 & $\begin{array}{l}2 \\
2\end{array}$ & $\begin{array}{l}\text { GP } \\
\text { GP }\end{array}$ & & & & & & & $\mathrm{pCi} / \mathrm{L}$ \\
\hline
\end{tabular}

Measured Radionuclides

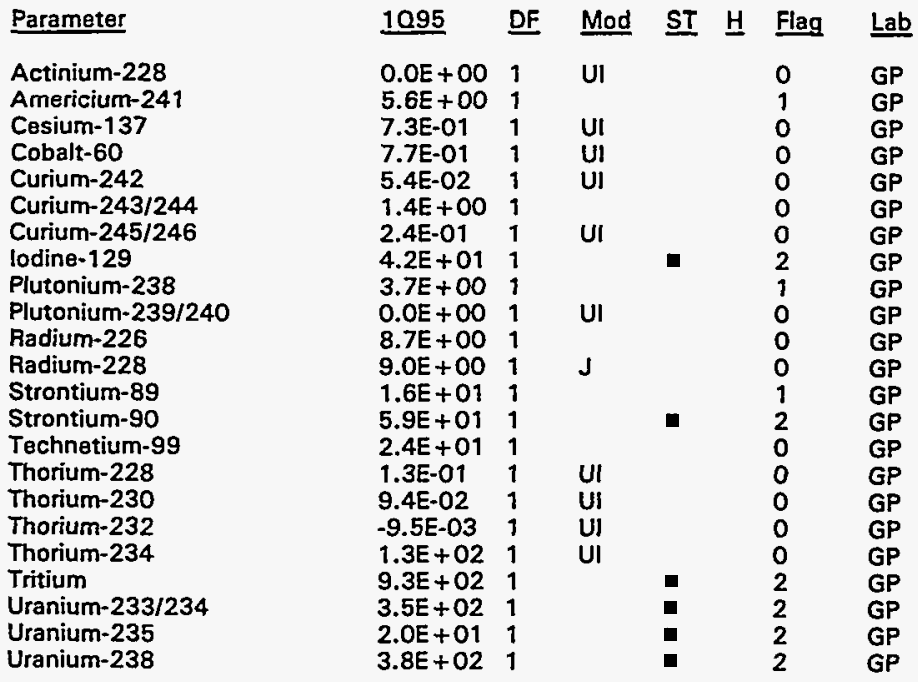

2095 DF Mod ST H Flag Lab Unit

Uranium-238

$\begin{array}{lcc}1095 & \text { DF Mod ST H Flag Lab } \\ 4.2 E-03 & & 0 \\ 7.7 E-01 & & 0 \\ 0.0 E+00 & & 0 \\ 0.0 E+00 & & 0\end{array}$

Unit

pCi/L

PCi/L

Nickel-63

Plutonium-241

$0.0 E+00$

Plutonium-242 
WELL FSB118D

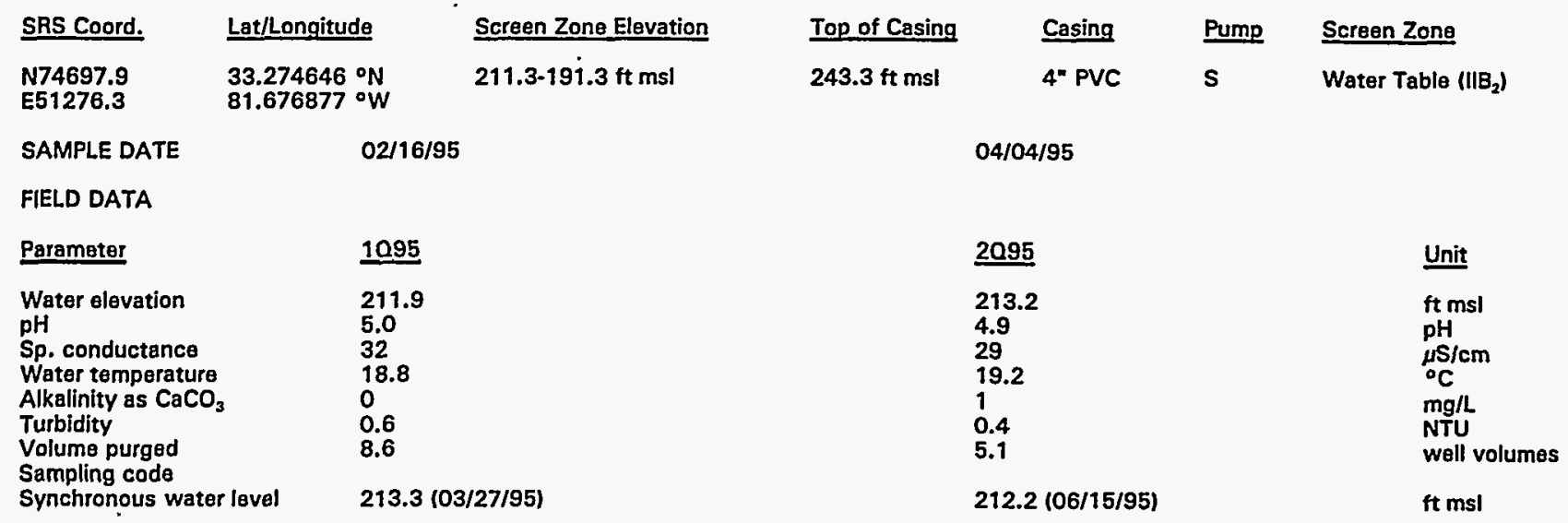

Hazardous Constituents (264.94, Table 1)

\begin{tabular}{|c|c|c|c|c|c|c|c|c|c|c|c|c|c|}
\hline Parameter & 1095 & DF & Mod & ST $\underline{H}$ & Flag & $\underline{\text { Lab }}$ & $\underline{2095}$ & DF & Mod & $\underline{\text { ST }} \underline{H}$ & Flag & Lab & Unit \\
\hline $\begin{array}{l}\text { Arsenic, total recoverable } \\
\text { Barium, total recoverable } \\
\text { Cadmium, total recoverable } \\
\text { Chromium, total recoverable } \\
\text { Lead, total recoverable } \\
\text { Mercury, total recoverable } \\
\text { Selenium, total recoverable } \\
\text { Silver, total recoverable }\end{array}$ & $\begin{array}{l}<3.3 \\
10 \\
<3.3 \\
<6.7 \\
<5.0 \\
<0.33 \\
<3.3 \\
<3.3\end{array}$ & $\begin{array}{l}1 \\
1 \\
1 \\
1 \\
1 \\
1 \\
1 \\
1\end{array}$ & & & $\begin{array}{l}0 \\
0 \\
0 \\
0 \\
0 \\
0 \\
0 \\
0\end{array}$ & $\begin{array}{l}\mathrm{GE} \\
\mathrm{GE} \\
\mathrm{GE} \\
\mathrm{GE} \\
\mathrm{GE} \\
\mathrm{GE} \\
\mathrm{GE} \\
\mathrm{GE}\end{array}$ & $\begin{array}{r}<2.0 \\
2.8\end{array}$ & 1 & JWV & & $\begin{array}{l}0 \\
0\end{array}$ & $\begin{array}{l}\text { GE } \\
\text { GE }\end{array}$ & $\begin{array}{l}\mu \mathrm{g} / \mathrm{L} \\
\mu \mathrm{g} / \mathrm{L} \\
\mu \mathrm{g} / \mathrm{L} \\
\mu \mathrm{g} / \mathrm{L} \\
\mu \mathrm{g} / \mathrm{L} \\
\mu \mathrm{g} / \mathrm{L} \\
\mu \mathrm{g} / \mathrm{L} \\
\mu \mathrm{g} / \mathrm{L}\end{array}$ \\
\hline
\end{tabular}

Hazardous Constituents (261 App. VIII/264 App. IX)

$\begin{array}{lccccccc}\text { Parameter } & 1095 & \text { DF } & \text { Mod } & \text { ST } & \text { Flag } & \text { Lab } \\ \text { Antimony, total recoverable } & <3.3 & 1 & & & 0 & \text { GE } \\ \text { Benzene } & <1.7 & 1 & & & 0 & \text { GE } \\ \text { Cobalt, total recoverable } & <6.7 & 1 & & & 0 & \text { GE } \\ \text { Copper, total recoverable } & 21 & 1 & & & 0 & \text { GE } \\ \text { Cyanide } & <8.3 & 1 & & & 0 & \text { GE } \\ \text { Nickel, total recoverable } & <6.7 & 1 & & & 0 & \text { GE } \\ \text { Phenols } & <8.3 & 1 & & & 0 & \text { GE } \\ \text { Tetrachloroethylene } & 1.3 & 1 & J & & 0 & \text { GE } \\ \text { Thallium, total recoverable } & <3.3 & 1 & & & 0 & \text { GE } \\ \text { Trichlorosthylene } & <1.7 & 1 & & & 0 & \text { GE } \\ \text { Trichlorofluoromethane } & 1.4 & 1 & J & & 0 & \text { GE } \\ \text { Vanadium, total recoverable } & <13 & 1 & & & 0 & \text { GE } \\ \text { Zinc, total recoverable } & 31 & 1 & & & 0\end{array}$

2095 DF Mod ST $\underline{H}$ Flag Lab Unit

Water Quality Indicator Parameters

\begin{tabular}{|c|c|c|c|c|c|c|c|c|c|c|c|c|c|c|c|}
\hline Parameter & 10.95 & $\underline{\mathrm{DF}}$ & Mod & $\underline{\mathbf{S T}}$ & $\underline{\mathbf{H}}$ & Flag & $\underline{\text { Lab }}$ & 2095 & DF & Mod & $\underline{\underline{S T}}$ & $\underline{\mathrm{H}}$ & Flag & Lab & Unit \\
\hline $\begin{array}{l}\text { Aluminum, total recoverable } \\
\text { Nitrate as nitrogen } \\
\text { pH }\end{array}$ & $\begin{array}{l}28 \\
950 \\
5.1\end{array}$ & $\begin{array}{l}1 \\
1 \\
1\end{array}$ & $\begin{array}{l}J \\
\text { J1 }\end{array}$ & - & - & $\begin{array}{l}1 \\
0 \\
0\end{array}$ & $\begin{array}{l}\mathrm{GE} \\
\mathrm{GE} \\
\mathrm{GE}\end{array}$ & $\begin{array}{l}920 \\
5.9\end{array}$ & $\begin{array}{l}1 \\
1\end{array}$ & J1 & - & & $\begin{array}{l}0 \\
0\end{array}$ & $\begin{array}{l}\mathrm{GE} \\
\mathrm{GE}\end{array}$ & $\begin{array}{l}\mu g / L \\
\mu g / L \\
\mathrm{pH}\end{array}$ \\
\hline \multicolumn{16}{|c|}{ Common Laboratory Contaminants } \\
\hline Parameter & 1095 & $\underline{\mathrm{DF}}$ & Mod & $\underline{\text { ST }}$ & $\underline{\text { H}}$ & Flag & $\underline{\text { Lab }}$ & 2095 & DF & Mod & $\underline{\mathbf{S T}}$ & 보 & Flag & $\underline{L a b}$ & Unit \\
\hline $\begin{array}{l}\text { Bis(2-ethylhexyl) phthalate } \\
\text { Dichloromethane }\end{array}$ & $\begin{array}{l}<17 \\
<1.7\end{array}$ & $\begin{array}{l}1 \\
1\end{array}$ & $\mathbf{V}$ & & & $\begin{array}{l}0 \\
0\end{array}$ & $\begin{array}{l}\text { GE } \\
\text { GE }\end{array}$ & & & & & & & & \\
\hline
\end{tabular}

Note: See Appendix B for flagging criteria. Synchronous water levels are measured over a 3-5 day period.

- = exceeded holding time.

- = exceeded groundwater protection standard. 
WELL FSB118D (cont.)

Radiological Indicator Parameters

\begin{tabular}{|c|c|c|c|c|c|c|c|c|c|c|c|c|c|c|c|}
\hline Parameter & 1095 & $\underline{\mathrm{DF}}$ & Mod & $\underline{\text { ST }}$ & H & Fiag & $\underline{\text { Lab }}$ & $\underline{2095}$ & $\underline{D F}$ & Mod & $\underline{\text { ST }}$ & $\underline{\mathrm{H}}$ & Flag & $\underline{\text { Lab }}$ & Unit \\
\hline $\begin{array}{l}\text { Gross alpha } \\
\text { Nonvolatile beta }\end{array}$ & $\begin{array}{l}1.5 \mathrm{E}+00 \\
7.0 \mathrm{E}-01\end{array}$ & $\begin{array}{l}1 \\
1\end{array}$ & UI & & & $\begin{array}{l}0 \\
0\end{array}$ & $\begin{array}{l}\text { GP } \\
\text { GP }\end{array}$ & $\begin{array}{l}2.2 E+\infty \\
1.9 E+00\end{array}$ & 1 & $J$ & & & $\begin{array}{l}0 \\
0\end{array}$ & $\begin{array}{l}\text { GP } \\
\text { GP }\end{array}$ & $\begin{array}{l}\text { pCi/L } \\
\text { pCi/L }\end{array}$ \\
\hline \multicolumn{16}{|c|}{ Measured Radionuclides } \\
\hline Parameter & 1095 & $\underline{\mathrm{DF}}$ & Mod & $\underline{\text { ST }}$ & $\underline{\mathrm{H}}$ & Flag & $\underline{\text { Lab }}$ & $\underline{2095}$ & $\underline{\text { DF }}$ & Mod & $\underline{\text { ST }}$ & $\underline{H}$ & Flag & $\underline{\text { Lab }}$ & Unit \\
\hline $\begin{array}{l}\text { Actinium-228 } \\
\text { Americium-241 } \\
\text { Cesium-137 } \\
\text { Cobalt-60 } \\
\text { Curium-242 } \\
\text { Curium-243/244 } \\
\text { Curium-245/246 } \\
\text { lodine-129 } \\
\text { Plutonium-238 } \\
\text { Plutonium-239/240 } \\
\text { Radium-226 } \\
\text { Radium-228 } \\
\text { Strontium-89 } \\
\text { Strontium-90 } \\
\text { Technetium-99 } \\
\text { Thorium-228 } \\
\text { Thorium-230 } \\
\text { Thorium-232 } \\
\text { Thorium-234 } \\
\text { Tritium } \\
\text { Uranium-233/234 } \\
\text { Uranium-235 } \\
\text { Uranium-238 }\end{array}$ & $\begin{array}{l}1.6 E+00 \\
8.7 E-02 \\
1.9 E+00 \\
-2.7 E-02 \\
0.0 E+00 \\
2.3 E-02 \\
0.0 E+00 \\
1.1 E+00 \\
-2.4 E-02 \\
8.4 E-02 \\
1.1 E+00 \\
-1.1 E+00 \\
-5.4 E-01 \\
-9.3 E-02 \\
-1.3 E+01 \\
0.0 E+00 \\
5.5 E-02 \\
0.0 E+00 \\
7.6 E+01 \\
1.2 E+01 \\
5.4 E-02 \\
0.0 E+00 \\
6.3 E-02\end{array}$ & $\begin{array}{l}1 \\
1 \\
1 \\
1 \\
1 \\
1 \\
1 \\
1 \\
1 \\
1 \\
1 \\
1 \\
1 \\
1 \\
1 \\
1 \\
1 \\
1 \\
1 \\
1 \\
1 \\
1 \\
1\end{array}$ & $\begin{array}{l}U I \\
U I \\
\text { UI } \\
\text { UI } \\
\text { UI } \\
\text { UI } \\
\text { UI } \\
\text { UI } \\
\text { UI } \\
\text { UI } \\
\text { UII } \\
\text { UI } \\
\text { UI } \\
\text { UI } \\
\text { UI } \\
\text { UI } \\
\text { UI } \\
\text { UI } \\
\text { UI } \\
\text { UI } \\
\text { UI }\end{array}$ & & & $\begin{array}{l}0 \\
0 \\
0 \\
0 \\
0 \\
0 \\
0 \\
0 \\
0 \\
0 \\
0 \\
0 \\
0 \\
0 \\
0 \\
0 \\
0 \\
0 \\
0 \\
1 \\
0 \\
0 \\
0\end{array}$ & $\begin{array}{l}\text { GP } \\
G P \\
G P \\
G P \\
G P \\
G P \\
G P \\
G P \\
G P \\
G P \\
G P \\
G P \\
G P \\
G P \\
G P \\
G P \\
G P \\
G P \\
G P \\
G P \\
G P \\
G P \\
G P\end{array}$ & $4.8 E+02$ & 1 & & - & & 2 & GP & 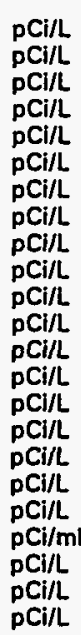 \\
\hline \multicolumn{16}{|c|}{ Calculated Radionuclides } \\
\hline Parameter & $\underline{1095}$ & $\underline{\mathrm{DF}}$ & Mod & $\underline{\text { ST }}$ & $\underline{H}$ & Flag & $\underline{\text { Lab }}$ & $\underline{2095}$ & $\underline{D F}$ & Mod & $\underline{\mathbf{S T}}$ & $\underline{\mathrm{H}}$ & Flag & $\underline{\text { Lab }}$ & Unit \\
\hline $\begin{array}{l}\text { Americium-243 } \\
\text { Nicke!-63 } \\
\text { Plutonium-241 } \\
\text { Plutonium-242 }\end{array}$ & $\begin{array}{l}7.0 \mathrm{E}-05 \\
-2.7 \mathrm{E}-02 \\
3.3 \mathrm{E}-01 \\
1.7 \mathrm{E}-06\end{array}$ & & & & & $\begin{array}{l}0 \\
0 \\
0 \\
0\end{array}$ & & & & & & & & & $\begin{array}{l}\mathrm{pCi} / \mathrm{L} \\
\mathrm{pCi} / \mathrm{L} \\
\mathrm{pCi} / \mathrm{L} \\
\mathrm{pCi} / \mathrm{L}\end{array}$ \\
\hline
\end{tabular}

Note: See Appendix B for flagging criteria. Synchronous water levels are measured over a 3-5 day period.

$\bullet=$ exceeded holding time.

- = exceeded groundwater protection standard. 
WELL FSB119D

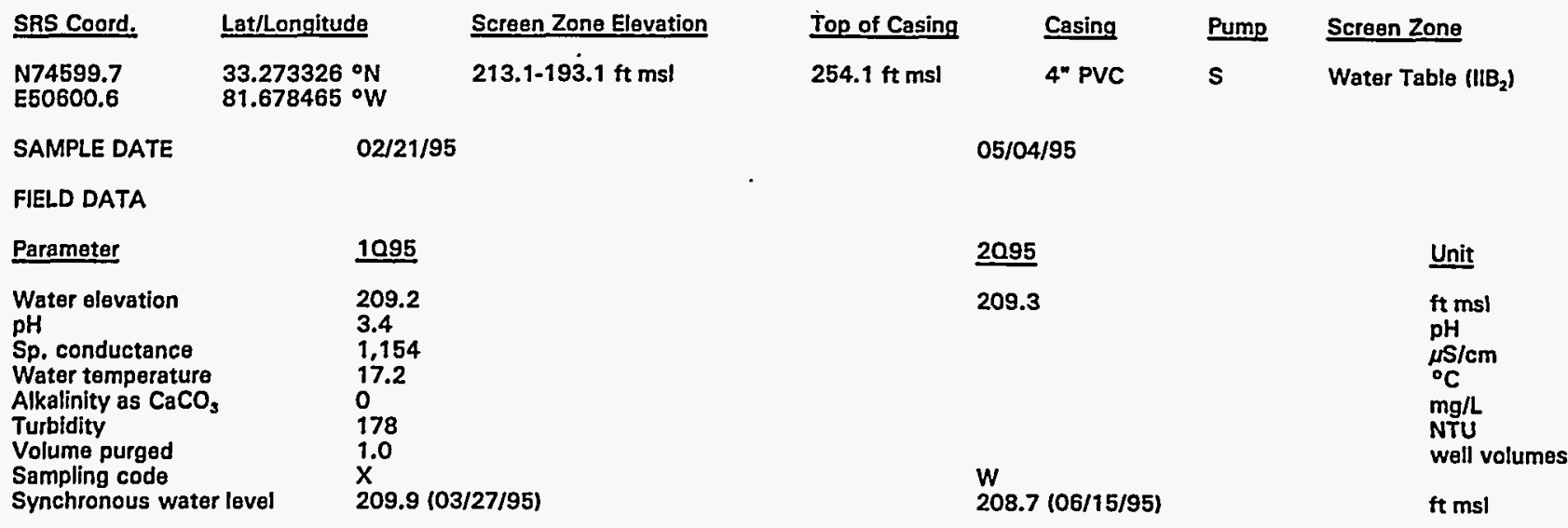

\section{Hazardous Constituents (264.94, Table 1$)$}

Parameter

Arsenic, total recoverable Barium, total recoverable

Cadmium, total recoverable

Chromium, total recoverable

Lead, total recoverable

Mercury, total recoverable

Selenium, total recoverable

Silver, total recoverable

\begin{tabular}{c}
1095 \\
\hline$<5.0$ \\
370 \\
5.6 \\
2.5 \\
9.2 \\
0.48 \\
$<5.0$ \\
$<0.65$
\end{tabular}

Lab
$\mathrm{GE}$
$\mathrm{GE}$
$\mathrm{GE}$
$\mathrm{GE}$
$\mathrm{GE}$
$\mathrm{GE}$
$\mathrm{GE}$
$\mathrm{GE}$

$\underline{2095}$

DF Mod ST $\underline{H}$ Flag Lab Unit

$\begin{array}{llll}1 & & 0 & \mathrm{GE} \\ 1 & \mathrm{~V} & 0 & \mathrm{GE} \\ 1 & & 2 & \mathrm{GE} \\ 1 & \mathrm{~J} & 0 & \mathrm{GE} \\ 1 & & 0 & \mathrm{GE} \\ 1 & \mathrm{~V} & 0 & \mathrm{GE} \\ 1 & & 0 & \mathrm{GE} \\ 1 & & 0 & \mathrm{GE}\end{array}$

Hazardous Constituents (261 App. Vill/264 App. IX)

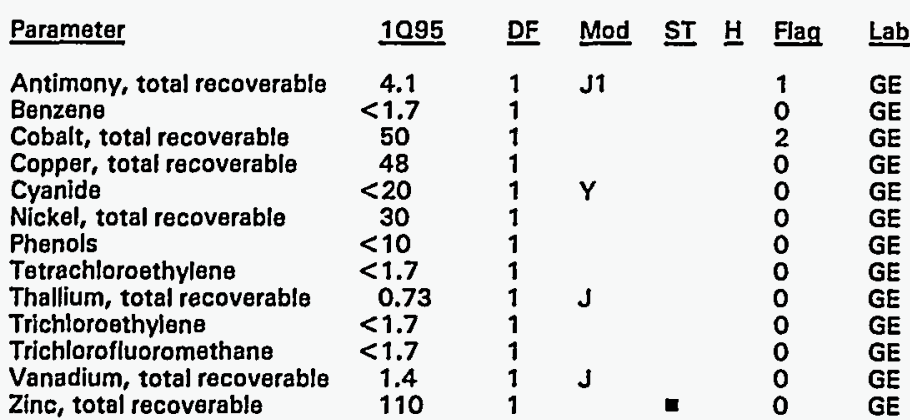

Water Quality Indicator Parameters

$\begin{array}{llllllll}\text { Parameter } & 1095 & \text { DF } & \text { Mod } & \text { ST } & \text { H } & \text { Flag } & \text { Lab } \\ \text { Aluminum, total recoverable } & 54,000 & 3 & \mathrm{~V} & & & 2 & \text { GE } \\ \text { Nitrate as nitrogen } & 130,000 & 80 & & & & 2 & \text { GE } \\ \text { pH } & 3.4 & 1 & \mathrm{~J} 1 & & \bullet & 1 & \text { GE }\end{array}$

$\mathrm{pH}$

3.4

Lab

2095 DF Mad ST $\underline{\text { Flag Lab }} \underline{\underline{\text { Unit }}}$

$\mu \mathrm{g} / \mathrm{L}$

$\mu g / L$

$\mu g / L$

$\mu g / L$
$\mu g / L$
$\mu g / L$

$\mu g / L$

$\mu \mathrm{g} / \mathrm{L}$

$\mu g / L$

$\mu \mathrm{g} / \mathrm{L}$

$\mu g / L$

$\mu g / L$

Common Laboratory Contaminants

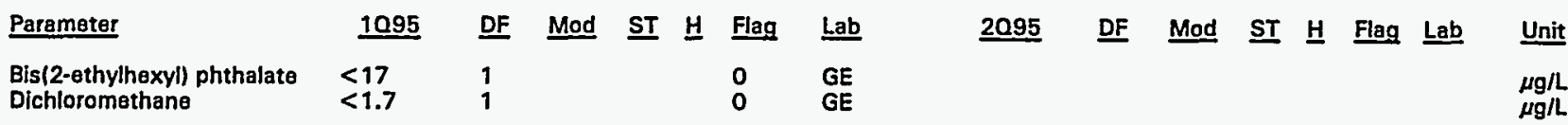

Note: See Appendix B for flagging criteria. Synchronous water levels are measured over a 3-5 day period.

- = exceeded holding time.

= exceeded groundwater protection standard. 
WELL FSB119D (cont.)

Radiological Indicator Parameters

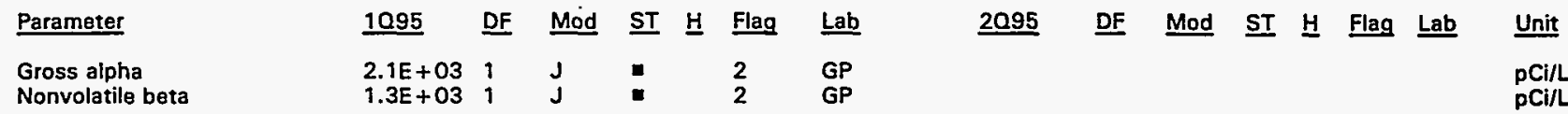

Measured Radionuclides

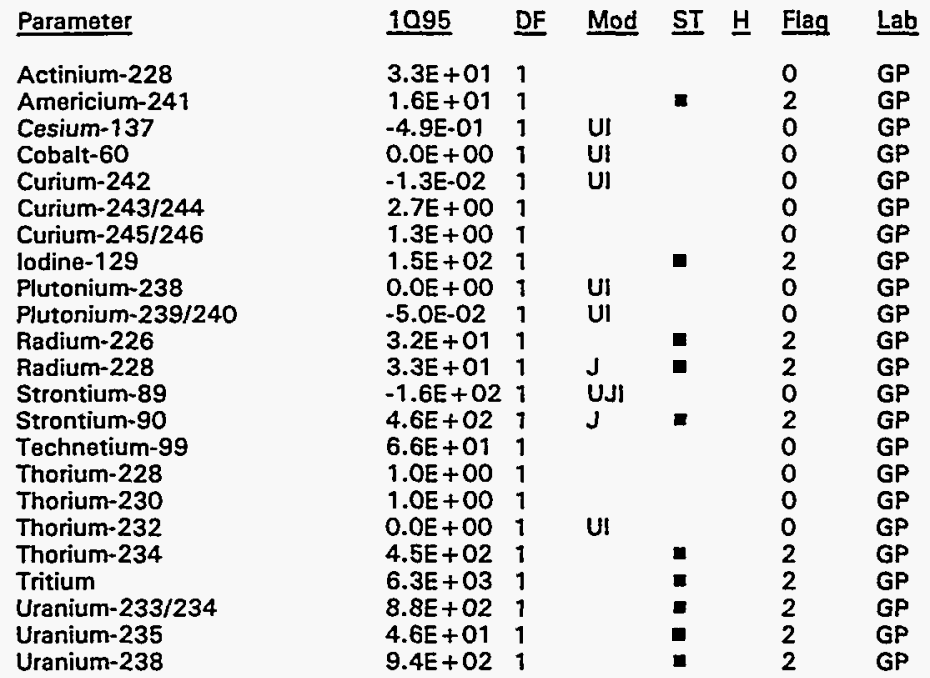

Uranium-238

$9.4 \mathrm{E}+021$

Calculated Radionuclides

Parameter

Americium-243

Nickel-63

Plutonium-241

Plutonium-242
1095

DF

8.0E-03

$0.0 \mathrm{E}+00$

$-2.0 E-01$

$-1.0 E-06$
2095 DF Mod ST H Flag Lab Unit

$\mathrm{pCi} / \mathrm{L}$

$\mathrm{PCi} / L$

$\mathrm{pCi} / \mathrm{L}$

pCill

pCi/L

pCi/L

pCi/L

pCi/L

pCi/L

pCi/L

PCI/L

pCI/L

$p C i / L$

$\mathrm{pCi} / \mathrm{L}$

pCi/L

pCi/L

$\mathrm{pCi} / \mathrm{L}$

pCi/mL

$\mathrm{pCi} / \mathrm{L}$

pCi/L

$\mathrm{pCi} / \mathrm{L}$

2095 DF Mod ST H Flag Lab Unit

pCil

$\mathrm{pCi} / \mathrm{L}$

pCI/L

pCilL

Note: See Appendix $B$ for flagging criteria. Synchronous water levels are measured over a 3-5 day period.

- = exceeded holding time.

- = exceeded groundwater protection standard. 


\section{WELL FSB120A}

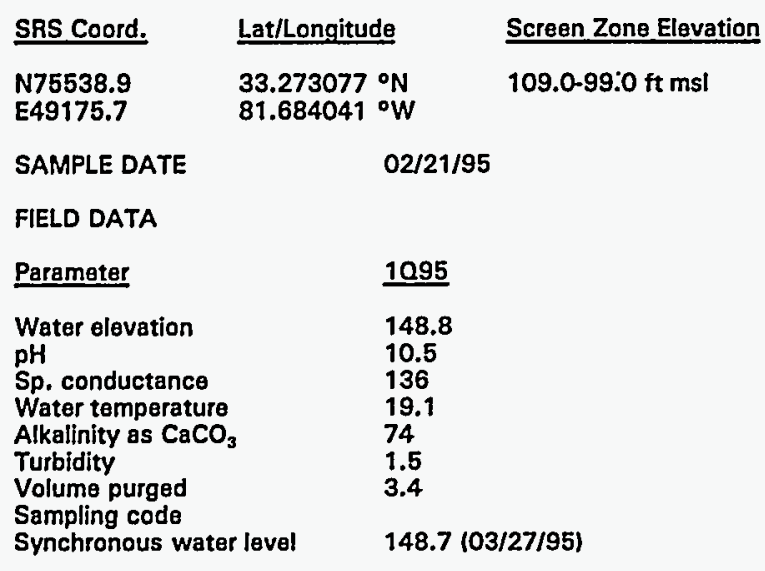

$\begin{array}{lrlll}\text { Top of Casing } & \text { Casing } & \text { Pump } & \text { Screen Zone } \\ 280.1 \mathrm{ft} \text { msl } & 4^{\text {" PVC }} & \mathrm{S} & \text { U. Congares (IIA) } \\ & 04 / 04 / 95 & & \end{array}$

Hazardous Constituents (264.94, Table 1)

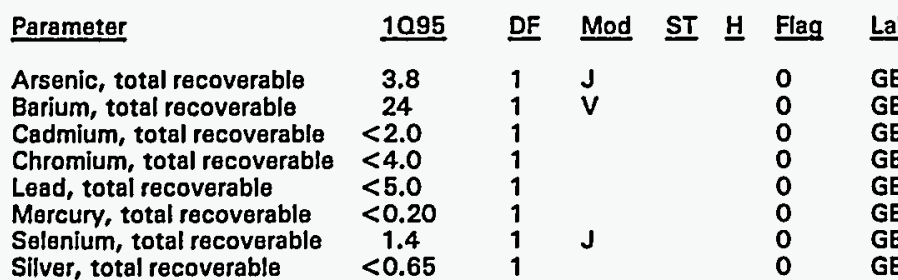

$\begin{array}{ll}2095 & \text { Unit } \\ 148.8 & \mathrm{ft} \mathrm{msl} \\ 10.6 & \mathrm{pH}^{\mathrm{jS} / \mathrm{cm}} \\ 151 & { }^{\circ} \mathrm{C} \\ 19.9 & \mathrm{mg} / \mathrm{L} \\ 33 & \mathrm{NTU} \\ 1 & \text { well volumes } \\ 3.1 & \text { ft ms! } \\ 148.6(06 / 15 / 95) & \end{array}$

Hazardous Constituents (261 App. VIII/264 App. IX)

\begin{tabular}{|c|c|c|c|c|c|c|c|c|c|c|c|c|c|c|c|}
\hline Parameter & 1095 & DF & Mod & $\underline{\text { ST }}$ & $\underline{H}$ & Flag & Lab & 2095 & DF & Mod & $\underline{\text { ST }}$ & $\underline{\mathrm{H}}$ & Flag & Lab & Unit \\
\hline $\begin{array}{l}\text { Antimony, total recoverable } \\
\text { Benzene } \\
\text { Cobalt, total recoverable } \\
\text { Copper, total recoverable } \\
\text { Cyanide } \\
\text { Nickel, total recoverable } \\
\text { Phenols } \\
\text { Tetrachloroethylene } \\
\text { Thallium, total recoverable } \\
\text { Trichloroethylene } \\
\text { Trichlorofluoromethane } \\
\text { Vanadium, total recoverable } \\
\text { Zinc, total recoverable }\end{array}$ & $\begin{aligned} & 3.7 \\
&< 1.7 \\
&<4.0 \\
&<4.0 \\
&<20 \\
&<10 \\
&<10 \\
&<10 \\
&<1.7 \\
&<5.0 \\
&<1.7 \\
&<1.7 \\
& \quad 6.5 \\
&<5.0\end{aligned}$ & $\begin{array}{l}1 \\
1 \\
1 \\
1 \\
1 \\
1 \\
1 \\
1 \\
1 \\
1 \\
1 \\
1 \\
1\end{array}$ & $\mathbf{J}$ & & & $\begin{array}{l}1 \\
0 \\
0 \\
0 \\
0 \\
0 \\
0 \\
0 \\
0 \\
0 \\
0 \\
0 \\
0\end{array}$ & $\begin{array}{l}\text { GE } \\
\text { GE } \\
\text { GE } \\
\text { GE } \\
\text { GE } \\
\text { GE } \\
\text { GE } \\
\text { GE } \\
\text { GE } \\
\text { GE } \\
\text { GE } \\
\text { GE } \\
\text { GE }\end{array}$ & & & & & & & & $\begin{array}{l}\mu g / L \\
\mu g / L \\
\mu g / L \\
\mu g / L \\
\mu g / L \\
\mu g / L \\
\mu g / L \\
\mu g / L \\
\mu g / L \\
\mu g / L \\
\mu g / L \\
\mu g / L \\
\mu g / L\end{array}$ \\
\hline \multicolumn{16}{|c|}{ Water Quality Indicator Parameters } \\
\hline Parameter & 1095 & DF & Mod & $\underline{\text { ST }}$ & $\underline{H}$ & Flag & $\underline{\text { Lab }}$ & $\underline{2095}$ & $\underline{D F}$ & Mod & $\underline{\text { ST }}$ & $\underline{H}$ & Flag & $\underline{\text { Lab }}$ & Unit \\
\hline \multicolumn{16}{|c|}{ Common Laboratory Contaminants } \\
\hline Parameter & 1095 & DF & Mod & $\underline{\mathbf{S T}}$ & $\underline{\mathrm{H}}$ & Flag & $\underline{\text { Lab }}$ & $\underline{2095}$ & DF & Mod & $\underline{\mathbf{S T}}$ & $\underline{\mathbf{H}}$ & Flag & Lab & Unit \\
\hline $\begin{array}{l}\text { Bis(2-ethylhexyl) phthalate } \\
\text { Dichloromethane }\end{array}$ & $\begin{array}{l}<17 \\
<1.7\end{array}$ & $\begin{array}{l}1 \\
1\end{array}$ & & & & $\begin{array}{l}0 \\
0\end{array}$ & $\begin{array}{l}\text { GE } \\
\text { GE }\end{array}$ & & & & & & & & $\begin{array}{l}\mu g / L \\
\mu g / L\end{array}$ \\
\hline
\end{tabular}

Note: See Appendix B for flagging criteria. Synchronous water levels are measured over a 3-5 day period.

$\bullet=$ exceeded holding time.

- = exceeded groundwater protection standard. 
WELL FSB120A (cont.)

Radiological Indicator Parameters

\begin{tabular}{|c|c|c|c|c|c|c|c|c|c|c|c|c|c|c|}
\hline Parameter & 1095 & $\underline{D F}$ & Mod & $\underline{\text { ST }} \underline{H}$ & Flag & $\underline{\text { Lab }}$ & 2095 & $\underline{\mathrm{DF}}$ & Mod & $\underline{\text { ST }}$ & H & Flag & Lab & Unit \\
\hline $\begin{array}{l}\text { Gross alpha } \\
\text { Nonvolatile beta }\end{array}$ & $\begin{array}{l}1.6 E+00 \\
3.5 E+00\end{array}$ & $\begin{array}{l}1 \\
1\end{array}$ & & & $\begin{array}{l}0 \\
0\end{array}$ & $\begin{array}{l}\text { GP } \\
\text { GP }\end{array}$ & $\begin{array}{l}6.6 \mathrm{E}-01 \\
4.4 \mathrm{E}+00\end{array}$ & $\begin{array}{l}1 \\
1\end{array}$ & UI & & & $\begin{array}{l}0 \\
0\end{array}$ & $\begin{array}{l}\text { GP } \\
\text { GP }\end{array}$ & $\begin{array}{l}\mathrm{pCi} / \mathrm{L} \\
\mathrm{pCi} / \mathrm{L}\end{array}$ \\
\hline
\end{tabular}

Measured Radionuclides

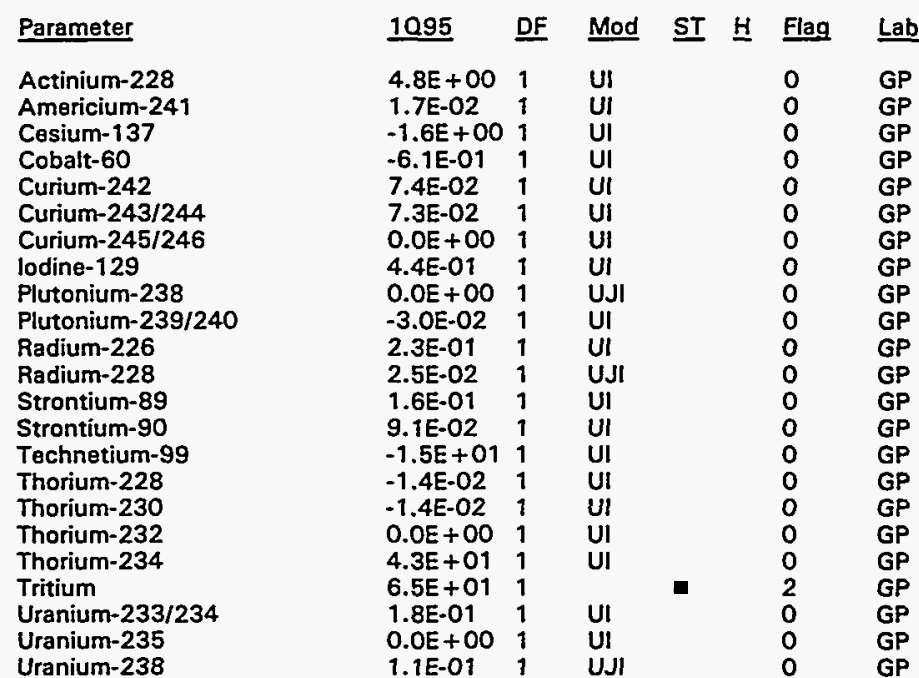

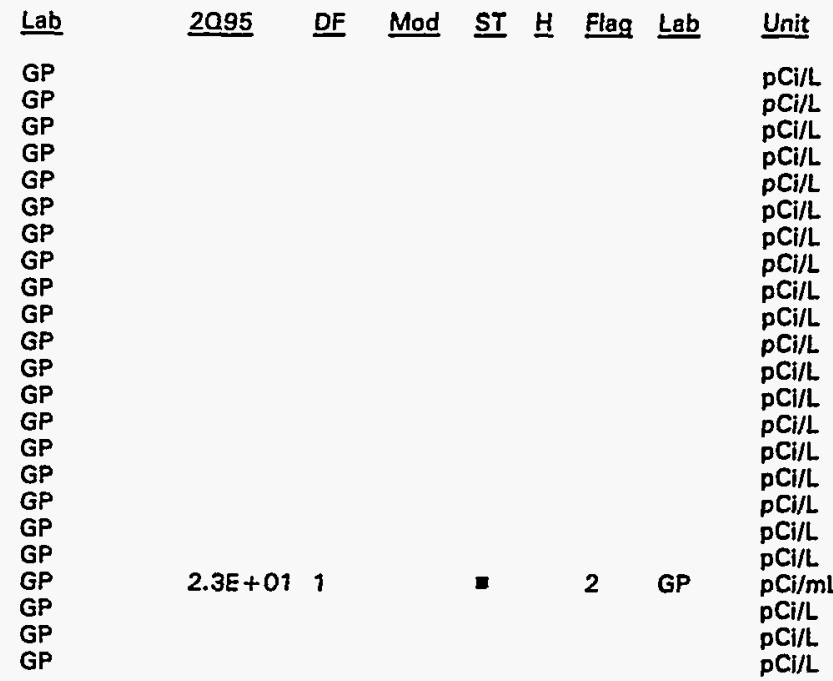

Calculated Radionuclides

Parameter

Americium-243

Nickel-63

Plutonium-241

Plutonium-242 $\underline{1095}$

$-6.1 E-01$

$-1.2 E-01$

$-5.9 E-07$
DF Mod ST $\underline{\text { H Flag Lab }}$

0

2095
DF Mod ST H Fiag Lab Unit

pCi/L

$\mathrm{pCi} / \mathrm{L}$

pCi/L

pCi/L

Note: See Appendix B for flagging criteria. Synchronous water levels are measured over a 3-5 day period.

$\bullet=$ exceeded holding time.

a = exceeded groundwater protection standard. 
WELL FSB120C

\begin{tabular}{|c|c|c|c|c|c|c|c|c|c|c|c|c|c|c|c|}
\hline sis Coord. & Lat/Longitude & \multicolumn{5}{|c|}{ Screen Zone Elevation } & Top of Casing & & \multicolumn{2}{|c|}{ Casing } & \multicolumn{2}{|l|}{ Pump } & \multicolumn{3}{|c|}{ Screen Zone } \\
\hline $\begin{array}{l}33.273094 \\
81.684074\end{array}$ & $\begin{array}{l}33.273094^{\circ} \mathrm{N} \\
81.684074^{\circ} \mathrm{W}\end{array}$ & \multicolumn{5}{|c|}{$160.7-150.7 \mathrm{ft} \mathrm{msl}$} & $279.7 \mathrm{ft} \mathrm{msl}$ & & \multicolumn{2}{|c|}{ 4" PVC } & \multicolumn{2}{|l|}{$\mathbf{S}$} & \multicolumn{3}{|c|}{ Barnwell (II, ) } \\
\hline SAMPLE DATE & \multicolumn{6}{|c|}{$02 / 21 / 95$} & & \multicolumn{3}{|c|}{$04 / 04 / 95$} & & & & & \\
\hline \multicolumn{16}{|l|}{ FIELD DATA } \\
\hline Parameter & \multicolumn{6}{|l|}{1095} & \multicolumn{5}{|c|}{$\underline{2095}$} & & \multicolumn{3}{|c|}{$\underline{\text { Unit }}$} \\
\hline $\begin{array}{l}\text { Water elevation } \\
\text { pH } \\
\text { Sp. conductance } \\
\text { Water temperature } \\
\text { Alkalinity as } \mathrm{CaCO}_{3} \\
\text { Turbidity } \\
\text { Volume purged } \\
\text { Sampling code } \\
\text { Synchronous water level }\end{array}$ & \multicolumn{6}{|l|}{$\begin{array}{l}205.6 \\
7.0 \\
306 \\
19.4 \\
20 \\
2.9 \\
3.5\end{array}$} & \multicolumn{5}{|c|}{$\begin{array}{l}206.1 \\
6.5 \\
326 \\
19.3 \\
7 \\
0.7 \\
4.5\end{array}$} & & \multicolumn{3}{|c|}{$\begin{array}{l}\mathrm{ft} \mathrm{msl} \\
\mathrm{pH} \\
\mu \mathrm{S} / \mathrm{cm} \\
{ }^{\circ} \mathrm{C} \\
\mathrm{mg} / \mathrm{L} \\
\mathrm{NTU} \\
\text { well volumes }\end{array}$} \\
\hline \multicolumn{16}{|c|}{ Hazardous Constituents (264.94, Table 1) } \\
\hline Parameter & 1095 & $\underline{D F}$ & Mod & $\underline{\text { ST }}$ & $\underline{H}$ & Flag & $\underline{\text { Lab }}$ & $\underline{2095}$ & $\underline{\mathrm{DF}}$ & Mod & $\underline{\text { ST }}$ & $\underline{H}$ & Flag & $\underline{\text { Lab }}$ & $\underline{\text { Unit }}$ \\
\hline $\begin{array}{l}\text { Arsenic, total recoverable } \\
\text { Barium, total recoverable } \\
\text { Cadmium, total recoverable } \\
\text { Chromium, total recoverable } \\
\text { Lead, total recoverable } \\
\text { Mercury, total recoverable } \\
\text { Selenium, total recoverable } \\
\text { Silver, total recoverable }\end{array}$ & $\begin{array}{l}<5.0 \\
100 \\
0.79 \\
<4.0 \\
<5.0 \\
<0.20 \\
<5.0 \\
<0.65\end{array}$ & $\begin{array}{l}1 \\
1 \\
1 \\
1 \\
1 \\
1 \\
1 \\
1\end{array}$ & $\begin{array}{l}V \\
J\end{array}$ & & & $\begin{array}{l}0 \\
0 \\
0 \\
0 \\
0 \\
0 \\
0 \\
0\end{array}$ & $\begin{array}{l}\mathrm{GE} \\
\mathrm{GE} \\
\mathrm{GE} \\
\mathrm{GE} \\
\mathrm{GE} \\
\mathrm{GE} \\
\mathrm{GE} \\
\mathrm{GE}\end{array}$ & $\begin{array}{l}82 \\
0.61 \\
1.8 \\
0.58\end{array}$ & $\begin{array}{l}5 \\
1 \\
1\end{array}$ & $\begin{array}{l}V \\
J \\
J W V\end{array}$ & & & $\begin{array}{l}0 \\
0 \\
0\end{array}$ & $\begin{array}{l}\text { GE } \\
\text { GE } \\
\text { GE } \\
\text { GE }\end{array}$ & $\begin{array}{l}\mu \mathrm{g} / \mathrm{L} \\
\mu g / \mathrm{L} \\
\mu \mathrm{g} / \mathrm{L} \\
\mu g / \mathrm{L} \\
\mu \mathrm{g} / \mathrm{L} \\
\mu \mathrm{g} / \mathrm{L} \\
\mu \mathrm{g} / \mathrm{L} \\
\mu \mathrm{g} / \mathrm{L}\end{array}$ \\
\hline \multicolumn{16}{|c|}{ Hazardous Constituents (261 App. VIII/264 App. IX) } \\
\hline Parameter & $\underline{1095}$ & $\underline{\mathrm{DF}}$ & Mod & $\underline{\mathbf{S T}}$ & 브 & Flag & $\underline{\text { Lab }}$ & $\underline{2095}$ & $\underline{D F}$ & Mod & $\underline{\text { ST }}$ & $\underline{H}$ & Flag & $\underline{\text { Lab }}$ & Unit \\
\hline $\begin{array}{l}\text { Antimony, total recoverable } \\
\text { Benzene } \\
\text { Cobalt, total recoverable } \\
\text { Copper, total recoverable } \\
\text { Cyanide } \\
\text { Nickel, total recoverable } \\
\text { Phenols } \\
\text { Tetrachloroethylene } \\
\text { Thallium, total recoverable } \\
\text { Trichloroethylene } \\
\text { Trichlorofluoromethane } \\
\text { Vanadium, total recoverable } \\
\text { Zinc, total recoverable }\end{array}$ & $\begin{aligned}<5.0 \\
<1.7 \\
5.5 \\
<4.0 \\
<20 \\
7.4 \\
<10 \\
<1.7 \\
<5.0 \\
<1.7 \\
<1.7 \\
<10 \\
12\end{aligned}$ & $\begin{array}{l}1 \\
1 \\
1 \\
1 \\
1 \\
1 \\
1 \\
1 \\
1 \\
1 \\
1 \\
1 \\
1\end{array}$ & $J$ & & & $\begin{array}{l}0 \\
0 \\
0 \\
0 \\
0 \\
0 \\
0 \\
0 \\
0 \\
0 \\
0 \\
0 \\
0\end{array}$ & $\begin{array}{l}\mathrm{GE} \\
\mathrm{GE} \\
\mathrm{GE} \\
\mathrm{GE} \\
\mathrm{GE} \\
\mathrm{GE} \\
\mathrm{GE} \\
\mathrm{GE} \\
\mathrm{GE} \\
\mathrm{GE} \\
\mathrm{GE} \\
\mathrm{GE} \\
\mathrm{GE}\end{array}$ & 5.4 & 1 & $\mathbf{J}$ & & & 0 & GE & $\begin{array}{l}\mu g / L \\
\mu g / L \\
\mu g / L \\
\mu g / L \\
\mu g / L \\
\mu g / L \\
\mu g / L \\
\mu g / L \\
\mu g / L \\
\mu g / L \\
\mu g / L \\
\mu g / L \\
\mu g / L\end{array}$ \\
\hline \multicolumn{16}{|c|}{ Water Quality Indicator Parameters } \\
\hline Parameter & 1095 & DF & Mod & $\underline{\mathbf{s T}}$ & $\underline{H}$ & Flag & $\underline{\text { Lab }}$ & $\underline{2095}$ & DF & Mod & $\underline{\mathbf{S T}}$ & $\underline{H}$ & Flag & Lab & $\underline{\text { Unit }}$ \\
\hline $\begin{array}{l}\text { Aluminum, total recoverable } \\
\text { Nitrate as nitrogen } \\
\text { pH }\end{array}$ & $\begin{array}{l}180 \\
34,000 \\
6.7\end{array}$ & $\begin{array}{l}1 \\
20 \\
1\end{array}$ & J1 & : & $\bullet$ & $\begin{array}{l}2 \\
2 \\
0\end{array}$ & $\begin{array}{l}\mathrm{GE} \\
\mathrm{GE} \\
\mathrm{GE}\end{array}$ & $\begin{array}{l}37,000 \\
6.5\end{array}$ & $\begin{array}{l}20 \\
1\end{array}$ & $\mathrm{J1}$ & 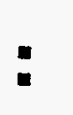 & $\bullet$ & $\begin{array}{l}2 \\
0\end{array}$ & $\begin{array}{l}\mathrm{GE} \\
\mathrm{GE}\end{array}$ & $\begin{array}{l}\mu \mathrm{g} / \mathrm{L} \\
\mu \mathrm{g} / \mathrm{L} \\
\mathrm{pH}\end{array}$ \\
\hline \multicolumn{16}{|c|}{ Common Laboratory Contaminants } \\
\hline Parameter & $\underline{1095}$ & DF & Mod & $\underline{\mathbf{S T}}$ & 브 & Flag & $\underline{\text { Lab }}$ & $\underline{2095}$ & DF & $\underline{\text { Mod }}$ & $\underline{\mathbf{S I}}$ & 보 & Flag & $\underline{\text { Lab }}$ & $\underline{\text { Unit }}$ \\
\hline $\begin{array}{l}\text { Bis(2-ethylhexyl) phthalate } \\
\text { Dichloromethane }\end{array}$ & $\begin{array}{l}<17 \\
<1.7\end{array}$ & $\begin{array}{l}1 \\
1\end{array}$ & & & & $\begin{array}{l}0 \\
0\end{array}$ & $\begin{array}{l}\mathrm{GE} \\
\mathrm{GE}\end{array}$ & & & & & & & & $\underset{\mu g / L}{\mu g / L}$ \\
\hline
\end{tabular}

Note: See Appendix B for flagging criteria. Synchronous water levels are measured over a 3-5 day period.

- = exceeded holding time.

- = exceeded groundwater protection standard. 
WELL FSB120C (cont.)

Radiological Indicator Parameters

\begin{tabular}{|c|c|c|c|c|c|c|c|c|c|c|c|c|c|c|c|}
\hline Parameter & 1095 & DF & Mod & $\underline{S T}$ & $\underline{\mathrm{H}}$ & Flag & Lab & $\underline{2095}$ & DF & Mod & $\underline{\mathbf{S T}}$ & $\underline{H}$ & Flag & Lab & Unit \\
\hline $\begin{array}{l}\text { Gross alpha } \\
\text { Nonvolatile beta }\end{array}$ & $\begin{array}{l}1.7 E+01 \\
5.0 E+01\end{array}$ & $\begin{array}{l}1 \\
1\end{array}$ & $J$ & घ & & $\begin{array}{l}2 \\
1\end{array}$ & $\begin{array}{l}\text { GP } \\
\text { GP }\end{array}$ & $\begin{array}{l}1.5 E+01 \\
4.4 E+01\end{array}$ & $\begin{array}{l}1 \\
1\end{array}$ & & & & $\begin{array}{l}1 \\
1\end{array}$ & $\begin{array}{l}\text { GP } \\
\text { GP }\end{array}$ & $\begin{array}{l}\mathrm{pCi} / \mathrm{L} \\
\mathrm{pCi} / \mathrm{L}\end{array}$ \\
\hline Parameter & 1095 & $\underline{\text { DF }}$ & Mod & $\underline{\text { ST }}$ & $\underline{H}$ & Flag & $\underline{\text { Lab }}$ & $\underline{2095}$ & $\underline{\mathrm{DF}}$ & Mod & $\underline{\text { ST }}$ & 브 & Flag & Lab & Unit \\
\hline $\begin{array}{l}\text { Actinium-228 } \\
\text { Americium-241 } \\
\text { Cesium-137 } \\
\text { Cobalt-60 } \\
\text { Curium-242 } \\
\text { Curium-243/244 } \\
\text { Curium-245/246 } \\
\text { lodine-129 } \\
\text { Plutonium-238 } \\
\text { Plutonium-239/240 } \\
\text { Radium-226 } \\
\text { Radium-228 } \\
\text { Strontium-89 } \\
\text { Strontium-90 } \\
\text { Technetium-99 } \\
\text { Thorium-228 } \\
\text { Thorium-230 } \\
\text { Thorium-232 } \\
\text { Thorium-234 } \\
\text { Tritium } \\
\text { Uranium-233/234 } \\
\text { Uranium-235 } \\
\text { Uranium-238 }\end{array}$ & $\begin{array}{l}3.7 E+00 \\
8.4 E-02 \\
2.1 E+00 \\
1.9 E+00 \\
-2.8 E-02 \\
-2.2 E-02 \\
0.0 E+00 \\
1.4 E+01 \\
1.0 E-01 \\
3.6 E-02 \\
5.1 E-01 \\
1.7 E+00 \\
-7.2 E-01 \\
4.5 E+00 \\
4.6 E+01 \\
0.0 E+00 \\
2.1 E-01 \\
-6.6 E-03 \\
4.2 E+00 \\
1.1 E+03 \\
3.7 E-01 \\
0.0 E+00 \\
2.2 E-01\end{array}$ & $\begin{array}{l}1 \\
1 \\
1 \\
1 \\
1 \\
1 \\
1 \\
1 \\
1 \\
1 \\
1 \\
1 \\
1 \\
1 \\
1 \\
1 \\
1 \\
1 \\
1 \\
1 \\
1 \\
1 \\
1\end{array}$ & $\begin{array}{l}\text { UI } \\
\text { UI } \\
\text { UI } \\
\text { UI } \\
\text { UI } \\
\text { UI } \\
\text { UI } \\
\text { UI } \\
\text { UI } \\
\text { UI } \\
\text { UI } \\
\text { UI } \\
\text { UI } \\
\text { UI } \\
\text { UI } \\
\text { UI } \\
\text { UI } \\
\text { UI } \\
\text { UI }\end{array}$ & 口 & & $\begin{array}{l}0 \\
0 \\
0 \\
0 \\
0 \\
0 \\
0 \\
2 \\
0 \\
0 \\
0 \\
0 \\
0 \\
1 \\
0 \\
0 \\
0 \\
0 \\
0 \\
2 \\
0 \\
0 \\
0\end{array}$ & $\begin{array}{l}\text { GP } \\
\text { GP } \\
\text { GP } \\
\text { GP } \\
\text { GP } \\
\text { GP } \\
\text { GP } \\
\text { GP } \\
\text { GP } \\
\text { GP } \\
\text { GP } \\
\text { GP } \\
\text { GP } \\
\text { GP } \\
\text { GP } \\
\text { GP } \\
\text { GP } \\
\text { GP } \\
\text { GP } \\
\text { GP } \\
\text { GP } \\
\text { GP } \\
\text { GP }\end{array}$ & $1.0 \mathrm{E}+03$ & 1 & & - & & 2 & GP & 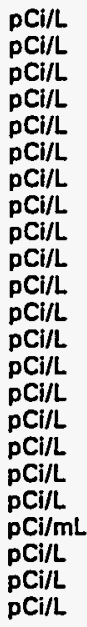 \\
\hline \multicolumn{16}{|c|}{ Calculated Radionuclides } \\
\hline Parameter & $\underline{1095}$ & $\underline{D F}$ & Mod & $\underline{\text { ST }}$ & 보 & Flag & Lab & 2095 & $\underline{D F}$ & Mod & $\underline{\underline{S T}}$ & 브 & Flag & $\underline{L a b}$ & Unit \\
\hline $\begin{array}{l}\text { Americium-243 } \\
\text { Nickel-63 } \\
\text { Plutonium-241 } \\
\text { Plutonium-242 }\end{array}$ & $\begin{array}{l}-6.7 E-05 \\
1.9 E+00 \\
1.5 E-01 \\
7.3 E-07\end{array}$ & & & & & $\begin{array}{l}0 \\
0 \\
0 \\
0\end{array}$ & & & & & & & & & $\begin{array}{l}\mathrm{pCi} / \mathrm{L} \\
\mathrm{pCi} / \mathrm{L} \\
\mathrm{pCi} / \mathrm{L} \\
\mathrm{pCi} / \mathrm{L}\end{array}$ \\
\hline
\end{tabular}

Note: See Appendix B for flagging criteria. Synchronous water levels are measured over a 3-5 day period.

- = exceeded holding time.

- = exceeded groundwater protection standard. 


\section{WELL FSB120D}

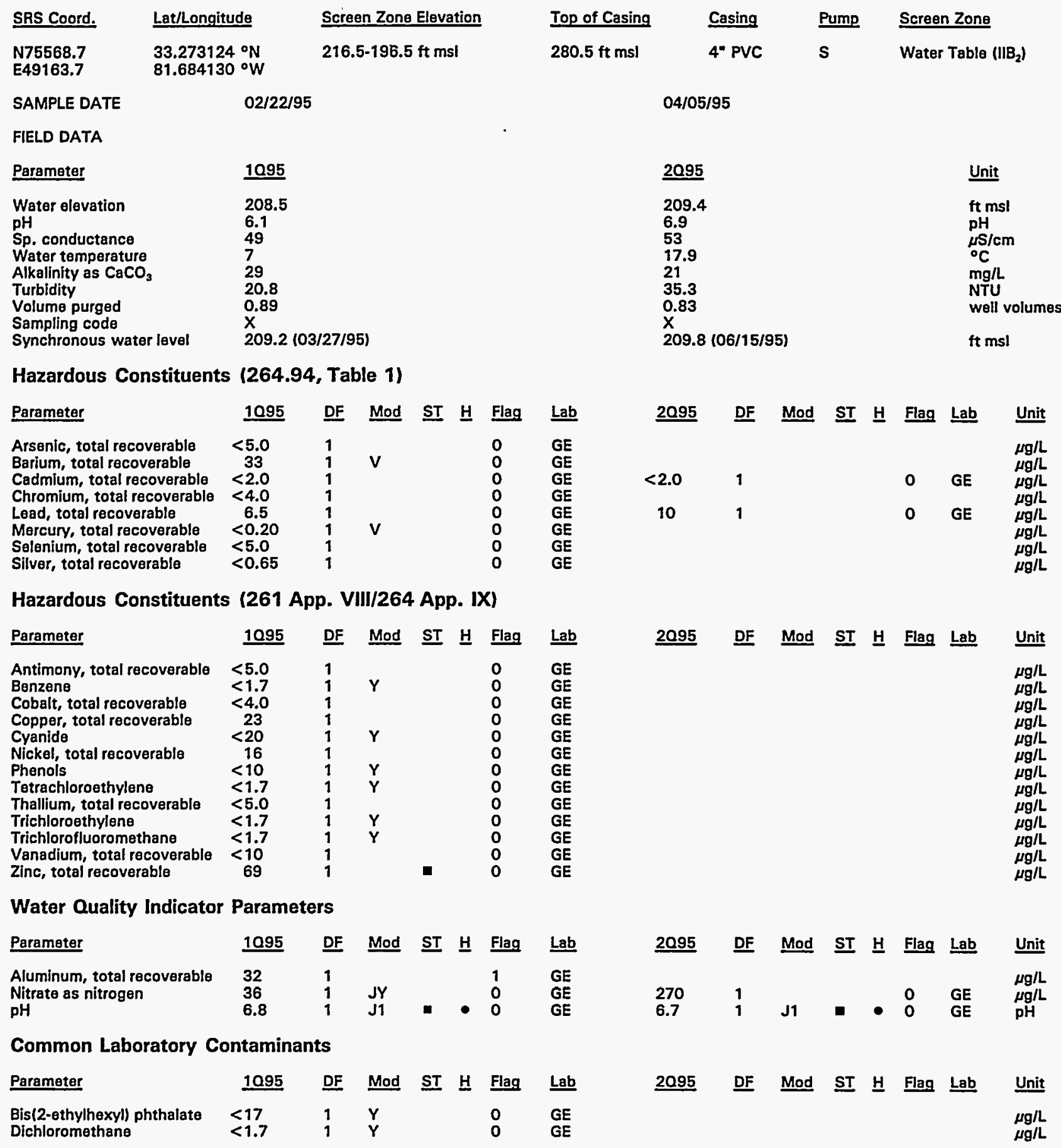

Note: See Appendix B for flagging criteria. Synchronous water levels are measured over a 3-5 day period.

$-=$ exceeded holding time.

- = exceeded groundwater protection standard. 
WELL FSB120D (cont.)

Radiological Indicator Parameters

\begin{tabular}{|c|c|c|c|c|c|c|}
\hline Parameter & 1095 & DF & Mod & $\underline{\text { ST }}$ & 브 & Flag \\
\hline $\begin{array}{l}\text { Gross alpha } \\
\text { Nonvolatile beta }\end{array}$ & $\begin{array}{l}1.8 E+00 \\
2.6 E+00\end{array}$ & $\begin{array}{l}1 \\
1\end{array}$ & $J$ & & & $\begin{array}{l}0 \\
0\end{array}$ \\
\hline \multicolumn{7}{|c|}{ Measured Radionuclides } \\
\hline Parameter & 1095 & $\underline{D F}$ & Mod & $\underline{\text { ST }}$ & 브 & Flag \\
\hline Actinium-228 & $-7.6 E-01$ & 1 & UI & & & 0 \\
\hline Americium-241 & 4.0E-02 & 1 & Ui & & & 0 \\
\hline Cesium-137 & $-8.3 E-01$ & $i$ & Ui & & & 0 \\
\hline Cobalt -60 & $-2.4 E-01$ & 1 & UI & & & 0 \\
\hline Curium-242 & $-4.4 E-02$ & 1 & UI & & & 0 \\
\hline Curium-243/244 & $-2.9 E-02$ & 1 & Ui & & & 0 \\
\hline Curium-245/246 & $0.0 E+00$ & 1 & Ui & & & 0 \\
\hline lodine-129 & $-3.5 \mathrm{E}-02$ & 1 & Ui & & & 0 \\
\hline Plutonium-238 & $1.7 E-01$ & 1 & UI & & & 0 \\
\hline Plutonium-239/240 & 4.4E-02 & 1 & UI & & & 0 \\
\hline Radium-226 & $1.9 E-01$ & 1 & UI & & & 0 \\
\hline Radium-228 & $-8.5 E-02$ & 1 & UJI & & & 0 \\
\hline Strontium-89 & 3.6E-01 & 1 & III & & & 0 \\
\hline Technetium-99 & $\begin{array}{l}-1.3 E-01 \\
-3.4 E+00\end{array}$ & 1 & UI & & & 0 \\
\hline Thorium-228 & $-6.3 \mathrm{E}-02$ & 1 & Ui & & & 0 \\
\hline Thorium-230 & 2.7E-01 & 1 & UI & & & 0 \\
\hline Thorium-232 & $2.8 \mathrm{E}-02$ & 1 & UI & & & 0 \\
\hline Thorium-234 & $0.0 E+00$ & 1 & UI & & & 0 \\
\hline Tritium & $1.1 E+01$ & 1 & & & & 1 \\
\hline Uranium-233/234 & 1.2E-02 & 1 & UI & & & 0 \\
\hline Uranium-235 & $-6.7 \mathrm{E}-03$ & 1 & UI & & & 0 \\
\hline Uranium-238 & $8.6 \mathrm{E}-02$ & 1 & UI & & & 0 \\
\hline
\end{tabular}

\begin{tabular}{|c|c|c|c|c|c|c|c|}
\hline Lab & $\underline{2095}$ & DF & Mod & $\underline{\text { ST }} \underline{H}$ & Flag & Lab & Unit \\
\hline $\begin{array}{l}\text { GP } \\
\text { GP }\end{array}$ & $\begin{array}{l}2.1 E+\infty \\
2.5 E+\infty\end{array}$ & $\begin{array}{l}1 \\
1\end{array}$ & $J$ & & $\begin{array}{l}0 \\
0\end{array}$ & $\begin{array}{l}\text { GP } \\
\text { GP }\end{array}$ & $\begin{array}{l}\mathrm{pCi} / \mathrm{L} \\
\mathrm{pCi} / \mathrm{L}\end{array}$ \\
\hline
\end{tabular}

Calculated Radionuclides

Parameter

Americium-243

Nickel-63

Plutonium-241

Plutonium-242
1095

$-2.4 \mathrm{E}-01$

1.7E-01

8.7E-07
Lab
GP
$G P$
$G P$
$G P$
$G P$
$G P$
$G P$
$G P$
$G P$
$G P$
$G P$
$G P$
$G P$
$G P$
$G P$
$G P$
$G P$
$G P$
$G P$
$G P$
$G P$
$G P$
$G P$
$G P$

$\underline{2095}$

DF Mod ST $\underline{H}$ Flag Lab

Unit

$\mathrm{pCi} / \mathrm{L}$

$\mathrm{pCi} / \mathrm{L}$

$\mathrm{pCi} / \mathrm{L}$

pCi/L

pCi/L

$\mathrm{pCi} / \mathrm{L}$

pCi/l

pCill

$\mathrm{pCI/L}$

pCill

pCill

$\mathrm{pCi} / \mathrm{L}$

$\mathrm{pCi} / \mathrm{L}$

pCi/l

pCi/L

pCi/L

$\mathrm{pCill}$

$\begin{array}{ccccc}1.1 E+01 & 1 & 1 & \mathrm{GP} & \mathrm{PCl} / \mathrm{L} \\ \mathrm{PCl} / \mathrm{mL}\end{array}$

$\mathrm{PCi} / \mathrm{L}$

$\mathrm{pCi} / \mathrm{L}$

pCi/L

2095 DF Mod ST H Flag Lab Unit

$\mathrm{pCi/L}$

pCilL

$\mathrm{pCi} / \mathrm{L}$

$\mathrm{pCi} / \mathrm{L}$

Note: See Appendix B for flagging criteria. Synchronous water levels are measured over a 3-5 day period.

- = exceeded holding time.

m = exceeded groundwater protection standard. 
WELL FSB121C

\begin{tabular}{|c|c|c|c|c|c|c|}
\hline SRS Coord. & Lat/Longitude & Screen Zone Elevation & Top of Casing & Casing & Pump & Screen Zone \\
\hline $\begin{array}{l}\text { N75155.7 } \\
\text { E48413.1 }\end{array}$ & $\begin{array}{l}33.270985 \circ \mathrm{o} \\
81.685304^{\circ} \mathrm{W}\end{array}$ & $158.4-148.4 \mathrm{ft} \mathrm{msl}$ & $256.5 \mathrm{ft} \mathrm{msl}$ & 4" PVC & $\mathbf{s}$ & Barnwell (\|B, \\
\hline SAMPLE DATE & 021 & & & 4/95 & & \\
\hline
\end{tabular}

FIELD DATA

\begin{tabular}{ll} 
Parameter & 1095 \\
\cline { 1 - 2 } Water elevation & \\
pH & 203.6 \\
Sp. conductance & 5.8 \\
Water temperature & 45 \\
Alkalinity as CaCO & 18.7 \\
Turbidity & 6 \\
Volume purged & 0.4 \\
Sampling code & 3.5 \\
Synchronous water Jevel & $204.4(03 / 27 / 95)$
\end{tabular}

$\begin{array}{ll}2095 & \text { Unit } \\ 204.5 & \mathrm{ft} \mathrm{msl} \\ 6.5 & \mathrm{pH} \\ 46 & \mu \mathrm{S} / \mathrm{cm} \\ 19 & { }^{\circ} \mathrm{C} \\ 6 & \mathrm{mg} / \mathrm{L} \\ 0.5 & \mathrm{NTU} \\ 3.7 & \text { well volumes } \\ 204.6(06 / 15 / 95) & \mathrm{ft} \mathrm{msl}\end{array}$

Hazardous Constituents (264.94, Table 1)

\begin{tabular}{|c|c|c|c|c|c|c|c|c|c|c|c|c|c|}
\hline Parameter & 1095 & $\underline{\mathrm{DF}}$ & Mod & ST H & Flag & Lab & 2095 & $\underline{D F}$ & Mod & ST & 브 & Flag & Lab \\
\hline $\begin{array}{l}\text { Arsenic, total recoverable } \\
\text { Barium, total recoverable } \\
\text { Cadmium, total recoverable } \\
\text { Chromium, total recoverable } \\
\text { Lead, total recoverable } \\
\text { Mercury, total recoverable } \\
\text { Selenium, total recoverable } \\
\text { Silver, total recoverable }\end{array}$ & $\begin{array}{l}<3.3 \\
29 \\
<3.3 \\
<6.7 \\
<5.0 \\
<0.33 \\
<3.3 \\
<3.3\end{array}$ & $\begin{array}{l}1 \\
1 \\
1 \\
1 \\
1 \\
1 \\
1 \\
1\end{array}$ & V & & $\begin{array}{l}0 \\
0 \\
0 \\
0 \\
0 \\
0 \\
0 \\
0\end{array}$ & $\begin{array}{l}\text { GE } \\
\text { GE } \\
\text { GE } \\
\text { GE } \\
\text { GE } \\
\text { GE } \\
\text { GE } \\
\text { GE }\end{array}$ & $\begin{array}{r}<2.0 \\
0.78\end{array}$ & $\begin{array}{l}1 \\
1\end{array}$ & JVV & & & $\begin{array}{l}0 \\
0\end{array}$ & $\begin{array}{l}\text { GE } \\
\text { GE }\end{array}$ \\
\hline
\end{tabular}

Hazardous Constituents (261 App. VIII/264 App. IX)

\begin{tabular}{|c|c|c|c|c|c|c|c|c|c|c|c|c|c|c|c|}
\hline Parameter & 1095 & $\underline{D F}$ & Mod & $\underline{\mathbf{S T}}$ & 브 & Flag & $\underline{\text { Lab }}$ & 2095 & DF & Mod & ST & $\underline{H}$ & Flag & $\underline{\text { Lab }}$ & Unit \\
\hline $\begin{array}{l}\text { Antimony, total recoverable } \\
\text { Benzene } \\
\text { Cobalt, total recoverable } \\
\text { Copper, total recoverable } \\
\text { Cyanide } \\
\text { Nickel, totel recoverable } \\
\text { Phenols } \\
\text { Tetrachloroethylene } \\
\text { Thallium, total recoverable } \\
\text { Trichloroethylene } \\
\text { Trichlorofluoromethane } \\
\text { Vanadium, total recoverable } \\
\text { Zinc, total recoverable }\end{array}$ & $\begin{aligned}<3.3 \\
<1.7 \\
<6.7 \\
<6.7 \\
<8.3 \\
<6.7 \\
<8.3 \\
<1.7 \\
<3.3 \\
<1.7 \\
<1.7 \\
<13 \\
\quad 4.6\end{aligned}$ & $\begin{array}{l}1 \\
1 \\
1 \\
1 \\
1 \\
1 \\
1 \\
1 \\
1 \\
1 \\
1 \\
1 \\
1\end{array}$ & J1 & - & $\bullet$ & $\begin{array}{l}0 \\
0 \\
0 \\
0 \\
0 \\
0 \\
0 \\
0 \\
0 \\
0 \\
0 \\
0 \\
0\end{array}$ & $\begin{array}{l}\text { GE } \\
\text { GE } \\
\text { GE } \\
\text { GE } \\
\text { GE } \\
\text { GE } \\
\text { GE } \\
\text { GE } \\
\text { GE } \\
\text { GE } \\
\text { GE } \\
\text { GE } \\
\text { GE }\end{array}$ & & & & & & & & $\begin{array}{l}\mu g / L \\
\mu g / L \\
\mu g / L \\
\mu g / L \\
\mu g / L \\
\mu g / L \\
\mu g / L \\
\mu g / L \\
\mu g / L \\
\mu g / L \\
\mu g / L \\
\mu g / L \\
\mu g / L\end{array}$ \\
\hline \multicolumn{16}{|c|}{ Water Quality Indicator Parameters } \\
\hline Parameter & 1095 & DF & Mod & ST & 브 & Flag & $\underline{L a b}$ & 2095 & DF & Mod & $\underline{\underline{S T}}$ & 브 & Flag & Lab & Unit \\
\hline $\begin{array}{l}\text { Aluminum, total recoverable } \\
\text { Nitrate as nitrogen } \\
\text { pH }\end{array}$ & $\begin{array}{l}<33 \\
1.400 \\
5.8\end{array}$ & $\begin{array}{l}1 \\
1 \\
1\end{array}$ & $j 1$ & v & $\bullet$ & $\begin{array}{l}\mathbf{0} \\
\mathbf{0} \\
\mathbf{0}\end{array}$ & $\begin{array}{l}\text { GE } \\
\mathbf{G E} \\
\mathbf{G E}\end{array}$ & $\begin{array}{l}1,500 \\
6.0\end{array}$ & $\begin{array}{l}1 \\
1\end{array}$ & J1 & $\boldsymbol{\square}$ & - & $\begin{array}{l}0 \\
0\end{array}$ & $\begin{array}{l}\text { GE } \\
\text { GE }\end{array}$ & $\begin{array}{l}\mu g / L \\
\mu g / L \\
\mathrm{pH}\end{array}$ \\
\hline \multicolumn{16}{|c|}{ Common Laboratory Contaminants } \\
\hline Parameter & 1095 & DF & Mod & ST & H & Flag & Lab & 2095 & DF & Mod & $\underline{\text { ST }}$ & 브 & Flag & $\underline{\underline{L a b}}$ & Unit \\
\hline $\begin{array}{l}\text { Bis(2-ethylhexyl) phthalate } \\
\text { Dichloromethane }\end{array}$ & $\begin{array}{l}<17 \\
<1.7\end{array}$ & $\begin{array}{l}1 \\
1\end{array}$ & & & & $\begin{array}{l}\mathbf{0} \\
\mathbf{0}\end{array}$ & $\begin{array}{l}\text { GE } \\
\text { GE }\end{array}$ & & & & & & & & $\begin{array}{l}\mu g / L \\
\mu g / L\end{array}$ \\
\hline
\end{tabular}

Note: See Appendix B for flagging criteria. Synchronous water levels are measured over a 3-5 day period. - = exceeded holding time.

- = exceeded groundwater protection standard. 
WELL FSB 121C (cont.)

Radiological Indicator Parameters

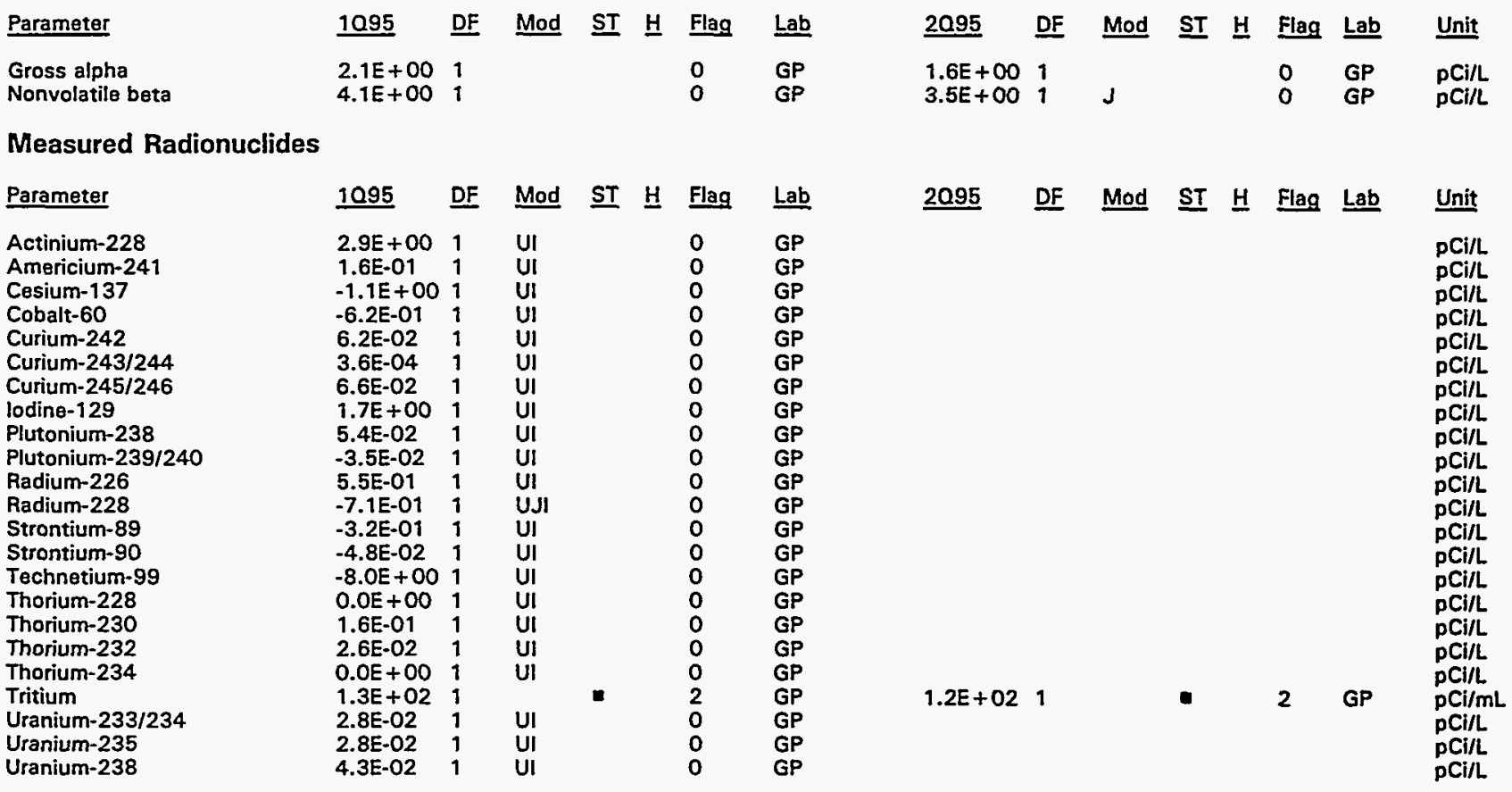

Calculated Radionuclides

Parameter

Americium-243

Nickel-63

Plutonium-241

Plutonium-242
109

1.1E-06

$-6.2 E-01$

$-1.4 E-01$

$-6.9 E-07$
DF Mod ST $\underline{H}$ Flag $\underline{\text { Lab }}$

0

0
0
0
0
2095 DF Mod ST $\underline{\text { H Flag Lab Uni }}$

pCi/L

pCi/L

pCi/L

Note: See Appendix B for flagging criteria. Synchronous water levels are measured over a 3-5 day period.

- = exceeded holding time.

- exceeded groundwater protection standard. 


\section{WELL FSB121DR}

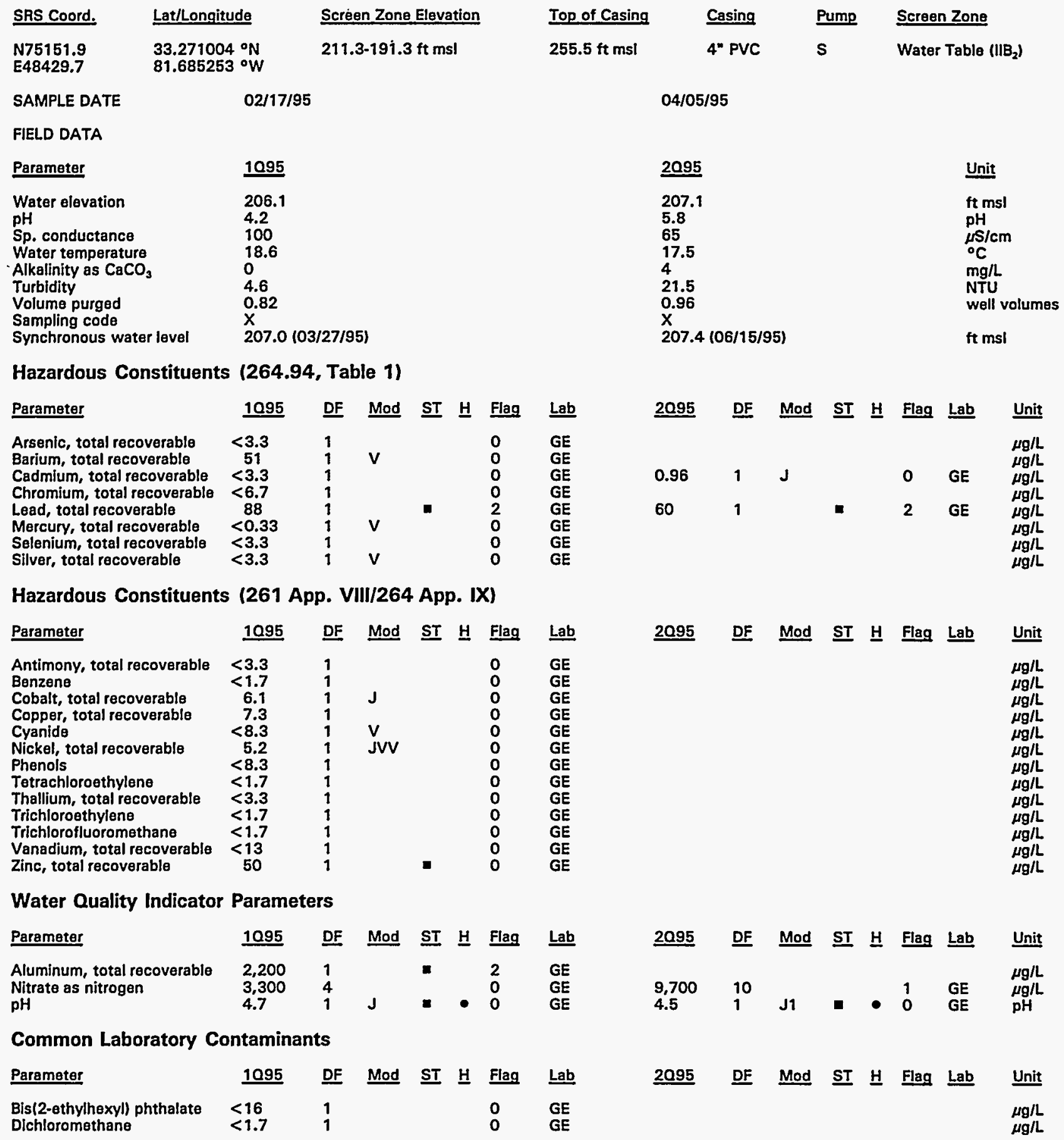

Note: See Appendix B for flagging criteria. Synchronous water levels are measured over a $3-5$ day period. - = exceeded holding time.

- = exceeded groundwater protection standard. 
WELL FSB121DR (cont.)

Radiological Indicator Parameters

Parameter

Gross alpha Nonvolatile beta
1095

DF

3. $O E+011$ 8.6E+01
Mod

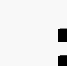

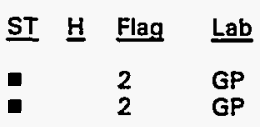

GP
2095 DF Mod ST 브 Flag Lab Unit

$\begin{array}{lllllll}2.6 E+01 & 1 & J & * & 2 & \mathrm{GP} & \mathrm{pCi} / \mathrm{L} \\ 9.2 E+01 & 1 & & * & 2 & \mathrm{GP} & \mathrm{pCi} / \mathrm{L}\end{array}$

Measured Radionuclides

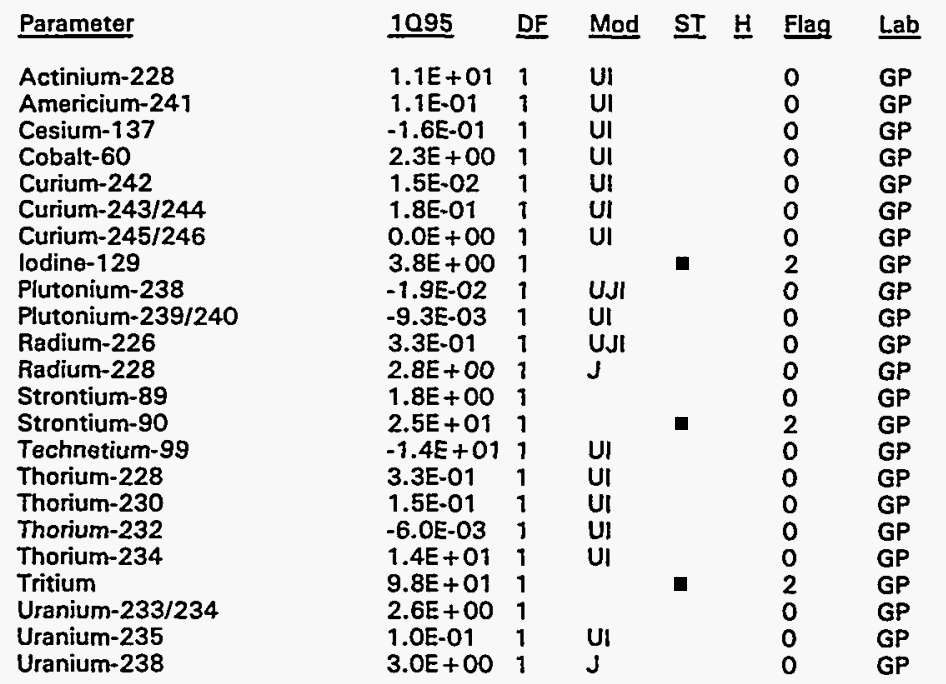

$\underline{D F}$ Mod ST $\underline{\text { H }}$ Flag Lab Unit

pCi/L

$\mathrm{pCi} / \mathrm{L}$

$\mathrm{pCi} / \mathrm{L}$

$\mathrm{pCi} / \mathrm{L}$

PCIL

pCin

$\mathrm{pCi} / \mathrm{L}$

$\mathrm{pCi} / \mathrm{L}$

$\mathrm{pCi} / \mathrm{L}$

$\mathrm{pCi} / \mathrm{L}$

$\mathrm{PCi} / \mathrm{L}$
$\mathrm{PCI} / \mathrm{L}$

$\mathrm{pCi} / \mathrm{L}$

$\mathrm{PCi} / \mathrm{L}$

$\mathrm{pCi} / \mathrm{L}$

$\mathrm{pCi} / \mathrm{L}$

$\mathrm{pCi} / \mathrm{L}$

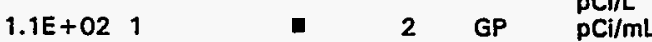

pCi/m

$\mathrm{pCi} / \mathrm{L}$

$\mathrm{PCi} / \mathrm{L}$

Calculated Radionuclides

Parameter

Americium-243

Nickel-63

Plutonium-241

Plutonium-242
5.3E-04

$2.3 E+00$

$-3.7 \mathrm{E}-02$

$-1.9 \mathrm{E}-07$

DF Mod ST 브 Flag Lab

$\underline{2095}$

DF Mod ST 브 Flag Lab

Unit

0
0
0
0

$\mathrm{pCi} / \mathrm{L}$

PCi/L

pCi/L

Note: See Appendix B for flagging criteria. Synchronous water levels are measured over a 3-5 day period.

$-=$ exceeded holding time.

- = exceeded groundwater protection standard. 


\section{WELL FSB122C}

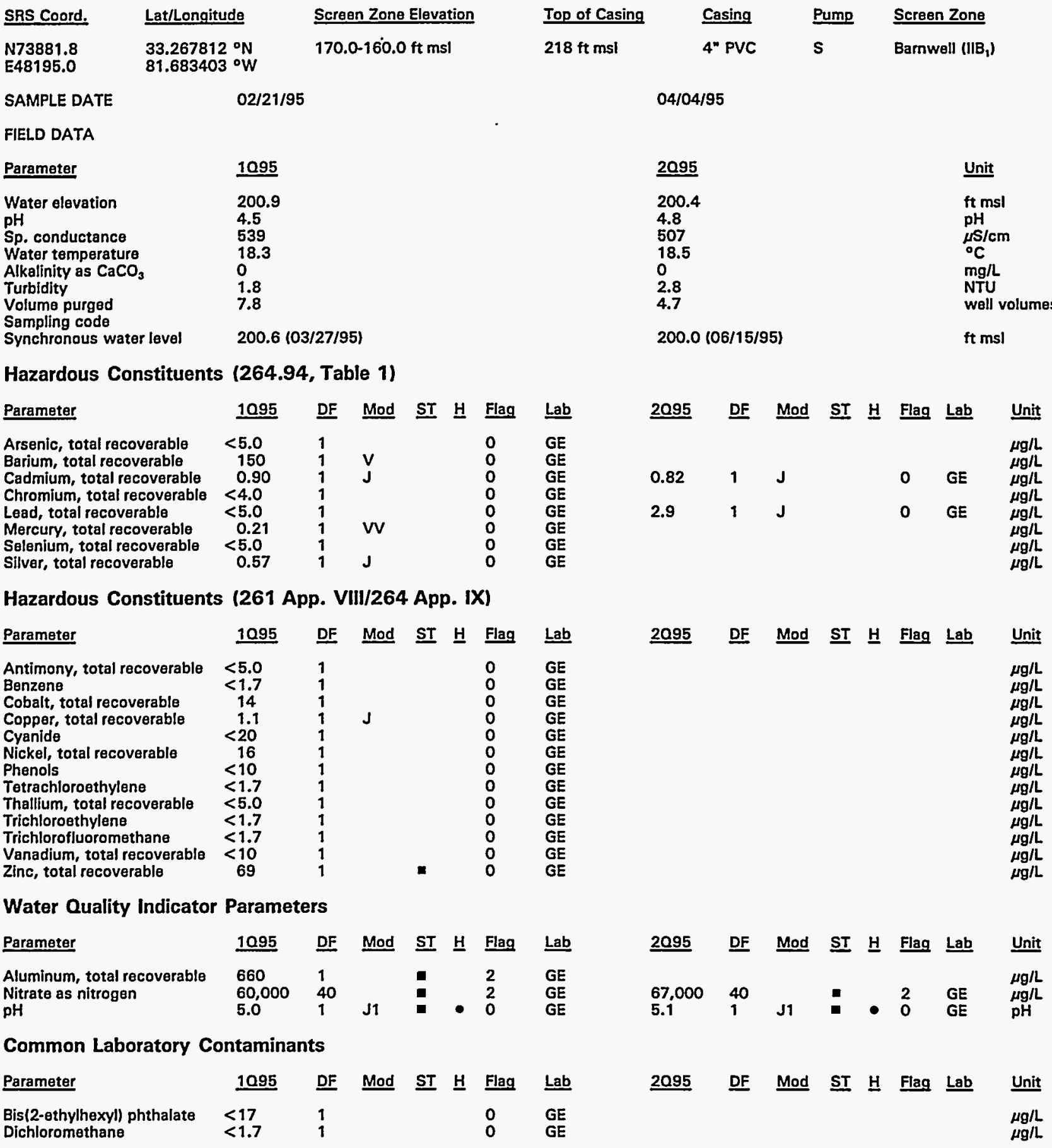

Note: See Appendix B for flagging criteria. Synchronous water levels are measured over a 3-5 day period.

- = exceeded holding time.

a = exceeded groundwater protection standard. 
WELL FSB122C (cont.)

Radiological Indicator Parameters

Parameter

Gross alpha

Nonvolatile beta

109

$2.0 E+01$

$6.5 E+011$
DF

od

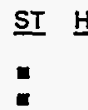

Flag
2
2

$\underline{\text { Lab }}$

GP $\underline{2095}$

$1.8 \mathrm{E}+011$

$9.2 E+01 ?$
Unit

pCi/L $\mathrm{pCi} / \mathrm{L}$

Measured Radionuclides

$\begin{array}{llllllll}\text { Parameter } & 1095 & \text { DF } & \text { Mod } & \text { ST } & \text { H } & \text { Flag } & \text { La } \\ \text { Actinium-228 } & 1.2 E+01 & 1 & \text { UI } & & & & \text { GP } \\ \text { Americium-241 } & -1.7 E-03 & 1 & \text { UI } & & 0 & \text { GP } \\ \text { Cesium-137 } & 1.3 E+00 & 1 & \text { UI } & & 0 & \text { GP } \\ \text { Cobalt-60 } & 3.7 E-01 & 1 & \text { UI } & & 0 & \text { GP } \\ \text { Curium-242 } & -2.2 E-02 & 1 & \text { UI } & & 0 & \text { GP } \\ \text { Curium-243/244 } & -3.0 E-02 & 1 & \text { UI } & & 0 & \text { GP } \\ \text { Curium-245/246 } & -5.0 E-03 & 1 & \text { UI } & & 0 & \text { GP } \\ \text { lodine-129 } & 2.2 E+01 & 1 & & & 2 & \text { GP } \\ \text { Plutonium-238 } & 1.1 E-01 & 1 & \text { UI } & & 0 & \text { GP } \\ \text { Plutonium-239/240 } & 1.3 E-01 & 1 & \text { UI } & & 0 & \text { GP } \\ \text { Radium-226 } & 5.6 E+00 & 1 & & & 0 & \text { GP } \\ \text { Radium-228 } & 8.6 E-01 & 1 & \text { J } & & 0 & \text { GP } \\ \text { Strontium-89 } & 1.5 E+00 & 1 & & & 0 & \text { GP } \\ \text { Strontium-90 } & -7.9 E-02 & 1 & \text { UI } & & 0 & \text { GP } \\ \text { Technetium-99 } & 1.1 E+02 & 1 & & & 0 & \text { GP } \\ \text { Thorium-228 } & -1.1 E-02 & 1 & \text { UI } & & 0 & \text { GP } \\ \text { Thorium-230 } & 2.6 E-01 & 1 & \text { UI } & & 0 & \text { GP } \\ \text { Thorium-232 } & 0.0 E+00 & 1 & \text { UI } & & 0 & \text { GP } \\ \text { Thorium-234 } & 1.8 E+01 & 1 & \text { UI } & & 0 & \text { GP } \\ \text { Tritium } & 1.9 E+03 & 1 & & & 2 & \text { GP } \\ \text { Uranium-233/234 } & 2.9 E-01 & 1 & \text { UI } & & 0 & \text { GP } \\ \text { Uranium-235 } & 0.0 E+00 & 1 & \text { UI } & & 0 & \text { GP } \\ \text { Uranium-238 } & 3.8 E-01 & 1 & \text { UI } & & 0 & \text { GP }\end{array}$

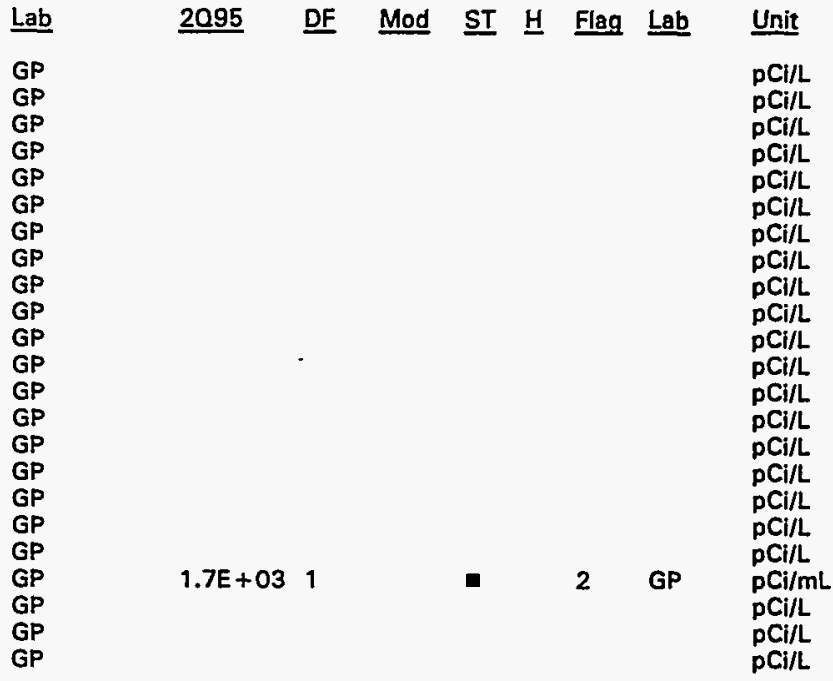

Calculated Radionuclides

Parameter

Americium-243

Nickel-63

Plutonium-241

Plutonium-242
1095

$-9.1 E-05$

3.7E-01

5.2E-01

2.6E-06
DF Mod ST $\underline{H}$ Flag Lab

0

0
0
0
2095 DF Mod ST $\underline{\text { H Flag Lab Unit }}$

$\mathrm{pCi} / \mathrm{L}$

$\mathrm{PCj} / \mathrm{L}$

pCi/L

$\mathrm{pCi} / \mathrm{L}$

Note: See Appendix B for flagging criteria. Synchronous water levels are measured over a 3-5 day period.

- = exceeded holding time.

- = exceeded groundwater protection standard. 


\section{WELL FSB122D}

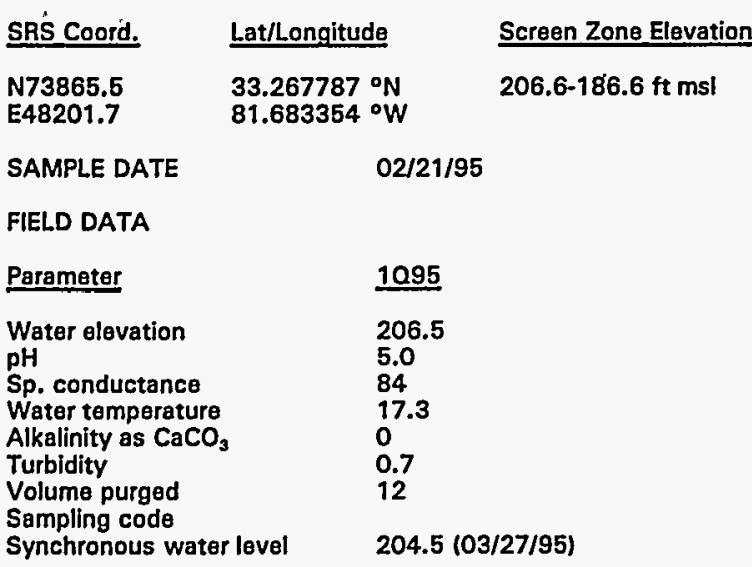

Hazardous Constituents (264.94, Table 1)

\begin{tabular}{|c|c|c|c|c|c|c|}
\hline Parameter & 1095 & DF & Mod & ST & $\underline{H}$ & Flag \\
\hline $\begin{array}{l}\text { Arsenic, total recoverable } \\
\text { Barium, total recoverable } \\
\text { Cadmium, total recoverable } \\
\text { Chromium, total recoverable } \\
\text { Lead, total recoverable } \\
\text { Mercury, total recoverable } \\
\text { Selenium, total recoverable } \\
\text { Silver, total recoverable }\end{array}$ & $\begin{array}{c}1.4 \\
31 \\
<2.0 \\
<4.0 \\
2.8 \\
<0.20 \\
2.7 \\
0.23\end{array}$ & $\begin{array}{l}1 \\
1 \\
1 \\
1 \\
1 \\
1 \\
1 \\
1\end{array}$ & $\begin{array}{l}\mathrm{J} 3 \\
\mathrm{~V} \\
\mathrm{~J} \\
\mathrm{~J}\end{array}$ & & & $\begin{array}{l}0 \\
0 \\
0 \\
0 \\
0 \\
0 \\
0 \\
0\end{array}$ \\
\hline
\end{tabular}

Hazardous Constituents (261 App. VIII/264 App. (X)

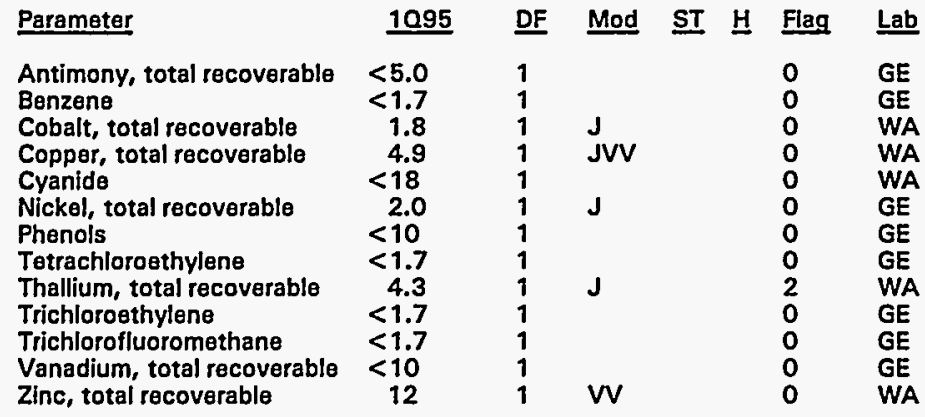

$\begin{array}{llll}\text { Top of Casing } & \text { Casing } & \text { Pump } & \text { Screan Zone } \\ 217.6 \mathrm{ft} \mathrm{msl} & \text { 4" PVC } & \text { S } & \text { Water Table (IIB })\end{array}$

$04 / 04 / 95$

$\begin{array}{ll}2095 & \text { Unit } \\ 204.1 & \mathrm{ft} \mathrm{msl} \\ 5.2 & \mathrm{pH} \\ 103 & \mu \mathrm{S} / \mathrm{cm} \\ 17 & { }^{\circ} \mathrm{C} \\ 0 & \mathrm{mg} / \mathrm{L} \\ 2.2 & \mathrm{NTU} \\ 9.0 & \text { well volumes } \\ 203.8(06 / 15 / 95) & \mathrm{ft} \mathrm{msl}\end{array}$

Water Quality Indicator Parameters

\begin{tabular}{|c|c|c|c|c|c|c|c|c|c|c|c|c|c|c|c|}
\hline Parameter & 1095 & DF & Mod & $\underline{\mathbf{S T}}$ & 브 & Flag & $\underline{\text { Lab }}$ & $\underline{2095}$ & $\underline{\mathrm{DF}}$ & Mod & $\underline{\text { ST }}$ & 브 & Flag & $\underline{L a b}$ & Unit \\
\hline $\begin{array}{l}\text { Aluminum, total recoverable } \\
\text { Nitrate as nitrogen } \\
\text { pH }\end{array}$ & $\begin{array}{l}110 \\
7.900 \\
5.4\end{array}$ & $\begin{array}{l}1 \\
25 \\
1\end{array}$ & $\begin{array}{l}V \\
J\end{array}$ & घ & $\bullet$ & $\begin{array}{l}2 \\
1 \\
0\end{array}$ & $\begin{array}{l}\text { WA } \\
\text { WA } \\
\text { WA }\end{array}$ & $\begin{array}{l}10,000 \\
5.1\end{array}$ & $\begin{array}{l}20 \\
1\end{array}$ & $\mathbf{J 1}$ & a & $\bullet$ & $\begin{array}{l}2 \\
0\end{array}$ & $\begin{array}{l}\text { GE } \\
\text { GE }\end{array}$ & $\begin{array}{l}\mu \mathrm{g} / \mathrm{L} \\
\mu \mathrm{g} / \mathrm{L} \\
\mathrm{pH}\end{array}$ \\
\hline \multicolumn{16}{|c|}{ Common Laboratory Contaminants } \\
\hline Parameter & 1095 & DF & Mod & ST & $\underline{H}$ & Flag & $\underline{\text { Lab }}$ & 2095 & DF & Mod & ST & 브 & Flag & Lab & Unit \\
\hline
\end{tabular}

Note: See Appendix B for flagging criteria. Synchronous water levels are measured over a 3-5 day period.

- = exceeded holding time.

- exceeded groundwater protection standard. 


\section{WELL FSB122D (cont.)}

Radiological Indicator Parameters

\section{Parameter}

Gross aipha Nonvolatile beta
$21 E+00$ $2.1 E+001$
$2.0 E+01$

Mod ST $\underline{H}$ Flag Lab

$\begin{array}{ll}0 & T M \\ 0 & T M\end{array}$

Measured Radionuclides

\section{Parameter}

Actinium-228

Americium-241

Cesium-137

Cobalt-60

Curium-242

Curium-243/244

Curium-245/246

lodine-129

Plutonium-238

Plutonium-239/240

Radium-226

Radium-228

Strontium-89

Strontium-90

Technetium-99

Thorium-228

Thorium-230

Thorium-232

Thorium-234

Tritium

Uranium-233/234

Uranium-235

Uranium-238
1095

$2.3 E+00$

2.3E-01 1

$-5.4 \mathrm{E}-01$

2.2E-01

$-1.8 \mathrm{E}-02$

$-4.1 \mathrm{E}-02$

$-5.3 E-03 \quad 1$

$8.8 E-01$

7.OE-O2

$-4.7 E-03$

$1.1 \mathrm{E}+00$

$1.7 E+001$

$0.0 E+001$

$0.0 E+001$

2.1E+01 1

$-1.0 \mathrm{E}-02 \quad 1$

2.0E-O2

$1.0 \mathrm{E}-02$

8.7E +011

$3.5 \mathbf{E}+021$

1.0E-01 1

$0.0 E+001$

F

$\begin{array}{cccc}\text { Mod ST } & \text { Flag } & \text { Lab } \\ \text { UI } & & 0 & \text { TM } \\ \text { U } & & 0 & \text { TM } \\ \text { UI } & & 0 & \text { TM } \\ \text { UI } & & 0 & \text { TM } \\ \text { UI } & & 0 & \text { GP } \\ \text { UI } & & 0 & \text { GP } \\ \text { UI } & & 0 & \text { GP } \\ \text { UI } & & 0 & \text { GP } \\ \text { UI } & & 0 & \text { TM } \\ \text { UI } & & 0 & \text { GP } \\ & & 0 & \text { GP } \\ \text { UI } & & 0 & \text { TM } \\ \text { UI } & & 0 & \text { TM } \\ \text { UI } & & 0 & \text { TM } \\ \text { UI } & & 0 & \text { TM } \\ \text { UI } & & 0 & \text { TM } \\ \text { UI } & & 0 & \text { TM } \\ \text { JI } & & 0 & \text { TM } \\ \text { UI } & & 2 & \text { TM } \\ \text { UI } & & 0 & \text { GP } \\ \text { UI } & & 0 & \text { GP } \\ \text { UI } & & 0 & \text { GP }\end{array}$

\section{$\underline{2095}$}

DF Mod ST

$1.8 E+\infty \quad 1$

$1.1 E+011$

\rfloor

$\begin{array}{ll}0 & \text { GP } \\ 0 & \text { GP }\end{array}$

Unit

$\mathrm{pCi} / \mathrm{L}$
$\mathrm{pCi} / \mathrm{L}$

Calculated Radionuclides

Parameter

1095

$-1.2 E-04$

$-1.9 \mathrm{E}-02$

$-9.5 \mathrm{E}-08$

DF Mod ST $\underline{H}$ Flag Lab

Nickel-63

Plutonium-241

Plutonium-242 $\underline{2095}$

DF Mad ST $\underline{H}$ Flag Lab

Unit

2095 DF Mod ST $\underline{H}$ Flag Lab Unit

pCill

$\mathrm{pCi} / \mathrm{L}$

$\mathrm{pCj} / \mathrm{L}$

pCi/L

$\mathrm{pCi} / \mathrm{L}$

$\mathrm{pCi} / \mathrm{L}$

$\mathrm{pCi} / \mathrm{L}$

Pill

PCill

pCi/L

$\mathrm{pCi} / \mathrm{L}$

pCi/L

$\mathrm{pCi} / \mathrm{L}$

$\mathrm{pCi} / \mathrm{L}$

$\mathrm{pCi} / \mathrm{L}$

pCi/L

$3.4 E+021$

a $2 \mathrm{GP}$

$\mathrm{pCi} / \mathrm{mL}$

pCi/L

pCi/L

$\mathrm{pCi} / \mathrm{L}$

$\mathrm{pCi} / \mathrm{L}$

$\mathrm{pCi} / \mathrm{L}$

$\mathrm{PCi} / \mathrm{L}$

Note: See Appendix B for flagging criteria. Synchronous water levels are measured over a 3-5 day period.

- = exceeded holding time.

= exceeded groundwater protection standard. 
WELL FSB123C

\begin{tabular}{|c|c|c|c|c|c|c|}
\hline SRS Coord. & Lat/Longitude & Screen Zone Elevation & Top of Casing & Casing & Pump & Screen Zone \\
\hline $\begin{array}{l}\text { N74566.7 } \\
E 51750.5\end{array}$ & $\begin{array}{l}33.275129^{\circ} \mathrm{N} \\
81.675374^{\circ} \mathrm{W}\end{array}$ & $165.3-155.3 \mathrm{ft} \mathrm{msl}$ & $238.1 \mathrm{ft} \mathrm{msl}$ & 4" PVC & $\mathbf{S}$ & Barnwell $\left(I 1 B_{1}\right)$ \\
\hline SAMPLE DATE & $02 J$ & & & $2 / 95$ & & \\
\hline
\end{tabular}

FIELD DATA

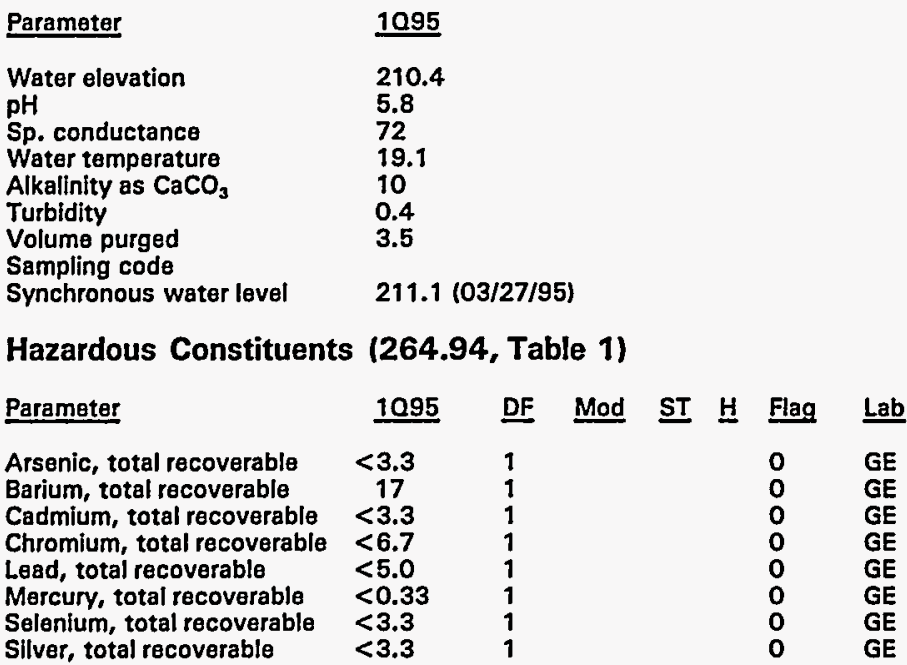

$\begin{array}{ll}\underline{2095} & \text { Unit } \\ 211.0 & \mathrm{ftmsl} \\ 5.0 & \mathrm{pH} \\ 61 & \mu \mathrm{JS} / \mathrm{cm} \\ 18.8 & { }^{\circ} \\ 7 & \mathrm{mg} / \mathrm{L} \\ 0.2 & \mathrm{NTU} \\ 3.9 & \text { well volumes } \\ 210.7(06 / 15 / 95) & \mathrm{ft} \mathrm{msl}\end{array}$

Hazardous Constituents (261 App. VIII/264 App. IX)

\begin{tabular}{|c|c|c|c|c|c|c|c|c|c|c|c|c|c|c|c|}
\hline Parameter & 1095 & DF & Mod & $\underline{\mathbf{S T}}$ & $\underline{\mathbf{H}}$ & Flag & $\underline{\text { Lab }}$ & $\underline{2095}$ & DF & Mod & $\underline{\text { sT }}$ & $\underline{\text { H}}$ & Flag & $\underline{\text { Lab }}$ & Unit \\
\hline $\begin{array}{l}\text { Antimony, total recoverable } \\
\text { Benzene } \\
\text { Cobalt, total recoverable } \\
\text { Copper, total recoverable } \\
\text { Cyanide } \\
\text { Nickel, total recoverable } \\
\text { Phenols } \\
\text { Tetrachloroethylene } \\
\text { Thallium, total recoverable } \\
\text { Trichloroethylene } \\
\text { Trichlorofluoromethane } \\
\text { Vanadium, total recoverable } \\
\text { Zinc, total recoverable }\end{array}$ & $\begin{aligned} & 2.8 \\
&<1.7 \\
&<6.7 \\
&<6.7 \\
&<8.3 \\
&<6.7 \\
&<8.3 \\
&<1.7 \\
&<3.3 \\
&<1.7 \\
&<1.7 \\
&<13 \\
& 3.6\end{aligned}$ & $\begin{array}{l}1 \\
1 \\
1 \\
1 \\
1 \\
1 \\
1 \\
1 \\
1 \\
1 \\
1 \\
1 \\
1\end{array}$ & J & & & $\begin{array}{l}0 \\
0 \\
0 \\
0 \\
0 \\
0 \\
0 \\
0 \\
0 \\
0 \\
0 \\
0 \\
0\end{array}$ & $\begin{array}{l}\mathrm{GE} \\
\mathrm{GE} \\
\mathrm{GE} \\
\mathrm{GE} \\
\mathrm{GE} \\
\mathrm{GE} \\
\mathrm{GE} \\
\mathrm{GE} \\
\mathrm{GE} \\
\mathrm{GE} \\
\mathrm{GE} \\
\mathrm{GE} \\
\mathrm{GE}\end{array}$ & & & & & & & & $\begin{array}{l}\mu g / L \\
\mu g / L \\
\mu g / L \\
\mu g / L \\
\mu g / L \\
\mu g / L \\
\mu g / L \\
\mu g / L \\
\mu g / L \\
\mu g / L \\
\mu g / L \\
\mu g / L \\
\mu g / L\end{array}$ \\
\hline \multicolumn{16}{|c|}{ Water Quality Indicator Parameters } \\
\hline Parameter & 1095 & DF & Mod & $\underline{S T}$ & $\underline{H}$ & Flag & $\underline{\text { Lab }}$ & $\underline{2095}$ & $\underline{\text { DF }}$ & Mod & $\underline{\text { ST }}$ & H & Flag & $\underline{\text { Lab }}$ & Unit \\
\hline $\begin{array}{l}\text { Aluminum, total recoverable } \\
\text { Nitrate as nitrogen } \\
\text { pH }\end{array}$ & $\begin{array}{l}<33 \\
2,900 \\
5.9\end{array}$ & $\begin{array}{l}1 \\
2 \\
1\end{array}$ & J1 & - & & $\begin{array}{l}0 \\
0 \\
0\end{array}$ & $\begin{array}{l}\text { GE } \\
\text { GE } \\
\text { GE }\end{array}$ & $\begin{array}{l}3,200 \\
6.0\end{array}$ & $\begin{array}{l}5 \\
1\end{array}$ & J1 & - & $\bullet$ & $\begin{array}{l}0 \\
0\end{array}$ & $\begin{array}{l}\text { WA } \\
\text { GE }\end{array}$ & $\begin{array}{l}\mu \mathrm{g} / \mathrm{L} \\
\mu \mathrm{g} / \mathrm{L} \\
\mathrm{pH}\end{array}$ \\
\hline \multicolumn{16}{|c|}{ Common Laboratory Contaminants } \\
\hline Parameter & 1095 & $\underline{D F}$ & Mod & $\underline{\text { ST }}$ & $\underline{H}$ & Flag & $\underline{\text { Lab }}$ & $\underline{2095}$ & $\underline{D F}$ & Mod & $\underline{\mathbf{S T}}$ & H & Flag & Lab & $\underline{\text { Unit }}$ \\
\hline $\begin{array}{l}\text { Bis(2-ethylhexyl) phthalate } \\
\text { Dichloromethene }\end{array}$ & $\begin{array}{l}<16 \\
<1.7\end{array}$ & $\begin{array}{l}1 \\
1\end{array}$ & $v$ & & & $\begin{array}{l}0 \\
0\end{array}$ & $\begin{array}{l}\mathrm{GE} \\
\mathrm{GE}\end{array}$ & & & & & & & & $\underset{\mu g / L}{\mu g / L}$ \\
\hline
\end{tabular}

Note: See Appendix B for flagging criteria. Synchronous water levels are measured over a 3-5 day period.

- = exceeded holding time.

- = exceeded groundwater protection standard. 
WELL FSB $123 \mathrm{C}$ (cont.)

Radiological Indicator Parameters

\begin{tabular}{|c|c|c|c|c|c|c|c|c|c|c|c|c|c|c|c|}
\hline Parameter & 1095 & $\underline{\mathrm{DF}}$ & Mod & $\underline{\mathbf{S T}}$ & $\underline{H}$ & Flag & $\underline{\text { Lab }}$ & $\underline{2095}$ & $\underline{\text { DF }}$ & Mod & $\underline{\text { ST }}$ & $\underline{H}$ & Flag & $\underline{\text { Lab }}$ & Unit \\
\hline $\begin{array}{l}\text { Gross alpha } \\
\text { Nonvolatile beta }\end{array}$ & $\begin{array}{l}2.0 \mathrm{E}-02 \\
8.4 \mathrm{E}-01\end{array}$ & $\begin{array}{l}1 \\
1\end{array}$ & UI & & & $\begin{array}{l}0 \\
0\end{array}$ & $\begin{array}{l}\text { GP } \\
\text { GP }\end{array}$ & $\begin{array}{l}\text { 7.7E-01 } \\
-1.0 E-01\end{array}$ & $\begin{array}{l}1 \\
1\end{array}$ & UI & & & $\begin{array}{l}0 \\
0\end{array}$ & $\begin{array}{l}\text { GP } \\
\text { TM }\end{array}$ & $\begin{array}{l}\mathrm{pCi} / \mathrm{L} \\
\mathrm{pCi} / \mathrm{L}\end{array}$ \\
\hline \multicolumn{16}{|c|}{ Measured Radionuclides } \\
\hline Parameter & 1095 & $\underline{D F}$ & Mod & $\underline{\text { ST }}$ & $\underline{\mathrm{H}}$ & Flag & $\underline{\text { Lab }}$ & $\underline{2095}$ & $\underline{\mathrm{DF}}$ & Mod & $\underline{\text { ST }}$ & $\underline{H}$ & Flag & Lab & Unit \\
\hline $\begin{array}{l}\text { Actinium-228 } \\
\text { Americium-241 } \\
\text { Cesium-137 } \\
\text { Cobalt-60 } \\
\text { Curium-242 } \\
\text { Curium-243/244 } \\
\text { Curium-245/246 } \\
\text { lodine-129 } \\
\text { Plutonium-238 } \\
\text { Plutonium-239/240 } \\
\text { Radium-226 } \\
\text { Radium-228 } \\
\text { Strontium-89 } \\
\text { Strontium-90 } \\
\text { Technetium-99 } \\
\text { Thorium-228 } \\
\text { Thorium-230 } \\
\text { Thorium-232 } \\
\text { Thorium-234 } \\
\text { Tritium } \\
\text { Uranium-233/234 } \\
\text { Uranium-235 } \\
\text { Uranium-238 }\end{array}$ & $\begin{array}{l}5.2 E+00 \\
8.7 E-02 \\
-1.9 E+00 \\
6.1 E-01 \\
2.6 E-02 \\
-1.3 E-02 \\
0.0 E+00 \\
1.7 E-01 \\
2.9 E-01 \\
1.1 E-01 \\
1.2 E-01 \\
1.8 E-01 \\
-6.3 E-01 \\
-3.9 E-01 \\
-2.6 E+01 \\
1.0 E-01 \\
6.9 E-02 \\
-2.6 E-02 \\
7.7 E+01 \\
2.3 E+00 \\
2.2 E-02 \\
0.0 E+00 \\
1.3 E-01\end{array}$ & $\begin{array}{l}1 \\
1 \\
1 \\
1 \\
1 \\
1 \\
1 \\
1 \\
1 \\
1 \\
1 \\
1 \\
1 \\
1 \\
1 \\
1 \\
1 \\
1 \\
1 \\
1 \\
1 \\
1 \\
1\end{array}$ & $\begin{array}{l}U I \\
U I \\
U I \\
U I \\
U I \\
U I \\
U I \\
U I \\
U J ! \\
U I \\
U I \\
U J I \\
U I \\
U I \\
U I \\
U I \\
U I \\
U I \\
U I \\
U ! \\
U I \\
U I\end{array}$ & & & $\begin{array}{l}0 \\
0 \\
0 \\
0 \\
0 \\
0 \\
0 \\
0 \\
0 \\
0 \\
0 \\
0 \\
0 \\
0 \\
0 \\
0 \\
0 \\
0 \\
0 \\
0 \\
0 \\
0 \\
0\end{array}$ & $\begin{array}{l}\text { GP } \\
\text { GP } \\
\text { GP } \\
\text { GP } \\
\text { GP } \\
\text { GP } \\
\text { GP } \\
\text { GP } \\
\text { GP } \\
\text { GP } \\
\text { GP } \\
\text { GP } \\
\text { GP } \\
\text { GP } \\
\text { GP } \\
\text { GP } \\
\text { GP } \\
\text { GP } \\
\text { GP } \\
\text { GP } \\
\text { GP } \\
\text { GP } \\
\text { GP }\end{array}$ & $2.9 E+00$ & 1 & & & & 0 & $T M$ & 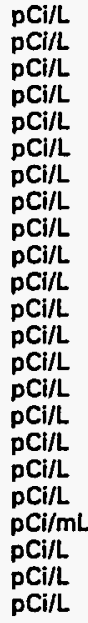 \\
\hline \multicolumn{16}{|c|}{ Calculated Radionuclides } \\
\hline Parameter & 1095 & $\underline{\text { DF }}$ & Mod & $\underline{\text { ST }}$ & H & Flag & $\underline{\text { Lab }}$ & $\underline{2095}$ & $\underline{\text { DF }}$ & Mod & $\underline{\text { ST }}$ & $\underline{H}$ & Flag & $\underline{\text { Lab }}$ & Unit \\
\hline $\begin{array}{l}\text { Americium-243 } \\
\text { Nickel-63 } \\
\text { Plutonium-241 } \\
\text { Plutonium-242 }\end{array}$ & $\begin{array}{l}-4.0 E-05 \\
6.1 E-01 \\
4.3 E-01 \\
2.1 E-06\end{array}$ & & & & & $\begin{array}{l}0 \\
0 \\
0 \\
0\end{array}$ & & & & & & & & & $\begin{array}{l}\mathrm{pCi} / \mathrm{L} \\
\mathrm{pCi} / \mathrm{L} \\
\mathrm{pCi} / \mathrm{L} \\
\mathrm{pCi} / \mathrm{L}\end{array}$ \\
\hline
\end{tabular}

Note: See Appendix B for flagging criteria. Synchronous water levels are measured over a 3-5 day period.

- = exceeded holding time.

- = exceeded groundwater protection standard. 


\section{WELL FSB123D}

\begin{tabular}{lll} 
SRS Coord. & Lat/Longitude & Screen Zone Elevation \\
\cline { 2 - 3 } $\begin{array}{l}\text { N74562.7 } \\
\text { E51734.8 }\end{array}$ & $\begin{array}{c}33.275095{ }^{\circ} \mathrm{N} \\
\text { S1.675407 } \mathrm{W}\end{array}$ & $214.1-194.1 \mathrm{ft} \mathrm{msl}$ \\
SAMPLE DATE & $02 / 16 / 95$ \\
FIELD DATA & \\
Parameter & $\underline{ }$ \\
\hline Water elevation & 213.0 \\
pH & 4.8 \\
Sp. conductance & 42 \\
Water temperature & 18.9 \\
Alkalinity as CaCO & 0 \\
Turbidity & 0.3 \\
Volume purged & 8.0 \\
Sampling code & \\
Synchronous water level & 213.5 (03/27/95)
\end{tabular}

Hazardous Constituents (264.94, Table 1)

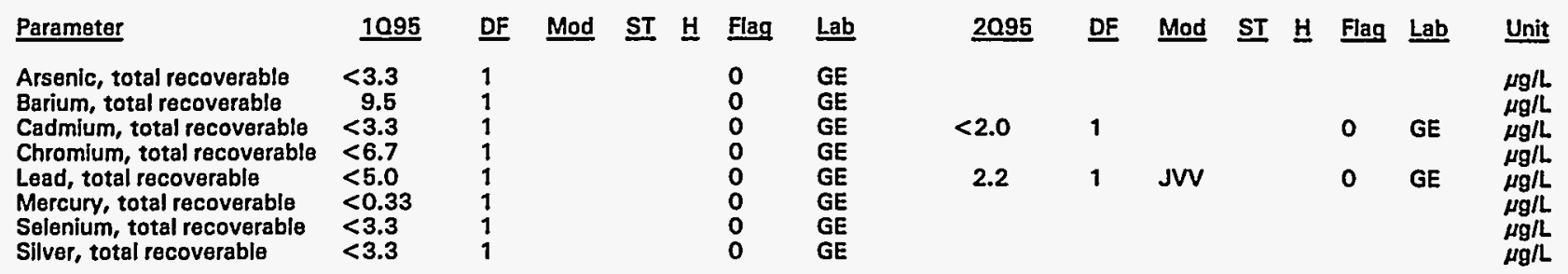

Hazardous Constituents (261 App. VIII/264 App. IX)

\begin{tabular}{|c|c|c|c|c|c|c|c|c|c|c|c|c|c|c|c|}
\hline Paramater & $\underline{1095}$ & DF & Mod & ST & 보 & Flag & $\underline{\text { Lab }}$ & $\underline{2095}$ & $\underline{D F}$ & Mod & $\underline{S T}$ & H & Flag & Lab & Unit \\
\hline $\begin{array}{l}\text { Antimony, total recoverable } \\
\text { Benzene } \\
\text { Cobalt, total recoverable } \\
\text { Copper, total recoverable } \\
\text { Cyanide } \\
\text { Nickel, total recoverable } \\
\text { Phenols } \\
\text { Tetrachloroethylene } \\
\text { Thallium, total recoverable } \\
\text { Trichloroethylene } \\
\text { Trichlorofluoromethane } \\
\text { Vanadium, total recoverable } \\
\text { Zinc, total recoverable }\end{array}$ & $\begin{aligned}<3.3 \\
<1.7 \\
<6.7 \\
<6.7 \\
<8.3 \\
<6.7 \\
<8.3 \\
<1.7 \\
<3.3 \\
<1.7 \\
<1.7 \\
<13 \\
3.6\end{aligned}$ & $\begin{array}{l}1 \\
1 \\
1 \\
1 \\
1 \\
1 \\
1 \\
1 \\
1 \\
1 \\
1 \\
1 \\
1\end{array}$ & & & & $\begin{array}{l}0 \\
0 \\
0 \\
0 \\
0 \\
0 \\
0 \\
0 \\
0 \\
0 \\
0 \\
0 \\
0\end{array}$ & $\begin{array}{l}\mathrm{GE} \\
\mathrm{GE} \\
\mathrm{GE} \\
\mathrm{GE} \\
\mathrm{GE} \\
\mathrm{GE} \\
\mathrm{GE} \\
\mathrm{GE} \\
\mathrm{GE} \\
\mathrm{GE} \\
\mathrm{GE} \\
\mathrm{GE} \\
\mathrm{GE}\end{array}$ & & & & & & & & $\begin{array}{l}\mu \mathrm{g} / \mathrm{L} \\
\mu \mathrm{g} / \mathrm{L} \\
\mu \mathrm{g} / \mathrm{L} \\
\mu \mathrm{g} / \mathrm{L} \\
\mu \mathrm{g} / \mathrm{L} \\
\mu \mathrm{g} / \mathrm{L} \\
\mu \mathrm{g} / \mathrm{L} \\
\mu \mathrm{g} / \mathrm{L} \\
\mu \mathrm{g} / \mathrm{L} \\
\mu \mathrm{g} / \mathrm{L} \\
\mu \mathrm{g} / \mathrm{L} \\
\mu \mathrm{g} / \mathrm{L} \\
\mu \mathrm{g} / \mathrm{L}\end{array}$ \\
\hline \multicolumn{16}{|c|}{ Water Quality Indicator Parameters } \\
\hline Parameter & 1095 & DF & Mod & $\underline{\mathbf{S T}}$ & 브 & Flag & $\underline{\text { Lab }}$ & $\underline{2095}$ & $\underline{\text { DF }}$ & Mod & $\underline{\mathbf{S T}}$ & 브 & Flag & $\underline{\text { Lab }}$ & $\underline{\text { Unit }}$ \\
\hline $\begin{array}{l}\text { Aluminum, total recoverable } \\
\text { Nitrate as nitrogen } \\
\text { pH }\end{array}$ & $\begin{array}{l}43 \\
1,900 \\
4.9\end{array}$ & $\begin{array}{l}1 \\
1 \\
1\end{array}$ & s1 & $=$ & & $\begin{array}{l}1 \\
0 \\
0\end{array}$ & $\begin{array}{l}\text { GE } \\
\text { GE } \\
\text { GE }\end{array}$ & $\begin{array}{l}1,900 \\
5.1\end{array}$ & $\begin{array}{l}1 \\
1\end{array}$ & J1 & - & - & $\begin{array}{l}0 \\
0\end{array}$ & $\begin{array}{l}\mathrm{GE} \\
\mathrm{GE}\end{array}$ & $\underset{\mathrm{pH}}{\mu \mathrm{g} / \mathrm{L}}$ \\
\hline \multicolumn{16}{|c|}{ Common Laboratory Contaminants } \\
\hline Parameter & 1095 & $\underline{\mathrm{DF}}$ & Mod & $\underline{\mathbf{S T}}$ & 보 & Flag & $\underline{\text { Lab }}$ & 2095 & DF & Mod & $\underline{\text { ST }}$ & H & Flag & $\underline{L a b}$ & Unit \\
\hline $\begin{array}{l}\text { Bis(2-ethylhexyl) phthalate } \\
\text { Dichloromethane }\end{array}$ & $\begin{array}{l}<17 \\
<1.7\end{array}$ & 1 & $v$ & & & $\begin{array}{l}0 \\
0\end{array}$ & $\begin{array}{l}G E \\
G E\end{array}$ & & & & & & & & $\underset{\mu g / L}{\mu g / L}$ \\
\hline
\end{tabular}

Note: See Appendix B for flagging criteria. Synchronous water levels are measured over a 3-5 day period.

- = exceeded holding time.

- = exceeded groundwater protection standard. 
WELL FSB123D (cont.)

Radiological Indicator Parameters

\begin{tabular}{|c|c|c|c|c|c|c|c|c|c|c|c|c|}
\hline Parameter & 1095 & DF & Mod & $\underline{\text { ST }} \underline{H}$ & Flag & $\underline{\text { Lab }}$ & 2095 & $\underline{\text { DF }}$ & Mad & $\underline{\text { ST }} \underline{H}$ & Flag & $\underline{\text { Lab }}$ \\
\hline $\begin{array}{l}\text { Gross alpha } \\
\text { Nonvolatile beta }\end{array}$ & $\begin{array}{l}1.6 E+\infty 0 \\
1.2 E+\infty\end{array}$ & $\begin{array}{l}1 \\
1\end{array}$ & Ui & & $\begin{array}{l}0 \\
0\end{array}$ & $\begin{array}{l}\text { GP } \\
G P\end{array}$ & $\begin{array}{l}2.1 E+\infty \\
1.1 E+\infty\end{array}$ & $\begin{array}{l}1 \\
1\end{array}$ & UJI & & $\begin{array}{l}0 \\
0\end{array}$ & $\begin{array}{l}\text { GP } \\
\text { GP }\end{array}$ \\
\hline
\end{tabular}

Measured Radionuclides

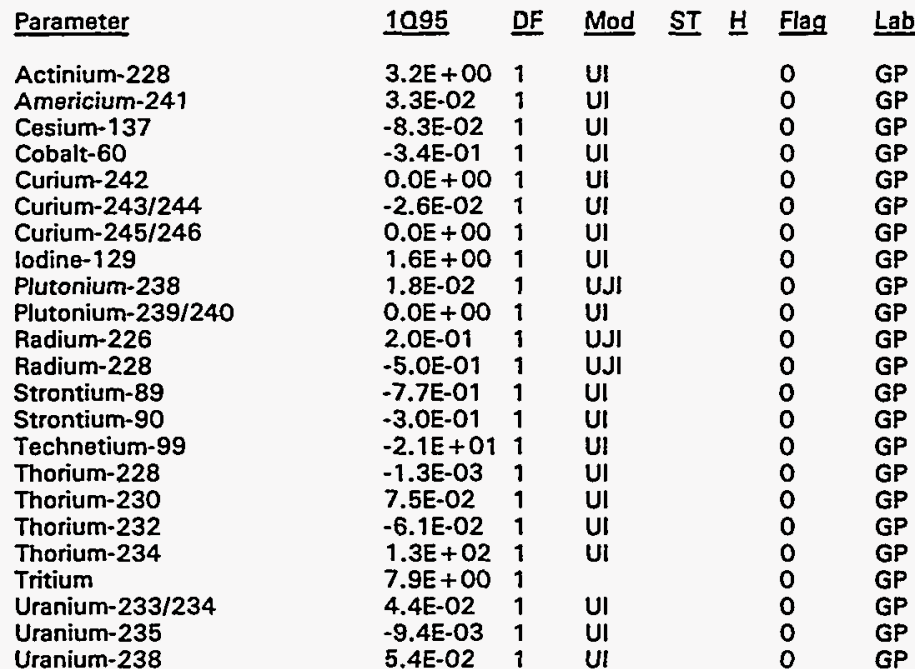

2095 DF Mad ST $\underline{H}$ Flag Lab Unit

Uranium-238

$5.4 E-02 \quad i$

Lab
GP
GP
GP
GP
GP
GP
GP
GP
GP
GP
GP
GP
GP
GP
GP
GP
GP
GP
GP
GP
GP
GP
GP

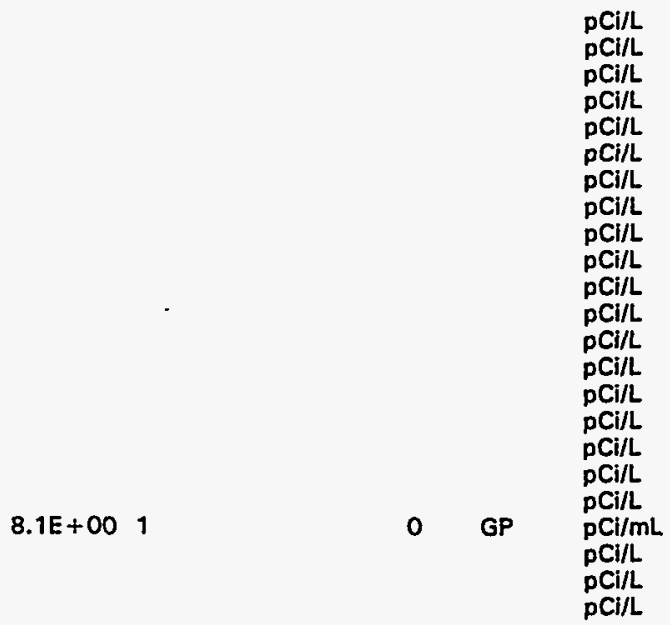

Calculated Radionuclides

\section{Parameter}

Americium-243

Nickel-63

Plutonium-241

Plutonium-242
1095

$-7.9 \mathrm{E}-05$

$-3.4 \mathrm{E}-01$

$0.0 E+00$

$0.0 E+00$
DF Mod ST H Flag Lab

0

$\stackrel{0}{0}$ $\underline{2095}$ DF $\underline{\text { Mod }} \underline{\text { ST }} \underline{H}$ Flag Lab

$\underline{\text { Unit }}$

pCi/L

pCi/L

$\mathrm{pCi} / \mathrm{L}$

Note: See Appendix B for flagging criteria. Synchronous water levels are measured over a $3-5$ day period. - = exceeded holding time.

a = exceeded groundwater protection standard. 
WELL FSL 1D

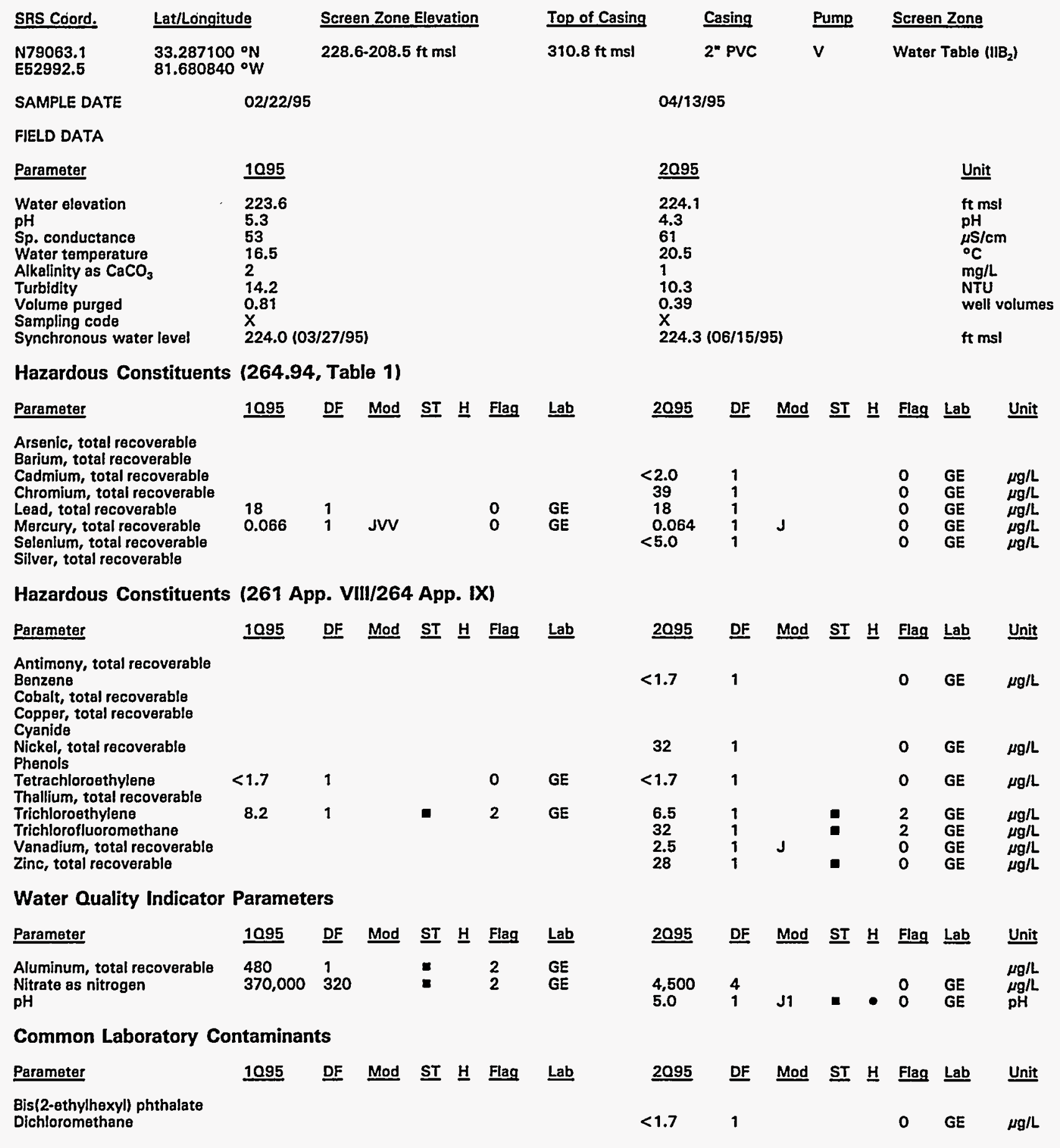

Note: See Appendix B for flagging criteria. Synchronous water levels are measured over a 3-5 day period.

- = exceeded holding time.

= exceeded groundwater protection standard. 
WELL FSL 1D (cont.)

Radiological Indicator Parameters

Parameter

Gross alpha Nonvolatile beta

\begin{tabular}{|c|c|c|c|c|c|}
\hline 1095 & $\underline{D F}$ & Mod & $\underline{S T}$ & 브 & Fle \\
\hline $\begin{array}{l}4.3 E+00 \\
4.0 E+00\end{array}$ & $\begin{array}{l}1 \\
1\end{array}$ & & & & $\begin{array}{l}0 \\
0\end{array}$ \\
\hline
\end{tabular}

$\underline{1095}$

Parameter

Actinium-228

Americium-241

Cesium-137

Cobalt-60

Curium-242

Curium-243/244

Curium-245/246

lodine-129

Plutonium-238

Plutonium-239/240

Radium-226

Radium-228

Strontium-89

Strontium-90

Technetium-99

Thorium-228

Thorium-230

Thorium-232

Thorium-234

Tritium

Uranium-233/234

Uranium-235

Uranium-238

Calculated Radionuclides

Parameter

\begin{tabular}{|c|c|c|c|c|c|}
\hline 2095 & DF & Mod & $\underline{\mathbf{S T}} \underline{H}$ & Flag & $\underline{\text { Lab }}$ \\
\hline & 1 & J & & $\begin{array}{l}0 \\
0\end{array}$ & $\underset{G P}{G P}$ \\
\hline
\end{tabular}

$\underline{2095}$ DF Mod ST $\underline{H}$ Flag Lab Unit

Americium-243

Nickel-63

Plutonium-241

Plutonium-242

Note: See Appendix B for flagging criteria. Synchronous water levels are measured over a 3-5 day period.

- = exceeded holding time.

- = exceeded groundwater protection standard. 
WELL FSL 2D

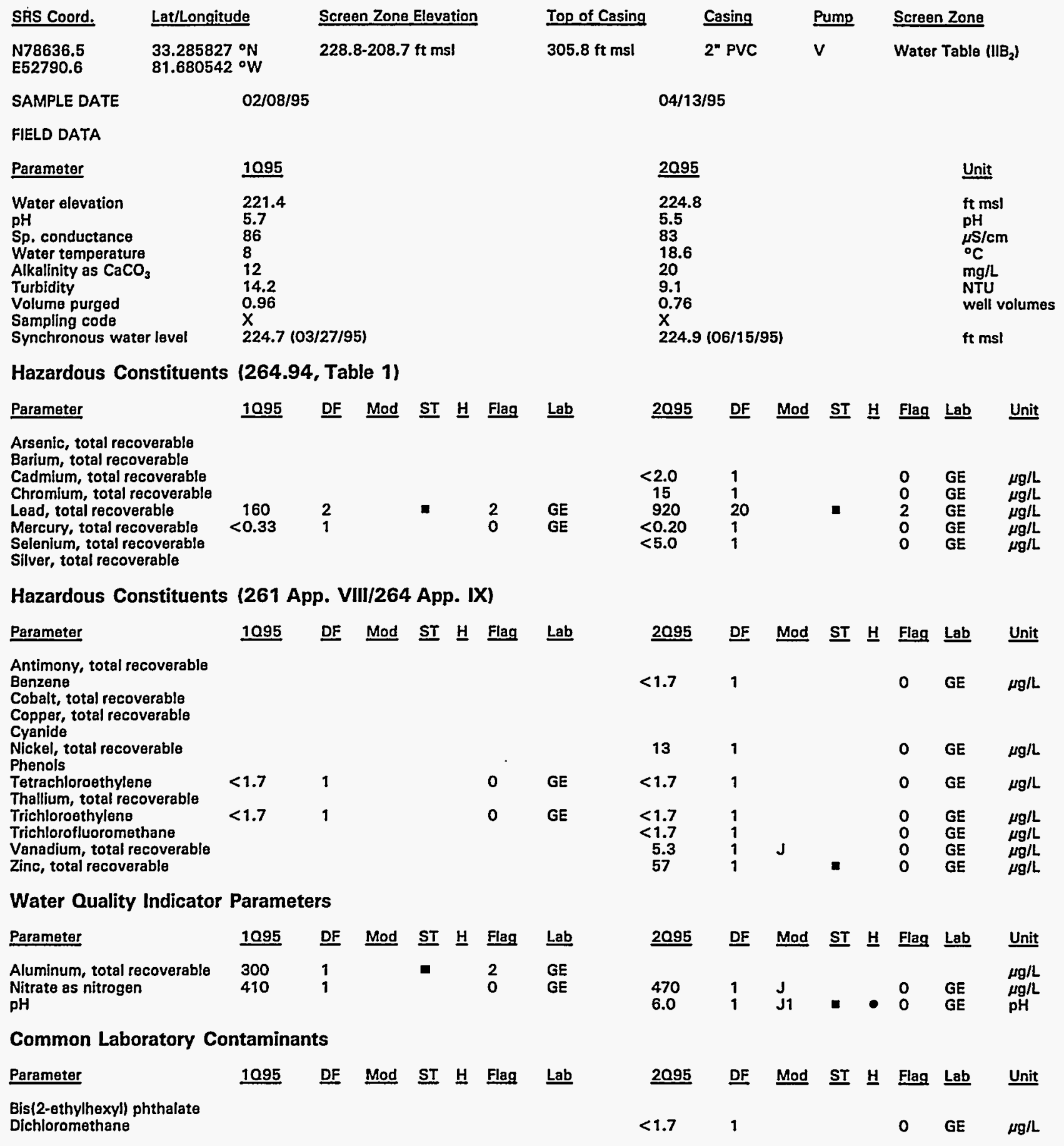

Note: See Appendix B for flagging criteria. Synchronous water levels are measured over a 3-5 day period.

- = exceeded holding time.

- = exceeded groundwater protection standard. 
WELL FSL 2D (cont.)

Radiological Indicator Parameters

\begin{tabular}{|c|c|c|c|c|c|c|c|c|c|c|c|c|c|c|c|}
\hline Parameter & 1095 & DF & Mod & $\underline{\mathrm{ST}}$ & H & Flag & $\underline{\text { Lab }}$ & 2095 & DF & Mod & $\underline{\mathbf{S T}}$ & H & Flag & Lab & Unit \\
\hline $\begin{array}{l}\text { Gross alpha } \\
\text { Nonvolatile beta }\end{array}$ & $\begin{array}{l}5.6 \mathrm{E}-01 \\
1.2 \mathrm{E}+00\end{array}$ & $\begin{array}{l}1 \\
1\end{array}$ & $\begin{array}{l}\text { UI } \\
\text { UI }\end{array}$ & & & $\begin{array}{l}0 \\
0\end{array}$ & $\begin{array}{l}\text { GP } \\
\text { GP }\end{array}$ & $\begin{array}{l}\text { 8.2E-01 } \\
2.2 E+00\end{array}$ & $\begin{array}{l}1 \\
1\end{array}$ & $J$ & & & $\begin{array}{l}0 \\
0\end{array}$ & $\begin{array}{l}\text { GP } \\
\text { GP }\end{array}$ & $\begin{array}{l}\mathrm{pCi} / \mathrm{L} \\
\mathrm{pCi} / \mathrm{L}\end{array}$ \\
\hline \multicolumn{16}{|c|}{ Measured Radionuclides } \\
\hline Parameter & 1095 & DF & Mod & $\underline{S T}$ & $\underline{H}$ & Flag & Lab & 2095 & DF & Mod & ST & $\underline{H}$ & Flag & $\underline{\text { Lab }}$ & Unit \\
\hline \multicolumn{16}{|l|}{$\begin{array}{l}\text { Actinium-228 } \\
\text { Americium-241 } \\
\text { Cesium-137 } \\
\text { Cobalt-60 } \\
\text { Curium-242 } \\
\text { Curium-243/244 } \\
\text { Curium-245/246 } \\
\text { lodine-129 } \\
\text { Plutonium-238 } \\
\text { Plutonium-239/24 } \\
\text { Radium-226 } \\
\text { Radium-228 } \\
\text { Strontium-89 } \\
\text { Strontium-90 } \\
\text { Technetium-99 } \\
\text { Thorium-228 } \\
\text { Thorium-230 } \\
\text { Thorium-232 } \\
\text { Thorium-234 }\end{array}$} \\
\hline $\begin{array}{l}\text { Tritium } \\
\text { Uranium-233/23 } \\
\text { Uranium-235 } \\
\text { Uranium-238 }\end{array}$ & $1.4 E+01$ & 1 & & & & 1 & GP & $1.4 E+01$ & 1 & & & & 1 & GP & $\mathrm{pCi} / \mathrm{mL}$ \\
\hline \multicolumn{16}{|c|}{ Calculated Radionuclides } \\
\hline Parameter & 1095 & DF & Mod & $\underline{S T}$ & $\underline{\mathrm{H}}$ & Flag & $\underline{L a b}$ & 2095 & DF & Mod & $\underline{\text { ST }}$ & 브 & Flag & $\underline{\text { Lab }}$ & Unit \\
\hline $\begin{array}{l}\text { Americium-243 } \\
\text { Nickel-63 } \\
\text { Plutonium-241 } \\
\text { Plutonium-242 }\end{array}$ & & & & & & & & & & & & & & & \\
\hline
\end{tabular}

Note: See Appendix B for flagging criteria. Synchronous water levels are measured over a 3-5 day period. $\checkmark=$ exceeded holding time.

- = exceeded groundwater protection standard. 


\section{WELL FSL 3D}

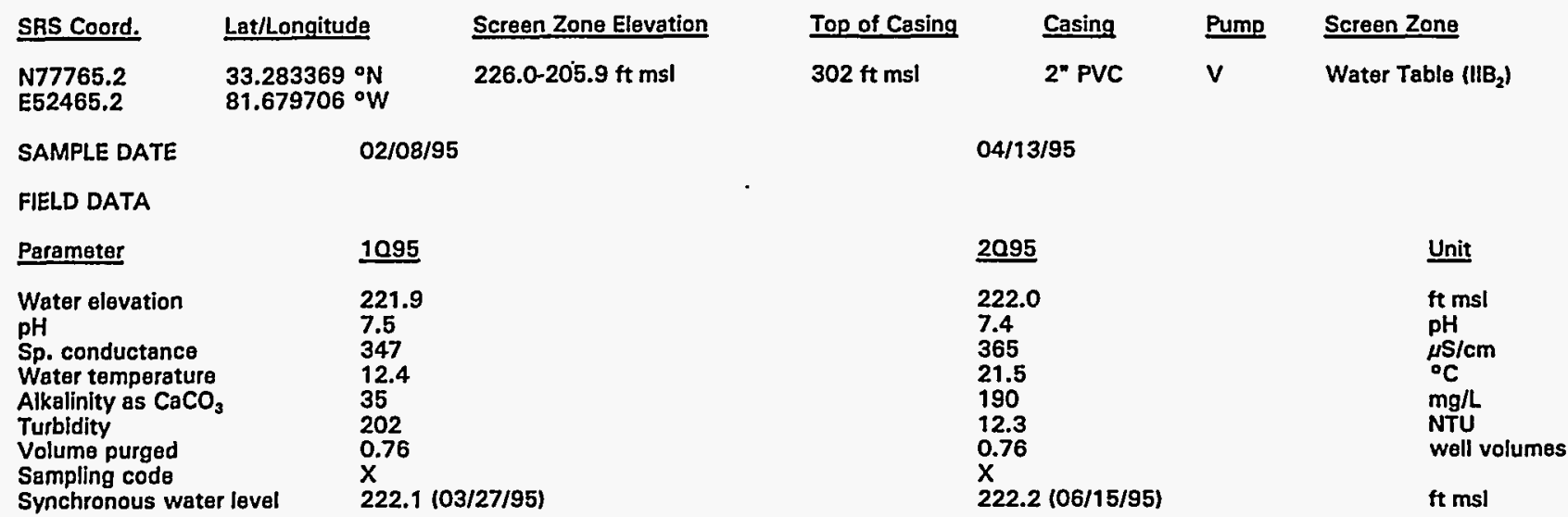

Hazardous Constituents (264.94, Table 1)

1095 DF Mod ST $\underline{H}$ Flag Lab

$\underline{2095}$

DF Mod ST $\underline{H}$ Flag Lab Unit

Arsenic, total recoverable

Barium, total recoverable

Cadmium, total recoverable

Chromium, total recoverable

Lead, total recoverable

Mercury, total recoverable

Mercury, total recoverable
Selenium, total recoverable

$\begin{array}{ll}<5.0 & 1 \\ <0.33 & 1\end{array}$

$\begin{array}{ll}0 & \text { GE } \\ 0 & \text { GE }\end{array}$

$\begin{array}{ll}<2.0 & 1 \\ 24 & 1 \\ 7.5 & 1 \\ <0.20 & 1\end{array}$

Silver, total recoverable

Hazardous Constituents (261 App. VIII/264 App. IX)

Parameter

1095 DF Mod ST $\underline{H}$ Flag Lab

2095 DF Mod ST $\underline{H}$ Flag Lab Unit

Antimony, total recoverable

Benzene

Cobalt, total recoverable

Copper, total recoverable

Cyanide

Nickel, total recoverablo

Phenols

Tetrachloroethylene

Thallium, total recoverable

Trichloroethylene

Trichlorofluoromethane

Vanadium, total recoverable

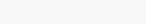

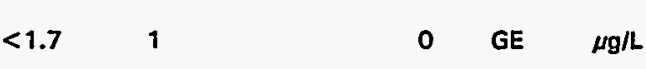

Zinc, total recoverable

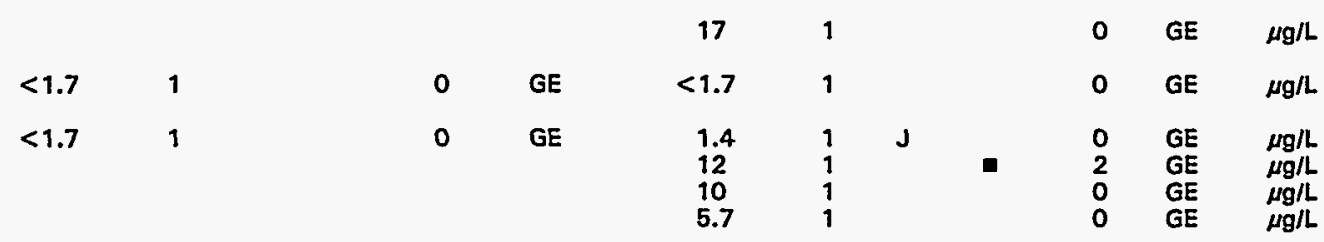

Water Quality Indicator Parameters

\begin{tabular}{|c|c|c|c|c|c|c|c|c|c|c|c|c|c|c|}
\hline Parameter & 1095 & $\underline{D F}$ & Mod & $\underline{\mathbf{S T}}$ & $\underline{\mathrm{H}}$ & Flag & Lab & 2095 & DF & Mod & $\underline{\mathrm{ST}}$ & 브 & Flag & Lab \\
\hline $\begin{array}{l}\text { Aluminum, total recoverable } \\
\text { Nitrate as nitrogen } \\
\text { pH }\end{array}$ & $\begin{array}{l}180 \\
260,000\end{array}$ & $\begin{array}{l}1 \\
250\end{array}$ & & E & & $\begin{array}{l}2 \\
2\end{array}$ & $\begin{array}{l}\text { GE } \\
\text { GE }\end{array}$ & $\begin{array}{l}930 \\
7.7\end{array}$ & $\begin{array}{l}1 \\
1\end{array}$ & $\begin{array}{l}\mathrm{J} 1 \\
\mathrm{j} 1\end{array}$ & & & $\begin{array}{l}0 \\
0\end{array}$ & $\begin{array}{l}\text { GE } \\
\text { GE }\end{array}$ \\
\hline
\end{tabular}

Common Laboratory Contaminants

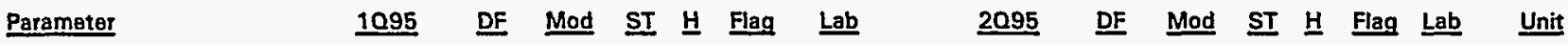

Bis(2-ethylhexyl) phthalate

Dichloromethane

$\begin{array}{lllll}<1.7 & 1 & \mathrm{GE} \quad \mu \mathrm{g} / \mathrm{L}\end{array}$

Note: See Appendix B for flagging criteria. Synchronous water levels are measured over a 3-5 day period.

- = exceeded holding time.

- = exceeded groundwater protection standard. 
WELL FSL 3D (cont.)

Radiological Indicator Parameters

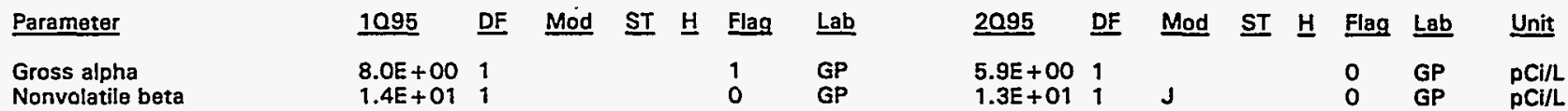

\section{Measured Radionuclides}

Parameter

1095 DF Mod ST $\underline{H}$ Flag Lab

$\underline{2095}$

Unit

Actinium-228

Americium-241

Cesium-137

Cobalt 60

Curium-242

Curium-243/244

Curium-245/246

lodine-129

Plutonium-238

Plutonium-239/240

Radium-226

Radium-228

Strontium-89

Strontium-90

Technetium-99

Thorium-228

Thorium-230

Thorium-232

Thorium-234

Tritium
Uranium-233/234

Uranium-235

Uranium-238

Calculated Radionuclides

Parameter

Americium-243

Nicke!-63

Plutonium-241

Plutonium-242

Note: See Appendix B for flagging criteria. Synchronous water levels are measured over a 3-5 day period. - = exceeded holding time.

- = exceeded groundwater protection standard. 
WELL FSL 4D

\begin{tabular}{|c|c|c|c|c|c|c|}
\hline SRS Coord. & Lat/Longitude & Screan Zone Elevation & Top of Casing & Casing & Pump & Screen Zone \\
\hline $\begin{array}{l}\text { N77452.4 } \\
\text { E52230.4 }\end{array}$ & $\begin{array}{l}33.282294^{\circ} \mathrm{N} \\
81.679717^{\circ} \mathrm{W}\end{array}$ & $224.1-204.0 \mathrm{ft} \mathrm{msl}$ & $294.1 \mathrm{ft} \mathrm{msl}$ & 2" PVC & $v$ & Water Table $\left(1 \mathrm{~B}_{2}\right)$ \\
\hline SAMPLE DATE & 02 & & & $3 / 95$ & & \\
\hline
\end{tabular}

FIELD DATA

\begin{tabular}{ll} 
Parameter & $\underline{1095}$ \\
\hline Water elevation & 216.3 \\
pH & 7.5 \\
Sp. conductance & 51 \\
Water temperature & 12.4 \\
Alkalinity as $\mathrm{CaCO}_{3}$ & 22 \\
Turbidity & 10 \\
Volume purged & 0.50 \\
Sampling code & $\times$ \\
Synchronous water level & $216.8(03 / 27 / 95$
\end{tabular}

$\begin{array}{ll}\underline{2095} & \text { Unit } \\ 217.0 & \mathrm{ft} \mathrm{msl} \\ 5.3 & \mathrm{pH} \\ 60 & \mu \mathrm{S} / \mathrm{cm} \\ 21.5 & { }^{\circ} \mathrm{C} \\ 8 & \mathrm{mg} / \mathrm{L} \\ 7 & \mathrm{NTU} \\ 0.47 & \text { well volumes } \\ \mathrm{X} & \mathrm{ft} \mathrm{msl} \\ 217.0(06 / 15 / 95) & \end{array}$

Hazardous Constituents (264.94, Table 1)

Parameter 1095 DF Mod ST H Flag Lab

$\underline{2095}$

DF Mod ST $\underline{H}$ Flag Lab Unit

Arsenic, total recoverable

Barium, total recoverable

Cadmium, total recoverable

Chromium, total recoverable

Lead, total recoverable

$\begin{array}{cccc}32 & 1 & 1 & G E\end{array}$

Mercury, total recoverable
Selenium, total recoverable

Silver, total recoverable

Hazardous Constituents (261 App. VIII/264 App. IX)

Parameter

1095 DF Mod ST H Flag Lab

$\underline{2095}$

DF Mod ST $\underline{\text { H Flag Lab }} \underline{\text { Unit }}$

Antimony, total recoverable

Benzene

Cobalt, total recoverable

Copper, total recoverable

Cyanide

Nickel, total recoverable

Phenols

Thallium, total recoverable

Trichloroethylene

$<1.7 \quad 1$

$0 \quad$ GE

$<2.0$
17
13
$<0.20$

$<5.0$

0 GE

Trichlorofluoromethane

Vanadium, total recoverable

$<1.7 \quad 1$

Zinc, total recoverable

\section{Water Quality Indicator Parameters}

\begin{tabular}{|c|c|c|c|c|c|c|c|c|c|c|c|c|c|c|c|}
\hline Parameter & 1095 & $\underline{D F}$ & Mod & $\underline{\underline{\mathbf{S T}}}$ & $\underline{\mathrm{H}}$ & Flag & $\underline{\text { Lab }}$ & $\underline{2095}$ & DF & Mod & $\underline{\text { ST }}$ & H & Flag & $\underline{\text { Lab }}$ & $\underline{\text { Unit }}$ \\
\hline $\begin{array}{l}\text { Aluminum, total recoverable } \\
\text { Nitrate as nitrogen } \\
\text { pH }\end{array}$ & $\begin{array}{l}580 \\
1,300\end{array}$ & $\begin{array}{l}1 \\
2\end{array}$ & & - & & $\begin{array}{l}2 \\
0\end{array}$ & $\begin{array}{l}\mathrm{GE} \\
\mathrm{GE}\end{array}$ & $\begin{array}{l}1,300 \\
5.7\end{array}$ & $\begin{array}{l}1 \\
1\end{array}$ & $\begin{array}{l}\mathrm{J} \\
\mathrm{J} 1\end{array}$ & 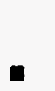 & - & $\begin{array}{l}0 \\
0\end{array}$ & $\begin{array}{l}\text { GE } \\
\text { GE }\end{array}$ & $\begin{array}{l}\mu \mathrm{g} / \mathrm{L} \\
\mu \mathrm{g} / \mathrm{L} \\
\mathrm{pH}\end{array}$ \\
\hline \multicolumn{16}{|c|}{ Common Laboratory Contaminants } \\
\hline Parameter & 1095 & DF & Mod & $\underline{\mathbf{S T}}$ & $\underline{H}$ & Flag & $\underline{\text { Lab }}$ & $\underline{2095}$ & $\underline{\mathrm{DF}}$ & Mod & ST & $\underline{\text { H}}$ & Flag & $\underline{\text { Lab }}$ & Unit \\
\hline $\begin{array}{l}\text { Bis(2-ethylhexyl) phthalate } \\
\text { Dichloromethane }\end{array}$ & & & & & & & & $<1.7$ & 1 & & & & 0 & GE & $\mu \mathrm{g} / \mathrm{L}$ \\
\hline
\end{tabular}

Note: See Appendix B for flagging criteria. Synchronous water levels are measured over a 3-5 day period.

- = exceeded holding time.

- exceeded groundwater protection standard. 
WELL FSL 4D (cont.)

Radiological Indicator Parameters

\begin{tabular}{|c|c|c|c|c|c|c|c|c|c|c|c|c|c|c|c|}
\hline Parameter & 1095 & DF & Mod & $\underline{S T}$ & $\underline{H}$ & Flag & $\underline{L a b}$ & $\underline{2095}$ & DF & Mod & $\underline{\text { ST }}$ & $\underline{H}$ & Flag & $\underline{L a b}$ & Unit \\
\hline $\begin{array}{l}\text { Gross alpha } \\
\text { Nonvolatile beta }\end{array}$ & $\begin{array}{l}1.2 E+00 \\
4.5 E+00\end{array}$ & $\begin{array}{l}1 \\
1\end{array}$ & & & & $\begin{array}{l}0 \\
0\end{array}$ & $\begin{array}{l}\text { GP } \\
\text { GP }\end{array}$ & $\begin{array}{l}2.1 E+00 \\
6.1 E+00\end{array}$ & $\begin{array}{l}1 \\
1\end{array}$ & $J$ & & & $\begin{array}{l}0 \\
0\end{array}$ & $\begin{array}{l}\text { GP } \\
G P\end{array}$ & $\begin{array}{l}\mathrm{pCi} / \mathrm{L} \\
\mathrm{pCi} / \mathrm{L}\end{array}$ \\
\hline Parameter & 1095 & $\underline{D F}$ & Mod & $\underline{\text { ST }}$ & 브 & Flag & Lab & 2095 & $\underline{D F}$ & Mod & $\underline{\text { ST }}$ & $\underline{\mathbf{H}}$ & Flag & $\underline{\text { Lab }}$ & Unit \\
\hline \multicolumn{16}{|l|}{$\begin{array}{l}\text { Actinium-228 } \\
\text { Americium-241 } \\
\text { Cesium-137 } \\
\text { Cobalt-60 } \\
\text { Curium-242 } \\
\text { Curium-243/244 } \\
\text { Curium-245/246 } \\
\text { lodine-129 } \\
\text { Plutonium-238 } \\
\text { Plutonium-239/240 } \\
\text { Radium-226 } \\
\text { Radium-228 } \\
\text { Strontium-89 } \\
\text { Strontium-90 } \\
\text { Technetium-99 } \\
\text { Thorium-228 } \\
\text { Thorium-230 } \\
\text { Thorium-232 } \\
\text { Thorium-234 }\end{array}$} \\
\hline $\begin{array}{l}\text { Tritium } \\
\text { Uranium-233/234 } \\
\text { Uranium-235 } \\
\text { Uranium-238 }\end{array}$ & $5.1 E+00$ & 1 & & & & 0 & GP & $6.2 E+00$ & 1 & & & & 0 & GP & $\mathrm{pCi} / \mathrm{mL}$ \\
\hline \multicolumn{16}{|c|}{ Calculated Radionuclides } \\
\hline Parameter & 1095 & $\underline{D F}$ & Mod & $\underline{\underline{S T}}$ & $\underline{H}$ & Flag & Lab & 2095 & $\underline{D F}$ & Mod & $\underline{\mathbf{S T}}$ & $\underline{H}$ & Flag & Lab & Unit \\
\hline
\end{tabular}

Note: See Appendix B for flagging criteria. Synchronous water levels are measured over a 3-5 day period. - = exceeded holding time.

- = exceeded groundwater protection standard. 
WSRC-TR-95-0146-2

Unclassified

\section{WELL FSL 5D}

\begin{tabular}{|c|c|c|c|c|c|c|}
\hline SRS Coord. & at/Longitude & Screen Zone Elevation & Top of Casing & Casing & Pump & Screen Zone \\
\hline $\begin{array}{l}\text { N77047.7 } \\
\text { E51903.3 }\end{array}$ & $\begin{array}{l}33.2808655^{\circ} \mathrm{N} \\
31.679791\end{array}$ & $223.7-203.5 \mathrm{ft} \mathrm{msl}$ & $291.8 \mathrm{ft} \mathrm{msl}$ & 2" PVC & v & Water Table $\left(\| B_{2}\right)$ \\
\hline SAMPLE DATE & \multicolumn{2}{|c|}{$02 / 08 / 95$} & & \multicolumn{3}{|l|}{$04 / 13 / 95$} \\
\hline \multicolumn{7}{|l|}{ FIELD DATA } \\
\hline Parameter & \multicolumn{2}{|c|}{1095} & \multicolumn{2}{|r|}{$\underline{2095}$} & & Unit \\
\hline $\begin{array}{l}\text { Water elevation } \\
\text { pH } \\
\text { Sp. conductance } \\
\text { Water temperature } \\
\text { Alkalinity as } \mathrm{CaCO}_{3} \\
\text { Turbidity } \\
\text { Volume purged } \\
\text { Sampling code } \\
\text { Synchronous water le }\end{array}$ & \multicolumn{2}{|c|}{$\begin{array}{l}219.8 \\
6.2 \\
224 \\
11.5 \\
28 \\
69.9 \\
0.86 \\
x\end{array}$} & \multicolumn{3}{|c|}{$\begin{array}{l}220.2 \\
5.9 \\
221 \\
21.7 \\
29 \\
11.9 \\
1.2 \\
\times \\
220.4(06 / 15 / 95)\end{array}$} & $\begin{array}{l}\mathrm{ft} \mathrm{msl} \\
\mathrm{pH} \\
\text { uS/cm } \\
{ }^{\circ} \mathrm{C} \\
\mathrm{mg} / \mathrm{L} \\
\text { NTU } \\
\text { well volumes } \\
\text { ft } \mathrm{msl}\end{array}$ \\
\hline
\end{tabular}

Hazardous Constituents (264.94, Table 1)

Parameter $\quad \underline{095} \underline{\text { DF }} \underline{\text { Mod }} \underline{\text { ST }} \underline{H} \underline{\text { Flag }} \underline{\text { Lab }} \quad \underline{2095} \underline{\text { DF }} \underline{\text { Mod }} \underline{\text { ST }} \underline{H} \underline{\text { Flag Lab }} \underline{\text { Unit }}$

Arsenic, total recoverable

Barium, total recoverable

Cadmium, total recoverable

Chromium, total recoverable

Lead, total recoverable

Mercury, total recoverable

$\begin{array}{cccc}360 & 5 & 2 & \text { GE }\end{array}$

Selenium, total recoverable

$\begin{array}{llll}<0.33 & 1 & 0 & G E\end{array}$

Silver, total recoverable

Hazardous Constituents (261 App. VIII/264 App. IX)

Parameter

1095 DF Mod ST H Flag Lab

2095 DF Mod ST $\underline{H}$ Flag LLab Unit

Antimony, total recoverable

Benzene

Cobalt, total recoverable

Copper, total recoverable

Cyanide

Nickel, total recoverable

Phenols

Tetrachloroethylene

Thallium, total recoverable

Trichloroethylene

Trichlorofluoromethane

Vanadium, total recoverable

\begin{tabular}{|c|c|c|c|c|c|}
\hline $\begin{array}{c}<2.0 \\
5.7 \\
100 \\
0.12 \\
<5.0\end{array}$ & $\begin{array}{l}1 \\
1 \\
1 \\
1 \\
1\end{array}$ & $\begin{array}{l}V \\
j\end{array}$ & $=$ & $\begin{array}{l}0 \\
0 \\
2 \\
0 \\
0\end{array}$ & $\begin{array}{l}G E \\
G E \\
G E \\
G E \\
G E\end{array}$ \\
\hline
\end{tabular}

Zinc, total recoverable

\begin{tabular}{|c|c|c|c|c|c|c|c|c|}
\hline & & & & 2.2 & 1 & $\mathrm{~J}$ & 0 & GE \\
\hline$<1.7$ & 1 & 0 & GE & $<1.7$ & 1 & & 0 & GE \\
\hline 3.1 & 1 & 1 & GE & $\begin{array}{l}2.8 \\
1.5 \\
6.3 \\
26\end{array}$ & $\begin{array}{l}1 \\
1 \\
1 \\
1\end{array}$ & ${ }_{J V}^{J}$ & $\begin{array}{l}1 \\
0 \\
0 \\
0\end{array}$ & $\begin{array}{l}\mathrm{GE} \\
\mathrm{GE} \\
\mathrm{GE} \\
\mathrm{GE}\end{array}$ \\
\hline
\end{tabular}

Water Quality Indicator Parameters

\begin{tabular}{|c|c|c|c|c|c|c|c|c|c|c|c|c|c|}
\hline Parameter & 1095 & DF & Mod & $\underline{\mathbf{S T}}$ & 브 & Flag & Lab & $\underline{2095}$ & DF & Mod & $\underline{\text { ST }}$ 브 & Flag & $\underline{\text { La }}$ \\
\hline $\begin{array}{l}\text { Aluminum, total recoverab } \\
\text { Nitrate as nitrogen }\end{array}$ & $\begin{array}{l}450 \\
21,000\end{array}$ & $\begin{array}{l}1 \\
20\end{array}$ & & 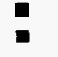 & & $\begin{array}{l}2 \\
2\end{array}$ & $\begin{array}{l}\text { GE } \\
\text { GE }\end{array}$ & 16,000 & 40 & $J$ & & 2 & \\
\hline
\end{tabular}

pH

Common Laboratory Contaminants

Parameter

1095 DF Mod ST H Flag Lab

$\underline{2095}$ DF Mad ST $\underline{H}$ Flag Lab Unit

Bis(2-ethylhexyl) phthalate

Dichloromethane

$\begin{array}{lllll}<1.7 & 1 & 0 & \text { GE } & \mu \mathrm{g} / \mathrm{L}\end{array}$

Note: See Appendix B for flagging criteria. Synchronous water levels are measured over a 3-5 day period.

- = exceeded holding time.

- = exceeded groundwater protection standard. 
WELL FSL 5D (cont.)

Radiological Indicator Parameters

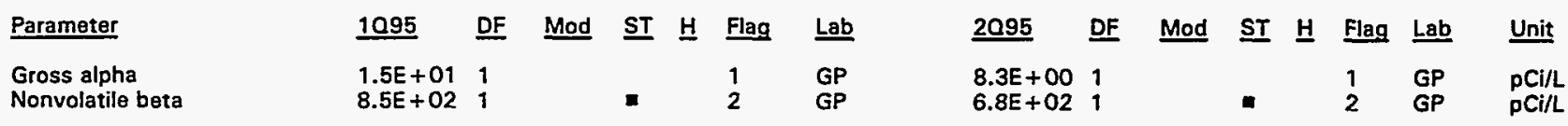

Measured Radionuclides

Parameter

1095

DF Mod ST $\underline{H}$ Flag Lab

DF Mod ST $\underline{\text { H Flag Lab }}$

Unit

Actinium-228

Americium-241

Cesium-137

Cobalt-60

Curium-242

Curium-243/244

Curium-245/246

lodine-129

Plutonium-238

Plutonium-239/240

Radium-226

Radium-228

Strontium-89

Strontium-90

Technetium-99

Thorium-228

Thorium-230

Thorium-232

Thorium-234

Tritium

Uranium-233/234

$4.8 E+021$

- 2

GP

$6.4 E+021$

- 2 GP

pCi/mL

Uranium-235

Uranium-238

Calculated Radionuclides

Parameter

1095

DF Mod ST $\underline{\text { H Flag Lab }}$

$\underline{2095}$

DF Mod ST $\underline{\text { H Flag Lab }}$

Unit

Americium-243

Nickel-63

Plutonium-241

Plutonium-242

Note: See Appendix B for flagging criteria. Synchronous water levels are measured over a 3-5 day period.

- = exceeded holding time.

- exceeded groundwater protection standard. 


\section{WELL FSL 6D}

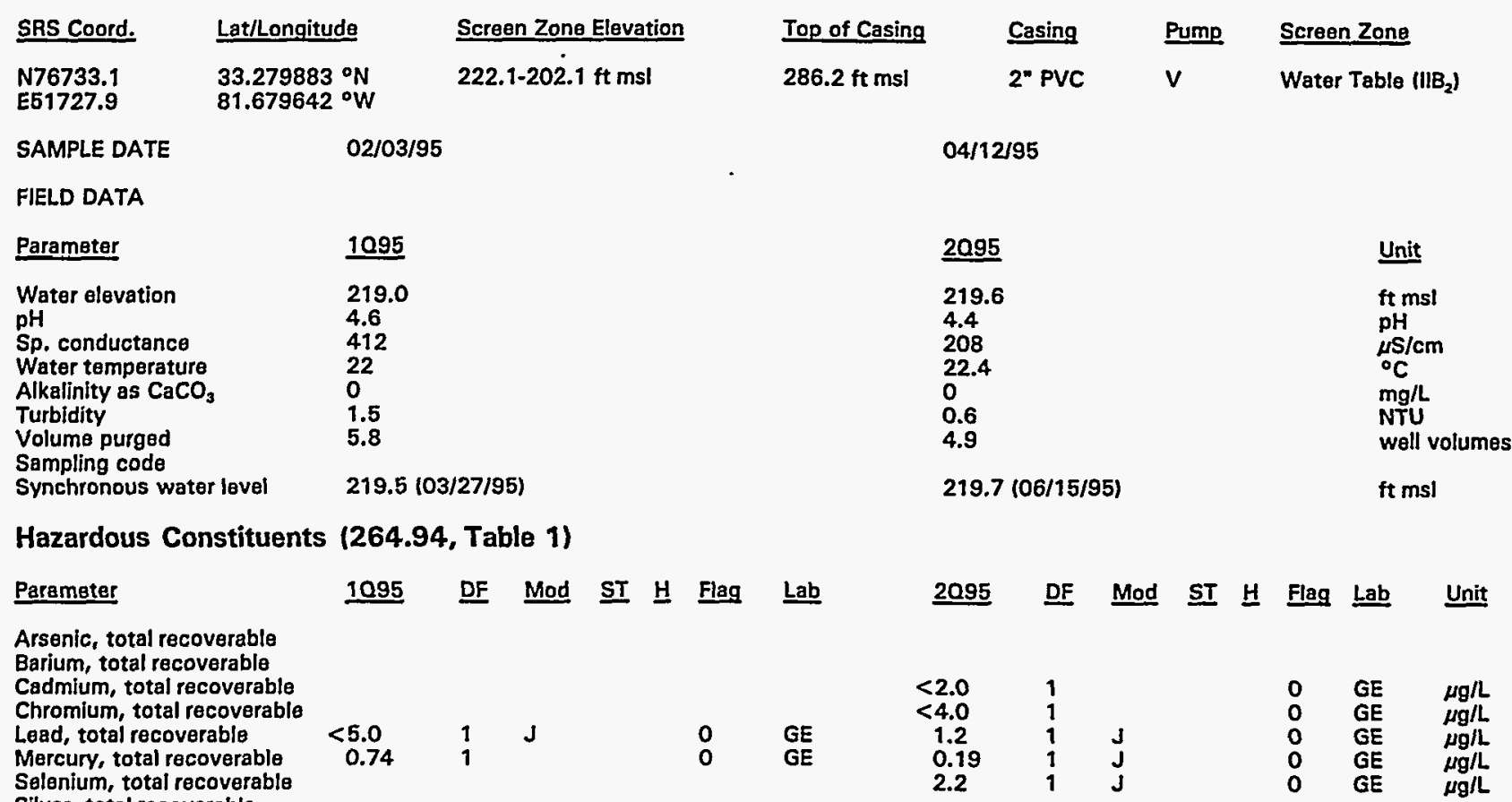

, total recoverable

Silver, total recoverable

Hazardous Constituents (261 App. VIII/264 App. IX)

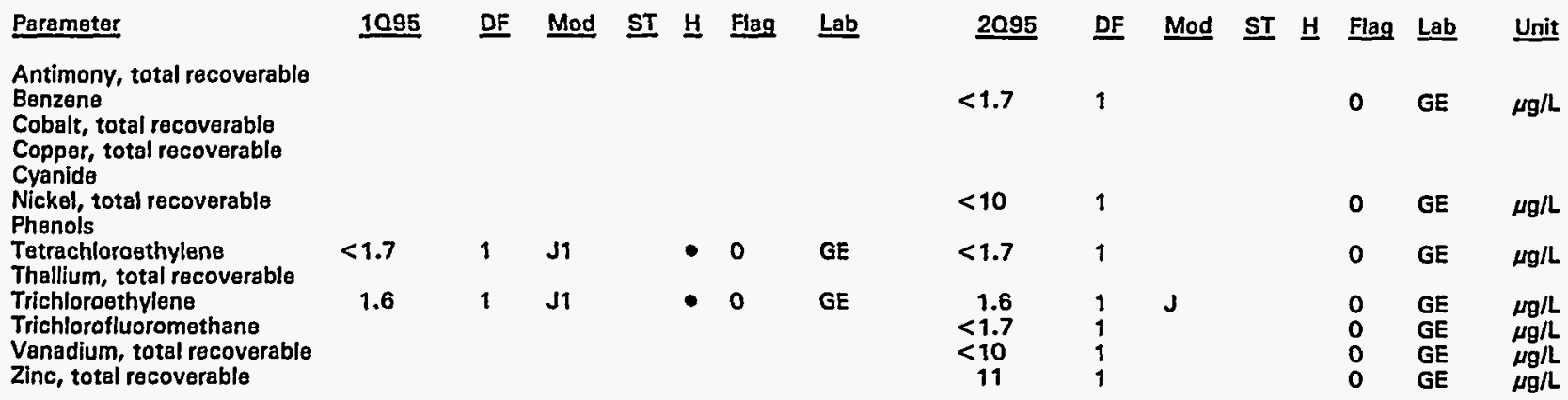

Water Quality Indicator Parameters

\begin{tabular}{|c|c|c|c|c|c|c|c|c|c|c|c|c|c|c|c|}
\hline Parameter & 1095 & $\underline{D F}$ & Mod & $\underline{\mathbf{S T}}$ & $\underline{\mathbf{H}}$ & Flag & $\underline{\text { Lab }}$ & $\underline{2095}$ & DF & Mod & ST & $\underline{\mathrm{H}}$ & Flag & $\underline{\text { Lab }}$ & $\underline{\text { Unit }}$ \\
\hline $\begin{array}{l}\text { Aluminum, total recoverable } \\
\text { Nitrate as nitrogen } \\
\text { pH }\end{array}$ & $\begin{array}{l}420 \\
38,000\end{array}$ & $\begin{array}{l}1 \\
40\end{array}$ & & 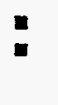 & & $\begin{array}{l}2 \\
2\end{array}$ & $\begin{array}{l}\text { GE } \\
\text { GE }\end{array}$ & $\begin{array}{l}27,000 \\
5.1\end{array}$ & $\begin{array}{l}20 \\
1\end{array}$ & J1 & 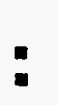 & • & $\begin{array}{l}2 \\
0\end{array}$ & $\begin{array}{l}\text { GE } \\
\text { GE }\end{array}$ & $\begin{array}{l}\mu \mathrm{g} / \mathrm{L} \\
\mu \mathrm{g} / \mathrm{L} \\
\mathrm{pH}\end{array}$ \\
\hline \multicolumn{16}{|c|}{ Common Laboratory Contaminants } \\
\hline Parameter & 1095 & DF & Mod & $\underline{\text { ST }}$ & $\underline{\text { H}}$ & Flag & Lab & $\underline{2095}$ & DF & Mod & $\underline{\mathbf{S I}}$ & $\underline{H}$ & Flag & $\underline{\text { Lab }}$ & $\underline{\text { Unit }}$ \\
\hline $\begin{array}{l}\text { Bis(2-ethylhexyl) phthalate } \\
\text { Dichloromethane }\end{array}$ & & & & & & & & $<1.7$ & 1 & & & & 0 & GE & $\mu g / L$ \\
\hline
\end{tabular}

Note: See Appendix B for flagging criteria. Synchronous water levels are measured over a 3-5 day period.

$-=$ exceeded holding time.

- = exceeded groundwater protection standard. 
WELL FSL 6D (cont.)

Radiological Indicator Parameters

Parameter

Gross alpha

Nonvolatile beta

\begin{tabular}{|c|c|c|c|c|}
\hline 1095 & $\mathrm{DF}$ & Mod & $\underline{\text { ST }} \quad \underline{H}$ & Flag \\
\hline $\begin{array}{l}1.1 E+01 \\
3.7 E+01\end{array}$ & $\begin{array}{l}1 \\
1\end{array}$ & $J$ & & $\begin{array}{l}1 \\
1\end{array}$ \\
\hline
\end{tabular}

1095

Actinium-228

Americium-241

Cesium-137

Cobalt- 60

Curium-242

Curium-243/244

Curium-245/246

lodine-129

Plutonium-238

Plutonium-239/240

Radium-226

Radium-228

Strontium-89

Strontium-90

Technetium-99

Thorium-228

Thorium-230

Thorium-232

Thorium-234

Tritium

Uranium-233/234

$8.7 E+02 \quad 1$

- 2

GP

$3.1 E+021$

- 2 GP

$\mathrm{pCi} / \mathrm{mL}$

Uranium-235

Uranium-238

Calculated Radionuclides

Parameter

Americium-243

Nickel-63

Plutonium-241

Plutonium-242

Note: See Appendix B for flagging criteria. Synchronous water levels are measured over a 3-5 day period.

- = exceeded holding time.

- = exceeded groundwater protection standard. 


\section{WELL FSL 7D}

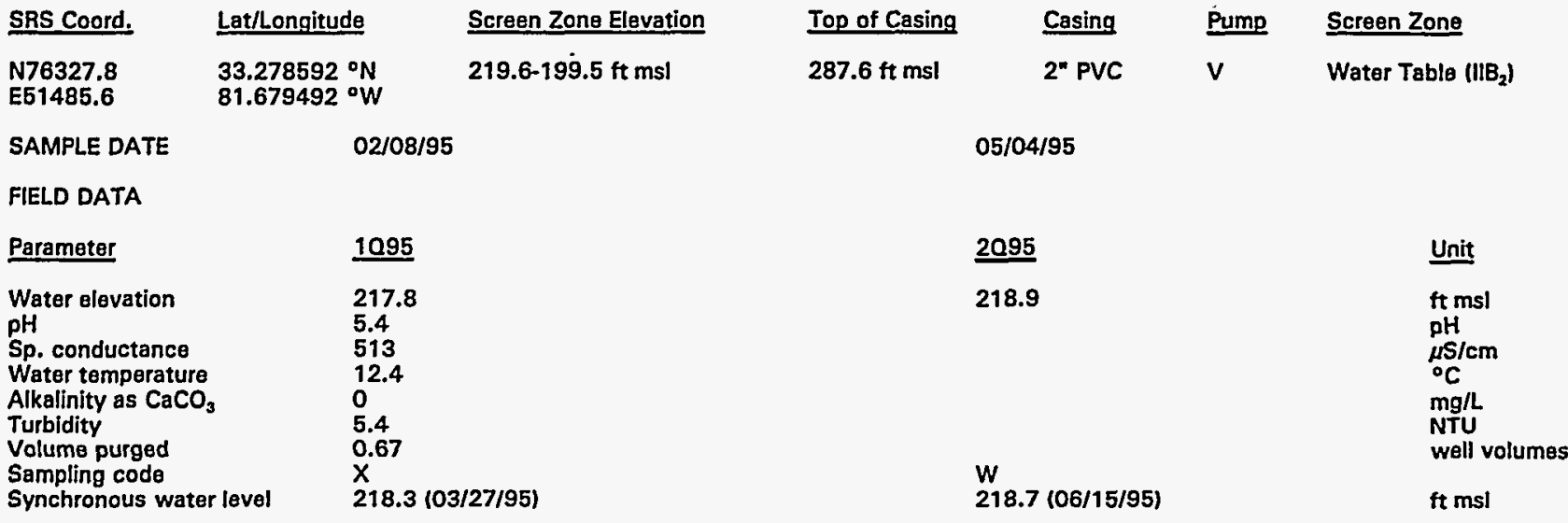

Hazardous Constituents (264.94, Table 1)

Parameter $\quad \underline{1095}$ DF Mod $\underline{\text { ST }} \underline{H} \underline{\text { Flag }} \underline{\text { Lab }} \quad \underline{2095} \underline{\text { DF }} \underline{\text { Mod }} \underline{\text { ST }} \underline{\text { Flag Lab }} \underline{\text { Unit }}$

Arsenic, total recoverable

Barium, total recoverable

Cadmium, total recoverable

Chromium, total recoverable

Lead, total recoverable

$\begin{array}{cccc}5.8 & 1 & 0 & \text { GE } \\ <0.33 & 1 & 0 & \text { GE }\end{array}$

$\mu g / L$

Mercury, total recoverable

Selenium, total recoverable

Silver, total recoverable

Hazardous Constituents (261 App. VIII/264 App. IX)

Parameter

1095 DF Mod ST H Flag Lab

$\underline{2095}$

DF Mod ST $\underline{H}$ Flag Lab Unit

Antimony, total recoverable

Benzene

Cobalt, total recoverable

Copper, total recoverable

Cyanide

Nickel, total recoverable

Phenols

Thallium, total recoverable

Trichloroethylene

$<1.7 \quad 1 \quad Y$

O GE

$\mu \mathrm{g} / \mathrm{L}$

Trichlorofluoromethane

$\begin{array}{lllll}<1.7 & 1 & Y & 0 & G E\end{array}$

$\mu \mathrm{g} / \mathrm{L}$

Vanam, total recoverable

Zinc, total recoverable

Water Quality Indicator Parameters

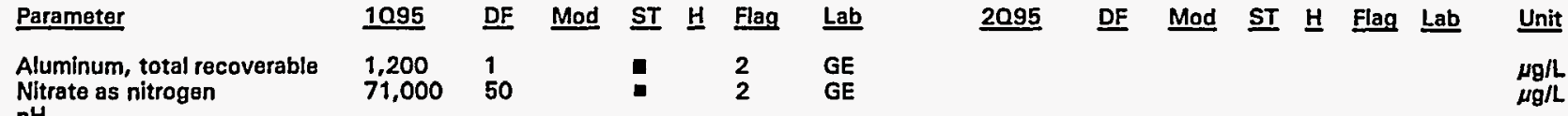

pH

Common Laboratory Contaminants

Paramater $\quad \underline{1095}$ DF Mod ST $\underline{H}$ Flag Lab $\underline{\text { Lab }} \underline{2095} \underline{\text { DF }} \underline{\text { Mod }} \underline{\text { ST }} \underline{H}$ Flag Lab $\underline{\text { Unit }}$

Bis(2-ethylhexyl) phthalate

Dichloromethane

Note: See Appendix B for flagging criteria. Synchronous water levels are measured over a 3-5 day period.

- = exceeded holding time.

= exceeded groundwater protection standard. 
WSRC-TR-95-0146-2

Unclassified

WELL FSL 7D (cont.)

Radiological Indicator Parameters

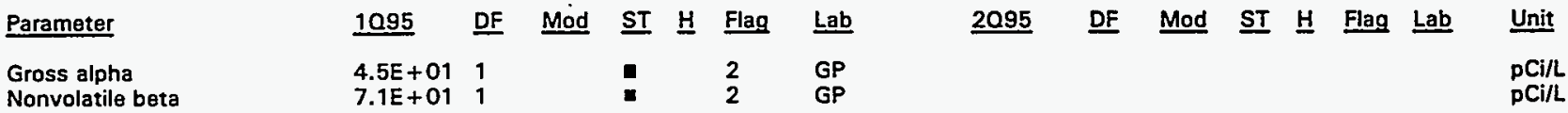

Measured Radionuclides

Parameter

$$
1095
$$

Actinium-228

Americium-241

Cesium-137

Cobalt- 60

Curium-242

Curium-243/244

Curium-245/246

lodine-129

Plutonium-238

Plutonium-239/240

Radium-226

Radium-228

Strontium-89

Strontium-90

Technetium-99

Thorium-228

Thorium-230

Thorium-232

Thorium-234

Tritium

Uritium

$1.9 E+03 \quad 1$

$\underline{\text { Mod ST }} \underline{\text { H Flag }} \underline{\underline{L a b}}$

\section{(1)}

DF

Uranium-235

Uranium-238

\section{Calculated Radionuclides}

Parameter

1095 DF Mod ST $\underline{H}$ Flag Lab

$\underline{2095}$

DF Mod ST $\underline{H}$ Flag Lab

Unit

Americium-243

Nickel-63

Plutonium-241

Plutonium-242

Note: See Appendix B for flagging criteria. Synchronous water levels are measured over a 3-5 day period.

- = exceeded holding time.

w = exceeded groundwater protection standard. 


\section{WELL FSL 8D}

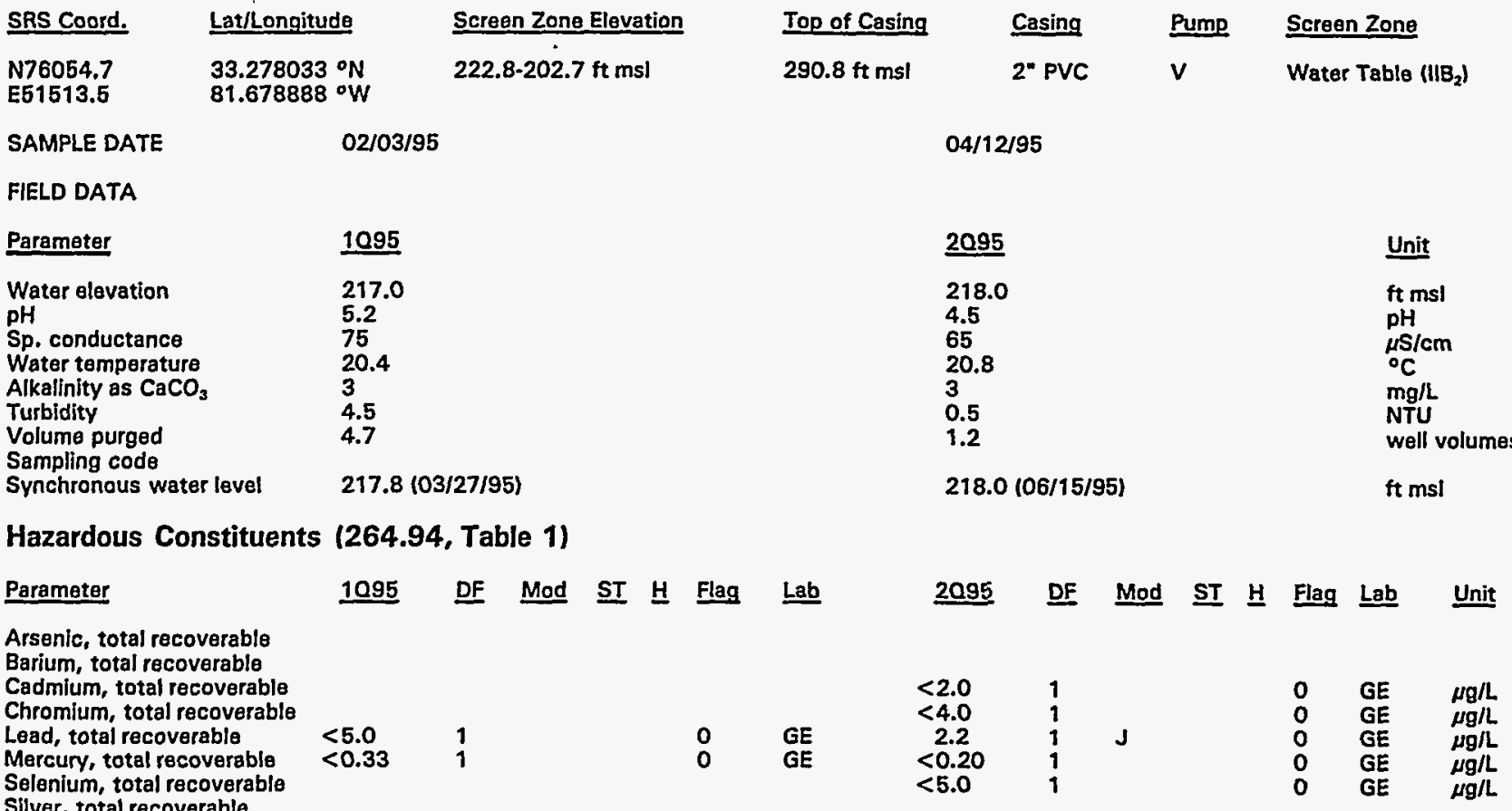

\section{Hazardous Constituents (261 App. VIII/264 App. IX)}

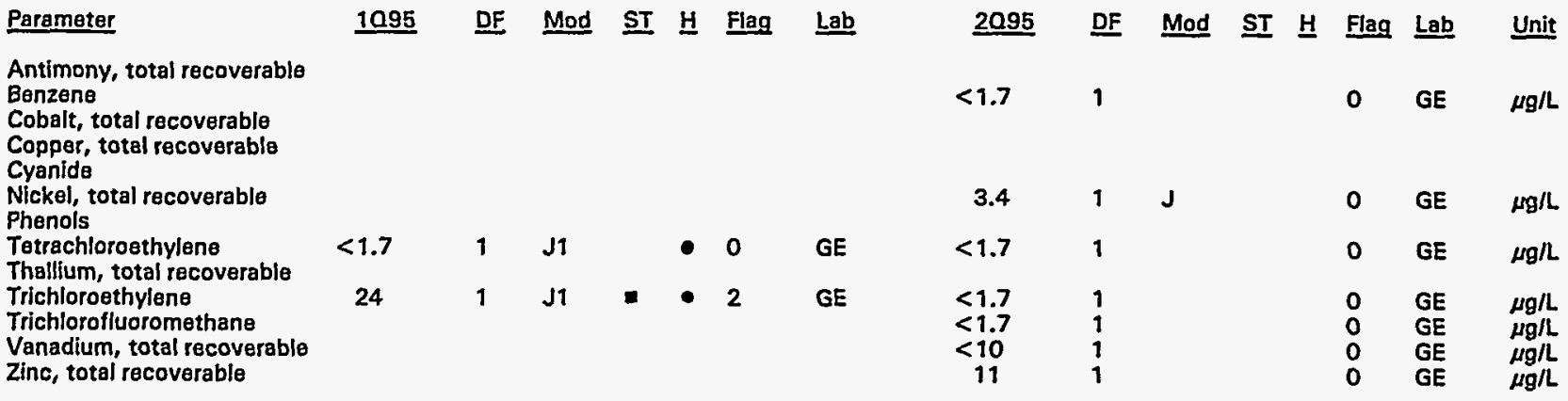

Water Quality Indicator Parameters

\begin{tabular}{|c|c|c|c|c|c|c|c|c|c|c|c|c|c|c|}
\hline Parameter & 1095 & DF & Mod & $\underline{\text { ST }} \underline{H}$ & Flag & Lab & 2095 & DF & Mod & & 브 & Flag & Lab & Unit \\
\hline $\begin{array}{l}\text { Aluminum, total recoverable } \\
\text { Nitrate as nitrogen } \\
\text { pH }\end{array}$ & $\begin{array}{r}<33 \\
5,800\end{array}$ & $\begin{array}{l}1 \\
4\end{array}$ & & & $\begin{array}{l}0 \\
1\end{array}$ & $\begin{array}{l}\mathrm{GE} \\
\mathrm{GE}\end{array}$ & $\begin{array}{l}5,300 \\
5.2\end{array}$ & $\begin{array}{l}4 \\
1\end{array}$ & J1 & n & & $\begin{array}{l}1 \\
0\end{array}$ & $\begin{array}{l}\mathrm{GE} \\
\mathrm{GE}\end{array}$ & $\begin{array}{l}\mu \mathrm{g} / \mathrm{L} \\
\mu \mathrm{g} / \mathrm{L} \\
\mathrm{pH}\end{array}$ \\
\hline
\end{tabular}

Common Laboratory Contaminants

Parameter $\quad \underline{1095}$ DF Mod ST $\underline{H}$ Flag Lab $\underline{2095}$ DF Mad ST $\underline{H} \underline{\text { Flag Lab }} \underline{\underline{\text { Lnit }}}$

Bis(2-ethylhexyl) phthalate

Dichloromethane

$\begin{array}{lllll}<1.7 & 1 & \mathrm{GE} & \mu \mathrm{g} / \mathrm{L}\end{array}$

Note: See Appendix B for flagging criteria. Synchronous water levels are measured over a 3-5 day period.

- = exceeded holding time.

= exceeded groundwater protection standard. 
WELL FSL 8D (cont.)

Radiological Indicator Parameters

\begin{tabular}{|c|c|c|c|c|c|c|c|c|c|c|c|c|c|c|c|}
\hline Parameter & 1095 & DF & Mod & $\underline{\mathrm{ST}}$ & $\underline{\mathrm{H}}$ & Flag & Lab & $\underline{2095}$ & DF & Mod & $\underline{\mathbf{S T}}$ & 브 & Flag & $\underline{\text { Lab }}$ & Unit \\
\hline $\begin{array}{l}\text { Gross alpha } \\
\text { Nonvolatile beta }\end{array}$ & $\begin{array}{l}2.2 E+00 \\
1.8 E+00\end{array}$ & $\begin{array}{l}1 \\
1\end{array}$ & & & & $\begin{array}{l}0 \\
0\end{array}$ & $\begin{array}{l}\text { GP } \\
\text { GP }\end{array}$ & $\begin{array}{l}2.3 E+00 \\
2.5 E+00\end{array}$ & $\begin{array}{l}1 \\
1\end{array}$ & & & & $\begin{array}{l}0 \\
0\end{array}$ & $\begin{array}{l}\text { GP } \\
\text { GP }\end{array}$ & $\begin{array}{l}\mathrm{pCi} / \mathrm{L} \\
\mathrm{pCi} / \mathrm{L}\end{array}$ \\
\hline \multicolumn{16}{|c|}{ Measured Radionuclides } \\
\hline Parameter & 1095 & $\underline{D F}$ & Mod & $\underline{\text { ST }}$ & $\underline{H}$ & Flag & Lab & $\underline{2095}$ & $\underline{D F}$ & Mod & $\underline{\mathbf{S T}}$ & $\underline{\mathbf{H}}$ & Flag & Lab & Unit \\
\hline $\begin{array}{l}\text { Actinium-228 } \\
\text { Americium-241 } \\
\text { Cesium-137 } \\
\text { Cobait-60 } \\
\text { Curium-242 } \\
\text { Curium-243/244 } \\
\text { Curium-245/246 } \\
\text { lodine-129 } \\
\text { Plutonium-238 } \\
\text { Plutonium-239/2 } \\
\text { Radium-226 } \\
\text { Radium-228 } \\
\text { Strontium-89 } \\
\text { Strontium-90 } \\
\text { Technetium-99 } \\
\text { Thorium-228 } \\
\text { Thorium-230 } \\
\text { Thorium-232 } \\
\text { Thorium-234 }\end{array}$ & & & & & & & & & & & & & & & \\
\hline $\begin{array}{l}\text { Tritium } \\
\text { Uranium-233/23 } \\
\text { Uranium-235 } \\
\text { Uranium-238 }\end{array}$ & $3.0 \mathrm{E}+01$ & 1 & & 口 & & 2 & GP & $3.9 E+01$ & 1 & & $\mathbf{0}$ & & 2 & GP & $\mathrm{pCi} / \mathrm{mL}$ \\
\hline \multicolumn{16}{|c|}{ Calculated Radionuclides } \\
\hline Parameter & $\underline{1095}$ & $\underline{D F}$ & Mod & $\underline{\text { ST }}$ & $\underline{\mathrm{H}}$ & Flag & $\underline{\text { Lab }}$ & 2095 & $\underline{\text { DF }}$ & Mod & $\underline{\text { ST }}$ & $\underline{H}$ & Flag & $\underline{\text { Lab }}$ & Unit \\
\hline $\begin{array}{l}\text { Americium-243 } \\
\text { Nickel-63 } \\
\text { Plutonium-241 } \\
\text { Plutonium-242 }\end{array}$ & & & & & & & & & & & & & & & \\
\hline
\end{tabular}

Note: See Appendix B for flagging criteria. Synchronous water levels are measured over a 3-5 day period. - = exceeded holding time.

- = exceeded groundwater protection standard. 


\section{WELL FSL 9D}

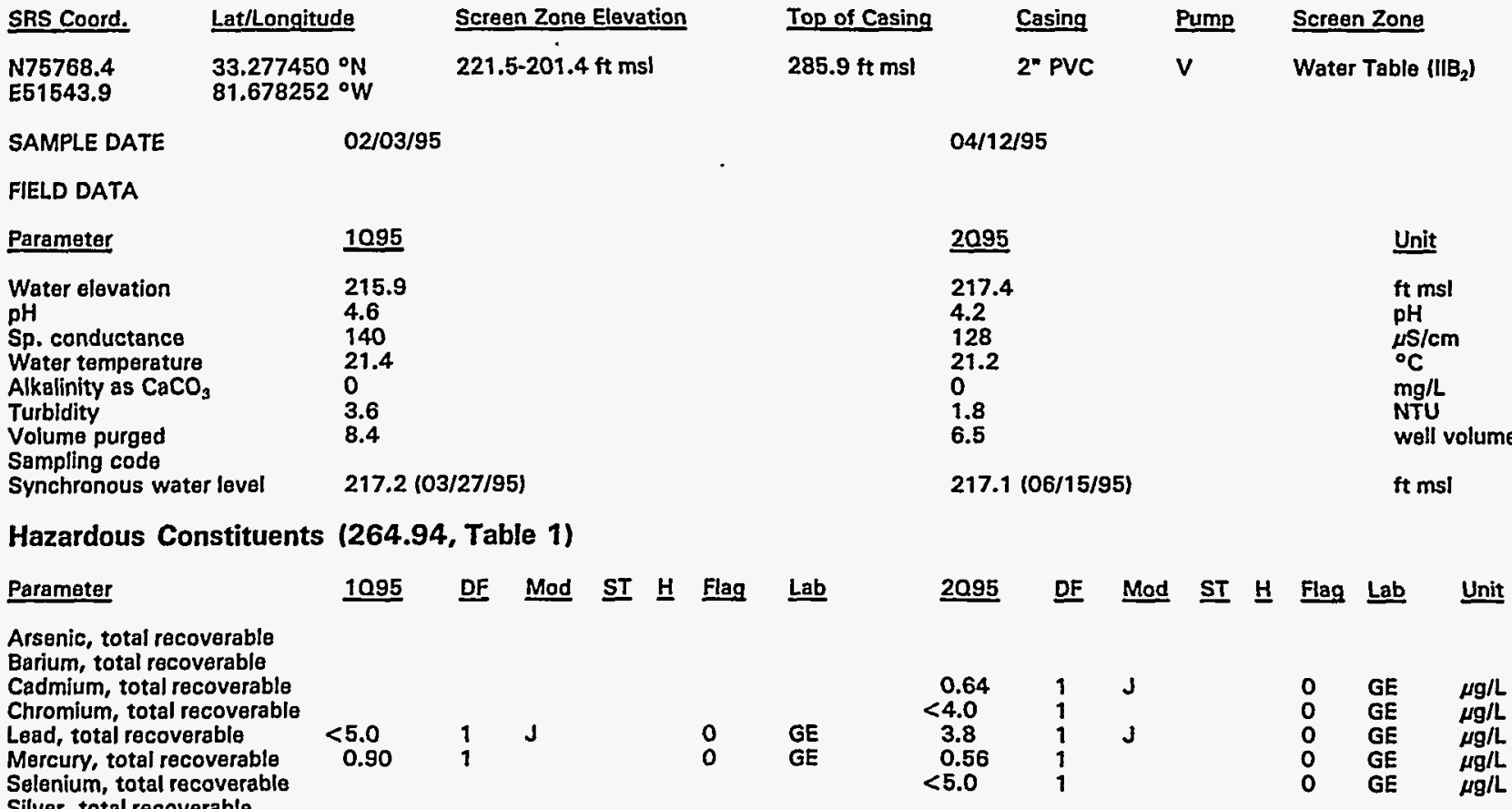

\section{Hazardous Constituents (261 App. VIII/264 App. IX)}

Parameter

Antimony, total recoverable

Benzene

Cobalt, total recoverable

Copper, total recoverable

Cyanide

Nickel, total recoverable

Phenois

Tetrachloroethylene

Thallium, total recoverable

Trichloroethylene

Trichlorofluoromethane

Vanadium, total recoverable

Zinc, total recoverable

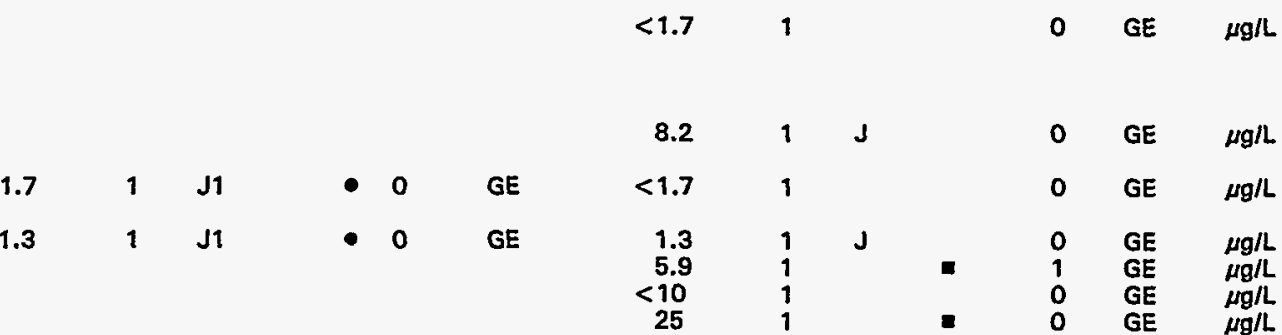

Water Quality Indicator Parameters

\begin{tabular}{|c|c|c|c|c|c|c|c|c|c|c|c|c|c|}
\hline Parameter & 1095 & DF & Mod & $\underline{\text { ST }} \underline{H}$ & Flag & $\underline{\text { Lab }}$ & $\underline{2095}$ & DF & Mod & $\underline{\text { ST }}$ & 브 & Flag & Lab \\
\hline $\begin{array}{l}\text { Aluminum, total recoverable } \\
\text { Nitrate as nitrogen } \\
\text { pH }\end{array}$ & $\begin{array}{l}2,600 \\
13,000\end{array}$ & $\begin{array}{l}1 \\
20\end{array}$ & & $=$ & $\begin{array}{l}2 \\
2\end{array}$ & $\begin{array}{l}\mathrm{GE} \\
\mathrm{GE}\end{array}$ & $\begin{array}{l}14,000 \\
4.7\end{array}$ & $\begin{array}{l}20 \\
1\end{array}$ & J1 & 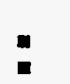 & & $\begin{array}{l}2 \\
0\end{array}$ & $\begin{array}{l}\text { GE } \\
\text { GE }\end{array}$ \\
\hline
\end{tabular}

\section{Common Laboratory Contaminants}

Parameter

1095 DF Mod ST H Flag Lab

2095 DF Mod ST H Flag Lab Unit

Bis(2-ethylhexyl) phthalate

Dichloromethane

$\begin{array}{lllll}<1.7 & 1 & 0 & \text { GE } & \mu \mathrm{g} / \mathrm{L}\end{array}$

Note: See Appendix B for flagging criteria. Synchronous water levels are measured over a 3-5 day period.

$\bullet=$ exceeded holding time.

- = exceeded groundwater protection standard. 
WELL FSL 9D (cont.)

Radiological Indicator Parameters

\begin{tabular}{|c|c|c|c|c|c|c|c|c|c|c|c|c|c|}
\hline Parameter & 1095 & $\underline{D F}$ & Mod & $\underline{\text { ST }} \underline{H}$ & Flag & Lab & 2095 & $\underline{\text { DF }}$ & Mod & $\underline{\text { ST }} \underline{H}$ & Flag & $\underline{\text { Lab }}$ & Unit \\
\hline $\begin{array}{l}\text { Gross alpha } \\
\text { Nonvolatile beta }\end{array}$ & $\begin{array}{l}8.1 E+01 \\
2.6 E+02\end{array}$ & $\begin{array}{l}1 \\
1\end{array}$ & $J$ & 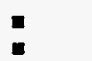 & $\begin{array}{l}2 \\
2\end{array}$ & $\begin{array}{l}\text { GP } \\
\text { GP }\end{array}$ & $\begin{array}{l}1.3 E+02 \\
3.2 E+02\end{array}$ & $\begin{array}{l}1 \\
1\end{array}$ & $\mathrm{~J}$ & ! & $\begin{array}{l}2 \\
2\end{array}$ & $\begin{array}{l}\text { GP } \\
\text { GP }\end{array}$ & \\
\hline
\end{tabular}

Measured Radionuclides

Parameter

Unit

Actinium-228

Americium-241

Cesium-137

Cobalt -60

Curium-242

Curium-243/244

Curium-245/246

lodine-129

Plutonium-238

Plutonium-239/240

Radium-226

Radium-228

Strontium-89

Strontium-90

Technetium-99

Thorium-228

Thorium-230

Thorium

Thorium-234

Tritium

4.7E+02 1

- 2 GP

$4.0 E+021$

Uranium-235

Uranium-238

Calculated Radionuclides

Parameter

$\underline{1095}$

DF Mod ST $\underline{H}$ Flag Lab

$\underline{2095}$

DF Mod ST H Flag Lab

Unit

Americium-243

Nickel-63

Plutonium-241

Plutonium-242

Note: See Appendix B for flagging criteria. Synchronous water levels are measured over a 3-5 day period.

- = exceeded holding time.

- =xceeded groundwater protection standard. 


\section{WELL HSB 85A}

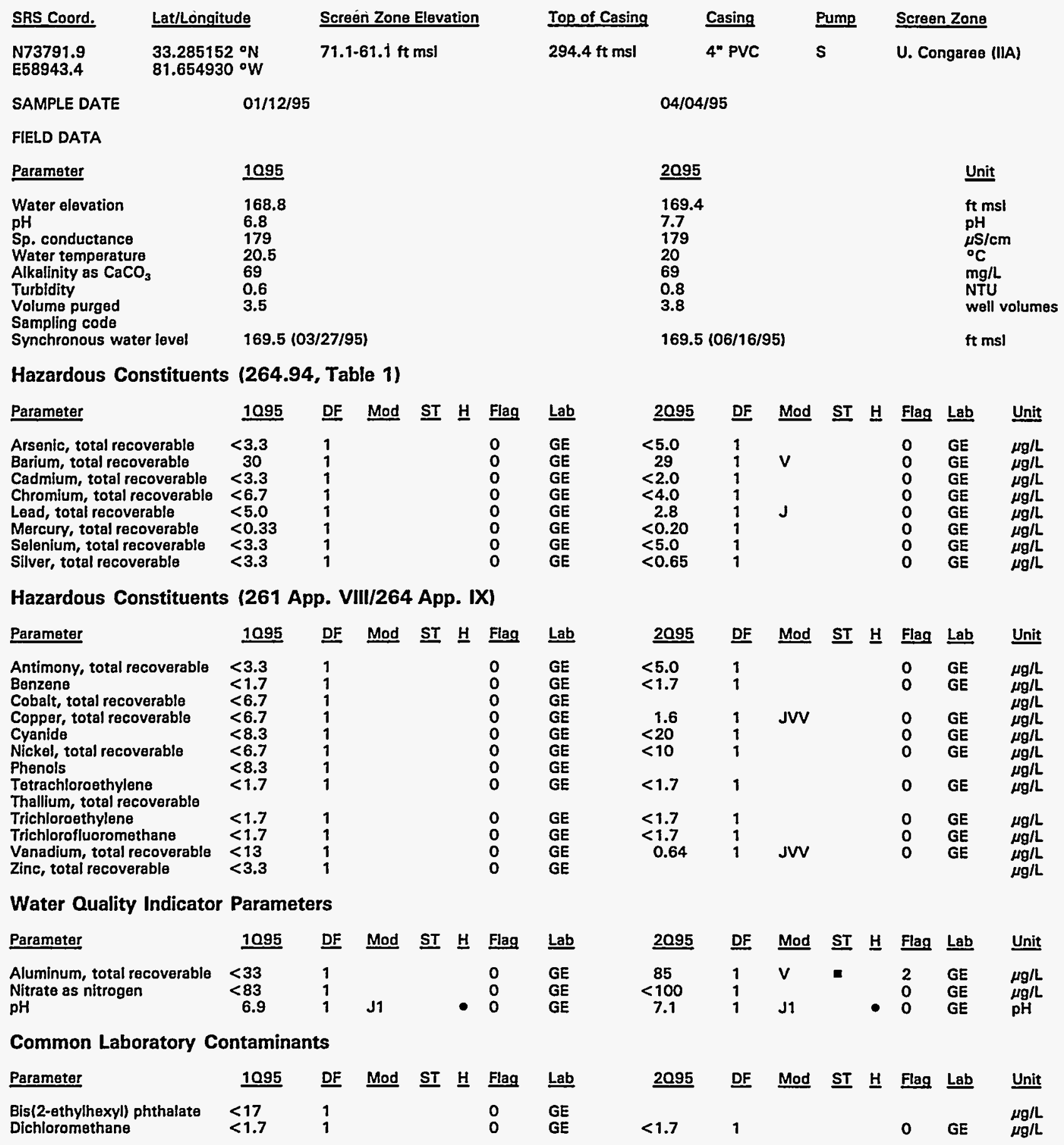

Note: See Appendix B for flagging criteria. Synchronous water levels are measured over a 3-5 day period.

- = exceeded holding time.

m = exceeded groundwater protection standard. 
WELL HSB 85A (cont.)

Radiological Indicator Parameters

\section{Parameter}

Gross alpha Nonvolatile beta

1095

$\begin{array}{ll}\text { 5.9E-01 } & 1 \\ 9.3 E-01 & 1\end{array}$

Mod

UI

109

Parameter

Actinium-228

Americium-241

Cesium-137

Cobalt -60

Curium-242

Curium-243/244

Curium-245/246

lodine-129

Plutonium-238

Plutonium-239/240

Radium-226

Radium-228

Strontium-89

Technetium-99

Thorium-228

Tharium-230

Thorium-232

Thorium-234

Tritium

Uranium-233/234

Uranium-235

Uranium-238

$\begin{array}{lll}\frac{1095}{5.9 E} & \text { DF } & \text { Mod } \\ 2.0 E+00 & 1 & U I \\ 3.4 E-01 & 1 & U I \\ 1.4 E+00 & 1 & U I \\ -4.9 E-02 & 1 & U I \\ -1.2 E-01 & 1 & U I \\ 0.0 E+00 & 1 & U I \\ 5.3 E-01 & 1 & U I \\ 2.3 E-01 & 1 & U I \\ -2.6 E-02 & 1 & U I \\ 8.0 E-02 & 1 & U I \\ -3.4 E-01 & 1 & U I \\ -3.8 E-01 & 1 & U I \\ 2.6 E+00 & 1 & \\ 5.3 E+00 & 1 & U I \\ 2.2 E-02 & 1 & U I \\ 1.5 E-01 & 1 & U I \\ 0.0 E+00 & 1 & U I \\ 9.2 E+00 & 1 & U I \\ -2.3 E-01 & 1 & U I \\ 9.7 E-02 & 1 & U I \\ -8.2 E-03 & 1 & U I \\ 4.9 E-02 & 1 & U I\end{array}$

Strontium-90

ST

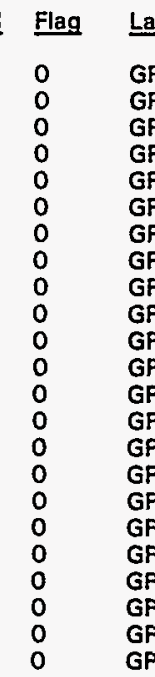

Calculated Radionuclides

Parameter

Americium-243

Nickel-63

Plutonium-241

Plutonium-242
1095 DF Mod ST $\underline{H}$ Flag Lab

\section{0}

$-3.6 E-04$

$-1.4 E+00$
$-1.0 \mathrm{E}-01$

$-5.2 E-07$

$$
-4.1 E-011 \text { UI }
$$

$\begin{array}{lll}8.3 E-01 & 1 & \text { UI } \\ 2.2 E+00 & 1 & J\end{array}$

ST $\underline{H}$ Flag Lab Unit

$\begin{array}{lll}0 & \mathrm{GP} & \mathrm{pCi} / \mathrm{L} \\ 0 & \mathrm{GP} & \mathrm{PCi} / \mathrm{L}\end{array}$

2095 DF Mad ST $\underline{H}$ Flag Lab Unit

$\mathrm{pCi} / \mathrm{L}$

pCi/L

$\mathrm{pCi} / \mathrm{L}$

pCi/L

$\mathrm{PCI} / \mathrm{L}$

$\mathrm{pCi} / \mathrm{L}$

$\mathrm{pCi} / \mathrm{L}$

$\mathrm{PCI} / \mathrm{L}$

pCi/L

pCinl

$\mathrm{pCi} / \mathrm{L}$

$\mathrm{pCi} / \mathrm{L}$

$\mathrm{pCi} / \mathrm{L}$

pCill

pCi/L

PCill

PCi/L

$\mathrm{pCi} / \mathrm{L}$

$\mathrm{pCi} / \mathrm{mL}$

PCi/L

$\mathrm{pCi} / \mathrm{L}$

$\mathrm{PCi} / \mathrm{L}$

$\underline{2095} \underline{\text { DF Mod }} \underline{\text { ST }} \underline{\mathrm{H}} \underline{\mathrm{Flag}} \underline{\text { Lab }} \underline{\text { Unit }}$

$\mathrm{pCi} / \mathrm{L}$

$\mathrm{PCi} / \mathrm{L}$

$\mathrm{pCi} / L$

pCi/L

Note: See Appendix B for flagging criteria. Synchronous water levels are measured over a 3-5 day period. $\checkmark=$ exceeded holding time.

= exceeded groundwater protection standard. 
WSRC-TR-95-0146-2

Unclassified

\section{Appendix E}

\section{Data Quality/Usability Assessment}


THIS PAGE LEFT BLANK INTENTIONALLY. 


\section{Data Quality/Usability Assessment}

Quality assurance/quality control (QAQC) procedures relating to accuracy and precision of analyses performed on groundwater samples are followed in the field and laboratory and are reviewed prior to publication of results. The review by the Environmental Protection Department/ Environmental Monitoring Section (EPD/EMS) of the volume of analytical data acquired each quarter and presented in various reports is an ongoing process; EPD/EMS' review of the QAVQC data cannot be completed in time to meet the deadlines for the groundwater monitoring reports required by the Resource Conservation and Recovery Act and associated regulations. Other site and regulatory personnel can obtain further information on the data quality and usability in a variety of ways, including those described below.

\section{Results below Detection}

For radiological analyses, the analytical result field contains the result recorded on the analytical instrument and reported by the laboratory, even if it is negative. For non-radiological analyses, if the analyte is not detected, the sample-specific estimated quantitative limit (EQL) is entered into the result field (with a less than [ $<]$ sign). The EQL is defined as the lowest concentration that can be achieved reliably within specified limits of precision and accuracy during routine laboratory operating conditions. The sample-specific EQL is modified for sample concentration or dilution or unusual aliquot size that affects analytical sensitivity.

\section{Data Qualification}

The contract laboratories continually assess their own accuracy and precision according to U.S. Environmental Protection Agency (EPA) guidelines. They submit sample- or batch-specific QAV QC information either at the same time as analytical results or in quarterly summaries. Properly defined and used modifiers (also referred to as qualifiers) can be a key component in assessing data usability. Modifiers designed by EPD/EMS and used by the primary laboratories are presented in Appendix D.

\section{Assessment of Accuracy of the Data}

Accuracy, or the nearness of the reported result to the true concentration of a constituent in a sample, can be assessed in several ways.

A laboratory's general accuracy can be judged by analysis of results obtained from known samples. The non-radionuclide contract laboratories analyze commercial reference samples every quarter at EPD/EMS' request. The results of these analyses are presented in the EPD/EMS groundwater monitoring quarterly reports. The primary laboratories also seek or maintain state certification by participating periodically in performance studies; reference samples and analysis of results are provided by EPA. Results of these studies also are published in the EPD/EMS quarterly reports.

Analysis of blanks provides a tool for assessing the accuracy of both sampling and laboratory analysis. Results for all field blanks for the quarter can be found in the EPD/EMS quarterly reports. Any field or laboratory blanks that exceed established minimums are identified in the same reports, in tables associating them with groundwater samples analyzed in the same batches. In these regulatory reports, the modifier $V$ is applied to every result that is run in the same batch as a laboratory blank with positive results for that analyte. A second $V$ qualifier is added if the analyte concentration is less than five times the concentration in the blank (or, for common laboratory contaminants, less than ten times.) 
Method Used to Analyze

EPA7060

EPA7421

EPA7470

EPA7740

EPA7841

EPA8010

EPA8080

EPA8150

EPA8240

EPA8270

EPA8280

EPA9012

EPA9020

EPA9020A

EPA9030

EPA9060

Lead

Mercury

Selenium

Thallium

GCMS volatiles

Cyanide

Sulfide
Arsenic

Chlorinated volatile organics

Organochlorine pesticides and PCBs

Chlorinated herbicides

GCMS semivolatiles

Dioxins and furans

Total organic halogens

Total organic halogens

Dissolved organic carbon, total inorganic carbon, total organic carbon
Source

EPA, 1986
EPA, 1986
EPA, 1986
EPA, 1986
EPA, 1986
EPA, 1986
EPA, 1986
EPA, 1986
EPA, 1986
EPA, 1986
EPA, 1986
EPA, 1986
EPA, 1986
EPA, 1986
EPA, 1986
EPA, 1986

An example of available method-specific QAVC information is that for the analysis of metals by EPA Method 6010/200.7 (EPA, 1986/EPA EMSL, 1983). The primary laboratories, General Engineering Laboratories (GE) and Roy F. Weston, Inc. (Weston), use this inductively coupled plasma (ICP) atomic emission spectrometric method.

The following precision and accuracy data are based on the experience of seven laboratories that applied the ICP technique to acid-distilled water matrices that had been spiked with various metal concentrates. (Note: Not all seven laboratories analyzed all 14 elements.) The references give results for samples having three concentration ranges; the results here are for samples having the lowest values, similar to actual groundwater results for the Savannah River Site.

ICP Precision and Accuracy Data

\begin{tabular}{|c|c|c|c|}
\hline Element & True value $(\mu \mathrm{g} / \mathrm{L})$ & $\begin{array}{l}\text { Mean reported } \\
\text { value }(\mu \mathrm{g} / \mathrm{L})\end{array}$ & $\begin{array}{l}\text { Mean percent } \\
\underline{\mathrm{RSD}}^{\mathrm{a}}\end{array}$ \\
\hline Aluminum & 60 & 62 & 33 \\
\hline Arsenic & 22 & 19 & 23 \\
\hline Beryllium & 20 & 20 & 9.8 \\
\hline Cadmium & 2.5 & 2.9 & 16 \\
\hline Chromium & 10 & 10 & 18 \\
\hline Cobalt & 20 & 20 & 4.1 \\
\hline Copper & 11 & 11 & 40 \\
\hline Iron & 20 & 19 & 15 \\
\hline Lead & 24 & 30 & 32 \\
\hline Manganese & 15 & 15 & 6.7 \\
\hline Nickel & 30 & 28 & 11 \\
\hline Selenium & 6 & 8.5 & 42 \\
\hline Vanadium & 70 & 69 & 2.9 \\
\hline Zinc & 16 & 19 & 45 \\
\hline
\end{tabular}

a Relative standard deviation. In EPA (1986), the column heading is Mean Standard Deviation (\%). 
As another example, EPA Method 601/8010 (EPA, 1991/EPA, 1986) is used by both GE and Weston for analyses of halogenated volatile organics. In the presentation of the method in both references, the following table gives method-specific accuracy and precision as functions of concentration. Contract laboratories are expected to achieve or at least approach these limits.

\section{Accuracy and Precision as Functions of Concentration for EPA Method 601/8010}

\section{Parameter}

Bromodichloromethane

Bromoform

Bromomethane

Carbon tetrachloride

Chlorobenzene

Chloroethane

2-Chloroethyl vinyl ether ${ }^{f}$

Chloroform

Chloromethane

Dibromochloromethane

1,2-Dichlorobenzene

1,3-Dichlorobenzene

1,4-Dichlorobenzene

1,1-Dichloroethane

1,2-Dichloroethane

1,1-Dichloroethene

trans-1,2-Dichloroethene

Dichloromethane

1,2-Dichloropropane ${ }^{f}$ cis-1,3-Dichloropropene ${ }^{f}$ trans-1,3-Dichloropropene $f$

1,1,2,2-Tetrachloroethane

Tetrachloroethylene

1,1,1-Trichloroethane

1,1,2-Trichloroethane

Trichloroethylene

Trichlorofluoromethane

Vinyl chloride
Accuracy as

recovery, $X^{a \mathrm{a}}(\mu \mathrm{g} / \mathrm{L})$

$1.12 C-1.02^{d}$

$0.96 C-2.05$

$0.76 C-1.27$

$0.98 C-1.04$

$1.00 C-1.23$

$0.99 C-1.53$

$1.00 \mathrm{C}$

$0.93 C-0.39$

$0.77 C+0.18$

$0.94 C+2.72$

$0.93 C+1.70$

$0.95 C+0.43$

0.93C-0.09

$0.95 C-1.08$

$1.04 C-1.06$

$0.98 C-0.87$

$0.97 C-0.16$

$0.91 C-0.93$

$1.00 \mathrm{C}$

$1.00 \mathrm{C}$

$1.00 \mathrm{C}$

$0.95 C+0.19$

$0.94 C+0.06$

$0.90 C-0.16$

$0.86 C+0.30$

$0.87 C+0.48$

$0.89 C-0.07$

$0.97 C-0.36$

\section{Single analyst}

${\text { precision }(\mu \mathrm{g} / \mathrm{L})^{b}}^{b}$

$0.11 \bar{X}+0.04^{e}$

$0.12 \bar{X}+0.58$

$0.28 \bar{X}+0.27$

$0.15 \bar{X}+0.38$

$0.15 \bar{X}-0.02$

$0.14 \bar{X}-0.13$

$0.20 \bar{X}$

$0.13 \bar{X}+0.15$

$0.28 \bar{X}-0.31$

$0.11 \bar{X}+1.10$

$0.20 \bar{X}+0.97$

$0.14 \bar{X}+2.33$

$0.15 \bar{X}+0.29$

$0.09 \bar{X}+0.17$

$0.11 \bar{X}+0.70$

$0.21 \bar{X}-0.23$

$0.11 \bar{X}+1.46$

$0.11 \bar{X}+0.33$

$0.13 \bar{X}$

$0.18 \bar{X}$

$0.18 \bar{X}$

$0.14 \bar{X}+2.41$

$0.14 \bar{X}+0.38$

$0.15 \bar{X}+0.04$

$0.13 \bar{X}-0.14$

$0.13 \bar{X}-0.03$

$0.15 \bar{X}+0.67$

$0.13 \bar{X}+0.65$
Overall ${\text { precision }(\mu \mathrm{g} / \mathrm{L})^{c}}^{c}$

$0.20 \bar{X}+1.00$

$0.21 \bar{X}+2.41$

$0.36 \bar{X}+0.94$

$0.20 \bar{X}+0.39$

$0.18 \bar{X}+1.21$

$0.17 \bar{X}+0.63$

$0.35 \bar{X}$

$0.19 \bar{X}-0.02$

$0.52 \bar{X}+1.31$

$0.24 \bar{X}+1.68$

$0.13 \bar{X}+6.13$

$0.26 \bar{X}+2.34$

$0.20 \bar{X}+0.41$

$0.14 \bar{X}+0.94$

$0.15 \bar{X}+0.94$

$0.29 \bar{X}-0.40$

$0.17 \bar{X}+1.46$

$0.21 \bar{X}+1.43$

$0.23 \bar{X}$

$0.32 \bar{X}$

$0.32 \bar{X}$

$0.23 \bar{X}+2.79$

$0.18 \bar{X}+2.21$

$0.20 \bar{X}+0.37$

$0.19 \bar{X}+0.67$

$0.23 \bar{X}+0.30$

$0.26 \bar{X}+0.91$

$0.27 \bar{X}+0.40$

a $X^{\prime}=$ expected recovery for one or more measurements of a sample containing a concentration of $C$, in $\mu \mathrm{g} / \mathrm{L}$.

b Expected single analyst standard deviation of measurements.

c Expected interlaboratory standard deviation of measurements.

d $C=$ true value for the concentration, in $\mu \mathrm{g} / \mathrm{L}$.

e $\bar{X}=$ average recovery found for measurements of samples containing a concentration of $C$, in $\mu \mathrm{g} / \mathrm{L}$.

$f$ Estimates based on performance of a single laboratory. 


\section{References Cited}

EPA (U.S. Environmental Protection Agency), 1986. Test Methods for Evaluating Solid Waste (SW-846), Volumes IA-IC. Washington, DC.

EPA (U.S. Environmental Protection Agency), 1991. Guidelines Establishing Test Procedures for the Analysis of Pollutants, Code of Federal Regulations, Title 40, Part 136, Appendix A. Washington, DC.

EPA EMSL (U.S. Environmental Protection Agency, Environmental Monitoring and Systems Laboratory), 1980. Prescribed Procedures for Measurement of Radioactivity in Drinking Water, EPA-600/4-80-032. Cincinnati, OH.

EPA EMSL (U.S. Environmental Protection Agency, Environmental Monitoring and Systems Laboratory), 1983. Methods for Chemical Analysis of Water and Wastes. Cincinnati, OH.

EPA EMSL (U.S. Environmental Protection Agency, Environmental Monitoring and Systems Laboratory), 1991. Test Method, The Determination of Inorganic Anions in Water by lon Chromatography-Method 300.0. Cincinnati, $\mathrm{OH}$. 
WSRC-TR-95-0146-2

Unclassified

\section{Appendix F}

Time Series Plots 
THIS PAGE LEFT BLANK INTENTIONALLY. 


\section{Cadmium Concentrations} Well Cluster FSB 79

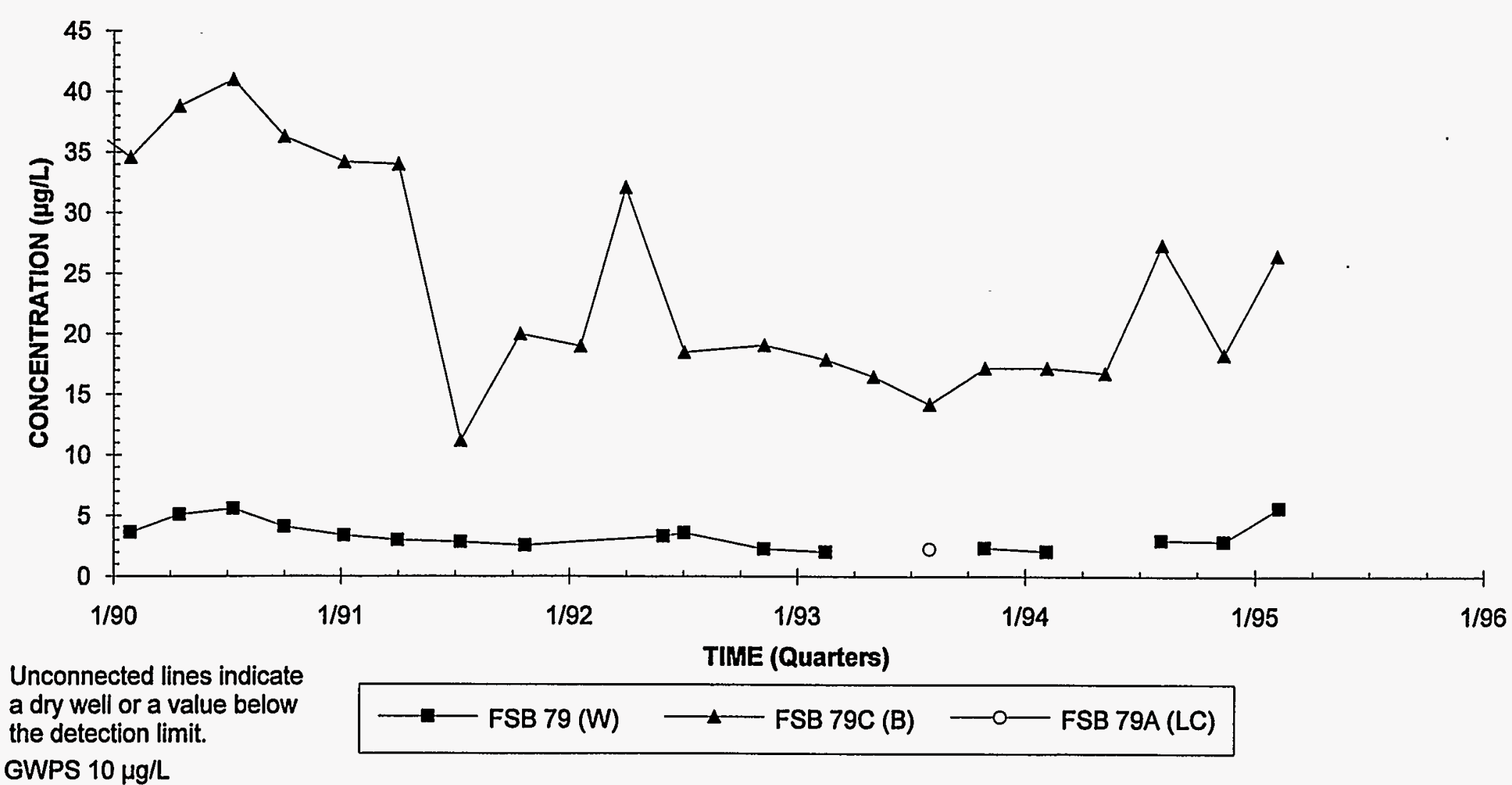

GWPS $10 \mu \mathrm{g} / \mathrm{L}$ 


\section{Cadmium Concentrations}

Well FSB 91D

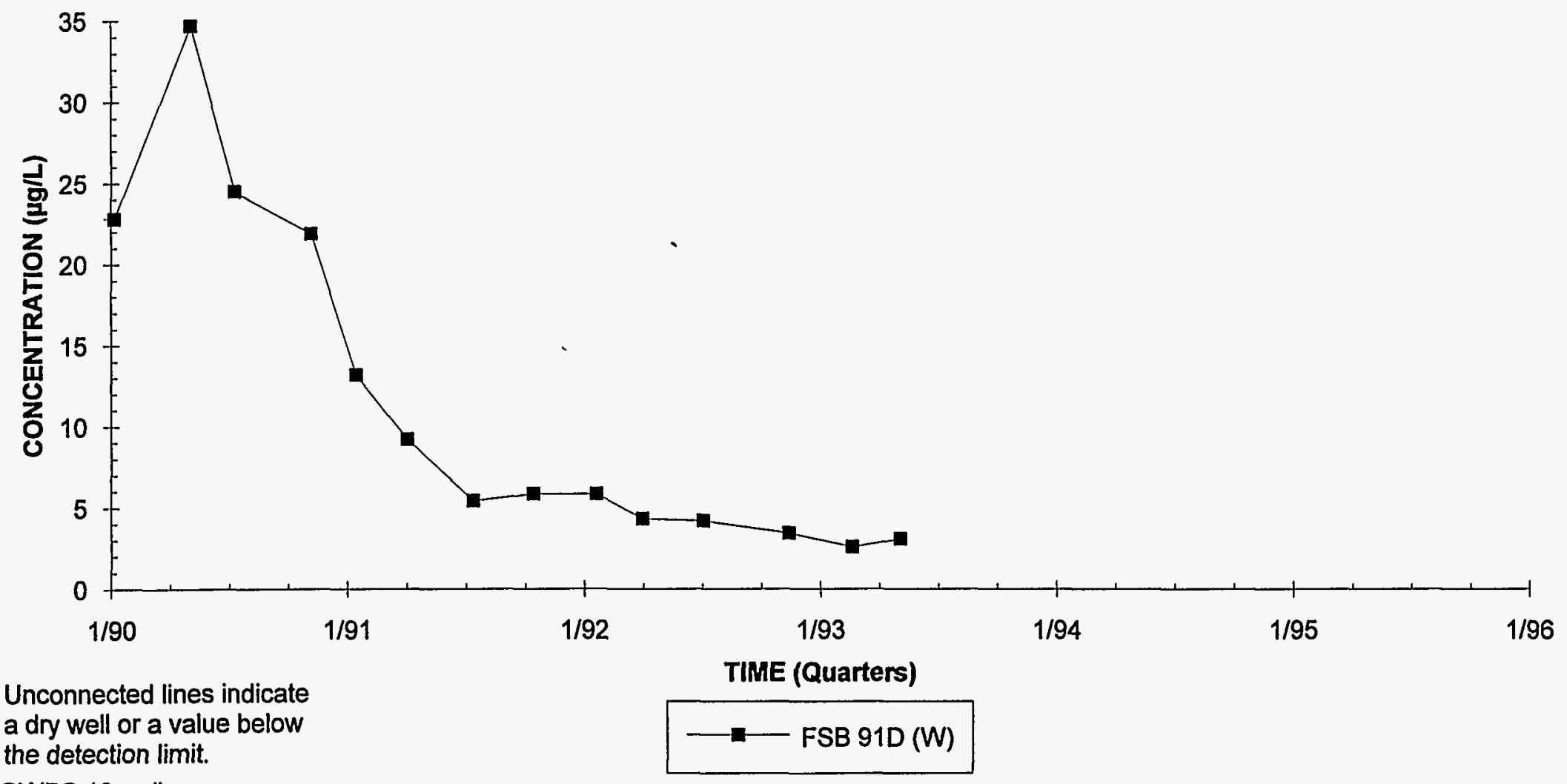

GWPS $10 \mu \mathrm{g} / \mathrm{L}$ 


\section{Cadmium Concentrations}

Well FSB 93D

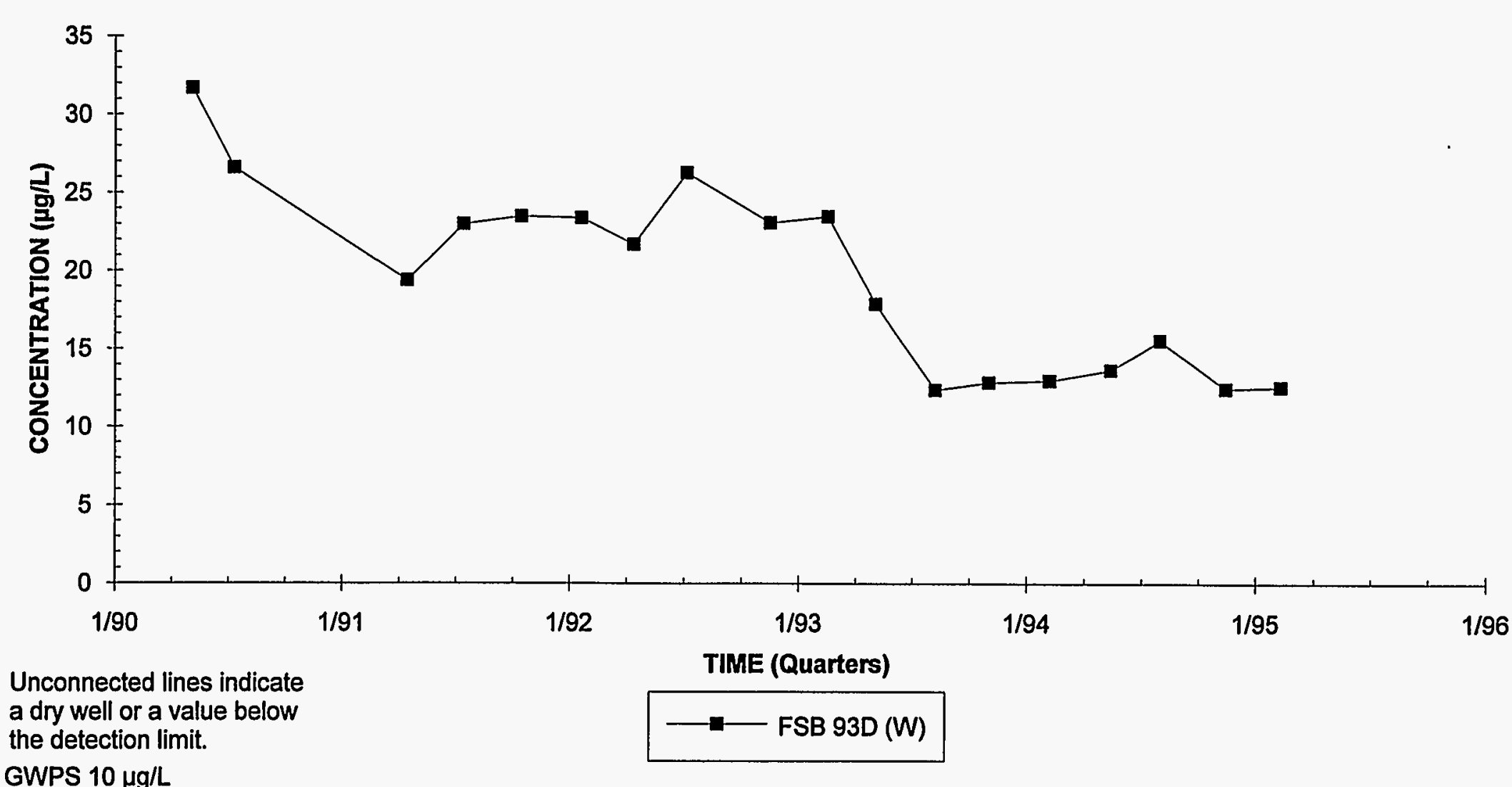

Note: $W=$ Water Table (IIB2); B=Barnwell (IIB1); M=McBean (IIB1); UC=Upper Congaree (IIA); MC=Middle Congaree (IIA); LC=Lower Congaree (IIA) 


\section{Cadmium Concentrations Well FSB 95CR}

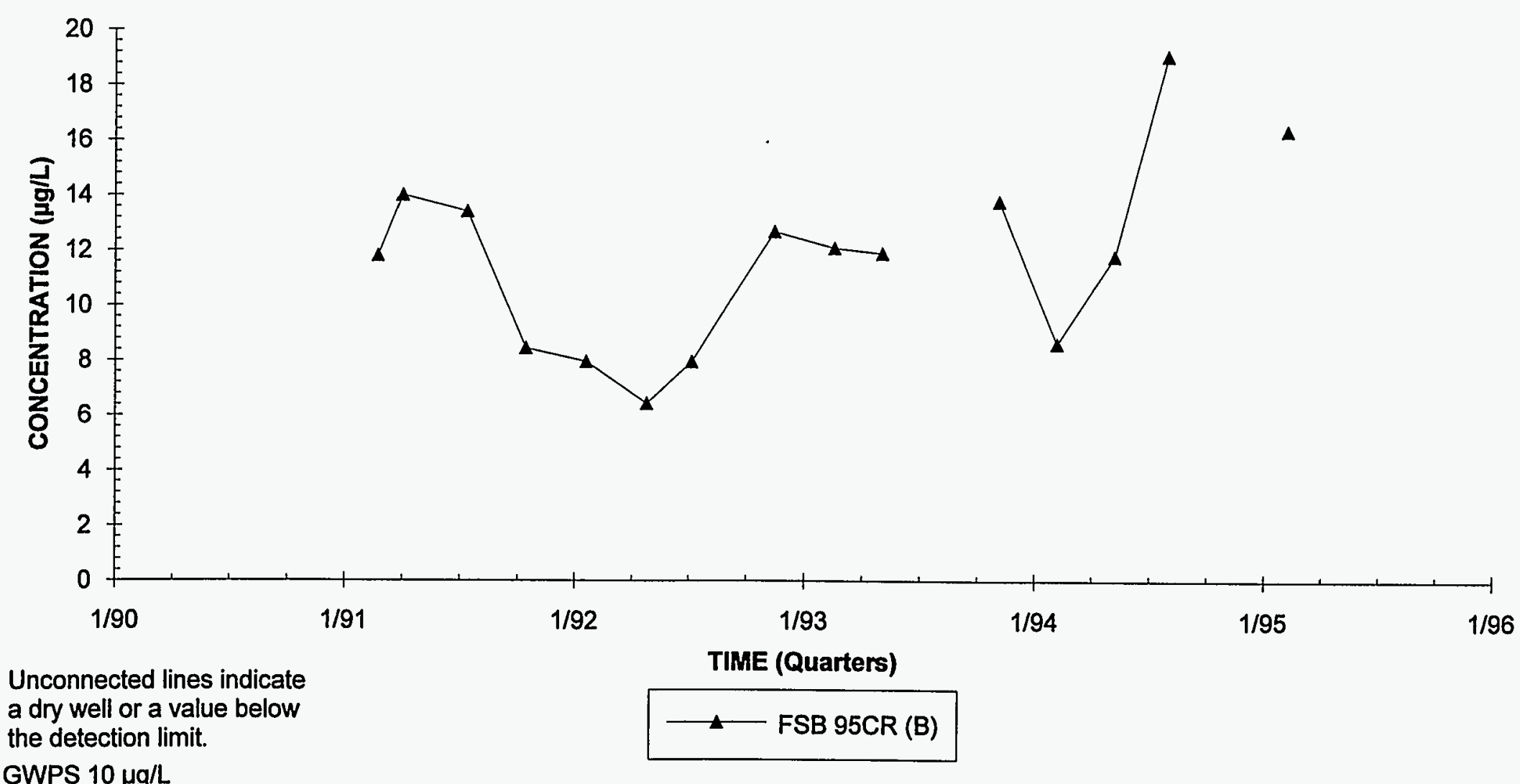

Note: W=Water Table (IIB2); B=Barnwell (IIB1); M=McBean (IIB1); UC=Upper Congaree (IIA); MC=Middle Congaree (IIA); LC=Lower Congaree (IIA) 

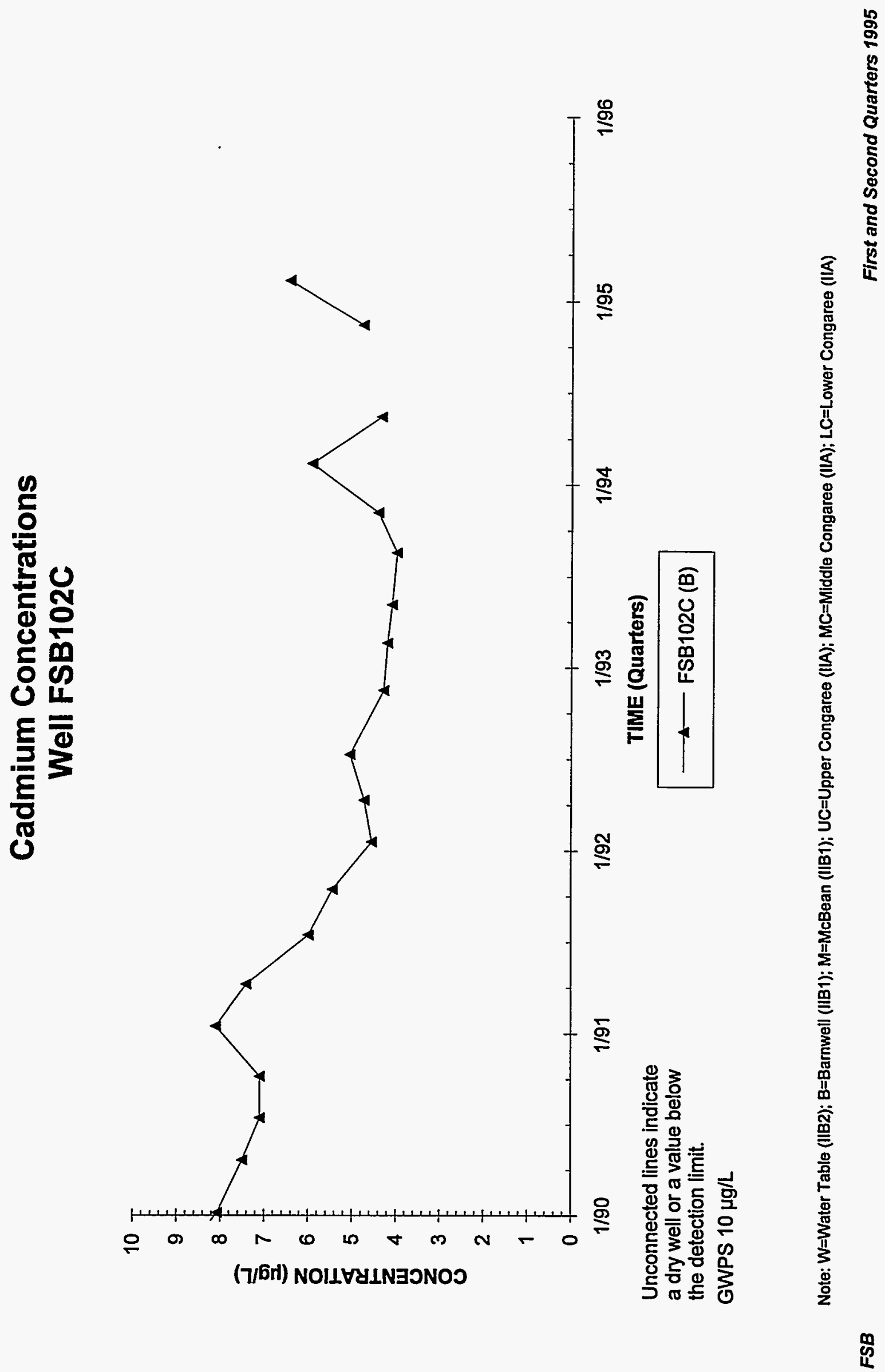


\section{Cadmium Concentrations \\ Well Cluster FSB104}

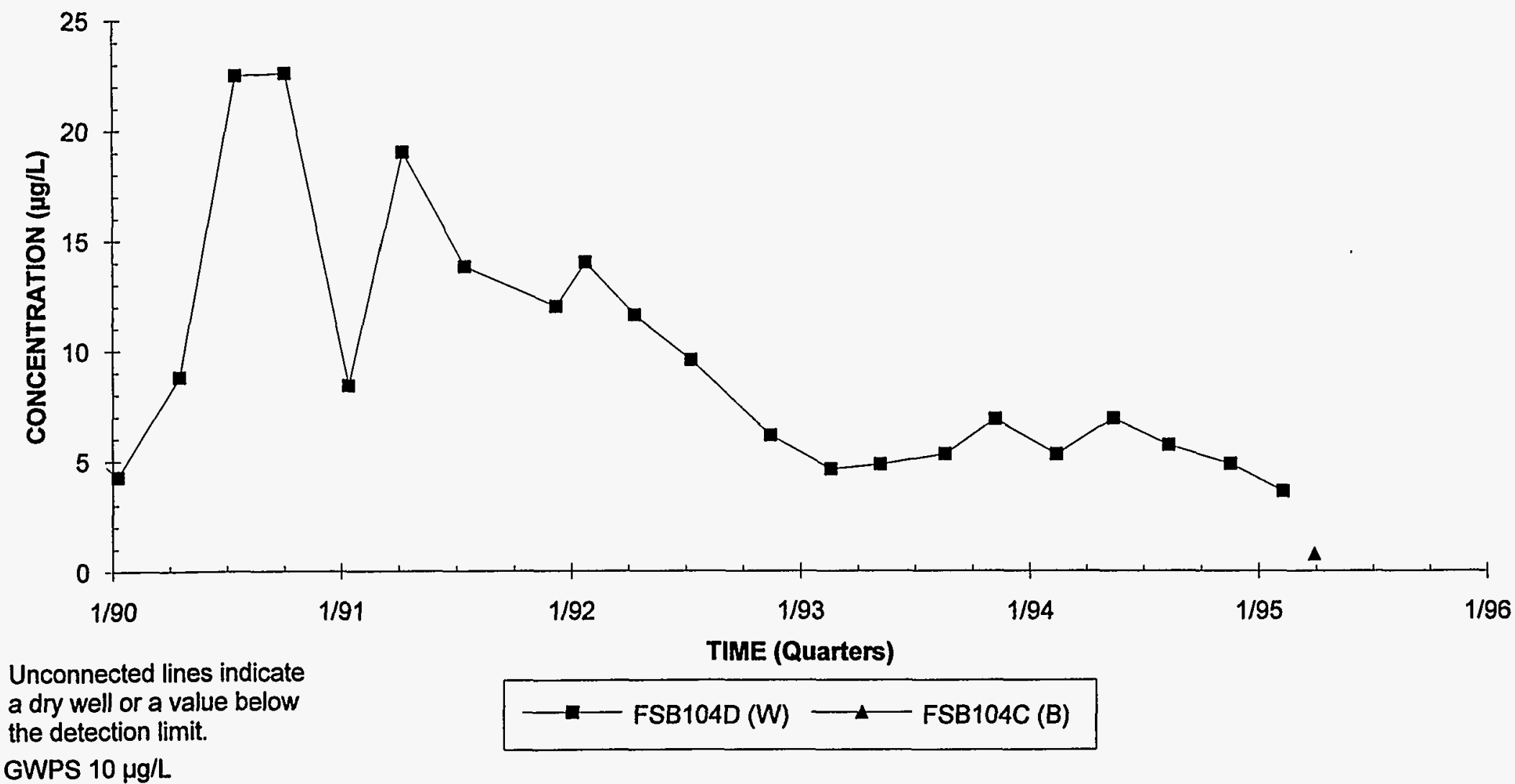

Note: W=Water Table (IIB2); B=Barnwell (IIB1); $M=$ MCBean (IIB1); UC=Upper Congaree (IIA); MC=Middle Congaree (IIA); LC=Lower Congaree (IIA) 


\section{Cadmium Concentrations}

\section{Well Cluster FSB106}

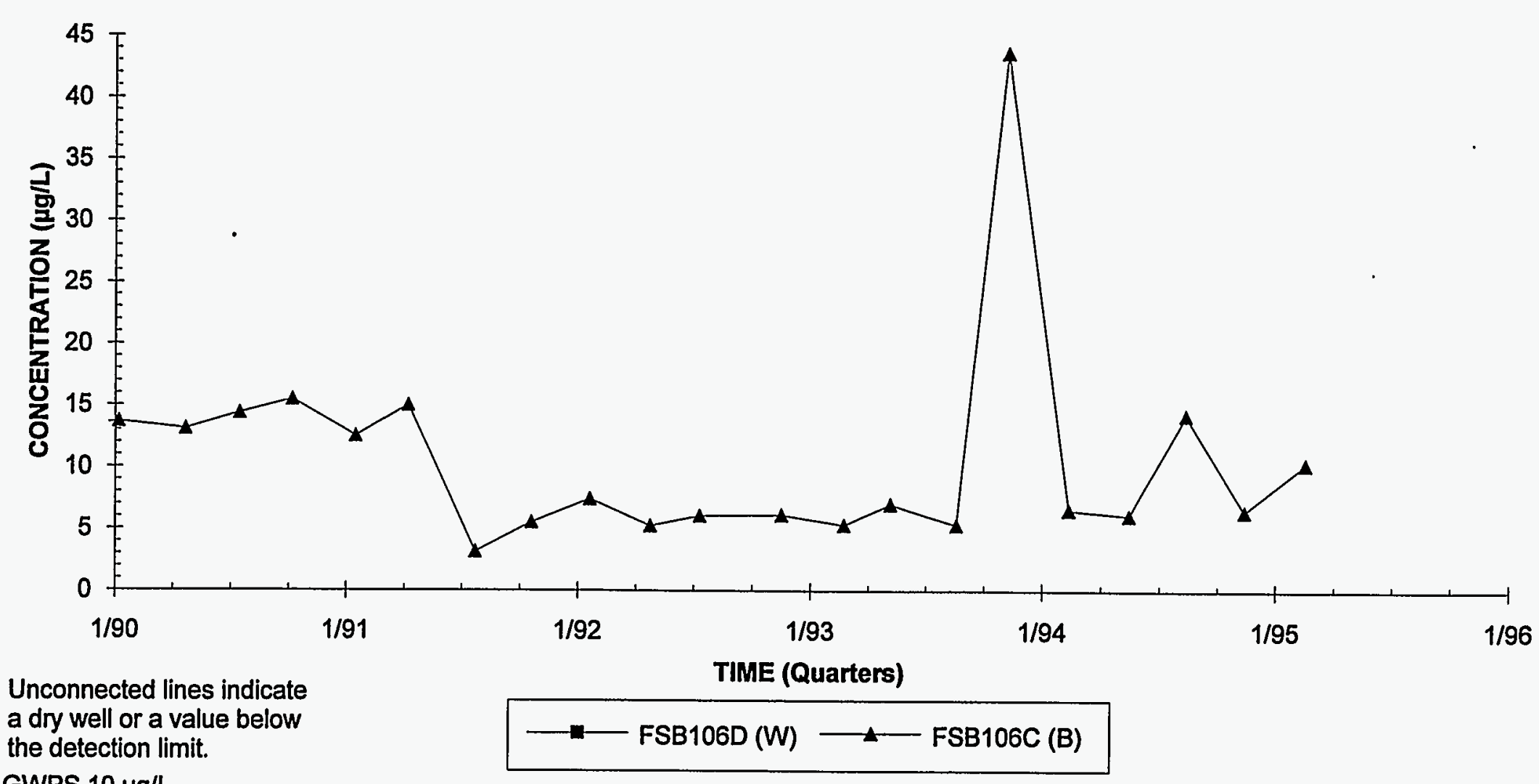

GWPS $10 \mu \mathrm{g} / \mathrm{L}$ 


\section{Cadmium Concentrations Well FSB112C}

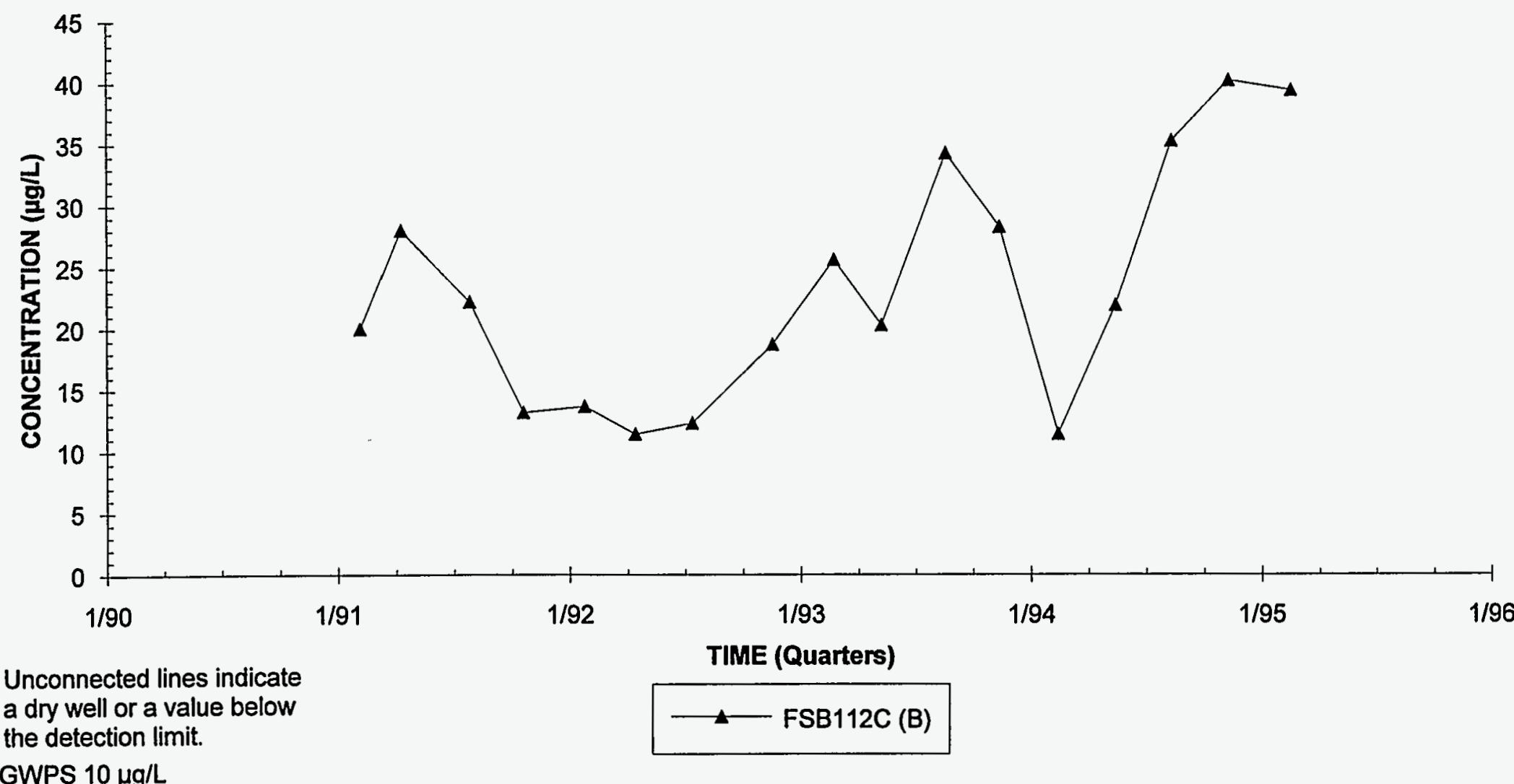

Note: W=Water Table (IIB2); B=Barnwell (IIB1); M=McBean (IIB1); UC=Upper Congaree (IIA); MC=Middle Congaree (IIA); LC=Lower Congaree (IIA) 


\section{Cadmium Concentrations Well FSB119D}

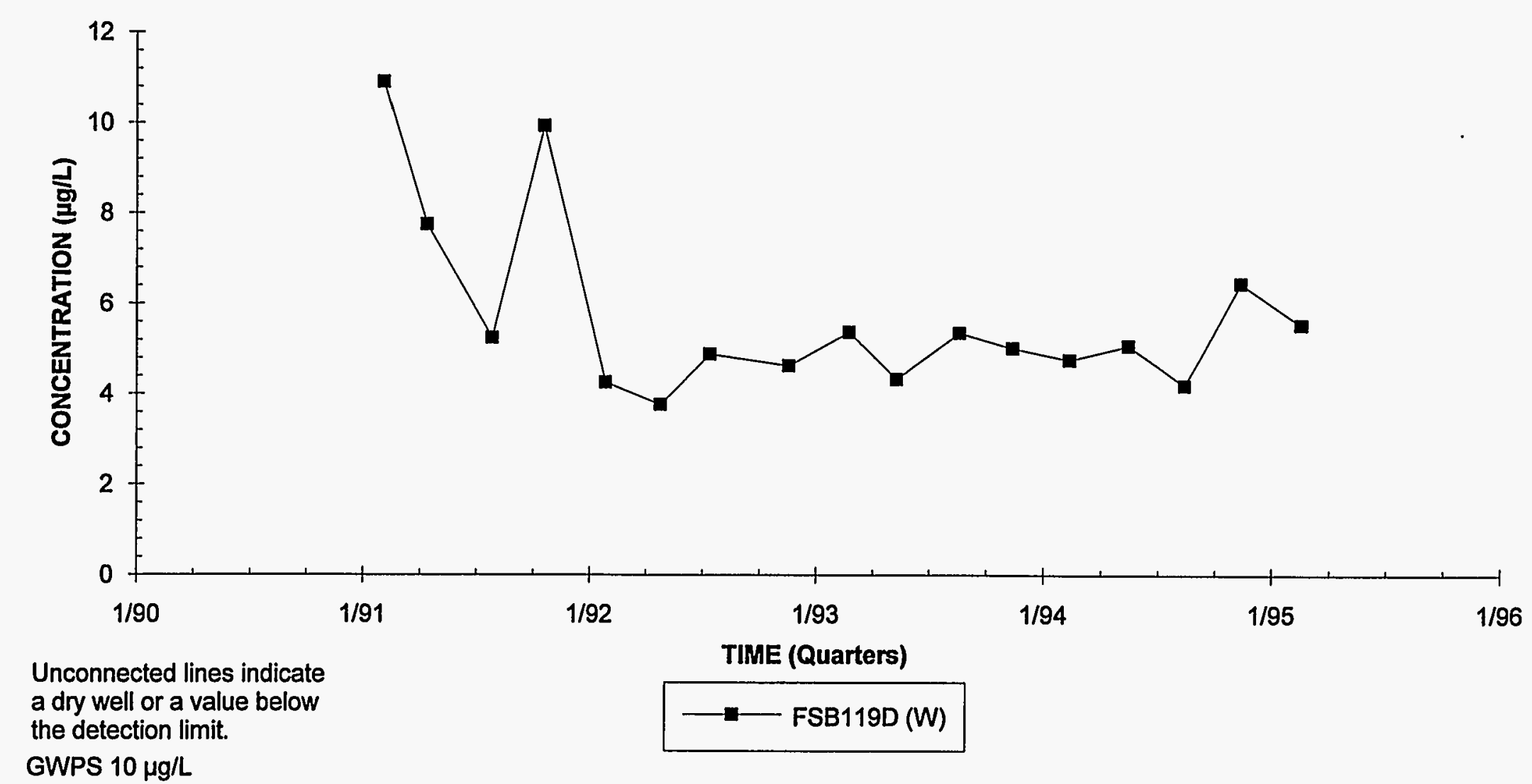

Note: W=Water Table (IIB2); B=Bamwell (IIB1); M=McBean (IIB1); UC=Upper Congaree (IIA); MC=Middle Congaree (IIA); LC=Lower Congaree (IIA) 


\section{Gross Alpha Activities}

Well FSB 77

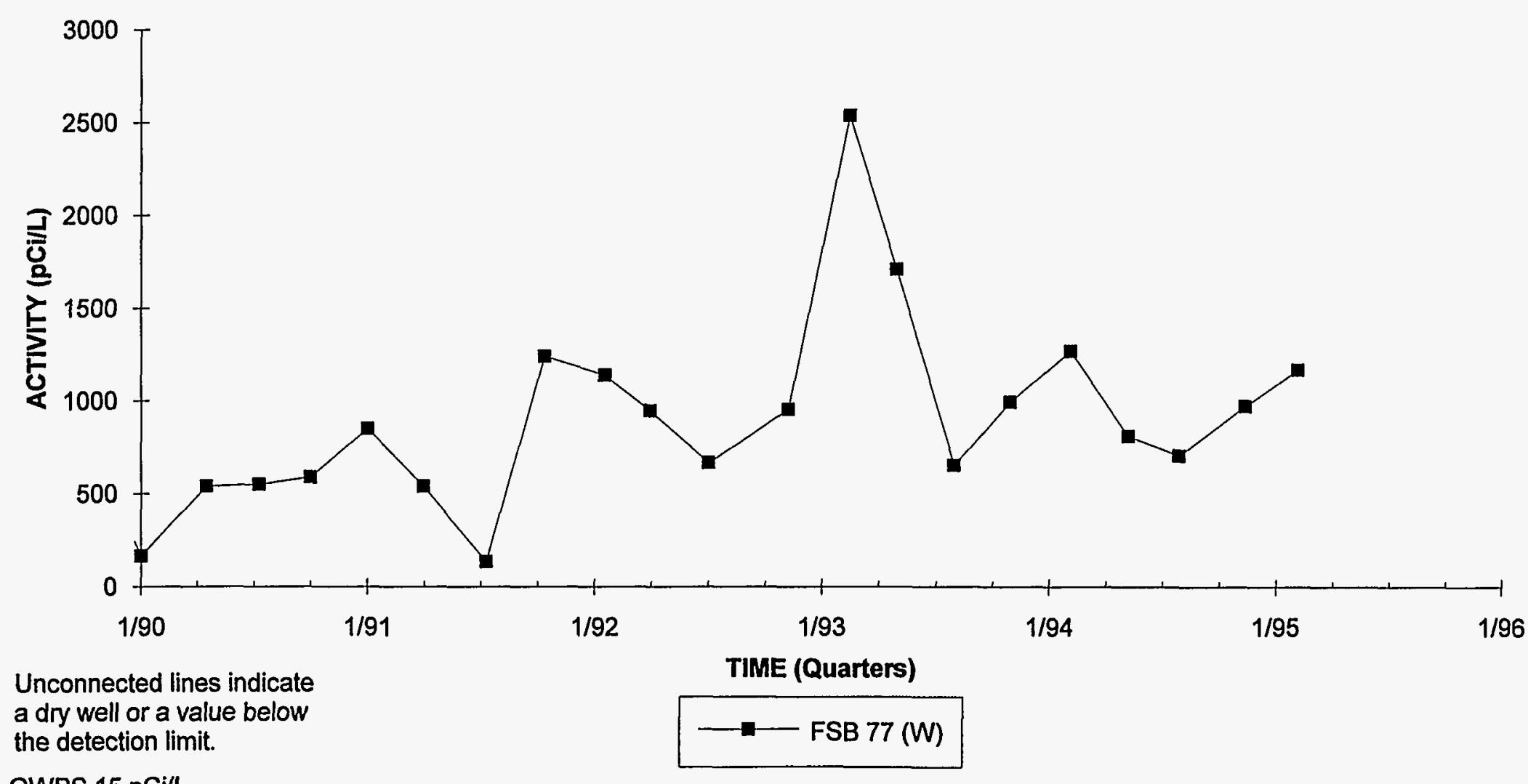

GWPS $15 \mathrm{pCi} / \mathrm{L}$

Note: W=Water Table (IIB2); B=Barnwell (IIB1); M=McBean (IIB1); UC=Upper Congaree (IIA); MC=Middle Congaree (IIA); LC=Lower Congaree (IIA) 


\section{Gross Alpha Activities \\ Well Cluster FSB 79}

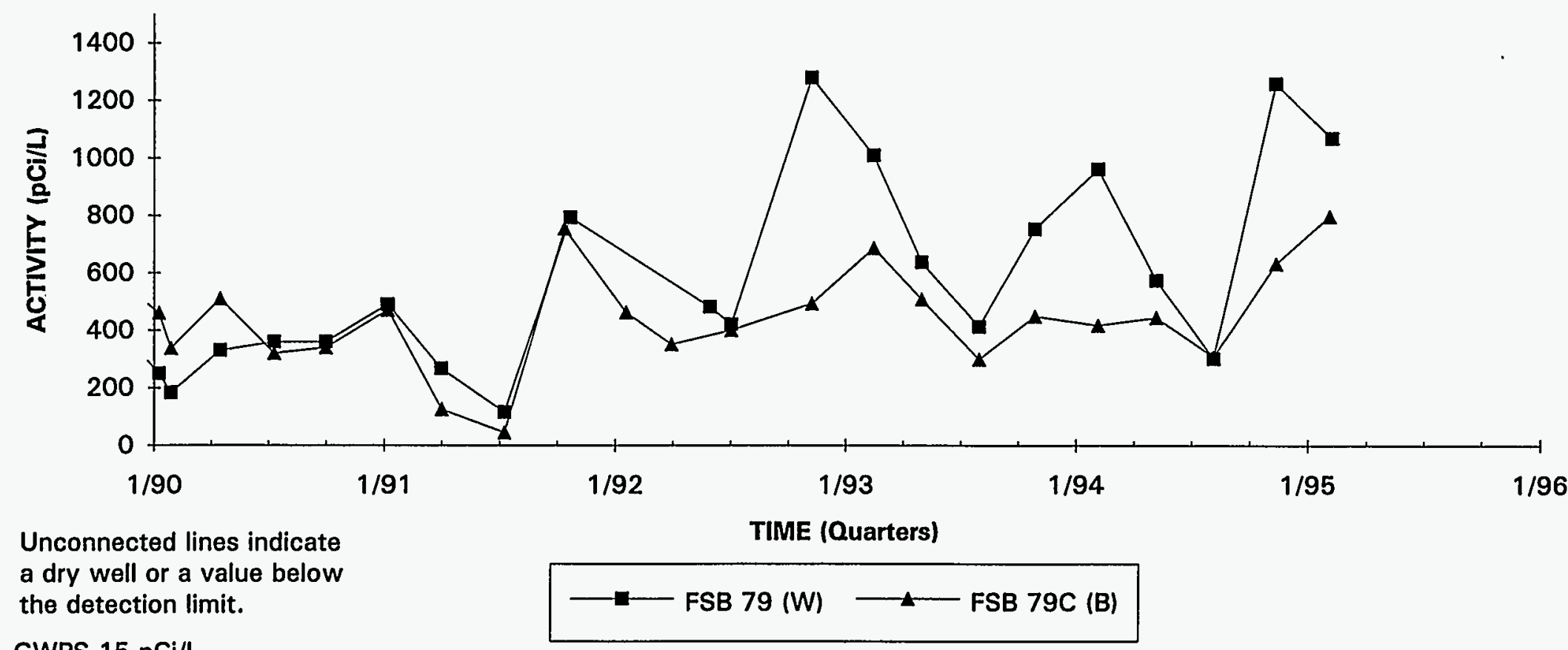

GWPS $15 \mathrm{pCi} / \mathrm{L}$

Note: W=Water Table (IIB2); B=Barnwell (IIB1); M=McBean (IIB1); UC=Upper Congaree (IIA); MC=Middle Congaree (IIA); LC=Lower Congaree (IIA) 


\section{Gross Alpha Activities \\ Well FSB 88D}

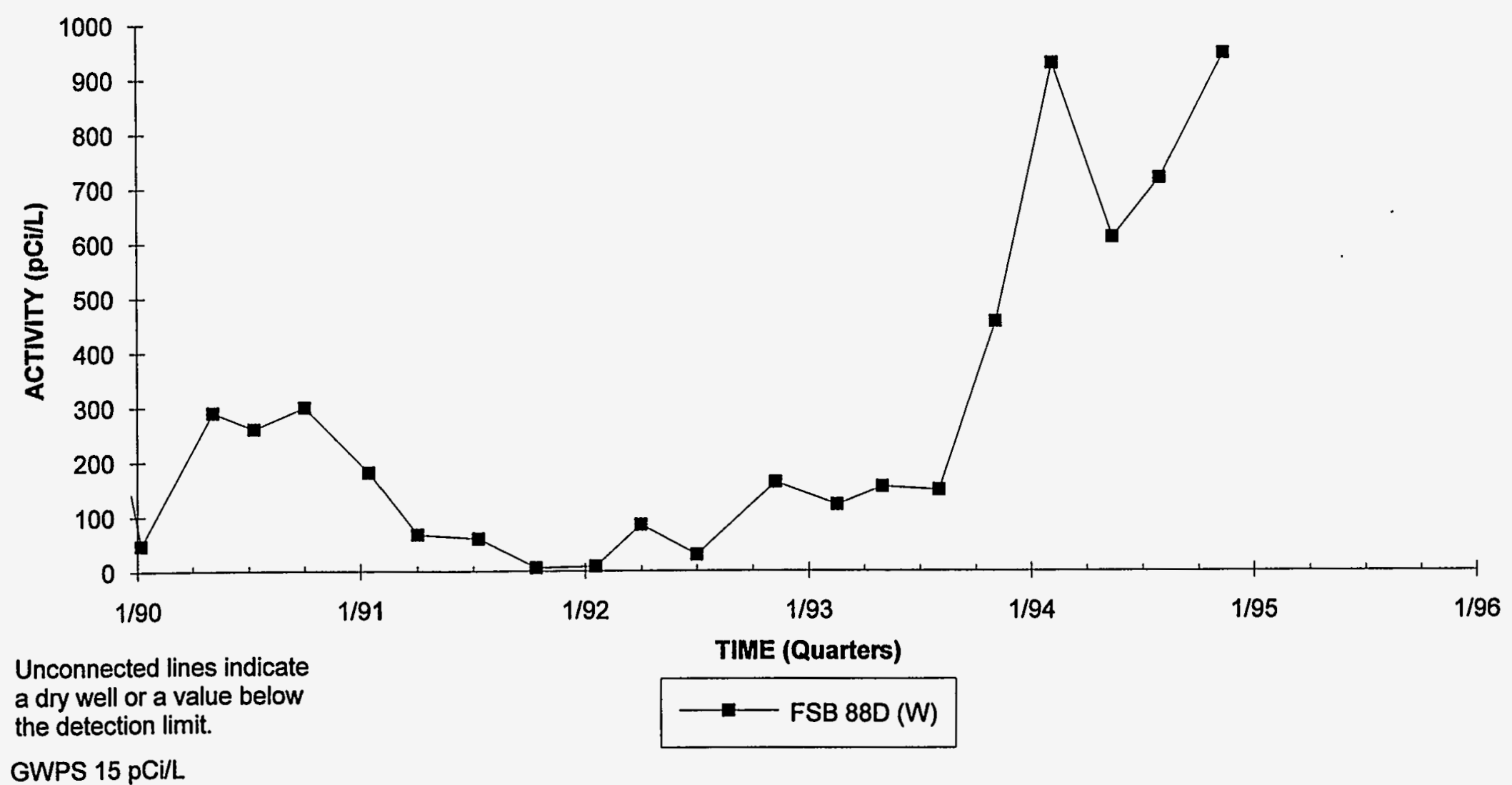

Note: W=Water Table (IIB2); B=Barnwell (IIB1); M=McBean (IIB1); UC=Upper Congaree (IIA); MC=Middle Congaree (IIA); LC=Lower Congaree (IIA) 


\section{Gross Alpha Activities \\ Well Cluster FSB 94}

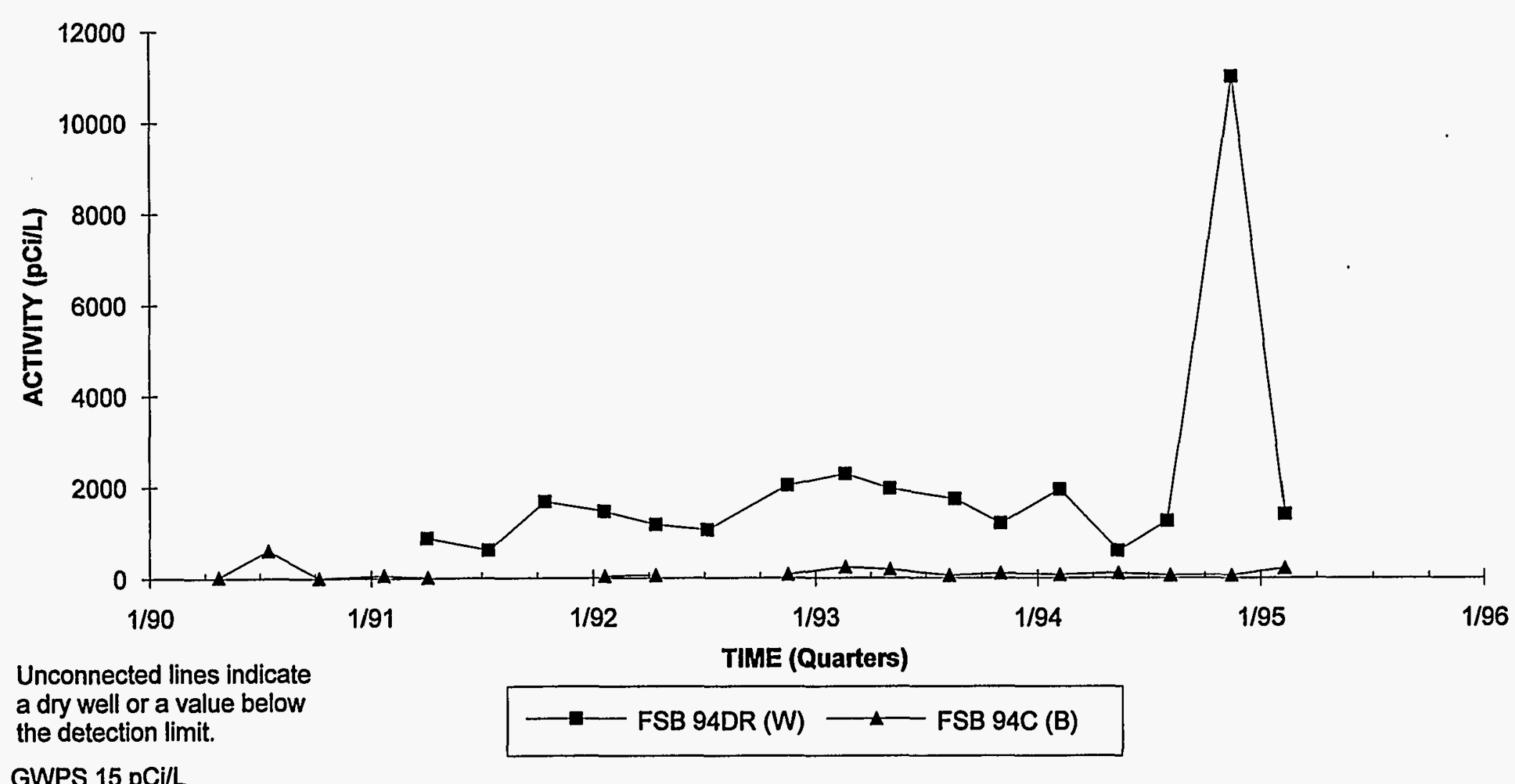

Note: W=Water Table (IIB2); B=Barnwell (IIB1); $M=$ McBean (IIB1); UC=Upper Congaree (IIA); MC=Middle Congaree (IIA); LC=Lower Congaree (IIA) 


\section{Gross Alpha Activities \\ Well Cluster FSB 95}

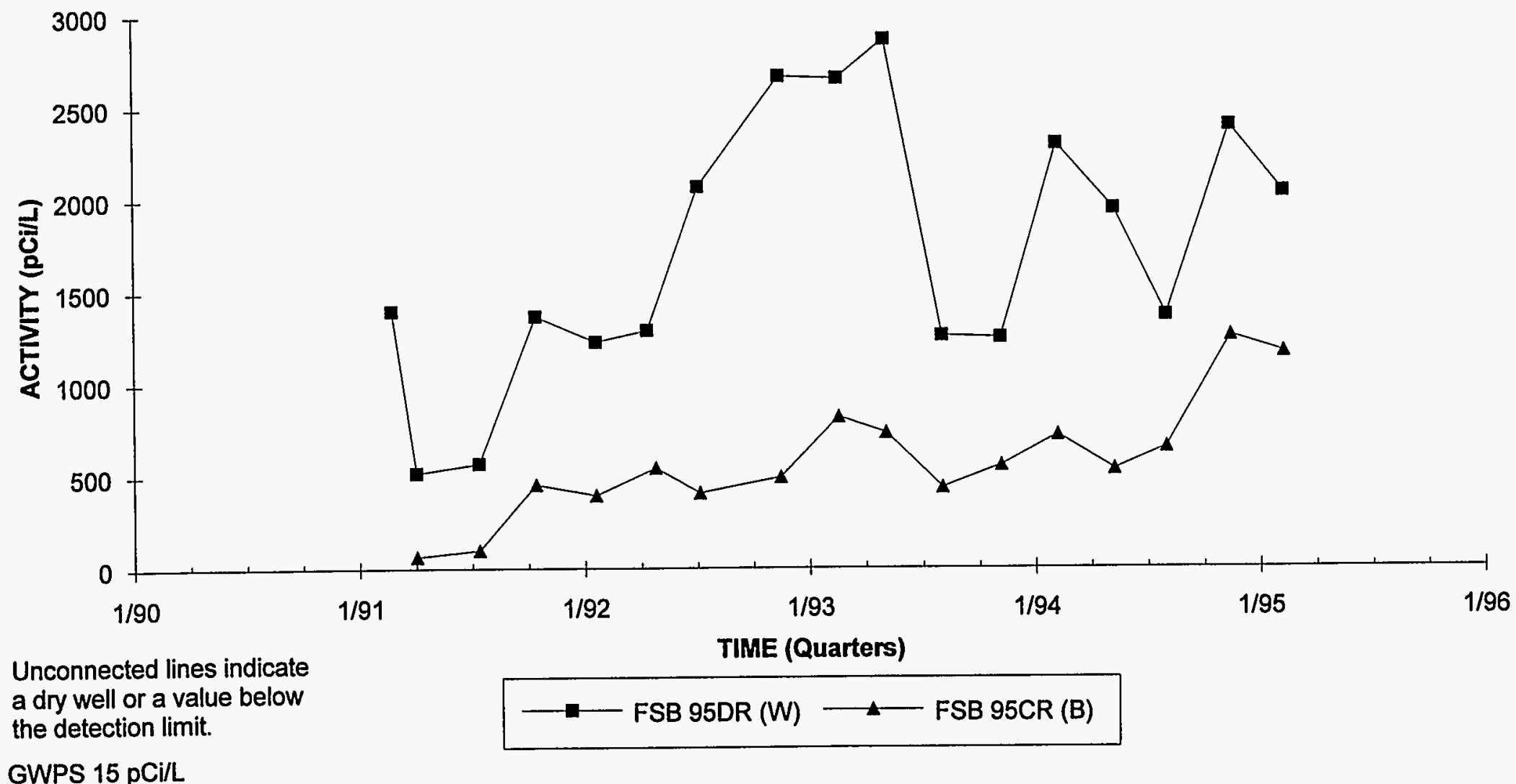

Note: W=Water Table (IIB2); B=Barnwell (IIB1); M=McBean (IIB1); UC=Upper Congaree (IIA); MC=Middle Congaree (IIA); LC=Lower Congaree (IIA) 


\section{Gross Alpha Activities \\ Well Cluster FSB 98}

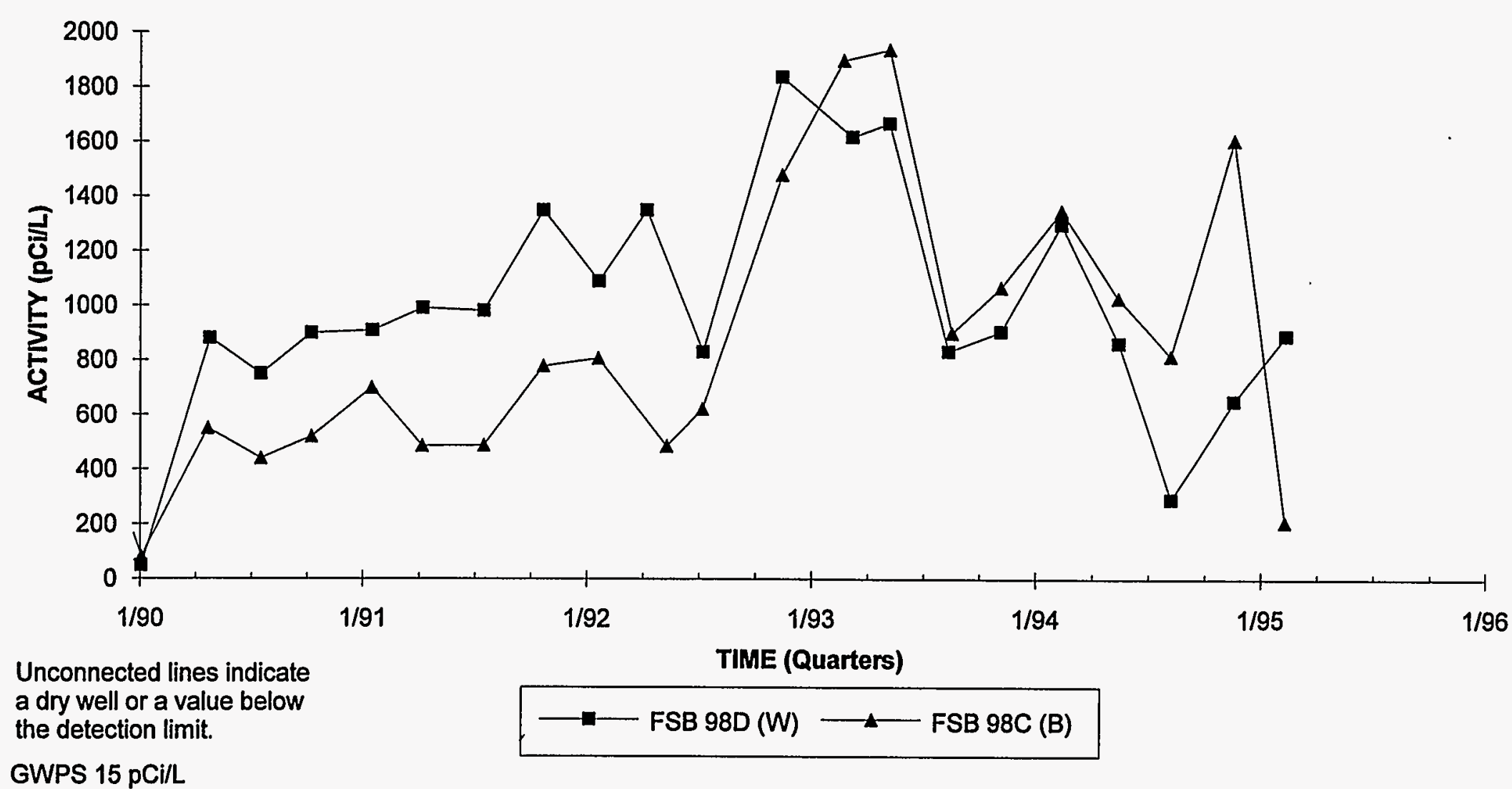

Note: W=Water Table (IIB2); B=Barnwell (IIB1); M=McBean (IIB1); UC=Upper Congaree (IIA); MC=Middle Congaree (IIA); LC=Lower Congaree (IIA) 


\section{Gross Alpha Activities \\ Well Cluster FSB 99}

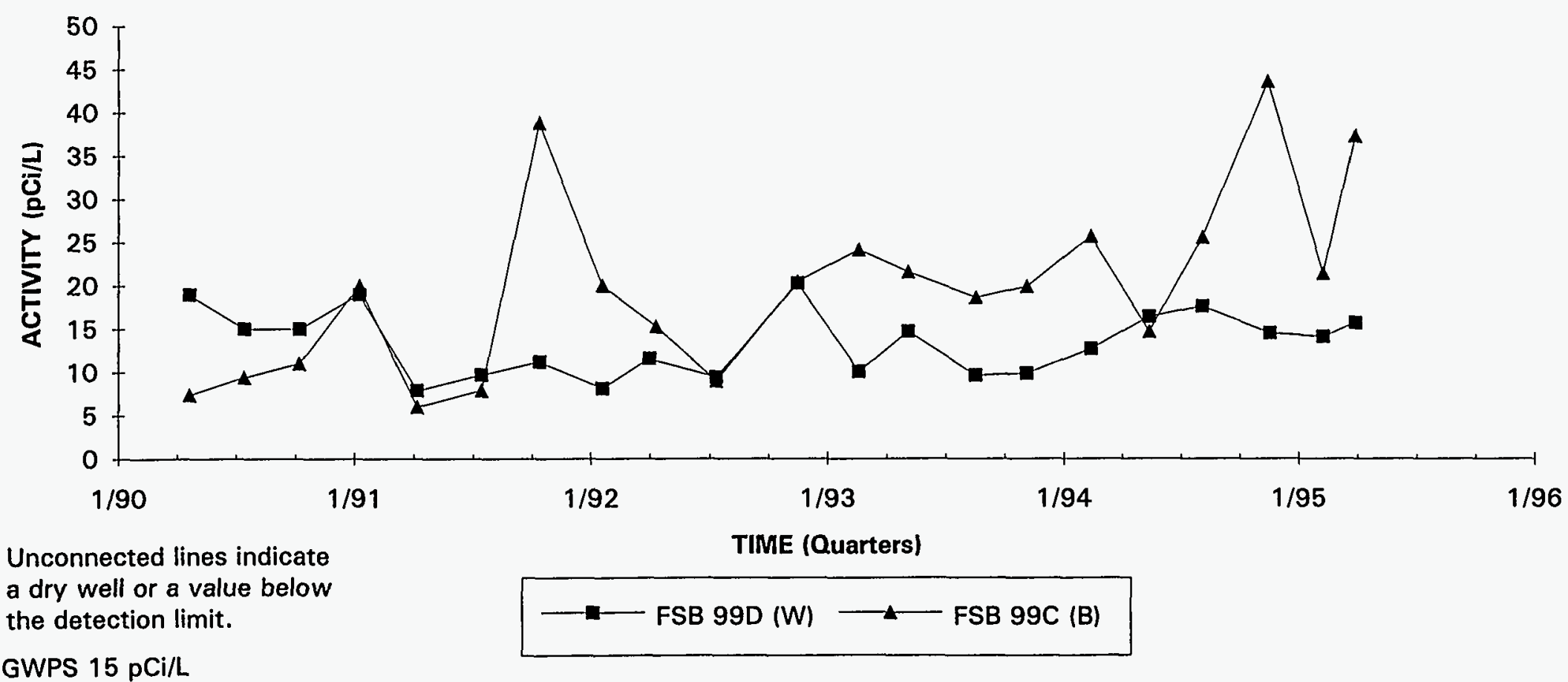

Note: $W=$ Water Table (IIB2); B=Barnwell (IIB1); $M=M c B e a n$ (IIB1); UC=Upper Congaree (IIA); MC=Middle Congaree (IIA); LC=Lower Congaree (IIA) 


\section{Gross Alpha Activities}

Well Cluster FSB104

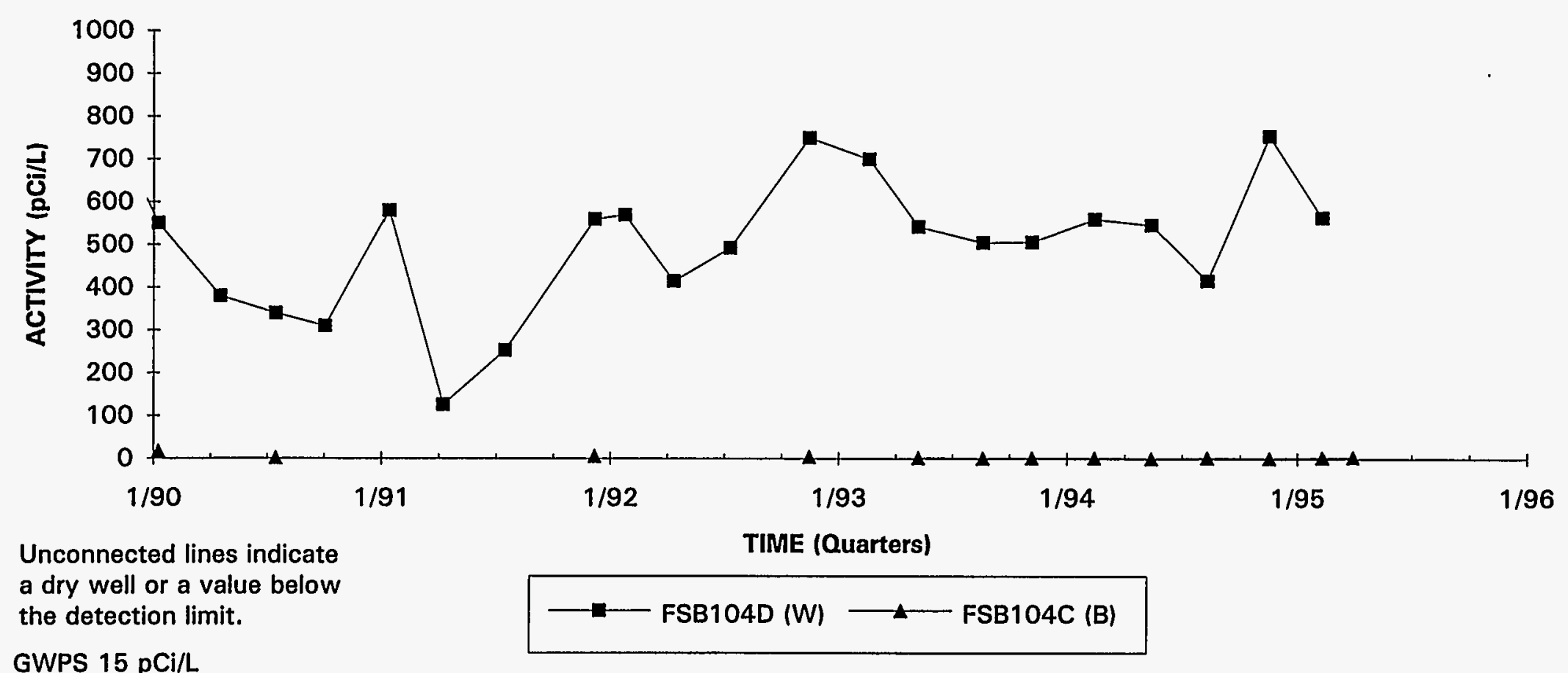

GWPS $15 \mathrm{pCi} / \mathrm{L}$

Note: W=Water Table (IIB2); B=Barnwell (IIB1); M=McBean (IIB1); UC=Upper Congaree (IIA); MC=Middle Congaree (IIA); LC=Lower Congaree (IIA) 


\section{Gross Alpha Activities} Well Cluster FSB107

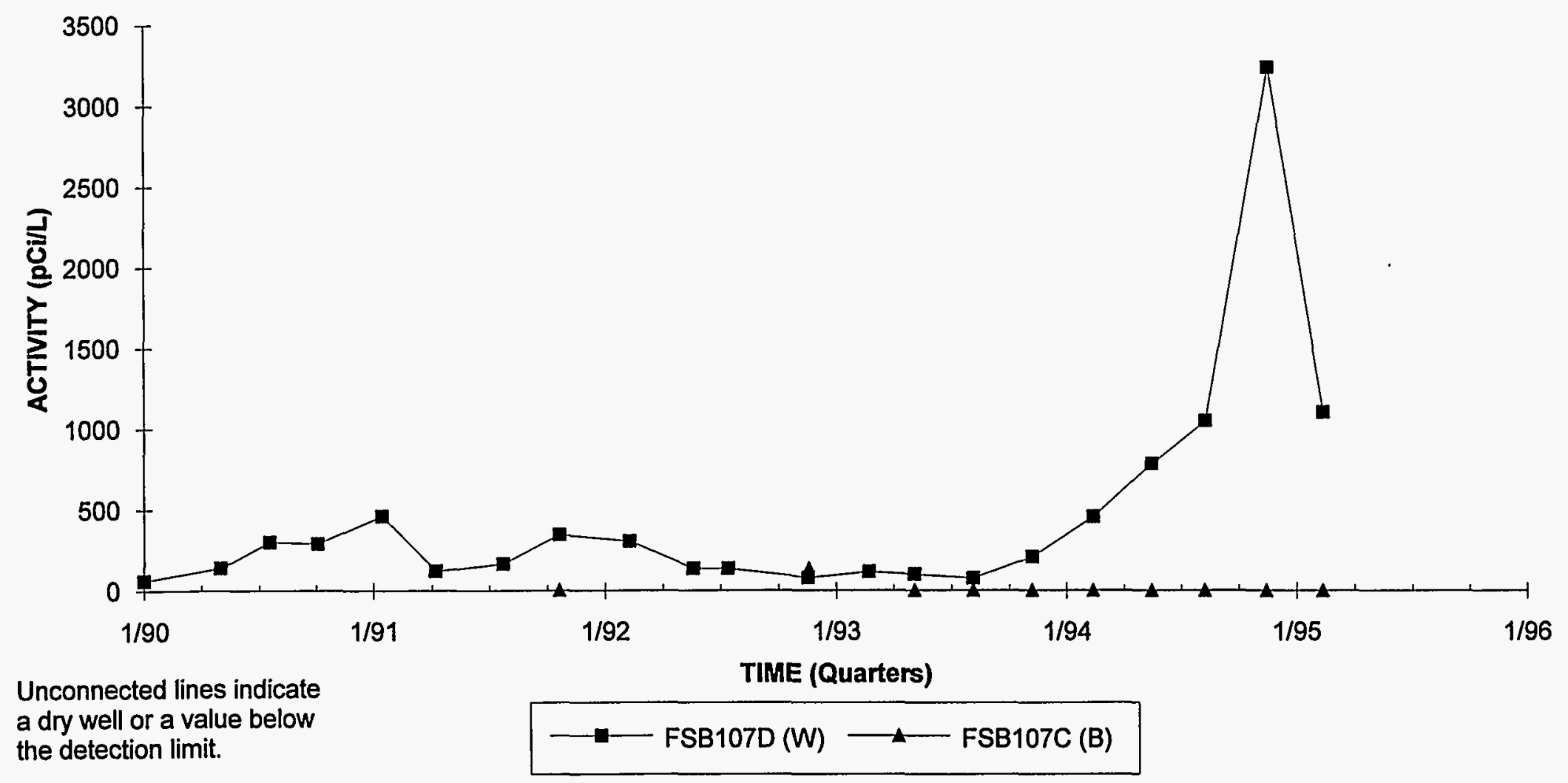

GWPS $15 \mathrm{pCi/L}$

Note: W=Water Table (IIB2); B=Barnwell (IIB1); M=McBean (IIB1); UC=Upper Congaree (IIA); MC=Middle Congaree (IIA); LC=Lower Congaree (IIA) 


\section{Lead Concentrations}

Well FSB 76

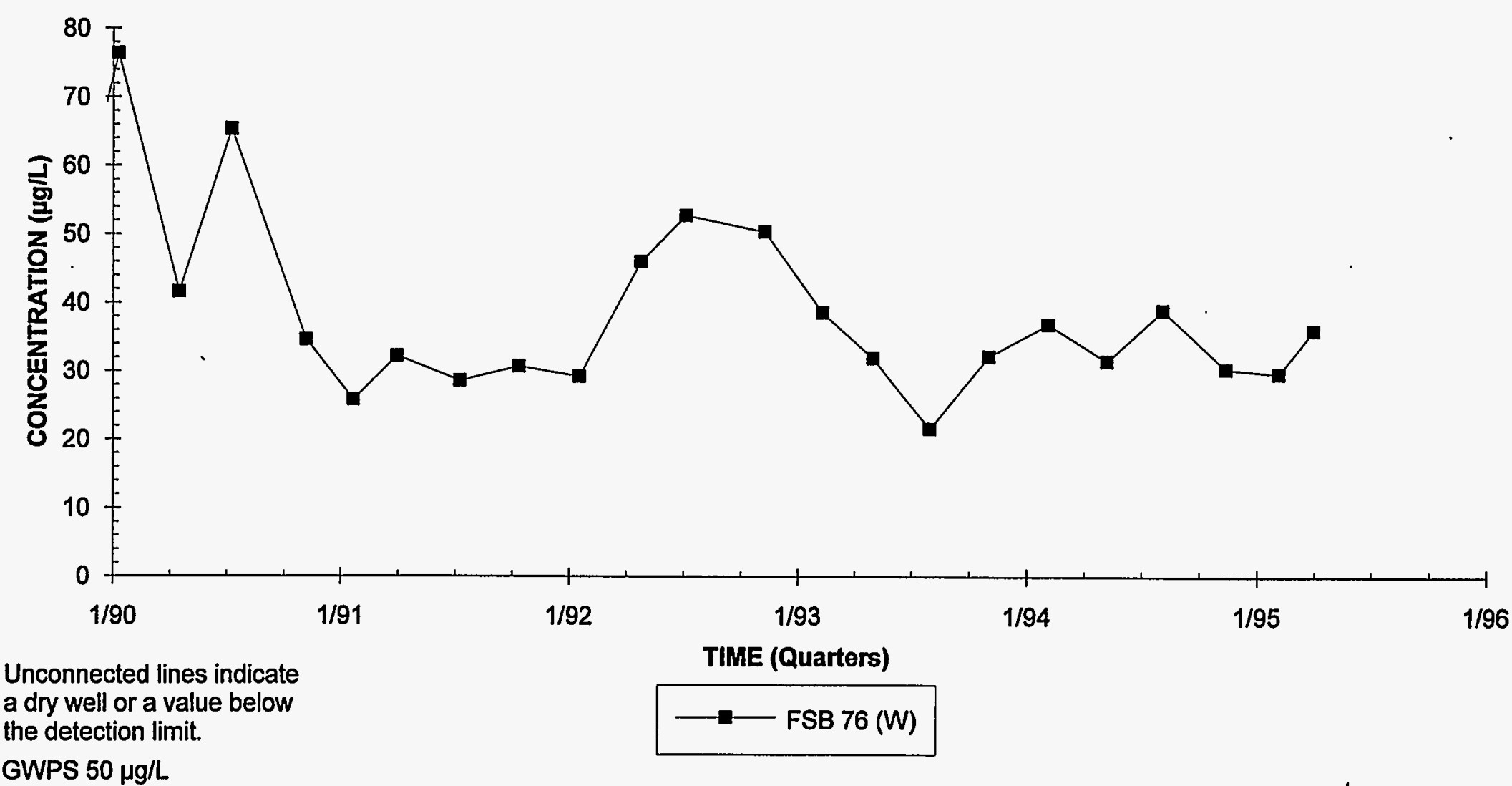

Note: W=Water Table (IIB2); B=Barnwell (IIB1); M=McBean (IIB1); UC=Upper Congaree (IIA); MC=Middle Congaree (IIA); LC=Lower Congaree (IIA) 


\section{Lead Concentrations \\ Well FSB 77}

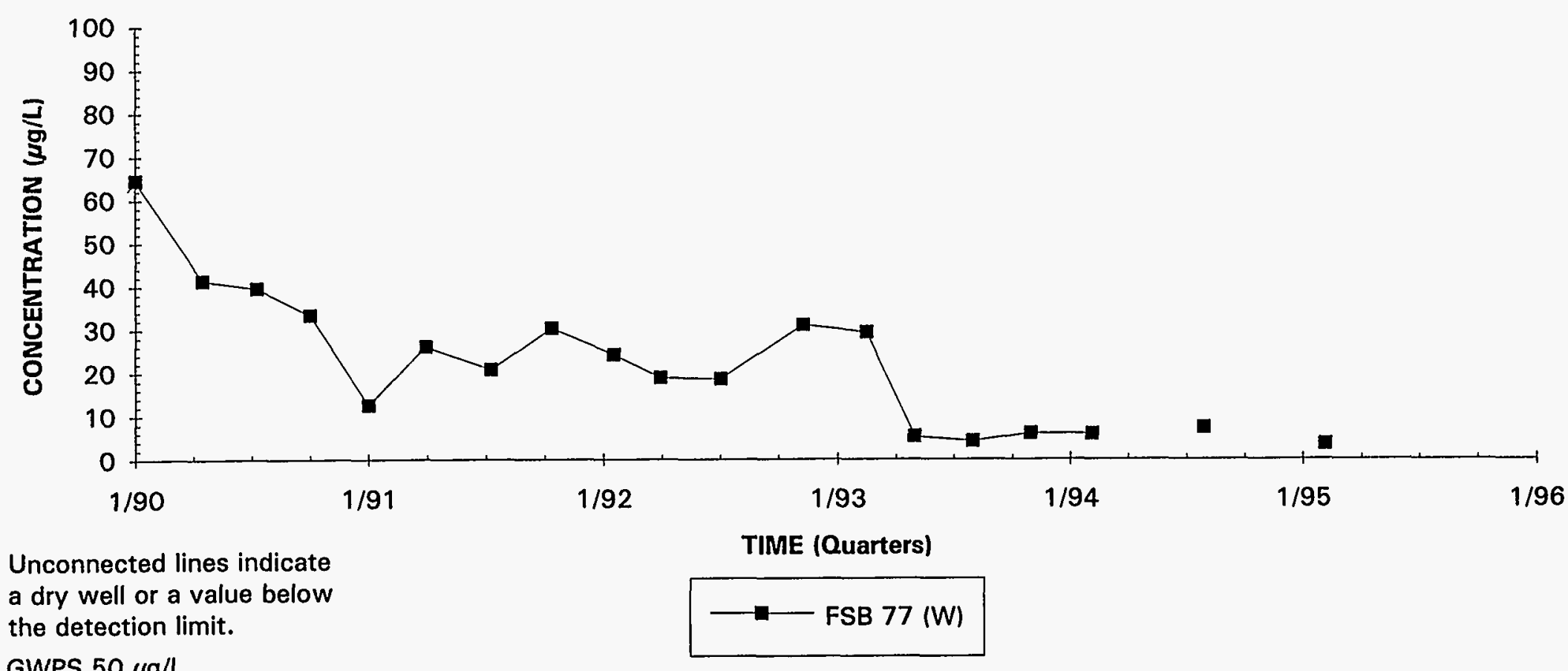

GWPS $50 \mu \mathrm{g} / \mathrm{L}$ 


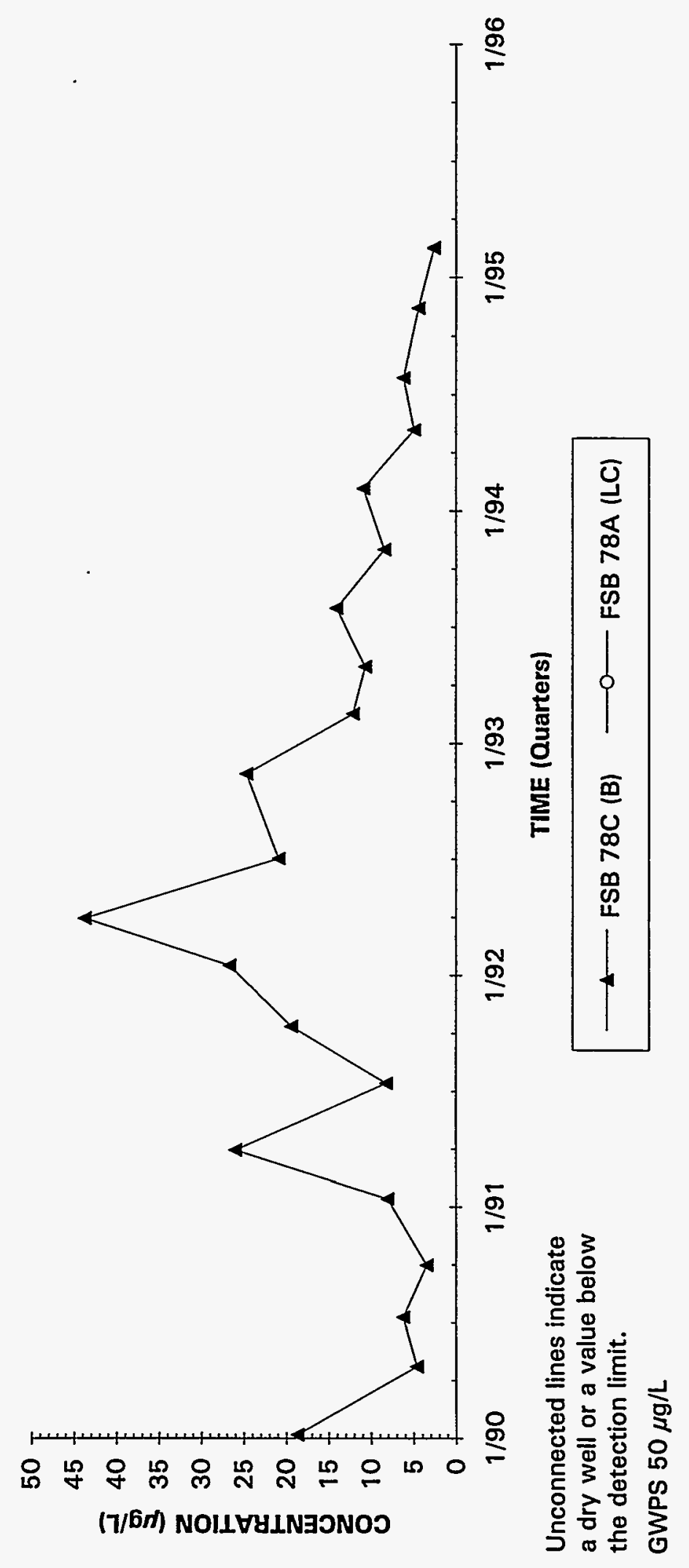




\section{Lead Concentrations}

Well FSB 90D

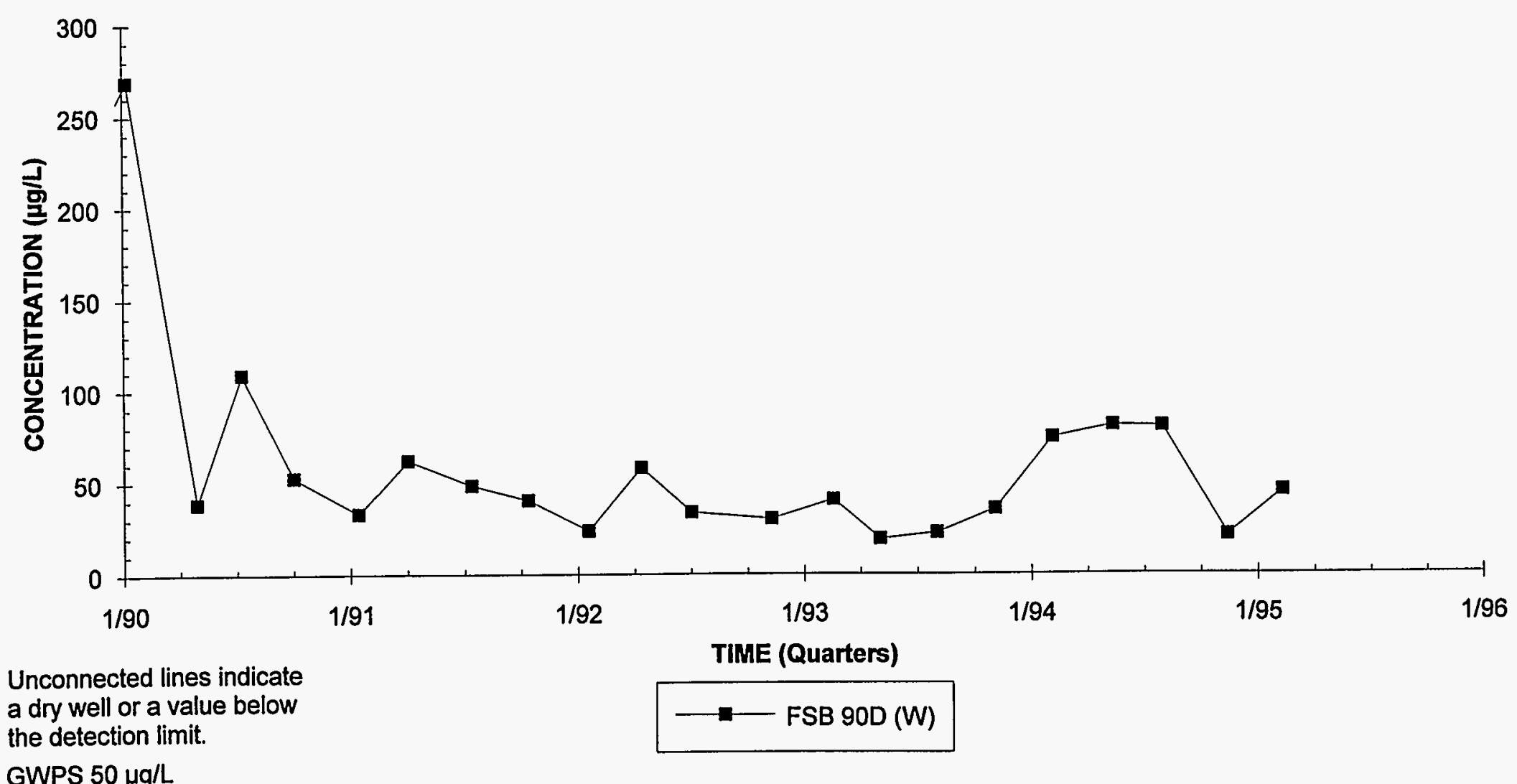

GWPS $50 \mu \mathrm{g} / \mathrm{L}$ 


\section{Lead Concentrations}

Well Cluster FSB 94

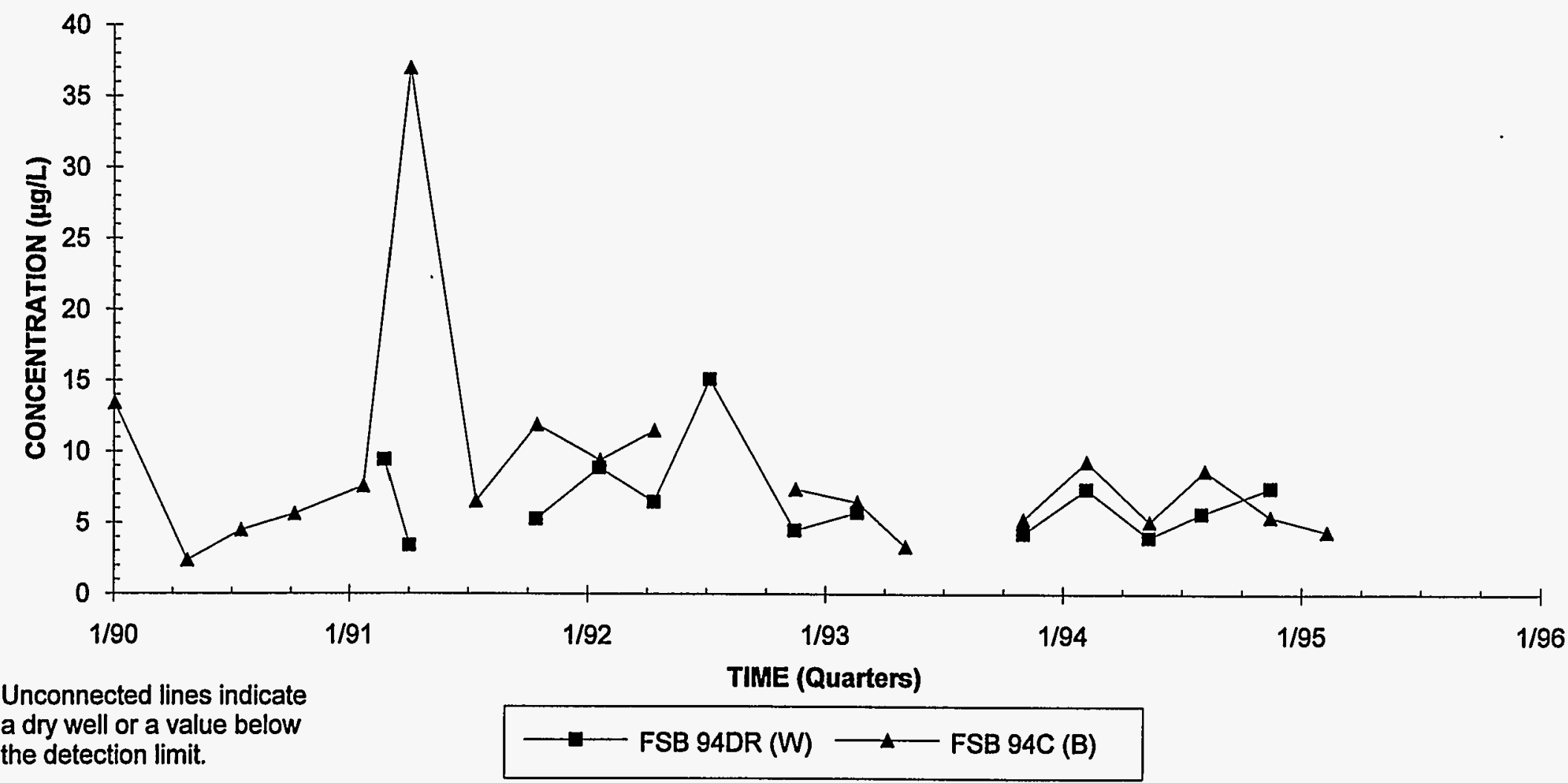

GWPS $50 \mu \mathrm{g} / \mathrm{L}$ 


\section{Lead Concentrations Well FSB 95DR}

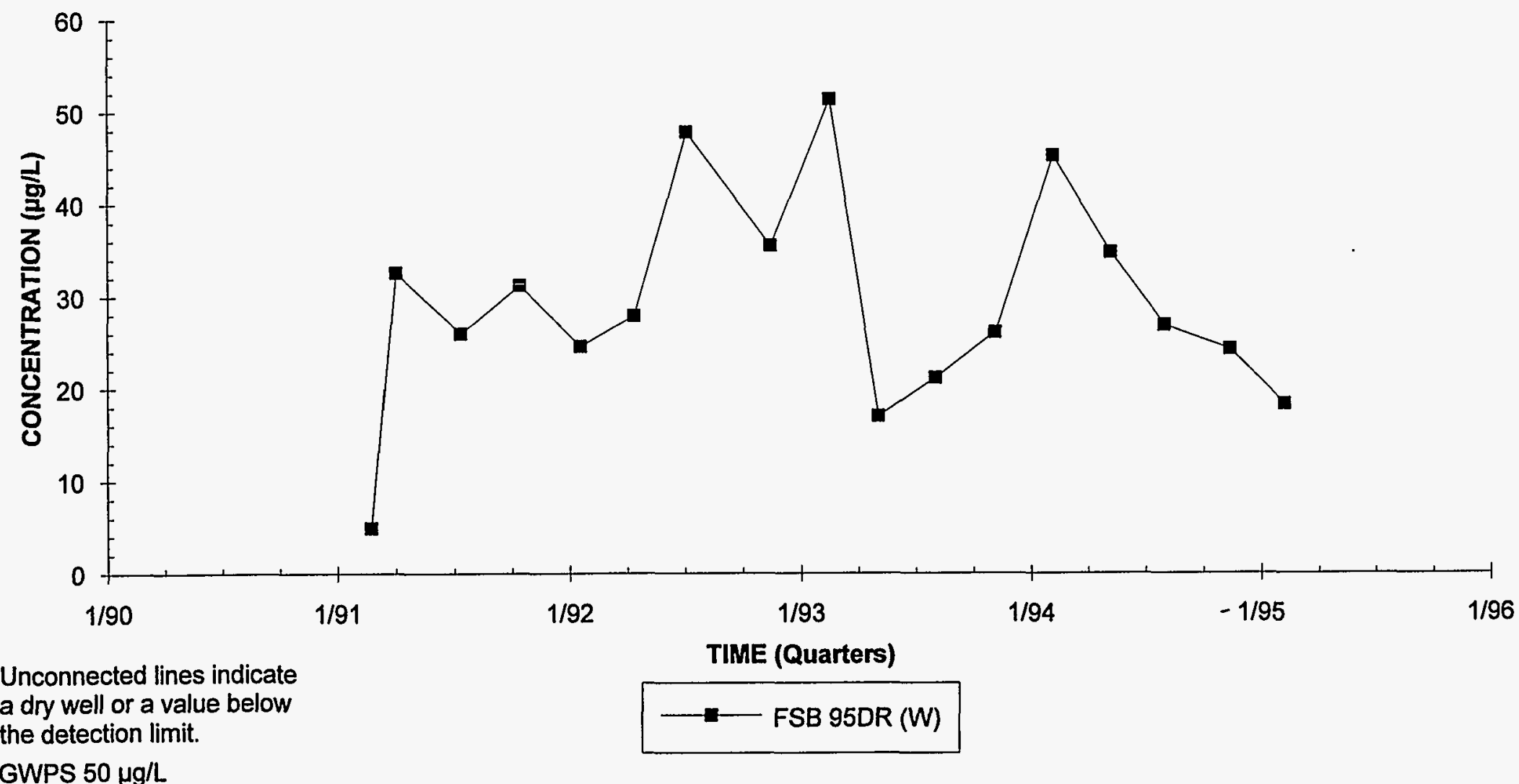

Note: W=Water Table (IIB2); B=Barnwell (IIB1); M=McBean (IIB1); UC=Upper Congaree (IIA); MC=Middle Congaree (IIA); LC=Lower Congaree (IIA) 

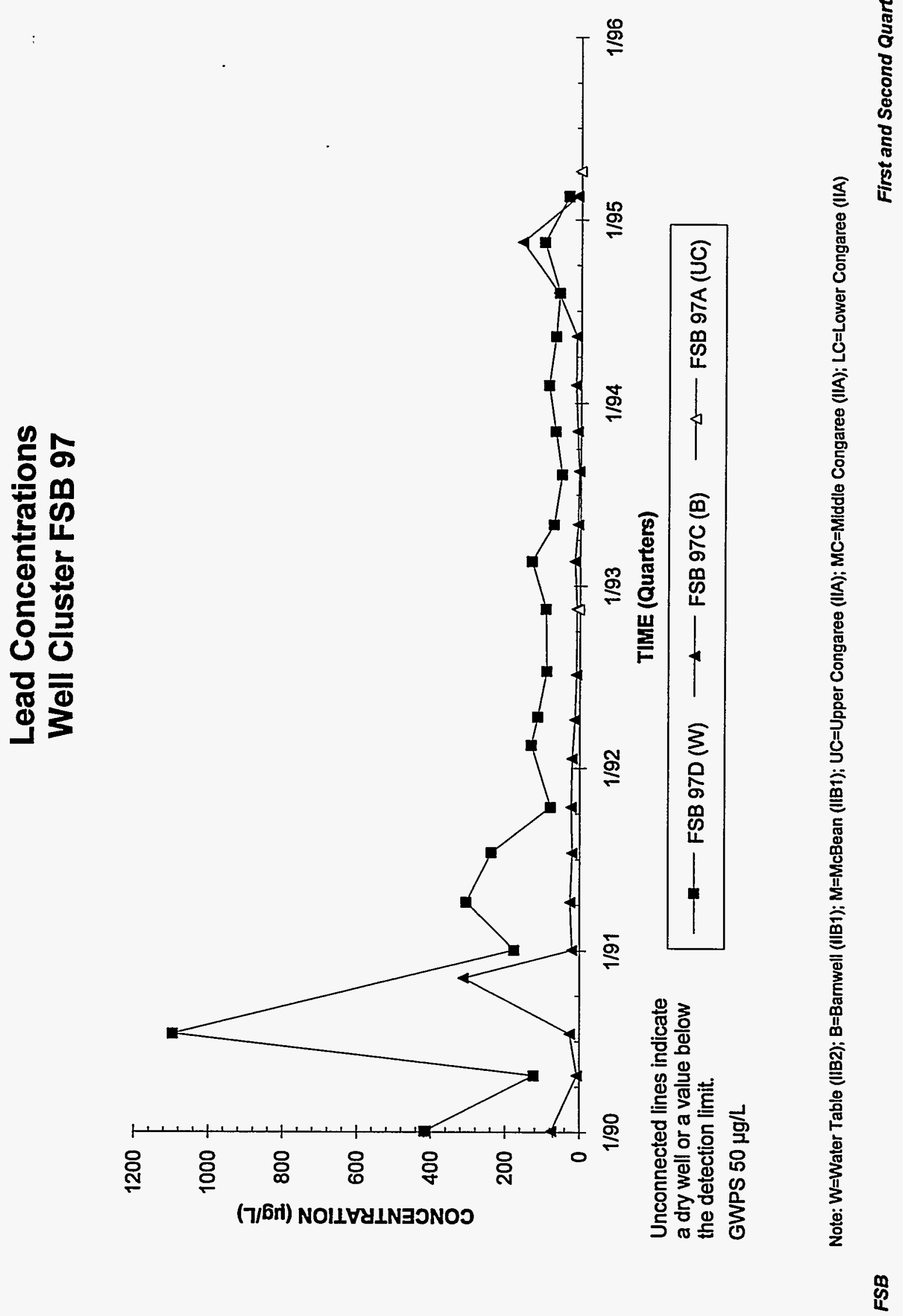


\section{Lead Concentrations \\ Well FSB108D}

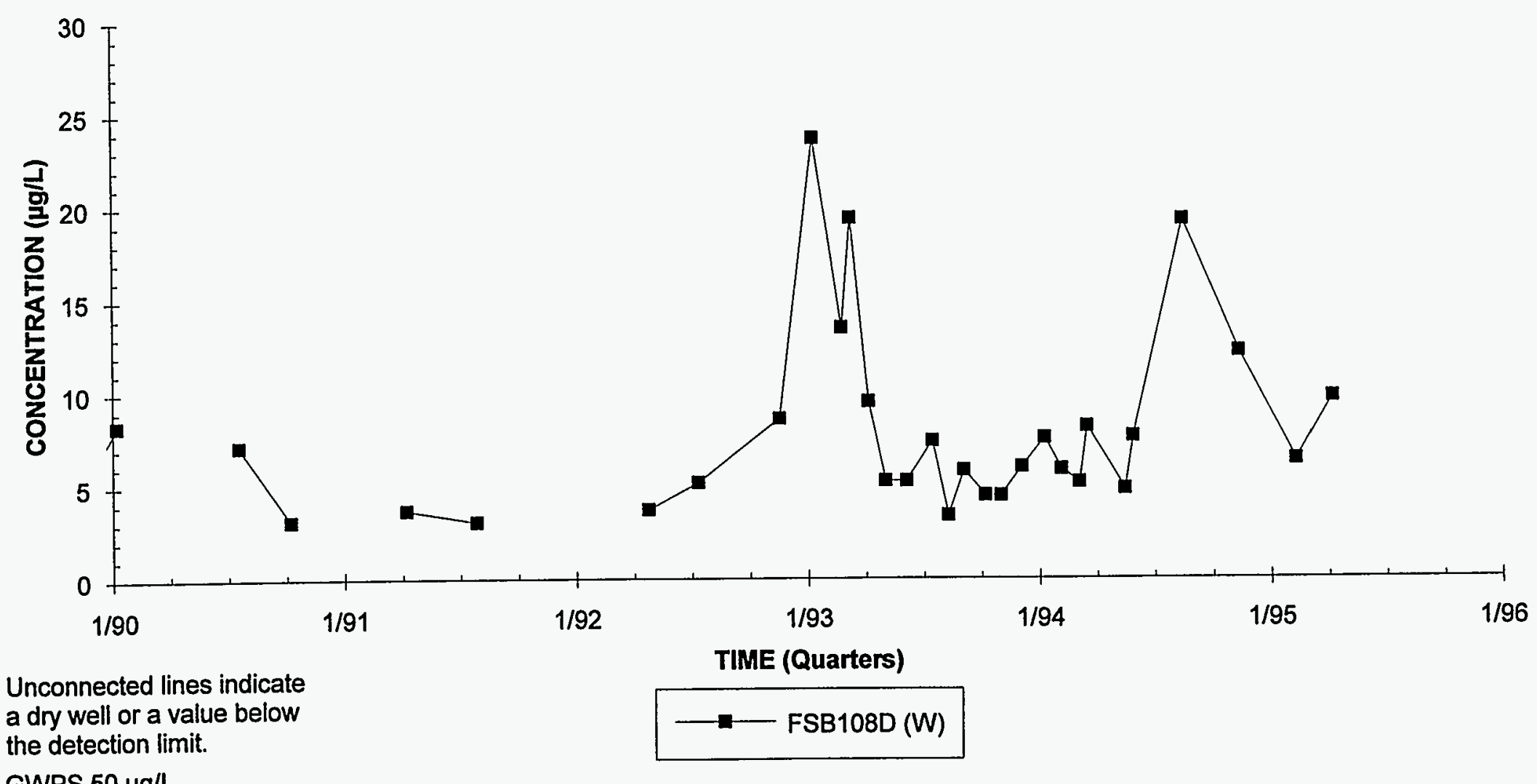

GWPS $50 \mu \mathrm{g} / \mathrm{L}$

Note: W=Water Table (IIB2); B=Barnwell (IIB1); M=McBean (IIB1); UC=Upper Congaree (IIA); MC=Middle Congaree (IIA); LC=Lower Congaree (IIA) 


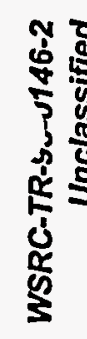
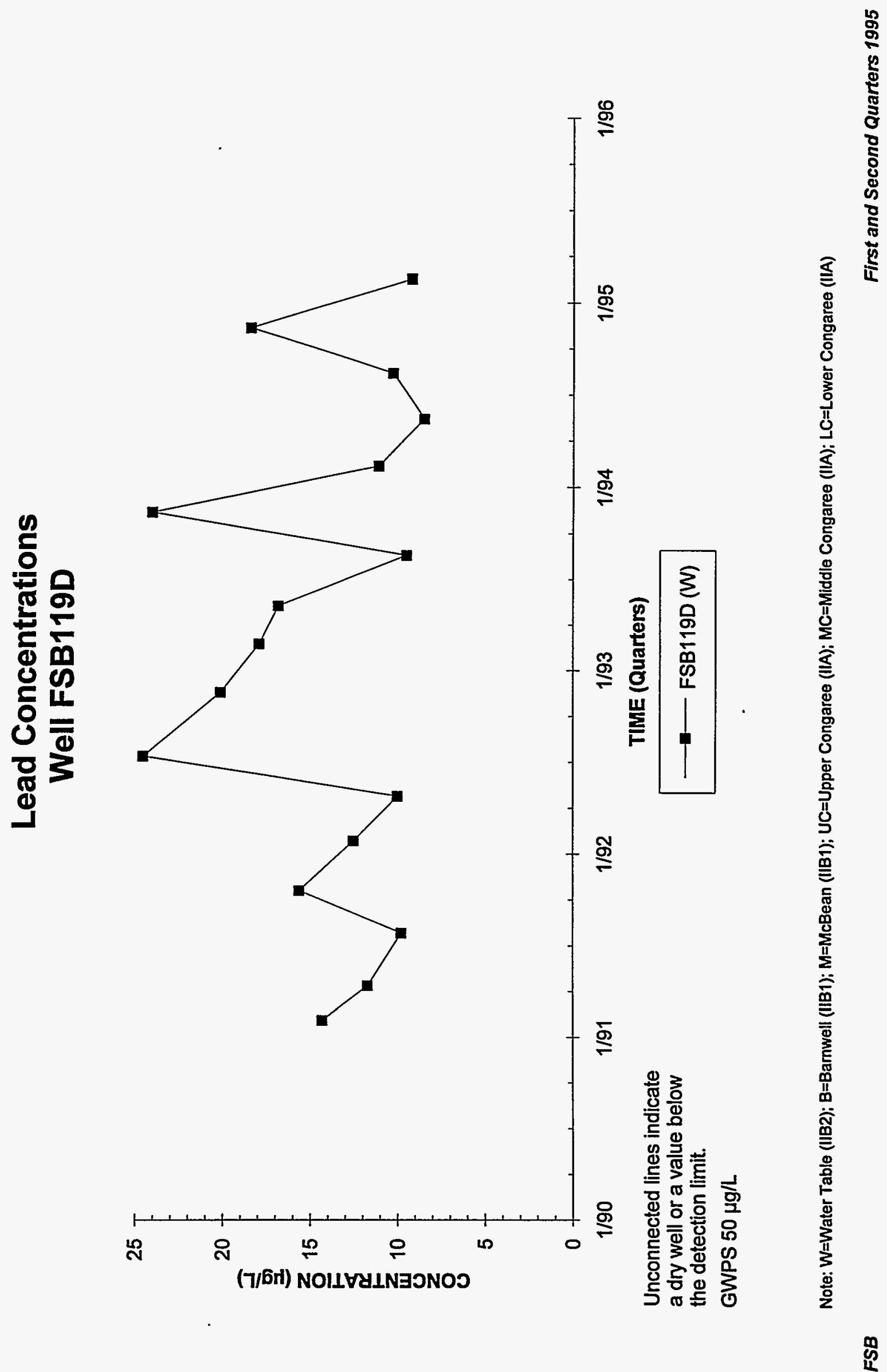


\section{Nitrate Concentrations \\ Well Cluster FSB 87}

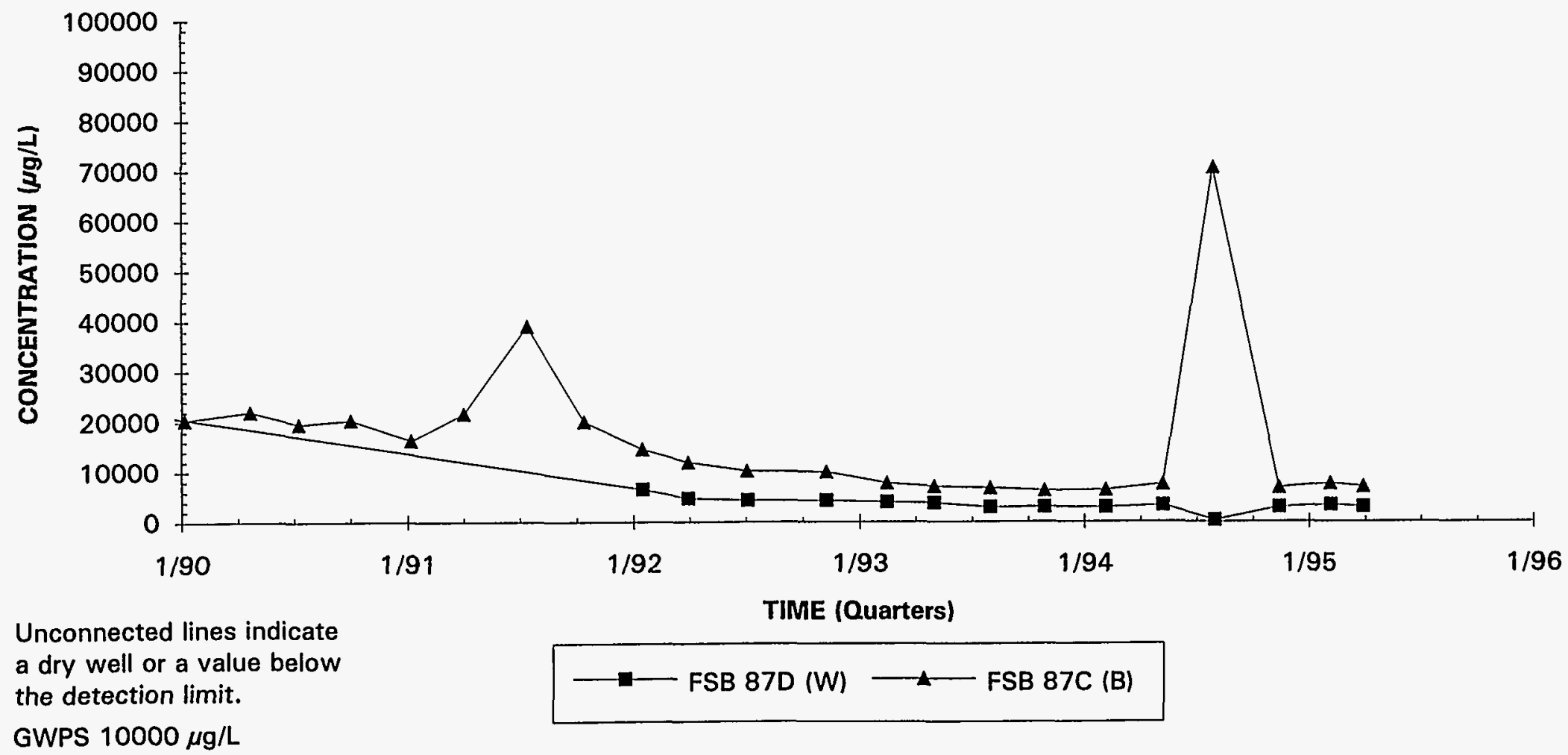

Note: W=Water Table (IIB2); B=Barnwell (IIB1); M=McBean (IIB1); UC=Upper Congaree (IIA); MC=Middle Congaree (IIA); LC=Lower Congaree (IIA) 

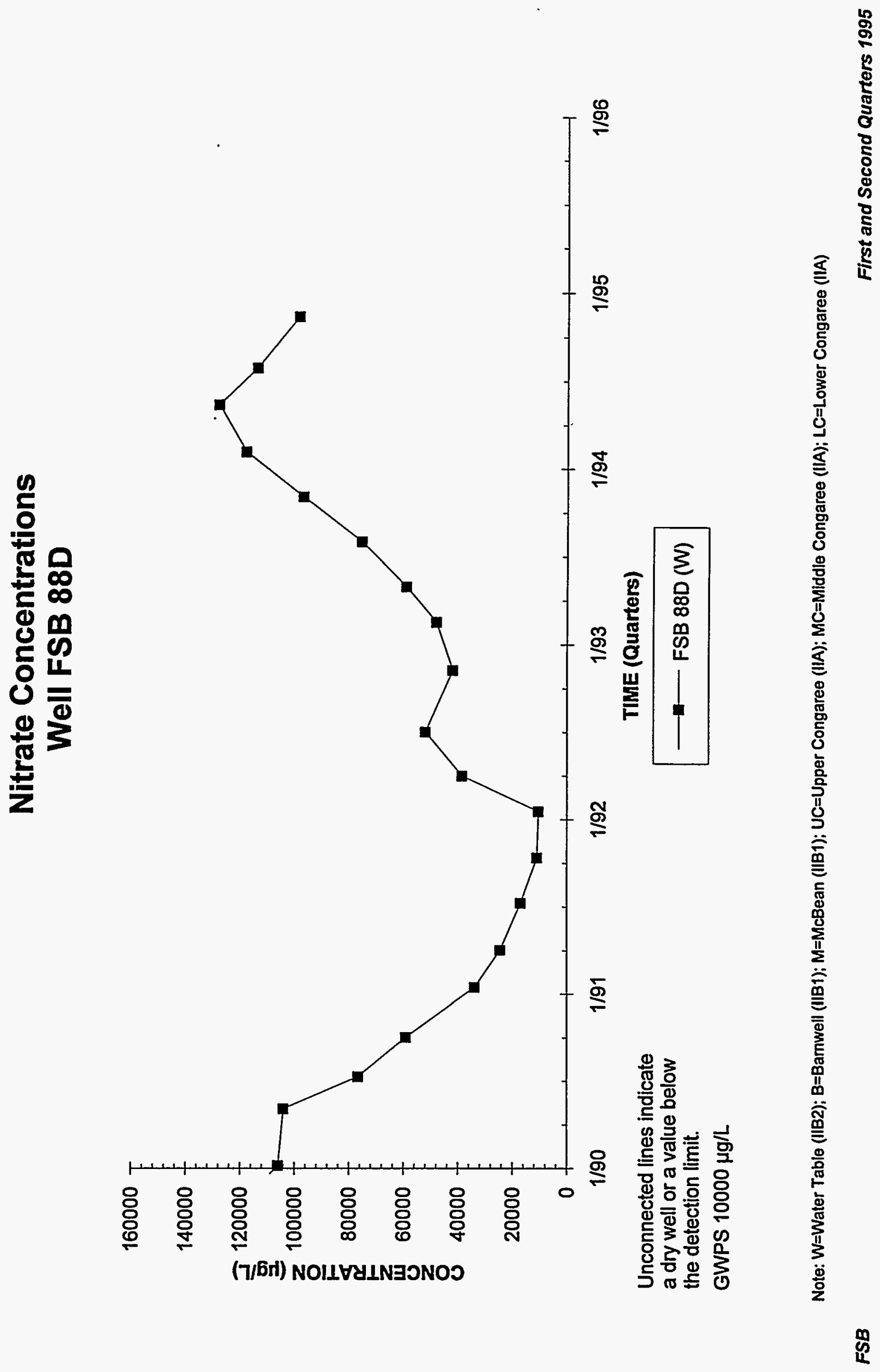


\section{Nitrate Concentrations \\ Well FSB 91D}

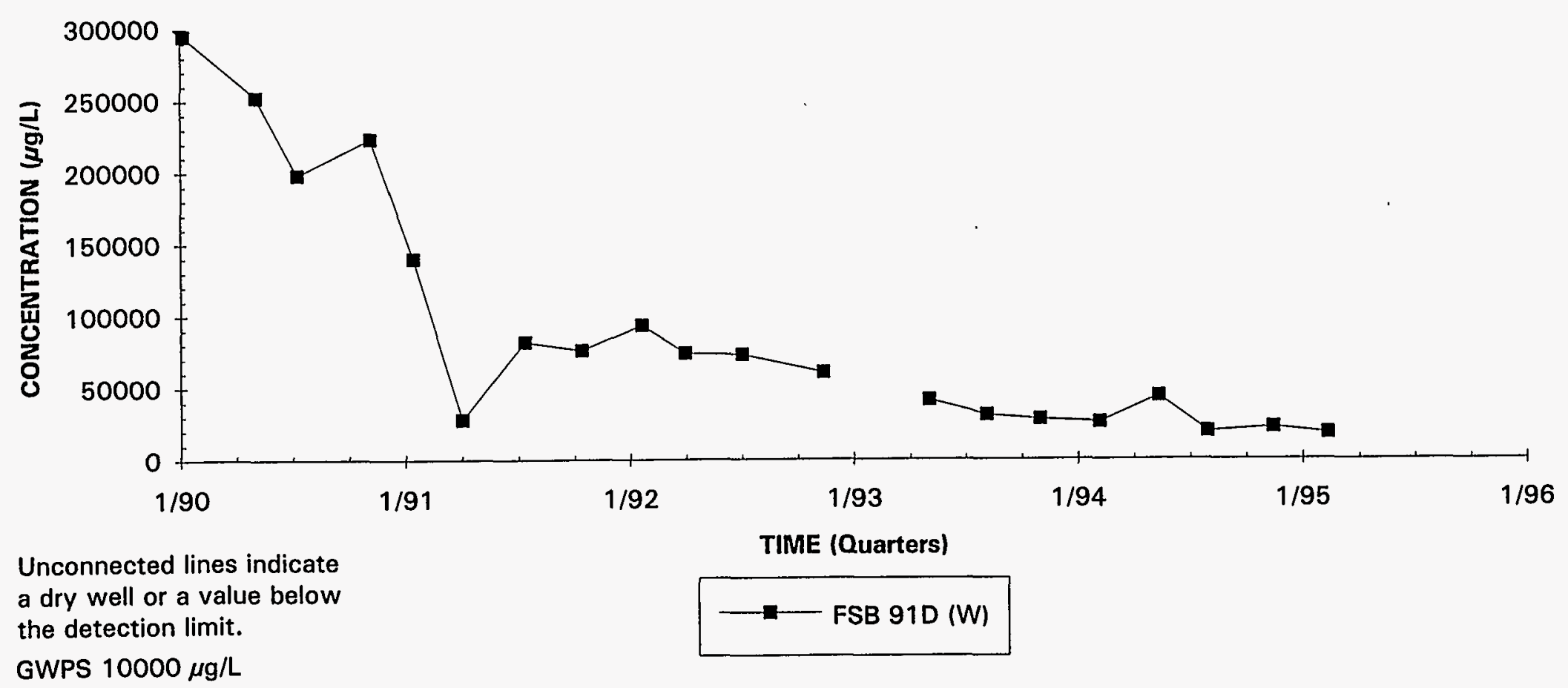

Note: W=Water Table (IIB2); B=Barnwell (IIB1); M=McBean (IIB1); UC=Upper Congaree (IIA); MC=Middle Congaree (IIA); LC=Lower Congaree (IIA) 
范
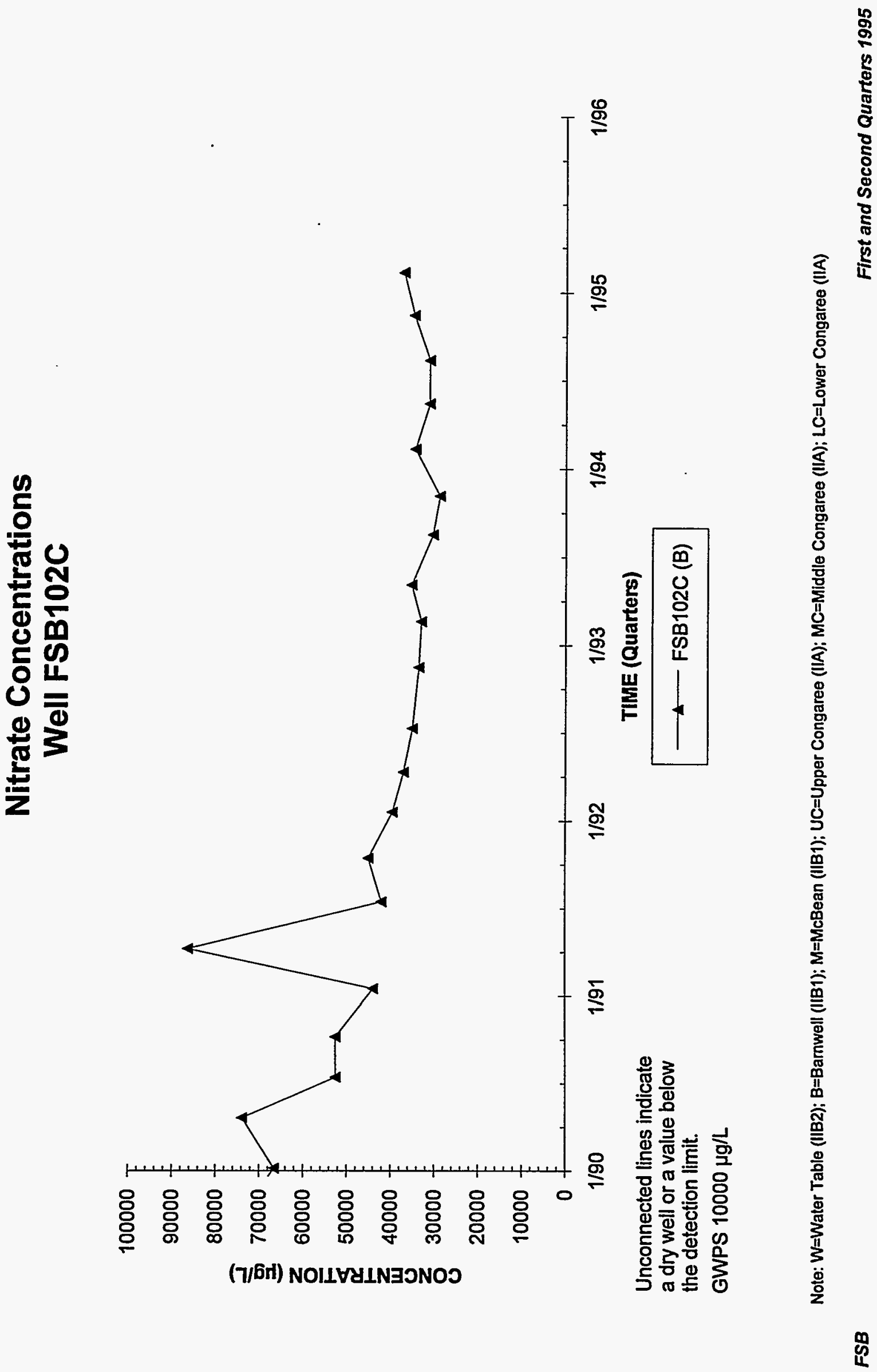


\section{Nitrate Concentrations Well Cluster FSB104}

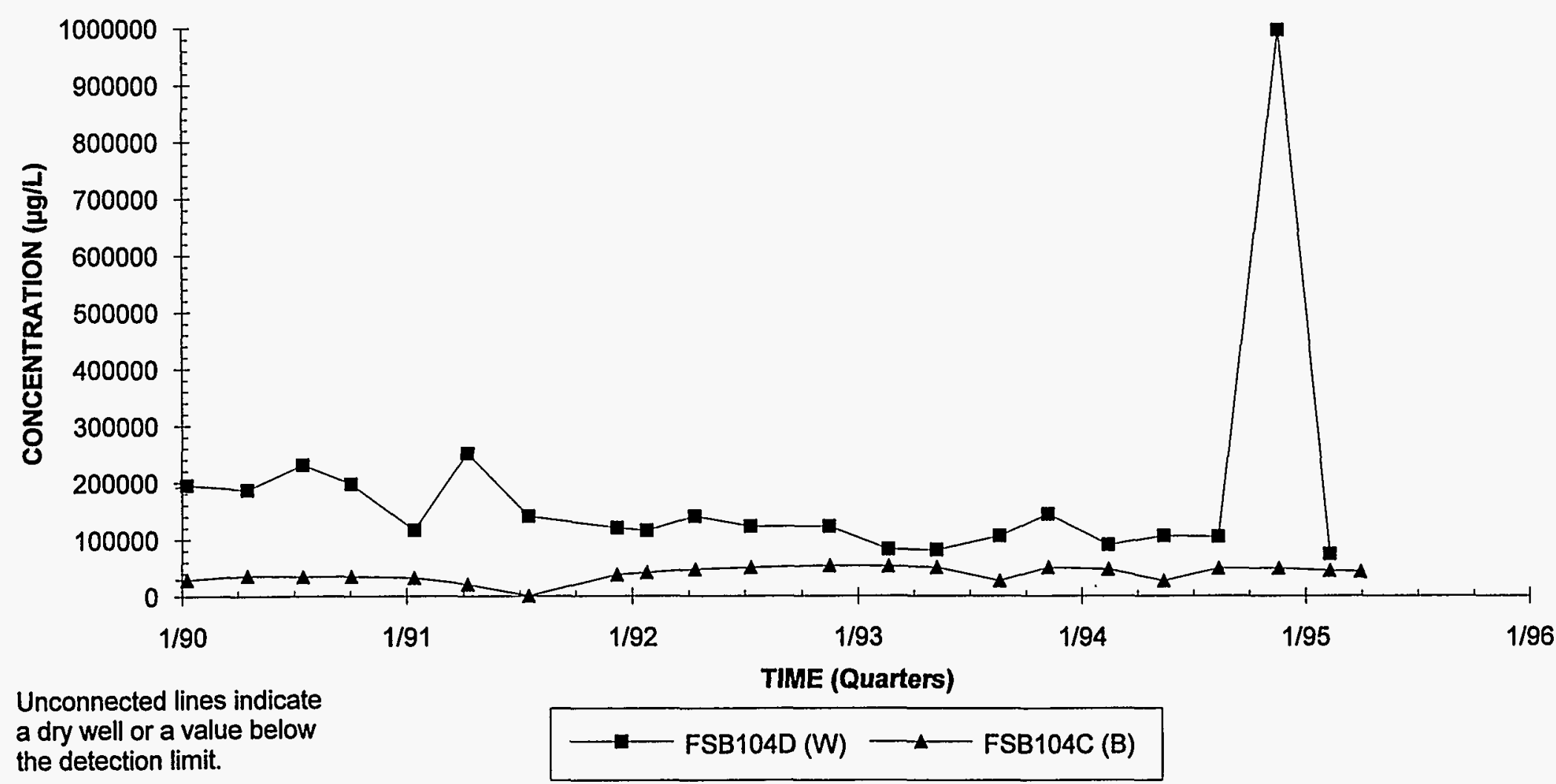

GWPS $10000 \mu \mathrm{g} / \mathrm{L}$ 
Nitrate Concentrations Well FSB110D

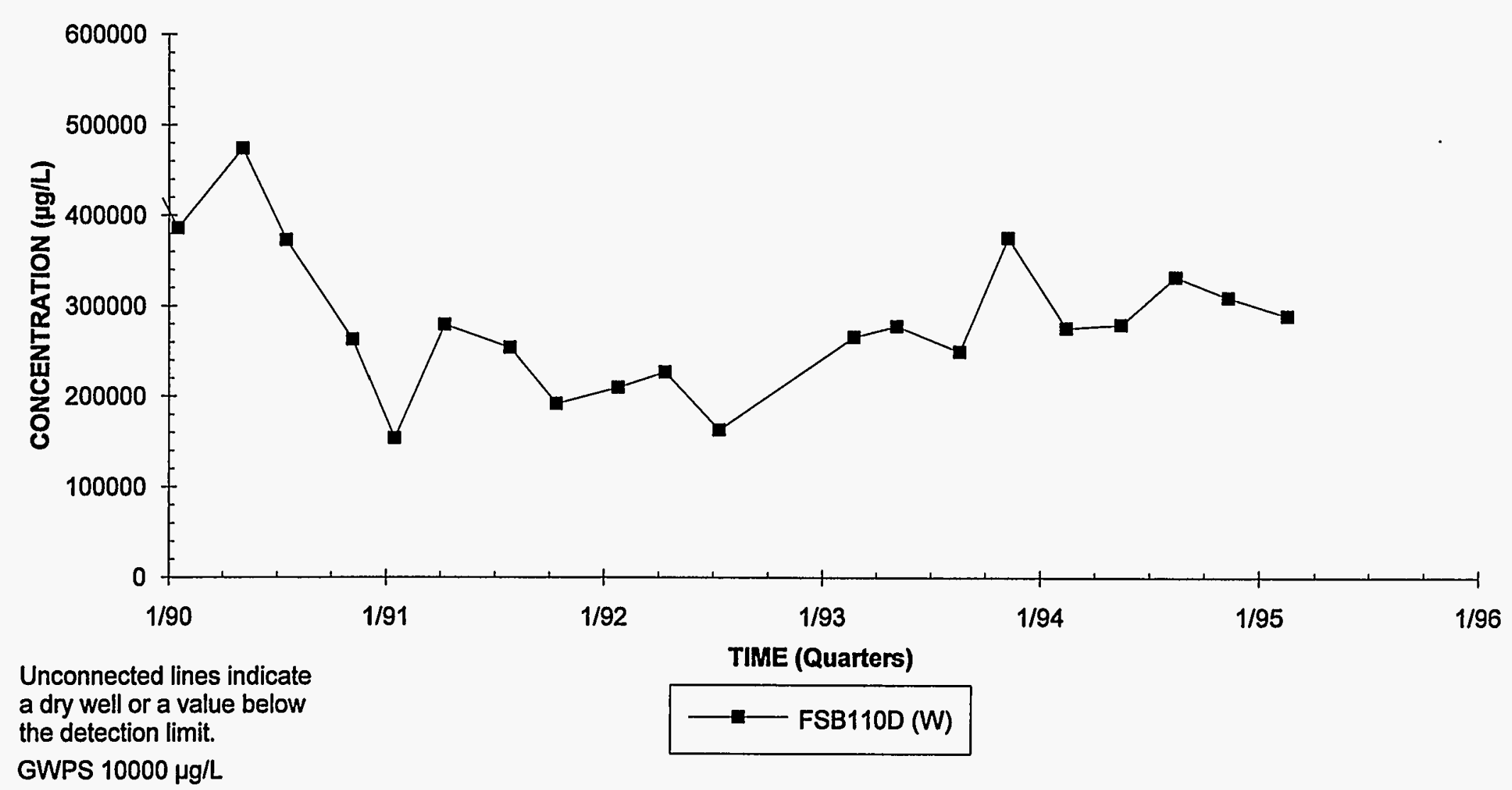

Note: W=Water Table (IIB2); B=Barnwell (IIB1); M=McBean (IIB1); UC=Upper Congaree (IIA); MC=Middle Congaree (IIA); LC=Lower Congaree (IIA) 


\section{Nitrate Concentrations \\ Well FSB117D}

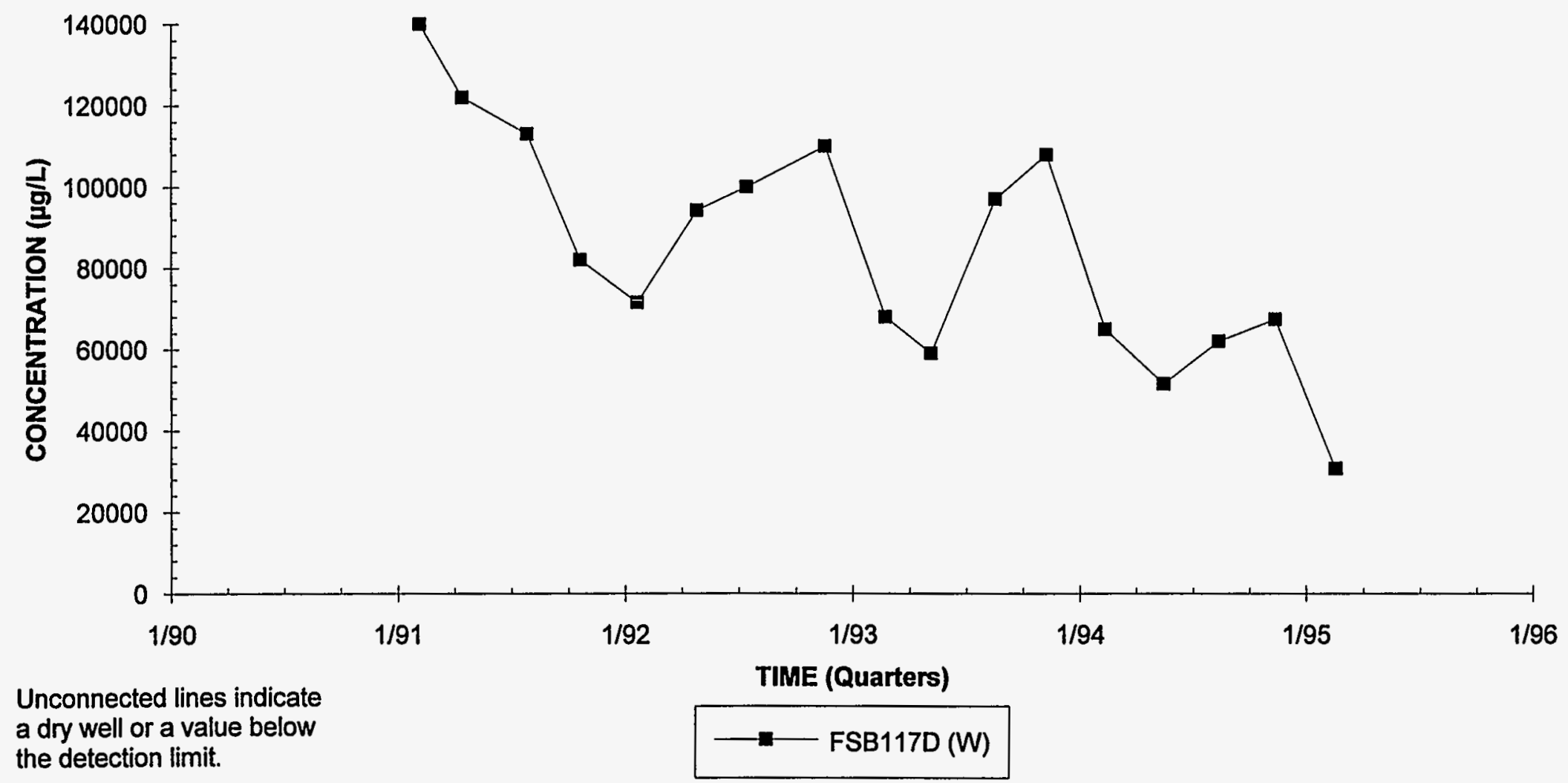

GWPS $10000 \mu \mathrm{g} / \mathrm{L}$ 


\section{Nitrate Concentrations}

Well FSB119D

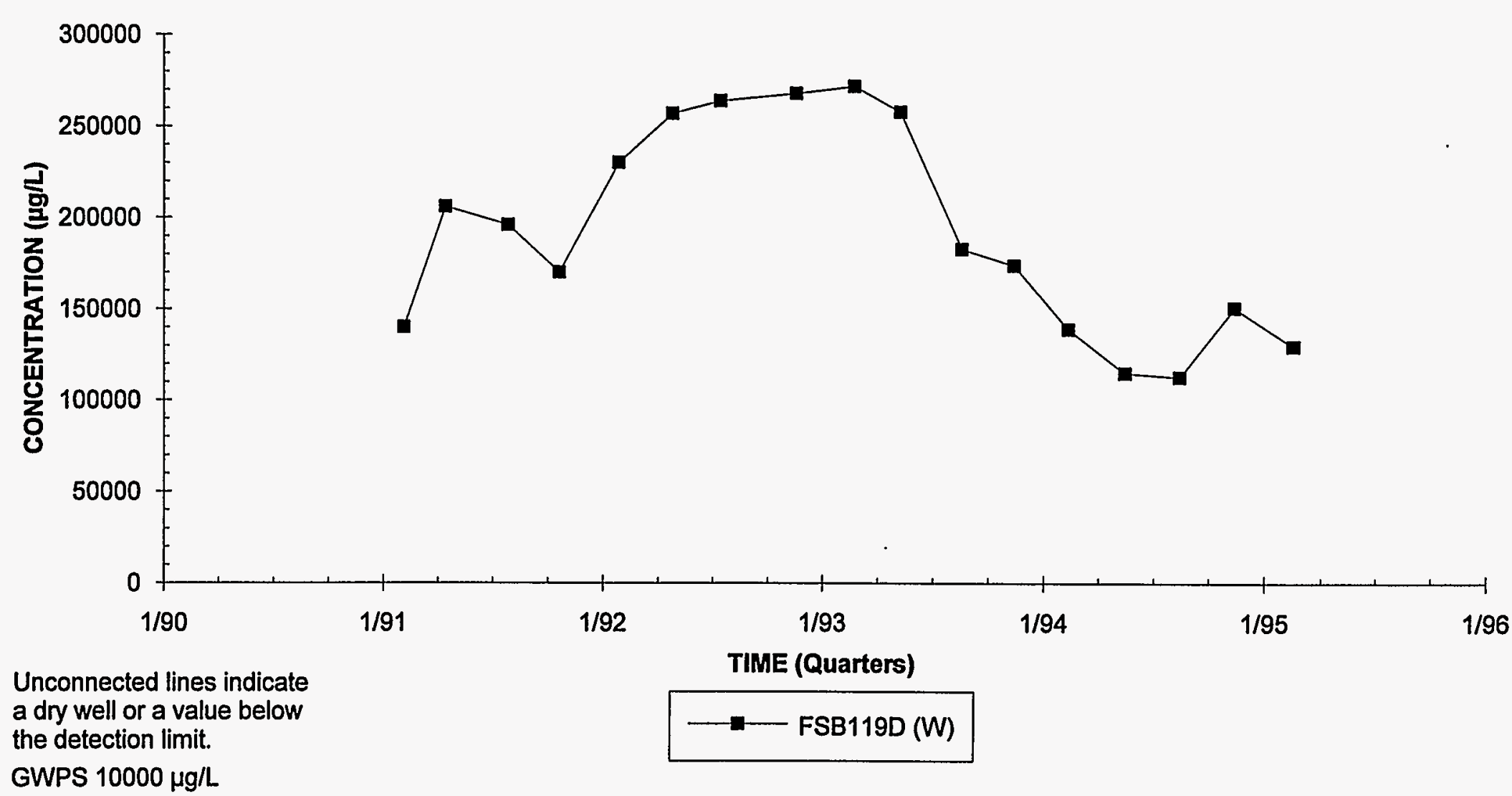

Note: $W=$ Water Table (IIB2); B=Barnwell (IIB1); $M=$ McBean (IIB1); UC=Upper Congaree (IIA); MC=Middle Congaree (IIA); LC=Lower Congaree (IIA) 


\section{Nitrate Concentrations \\ Well Cluster FSB120}

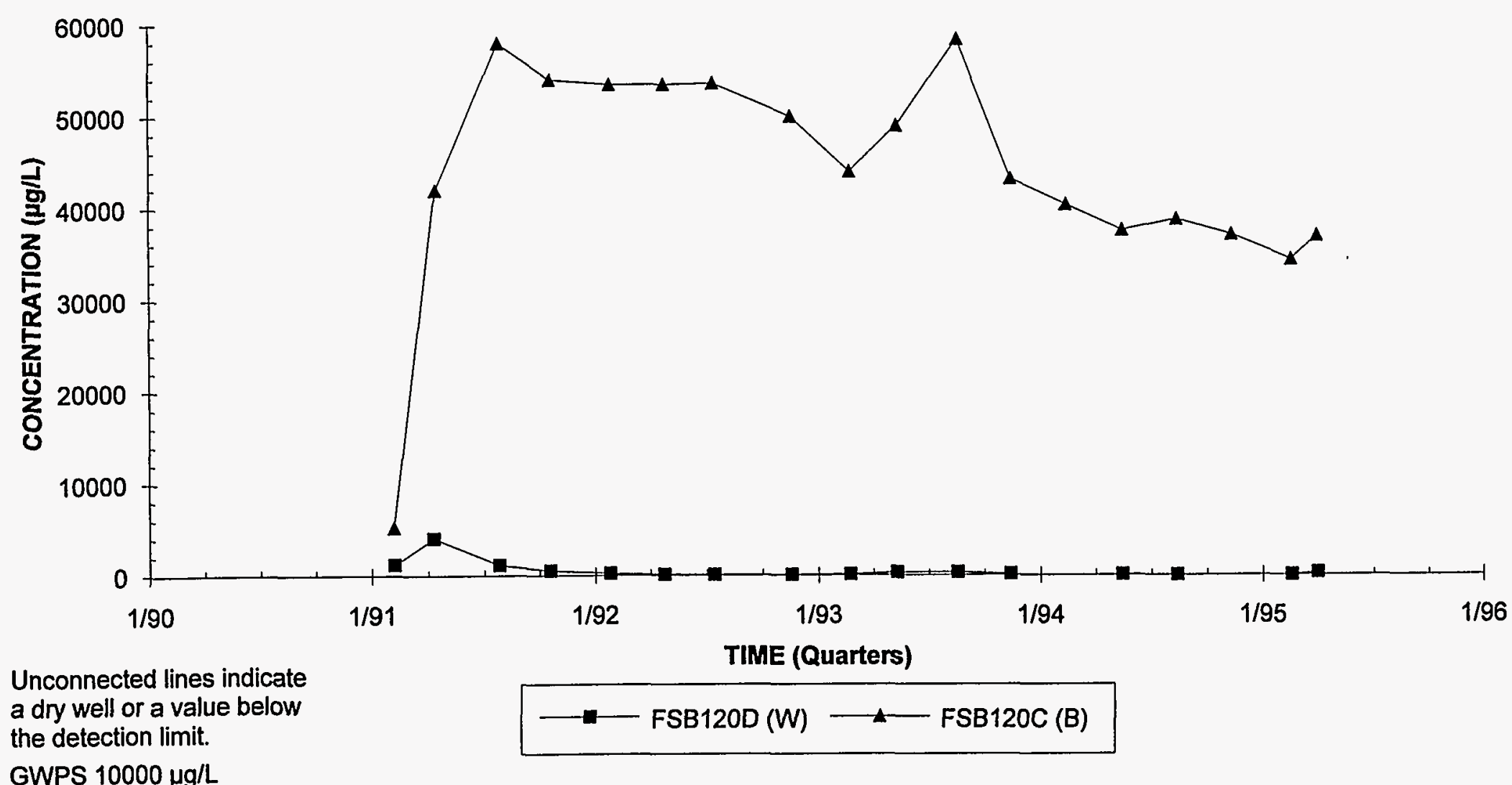

Note: $W=W a t e r$ Table (IIB2); B=Barnwell (IIB1); $M=M c B e a n$ (IIB1); UC=Upper Congaree (IIA); MC=Middle Congaree (IIA); LC=Lower Congaree (IIA) 


\section{Nitrate Concentrations}

Well Cluster FSB122

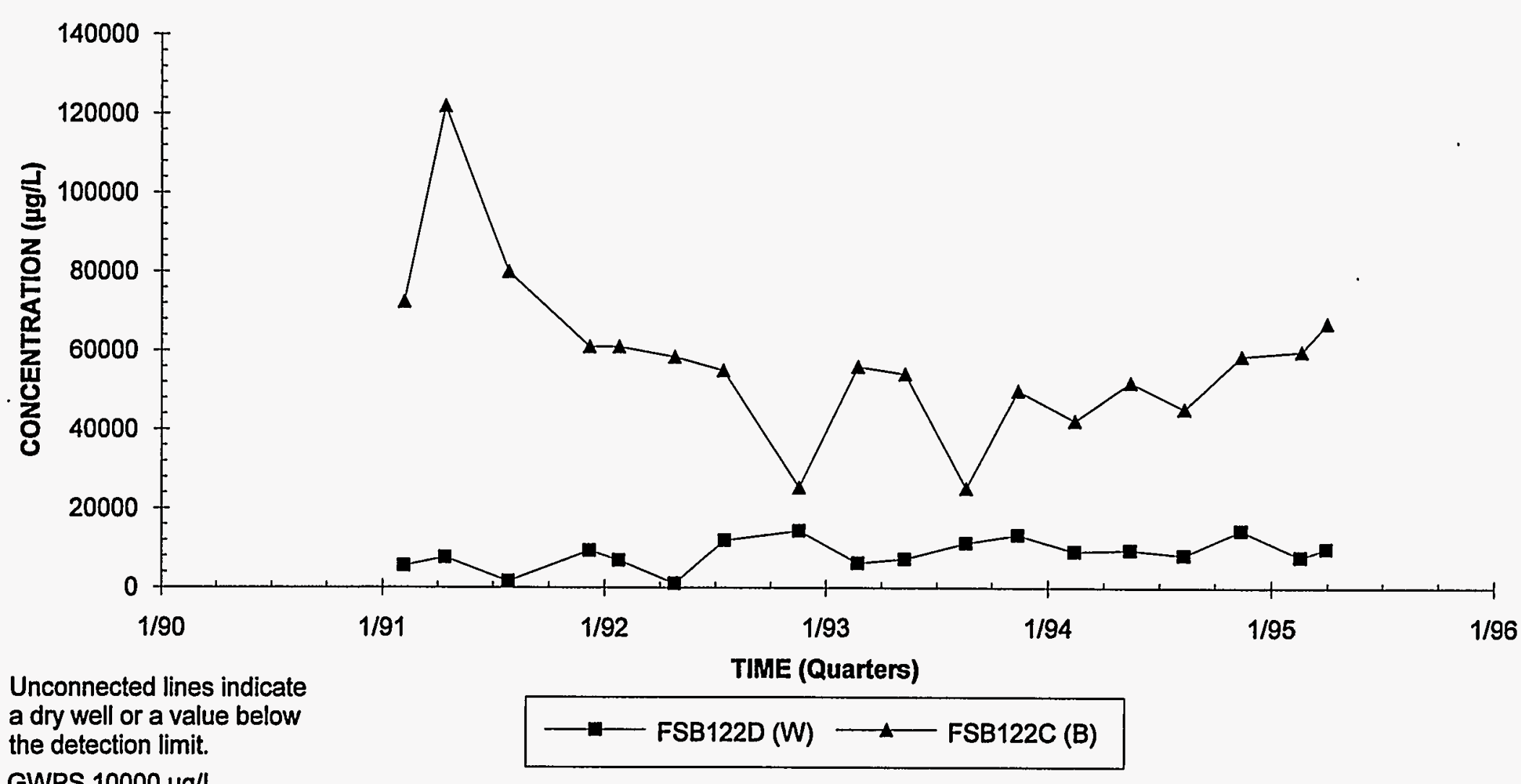

Note: $W=$ Water Table (IIB2); B=Barnwell (IIB1); M=McBean (IIB1); UC=Upper Congaree (IIA); MC=Middle Congaree (IIA); LC=Lower Congaree (IIA) 


\section{Nonvolatile Beta Activities}

\section{Well Cluster FSB 90}

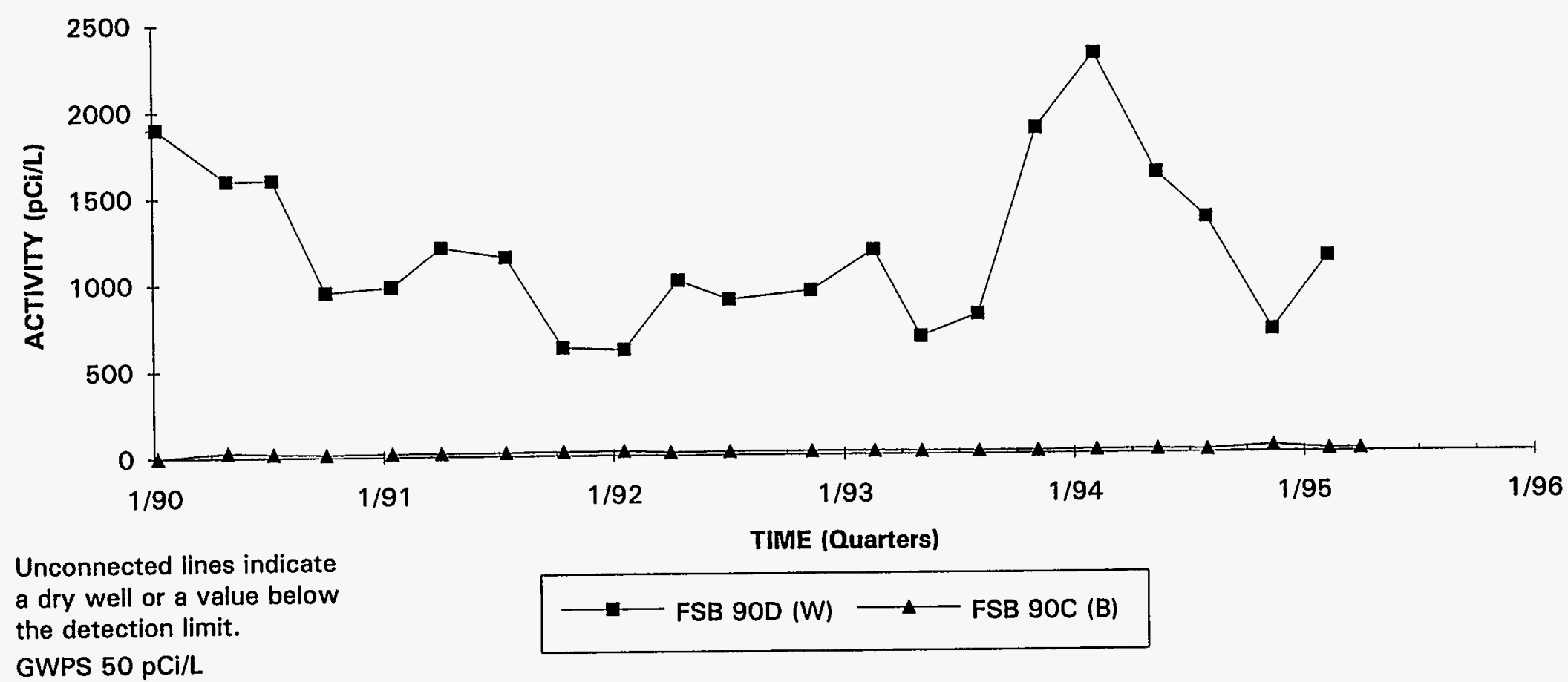

Note: W=Water Table (IIB2); B=Barnwell (IIB1); M=McBean (IIB1); UC=Upper Congaree (IIA); MC=Middle Congaree (IIA); LC=Lower Congaree (IIA) 

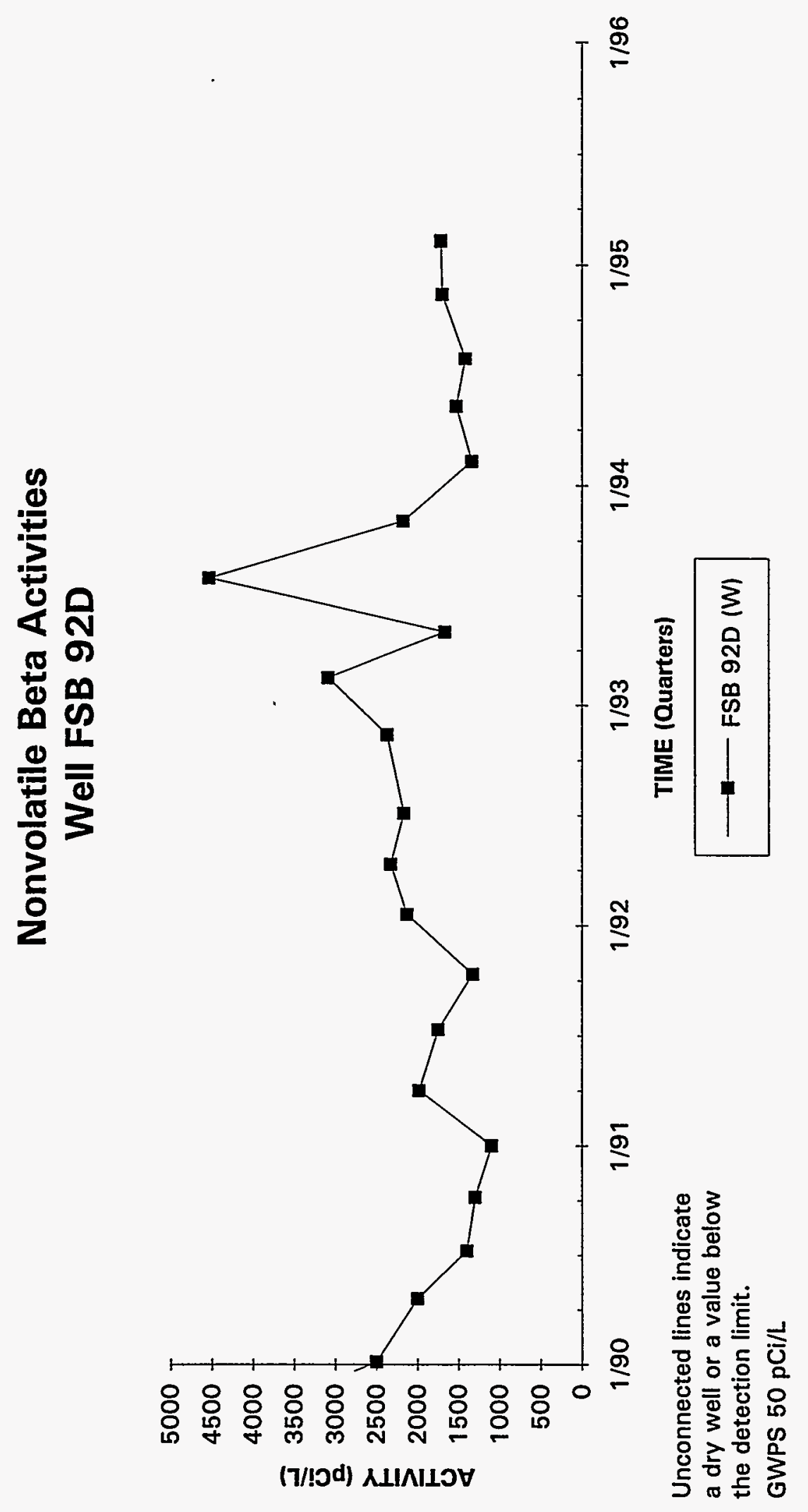

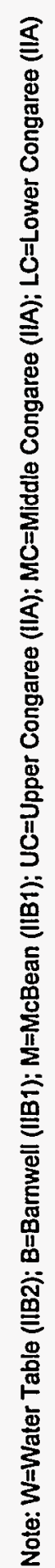

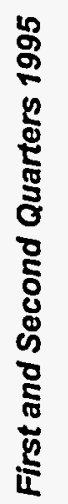




\section{Nonvolatile Beta Activities \\ Well Cluster FSB 94}

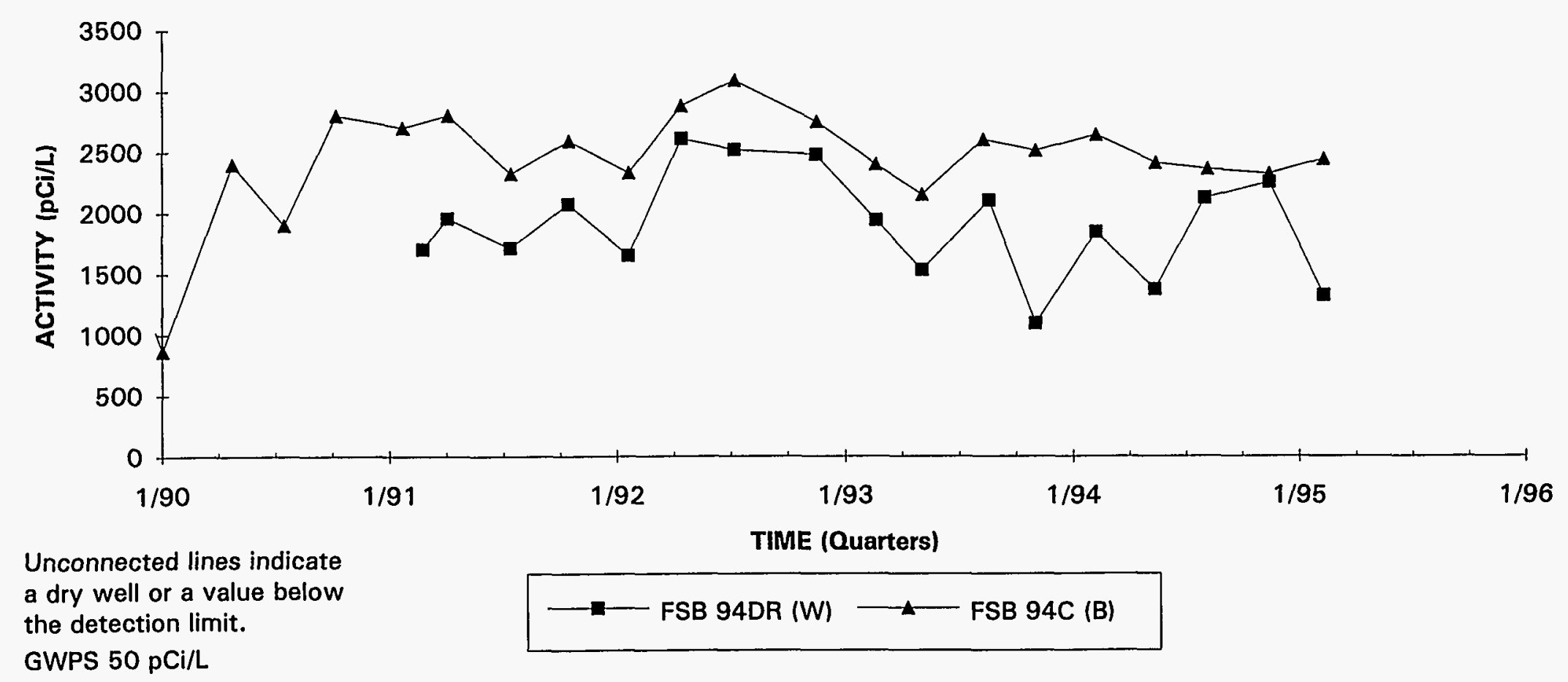

Note: W=Water Table (IIB2); B=Barnwell (IIB1); M=MCBean (IIB1); UC=Upper Congaree (IIA); MC=Middle Congaree (IIA); LC=Lower Congaree (IIA) 


\section{Nonvolatile Beta Activities \\ Well Cluster FSB 95}

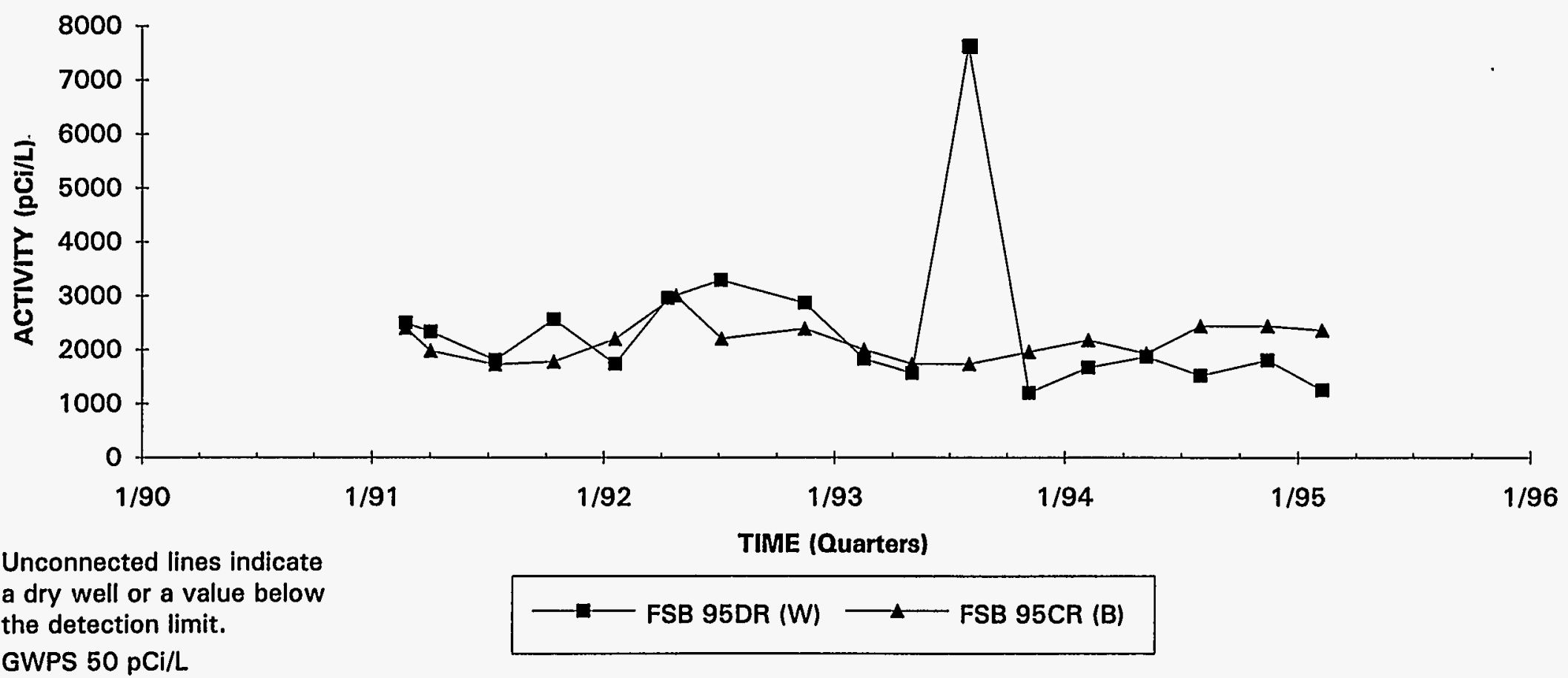

Note: W=Water Table (IIB2); B=Barnwell (IIB1); M=McBean (IIB1); UC=Upper Congaree (IIA); MC=Middle Congaree (IIA); LC=Lower Congaree (IIA) 


\section{Nonvolatile Beta Activities Well Cluster FSB 98}

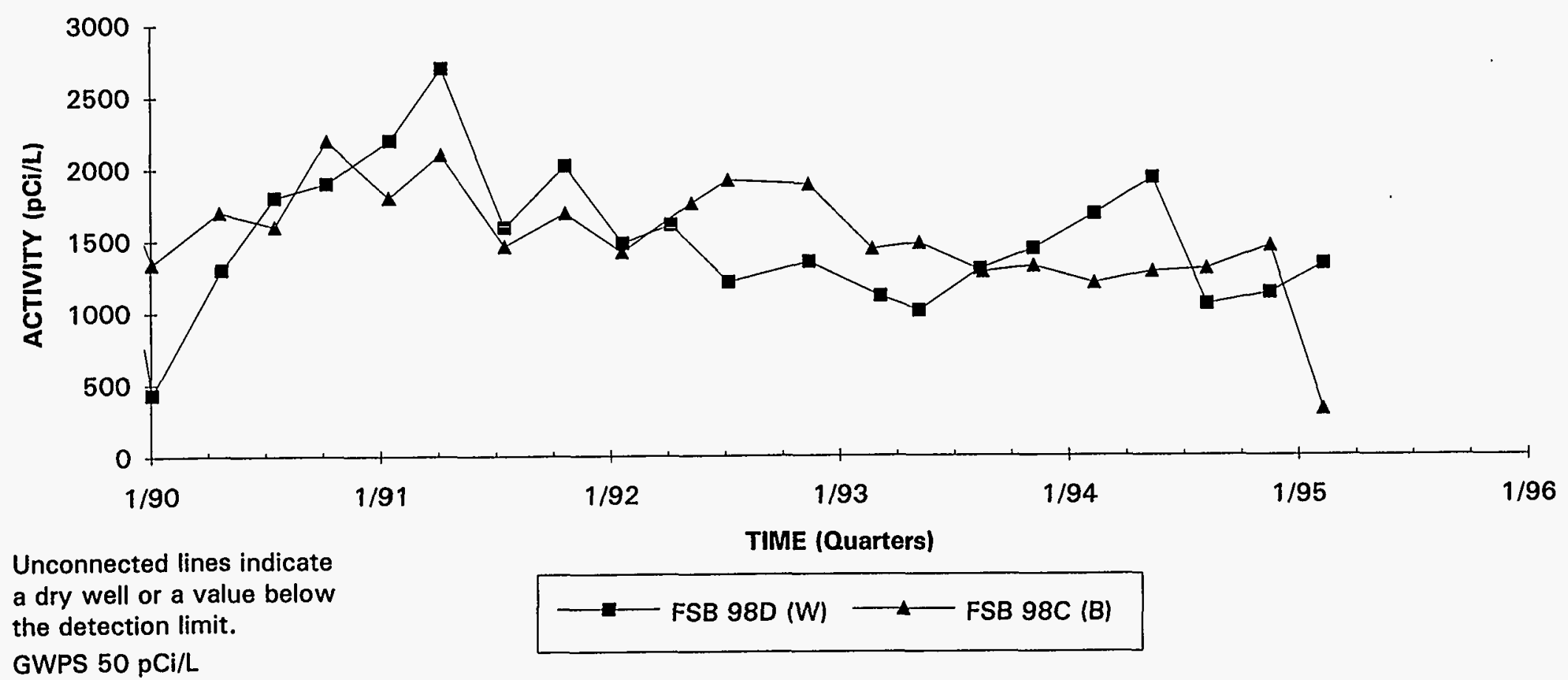

Note: W=Water Table (IIB2); B=Barnwell (IIB1); M=McBean (IIB1); UC=Upper Congaree (IIA); MC=Middle Congaree (IIA); LC=Lower Congaree (IIA) 


\section{Nonvolatile Beta Activities \\ Well Cluster FSB 99}

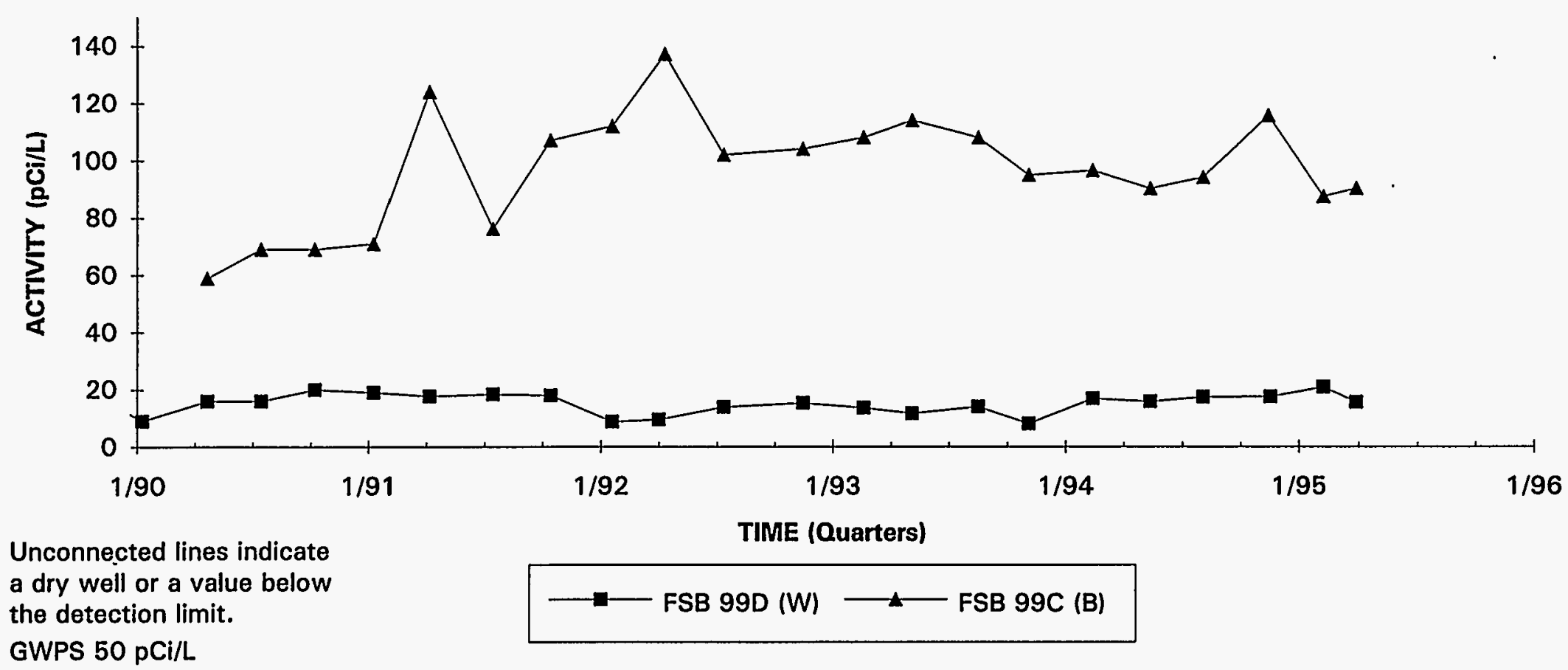

Note: W=Water Table (IIB2); B=Barnwell (IIB1); M=McBean (IIB1); UC=Upper Congaree (IIA); MC=Middle Congaree (IIA); LC=Lower Congaree (IIA) 

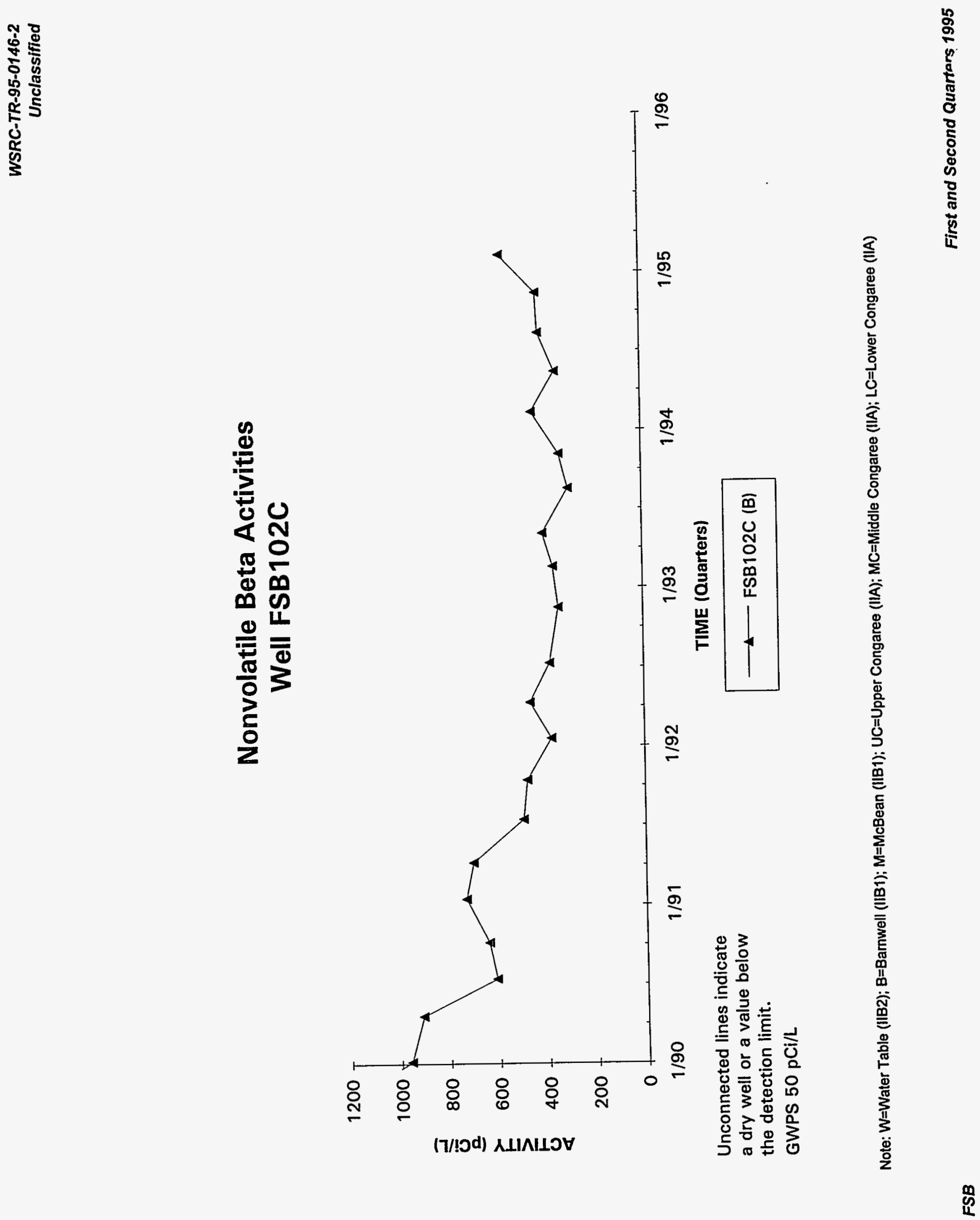


\section{Nonvolatile Beta Activities}

Well Cluster FSB104

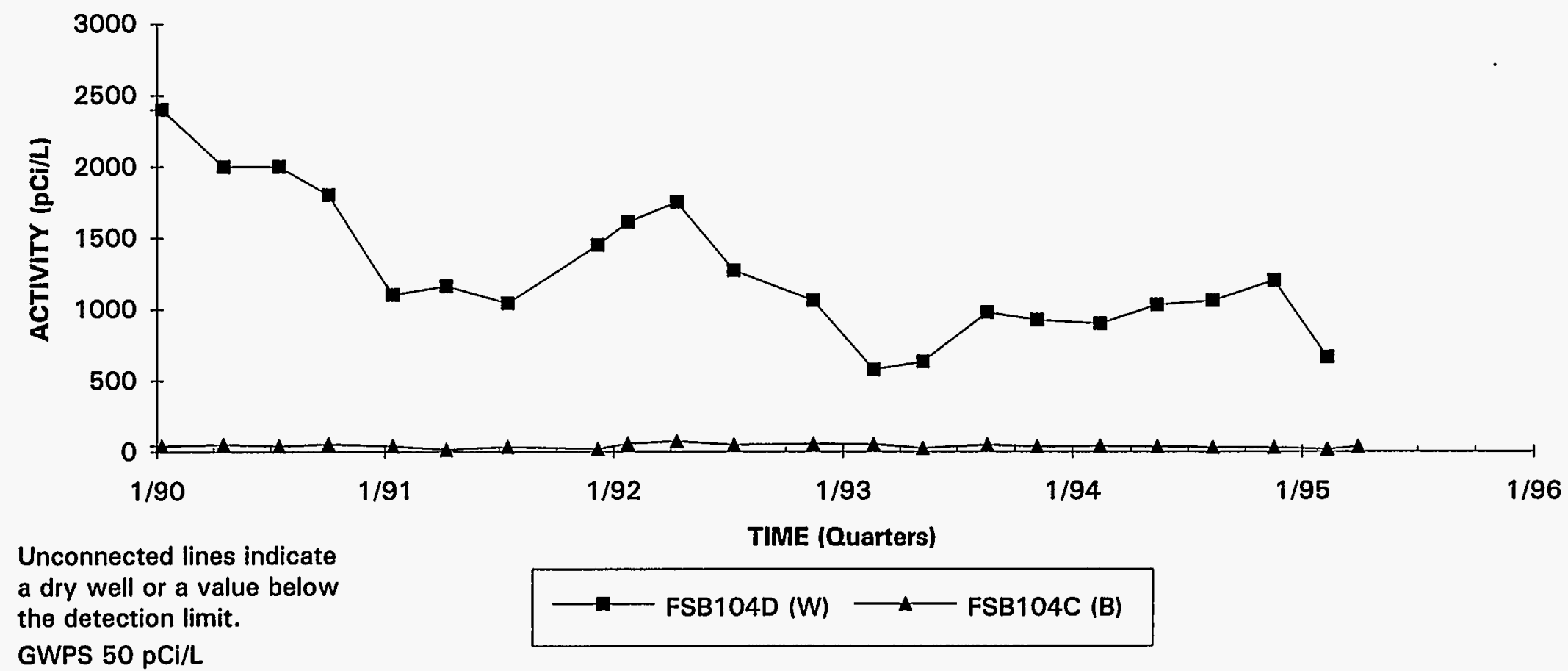

Note: W=Water Table (IIB2); B=Barnwell (IIB1); M=McBean (IIB1); UC=Upper Congaree (IIA); MC=Middle Congaree (IIA); LC=Lower Congaree (IIA) 


\section{Nonvolatile Beta Activities \\ Well Cluster FSB110}

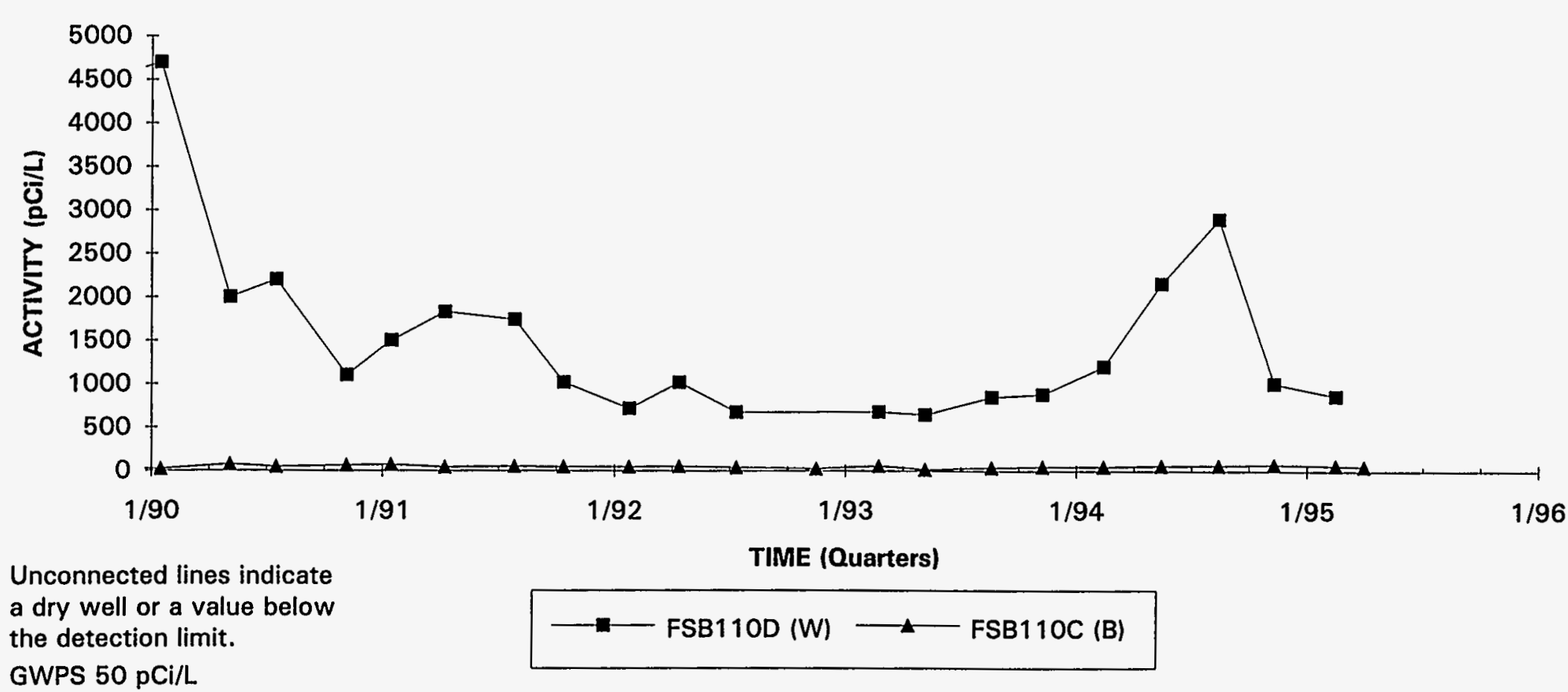

Note: W=Water Table (IIB2); B=Barnwell (IIB1); M=McBean (IIB1); UC=Upper Congaree (IIA); MC=Middle Congaree (IIA); LC=Lower Congaree (IIA) 


\section{Nonvolatile Beta Activities}

Well Cluster FSB122

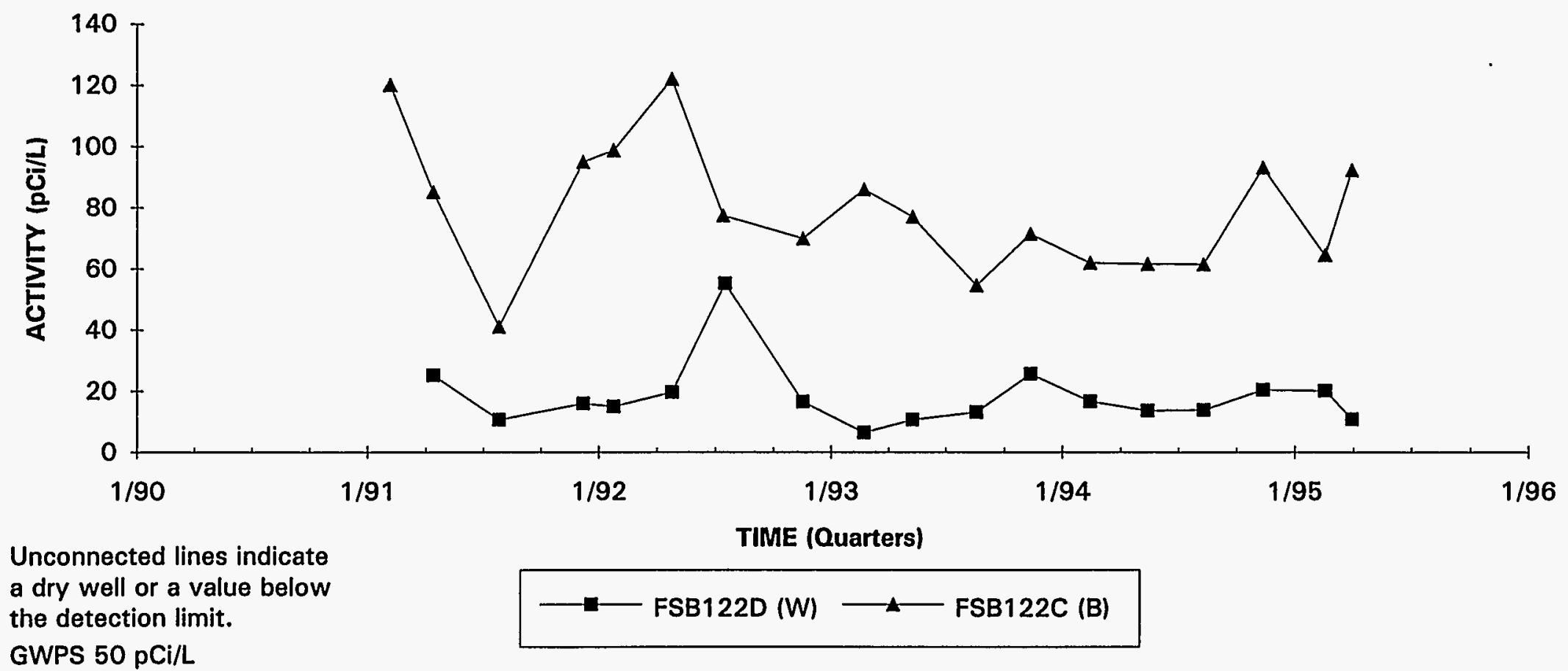

Note: W=Water Table (IIB2); B=Barnwell (IIB1); $M=$ McBean (IIB1); UC=Upper Congaree (IIA); MC=Middle Congaree (IIA); LC=Lower Congaree (IIA) 


\section{Radium-226 Activities \\ Well Cluster FSB 78}

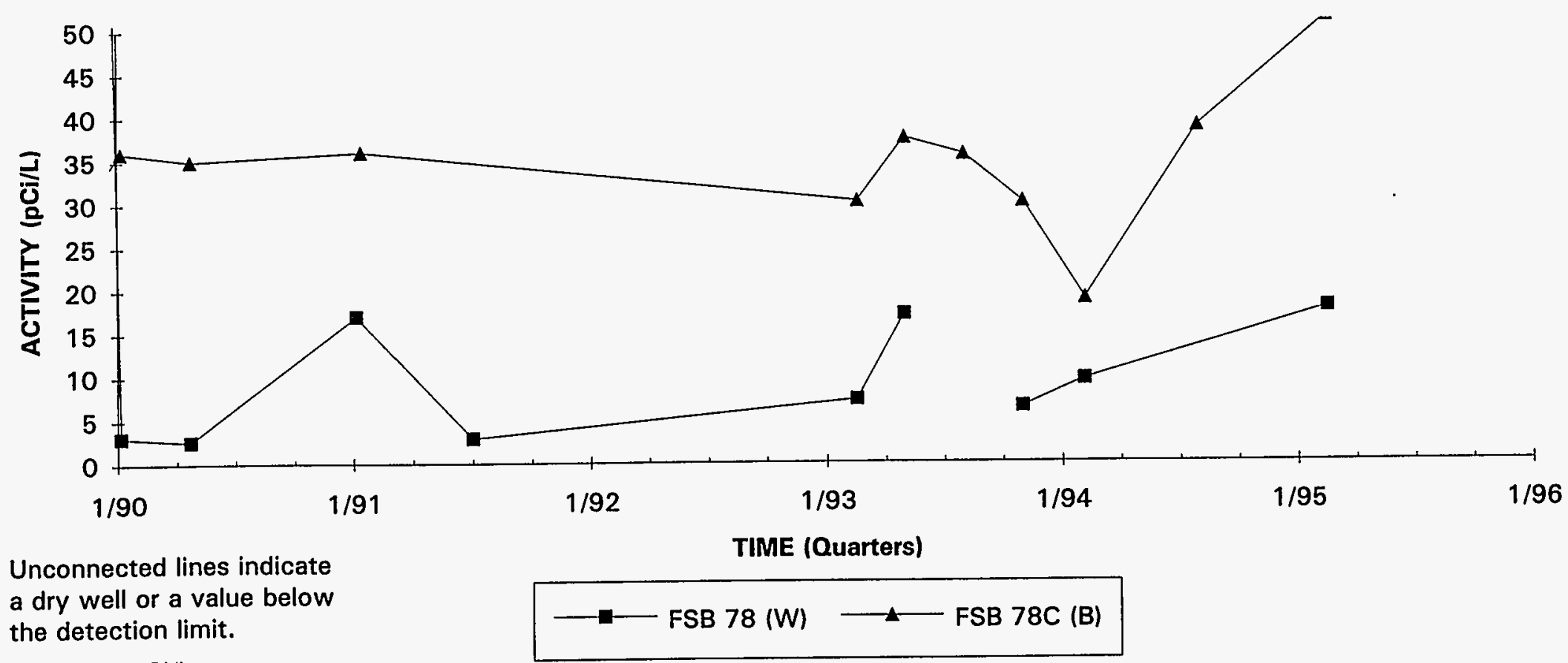

GWPS $20 \mathrm{pCi} / \mathrm{L}$ 


\section{Radium-226 Activities \\ Well Cluster FSB 79}

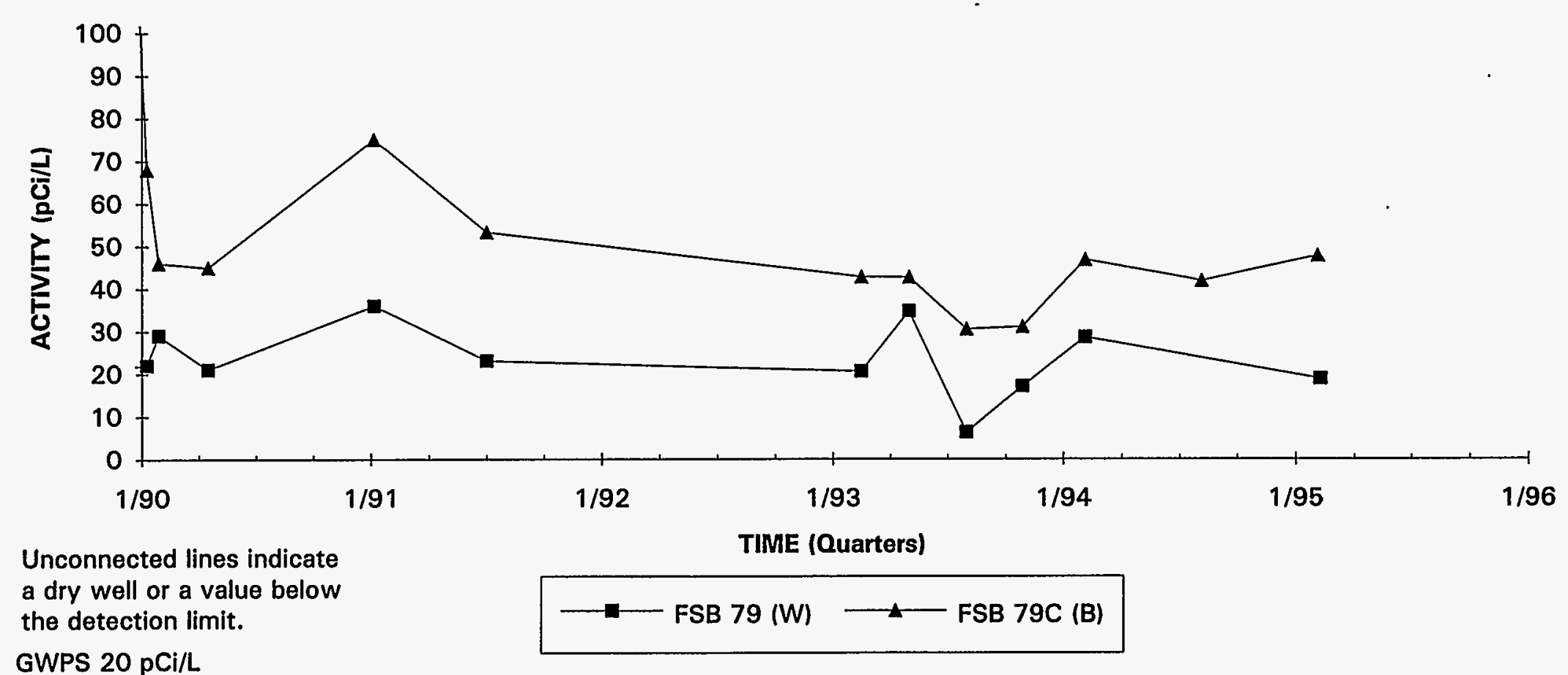

Note: $W=$ Water Table (IIB2); B=Barnwell (IIB1); M=McBean (IIB1); UC=Upper Congaree (IIA); MC=Middle Congaree (IIA); LC=Lower Congaree (IIA) 


\section{Radium-226 Activities \\ Well FSB 90D}

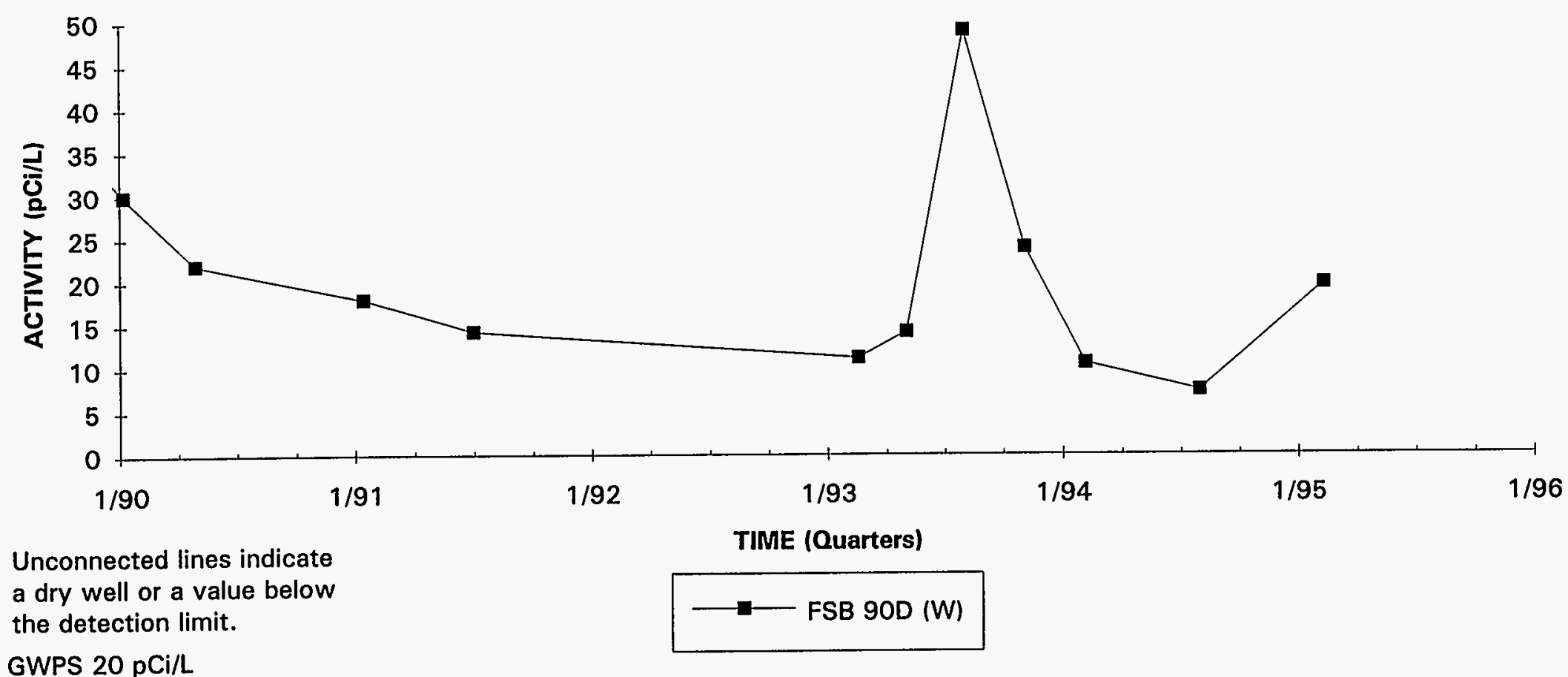

GWPS $20 \mathrm{pCi} / \mathrm{L}$ 


\section{Radium-226 Activities \\ Well FSB 92D}

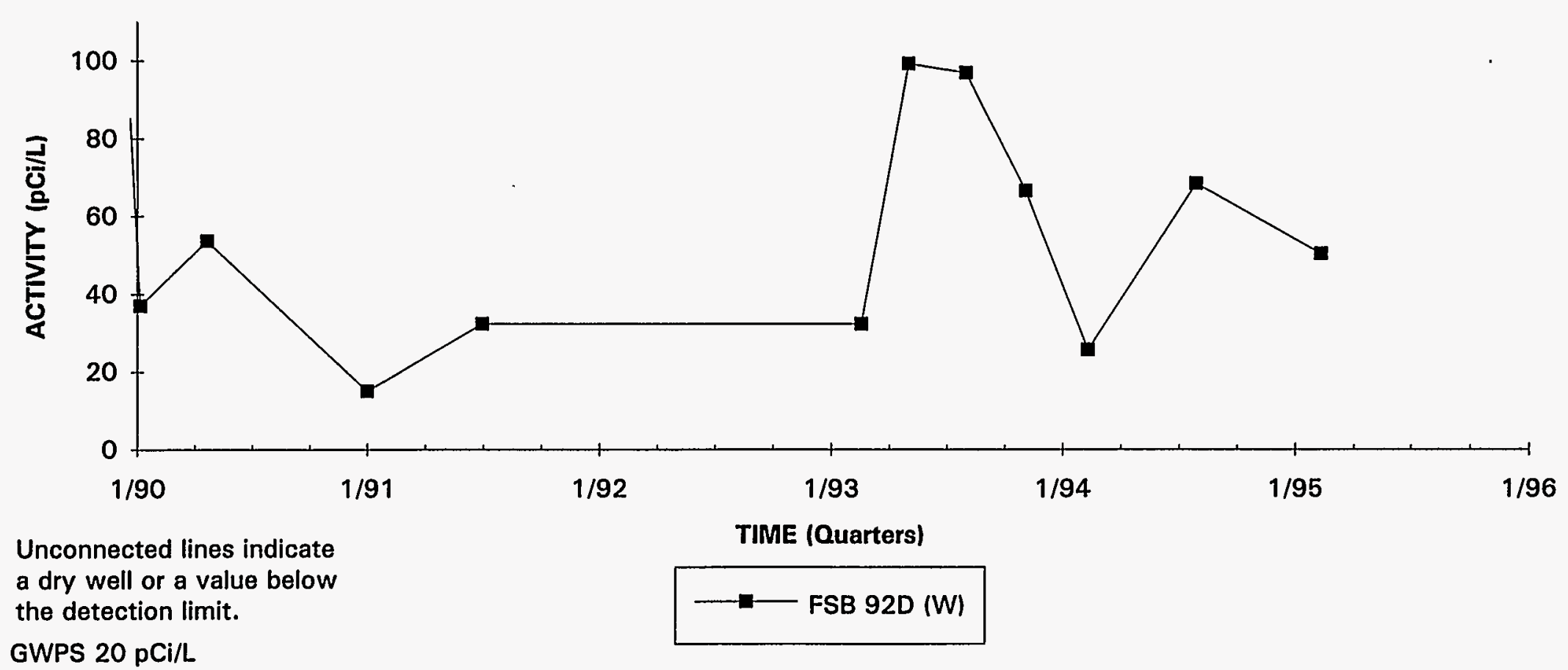

Note: W=Water Table (IIB2); B=Barnwell (IIB1); M=McBean (IIB1); UC=Upper Congaree (IIA); MC=Middle Congaree (IIA); LC=Lower Congaree (IIA) 


\section{Radium-226 Activities \\ Well FSB102C}

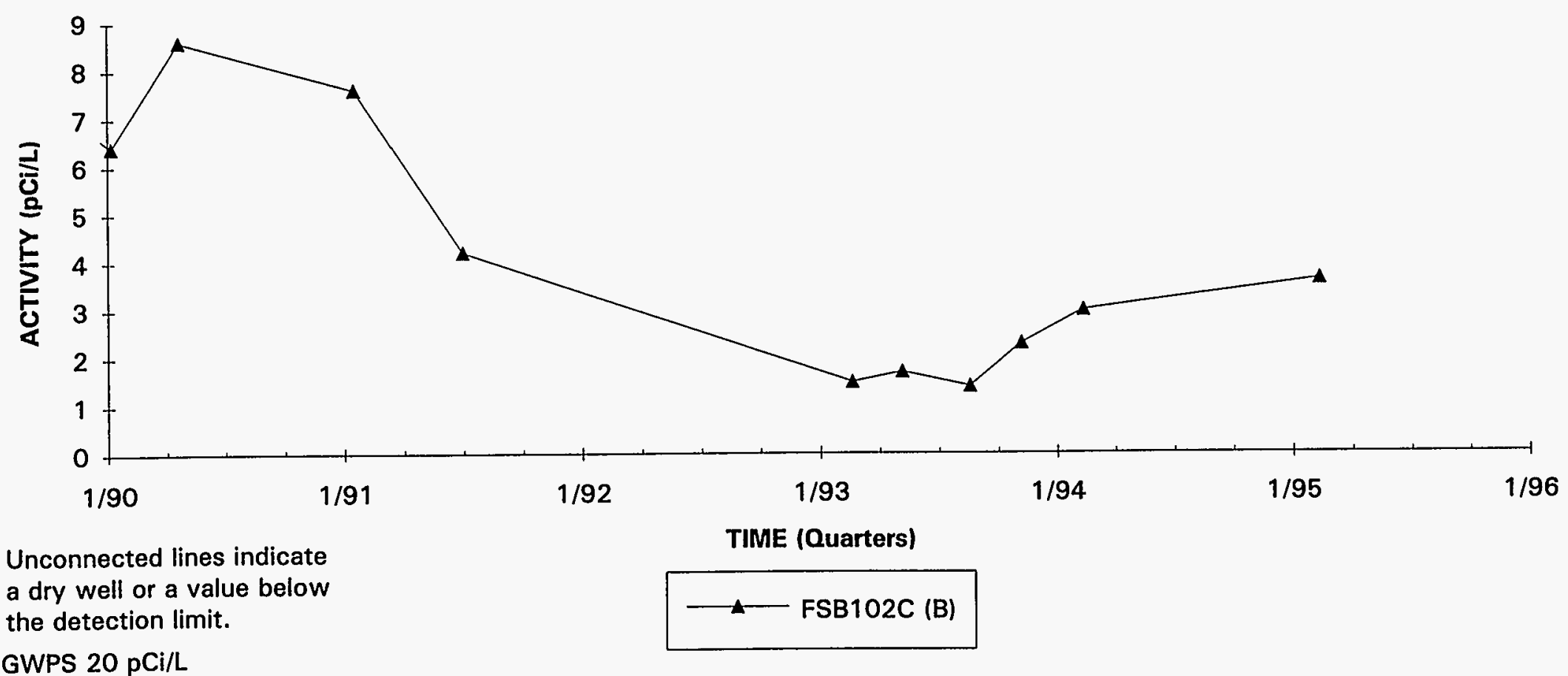

GWPS $20 \mathrm{pCi} / \mathrm{L}$ 


\section{Radium-226 Activities}

Well Cluster FSB112

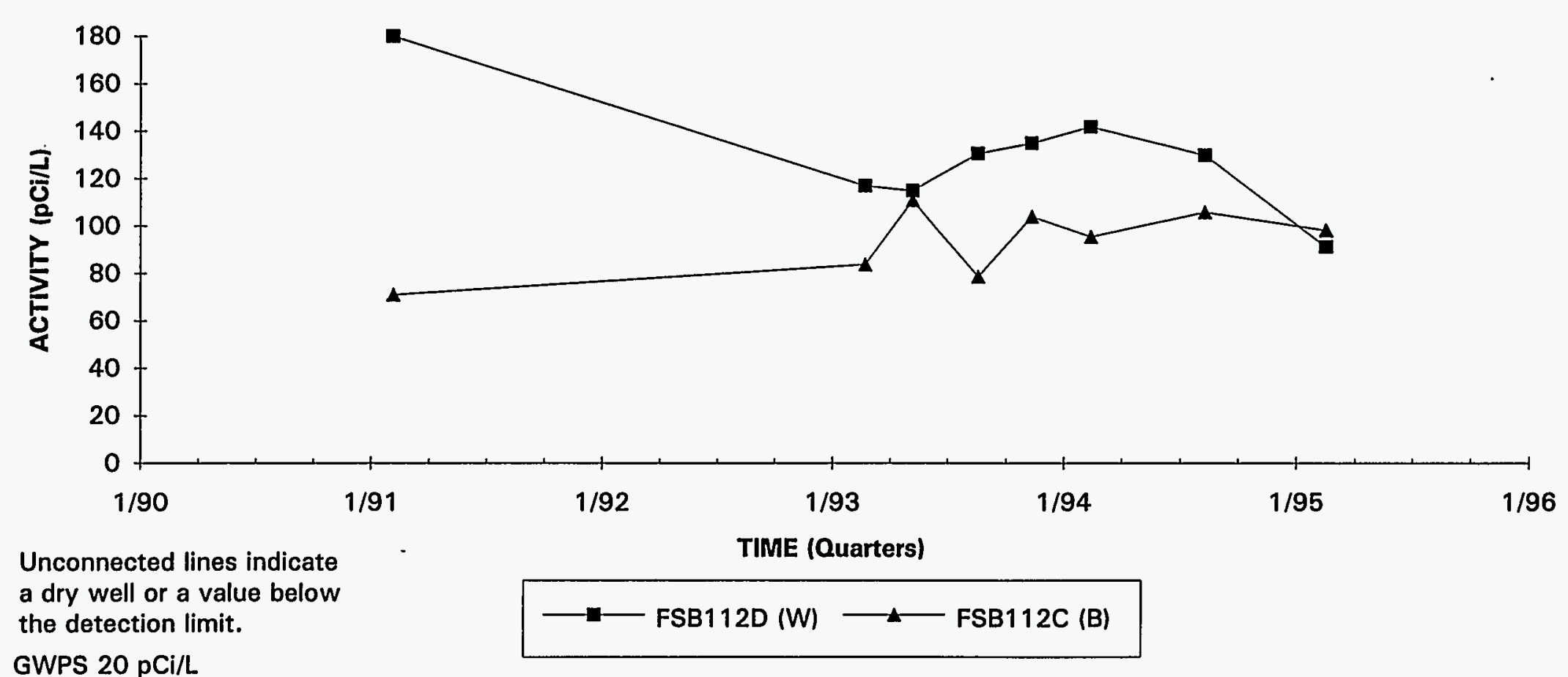

GWPS $20 \mathrm{pCi} / \mathrm{L}$ 


\section{Radium-226 Activities \\ Well FSB117D}

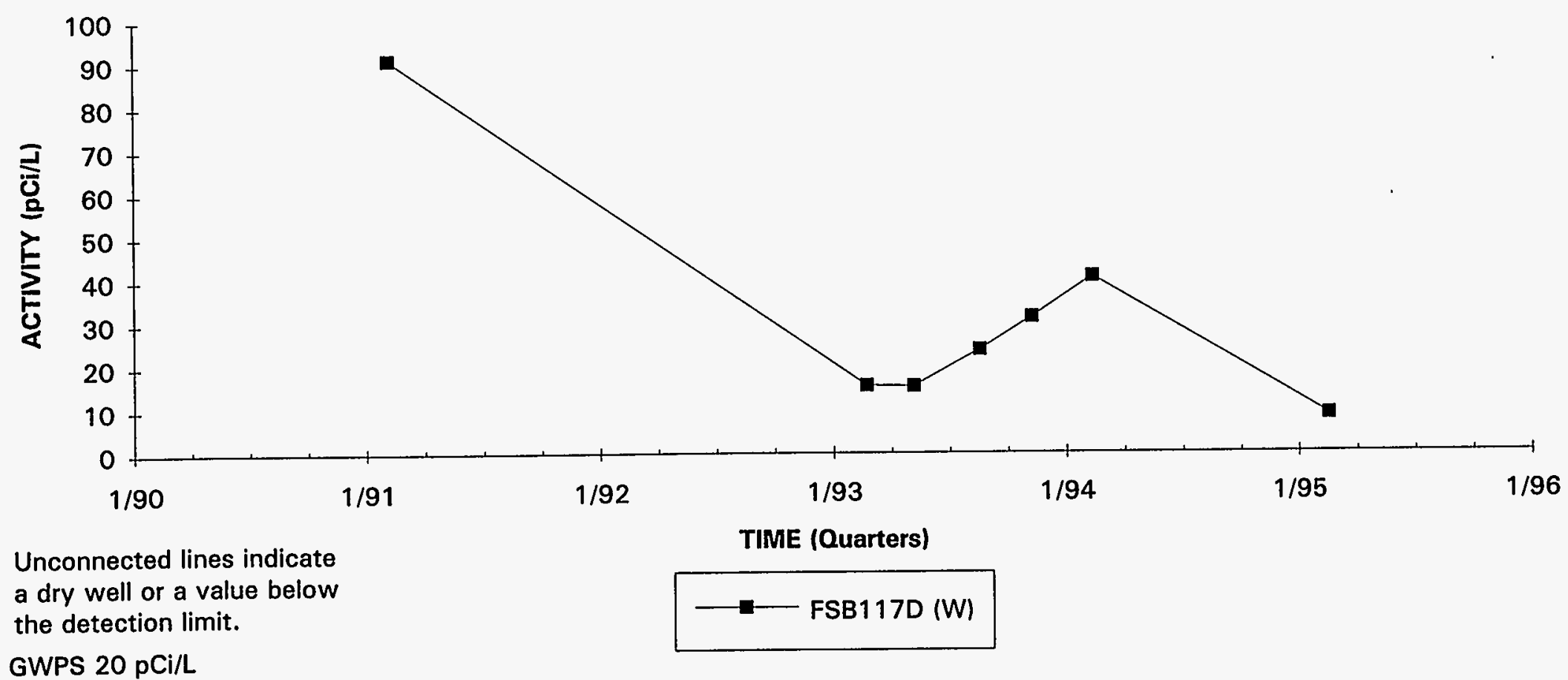

Note: W=Water Table (IIB2); B=Barnwell (IIB1); M=McBean (IIB1); UC=Upper Congaree (IIA); MC=Middle Congaree (IIA); LC=Lower Congaree (IIA) 


\section{Radium-226 Activities}

Well FSB1 19D

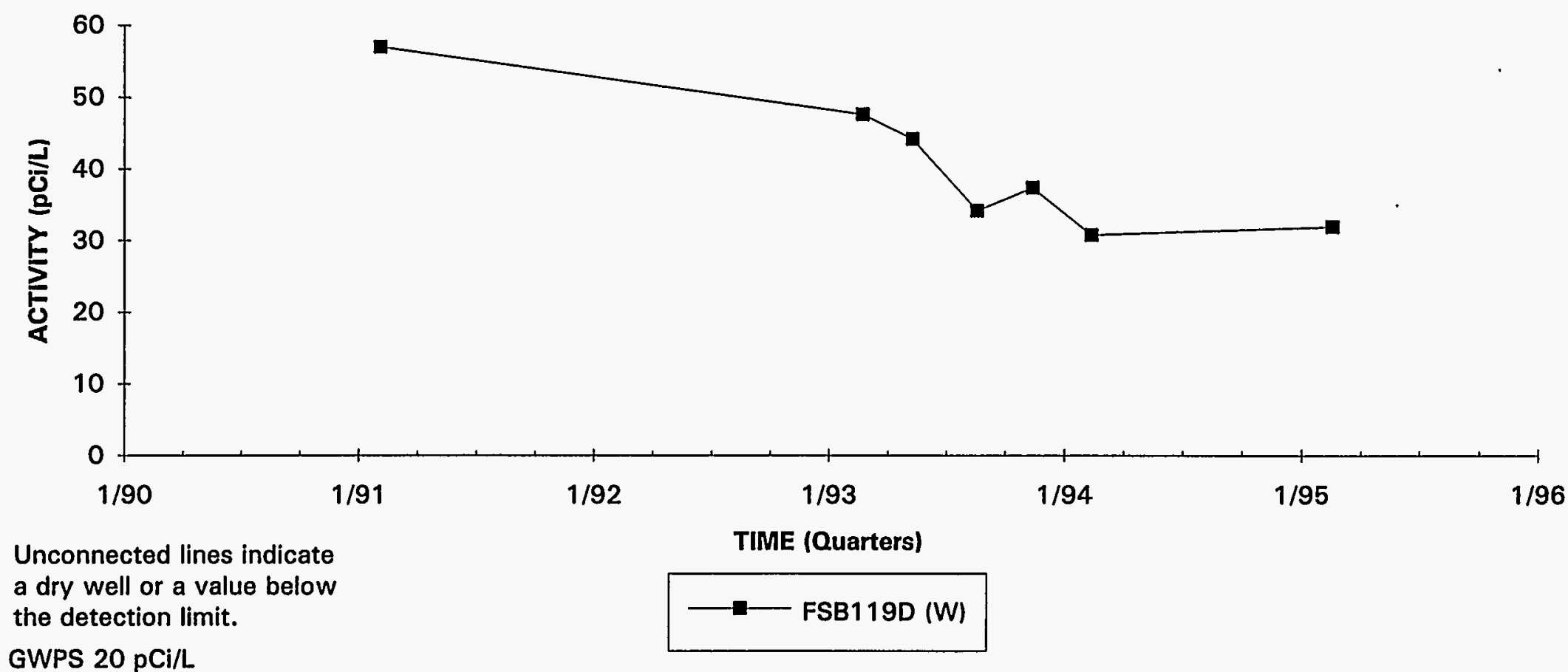

Note: W=Water Table (IIB2); B=Barnwell (IIB1); M=McBean (IIB1); UC=Upper Congaree (IIA); MC=Middle Congaree (IIA); LC=Lower Congaree (IIA) 


\section{Radium-228 Activities \\ Well Cluster FSB 78}

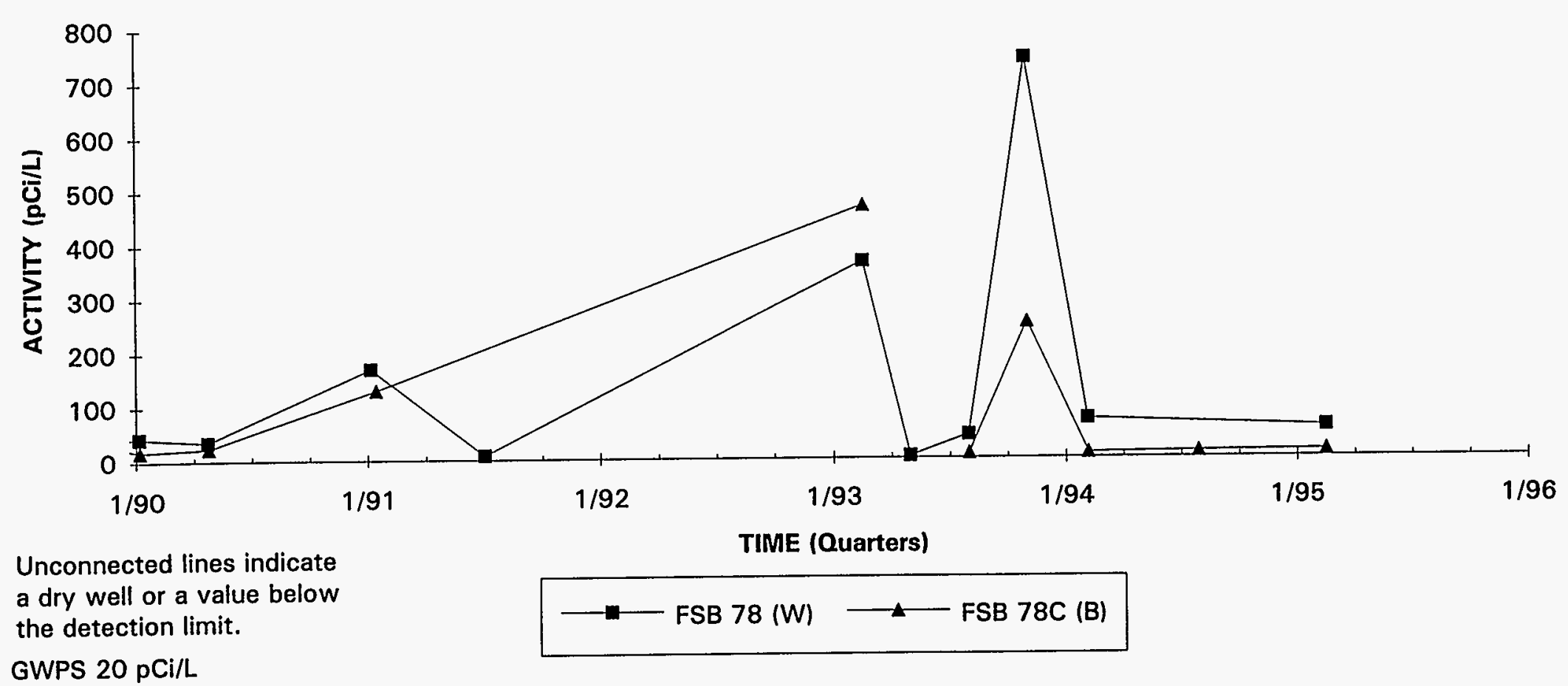

Note: $W=$ Water Table (IIB2); B=Barnwell (IIB1); $M=$ McBean (IIB1); UC=Upper Congaree (IIA); MC=Middle Congaree (IIA); LC=Lower Congaree (IIA) 


\section{Radium-228 Activities \\ Well Cluster FSB 79}

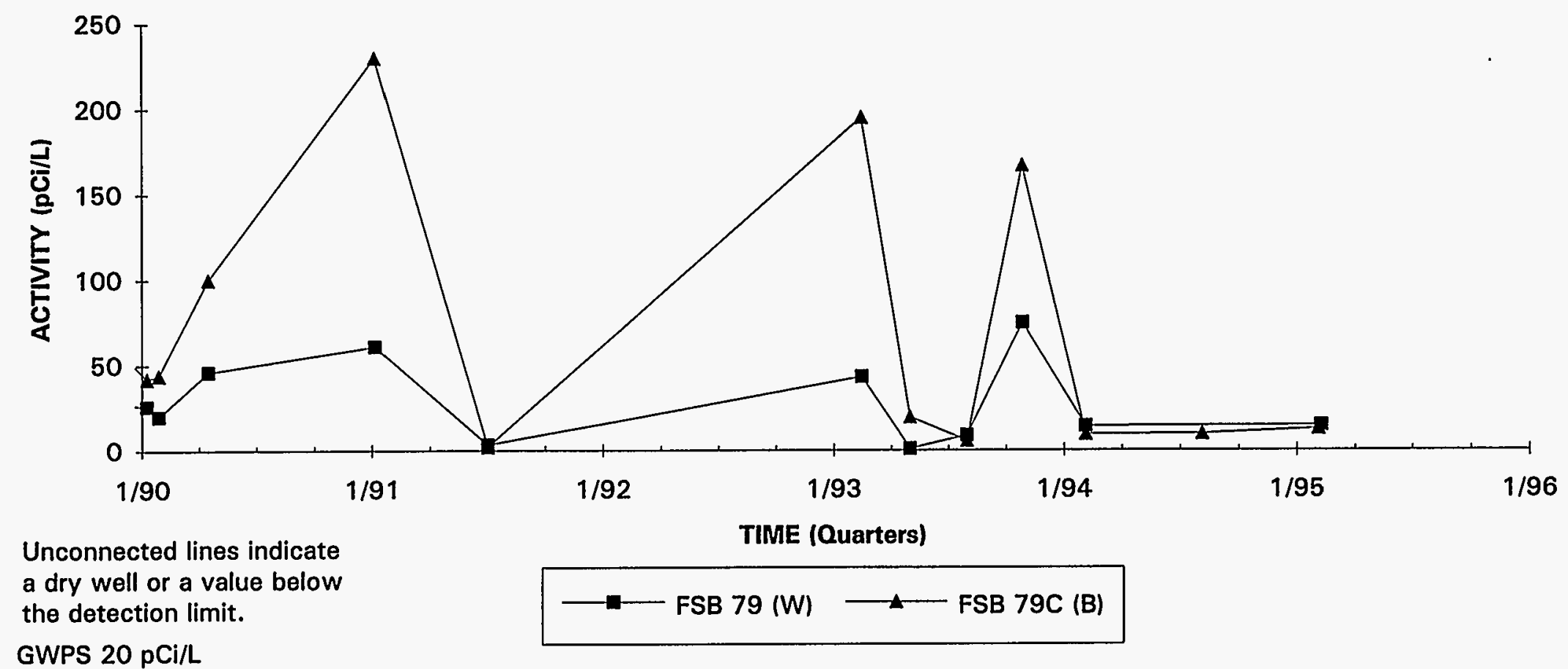

GWPS $20 \mathrm{pCi} / \mathrm{L}$

Note: W=Water Table (IIB2); B=Barnwell (IIB1); M=McBean (IIB1); UC=Upper Congaree (IIA); MC=Middle Congaree (IIA); LC=Lower Congaree (IIA) 


\section{Radium-228 Activities}

Well FSB 89D

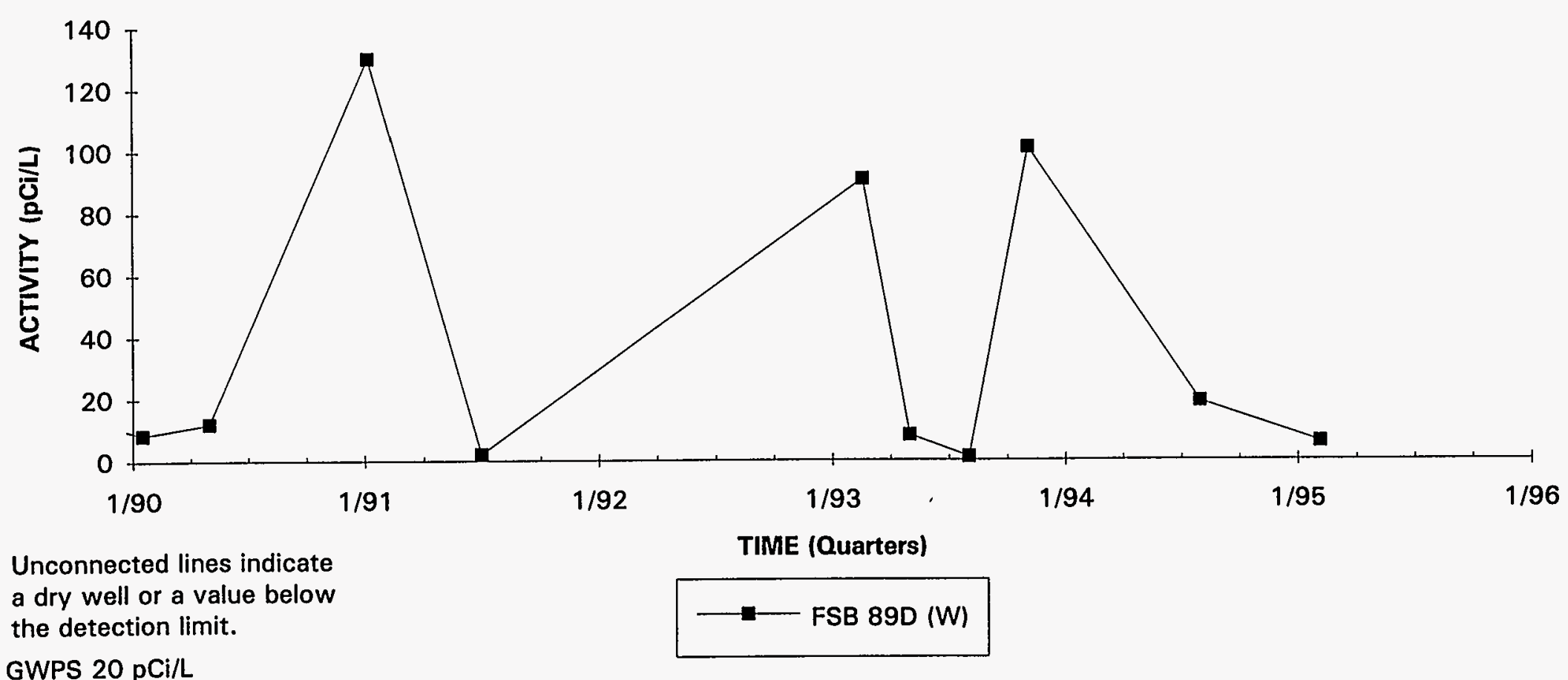

GWPS $20 \mathrm{pCi} / \mathrm{L}$

Note: $W=$ Water Table (IIB2); B=Barnwell (IIB1); M=McBean (IIB1); UC=Upper Congaree (IIA); MC=Middle Congaree (IIA); LC=Lower Congaree (IIA) 


\section{Radium-228 Activities}

Well FSB 90D

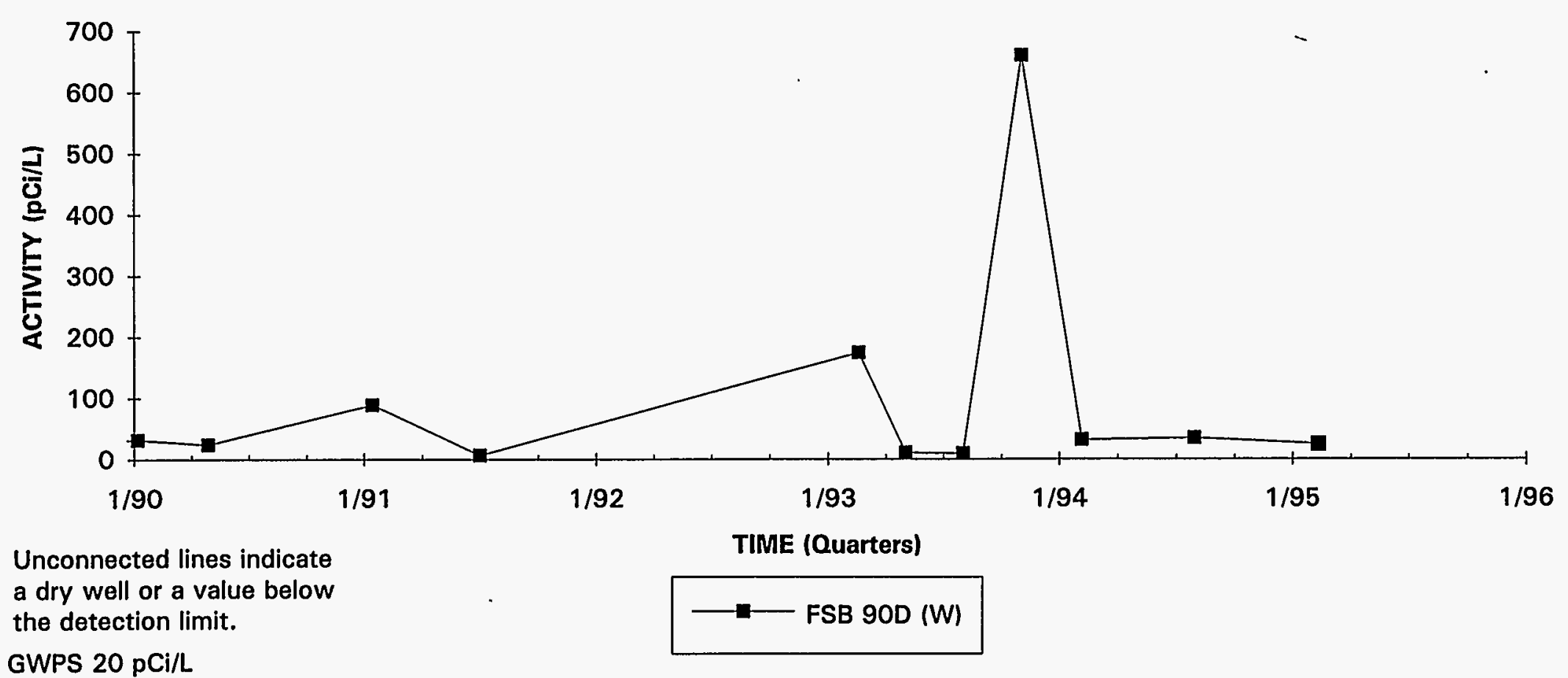

Note: W=Water Table (IIB2); B=Barnwell (IIB1); $M=$ McBean (IIB1); UC=Upper Congaree (IIA); MC=Middle Congaree (IIA); LC=Lower Congaree (IIA) 


\section{Radium-228 Activities \\ Well Cluster FSB 91}

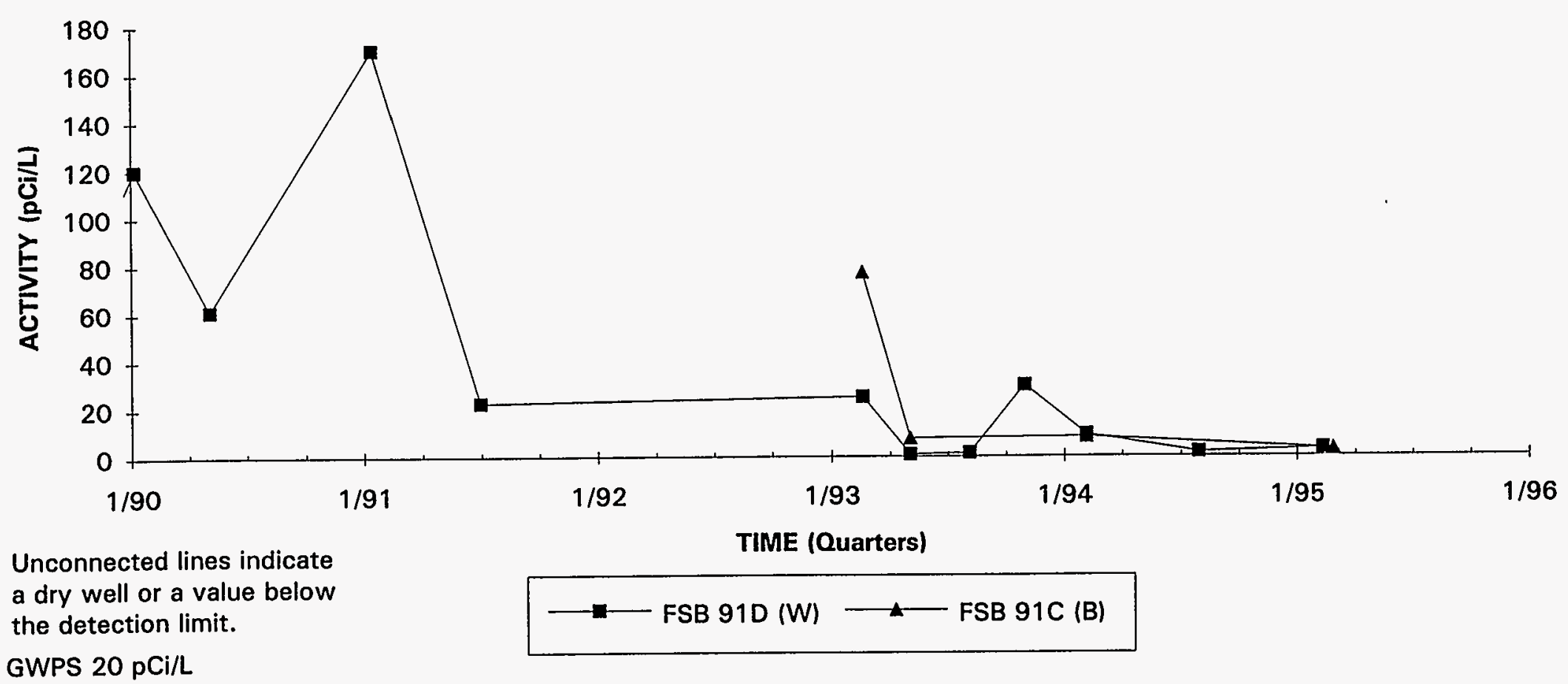

Note: $W=$ Water Table (IIB2); B=Barnwell (IIB1); $M=M c B e a n$ (IIB1); UC=Upper Congaree (IIA); MC=Middle Congaree (IIA); LC=Lower Congaree (IIA) 


\section{Radium-228 Activities}

\section{Well FSB 92D}

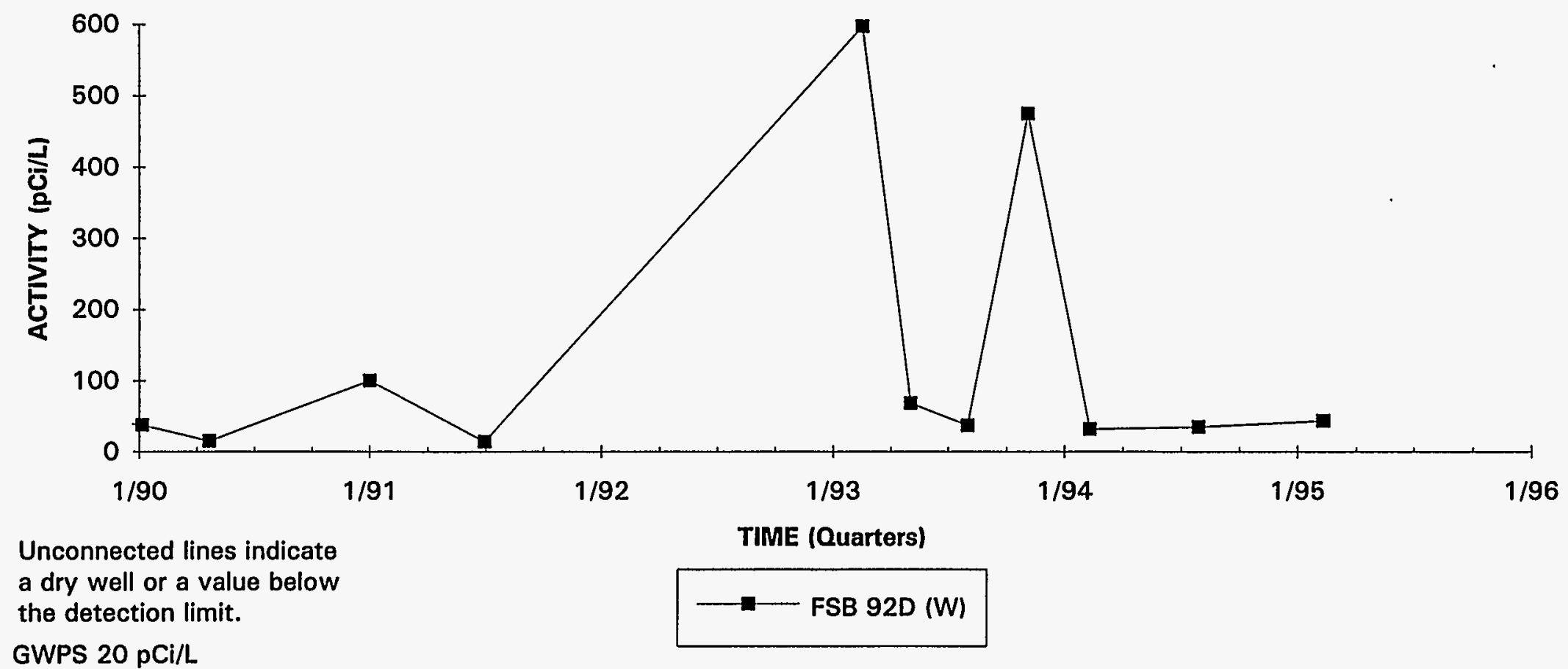

Note: W=Water Table (IIB2); B=Barnwell (IIB1); M=McBean (IIB1); UC=Upper Congaree (IIA); MC=Middle Congaree (IIA); LC=Lower Congaree (IIA) 


\section{Radium-228 Activities}

\section{Well FSB106C}

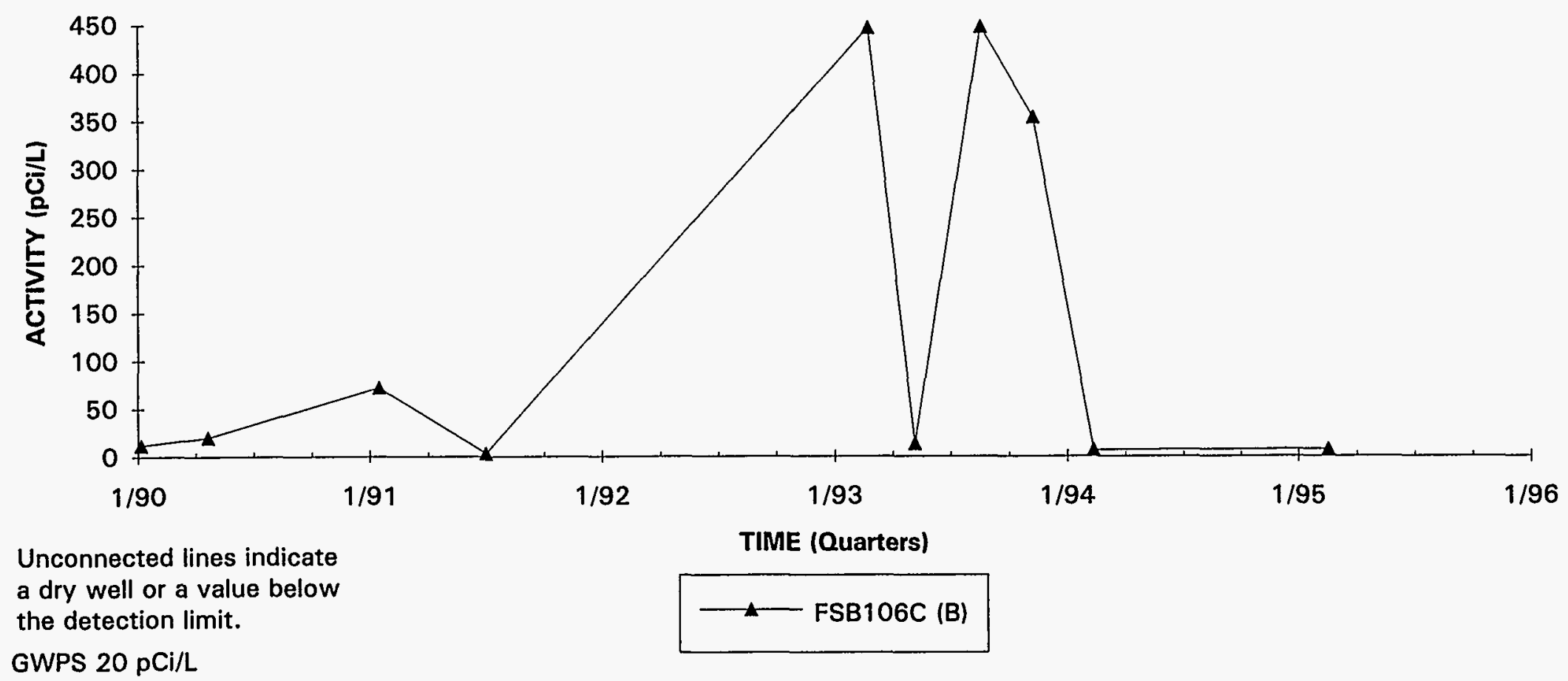

Note: W=Water Table (IIB2); B=Barnwell (IIB1); M=McBean (IIB1); UC=Upper Congaree (IIA); MC=Middle Congaree (IIA); LC=Lower Congaree (IIA) 


\section{Radium-228 Activities}

Well FSB1 10D

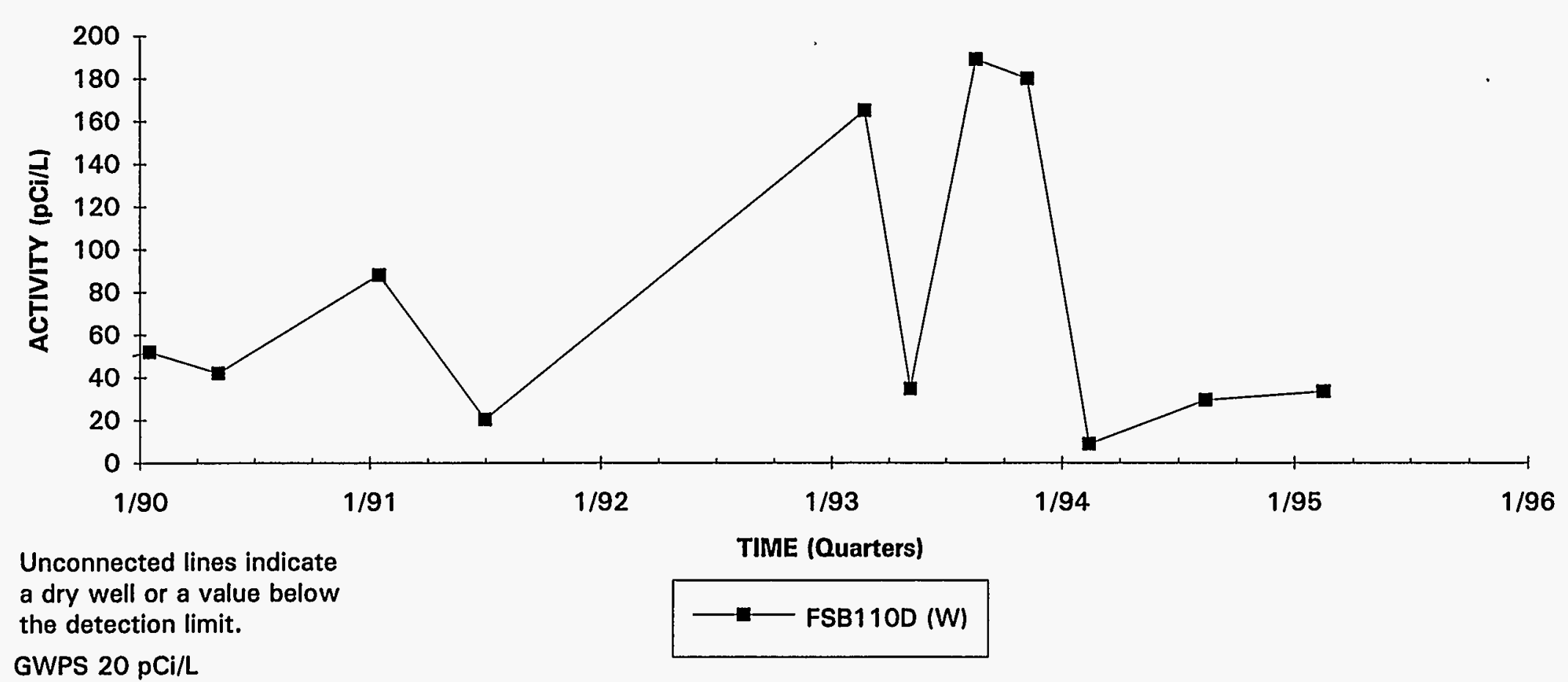

GWPS $20 \mathrm{pCi} / \mathrm{L}$ 


\section{Radium-228 Activities \\ Well Cluster FSB112}

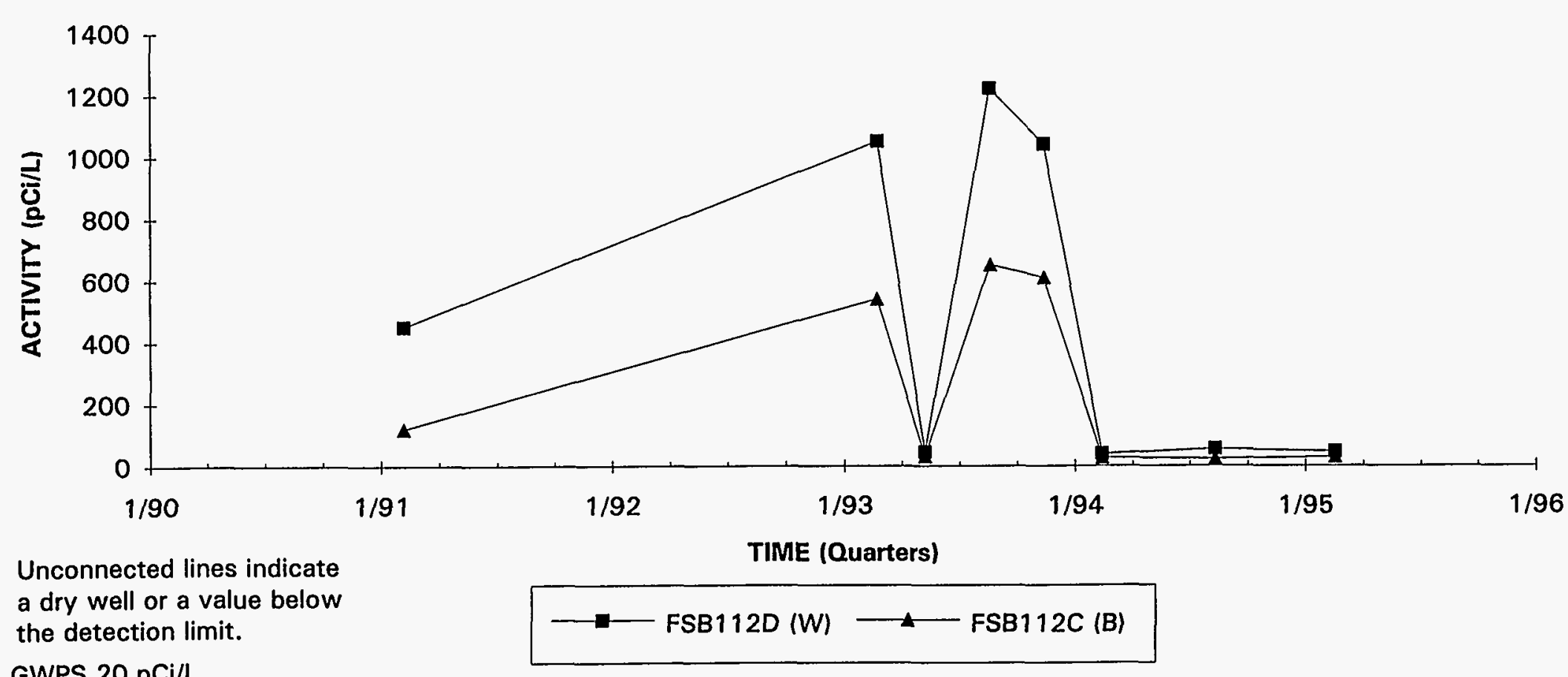

GWPS $20 \mathrm{pCi} / \mathrm{L}$ 


\section{Tritium Activities Well Cluster FSB 79}

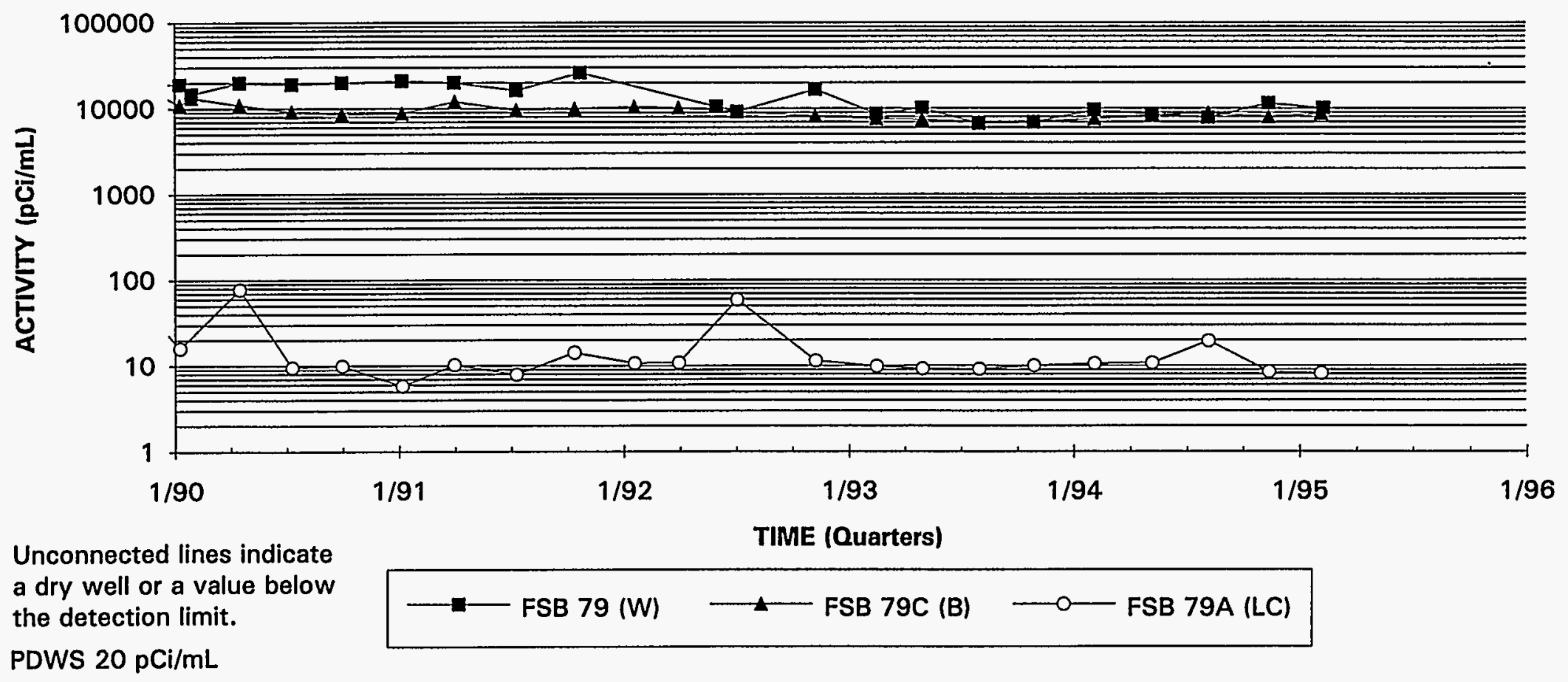

Note: W=Water Table (IIB2); B=Barnwell (IIB1); M=McBean (IIB1); UC=Upper Congaree (IIA); MC=Middle Congaree (IIA); LC=Lower Congaree (IIA) 


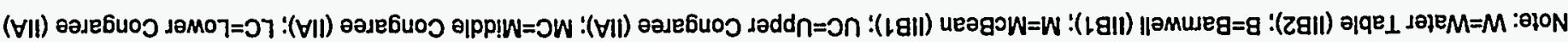

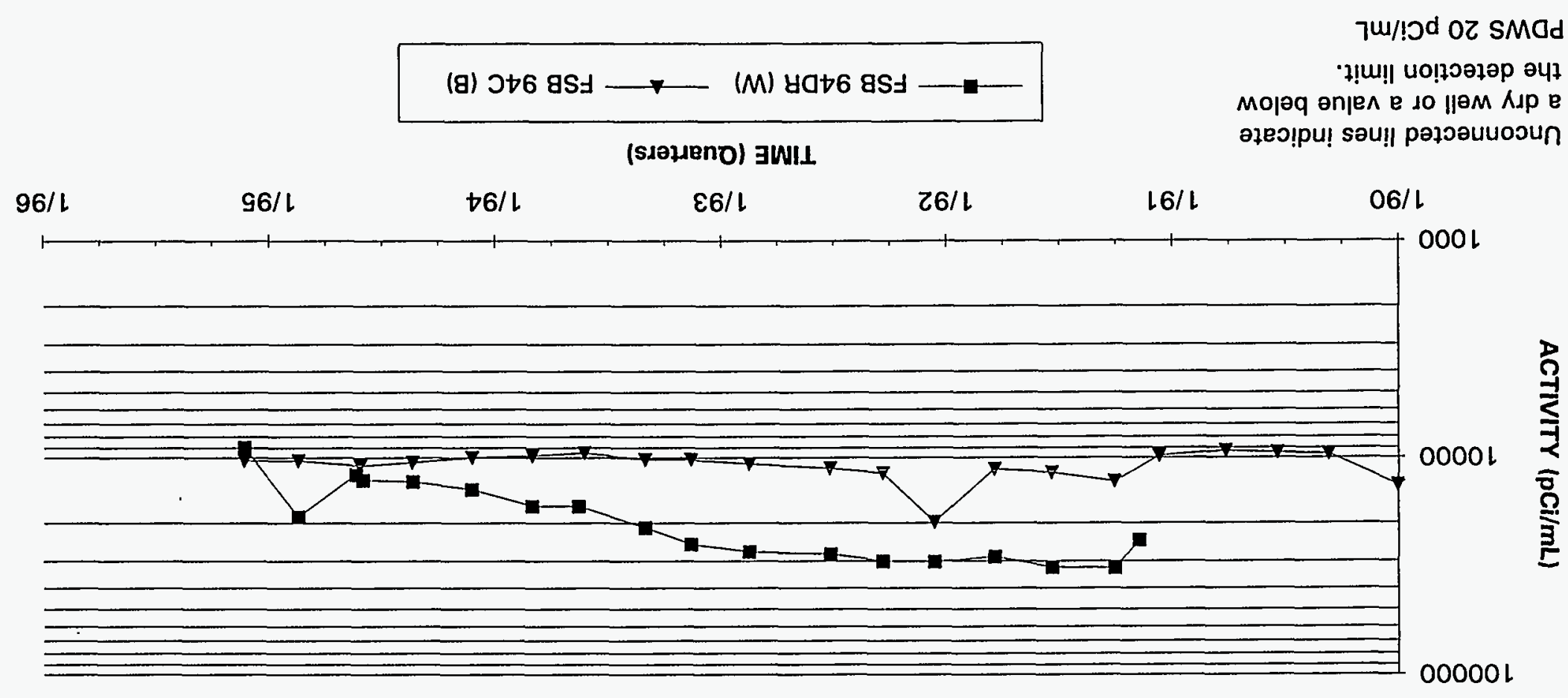

\section{†6 9S」 גəłsn|כ ॥əM Sə!!!ก!ฺ०}




\section{Tritium Activities \\ Well Cluster FSB 97}

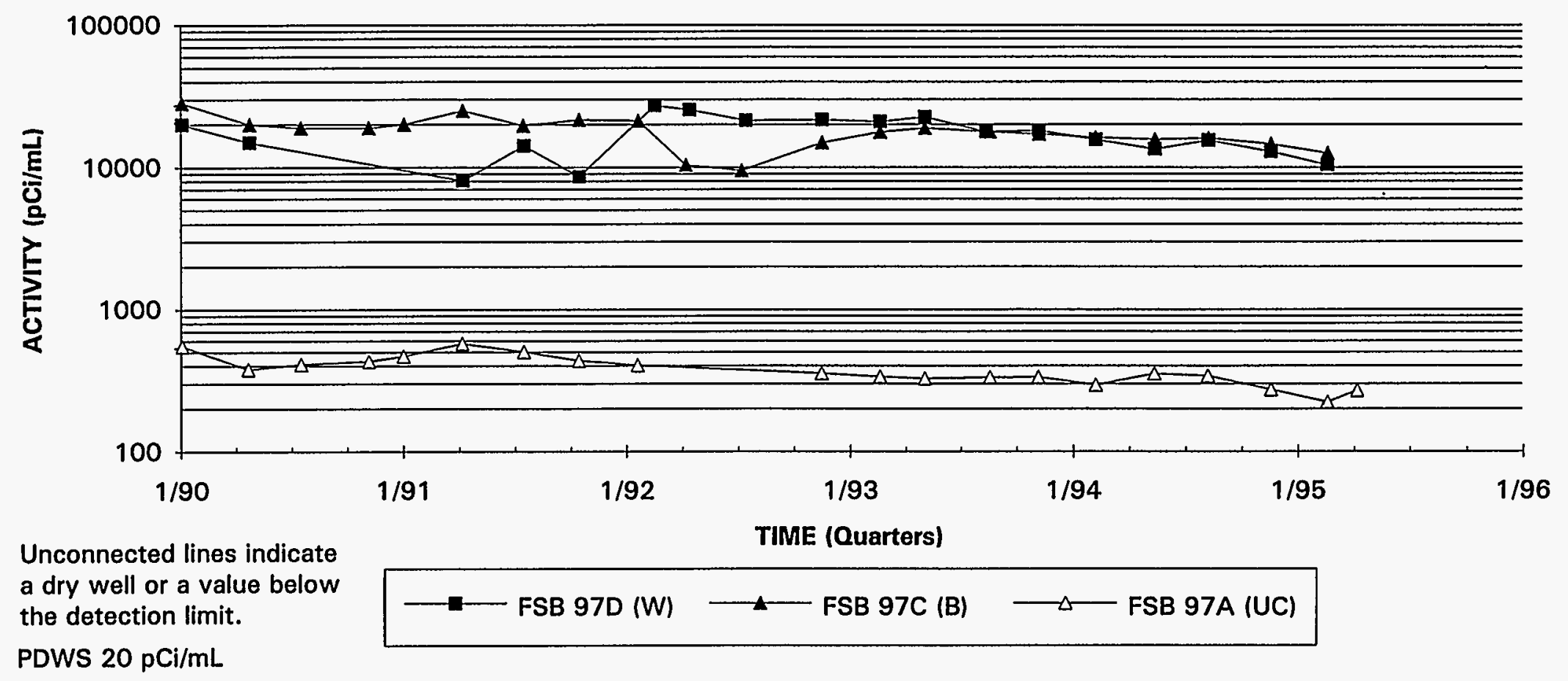

Note: W=Water Table (IIB2); B=Barnwell (IIB1); M=McBean (IIB1); UC=Upper Congaree (IIA); MC=Middle Congaree (IIA); LC=Lower Congaree (IIA) 


\section{Tritium Activities Well Cluster FSB 99}

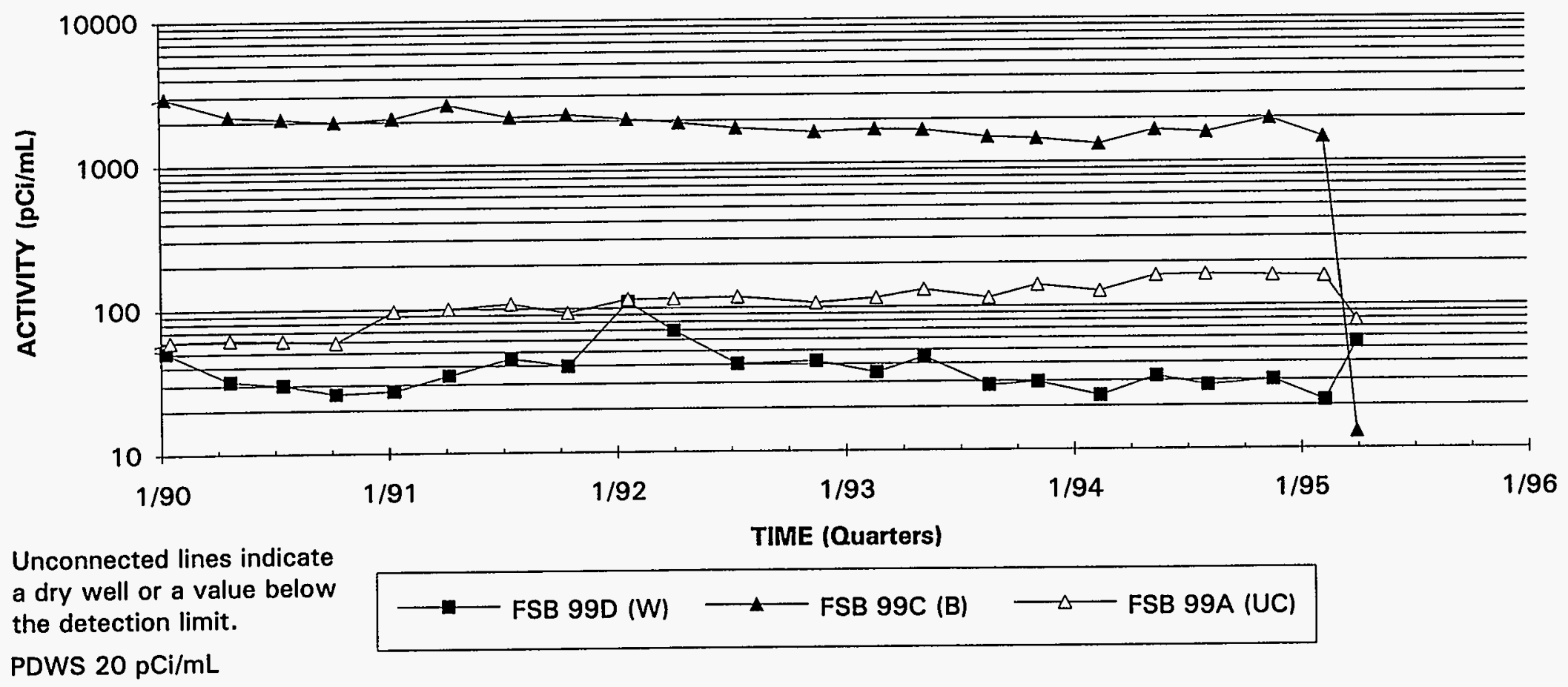

Note: $W=$ Water Table (IIB2); B=Bamwell (IIB1); M=McBean (IIB1); UC=Upper Congaree (IIA); MC=Middle Congaree (IIA); LC=Lower Congaree (IIA) 


\section{Tritium Activities \\ Well FSB102C}

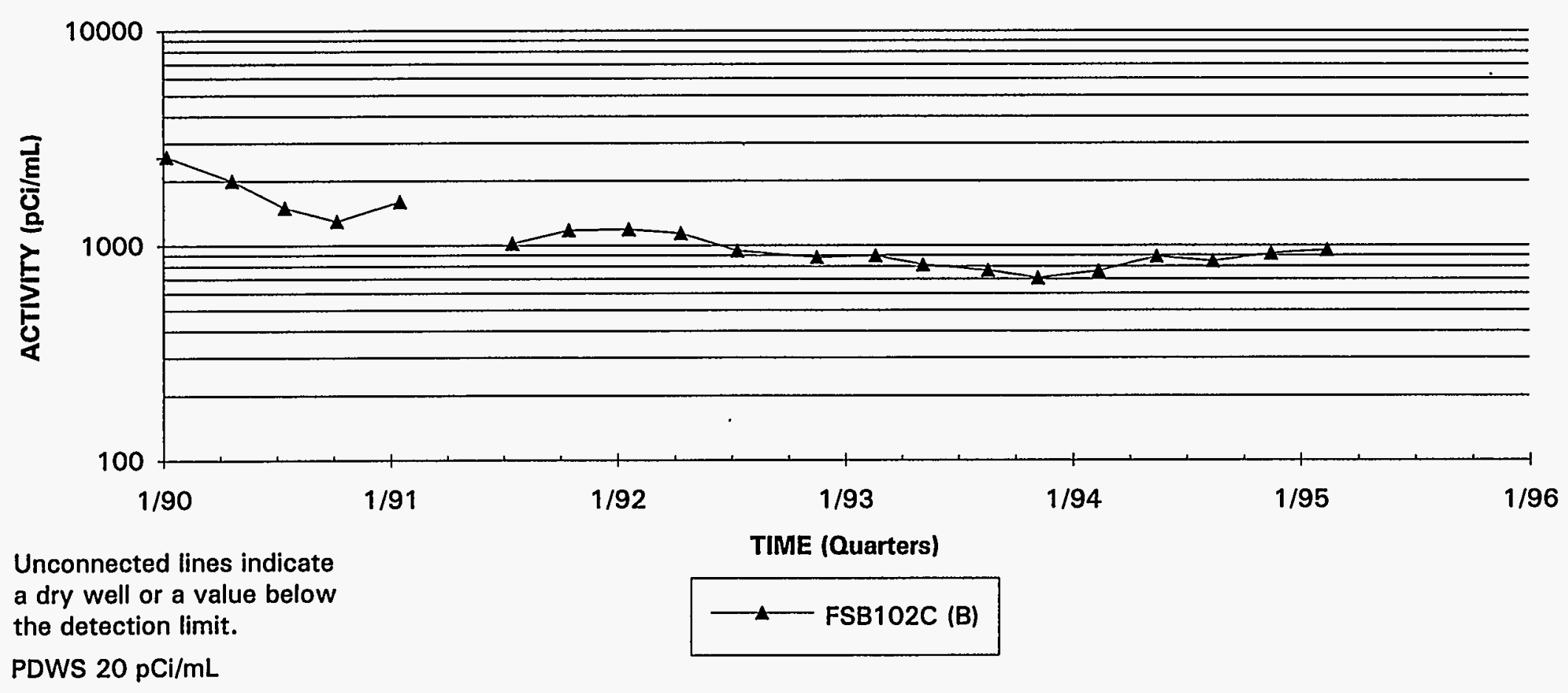

Note: W=Water Table (IIB2); B=Barnwell (IIB1); M=McBean (IIB1); UC=Upper Congaree (IIA); MC=Middle Congaree (IIA); LC=Lower Congaree (IIA) 


\section{Tritium Activities Well Cluster FSB107}

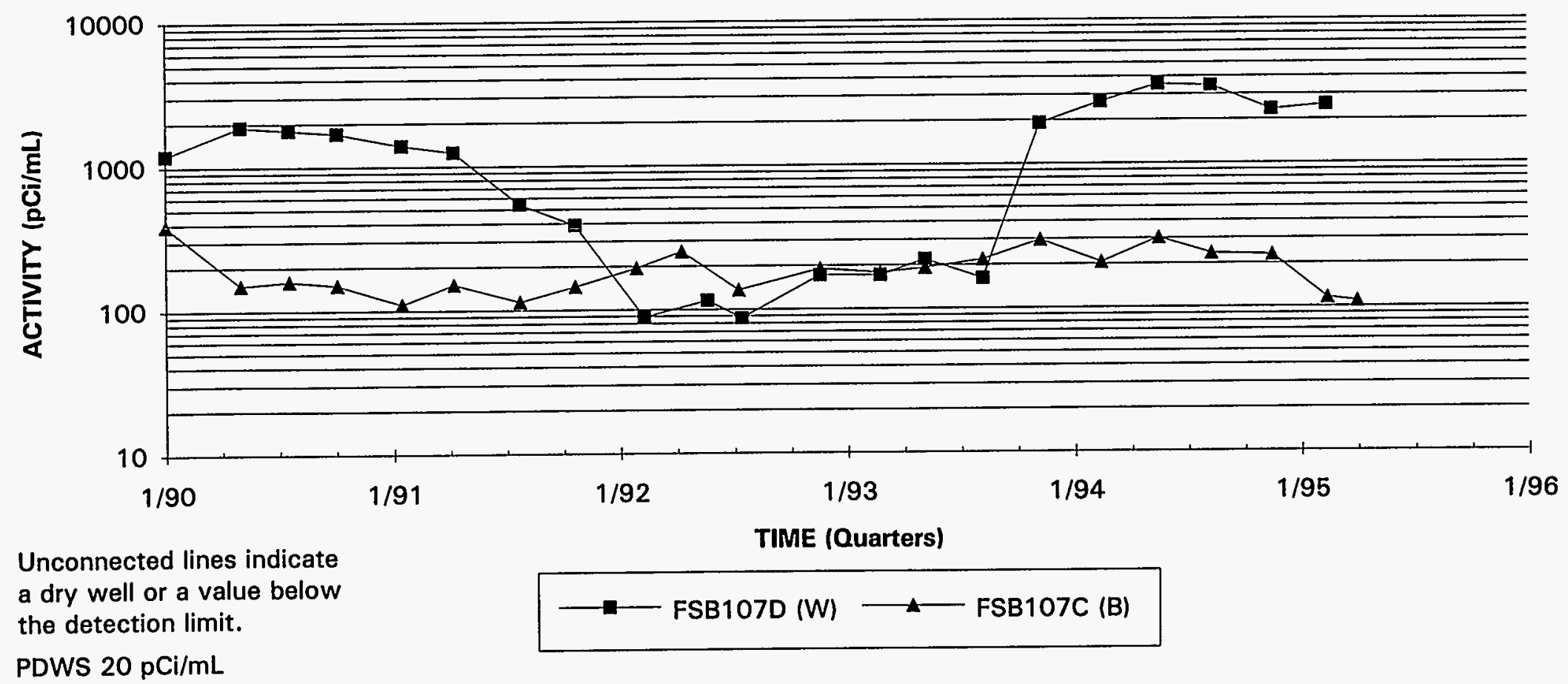




\section{Tritium Activities}

Well FSB108D

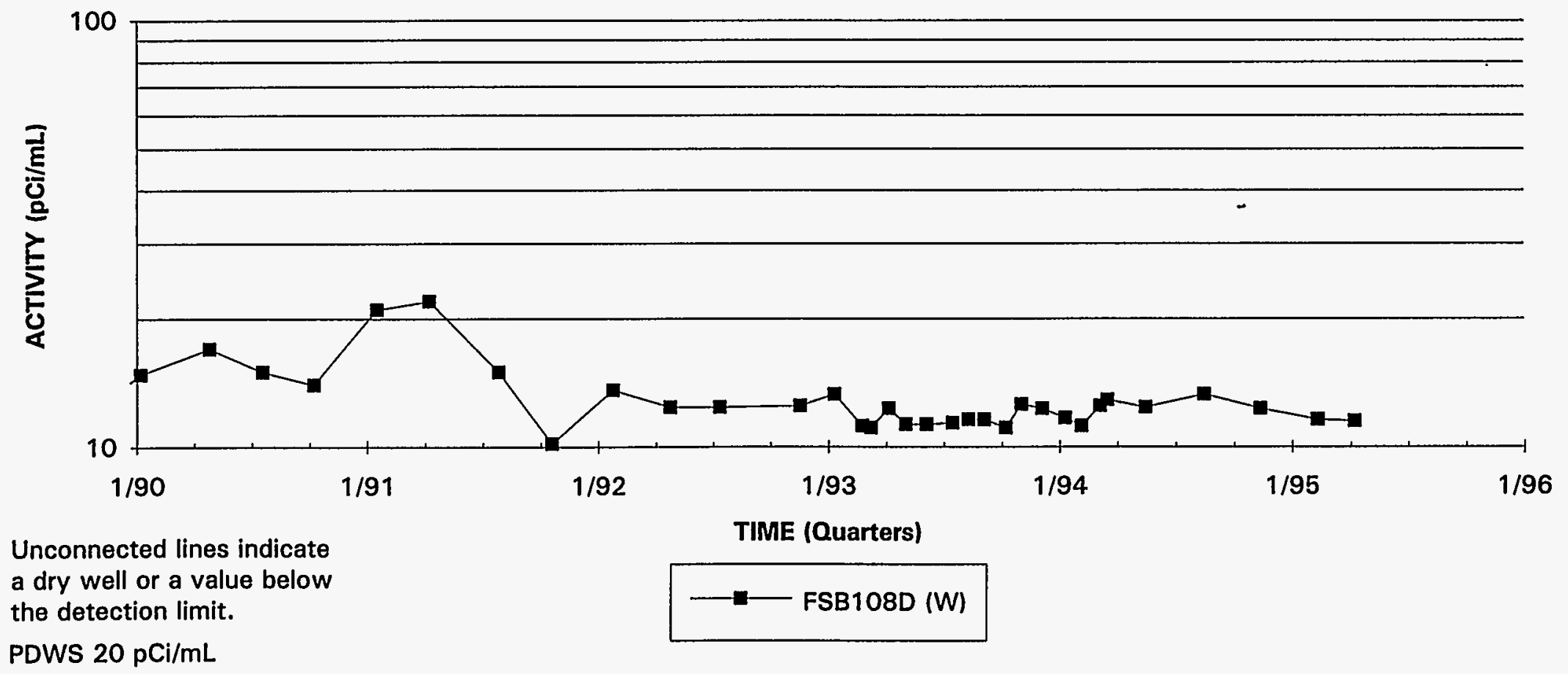

Note: W=Water Table (IIB2); B=Barnwell (IIB1); M=McBean (IIB1); UC=Upper Congaree (IIA); MC=Middle Congaree (IIA); LC=Lower Congaree (IIA) 


\section{Tritium Activities}

\section{Well Cluster FSB110}

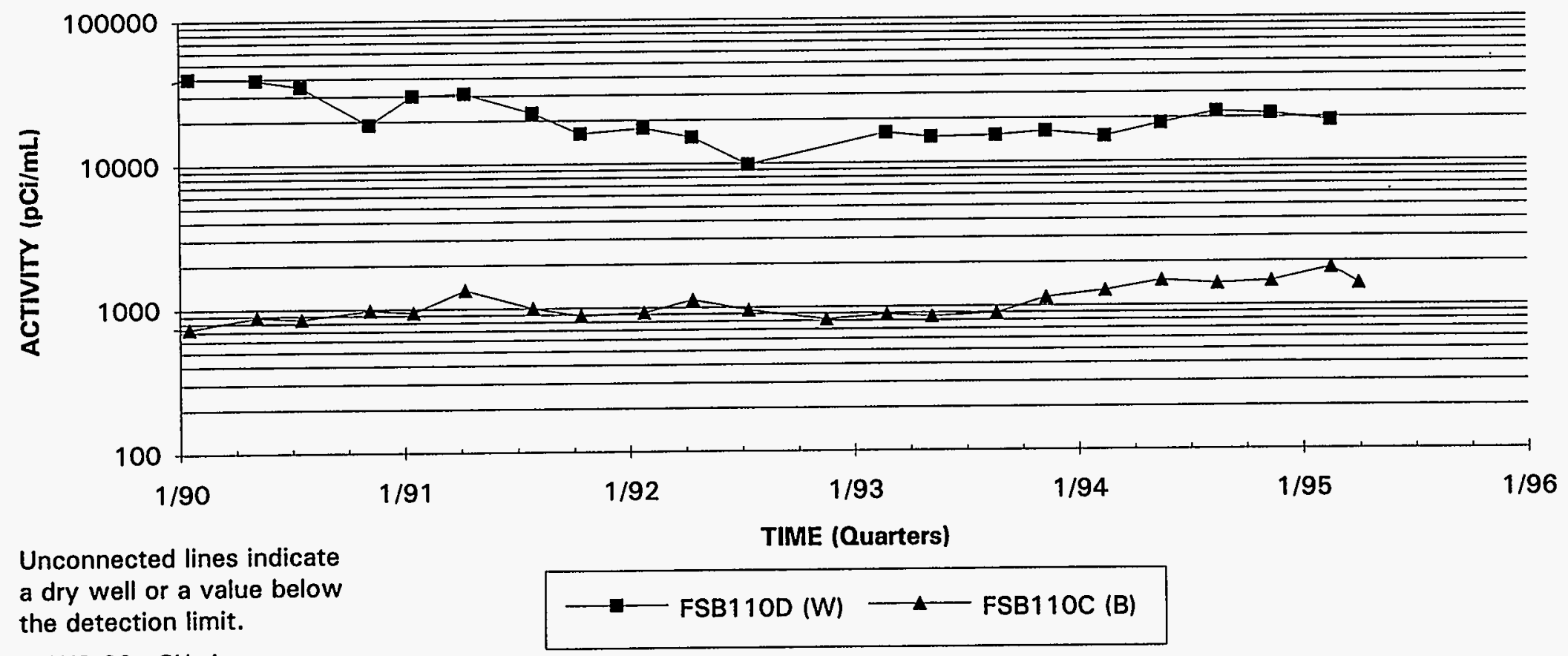

PDWS $20 \mathrm{pCi} / \mathrm{mL}$ 


\section{Tritium Activities Well Cluster FSB120}

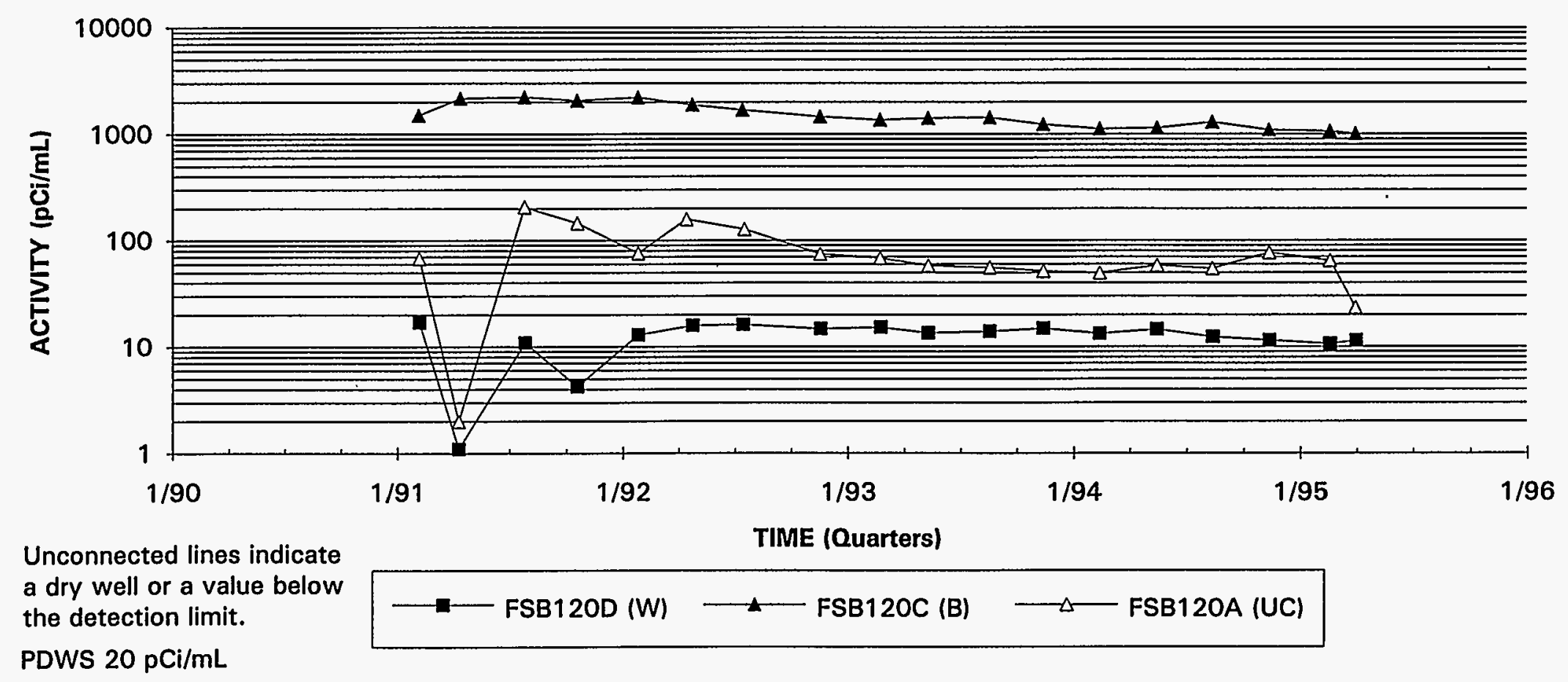

Note: $W=$ Water Table (IIB2); B=Barnwell (IIB1); M=McBean (IIB1); UC=Upper Congaree (IIA); MC=Middle Congaree (IIA); LC=Lower Congaree (IIA) 


\section{Tritium Activities Well Cluster FSB122}

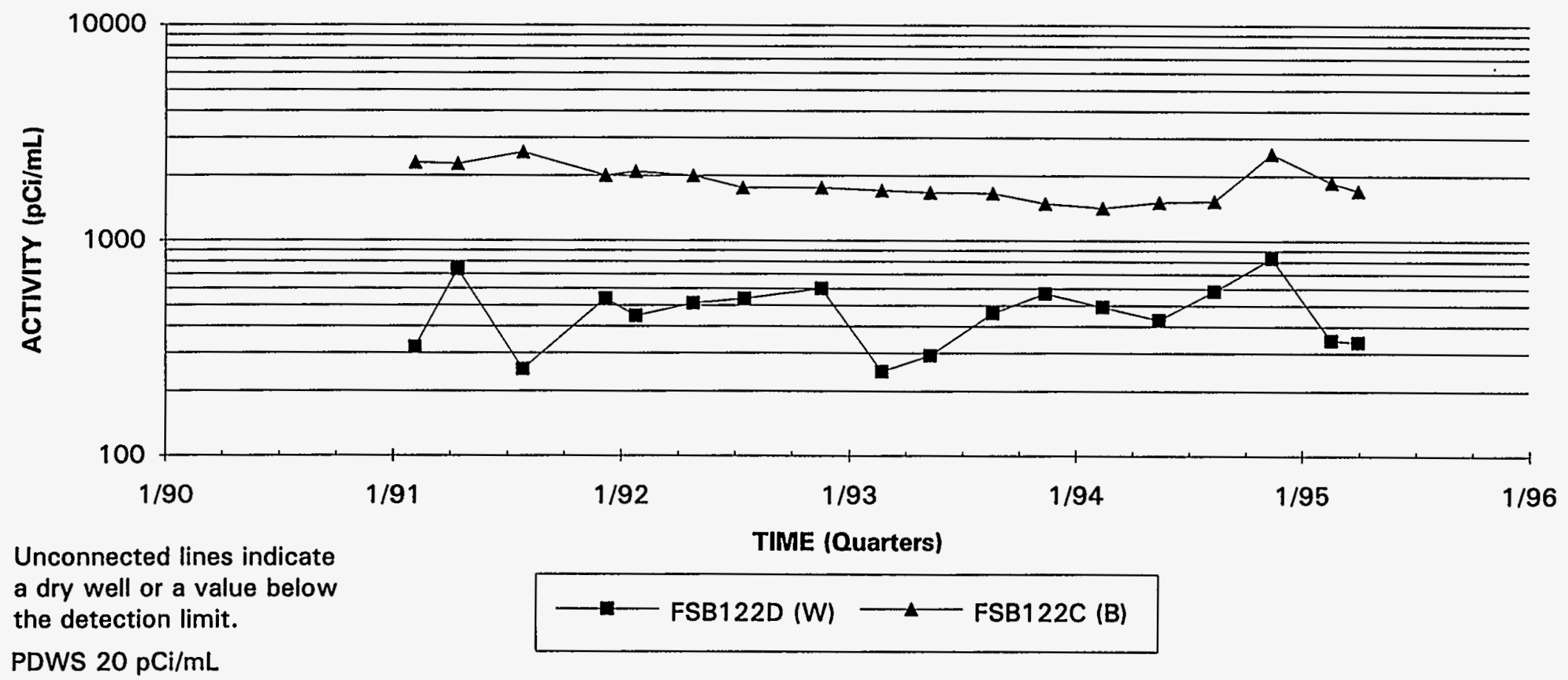

Note: W=Water Table (IIB2); B=Barnwell (IIB1); M=McBean (IIB1); UC=Upper Congaree (IIA); MC=Middle Congaree (IIA); LC=Lower Congaree (IIA) 
WSRC-TR-95-0146-2

Unclassified

\section{Appendix G}

Hydrographs 
WSRC-TR-95-0146-2

Unclassified

THIS PAGE LEFT BLANK INTENTIONALLY. 


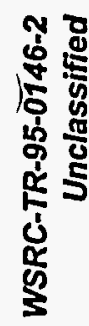
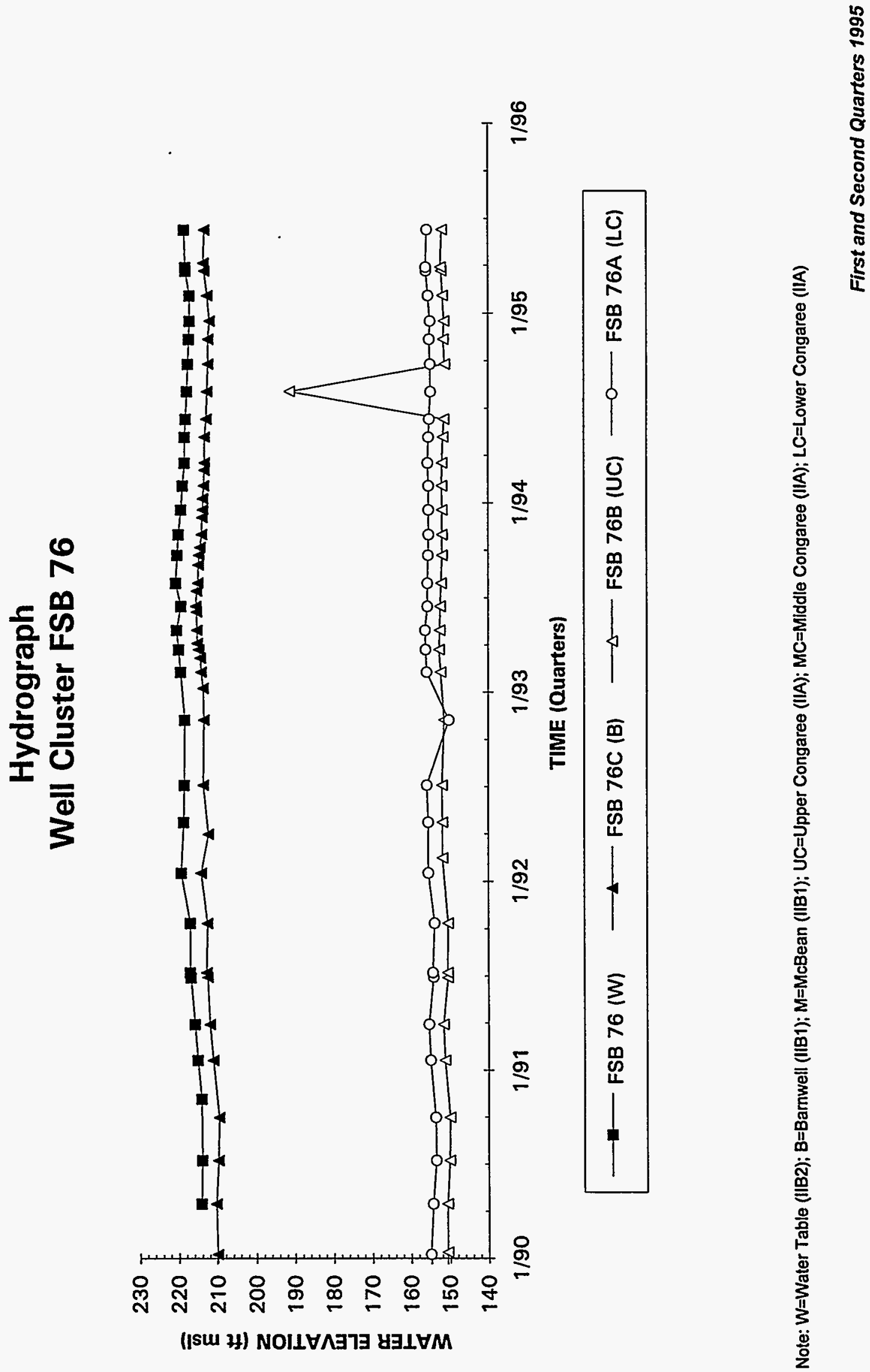


\section{Hydrograph}

\section{Well FSB 77}

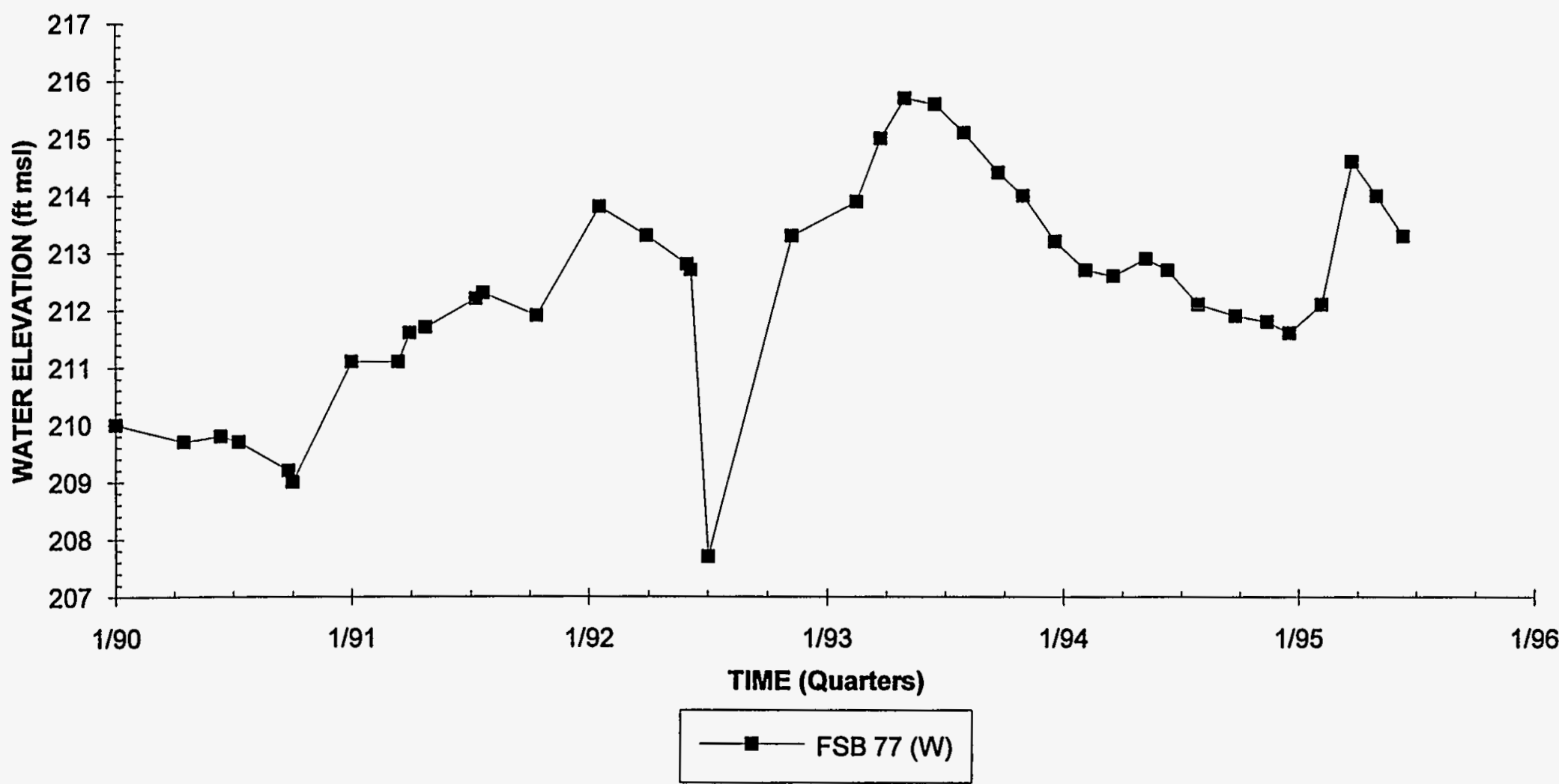

Note: W=Water Table (IIB2); B=Barnwell (IIB1); M=MCBean (IIB1); UC=Upper Congaree (IIA); MC=Middle Congaree (IIA); LC=Lower Congaree (IIA) 

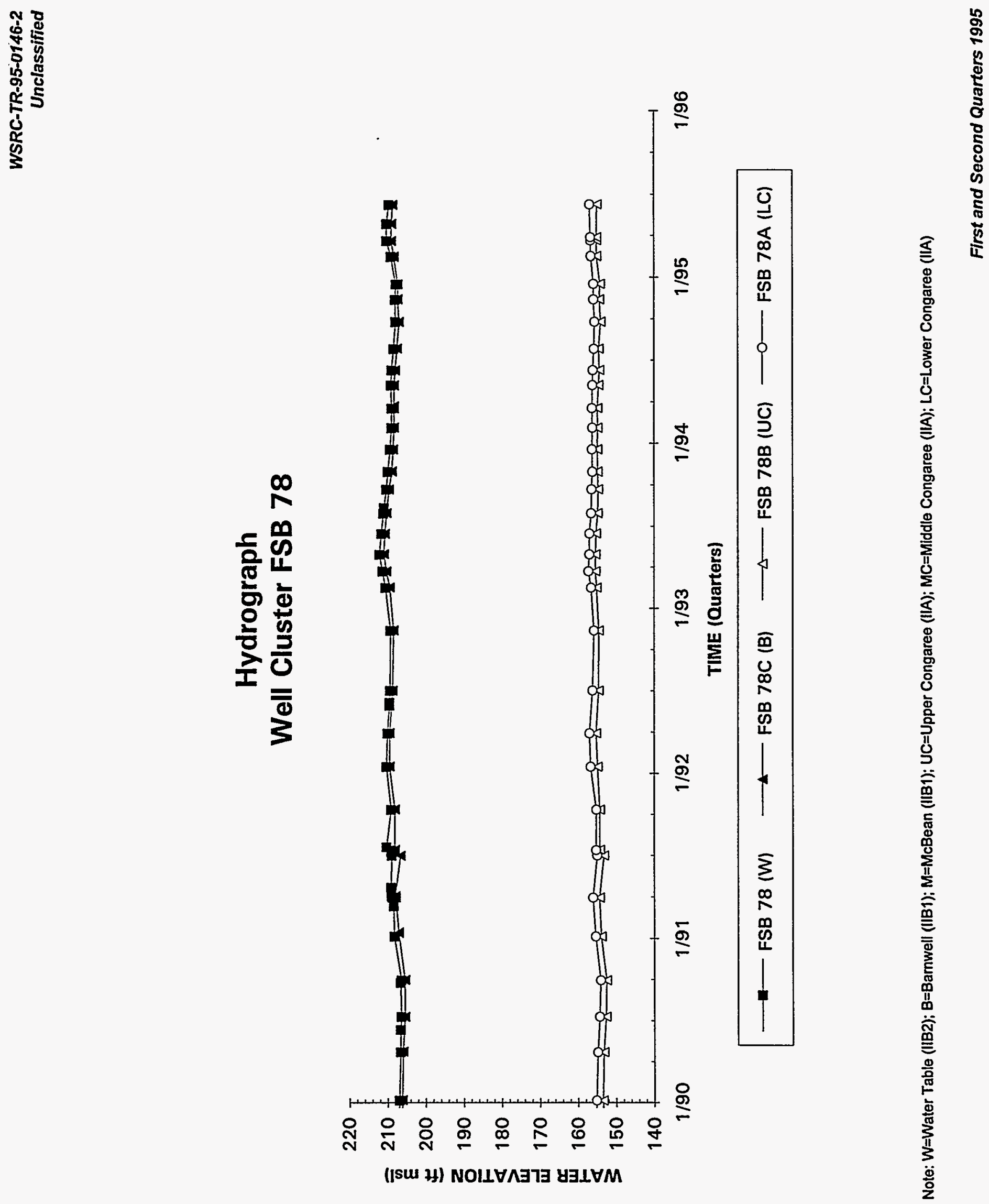
预

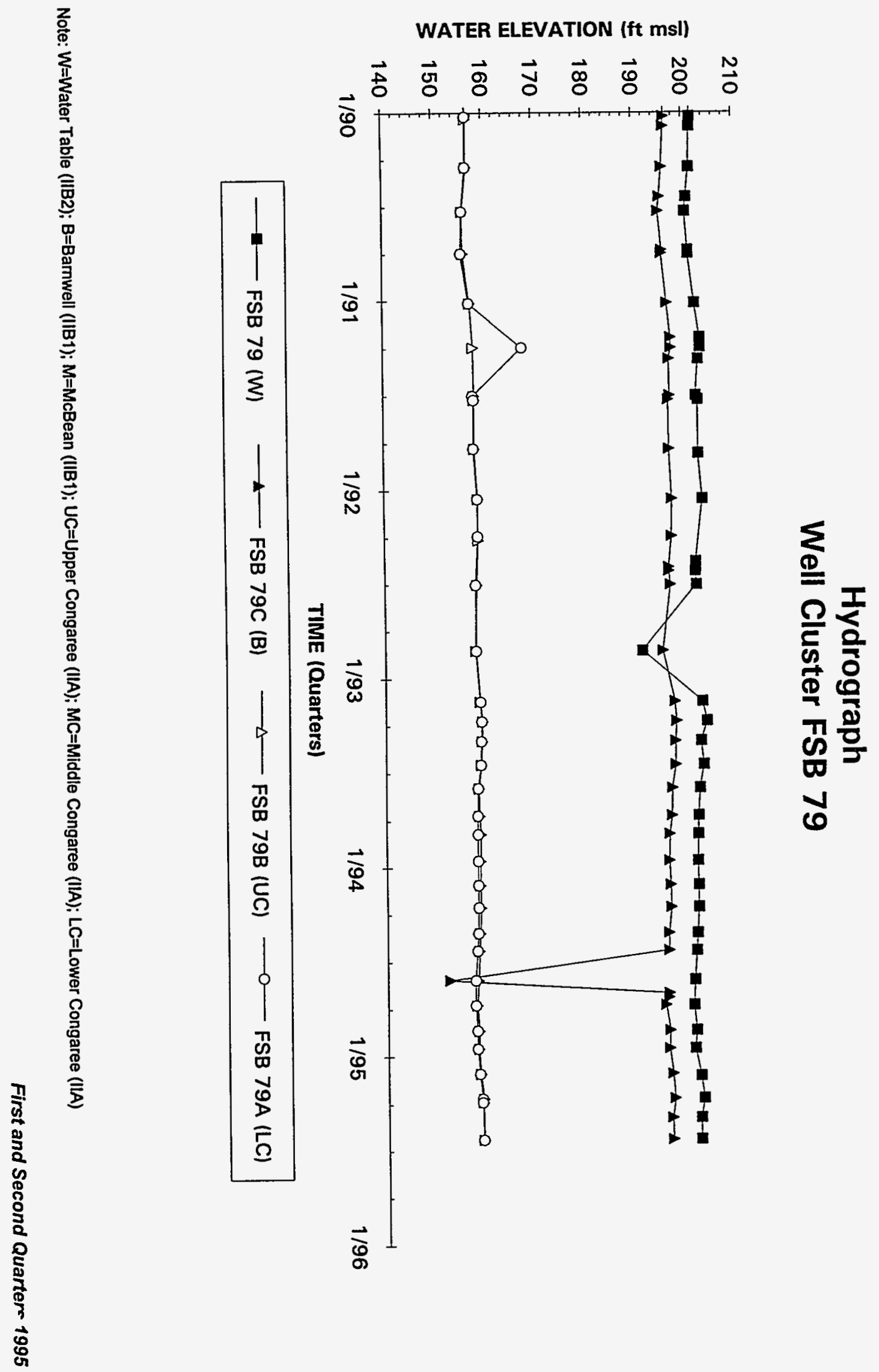

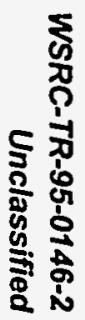



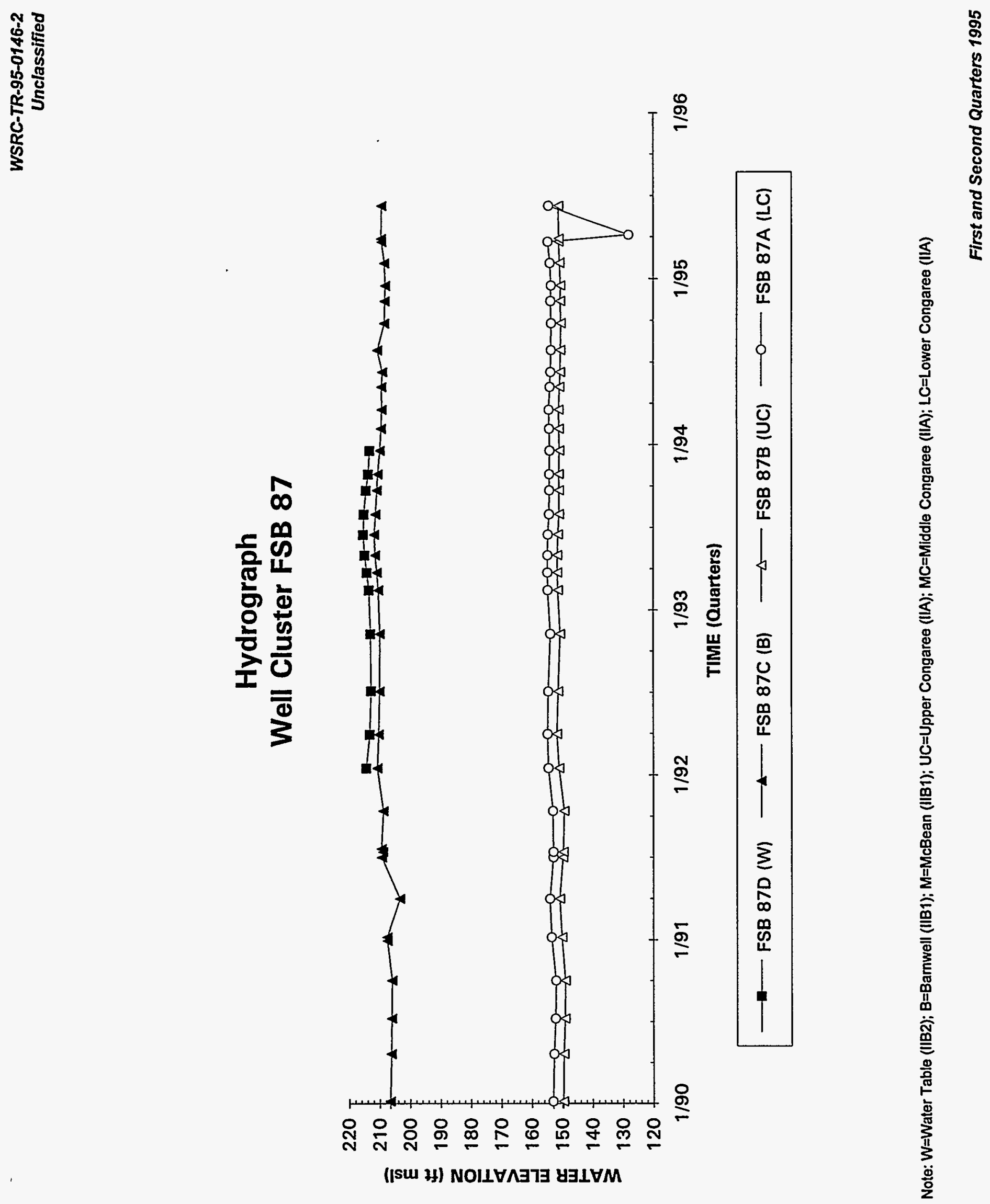


\section{Hydrograph \\ Well Cluster FSB 88}

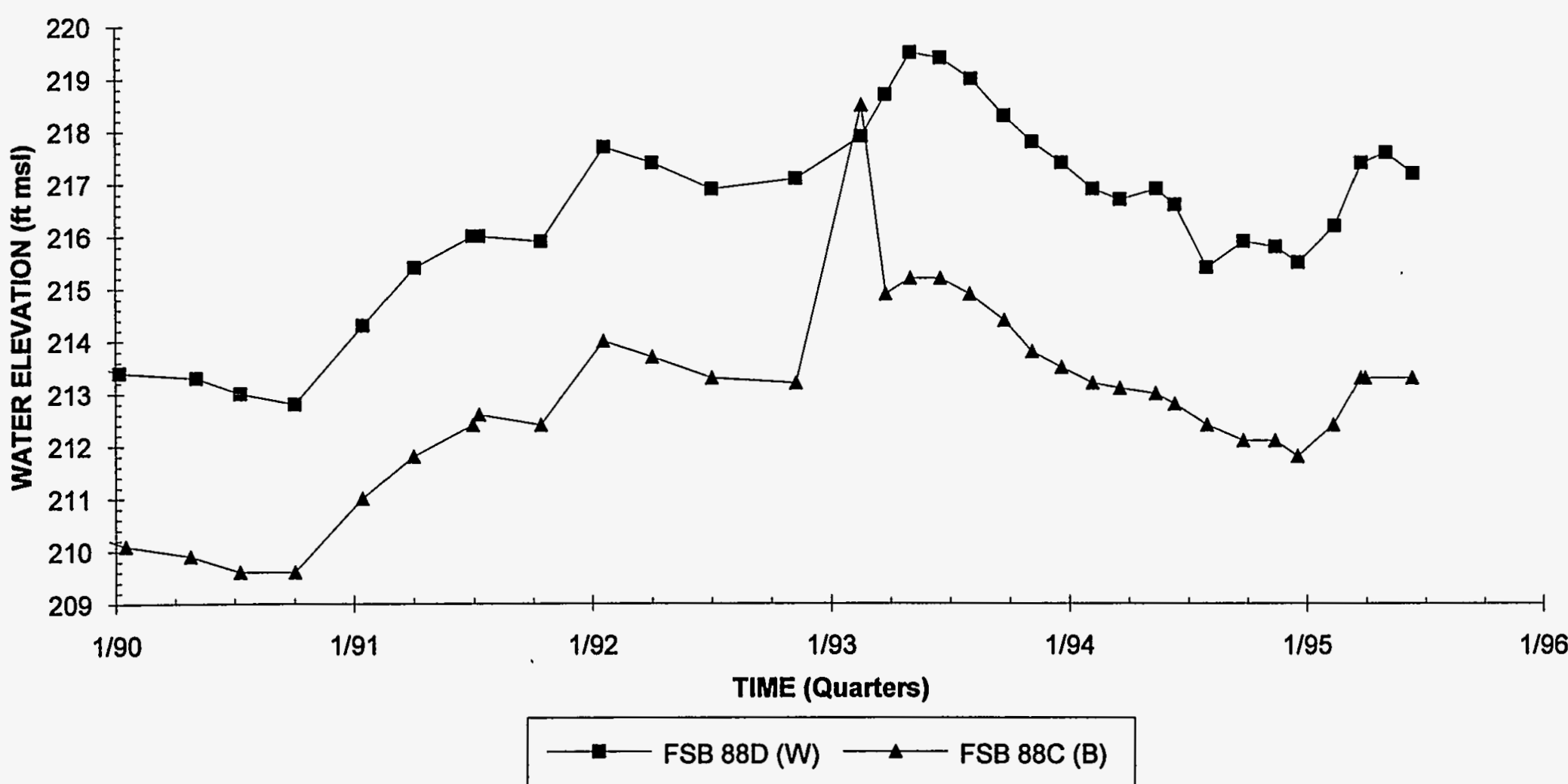

Note: W=Water Table (IIB2); B=Barnwell (IIB1); M=MCBean (IIB1); UC=Upper Congaree (IIA); MC=Middle Congaree (IIA); LC=Lower Congaree (IIA) 


\section{Hydrograph \\ Well Cluster FSB 89}

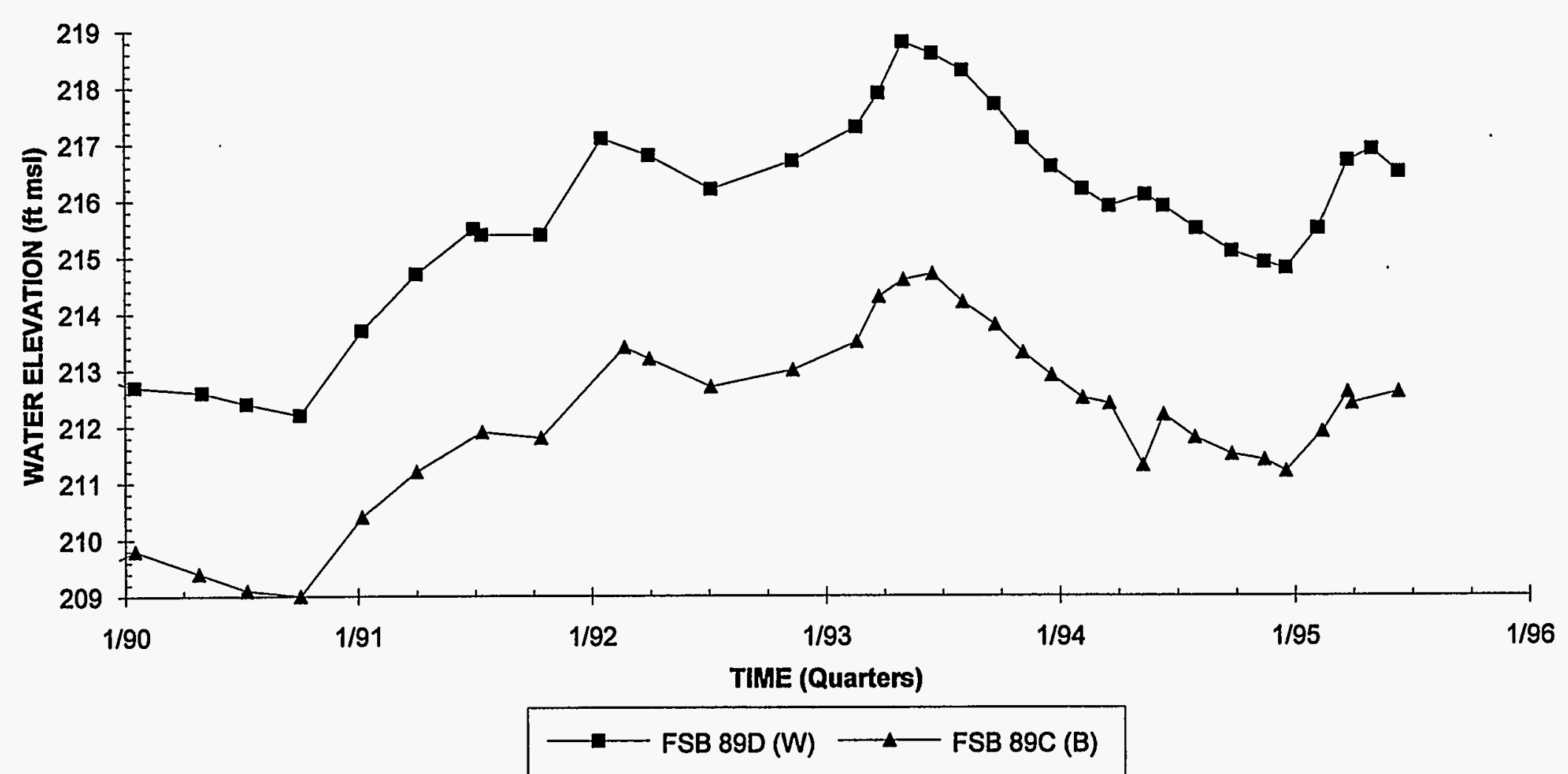

Note: W=Water Table (IIB2); B=Barnwell (IIB1); M=McBean (IIB1); UC=Upper Congaree (IIA); MC=Middle Congaree (IIA); LC=Lower Congaree (IIA) 


\section{Hydrograph \\ Well Cluster FSB 90}

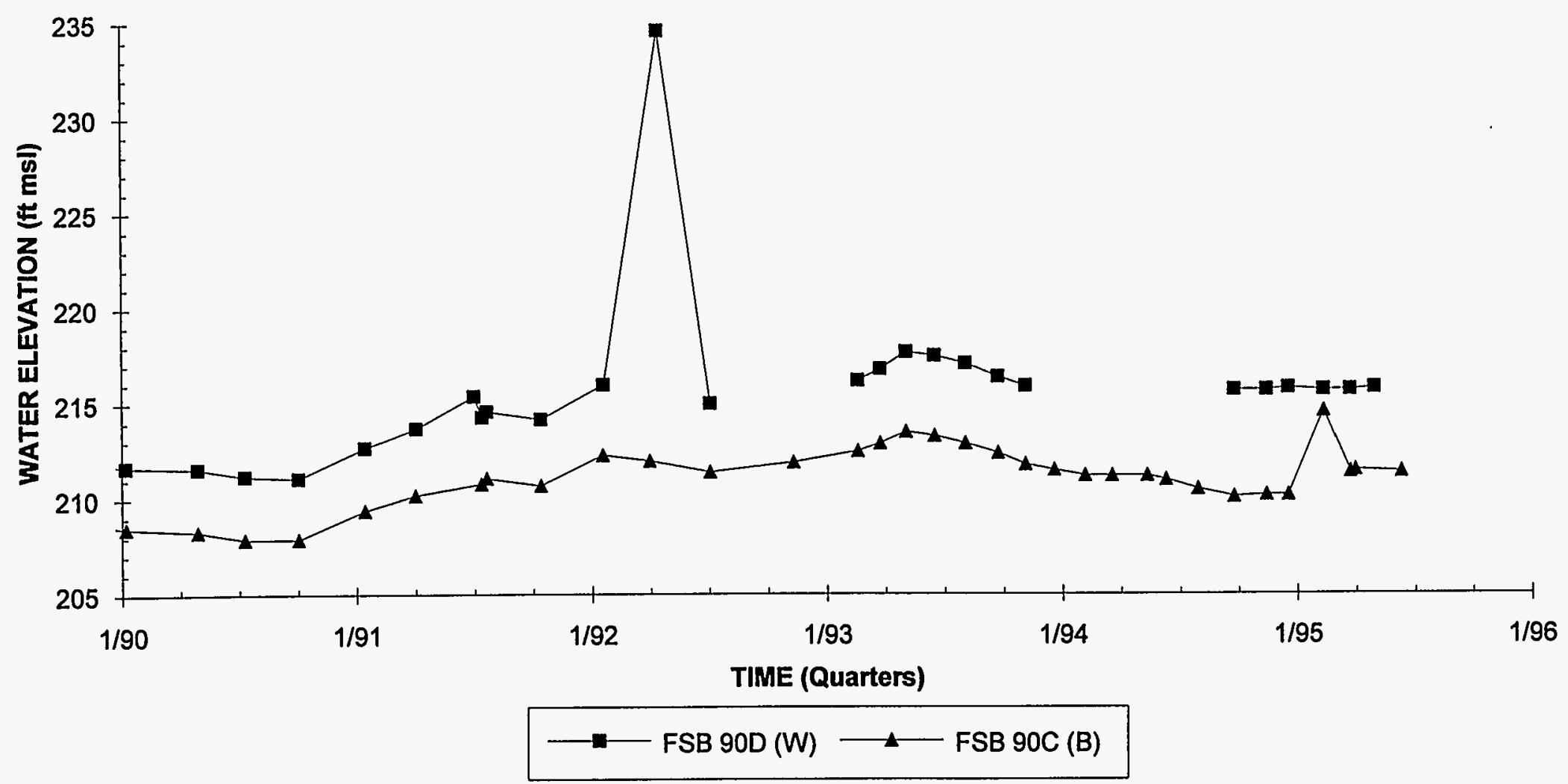

Note: $W=$ Water Table (IIB2); B=Barnwell (IIB1); M=McBean (IIB1); UC=Upper Congaree (IIA); MC=Middle Congaree (IIA); LC=Lower Congaree (IIA) 
告
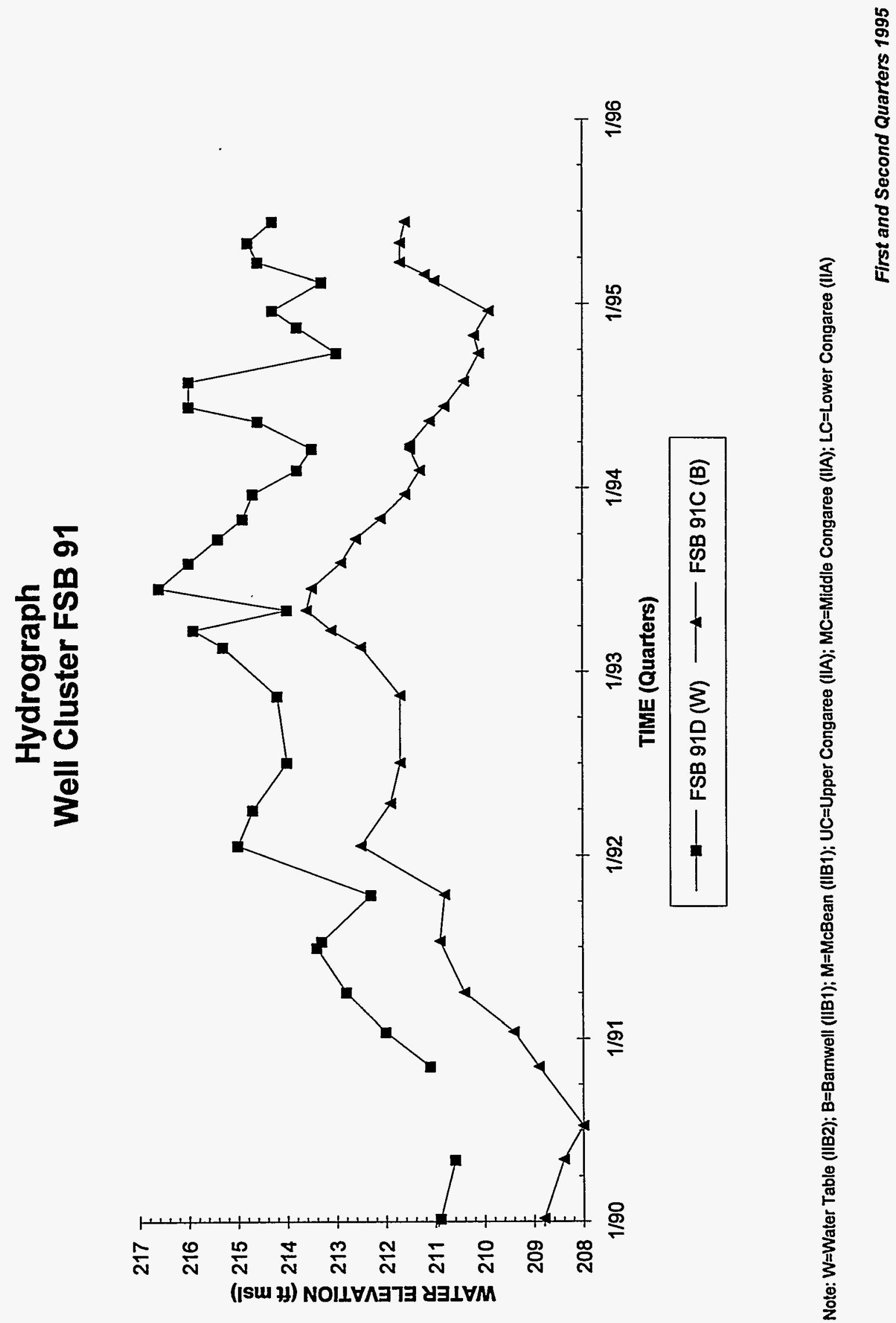


\section{Hydrograph \\ Well Cluster FSB 92}

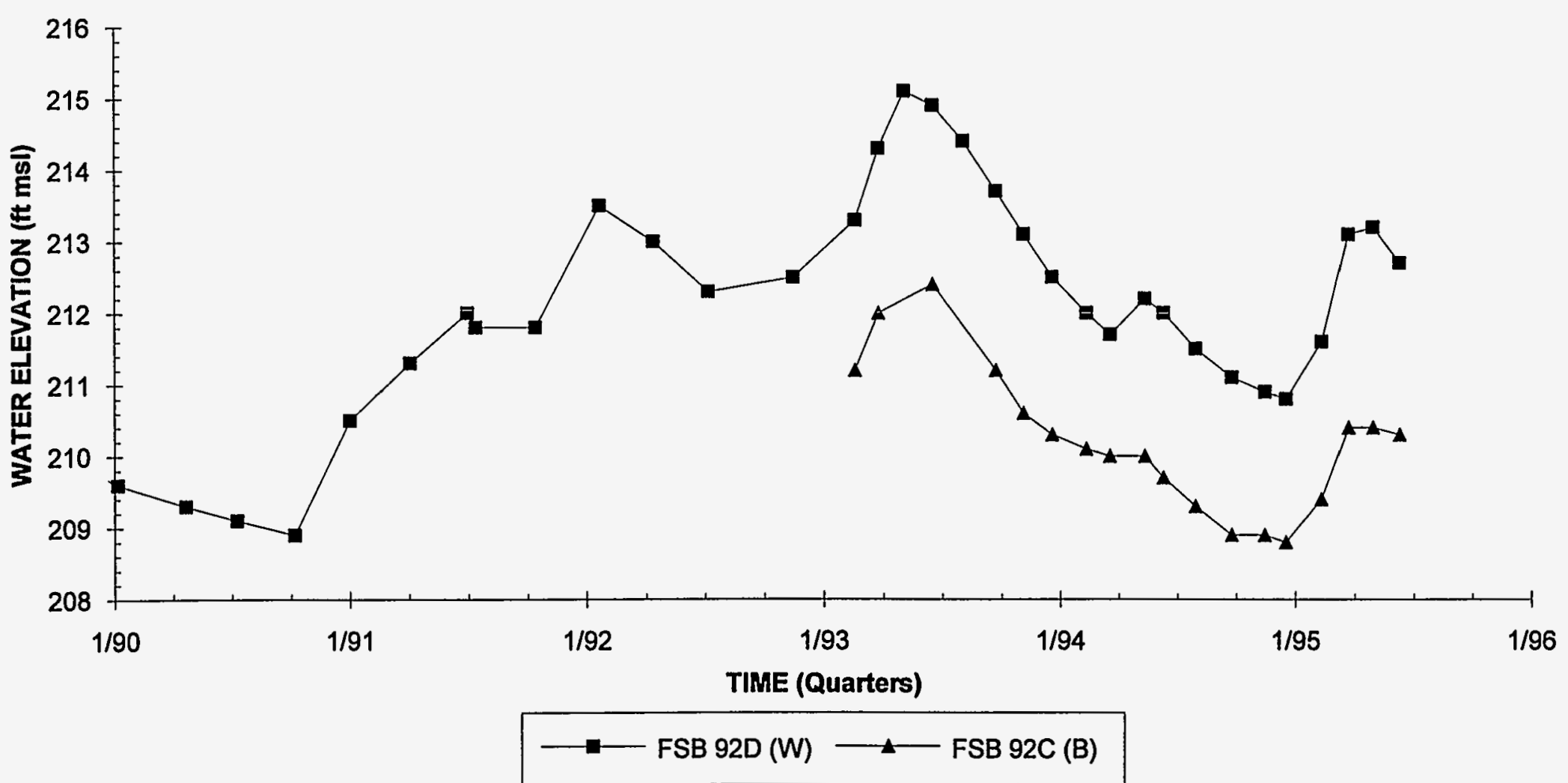

Note: W=Water Table (IIB2); B=Barnwell (IIB1); M=McBean (IIB1); UC=Upper Congaree (IIA); MC=Middle Congaree (IIA); LC=Lower Congaree (IIA) 


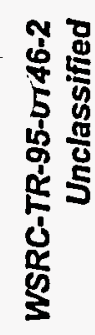
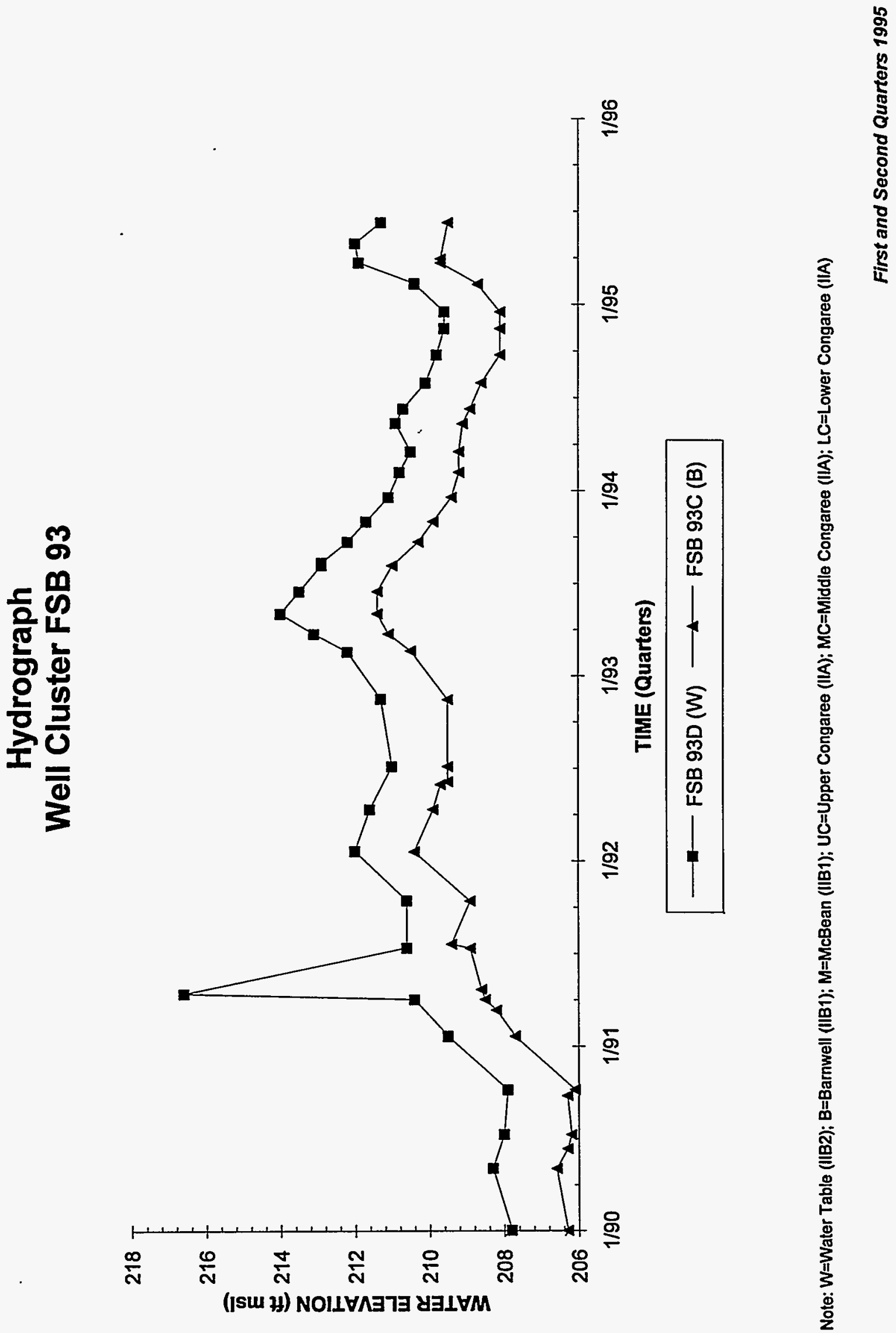

: 


\section{Hydrograph \\ Well Cluster FSB 94}

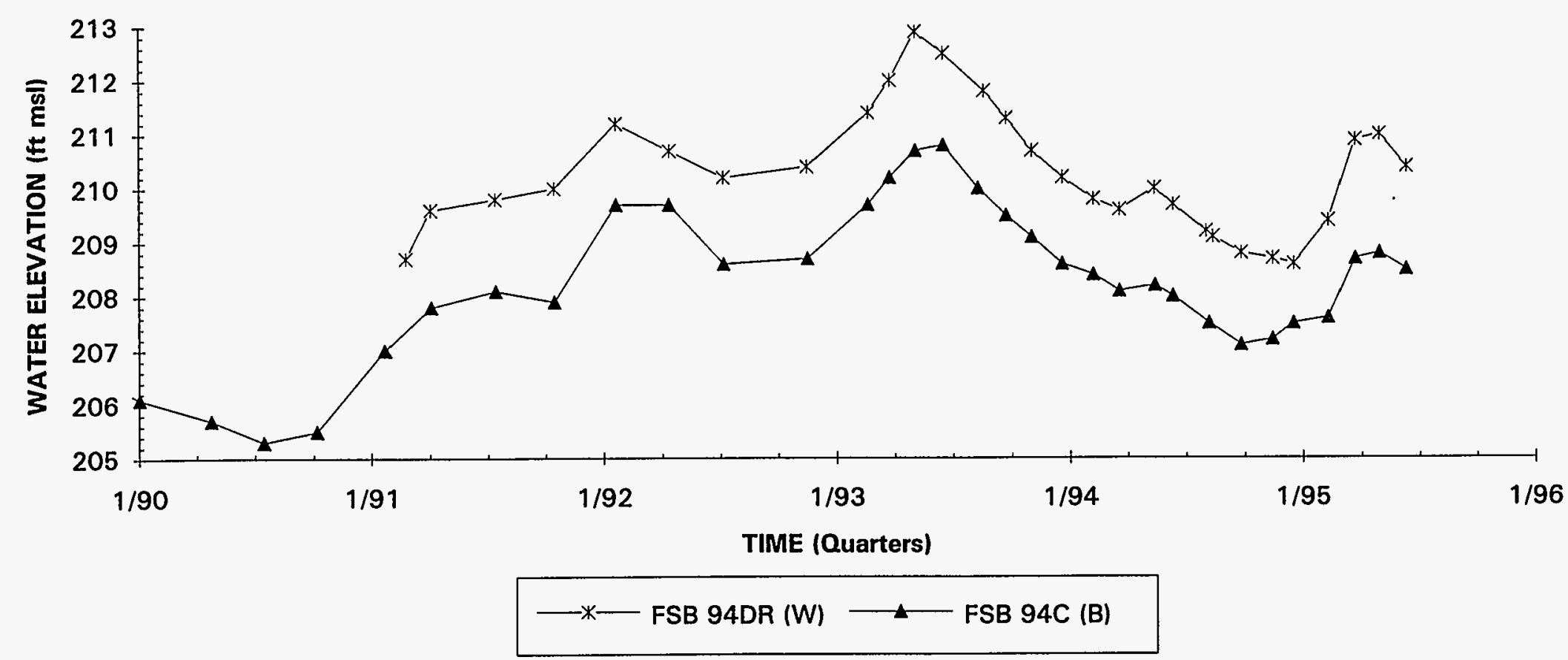

Note: W=Water Table (IIB2); B=Barnwell (IIB1); M=McBean (IIB1); UC=Upper Congaree (IIA); MC=Middle Congaree (IIA); LC=Lower Congaree (IIA) 


\section{Hydrograph \\ Well Cluster FSB 95}

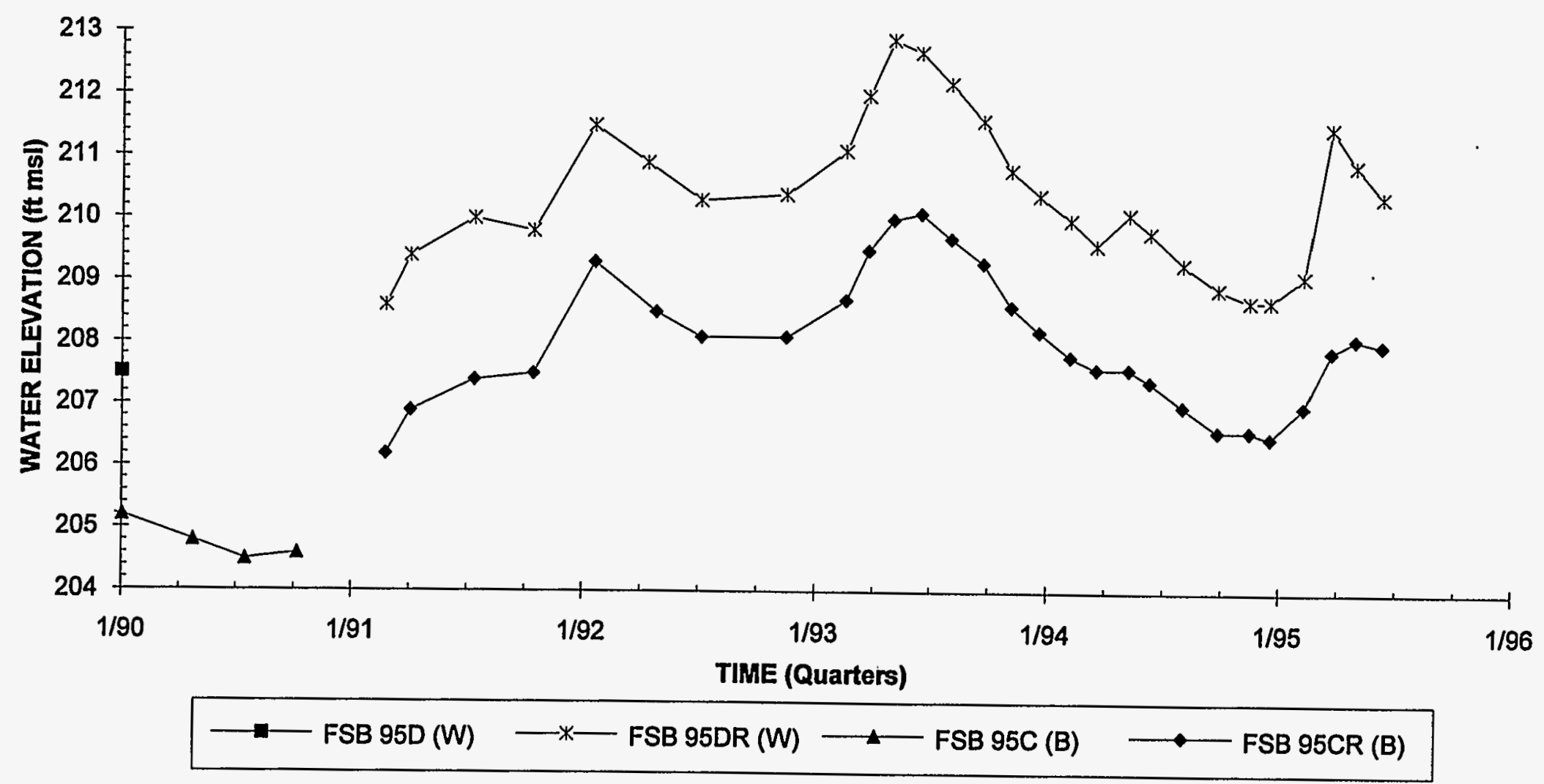

Note: W=Water Table (IIB2); B=Barnwell (IIB1); M=MCBean (IIB1); UC=Upper Congaree (IIA); MC=Middle Congaree (IIA); LC=Lower Congaree (IIA) 
总

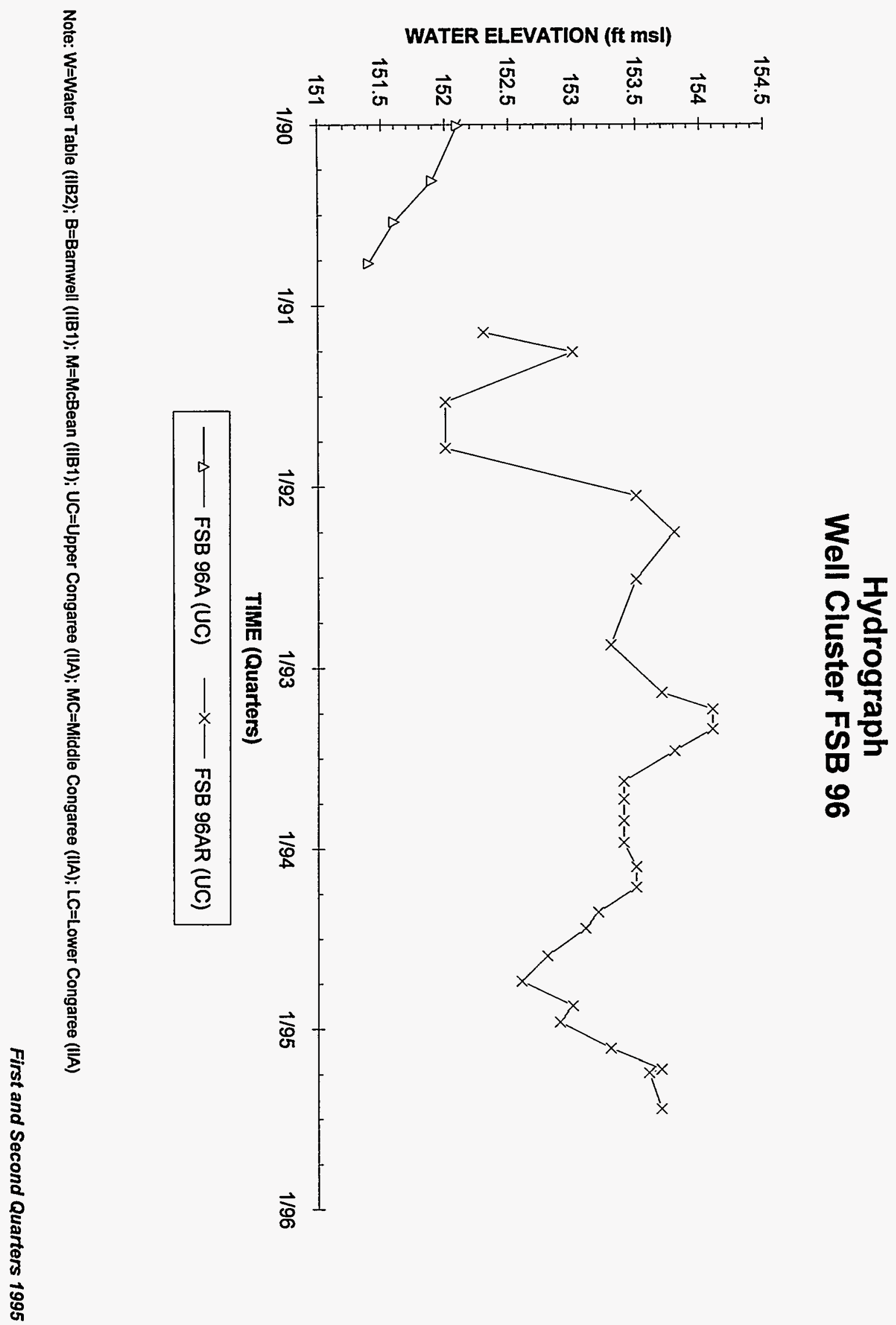




\section{Hydrograph \\ Well Cluster FSB 97}

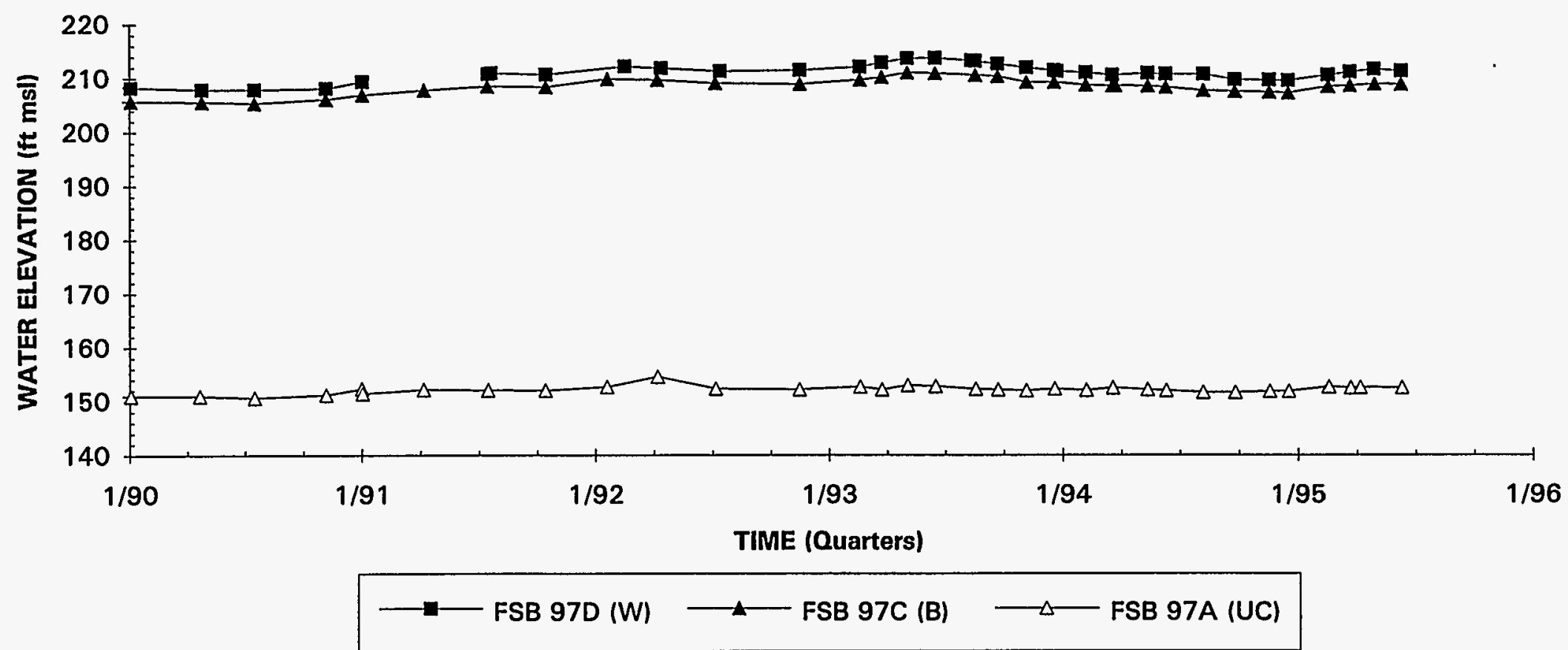

Note: $W=$ Water Table (IIB2); B=Barnwell (IIB1); M=McBean (IIB1); UC=Upper Congaree (IIA); MC=Middle Congaree (IIA); LC=Lower Congaree (IIA) 


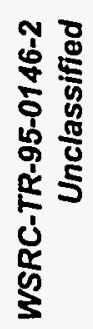
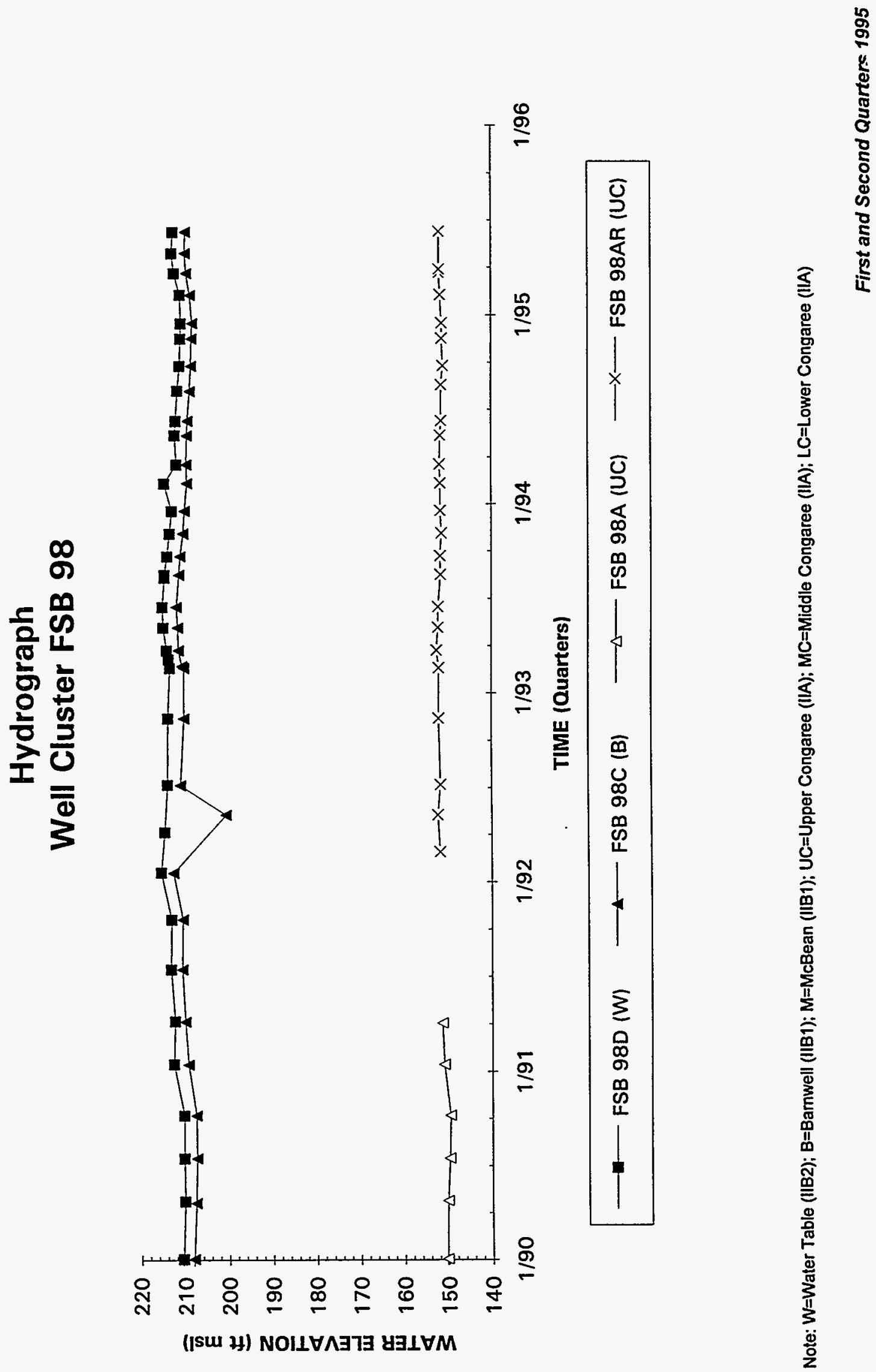

品 


\section{Hydrograph \\ Well Cluster FSB 99}

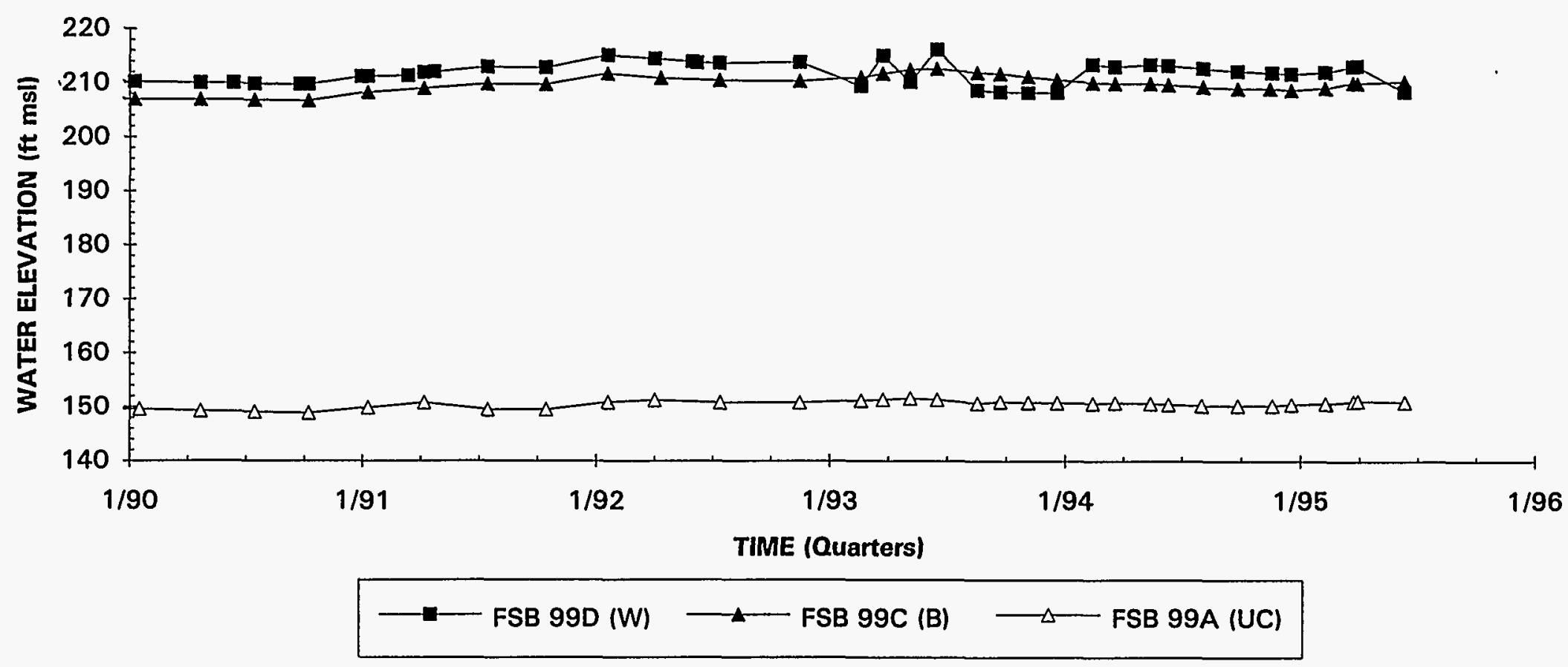

Note: W=Water Table (IIB2); B=Bamwell (IIB1); M=McBean (IIB1); UC=Upper Congaree (IIA); MC=Middle Congaree (IIA); LC=Lower Congaree (IIA) 


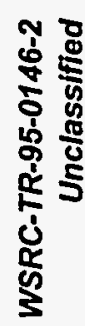
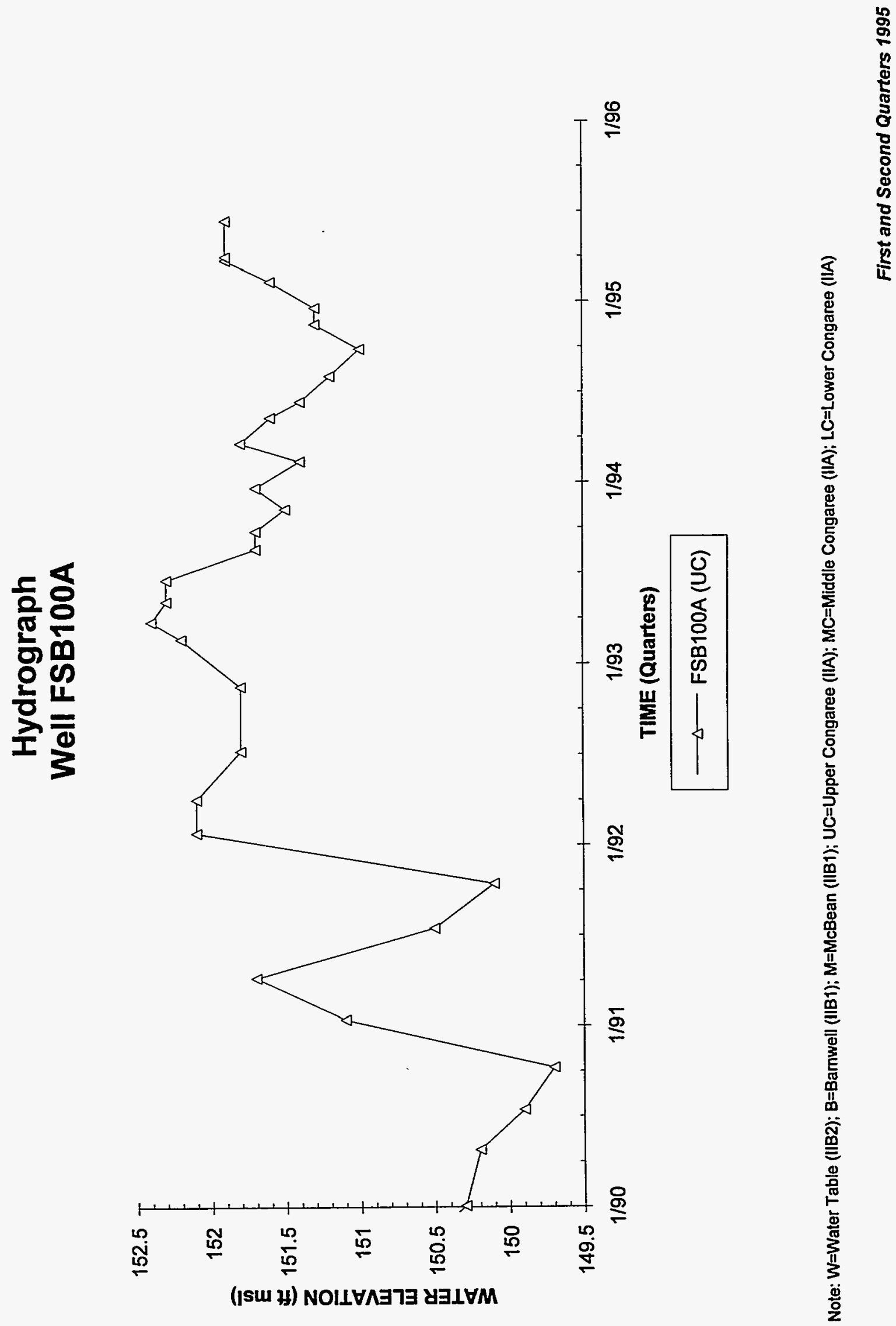

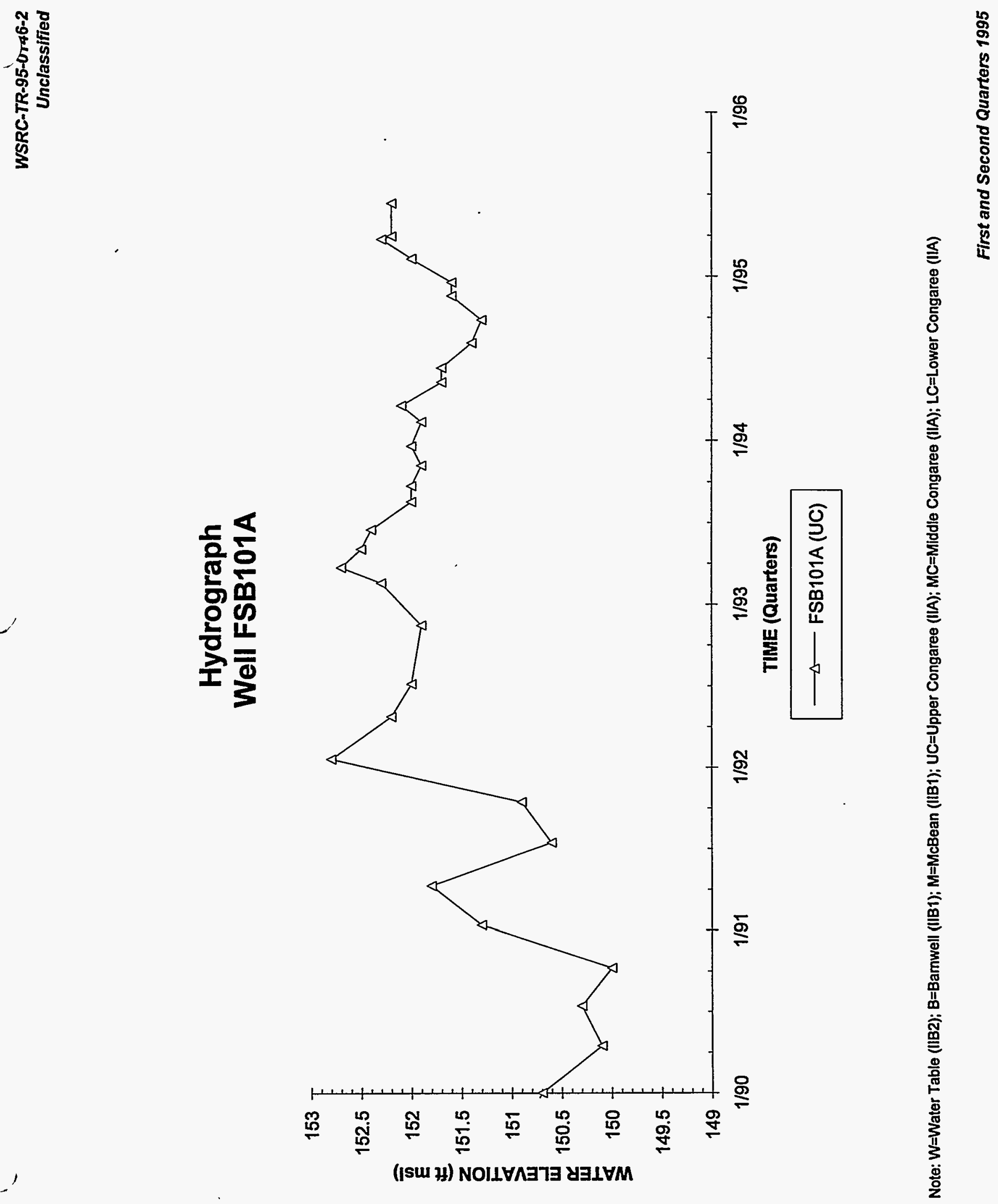


\section{Hydrograph \\ Well FSB102C}

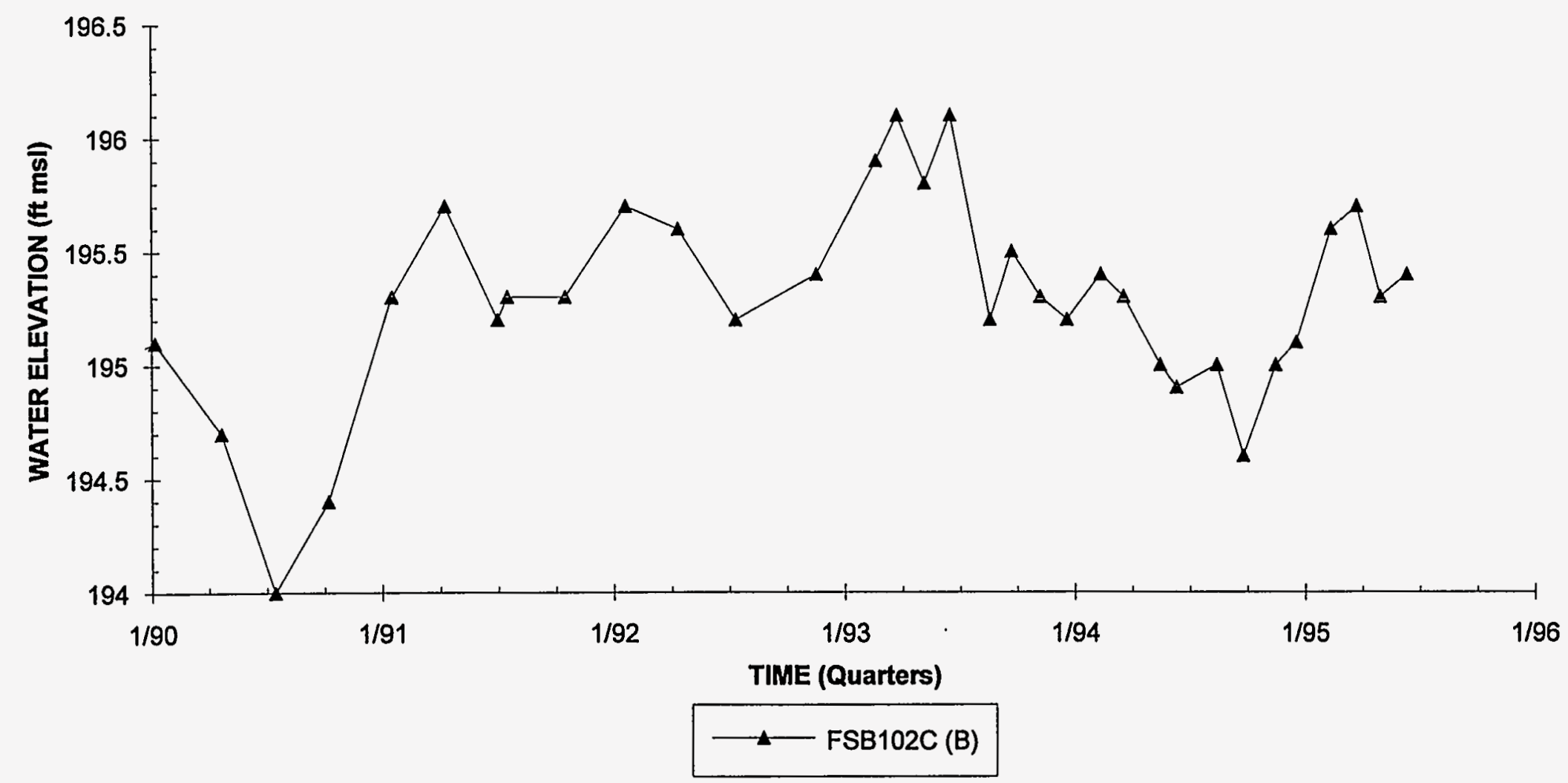

Note: W=Water Table (IIB2); B=Barnwell (IIB1); M=McBean (IIB1); UC=Upper Congaree (IIA); MC=Middle Congaree (IIA); LC=Lower Congaree (IIA) 


\section{Hydrograph}

Well FSB103C

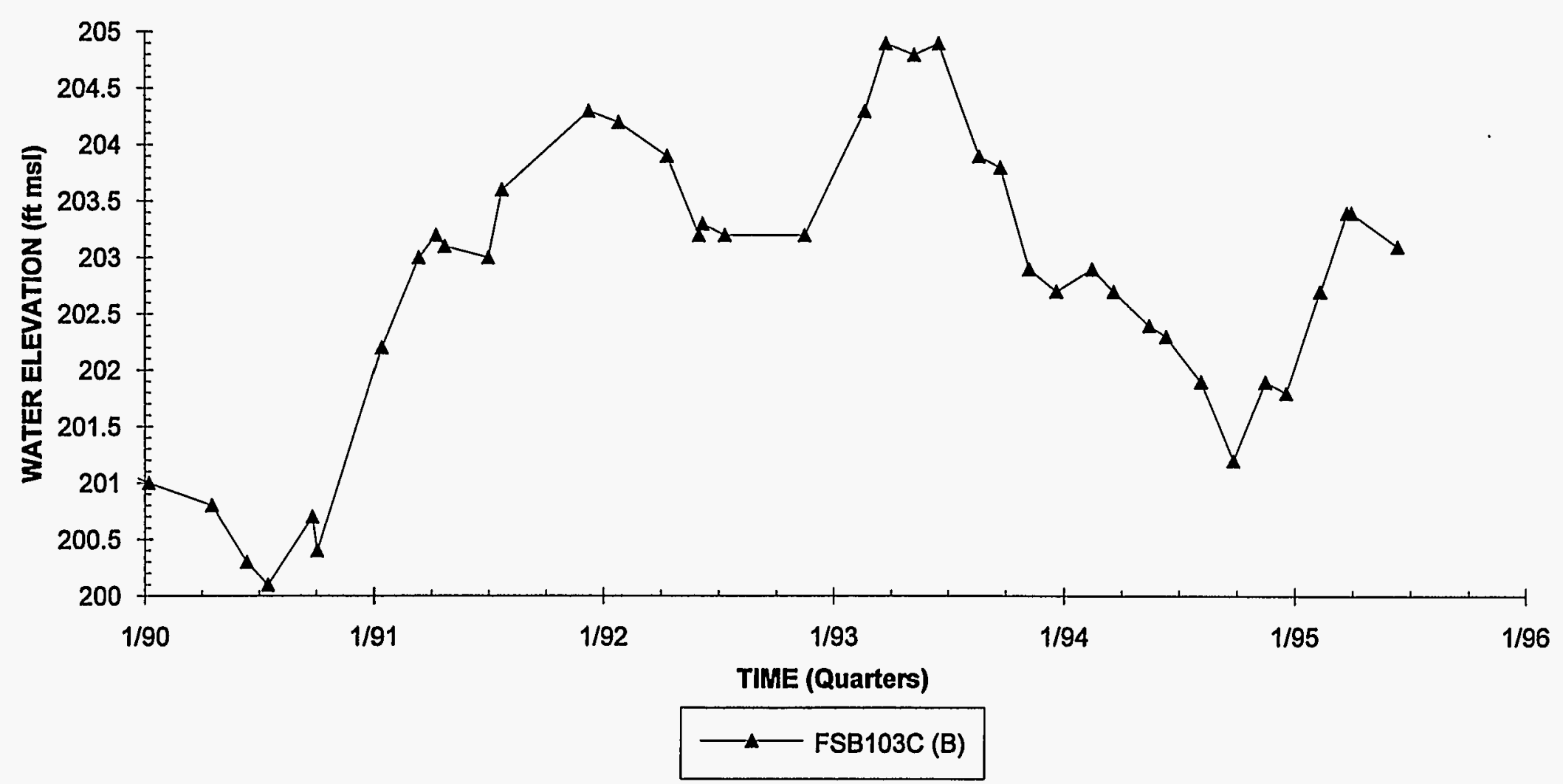

Note: W=Water Table (IIB2); B=Barnwell (IIB1); $M=$ McBean (IIB1); UC=Upper Congaree (IIA); MC=Middle Congaree (IIA); LC=Lower Congaree (IIA) 


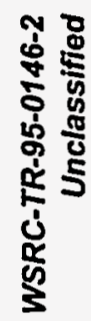
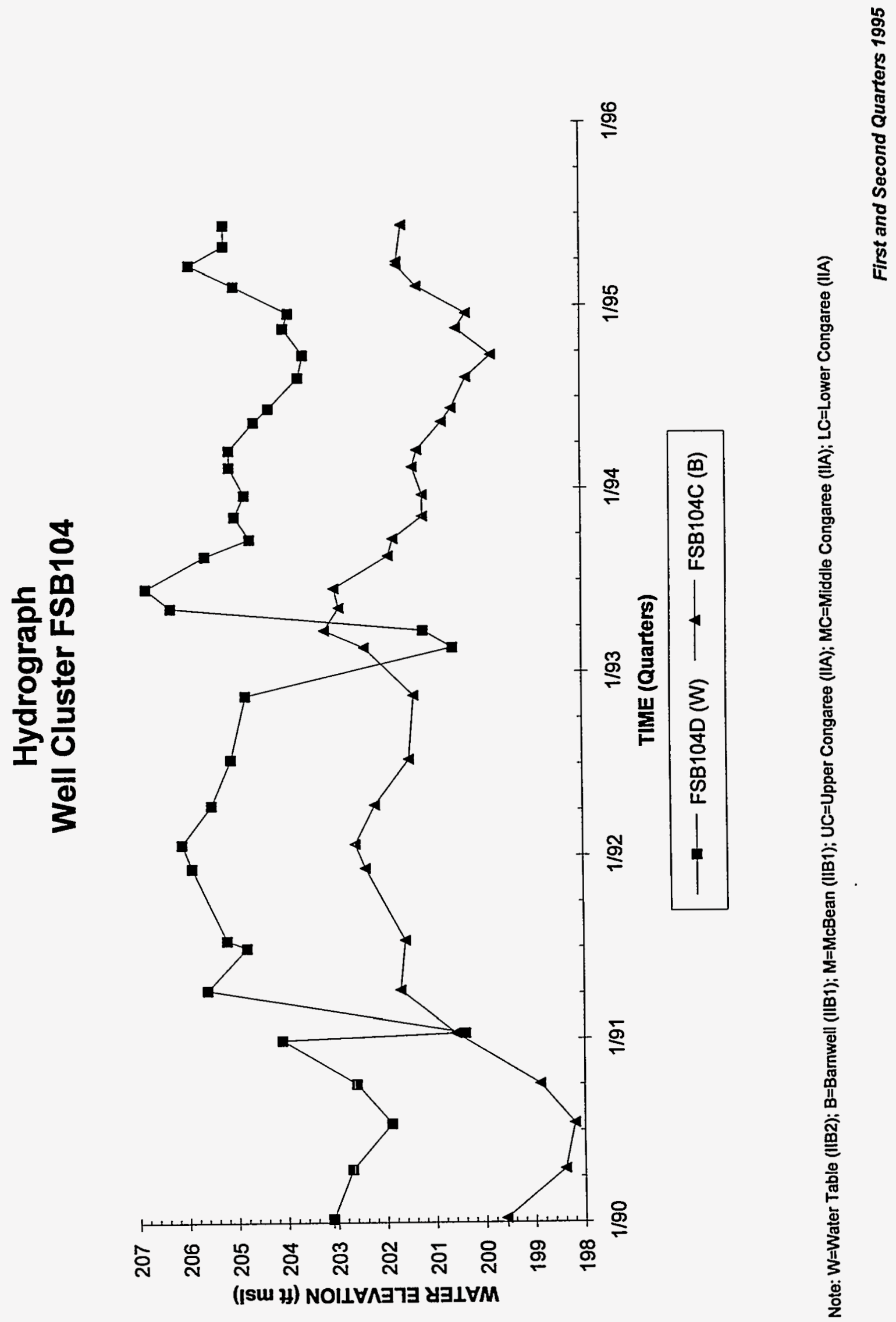


\section{Hydrograph \\ Well Cluster FSB105}

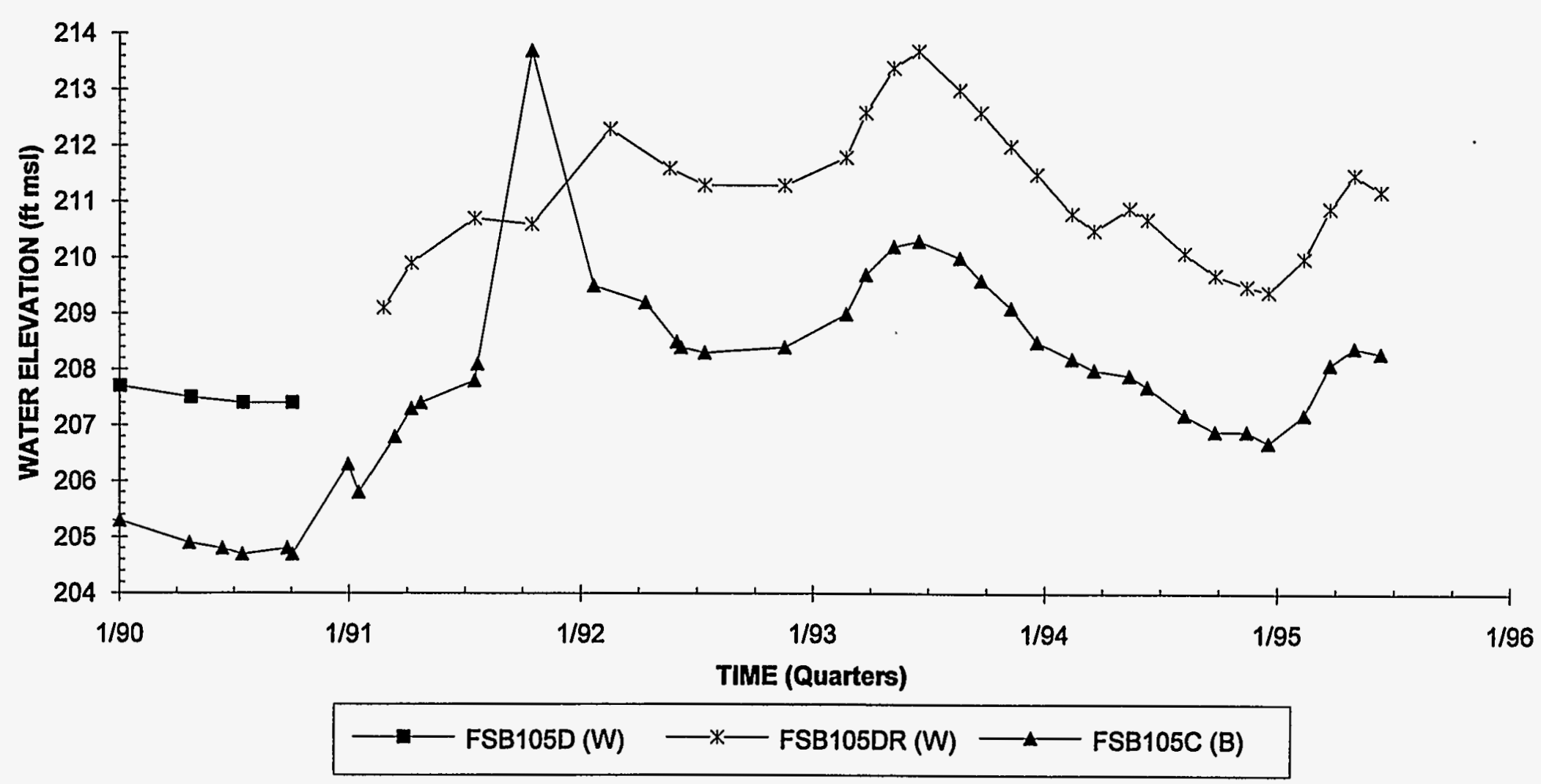

Note: W=Water Table (IIB2); B=Barnwell (IIB1); M=McBean (IIB1); UC=Upper Congaree (IIA); MC=Middle Congaree (IIA); LC=Lower Congaree (IIA) 


\section{Hydrograph \\ Well Cluster FSB106}

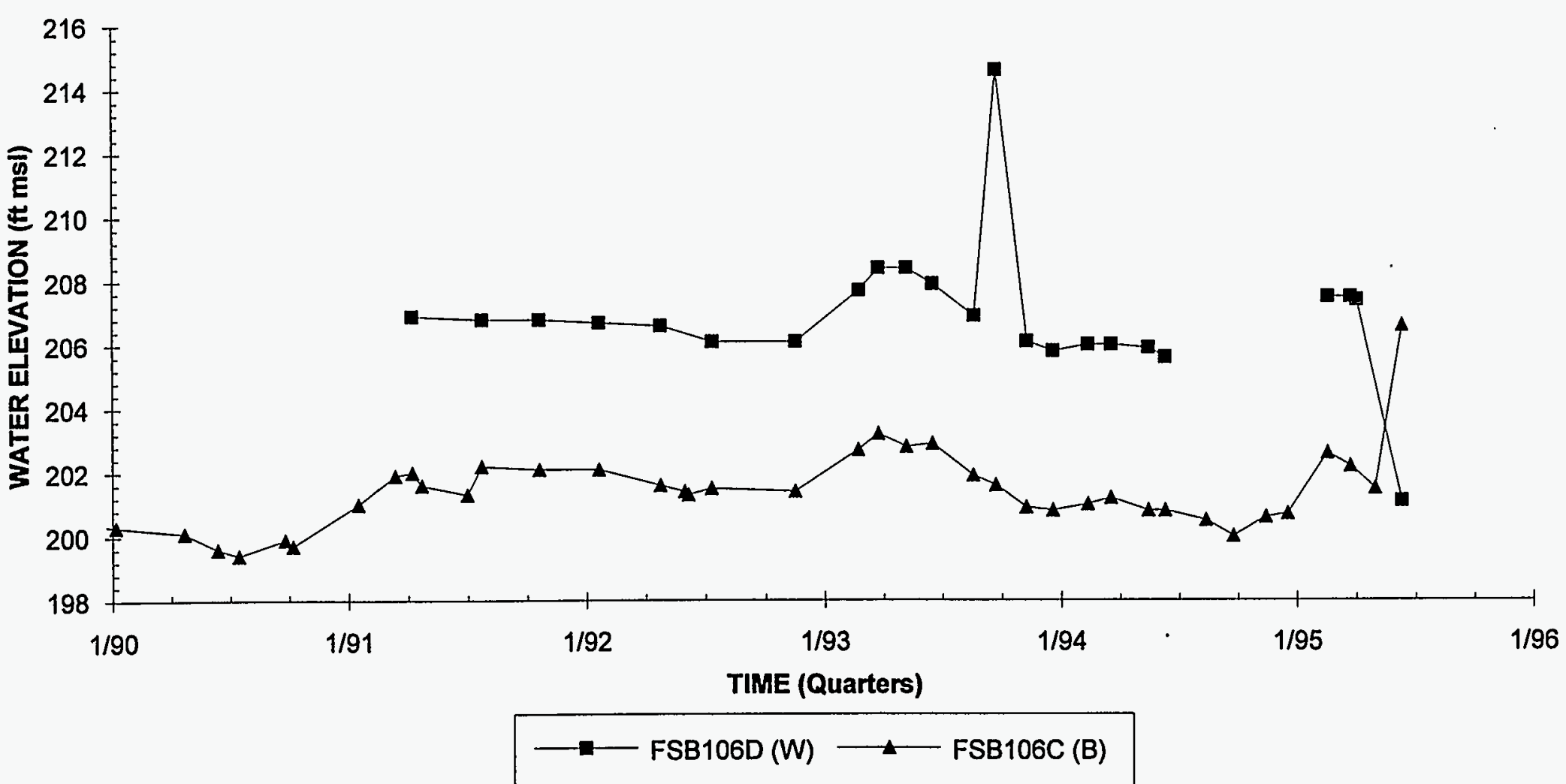

Note: W=Water Table (IIB2); B=Barnwell (IIB1); M=MCBean (IIB1); UC=Upper Congaree (IIA); MC=Middle Congaree (IIA); LC=Lower Congaree (IIA) 


\section{Hydrograph \\ Well Cluster FSB107}

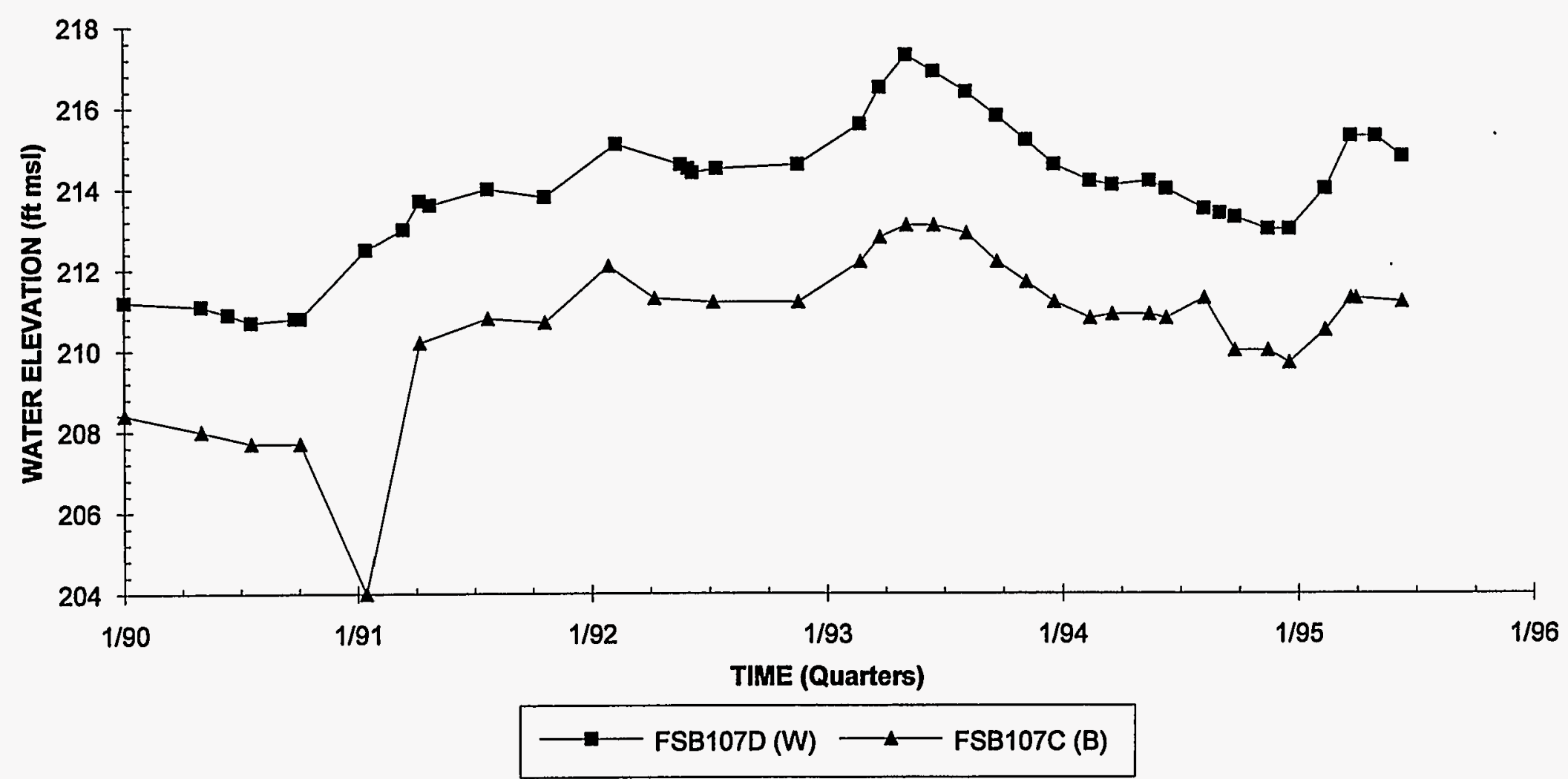

Note: W=Water Table (IIB2); B=Barnwell (IIB1); M=MCBean (IIB1); UC=Upper Congaree (IIA); MC=Middle Congaree (IIA); LC=Lower Congaree (IIA) 


\section{Hydrograph \\ Well FSB108D}

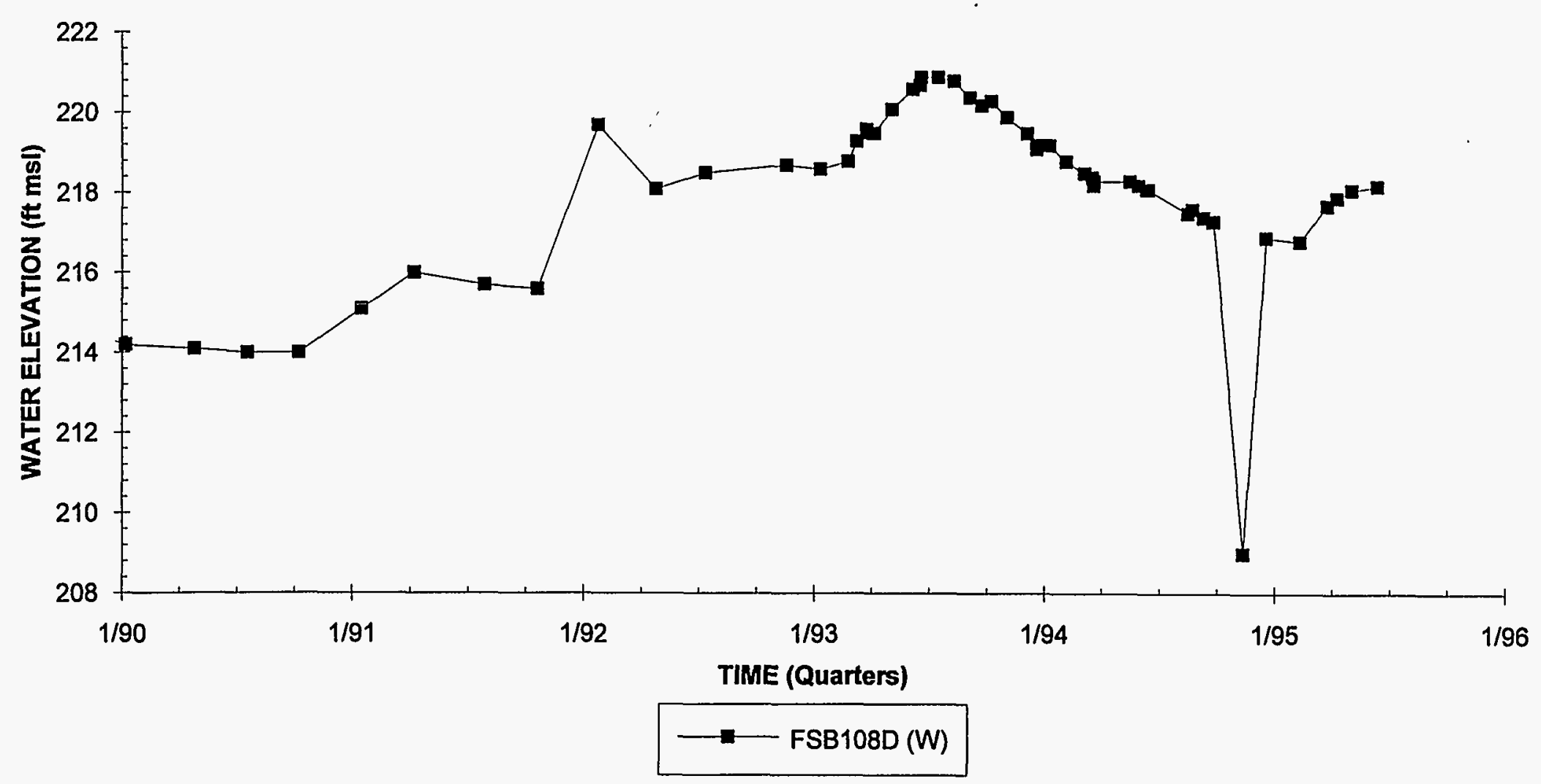

Note: W=Water Table (IIB2); B=Barnwell (IIB1); M=McBean (IIB1); UC=Upper Congaree (IIA); MC=Middle Congaree (IIA); LC=Lower Congaree (IIA) 


\section{Hydrograph \\ Well FSB109D}

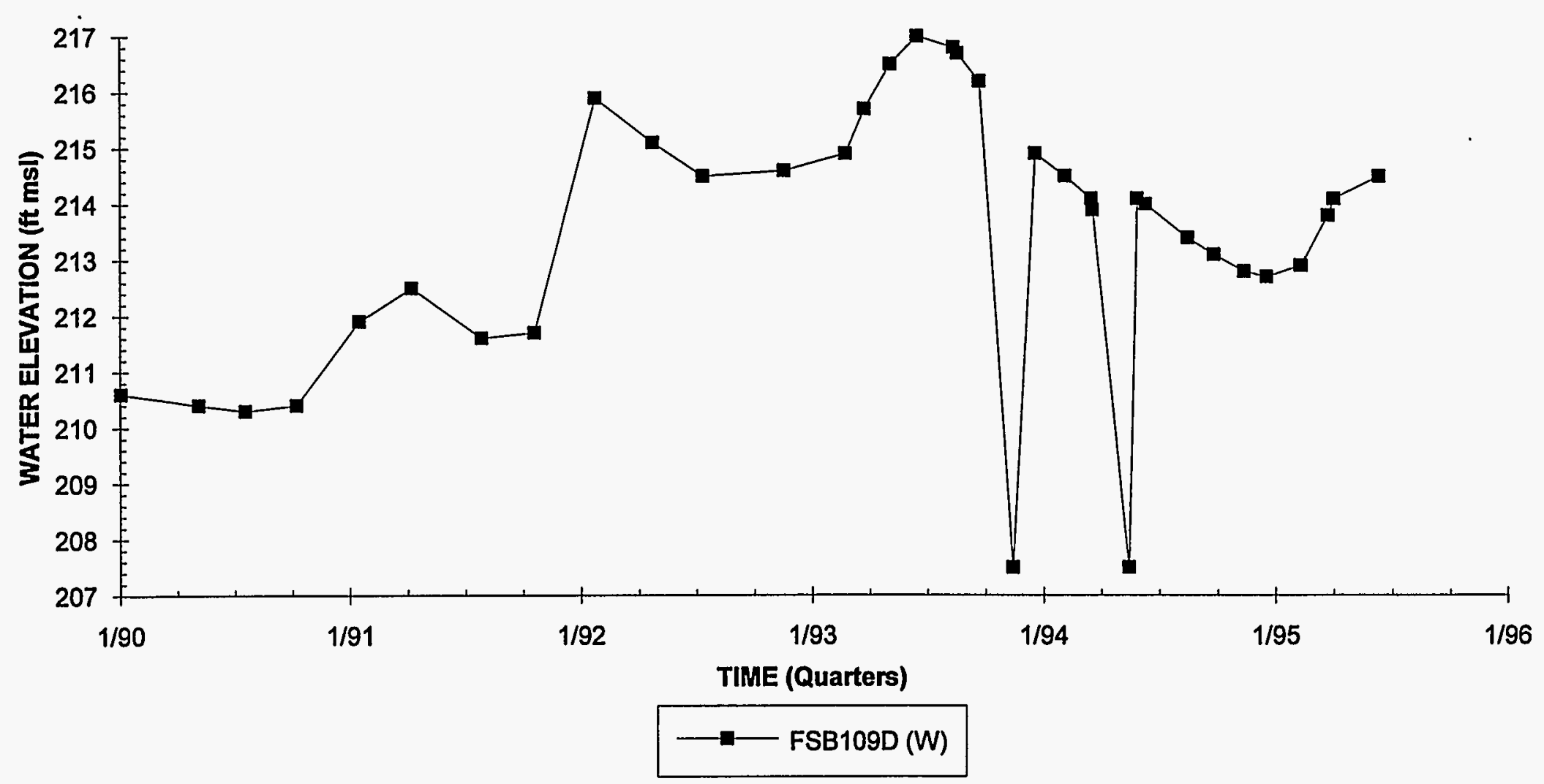

Note: W=Water Table (IIB2); B=Barnwell (IIB1); M=McBean (IIB1); UC=Upper Congaree (IIA); MC=Middle Congaree (IIA); LC=Lower Congaree (IIA) 


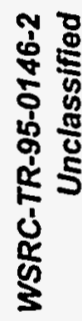
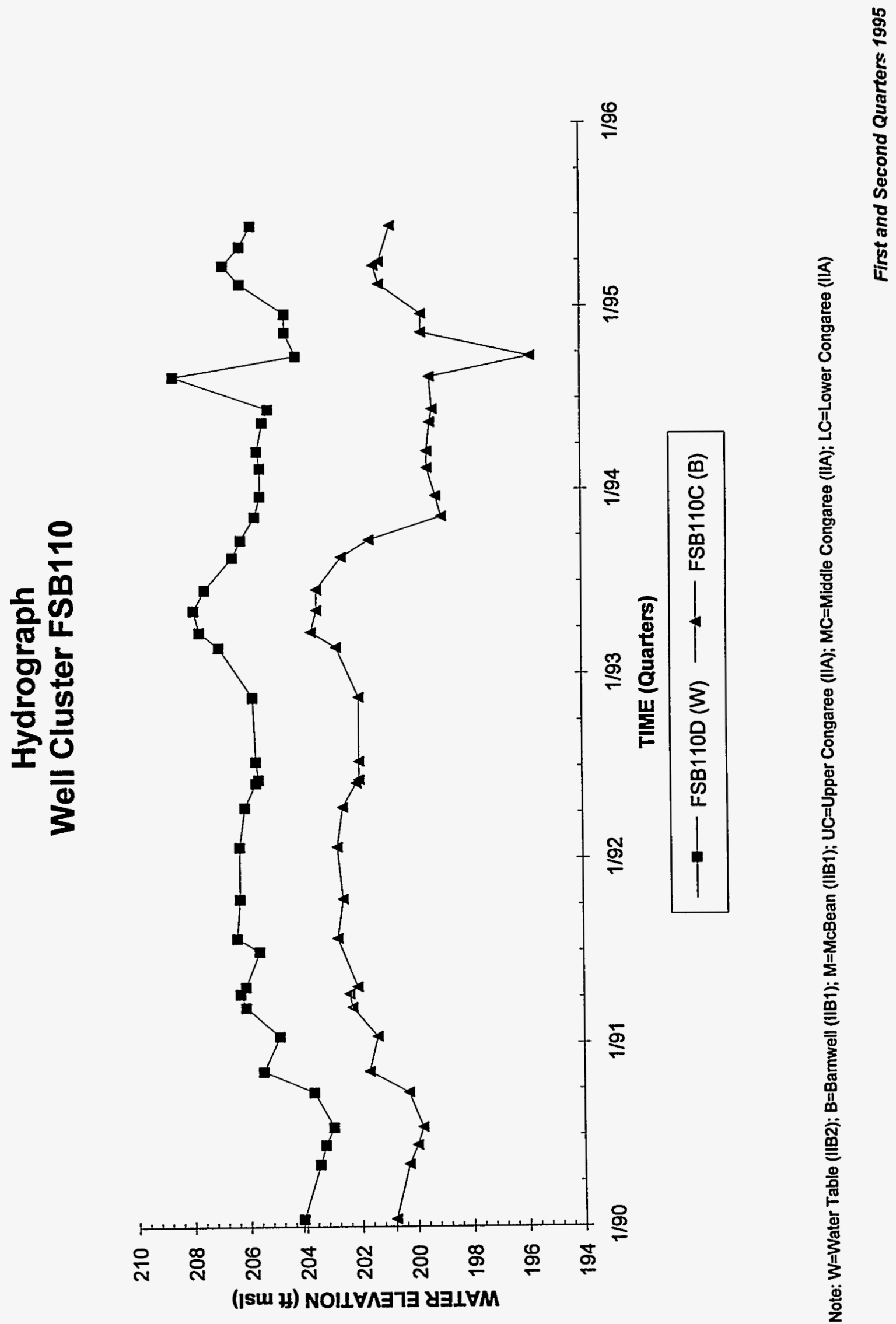

总 


\section{Hydrograph \\ Well Cluster FSB111}

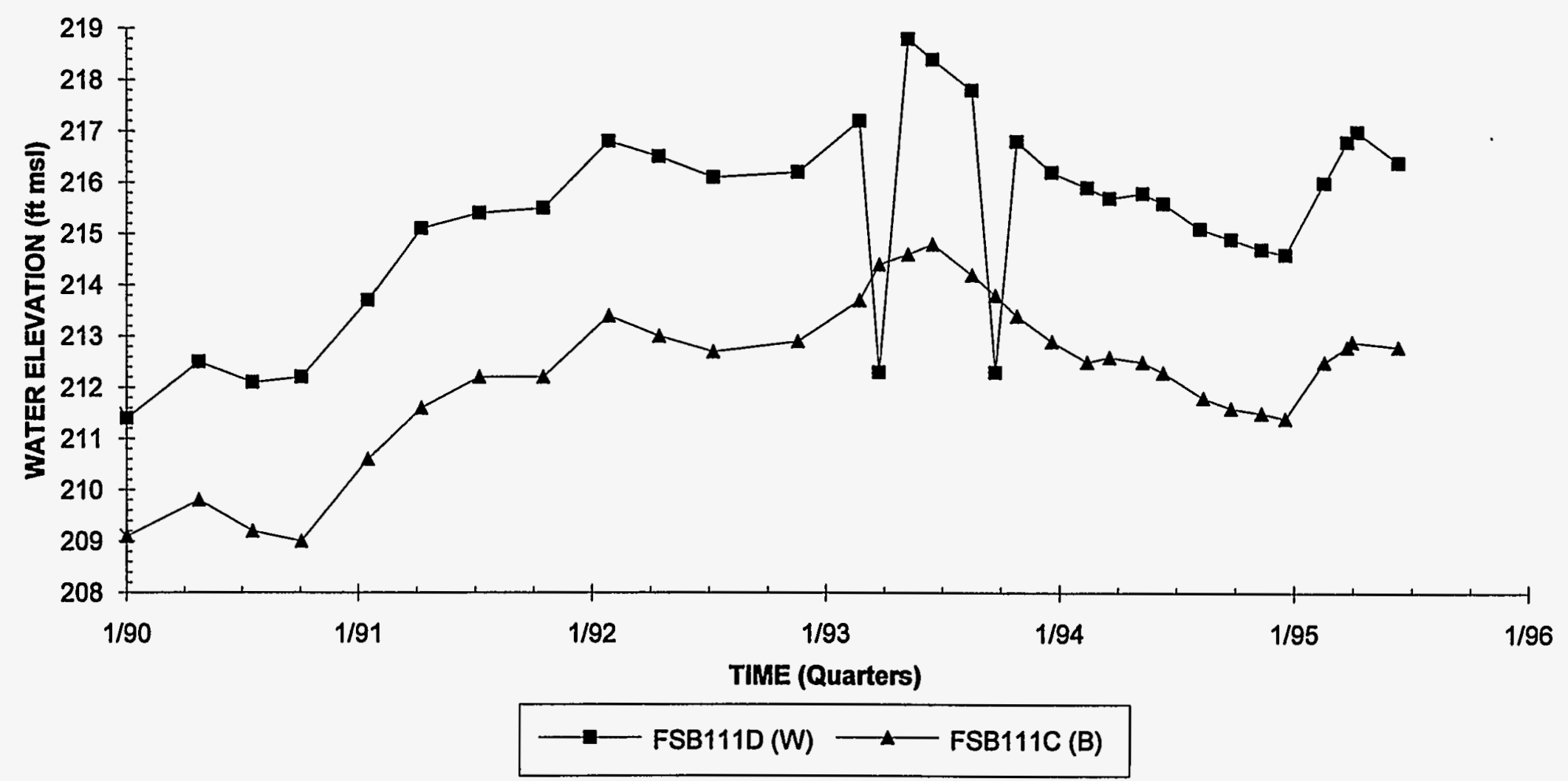

Note: W=Water Table (IIB2); B=Barnwell (IIB1); M=McBean (IIB1); UC=Upper Congaree (IIA); MC=Middle Congaree (IIA); LC=Lower Congaree (IIA) 


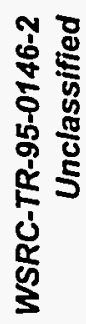
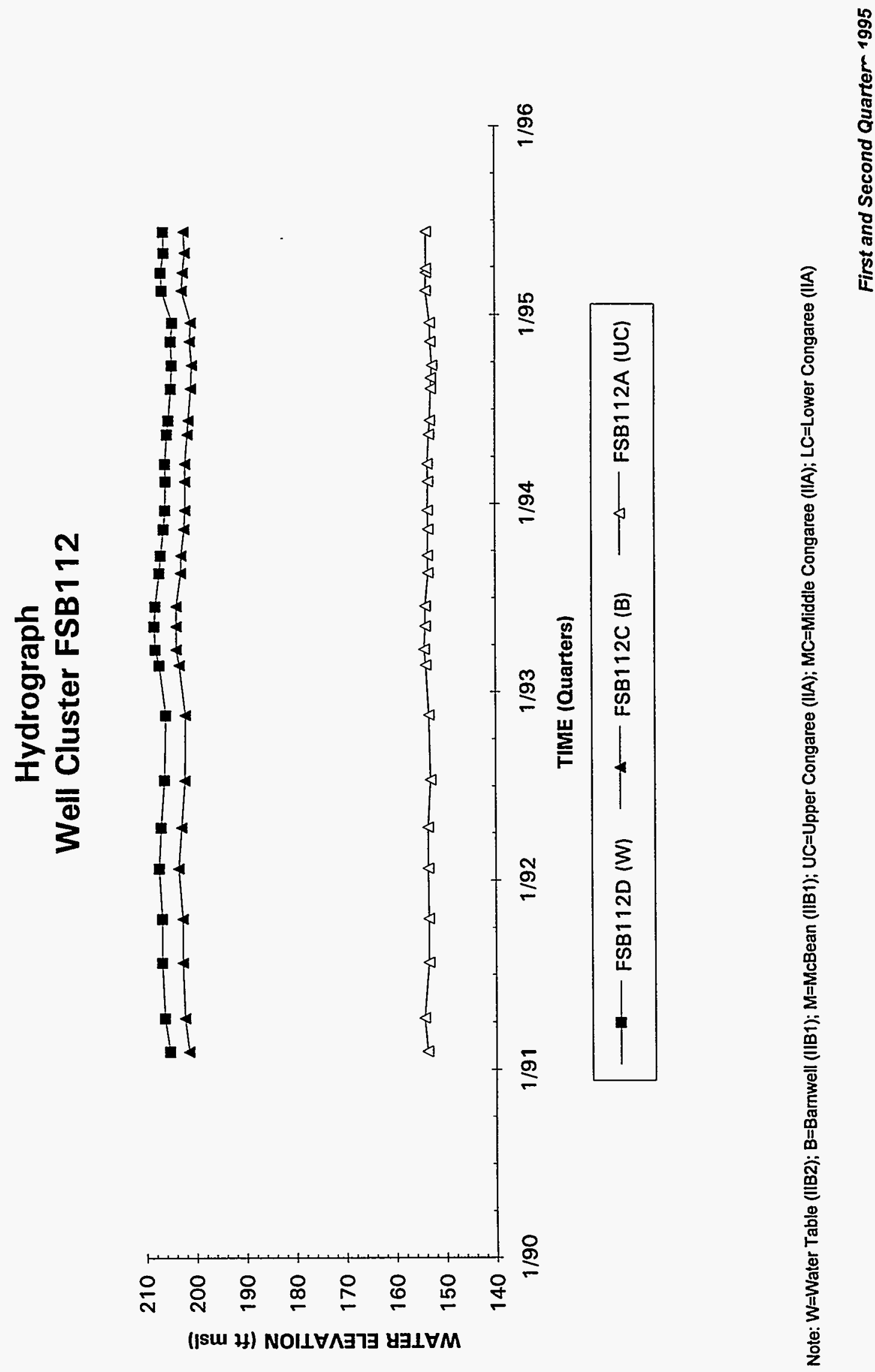

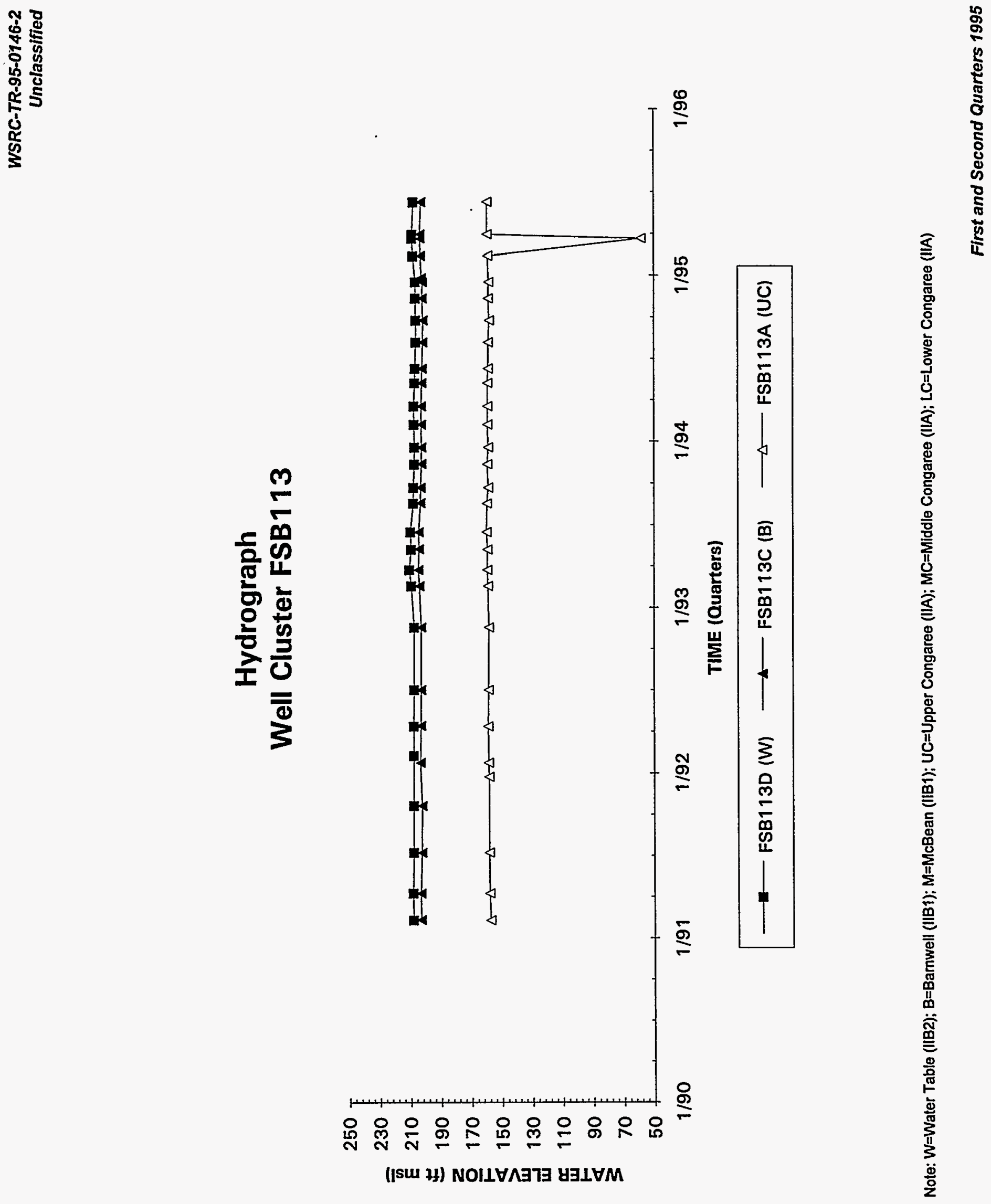


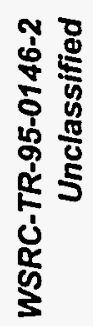

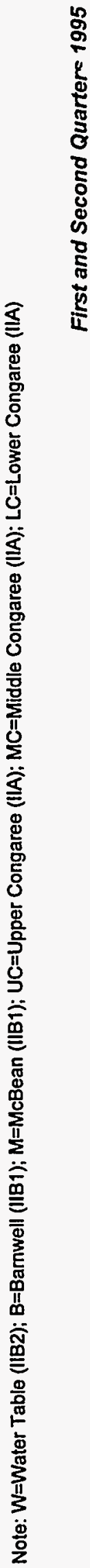



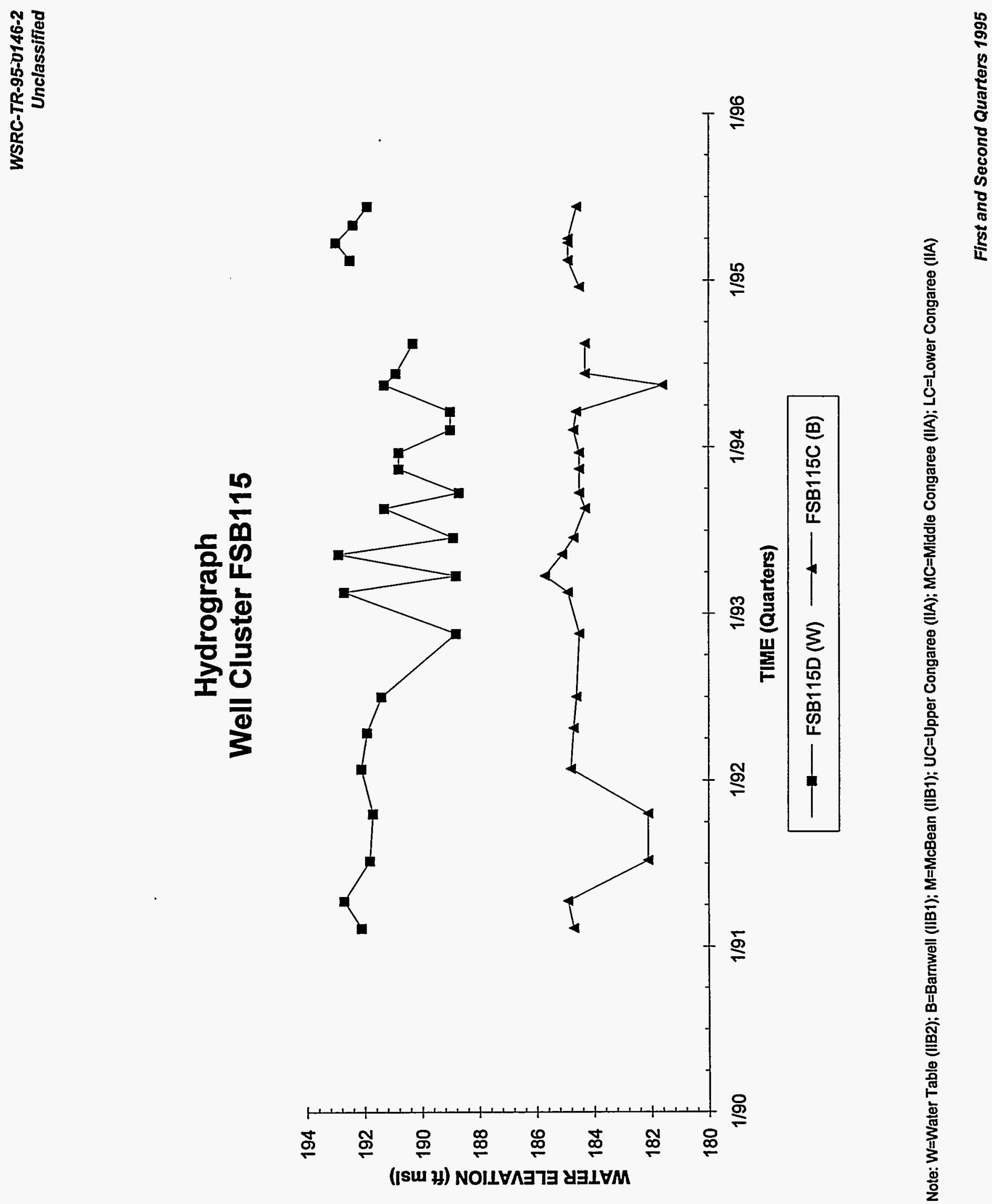


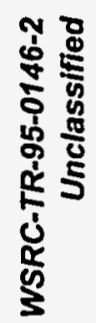
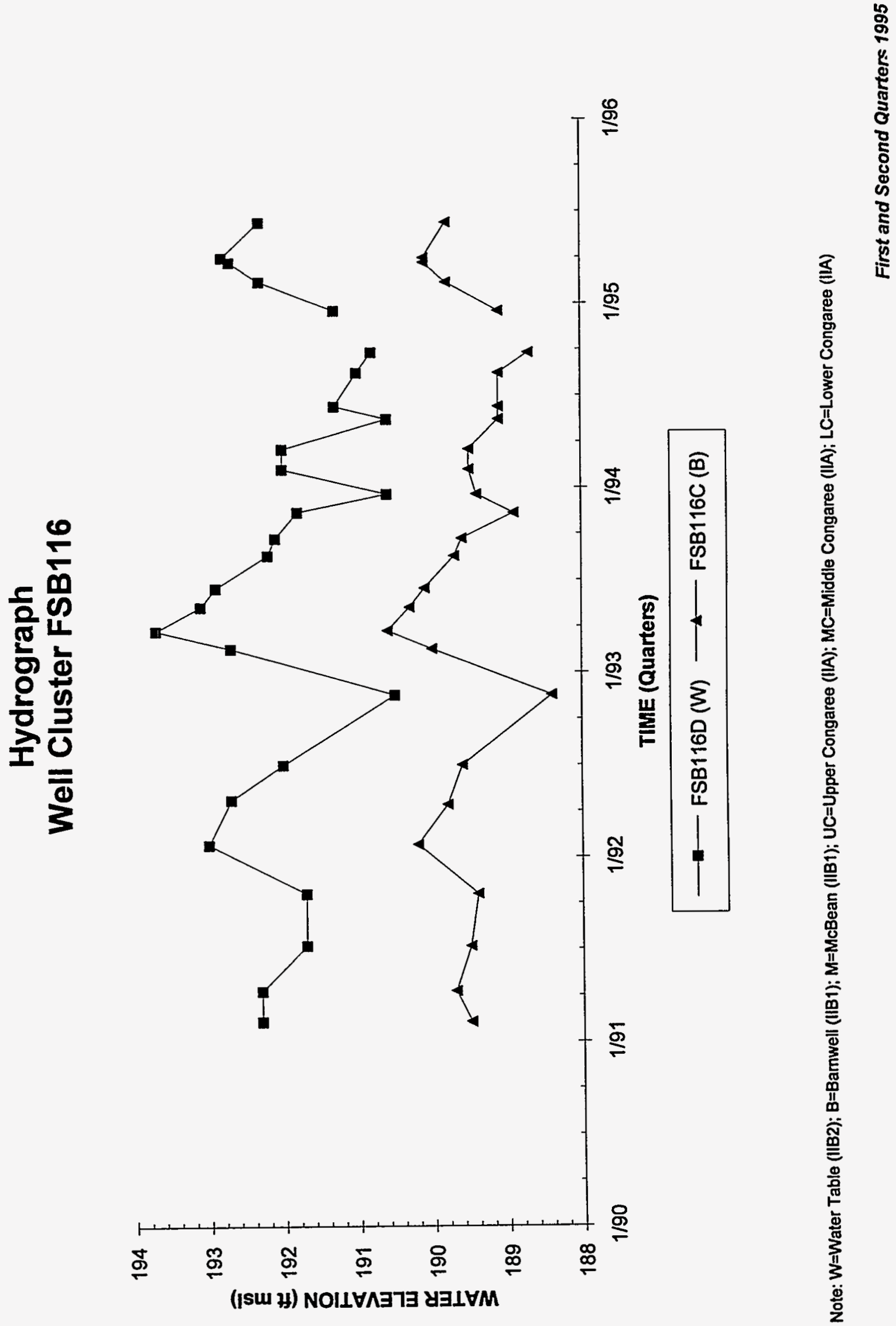


\section{Hydrograph \\ Well FSB117D}

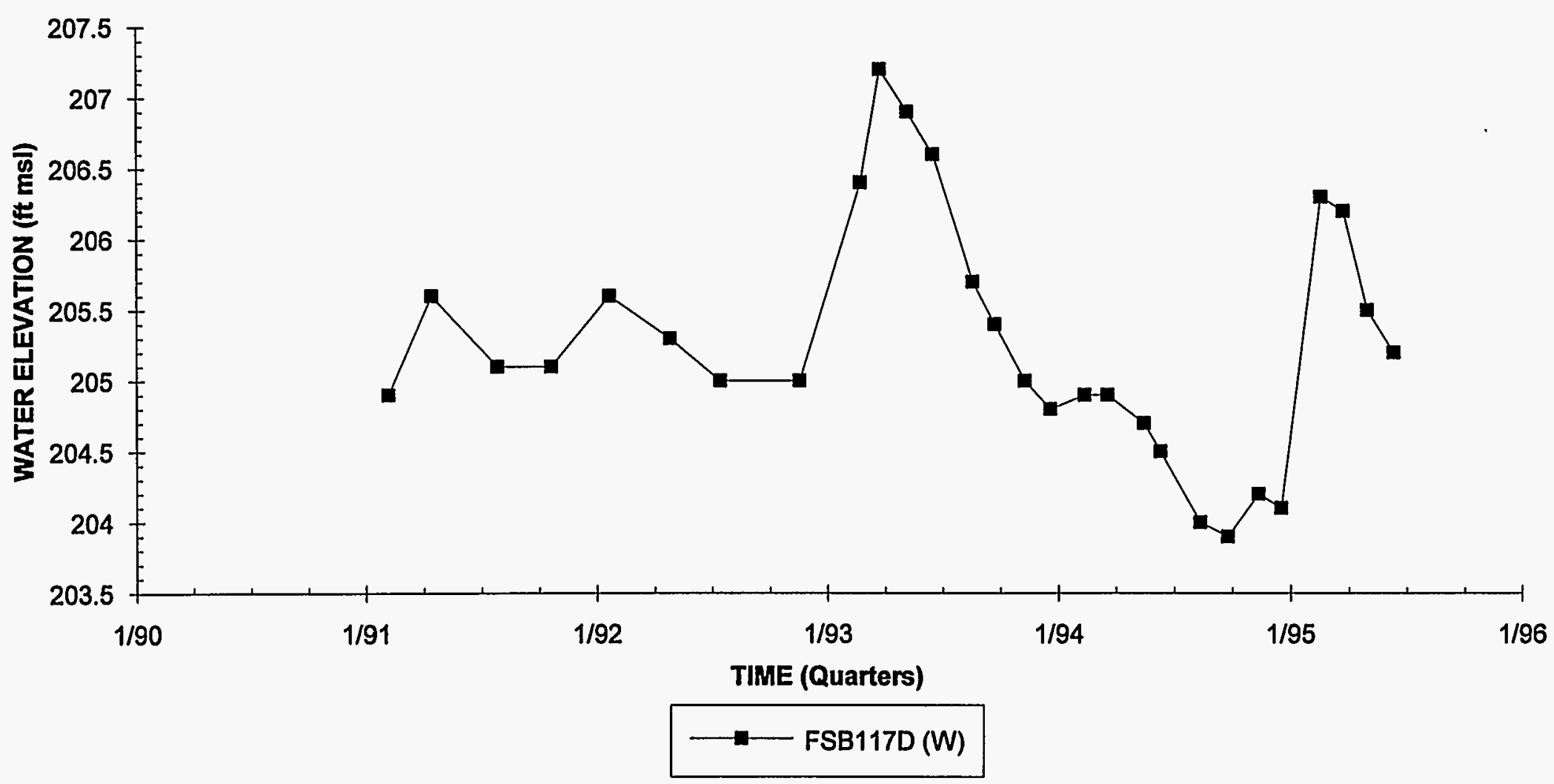

Note: W=Water Table (IIB2); B=Barnwell (IIB1); M=McBean (IIB1); UC=Upper Congaree (IIA); MC=Middle Congaree (IIA); LC=Lower Congaree (IIA) 


\section{Hydrograph \\ Well FSB118D}

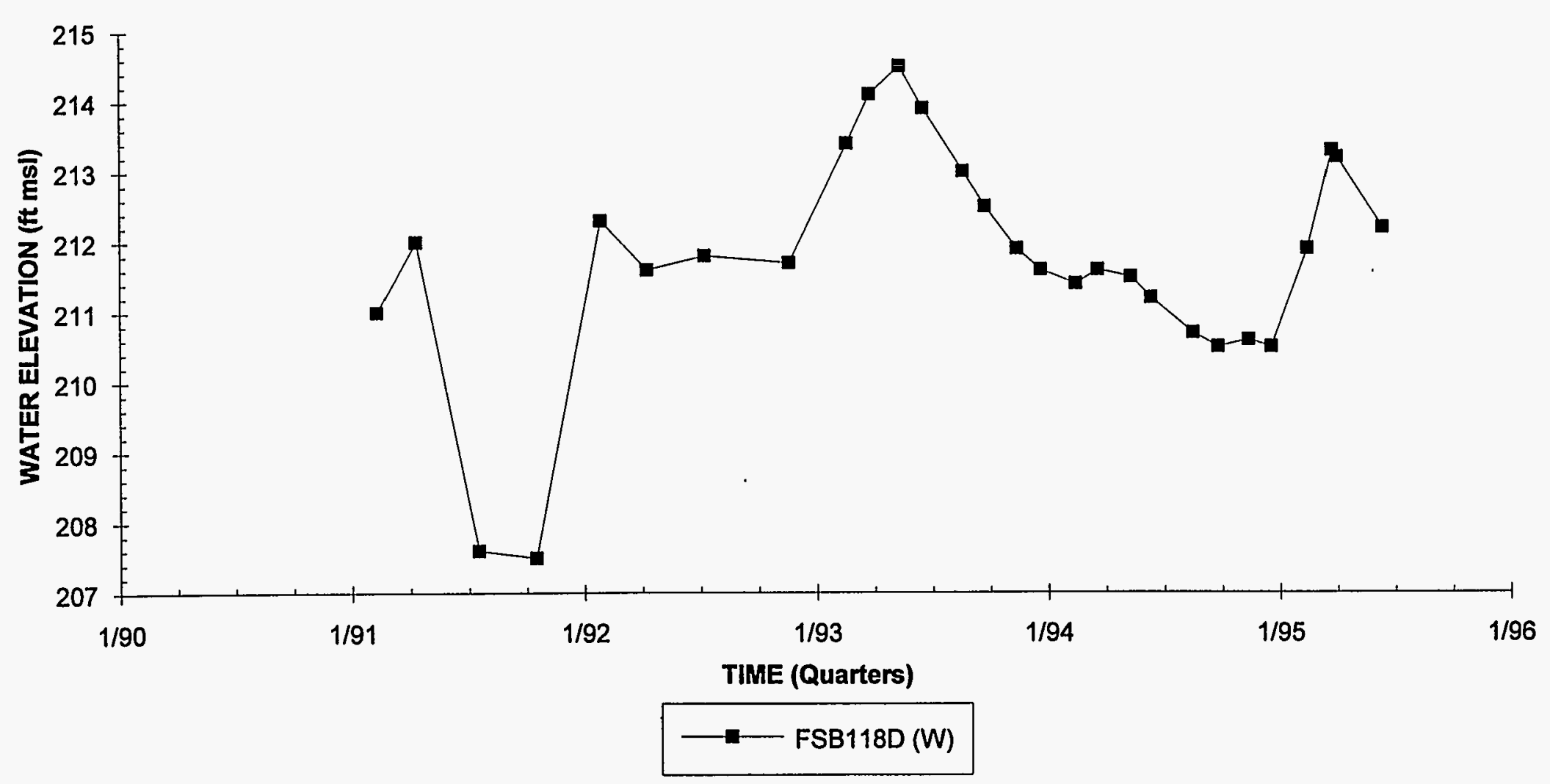

Note: $W=$ Water Table (IIB2); B=Barnwell (IIB1); M=MCBean (IIB1); UC=Upper Congaree (IIA); MC=Middle Congaree (IIA); LC=Lower Congaree (IIA) 

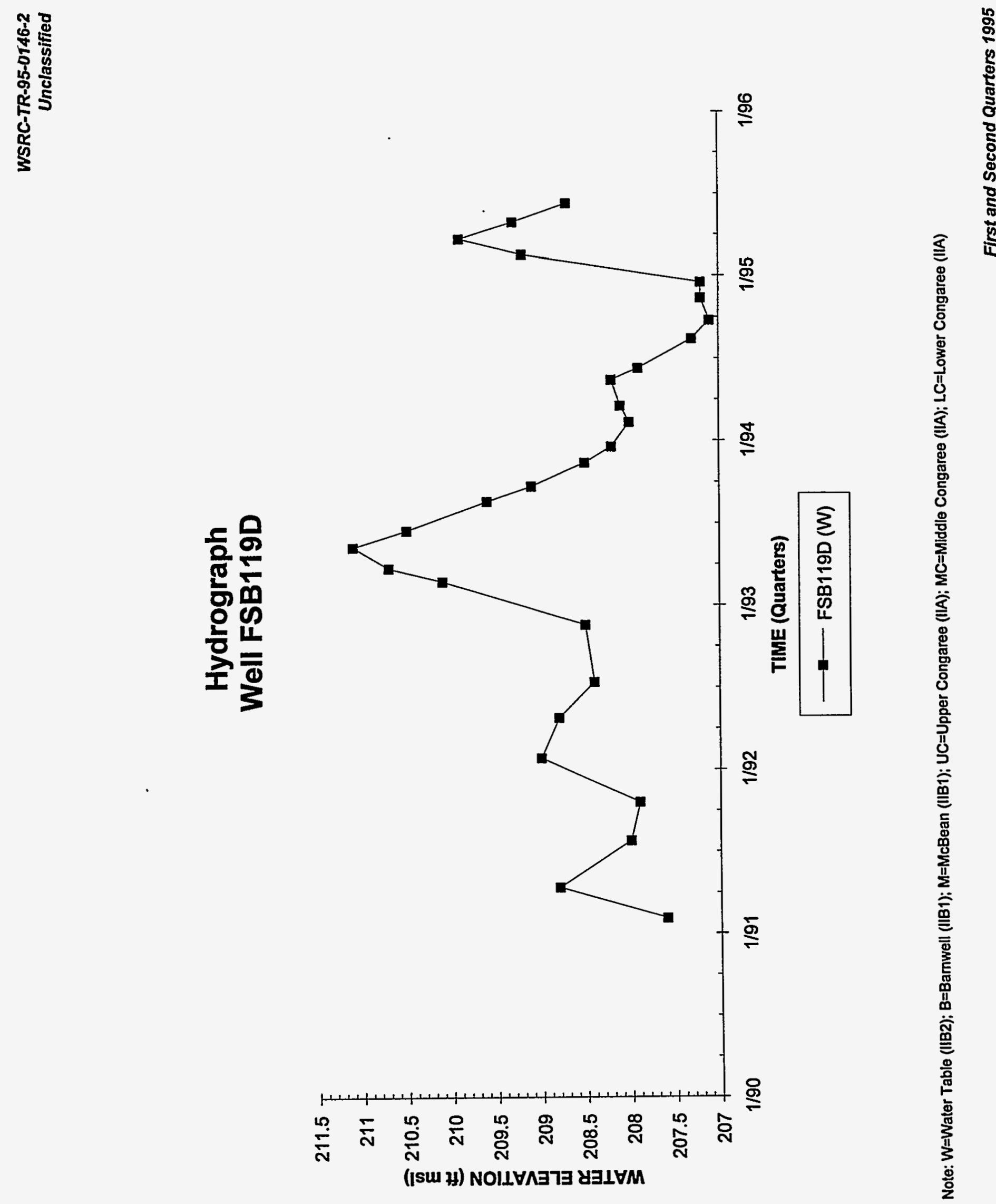


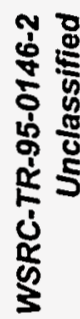
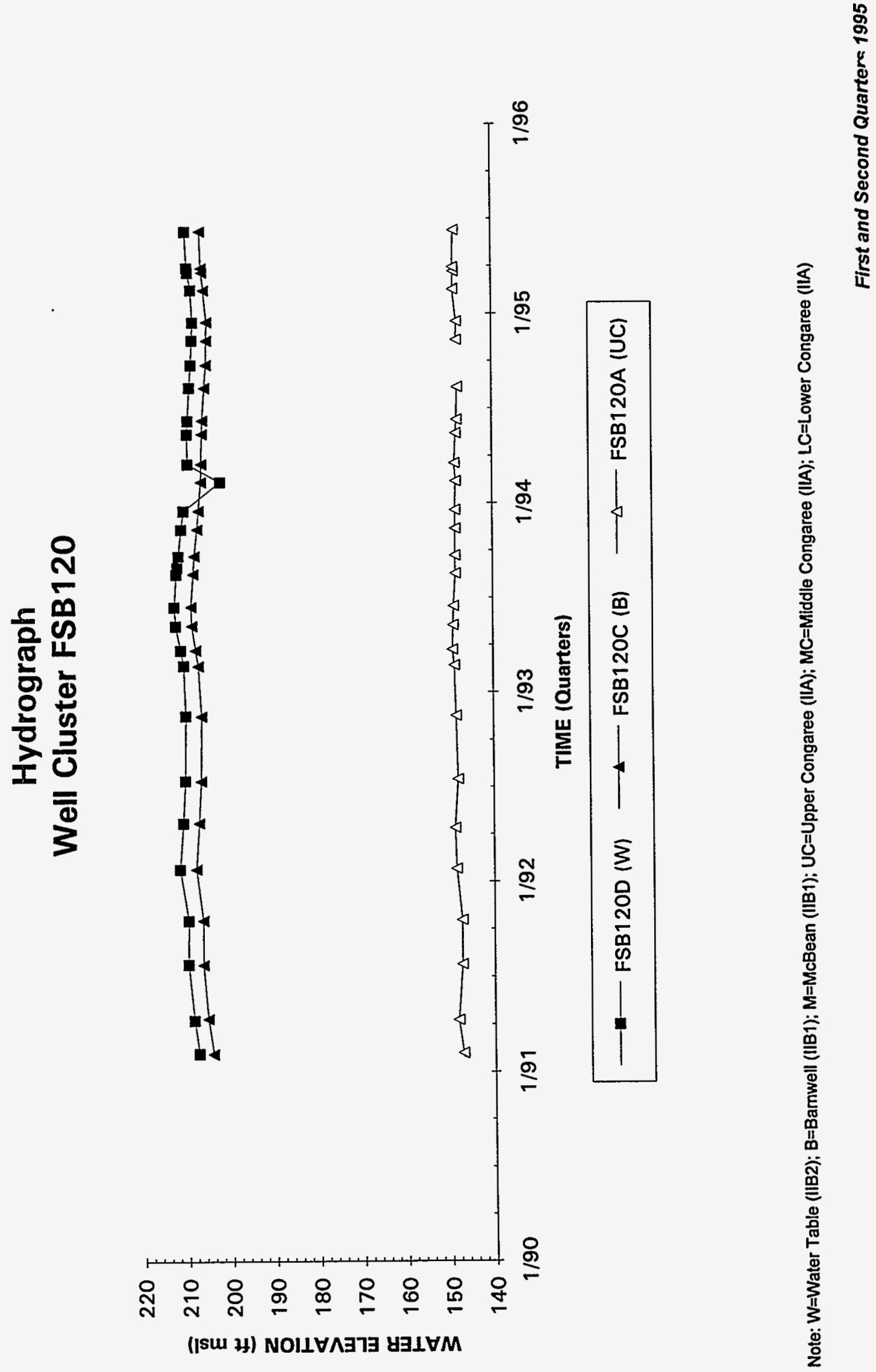


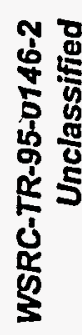
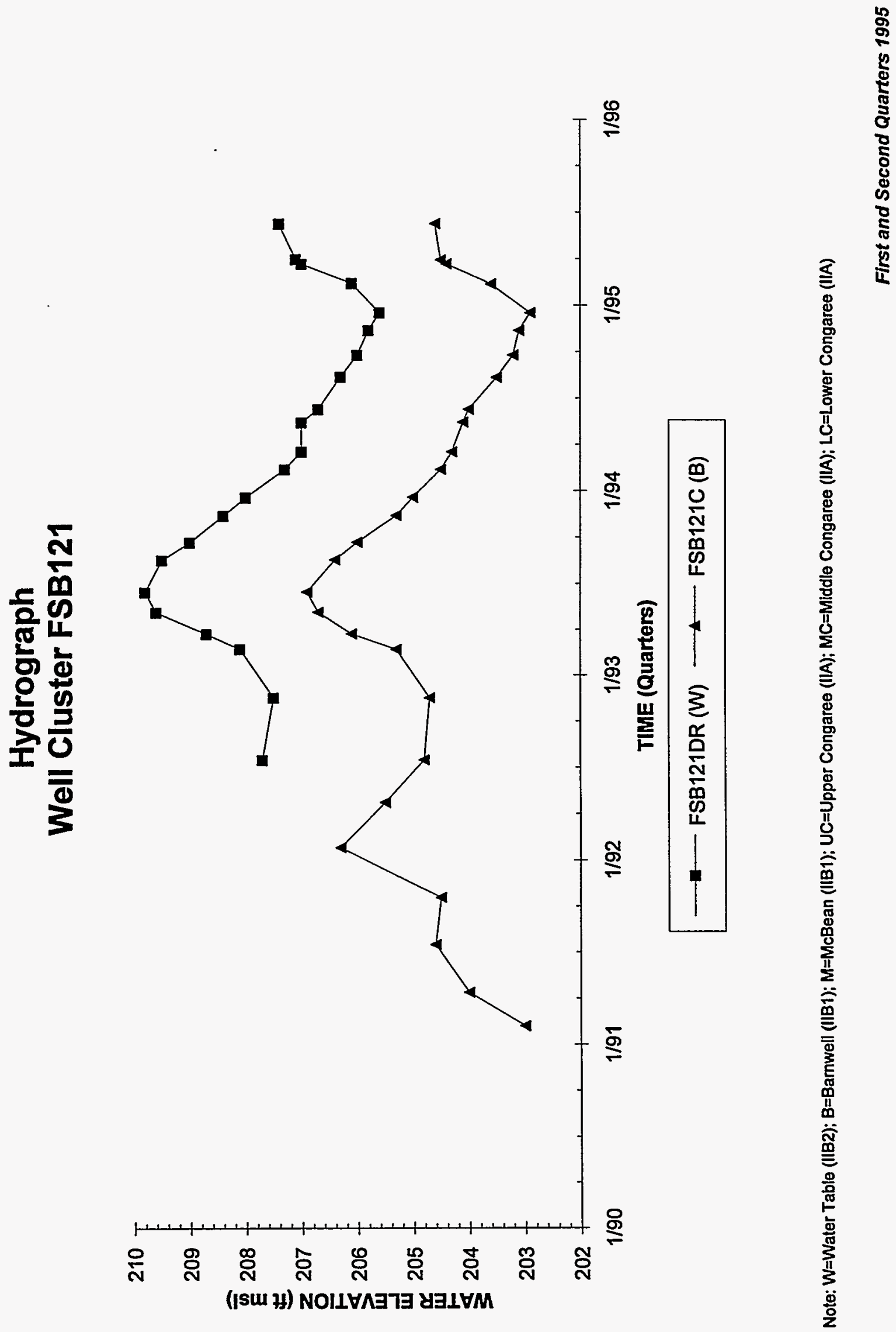


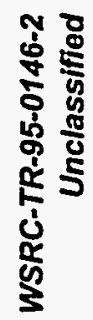
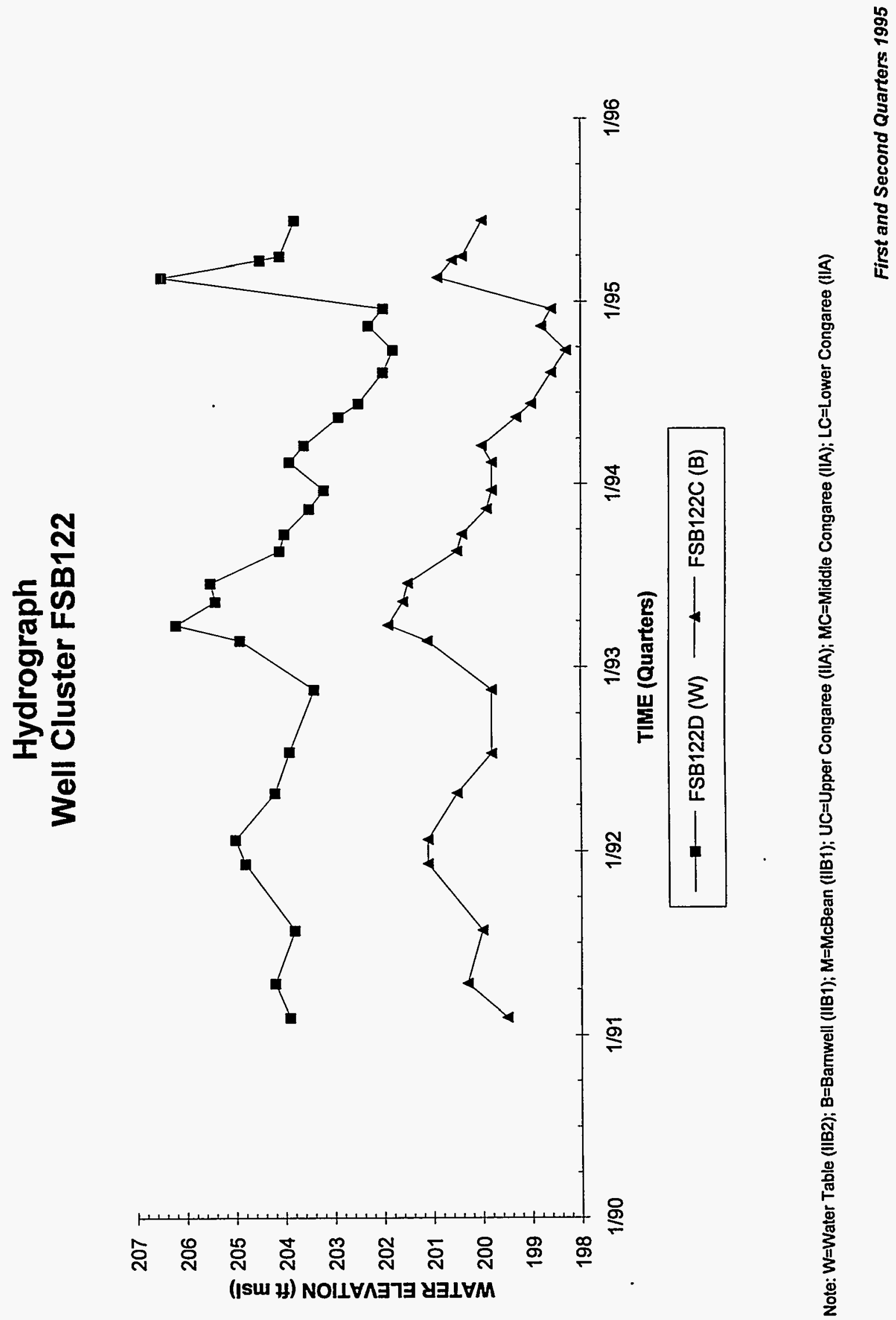


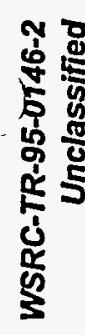
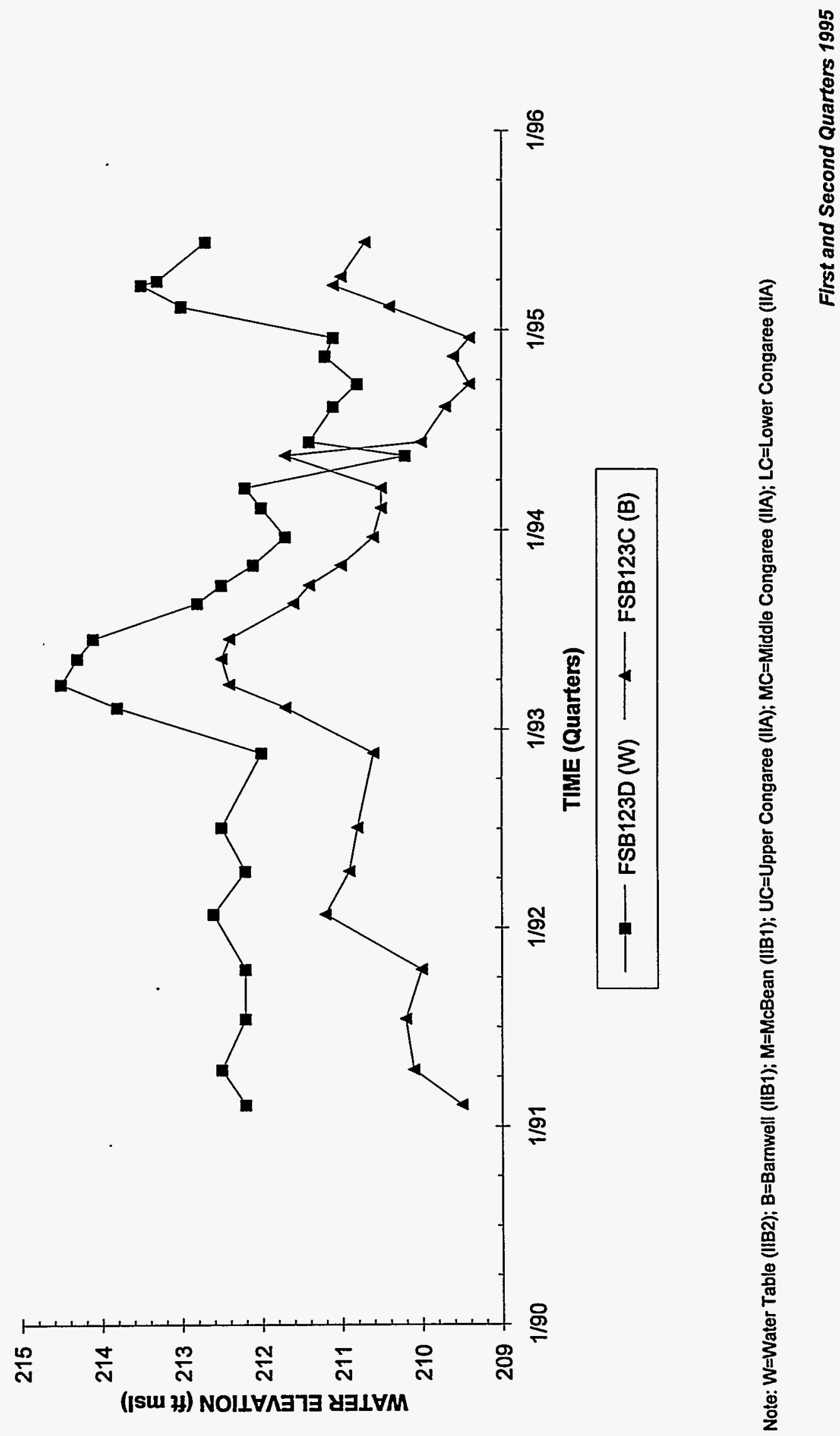


\section{Hydrograph \\ Well FSL 1D}

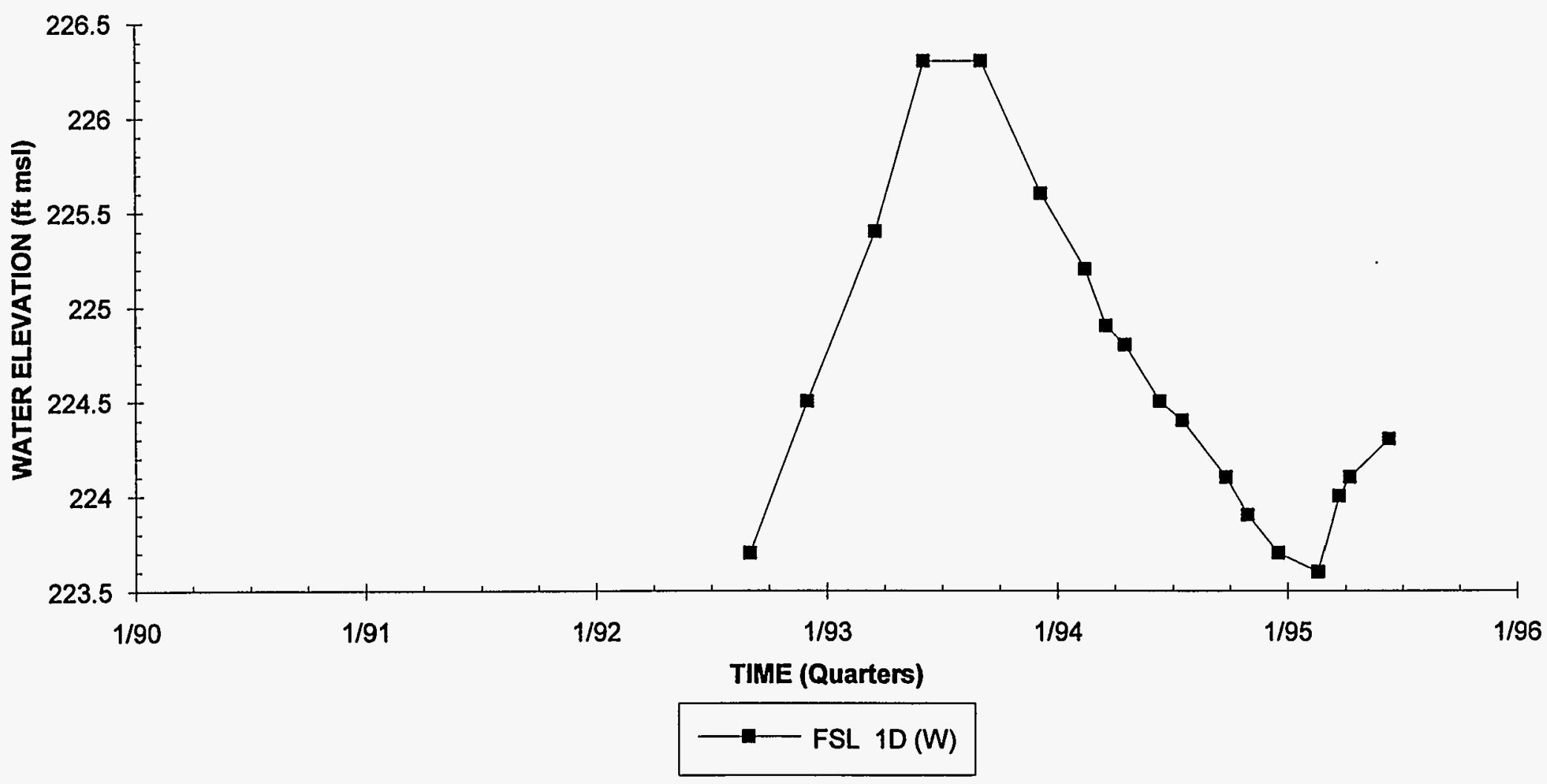

Note: W=Water Table (IIB2); B=Barnwell (IIB1); M=McBean (IIB1); UC=Upper Congaree (IIA); MC=Middle Congaree (IIA); LC=Lower Congaree (IIA) 


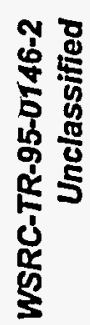
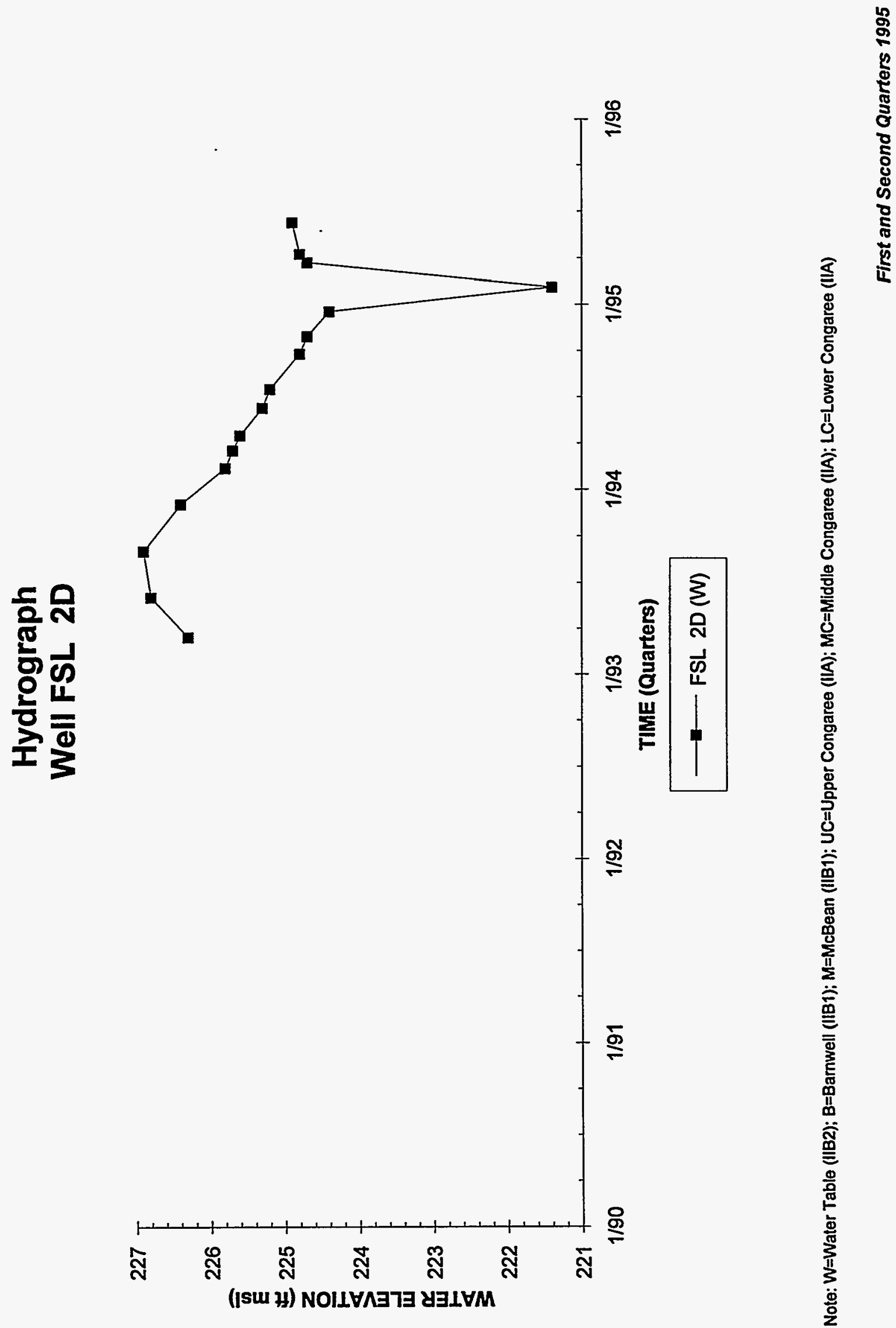


\section{Hydrograph}

Well FSL 3D

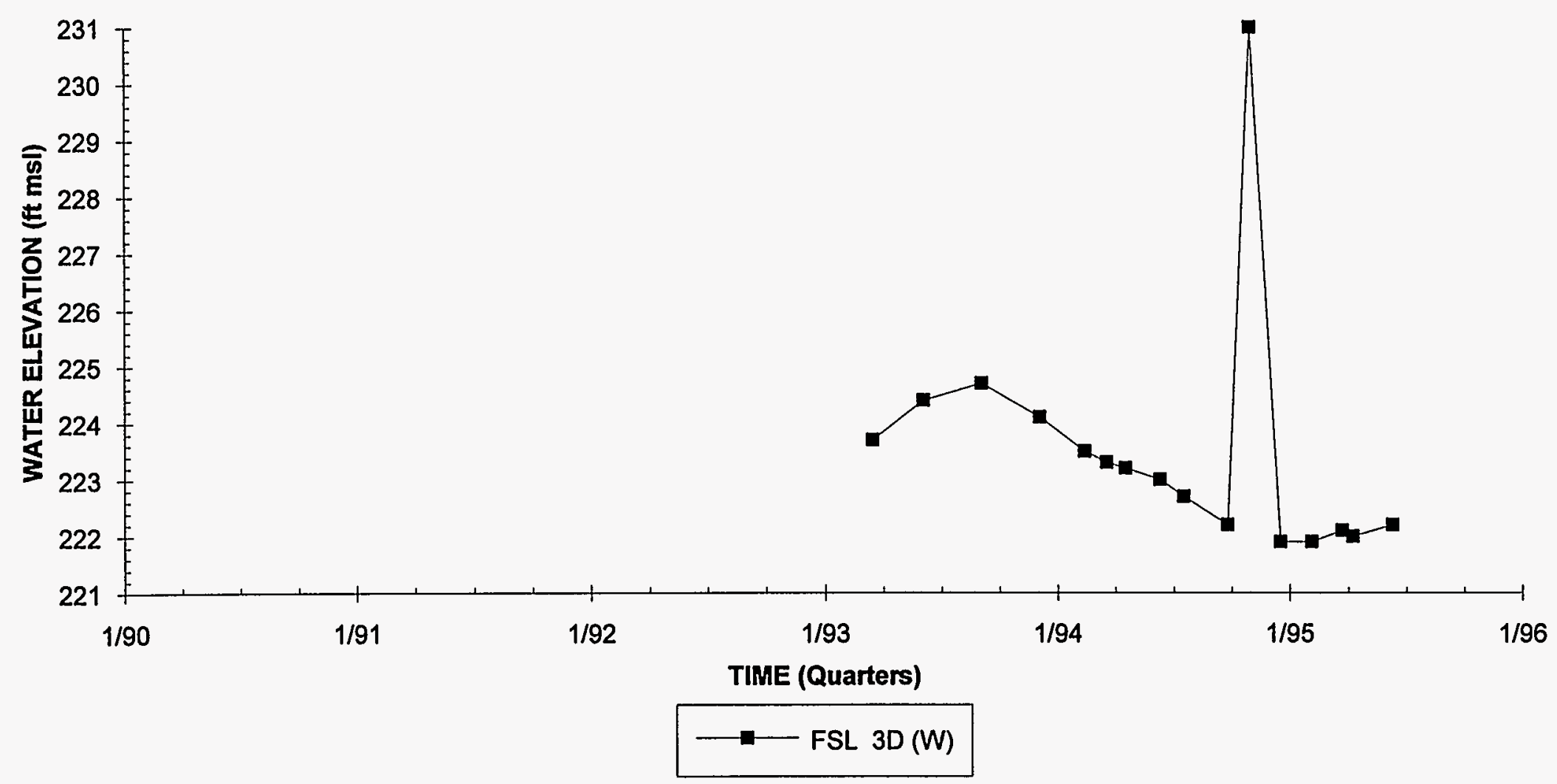

Note: W=Water Table (IIB2); B=Barnwell (IIB1); M=McBean (IIB1); UC=Upper Congaree (IIA); MC=Middle Congaree (IIA); LC=Lower Congaree (IIA) 

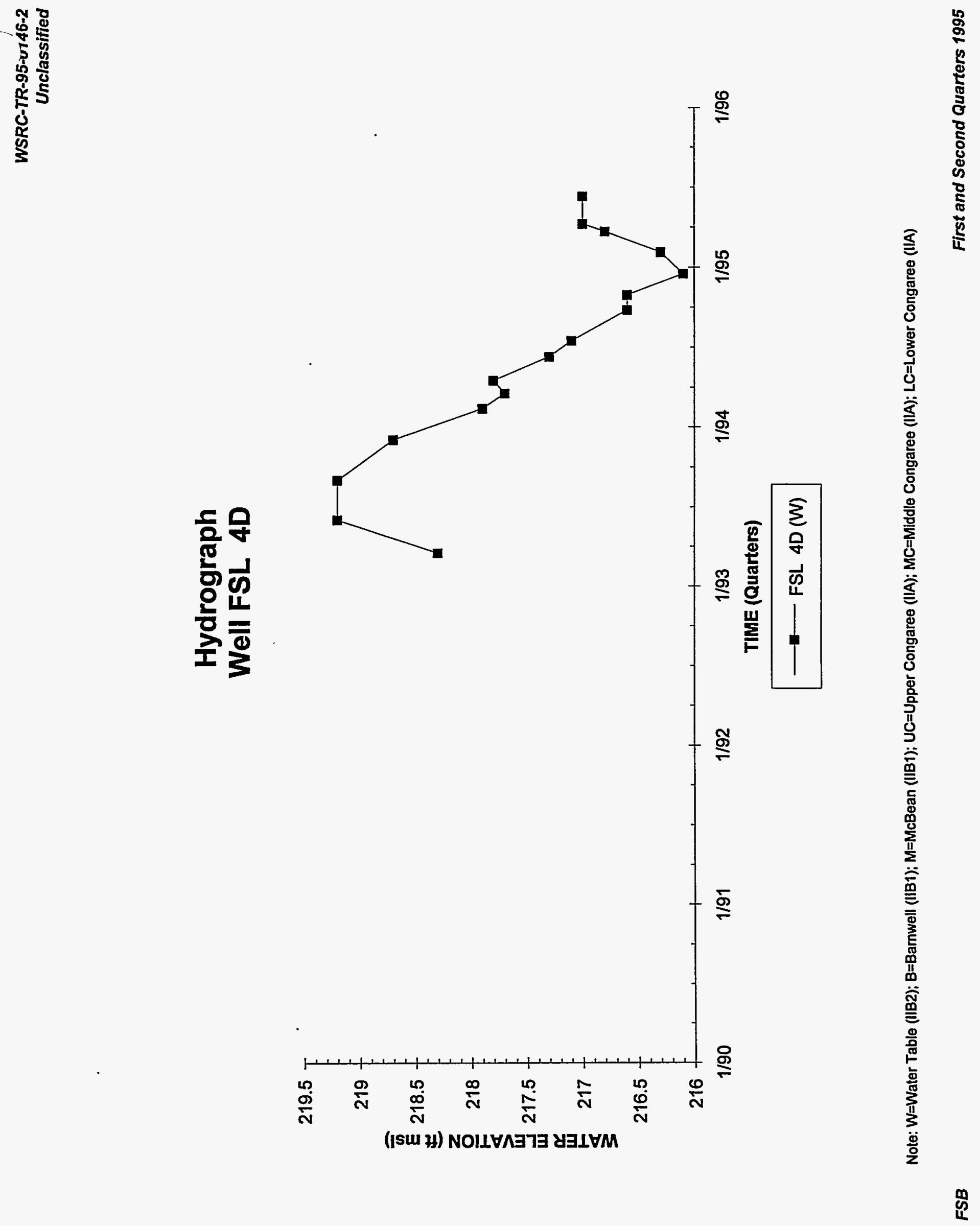

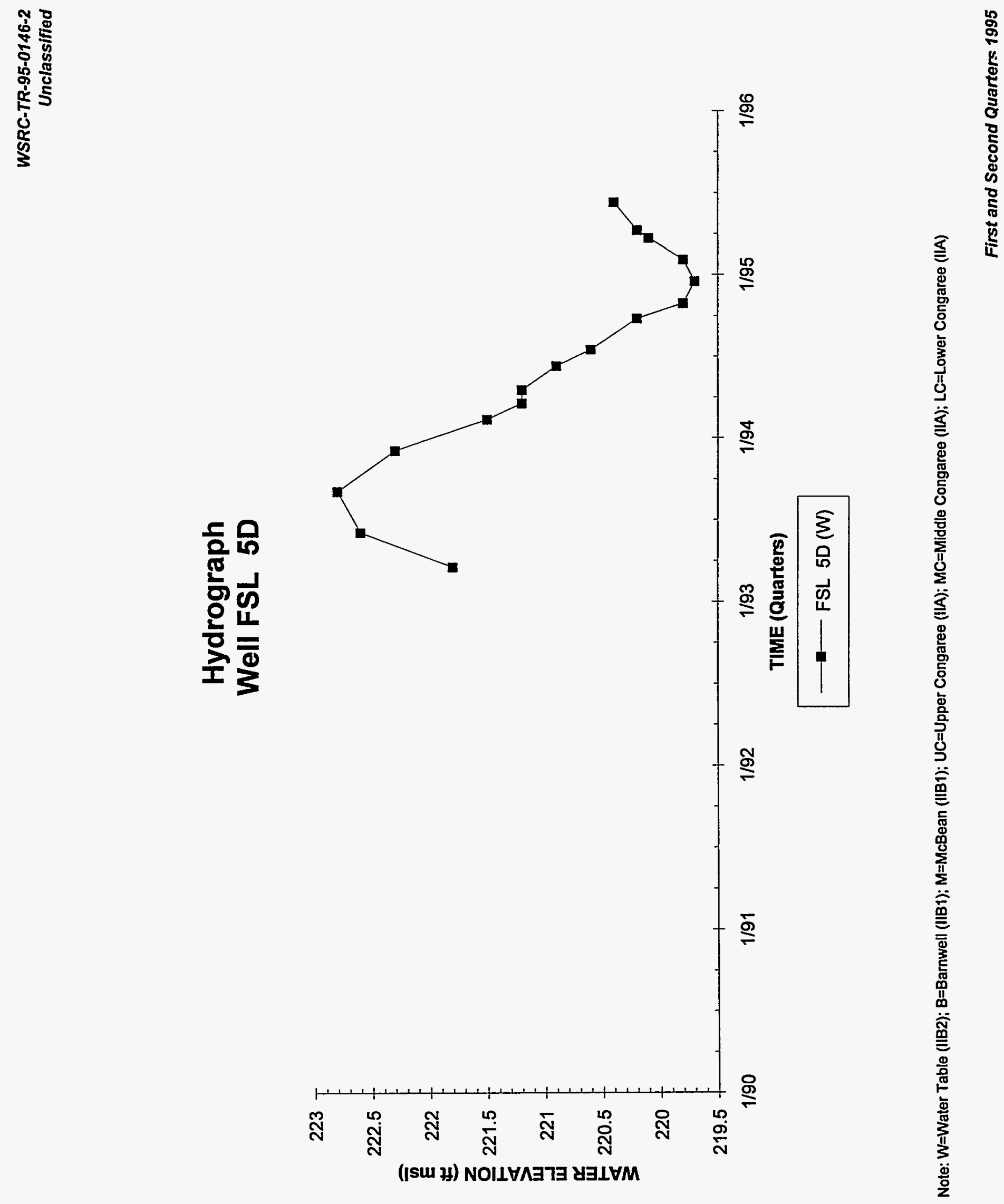


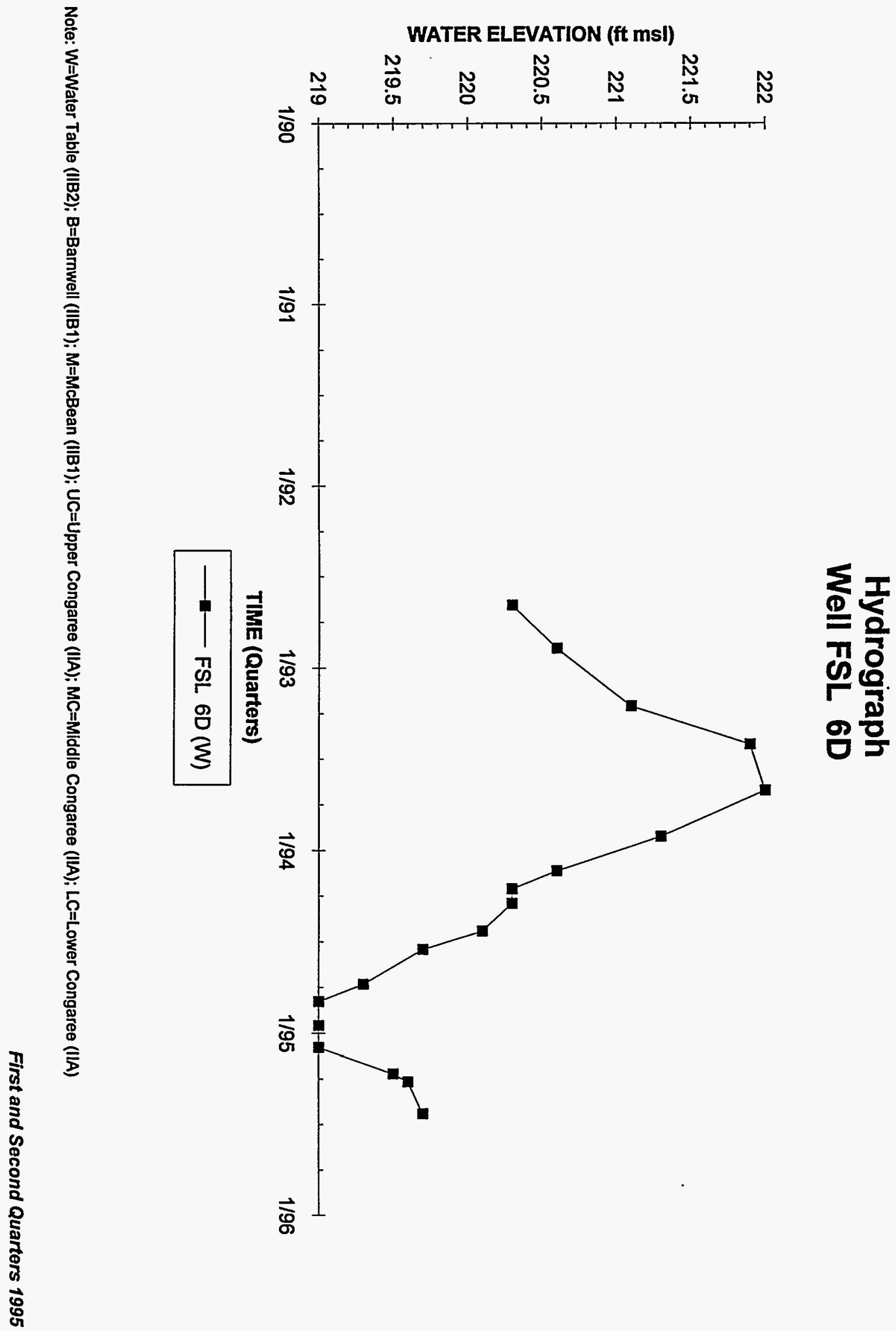




\section{Hydrograph}

Well FSL 7D

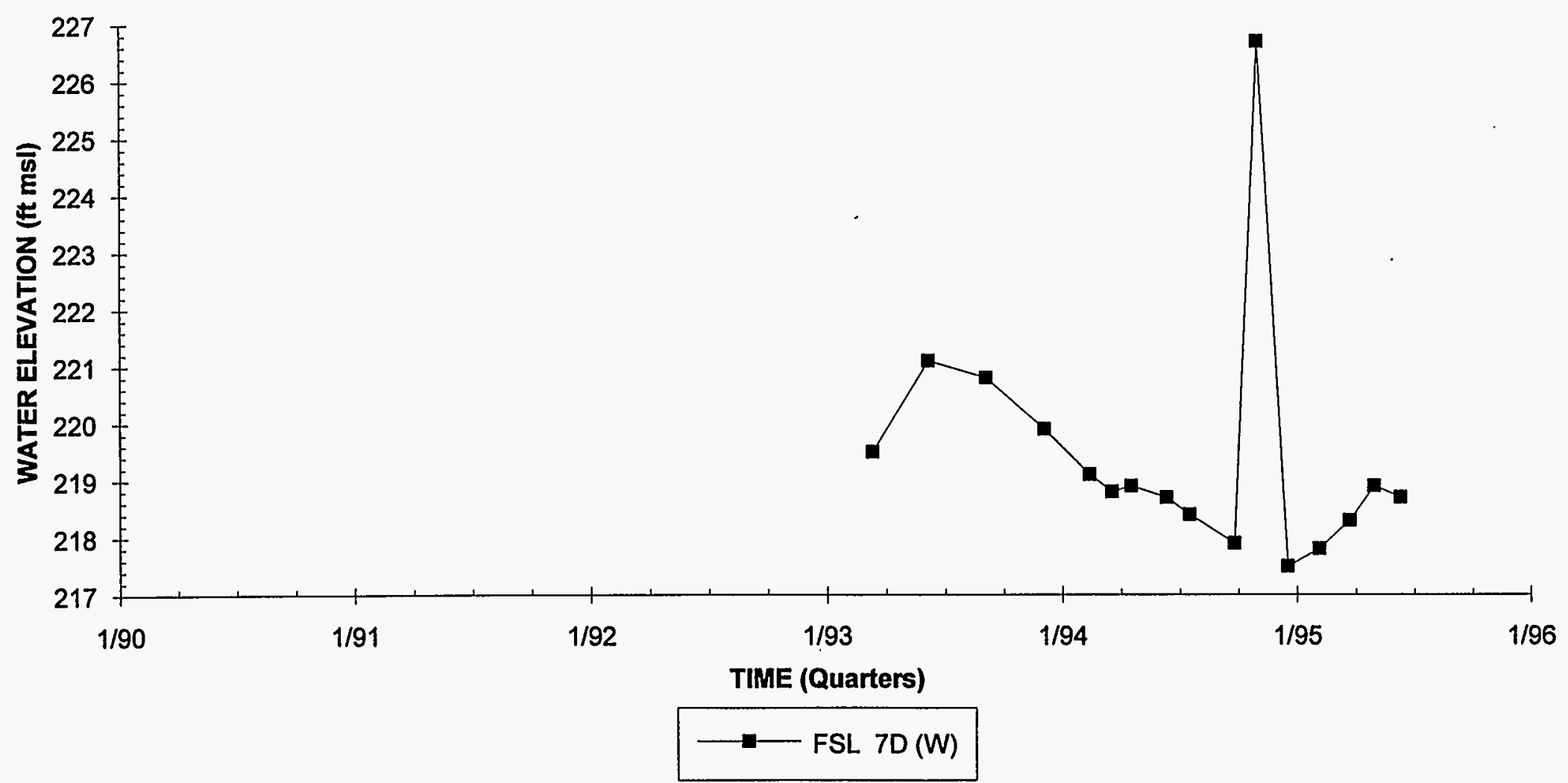

Note: W=Water Table (IIB2); B=Barnwell (IIB1); M=McBean (IIB1); UC=Upper Congaree (IIA); MC=Middle Congaree (IIA); LC=Lower Congaree (IIA) 

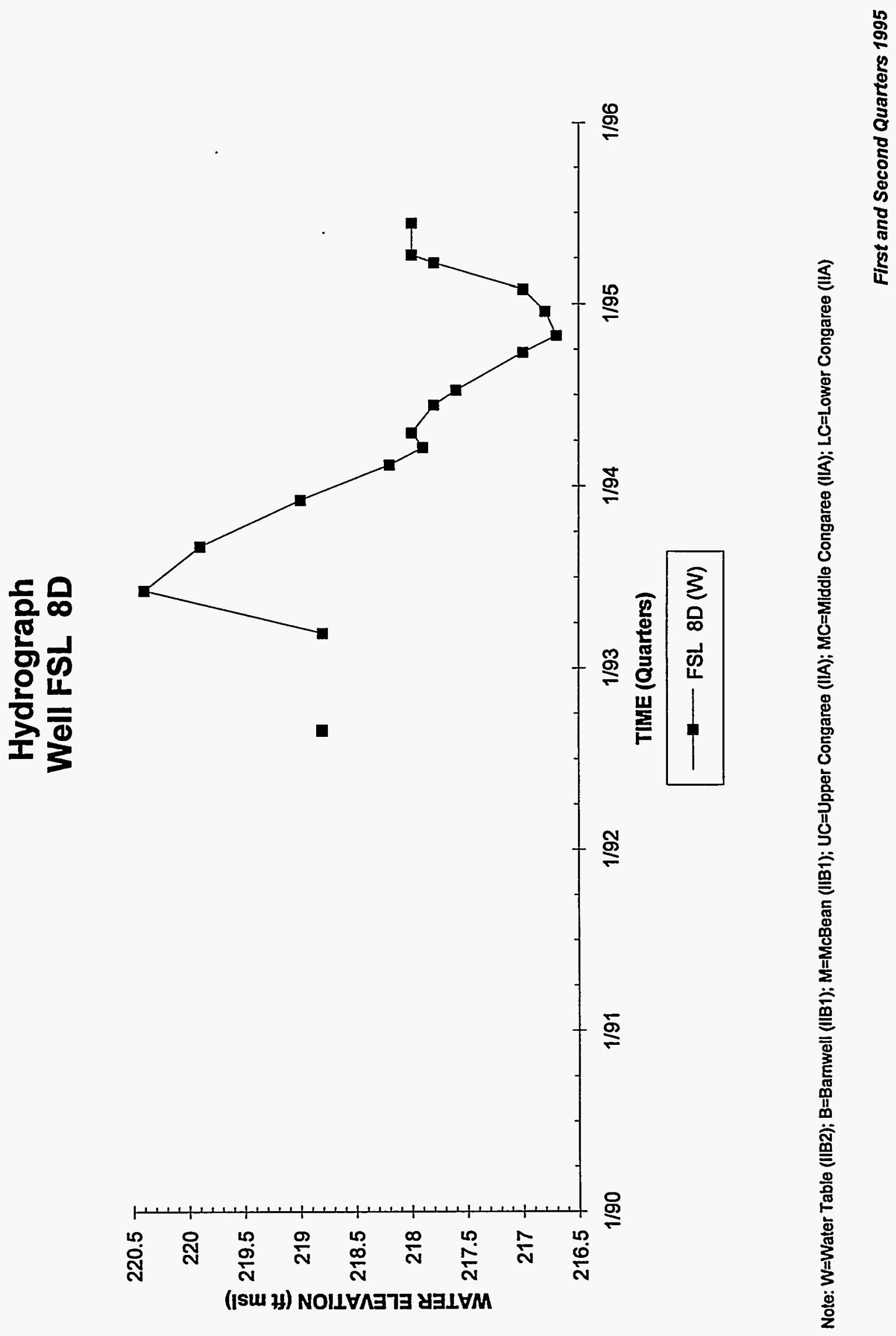


\section{Hydrograph \\ Well FSL 9D}

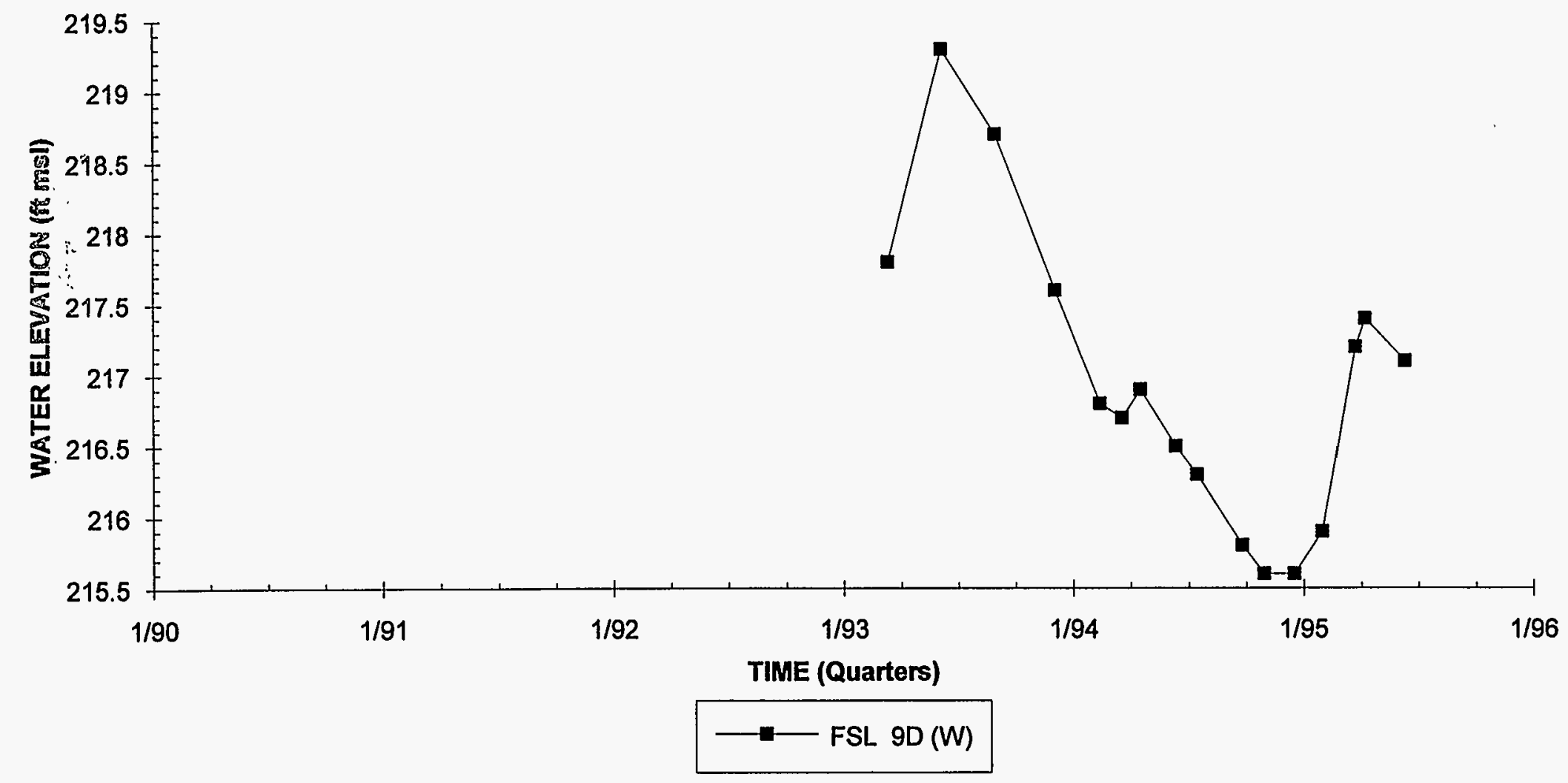

Note: W=Water Table (IIB2); B=Barnwell (IIB1); M=McBean (IIB1); UC=Upper Congaree (IIA); MC=Middle Congaree (IIA); LC=Lower Congaree (IIA) 